
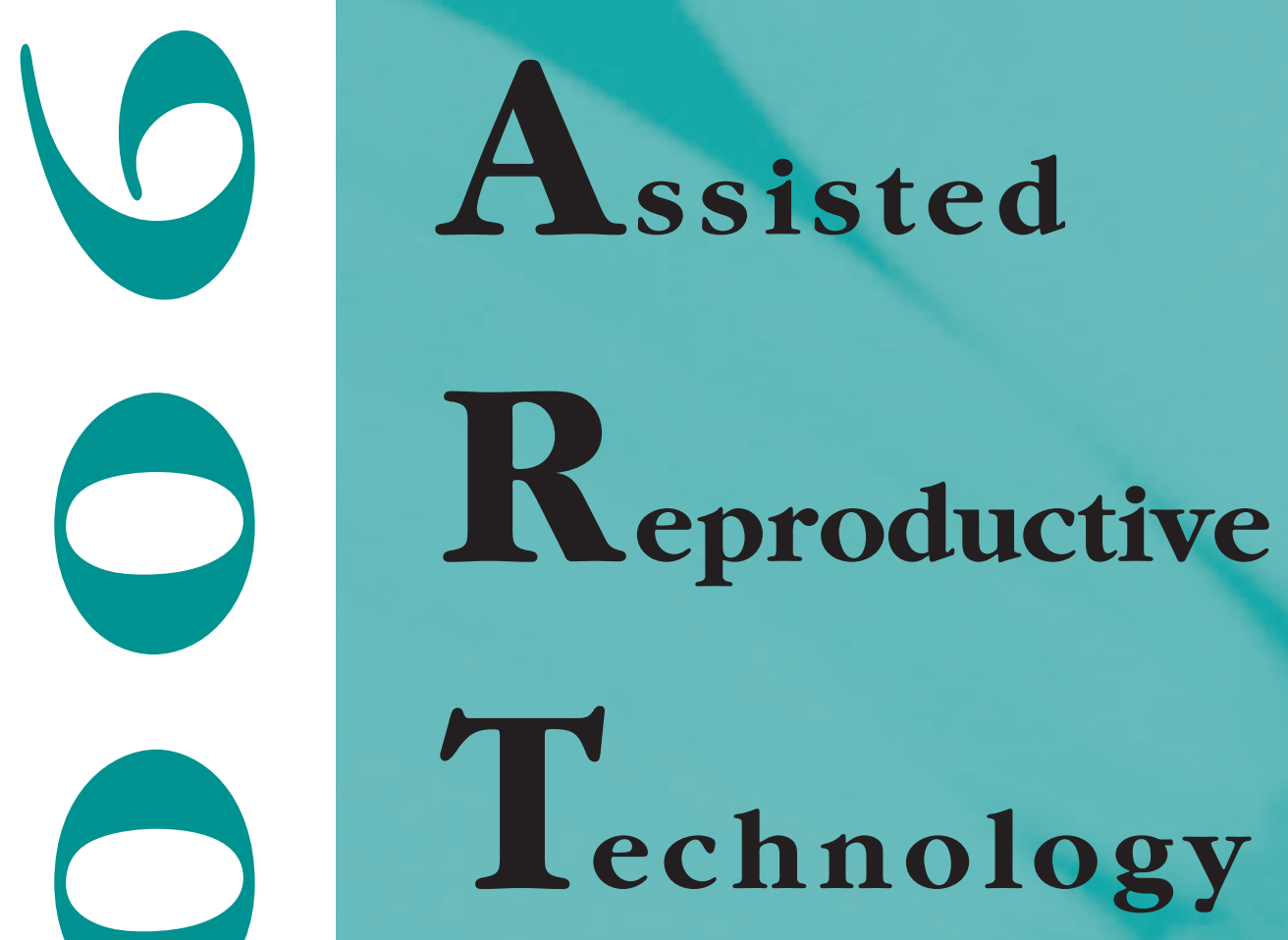

\title{
Success Rates
}

National Summary and Fertility Clinic Reports

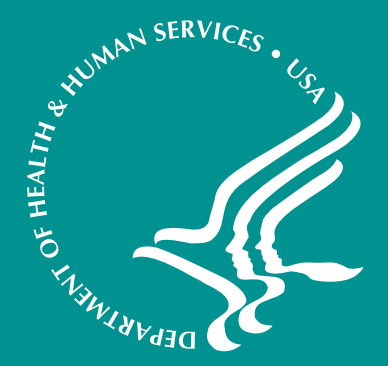

U.S. Department of Health and Human Services Centers for Disease Control and Prevention

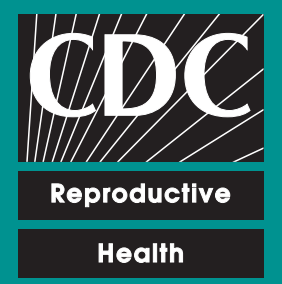


Updates to this report will be posted on the CDC Web site at the following address:

http://www.cdc.gov/ART/ART2006

For additional information, send an e-mail to ccdinfo@cdc.gov (Subject: ART)

Or write to CDC, ATTN: ARTE Unit; 4770 Buford Highway, N.E.;

Mail Stop K-34; Atlanta, GA 30341-3717. 


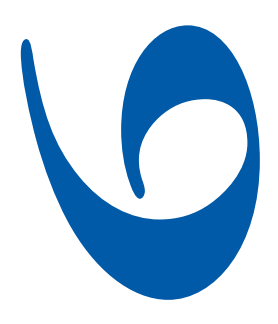

Assisted

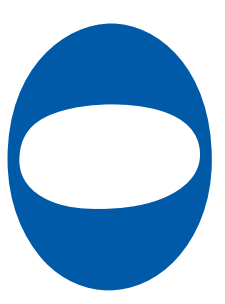

Reproductive
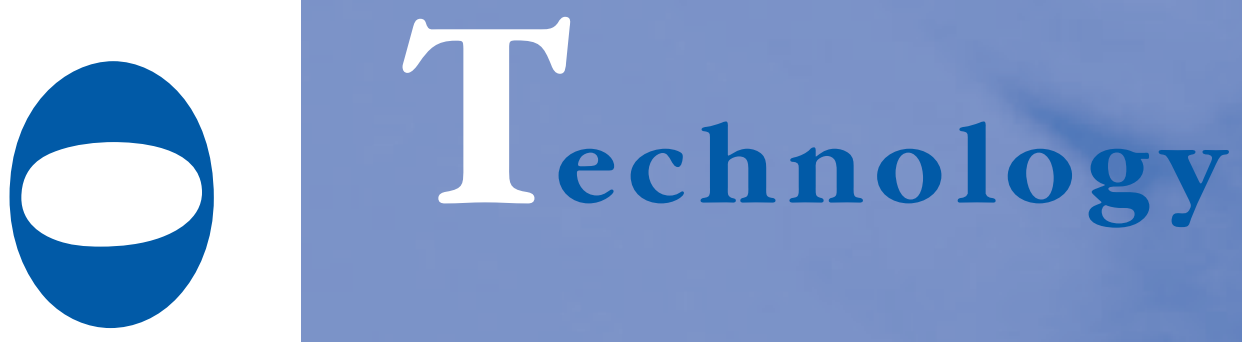

Success Rates

\section{National Summary and Fertility Clinic Reports}

Centers for Disease Control and Prevention

Coordinating Center for Health Promotion

National Center for Chronic Disease Prevention and Health Promotion

Division of Reproductive Health

Atlanta, Georgia

American Society for Reproductive Medicine

Society for Assisted Reproductive Technology

Birmingham, Alabama

November 2008

U.S. Department of Health and Human Services Centers for Disease Control and Prevention 
This publication was developed and produced by the National Center for Chronic Disease Prevention and Health Promotion of the Centers for Disease Control and Prevention in consultation with the American Society for Reproductive Medicine and the Society for Assisted Reproductive Technology.

\title{
Centers for Disease Control and Prevention
}

Coordinating Center for Health Promotion

National Center for Chronic Disease

Prevention and Health Promotion

Janet Collins, PhD, Director

Division of Reproductive Health

John R. Lehnherr, Acting Director

Kelly Brumbaugh, MPH, CHES

Women's Health and Fertility Branch

\author{
Maurizio Macaluso, MD, DrPH, Chief \\ Jeani Chang, MPH \\ Sherry L. Farr, PhD \\ Gary Jeng, PhD \\ Glenda Sentelle, MA, MSHS \\ Mithi Sunderam, PhD, MA \\ Daniel M. Wallace
}

\section{American Society for Reproductive Medicine}

Robert Rebar, MD, Executive Director

Society for Assisted Reproductive Technology

Mark Hornstein, MD, MPH, President

Joyce G. Zeitz

The data included in this report and publication support were provided by Westat under Contract

No. 200-2004-06702 for the National Center for Chronic Disease Prevention and Health Promotion, Centers for Disease Control and Prevention, U.S. Department of Health and Human Services.

Suggested Citation: Centers for Disease Control and Prevention, American Society for Reproductive Medicine, Society for Assisted Reproductive Technology. 2006 Assisted Reproductive Technology Success Rates: National Summary and Fertility Clinic Reports, Atlanta: U.S. Department of Health and Human Services, Centers for Disease Control and Prevention; 2008. 


\section{Acknowledgments}

The Centers for Disease Control and Prevention (CDC), the Society for Assisted Reproductive Technology, and the American Society for Reproductive Medicine thank RESOLVE: The National Infertility Association and The American Fertility Association for their commitment to assisted reproductive technology (ART) surveillance. Their assistance in making this report informative and helpful to people considering an ART procedure is greatly appreciated. Appendix D has current contact information for these national consumer organizations.

The Division of Reproductive Health in CDC's National Center for Chronic Disease Prevention and Health Promotion also thanks the following individuals for their many contributions and support of this effort:

Connie Butler, Science Applications International Corporation

Amanda Crowell, Writer-Editor Services Branch, CDC

Valerie Jackson, Science Applications International Corporation 



\section{Table of Contents}

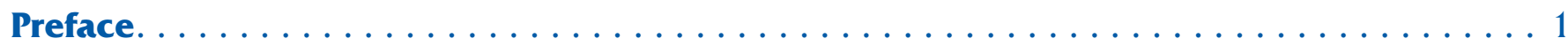

Commonly Asked Questions About the U.S. Clinic Reporting System . . . . . . . . . . . 3

2006 National Report . . . . . . . . . . . . . . . . . . . . . . . 9

Introduction to the 2006 National Report $\ldots \ldots \ldots \ldots \ldots \ldots \ldots \ldots \ldots$

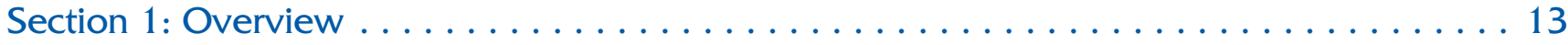

Section 2: ART Cycles Using Fresh Nondonor Eggs or Embryos . . . . . . . . . . . . . . 17

Section 3: ART Cycles Using Frozen Nondonor Embryos . . . . . . . . . . . . . . . . . . . . . 54

Section 4: ART Cycles Using Donor Eggs . . . . . . . . . . . . . . . . . . . . . 56

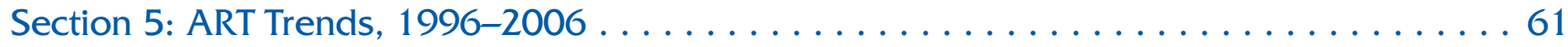

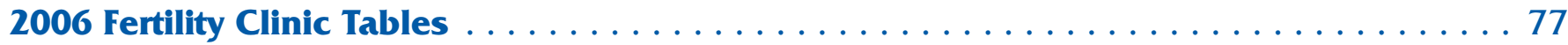

Introduction to Fertility Clinic Tables . . . . . . . . . . . . . . . . . . . . . . . . . . 79

Important Factors to Consider When Using These Tables to Assess a Clinic . . . . . . . . 79

How to Read a Fertility Clinic Table. . . . . . . . . . . . . . . . . . . 83

2006 National Summary . . . . . . . . . . . . . . . . . . . . . . . . . . . . 89

Alabama . . . . . . . . . . . . . . . . . . . . . . . . . 91

Alaska . . . . . . . . . . . . . . . . . . . . . . . . . . . . 97

Arizona . . . . . . . . . . . . . . . . . . . . . . . . . . 98

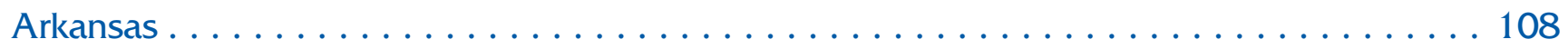

California. . . . . . . . . . . . . . . . . . . . . . . . . . . . . . . . . 109

Colorado . . . . . . . . . . . . . . . . . . . . . . . . . . . . . . 172

Connecticut. . . . . . . . . . . . . . . . . . . . . . . . . . . . . . . . . . 179

Delaware. . . . . . . . . . . . . . . . . . . . . . . . . . . . . . 186

District of Columbia . . . . . . . . . . . . . . . . . . . . . . . . . . . 187

Florida . . . . . . . . . . . . . . . . . . . . . . . . . . . . . . 191

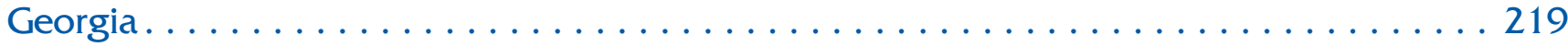

Hawaii. . . . . . . . . . . . . . . . . . . . . . . . . . . . . . 228

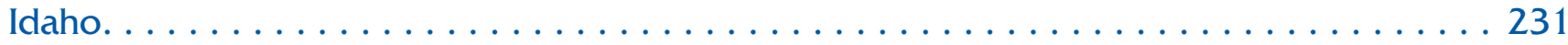

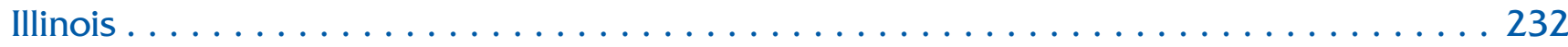

Indiana . . . . . . . . . . . . . . . . . . . . . . . . . . . . . . 260

lowa . . . . . . . . . . . . . . . . . . . . . . . . . . . . . 272

Kansas. . . . . . . . . . . . . . . . . . . . . . . . . . . . . . . . 274

Kentucky. . . . . . . . . . . . . . . . . . . . . . . . . . . . . . . . . . . . . . . . . 279

Louisiana. . . . . . . . . . . . . . . . . . . . . . . . . . . . . . 282

Maine . . . . . . . . . . . . . . . . . . . . . . . . . . . 287

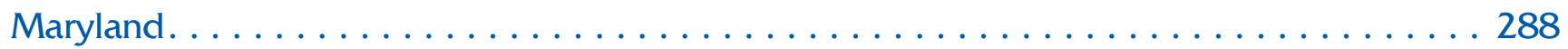


Massachusetts. . . . . . . . . . . . . . . . . . . . . . . . . . . . . . . 295

Michigan.......................................... 301

Minnesota........................................ 313

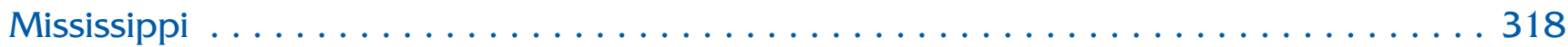

Missouri ....................................... 320

Nebraska.......................................... 328

Nevada ................................................... 330

New Hampshire . . . . . . . . . . . . . . . . . . . . . . . . . . . . . . . . . . . . . . . . 334

New Jersey .......................................... 335

New Mexico . . . . . . . . . . . . . . . . . . . . . . . . . . . . . . . . . . . . . . . 357

New York . . . . . . . . . . . . . . . . . . . . . . . . . . . . . . . . . . . 358

North Carolina. . . . . . . . . . . . . . . . . . . . . . . . . . . . . . . . . . . . . 392

North Dakota. ........................................... 400

Ohio ........................................... 401

Oklahoma .........................................4 412

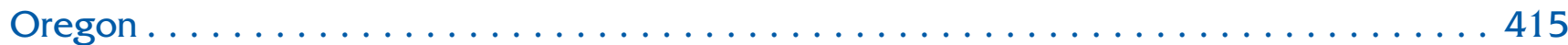

Pennsylvania . . . . . . . . . . . . . . . . . . . . . . . . . . . . . . . . . . . . . . . 419

Puerto Rico . . . . . . . . . . . . . . . . . . . . . . . . . . . . . . . . . . . . 438

Rhode Island . . . . . . . . . . . . . . . . . . . . . . . . . . . . . . . . . . . . . . . . . . . 440

South Carolina. . . . . . . . . . . . . . . . . . . . . . . . . . . . . . . . . . . . 441

South Dakota. . . . . . . . . . . . . . . . . . . . . . . . . . . . . . . . . . . . . . . 445

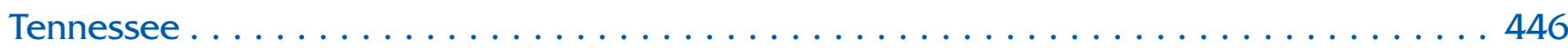

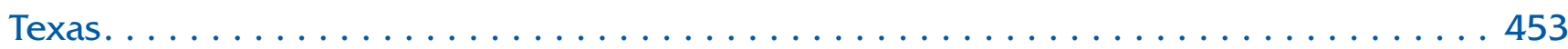

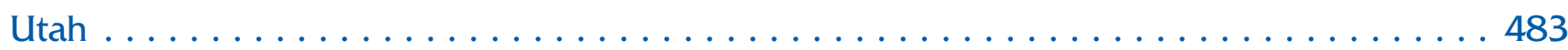

Vermont .............................................. 485

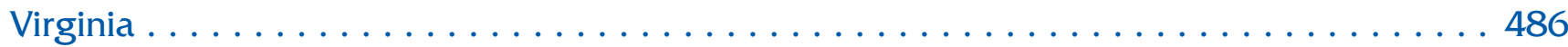

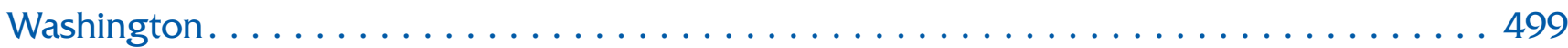

West Virginia...................................... 508

Wisconsin ....................................... 510

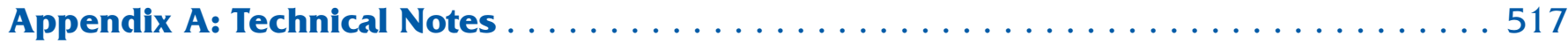

How to Interpret a Confidence Interval . . . . . . . . . . . . . . . . . . . . . . 519

Findings from Validation Visits for 2006 ART Data . . . . . . . . . . . . . . . 521

Appendix B: Glossary of Terms Used in This Report . . . . . . . . . . . . . . . . 523

Appendix C: ART Clinics, 2006. . . . . . . . . . . . . . . . . . . . . . . . . . . . . . . . . . . . 529

Reporting ART Clinics for 2006, by State . . . . . . . . . . . . . . . . . . . 531

Nonreporting ART Clinics for 2006, by State . . . . . . . . . . . . . . . . . . . . . 567

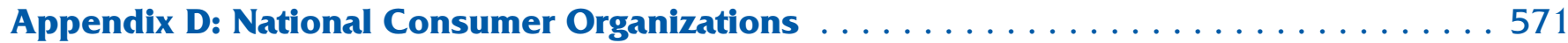




\section{Preface}

For many people who want to start a family, the dream of having a child is not easily realized; about $12 \%$ of women of childbearing age in the United States have used an infertility service. Assisted reproductive technology (ART) has been used in the United States since 1981 to help women become pregnant, most commonly through the transfer of fertilized human eggs into a woman's uterus. However, for many people, deciding whether to undergo this expensive and time-consuming treatment can be difficult.

The goal of this report is to help potential ART users make informed decisions about ART by providing some of the information needed to answer the following questions:

- What are my chances of having a child by using ART?

- Where can I go to get this treatment?

The Society for Assisted Reproductive Technology (SART), an organization of ART providers affiliated with the American Society for Reproductive Medicine (ASRM), has been collecting data and publishing annual reports of pregnancy success rates for fertility clinics in the United States and Canada since 1989. In 1992, the U.S. Congress passed the Fertility Clinic Success Rate and Certification Act. This law requires the Centers for Disease Control and Prevention (CDC) to publish pregnancy success rates for ART in fertility clinics in the United States. Since 1995, CDC has worked in consultation with SART and ASRM to report ART success rates.

The 2006 report of pregnancy success rates is the twelfth to be issued under the law. This report is based on the latest available data on the type, number, and outcome of ART cycles performed in U.S. clinics.

The 2006 ART report has four major sections:

- Commonly asked questions about the U.S. ART clinic reporting system. This section provides background information on infertility and ART and an explanation of the data collection, analysis, and publication processes.

- A national report. The national report section presents overall success rates and shows how they are affected by certain patient and treatment characteristics. Because the national report summarizes findings from all 426 fertility clinics that reported data, it can give people considering ART a good idea of the average chance of having a child by using ART.

- Fertility clinic tables. Success also is related to the expertise of a particular clinic's staff, the quality of its laboratory, and the characteristics of the patient population. The fertility clinic table section displays ART results and success rates for individual U.S. fertility clinics in 2006.

\section{- Appendixes:}

Appendix A contains technical notes on the interpretation of $95 \%$ confidence intervals and findings from the data validation visits to selected fertility clinics.

Appendix B (Glossary) provides definitions for technical and medical terms used throughout the report. 
Appendix C includes the current names and addresses of all reporting clinics along with a list of clinics known to be in operation in 2006 that did not report their success rate data to CDC as required by law.

Appendix D includes the names and addresses of national consumer organizations that offer support to people experiencing infertility.

Success rates can be reported in a variety of ways, and the statistical aspects of these rates can be difficult to interpret. As a result, presenting information about ART success rates is a complex task. This report is intended for the general public, and the emphasis is on presenting the information in an easily understandable form. CDC hopes that this report is informative and helpful to people considering an ART procedure. We welcome any suggestions for improving the report and making it easier to use. (See contact information, inside front cover.) 


\section{Commonly Asked Questions About the U.S. ART Clinic Reporting System}

\section{Background Information, Data Collection Methods, Content and Design of the Report, and Additional Information About ART in the United States}

\section{How many people in the United States have infertility problems?}

The latest data on infertility available to the Centers for Disease Control and Prevention (CDC) are from the 2002 National Survey of Family Growth.

- Of the approximately 62 million women of reproductive age in 2002 , about 1.2 million, or $2 \%$, had had an infertility-related medical appointment within the previous year and an additional $10 \%$ had received infertility services at some time in their lives. (Infertility services include medical tests to diagnose infertility, medical advice and treatments to help a woman become pregnant, and services other than routine prenatal care to prevent miscarriage.)

- Additionally, 7\% of married couples in which the woman was of reproductive age $(2.1$ million couples) reported that they had not used contraception for 12 months and the woman had not become pregnant.

\section{What is assisted reproductive technology (ART)?}

Although various definitions have been used for ART, the definition used in this report is based on the 1992 law that requires CDC to publish this report. According to this definition, ART includes all fertility treatments in which both eggs and sperm are handled. In general, ART procedures involve surgically removing eggs from a woman's ovaries, combining them with sperm in the laboratory, and returning them to the woman's body or donating them to another woman. They do NOT include treatments in which only sperm are handled (i.e., intrauterine-or artificial-insemination) or procedures in which a woman takes drugs only to stimulate egg production without the intention of having eggs retrieved.

The types of ART include the following:

- IVF (in vitro fertilization). Involves extracting a woman's eggs, fertilizing the eggs in the laboratory, and then transferring the resulting embryos into the woman's uterus through the cervix. For some IVF procedures, fertilization involves a specialized technique known as intracytoplasmic sperm injection (ICSI). In ICSI, a single sperm is injected directly into the woman's egg.

- GIFT (gamete intrafallopian transfer). Involves using a fiber-optic instrument called a laparoscope to guide the transfer of unfertilized eggs and sperm (gametes) into the woman's fallopian tubes through small incisions in her abdomen.

- ZIFT (zygote intrafallopian transfer). Involves fertilizing a woman's eggs in the laboratory and then using a laparoscope to guide the transfer of the fertilized eggs (zygotes) into her fallopian tubes. 
In addition, ART often is categorized according to whether the procedure used a woman's own eggs (nondonor) or eggs from another woman (donor) and according to whether the embryos used were newly fertilized (fresh) or previously fertilized, frozen, and then thawed (frozen). Because an ART procedure includes several steps, it is typically referred to as a cycle of treatment. (See What is an ART cycle? below.)

\section{What is an ART cycle?}

Because ART consists of several steps over an interval of approximately 2 weeks, an ART procedure is more appropriately considered a cycle of treatment rather than a procedure at a single point in time. The start of an ART cycle is considered to be when a woman begins taking drugs to stimulate egg production or starts ovarian monitoring with the intent of having embryos transferred. (See Figure 5, page 17, for a full description of the steps in an ART cycle.) For the purposes of this report, data on all cycles that were started, even those that were discontinued before all steps were undertaken, are submitted to CDC through a Web-based data collection system called the National ART Surveillance System (NASS) and are counted in the clinic's success rates.

\section{How do U.S. ART clinics report data to CDC about their success rates?}

CDC contracts with a statistical survey research organization, Westat, to obtain the data published in the ART success rates report. Westat maintains a list of all ART clinics known to be in operation and tracks clinic reorganizations and closings. This list includes clinics and individual providers that are members of the Society for Assisted Reproductive Technology (SART) as well as clinics and providers that are not SART members. Westat actively follows up reports of ART physicians or clinics not on its list to update the list as needed. Westat maintains NASS, the Web-based data collection system that all ART clinics use. Clinics either electronically enter or import data into NASS for each ART procedure they start in a given reporting year. The data collected include information on the client's medical history (such as infertility diagnoses), clinical information pertaining to the ART procedure, and information on resulting pregnancies and births.

See below (Why is the report of 2006 success rates being published in 2008?) for a complete description of the reporting process.

\section{Why is the report of $\mathbf{2 0 0 6}$ success rates being published in 2008 ?}

Before success rates based on live births can be calculated, every ART pregnancy must be followed up to determine whether a birth occurred. Therefore, the earliest that clinics can report complete annual data is late in the year after ART treatment was initiated (about 9 months past year-end, when all the births have occurred). Accordingly, the results of all the cycles initiated in 2006 were not known until October 2007. After ART outcomes are known, the following occurs before the report is published:

- Clinics enter their data into NASS and verify the data's accuracy before sending the data to Westat.

- Westat compiles a national data set from the data submitted by individual clinics.

- CDC data analysts conduct comprehensive checks of the numbers reported for every clinic.

- Clinic tables, national figures, and accompanying text for both the printed and Internet versions of the report are compiled and laid out. 
- $\mathrm{CDC}$ and Westat review the report.

- Necessary changes are incorporated and proofread.

- The report is submitted to the Government Printing Office to begin the printing and production process.

These steps are time-consuming but essential for ensuring that the report provides the public with correct information particularly regarding each clinic's success rates.

\section{Which clinics are represented in this report?}

The data in both the national report and the individual fertility clinic tables come from 426 fertility clinics that provided and verified information about the outcomes of the ART cycles started in their clinics in 2006.

Although we believe that almost all clinics that provided ART services in the United States throughout 2006 are represented in this report, data for a few clinics or practitioners are not included because they either were not in operation throughout 2006 or did not report as required. Clinics and practitioners known to have been in operation throughout 2006 that did not report and verify their data are listed in this report as nonreporters, as required by law (see Appendix C, Nonreporting ART Clinics for 2006, by State, on pages 567-570). We will continue to make every effort to include in future reports all clinics and practitioners providing ART services.

\section{Why doesn't CDC rank the clinics?}

Because the decision to undergo ART treatment is a very personal decision, this report may not contain all of the information that a woman or a couple needs to decide which ART clinic or procedure is best for their treatment. Many factors contribute to the success rate of an ART procedure in particular patients, and a difference in success rates between two ART programs may reflect differences in the groups of patients treated, the types of procedures used, or other factors. More explanations on how to use the success rates and other statistics published in this report are in the Introduction to Fertility Clinic Tables (pages 79-88). The report should be used to help people considering an ART procedure find clinics where they can meet personally with ART providers to discuss their specific medical situation and their likelihood of success using ART. Contacting a clinic also may provide additional information that could be helpful in deciding whether or not to use ART. Because ART offers several treatment options for infertility, there are many other factors that may affect the decision. Going through repeated ART cycles requires substantial commitments of time, effort, money, and emotional energy. Therefore, this report may be a helpful starting point for consumers to obtain information and consider their options.

\section{Does this report include all ART cycles performed by the reporting clinics?}

This report includes data for the 138,198 cycles performed in 2006 by the 426 clinics that reported their data as required. A small number of ART cycles are not included in either the national data or the individual fertility clinic tables. These were cycles in which a new treatment procedure was being evaluated. Only 69 ART cycles fell into this category in 2006. 


\section{How are the success rates determined?}

Three measures of success are presented in this report: (1) pregnancy, (2) birth of one or more living infants (the delivery of multiple infants is counted as one live birth), and (3) birth of a singleton live-born infant. The pregnancies reported here were diagnosed using an ultrasound procedure. All live-birth deliveries were reported to the ART physician by either the patient or her obstetric provider. Because this report is geared toward patients, the focus is on the percentage of cycles resulting in live births. Singleton live births are presented as a separate measure of success because they have a much lower risk than multiple-infant births for adverse infant health outcomes, including prematurity, low birth weight, disability, and death. Pregnancies, live births, and singleton live births were calculated based on all cycles started. As noted throughout the report, success rates were additionally calculated at various steps of the ART cycle to provide a complete picture of the chances for success as the cycle progresses.

\section{What are my chances of getting pregnant using ART?}

This report presents several measures of success for ART (see Figure 7, page 19), including the proportion of ART cycles that result in a pregnancy. Many women ask this question because they assume that the pregnancy will lead to a live birth. Unfortunately, not all ART procedures that result in a pregnancy lead to the delivery of a live infant. For example, in 2006, 99,199 fresh-nondonor ART cycles were started. Of those, 34,719 (35\%) led to a pregnancy, but only 28,404 (29\%) resulted in a live birth. In other words, $18 \%$ of ART pregnancies did not result in a live birth. The percentage of cycles resulting in live births will give a more accurate answer to the question, "If I have an ART procedure, what is my chance that I will have a baby?"

It is important to note that multiple-infant pregnancies and multiple-infant births are common with ART (see Figure 10, page 22). Multiple-infant births are associated with greater risk for adverse health outcomes for both the mother and the infants (see Figures 11 and 12 on preterm deliveries and low birth weight, pages 23 and 24). This report also includes singleton live births as a measure of success because they have a lower risk of adverse health outcomes.

\section{If a woman has had more than one ART treatment cycle, how is the success rate calculated? Alternatively, how many cycles does a woman usually go through before getting pregnant?}

As required by law, this report presents ART success rates in terms of how many cycles were started each year, rather than in terms of how many women were treated. (A cycle starts when a woman begins taking fertility drugs or having her ovaries monitored for follicle production.) Clinics do not report to CDC the number of women treated at each facility. Because clinics report information only on outcomes for each cycle started, it is not possible to compute the success rates on a "per woman" basis, or the number of cycles that an average woman may undergo before achieving success. 


\section{What factors that influence success rates are presented in this report?}

The national report presents a more in-depth picture of ART than can be shown for each individual clinic. Success rates are presented in the context of various patient and treatment characteristics that may influence success. These characteristics include age, infertility diagnosis, history of previous births, previous miscarriages, previous ART cycles, number of embryos transferred, type of ART procedure, use of techniques such as ICSI, and clinic size.

\section{What quality control steps are used to ensure data accuracy?}

To have their success rates published in this annual report, clinics have to submit their data in time for analysis and the clinics' medical directors have to verify by signature that the tabulated success rates are accurate. Then, Westat conducts an in-house review and contacts the clinics if corrections are necessary. After the data have been verified, a quality control process called validation begins. This year, 35 of 426 reporting clinics were randomly selected for site visits. Two members of the Westat Validation Team visited these clinics and reviewed medical record data for a sample of the clinic's ART cycles. For each cycle, the validation team abstracted information from the patient's medical record. The abstracted information was then reviewed on site and compared with the data submitted for the report. CDC staff members participated as observers in some of the visits. For each clinic, the sample of cycles validated included all cycles that were reported to have ended in a live birth and a random sample of up to 50 additional cycles. In almost all cases, data on pregnancies and births in the medical records were consistent with reported data. Validation primarily helps ensure that clinics are being careful to submit accurate data. It also serves to identify any systematic problems that could cause data collection to be inconsistent or incomplete.

The data validation process does not include any assessment of clinical practice or overall record keeping. See Appendix A, Technical Notes (pages 519-522), for a more detailed presentation of findings from the validation visits.

\section{Why doesn't the report contain specific medical information about ART?}

This report describes a woman's average chances of success using ART. Although the report provides some information about factors such as age and infertility diagnosis, individual couples face many unique medical situations. This population-based registry of ART procedures cannot capture detailed information about specific medical conditions associated with infertility. A physician in clinical practice should be consulted for the individual evaluation that will help a woman or couple understand their specific medical situation and their chances of success using ART.

\section{Does CDC have any information on the age, race, income, and education levels of women who donate eggs?}

CDC does not collect information on egg donors beyond what is presented in this report. Success rates for cycles using donor eggs or using embryos derived from donor eggs are presented separately based on the ART patient's age. 


\section{Are there any medical guidelines for ART performed in the United States?}

The American Society for Reproductive Medicine (ASRM) and SART issue guidelines dealing with specific ART practice issues, such as the number of embryos to be transferred in an ART procedure. Further information can be obtained from ASRM or SART (both at telephone 205-978-5000 or Web sites www.asrm.org and www.sart.org).

\section{What is CDC doing to ensure that the report is helpful to the public?}

We continually review comments from patients and providers about things to consider including in future reports. In early 2007, we asked ART clinic staff about their experiences using the report. They suggested specific ways to improve the report and specific analyses that might be beneficial. We also conducted in-depth interviews with patients who have used the report in the past and with patients who were currently seeking ART services. If you have any suggestions for improving the report and making it easier to use, go to www.cdc.gov/art and click on the Contact Us link or email your suggestions directly to ccdinfo@cdc.gov. The information will be used to improve future ART Success Rates reports.

\section{Where can I get additional information on U.S. fertility clinics?}

For further information on specific clinics, contact the clinic directly (see Appendix C for current contact information). In addition, SART can provide general information on its member clinics (telephone 205-978-5000, extension 109).

\section{What's new in the 2006 report?}

Overall, the content and format of this report are similar to those used in previous years. New information includes the following:

National Report, Section 5, ART Trends, 1996-2006 (Figures 50-55):

- These figures present summary statistics separately for intracytoplasmic sperm injection (ICSI) and non-ICSI cycles.

National Summary Table:

- The ART cycle profile now includes summary statistics for the use of Preimplantation Genetic Diagnosis (PGD).

Individual Fertility Clinic Tables:

- These tables now identify clinics that performed more than 50 cycles with PGD in 2006 and among them more than 10 cycles specifically for the purpose of prevention of genetic disorders. 
National Report 



\section{INTRODUCTION TO THE 2006 NATIONAL REPORT}

Data provided by U.S. clinics that use assisted reproductive technology (ART) to treat infertility are a rich source of information about the factors that contribute to a successful ART treatment-the delivery of a live-born infant. Pooling the data from all reporting clinics provides an overall national picture that could not be obtained by examining data from an individual clinic.

A woman's chances of having a pregnancy and a live birth by using ART are influenced by many factors, some of which are patient-related and outside a clinic's control (e.g., the woman's age, the cause of infertility). Because the national data set includes information on many of these factors, it can give potential ART users an idea of their average chances of success. Average chances, however, do not necessarily apply to a particular individual or couple. People considering ART should consult their physician to discuss all the factors that apply in their particular case.

The data for this national report come from the 426 fertility clinics in operation in 2006 that provided and verified data on the outcomes of all ART cycles started in their clinics. The 138,198 ART cycles performed at these reporting clinics in 2006 resulted in 41,343 live births (deliveries of one or more living infants) and 54,656 infants.

The national report consists of graphs and charts that use 2006 data to answer specific questions related to ART success rates. These figures are organized according to the type of ART procedure used. Some ART procedures use a woman's own eggs, and others use donated eggs or embryos. (Although sperm used to create an embryo also may be either from a woman's partner or from a sperm donor, information in this report is presented according to the source of the egg.) In some procedures, the embryos that develop are transferred back to the woman (fresh embryo transfer); in others, the embryos are frozen (cryopreserved) for transfer at a later date. This report includes data on frozen embryos that were thawed and transferred in 2006.

The national report has five sections:

- Section 1 (Figures 1 through 4) presents information from all ART procedures reported.

- Section 2 (Figures 5 through 41) presents information on the ART cycles that used only fresh embryos from nondonor eggs or, in a few cases, a mixture of fresh and frozen embryos from nondonor eggs $(99,199$ cycles resulting in 80,313 transfers).

- Section 3 (Figures 42 and 43) presents information on the ART cycles that used only frozen embryos from nondonor eggs (22,023 cycles resulting in 20,057 transfers).

- Section 4 (Figures 44 through 48) presents information on the ART cycles that used only donated eggs or embryos (16,976 cycles resulting in 15,505 transfers).

- Section 5 (Figures 49 through 64) presents trends in the number of ART procedures and success rates from 1996 through 2006.

The 2006 national summary table, which is based on data from all clinics included in this report, is on page 89 , immediately preceding the individual clinic tables. An explanation of how to read these tables is on pages 83-88. 



\section{SECTION I: OVERVIEW}

\section{Where are U.S. ART clinics located, how many ART cycles did they perform in 2006, and how many infants were born?}

Although ART clinics are located throughout the United States, generally in or near major cities, the greatest number of clinics is in the eastern United States. Figure 1 shows the locations of the 426 reporting clinics. The fertility clinic section of this report, arranged in alphabetical order by state, city, and clinic name, provides specific information on each of these clinics. The number of clinics, cycles performed, live-birth deliveries, and infants born as a result of ART all have increased steadily since CDC began collecting this information in 1995 (see Section 5, pages 61-76). Because in some cases more than one infant is born during a live-birth delivery (e.g., twins), the total number of infants born is greater than the number of live-birth deliveries. CDC estimates that ART accounts for slightly more than $1 \%$ of total U.S. births.

\section{Figure 1}

\section{Location of ART Clinics in the United States and Puerto Rico, 2006}

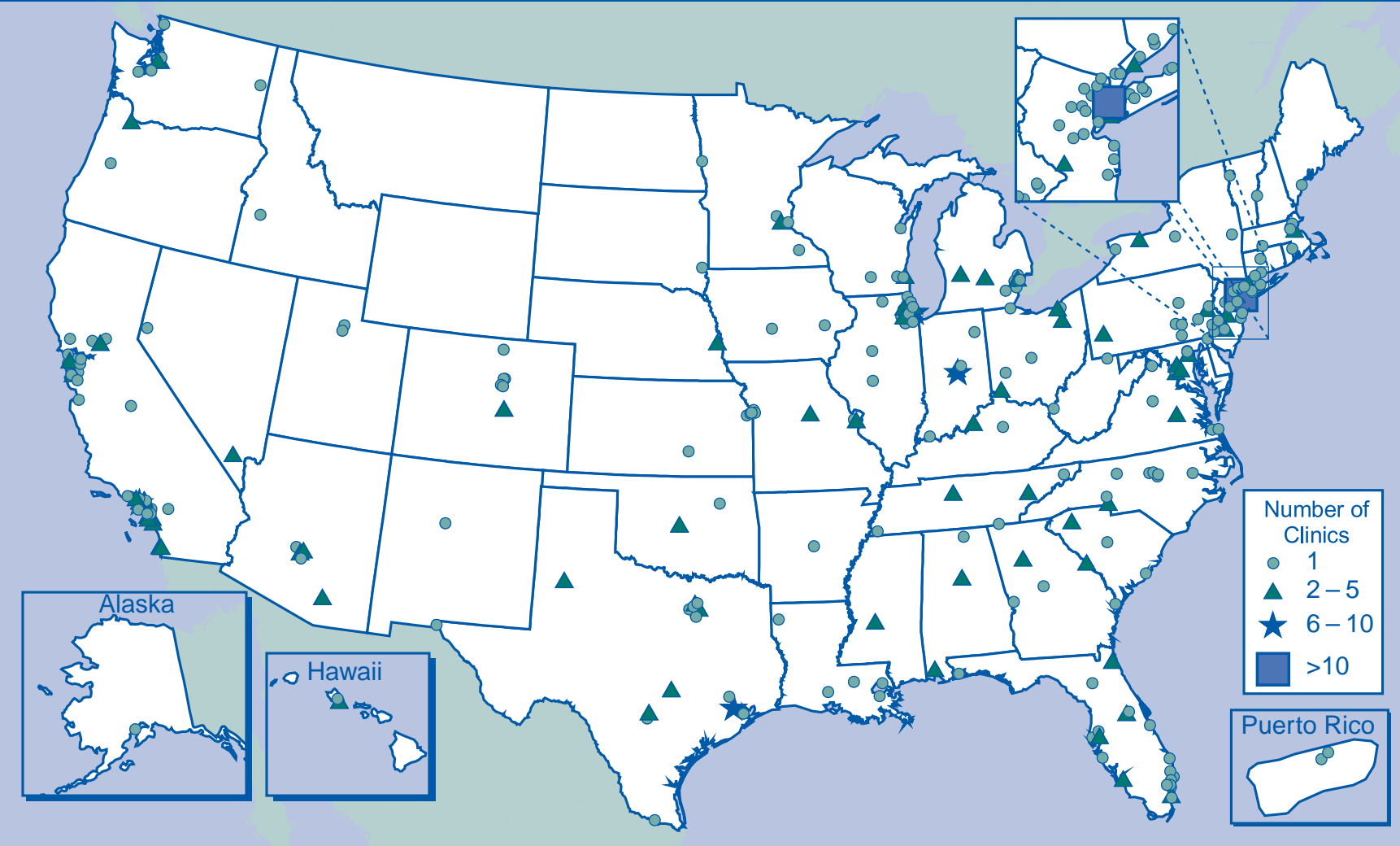

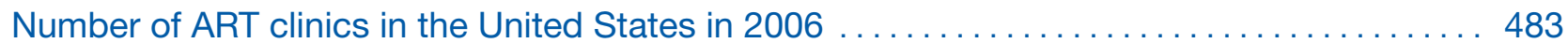

Number of ART clinics that submitted data in $2006 \ldots \ldots \ldots \ldots \ldots \ldots \ldots \ldots \ldots \ldots \ldots \ldots \ldots$

Number of ART cycles reported in $2006 \ldots \ldots \ldots \ldots \ldots \ldots \ldots \ldots \ldots \ldots \ldots \ldots \ldots \ldots \ldots \ldots{ }^{*}$

Number of live-birth deliveries resulting from ART cycles started in $2006 \ldots \ldots \ldots \ldots \ldots 41,343$

Number of infants born as a result of ART cycles carried out in $2006 \ldots \ldots \ldots \ldots \ldots \ldots 4,656$

* Note: This number does not include 69 cycles in which a new treatment procedure was being evaluated (see Figure 2, page 14). 


\section{What types of ART cycles were used in the United States in 2006?}

For $72 \%$ of ART cycles carried out in 2006, fresh nondonor eggs or embryos were used. ART cycles that used frozen nondonor embryos were the next most common type, accounting for approximately $16 \%$ of the total. In about $12 \%$ of cycles, eggs or embryos were donated by another woman. A very small number of cycles (less than $0.1 \%$ of the ART cycles carried out in 2006) involved the evaluation of a new treatment procedure. Because of the small number, cycles in which a new treatment procedure was being evaluated are not included in the total number of cycles reported in the national report or in the individual fertility clinic tables. Thus, data presented in subsequent figures in this report and in the individual fertility clinic tables are based on 138,198 ART cycles.

Figure 2

Types of ART Cycles-United States, ${ }^{*} 2006$

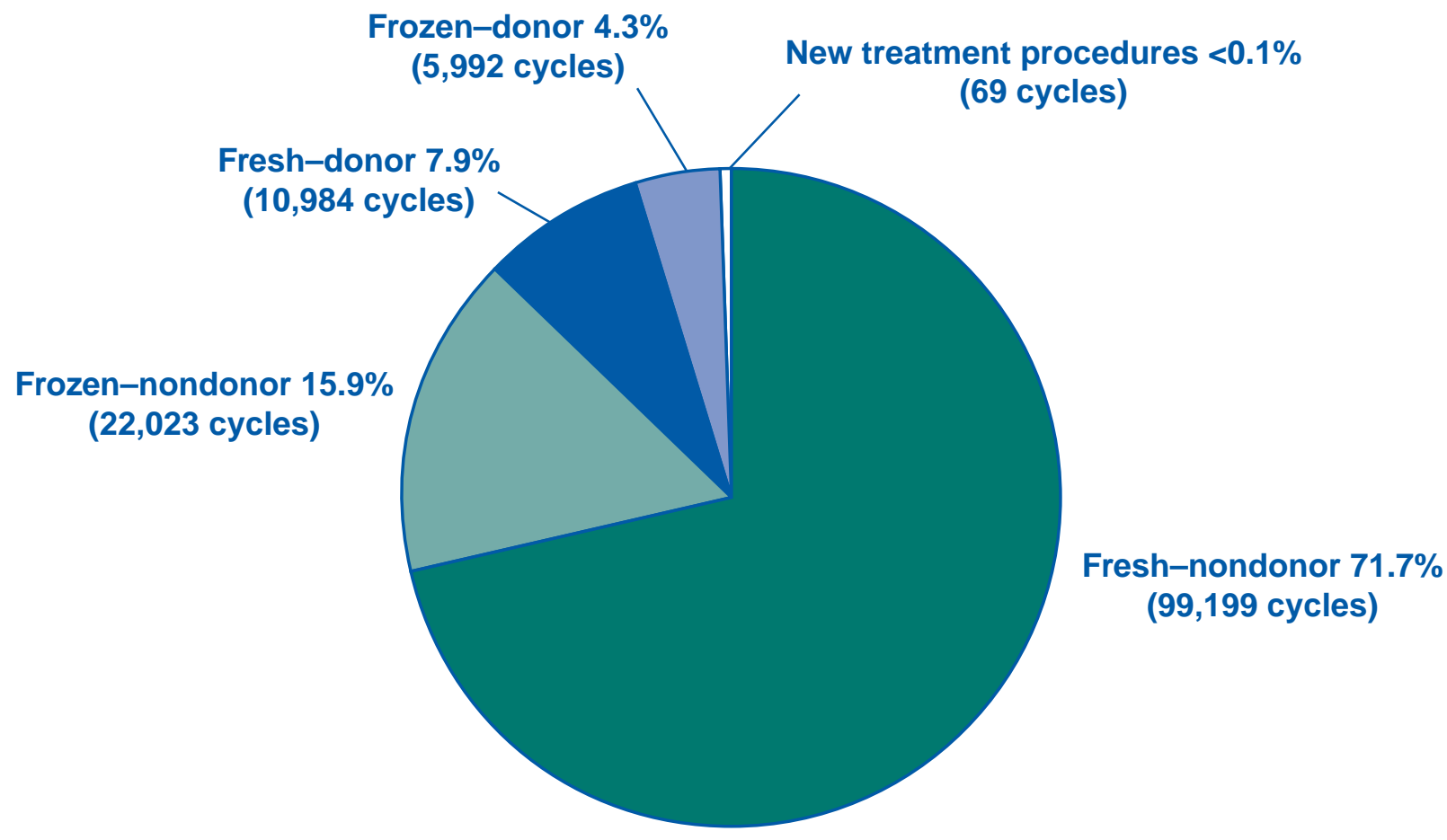

*Total does not equal $100 \%$ due to rounding. 


\section{How old were the women who used ART in the United States in 2006?}

The average age of women using ART services in 2006 was 36. The largest group of women using ART services were women younger than 35, representing 39\% of all ART cycles carried out in 2006. Twenty-three percent of ART cycles were carried out among women aged 35-37, 19\% among women aged 38-40, 10\% among women aged 41-42, and 10\% among women older than 42 .

\section{Figure 3}

ART Use by Age Group-United States, ${ }^{*} 2006$

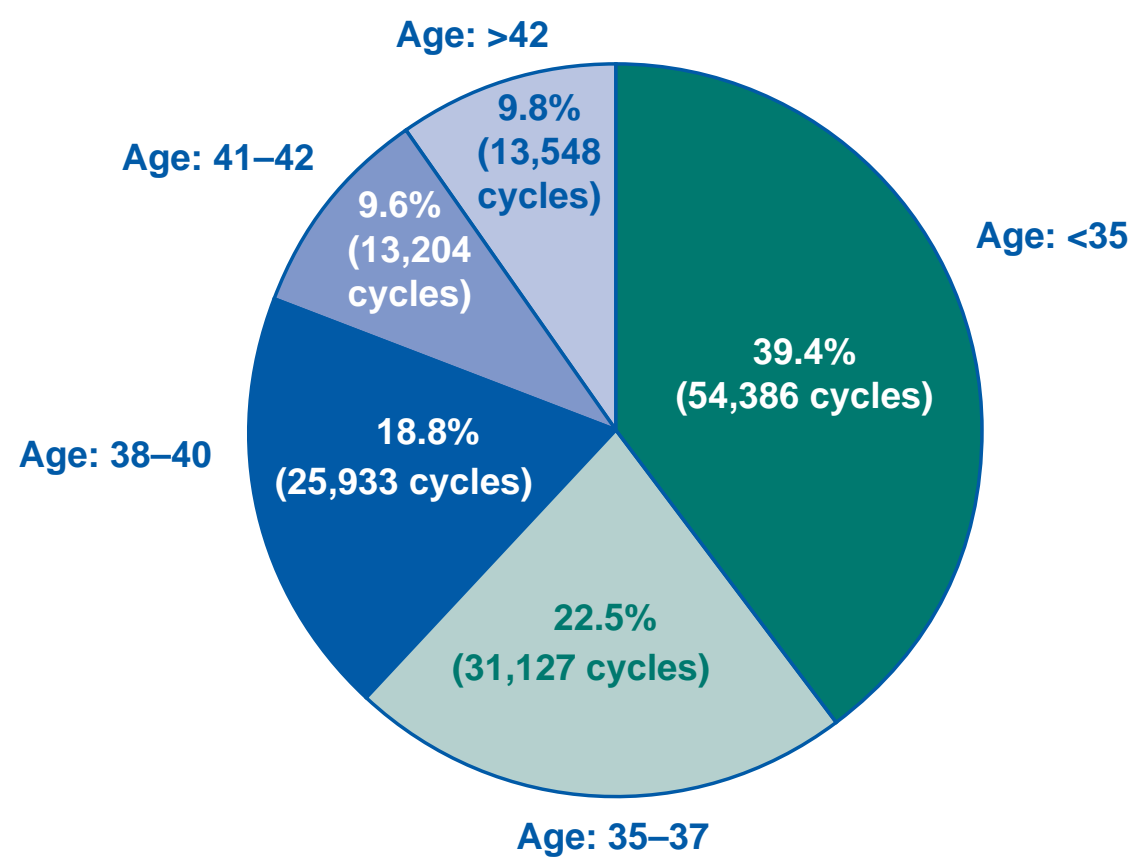

*Total does not equal $100 \%$ due to rounding. 


\section{How did the types of ART cycles used in the United States in 2006 differ among women of different ages?}

Figure 4 shows that, in 2006, the type of ART cycles varied by the woman's age. The vast majority $(96 \%)$ of women younger than 35 used their own eggs, whereas only $4 \%$ used donor eggs. In contrast, $21 \%$ of women aged 41 to 42 and more than half (55\%) of women older than 42 used donor eggs. Across all age groups, more ART cycles using fresh eggs or embryos were performed than cycles using frozen embryos.

\section{Figure 4}

Types of ART Cycles by Age Group-United States, 2006

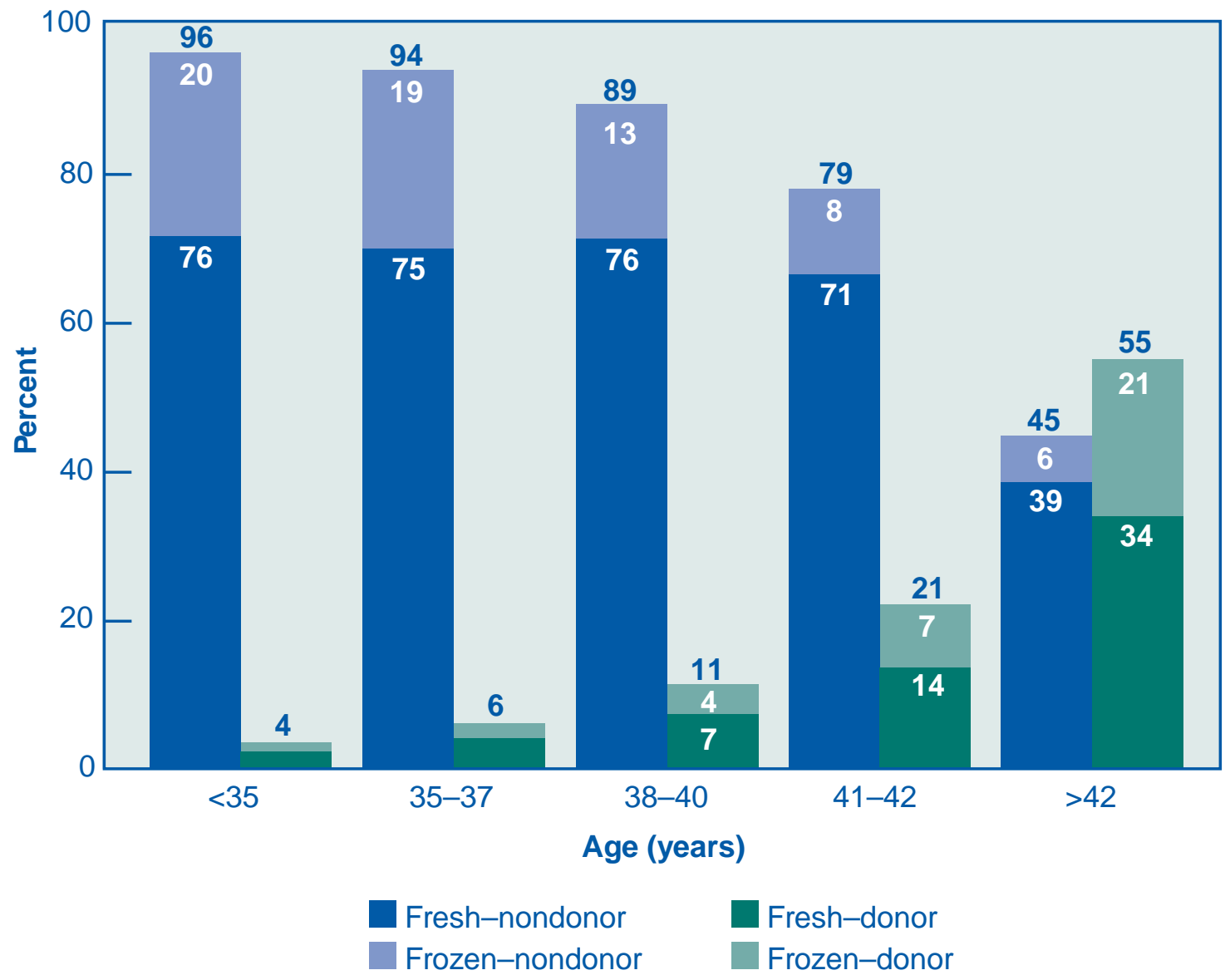




\section{SECTION 2: ART CYCLES USING FRESH NONDONOR EGGS OR EMBRYOS}

\section{What are the steps for an ART cycle using fresh nondonor eggs or embryos?}

Figure 5 presents the steps for an ART cycle using fresh nondonor eggs or embryos and shows how ART users in 2006 progressed through these stages toward pregnancy and live birth.

An ART cycle is started when a woman begins taking medication to stimulate the ovaries to develop eggs or, if no drugs are given, when the woman begins having her ovaries monitored (using ultrasound or blood tests) for natural egg production.

If eggs are produced, the cycle then progresses to egg retrieval, a surgical procedure in which eggs are collected from a woman's ovaries.

Once retrieved, eggs are combined with sperm in the laboratory. If fertilization is successful, one or more of the resulting embryos are selected for transfer, most often into a woman's uterus through the cervix (IVF), but sometimes into the fallopian tubes (e.g., GIFT, ZIFT; see pages 526 and 527 for definitions).

If one or more of the transferred embryos implant within the woman's uterus, the cycle then may progress to clinical pregnancy.

Finally, the pregnancy may progress to a live birth, the delivery of one or more live-born infants. (The birth of twins, triplets, or more is counted as one live birth.)

A cycle may be discontinued at any step for specific medical reasons (e.g., no eggs are produced, the embryo transfer was not successful) or by patient choice.

\section{Figure 5}

Outcome of ART Cycles Using Fresh Nondonor Eggs or Embryos, by Stage, 2006

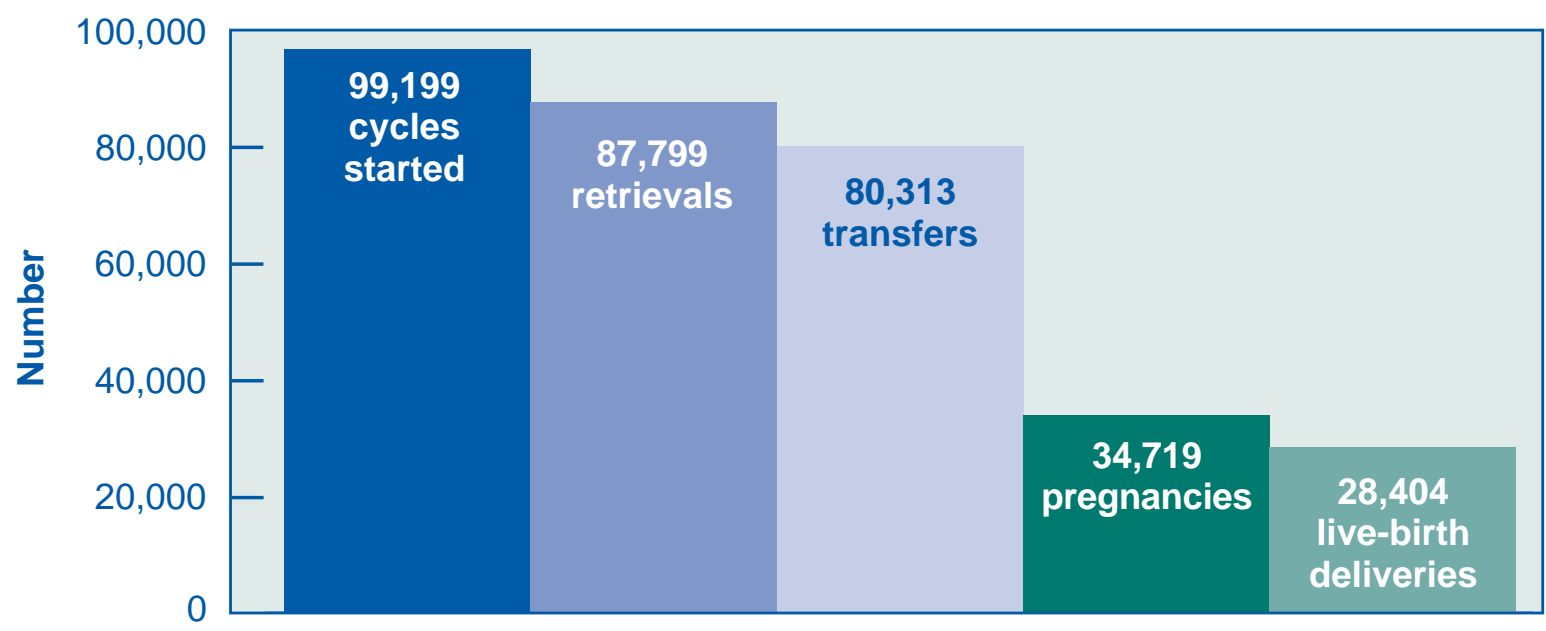




\section{Why are some ART cycles discontinued?}

In 2006, 11,400 ART cycles (about 11\%) were discontinued before the egg retrieval step (see Figure 5, page 17). Figure 6 shows reasons that the cycles were stopped. For approximately $84 \%$ of these cycles, there was no or inadequate egg production. Other reasons included too high a response to ovarian stimulation medications (i.e., potential for ovarian hyperstimulation syndrome), concurrent medical illness, or a patient's personal reasons.

\section{Figure 6}

Reasons ART Cycles Using Fresh Nondonor Eggs or Embryos

Were Discontinued, ${ }^{* \dagger} 2006$

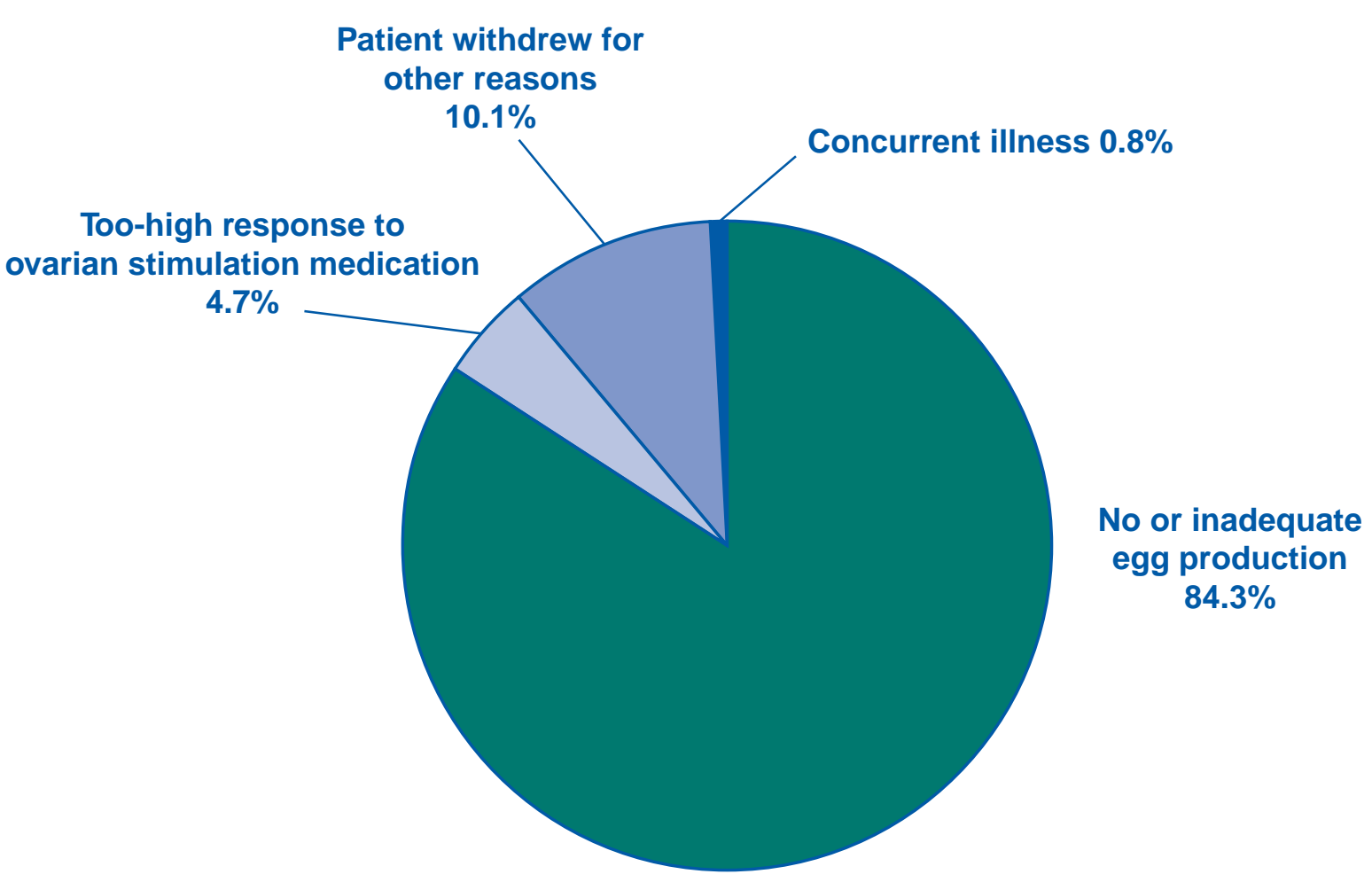

*Based on 11,400 ART cycles.

tTotal does not equal $100 \%$ due to rounding. 


\section{How is the success of ART measured?}

Figure 7 shows ART success rates using six different measures, each providing slightly different information about this complex process. The vast majority of success rates have increased slightly each year since CDC began monitoring them in 1995 (see Section 5, pages 61-76).

- Percentage of ART cycles started that produced a pregnancy: This is higher than the percentage of cycles that resulted in a live birth because some pregnancies end in miscarriage, induced abortion, or stillbirth (see Figure 9, page 21).

- Percentage of ART cycles started that resulted in a live birth (a delivery of one or more live-born infants): This is the one many people are most interested in because it represents the average chance of having a live-born infant by using ART. This is referred to as the basic live birth rate in the Fertility Clinic Success Rate and Certification Act of 1992.

- Percentage of ART cycles in which eggs were retrieved that resulted in a live birth: This is generally higher than the percentage of cycles that resulted in a live birth because it excludes cycles that were canceled before eggs were retrieved. In 2006, about $11 \%$ of all cycles using fresh nondonor eggs or embryos were canceled for a variety of reasons (see Figure 6, page 18). This is referred to as the live birth rate per successful oocyte (egg) retrieval in the Fertility Clinic Success Rate and Certification Act of 1992.

- Percentage of ART cycles in which an embryo or egg and sperm transfer occurred that resulted in a live birth: This is the highest of these six measures of ART success.

- Percentage of ART cycles started that resulted in a singleton live birth: Overall, singleton live births have a much lower risk than multiple-infant births for adverse infant health outcomes, including prematurity, low birth weight, disability, and death.

- Percentage of ART cycles in which an embryo or egg and sperm transfer occurred that resulted in a singleton live birth: This is higher than the percentage of ART cycles started that resulted in a singleton live birth because not all ART cycles proceed to embryo transfer.

\section{Figure 7}

Success Rates for ART Cycles Using Fresh Nondonor Eggs or Embryos, by Different Measures, 2006

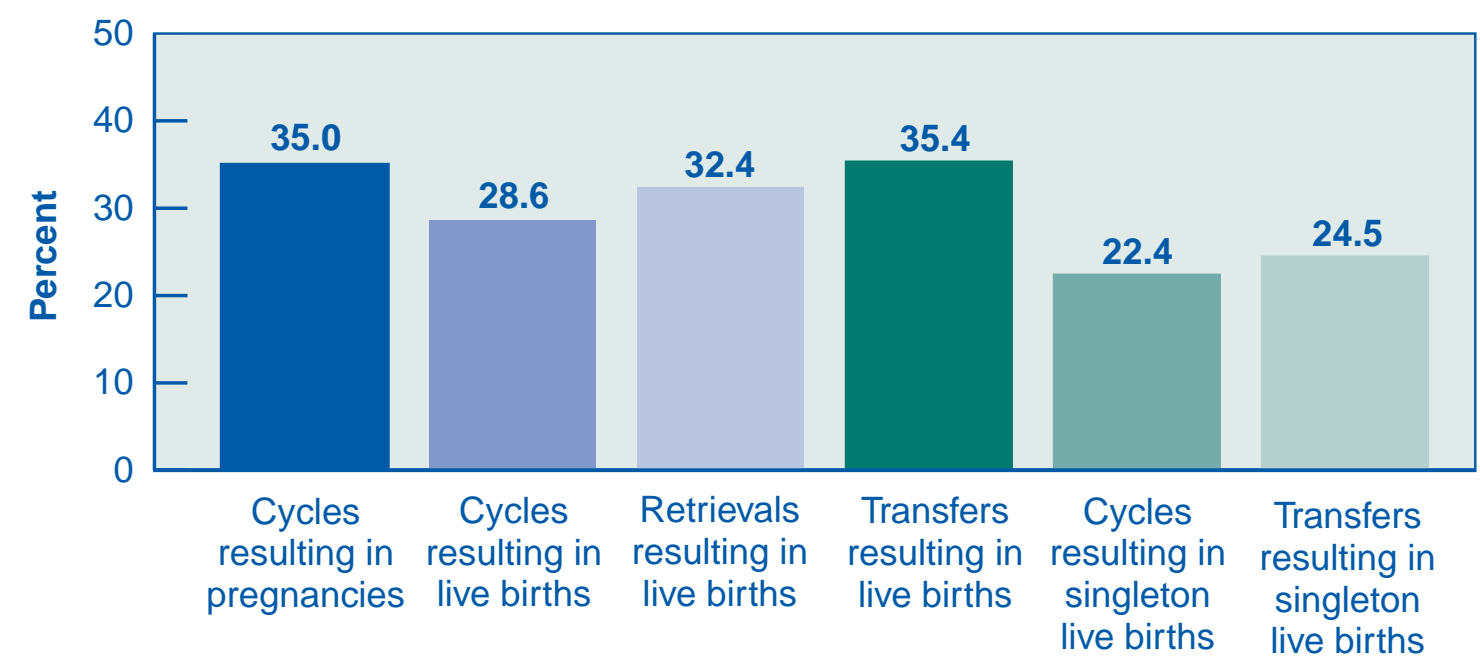




\section{What percentage of ART cycles results in a pregnancy?}

Figure 8 shows the results of ART cycles in 2006 that used fresh nondonor eggs or embryos. Most of these cycles (64\%) did not produce a pregnancy; a very small proportion $(0.7 \%)$ resulted in an ectopic pregnancy (the embryo implanted outside the uterus), and 35\% resulted in clinical pregnancy. Clinical pregnancies can be further subdivided as follows:

- $21.5 \%$ resulted in a single-fetus pregnancy.

- $11.1 \%$ resulted in a multiple-fetus pregnancy.

- $2.3 \%$ ended in miscarriage before the number of fetuses could be accurately determined.

\section{Figure 8}

Results of ART Cycles Using Fresh Nondonor Eggs or Embryos, ${ }^{*} 2006$

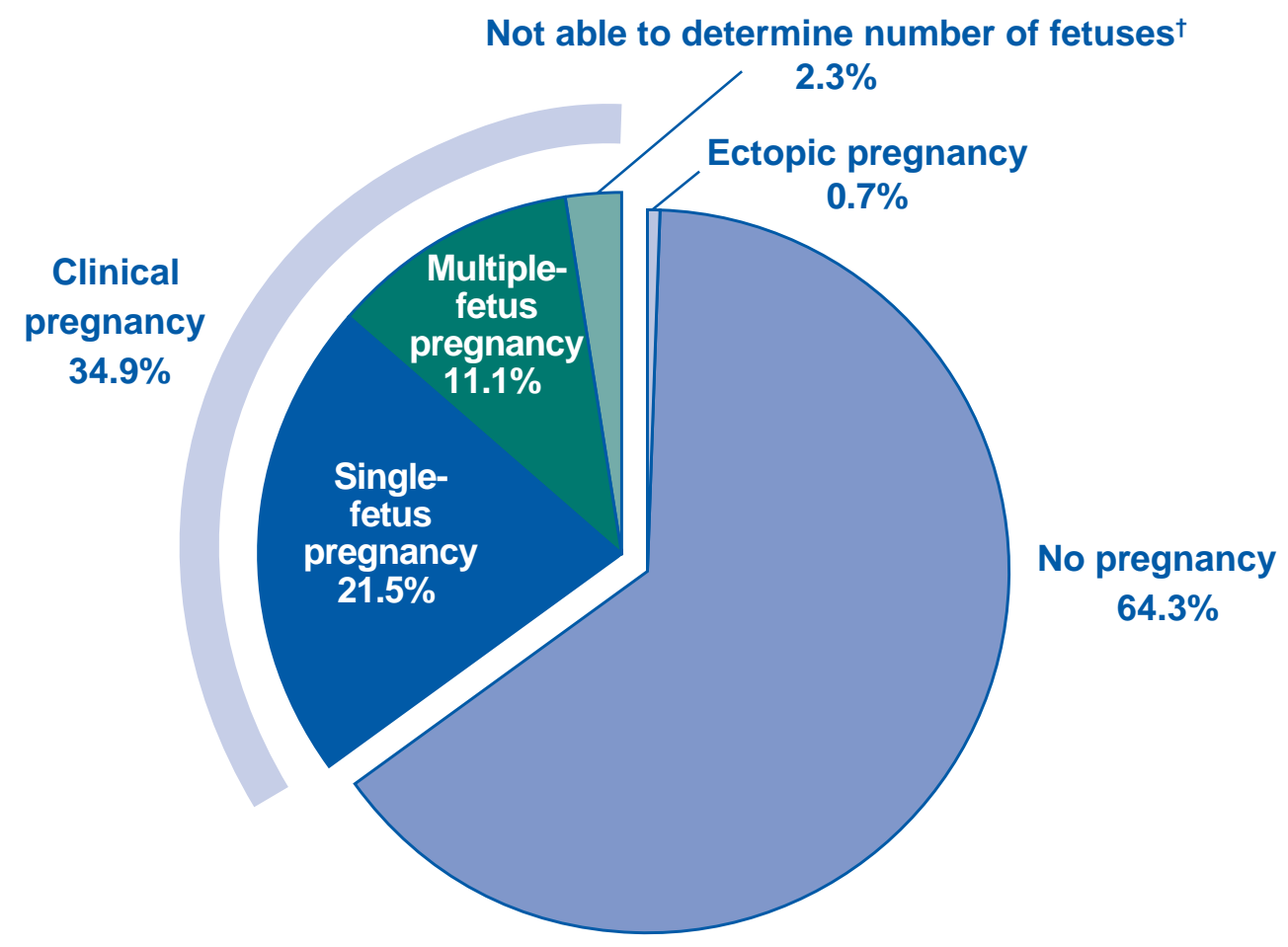

${ }^{*}$ Total does not equal $100 \%$ due to rounding.

†Number of fetuses not known because the pregnancy ended in an early miscarriage. 


\section{What percentage of pregnancies results in a live birth?}

Figure 9 shows the outcomes of pregnancies resulting from ART cycles in 2006 (see Figure 8, page 20 ). Approximately $82 \%$ of the pregnancies resulted in a live birth (57\% in a singleton birth and $25 \%$ in a multiple-infant birth). About $18 \%$ of pregnancies resulted in an adverse outcome (miscarriage, stillbirth, induced abortion, or maternal death). For $0.6 \%$ of pregnancies, the outcome was unknown.

Although the birth of more than one infant is counted as one live birth, multiple-infant births are presented here as a separate category because they often are associated with problems for both mothers and infants. Infant deaths and birth defects are not included as adverse outcomes because the available information for these outcomes is incomplete.

\section{Figure 9}

Outcomes of Pregnancies Resulting from ART Cycles Using Fresh Nondonor Eggs or Embryos, ${ }^{*} 2006$

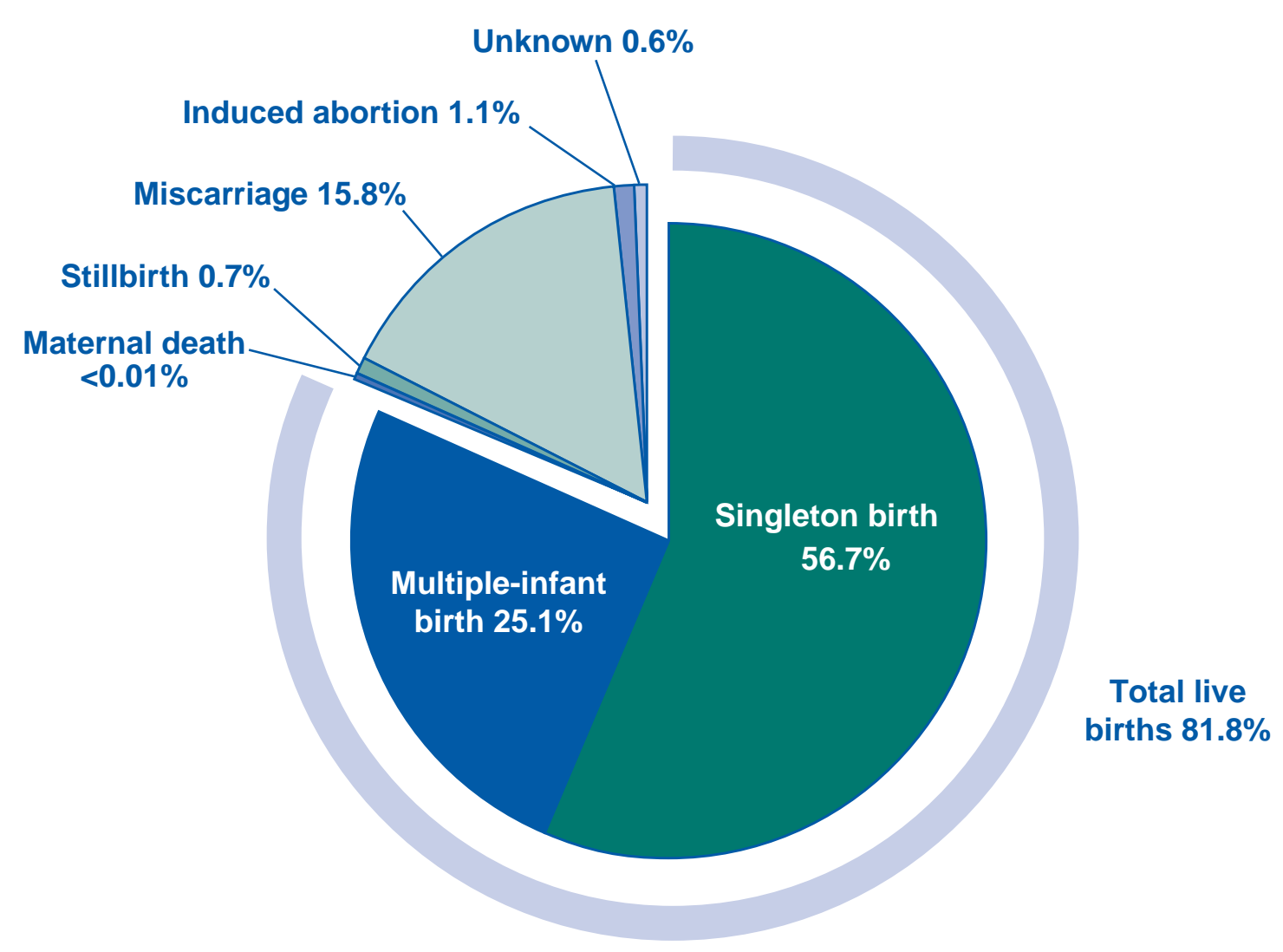

*Total does not equal $100 \%$ due to rounding. 


\section{Using ART, what is the risk of having a multiple-fetus pregnancy or multiple-infant live birth?}

Multiple-infant births are associated with greater problems for both mothers and infants, including higher rates of caesarean section, prematurity, low birth weight, and infant disability or death.

Part A of Figure 10 shows that among the 34,719 pregnancies that resulted from ART cycles using fresh nondonor eggs or embryos, 62\% were singleton pregnancies, $28 \%$ were twins, and about $4 \%$ were triplets or more. Seven percent of pregnancies ended in miscarriage in which the number of fetuses could not be accurately determined. Therefore, the percentage of pregnancies with more than one fetus might have been higher than what was reported (about 32\%).

In 2006, 6,117 pregnancies resulting from ART cycles ended in either miscarriage, stillbirth, induced abortion, or maternal death, and 198 pregnancy outcomes were not reported. The remaining 28,404 pregnancies resulted in live births. Part B of Figure 10 shows that approximately $31 \%$ of these live births produced more than one infant (29\% twins and approximately $2 \%$ triplets or more). This compares with a multiple-infant birth rate of slightly more than $3 \%$ in the general U.S. population. Although the total rates for multiples were similar between pregnancies and live births, there were more triplet-or-more pregnancies than births. Triplet-or-more pregnancies may be reduced to twins or singletons by the time of birth. This can happen naturally (e.g., fetal death), or a woman and her doctor may decide to reduce the number of fetuses using a procedure called multifetal pregnancy reduction. CDC does not collect information on multifetal pregnancy reductions.

\section{Figure 10}

Risks of Having Multiple-Fetus Pregnancy and Multiple-Infant Live Birth from ART Cycles Using Fresh Nondonor Eggs or Embryos, 2006

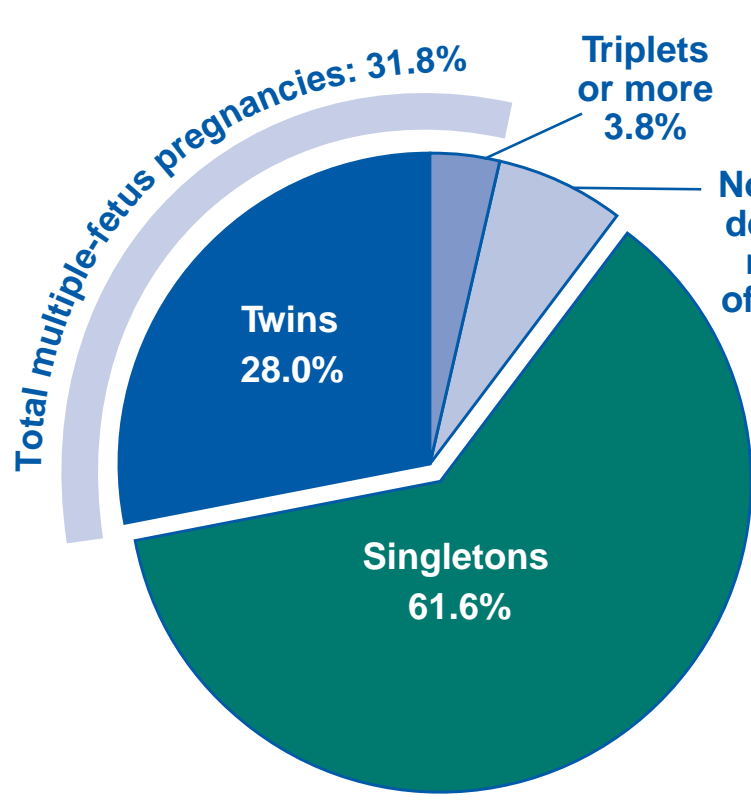

A. 34,719 Pregnancies

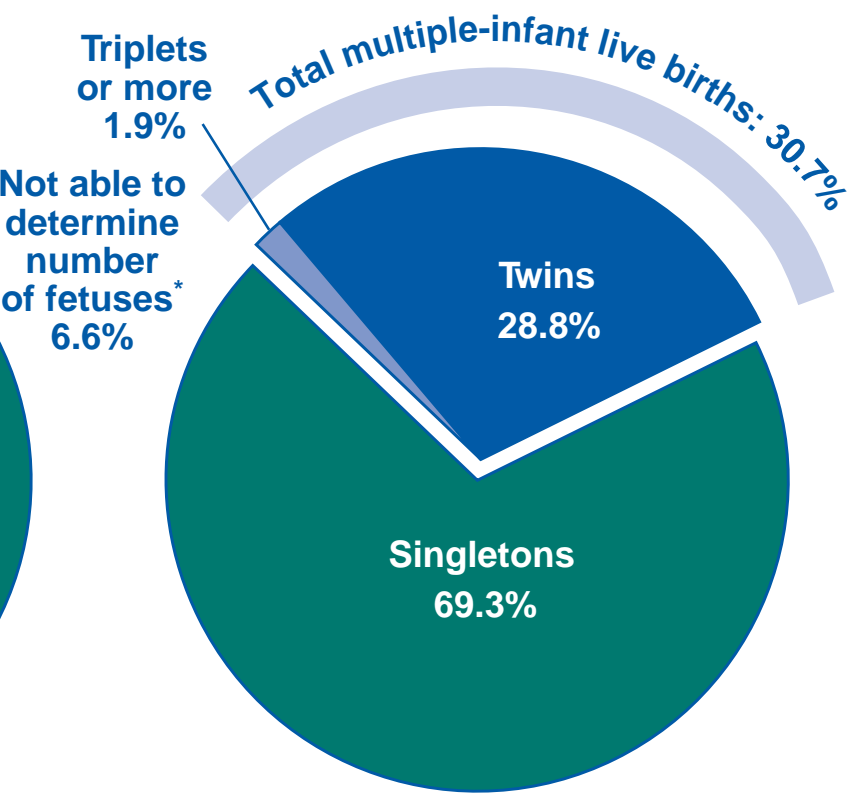

B. 28,404 Live births

*Number of fetuses not known because the pregnancy ended in an early miscarriage. 


\section{Using ART, what is the risk for preterm birth?}

Preterm birth occurs when a woman gives birth before 37 full weeks of pregnancy. Infants born preterm are at greater risk for death in the first few days of life, as well as other adverse health outcomes including mental retardation, visual and hearing impairments, learning disabilities, and behavioral and emotional problems throughout life. Preterm births also cause substantial emotional and economic burdens for families.

Figure 11 shows percentages of preterm births resulting from ART cycles that used fresh nondonor eggs or embryos, by the number of infants born. For singletons, it shows separately the preterm percentage for pregnancies that started with one fetus (single-fetus pregnancies) or more than one (multiple-fetus pregnancies).

Among singletons, the percentage of preterm births was higher for those from multiple-fetus pregnancies (18\%) than those from single-fetus pregnancies (12\%). In the general U.S. population, where singletons are almost always the result of a single-fetus pregnancy, 13\% were born preterm in 2005 (most recent available data).

Among ART births, $63 \%$ of twins and $95 \%$ of triplets or more were born preterm. A comparison of preterm births between ART twins and triplets or more and similar births in the general population is not meaningful because the vast majority of multiple-infant births in the United States are due to infertility treatments (both ART and non-ART).

These data indicate that the risk for preterm birth is higher among infants conceived through ART than for infants in the general population. This increase in risk is, in large part, due to the higher rate of multiple-infant pregnancies resulting from ART cycles.

\section{Figure 11}

Percentages of Preterm Births from ART Cycles Using Fresh Nondonor Eggs or Embryos, by Number of Infants Born, 2006

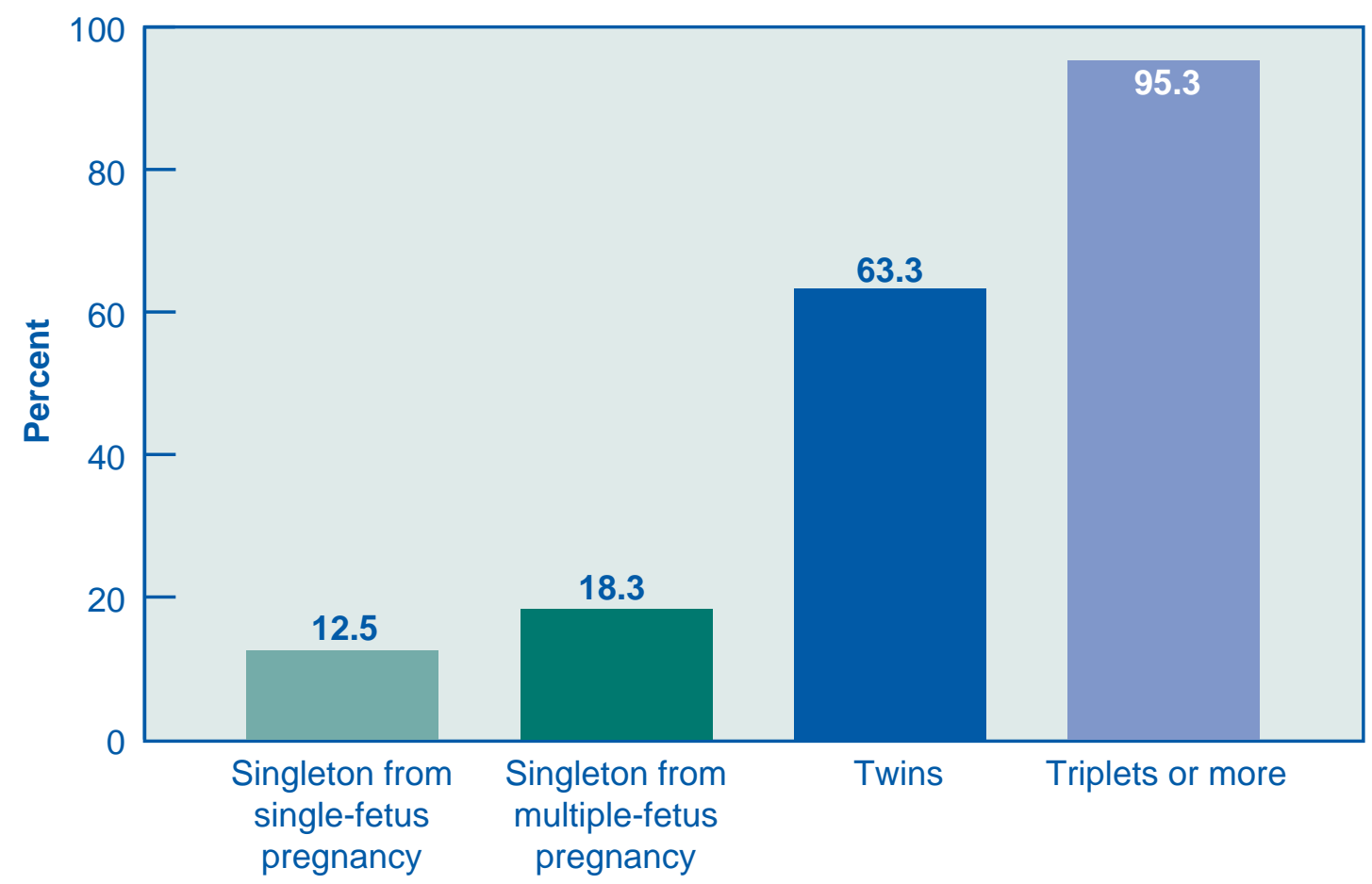

Preterm Birth 


\section{Using ART, what is the risk of having low-birth-weight infants?}

Low-birth-weight infants (less than 2,500 grams, or 5 pounds, 9 ounces) are at increased risk for death and short- and long-term disabilities such as cerebral palsy, mental retardation, and limitations in motor and cognitive skills.

Figure 12 presents percentages of low-birth-weight infants resulting from ART cycles that used fresh nondonor eggs or embryos, by number of infants born. For singletons, it shows separately the percentages of low birth weight among infants born from pregnancies that started with one fetus (singlefetus pregnancies) and with more than one fetus (multiple-fetus pregnancies).

Among singletons born through ART, the percentage of low-birth-weight infants was higher for those from multiple-fetus pregnancies (17\%) than those from single-fetus pregnancies (8\%). In the general U.S. population, where singletons are almost always the result of a single-fetus pregnancy, $8 \%$ of infants born in 2005 (most recent available data) had low birth weights.

Approximately $57 \%$ of twins and $90 \%$ of triplets or more had low birth weights. Comparing percentages of low birth weight between ART twins and triplets or more and the general population is not meaningful because the vast majority of multiple births in the United States are due to infertility treatments (both ART and non-ART).

These data indicate that the risk for low birth weight is higher for infants conceived through ART than for infants in the general population. The increase in risk is due, in large part, to the higher percentage of multiple-infant pregnancies resulting from ART cycles.

\section{Figure 12}

Percentages of Low-Birth-Weight Infants from ART Cycles Using Fresh Nondonor Eggs or Embryos, by Number of Infants Born, 2006

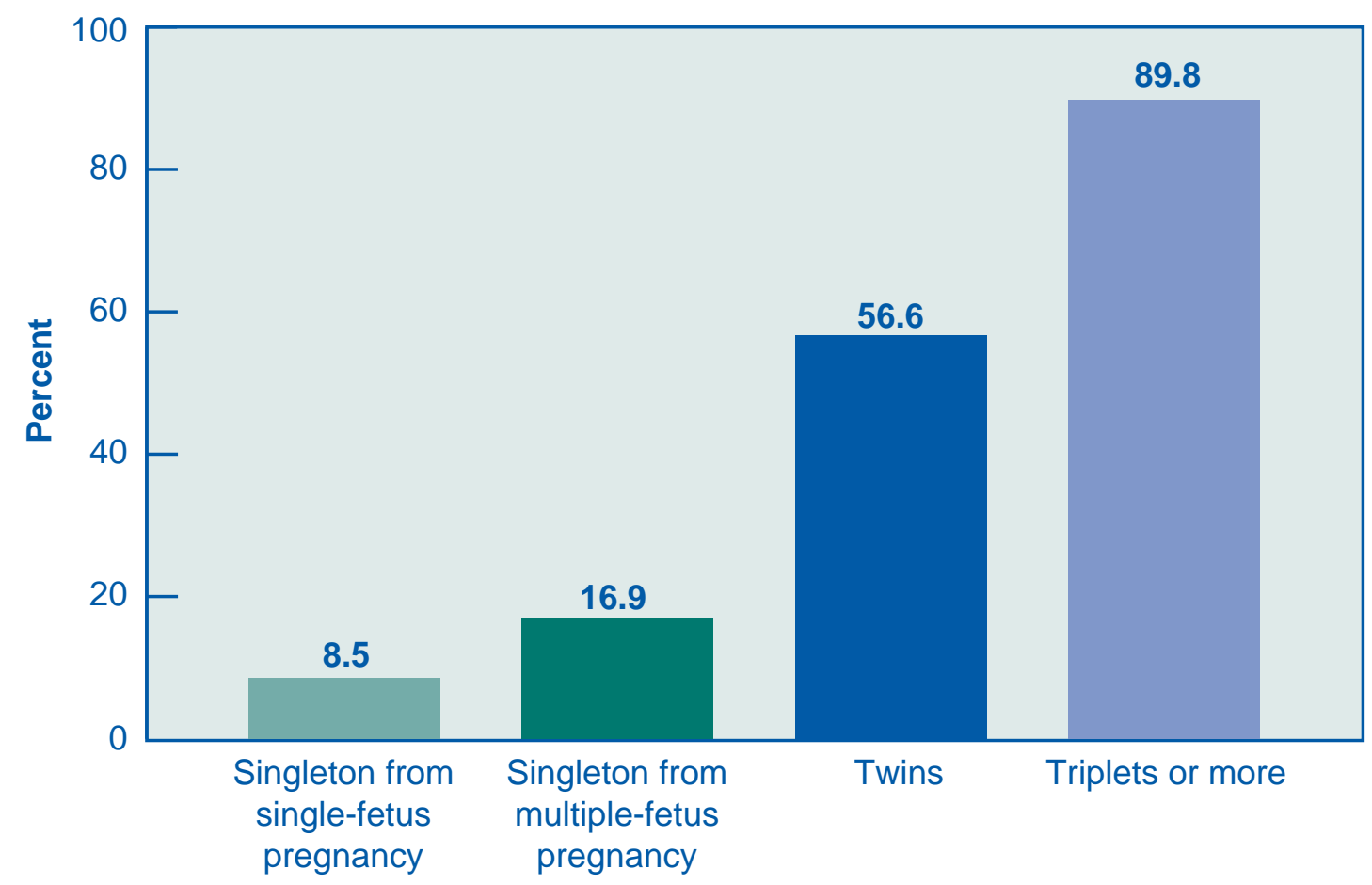

Low Birth Weight 


\section{What are the ages of women who use ART?}

Figure 13 presents ART cycles using fresh nondonor eggs or embryos according to the age of the woman who had the procedure. About $12 \%$ of these cycles were among women younger than age $30,67 \%$ were among women aged $30-39$, and approximately $21 \%$ were among women aged 40 and older.

\section{Figure 13}

Age Distribution of Women Who Had ART Cycles Using

Fresh Nondonor Eggs or Embryos, 2006

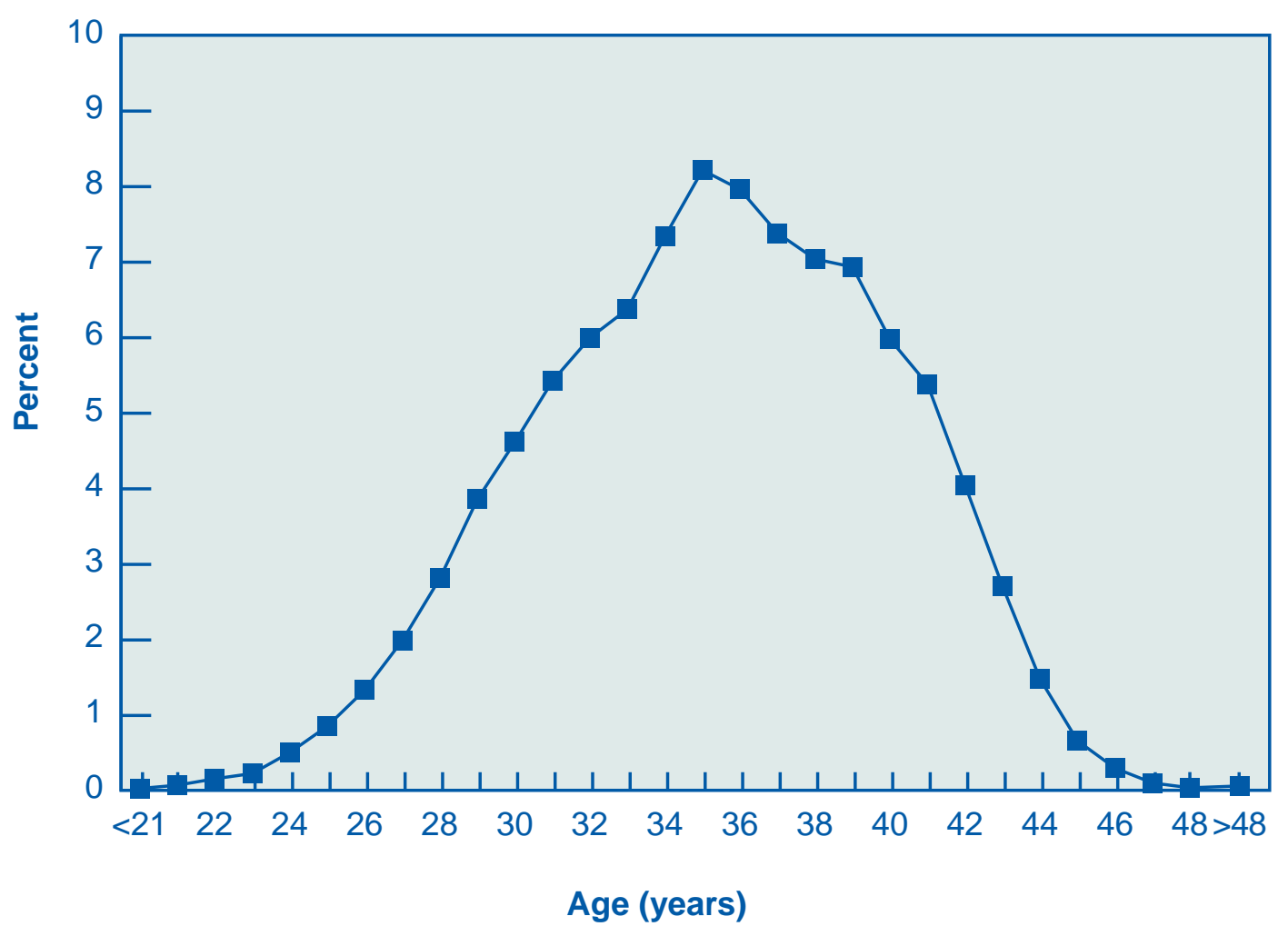




\section{Do ART success rates differ among women of different ages?}

A woman's age is the most important factor affecting the chances of a live birth when her own eggs are used. Figure 14 shows the percentages of pregnancies, live births, and singleton live births for women of different ages who had ART procedures using fresh nondonor eggs or embryos in 2006. The percentages of ART cycles resulting in live births and singleton live births are different because of the high percentage of multiple-infant deliveries counted among the total live births. The percentage of multiple-infant births is particularly high among women younger than 35 (see Figure 34, page 46). Among women in their 20s, the percentages of ART cycles resulting in pregnancies, live births, and singleton live births were relatively stable; however, success rates declined steadily from the mid-30s onward. For additional detail on success rates among women aged 40 or older, see Figure 15 on page 27.

\section{Figure 14}

Percentages of ART Cycles Using Fresh Nondonor Eggs or Embryos That Resulted in Pregnancies, Live Births, and Singleton Live Births, by Age of Woman, * 2006

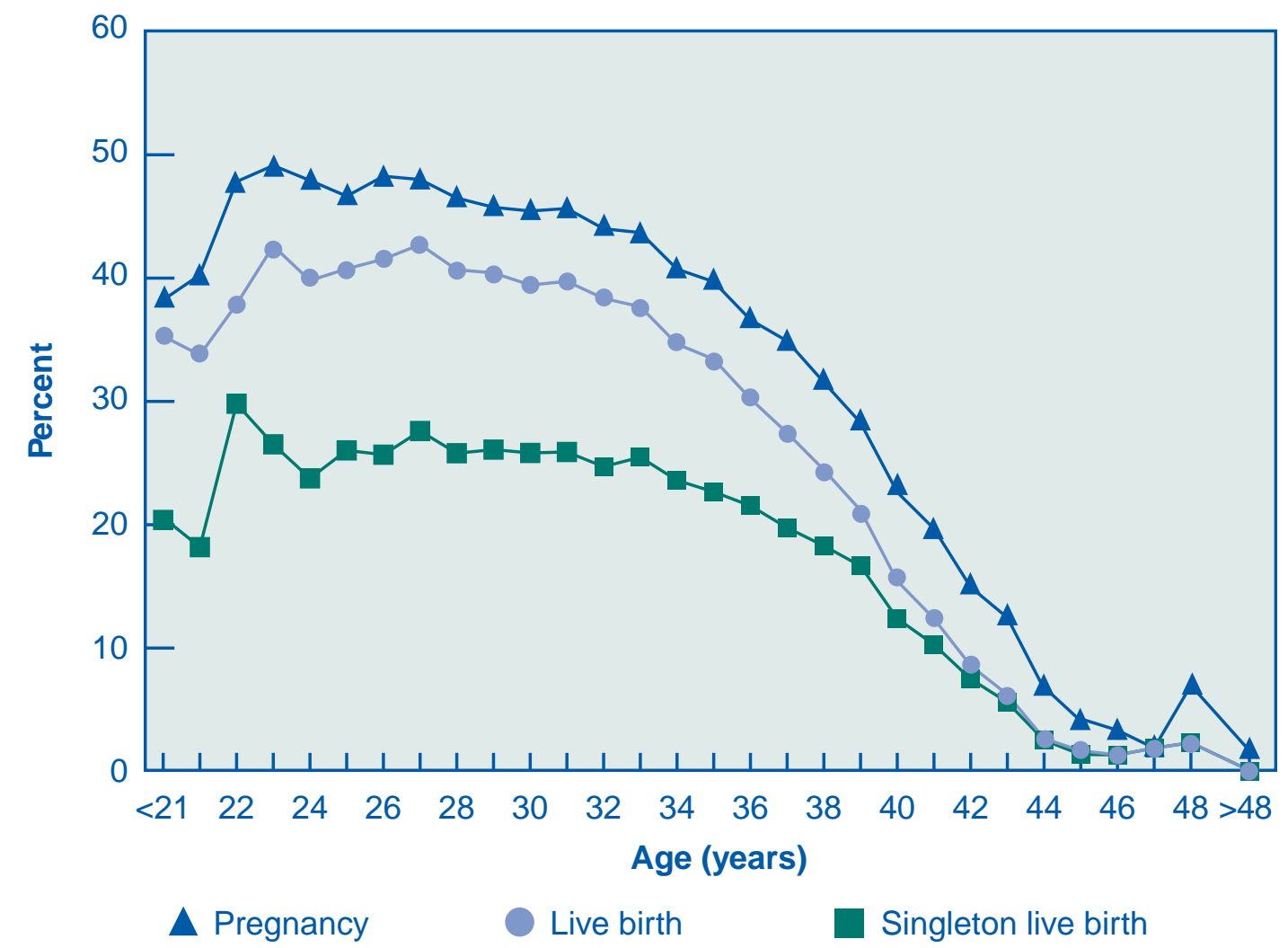

${ }^{*}$ For consistency, all percentages are based on cycles started. 


\section{How do ART success rates differ for women who are $\mathbf{4 0}$ or older?}

Success rates decline with each year of age and are particularly low for women 40 or older. Figure 15 shows the percentages of pregnancies, live births, and singleton live births in 2006 for women 40 or older who used fresh nondonor eggs or embryos. The average chance for pregnancy was $23 \%$ for women age 40; the percentage of ART cycles resulting in live births for this age was about $15 \%$, and the percentage of ART cycles resulting in singleton live births was about $12 \%$. All percentages dropped steadily with each 1 -year increase in age. For women older than 44 , the percentages of live births and singleton live births were both a little more than $1 \%$. Women 40 or older generally have much higher success rates using donor eggs (see Figure 45, page 57).

\section{Figure 15}

Percentages of ART Cycles Using Fresh Nondonor Eggs or Embryos That Resulted in Pregnancies, Live Births, and Singleton Live Births Among Women Aged 40 or Older, 2006

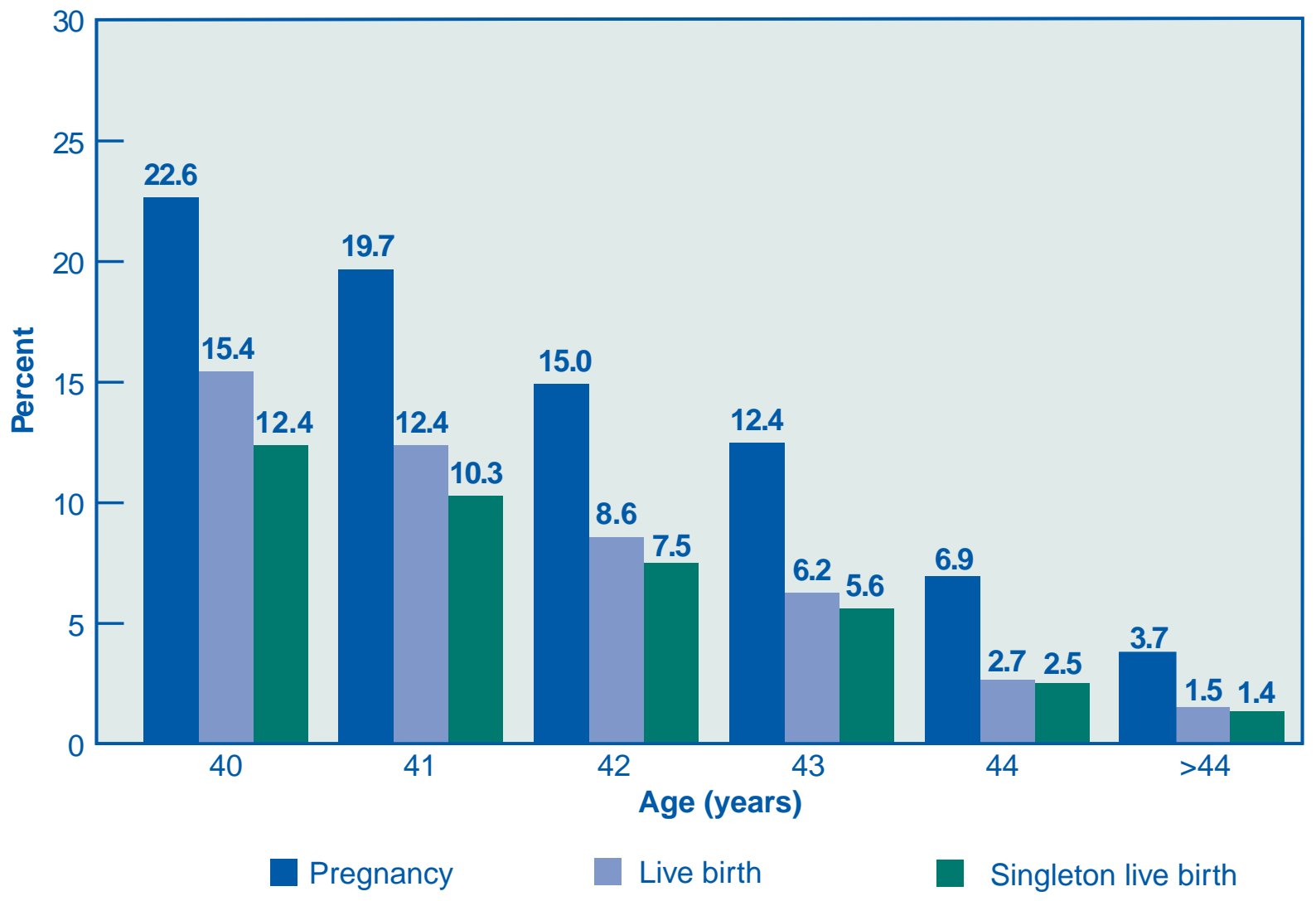

${ }^{*}$ For consistency, all percentages are based on cycles started. 


\section{How does the risk for miscarriage differ among women of different ages?}

A woman's age not only affects the chance for pregnancy when her own eggs are used, but also affects her risk for miscarriage. Figure 16 shows the percentages of ART cycles started in 2006 that resulted in miscarriage for women of different ages. The percentages of ART cycles that resulted in miscarriage were below $14 \%$ among women younger than 35 . The percentages of ART cycles that resulted in miscarriages began to increase among women in their mid- to late 30s and continued to increase with age, reaching 28\% at age 40 and 56\% among women older than 43 .

The risk for miscarriage observed among women undergoing ART procedures using fresh nondonor eggs or embryos appear to be similar to those reported in various studies of other pregnant women in the United States.

\section{Figure 16}

Percentages of ART Cycles Using Fresh Nondonor Eggs or Embryos

That Resulted in Miscarriage, by Age of Woman, 2006

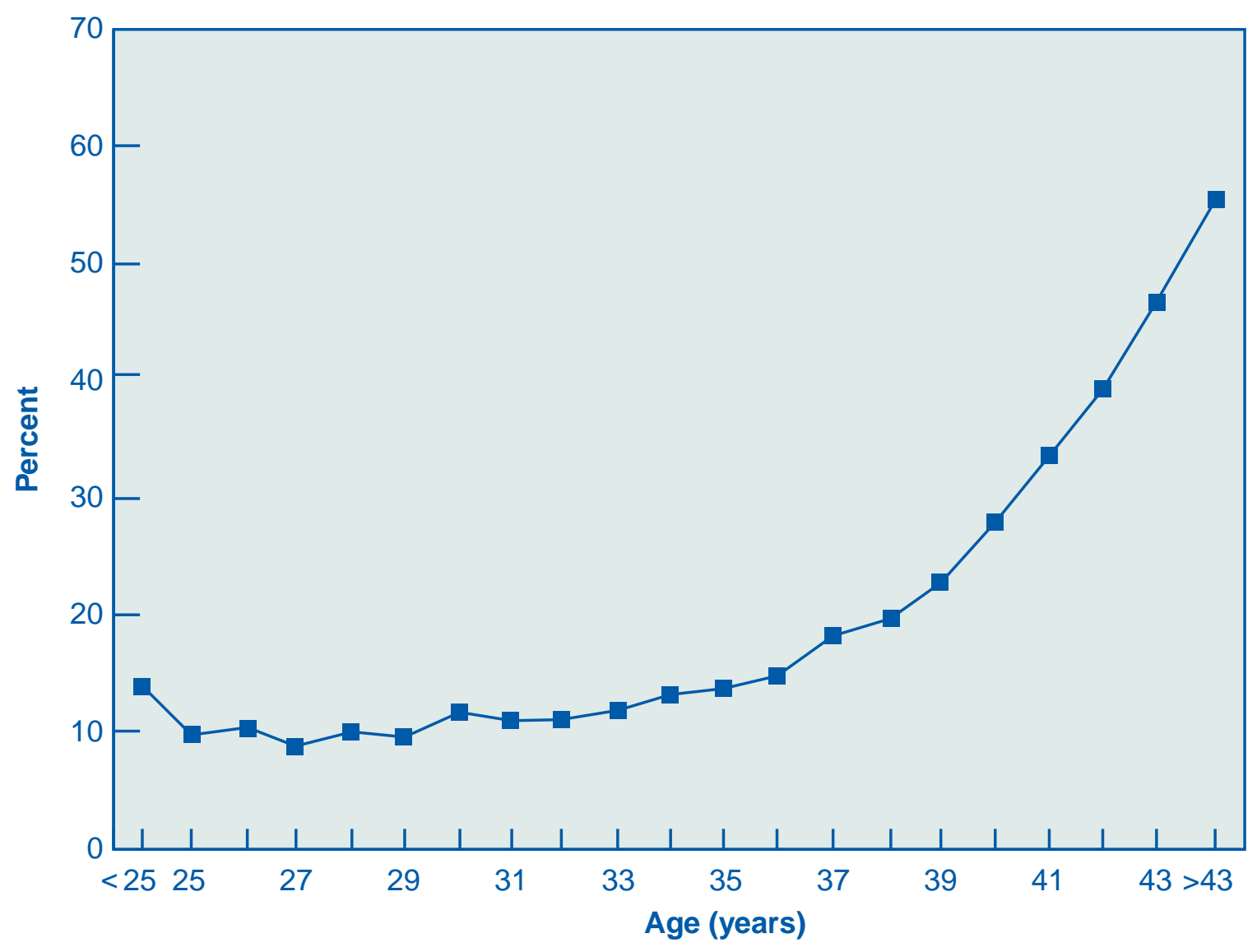




\section{What is the risk for pregnancy loss at different times during pregnancy among women of different ages?}

A woman's risk for pregnancy loss (loss of an entire pregnancy, or all fetuses in a multiple-fetus pregnancy) is affected by the duration of her pregnancy and her age. Figure 17 shows that between $13 \%$ and $52 \%$ of clinically-detected pregnancies (clinical detection through ultrasound performed between 4 and 6 weeks after the day of embryo transfer) are lost at some later point during the pregnancy, depending on the woman's age. Among women younger than 35, 13\% of pregnancies were lost and $87 \%$ continued through week 24 . In contrast, among women older than $42,52 \%$ of pregnancies were lost and only $48 \%$ continued through week 24 . In all age groups, most pregnancy losses occurred before week 14 (i.e., during the first trimester). The risk of pregnancy loss after 24 weeks was less than $1 \%$ for all age groups because most pregnancies that progress beyond week 24 lead to live births.

\section{Figure 17}

Percentages of Pregnancies That Continued Past a Given Gestational Week Among Women Who Had ART Cycles Using Fresh Nondonor Eggs or Embryos, by Age of Woman, 2006

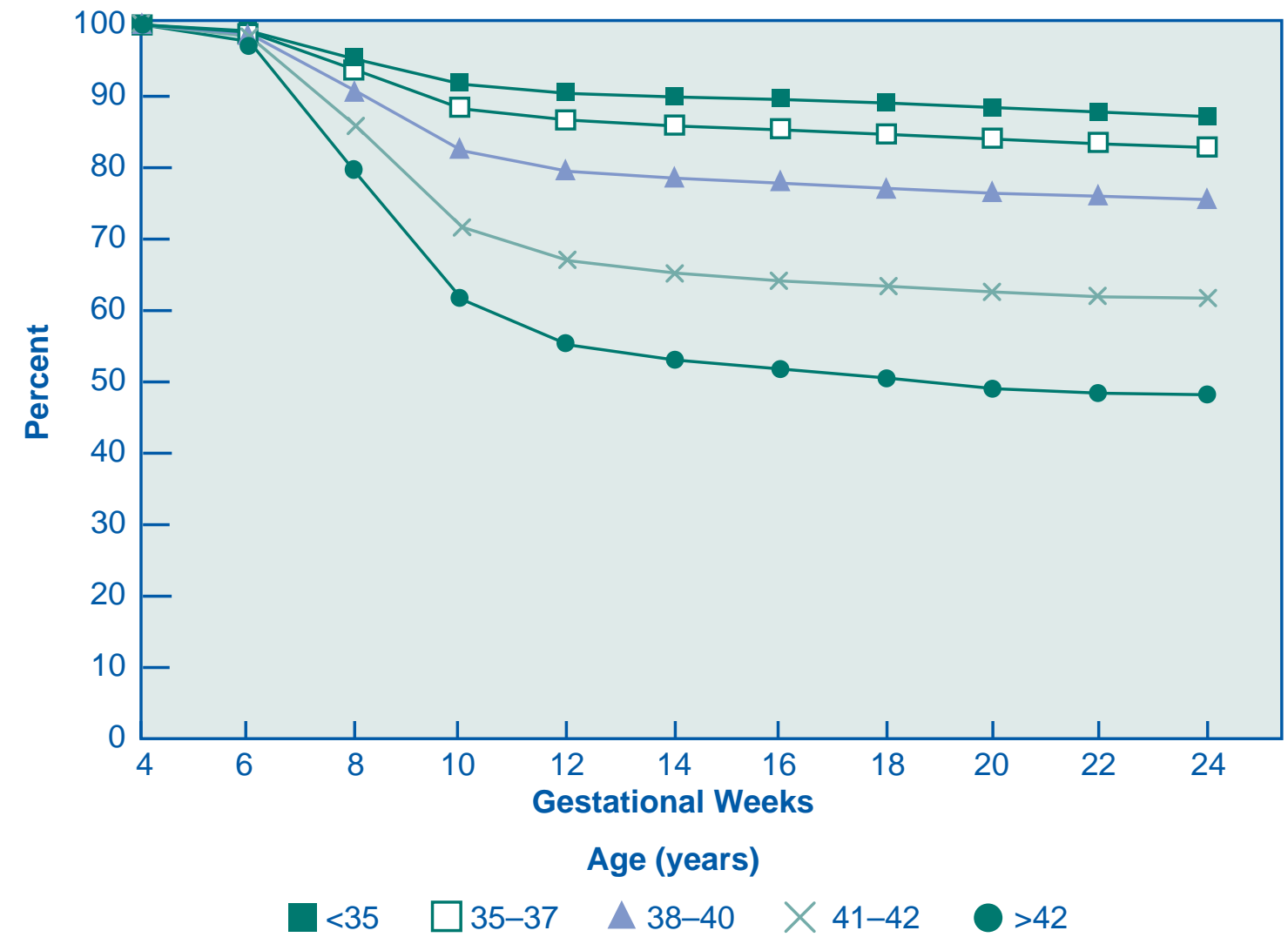




\section{How does a woman's age affect her chances of progressing through the various stages of ART?}

In 2006, a total of 99,199 cycles using fresh nondonor eggs or embryos were started:

- 41,369 in women younger than 35

- 23,376 in women 35-37

- 19,775 in women 38-40
- 9,346 in women 41-42

- 5,333 in women older than 42

Figure 18 shows that a woman's chance of progressing from the beginning of ART to pregnancy and live birth (using her own eggs) decreases at every stage of ART as her age increases.

- As women get older, the likelihood of a successful response to ovarian stimulation and progression to egg retrieval decreases.

- As women get older, cycles that have progressed to egg retrieval are slightly less likely to reach transfer.

- The percentage of cycles that progress from transfer to pregnancy also decreases as women get older.

- As women get older, cycles that have progressed to pregnancy are less likely to result in a live birth because the risk for miscarriage is greater (see Figure 16, page 28).

Overall, 39\% of cycles started in 2006 among women younger than 35 resulted in live births. This percentage decreased to 30\% among women 35-37 years of age, 21\% among women 38-40, 11\% among women 41-42, and 4\% among women older than 42. As noted in Figures 14 and 15 (see pages 26 and 27), the proportion of cycles that resulted in singleton live births is even lower for each age group.

\section{Figure 18}

Outcomes of ART Cycles Using Fresh Nondonor Eggs or Embryos,

by Stage and Age Group, 2006

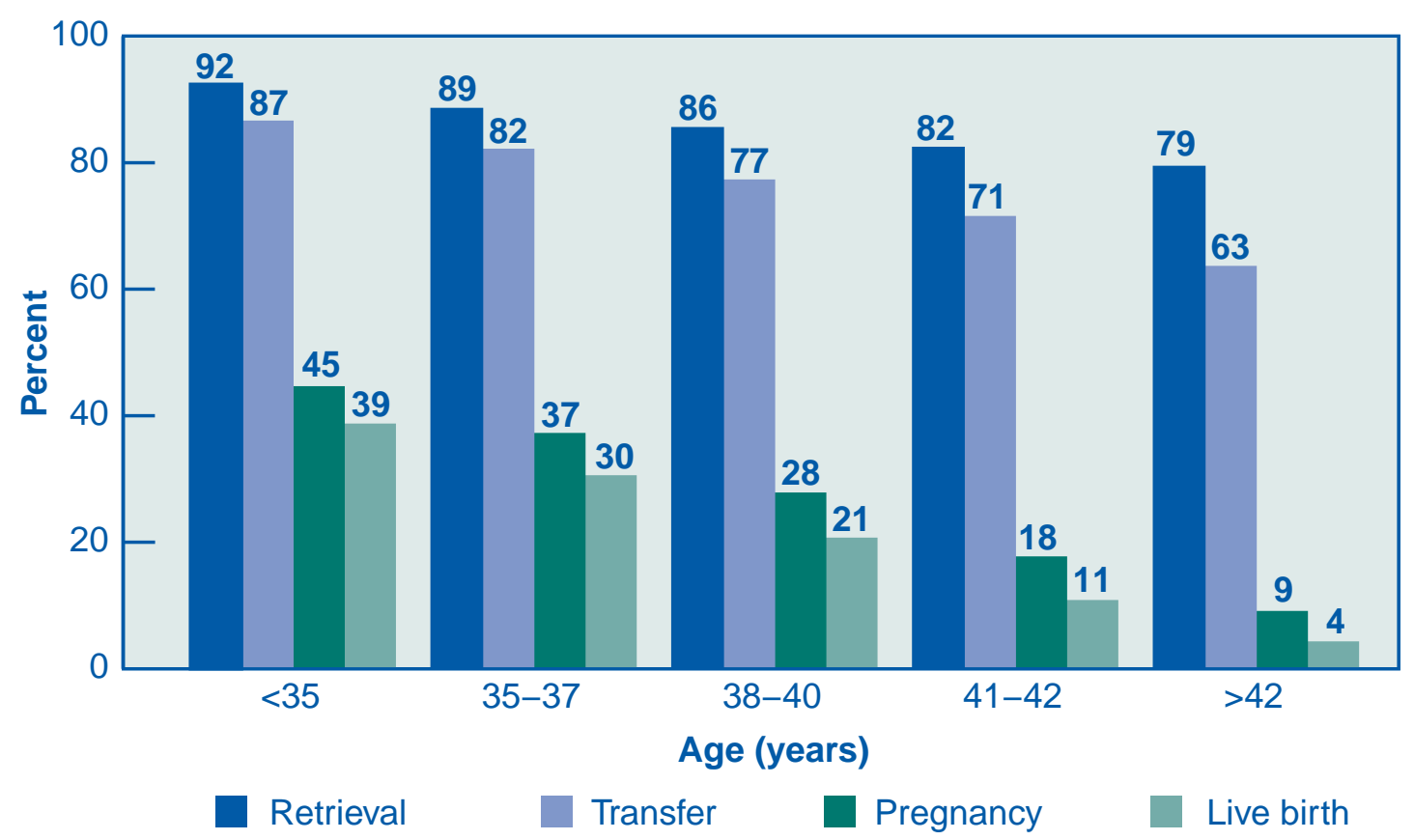




\section{What are the causes of infertility among couples who use ART?}

Figure 19 shows the infertility diagnoses reported among couples who had an ART procedure using fresh nondonor eggs or embryos in 2006. Diagnoses range from one infertility factor in one partner to multiple factors in either one or both partners. However, diagnostic procedures may vary from one clinic to another, so the categorization may also vary.

- Tubal factor means that the woman's fallopian tubes are blocked or damaged, making it difficult for the egg to be fertilized or for an embryo to travel to the uterus.

- Ovulatory dysfunction means that the ovaries are not producing eggs normally. Such dysfunctions include polycystic ovary syndrome and multiple ovarian cysts.

- Diminished ovarian reserve means that the ability of the ovary to produce eggs is reduced. Reasons include congenital, medical, or surgical causes or advanced age.

- Endometriosis involves the presence of tissue similar to the uterine lining in abnormal locations. This condition can affect both fertilization of the egg and embryo implantation.

- Uterine factor means a structural or functional disorder of the uterus that results in reduced fertility.

- Male factor refers to a low sperm count or problems with sperm function that make it difficult for a sperm to fertilize an egg under normal conditions.

- Other causes of infertility include immunological problems, chromosomal abnormalities, cancer chemotherapy, and serious illnesses.

- Unexplained cause means that no cause of infertility was found in either the woman or the man.

- Multiple factors, female only, means that more than one female cause was diagnosed.

- Multiple factors, female and male, means that one or more female causes and male factor infertility were diagnosed.

\section{Figure 19}

Diagnoses Among Couples Who Had ART Cycles Using Fresh Nondonor Eggs or Embryos, ${ }^{*} 2006$

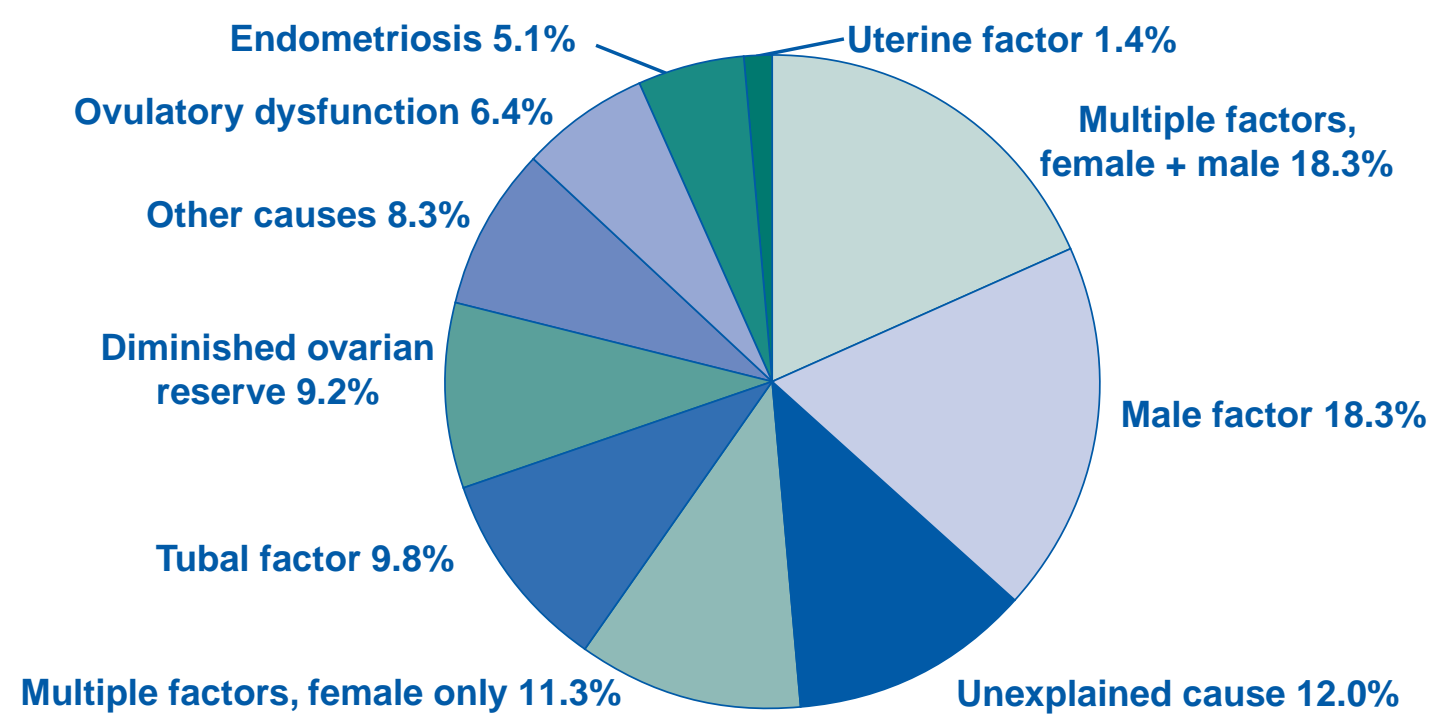

${ }^{*}$ Total does not equal $100 \%$ due to rounding. 


\section{Does the cause of infertility affect the chances of success using ART?}

Figure 20 shows the percentage of ART cycles that resulted in live births according to the causes of infertility. (See Figure 19, page 31, or the Glossary in Appendix B for an explanation of the diagnoses.) Although the national average success rate was about 29\% (see Figure 7, page 19), success rates varied somewhat depending on the couple's diagnosis; however, the definitions of these diagnoses may vary from clinic to clinic. In general, couples diagnosed with tubal factor, ovulatory dysfunction, endometriosis, male factor, or unexplained infertility had success rates above the national average. The lowest success rate was observed for those with diminished ovarian reserve. Additionally, couples with uterine factor, "other" causes, or multiple infertility factors had below-average success rates. Please note, however, that a review of select clinical records revealed that reporting of infertility causes may be incomplete. Therefore, differences in success rates by causes of infertility should be interpreted with caution. (See Findings from Validation Visits for 2006 ART Data in Appendix A for additional information.)

\section{Figure 20}

Percentages of ART Cycles Using Fresh Nondonor Eggs or Embryos

That Resulted in Live Births, by Diagnosis, 2006

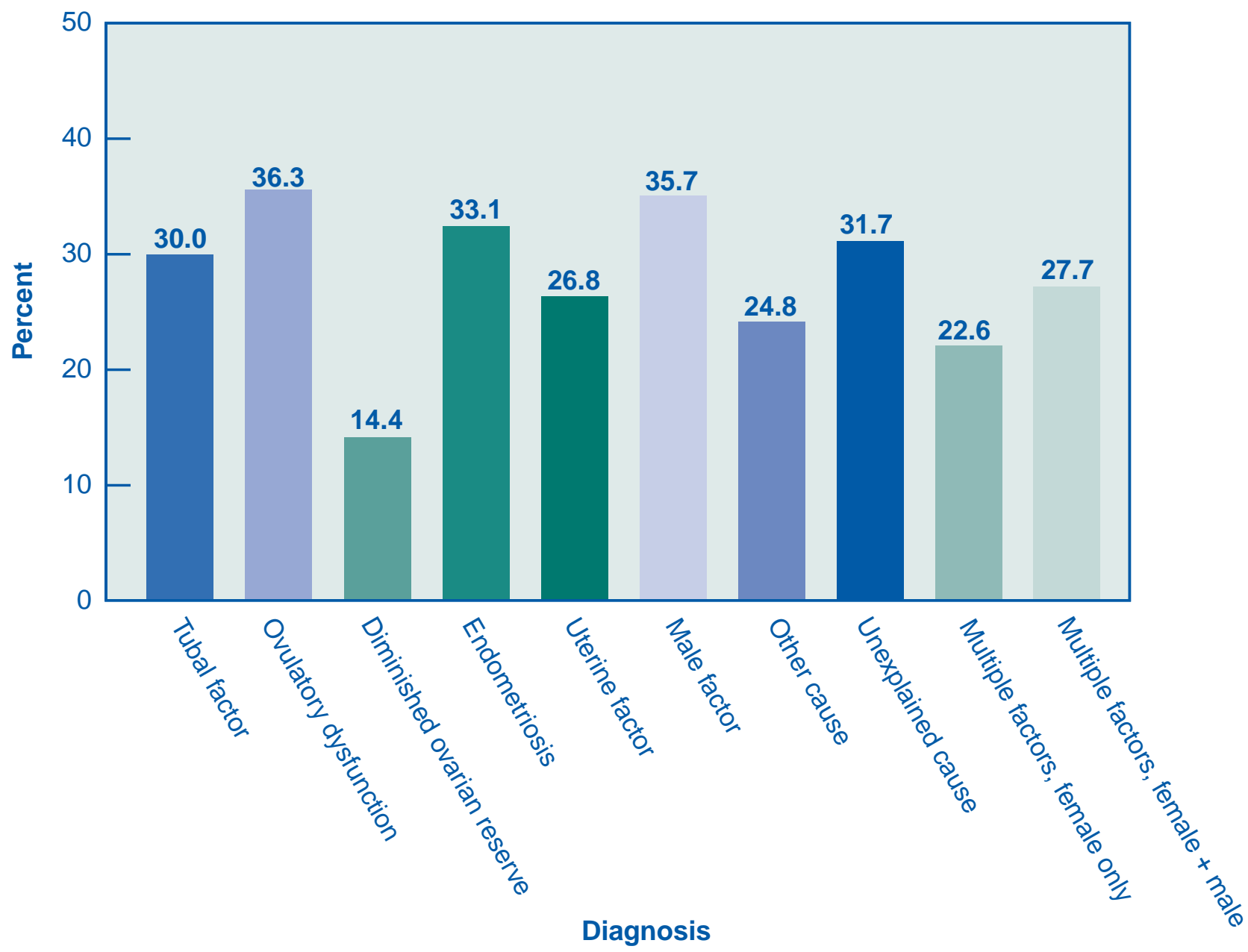




\section{How many women who use ART have previously given birth?}

Figure 21 shows the number of previous births among women who had an ART procedure using fresh nondonor eggs or embryos in 2006. Most of these women (71\%) had no previous births, although they may have had a pregnancy that resulted in a miscarriage or an induced abortion. Twenty-one percent of women using ART in 2006 reported one previous birth, and about 8\% reported two or more previous births. However, we do not have information about how many of these were ART births and how many were not. These data nonetheless point out that women who have previously had children can still face infertility problems.

\section{Figure 21}

Number of Previous Births Among Women Who Had ART Cycles Using Fresh Nondonor Eggs or Embryos, 2006

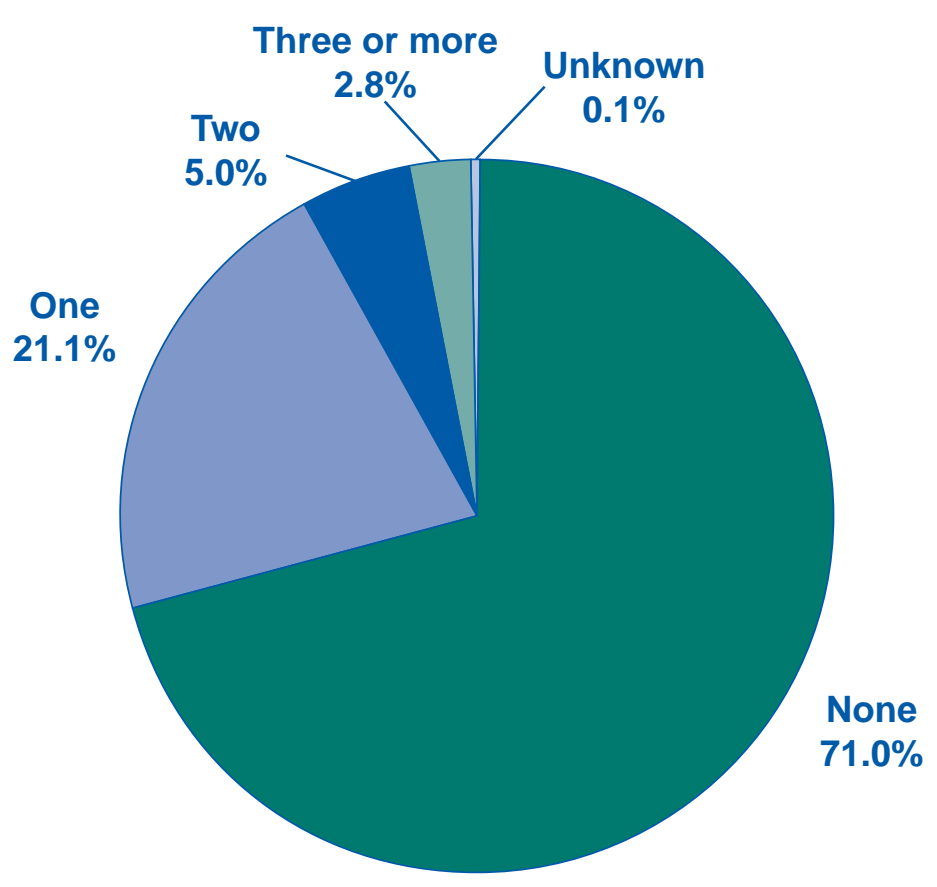




\section{Do women who have previously given birth have higher ART success rates?}

Figure 22 shows the relationship between the success of an ART cycle and the woman's history of previous births. Previous live-born infants were conceived naturally in some cases and through ART in others. In all age groups, women who had a previous live birth were more likely to have a successful ART procedure.

\section{Figure 22}

Percentages of ART Cycles Using Fresh Nondonor Eggs or Embryos That

Resulted in Live Births, by Woman's Age and Number of Previous Live Births, 2006

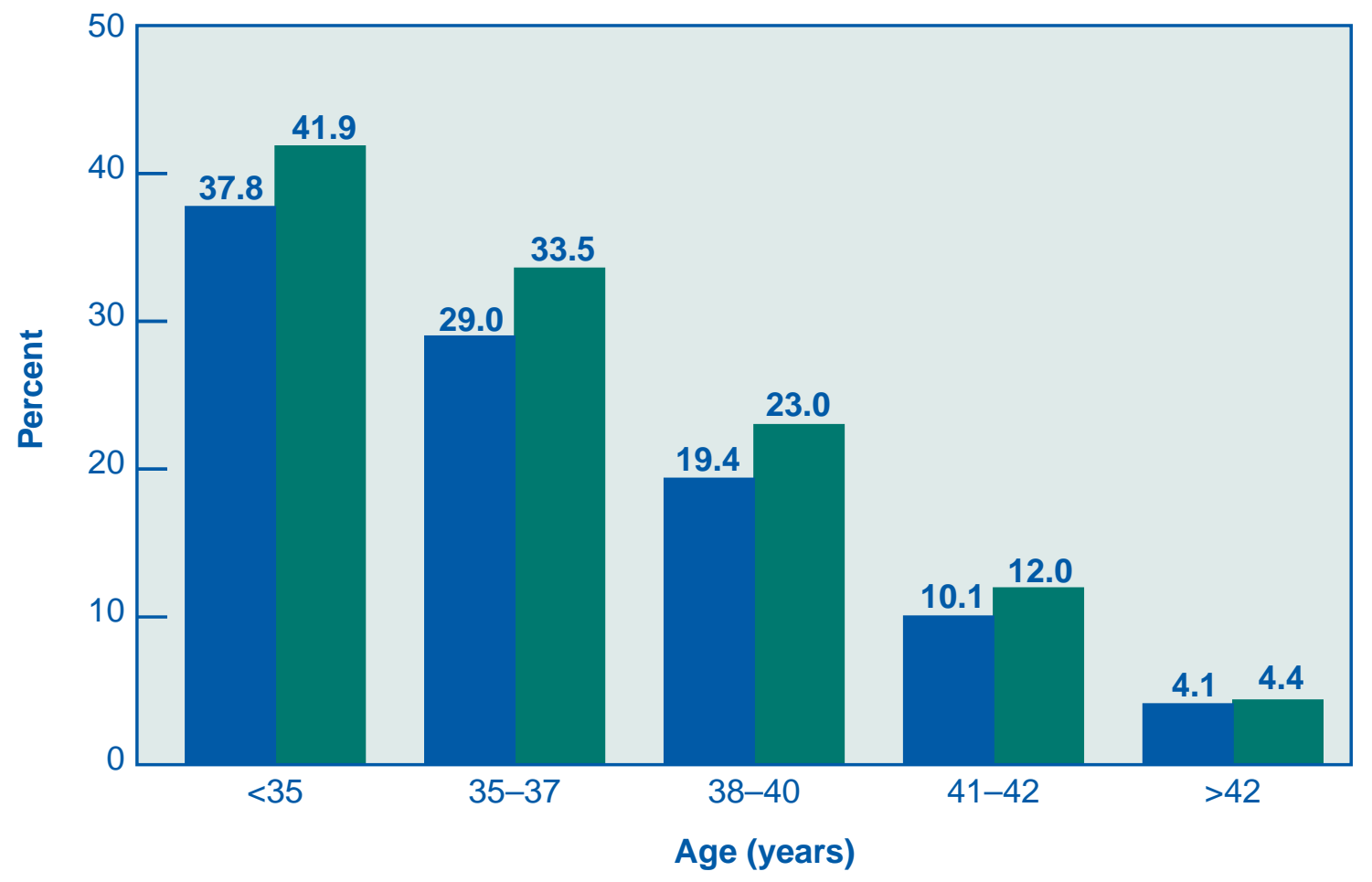




\section{Is there a difference in ART success rates between women with previous miscarriages and women who have never been pregnant?}

In 2006, 70,428 ART cycles were performed among women who had not previously given birth. However, about $27 \%$ of those cycles were reported by women with one or more previous pregnancies that had ended in miscarriage - we do not have information on whether these pregnancies ending in miscarriage were the result of ART or were conceived naturally. Figure 23 shows the relationship between the success of an ART cycle and the history of previous miscarriage. In all age groups, women who had a previous miscarriage were as likely to have a live birth as women who had never been pregnant. Thus, a history of unsuccessful pregnancy does not appear to be associated with lower chances for success during ART.

\section{Figure 23}

Percentages of ART Cycles Using Fresh Nondonor Eggs or Embryos That Resulted in Live Births, by Woman's Age and History of Miscarriage, Among Women with No Previous Births, ${ }^{*} 2006$

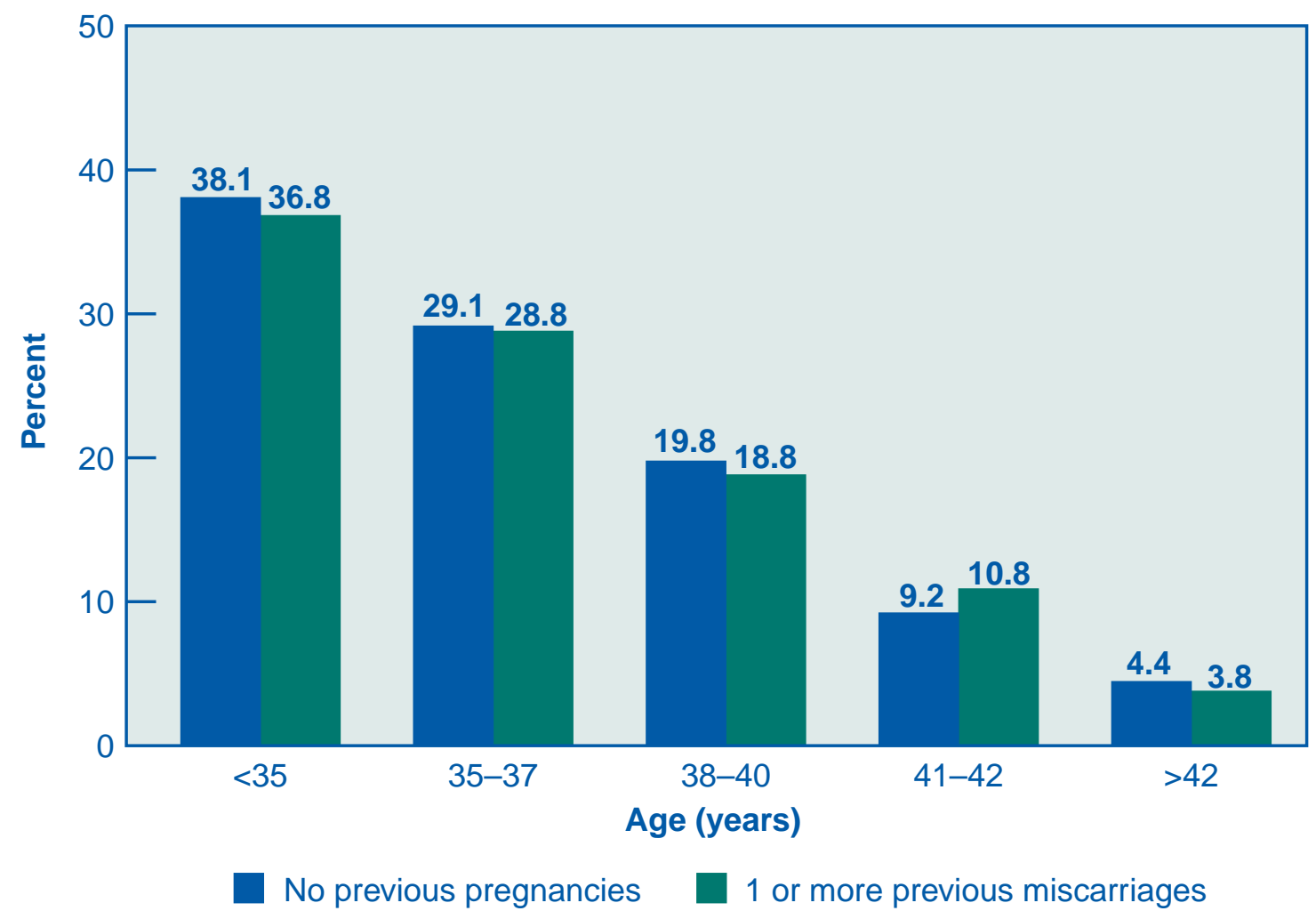

*Women reporting only previous ectopic pregnancies or pregnancies that ended in induced abortion were not included in the above statistics. 


\section{How many current ART users have undergone previous ART cycles?}

Figure 24 presents ART cycles that used fresh nondonor eggs or embryos in 2006 according to whether previous ART cycles had been performed. For about $43 \%$, one or more previous cycles were reported. (This percentage includes previous cycles using either fresh or frozen embryos.) This finding illustrates that it is not uncommon for a couple to undergo multiple ART cycles. We do not have information on when previous cycles were performed, nor do we have information on the outcomes of those previous cycles.

\section{Figure 24}

Number of Previous ART Cycles Among Women Undergoing ART with Fresh Nondonor Eggs or Embryos, ${ }^{*} 2006$

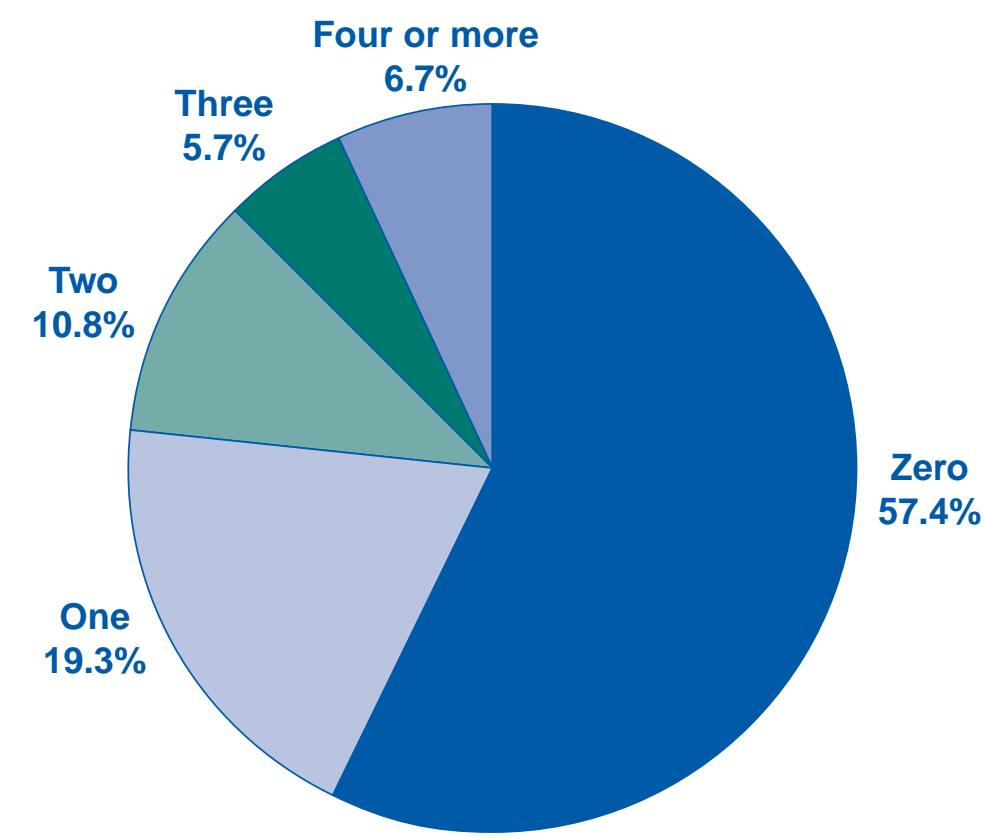

*Total does not equal $100 \%$ due to rounding. 


\section{Are success rates different for women using ART for the first time and women who previously used ART but did not give birth?}

Figure 25 shows the relationship between the success of ART cycles performed in 2006 using fresh nondonor eggs or embryos and a history of previous ART cycles among women with no previous births. In all age groups up to age 42 , success rates were lower for women who had previously undergone an unsuccessful ART cycle.

\section{Figure 25}

Percentages of ART Cycles Using Fresh Nondonor Eggs or Embryos That Resulted in Live Births, by Woman's Age and History of Previous ART Cycles, Among Women with No Previous Births, 2006

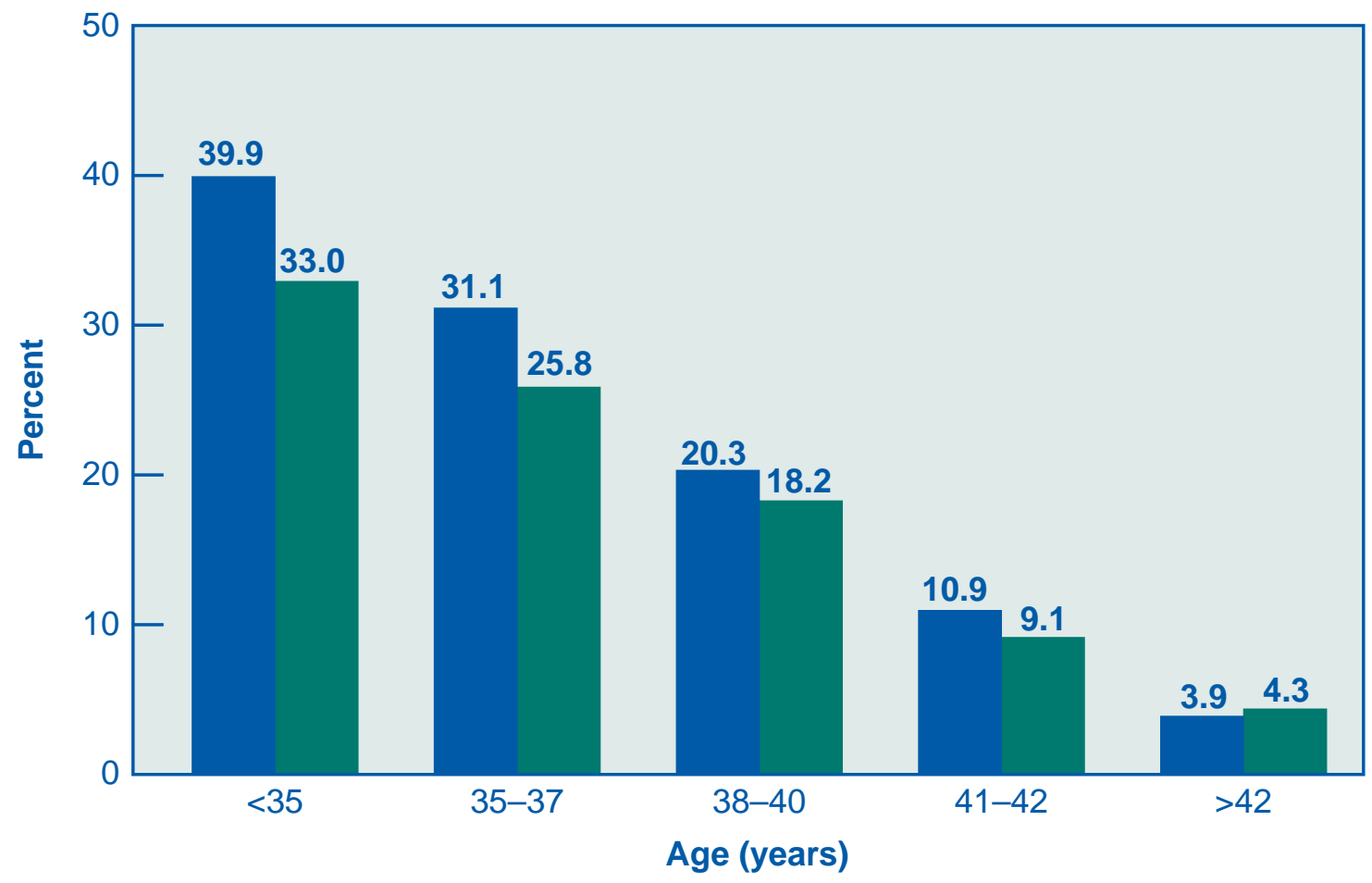

No previous ART and no previous births

1 or more previous ART cycles and no previous births 


\section{What are the success rates for women who have had both previous ART and previous births?}

Figure 26 shows the relationship between the success of ART cycles performed in 2006 using fresh nondonor eggs or embryos and a history of both previous ART cycles and previous births. We do not have information on whether the previous births were the result of ART or were conceived naturally. However, among women with previous births, success rates among women who did not undergo a previous ART procedure were comparable to success rates among women who had undergone previous ART cycles.

Taken together, Figures 25 (see page 37) and 26 show that having undergone previous ART cycles may be related to the success of the current ART cycle. However, it is important to consider the outcomes of previous cycles and whether the woman has given birth in the past.

\section{Figure 26}

Percentages of ART Cycles Using Fresh Nondonor Eggs or Embryos That Resulted in Live Births, by Woman's Age and History of Previous ART Cycles, Among Women with One or More Previous Births, 2006

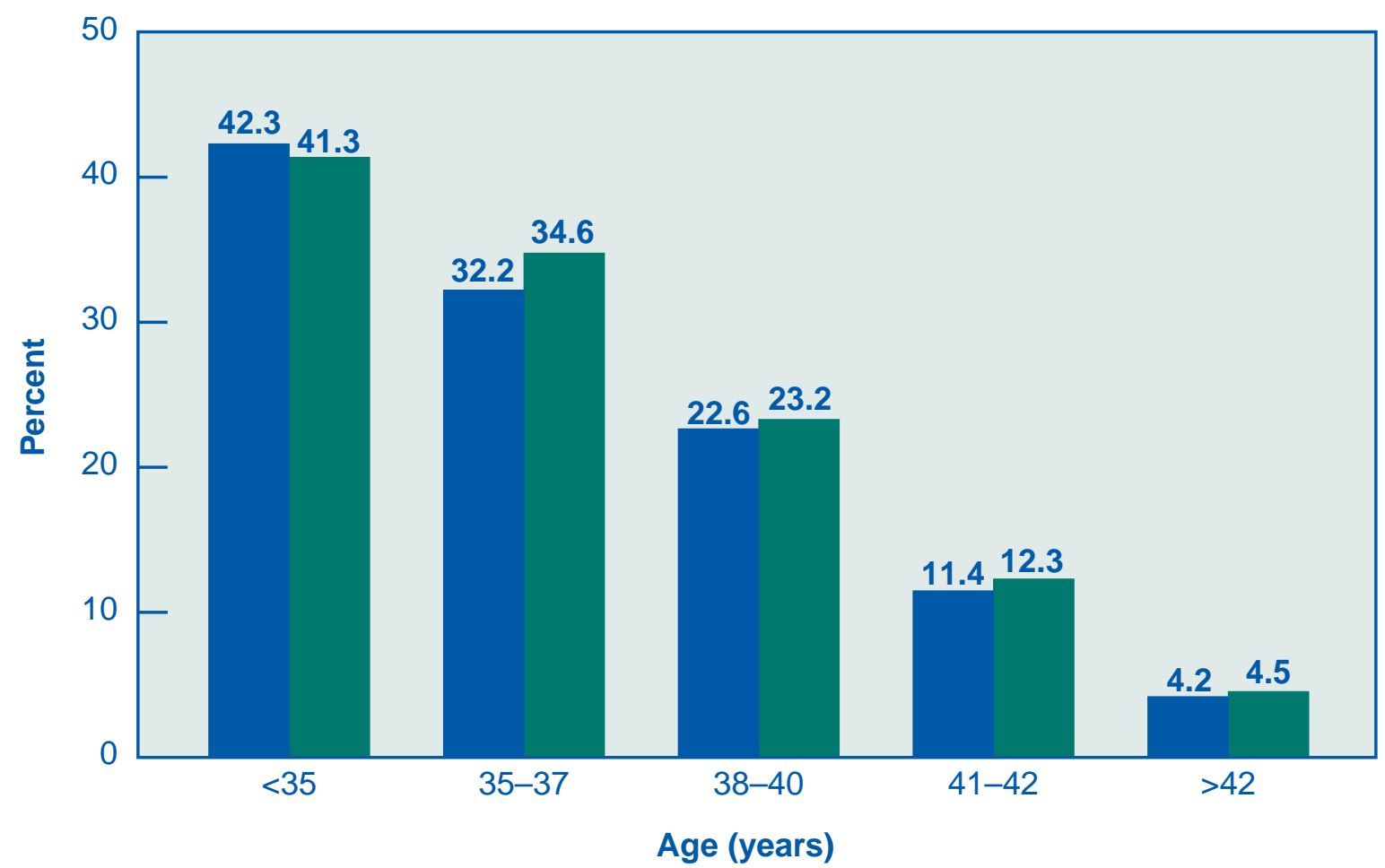

No previous ART and 1 or more previous births

1 or more previous ART cycles and 1 or more previous births 


\section{What were the specific types of ART performed among women who used fresh nondonor eggs or embryos in 2006?}

For about 38\% of ART procedures that used fresh nondonor eggs or embryos in 2006, standard IVF (in vitro fertilization) techniques were used: eggs and sperm were combined in the laboratory, the resulting embryos were cultured for 2 or more days, and one or more embryos were then transferred into the woman's uterus through the cervix.

For most of the remaining ART procedures (62\%), fertilization was accomplished using intracytoplasmic sperm injection (ICSI). This technique involves injecting a single sperm directly into an egg; the embryos are then cultured and transferred as in standard IVF.

For a small proportion of ART procedures, unfertilized eggs and sperm (gametes) or early embryos (zygotes) were transferred into the woman's fallopian tubes. These procedures are known as gamete and zygote intrafallopian transfer (GIFT and ZIFT). Some women with tubal infertility are not suitable candidates for GIFT and ZIFT. GIFT and ZIFT are more invasive procedures than IVF because they involve inserting a laparoscope into a woman's abdomen to transfer the embryos or gametes into the fallopian tubes. In contrast, IVF involves transferring embryos or gametes into a woman's uterus through the cervix without surgery.

\section{Figure 27}

Types of ART Procedures Using Fresh Nondonor Eggs or Embryos, ${ }^{* \dagger} 2006$

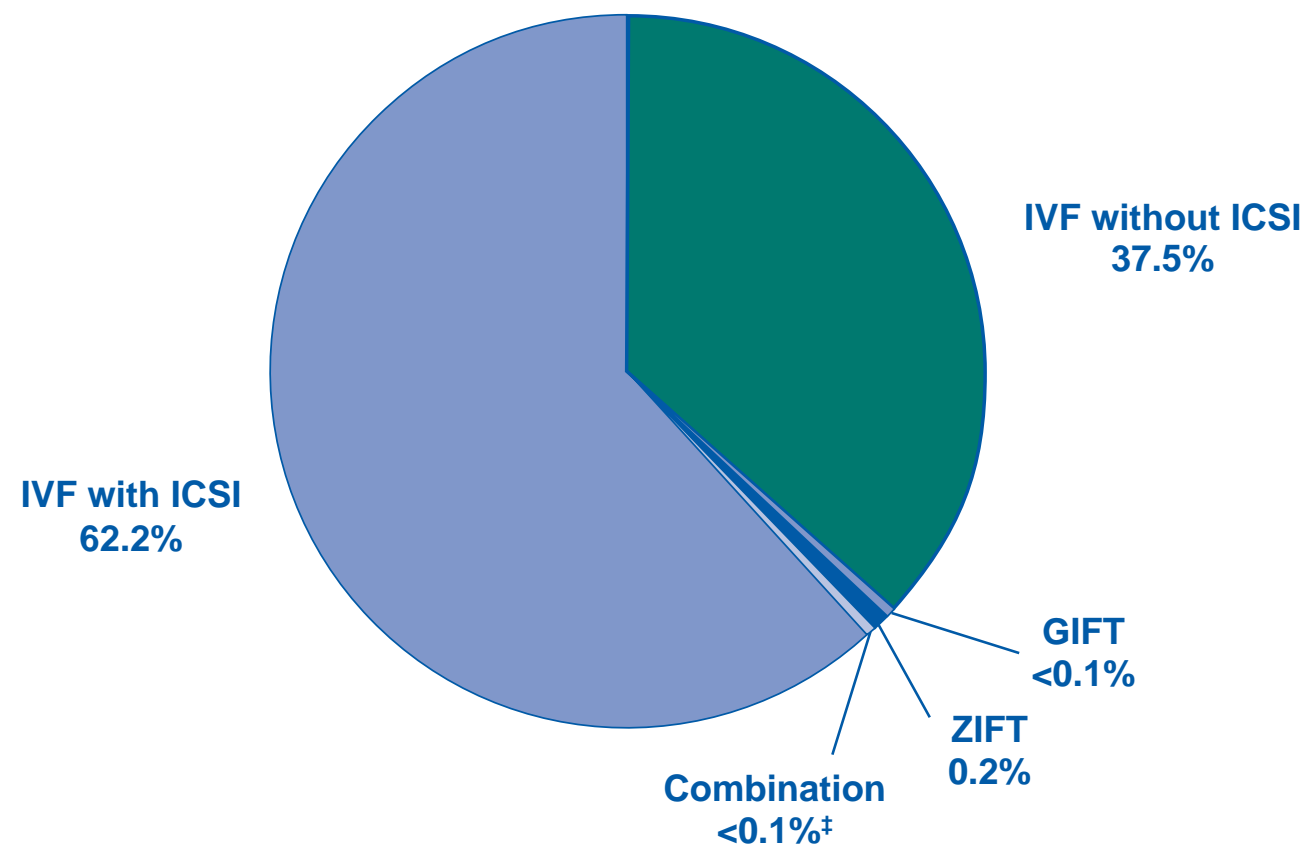

${ }^{*}$ Cycles that were canceled before egg retrieval were classified as IVF, GIFT, or ZIFT based on the intended ART method.

†Total does not equal $100 \%$ due to rounding.

₹ Combination of IVF with or without ICSI and either GIFT or ZIFT. 


\section{What are the success rates for different types of ART procedures?}

Figure 28 shows the percentage of egg retrievals that resulted in a live birth for each type of ART procedure started in 2006. Success rates for the two predominant types of ART, IVF without ICSI and IVF with ICSI, were similar. The success rates for cycles that used GIFT, ZIFT, or a combination of IVF were much lower than for cycles that used other ART procedures. See Figures 29-31 (pages 41-43) and Figures 50-55 (pages 62-67) for further details on IVF procedures that used ICSI.

\section{Figure 28}

Percentages of Egg Retrievals That Resulted in Live Births, by Type of ART Procedure, 2006

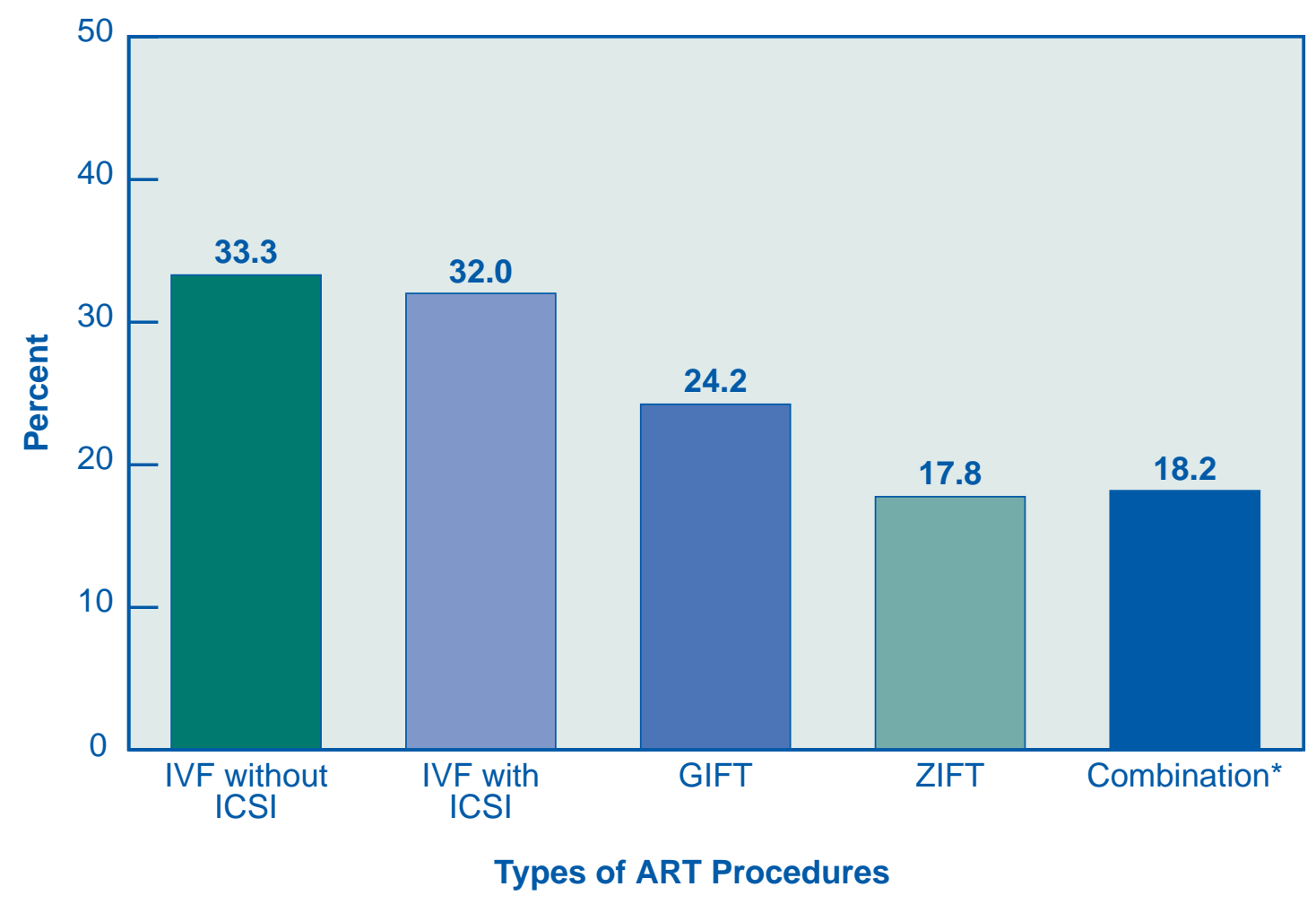

* Combination of IVF with or without ICSI and either GIFT or ZIFT. 


\section{Is ICSI used only for couples diagnosed with male factor infertility?}

ICSI was developed to overcome problems with fertilization that sometimes occur in couples diagnosed with male factor infertility. In 2006, 61,722 ICSI cycles were performed. Approximately half of the ICSI cycles were performed for couples with a diagnosis of male factor infertility. However, diagnostic procedures may vary from one clinic to another, so the categorization of causes of infertility may also vary.

\section{Figure 29}

Use of ICSI* in Fresh-Nondonor Cycles Among Couples With and Without Diagnoses of Male Factor Infertility, ${ }^{\dagger} 2006$

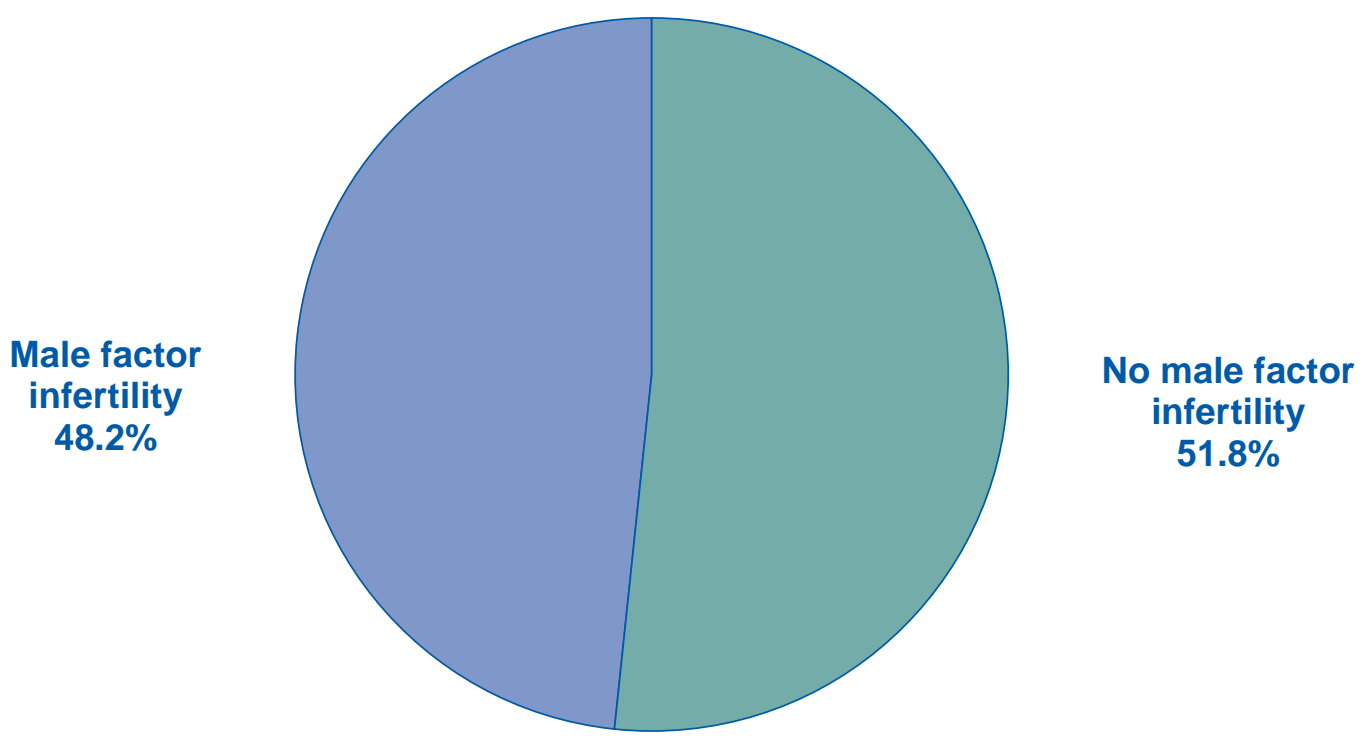

* Intracytoplasmic sperm injection.

† Based on 61,722 cycles that used IVF with ICSI. 


\section{What are the success rates for couples with male factor infertility when ICSI is used?}

ICSI was developed to overcome problems with fertilization that sometimes occur among couples diagnosed with male factor infertility. In $2006,82 \%$ of couples diagnosed with male factor infertility used IVF with ICSI. Figure 30 presents the success rates for these ICSI procedures among couples diagnosed with male factor infertility. For comparison, these rates are presented alongside the success rates for ART cycles that used standard IVF without ICSI. This standard IVF comparison group includes couples with all diagnoses except male factor. Because ICSI can be performed only when at least one egg has been retrieved, the percentage of egg retrievals that resulted in live births are presented.

In every age group, success rates for the IVF with ICSI group were similar to the success rates for the groups that used standard IVF without ICSI. These results show that when ICSI was used for couples diagnosed with male factor infertility, their success rates were close to those achieved by couples who were not diagnosed with male factor infertility. Please note, however, that review of select clinical records revealed that reporting of infertility causes may be incomplete. Therefore, differences in success rates by causes of infertility should be interpreted with caution. (See Findings from Validation Visits for 2006 ART Data in Appendix A for additional information.)

\section{Figure 30}

Percentages of Retrievals That Resulted in Live Births Among Couples

Diagnosed with Male Factor Infertility Who Used IVF with ICSI, ${ }^{\star}$ Compared with Couples Not Diagnosed with Male Factor Infertility Who Used IVF Without ICSI, by Woman's Age, ${ }^{\dagger} 2006$

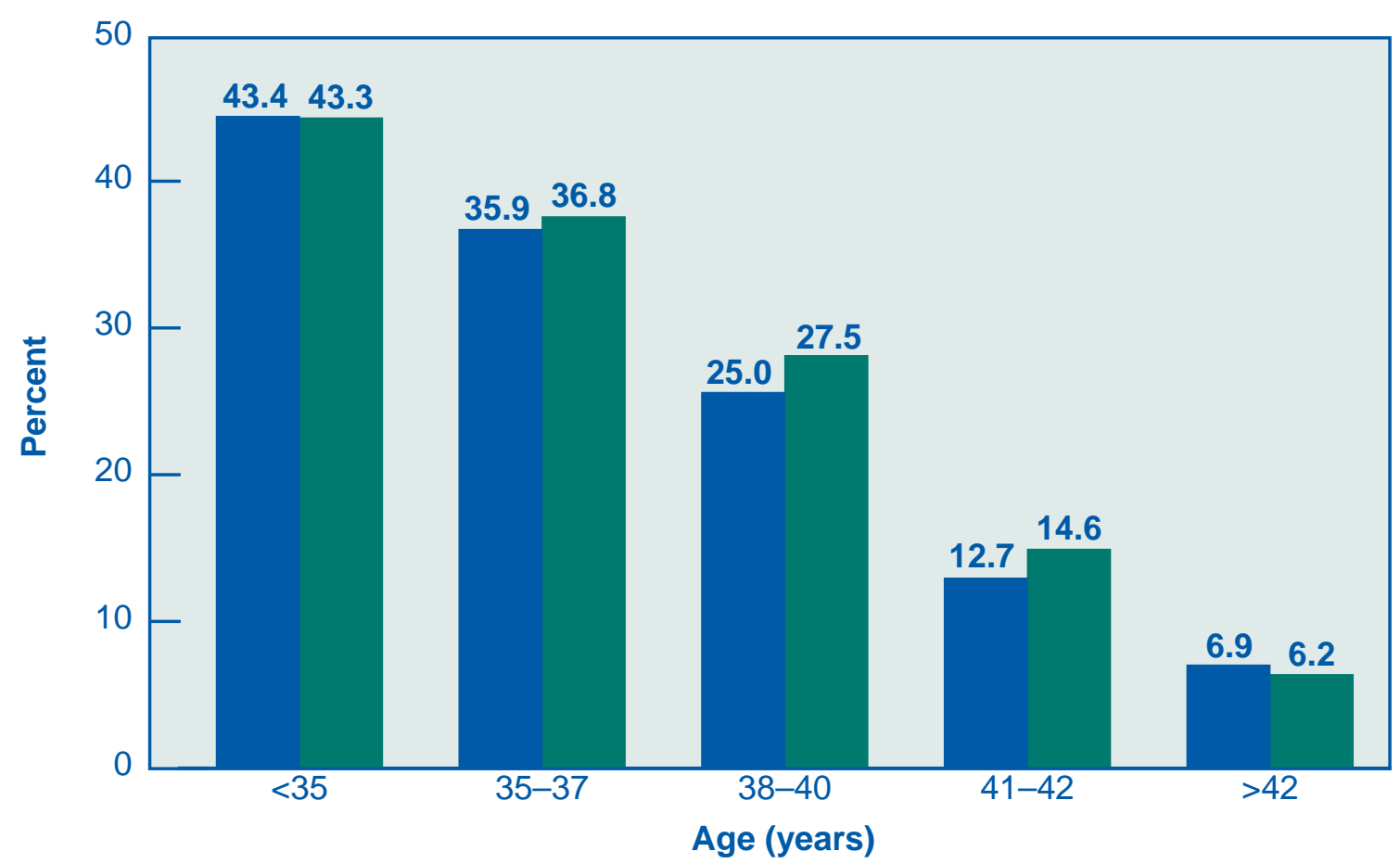

IVF with ICSI among couples with male factor infertility

IVF without ICSI among couples with no male factor infertility

* Intracytoplasmic sperm injection.

† Cycles using donor sperm and cycles using GIFT or ZIFT are excluded. 


\section{What are the success rates for couples without a diagnosis of male factor infertility when ICSI is used?}

As shown in Figure 29 (page 41), a large number of ICSI procedures are now performed even when couples are not diagnosed with male factor infertility. Figure 31 presents percentages of egg retrievals that resulted in live births for those cycles compared with ART cycles among couples who used IVF without ICSI. For every age group, the ICSI procedures were less successful. Please note, however, that review of select clinical records revealed that reporting of infertility causes may be incomplete.

Therefore, differences in success rates by causes of infertility should be interpreted with caution. (See Findings from Validation Visits for 2006 ART Data in Appendix A for additional information.)

Additionally, information was not available to completely determine whether this finding was directly related to the ICSI procedure or whether the patients who used ICSI were somehow different from those who use IVF alone. However, separate evaluation of various groups of patients with an indication of being difficult to treat revealed a pattern of results consistent with those presented below. These difficult-to-treat groups included couples with previous failed ART cycles, couples diagnosed with diminished ovarian reserve, and couples with a low number of eggs retrieved (fewer than five). Within each of these groups, ART cycles that used IVF with ICSI had lower success rates compared with cycles that used IVF without ICSI.

\section{Figure 31}

Percentages of Retrievals That Resulted in Live Births Among Couples Not Diagnosed with Male Factor Infertility, by Use of ICSI* and Woman's Age, ${ }^{\dagger} 2006$

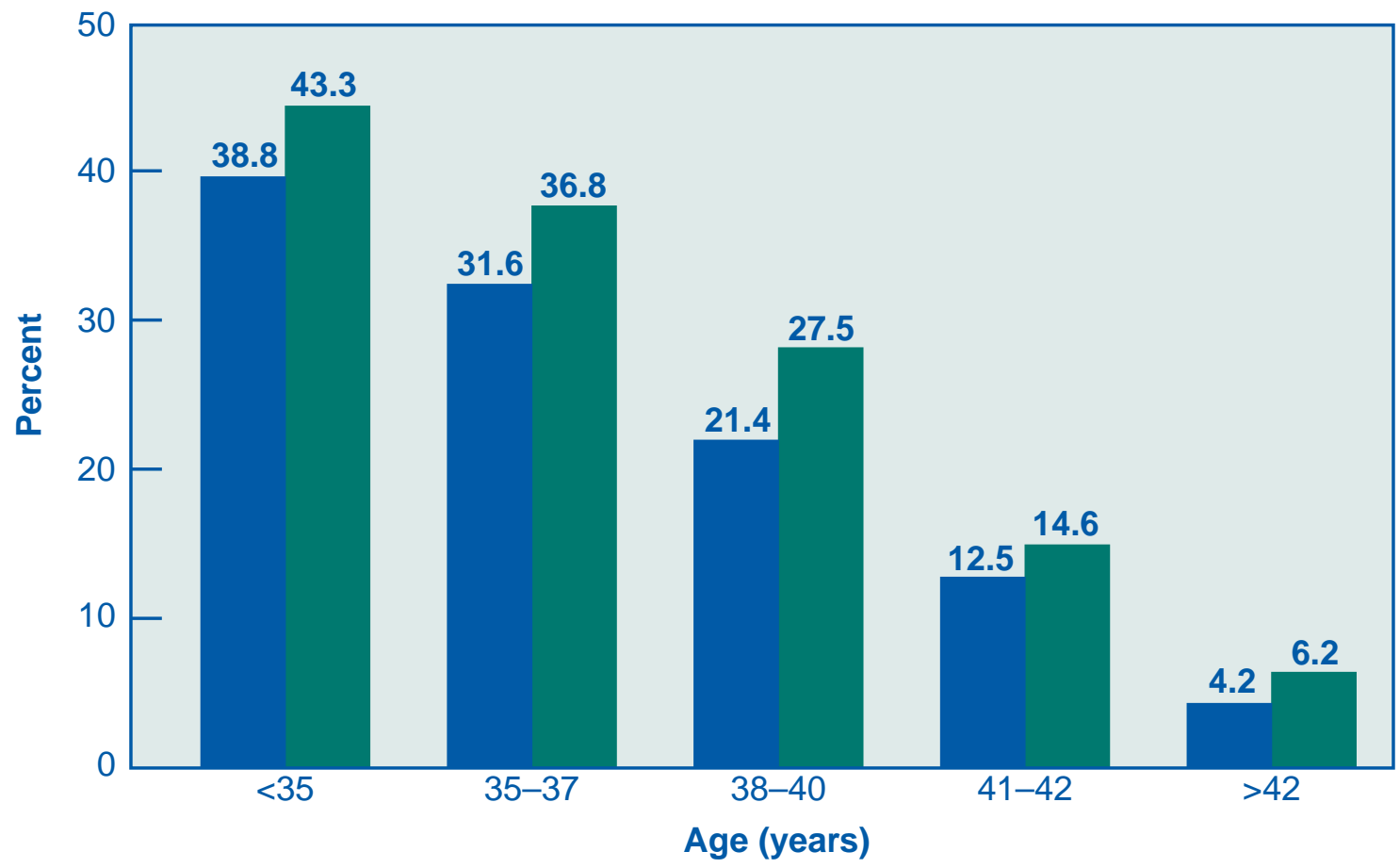

IVF with ICSI among couples with no male factor infertility

IVF without ICSI among couples with no male factor infertility

* Intracytoplasmic sperm injection.

† Cycles using donor sperm and cycles using GIFT or ZIFT are excluded. 


\section{How many embryos are transferred in an ART procedure?}

Figure 32 shows that approximately $43 \%$ of ART cycles that used fresh nondonor eggs or embryos and progressed to the embryo transfer stage in 2006 involved the transfer of three or more embryos, about $16 \%$ of cycles involved the transfer of four or more, and approximately $5 \%$ of cycles involved the transfer of five or more embryos.

\section{Figure 32}

Number of Embryos Transferred During ART Cycles

Using Fresh Nondonor Eggs or Embryos, ${ }^{*} 2006$

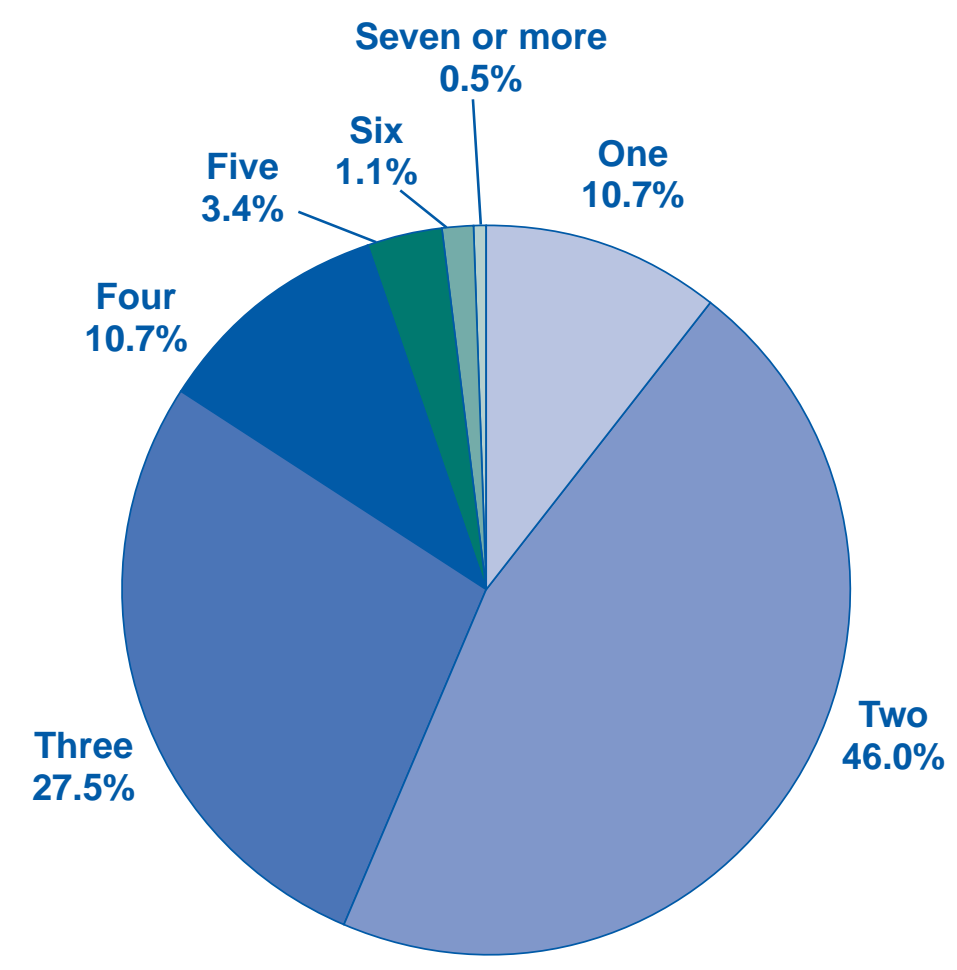

* Total does not equal $100 \%$ due to rounding. 


\section{In general, is an ART cycle more likely to be successful if more embryos are transferred?}

Figure 33 shows the relationship between the number of embryos transferred during an ART procedure in 2006 and the number of infants born alive as a result of that procedure. The success rate increased when two or more embryos were transferred; however, transferring multiple embryos also poses a risk of having a multiple-infant birth. Multiple-infant births cause concern because of the additional health risks they create for both mothers and infants. Also, pregnancies with multiple fetuses are potentially subject to multifetal reduction. Multifetal reduction can happen naturally (e.g., fetal death), or a woman or couple may decide to reduce the number of fetuses using a procedure called multifetal pregnancy reduction. Information on multifetal pregnancy reductions is incomplete and therefore is not provided here.

The relationships between number of embryos transferred, success rates, and multiple-infant births are complicated by several factors, such as the woman's age and embryo quality. See Figure 34 (page 46) for more details on women most at risk for multiple births.

\section{Figure 33}

Percentages of Transfers That Resulted in Live Births and Percentages of Multiple-Infant Live Births for ART Cycles Using Fresh Nondonor Eggs or Embryos, by Number of Embryos Transferred, 2006

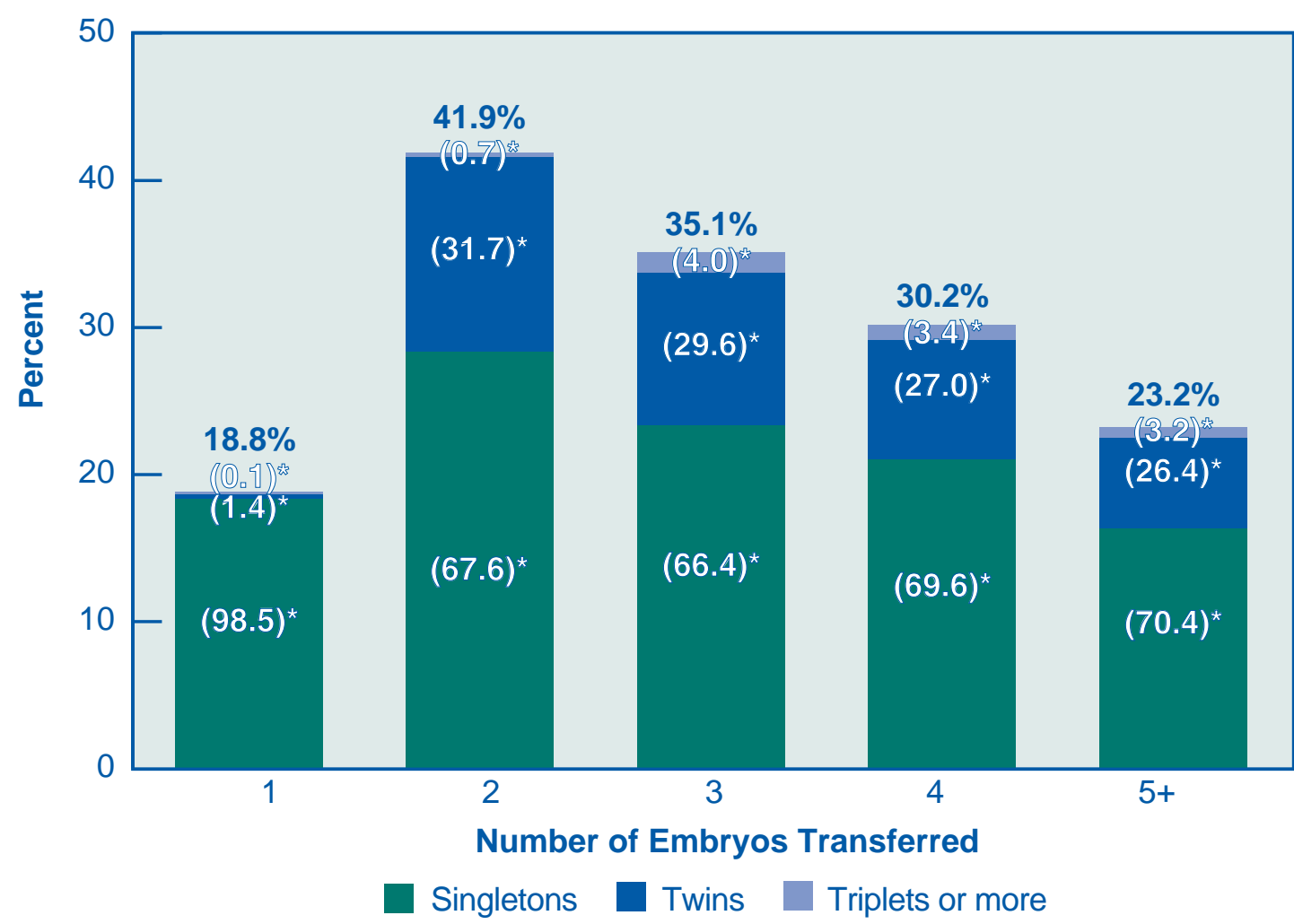

${ }^{*}$ Percentages of live births that were singletons, twins, and triplets or more are in parentheses. Note: In rare cases a single embryo may divide and thus produce twins. For this reason, a small percentage of twins resulted from a single embryo transfer, and a small percentage of triplets resulted when two embryos were transferred. 


\section{Are success rates affected by the number of embryos transferred for women who have more embryos available than they choose to transfer?}

Although, in general, transferring more than one embryo tends to improve the chance for a successful ART procedure (see Figure 33, page 45), other factors are also important. Previous research suggests that the number of embryos fertilized and thus available for ART is just as, if not more, important in predicting success as the number of embryos transferred. Additionally, younger women tend to have both higher success rates and higher likelihood of multiple-infant births. Figure 34 shows the relationship between the number of embryos transferred, success rates, and multiple-infant births for a subset of ART procedures in which the woman was younger than 35 and the couple chose to set aside some embryos for future cycles rather than transfer all available embryos at one time.

For this group, the chance for a live birth using ART was about $49 \%$ when only one embryo was transferred. If one measures success as the percentage of transfers resulting in singleton live births, the highest likelihood of live birth was observed with only one embryo transferred.

The proportion of live births that were multiple-infant births was about 39\% with two embryos and about $46 \%$ with three embryos. Transferring three or more embryos also created an additional risk for higher-order multiple births (i.e., triplets or more).

\section{Figure 34}

Percentages of Transfers That Resulted in Live Births and Percentages of Multiple-Infant Live Births for ART Cycles in Women Who Were Younger Than 35, Used Fresh Nondonor Eggs or Embryos, and Set Aside Extra Embryos for Future Use, by Number of Embryos Transferred, 2006

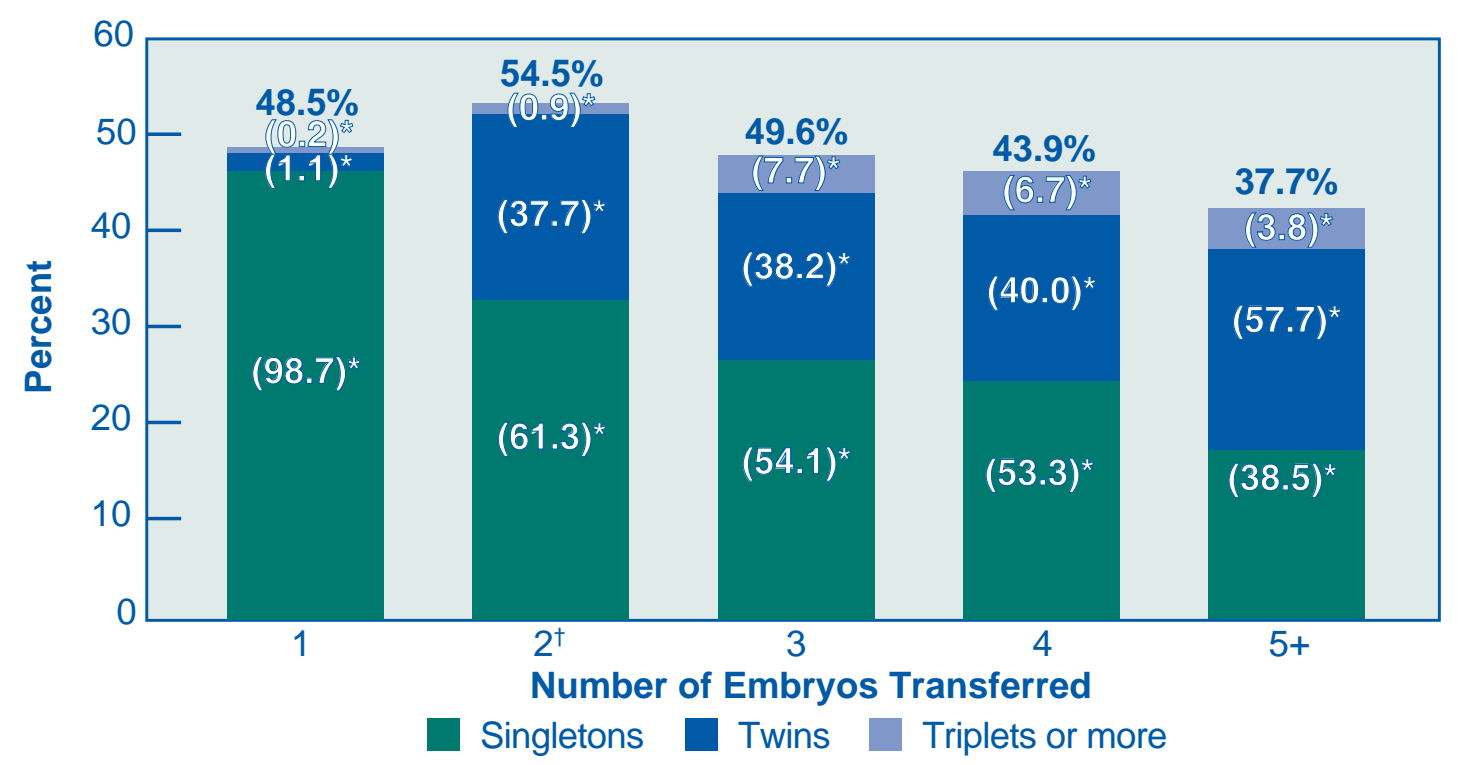

* Percentages of live births that were singleton, twins, and triplets or more are in parentheses.

Note: In rare cases a single embryo may divide and thus produce twins. For this reason, a small percentage of twins resulted from a single embryo transfer, and a small percentage of triplets resulted when two embryos were transferred.

† Total does not equal $100 \%$ due to rounding. 


\section{How long after egg retrieval does embryo transfer occur?}

Once an ART cycle has progressed from egg retrieval to fertilization, the embryo(s) can be transferred into the woman's uterus in the subsequent 1 to 6 days. Figure 35 shows that in 2006 approximately $64 \%$ of embryo transfers occurred on day 3. Day 5 embryo transfers were the next most common, accounting for about $27 \%$ of ART procedures that progressed to the embryo transfer stage.

\section{Figure 35}

Day of Embryo Transfer* Among ART Cycles Using

Fresh Nondonor Eggs or Embryos, ${ }^{\dagger} 2006$

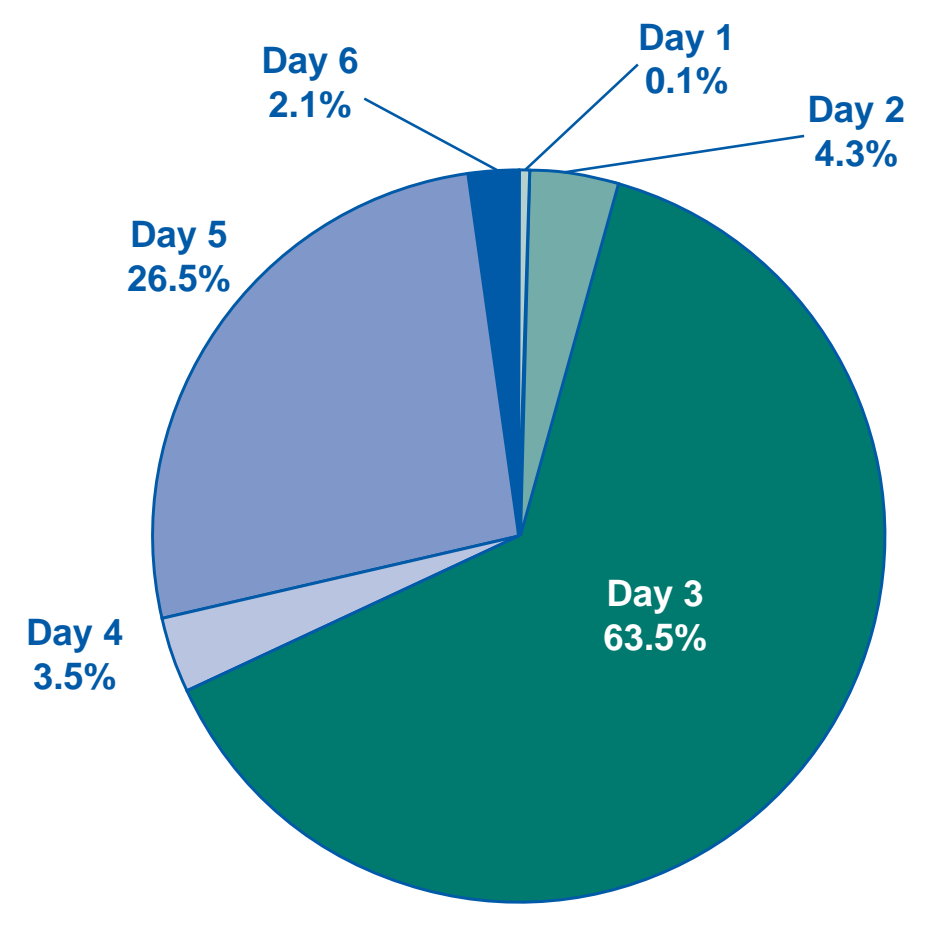

* Number of days following egg retrieval.

+ Cycles using GIFT or ZIFT are excluded. Missing or implausible values for day of embryo transfer (i.e., 0 or $>6$ ) are not included. 


\section{In general, is an ART cycle more likely to be successful if embryos are transferred on day 5 ?}

As shown in Figure 35 (page 47), in the vast majority of ART procedures, embryos were transferred on day $3(64 \%)$ or day 5 (27\%). Figure 36 compares success rates for day 3 embryo transfers with those for day 5 embryo transfers. In all age groups, the success rates were higher for day 5 embryo transfers than for day 3 transfers. However, some cycles do not progress to the embryo transfer stage because of embryo arrest (interruption in embryo development) between day 3 and day 5 . These cycles are not accounted for in the success rates for day 5 transfers. Therefore, differences in success rates for day 3 and day 5 transfers should be interpreted with caution.

\section{Figure 36}

Percentages of Day 3 and Day 5 Embryo Transfers (Using Fresh Nondonor Eggs or Embryos) That Resulted in Live Births, by Woman's Age, ${ }^{*} 2006$

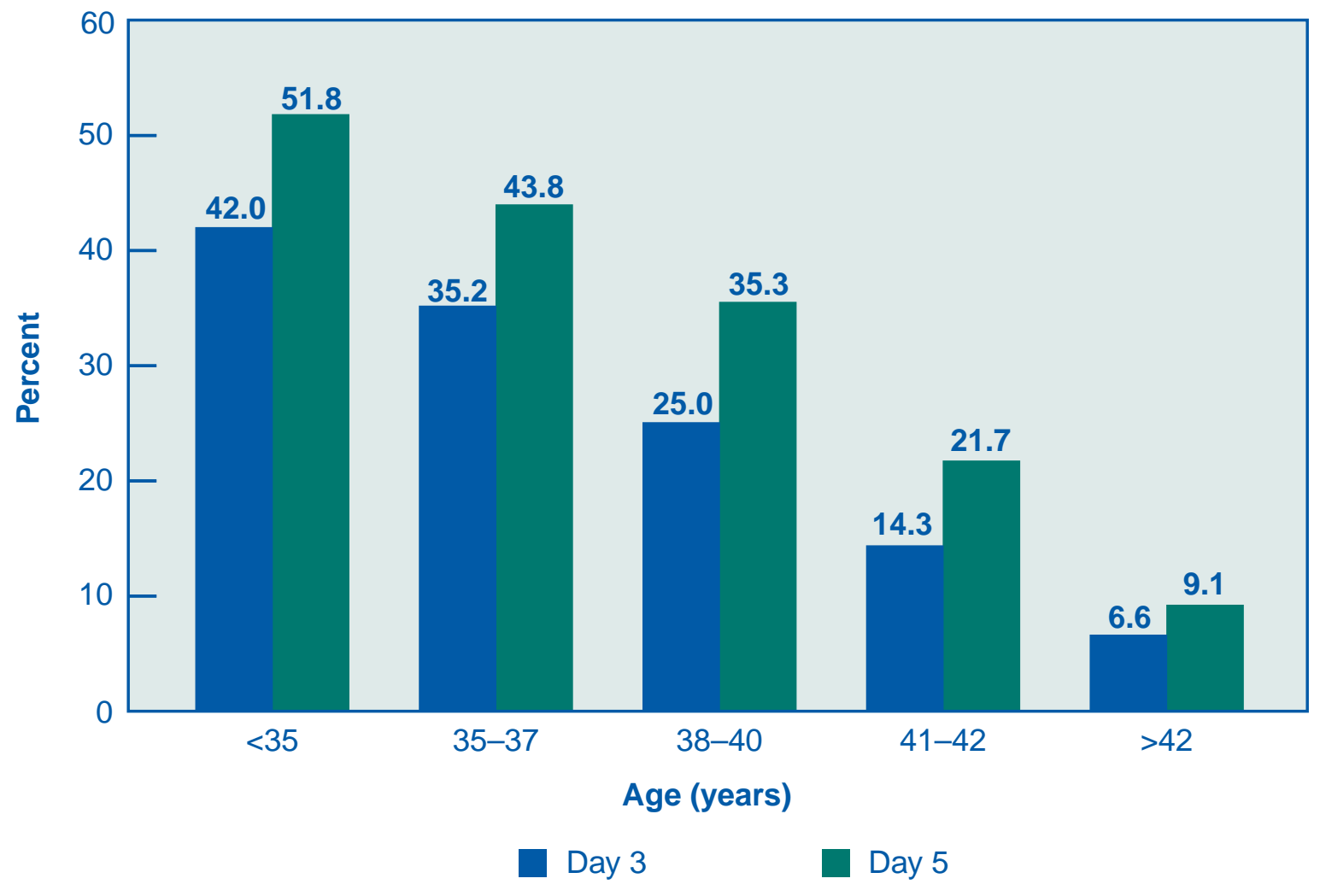

* Cycles using GIFT or ZIFT are excluded. This comparison is limited to transfers on day 3 and day 5. Embryo transfers performed on days 1, 2, 4, and 6 are not included because each of these accounted for a small proportion of procedures. 


\section{Does the number of embryos transferred differ for day 3 and day 5 embryo transfers?}

Figure 37 shows the number of embryos transferred on day 3 and day 5 . Overall, fewer embryos were transferred on day 5 than on day 3. Approximately $53 \%$ of day 3 embryo transfers and $21 \%$ of day 5 embryo transfers involved the transfer of three or more embryos. The decrease in the number of embryos transferred on day 5, however, did not translate into a lower risk for multiple-infant births. See Figure 38 (page 50) for more details on the relationship between multiple-infant birth risk and day of embryo transfer.

\section{Figure 37}

Number of Embryos Transferred During ART Cycles Using

Fresh Nondonor Eggs or Embryos for Day 3 and Day 5 Embryo Transfers, ${ }^{*} 2006$

Day $3^{\dagger}$

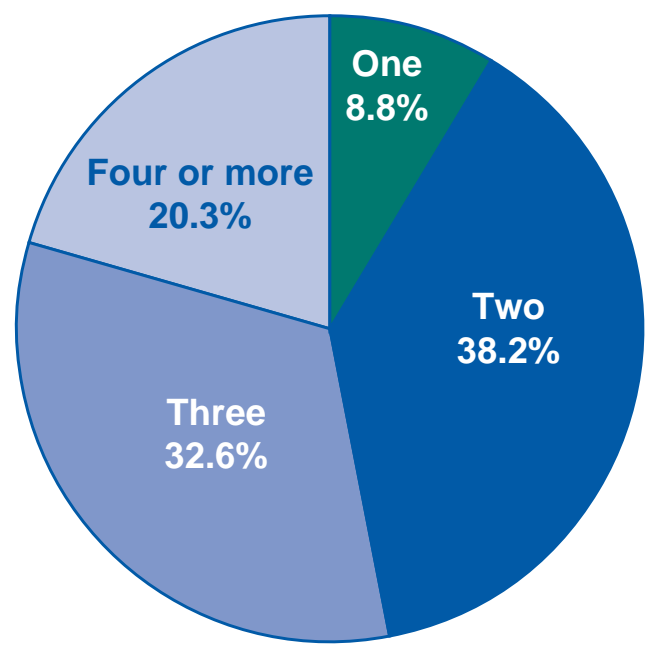

Day 5

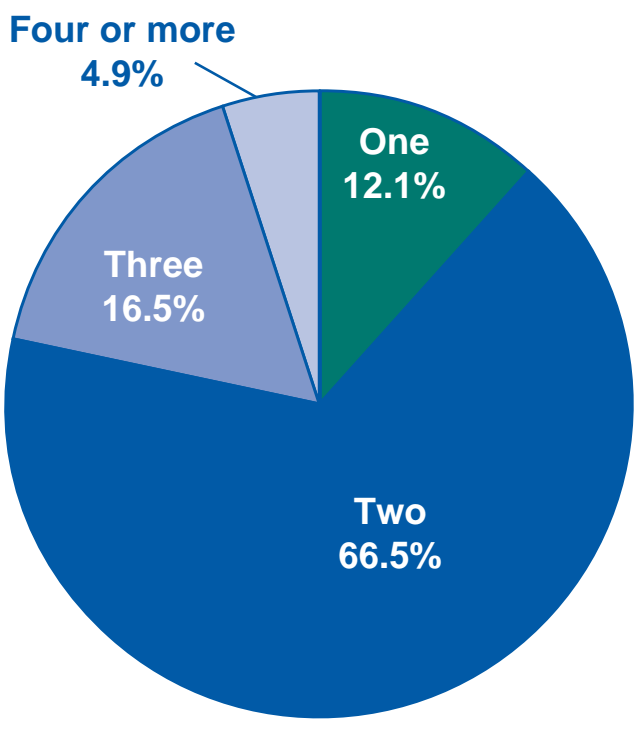

* Cycles using GIFT or ZIFT are excluded. This comparison is limited to transfers on day 3 and day 5. Embryo transfers performed on days 1, 2, 4, and 6 are not included because each of these accounted for a small proportion of procedures.

† Total does not equal $100 \%$ due to rounding. 


\section{In general, how does the multiple-infant birth risk vary by the day of embryo transfer?}

Multiple-infant births are associated with greater problems for both mothers and infants, including higher rates of caesarean section, prematurity, low birth weight, and infant disability or death.

Part A of Figure 38 shows that among the 16,519 live births that occurred following day 3 embryo transfer, $71 \%$ were singletons, $27 \%$ were twins, and about $2 \%$ were triplets or more. Thus, approximately $29 \%$ of these live births produced more than one infant.

In 2006, 9,567 live births occurred following day 5 embryo transfer. Part B of Figure 38 shows that 35\% of these live births produced more than one infant (approximately 33\% twins and $2 \%$ triplets or more).

As shown in Figure 37 (page 49), fewer embryos were transferred on day 5 than on day 3. While the reduction in the number of embryos transferred on day 5 was associated with a decrease in triplet-or-more births, it also was associated with an increase in twin births. Thus, the risk of having a multiple-infant birth was higher for day 5 embryo transfers. The likelihood of multiple-infant births for both day 3 and day 5 embryo transfers is much higher overall than for multiple-infant births in the general U.S. population (about 3\%).

\section{Figure 38}

Risks of Having Multiple-Infant Live Birth for ART Cycles Using Fresh Nondonor Eggs or Embryos for Day 3 and Day 5 Embryo Transfers, ${ }^{\star} 2006$

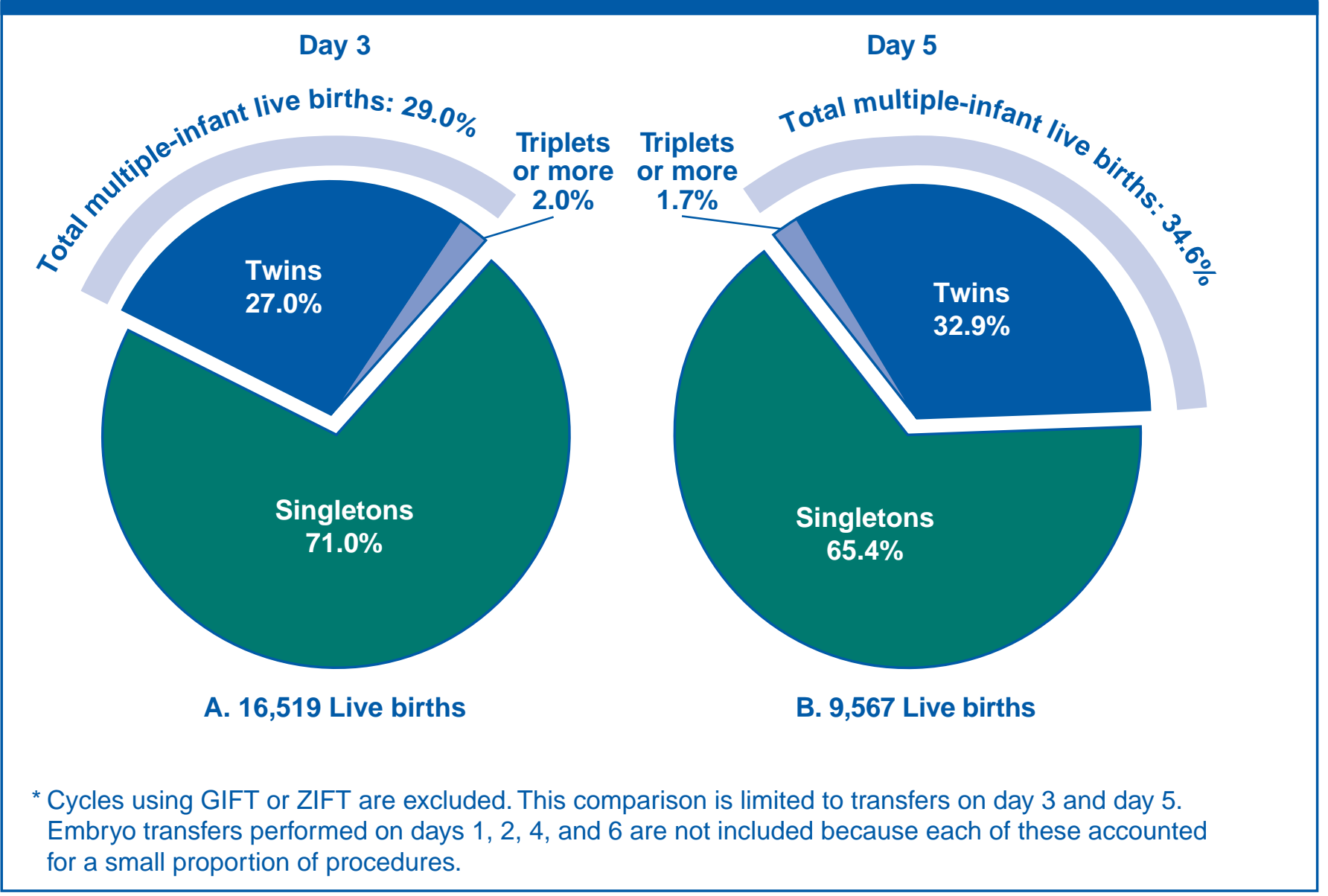




\section{For day 5 embryo transfers, are success rates affected by the number of embryos transferred for women who have more embryos available than they choose to transfer?}

As shown in Figure 37 (page 49), embryos transferred on day 5 result in more multiple-infant births compared with embryos transferred on day 3, despite the smaller number of embryos transferred on day 5. Figure 39 shows the relationship between the number of embryos transferred, the percentage of transfers resulting in live births, and the percentage of multiple-infant births for day 5 embryo transfer procedures in which the woman was younger than 35 and the couple decided to set aside some embryos for future cycles rather than transfer all available embryos at one time.

The percentage of transfers resulting in live births was 53\% when only one embryo was transferred on day 5 . The percentage of transfers resulting in live births was higher (59\%) when two embryos were transferred; however, the proportion of live births that were multiples (twins or more) - which presents a higher risk for poor health outcomes - was 44\%. The chance for a live birth was lower (50\%) when 3 or more embryos were transferred on day 5 , and the percentage of live births that were higher-order multiples (triplets or more) was much higher for these transfers (10\%) than for those involving the transfer of just two embryos on day 5 (1.4\%).

If one measures success as the percentage of transfers resulting in singleton live births, the highest rate (53\%) was observed with the transfer of a single embryo on day 5.

\section{Figure 39}

Percentages of Transfers That Resulted in Live Births and Percentages of

Multiple-Infant Live Births for Day 5 Embryo Transfers Among Women Who Were Younger Than 35, Used Fresh Nondonor Eggs or Embryos, and Set Aside Extra Embryos for Future Use, by Number of Embryos Transferred, 2006

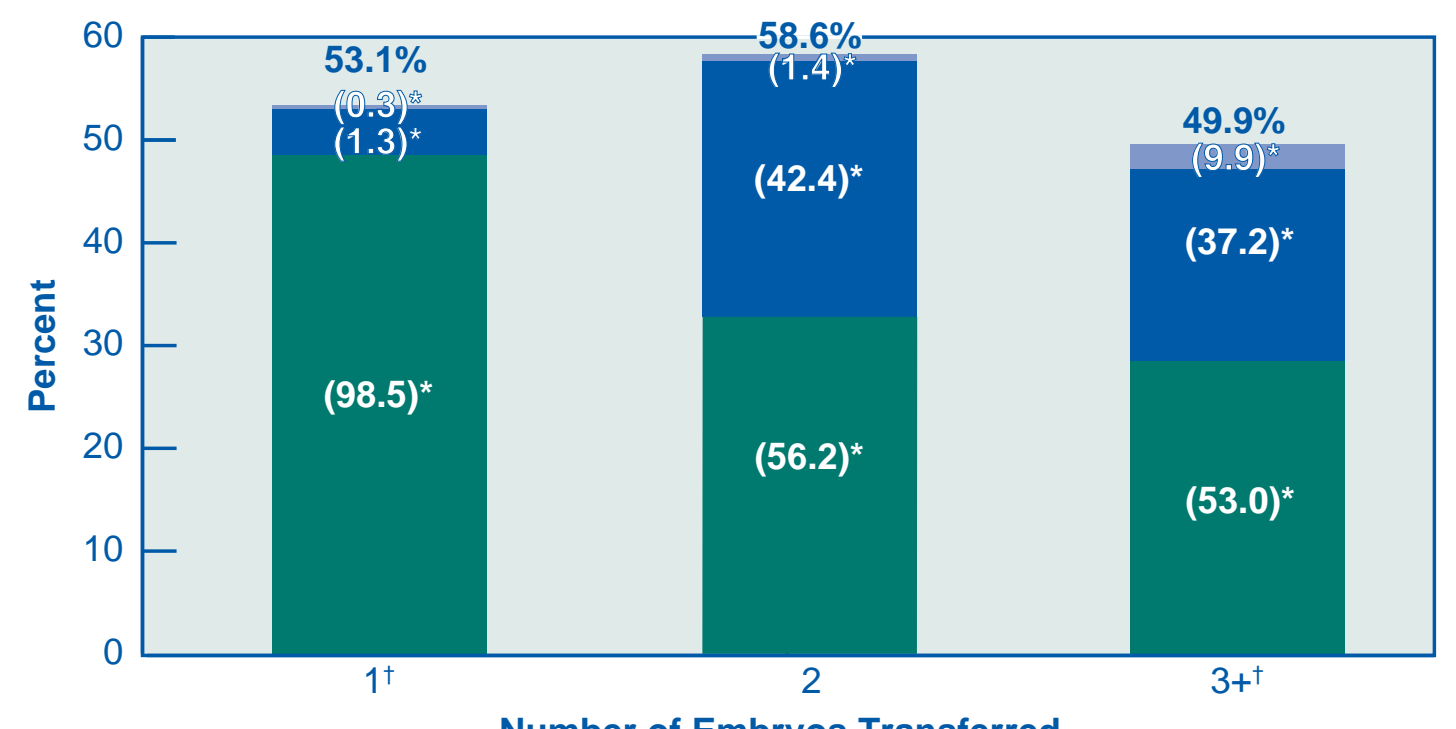

Number of Embryos Transferred

\section{Singletons $\square$ Twins $\square$ Triplets or more}

*Percentages of live births that were singletons, twins, and triplets or more are in parentheses.

Note: In rare cases a single embryo may divide and thus produce twins. For this reason a small percentage of twins resulted from a single embryo transfer and a small percentage of triplets resulted when two embryos were transferred.

†Totals do not equal $100 \%$ due to rounding. 


\section{What are the success rates for women who use gestational carriers?}

In some cases a woman has trouble carrying a pregnancy. In such cases the couple may use ART with a gestational carrier, sometimes called a surrogate. A gestational carrier is a woman who agrees to carry the developing embryo for a couple with infertility problems. Gestational carriers were used in $1 \%$ of ART cycles using fresh nondonor embryos in 2006 (1,042 cycles). Figure 40 compares success rates per transfer for ART cycles that used a gestational carrier in 2006 with cycles that did not. In all age groups, success rates for ART cycles that used gestational carriers were higher than success rates for those cycles that did not.

\section{Figure 40}

Comparison of Percentages of Transfers That Resulted in Live Births Between Cycles That Used Gestational Carriers and Those That Did Not (Both Using Fresh Nondonor Embryos), by ART Patient's Age, ${ }^{*} 2006$

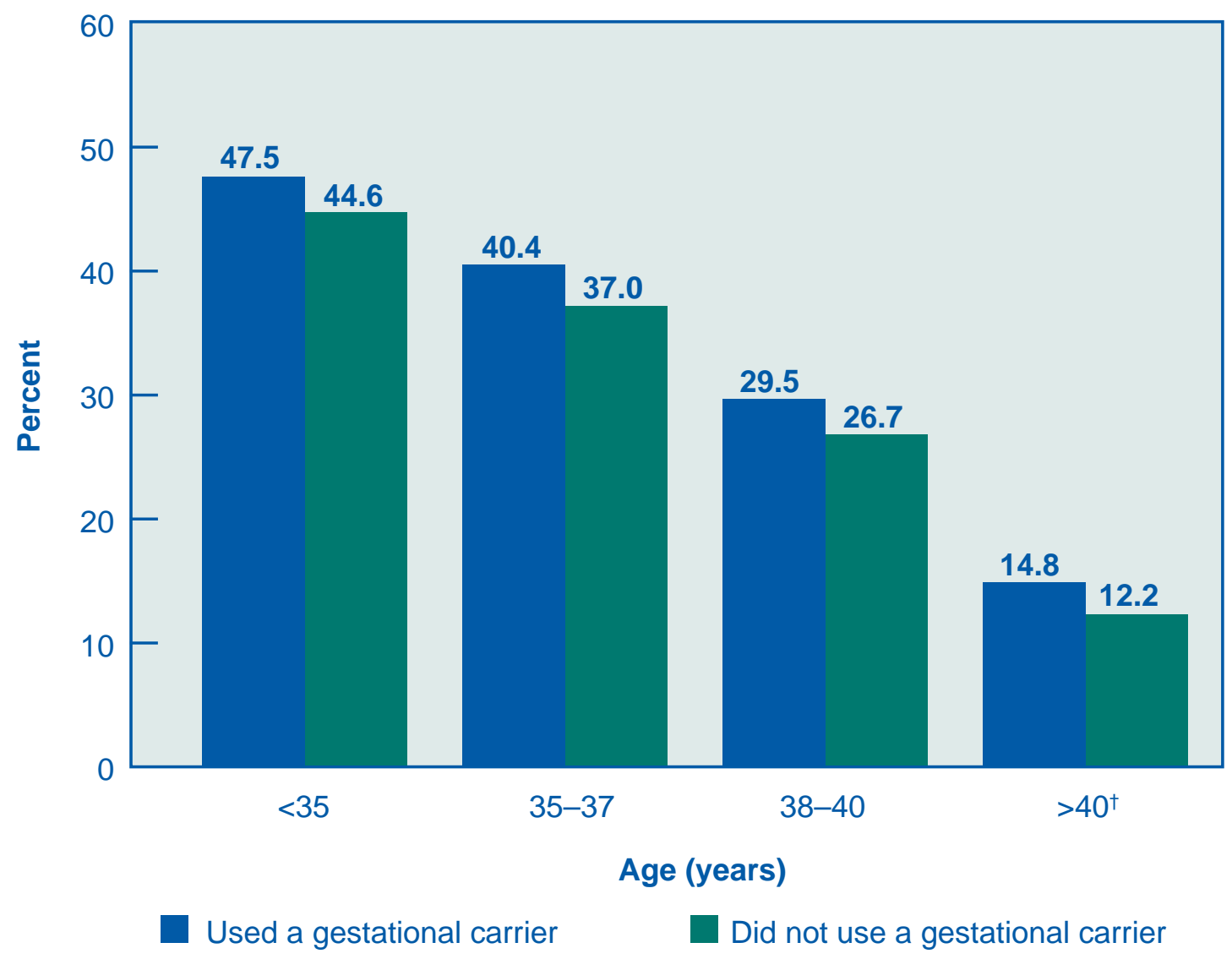

${ }^{*}$ Age categories reflect the age of the ART patient, not the age of the gestational carrier.

+ We were unable to further subdivide ages $>40$ because the number of such cycles is very small. 


\section{How is clinic size related to success rates?}

The number of ART procedures carried out every year varies among fertility clinics in the United States. In 2006, success rates were similar for all 426 clinics regardless of the number of cycles performed. For Figure 41, clinics were divided equally into four groups (called quartiles) based on the size of the clinic as determined by the number of cycles it carried out. The percentage for each quartile represents the average success rate for clinics in that quartile. For the exact number of cycles and success rates at an individual clinic, refer to the clinic table section of this report.

\section{Figure 41}

Percentages of ART Cycles (Using Fresh Nondonor Eggs

or Embryos) That Resulted in Live Births, by Clinic Size, 2006

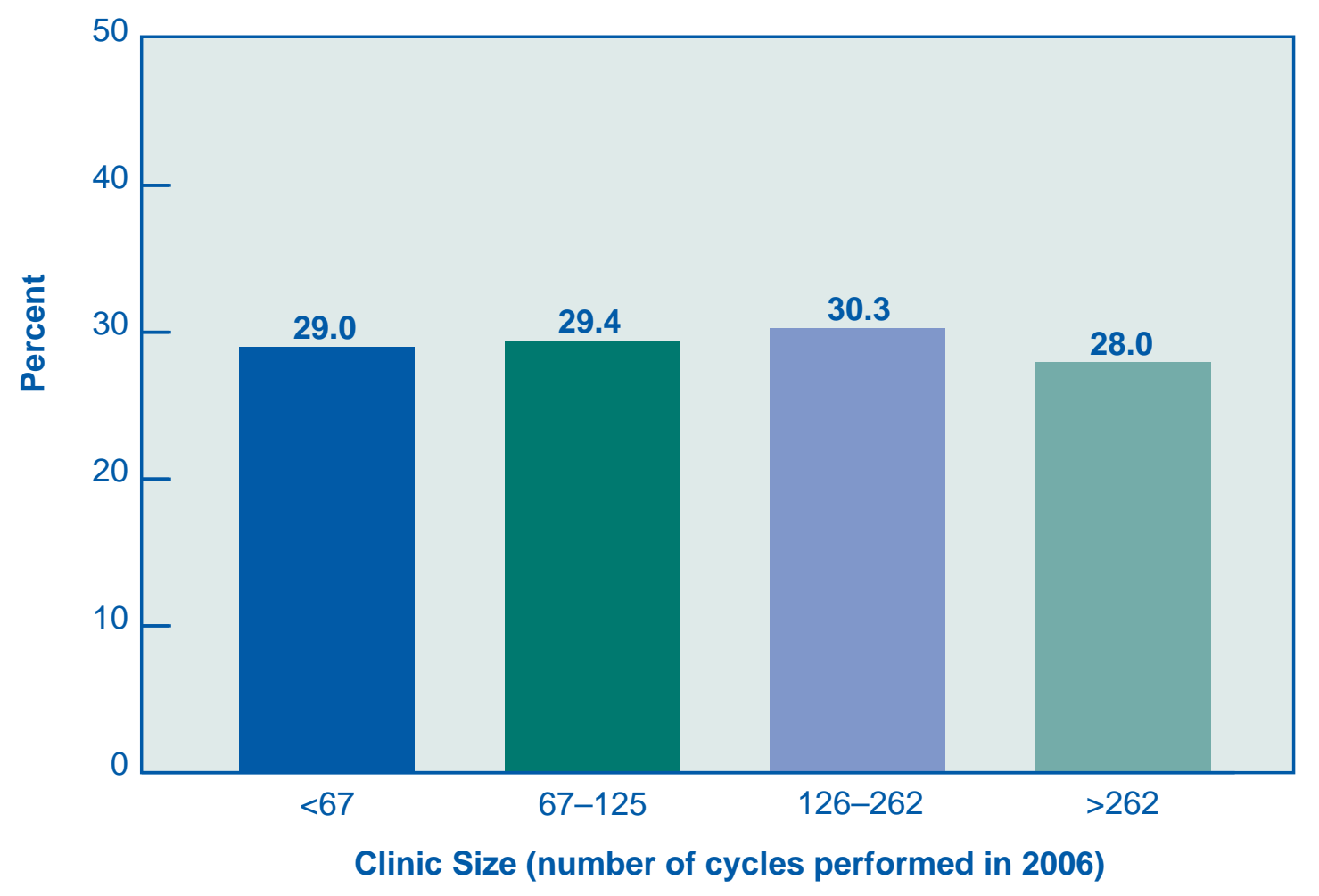




\title{
SECTION 3: ART CYCLES USING FROZEN NONDONOR EMBRYOS
}

\author{
What are the success rates for ART cycles \\ using frozen nondonor embryos?
}

Frozen embryos were used in approximately $16 \%$ of all ART cycles performed in 2006 (22,023 cycles). Figure 42 compares the success rates for frozen embryos with the success rates for fresh embryos among women using their own eggs. Because some embryos do not survive the thawing process, the percentage of thawed embryos that result in live births is usually lower than the percentage of transfers resulting in live births. In 2006, the success rates for frozen embryos were lower than the success rates for fresh embryos. However, the average number of embryos transferred was similar for cycles using both frozen embryos and fresh embryos (see the national summary table on page 89 for information on the average number of embryos transferred for these cycles). It is important to note that cycles using frozen embryos are both less expensive and less invasive than those using fresh embryos because the woman does not have to go through the fertility drug stimulation and egg retrieval steps again.

\section{Figure 42}

Success Rates for ART Cycles Using Frozen Embryos and Fresh Embryos, 2006

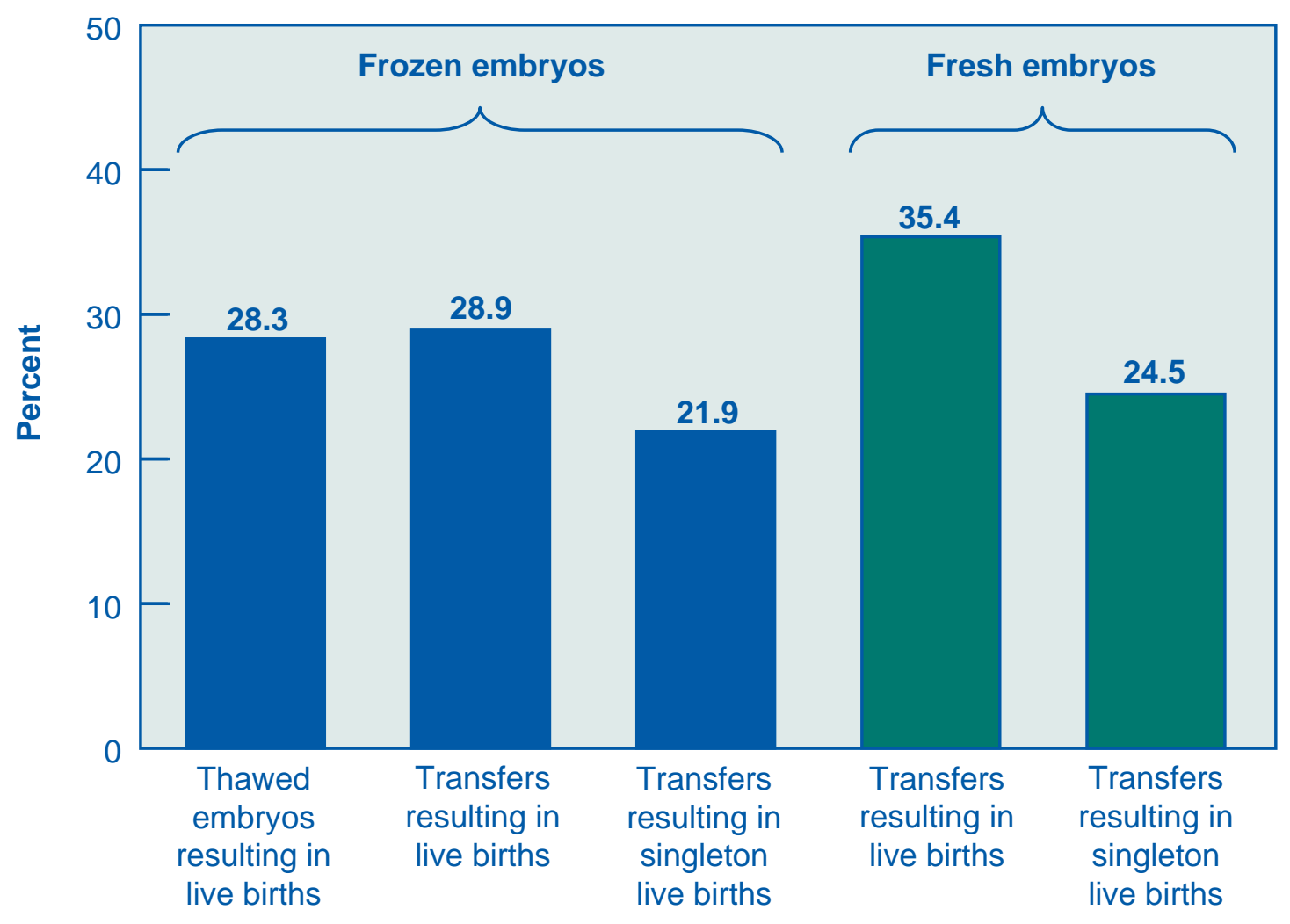




\section{What is the risk of having a multiple-fetus pregnancy or multiple-infant live birth from an ART cycle using frozen nondonor embryos?}

Multiple-infant births are associated with greater problems for both mothers and infants, including higher rates of caesarean section, prematurity, low birth weight, and infant disability or death.

Part A of Figure 43 shows that among the 7,401 pregnancies that resulted from ART cycles using frozen nondonor embryos, $66 \%$ were singleton pregnancies, $21 \%$ were twins, and $3 \%$ were triplets or more. Ten percent of pregnancies ended in miscarriage before the number of fetuses could be accurately determined. Therefore, the percentage of pregnancies with more than one fetus might have been higher than what was reported (24\%).

In 2006, 5,797 pregnancies from ART cycles that used frozen nondonor embryos resulted in live births. Part B of Figure 43 shows that approximately $24 \%$ of these live births produced more than one infant. This compares with a multiple-infant birth rate of slightly more than $3 \%$ in the general U.S. population.

Although the total rates for multiples were similar for pregnancies and live births, there were more triplet-or-more pregnancies than births. Triplet-or-more pregnancies may be reduced to twins or singletons by the time of birth. This can happen naturally (e.g., fetal death), or a woman and her doctor may decide to reduce the number of fetuses using a procedure called multifetal pregnancy reduction. CDC does not collect information on multifetal pregnancy reductions.

\section{Figure $4 \mathbf{3}$}

Risks of Having Multiple-Fetus Pregnancy and Multiple-Infant

Live Birth from ART Cycles Using Frozen Nondonor Embryos, 2006

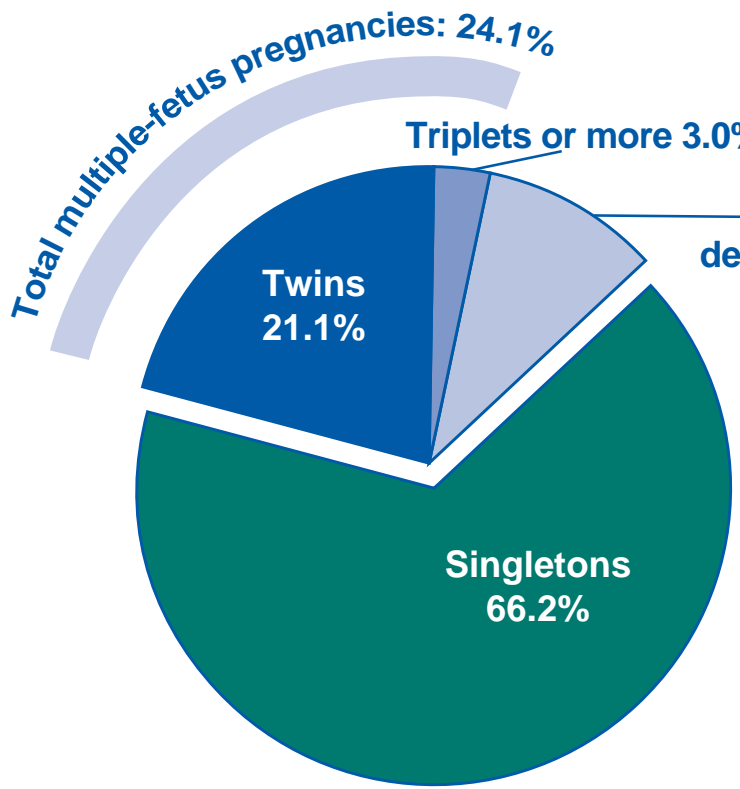

A. 7,401 Pregnancies ${ }^{\dagger}$

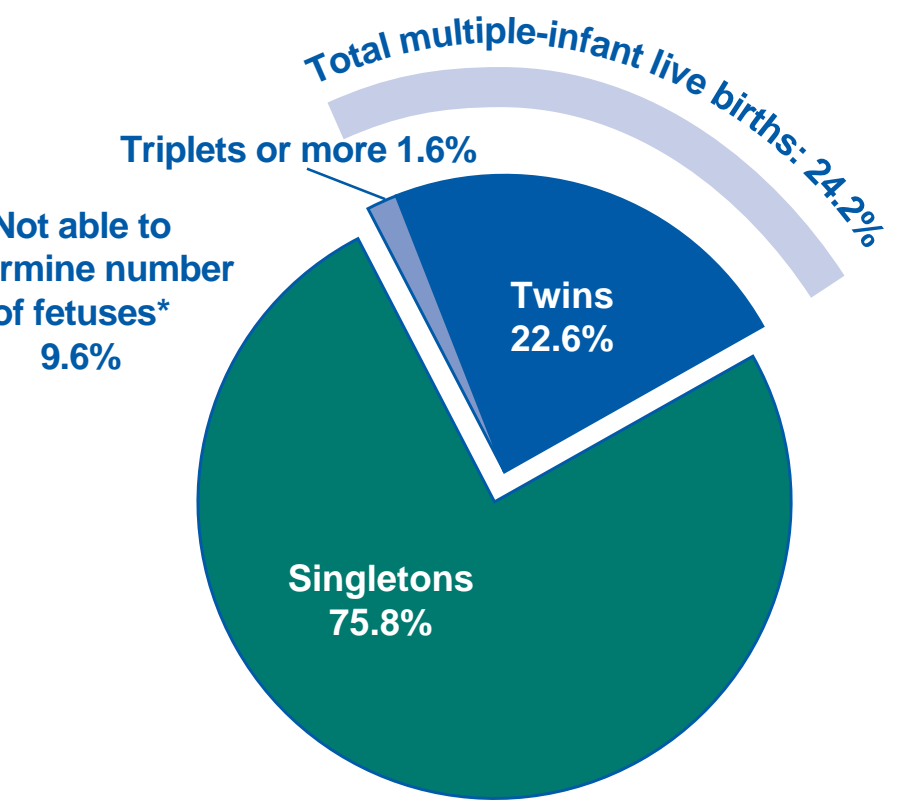

B. 5,797 Live births

* Number of fetuses not known because the pregnancy ended in an early miscarriage.

† Total does not equal $100 \%$ due to rounding. 


\section{SECTION 4: ART CYCLES USING DONOR EGGS}

\section{Are older women undergoing ART more likely to use donor eggs or embryos?}

As shown in Figures 14-16 (pages 26-28), eggs produced by women in older age groups form embryos that are less likely to implant and more likely to result in miscarriage if they do implant. As a result, ART using donor eggs is much more common among older women than among younger women. Donor eggs or embryos were used in approximately 12\% of all ART cycles carried out in 2006 (16,976 cycles). Figure 44 shows the percentage of ART cycles using donor eggs in 2006 according to the woman's age. Few women younger than age 39 used donor eggs; however, the percentage of cycles carried out with donor eggs increased sharply starting at age 39. Among women older than age 47 , for example, about $89 \%$ of all ART cycles used donor eggs.

\section{Figure 44}

Percentage of ART Cycles Using Donor Eggs, by ART Patient's Age, 2006

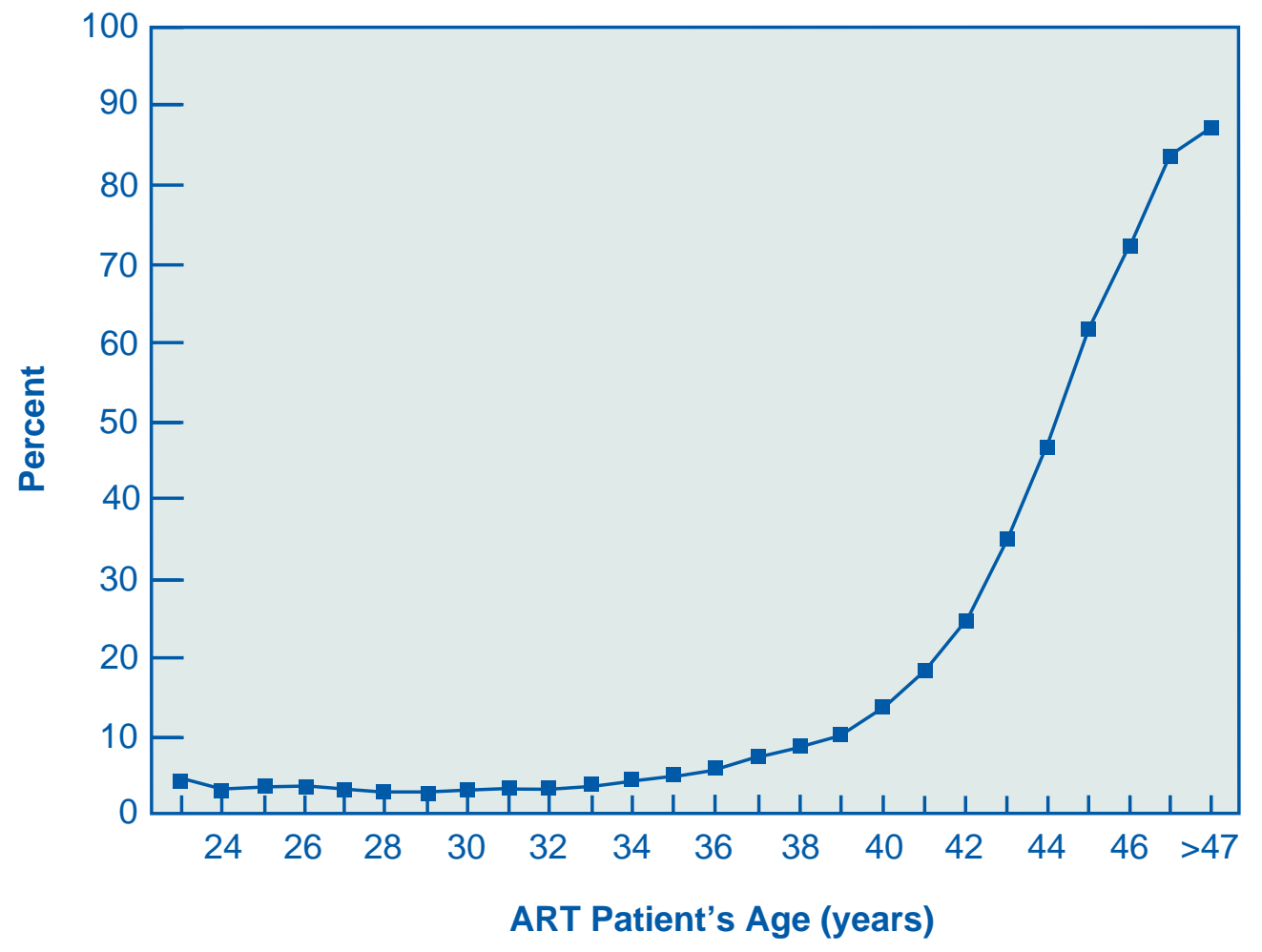




\section{Do success rates differ by age for women who used ART with donor eggs compared with women who used ART with their own eggs?}

Figure 45 compares percentages of transfers resulting in live births for ART cycles using fresh embryos from donor eggs with those for ART cycles using a woman's own eggs, among women of different ages. The likelihood of a fertilized egg implanting is related to the age of the woman who produced the egg. Thus, the percentage of transfers resulting in live births for cycles using embryos from women's own eggs declines as women get older. In contrast, since egg donors are typically in their 20s or early 30s, the percentage of transfers resulting in live births for cycles using embryos from donor eggs remained consistently high at above $40 \%$.

\section{Figure 45}

Percentages of Transfers That Resulted in Live Births for ART Cycles Using Fresh Embryos from Own and Donor Eggs, by ART Patient's Age, 2006

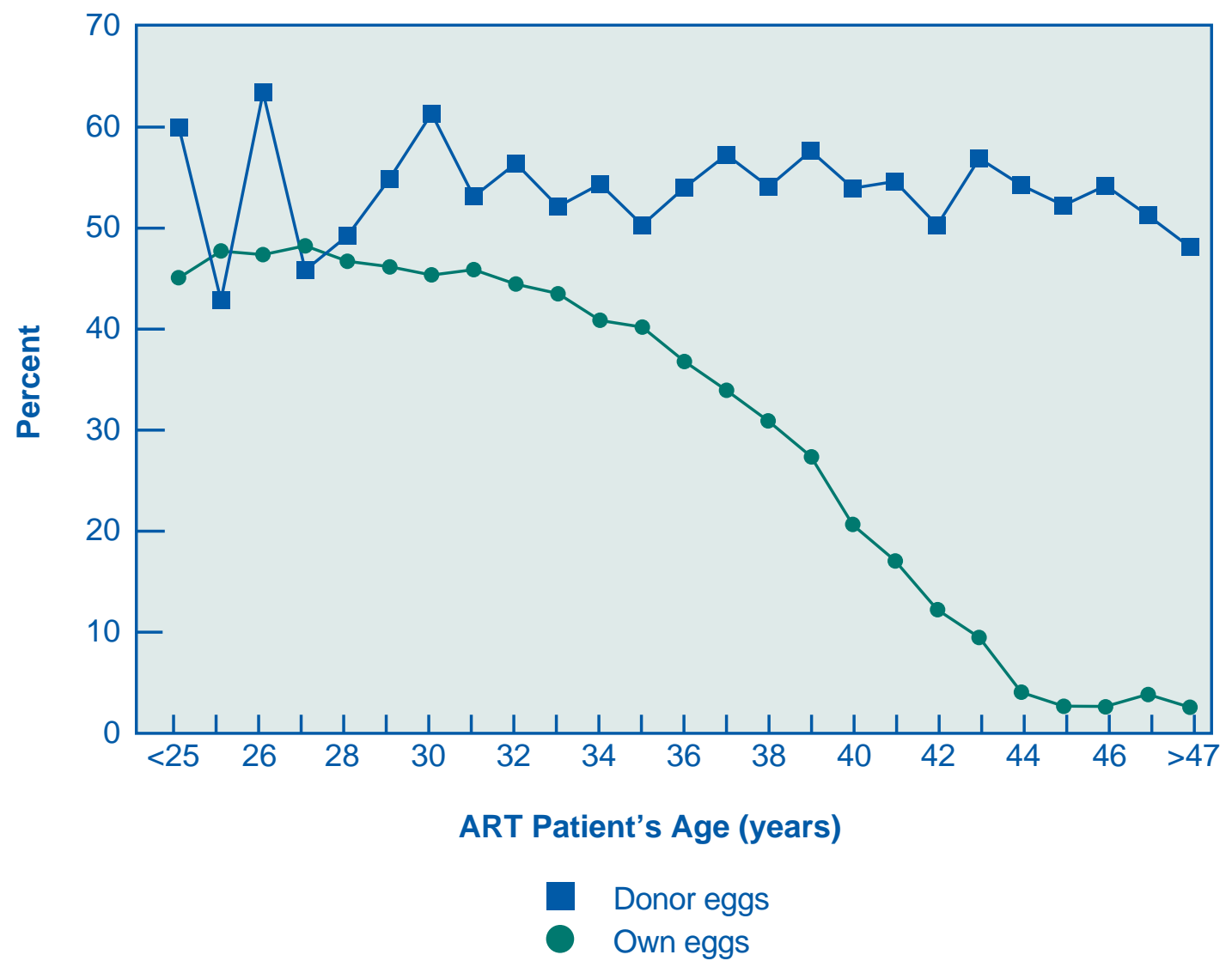




\section{How successful is ART when donor eggs are used?}

Figure 46 shows percentages of transfers resulting in live births and singleton live births for ART cycles using fresh embryos from donor eggs among women of different ages. For all ages, the percentage of transfers resulting in singleton live births (average 33\%) was lower than the percentage of transfers resulting in live births (average 54\%). Singleton live births are an important measure of success because they have a much lower risk than multiple-infant births for adverse infant health outcomes, including prematurity, low birth weight, disability, and death.

\section{Figure 46}

Percentages of Transfers That Resulted in Live Births and Singleton Live Births for ART Cycles Using Fresh Embryos from Donor Eggs, by ART Patient's Age, 2006

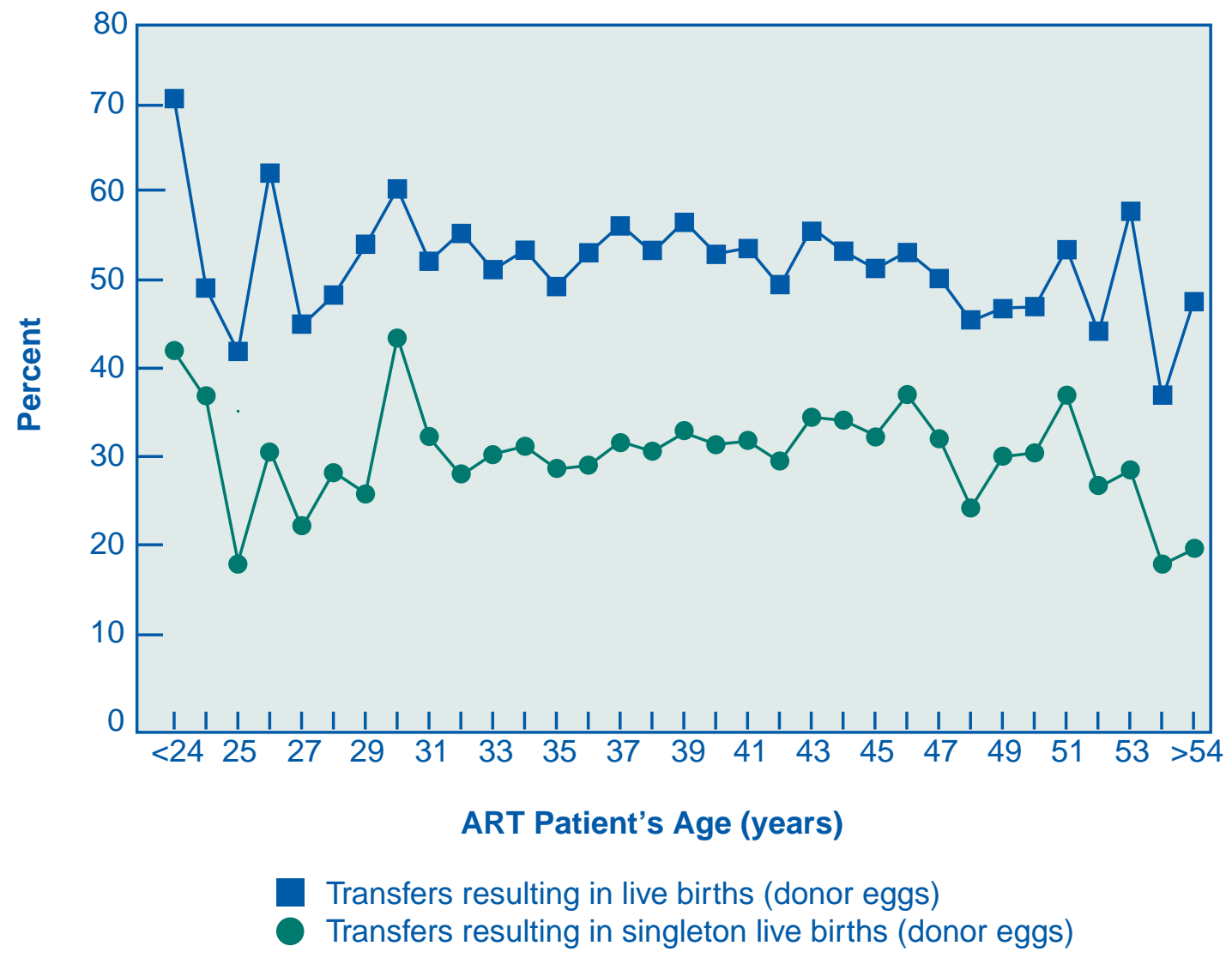




\section{What is the risk of having a multiple-fetus pregnancy or multiple- infant live birth from an ART cycle using fresh donor eggs?}

Multiple-infant births are associated with greater problems for both mothers and infants, including higher rates of caesarean section, prematurity, low birth weight, and infant disability or death.

Part A of Figure 47 shows that among the 6,315 pregnancies that resulted from ART cycles using fresh embryos from donor eggs, about 54\% were singleton pregnancies, about 37\% were twins, and nearly $4 \%$ were triplets or more. About $5 \%$ of pregnancies ended in miscarriage before the number of fetuses could be accurately determined. Therefore, the percentage of pregnancies with more than one fetus might have been higher than what was reported (about 41\%).

In 2006, 5,393 pregnancies from ART cycles that used fresh embryos from donor eggs resulted in live births. Part B of Figure 47 shows that 39\% of these live births produced more than one infant. This compares with a multiple-infant birth rate of slightly more than $3 \%$ in the general population.

Although the total rates for multiples were similar for pregnancies and live births, there were more triplet-or-more pregnancies than births. Triplet-or-more pregnancies may be reduced to twins or singletons by the time of birth. This can happen naturally (e.g., fetal death), or a woman and her doctor may decide to reduce the number of fetuses using a procedure called multifetal pregnancy reduction. CDC does not collect information on multifetal pregnancy reductions.

\section{Figure 47}

Risks of Having Multiple-Fetus Pregnancy and Multiple-Infant Live Birth from ART Cycles Using Fresh Embryos from Donor Eggs, 2006

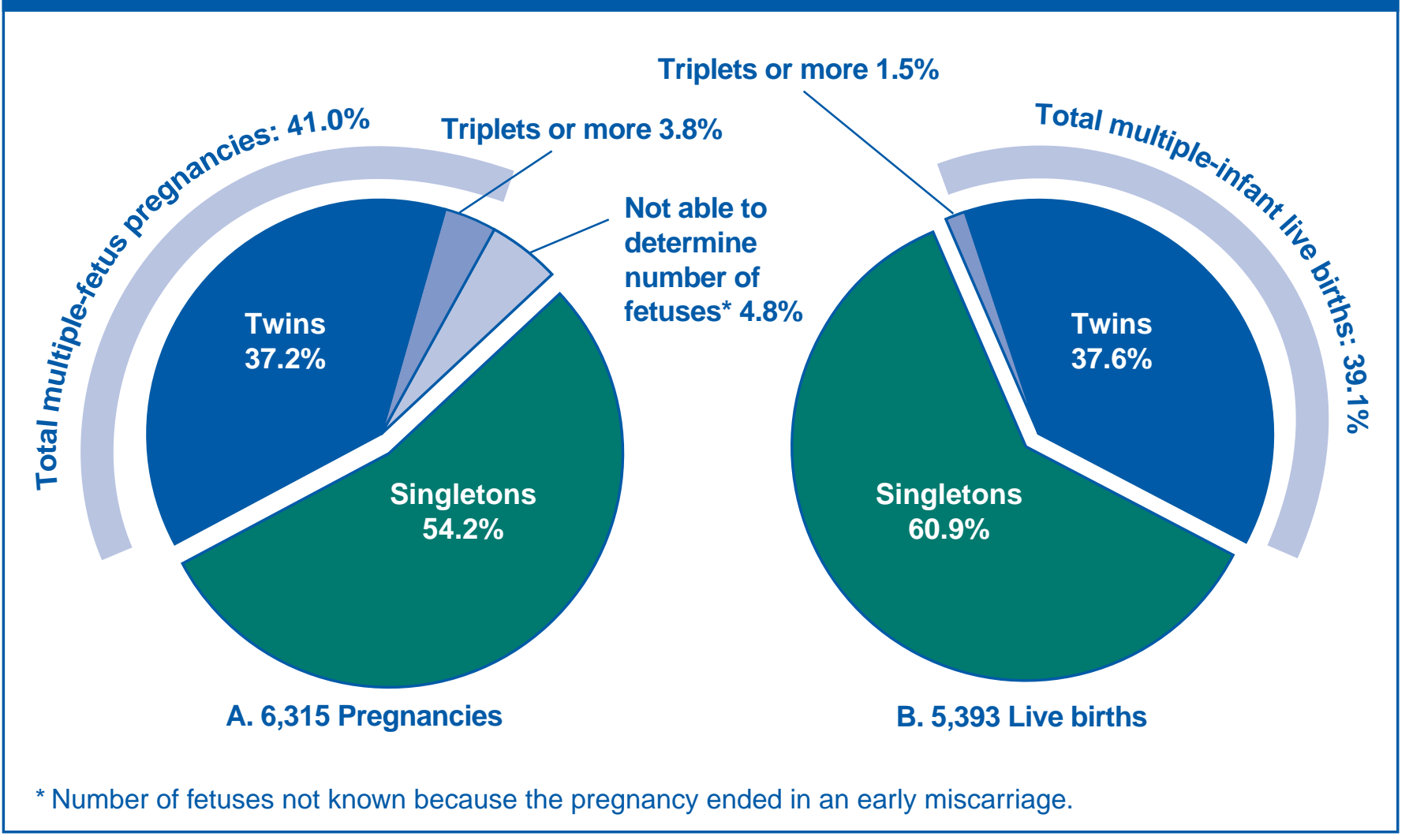




\section{How do success rates differ between \\ women who use frozen donor embryos and those who use fresh donor embryos?}

Figure 48 shows that the success rates resulting from the transfer of frozen donor embryos were substantially lower than the success rates resulting from the transfer of fresh donor embryos. This is similar to the findings for frozen nondonor embryos (see Figure 42, page 54). The average number of embryos transferred was similar for cycles using frozen donor embryos and those using fresh donor embryos. (See the national summary table on page 89 for information on the average number of embryos transferred for these cycles.)

\section{Figure 48}

Success Rates for ART Cycles Using Frozen Donor and

Fresh Donor Embryos, 2006

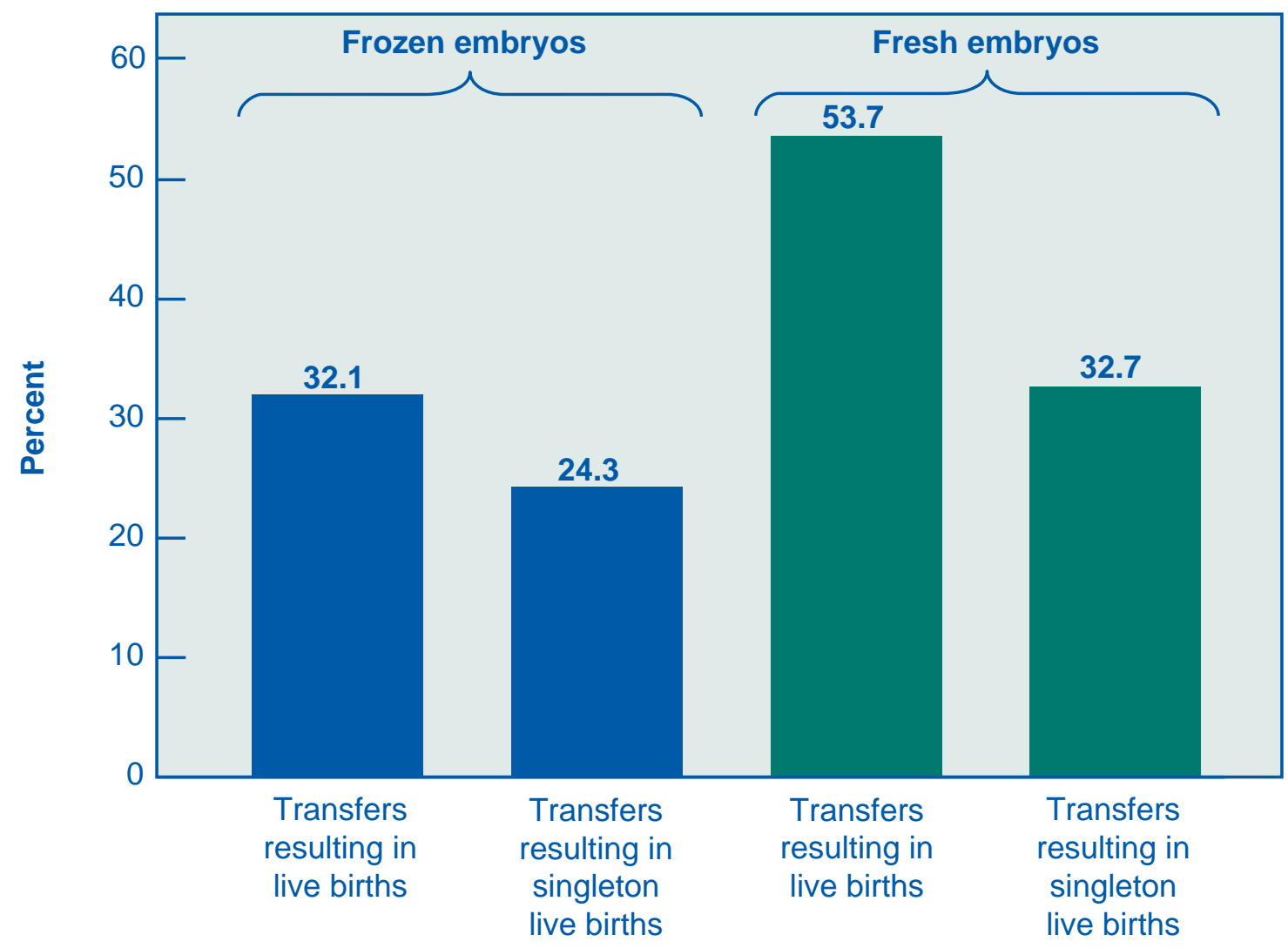




\section{SECTION 5: ART TRENDS, 1996-2006}

This report marks the twelfth consecutive year that $C D C$ has published an annual report detailing the success rates for ART clinics in the United States. Having several years of data provides us with the opportunity to examine trends in ART use and success rates over time. Because the first year of data collection, 1995, did not include non-SART member clinics, we limit our examination of trends to the years 1996-2006.

\section{Is the use of ART increasing?}

Figure 49 shows the numbers of ART cycles performed, live-birth deliveries, and infants born using ART from 1996 through 2006. The number of ART cycles performed in the United States has more than doubled, from 64,681 cycles in 1996 to 138,198 in 2006. The number of live-birth deliveries in $2006(41,343)$ was more than two and a half times higher than in $1996(14,507)$. The number of infants born who were conceived using ART also increased steadily between 1996 and 2006. In 2006, 54,656 infants were born, which was more than two and a half times the 20,840 born in 1996. Because in some cases more than one infant is born during a live-birth delivery (e.g., twins), the total number of infants born is greater than the number of live-birth deliveries.

\section{Figure 49}

Numbers of ART Cycles Performed, Live-Birth Deliveries, and Infants Born Using ART, 1996-2006

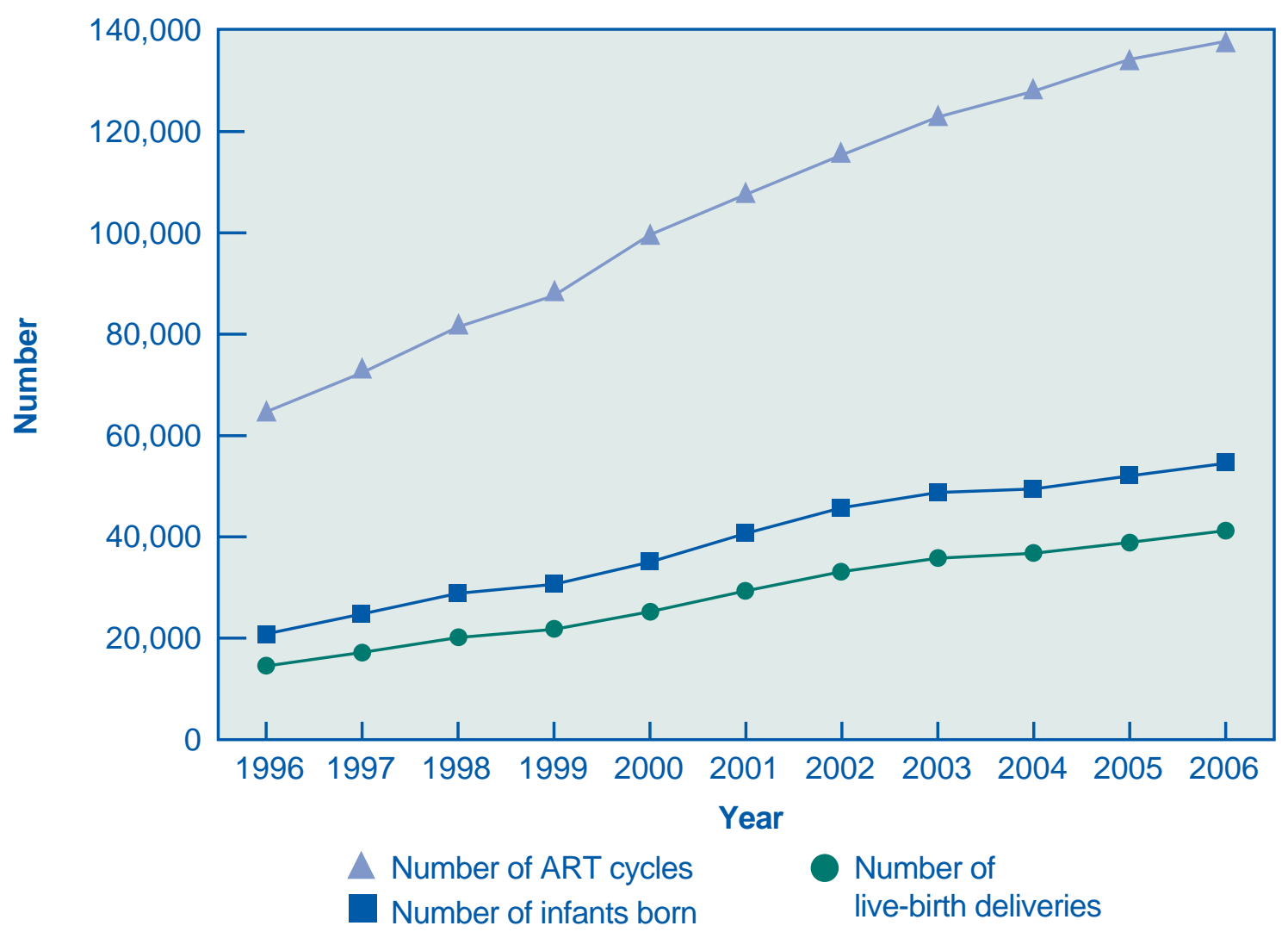




\section{Have there been changes in the type of ART cycles performed among women who used fresh or frozen nondonor eggs or embryos?}

Intracytoplasmic sperm injection (ICSI) was originally developed to use in ART cycles to improve fertilization rates when severe male factor infertility was the indication for using ART. Today, this procedure is widely used even among couples without a diagnosis of male factor infertility.

Figure 50 shows the numbers of ART cycles performed using fresh nondonor eggs or embryos with or without ICSI and the numbers of cycles using frozen nondonor eggs or embryos from 1996 through 2006. During the past 11 years, while the number of fresh-nondonor cycles performed without ICSI remained stable, the number of fresh-nondonor cycles performed with ICSI increased four times from 14,885 in 1996 to 61,835 in 2006. The number of frozen-nondonor cycles more than doubled, from 9,445 in 1996 to 22,023 in 2006.

Note that the information on use of ICSI was not collected for ART cycles using frozen embryos; therefore, cycles using frozen embryos are presented together as one group.

\section{Figure 50}

Numbers of ART Cycles Using Fresh or Frozen Nondonor Eggs or Embryos,

by ICSI, ${ }^{*}$ 1996-2006

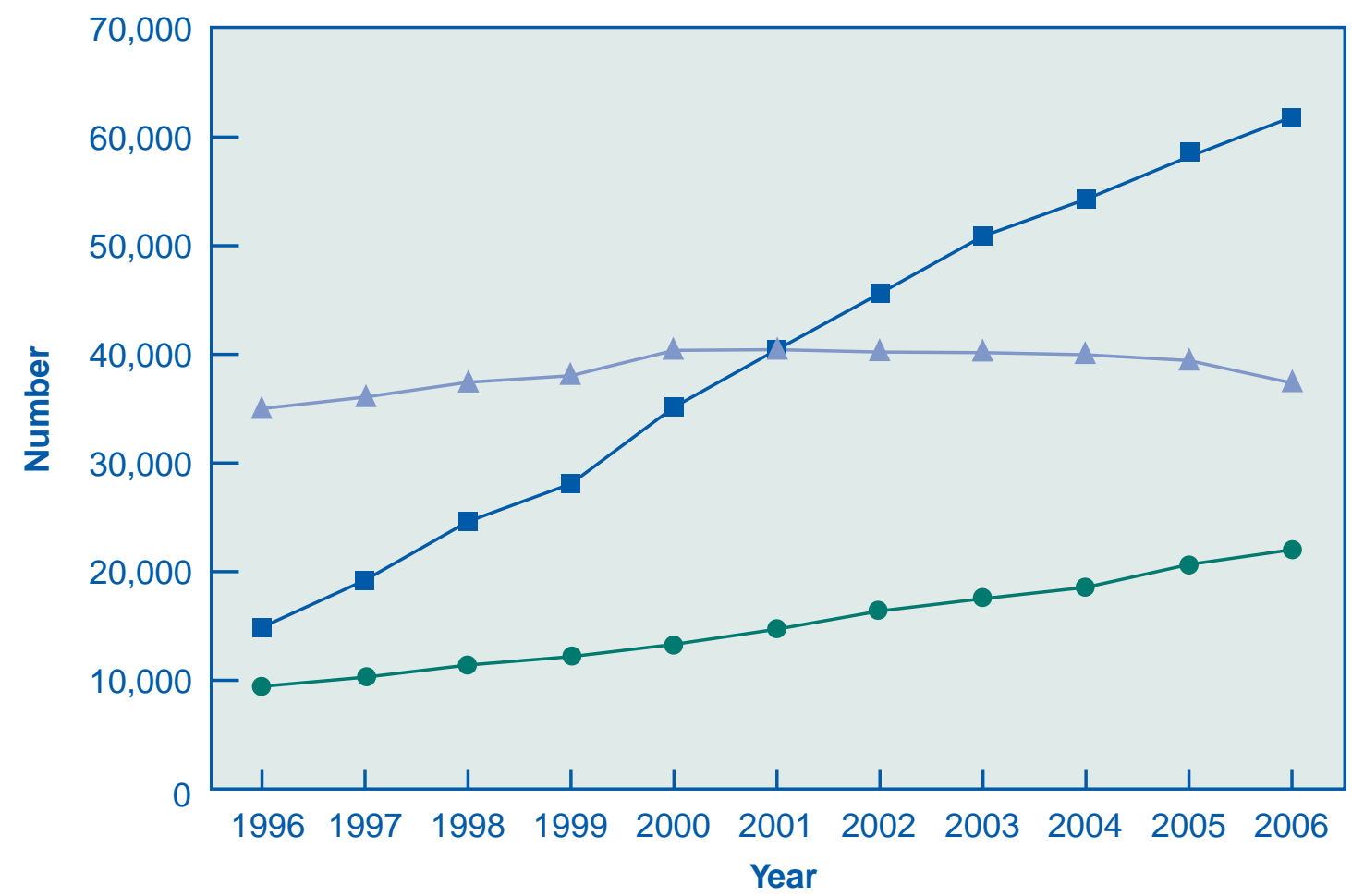

A Fresh-nondonor without ICSI

Frozen-nondonor

Fresh-nondonor with ICSI

* Intracytoplasmic sperm injection. 


\section{Have there been changes in the types of ART cycles performed among women who used fresh or frozen donor eggs or embryos?}

Figure 51 shows the numbers of ART cycles performed using fresh donor eggs or embryos with or without ICSI and cycles using frozen donor eggs or embryos. While the number of fresh-donor cycles performed without ICSI remained fairly stable during the past 11 years, the number of fresh-donor cycles performed with ICSI increased from 857 in 1996 to 7,039 in 2006. The number of frozen-donor cycles increased from 1,118 in 1996 to 5,992 in 2006. In particular, during reporting year 2006, fresh donor eggs with ICSI were used the most among all donor cycles.

Note that the information on use of ICSI was not collected for ART cycles using frozen embryos; therefore, cycles using frozen embryos are presented together as one group.

\section{Figure 51}

Numbers of ART Cycles Using Fresh or Frozen Donor Eggs or Embryos,

by ICSI, ${ }^{*} 1996-2006$

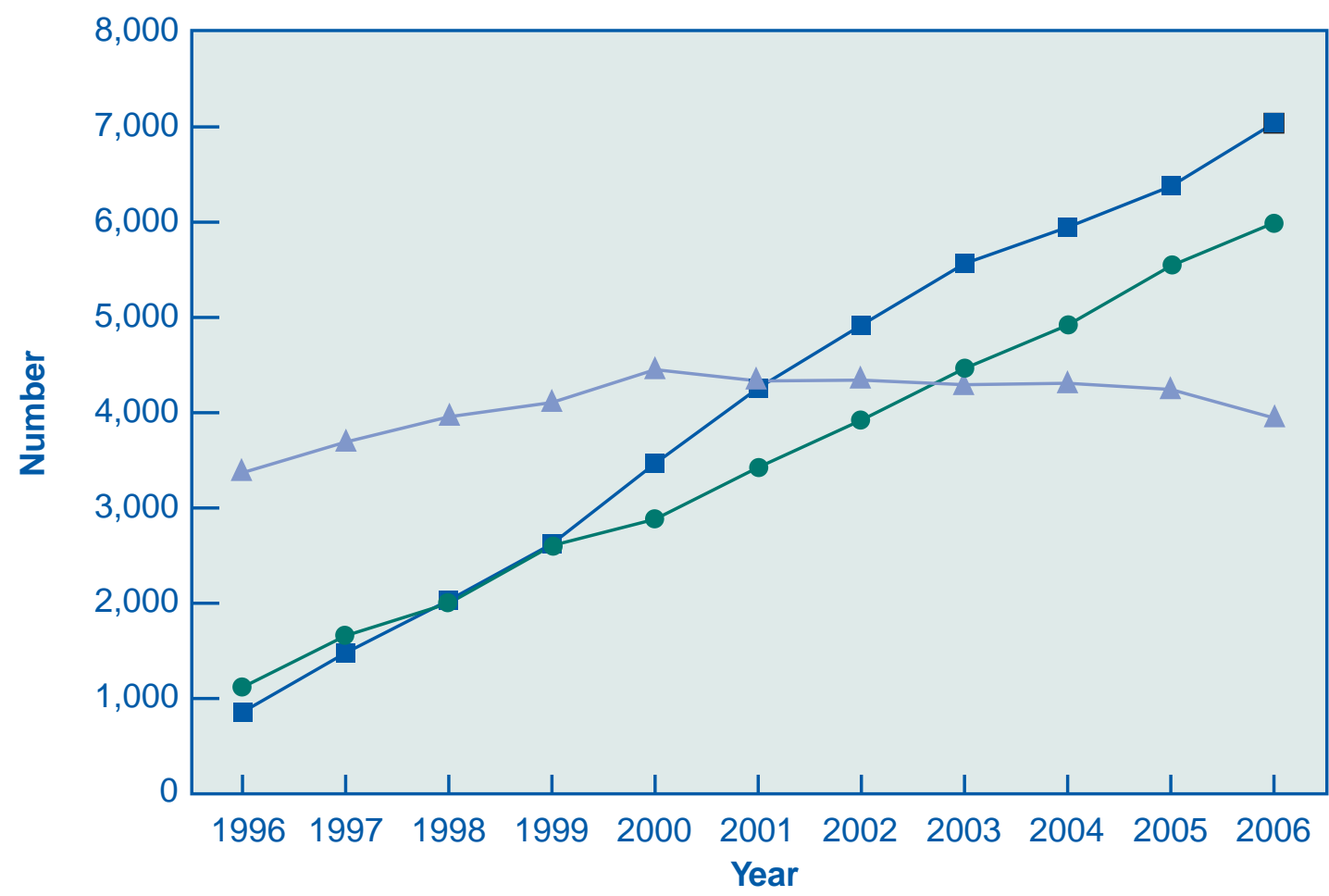

$\Delta$ Fresh-donor without ICSI

Frozen-donor

Fresh-donor with ICSI

*Intracytoplasmic sperm injection. 


\section{Have there been improvements in the percentage of transfers that result in live births among women who used fresh or frozen nondonor eggs or embryos?}

Figure 52 presents percentages of transfers that resulted in live births for ART cycles using fresh nondonor eggs or embryos with or without ICSI and for cycles using frozen nondonor eggs or embryos. Percentages of transfers that resulted in live births are presented rather than percentages of cycles that resulted in live births because this is the only way to directly compare cycles using fresh embryos with those using frozen embryos.

Overall, higher success rates were consistently observed among fresh-nondonor cycles than frozennondonor cycles. The percentage of transfers that resulted in live births for fresh-nondonor cycles performed without ICSI increased from 28\% in 1996 to 37\% in 2006. Over the same period, the percentage of transfers that resulted in live births for cycles using fresh nondonor embryos performed with ICSI remained slightly lower than without ICSI, but steadily increased. The percentage of transfers that resulted in live births for cycles using frozen nondonor embryos increased from 17\% in 1996 to $29 \%$ in 2006 , but was generally lower than the percentage of transfers that resulted in live births for cycles using fresh nondonor embryos.

Note that the information on use of ICSI was not collected for ART cycles using frozen embryos; therefore, such cycles are presented together as one group.

\section{Figure 52}

Percentages of Transfers That Resulted in Live Births Using Fresh or Frozen Nondonor Eggs or Embryos, by ICSI, * 1996-2006

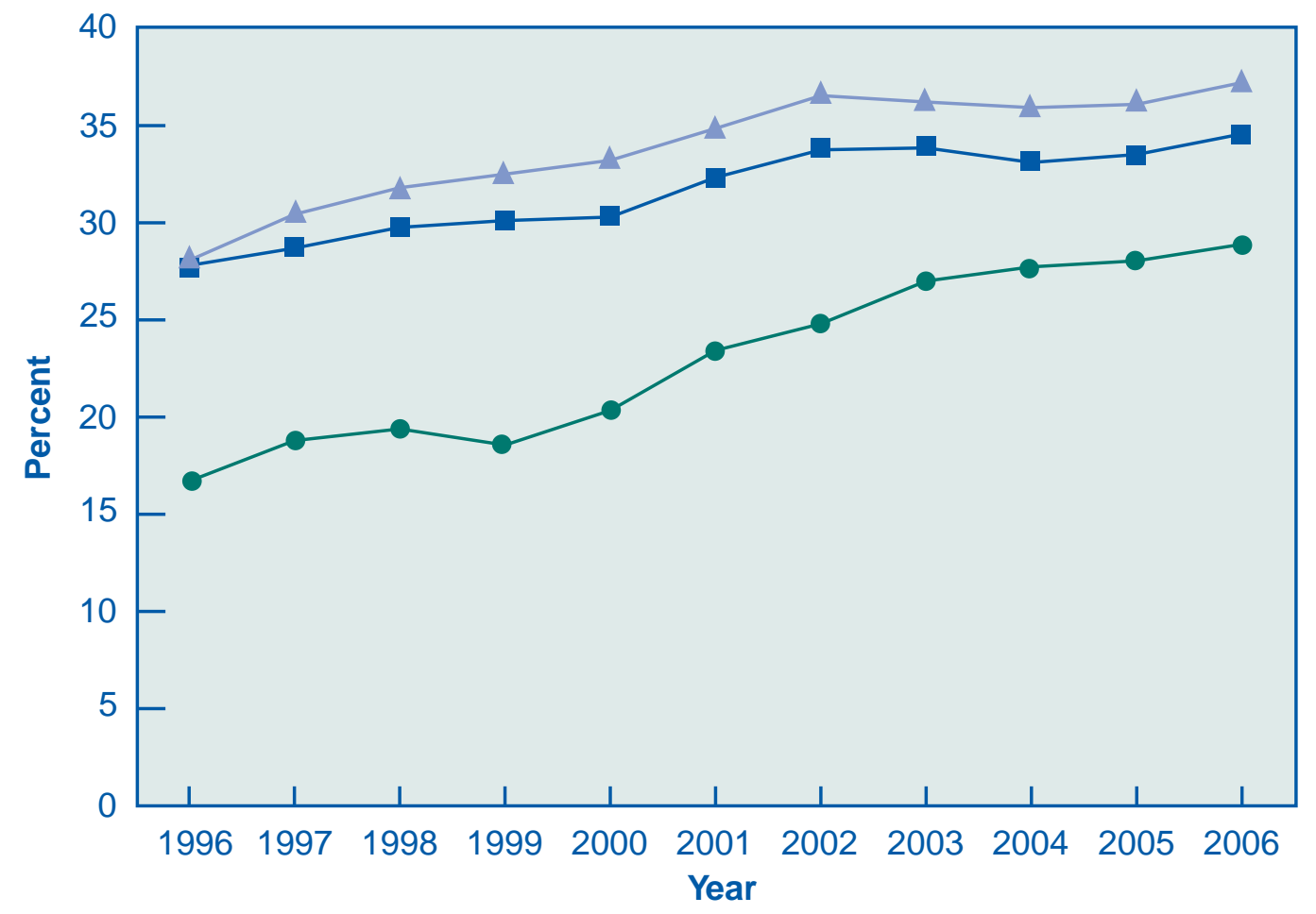




\section{Have there been improvements in the percentage of transfers that result in live births among women who used fresh or frozen donor eggs or embryos?}

Figure 53 presents the percentages of transfers that resulted in live births for ART cycles using fresh donor eggs or embryos with or without ICSI and for cycles using frozen donor eggs or embryos. Percentages of transfers that resulted in live births are presented rather than percentages of cycles that resulted in live births because that is the only way to directly compare cycles using fresh embryos with those using frozen embryos.

Similar to the trends shown in Figure 52 (page 64) for nondonor cycles, the success rates for cycles using fresh donor eggs or embryos were generally higher than for cycles using frozen donor eggs or embryos during 1996-2006. The percentage of transfers that resulted in live births for cycles that used fresh donor eggs or embryos performed without ICSI increased from 39\% in 1996 to 56\% in 2006. Over the same period, the percentage of transfers resulting in live births increased from 39\% to 53\% for cycles that used fresh donor eggs or embryos and were performed with ICSI, and from $21 \%$ to $32 \%$ for cycles that used frozen donor eggs or embryos.

Note that the information on use of ICSI was not collected for ART cycles using frozen embryos; therefore, such cycles are presented together as one group.

\section{Figure 53}

Percentages of Transfers That Resulted in Live Births Using Fresh or Frozen Donor Eggs or Embryos, by ICSI, * 1996-2006

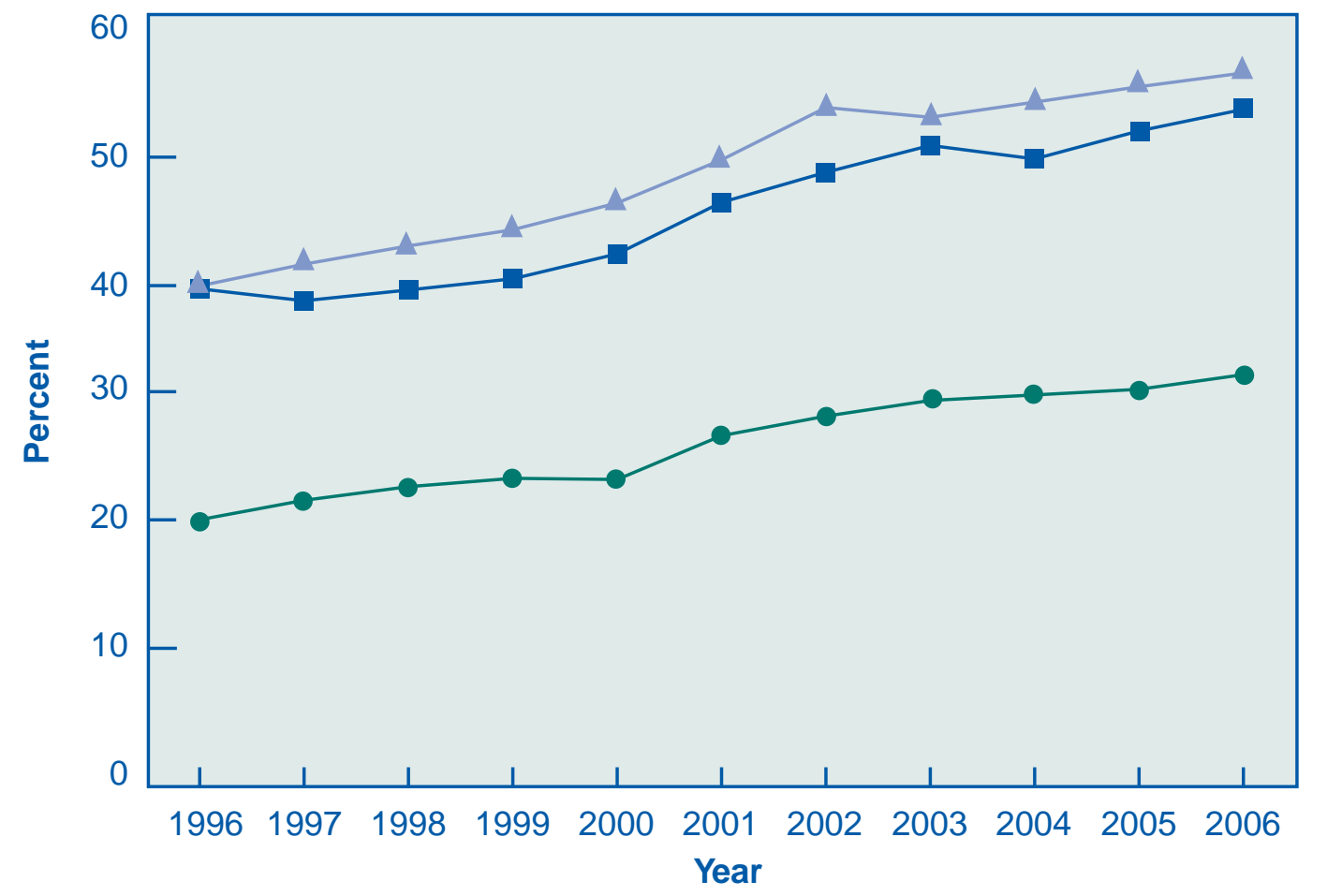

$\Delta$ Fresh-donor without ICSI

Frozen-donor

Fresh-donor with ICSI 


\section{Have there been improvements in the percentage of transfers that result in singleton live births among women who used fresh or frozen nondonor eggs or embryos?}

Singleton live births are an important measure of success because they entail a much lower risk than multiple-infant births for adverse infant health outcomes, including prematurity, low birth weight, disability, and death. Figure 54 presents percentages of transfers that resulted in singleton live births for ART cycles performed using fresh nondonor eggs or embryos with or without ICSI or for cycles using frozen nondonor eggs or embryos.

While the total numbers of nondonor cycles using ICSI greatly increased over the past 11 years (see Figure 50, page 62), the percentage of transfers that resulted in singleton live births from these cycles were not any higher than those without ICSI: $17 \%$ to $24 \%$ with ICSI versus $17 \%$ to $25 \%$ without ICSI.

Over the same period, the percentage of transfers that resulted in singleton live births among frozennondonor cycles increased from $12 \%$ to $22 \%$.

Note that the information on use of ICSI was not collected for ART cycles using frozen embryos; therefore, such cycles are presented together as one group.

\section{Figure 54}

Percentages of Transfers That Resulted in Singleton Live Births Among Women Using Fresh or Frozen Nondonor Eggs or Embryos, by ICSI, ${ }^{*}$ 1996-2006

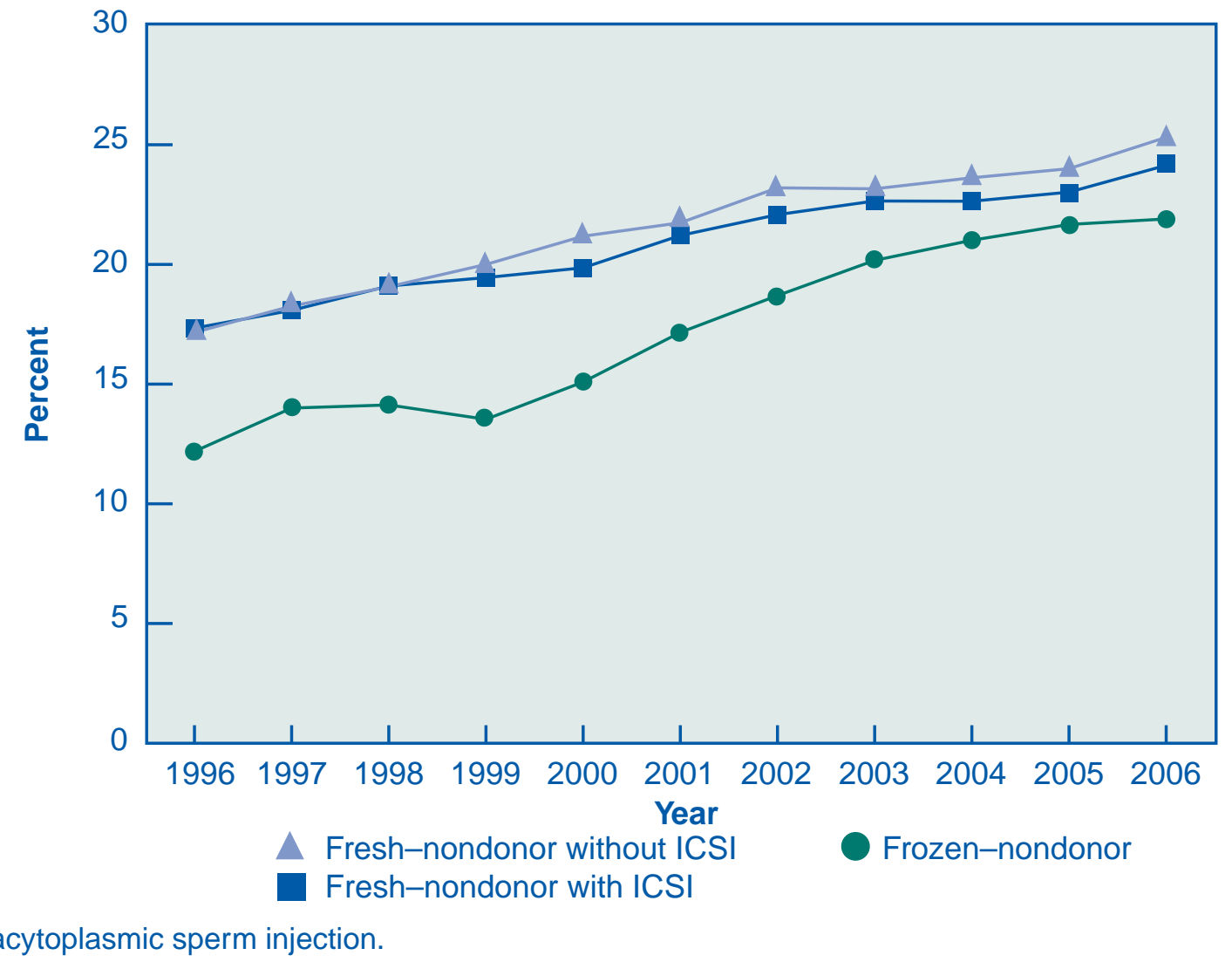




\section{Have there been improvements in the percentage of transfers that result in singleton live births among women who used fresh or frozen donor eggs or embryos?}

Singleton live births are an important measure of success because they entail a much lower risk than multiple-infant births for adverse infant health outcomes, including prematurity, low birth weight, disability, and death. Figure 55 presents percentages of transfers that resulted in singleton live births for ART cycles performed using fresh donor eggs or embryos with or without ICSI or for cycles using frozen donor eggs or embryos.

The percentage of transfers that resulted in singleton live births were consistently higher for freshdonor cycles than for frozen-donor cycles. Percentages increased for fresh-donor cycles without ICSI from 22\% in 1996 to 33\% in 2006; a similar increase from 24\% to 33\% was observed for cycles with ICSI. Over the same period, the percentage of transfers that resulted in singleton live births increased from $15 \%$ to $24 \%$ for frozen-donor cycles.

Note that the information on use of ICSI was not collected for ART cycles using frozen embryos; therefore,such cycles are presented together as one group.

\section{Figure 55}

Percentages of Transfers That Resulted in Singleton Live Births Among Women Using Fresh or Frozen Donor Eggs or Embryos, by ICSI, * 1996-2006

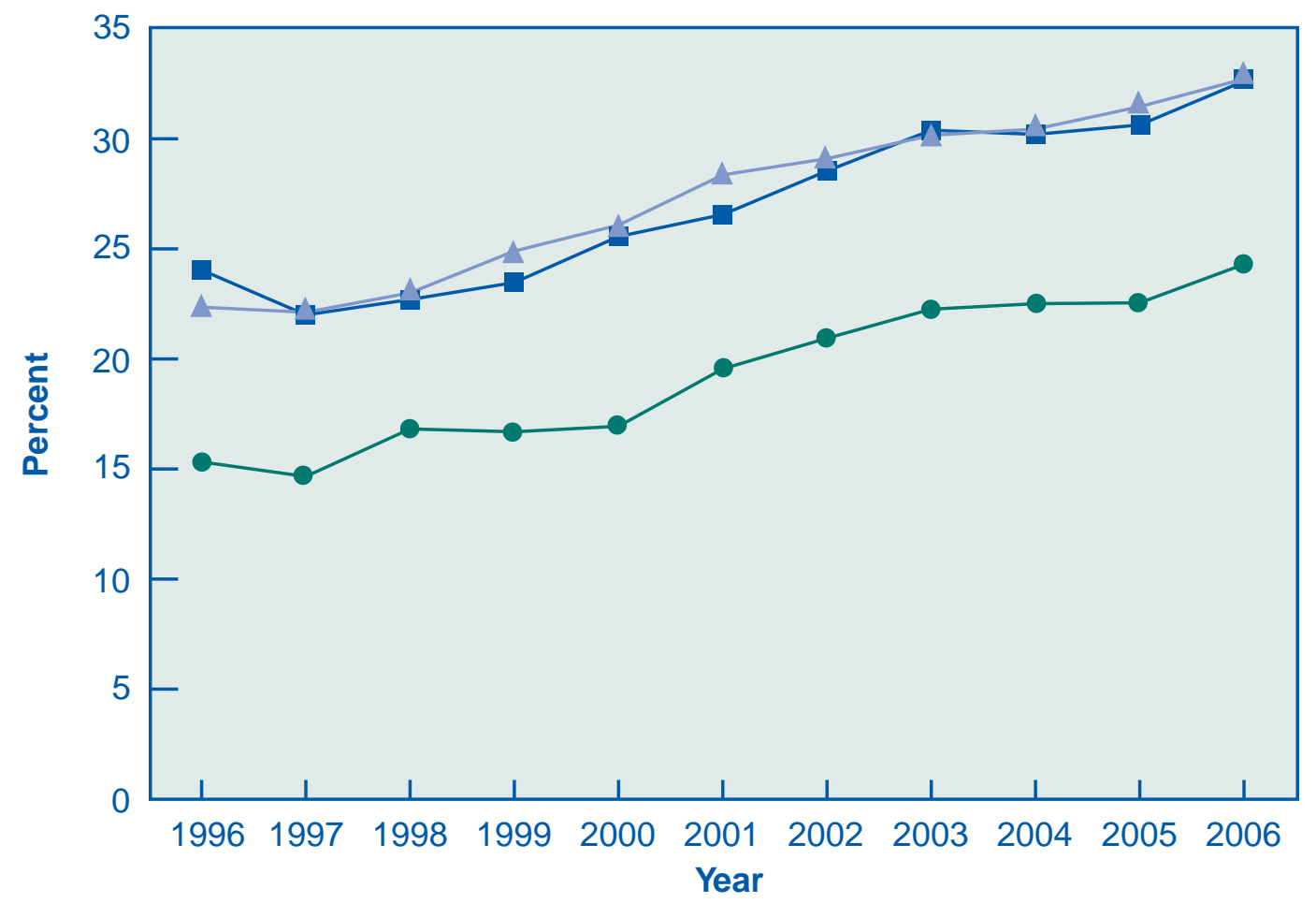
$\Delta$ Fresh-donor without ICSI
Fresh-donor with ICSI

* Intracytoplasmic sperm injection. 


\section{Have there been improvements in the percentage of transfers that result in live births for all ART patients or only for those in particular age groups?}

Figure 56 presents percentages of transfers that resulted in live births, by woman's age, for ART cycles using fresh nondonor eggs or embryos.

From 1996 through 2006, the percentage of transfers that resulted in live births for women younger than 35 increased 33\%, from 34\% in 1996 to 45\% in 2006. Over the same period, the percentage of transfers that resulted in live births increased $28 \%$ for women 35-37, 24\% for women 38-40, 31\% for women $41-42$, and $22 \%$ for women older than 42 .

\section{Figure 56}

Percentages of Transfers That Resulted in Live Births for ART Cycles Using Fresh Nondonor Eggs or Embryos, by Woman's Age, 1996-2006

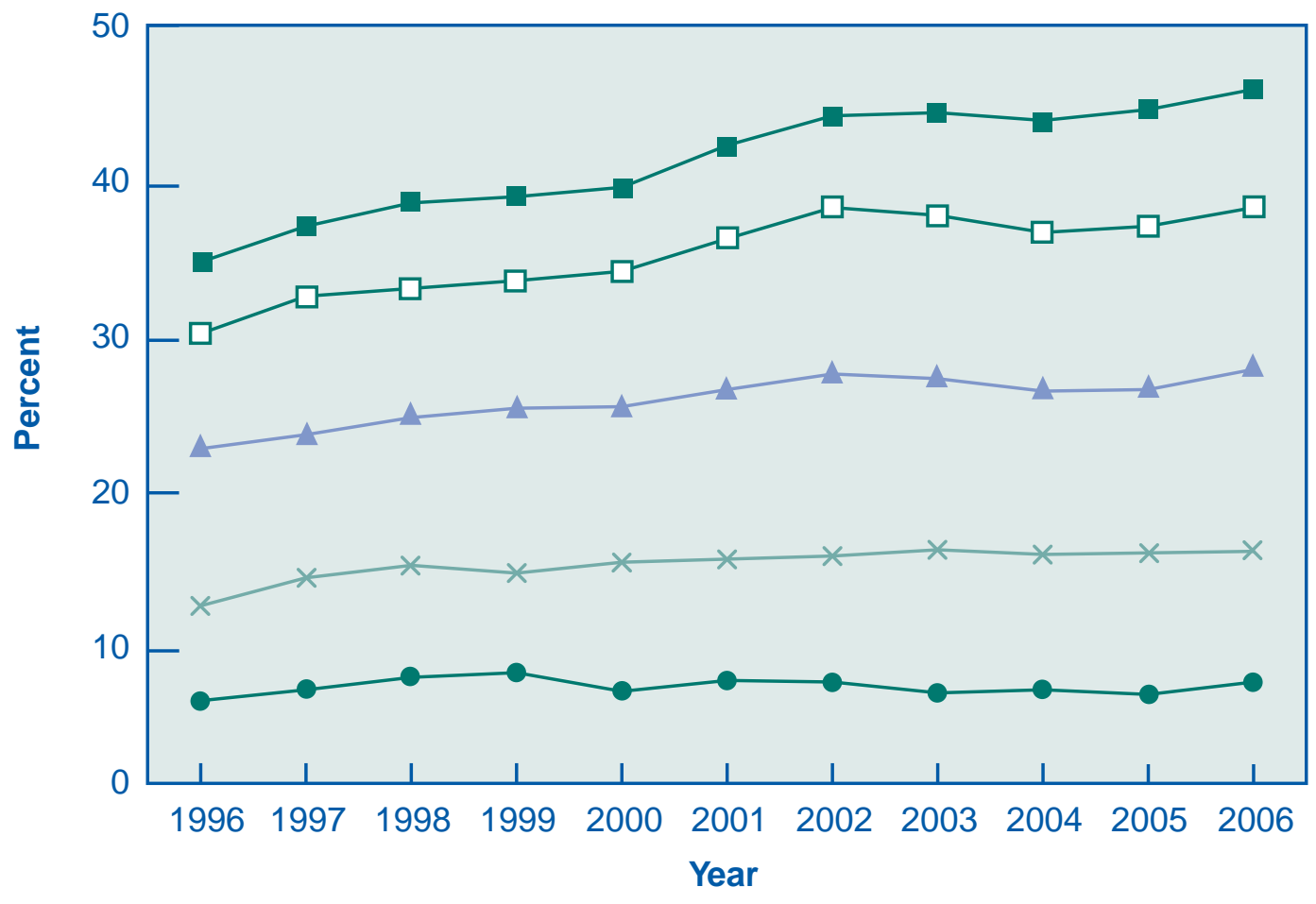

Age Group: $\square<35 \square 35-37 \quad \triangle 38-40 \quad \times 41-42 \quad>42$ 


\section{Have there been improvements in the percentage of transfers that result in singleton live births for all ART patients or only for those in particular age groups?}

Singleton live births are an important measure of success because they have a much lower risk than multiple-infant births for adverse infant health outcomes, including prematurity, low birth weight, disability, and death. Figure 57 presents percentages of transfers that resulted in singleton live births, by woman's age, for ART cycles using fresh nondonor eggs or embryos.

From 1996 through 2006, the percentage of transfers that resulted in singleton live births for women younger than 35 increased about 52\%, from 19\% in 1996 to 29\% in 2006. Over the same period, the percentage of transfers that resulted in singleton live births increased $42 \%$ for women $35-37$, 34\% for women 38-40, 34\% for women 41-42, and 30\% for women older than 42.

\section{Figure 57}

Percentages of Transfers (Using Fresh Nondonor Eggs or Embryos) That Resulted in Singleton Live Births, by Woman's Age, 1996-2006

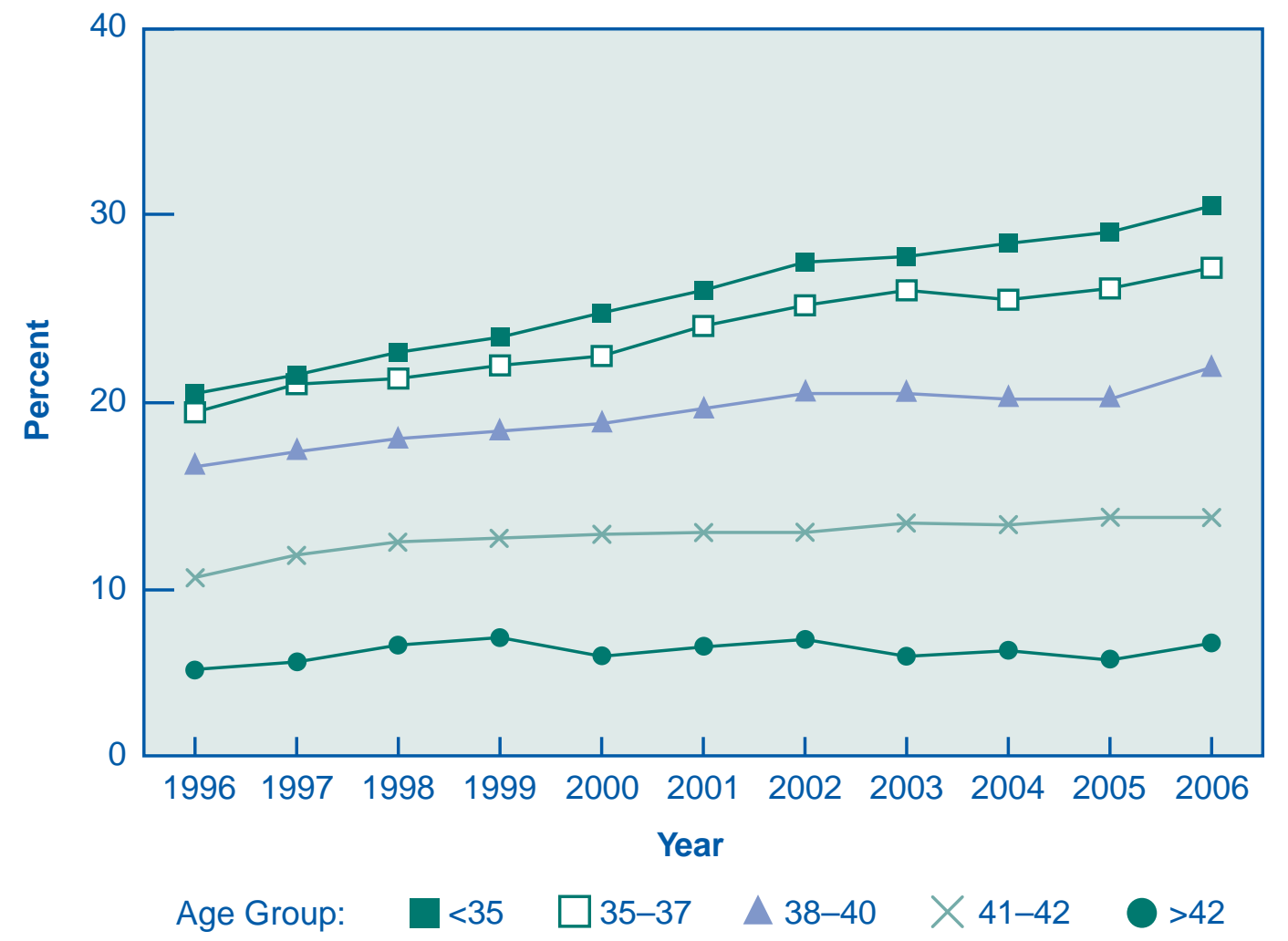




\section{Has the number of embryos transferred in fresh-nondonor cycles changed?}

Figure 58 presents the trends for number of embryos transferred in fresh-nondonor cycles that progressed to the embryo transfer stage. From 1996 through 2006, cycles that involved the transfer of one embryo increased slightly, from $6 \%$ to $11 \%$; cycles that involved the transfer of two embryos increased dramatically, from 10\% in 1996 to $46 \%$ in 2006. Cycles that involved the transfer of three embryos increased from $23 \%$ in 1996 to $28 \%$ in 2006, and cycles that involved the transfer of four or more embryos decreased from 62\% in 1996 to $16 \%$ in 2006.

\section{Figure 58}

Percentages of Fresh-Nondonor Cycles That Involved the Transfer of One, Two, Three, or Four or More Embryos, 1996-2006

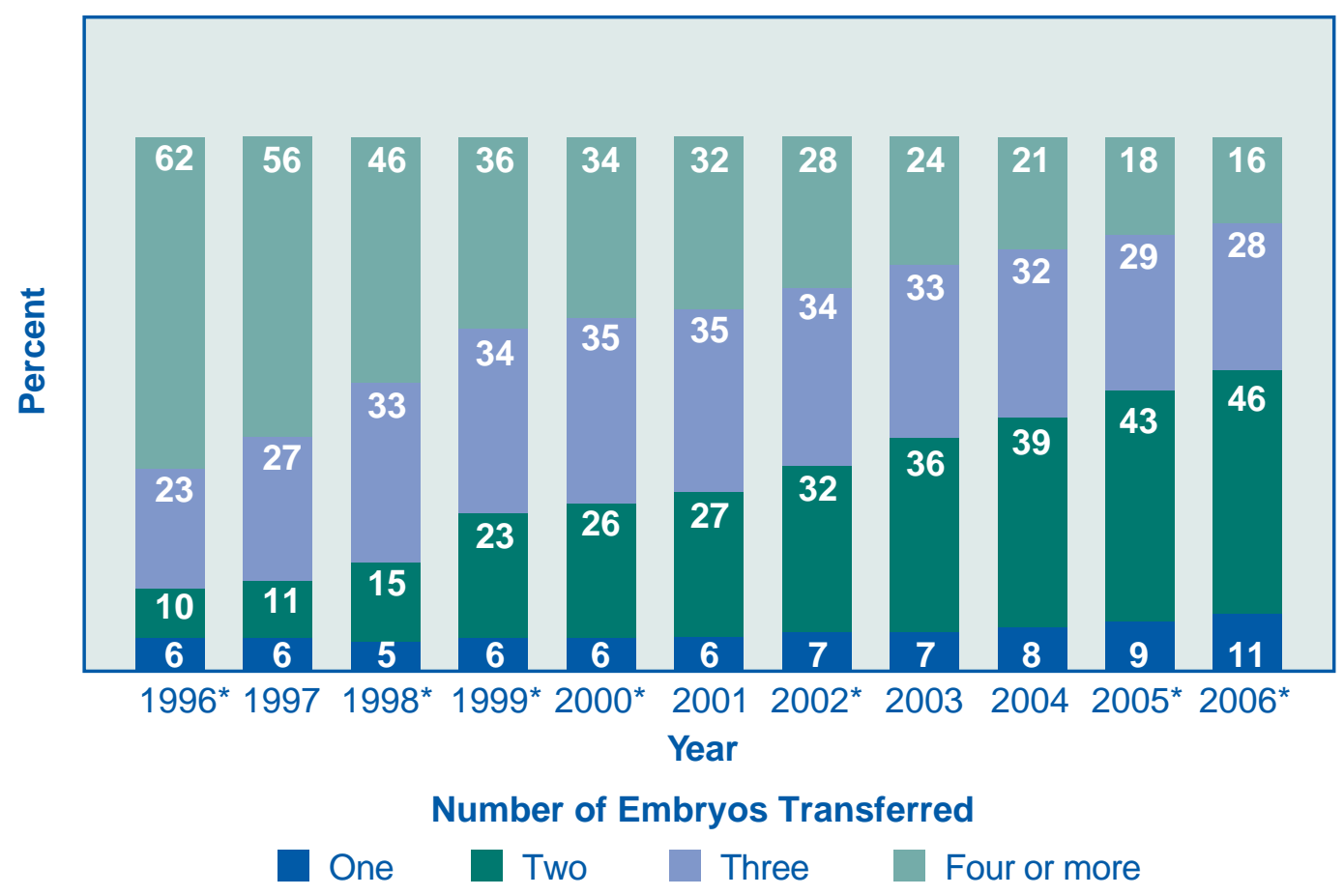

*Totals do not equal $100 \%$ due to rounding. 


\section{Has the number of embryos transferred in each ART cycle changed for women younger than 35 who have more embryos available than they choose to transfer?}

As shown in Figure 58 (page 70), the number of embryos transferred in fresh-nondonor cycles has decreased during the past 11 years. Figure 59 shows the change over time in the number of embryos transferred for ART procedures in which the woman was younger than 35 and the couple chose to set aside some embryos for future cycles rather than transfer all available embryos at one time. Previous research suggests that the number of embryos available for an ART cycle is important in predicting success. Younger women also tend to have higher success rates (see Figure 14, page 26).

Overall, the number of embryos transferred decreased among couples who chose to transfer fewer embryos than were available. In 1996, almost two-thirds (64\%) of ART cycles involved the transfer of four or more embryos; 33\%, three embryos; 3\%, two embryos; and less than 1\%, one embryo. By 1998, the percentage of cycles in which four or more embryos were transferred had decreased to $33 \%$; half of all ART cycles involved the transfer of three embryos; $16 \%$ of cycles, two embryos; and less than $1 \%$, one embryo. By 2006, four or more embryos were transferred in only $3 \%$ of cycles, three in $16 \%$ of cycles, two in $75 \%$ of cycles, and one in $7 \%$ of cycles.

\section{Figure 59}

Percentages of Fresh-Nondonor Cycles That Involved the Transfer of One, Two, Three, or Four or More Embryos in Women Who Were Younger Than 35 and Set Aside Extra Embryos for Future Use, 1996-2006

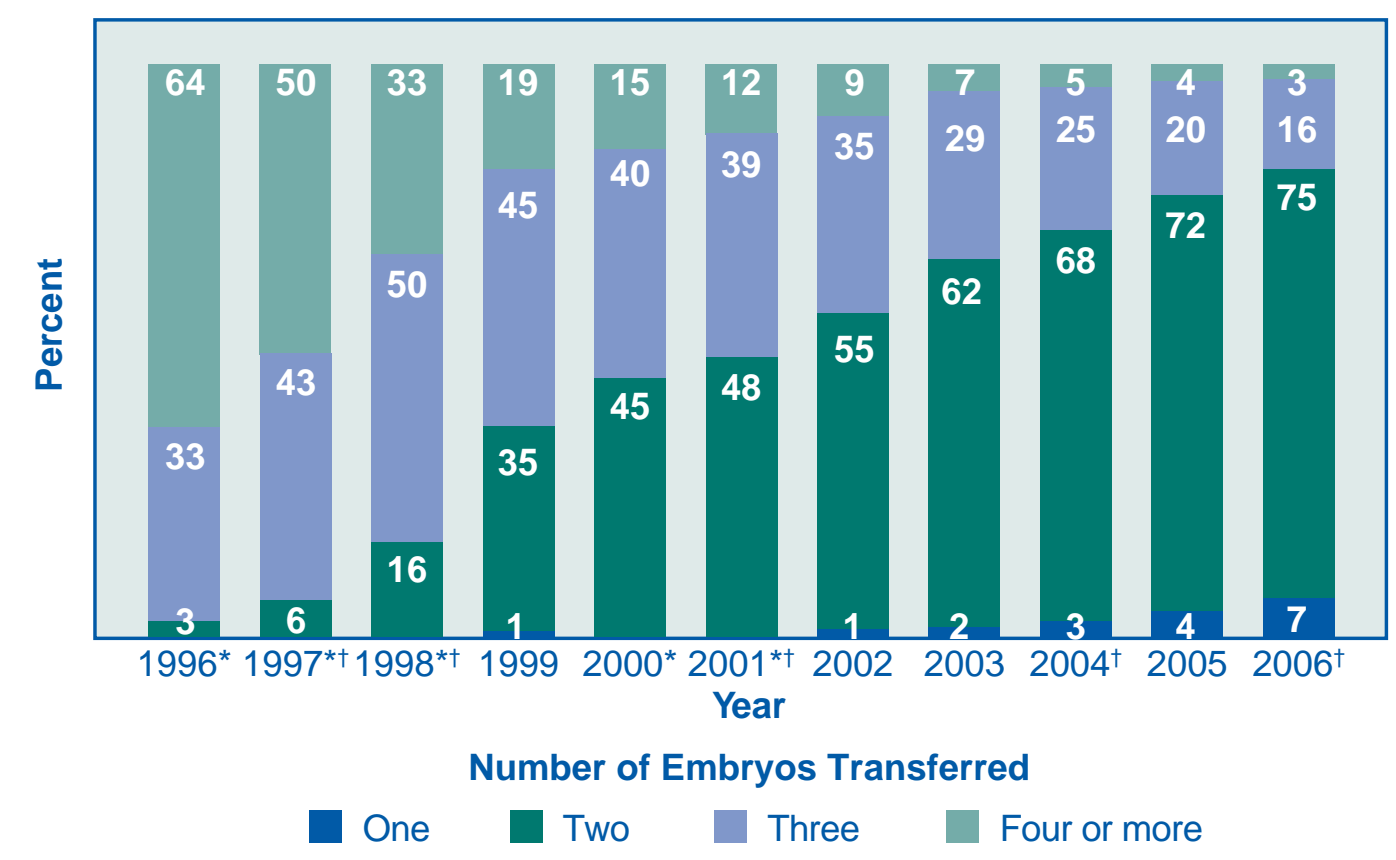

${ }^{*}$ Cycles involving the transfer of one embryo were not included because of the small number of cycles where one embryo was transferred and extra embryos were set aside for future use.

'Totals do not equal $100 \%$ due to rounding. 


\section{Have there been improvements in ART success rates, by number of embryos transferred?}

Figure 60 presents success rates by the number of embryos transferred for ART cycles using fresh nondonor eggs or embryos from 1996 through 2006. In general, success rates were higher when two or more embryos were transferred. From 1996 through 2006, the success rates tripled, from 14\% to $42 \%$, for ART cycles that involved the transfer of two embryos. The success rates also increased for ART cycles that involved the transfer of either one or three embryos; however, the success rates decreased $13 \%$, from $32 \%$ to $28 \%$, for ART cycles that involved the transfer of four or more embryos.

The relationship between number of embryos transferred and success rates is complicated by several factors, such as the woman's age and embryo quality. Trends over time may reflect changes in these factors.

\section{Figure 60}

Percentages of Transfers (Using Fresh Nondonor Eggs or Embryos) That Resulted in Live Births, by Number of Embryos Transferred, 1996-2006

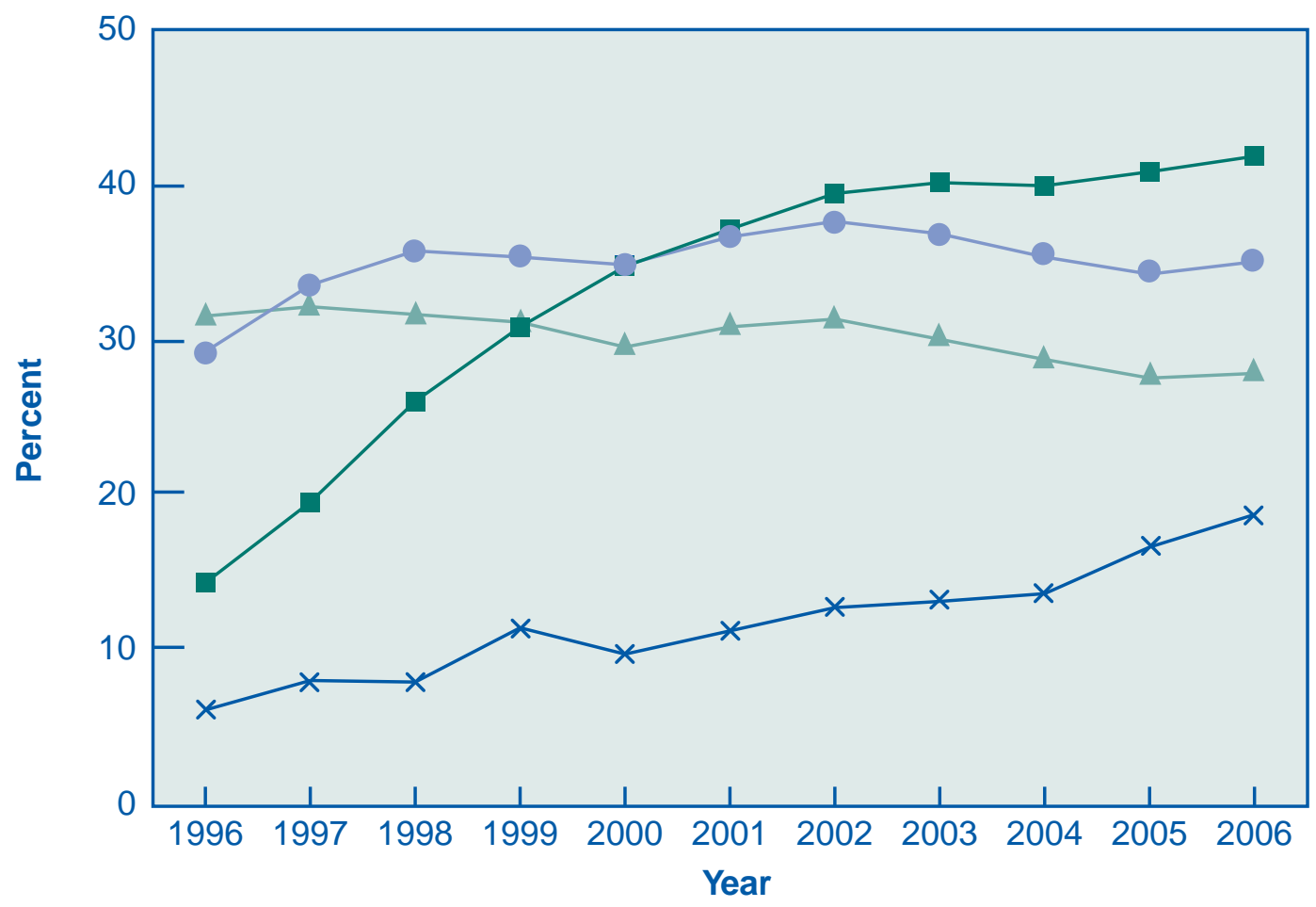

Number of Embryos Transferred
X One
Two
Three
$\Delta$ Four or more 


\section{Have there been improvements in the percentage of transfers that resulted in live births for women younger than 35 who have more embryos available than they choose to transfer?}

Figure 61 shows changes over time in the number of embryos transferred and the percentage of transfers that resulted in live births for ART cycles in which the woman was younger than 35 and chose to set aside some embryos for future cycles rather than transfer all available embryos at one time. Previous research suggests that the number of embryos available for an ART cycle is an important predictor of success. Younger women also tend to have higher success rates (see Figure 14, page 26).

For this group of women, the percentage of transfers that resulted in live births generally increased over time, regardless of the number of embryos transferred. The biggest increase was for cycles in which two embryos were transferred. In 1996, the chance for a live birth was highest (41\%) when three embryos were transferred; however, in 2006, the chance for a live birth was highest (55\%) when two embryos were transferred.

Success rates for cycles involving the transfer of one embryo were comparable to those that involved multiple embryos. Elective single-embryo transfer minimizes the risk for multiple-infant pregnancy and related adverse outcomes. Recently, the Society for Assisted Reproductive Technology (SART) revised its embryo transfer guidelines to encourage single-embryo transfer among patients with good prognoses. ${ }^{* *}$

\section{Figure 61}

Percentages of Transfers That Resulted in Live Births Among Women Who Were Younger Than 35 and Set Aside Extra Embryos for Future Use, by Number of Embryos Transferred, 1996-2006

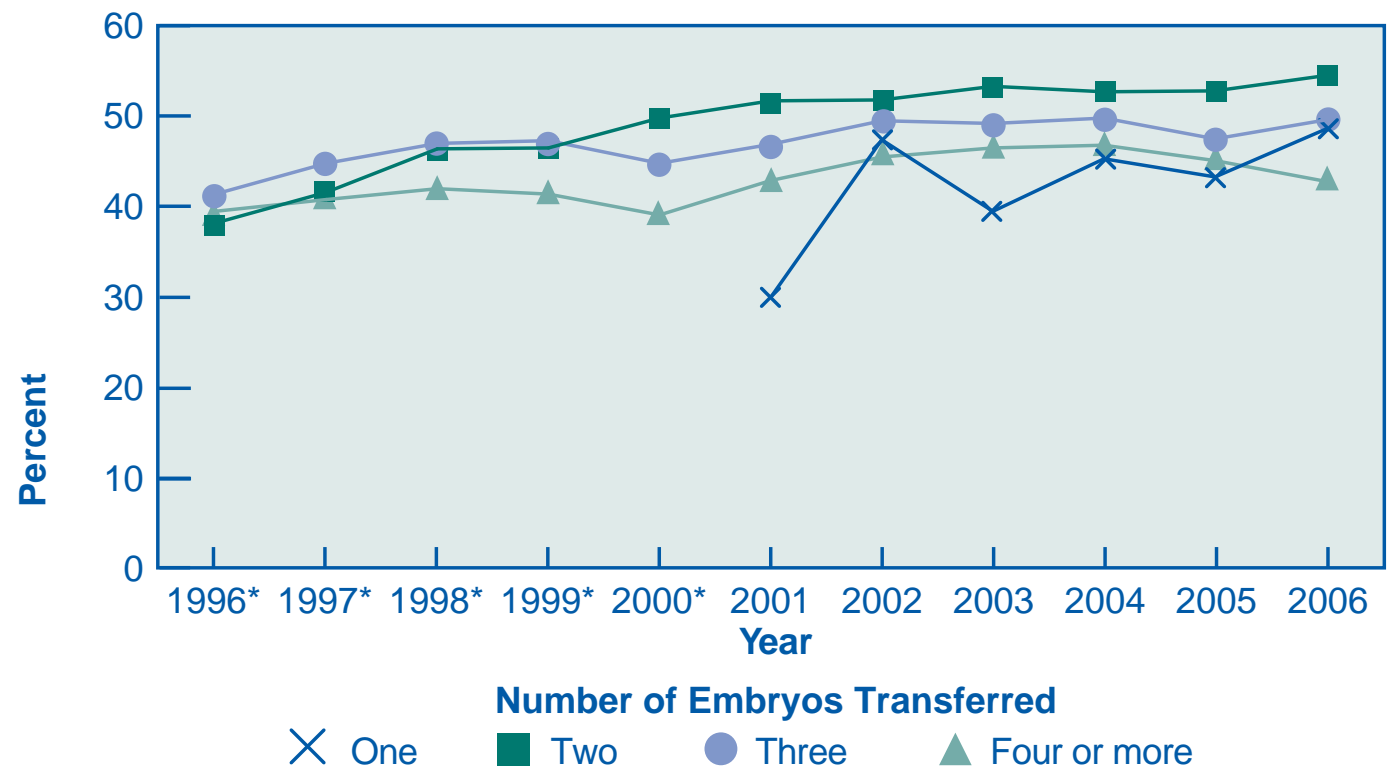

${ }^{*}$ Cycles involving the transfer of one embryo were not included because of the small number of cycles where one embryo was transferred and extra embryos were set aside for future use.

${ }^{* *}$ For more information, contact SART (by telephone at 205-978-5000 or online at www.sart.org). 


\section{Has the percentage of multiple-infant live births changed?}

Multiple-infant births are associated with greater problems for both mothers and infants, including higher rates of caesarean section, prematurity, low birth weight, and infant disability or death. Figure 62 shows the percentages of multiple-infant live births for the four primary types of ART procedures.

For fresh-nondonor cycles, the percentage of multiple-infant live births decreased $20 \%$ since 1996 , from $38 \%$ of all live births in 1996 to $31 \%$ in 2006 . Over the same period, the percentage of multipleinfant live births decreased $10 \%$ for frozen-nondonor cycles and $6 \%$ for fresh-donor cycles. In all years except 1997, the percentage of multiple-infant live births remained stable for frozen-donor cycles.

\section{Figure 62}

Percentages of Cycles That Resulted in Multiple-Infant Live Births, by Type of ART Cycle, 1996-2006

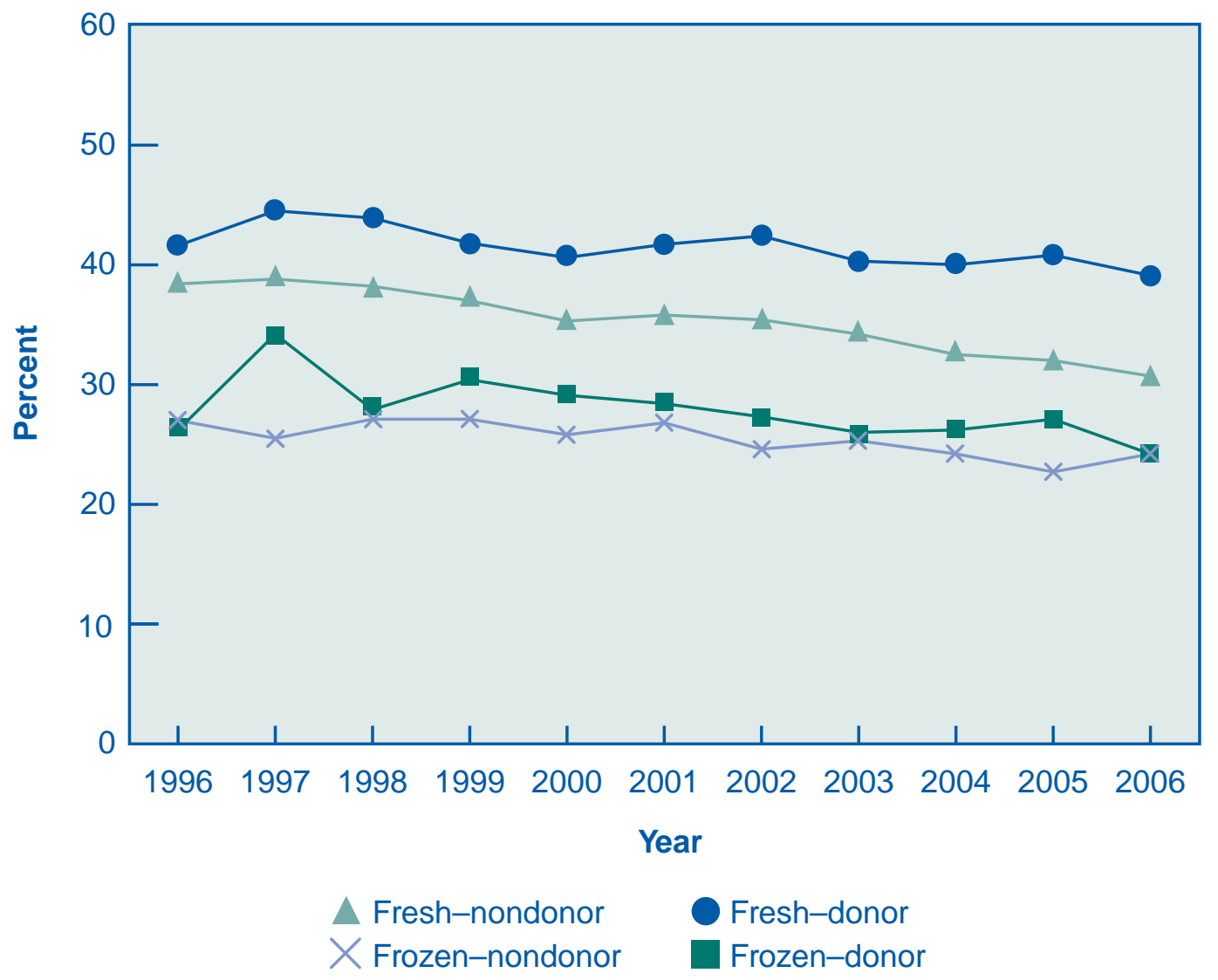




\section{Have multiple-infant live births for cycles using fresh nondonor eggs or embryos changed for all ART patients or only for those in particular age groups?}

Figure 63 shows that the percentages of multiple-infant live births decreased between 1996 and 2006 for women in all age groups. In 1996, 43\% of live-birth deliveries to women younger than 35 were multiple-infant births, compared with 34\% in 2006. Among women older than 42, the percentages of multiple-infant live births decreased from 14\% in 1996 to 9\% in 2006.

\section{Figure 63}

Percentages of Multiple-Infant Live Births,

for Fresh-Nondonor Cycles, by ART Patient's Age, 1996-2006

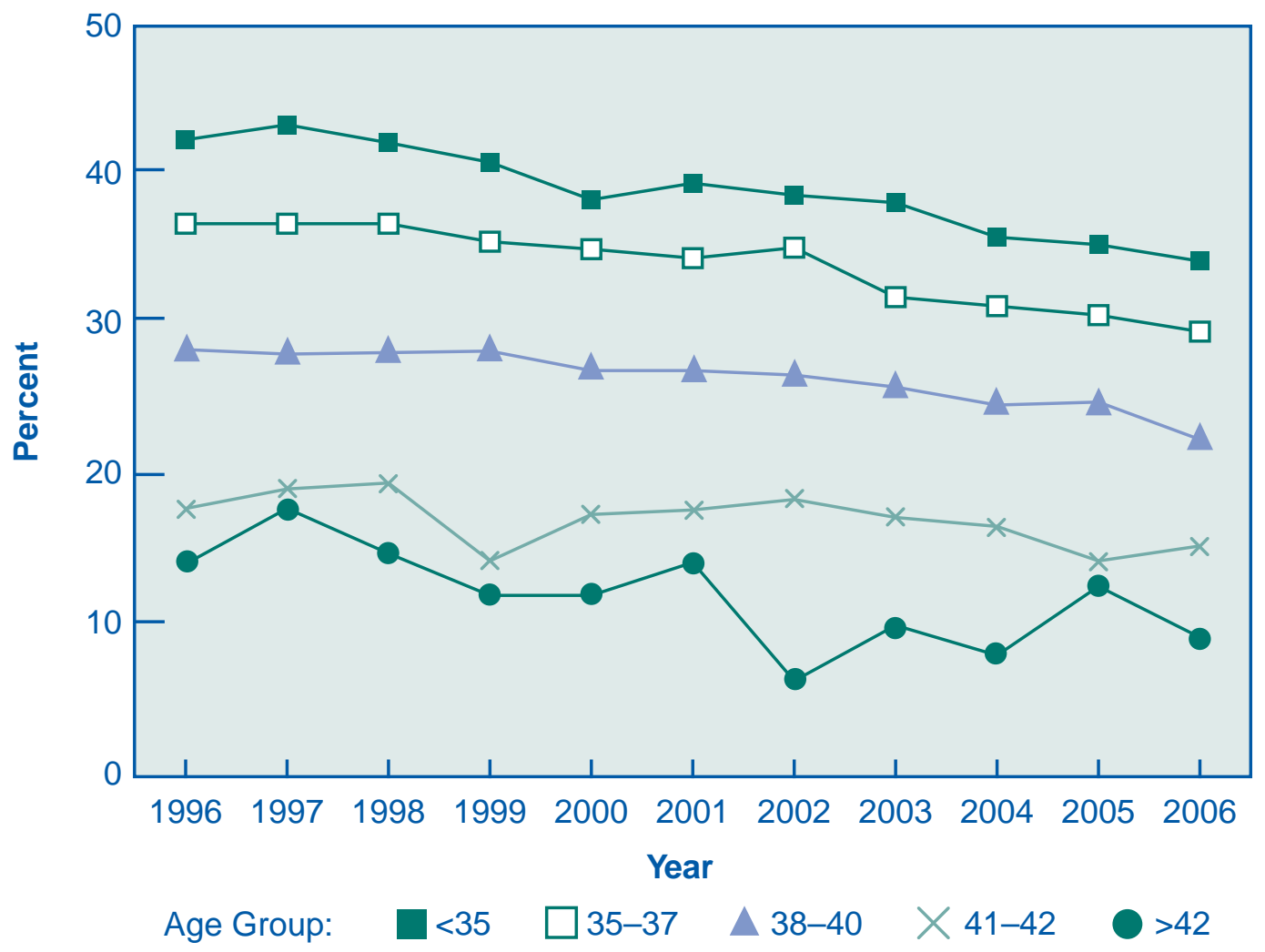




\section{Have the percentages of singletons, twins, and triplets or more changed for ART cycles using fresh nondonor eggs or embryos?}

Figure 64 presents the trends in percentages of transfers that resulted in live births and percentages of multiple-infant live births for ART cycles using fresh nondonor eggs or embryos. Overall, the percentage of transfers that resulted in live births increased from 28\% in 1996 to 35\% in 2006. From 1996 through 2006, the percentage of singleton live births increased from $62 \%$ to $69 \%$; the percentage of twin births remained stable, ranging from $29 \%$ to $32 \%$; and the percentage of triplet-ormore births decreased from 7\% in 1996 to $2 \%$ in 2006.

It is important to note that twins, albeit to a lesser extent than triplets or more, are still at substantially greater risk for illness and death than singletons. These risks include low birth weight, preterm birth, and neurological impairments such as cerebral palsy. Both the percentages of twin and triplet-or-more births remain significantly higher for ART births than for births resulting from natural conception.

\section{Figure 64}

Percentages of Transfers That Resulted in Live Births and Percentages of Multiple-Infant Live Births for ART Cycles Using Fresh Nondonor Eggs or Embryos, 1996-2006

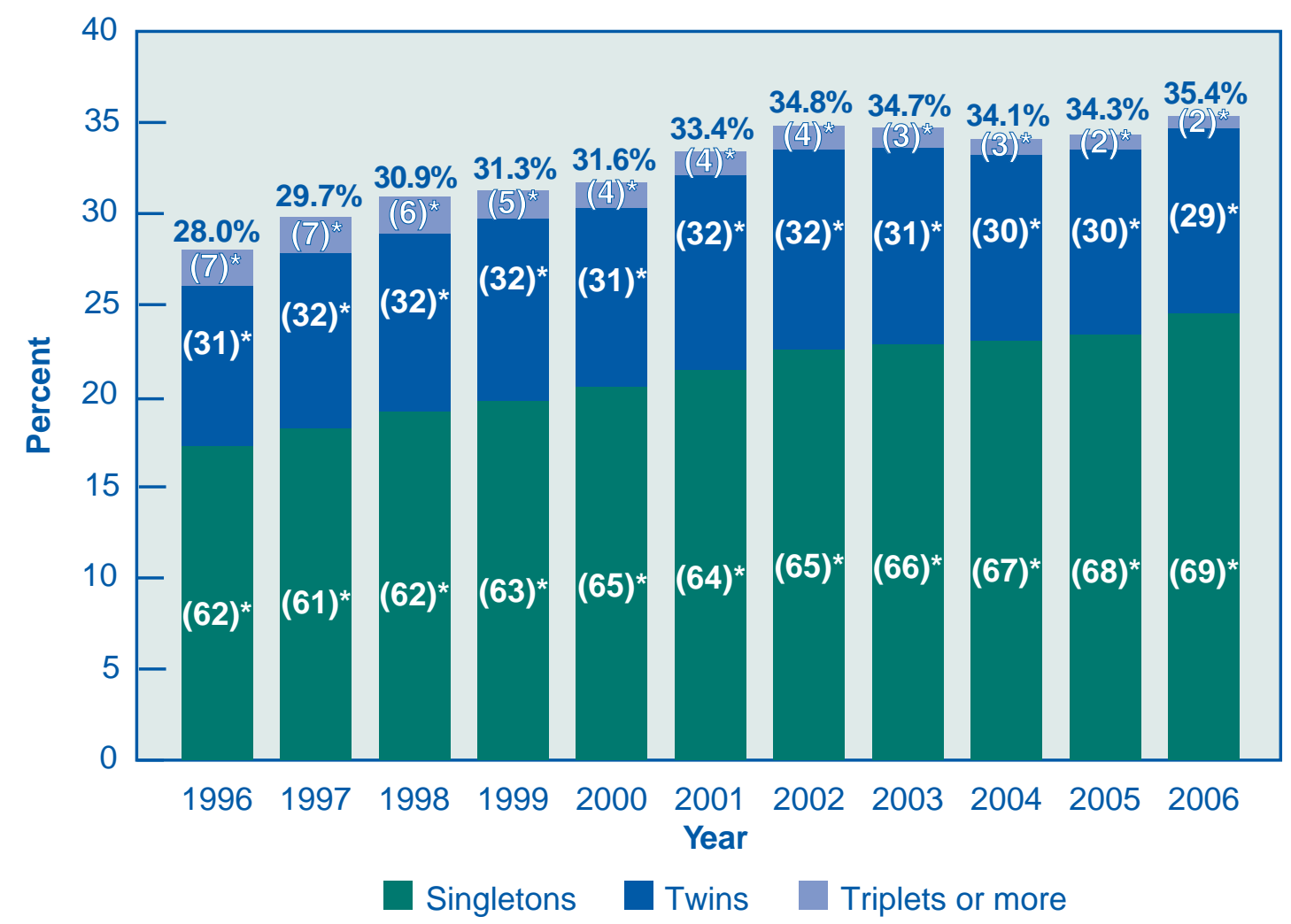

*Percentages of live births that were singletons, twins, and triplets or more are in parentheses.

tTotal does not equal $100 \%$ due to rounding. 
Fertility Clinic Tables 



\section{INTRODUCTION TO FERTILITY CLINIC TABLES}

The first table in this section is the national summary of combined data from all clinics. Individual clinic tables follow, with each clinic's data presented in a one-page table that includes the types of ART used, patient diagnoses, success rates that each clinic reported and verified for 2006, and individual program characteristics. Clinics are listed in alphabetical order by state, city, and clinic name.

Many people considering ART will want to use this report to find the "best" clinic. However, comparisons between clinics must be made with caution. Many factors contribute to the success of an ART procedure. Some factors are related to the training and experience of the ART clinic and laboratory professionals and the quality of services they provide. Other factors are related to the patients themselves, such as their age and the cause of their infertility. Some clinics may be more willing than others to accept patients with low chances of success or may specialize in various ART treatments that attract particular types of patients. These and other factors to consider when interpreting clinic data are discussed below.

\section{Important Factors to Consider When Using These Tables to Assess a Clinic}

- These statistics are for 2006. Data for cycles started in 2006 could not be published until 2008 because the final outcomes of pregnancies conceived in December 2006 were not known until October 2007. Additional time was then required to collect and analyze the data and prepare the report. Many factors that contribute to a clinic's success rate may have changed in the 2 years since these procedures were performed. Personnel may be different. Equipment and training may or may not have been updated. As a result, success rates for 2006 may differ from current rates.

- No reported success rate is absolute. A clinic's success rates vary from year to year even if all determining factors remain the same. The more cycles that a clinic carries out, the less the rate is likely to vary. Conversely, clinics that carry out fewer cycles are likely to have more variability in success rates from year to year. As an extreme example, if a clinic reports only one ART cycle in a given category, as is sometimes the case in the data presented here, the clinic's success rate in that category would be either $0 \%$ or $100 \%$. For further detail, see the explanation of confidence intervals on pages 519-520.

- Some clinics see more than the average number of patients with difficult infertility problems. Some clinics are willing to offer ART to most potential users, even those who have a low probability of success. Others discourage such patients or encourage them to use donor eggs, practices that result in higher success rates among older women. Clinics that accept a higher percentage of women who previously have had multiple unsuccessful ART cycles will generally have lower success rates. In contrast, clinics that offer ART procedures to patients who might have become pregnant with less technologically advanced treatment will have higher success rates.

A related issue is that success rates shown in this report are presented in terms of cycles, as required by law, rather than in terms of women. As a result, women who had more than one ART cycle in 2006 are represented in multiple cycles that cannot be linked. If a woman who underwent several ART cycles at a given clinic either never had a successful cycle or had a successful cycle only after numerous attempts, the clinic's success rates would be lowered. 
- Cancellation rates affect a clinic's success rate. Cancellation rates for cycles using fresh nondonor eggs or embryos vary among clinics from less than $1 \%$ to, in a few cases, more than $30 \%$. A high percentage of cancellations tends to lower the percentage of cycles resulting in live births but may increase the percentage of retrievals resulting in live births and the percentage of transfers resulting in live births.

- Success rates for unstimulated (or "natural") cycles are included with those for stimulated cycles. In an unstimulated cycle, the woman ovulates naturally rather than through the daily injections used in stimulated cycles. Unstimulated cycles are less expensive because they require no daily injections and fewer ultrasounds and blood tests. However, women who use natural or mild stimulation produce only one or two follicles, thus reducing the potential number of embryos for transfer. As a result, unstimulated cycles are less successful, and clinics that carry out a relatively high proportion of unstimulated cycles will have lower success rates. Nationally, fewer than $1 \%$ of ART cycles using fresh nondonor eggs or embryos in 2006 were unstimulated. In a very few clinics, more than $2 \%$ of cycles were unstimulated.

- Success rates are calculated per cycle rather than per patient. Therefore, for patients who undergo both fresh and frozen cycles, success rates are calculated separately for each cycle. Clinics that have a very high percentage of cycles resulting in live births with frozen embryos would have higher ART success rates if these births were included as successes from the original stimulated cycle. Consumers should look at both rates (for cycles using fresh embryos and for those using frozen embryos) when assessing a clinic's success rates.

- The number of embryos transferred varies from clinic to clinic. In 2006, the average number of embryos that a clinic transferred to women younger than age 35 ranged from two to six for freshnondonor cycles. The American Society for Reproductive Medicine and the Society for Assisted Reproductive Technology discourage the transfer of a large number of embryos because it increases the likelihood of multiple gestations. Multiple gestations, in turn, increase both the probability of premature birth and its related problems and the need for multifetal pregnancy reductions.

In addition, success rates can be affected by many other factors, including

- Quality of eggs.

- Quality of sperm (including motility and ability to penetrate the egg).

- Skill and competence of the treatment team.

- General health of the woman.

- Genetic factors.

We encourage consumers considering ART to contact clinics to discuss their specific medical situations and their potential for success using ART. Because clinics did not have the opportunity to provide narratives to explain their data, such conversations could provide additional information to help people decide whether to use ART. 
Although ART offers important options for the treatment of infertility, the decision to use ART involves many factors in addition to success rates. Undergoing repeated ART cycles requires substantial

commitments of time, effort, money, and emotional energy. Therefore, consumers should carefully examine all related financial, psychological, and medical issues before beginning treatment. They also will want to consider the location of the clinic, the counseling and support services available, and the rapport that staff members have with their patients.

An explanation of how to read a fertility clinic table begins on page 83 . 
A comparison of clinic success rates may not be meaningful because patient medical characteristics and treatment approaches vary from clinic to clinic. For more details about this, along with information on how to interpret the statistics in this table, see pages 79-88.

\section{ART CYCLE PROFILE}

\section{Type of ART ${ }^{\mathrm{a}}$}

IVF

GIFT

ZIFT $>99 \%$ Procedural Factors:

Combination $<1 \%$ With ICSI

$<1 \%$ Unstimulated

$53 \%$

$<1 \%$

$<1 \%$

\section{Patient Diagnosis}

Tubal factor $\quad 13 \%$ Other factor

Ovulatory dysfunction

$6 \%$ Unknown factor

Diminished ovarian reserve

9\% Multiple Factors:

Endometriosis

$6 \%$ Female factors only $13 \%$

Uterine factor

$1 \%$ Female \& male factors $18 \%$

Male factor

$17 \%$
42006 PREGNANCY SUCCESS RATES

$$
\text { Type of Cycle }
$$

\section{A Fresh Embryos from Nondonor Eggs}

Number of cycles

Percentage of cycles resulting in pregnancies ${ }^{b}$

Percentage of cycles resulting in live births $s^{b, c}$

6 (Confidence Interval)

Percentage of retrievals resulting in live births $s^{b, c}$

Percentage of transfers resulting in live births ${ }^{\mathrm{b}, \mathrm{c}}$

Percentage of transfers resulting in singleton live births ${ }^{b}$

Percentage of cancellations ${ }^{b}$

Average number of embryos transferred

Percentage of pregnancies with twins ${ }^{b}$

Percentage of pregnancies with triplets or more ${ }^{b}$

Percentage of live births having multiple infants ${ }^{b, c}$

4B Frozen Embryos from Nondonor Eggs

Number of transfers

Percentage of transfers resulting in live births ${ }^{\mathrm{b}, \mathrm{c}}$

Average number of embryos transferred

4C Donor Eggs

Number of transfers

Percentage of transfers resulting in live births ${ }^{\mathrm{b}, \mathrm{c}}$

Average number of embryos transferred

Data verified by X. Y. Zee, MD

\section{Age of Woman}

35-37

38-40

$41-42^{d}$

$<35$

106

68

19

115

37.7

23.5

$5 / 19$

37.4

31.1

20.6

$2 / 19$

(28.5-46.2)

(22.3-39.9)

(11.0-30.2)

42.6

33.3

23.7

$2 / 17$

52.4

34.7

24.1

$2 / 15$

29.3

29.5

19.0

$2 / 15$

12.2

6.6

13.2

$2 / 19$

2.0

2.5

3.8

2.9

38.5

12.5

$4 / 16$

$1 / 5$

3.8

2.5

$1 / 16$

$0 / 5$

44.2

15.2

$3 / 14$

$0 / 2$

$\begin{array}{cccc}62 & 25 & 20 & 14 \\ 27.4 & 24.0 & 20.0 & 2 / 14\end{array}$

2.1

2.0

2.7

3.1

All Ages Combined

Fresh Embryos

49

51.0

2.1
Frozen Embryos

14

$4 / 14$

3.4

\section{CURRENT CLINIC SERVICES AND PROFILE}

Current Name: ART Clinic of the United States

\begin{tabular}{ll|lllll} 
Donor egg? & Yes & Gestational carriers? & Yes & SART member? & Yes \\
\hline Donor embryo? & Yes & Cryopreservation? & Yes & Verified lab accreditation? & Yes \\
Single women? & No & & & (See Appendix C for details.) &
\end{tabular}

${ }^{a}$ Reflects patient and treatment characteristics of ART cycles performed in 2006 using fresh nondonor eggs or embryos.

${ }^{b}$ When fewer than 20 cycles are reported in an age category, rates are shown as a fraction and confidence intervals are not given. Calculating percentages from fractions may be misleading and is not encouraged.

${ }^{c}$ A multiple-infant birth is counted as one live birth.

${ }^{d}$ Clinic-specific outcome rates are unreliable for women older than 42 undergoing ART cycles using fresh or frozen embryos with nondonor eggs. Readers are urged to review national outcomes for these age groups (see page 27).

e All ages (including ages $>42$ ) are reported together because previous data show that patient age does not materially affect success with donor eggs. 


\section{How to Read a Fertility Clinic Table}

This section is provided to help consumers understand the information presented in the fertility clinic tables. The number before each heading refers to the number of the corresponding section in the sample clinic table on the opposite page. Technical terms are defined in the Glossary of Terms (Appendix B).

\section{Type of ART used}

This section gives the breakdown of ART cycle types that each clinic performed using fresh nondonor eggs or embryos (IVF, GIFT, ZIFT, or combinations thereof). It also lists the percentage of procedures that involved intracytoplasmic sperm injection (ICSI), which was not performed by all clinics in 2006; the percentage of cycles that were unstimulated; and the percentage of cycles that used a gestational carrier. (See Glossary of Terms in Appendix B for definitions of IVF, GIFT, ZIFT, ICSI, and gestational carrier.)

\section{ART patient diagnosis}

Consumers may want to know what percentage of a particular clinic's patients have the same diagnosis as they do. (See Glossary of Terms in Appendix B for definitions of diagnoses.) In addition, patients' diagnoses may affect a clinic's success rates. However, the use of these diagnostic categories may vary somewhat from clinic to clinic.

\section{Verification}

To have success rates published in the annual report, a clinic's medical director must verify the accuracy of the tabulated success rates. The name of the individual who verified the clinic's data is shown.

\section{Success rates by type of cycle}

Success rates are given for the three categories of cycles described in 4A-C below: cycles using fresh embryos from nondonor eggs, cycles using frozen embryos from nondonor eggs, and cycles using donor eggs. The ART success rates shown were calculated based on data from all ART cycle types (IVF, both with and without ICSI; GIFT; and ZIFT). Data from these procedures were combined because there was little difference in success rates when we examined each type of ART procedure separately.

The success rates indicate the average chance of success for the given procedure at the clinic in 2006 for each of four age groups. Success rates are calculated as the percentage of cycles started, egg retrievals, or embryo transfers that resulted in either pregnancies or live births at the ART clinic in 2006. For example, if a clinic started a total of 50 cycles in 2006 and these resulted in 15 live births, the average success rate for cycles started at that clinic would be

$$
15 \text { (births) } \div 50 \text { (cycles) }=0.3 \text { or } 30 \% \text {. }
$$

Thus, the success rate at that clinic in 2006 was 30\%, meaning that 30\% of cycles started that year resulted in a live birth.

Success rate calculations are very unstable if they are based on a small number of cycles. Therefore, when fewer than 20 cycles are reported in a given category, the rates are shown as fractions rather than percentages. For example, the sample clinic carried out only 19 fresh embryo cycles using 
nondonor eggs among women aged 41-42 years. Of these 19 cycles, 2 -or $10 \%$-were successful. However, because of the small number of cycles, $10 \%$ is not a statistically reliable success rate, so the success rate is presented as $2 / 19$, meaning 2 out of 19 .

\section{A. Cycles using fresh embryos from nondonor eggs}

This section includes IVF, ICSI, GIFT, and ZIFT cycles that used a woman's own eggs. Cycles that used frozen embryos or donor eggs or embryos are not included here.

\section{- Percentage of cycles resulting in pregnancies}

(Number of pregnancies divided by number of cycles started, expressed as a percentage of cycles)

A stimulated cycle is started when a woman begins taking fertility drugs; an unstimulated cycle is started when egg production begins being monitored. The number of cycles that a clinic starts is not the same as the number of patients that it treats because some women start more than one cycle in a year. Because some pregnancies end in a miscarriage, induced abortion, or stillbirth, the percentage of cycles resulting in pregnancies is usually higher than the percentage of cycles resulting in live births.

\section{- Percentage of cycles resulting in live births}

(Number of live births divided by number of cycles started, expressed as a percentage of cycles)

This number represents the cycles that resulted in a live birth out of all ART cycles started. One live birth may include one or more children born alive; that is, a multiple-infant birth (e.g., twins, triplets) is counted as one live birth.

\section{- Percentage of retrievals resulting in live births}

(Number of live births divided by number of egg retrieval procedures, expressed as a percentage of retrievals)

This number represents the cycles that resulted in a live birth out of all cycles in which an egg retrieval was performed. The number of egg retrievals a clinic performs often is smaller than the number of cycles started because some cycles are canceled before the woman has an egg retrieved. As a result, the percentage of retrievals resulting in live births is usually higher than the percentage of cycles resulting in live births. Cycles are canceled for many reasons: eggs may not develop, the patient may become ill, or the patient may choose to stop treatment (see Figure 6, page 18).

\section{- Percentage of transfers resulting in live births}

(Number of live births divided by number of embryo transfer procedures, expressed as a percentage of transfers)

This number represents the cycles that resulted in a live birth out of all cycles in which one or more embryos were transferred into the woman's uterus or, in the case of GIFT and ZIFT, egg and sperm or embryos were transferred into the woman's fallopian tubes. A clinic may carry out more egg retrievals than embryo transfers because not every retrieval results in egg fertilization and embryo transfer. For this reason, the percentage of transfers resulting in live births generally will be higher than those reported for egg retrievals and for cycles started. 


\section{- Percentage of transfers resulting in singleton live births}

(Number of singleton live births divided by number of embryo transfer procedures, expressed as a percentage of transfers)

This number represents the cycles that resulted in the birth of a single infant out of all cycles in which one or more embryos were transferred into the woman's uterus or, in the case of GIFT and ZIFT, egg and sperm or embryos were transferred into the woman's fallopian tubes. Singleton births have a much lower risk than multiple-infant births for adverse infant health outcomes, including prematurity, low birth weight, disability, and death.

\section{- Percentage of cancellations}

(Number of cycles canceled divided by the total number of cycles, expressed as a percentage of cycles)

This number refers to the cycles that were stopped before an egg was retrieved. A cycle may be canceled if a woman's ovaries do not respond to fertility medications and thus do not produce a sufficient number of follicles. Cycles also may be canceled because of illness or other medical or personal reasons.

\section{- Average number of embryos transferred}

(Average number of embryos per embryo transfer procedure)

The average number of embryos transferred varies from clinic to clinic. The American Society for Reproductive Medicine (ASRM) and the Society for Assisted Reproductive Technology (SART) have practice guidelines that address this issue.

\section{- Percentage of pregnancies with twins}

(Number of pregnancies with two fetuses divided by the total number of pregnancies, expressed as a percentage of pregnancies)

A pregnancy with two fetuses is counted as one pregnancy.

\section{- Percentage of pregnancies with triplets or more}

(Number of pregnancies with three or more fetuses divided by the total number of pregnancies, expressed as a percentage of pregnancies)

Pregnancies with multiple fetuses can be associated with increased risk for mothers and infants (e.g., higher rates of caesarean section, prematurity, low birth weight, infant death) and the possibility of multifetal pregnancy reduction.

A pregnancy with three or more fetuses is counted as one pregnancy.

\section{- Percentage of live births having multiple infants}

(Number of deliveries resulting in a birth of more than one infant divided by the number of live births, expressed as a percentage of live births)

A delivery of one or more live-born infants is counted as one live birth. 


\section{B. Cycles using frozen embryos from nondonor eggs}

Frozen (cryopreserved) embryo cycles are those in which previously frozen embryos are thawed and then transferred. Because frozen embryo cycles use embryos formed from a previous stimulated cycle, no stimulation or retrieval is involved. As a result, these cycles usually are less expensive and less invasive than cycles using fresh embryos. In addition, freezing some of the embryos from a retrieval procedure may increase a woman's overall chances of having a child from a single retrieval.

\section{C. Cycles using donor eggs}

Success rates are presented separately for cycles using fresh donor eggs or embryos and those using frozen donor embryos. Older women, women with premature ovarian failure (early menopause), women whose ovaries have been removed, and women with a genetic concern about using their own eggs may consider using eggs that are donated by a young, healthy woman. Embryos donated by couples who previously had ART also may be available. Many clinics provide services for donor egg and embryo cycles. For these cycle types, results from women in all age groups (including older than 42) are reported together because previous data show that patient age does not affect success rates with donor eggs (see Figures 45 and 46 on pages 57 and 58).

\section{Age of woman}

Because a woman's fertility declines with age, clinics report lower success rates for older women attempting to become pregnant with their own eggs. For this reason, rates for women using nondonor eggs or embryos are reported separately for women younger than age 35, for women 35-37, for women 38-40, and for women 41-42. Clinic-specific outcome rates are not shown for women older than 42 who undergo ART using their own eggs because the number of women in this age group at each clinic is small; therefore, a calculation of the percentage of cycles resulting in live births in older age groups may not be meaningful. Readers are encouraged to review national outcomes for these age groups shown on page 27. The sample clinic table illustrates the decline in ART success rates among older women. For example, for cycles that used fresh embryos from nondonor eggs, the percentage of cycles resulting in live births among women younger than 35 was 37.4\%, whereas the percentage of cycles resulting in live births among women aged 38-40 was 20.6\%.

\section{Confidence interval}

The tables show a range, called the $\mathbf{9 5 \%}$ confidence interval, that conveys the reliability of a clinic's demonstrated success rate. This range is calculated only if 20 or more cycles are reported in an age category. (When fewer than 20 cycles are reported in a given category, success rates are shown as fractions rather than percentages; see paragraph 4, Success rates by type of cycle, page 83.) In general, the more cycles that a clinic performs, the narrower the range. A narrow range means we are more confident that a clinic would have a similar success rate if it treated other similar groups of patients under similar clinical conditions. On the other hand, a wide range tells us that a clinic's success rate is more likely to vary under similar circumstances because we had less information (fewer cycles) on which to base our estimates. Even though one clinic's success rate may appear higher than another's based on the confidence intervals, these confidence intervals are only one indication that the success rate may be better. Other factors also must be considered when comparing rates from two clinics. For example, some clinics see more than the average number of patients with difficult 
infertility problems, whereas others discourage patients with a low probability of success. For further information on important factors to consider when using the tables to assess a clinic, refer to pages $79-81$.

For a more detailed explanation and examples of confidence intervals, see pages 519-520 in Appendix A.

\section{Clinic services and profile}

- Current name. This name reflects name changes that may have occurred since 2006, whereas the clinic name at the top of the table was the name of the ART clinic as it existed in 2006. Some clinics not only have changed their names but have reorganized as well. Reorganization is defined as a change in ownership or affiliation or a change in at least two of the three key staff positions (practice director, medical director, or laboratory director). In such cases, no current name will be listed, but a statement will be included that the clinic has undergone reorganization since 2006. Also, in such cases, no current clinic services or profile will be listed.

- Donor egg program. Some clinics have programs for ART using donor eggs. Donor eggs are eggs that have been retrieved from one woman (the donor) and then transferred to another woman who is unable to conceive with her own eggs (the recipient). Policies regarding sharing of donor eggs vary from clinic to clinic.

- Donor embryo. These are embryos that were donated by another couple who previously underwent ART treatment and had extra embryos available.

- Single women. Clinics have varying policies regarding ART services for single (unmarried) women.

- Gestational carriers. A gestational carrier is a woman who carries a child for another woman; sometimes such women are referred to as gestational surrogates. Policies regarding ART services using gestational carriers vary from clinic to clinic. Some states do not permit clinics to offer this service.

- Cryopreservation. This item refers to whether the clinic has a program for freezing extra embryos that may be available from a couple's ART cycle.

- SART member. In 2006, 378 of the 426 reporting clinics were Society for Assisted Reproductive Technology (SART) members.

- Verified lab accreditation. If "yes" appears next to this item, the ART clinic uses an embryo laboratory accredited by one of the following organizations:

- College of American Pathologists (CAP)/American Society for Reproductive Medicine (ASRM), Reproductive Laboratory Accreditation Program.

- Joint Commission on Accreditation of Healthcare Organizations (JCAHO).

- New York State Tissue Bank Program (NYSTB).

If "pending" appears here, it means that the clinic has submitted an application for accreditation to one of the above organizations and has provided proof of such application to Westat. "No" indicates that the embryo laboratory has not been accredited by any of these three organizations. 
CDC provides this information as a public service. Please note that CDC does not oversee any of these accreditation programs. They are all nonfederal programs. To become certified, laboratories must have in place systems and processes that comply with the accrediting organization's standards. Depending on the organization, standards may include those for personnel, quality control and quality assurance, specimen tracking, results reporting, and the performance of technical procedures.

Compliance with these standards is confirmed by documentation provided by the laboratory and by on-site inspections. For further information, consumers may contact the following accrediting organizations directly:

- CAP/ASRM, Reproductive Laboratory Accreditation Program: For a list of accredited laboratories, call 800-323-4040 and ask for Laboratory Accreditation.

- JCAHO: Call 630-792-5000 to inquire about the status of individual laboratories.

- New York State: Call 518-485-5341 to find out which laboratories are certified under the tissue bank regulations.

Further information on laboratory accreditation is provided in Appendix C. 


\section{NATIONAL SUMMARY}

A comparison of clinic success rates may not be meaningful because patient medical characteristics and treatment approaches vary from clinic to clinic. For more details about this, along with information on how to interpret the statistics in this table, see pages 79-88.

\section{ART CYCLE PROFILE}

\section{Type of ART}

IVF $\quad>99 \%$ Procedural Factors:

ZIFT

Combination
GIFT $\quad<1 \%$ With ICSI
$<1 \%$ Unstimulated

$<1 \%$ Used gestational carrier Used PGD

$\begin{aligned} & \text { Tubal factor } \\ 62 \% & \text { Ovulatory dysfunction } \\ <1 \% & \text { Diminished ovarian reserve } \\ 1 \% & \text { Endometriosis } \\ 5 \% & \text { Uterine factor } \\ & \text { Male factor }\end{aligned}$

\section{Patient Diagnosis}

$9 \%$ Other factor

6\% Unknown factor

12\% Multiple Factors:

$5 \%$ Female factors only $12 \%$

$1 \%$ Female \& male factors $18 \%$

\section{PREGNANCY SUCCESS RATES}

\section{Type of Cycle}

\section{Fresh Embryos from Nondonor Eggs}

Number of cycles

Percentage of cycles resulting in pregnancies

Percentage of cycles resulting in live births ${ }^{b}$

Percentage of retrievals resulting in live births ${ }^{b}$

Percentage of transfers resulting in live births ${ }^{b}$

Percentage of transfers resulting in singleton live births ${ }^{b}$

Percentage of cancellations

Average number of embryos transferred

Percentage of pregnancies with twins

Percentage of pregnancies with triplets or more

Percentage of live births having multiple infants ${ }^{b}$

Number of transfers

Percentage of transfers resulting in live births ${ }^{b}$

Average number of embryos transferred

\section{Donor Eggs}

Number of transfers

Percentage of transfers resulting in live births ${ }^{b}$

Average number of embryos transferred

\section{Frozen Embryos from Nondonor Eggs}

\section{Age of Woman}

$\begin{array}{cccc}<35 & \mathbf{3 5 - 3 7} & \mathbf{3 8 - 4 0} & \mathbf{4 1 - 4 2}^{\mathbf{C}} \\ 41,369 & 23,376 & 19,775 & 9,346 \\ 44.6 & 37.1 & 27.7 & 17.7 \\ 38.7 & 30.4 & 20.6 & 10.8 \\ 41.8 & 34.4 & 24.1 & 13.0 \\ 44.7 & 37.1 & 26.7 & 15.1 \\ 29.3 & 26.0 & 20.7 & 12.7 \\ 7.6 & 11.4 & 14.5 & 17.6 \\ 2.3 & 2.5 & 2.9 & 3.2 \\ 32.4 & 27.0 & 20.9 & 13.3 \\ 3.8 & 4.3 & 3.8 & 2.5 \\ 34.4 & 29.8 & 22.6 & 15.4 \\ & & & \\ 10,154 & 5,299 & 3,006 & 963 \\ 33.0 & 27.7 & 23.2 & 20.5 \\ 2.3 & 2.4 & 2.4 & 2.6\end{array}$

All Ages Combined ${ }^{\text {d }}$

\section{Fresh Embryos}

10,049

53.7

2.3

\section{CURRENT CLINIC SERVICES AND PROFILE}

Total number of reporting clinics: 426
Percentage of clinics that offer the following services:

Donor egg $\quad 93$

Donor embryo 65

Single women 90
Gestational carriers $\quad 81$

Cryopreservation $\quad 100$
Clinic profile:

SART member

Verified lab accreditation

Yes

91

No

Pending
5,456

32.1

${ }^{a}$ Reflects patient and treatment characteristics of ART cycles performed in 2006 using fresh nondonor eggs or embryos.

${ }^{\mathrm{b}}$ A multiple-infant birth is counted as one live birth.

${ }^{\mathrm{C}}$ See page 27 for national summary statistics for women older than 42 .

${ }^{d}$ All ages (including ages $>42$ ) are reported together because previous data show that patient age does not materially affect success with donor eggs. 



\section{ALABAMA FERTILITY SPECIALISTS BIRMINGHAM, ALABAMA}

A comparison of clinic success rates may not be meaningful because patient medical characteristics and treatment approaches vary from clinic to clinic. For more details about this, along with information on how to interpret the statistics in this table, see pages 79-88.

\section{ART CYCLE PROFILE}

\begin{tabular}{|c|c|c|c|c|c|c|c|}
\hline \multicolumn{4}{|c|}{ Type of ART ${ }^{a}$} & \multicolumn{4}{|c|}{ Patient Diagnosis } \\
\hline IVF & $100 \%$ & Procedural Factors: & & Tubal factor & $12 \%$ & Other factor & $1 \%$ \\
\hline GIFT & $0 \%$ & With ICSI & $46 \%$ & Ovulatory dysfunction & $11 \%$ & Unknown factor & $3 \%$ \\
\hline ZIFT & $0 \%$ & Unstimulated & $0 \%$ & Diminished ovarian reserve & $4 \%$ & Multiple Factors: & \\
\hline \multirow[t]{3}{*}{ Combination } & $0 \%$ & Used gestational carrier & $0 \%$ & Endometriosis & $8 \%$ & Female factors only & $19 \%$ \\
\hline & & & & Uterine factor & $0 \%$ & Female \& male factors & $32 \%$ \\
\hline & & & & Male factor & $10 \%$ & & \\
\hline
\end{tabular}

2006 PREGNANCY SUCCESS RATES

Data verified by Michael P. Steinkampf, MD

\section{Type of Cycle}

\section{Fresh Embryos from Nondonor Eggs}

Number of cycles

Percentage of cycles resulting in pregnancies ${ }^{b}$

Percentage of cycles resulting in live births ${ }^{b, c}$

(Confidence Interval)

Percentage of retrievals resulting in live births $s^{b, c}$

Percentage of transfers resulting in live births $s^{b, c}$

Percentage of transfers resulting in singleton live births ${ }^{b}$

Percentage of cancellations ${ }^{b}$

Average number of embryos transferred

Percentage of pregnancies with twins ${ }^{b}$

Percentage of pregnancies with triplets or more ${ }^{b}$

Percentage of live births having multiple infants ${ }^{b, c}$

\section{Frozen Embryos from Nondonor Eggs}

Number of transfers

Percentage of transfers resulting in live births $s^{b, c}$

Average number of embryos transferred

\section{Donor Eggs}

Number of transfers

Percentage of transfers resulting in live births $s^{b, c}$

Average number of embryos transferred

\section{Age of Woman}

35-37 38-40 $41-42^{d}$

\section{$<35$}

8

$1 / 8$

5

38.2

35.3

$1 / 8$

$0 / 5$

$0 / 5$

$(19.7-53.5)$

38.7

40.0

$1 / 7$

$0 / 4$

26.7

$1 / 6$

$0 / 4$

$0 / 6$

$0 / 4$

8.8

$1 / 8$

$1 / 5$

3.3

$5 / 13$

2.8

$1 / 1$

$0 / 13$

$0 / 1$

$4 / 12$

$1 / 1$

8

$1 / 8$

2.3

\section{4}

$0 / 4$

2.3
1

$0 / 1$

$0 / 1$

$\%$

$3 \%$

$9 \%$

$32 \%$

\section{CURRENT CLINIC SERVICES AND PROFILE}

Current Name: Alabama Fertility Specialists
Donor egg?
Yes
Donor embryo? No
Gestational carriers?
No
Cryopreservation?
Yes
Single women? Yes
${ }^{a}$ Reflects patient and treatment characteristics of ART cycles performed in 2006 using fresh nondonor eggs or embryos.
${ }^{b}$ When fewer than 20 cycles are reported in an age category, rates are shown as a fraction and confidence intervals are not given. Calculating percentages from fractions may be misleading and is not encouraged.
c A multiple-infant birth is counted as one live birth.
d Clinic-specific outcome rates are unreliable for women older than 42 undergoing ART cycles using fresh or frozen embryos with nondonor eggs. Readers are urged to review national outcomes for these age groups (see page 27).
e All ages (including ages $>42$ ) are reported together because previous data show that patient age does not materially affect success with donor eggs.

Fresh Embryos

All Ages Combined ${ }^{\mathrm{e}}$

$\begin{array}{cc}5 & 3 \\ 2 / 5 & 0 / 3 \\ 2.0 & 2.0\end{array}$

2
$0 / 2$
3.5
bined
Frozen Embryos
$\quad \begin{aligned} & 3 \\ & 0 / 3 \\ & 2.0\end{aligned}$

$1 / 1$ 


\section{ART FERTILITY PROGRAM OF ALABAMA \\ BIRMINGHAM, ALABAMA}

A comparison of clinic success rates may not be meaningful because patient medical characteristics and treatment approaches vary from clinic to clinic. For more details about this, along with information on how to interpret the statistics in this table, see pages 79-88.

\section{ART CYCLE PROFILE}

\section{Type of ART ${ }^{\mathrm{a}}$}

IVF

GIFT

100\% Procedural Factors:

ZIFT

Combination

\author{
$0 \%$ With ICSI
}

$0 \%$ Unstimulated

0\% Used gestational carrier

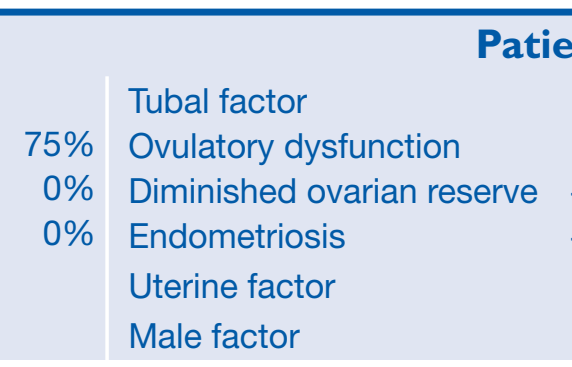

Patient Diagnosis

$\begin{array}{rlr}2 \% & \text { Other factor } & 2 \% \\ 2 \% & \text { Unknown factor } & 1 \% \\ <1 \% & \text { Multiple Factors: } & \\ <1 \% & \text { Female factors only } & 15 \% \\ 0 \% & \text { Female \& male factors } & 66 \% \\ 12 \% & & \end{array}$

2006 PREGNANCY SUCCESS RATES

Data verified by Kathryn L. Honea, MD

\section{Type of Cycle}

\section{Fresh Embryos from Nondonor Eggs}

Number of cycles

Percentage of cycles resulting in pregnancies ${ }^{b}$

Percentage of cycles resulting in live births $s^{b, c}$

(Confidence Interval)

Percentage of retrievals resulting in live births $s^{b, c}$

Percentage of transfers resulting in live births $s^{b, c}$

Percentage of transfers resulting in singleton live births ${ }^{b}$

Percentage of cancellations ${ }^{b}$

Average number of embryos transferred

Percentage of pregnancies with twins ${ }^{b}$

Percentage of pregnancies with triplets or more ${ }^{b}$

Percentage of live births having multiple infants ${ }^{b, c}$

\section{Frozen Embryos from Nondonor Eggs}

Number of transfers

Percentage of transfers resulting in live births $s^{b, c}$

Average number of embryos transferred

\section{Donor Eggs}

Number of transfers

Percentage of transfers resulting in live births $s^{b, c}$

Average number of embryos transferred

\section{Age of Woman}

35-37 38-40

$41-42^{d}$

$<35$

56

45

11

179

37.5

33.3

$2 / 11$

38.5

28.6

26.7

$0 / 11$

(31.4-46.1)

$(17.3-42.2)$

(14.6-41.9)

44.2

30.8

33.3

$0 / 10$

46.3

34.0

35.3

$0 / 10$

30.9

27.7

32.4

$0 / 10$

12.8

7.1

20.0

$1 / 11$

2.0

2.1

32.9

28.6

2.7

0.0

2.8

2.9

33.3

$3 / 16$

$3 / 15$

$0 / 2$

$0 / 15$

$0 / 2$

$1 / 12$

22

22.7

8

$2 / 8$

2

$0 / 2$

2.2

2.0

1.0

All Ages Combined ${ }^{\mathrm{e}}$

Fresh Embryos

Frozen Embryos

35

68.6

9

2.1

$3 / 9$

2.0

\section{CURRENT CLINIC SERVICES AND PROFILE}

Current Name: ART Fertility Program of Alabama

Donor egg? Yes

Donor embryo? Yes

Single women? No

\begin{abstract}
Gestational carriers? Yes
Cryopreservation? Yes
\end{abstract}

SART member?

Yes

Verified lab accreditation?

(See Appendix C for details.)

${ }^{a}$ Reflects patient and treatment characteristics of ART cycles performed in 2006 using fresh nondonor eggs or embryos.

${ }^{b}$ When fewer than 20 cycles are reported in an age category, rates are shown as a fraction and confidence intervals are not given. Calculating percentages from fractions may be misleading and is not encouraged.

${ }^{c}$ A multiple-infant birth is counted as one live birth.

d Clinic-specific outcome rates are unreliable for women older than 42 undergoing ART cycles using fresh or frozen embryos with nondonor eggs. Readers are urged to review national outcomes for these age groups (see page 27).

${ }^{e}$ All ages (including ages $>42$ ) are reported together because previous data show that patient age does not materially affect success with donor eggs. 


\section{UNIVERSITY OF ALABAMA AT BIRMINGHAM BIRMINGHAM, ALABAMA}

A comparison of clinic success rates may not be meaningful because patient medical characteristics and treatment approaches vary from clinic to clinic. For more details about this, along with information on how to interpret the statistics in this table, see pages 79-88.

\section{ART CYCLE PROFILE}

\section{Type of ART ${ }^{\mathrm{a}}$}

IVF

GIFT

ZIFT

Combination
98\% Procedural Factors:

$2 \%$ With ICSI

$0 \%$ Unstimulated

$0 \%$ Used gestational carrier

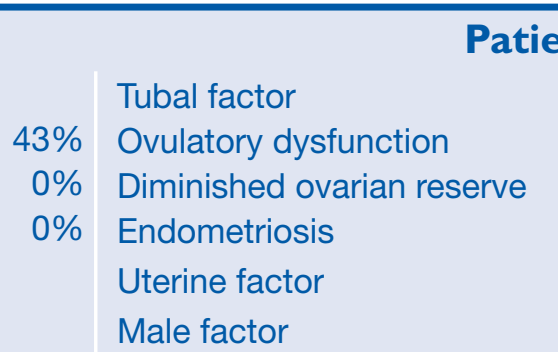

Patient Diagnosis

$\begin{array}{rlr}15 \% & \text { Other factor } & 9 \% \\ 5 \% & \text { Unknown factor } & 12 \% \\ 7 \% & \text { Multiple Factors: } & \\ 7 \% & \text { Female factors only } & 3 \% \\ 0 \% & \text { Female \& male factors } & 12 \% \\ 31 \% & & \end{array}$

Data verified by John A. Lucas, MD

\section{PREGNANCY SUCCESS RATES}

\section{Type of Cycle}

\section{Fresh Embryos from Nondonor Eggs}

Number of cycles

Percentage of cycles resulting in pregnancies ${ }^{b}$

Percentage of cycles resulting in live births ${ }^{b, c}$

(Confidence Interval)

Percentage of retrievals resulting in live births ${ }^{b, c}$

Percentage of transfers resulting in live births $s^{b, c}$

Percentage of transfers resulting in singleton live births ${ }^{b}$

Percentage of cancellations ${ }^{b}$

Average number of embryos transferred

Percentage of pregnancies with twins ${ }^{b}$

Percentage of pregnancies with triplets or more ${ }^{b}$

Percentage of live births having multiple infants ${ }^{b, c}$

\section{Frozen Embryos from Nondonor Eggs}

Number of transfers

Percentage of transfers resulting in live births $s^{b, c}$

Average number of embryos transferred

\section{Donor Eggs}

Number of transfers

Percentage of transfers resulting in live births $s^{b, c}$

Average number of embryos transferred

\section{Age of Woman}

35-37 $38-40 \quad 41-42^{d}$

\section{$<35$}

12

2

30
36.7

9

$2 / 12$

$0 / 2$

36.7

$(19.9-56.1)$

42.3

44.0

28.0

$1 / 9$

$1 / 12$

$0 / 2$

\section{3}

2.3

$3 / 11$

$1 / 11$

$4 / 11$

$1 / 7$

$1 / 10$

$1 / 7$

$1 / 10$

$0 / 7$

$1 / 10$

$2 / 9$

$0 / 1$
$2 / 12$

3.0

$1 / 1$

$1 / 2$

$0 / 2$

$0 / 1$

$2 / 2$

$1 / 1$

0

0

$\begin{array}{cc}0 / 1 & 0 / 2 \\ 3.0 & 1.0\end{array}$

\section{CURRENT CLINIC SERVICES AND PROFILE}

Current Name: University of Alabama at Birmingham

Donor egg? Yes

Donor embryo? No

Single women? Yes

\section{Gestational carriers? No}

Cryopreservation?

Yes
Fresh Embryos

2

$1 / 2$

2.5
All Ages Combined ${ }^{\mathrm{e}}$

Frozen Embryos

\section{1}

$0 / 1$

1.0

${ }^{a}$ Reflects patient and treatment characteristics of ART cycles performed in 2006 using fresh nondonor eggs or embryos.

${ }^{b}$ When fewer than 20 cycles are reported in an age category, rates are shown as a fraction and confidence intervals are not given. Calculating percentages from fractions may be misleading and is not encouraged.

${ }^{c}$ A multiple-infant birth is counted as one live birth.

d Clinic-specific outcome rates are unreliable for women older than 42 undergoing ART cycles using fresh or frozen embryos with nondonor eggs. Readers are urged to review national outcomes for these age groups (see page 27).

e All ages (including ages $>42$ ) are reported together because previous data show that patient age does not materially affect success with donor eggs. 


\section{HUNTSVILLE REPRODUCTIVE MEDICINE, PC HUNTSVILLE, ALABAMA}

A comparison of clinic success rates may not be meaningful because patient medical characteristics and treatment approaches vary from clinic to clinic. For more details about this, along with information on how to interpret the statistics in this table, see pages 79-88.

\section{ART CYCLE PROFILE}

$$
\text { Type of ART }{ }^{\mathrm{a}}
$$

IVF

$$
\text { 100\% Procedural Factors: }
$$

GIFT

$0 \%$ With ICSI

ZIFT

$0 \%$ Unstimulated

Combination

0\% Used gestational carrier

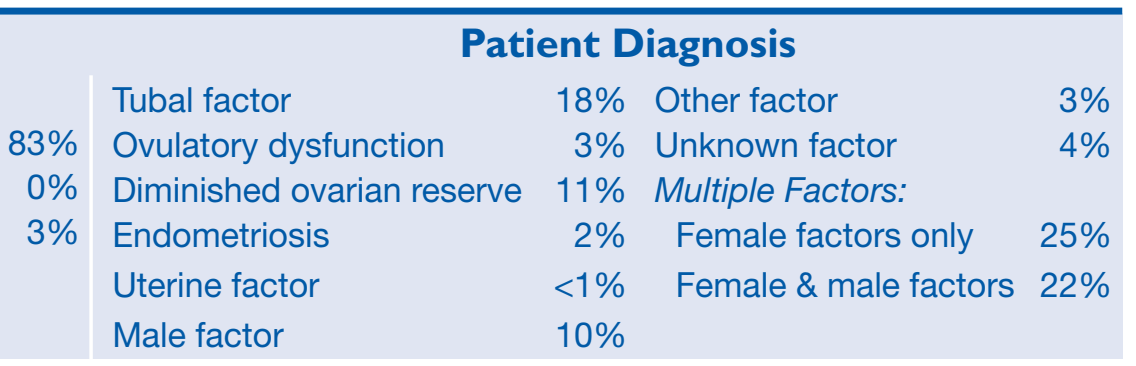

2006 PREGNANCY SUCCESS RATES

Data verified by Andrew J. Harper, MD

\section{Type of Cycle}

\section{Fresh Embryos from Nondonor Eggs}

Number of cycles

Percentage of cycles resulting in pregnancies ${ }^{b}$

Percentage of cycles resulting in live births $s^{b, c}$

(Confidence Interval)

Percentage of retrievals resulting in live births $s^{b, c}$

Percentage of transfers resulting in live births $s^{b, c}$

Percentage of transfers resulting in singleton live births ${ }^{b}$

Percentage of cancellations ${ }^{b}$

Average number of embryos transferred

Percentage of pregnancies with twins ${ }^{b}$

Percentage of pregnancies with triplets or more ${ }^{b}$

Percentage of live births having multiple infants ${ }^{b, c}$

\section{Frozen Embryos from Nondonor Eggs}

Number of transfers

Percentage of transfers resulting in live births ${ }^{\mathrm{b}, \mathrm{c}}$

Average number of embryos transferred

\section{Donor Eggs}

Number of transfers

Percentage of transfers resulting in live births ${ }^{\mathrm{b}, \mathrm{c}}$

Average number of embryos transferred

\section{Age of Woman}

35-37 38-40

$41-42^{d}$ $<35$

10

$\begin{array}{cc}52 & 10 \\ 51.9 & 2 / 10\end{array}$

46.2

$2 / 10$

12

$4 / 12$

$2 / 12$

1

(32.2-60.5)

49.0

50.0

$2 / 9$

$2 / 11$

$2 / 11$

$1 / 11$

$1 / 12$

3.2

$1 / 4$

$0 / 4$

$1 / 2$

$2 / 2$

2.6

$1 / 2$

$1 / 2$

12.5

12

$8 / 12$

1.9

\section{7}

$1 / 7$

2.6

2

$1 / 2$

2.5

All Ages Combined ${ }^{\mathrm{e}}$

Fresh Embryos 20

55.0

2.0
Frozen Embryos

\section{5}

$1 / 5$

2.0

\section{CURRENT CLINIC SERVICES AND PROFILE}

Current Name: Huntsville Reproductive Medicine, PC

Donor egg? Yes

Donor embryo? Yes

Single women? Yes

\section{Gestational carriers? \\ Yes}

Cryopreservation?
Yes
SART member?

Verified lab accreditation?

(See Appendix C for details.)
No

No

${ }^{a}$ Reflects patient and treatment characteristics of ART cycles performed in 2006 using fresh nondonor eggs or embryos.

${ }^{b}$ When fewer than 20 cycles are reported in an age category, rates are shown as a fraction and confidence intervals are not given. Calculating percentages from fractions may be misleading and is not encouraged.

${ }^{c}$ A multiple-infant birth is counted as one live birth.

d Clinic-specific outcome rates are unreliable for women older than 42 undergoing ART cycles using fresh or frozen embryos with nondonor eggs. Readers are urged to review national outcomes for these age groups (see page 27).

e All ages (including ages $>42$ ) are reported together because previous data show that patient age does not materially affect success with donor eggs. 


\section{CENTER FOR REPRODUCTIVE MEDICINE MOBILE, ALABAMA}

A comparison of clinic success rates may not be meaningful because patient medical characteristics and treatment approaches vary from clinic to clinic. For more details about this, along with information on how to interpret the statistics in this table, see pages 79-88.

\section{ART CYCLE PROFILE}

\begin{tabular}{|c|c|c|c|c|c|c|c|}
\hline \multicolumn{4}{|c|}{ Type of ART ${ }^{a}$} & \multicolumn{4}{|c|}{ Patient Diagnosis } \\
\hline IVF & $100 \%$ & Procedural Factors: & & Tubal factor & $21 \%$ & Other factor & $11 \%$ \\
\hline GIFT & $0 \%$ & With ICSI & $67 \%$ & Ovulatory dysfunction & $4 \%$ & Unknown factor & $0 \%$ \\
\hline ZIFT & $0 \%$ & Unstimulated & $0 \%$ & Diminished ovarian reserve & $3 \%$ & Multiple Factors: & \\
\hline \multirow[t]{3}{*}{ Combination } & $0 \%$ & Used gestational carrier & $0 \%$ & Endometriosis & $13 \%$ & Female factors only & $9 \%$ \\
\hline & & & & Uterine factor & $0 \%$ & Female \& male factors & $21 \%$ \\
\hline & & & & Male factor & $16 \%$ & & \\
\hline
\end{tabular}

2006 PREGNANCY SUCCESS RATES

Data verified by George T. Koulianos, MD

\section{Type of Cycle}

\section{Fresh Embryos from Nondonor Eggs}

Number of cycles

Percentage of cycles resulting in pregnancies ${ }^{b}$

Percentage of cycles resulting in live births $s^{b, c}$

(Confidence Interval)

Percentage of retrievals resulting in live births $s^{b, c}$

Percentage of transfers resulting in live births $s^{b, c}$

Percentage of transfers resulting in singleton live births ${ }^{b}$

Percentage of cancellations ${ }^{b}$

Average number of embryos transferred

Percentage of pregnancies with twins ${ }^{b}$

Percentage of pregnancies with triplets or more ${ }^{b}$

Percentage of live births having multiple infants ${ }^{b, c}$

\section{Frozen Embryos from Nondonor Eggs}

Number of transfers

Percentage of transfers resulting in live births $s^{b, c}$

Average number of embryos transferred

\section{Donor Eggs}

Number of transfers

Percentage of transfers resulting in live births $s^{b, c}$

Average number of embryos transferred

\section{Age of Woman}

35-37 38-40

$41-42^{d}$

\section{$<35$}

39

38.5

20

5

49.1

30.8

35.0

$1 / 5$

44.4

$(17.0-47.6)$

30.0

$0 / 5$

(34.9-54.3)

51.1

52.7

44.4

$(11.9-54.3)$

36.3

29.6

$6 / 13$

$0 / 3$

13.0

28.2

$6 / 13$

$0 / 3$

$2 / 13$

$0 / 3$

2.1

2.4

35.0

$2 / 5$

32.1

$5 / 15$

1.9

$0 / 15$

31.3

$4 / 12$

2.8

3.0

$0 / 1$

$0 / 1$

$1 / 7$

$4 / 6$

6

$2 / 6$

2

$0 / 2$

1

3.0

$0 / 1$

2.0

All Ages Combined ${ }^{\mathrm{e}}$

Fresh Embryos 25

40.0

2.2
Frozen Embryos 6

$2 / 6$

2.5

\section{CURRENT CLINIC SERVICES AND PROFILE}

Current Name: Center for Reproductive Medicine

Donor egg? Yes Gestational carriers? Yes

Donor embryo? Yes Cryopreservation? Yes

Single women? No

SART member? $\quad$ Yes

Verified lab accreditation? Yes

(See Appendix C for details.)

${ }^{a}$ Reflects patient and treatment characteristics of ART cycles performed in 2006 using fresh nondonor eggs or embryos.

${ }^{b}$ When fewer than 20 cycles are reported in an age category, rates are shown as a fraction and confidence intervals are not given. Calculating percentages from fractions may be misleading and is not encouraged.

${ }^{c}$ A multiple-infant birth is counted as one live birth.

${ }^{d}$ Clinic-specific outcome rates are unreliable for women older than 42 undergoing ART cycles using fresh or frozen embryos with nondonor eggs. Readers are urged to review national outcomes for these age groups (see page 27).

e All ages (including ages $>42$ ) are reported together because previous data show that patient age does not materially affect success with donor eggs. 


\section{UNIVERSITY OF SOUTH ALABAMA IVF AND ART PROGRAM MOBILE, ALABAMA}

A comparison of clinic success rates may not be meaningful because patient medical characteristics and treatment approaches vary from clinic to clinic. For more details about this, along with information on how to interpret the statistics in this table, see pages 79-88.

\section{ART CYCLE PROFILE}

$$
\text { Type of ART }{ }^{\mathrm{a}}
$$

IVF

$$
\text { 100\% Procedural Factors: }
$$

GIFT

$0 \%$ With ICSI

ZIFT

$0 \%$ Unstimulated

Combination

\begin{tabular}{|c|c|c|c|c|}
\hline & \multicolumn{4}{|c|}{ Patient Diagnosis } \\
\hline & Tubal factor & $22 \%$ & Other factor & $5 \%$ \\
\hline $32 \%$ & Ovulatory dysfunction & $10 \%$ & Unknown factor & $0 \%$ \\
\hline $0 \%$ & Diminished ovarian reserve & $7 \%$ & Multiple Factors: & \\
\hline $0 \%$ & Endometriosis & $0 \%$ & Female factors only & $24 \%$ \\
\hline & Uterine factor & $2 \%$ & Female \& male factors & $17 \%$ \\
\hline & Male factor & $12 \%$ & & \\
\hline
\end{tabular}

0\% Used gestational carrier

\section{PREGNANCY SUCCESS RATES}

Data verified by Botros M. Rizk, MD

\section{Type of Cycle}

\section{Fresh Embryos from Nondonor Eggs}

Number of cycles

Percentage of cycles resulting in pregnancies ${ }^{b}$

Percentage of cycles resulting in live births $s^{b, c}$

(Confidence Interval)

Percentage of retrievals resulting in live births $s^{b, c}$

Percentage of transfers resulting in live births $s^{b, c}$

Percentage of transfers resulting in singleton live births ${ }^{b}$

Percentage of cancellations ${ }^{b}$

Average number of embryos transferred

Percentage of pregnancies with twins ${ }^{b}$

Percentage of pregnancies with triplets or more ${ }^{b}$

Percentage of live births having multiple infants ${ }^{b, c}$

\section{Frozen Embryos from Nondonor Eggs}

Number of transfers

Percentage of transfers resulting in live births ${ }^{\mathrm{b}, \mathrm{c}}$

Average number of embryos transferred

\section{Age of Woman}

$\begin{array}{cccc}<35 & 35-37 & 38-40 & \mathbf{4 1 - 4 2}^{\text {d }} \\ 31 & 1 & 1 & 5 \\ 6.5 & 1 / 1 & 0 / 1 & 0 / 5 \\ 6.5 & 0 / 1 & 0 / 1 & 0 / 5\end{array}$

$(0.8-21.4)$

$\begin{array}{llll}7.1 & 0 / 1 & 0 / 1 & 0 / 4 \\ 7.7 & 0 / 1 & 0 / 1 & 0 / 4 \\ 7.7 & 0 / 1 & 0 / 1 & 0 / 4 \\ 9.7 & 0 / 1 & 0 / 1 & 1 / 5 \\ 2.2 & 3.0 & 1.0 & 2.0 \\ 0 / 2 & 0 / 1 & & \\ 0 / 2 & 0 / 1 & & \\ 0 / 2 & & & \end{array}$

\section{Donor Eggs}

2

$0 / 2$

2.5

Number of transfers

Percentage of transfers resulting in live births $s^{b, c}$

Average number of embryos transferred

\begin{tabular}{cc}
\multicolumn{2}{c}{ All Ages Combined } \\
Fresh Embryos & Frozen Embryos \\
1 & 0 \\
$0 / 1$ & \\
2.0 &
\end{tabular}

\section{CURRENT CLINIC SERVICES AND PROFILE}

Current Name: University of South Alabama IVF and ART Program

\begin{tabular}{ll|lll|l|l} 
Donor egg? & Yes & Gestational carriers? & No & SART member? & Yes \\
\hline Donor embryo? & No & Cryopreservation? & Yes & Verified lab accreditation? & Yes \\
Single women? & No & & & (See Appendix C for details.) &
\end{tabular}

${ }^{a}$ Reflects patient and treatment characteristics of ART cycles performed in 2006 using fresh nondonor eggs or embryos.

b When fewer than 20 cycles are reported in an age category, rates are shown as a fraction and confidence intervals are not given. Calculating percentages from fractions may be misleading and is not encouraged.

${ }^{c}$ A multiple-infant birth is counted as one live birth.

${ }^{d}$ Clinic-specific outcome rates are unreliable for women older than 42 undergoing ART cycles using fresh or frozen embryos with nondonor eggs. Readers are urged to review national outcomes for these age groups (see page 27).

e All ages (including ages $>42$ ) are reported together because previous data show that patient age does not materially affect success with donor eggs. 


\section{PENINSULA MEDICAL CENTER \\ JOHN NELS ANDERSON, MD \\ SOLDOTNA, ALASKA}

A comparison of clinic success rates may not be meaningful because patient medical characteristics and treatment approaches vary from clinic to clinic. For more details about this, along with information on how to interpret the statistics in this table, see pages 79-88.

\section{ART CYCLE PROFILE}

\section{Type of ART ${ }^{\mathrm{a}}$}

IVF

GIFT

100\% Procedural Factors:

ZIFT

Combination
$0 \%$ With ICSI

$0 \%$ Unstimulated

$0 \%$ Used gestational carrier

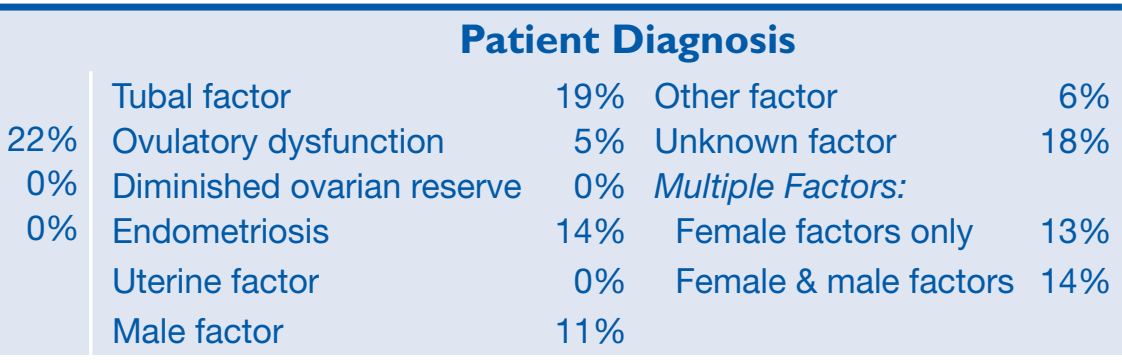

Data verified by John N. Anderson, MD

2006 PREGNANCY SUCCESS RATES

\section{Type of Cycle}

\begin{tabular}{|c|c|c|c|c|c|c|}
\hline \multirow{2}{*}{\multicolumn{3}{|c|}{ Froch Fmbrugc from Nandanor Fage }} & $<35$ & 35-37 & 38-40 & $41-42^{d}$ \\
\hline \multicolumn{6}{|c|}{ Fresh Embryos from Nondonor Eggs } & \\
\hline \multicolumn{3}{|c|}{ Number of cycles } & 28 & 7 & 15 & 5 \\
\hline \multicolumn{3}{|c|}{ Percentage of cycles resulting in pregnancies ${ }^{b}$} & 42.9 & $2 / 7$ & $6 / 15$ & $1 / 5$ \\
\hline \multicolumn{3}{|c|}{$\begin{array}{l}\text { Percentage of cycles resulting in live births }{ }^{\mathrm{b}, \mathrm{c}} \\
\text { (Confidence Interval) }\end{array}$} & $\begin{array}{c}32.1 \\
(15.9-52.4)\end{array}$ & $1 / 7$ & $4 / 15$ & $1 / 5$ \\
\hline \multicolumn{3}{|c|}{ Percentage of retrievals resulting in live births $s^{b, c}$} & 34.6 & $1 / 7$ & $4 / 14$ & $1 / 3$ \\
\hline \multicolumn{3}{|c|}{ Percentage of transfers resulting in live births ${ }^{b, c}$} & 40.9 & $1 / 5$ & $4 / 12$ & $1 / 1$ \\
\hline \multicolumn{3}{|c|}{ Percentage of transfers resulting in singleton live births ${ }^{b}$} & 18.2 & $0 / 5$ & $3 / 12$ & $1 / 1$ \\
\hline \multicolumn{3}{|c|}{ Percentage of cancellations ${ }^{b}$} & 7.1 & $0 / 7$ & $1 / 15$ & $2 / 5$ \\
\hline \multicolumn{3}{|c|}{ Average number of embryos transferred } & 2.2 & 2.6 & 3.0 & 9.0 \\
\hline \multicolumn{3}{|c|}{ Percentage of pregnancies with twins ${ }^{\mathrm{b}}$} & $5 / 12$ & $1 / 2$ & $2 / 6$ & $0 / 1$ \\
\hline \multicolumn{3}{|c|}{ Percentage of pregnancies with triplets or more } & $1 / 12$ & $0 / 2$ & $0 / 6$ & $0 / 1$ \\
\hline \multicolumn{3}{|c|}{ Percentage of live births having multiple infants ${ }^{b, c}$} & $5 / 9$ & $1 / 1$ & $1 / 4$ & $0 / 1$ \\
\hline \multicolumn{7}{|c|}{ Frozen Embryos from Nondonor Eggs } \\
\hline \multicolumn{3}{|c|}{ Number of transfers } & 2 & 0 & 1 & 0 \\
\hline \multicolumn{3}{|c|}{ Percentage of transfers resulting in live births $s^{b, c}$} & $0 / 2$ & & $0 / 1$ & \\
\hline \multicolumn{3}{|c|}{ Average number of embryos transferred } & 3.0 & & 1.0 & \\
\hline & \multicolumn{4}{|c|}{ All Ages Combined ${ }^{\mathrm{e}}$} \\
\hline & \multicolumn{2}{|c|}{ Donor Eggs } & \multicolumn{2}{|c|}{ Fresh Embryos } & \multicolumn{2}{|c|}{ Frozen Embryos } \\
\hline \multirow{3}{*}{\multicolumn{3}{|c|}{$\begin{array}{l}\text { Number of transfers } \\
\text { Percentage of transfers resulting in live births }{ }^{b, c} \\
\text { Average number of embryos transferred }\end{array}$}} & \multicolumn{2}{|c|}{1} & \multicolumn{2}{|c|}{0} \\
\hline & & & \multicolumn{2}{|c|}{$0 / 1$} & & \\
\hline & & & \multicolumn{2}{|c|}{3.0} & & \\
\hline \multicolumn{7}{|c|}{ CURRENT CLINIC SERVICES AND PROFILE } \\
\hline \multicolumn{7}{|c|}{ Current Name: Peninsula Medical Center, John Nels Anderson, MD } \\
\hline \multirow{3}{*}{$\begin{array}{l}\text { Donor egg? } \\
\text { Donor embryo? } \\
\text { Single women? }\end{array}$} & Yes & Gestational carriers? & Yes & SART $m$ & & No \\
\hline & Yes & Cryopreservation? & Yes & Verified l & creditation? & No \\
\hline & Yes & & & (See App & ( for details.) & \\
\hline \multicolumn{7}{|c|}{$\begin{array}{l}\text { a Reflects patient and treatment characteristics of ART cycles performed in } 2006 \text { using fresh nondonor eggs or embryos. } \\
\text { b When fewer than } 20 \text { cycles are reported in an age category, rates are shown as a fraction and confidence intervals are not given. Calculating } \\
\text { percentages from fractions may be misleading and is not encouraged. } \\
\text { d A multiple-infant birth is counted as one live birth. } \\
\text { d Clinic-specific outcome rates are unreliable for women older than } 42 \text { undergoing ART cycles using fresh or frozen embryos with nondonor } \\
\text { eggs. Readers are urged to review national outcomes for these age groups (see page } 27 \text { ). } \\
\text { e All ages (including ages }>42 \text { ) are reported together because previous data show that patient age does not materially affect } \\
\text { success with donor eggs. }\end{array}$} \\
\hline
\end{tabular}




\section{WEST VALLEY FERTILITY CENTER GLENDALE, ARIZONA}

A comparison of clinic success rates may not be meaningful because patient medical characteristics and treatment approaches vary from clinic to clinic. For more details about this, along with information on how to interpret the statistics in this table, see pages $79-88$.

\section{ART CYCLE PROFILE}

\begin{tabular}{|c|c|c|c|c|c|c|c|}
\hline \multicolumn{4}{|c|}{ Type of ART } & \multicolumn{4}{|c|}{ Patient Diagnosis } \\
\hline IVF & $100 \%$ & Procedural Factors: & & Tubal factor & $3 \%$ & Other factor & $2 \%$ \\
\hline GIFT & $0 \%$ & With ICSI & $88 \%$ & Ovulatory dysfunction & $1 \%$ & Unknown factor & $1 \%$ \\
\hline ZIFT & $0 \%$ & Unstimulated & $0 \%$ & Diminished ovarian reserve & $2 \%$ & Multiple Factors: & \\
\hline \multirow[t]{3}{*}{ Combination } & $0 \%$ & Used gestational carrier & $<1 \%$ & Endometriosis & $<1 \%$ & Female factors only & $4 \%$ \\
\hline & & & & Uterine factor & $0 \%$ & Female \& male factors & $54 \%$ \\
\hline & & & & Male factor & $31 \%$ & & \\
\hline
\end{tabular}

2006 PREGNANCY SUCCESS RATES

Data verified by Vladimir Troche, MD

\section{Type of Cycle}

\section{Fresh Embryos from Nondonor Eggs}

Number of cycles

Percentage of cycles resulting in pregnancies ${ }^{b}$

Percentage of cycles resulting in live births ${ }^{b, c}$

(Confidence Interval)

Percentage of retrievals resulting in live births $s^{b, c}$

Percentage of transfers resulting in live births $s^{b, c}$

Percentage of transfers resulting in singleton live births ${ }^{b}$

Percentage of cancellations ${ }^{b}$

Average number of embryos transferred

Percentage of pregnancies with twins ${ }^{b}$

Percentage of pregnancies with triplets or more ${ }^{b}$

Percentage of live births having multiple infants ${ }^{b, c}$

\section{Frozen Embryos from Nondonor Eggs}

Number of transfers

Percentage of transfers resulting in live births $s^{b, c}$

Average number of embryos transferred

\section{Donor Eggs}

Number of transfers

Percentage of transfers resulting in live births $s^{b, c}$

Average number of embryos transferred

\section{Age of Woman}

35-37 38-40

$41-42^{d}$

\section{$<35$}

48

39.6

23

10

94
42.6

36.2

31.3

34.8

$2 / 10$

$(26.5-46.7)$
37.0

$(18.7-46.3)$

30.4

$2 / 10$

41.5

34.9

$(13.2-52.9)$

$\begin{array}{ll}31.5 & 34.9\end{array}$

31.8

$2 / 10$

24.4

27.9

31.8

$2 / 9$

2.1

10.4

22.7

$2 / 9$

2.5

2.5

4.3

$0 / 10$

40.0

$6 / 19$

5.0

$0 / 19$

41.2

$3 / 15$

2.5

2.8

$2 / 8$

$0 / 2$

$0 / 8$

$0 / 2$

$2 / 7$

$0 / 2$

33

18.2

\section{9}

$1 / 9$

2

$2 / 2$

2.5

2.3

3.0

All Ages Combined ${ }^{\mathrm{e}}$

Fresh Embryos

22

54.5

2.3
Frozen Embryos 4

$2 / 4$

3.0

\section{CURRENT CLINIC SERVICES AND PROFILE}

Current Name: West Valley Fertility Center

Donor egg? Yes Gestational carriers? Yes

Donor embryo? Yes Cryopreservation? Yes

Single women? Yes

\section{SART member? Yes}

Verified lab accreditation? Yes

(See Appendix C for details.)

${ }^{a}$ Reflects patient and treatment characteristics of ART cycles performed in 2006 using fresh nondonor eggs or embryos.

${ }^{b}$ When fewer than 20 cycles are reported in an age category, rates are shown as a fraction and confidence intervals are not given. Calculating percentages from fractions may be misleading and is not encouraged.

${ }^{c}$ A multiple-infant birth is counted as one live birth.

d Clinic-specific outcome rates are unreliable for women older than 42 undergoing ART cycles using fresh or frozen embryos with nondonor eggs. Readers are urged to review national outcomes for these age groups (see page 27).

e All ages (including ages $>42$ ) are reported together because previous data show that patient age does not materially affect success with donor eggs. 


\section{ARIZONA REPRODUCTIVE MEDICINE SPECIALISTS \\ PHOENIX, ARIZONA}

A comparison of clinic success rates may not be meaningful because patient medical characteristics and treatment approaches vary from clinic to clinic. For more details about this, along with information on how to interpret the statistics in this table, see pages 79-88.

\section{ART CYCLE PROFILE}

\section{Type of ART ${ }^{\mathrm{a}}$}

IVF

GIFT

100\% Procedural Factors:

ZIFT

Combination
$0 \%$ With ICSI

$0 \%$ Unstimulated

$0 \%$ Used gestational carrier

\begin{tabular}{|l|l|} 
& \\
\hline & Tubal factor \\
\hline $49 \%$ & Ovulatory dysfunction \\
0\% & Diminished ovarian reserve \\
$0 \%$ & Endometriosis \\
& Uterine factor \\
& Male factor
\end{tabular}

Patient Diagnosis

$\begin{array}{lll}5 \% & \text { Other factor } & 5 \% \\ 5 \% & \text { Unknown factor } & 7 \% \\ 6 \% & \text { Multiple Factors: } & \\ 2 \% & \text { Female factors only } & 26 \% \\ 0 \% & \text { Female \& male factors } & 32 \% \\ 12 \% & & \end{array}$

2006 PREGNANCY SUCCESS RATES

Data verified by Drew V. Moffitt, MD

\section{Type of Cycle}

\section{Age of Woman}

$\begin{array}{cccc}<35 & \mathbf{3 5 - 3 7} & \mathbf{3 8 - 4 0} & \mathbf{4 1 - 4 2}^{\mathbf{d}} \\ & & & \\ 115 & 63 & 38 & 8 \\ 40.9 & 34.9 & 23.7 & 2 / 8 \\ 33.9 & 28.6 & 15.8 & 2 / 8 \\ 25.3-43.3) & (17.9-41.3) & (6.0-31.3) & \\ 35.8 & 36.0 & 19.4 & 2 / 6 \\ 37.1 & 36.7 & 21.4 & 2 / 5 \\ 28.6 & 24.5 & 17.9 & 2 / 5 \\ 5.2 & 20.6 & 18.4 & 2 / 8 \\ 2.2 & 2.1 & 2.6 & 2.8 \\ 31.9 & 27.3 & 1 / 9 & 0 / 2 \\ 4.3 & 0.0 & 1 / 9 & 0 / 2 \\ 23.1 & 6 / 18 & 1 / 6 & 0 / 2\end{array}$

$41-42^{d}$

57

37

32.4

10

3

22.8

2.1

$3 / 10$

$0 / 3$

2.1

All Ages Combined ${ }^{\mathrm{e}}$

Fresh Embryos 15

$5 / 15$

2.0
Frozen Embryos

6

$1 / 6$

2.0

Average number of embryos transferred

\section{CURRENT CLINIC SERVICES AND PROFILE}

Current Name: Arizona Reproductive Medicine Specialists

Donor egg? Yes Gestational carriers? No

Donor embryo? Yes Cryopreservation? Yes

Single women? Yes

SART member? Yes

Verified lab accreditation? Yes

(See Appendix C for details.)

${ }^{a}$ Reflects patient and treatment characteristics of ART cycles performed in 2006 using fresh nondonor eggs or embryos.

${ }^{b}$ When fewer than 20 cycles are reported in an age category, rates are shown as a fraction and confidence intervals are not given. Calculating percentages from fractions may be misleading and is not encouraged.

${ }^{c}$ A multiple-infant birth is counted as one live birth.

d Clinic-specific outcome rates are unreliable for women older than 42 undergoing ART cycles using fresh or frozen embryos with nondonor eggs. Readers are urged to review national outcomes for these age groups (see page 27).

All ages (including ages $>42$ ) are reported together because previous data show that patient age does not materially affect success with donor eggs. 


\section{IVF PHOENIX \\ PHOENIX, ARIZONA}

A comparison of clinic success rates may not be meaningful because patient medical characteristics and treatment approaches vary from clinic to clinic. For more details about this, along with information on how to interpret the statistics in this table, see pages $79-88$.

\section{ART CYCLE PROFILE}

\begin{tabular}{|c|c|c|c|c|c|c|c|}
\hline \multicolumn{4}{|c|}{ Type of ART ${ }^{a}$} & \multicolumn{4}{|c|}{ Patient Diagnosis } \\
\hline IVF & $100 \%$ & Procedural Factors: & & Tubal factor & $17 \%$ & Other factor & $5 \%$ \\
\hline GIFT & $0 \%$ & With ICSI & $70 \%$ & Ovulatory dysfunction & $4 \%$ & Unknown factor & $17 \%$ \\
\hline $\mathrm{ZIFT}$ & $0 \%$ & Unstimulated & $0 \%$ & Diminished ovarian reserve & $8 \%$ & Multiple Factors: & \\
\hline \multirow[t]{3}{*}{ Combination } & $0 \%$ & Used gestational carrier & $0 \%$ & Endometriosis & $6 \%$ & Female factors only & $7 \%$ \\
\hline & & & & Uterine factor & $5 \%$ & Female \& male factors & $5 \%$ \\
\hline & & & & Male factor & $28 \%$ & & \\
\hline
\end{tabular}

2006 PREGNANCY SUCCESS RATES

Data verified by John L. Couvaras, MD

\section{Type of Cycle}

\section{Fresh Embryos from Nondonor Eggs}

Number of cycles

Percentage of cycles resulting in pregnancies ${ }^{b}$

Percentage of cycles resulting in live births $s^{b, c}$

(Confidence Interval)

Percentage of retrievals resulting in live births $s^{b, c}$

Percentage of transfers resulting in live births $s^{b, c}$

Percentage of transfers resulting in singleton live births ${ }^{b}$

Percentage of cancellations ${ }^{b}$

Average number of embryos transferred

Percentage of pregnancies with twins ${ }^{b}$

Percentage of pregnancies with triplets or more ${ }^{b}$

Percentage of live births having multiple infants ${ }^{b, c}$

\section{Frozen Embryos from Nondonor Eggs}

Number of transfers

Percentage of transfers resulting in live births $s^{b, c}$

Average number of embryos transferred

\section{Donor Eggs}

Number of transfers

Percentage of transfers resulting in live births $s^{b, c}$

Average number of embryos transferred

\section{Age of Woman}

35-37 38-40

$41-42^{d}$

$<35$

18

$4 / 18$

$3 / 18$

9

9

27.3

27.3

(13.3-45.5)

27.3

28.1

$3 / 18$

$3 / 13$

$3 / 13$

$3 / 9$

$0 / 9$

18.8

$0 / 18$

$1 / 9$

$0 / 9$

0.0

3.5

$0 / 4$

$1 / 9$

$1 / 9$

$0 / 9$

2.6

$3 / 9$

$0 / 4$

$0 / 9$

$0 / 4$

$0 / 9$

$0 / 3$

$0 / 9$

$0 / 4$

$3 / 9$

4.1

$0 / 9$

3.3

$0 / 3$

$1 / 3$

$1 / 1$

\section{CURRENT CLINIC SERVICES AND PROFILE}

Current Name: IVF Phoenix

$\begin{array}{ll}\text { Donor egg? } & \text { Yes } \\ \text { Donor embryo? } & \text { No } \\ \text { Single women? } & \text { Yes }\end{array}$

$\begin{array}{cccc}12 & 11 & 2 & 2 \\ 1 / 12 & 1 / 11 & 0 / 2 & 0 / 2 \\ 3.2 & 3.5 & 5.5 & 4.0\end{array}$

All Ages Combined ${ }^{\mathrm{e}}$

Fresh Embryos

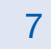

$2 / 7$

3.4
Frozen Embryos

3

$1 / 3$

4.3

\footnotetext{
${ }_{b}^{a}$ Reflects patient and treatment characteristics of ART cycles performed in 2006 using fresh nondonor eggs or embryos.

${ }^{b}$ When fewer than 20 cycles are reported in an age category, rates are shown as a fraction and confidence intervals are not given. Calculating percentages from fractions may be misleading and is not encouraged.

${ }^{c}$ A multiple-infant birth is counted as one live birth.

${ }^{d}$ Clinic-specific outcome rates are unreliable for women older than 42 undergoing ART cycles using fresh or frozen embryos with nondonor eggs. Readers are urged to review national outcomes for these age groups (see page 27).

${ }^{e}$ All ages (including ages $>42$ ) are reported together because previous data show that patient age does not materially affect success with donor eggs.
} 


\section{SOUTHWEST FERTILITY CENTER \\ PHOENIX, ARIZONA}

A comparison of clinic success rates may not be meaningful because patient medical characteristics and treatment approaches vary from clinic to clinic. For more details about this, along with information on how to interpret the statistics in this table, see pages 79-88.

\section{ART CYCLE PROFILE}

\begin{tabular}{|c|c|c|c|c|c|c|c|}
\hline \multicolumn{4}{|c|}{ Type of ART ${ }^{a}$} & \multicolumn{4}{|c|}{ Patient Diagnosis } \\
\hline IVF & $100 \%$ & Procedural Factors: & & Tubal factor & $6 \%$ & Other factor & $1 \%$ \\
\hline GIFT & $0 \%$ & With ICSI & $63 \%$ & Ovulatory dysfunction & $1 \%$ & Unknown factor & $2 \%$ \\
\hline ZIFT & $0 \%$ & Unstimulated & $0 \%$ & Diminished ovarian reserve & $11 \%$ & Multiple Factors: & \\
\hline \multirow[t]{3}{*}{ Combination } & $0 \%$ & Used gestational carrier & $0 \%$ & Endometriosis & $4 \%$ & Female factors only & $38 \%$ \\
\hline & & & & Uterine factor & $<1 \%$ & Female \& male factors & $28 \%$ \\
\hline & & & & Male factor & $7 \%$ & & \\
\hline
\end{tabular}

2006 PREGNANCY SUCCESS RATES

Data verified by Sujatha Gunnala, MD

\section{Type of Cycle}

\section{Fresh Embryos from Nondonor Eggs}

Number of cycles

Percentage of cycles resulting in pregnancies ${ }^{b}$

Percentage of cycles resulting in live births $s^{b, c}$

(Confidence Interval)

Percentage of retrievals resulting in live births $s^{b, c}$

Percentage of transfers resulting in live births $s^{b, c}$

Percentage of transfers resulting in singleton live births ${ }^{b}$

Percentage of cancellations ${ }^{b}$

Average number of embryos transferred

Percentage of pregnancies with twins ${ }^{b}$

Percentage of pregnancies with triplets or more ${ }^{b}$

Percentage of live births having multiple infants ${ }^{b, c}$

\section{Frozen Embryos from Nondonor Eggs}

Number of transfers

Percentage of transfers resulting in live births ${ }^{b, c}$

Average number of embryos transferred

\section{Donor Eggs}

Number of transfers

Percentage of transfers resulting in live births $s^{b, c}$

Average number of embryos transferred

\section{Age of Woman}

35-37 38-40

$41-42^{d}$

$<35$

21

16

2

33.3

33.3

$6 / 16$

$6 / 16$

$1 / 2$

28.3

28.6

$6 / 16$

$1 / 2$

(17.5-41.4)

(11.3-52.2)

28.3

28.6

$6 / 18$

$6 / 16$

$1 / 2$

28.3

$4 / 18$

$5 / 16$

$1 / 2$

21.7

0.0

$0 / 16$

$1 / 2$

0.0

2.1

$2 / 7$

2.1

$0 / 2$

2.3

$0 / 7$

$1 / 6$

2.5

5.0

$2 / 6$

$0 / 6$

$0 / 1$

$4 / 17$

$1 / 6$

$0 / 1$

$0 / 1$

$\begin{array}{cccc}16 & 4 & 2 & 2 \\ 6 / 16 & 2 / 4 & 2 / 2 & 1 / 2 \\ 2.2 & 2.0 & 2.5 & 2.0\end{array}$

\section{All Ages Combined}

Fresh Embryos

Frozen Embryos

9

$6 / 9$

5

2.2

$3 / 5$

2.0

\section{CURRENT CLINIC SERVICES AND PROFILE}

Current Name: Southwest Fertility Center

Donor egg? Yes Gestational carriers? Yes

Donor embryo? Yes Cryopreservation? Yes

Single women? Yes

SART member? Yes

Verified lab accreditation?

(See Appendix C for details.)

${ }^{a}$ Reflects patient and treatment characteristics of ART cycles performed in 2006 using fresh nondonor eggs or embryos.

${ }^{b}$ When fewer than 20 cycles are reported in an age category, rates are shown as a fraction and confidence intervals are not given. Calculating percentages from fractions may be misleading and is not encouraged.

${ }^{c}$ A multiple-infant birth is counted as one live birth.

d Clinic-specific outcome rates are unreliable for women older than 42 undergoing ART cycles using fresh or frozen embryos with nondonor eggs. Readers are urged to review national outcomes for these age groups (see page 27).

e All ages (including ages $>42$ ) are reported together because previous data show that patient age does not materially affect success with donor eggs. 


\section{ADVANCED FERTILITY CARE SCOTTSDALE, ARIZONA}

A comparison of clinic success rates may not be meaningful because patient medical characteristics and treatment approaches vary from clinic to clinic. For more details about this, along with information on how to interpret the statistics in this table, see pages $79-88$.

\section{ART CYCLE PROFILE}

\begin{tabular}{|c|c|c|c|c|c|c|c|}
\hline \multicolumn{4}{|c|}{ Type of ART ${ }^{\mathrm{a}}$} & \multicolumn{4}{|c|}{ Patient Diagnosis } \\
\hline IVF & $100 \%$ & Procedural Factors: & & Tubal factor & $14 \%$ & Other factor & $0 \%$ \\
\hline GIFT & $0 \%$ & With ICSI & $56 \%$ & Ovulatory dysfunction & $10 \%$ & Unknown factor & $0 \%$ \\
\hline ZIFT & $0 \%$ & Unstimulated & $0 \%$ & Diminished ovarian reserve & $19 \%$ & Multiple Factors: & \\
\hline \multirow[t]{3}{*}{ Combination } & $0 \%$ & Used gestational carrier & $0 \%$ & Endometriosis & $0 \%$ & Female factors only & $14 \%$ \\
\hline & & & & Uterine factor & $0 \%$ & Female \& male factors & $24 \%$ \\
\hline & & & & Male factor & $19 \%$ & & \\
\hline
\end{tabular}

2006 PREGNANCY SUCCESS RATES

Data verified by Nathaniel Zoneraich, MD

\section{Type of Cycle}

\section{Fresh Embryos from Nondonor Eggs}

Number of cycles

Percentage of cycles resulting in pregnancies ${ }^{b}$

Percentage of cycles resulting in live births ${ }^{b, c}$

(Confidence Interval)

Percentage of retrievals resulting in live births $s^{b, c}$

Percentage of transfers resulting in live births $s^{b, c}$

Percentage of transfers resulting in singleton live births ${ }^{b}$

Percentage of cancellations ${ }^{b}$

Average number of embryos transferred

Percentage of pregnancies with twins ${ }^{b}$

Percentage of pregnancies with triplets or more ${ }^{b}$

Percentage of live births having multiple infants ${ }^{b, c}$

\section{Frozen Embryos from Nondonor Eggs}

Number of transfers

Percentage of transfers resulting in live births ${ }^{\mathrm{b}, \mathrm{c}}$

Average number of embryos transferred

\section{Donor Eggs}

Number of transfers

Percentage of transfers resulting in live births $s^{b, c}$

Average number of embryos transferred

\section{Age of Woman}

$\begin{array}{cccc}\mathbf{3 5 5} & \mathbf{3 5 - 3 7} & \mathbf{3 8 - 4 0} & \mathbf{4 1 - 4 2}^{\mathbf{d}} \\ 12 & 1 & 2 & 0 \\ 6 / 12 & 1 / 1 & 1 / 2 & \\ 6 / 12 & 1 / 1 & 0 / 2 & \end{array}$

$\begin{array}{lll}6 / 12 & 1 / 1 & 0 / 2 \\ 6 / 11 & 1 / 1 & 0 / 2 \\ 1 / 11 & 1 / 1 & 0 / 2 \\ 0 / 12 & 0 / 1 & 0 / 2 \\ 2.4 & 2.0 & 2.5 \\ 5 / 6 & 0 / 1 & 0 / 1 \\ 0 / 6 & 0 / 1 & 0 / 1 \\ 5 / 6 & 0 / 1 & \end{array}$

2

$2 / 2$

0

1

$0 / 1$

2.0

2.0
$0 \%$

$0 \%$

$4 \%$

$24 \%$

(1)

\section{CURRENT CLINIC SERVICES AND PROFILE}

Current Name: Advanced Fertility Care

\begin{tabular}{ll|ll|ll} 
Donor egg? & Yes & Gestational carriers? & Yes & SART member? & No \\
Donor embryo? & No & Cryopreservation? & Yes & Verified lab accreditation? & Yes \\
Single women? & No & & & (See Appendix C for details.) &
\end{tabular}

${ }^{a}$ Reflects patient and treatment characteristics of ART cycles performed in 2006 using fresh nondonor eggs or embryos.

${ }^{b}$ When fewer than 20 cycles are reported in an age category, rates are shown as a fraction and confidence intervals are not given. Calculating percentages from fractions may be misleading and is not encouraged.

c A multiple-infant birth is counted as one live birth.

${ }^{d}$ Clinic-specific outcome rates are unreliable for women older than 42 undergoing ART cycles using fresh or frozen embryos with nondonor eggs. Readers are urged to review national outcomes for these age groups (see page 27).

${ }^{e}$ All ages (including ages $>42$ ) are reported together because previous data show that patient age does not materially affect success with donor eggs. 


\section{ARIZONA ASSOCIATES FOR REPRODUCTIVE HEALTH SCOTTSDALE, ARIZONA}

A comparison of clinic success rates may not be meaningful because patient medical characteristics and treatment approaches vary from clinic to clinic. For more details about this, along with information on how to interpret the statistics in this table, see pages 79-88.

\section{ART CYCLE PROFILE}

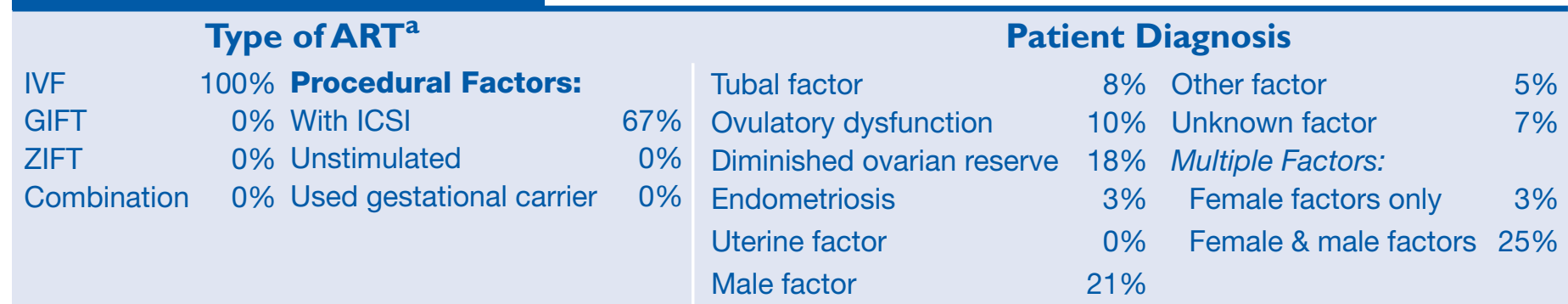

\section{PREGNANCY SUCCESS RATES}

Data verified by Ketan S. Patel, MD

\section{Type of Cycle}

\section{Fresh Embryos from Nondonor Eggs}

Number of cycles

Percentage of cycles resulting in pregnancies ${ }^{b}$

Percentage of cycles resulting in live births $s^{b, c}$

(Confidence Interval)

Percentage of retrievals resulting in live births $s^{b, c}$

Percentage of transfers resulting in live births $s^{b, c}$

Percentage of transfers resulting in singleton live births ${ }^{b}$

Percentage of cancellations ${ }^{b}$

Average number of embryos transferred

Percentage of pregnancies with twins ${ }^{b}$

Percentage of pregnancies with triplets or more ${ }^{b}$

Percentage of live births having multiple infants ${ }^{b, c}$

\section{Frozen Embryos from Nondonor Eggs}

Number of transfers

Percentage of transfers resulting in live births ${ }^{b, c}$

Average number of embryos transferred

\section{Donor Eggs}

Number of transfers

Percentage of transfers resulting in live births $s^{b, c}$

Average number of embryos transferred

\section{Age of Woman}

35-37 38-40

$41-42^{d}$

$<35$

13

$6 / 13$

$5 / 13$

11

4

44.8

41.4

$3 / 11$

$2 / 4$

(23.5-61.1)

50.0

52.2

$5 / 12$

$2 / 11$

$1 / 4$

\section{8}

17.2

2.3

$4 / 13$

$0 / 13$

$4 / 12$

\section{$5 / 11$}

$2 / 11$

$1 / 3$

$3 / 11$

$2 / 11$

$1 / 3$

$1 / 13$

$1 / 11$

$0 / 3$

2.5

$1 / 6$

$0 / 11$

$1 / 4$

4.0

2.5

$0 / 2$

$1 / 6$

$2 / 5$

$0 / 3$

$1 / 2$

$1 / 3$

$1 / 2$

$1 / 1$

\section{8}

$4 / 8$

4

2.4
0

3

$0 / 3$

3.0

\section{CURRENT CLINIC SERVICES AND PROFILE}

Current Name: Arizona Associates for Reproductive Health

Donor egg? Yes Gestational carriers? Yes

Donor embryo? Yes Cryopreservation? Yes

Single women? Yes

\section{Fresh Embryos}

All Ages Combined ${ }^{\mathrm{e}}$
Frozen Embryos 5

$3 / 10$

2.1
$2 / 5$

2.8

\footnotetext{
${ }^{a}$ Reflects patient and treatment characteristics of ART cycles performed in 2006 using fresh nondonor eggs or embryos.

b When fewer than 20 cycles are reported in an age category, rates are shown as a fraction and confidence intervals are not given. Calculating percentages from fractions may be misleading and is not encouraged.

${ }^{c}$ A multiple-infant birth is counted as one live birth.

d Clinic-specific outcome rates are unreliable for women older than 42 undergoing ART cycles using fresh or frozen embryos with nondonor eggs. Readers are urged to review national outcomes for these age groups (see page 27).

e All ages (including ages $>42$ ) are reported together because previous data show that patient age does not materially affect success with donor eggs.
} 


\section{ARIZONA CENTER FOR FERTILITY STUDIES \\ SCOTTSDALE, ARIZONA}

A comparison of clinic success rates may not be meaningful because patient medical characteristics and treatment approaches vary from clinic to clinic. For more details about this, along with information on how to interpret the statistics in this table, see pages $79-88$.

\section{ART CYCLE PROFILE}

\section{Type of ART ${ }^{\mathrm{a}}$}

$\begin{array}{llr}\text { IVF } & 41 \% \text { Procedural Factors: } & \\ \text { GIFT } & 0 \% \text { With ICSI } & 32 \% \\ \text { ZIFT } & 59 \% \text { Unstimulated } & <1 \% \\ \text { Combination } & 0 \% \text { Used gestational carrier } & 1 \%\end{array}$

2006 PREGNANCY SUCCESS RATES

\section{Type of Cycle}

\section{Donor Eggs}

Number of transfers

Percentage of transfers resulting in live births $s^{b, c}$

Average number of embryos transferred

\section{Frozen Embryos from Nondonor Eggs}

Number of transfers

Percentage of transfers resulting in live births $s^{b, c}$

Average number of embryos transferred

\section{CURRENT CLINIC SERVICES AND PROFILE}

Current Name: Arizona Center for Fertility Studies
Donor egg?
Yes
Donor embryo? Yes
Gestational carriers? Yes
Cryopreservation?
Single women? Yes

\footnotetext{
${ }_{b}^{a}$ Reflects patient and treatment characteristics of ART cycles performed in 2006 using fresh nondonor eggs or embryos.

b When fewer than 20 cycles are reported in an age category, rates are shown as a fraction and confidence intervals are not given. Calculating percentages from fractions may be misleading and is not encouraged.

${ }_{\mathrm{d}}^{\mathrm{c}}$ A multiple-infant birth is counted as one live birth.

${ }^{d}$ Clinic-specific outcome rates are unreliable for women older than 42 undergoing ART cycles using fresh or frozen embryos with nondonor eggs. Readers are urged to review national outcomes for these age groups (see page 27).

e All ages (including ages $>42$ ) are reported together because previous data show that patient age does not materially affect success with donor eggs.
}

Tubal factor

Patient Diagnosis

Ovulatory dysfunction

$5 \%$ Other factor

$35 \%$

Diminished ovarian reserve

0\% Unknown factor

Endometriosis

$11 \%$ Multiple Factors:

Uterine factor

$5 \%$ Female factors only $13 \%$

Male factor

$0 \%$ Female \& male factors

$7 \%$ $11 \%$

Data verified by Jay S. Nemiro, MD

\section{Age of Woman}

$\begin{array}{llll}<35 & 35-37 & 38-40 & 41-42^{d}\end{array}$

$\begin{array}{cccc}59 & 37 & 40 & 17 \\ 27.1 & 13.5 & 30.0 & 4 / 17 \\ 22.0 & 5.4 & 15.0 & 1 / 17\end{array}$

(12.3-34.7)

$(0.7-18.2)$

(5.7-29.8)

24.1

6.1

8.3

16.2

$1 / 17$

25.0

$1 / 12$

20.0

8.3

16.7

$1 / 12$

$8.5 \quad 10.8$

7.5

$0 / 17$

2.4

2.5

2.9

2.6

$4 / 16$

$0 / 5$

$2 / 12$

$0 / 4$

$1 / 16$

$1 / 5$

$0 / 12$

$0 / 4$

$4 / 13$

$0 / 2$

$2 / 6$

$0 / 1$

$\begin{array}{cccc}17 & 9 & 5 & 3 \\ 5 / 17 & 4 / 9 & 2 / 5 & 0 / 3 \\ 3.2 & 3.4 & 3.8 & 2.3\end{array}$

All Ages Combined

Fresh Embryos

Frozen Embryos

$\begin{array}{cc}26 & 10 \\ 38.5 & 0 / 10 \\ 2.5 & 2.6\end{array}$




\section{FERTILITY TREATMENT CENTER \\ TEMPE, ARIZONA}

A comparison of clinic success rates may not be meaningful because patient medical characteristics and treatment approaches vary from clinic to clinic. For more details about this, along with information on how to interpret the statistics in this table, see pages 79-88.

\section{ART CYCLE PROFILE}

\section{Type of ART ${ }^{\mathrm{a}}$}

IVF

GIFT

ZIFT

Combination
100\% Procedural Factors:

$0 \%$ With ICSI

$0 \%$ Unstimulated

$0 \%$ Used gestational carrier

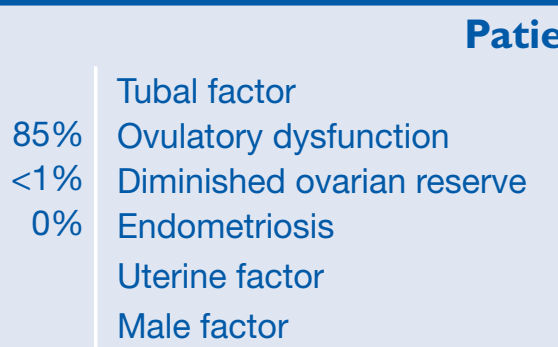

Patient Diagnosis

8\% Other factor

13\% Unknown factor

24\% Multiple Factors:

$6 \%$ Female factors only $12 \%$

$<1 \%$ Female \& male factors $12 \%$

$13 \%$

2006 PREGNANCY SUCCESS RATES

Data verified by H. Randall Craig, MD

\section{Type of Cycle}

\section{Age of Woman}

35-37 38-40

47

19.1

27.1

21.2

21.2

17.3

8.5

Percentage of cycles resulting in live births ${ }^{b, c}$

(Confidence Interval)

Percentage of retrievals resulting in live births ${ }^{b, c}$

Percentage of transfers resulting in live births ${ }^{b, c}$

$(14.2-29.7)$

22.9

36.2

23.2

7.6

2.4

34.4

3.1

36.0

(8.2-30.3)

19.6

(2.4-20.4)

11.4

15.4

11.5

25.5

2.5

11.5

2.2

$4 / 11$

$0 / 11$

$2 / 9$

81

54.3

2.4
40

37.5

2.4
$1 / 9$

$0 / 9$

$1 / 4$

17

$6 / 17$

2.3
$2 / 17$

$41-42^{d}$

17

$2 / 17$

$2 / 13$

$2 / 9$

$2 / 9$

$4 / 17$

2.6

$0 / 2$

$0 / 2$

$0 / 2$
Number of transfers

Average number of embryos transferred

All Ages Combined

Fresh Embryos 35

40.0

2.1
Frozen Embryos 54

48.1

2.4

Average number of embryos transferred

\section{CURRENT CLINIC SERVICES AND PROFILE}

Current Name: Fertility Treatment Center

Donor egg? Yes Gestational carriers? Yes

Donor embryo? Yes

Cryopreservation? Yes

Single women? Yes

SART member? Yes

Verified lab accreditation? Yes

(See Appendix C for details.)

${ }^{a}$ Reflects patient and treatment characteristics of ART cycles performed in 2006 using fresh nondonor eggs or embryos.

b When fewer than 20 cycles are reported in an age category, rates are shown as a fraction and confidence intervals are not given. Calculating percentages from fractions may be misleading and is not encouraged.

${ }^{\mathrm{C}}$ A multiple-infant birth is counted as one live birth.

d Clinic-specific outcome rates are unreliable for women older than 42 undergoing ART cycles using fresh or frozen embryos with nondonor eggs. Readers are urged to review national outcomes for these age groups (see page 27).

ell ages (including ages $>42$ ) are reported together because previous data show that patient age does not materially affect success with donor eggs. 


\section{ARIZONA CENTER FOR REPRODUCTIVE ENDOCRINOLOGY AND INFERTILITY TUCSON, ARIZONA}

A comparison of clinic success rates may not be meaningful because patient medical characteristics and treatment approaches vary from clinic to clinic. For more details about this, along with information on how to interpret the statistics in this table, see pages $79-88$.

\section{ART CYCLE PROFILE}

\begin{tabular}{|c|c|c|c|c|c|c|c|}
\hline \multicolumn{4}{|c|}{ Type of ART ${ }^{\mathrm{a}}$} & \multicolumn{4}{|c|}{ Patient Diagnosis } \\
\hline IVF & $100 \%$ & Procedural Factors: & & Tubal factor & $19 \%$ & Other factor & $18 \%$ \\
\hline GIFT & $0 \%$ & With ICSI & $28 \%$ & Ovulatory dysfunction & $2 \%$ & Unknown factor & $0 \%$ \\
\hline ZIFT & $0 \%$ & Unstimulated & $0 \%$ & Diminished ovarian reserve & $14 \%$ & Multiple Factors: & \\
\hline \multirow[t]{3}{*}{ Combination } & $0 \%$ & Used gestational carrier & $0 \%$ & Endometriosis & $25 \%$ & Female factors only & $5 \%$ \\
\hline & & & & Uterine factor & $<1 \%$ & Female \& male factors & $3 \%$ \\
\hline & & & & Male factor & $14 \%$ & & \\
\hline
\end{tabular}

2006 PREGNANCY SUCCESS RATES

Data verified by Timothy J. Gelety, MD

\section{Type of Cycle}

\section{Fresh Embryos from Nondonor Eggs}

Number of cycles

Percentage of cycles resulting in pregnancies ${ }^{b}$

Percentage of cycles resulting in live births ${ }^{b, c}$

(Confidence Interval)

Percentage of retrievals resulting in live births $s^{b, c}$

Percentage of transfers resulting in live births $s^{b, c}$

Percentage of transfers resulting in singleton live births ${ }^{b}$

Percentage of cancellations ${ }^{b}$

Average number of embryos transferred

Percentage of pregnancies with twins ${ }^{b}$

Percentage of pregnancies with triplets or more ${ }^{b}$

Percentage of live births having multiple infants ${ }^{b, c}$

\section{Frozen Embryos from Nondonor Eggs}

Number of transfers

Percentage of transfers resulting in live births $s^{b, c}$

Average number of embryos transferred

\section{Donor Eggs}

Number of transfers

Percentage of transfers resulting in live births ${ }^{\mathrm{b}, \mathrm{c}}$

Average number of embryos transferred

\section{Age of Woman}

35-37 38-40

$41-42^{d}$

$<35$

64

53.1

32

14

53.2

42.2

46.9

$6 / 14$

46.0

(29.9-55.2)

34.4

$3 / 14$

$(37.0-55.1)$

47.9

43.5

(18.6-53.2)

59.4

46.6

36.7

39.3

$3 / 14$

33.3

36.2

21.4

$3 / 14$

4.0

3.1

6.3

$3 / 14$

2.8

31.8

3.1

14.7

6.1

43.9

2.9

22.2

3.1

$0 / 14$

3.4

$5 / 15$

$0 / 6$

$0 / 15$

$0 / 6$

$5 / 11$

$0 / 3$

58

44.8

3.9

\section{4}

12.5

3.7

10
$1 / 10$

3.5
6

2 / 6

3.7

All Ages Combined

Fresh Embryos

Frozen Embryos

$\begin{array}{cc}11 & 20 \\ 6 / 11 & 30.0 \\ 2.6 & 3.9\end{array}$

\section{CURRENT CLINIC SERVICES AND PROFILE}

Current Name: Arizona Center for Reproductive Endocrinology and Infertility
Donor egg? Yes
Donor embryo? Yes
Gestational carriers? Yes
Cryopreservation?
SART member?
Verified lab accreditation?
(See Appendix $C$ for details.
Yes
Single women? Yes
Yes

${ }^{a}$ Reflects patient and treatment characteristics of ART cycles performed in 2006 using fresh nondonor eggs or embryos.

${ }^{b}$ When fewer than 20 cycles are reported in an age category, rates are shown as a fraction and confidence intervals are not given. Calculating percentages from fractions may be misleading and is not encouraged.

${ }^{c}$ A multiple-infant birth is counted as one live birth.

d Clinic-specific outcome rates are unreliable for women older than 42 undergoing ART cycles using fresh or frozen embryos with nondonor eggs. Readers are urged to review national outcomes for these age groups (see page 27).

${ }^{e}$ All ages (including ages $>42$ ) are reported together because previous data show that patient age does not materially affect success with donor eggs. 


\section{REPRODUCTIVE HEALTH CENTER \\ TUCSON, ARIZONA}

A comparison of clinic success rates may not be meaningful because patient medical characteristics and treatment approaches vary from clinic to clinic. For more details about this, along with information on how to interpret the statistics in this table, see pages 79-88.

\section{ART CYCLE PROFILE}

\begin{tabular}{|c|c|c|c|c|c|c|c|}
\hline \multicolumn{4}{|c|}{ Type of ART ${ }^{a}$} & \multicolumn{4}{|c|}{ Patient Diagnosis } \\
\hline IVF & $100 \%$ & Procedural Factors: & & Tubal factor & $19 \%$ & Other factor & $15 \%$ \\
\hline GIFT & $0 \%$ & With ICSI & $85 \%$ & Ovulatory dysfunction & $1 \%$ & Unknown factor & $18 \%$ \\
\hline ZIFT & $0 \%$ & Unstimulated & $0 \%$ & Diminished ovarian reserve & $<1 \%$ & Multiple Factors: & \\
\hline \multirow[t]{3}{*}{ Combination } & $0 \%$ & Used gestational carrier & $0 \%$ & Endometriosis & $4 \%$ & Female factors only & $18 \%$ \\
\hline & & & & Uterine factor & $<1 \%$ & Female \& male factors & $6 \%$ \\
\hline & & & & Male factor & $18 \%$ & & \\
\hline
\end{tabular}

\section{PREGNANCY SUCCESS RATES}

Data verified by Scot M. Hutchison, MD

\section{Type of Cycle}

\section{Fresh Embryos from Nondonor Eggs}

Number of cycles

Percentage of cycles resulting in pregnancies ${ }^{b}$

Percentage of cycles resulting in live births $s^{b, c}$

(Confidence Interval)

Percentage of retrievals resulting in live births $s^{b, c}$

Percentage of transfers resulting in live births $s^{b, c}$

Percentage of transfers resulting in singleton live births ${ }^{b}$

Percentage of cancellations ${ }^{b}$

Average number of embryos transferred

Percentage of pregnancies with twins ${ }^{b}$

Percentage of pregnancies with triplets or more ${ }^{b}$

Percentage of live births having multiple infants ${ }^{\mathrm{b}, \mathrm{c}}$

\section{Frozen Embryos from Nondonor Eggs}

Number of transfers

Percentage of transfers resulting in live births ${ }^{b, c}$

Average number of embryos transferred

\section{Donor Eggs}

Number of transfers

Percentage of transfers resulting in live births $s^{b, c}$

Average number of embryos transferred

\section{Age of Woman}

35-37 38-40

$41-42^{d}$

$<35$

22

22.7

17

7

25.0

18.2

$3 / 17$

$1 / 7$

23.2

$(5.2-40.3)$

(13.0-36.4)

$4 / 19$

$3 / 17$

$1 / 7$

24.1

26.0

$4 / 18$

$3 / 15$

$1 / 7$

24.0

$2 / 18$

$3 / 14$

$1 / 5$

3.6

13.6

$3 / 14$

$1 / 5$

2.4

2.9

$2 / 17$

$0 / 7$

$2 / 14$

$2 / 5$

2.9

3.8

$0 / 14$

$1 / 13$

$0 / 5$

$1 / 3$

$0 / 1$

$0 / 3$

$0 / 1$

$2 / 4$

$0 / 3$

$0 / 1$

$\begin{array}{cccc}7 & 3 & 6 & 1 \\ 2 / 7 & 1 / 3 & 1 / 6 & 0 / 1 \\ 1.7 & 3.0 & 3.7 & 5.0\end{array}$

All Ages Combined

Fresh Embryos

Frozen Embryos

9

$1 / 9$

$4 / 13$

2.2

\section{CURRENT CLINIC SERVICES AND PROFILE}

Current Name: Reproductive Health Center

Donor egg? Yes Gestational carriers? Yes

Donor embryo? Yes Cryopreservation? Yes

Single women? Yes

SART member? Yes

Verified lab accreditation? Yes

(See Appendix C for details.)

${ }^{a}$ Reflects patient and treatment characteristics of ART cycles performed in 2006 using fresh nondonor eggs or embryos.

${ }^{b}$ When fewer than 20 cycles are reported in an age category, rates are shown as a fraction and confidence intervals are not given. Calculating percentages from fractions may be misleading and is not encouraged.

${ }^{c}$ A multiple-infant birth is counted as one live birth.

${ }^{d}$ Clinic-specific outcome rates are unreliable for women older than 42 undergoing ART cycles using fresh or frozen embryos with nondonor eggs. Readers are urged to review national outcomes for these age groups (see page 27).

e All ages (including ages $>42$ ) are reported together because previous data show that patient age does not materially affect success with donor eggs. 


\section{ARKANSAS FERTILITY CENTER LITTLE ROCK, ARKANSAS}

A comparison of clinic success rates may not be meaningful because patient medical characteristics and treatment approaches vary from clinic to clinic. For more details about this, along with information on how to interpret the statistics in this table, see pages $79-88$.

\section{ART CYCLE PROFILE}

\begin{tabular}{|c|c|c|c|c|c|c|c|}
\hline & Type & of $A R T^{a}$ & & Pat & ent $D$ & iagnosis & \\
\hline IVF & $100 \%$ & Procedural Factors: & & Tubal factor & $20 \%$ & Other factor & $2 \%$ \\
\hline GIFT & $0 \%$ & With ICSI & $28 \%$ & Ovulatory dysfunction & $2 \%$ & Unknown factor & $31 \%$ \\
\hline ZIFT & $0 \%$ & Unstimulated & $0 \%$ & Diminished ovarian reserve & $8 \%$ & Multiple Factors: & \\
\hline Combination & $0 \%$ & Used gestational carrier & $1 \%$ & Endometriosis & $5 \%$ & Female factors only & $2 \%$ \\
\hline & & & & Uterine factor & $3 \%$ & Female \& male factors & $5 \%$ \\
\hline
\end{tabular}

2006 PREGNANCY SUCCESS RATES

Data verified by Dean M. Moutos, MD

\section{Type of Cycle}

\section{Fresh Embryos from Nondonor Eggs}

Number of cycles

Percentage of cycles resulting in pregnancies ${ }^{b}$

Percentage of cycles resulting in live births ${ }^{b, c}$

(Confidence Interval)

Percentage of retrievals resulting in live births $s^{b, c}$

Percentage of transfers resulting in live births $s^{b, c}$

Percentage of transfers resulting in singleton live births ${ }^{b}$

Percentage of cancellations ${ }^{b}$

Average number of embryos transferred

Percentage of pregnancies with twins ${ }^{b}$

Percentage of pregnancies with triplets or more ${ }^{b}$

Percentage of live births having multiple infants ${ }^{b, c}$

\section{Frozen Embryos from Nondonor Eggs}

Number of transfers

Percentage of transfers resulting in live births ${ }^{\mathrm{b}, \mathrm{c}}$

Average number of embryos transferred

\section{Donor Eggs}

Number of transfers

Percentage of transfers resulting in live births $s^{b, c}$

Average number of embryos transferred

\section{Age of Woman}

35-37 38-40

$41-42^{d}$

\section{$<35$}

34

41.2

21

4

44.1

41.2

14.3

$1 / 4$

42.3

(24.6-59.3)

9.5

$1 / 4$

(33.0-52.1)

47.5

48.5

45.2

$(1.2-30.4)$

$2 / 13$

$1 / 3$

50.0

$2 / 12$

$1 / 3$

24.7

32.1

$1 / 12$

$1 / 3$

10.8

8.8

38.1

$1 / 4$

2.4

2.3

2.7

2.7

49.0

$5 / 14$

$1 / 3$

$0 / 1$

6.1

$0 / 14$

$0 / 3$

$0 / 1$

48.9

$5 / 14$

$1 / 2$

$0 / 1$

38

15.8

2.3

\section{0}

$1 / 10$

2.1

\section{7 \\ $4 / 7$}

2.7
2

$0 / 2$

3.0

\section{CURRENT CLINIC SERVICES AND PROFILE}

Fresh Embryos

All Ages Combined ${ }^{\mathrm{e}}$

$\begin{array}{cc}12 & 5 \\ 8 / 12 & 1 / 5 \\ 2.3 & 2.4\end{array}$

Current Name: Arkansas Fertility Center

\begin{tabular}{ll|lll|l|l} 
Donor egg? & Yes & Gestational carriers? & Yes & SART member? & Yes \\
\hline Donor embryo? & Yes & Cryopreservation? & Yes & Verified lab accreditation? & Yes \\
Single women? & No & & & (See Appendix C for details.) &
\end{tabular}

${ }^{a}$ Reflects patient and treatment characteristics of ART cycles performed in 2006 using fresh nondonor eggs or embryos.

b When fewer than 20 cycles are reported in an age category, rates are shown as a fraction and confidence intervals are not given. Calculating percentages from fractions may be misleading and is not encouraged.

A multiple-infant birth is counted as one live birth.

${ }^{d}$ Clinic-specific outcome rates are unreliable for women older than 42 undergoing ART cycles using fresh or frozen embryos with nondonor eggs. Readers are urged to review national outcomes for these age groups (see page 27).

e All ages (including ages $>42$ ) are reported together because previous data show that patient age does not materially affect success with donor eggs. 


\section{LIFESTART FERTILITY CENTER \\ ANITA SINGH, MD \\ AGOURA HILLS, CALIFORNIA}

A comparison of clinic success rates may not be meaningful because patient medical characteristics and treatment approaches vary from clinic to clinic. For more details about this, along with information on how to interpret the statistics in this table, see pages 79-88.

\section{ART CYCLE PROFILE}

\section{Type of ART ${ }^{\mathrm{a}}$}

IVF

GIFT

100\% Procedural Factors:

ZIFT

Combination
$0 \%$ With ICSI

$0 \%$ Unstimulated

$0 \%$ Used gestational carrier

\begin{tabular}{r|l} 
& Tubal factor \\
\hline $77 \%$ & Ovulatory dysfunction \\
0\% & Diminished ovarian reserve \\
0\% & Endometriosis \\
& Uterine factor \\
& Male factor
\end{tabular}

Patient Diagnosis

$5 \%$ Other factor

5\% Unknown factor

10\% Multiple Factors:

$5 \%$ Female factors only $14 \%$

$5 \%$ Female \& male factors $48 \%$

$5 \%$
$0 \%$

$5 \%$

$14 \%$
$8 \%$

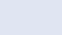

2006 PREGNANCY SUCCESS RATES

Data verified by Anita P. Singh, MD

\section{Type of Cycle}

\section{Fresh Embryos from Nondonor Eggs}

Number of cycles

Percentage of cycles resulting in pregnancies ${ }^{\mathrm{b}}$

Percentage of cycles resulting in live births ${ }^{b, c}$

(Confidence Interval)

Percentage of retrievals resulting in live births $s^{b, c}$

Percentage of transfers resulting in live births $s^{b, c}$

Percentage of transfers resulting in singleton live births ${ }^{b}$

Percentage of cancellations ${ }^{b}$

Average number of embryos transferred

Percentage of pregnancies with twins ${ }^{b}$

Percentage of pregnancies with triplets or more

Percentage of live births having multiple infants ${ }^{\mathrm{b}, \mathrm{c}}$

\section{Frozen Embryos from Nondonor Eggs}

Number of transfers

Percentage of transfers resulting in live births ${ }^{b, c}$

Average number of embryos transferred

\section{Donor Eggs}

Number of transfers

Percentage of transfers resulting in live births ${ }^{b, c}$

Average number of embryos transferred

\section{Age of Woman}

$<35$

35-37

38-40

$41-42^{d}$

7

$5 / 7$

3

$4 / 7$

$3 / 3$

5

1

$3 / 3$

$2 / 5$

$0 / 1$

$2 / 5$

$0 / 1$

$4 / 7$

$3 / 3$

$2 / 5$

$0 / 1$

$4 / 6$

$3 / 3$

$2 / 4$

$0 / 1$

$2 / 6$

$2 / 3$

$2 / 4$

$0 / 1$

$0 / 7$

$0 / 3$

$0 / 5$

$0 / 1$

2.2

2.7

2.3

4.0

$0 / 5$

$1 / 3$

$0 / 2$

$2 / 5$

$0 / 3$

$0 / 2$

$2 / 4$

$1 / 3$

$0 / 2$

3

$3 / 3$

2.0
0

\section{Fresh Embryos}

All Ages Combined

\section{CURRENT CLINIC SERVICES AND PROFILE}

Current Name: Lifestart Fertility Center, Anita Singh, MD

Donor egg? Yes

Donor embryo? Yes

Gestational carriers? Yes

Cryopreservation?

SART member?

No

Single women? Yes

Verified lab accreditation?

Yes

(See Appendix C for details.)

${ }^{a}$ Reflects patient and treatment characteristics of ART cycles performed in 2006 using fresh nondonor eggs or embryos.

b When fewer than 20 cycles are reported in an age category, rates are shown as a fraction and confidence intervals are not given. Calculating percentages from fractions may be misleading and is not encouraged.

${ }^{c}$ A multiple-infant birth is counted as one live birth.

d Clinic-specific outcome rates are unreliable for women older than 42 undergoing ART cycles using fresh or frozen embryos with nondonor eggs. Readers are urged to review national outcomes for these age groups (see page 27).

e All ages (including ages $>42$ ) are reported together because previous data show that patient age does not materially affect success with donor eggs. 


\section{GARFIELD FERTILITY CENTER ALHAMBRA, CALIFORNIA}

A comparison of clinic success rates may not be meaningful because patient medical characteristics and treatment approaches vary from clinic to clinic. For more details about this, along with information on how to interpret the statistics in this table, see pages 79-88.

\section{ART CYCLE PROFILE}

\section{Type of ART ${ }^{\mathrm{a}}$}

IVF

GIFT

100\% Procedural Factors:

ZIFT

Combination

\author{
$0 \%$ With ICSI
}

$0 \%$ Unstimulated

$0 \%$ Used gestational carrier

\begin{tabular}{|c|c|c|c|c|}
\hline & \multicolumn{4}{|c|}{ Patient Diagnosis } \\
\hline & Tubal factor & $4 \%$ & Other factor & $4 \%$ \\
\hline $33 \%$ & Ovulatory dysfunction & $8 \%$ & Unknown factor & $14 \%$ \\
\hline $0 \%$ & Diminished ovarian reserve & $17 \%$ & Multiple Factors: & \\
\hline $0 \%$ & Endometriosis & $0 \%$ & Female factors only & $25 \%$ \\
\hline & Uterine factor & $4 \%$ & Female \& male factors & $14 \%$ \\
\hline & Male factor & $12 \%$ & & \\
\hline
\end{tabular}

Data verified by Brian C. Su, MD

\section{PREGNANCY SUCCESS RATES}

\section{Type of Cycle}

\section{Fresh Embryos from Nondonor Eggs}

Number of cycles

Percentage of cycles resulting in pregnancies ${ }^{b}$

Percentage of cycles resulting in live births $s^{b, c}$

(Confidence Interval)

Percentage of retrievals resulting in live births $s^{b, c}$

Percentage of transfers resulting in live births $s^{b, c}$

Percentage of transfers resulting in singleton live births ${ }^{b}$

Percentage of cancellations ${ }^{b}$

Average number of embryos transferred

Percentage of pregnancies with twins ${ }^{b}$

Percentage of pregnancies with triplets or more ${ }^{b}$

Percentage of live births having multiple infants ${ }^{b, c}$

\section{Frozen Embryos from Nondonor Eggs}

Number of transfers

Percentage of transfers resulting in live births ${ }^{\mathrm{b}, \mathrm{c}}$

Average number of embryos transferred

$\begin{array}{cccc}<35 & 35-37 & 38-40 & \mathbf{4 1 - 4 2}^{\text {d }} \\ 14 & 10 & 6 & 4 \\ 6 / 14 & 3 / 10 & 2 / 6 & 1 / 4 \\ 4 / 14 & 3 / 10 & 2 / 6 & 1 / 4\end{array}$

$\begin{array}{cccc}4 / 13 & 3 / 9 & 2 / 6 & 1 / 4 \\ 4 / 10 & 3 / 8 & 2 / 5 & 1 / 4 \\ 2 / 10 & 3 / 8 & 1 / 5 & 0 / 4 \\ 1 / 14 & 1 / 10 & 0 / 6 & 0 / 4 \\ 2.8 & 2.9 & 2.8 & 3.0 \\ 1 / 6 & 1 / 3 & 0 / 2 & 1 / 1 \\ 1 / 6 & 0 / 3 & 1 / 2 & 0 / 1 \\ 2 / 4 & 0 / 3 & 1 / 2 & 1 / 1\end{array}$

\section{Donor Eggs}

\section{5}

$2 / 5$

2.2
0

0

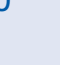
1
$0 / 1$
3.0

Number of transfers

Percentage of transfers resulting in live births $s^{b, c}$

Average number of embryos transferred

\begin{tabular}{cc}
\multicolumn{2}{c}{ All Ages Combined } \\
Fresh Embryos & $\begin{array}{c}\text { Frozen Embryos } \\
4\end{array}$ \\
$4 / 4$ & $1 / 1$ \\
2.0 & 1.0
\end{tabular}

\section{CURRENT CLINIC SERVICES AND PROFILE}

Current Name: Garfield Fertility Center

\begin{tabular}{ll|ll|l|l} 
Donor egg? & Yes & Gestational carriers? & Yes & SART member? & Yes \\
\hline Donor embryo? & Yes & Cryopreservation? & Yes & Verified lab accreditation? & Yes \\
Single women? & No & & & (See Appendix C for details.) &
\end{tabular}

${ }^{a}$ Reflects patient and treatment characteristics of ART cycles performed in 2006 using fresh nondonor eggs or embryos.

b When fewer than 20 cycles are reported in an age category, rates are shown as a fraction and confidence intervals are not given. Calculating percentages from fractions may be misleading and is not encouraged.

${ }^{c}$ A multiple-infant birth is counted as one live birth.

${ }^{d}$ Clinic-specific outcome rates are unreliable for women older than 42 undergoing ART cycles using fresh or frozen embryos with nondonor eggs. Readers are urged to review national outcomes for these age groups (see page 27).

e All ages (including ages $>42$ ) are reported together because previous data show that patient age does not materially affect success with donor eggs. 


\section{ALTA BATES IN VITRO FERTILIZATION PROGRAM BERKELEY, CALIFORNIA}

A comparison of clinic success rates may not be meaningful because patient medical characteristics and treatment approaches vary from clinic to clinic. For more details about this, along with information on how to interpret the statistics in this table, see pages 79-88.

\section{ART CYCLE PROFILE}

\section{Type of ART ${ }^{\mathrm{a}}$}

IVF

GIFT $100 \%$ Procedural Factors:

ZIFT

Combination
$0 \%$ With ICSI

$0 \%$ Unstimulated

$0 \%$ Used gestational carrier

\begin{tabular}{|c|c|c|c|c|}
\hline & \multicolumn{4}{|c|}{ Patient Diagnosis } \\
\hline & Tubal factor & $6 \%$ & Other factor & $9 \%$ \\
\hline $84 \%$ & Ovulatory dysfunction & $7 \%$ & Unknown factor & $4 \%$ \\
\hline $0 \%$ & Diminished ovarian reserve & $26 \%$ & Multiple Factors: & \\
\hline $3 \%$ & Endometriosis & $4 \%$ & Female factors only & $21 \%$ \\
\hline & Uterine factor & $2 \%$ & Female \& male factors & $16 \%$ \\
\hline & Male factor & $6 \%$ & & \\
\hline
\end{tabular}

Data verified by Ryszard J. Chetkowski, MD

2006 PREGNANCY SUCCESS RATES

Age of Woman

Type of Cycle

$\begin{array}{cccc}<35 & 35-37 & 38-40 & \mathbf{4 1 - 4 2}^{\mathbf{d}} \\ 18 & 10 & 17 & 13 \\ 7 / 18 & 5 / 10 & 6 / 17 & 6 / 13 \\ 6 / 18 & 5 / 10 & 3 / 17 & 3 / 13\end{array}$

Fresh Embryos from Nondonor Eggs

Number of cycles

$7 / 18$

$5 / 10$

$3 / 17$

$3 / 13$

Percentage of cycles resulting in live births ${ }^{b, c}$

(Confidence Interval)

Percentage of retrievals resulting in live births $s^{b, c}$

$6 / 14$

$5 / 9$

$3 / 17$

$3 / 13$

$6 / 12$

$5 / 8$

$3 / 16$

$3 / 12$

Percentage of transfers resulting in singleton live births ${ }^{b}$

$5 / 12$

$4 / 8$

$3 / 16$

$3 / 12$

$4 / 18$

$1 / 10$

$0 / 17$

$0 / 13$

2.7

$1 / 7$

2.5

$1 / 5$

3.1

4.1

$0 / 7$

$0 / 5$

$0 / 6$

$0 / 6$

$1 / 6$

$1 / 5$

$0 / 6$

$0 / 6$

Percentage of pregnancies with triplets or more
Percentage of live births having multiple infants ${ }^{b, c}$

Frozen Embryos from Nondonor Eggs

Number of transfers

Percentage of transfers resulting in live births ${ }^{b, c}$

Average number of embryos transferred

$\begin{array}{cccc}5 & 9 & 4 & 8 \\ 0 / 5 & 3 / 9 & 2 / 4 & 2 / 8 \\ 2.6 & 2.3 & 3.5 & 2.9\end{array}$

\section{Donor Eggs}

All Ages Combined

Number of transfers

Percentage of transfers resulting in live births $s^{b, c}$

Average number of embryos transferred

\section{Fresh Embryos}

15

$8 / 15$

2.3
Frozen Embryos

22

40.9

2.4

\section{CURRENT CLINIC SERVICES AND PROFILE}

Current Name: Alta Bates In Vitro Fertilization Program

Donor egg? Yes

Donor embryo? Yes

Single women? Yes

\begin{abstract}
Gestational carriers? Yes
Cryopreservation? Yes
\end{abstract}

SART member?

Yes

Verified lab accreditation?

(See Appendix C for details.)

${ }^{a}$ Reflects patient and treatment characteristics of ART cycles performed in 2006 using fresh nondonor eggs or embryos.

${ }^{b}$ When fewer than 20 cycles are reported in an age category, rates are shown as a fraction and confidence intervals are not given. Calculating percentages from fractions may be misleading and is not encouraged.

${ }^{c}$ A multiple-infant birth is counted as one live birth.

${ }^{d}$ Clinic-specific outcome rates are unreliable for women older than 42 undergoing ART cycles using fresh or frozen embryos with nondonor eggs. Readers are urged to review national outcomes for these age groups (see page 27).

e All ages (including ages $>42$ ) are reported together because previous data show that patient age does not materially affect success with donor eggs. 


\section{CENTER FOR REPRODUCTIVE HEALTH \& GYNECOLOGY \\ (CRH\&G) \\ BEVERLY HILLS, CALIFORNIA}

A comparison of clinic success rates may not be meaningful because patient medical characteristics and treatment approaches vary from clinic to clinic. For more details about this, along with information on how to interpret the statistics in this table, see pages 79-88.

\section{ART CYCLE PROFILE}

$$
\text { Type of ART }{ }^{\mathrm{a}}
$$

\begin{tabular}{|c|c|c|c|c|c|c|c|}
\hline IVF & $100 \%$ & Procedural Factors: & & Tubal factor & $8 \%$ & Other factor & $8 \%$ \\
\hline GIFT & $0 \%$ & With ICSI & $46 \%$ & Ovulatory dysfunction & $7 \%$ & Unknown factor & $11 \%$ \\
\hline ZIFT & $0 \%$ & Unstimulated & $0 \%$ & Diminished ovarian reserve & $25 \%$ & Multiple Factors: & \\
\hline \multirow{3}{*}{ Combination } & $0 \%$ & Used gestational carrier & $3 \%$ & Endometriosis & $9 \%$ & Female factors only & \\
\hline & & & & Uterine factor & $3 \%$ & Female \& male factors & \\
\hline & & & & Male factor & $19 \%$ & & \\
\hline
\end{tabular}

2006 PREGNANCY SUCCESS RATES

Data verified by Sam Najmabadi, MD

\section{Type of Cycle}

\section{Fresh Embryos from Nondonor Eggs}

Number of cycles

Percentage of cycles resulting in pregnancies ${ }^{b}$

Percentage of cycles resulting in live births ${ }^{b, c}$

(Confidence Interval)

Percentage of retrievals resulting in live births $s^{b, c}$

Percentage of transfers resulting in live births $s^{b, c}$

Percentage of transfers resulting in singleton live births ${ }^{b}$

Percentage of cancellations ${ }^{b}$

Average number of embryos transferred

Percentage of pregnancies with twins ${ }^{b}$

Percentage of pregnancies with triplets or more ${ }^{b}$

Percentage of live births having multiple infants ${ }^{b, c}$

\section{Frozen Embryos from Nondonor Eggs}

Number of transfers

Percentage of transfers resulting in live births $s^{b, c}$

Average number of embryos transferred

\section{Donor Eggs}

Number of transfers

Percentage of transfers resulting in live births ${ }^{\mathrm{b}, \mathrm{c}}$

Average number of embryos transferred

\section{Age of Woman}

35-37 38-40

$41-42^{d}$ $<35$

32

27

20

69.2

59.4

37.0

40.0

66.7

46.9

29.6

25.0

(49.8-80.9)

$(29.1-65.3)$

(13.8-50.2)

(8.7-49.1)

66.7

46.9

29.6

25.0

68.4

50.0

29.6

25.0

52.6

43.3

22.2

20.0

0.0

0.0

0.0

0.0

1.8

1.7

1.7

1.5

33.3

$3 / 19$

$2 / 10$

$1 / 8$

0.0

$0 / 19$

$0 / 10$

$0 / 8$

23.1

2/ 15

$2 / 8$

$1 / 5$

$\begin{array}{cccc}11 & 6 & 2 & 1 \\ 7 / 11 & 3 / 6 & 2 / 2 & 1 / 1 \\ 2.2 & 2.2 & 1.5 & 3.0\end{array}$

All Ages Combined ${ }^{\mathrm{e}}$ Fresh Embryos

Frozen Embryos

$\begin{array}{cc}11 & 4 \\ 9 / 11 & 4 / 4 \\ 1.9 & 2.0\end{array}$

\section{CURRENT CLINIC SERVICES AND PROFILE}

Current Name: Center for Reproductive Health \& Gynecology, (CRH\&G)

Donor egg? Yes Gestational carriers? Yes

Donor embryo? No Cryopreservation? Yes

Single women? Yes

$\begin{array}{ll}\text { SART member? } & \text { Yes } \\ \text { Verified lab accreditation? } & \text { Yes } \\ \text { (See Appendix C for details.) } & \end{array}$

${ }^{a}$ Reflects patient and treatment characteristics of ART cycles performed in 2006 using fresh nondonor eggs or embryos.

${ }^{b}$ When fewer than 20 cycles are reported in an age category, rates are shown as a fraction and confidence intervals are not given. Calculating percentages from fractions may be misleading and is not encouraged.

${ }^{c}$ A multiple-infant birth is counted as one live birth.

d Clinic-specific outcome rates are unreliable for women older than 42 undergoing ART cycles using fresh or frozen embryos with nondonor eggs. Readers are urged to review national outcomes for these age groups (see page 27).

${ }^{e}$ All ages (including ages $>42$ ) are reported together because previous data show that patient age does not materially affect success with donor eggs. 


\section{SOUTHERN CALIFORNIA REPRODUCTIVE CENTER \\ BEVERLY HILLS, CALIFORNIA}

A comparison of clinic success rates may not be meaningful because patient medical characteristics and treatment approaches vary from clinic to clinic. For more details about this, along with information on how to interpret the statistics in this table, see pages 79-88.

\section{ART CYCLE PROFILE}

\begin{tabular}{|c|c|c|c|c|c|c|c|}
\hline \multicolumn{4}{|c|}{ Type of ART ${ }^{a}$} & \multicolumn{4}{|c|}{ Patient Diagnosis } \\
\hline IVF & $100 \%$ & Procedural Factors: & & Tubal factor & $8 \%$ & Other factor & $10 \%$ \\
\hline GIFT & $0 \%$ & With ICSI & $50 \%$ & Ovulatory dysfunction & $5 \%$ & Unknown factor & $12 \%$ \\
\hline $\mathrm{ZIFT}$ & $0 \%$ & Unstimulated & $0 \%$ & Diminished ovarian reserve & $24 \%$ & Multiple Factors: & \\
\hline \multirow[t]{3}{*}{ Combination } & $0 \%$ & Used gestational carrier & $0 \%$ & Endometriosis & $8 \%$ & Female factors only & $10 \%$ \\
\hline & & & & Uterine factor & $1 \%$ & Female \& male factors & $6 \%$ \\
\hline & & & & Male factor & $17 \%$ & & \\
\hline
\end{tabular}

\section{PREGNANCY SUCCESS RATES}

Data verified by Hal Danzer, MD

\section{Type of Cycle}

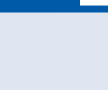

\section{Fresh Embryos from Nondonor Eggs}

Number of cycles

Percentage of cycles resulting in pregnancies ${ }^{b}$

Percentage of cycles resulting in live births ${ }^{\mathrm{b}, \mathrm{c}}$

(Confidence Interval)

Percentage of retrievals resulting in live births ${ }^{b, c}$

Percentage of transfers resulting in live births ${ }^{b, c}$

Percentage of transfers resulting in singleton live births ${ }^{b}$

Percentage of cancellations ${ }^{b}$

Average number of embryos transferred

Percentage of pregnancies with twins ${ }^{b}$

Percentage of pregnancies with triplets or more

Percentage of live births having multiple infants ${ }^{b, c}$

\section{Frozen Embryos from Nondonor Eggs}

Number of transfers

Percentage of transfers resulting in live births ${ }^{b, c}$

Average number of embryos transferred

\section{Donor Eggs}

Number of transfers

Percentage of transfers resulting in live births ${ }^{b, c}$

Average number of embryos transferred

\section{Age of Woman}

$<35 \quad 35-37 \quad 38-40 \quad 41-42^{\text {d }}$

31

67.7

29

31.0

42

17

67.7

31.0

31.0

$5 / 17$

(48.6-83.3)

(15.3-50.8)

70.0

33.3

23.8

$4 / 17$

70.0

34.6

(12.1-39.5)

25.0

27.0

$4 / 16$

26.9

18.9

$4 / 14$

56.7

6.9

4.8

$2 / 14$

1.9

2.3

$2 / 9$

2.8

$1 / 17$

23.8

4.8

19.0

$0 / 9$

$2 / 13$

3.1

$2 / 5$

$2 / 9$

$1 / 13$

$0 / 5$

$3 / 10$

$2 / 4$

9

$4 / 9$

6

$0 / 6$

4

$0 / 4$

3

2.3

2.2

2.8

$1 / 3$

2.7

All Ages Combined ${ }^{\mathrm{e}}$

Fresh Embryos

19

$10 / 19$

1.9
Frozen Embryos

\section{7}

$3 / 7$

2.7

\section{CURRENT CLINIC SERVICES AND PROFILE}

Current Name: Southern California Reproductive Center

Donor egg? Yes

Donor embryo? Yes

Single women? Yes
Gestational carriers? Yes

Cryopreservation? Yes
SART member?

Yes

Verified lab accreditation?

(See Appendix C for details.)

\footnotetext{
${ }^{a}$ Reflects patient and treatment characteristics of ART cycles performed in 2006 using fresh nondonor eggs or embryos.

b When fewer than 20 cycles are reported in an age category, rates are shown as a fraction and confidence intervals are not given. Calculating percentages from fractions may be misleading and is not encouraged.

${ }^{c}$ A multiple-infant birth is counted as one live birth.

d Clinic-specific outcome rates are unreliable for women older than 42 undergoing ART cycles using fresh or frozen embryos with nondonor eggs. Readers are urged to review national outcomes for these age groups (see page 27).

e All ages (including ages $>42$ ) are reported together because previous data show that patient age does not materially affect success with donor eggs.
} 


\section{SOUTHERN CALIFORNIA REPRODUCTIVE CENTER BEVERLY HILLS, CALIFORNIA}

A comparison of clinic success rates may not be meaningful because patient medical characteristics and treatment approaches vary from clinic to clinic. For more details about this, along with information on how to interpret the statistics in this table, see pages 79-88.

\section{ART CYCLE PROFILE}

\section{Type of ART ${ }^{\mathrm{a}}$}

IVF

GIFT

100\% Procedural Factors:

ZIFT

$0 \%$ With ICSI

Combination
$0 \%$ Unstimulated

0\% Used gestational carrier

\begin{tabular}{|c|c|}
\hline \multirow{5}{*}{$\begin{array}{r}55 \% \\
0 \% \\
3 \%\end{array}$} & Ovulatory dvsfunction \\
\hline & Diminished ovarian reserve \\
\hline & Endometriosis \\
\hline & Uterine factor \\
\hline & Male factor \\
\hline
\end{tabular}

$6 \%$ Other factor

5\% Unknown factor

4\% Multiple Factors:

$2 \%$ Female factors only $36 \%$

$1 \%$ Female \& male factors $26 \%$ $6 \%$

2006 PREGNANCY SUCCESS RATES

Data verified by Mark W. Surrey, MD

\section{Type of Cycle}

\section{Age of Woman}

\section{Fresh Embryos from Nondonor Eggs}

Number of cycles

Percentage of cycles resulting in pregnancies ${ }^{b}$

Percentage of cycles resulting in live births $s^{b, c}$

(Confidence Interval)

Percentage of retrievals resulting in live births $s^{b, c}$

Percentage of transfers resulting in live births $s^{b, c}$

Percentage of transfers resulting in singleton live births ${ }^{b}$

Percentage of cancellations ${ }^{b}$

Average number of embryos transferred

Percentage of pregnancies with twins ${ }^{b}$

Percentage of pregnancies with triplets or more ${ }^{b}$

Percentage of live births having multiple infants ${ }^{b, c}$

\section{Frozen Embryos from Nondonor Eggs}

Number of transfers

Percentage of transfers resulting in live births $s^{\mathrm{b}, \mathrm{c}}$

Average number of embryos transferred

\begin{tabular}{|c|c|c|c|c|}
\hline & 61 & 42 & 65 & 33 \\
\hline & 54.1 & 50.0 & 27.7 & 39.4 \\
\hline & 47.5 & 47.6 & 21.5 & 27.3 \\
\hline & $(34.6-60.7)$ & $(32.0-63.6)$ & $(12.3-33.5)$ & $(13.3-45.5)$ \\
\hline & 49.2 & 48.8 & 23.0 & 28.1 \\
\hline & 51.8 & 58.8 & 27.5 & 30.0 \\
\hline le births ${ }^{b}$ & 33.9 & 47.1 & 25.5 & 23.3 \\
\hline & 3.3 & 2.4 & 6.2 & 3.0 \\
\hline & 2.1 & 2.4 & 2.2 & 2.6 \\
\hline & 24.2 & 14.3 & $2 / 18$ & $3 / 13$ \\
\hline$e^{b}$ & 6.1 & 9.5 & $1 / 18$ & $0 / 13$ \\
\hline$b, c$ & 34.5 & 20.0 & $1 / 14$ & $2 / 9$ \\
\hline & 10 & 13 & 5 & 1 \\
\hline & $3 / 10$ & $4 / 13$ & $0 / 5$ & $0 / 1$ \\
\hline & 2.5 & 2.1 & 2.2 & 3.0 \\
\hline & \multicolumn{4}{|c|}{ All Ages Combined ${ }^{e}$} \\
\hline & \multicolumn{2}{|c|}{ Fresh Embryos } & \multicolumn{2}{|c|}{ Frozen Embryos } \\
\hline & \multicolumn{2}{|c|}{30} & \multicolumn{2}{|c|}{7} \\
\hline & \multicolumn{2}{|c|}{60.0} & \multicolumn{2}{|c|}{$4 / 7$} \\
\hline & \multicolumn{2}{|c|}{1.9} & \multicolumn{2}{|c|}{2.3} \\
\hline
\end{tabular}

\section{Donor Eggs} $<35$

35-37

38-40

$41-42^{d}$

Number of transfers

Percentage of transfers resulting in live births $s^{b, c}$

Average number of embryos transferred

1.9

\section{CURRENT CLINIC SERVICES AND PROFILE}

Current Name: Southern California Reproductive Center

\begin{tabular}{ll|ll} 
Donor egg? & Yes & Gestational carriers? & Yes \\
Donor embryo? & Yes & Cryopreservation? & Yes
\end{tabular}

Single women? Yes

\section{SART member? Yes}

Verified lab accreditation? Yes

(See Appendix C for details.)

${ }^{a}$ Reflects patient and treatment characteristics of ART cycles performed in 2006 using fresh nondonor eggs or embryos.

${ }^{b}$ When fewer than 20 cycles are reported in an age category, rates are shown as a fraction and confidence intervals are not given. Calculating percentages from fractions may be misleading and is not encouraged.

${ }^{c}$ A multiple-infant birth is counted as one live birth.

d Clinic-specific outcome rates are unreliable for women older than 42 undergoing ART cycles using fresh or frozen embryos with nondonor eggs. Readers are urged to review national outcomes for these age groups (see page 27).

e All ages (including ages $>42$ ) are reported together because previous data show that patient age does not materially affect success with donor eggs. 


\section{WEST COAST IVF CLINIC, INC. \\ BEVERLY HILLS, CALIFORNIA}

A comparison of clinic success rates may not be meaningful because patient medical characteristics and treatment approaches vary from clinic to clinic. For more details about this, along with information on how to interpret the statistics in this table, see pages 79-88.

\section{ART CYCLE PROFILE}

\section{Type of ART ${ }^{\mathrm{a}}$}

IVF

GIFT

100\% Procedural Factors:

ZIFT

Combination
$0 \%$ With ICSI

$0 \%$ Unstimulated

$0 \%$ Used gestational carrier

\section{$77 \%$ \\ $0 \%$ \\ $0 \%$}

Tubal factor

Ovulatory dysfunction

Diminished ovarian reserve

Endometriosis

Uterine factor

Male factor

\section{Patient Diagnosis}

$15 \%$ Other factor

$0 \%$ Unknown factor

0\% Multiple Factors:

$10 \%$ Female factors only

Female \& male factors $23 \%$

$15 \%$
$7 \%$

$18 \%$

$12 \%$

2006 PREGNANCY SUCCESS RATES

Data verified by Michael Kamrava, MD

\section{Type of Cycle}

\section{Fresh Embryos from Nondonor Eggs}

Number of cycles

Percentage of cycles resulting in pregnancies ${ }^{b}$

Percentage of cycles resulting in live births $s^{b, c}$

(Confidence Interval)

Percentage of retrievals resulting in live births $s^{b, c}$

Percentage of transfers resulting in live births $s^{b, c}$

Percentage of transfers resulting in singleton live births ${ }^{b}$

Percentage of cancellations ${ }^{b}$

Average number of embryos transferred

Percentage of pregnancies with twins ${ }^{b}$

Percentage of pregnancies with triplets or more ${ }^{b}$

Percentage of live births having multiple infants ${ }^{b, c}$

\section{Frozen Embryos from Nondonor Eggs}

Number of transfers

Percentage of transfers resulting in live births $s^{b, c}$

Average number of embryos transferred

\section{Donor Eggs}

Number of transfers

Percentage of transfers resulting in live births $s^{b, c}$

Average number of embryos transferred

\section{Age of Woman}

35-37 38-40

$41-42^{d}$ $<35$

9

13

10

20
20.0

$0 / 9$

$1 / 13$

$0 / 10$

10.0

$0 / 9$

$0 / 13$

$0 / 10$

(1.2-31.7)

$\begin{array}{cccc}2 / 18 & 0 / 7 & 0 / 12 & 0 / 8 \\ 2 / 15 & 0 / 6 & 0 / 8 & 0 / 8 \\ 1 / 15 & 0 / 6 & 0 / 8 & 0 / 8 \\ 10.0 & 2 / 9 & 1 / 13 & 2 / 10 \\ 3.5 & 2.3 & 2.3 & 2.5 \\ 1 / 4 & & 0 / 1 & \\ 0 / 4 & & 0 / 1 & \\ 1 / 2 & & & \end{array}$

$1 / 2$

0

0

0

0

\section{CURRENT CLINIC SERVICES AND PROFILE}

Current Name: West Coast IVF Clinic, Inc.
Donor egg?
Yes
Donor embryo? No
Gestational carriers?
Yes
Cryopreservation?
Yes
Single women? Yes
SART member? Yes
Verified lab accreditation?
(See Appendix C for details.)
${ }^{a}$ Reflects patient and treatment characteristics of ART cycles performed in 2006 using fresh nondonor eggs or embryos.
${ }^{b}$ When fewer than 20 cycles are reported in an age category, rates are shown as a fraction and confidence intervals are not given. Calculating percentages from fractions may be misleading and is not encouraged.
${ }^{c}$ A multiple-infant birth is counted as one live birth.
d Clinic-specific outcome rates are unreliable for women older than 42 undergoing ART cycles using fresh or frozen embryos with nondonor eggs. Readers are urged to review national outcomes for these age groups (see page 27).
e All ages (including ages $>42$ ) are reported together because previous data show that patient age does not materially affect success with donor eggs.

\section{Fresh Embryos}

3

$0 / 3$

3.0 


\section{FERTILITY CARE OF ORANGE COUNTY BREA, CALIFORNIA}

A comparison of clinic success rates may not be meaningful because patient medical characteristics and treatment approaches vary from clinic to clinic. For more details about this, along with information on how to interpret the statistics in this table, see pages 79-88.

\section{ART CYCLE PROFILE}

\section{Type of ART ${ }^{\mathrm{a}}$}

IVF

GIFT

100\% Procedural Factors:

ZIFT

Combination

\author{
$0 \%$ With ICSI
}

$0 \%$ Unstimulated

0\% Used gestational carrier

\section{Patient Diagnosis}

$7 \%$ Other factor $\quad 4 \%$

$3 \%$ Unknown factor $\quad 27 \%$

$66 \%$ Ovulatory dysfunction

$0 \%$ Diminished ovarian reserve $27 \%$ Multiple Factors:

$4 \%$

$2 \%$ Female factors only $2 \%$

$0 \%$ Female \& male factors $13 \%$

Uterine factor $\quad 0 \%$

Male factor $15 \%$

\section{PREGNANCY SUCCESS RATES}

\section{Type of Cycle}

\section{Fresh Embryos from Nondonor Eggs}

Number of cycles

Percentage of cycles resulting in pregnancies ${ }^{b}$

Percentage of cycles resulting in live births $s^{b, c}$

(Confidence Interval)

Percentage of retrievals resulting in live births $s^{b, c}$

Percentage of transfers resulting in live births $s^{b, c}$

Percentage of transfers resulting in singleton live births ${ }^{b}$

Percentage of cancellations ${ }^{b}$

Average number of embryos transferred

Percentage of pregnancies with twins ${ }^{b}$

Percentage of pregnancies with triplets or more ${ }^{b}$

Percentage of live births having multiple infants ${ }^{b, c}$

\section{Frozen Embryos from Nondonor Eggs}

Number of transfers

Percentage of transfers resulting in live births ${ }^{\mathrm{b}, \mathrm{c}}$

Average number of embryos transferred

\section{Donor Eggs}

Number of transfers

Percentage of transfers resulting in live births $s^{b, c}$

Average number of embryos transferred
Data verified by C. Terence Lee, MD

\section{Age of Woman}

$<35 \quad 35-37 \quad 38-40 \quad 41-42^{d}$

$\begin{array}{cccc}37 & 21 & 19 & 10 \\ 35.1 & 28.6 & 6 / 19 & 2 / 10 \\ 35.1 & 19.0 & 4 / 19 & 1 / 10\end{array}$

$(20.2-52.5)$

(5.4-41.9)

44.8

$4 / 14$

$4 / 14$

$1 / 9$

48.1

$4 / 14$

$4 / 14$

$1 / 9$

18.5

$2 / 14$

$3 / 14$

$1 / 9$

21.6

33.3

$5 / 19$

$1 / 10$

3.2

3.6

4.1

3.8

$7 / 13$

$1 / 6$

$1 / 6$

$0 / 2$

$1 / 13$

$1 / 6$

$1 / 6$

$0 / 2$

$8 / 13$

$2 / 4$

$1 / 4$

$0 / 1$

$\begin{array}{cccc}11 & 8 & 1 & 1 \\ 3 / 11 & 0 / 8 & 0 / 1 & 0 / 1 \\ 2.5 & 2.3 & 2.0 & 4.0\end{array}$

All Ages Combined

Fresh Embryos

Frozen Embryos

9

$5 / 9$

4

2.0

$0 / 4$

1.8

\section{CURRENT CLINIC SERVICES AND PROFILE}

Current Name: Fertility Care of Orange County

\begin{tabular}{|ll|ll|ll} 
Donor egg? & Yes & Gestational carriers? & Yes & SART member? & No \\
Donor embryo? & No & Cryopreservation? & Yes & Verified lab accreditation? & Yes \\
\hline Single women? & Yes & & & (See Appendix C for details.)
\end{tabular}

${ }^{a}$ Reflects patient and treatment characteristics of ART cycles performed in 2006 using fresh nondonor eggs or embryos.

${ }^{b}$ When fewer than 20 cycles are reported in an age category, rates are shown as a fraction and confidence intervals are not given. Calculating

percentages from fractions may be misleading and is not encouraged.

A multiple-infant birth is counted as one live birth.

${ }^{d}$ Clinic-specific outcome rates are unreliable for women older than 42 undergoing ART cycles using fresh or frozen embryos with nondonor eggs. Readers are urged to review national outcomes for these age groups (see page 27).

${ }^{e}$ All ages (including ages $>42$ ) are reported together because previous data show that patient age does not materially affect success with donor eggs.

\section{6}




\section{CENTRAL CALIFORNIA IVF PROGRAM \\ WOMEN'S SPECIALTY AND FERTILITY CENTER \\ CLOVIS, CALIFORNIA}

A comparison of clinic success rates may not be meaningful because patient medical characteristics and treatment approaches vary from clinic to clinic. For more details about this, along with information on how to interpret the statistics in this table, see pages 79-88.

\section{ART CYCLE PROFILE}

\section{Type of ART ${ }^{\mathrm{a}}$}

IVF

GIFT

ZIFT

Combination
96\% Procedural Factors:

$4 \%$ With ICSI

$0 \%$ Unstimulated

$0 \%$ Used gestational carrier

\section{$45 \%$ \\ $0 \%$ \\ $0 \%$}

\section{Patient Diagnosis}

Tubal factor

Ovulatory dysfunction

$13 \%$ Other factor

7\% Unknown factor

Diminished ovarian reserve $16 \%$ Multiple Factors:

Endometriosis

$1 \%$ Female factors only

$0 \%$ Female \& male factors $18 \%$
Uterine factor

Male factor
2006 PREGNANCY SUCCESS RATES

\section{Type of Cycle}

\section{Fresh Embryos from Nondonor Eggs}

Number of cycles

Percentage of cycles resulting in pregnancies ${ }^{b}$

Percentage of cycles resulting in live births $s^{b, c}$

(Confidence Interval)

Percentage of retrievals resulting in live births $s^{b, c}$

Percentage of transfers resulting in live births $s^{b, c}$

Percentage of transfers resulting in singleton live births ${ }^{b}$

Percentage of cancellations ${ }^{b}$

Average number of embryos transferred

Percentage of pregnancies with twins ${ }^{\mathrm{b}}$

Percentage of pregnancies with triplets or more ${ }^{b}$

Percentage of live births having multiple infants ${ }^{b, c}$

\section{Frozen Embryos from Nondonor Eggs}

Number of transfers

Percentage of transfers resulting in live births ${ }^{b, c}$

Average number of embryos transferred

\section{Donor Eggs}

Number of transfers

Percentage of transfers resulting in live births $s^{b, c}$

Average number of embryos transferred

Data verified by H. Michael Synn, MD

\section{Age of Woman}

35-37 $38-40 \quad 41-42^{d}$

$<35$

31

28

17

63
33.3

32.3

10.7

$0 / 17$

28.6

22.6

7.1

$0 / 17$

$(17.9-41.3)$

$(9.6-41.1)$

(0.9-23.5)

34.6

26.9

9.1

$0 / 9$

35.3

26.9

10.0

$0 / 9$

11.5

10.0

$0 / 9$

17.5

16.1

21.4

$8 / 17$

2.7

3.0

3.5

3.3

38.1

$5 / 10$

$1 / 3$

9.5

$0 / 10$

$0 / 3$

$8 / 18$

$4 / 7$

$0 / 2$

4

$0 / 4$

2.0

2

$0 / 2$

2

$0 / 2$

3.0

2.5

All Ages Combined ${ }^{\mathrm{e}}$

Fresh Embryos

Frozen Embryos

12

$2 / 12$

0

\section{CURRENT CLINIC SERVICES AND PROFILE}

Current Name: Central California IVF Program, Women's Specialty and Fertility Center

Donor egg? Yes

Donor embryo? Yes

Gestational carriers? Yes

Cryopreservation? Yes

3.2

$\begin{array}{ll}\text { SART member? } & \text { Yes } \\ \text { Verified lab accreditation? } & \text { Yes } \\ \text { (See Appendix C for details.) } & \end{array}$

(See Appendix C for details.)

${ }^{a}$ Reflects patient and treatment characteristics of ART cycles performed in 2006 using fresh nondonor eggs or embryos.

${ }^{b}$ When fewer than 20 cycles are reported in an age category, rates are shown as a fraction and confidence intervals are not given. Calculating percentages from fractions may be misleading and is not encouraged.

${ }^{c}$ A multiple-infant birth is counted as one live birth.

d Clinic-specific outcome rates are unreliable for women older than 42 undergoing ART cycles using fresh or frozen embryos with nondonor eggs. Readers are urged to review national outcomes for these age groups (see page 27).

e All ages (including ages $>42$ ) are reported together because previous data show that patient age does not materially affect success with donor eggs. 


\section{ZOUVES FERTILITY CENTER \\ DALY CITY, CALIFORNIA}

A comparison of clinic success rates may not be meaningful because patient medical characteristics and treatment approaches vary from clinic to clinic. For more details about this, along with information on how to interpret the statistics in this table, see pages 79-88.

\section{ART CYCLE PROFILE}

$$
\text { Type of ART }{ }^{\mathrm{a}}
$$

IVF

$$
\text { 100\% Procedural Factors: }
$$

GIFT

$0 \%$ With ICSI

ZIFT

$0 \%$ Unstimulated

Combination

\begin{tabular}{|c|c|}
\hline & Tubal factor \\
\hline $96 \%$ & Ovulatory dysfunction \\
\hline $0 \%$ & Diminished ovarian reserve \\
\hline $8 \%$ & Endometriosis \\
\hline & $\begin{array}{l}\text { Uterine factor } \\
\text { Male factor }\end{array}$ \\
\hline
\end{tabular}

0\% Used gestational carrier

\section{Patient Diagnosis}

$4 \%$ Other factor

$11 \%$

$11 \%$ Unknown factor

$5 \%$

21\% Multiple Factors:

$5 \% \quad$ Female factors only $\quad 15 \%$

$2 \%$ Female \& male factors $19 \%$ $9 \%$

2006 PREGNANCY SUCCESS RATES

Data verified by Christo Zouves, MD

\section{Type of Cycle}

\section{Age of Woman}

\section{Fresh Embryos from Nondonor Eggs}

Number of cycles 35-37

38-40

$41-42^{d}$

Percentage of cycles resulting in pregnancies ${ }^{b}$

Percentage of cycles resulting in live births ${ }^{b, c}$

(Confidence Interval)

Percentage of retrievals resulting in live births $s^{b, c}$

Percentage of transfers resulting in live births $s^{b, c}$ $<35$

78

92

52

68

43.6

37.0

17.3

45.6

38.5

30.4

11.5

Percentage of transfers resulting in singleton live births ${ }^{b}$

Percentage of cancellations ${ }^{b}$

$(33.5-58.1)$

$(27.7-50.2)$

(21.3-40.9)

$(4.4-23.4)$

46.3

39.0

31.8

11.8

47.0

41.1

36.8

14.0

27.3

26.0

21.1

14.0

1.5

1.3

4.3

1.9

2.9

3.2

3.1

2.3

Average number of embryos transferred

34.4

29.4

29.4

$1 / 9$

Percentage of pregnancies with triplets or more ${ }^{b}$

Percentage of live births having multiple infants ${ }^{b, c}$

9.4

2.9

8.8

$0 / 9$

41.9

36.7

42.9

$0 / 6$

\section{Frozen Embryos from Nondonor Eggs}

Number of transfers

Percentage of transfers resulting in live births $s^{\mathrm{b}, \mathrm{c}}$

39

Average number of embryos transferred

35.9

$$
19
$$

3.5

$4 / 19$

\section{7}

$1 / 7$

1

3.5

3.0

$0 / 1$

5.0

\section{Donor Eggs}

All Ages Combined

Number of transfers

Percentage of transfers resulting in live births $s^{b, c}$

Fresh Embryos

$$
84
$$

51.2

2.6

\section{Frozen Embryos} 62

33.9

Average number of embryos transferred

\section{CURRENT CLINIC SERVICES AND PROFILE}

Current Name: Zouves Fertility Center

\begin{tabular}{ll|ll} 
Donor egg? & Yes & Gestational carriers? & Yes \\
Donor embryo? & Yes & Cryopreservation? & Yes
\end{tabular}

Single women? Yes

\section{SART member? Yes}

Verified lab accreditation? Yes

(See Appendix C for details.)

${ }^{a}$ Reflects patient and treatment characteristics of ART cycles performed in 2006 using fresh nondonor eggs or embryos.

${ }^{b}$ When fewer than 20 cycles are reported in an age category, rates are shown as a fraction and confidence intervals are not given. Calculating percentages from fractions may be misleading and is not encouraged.

${ }^{c}$ A multiple-infant birth is counted as one live birth.

d Clinic-specific outcome rates are unreliable for women older than 42 undergoing ART cycles using fresh or frozen embryos with nondonor eggs. Readers are urged to review national outcomes for these age groups (see page 27).

${ }^{e}$ All ages (including ages $>42$ ) are reported together because previous data show that patient age does not materially affect success with donor eggs. 


\section{CALIFORNIA IVF: DAVIS FERTILITY CENTER, INC. DAVIS, CALIFORNIA}

A comparison of clinic success rates may not be meaningful because patient medical characteristics and treatment approaches vary from clinic to clinic. For more details about this, along with information on how to interpret the statistics in this table, see pages 79-88.

\section{ART CYCLE PROFILE}

\section{Type of ART ${ }^{\mathrm{a}}$}

IVF

GIFT

ZIFT

Combination
100\% Procedural Factors:

$0 \%$ With ICSI

$0 \%$ Unstimulated

$0 \%$ Used gestational carrier

\begin{tabular}{|c|c|}
\hline & Tubal factor \\
\hline $55 \%$ & Ovulatory dysfunction \\
\hline $0 \%$ & Diminished ovarian reserve \\
\hline $0 \%$ & Endometriosis \\
\hline & Uterine factor \\
\hline
\end{tabular}

\section{Patient Diagnosis}

$9 \%$ Other factor

$6 \%$ Unknown factor

16\% Multiple Factors:

$6 \%$ Female factors only $12 \%$

$<1 \%$ Female \& male factors $21 \%$

$23 \%$

2006 PREGNANCY SUCCESS RATES

Data verified by Ernest J. Zeringue, MD

\section{Type of Cycle}

\section{Age of Woman}

Number of cycles

Percentage of cycles resulting in pregnancies ${ }^{b}$

Percentage of cycles resulting in live births ${ }^{b, c}$

(Confidence Interval)

Percentage of retrievals resulting in live births ${ }^{b, c}$

Percentage of transfers resulting in live births ${ }^{\text {b,c }}$

Percentage of transfers resulting in singleton live births ${ }^{b}$

Percentage of cancellations ${ }^{b}$

Average number of embryos transferred

Percentage of pregnancies with twins ${ }^{b}$

Percentage of pregnancies with triplets or more ${ }^{b}$

Percentage of live births having multiple infants ${ }^{b, c}$

\section{Frozen Embryos from Nondonor Eggs}

Number of transfers

Percentage of transfers resulting in live births ${ }^{b, c}$

Average number of embryos transferred

\section{Donor Eggs}

Number of transfers

Percentage of transfers resulting in live births $s^{\mathrm{b}, \mathrm{c}}$

Average number of embryos transferred

$<35 \quad 35-37 \quad 38-40 \quad 41-42^{d}$

14

10

$\begin{array}{cccc}43 & 31 & 14 & 10 \\ 39.5 & 29.0 & 6 / 14 & 1 / 10 \\ 37.2 & 19.4 & 3 / 14 & 0 / 10\end{array}$

(23.0-53.3)

(7.5-37.5)

39.0

23.1

$3 / 14$

$0 / 7$

43.2

24.0

$3 / 13$

$0 / 6$

29.7

24.0

$2 / 13$

$0 / 6$

4.7

16.1

$0 / 14$

$3 / 10$

2.1

2.4

2.6

2.2

$5 / 17$

$0 / 9$

$3 / 6$

$0 / 1$

$1 / 17$

$0 / 9$

$0 / 6$

$0 / 1$

$5 / 16$

$0 / 6$

$1 / 3$

$\begin{array}{ccc}17 & 3 & 3 \\ 5 / 17 & 2 / 3 & 1 / 3 \\ 2.2 & 2.0 & 3.0\end{array}$

All Ages Combined

Fresh Embryos

Frozen Embryos

7

$5 / 7$

2.0

\section{CURRENT CLINIC SERVICES AND PROFILE}

Current Name: California IVF: Davis Fertility Center, Inc.

Donor egg? Yes Gestational carriers? Yes

Donor embryo? Yes Cryopreservation? Yes

Single women? Yes

SART member?

No

Verified lab accreditation?

No

(See Appendix C for details.)

${ }^{a}$ Reflects patient and treatment characteristics of ART cycles performed in 2006 using fresh nondonor eggs or embryos.

b When fewer than 20 cycles are reported in an age category, rates are shown as a fraction and confidence intervals are not given. Calculating percentages from fractions may be misleading and is not encouraged.

${ }^{c}$ A multiple-infant birth is counted as one live birth.

d Clinic-specific outcome rates are unreliable for women older than 42 undergoing ART cycles using fresh or frozen embryos with nondonor eggs. Readers are urged to review national outcomes for these age groups (see page 27).

e All ages (including ages $>42$ ) are reported together because previous data show that patient age does not materially affect success with donor eggs. 


\section{THE FERTILITY INSTITUTES-CALIFORNIA, NEVADA ENCINO, CALIFORNIA}

A comparison of clinic success rates may not be meaningful because patient medical characteristics and treatment approaches vary from clinic to clinic. For more details about this, along with information on how to interpret the statistics in this table, see pages 79-88.

\section{ART CYCLE PROFILE}

$$
\text { Type of ART }{ }^{\mathrm{a}}
$$

IVF

100\% Procedural Factors:

GIFT

$0 \%$ With ICSI

ZIFT

$0 \%$ Unstimulated

Combination

$0 \%$ Used gestational carrier

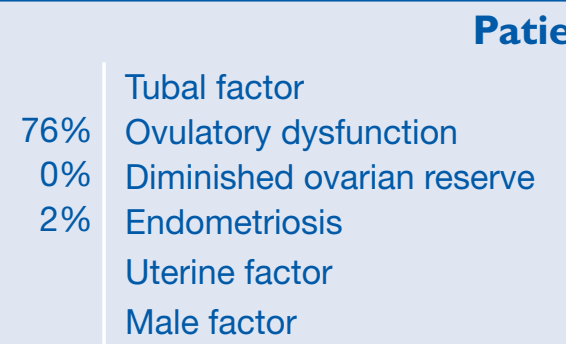

Patient Diagnosis

$\begin{array}{rlr}10 \% & \text { Other factor } & 51 \% \\ 1 \% & \text { Unknown factor } & 5 \% \\ 7 \% & \text { Multiple Factors: } & \\ 0 \% & \text { Female factors only } & 13 \% \\ 1 \% & \text { Female \& male factors } & 5 \% \\ 8 \% & & \end{array}$

2006 PREGNANCY SUCCESS RATES

Data verified by Jeffrey Steinberg, MD

\section{Type of Cycle}

\section{Age of Woman}

\section{Fresh Embryos from Nondonor Eggs}

Number of cycles

35-37

38-40

$41-42^{d}$

Percentage of cycles resulting in pregnancies ${ }^{b}$

Percentage of cycles resulting in live births ${ }^{b, c}$

(Confidence Interval)

Percentage of retrievals resulting in live births $s^{b, c}$

Percentage of transfers resulting in live births ${ }^{b, c}$ $<35$

40

57

59.6

65.0

28

2

Percentage of transfers resulting in singleton live births ${ }^{b}$

Percentage of cancellations ${ }^{b}$

54.4

57.5

42.9

$1 / 2$

Average number of embryos transferred

Percentage of pregnancies with twins ${ }^{b}$

$(40.7-67.6)$

$(40.9-73.0)$

39.3

$1 / 2$

Percentage of pregnancies with triplets or more ${ }^{b}$

Percentage of live births having multiple infants ${ }^{b, c}$

\section{4}

65.7

$(21.5-59.4)$

$57.4 \quad 65.7$

52.4

$1 / 2$

33.3

48.6

52.4

$1 / 2$

5.3

12.5

42.9

$1 / 2$

2.6

2.8

35.3

23.1

8.8

0.0

41.9

26.1

25.0

$0 / 2$

2.5

2.0

$1 / 12$

$0 / 1$

Frozen Embryos from Nondonor Eggs

Number of transfers

Percentage of transfers resulting in live births ${ }^{b, c}$

Average number of embryos transferred

\section{6}

$0 / 6$

2.8

\section{7}

$2 / 7$

2.6

$1 / 12$

$0 / 1$

$2 / 11$

$0 / 1$

All Ages Combined ${ }^{\mathrm{e}}$

\section{Donor Eggs}

Fresh Embryos

Number of transfers

Percentage of transfers resulting in live births $s^{b, c}$

Average number of embryos transferred

38

44.7

3.0
3

$0 / 3$

3.3

\section{CURRENT CLINIC SERVICES AND PROFILE}

Current Name: The Fertility Institutes-California, Nevada

Donor egg? Yes

Donor embryo? Yes

Single women? Yes
Gestational carriers? Yes

Cryopreservation? Yes
Frozen Embryos 15

$6 / 15$

3.2

\footnotetext{
${ }_{b}^{a}$ Reflects patient and treatment characteristics of ART cycles performed in 2006 using fresh nondonor eggs or embryos.

${ }^{b}$ When fewer than 20 cycles are reported in an age category, rates are shown as a fraction and confidence intervals are not given. Calculating percentages from fractions may be misleading and is not encouraged.

${ }^{c}$ A multiple-infant birth is counted as one live birth.

${ }^{d}$ Clinic-specific outcome rates are unreliable for women older than 42 undergoing ART cycles using fresh or frozen embryos with nondonor eggs. Readers are urged to review national outcomes for these age groups (see page 27).

${ }^{e}$ All ages (including ages $>42$ ) are reported together because previous data show that patient age does not materially affect success with donor eggs.
} 


\section{WEST COAST FERTILITY CENTERS FOUNTAIN VALLEY, CALIFORNIA}

A comparison of clinic success rates may not be meaningful because patient medical characteristics and treatment approaches vary from clinic to clinic. For more details about this, along with information on how to interpret the statistics in this table, see pages 79-88.

\section{ART CYCLE PROFILE}

\section{Type of ART ${ }^{\mathrm{a}}$}

IVF

GIFT

100\% Procedural Factors:

ZIFT

Combination
$0 \%$ With ICSI

$0 \%$ Unstimulated

$0 \%$ Used gestational carrier $<1 \%$
Tubal factor

91\% Ovulatory dysfunction

Diminished ovarian reserve

Endometriosis

Uterine factor

Male factor

\section{Patient Diagnosis}

$\begin{array}{rlr}11 \% & \text { Other factor } & <1 \% \\ 8 \% & \text { Unknown factor } & 4 \% \\ 5 \% & \text { Multiple Factors: } & \\ 2 \% & \text { Female factors only } & 32 \% \\ <1 \% & \text { Female \& male factors } & 27 \% \\ 10 \% & & \end{array}$

Data verified by David G. Diaz, MD

2006 PREGNANCY SUCCESS RATES

\section{Age of Woman}

\section{Type of Cycle}

35-37

43

53.5

48.8

43.8

(32.7-55.3)

43.8

45.5

27.3

0.0

3.1

22.0

22.0

40.0
(33.3-64.5)

52.5

53.8

35.9

7.0

3.5

34.8

8.7

33.3

$$
\text { 38-40 }
$$$$
41-42^{d}
$$

$41-42^{d}$

14

$2 / 14$

24.1

$1 / 14$

(9.3-31.4)

19.6

$1 / 14$

21.3

$1 / 9$

19.1

$1 / 9$

5.6

$0 / 14$

3.6

4.3

$0 / 13$

$0 / 2$

$1 / 13$

$0 / 2$

Percentage of pregnancies with triplets or more
Percentage of live births having multiple infants ${ }^{b, c}$

\section{Frozen Embryos from Nondonor Eggs}

Number of transfers

Percentage of transfers resulting in live births $s^{b, c}$

Average number of embryos transferred

29

34.5

15

$1 / 10$

$0 / 1$

4.3

\section{7}

$3 / 7$

2.9

\section{5}

$1 / 5$

2.8

\section{Donor Eggs}

Number of transfers

Percentage of transfers resulting in live births $s^{b, c}$

Average number of embryos transferred
3.1

All Ages Combined ${ }^{\mathrm{e}}$

\section{Fresh Embryos}

15

$10 / 15$

3.7
Frozen Embryos 20

45.0

4.1

\section{CURRENT CLINIC SERVICES AND PROFILE}

Current Name: West Coast Fertility Centers

Donor egg? Yes

Donor embryo? No

Single women? Yes
Gestational carriers? Yes

Cryopreservation? Yes
SART member?

Yes

Verified lab accreditation?

Yes

${ }^{a}$ Reflects patient and treatment characteristics of ART cycles performed in 2006 using fresh nondonor eggs or embryos.

${ }^{b}$ When fewer than 20 cycles are reported in an age category, rates are shown as a fraction and confidence intervals are not given. Calculating percentages from fractions may be misleading and is not encouraged.

${ }^{c}$ A multiple-infant birth is counted as one live birth.

d Clinic-specific outcome rates are unreliable for women older than 42 undergoing ART cycles using fresh or frozen embryos with nondonor eggs. Readers are urged to review national outcomes for these age groups (see page 27).

e All ages (including ages $>42$ ) are reported together because previous data show that patient age does not materially affect success with donor eggs. 


\section{KAISER PERMANENTE CENTER FOR REPRODUCTIVE HEALTH FREMONT, CALIFORNIA}

A comparison of clinic success rates may not be meaningful because patient medical characteristics and treatment approaches vary from clinic to clinic. For more details about this, along with information on how to interpret the statistics in this table, see pages 79-88.

\section{ART CYCLE PROFILE}

$$
\text { Type of ART }{ }^{\mathrm{a}}
$$

\begin{tabular}{|c|c|c|c|c|c|c|c|}
\hline IVF & $100 \%$ & Procedural Factors: & & Tubal factor & $9 \%$ & Other factor & $4 \%$ \\
\hline GIFT & $0 \%$ & With ICSI & $74 \%$ & Ovulatory dysfunction & $4 \%$ & Unknown factor & $4 \%$ \\
\hline ZIFT & $0 \%$ & Unstimulated & $0 \%$ & Diminished ovarian reserve & $27 \%$ & Multiple Factors: & \\
\hline \multirow[t]{3}{*}{ Combination } & $0 \%$ & Used gestational carrier & $0 \%$ & Endometriosis & $2 \%$ & Female factors only & \\
\hline & & & & Uterine factor & $<1 \%$ & Female \& male factors & \\
\hline & & & & Male factor & $13 \%$ & & \\
\hline
\end{tabular}

2006 PREGNANCY SUCCESS RATES

Data verified by Jon A. Proctor, MD

\section{Type of Cycle}

\section{Fresh Embryos from Nondonor Eggs}

Number of cycles

Percentage of cycles resulting in pregnancies ${ }^{b}$

Percentage of cycles resulting in live births $s^{b, c}$

(Confidence Interval)

Percentage of retrievals resulting in live births $s^{b, c}$

Percentage of transfers resulting in live births $s^{b, c}$

Percentage of transfers resulting in singleton live births ${ }^{b}$

Percentage of cancellations ${ }^{b}$

Average number of embryos transferred

Percentage of pregnancies with twins ${ }^{b}$

Percentage of pregnancies with triplets or more ${ }^{b}$

Percentage of live births having multiple infants ${ }^{b, c}$

\section{Frozen Embryos from Nondonor Eggs}

Number of transfers

Percentage of transfers resulting in live births $s^{\mathrm{b}, \mathrm{c}}$

Average number of embryos transferred

\section{Donor Eggs}

Number of transfers

Percentage of transfers resulting in live births $s^{b, c}$

Average number of embryos transferred

\section{Age of Woman}

35-37 38-40

$41-42^{d}$

$<35$

110

61

60

41

58.2

57.4

50.0

26.8

50.0

44.3

40.0

19.5

(40.3-59.7)

(31.5-57.6)

(27.6-53.5)

(8.8-34.9)

56.1

50.0

48.0

50.0

22.2

61.8

54.0

41.7

16.7

24.2

28.0

11.5

3.2

12.1

10.9

2.6

42.9

20.0

12.2

43.8

11.4

48.1

45.5

6.7

$3 / 11$

$1 / 11$

$4 / 8$

26

80.8

$$
7
$$

$4 / 7$

16.7

2.9

\section{7}

$2 / 7$

2

2.1

All Ages Combined

Fresh Embryos

18

$13 / 18$

2.0
Frozen Embryos

\section{5}

$3 / 5$

2.0

\section{CURRENT CLINIC SERVICES AND PROFILE}

Current Name: Kaiser Permanente Center for Reproductive Health

Donor egg? Yes Gestational carriers? No

Donor embryo? No Cryopreservation? Yes

Single women? Yes

SART member? Yes

Verified lab accreditation? Yes

(See Appendix C for details.)

${ }^{a}$ Reflects patient and treatment characteristics of ART cycles performed in 2006 using fresh nondonor eggs or embryos.

${ }^{b}$ When fewer than 20 cycles are reported in an age category, rates are shown as a fraction and confidence intervals are not given. Calculating percentages from fractions may be misleading and is not encouraged.

${ }^{c}$ A multiple-infant birth is counted as one live birth.

d Clinic-specific outcome rates are unreliable for women older than 42 undergoing ART cycles using fresh or frozen embryos with nondonor eggs. Readers are urged to review national outcomes for these age groups (see page 27).

e All ages (including ages $>42$ ) are reported together because previous data show that patient age does not materially affect success with donor eggs. 


\section{KATHLEEN L. KORNAFEL, MD, PhD GLENDALE, CALIFORNIA}

A comparison of clinic success rates may not be meaningful because patient medical characteristics and treatment approaches vary from clinic to clinic. For more details about this, along with information on how to interpret the statistics in this table, see pages 79-88.

\section{ART CYCLE PROFILE}

\section{Type of ART ${ }^{\mathrm{a}}$}

IVF

GIFT

ZIFT

Combination
100\% Procedural Factors:

$0 \%$ With ICSI

$0 \%$ Unstimulated

$0 \%$ Used gestational carrier $84 \%$
Tubal factor

Ovulatory dysfunction

Diminished ovarian reserve

Endometriosis

Uterine factor

Male factor

\section{Patient Diagnosis}

$1 \%$ Other factor

$1 \%$ Unknown factor

0\% Multiple Factors:

$0 \%$ Female factors only $1 \%$

$0 \%$ Female \& male factors $1 \%$

$11 \%$
2006 PREGNANCY SUCCESS RATES

\section{Type of Cycle}

\section{Fresh Embryos from Nondonor Eggs}

Number of cycles

Percentage of cycles resulting in pregnancies ${ }^{\mathrm{b}}$

Percentage of cycles resulting in live births ${ }^{b, c}$

(Confidence Interval)

Percentage of retrievals resulting in live births $s^{b, c}$

Percentage of transfers resulting in live births ${ }^{b, c}$

Percentage of transfers resulting in singleton live births ${ }^{b}$

Percentage of cancellations ${ }^{b}$

Average number of embryos transferred

Percentage of pregnancies with twins ${ }^{b}$

Percentage of pregnancies with triplets or more ${ }^{b}$

Percentage of live births having multiple infants ${ }^{b, c}$

\section{Frozen Embryos from Nondonor Eggs}

Number of transfers

Percentage of transfers resulting in live births $s^{b, c}$

Average number of embryos transferred

\section{Donor Eggs}

Number of transfers

Percentage of transfers resulting in live births ${ }^{b, c}$

Average number of embryos transferred
Data verified by Kathleen L. Kornafel, MD, PhD

\section{Age of Woman}

$<35$

35-37

38-40

$41-42^{d}$

16

19

15

9

$5 / 16$

$5 / 19$

$2 / 15$

$4 / 9$

$4 / 16$

$3 / 19$

$2 / 15$

$3 / 9$

$4 / 16$

$3 / 19$

$2 / 15$

$3 / 9$

$4 / 16$

$3 / 19$

$2 / 14$

$3 / 9$

$3 / 16$

$2 / 19$

$1 / 14$

$2 / 9$

$0 / 16$

$0 / 19$

$0 / 15$

$0 / 9$

2.6

3.3

2.9

3.0

$2 / 5$

$2 / 5$

$0 / 2$

$1 / 4$

$0 / 5$

$0 / 5$

$1 / 2$

$0 / 4$

$1 / 4$

$1 / 3$

$1 / 2$

$1 / 3$

$\begin{array}{cccc}4 & 5 & 6 & 1 \\ 0 / 4 & 0 / 5 & 2 / 6 & 0 / 1 \\ 2.0 & 2.4 & 2.2 & 2.0\end{array}$

All Ages Combined

Fresh Embryos

Frozen Embryos

\section{7}

$4 / 7$

8

2.9

$1 / 8$

3.0

\section{CURRENT CLINIC SERVICES AND PROFILE}

Current Name: Kathleen L. Kornafel, MD, PhD

Donor egg? Yes

Donor embryo? Yes

Gestational carriers? Yes

Cryopreservation? Yes

Single women? Yes

SART member?

No

Verified lab accreditation?

Yes

(See Appendix C for details.)

${ }^{a}$ Reflects patient and treatment characteristics of ART cycles performed in 2006 using fresh nondonor eggs or embryos.

b When fewer than 20 cycles are reported in an age category, rates are shown as a fraction and confidence intervals are not given. Calculating percentages from fractions may be misleading and is not encouraged.

${ }^{c}$ A multiple-infant birth is counted as one live birth.

d Clinic-specific outcome rates are unreliable for women older than 42 undergoing ART cycles using fresh or frozen embryos with nondonor eggs. Readers are urged to review national outcomes for these age groups (see page 27).

e All ages (including ages $>42$ ) are reported together because previous data show that patient age does not materially affect success with donor eggs. 


\section{SHER INSTITUTE OF REPRODUCTIVE MEDICINE-LOS ANGELES GLENDALE, CALIFORNIA}

A comparison of clinic success rates may not be meaningful because patient medical characteristics and treatment approaches vary from clinic to clinic. For more details about this, along with information on how to interpret the statistics in this table, see pages 79-88.

\section{ART CYCLE PROFILE}

$$
\text { Type of ART }{ }^{\mathrm{a}}
$$

IVF

100\% Procedural Factors:

GIFT

$0 \%$ With ICSI

ZIFT

$0 \%$ Unstimulated

$83 \%$

Combination
$0 \%$ Used gestational carrier $\quad 2 \%$
Tubal factor

\section{Patient Diagnosis}

$7 \%$ Other factor

$38 \%$

4\% Unknown factor

$5 \%$

Diminished ovarian reserve $3 \%$ Multiple Factors:

Endometriosis

$6 \%$ Female factors only $15 \%$

Uterine factor

$<1 \% \quad$ Female \& male factors

$16 \%$

2006 PREGNANCY SUCCESS RATES

Data verified by Brian Acacio, MD

\section{Type of Cycle}

\section{Fresh Embryos from Nondonor Eggs}

Number of cycles

Percentage of cycles resulting in pregnancies ${ }^{b}$

Percentage of cycles resulting in live births $s^{b, c}$

(Confidence Interval)

Percentage of retrievals resulting in live births $s^{b, c}$

Percentage of transfers resulting in live births $s^{b, c}$

Percentage of transfers resulting in singleton live births ${ }^{b}$

Percentage of cancellations ${ }^{b}$

Average number of embryos transferred

Percentage of pregnancies with twins ${ }^{b}$

Percentage of pregnancies with triplets or more ${ }^{b}$

Percentage of live births having multiple infants ${ }^{b, c}$

\section{Frozen Embryos from Nondonor Eggs}

Number of transfers

Percentage of transfers resulting in live births $s^{b, c}$

Average number of embryos transferred

\section{Donor Eggs}

Number of transfers

Percentage of transfers resulting in live births ${ }^{\mathrm{b}, \mathrm{c}}$

Average number of embryos transferred
Male factor

\section{Age of Woman}

35-37 38-40

$41-42^{d}$

$<35$

67

84

34

74
36.5

44.8

26.2

14.7

31.1

29.9

10.7

2.9

$(20.8-42.9)$

(19.3-42.3)

(5.0-19.4)

(0.1-15.3)

36.5

32.8

12.5

3.3

38.3

33.9

14.1

3.8

21.7

27.1

9.4

3.8

14.9

9.0

14.3

11.8

2.8

22.2

14.8

43.5

3.1

16.7

10.0

3.3

3.5

22.7

$0 / 5$

0.0

$0 / 5$

20.0

$3 / 9$

$0 / 1$

14

$6 / 14$

11

$1 / 11$

6

$0 / 6$

2.4

2.7

2.0

All Ages Combined ${ }^{\mathrm{e}}$

Fresh Embryos

\section{7}

$6 / 17$

3.2
Frozen Embryos

4

$2 / 4$

3.3

\section{CURRENT CLINIC SERVICES AND PROFILE}

Current Name: Sher Institute of Reproductive Medicine-Los Angeles

Donor egg? Yes Gestational carriers? Yes

Donor embryo? Yes Cryopreservation? Yes

Single women? Yes

SART member? No

Verified lab accreditation?

(See Appendix C for details.)

${ }^{a}$ Reflects patient and treatment characteristics of ART cycles performed in 2006 using fresh nondonor eggs or embryos.

${ }^{b}$ When fewer than 20 cycles are reported in an age category, rates are shown as a fraction and confidence intervals are not given. Calculating percentages from fractions may be misleading and is not encouraged.

${ }^{c}$ A multiple-infant birth is counted as one live birth.

d Clinic-specific outcome rates are unreliable for women older than 42 undergoing ART cycles using fresh or frozen embryos with nondonor eggs. Readers are urged to review national outcomes for these age groups (see page 27).

e All ages (including ages $>42$ ) are reported together because previous data show that patient age does not materially affect success with donor eggs. 


\section{MARIN REPRODUCTIVE MEDICAL ASSOCIATES, INC. GREENBRAE, CALIFORNIA}

A comparison of clinic success rates may not be meaningful because patient medical characteristics and treatment approaches vary from clinic to clinic. For more details about this, along with information on how to interpret the statistics in this table, see pages 79-88.

\section{ART CYCLE PROFILE}

\section{Type of ART ${ }^{\mathrm{a}}$}

IVF

GIFT 100\% Procedural Factors:

ZIFT

Combination
$0 \%$ With ICSI

$0 \%$ Unstimulated

$0 \%$ Used gestational carrier

\section{$38 \%$ \\ $0 \%$ \\ $0 \%$}

\section{Patient Diagnosis}

Tubal factor

Ovulatory dysfunction

Diminished ovarian reserve

Endometriosis

Uterine factor

Male factor

$\begin{array}{rlr}5 \% & \text { Other factor } & 3 \% \\ 7 \% & \text { Unknown factor } & 16 \% \\ 22 \% & \text { Multiple Factors: } & \\ 3 \% & \text { Female factors only } & 13 \% \\ 2 \% & \text { Female \& male factors } & 16 \% \\ 14 \% & & \end{array}$

Data verified by Sae H. Sohn, MD

2006 PREGNANCY SUCCESS RATES

\section{Age of Woman}

\section{Type of Cycle}

\section{Fresh Embryos from Nondonor Eggs}

Number of cycles

Percentage of cycles resulting in pregnancies ${ }^{b}$

Percentage of cycles resulting in live births ${ }^{b, c}$

(Confidence Interval)

Percentage of retrievals resulting in live births $s^{b, c}$

Percentage of transfers resulting in live births $s^{b, c}$

Percentage of transfers resulting in singleton live births ${ }^{b}$

Percentage of cancellations ${ }^{b}$

Average number of embryos transferred

Percentage of pregnancies with twins ${ }^{b}$

Percentage of pregnancies with triplets or more ${ }^{b}$

Percentage of live births having multiple infants ${ }^{b, c}$

\section{Frozen Embryos from Nondonor Eggs}

Number of transfers

Percentage of transfers resulting in live births ${ }^{\mathrm{b}, \mathrm{c}}$

Average number of embryos transferred

\section{Donor Eggs}

Number of transfers

Percentage of transfers resulting in live births $s^{b, c}$

Average number of embryos transferred

\begin{tabular}{|c|c|c|c|c|}
\hline & & Age of & Voman & \\
\hline & $<35$ & $35-37$ & $38-40$ & $41-42^{d}$ \\
\hline & 19 & 34 & 27 & 8 \\
\hline & $12 / 19$ & 29.4 & 25.9 & $1 / 8$ \\
\hline & $11 / 19$ & $\begin{array}{c}26.5 \\
(12.9-44.4)\end{array}$ & $\begin{array}{c}25.9 \\
(11.1-46.3)\end{array}$ & $0 / 8$ \\
\hline & $11 / 19$ & 30.0 & 26.9 & $0 / 7$ \\
\hline & $11 / 19$ & 32.1 & 31.8 & $0 / 7$ \\
\hline irths ${ }^{b}$ & $7 / 19$ & 17.9 & 9.1 & $0 / 7$ \\
\hline & $0 / 19$ & 11.8 & 3.7 & $1 / 8$ \\
\hline & 2.3 & 2.8 & 2.7 & 3.7 \\
\hline & $4 / 12$ & $4 / 10$ & $5 / 7$ & $0 / 1$ \\
\hline & $0 / 12$ & $1 / 10$ & $0 / 7$ & $0 / 1$ \\
\hline & $4 / 11$ & $4 / 9$ & $5 / 7$ & \\
\hline & 6 & 4 & 9 & 1 \\
\hline & $0 / 6$ & $0 / 4$ & $2 / 9$ & $0 / 1$ \\
\hline & 3.3 & 4.0 & 4.1 & 6.0 \\
\hline & & All Ages & ombined $^{\mathrm{e}}$ & \\
\hline & Fresh & nbryos & Frozen & nbryos \\
\hline & & & & \\
\hline & & & & \\
\hline & & & & \\
\hline
\end{tabular}

\begin{tabular}{|c|c|c|c|c|}
\hline & & Age of & Voman & \\
\hline & $<35$ & $35-37$ & $38-40$ & $41-42^{d}$ \\
\hline & 19 & 34 & 27 & 8 \\
\hline & $12 / 19$ & 29.4 & 25.9 & $1 / 8$ \\
\hline & $11 / 19$ & $\begin{array}{c}26.5 \\
(12.9-44.4)\end{array}$ & $\begin{array}{c}25.9 \\
(11.1-46.3)\end{array}$ & $0 / 8$ \\
\hline & $11 / 19$ & 30.0 & 26.9 & $0 / 7$ \\
\hline & $11 / 19$ & 32.1 & 31.8 & $0 / 7$ \\
\hline irths ${ }^{b}$ & $7 / 19$ & 17.9 & 9.1 & $0 / 7$ \\
\hline & $0 / 19$ & 11.8 & 3.7 & $1 / 8$ \\
\hline & 2.3 & 2.8 & 2.7 & 3.7 \\
\hline & $4 / 12$ & $4 / 10$ & $5 / 7$ & $0 / 1$ \\
\hline & $0 / 12$ & $1 / 10$ & $0 / 7$ & $0 / 1$ \\
\hline & $4 / 11$ & $4 / 9$ & $5 / 7$ & \\
\hline & 6 & 4 & 9 & 1 \\
\hline & $0 / 6$ & $0 / 4$ & $2 / 9$ & $0 / 1$ \\
\hline & 3.3 & 4.0 & 4.1 & 6.0 \\
\hline & & All Ages & ombined $^{\mathrm{e}}$ & \\
\hline & Fresh & nbryos & Frozen & nbryos \\
\hline & & & & \\
\hline & & & & \\
\hline & & & & \\
\hline
\end{tabular}

\section{CURRENT CLINIC SERVICES AND PROFILE}

Current Name: This clinic has closed or reorganized since 2006. Information on current clinic services and profile therefore is not provided here. Contact the NASS Help Desk for current information about this clinic.

\footnotetext{
${ }^{a}$ Reflects patient and treatment characteristics of ART cycles performed in 2006 using fresh nondonor eggs or embryos.

${ }^{b}$ When fewer than 20 cycles are reported in an age category, rates are shown as a fraction and confidence intervals are not given. Calculating percentages from fractions may be misleading and is not encouraged.

${ }^{\mathrm{C}}$ A multiple-infant birth is counted as one live birth.

d Clinic-specific outcome rates are unreliable for women older than 42 undergoing ART cycles using fresh or frozen embryos with nondonor eggs. Readers are urged to review national outcomes for these age groups (see page 27).

e All ages (including ages $>42$ ) are reported together because previous data show that patient age does not materially affect success with donor eggs.
} 


\section{COASTAL FERTILITY MEDICAL CENTER, INC. IRVINE, CALIFORNIA}

A comparison of clinic success rates may not be meaningful because patient medical characteristics and treatment approaches vary from clinic to clinic. For more details about this, along with information on how to interpret the statistics in this table, see pages 79-88.

\section{ART CYCLE PROFILE}

\section{Type of ART ${ }^{\mathrm{a}}$}

IVF

GIFT

100\% Procedural Factors:

ZIFT

Combination

\author{
$0 \%$ With ICSI
}

$0 \%$ Unstimulated

0\% Used gestational carrier

\begin{tabular}{|c|c|}
\hline $88 \%$ & Ovulatory dysfunction \\
\hline $0 \%$ & Diminished ovarian reserve \\
\hline $1 \%$ & Endometriosis \\
\hline & Uterine factor \\
\hline & Male factor \\
\hline
\end{tabular}

\begin{tabular}{|c|c|c|}
\hline $3 \%$ & Other factor & $8 \%$ \\
\hline $2 \%$ & Unknown factor & $23 \%$ \\
\hline $14 \%$ & Multiple Factors: & \\
\hline $4 \%$ & Female factors only & $8 \%$ \\
\hline$<1 \%$ & Female \& male factors & $17 \%$ \\
\hline $22 \%$ & & \\
\hline
\end{tabular}

2006 PREGNANCY SUCCESS RATES

Data verified by Lawrence B. Werlin, MD

\section{Type of Cycle}

\section{Age of Woman}

\section{Fresh Embryos from Nondonor Eggs}

Number of cycles

Percentage of cycles resulting in pregnancies ${ }^{b}$

Percentage of cycles resulting in live births ${ }^{b, c}$

(Confidence Interval)

Percentage of retrievals resulting in live births $s^{b, c}$

Percentage of transfers resulting in live births $s^{b, c}$

Percentage of transfers resulting in singleton live births ${ }^{b}$

Percentage of cancellations ${ }^{b}$

Average number of embryos transferred

Percentage of pregnancies with twins ${ }^{b}$

Percentage of pregnancies with triplets or more ${ }^{b}$

Percentage of live births having multiple infants ${ }^{b, c}$

\section{Frozen Embryos from Nondonor Eggs}

Number of transfers

Percentage of transfers resulting in live births $s^{b, c}$

Average number of embryos transferred

\section{Donor Eggs}

Number of transfers

Percentage of transfers resulting in live births $s^{b, c}$

Average number of embryos transferred

\begin{tabular}{|c|c|c|c|}
\hline \multicolumn{4}{|c|}{ 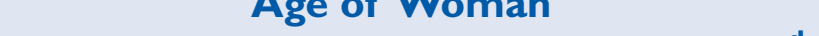 } \\
\hline$<35$ & $35-37$ & $38-40$ & $41-42^{d}$ \\
\hline 74 & 60 & 57 & 24 \\
\hline 39.2 & 33.3 & 29.8 & 16.7 \\
\hline 35.1 & 26.7 & 17.5 & 4.2 \\
\hline $4.4-47.1)$ & $(16.1-39.7)$ & (8.7-29.9) & $(0.1-21.1)$ \\
\hline 36.1 & 27.1 & 21.3 & 5.0 \\
\hline 36.1 & 28.6 & 27.8 & $1 / 19$ \\
\hline 20.8 & 23.2 & 13.9 & $0 / 19$ \\
\hline 2.7 & 1.7 & 17.5 & 16.7 \\
\hline 3.3 & 3.3 & 3.4 & 3.6 \\
\hline 37.9 & 25.0 & $7 / 17$ & $0 / 4$ \\
\hline 6.9 & 0.0 & $0 / 17$ & $1 / 4$ \\
\hline 42.3 & $3 / 16$ & $5 / 10$ & $1 / 1$ \\
\hline 24 & 18 & 12 & 4 \\
\hline 29.2 & $5 / 18$ & $2 / 12$ & $1 / 4$ \\
\hline 3.1 & 3.3 & 3.7 & 2.3 \\
\hline & All Ages & mbined $^{e}$ & \\
\hline Fresh & mbryos & Frozen & nbryos \\
\hline & 5 & & \\
\hline & .1 & & \\
\hline & & & \\
\hline
\end{tabular}

\section{CURRENT CLINIC SERVICES AND PROFILE}

Current Name: Coastal Fertility Medical Center, Inc.
Donor egg?
Yes
Donor embryo? Yes
Gestational carriers? Yes
Cryopreservation?
Yes
Single women? Yes

$\begin{array}{ll}\text { SART member? } & \text { Yes } \\ \text { Verified lab accreditation? } & \text { Yes } \\ \text { (See Appendix C for details.) } & \end{array}$
${ }^{a}$ Reflects patient and treatment characteristics of ART cycles performed in 2006 using fresh nondonor eggs or embryos.
b When fewer than 20 cycles are reported in an age category, rates are shown as a fraction and confidence intervals are not given. Calculating percentages from fractions may be misleading and is not encouraged.
c A multiple-infant birth is counted as one live birth.
${ }^{d}$ Clinic-specific outcome rates are unreliable for women older than 42 undergoing ART cycles using fresh or frozen embryos with nondonor eggs. Readers are urged to review national outcomes for these age groups (see page 27).
e All ages (including ages $>42$ ) are reported together because previous data show that patient age does not materially affect success with donor eggs. 


\section{FERTILITY CENTER OF SOUTHERN CALIFORNIA IRVINE, CALIFORNIA}

A comparison of clinic success rates may not be meaningful because patient medical characteristics and treatment approaches vary from clinic to clinic. For more details about this, along with information on how to interpret the statistics in this table, see pages 79-88.

\section{ART CYCLE PROFILE}

\begin{tabular}{|c|c|c|c|c|c|c|c|}
\hline \multicolumn{4}{|c|}{ Type of ART ${ }^{a}$} & \multicolumn{4}{|c|}{ Patient Diagnosis } \\
\hline IVF & $100 \%$ & Procedural Factors: & & Tubal factor & $10 \%$ & Other factor & $15 \%$ \\
\hline GIFT & $0 \%$ & With ICSI & $88 \%$ & Ovulatory dysfunction & $6 \%$ & Unknown factor & $21 \%$ \\
\hline ZIFT & $0 \%$ & Unstimulated & $0 \%$ & Diminished ovarian reserve & $24 \%$ & Multiple Factors: & \\
\hline \multirow[t]{3}{*}{ Combination } & $0 \%$ & Used gestational carrier & $<1 \%$ & Endometriosis & $4 \%$ & Female factors only & $2 \%$ \\
\hline & & & & Uterine factor & $0 \%$ & Female \& male factors & $5 \%$ \\
\hline & & & & Male factor & $13 \%$ & & \\
\hline
\end{tabular}

2006 PREGNANCY SUCCESS RATES

Data verified by llene E. Hatch, MD

\section{Type of Cycle}

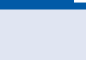

$\sqrt{2}$

\section{Fresh Embryos from Nondonor Eggs}

Number of cycles

Percentage of cycles resulting in pregnancies ${ }^{\mathrm{b}}$

Percentage of cycles resulting in live births ${ }^{b, c}$

(Confidence Interval)

Percentage of retrievals resulting in live births ${ }^{b, c}$

Percentage of transfers resulting in live births ${ }^{b, c}$

Percentage of transfers resulting in singleton live births ${ }^{b}$

Percentage of cancellations ${ }^{b}$

Average number of embryos transferred

Percentage of pregnancies with twins ${ }^{b}$

Percentage of pregnancies with triplets or more ${ }^{b}$

Percentage of live births having multiple infants ${ }^{b, c}$

\section{Frozen Embryos from Nondonor Eggs}

Number of transfers

Percentage of transfers resulting in live births ${ }^{\mathrm{b}, \mathrm{c}}$

Average number of embryos transferred

\section{Donor Eggs}

Number of transfers

Percentage of transfers resulting in live births ${ }^{b, c}$

Average number of embryos transferred

\section{Age of Woman}

35-37 38-40

$41-42^{d}$

\section{$<35$}

25

36.0

32

14

47.8

28.0

28.1

$2 / 14$

39.1

(12.1-49.4)

18.8

$1 / 14$

(25.1-54.6)

29.2

(7.2-36.4)

43.9

30.4

23.1

$1 / 12$

24.0

$1 / 12$

31.7

21.7

16.0

$1 / 12$

8.7

4.0

18.8

$2 / 14$

3.7

13.6

4.1

4.2

4.8

9.1

$5 / 18$

$2 / 9$

$1 / 9$

$0 / 2$

$1 / 9$

$1 / 9$

$0 / 2$

$2 / 6$

$0 / 1$

$\begin{array}{cccc}10 & 11 & 9 & 3 \\ 4 / 10 & 5 / 11 & 1 / 9 & 2 / 3 \\ 4.2 & 4.0 & 4.0 & 5.3\end{array}$

\section{All Ages Combined}

Fresh Embryos

16

$12 / 16$

2.9
Frozen Embryos 13

$2 / 13$

4.8

\section{CURRENT CLINIC SERVICES AND PROFILE}

Current Name: Fertility Center of Southern California

Donor egg? Yes

Donor embryo? Yes

Gestational carriers? Yes

Cryopreservation?

SART member?

Yes

Single women? Yes

Verified lab accreditation?

Yes

(See Appendix C for details.)

${ }^{\text {a }}$ Reflects patient and treatment characteristics of ART cycles performed in 2006 using fresh nondonor eggs or embryos.

${ }^{b}$ When fewer than 20 cycles are reported in an age category, rates are shown as a fraction and confidence intervals are not given. Calculating percentages from fractions may be misleading and is not encouraged.

${ }^{c}$ A multiple-infant birth is counted as one live birth.

d Clinic-specific outcome rates are unreliable for women older than 42 undergoing ART cycles using fresh or frozen embryos with nondonor eggs. Readers are urged to review national outcomes for these age groups (see page 27).

e All ages (including ages $>42$ ) are reported together because previous data show that patient age does not materially affect success with donor eggs. 


\section{REPRODUCTIVE FERTILITY CENTER \\ IRVINE, CALIFORNIA}

A comparison of clinic success rates may not be meaningful because patient medical characteristics and treatment approaches vary from clinic to clinic. For more details about this, along with information on how to interpret the statistics in this table, see pages 79-88.

\section{ART CYCLE PROFILE}

\section{Type of ART ${ }^{\mathrm{a}}$}

IVF

GIFT

100\% Procedural Factors:

ZIFT

$0 \%$ With ICSI

Combination
$0 \%$ Unstimulated

0\% Used gestational carrier

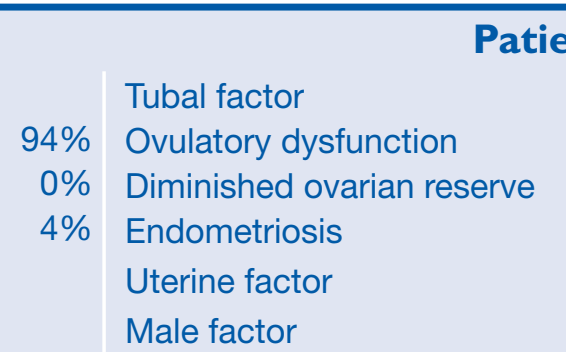

Patient Diagnosis

$\begin{array}{lll}5 \% & \text { Other factor } & 34 \% \\ 7 \% & \text { Unknown factor } & 10 \% \\ 7 \% & \text { Multiple Factors: } & \\ 4 \% & \text { Female factors only } & 6 \% \\ 2 \% & \text { Female \& male factors } & 9 \% \\ 16 \% & & \end{array}$

2006 PREGNANCY SUCCESS RATES

Data verified by James P. Lin, MD

\section{Type of Cycle}

\section{Age of Woman}

35-37 38-40

$41-42^{d}$

\section{$<35$}

$$
41
$$

65.9

51.2

(35.1-67.1)

51.2

52.5

30.0

0.0

2.4

29.6

7.4

42.9

Percentage of pregnancies with triplets or more ${ }^{b}$

Percentage of live births having multiple infants ${ }^{b, c}$

\section{Frozen Embryos from Nondonor Eggs}

Number of transfers

Percentage of transfers resulting in live births ${ }^{\mathrm{b}, \mathrm{c}}$

Average number of embryos transferred

$\begin{array}{cc}4 & 2 \\ 0 / 4 & 0 / 2\end{array}$

$\begin{array}{ccc}28 & 27 & 9 \\ 50.0 & 48.1 & 1 / 9 \\ 42.9 & 40.7 & 1 / 9\end{array}$

(24.5-62.8)

44.4

44.4

29.6

3.6

2.6

$4 / 14$

$0 / 14$

$4 / 12$

3.0
(22.4-61.2)

42.3

42.3

34.6

3.7

2.6

$4 / 13$

$0 / 13$

$2 / 11$

0

0

All Ages Combined ${ }^{\mathrm{e}}$

\section{Donor Eggs}

Fresh Embryos

Frozen Embryos

Number of transfers

Percentage of transfers resulting in live births $s^{b, c}$

Average number of embryos transferred

\section{2}

$1 / 2$

2.5

\section{CURRENT CLINIC SERVICES AND PROFILE}

Current Name: Reproductive Fertility Center

Donor egg? Yes

Donor embryo? No

Gestational carriers? Yes

Cryopreservation? Yes

$8 / 10$

Single women? Yes

SART member?

No

Verified lab accreditation?

(See Appendix C for details.)

${ }^{a}$ Reflects patient and treatment characteristics of ART cycles performed in 2006 using fresh nondonor eggs or embryos.

${ }^{b}$ When fewer than 20 cycles are reported in an age category, rates are shown as a fraction and confidence intervals are not given. Calculating percentages from fractions may be misleading and is not encouraged.

${ }^{c}$ A multiple-infant birth is counted as one live birth.

d Clinic-specific outcome rates are unreliable for women older than 42 undergoing ART cycles using fresh or frozen embryos with nondonor eggs. Readers are urged to review national outcomes for these age groups (see page 27).

e All ages (including ages $>42$ ) are reported together because previous data show that patient age does not materially affect success with donor eggs. 


\section{REPRODUCTIVE PARTNERS-UCSD REGIONAL FERTILITY CENTER LA JOLLA, CALIFORNIA}

A comparison of clinic success rates may not be meaningful because patient medical characteristics and treatment approaches vary from clinic to clinic. For more details about this, along with information on how to interpret the statistics in this table, see pages 79-88.

\section{ART CYCLE PROFILE}

\section{Type of ART ${ }^{\mathrm{a}}$}

IVF

GIFT

100\% Procedural Factors:

ZIFT

Combination
$0 \%$ With ICSI

$0 \%$ Unstimulated

$0 \%$ Used gestational carrier

\begin{tabular}{r|lrlr}
\multicolumn{4}{c}{ Patient Diagnosis } \\
\\
\hline $85 \%$ & Tubal factor & $4 \%$ & Other factor & $11 \%$ \\
$0 \%$ & Dimininished ovarian reserve & $13 \%$ & Mnknown factor & $5 \%$ \\
$3 \%$ & Endometriosis & $4 \%$ & Female factors only & $11 \%$ \\
& Uterine factor & $5 \%$ & Female \& male factors & $25 \%$ \\
& Male factor & $17 \%$ & & \\
\hline
\end{tabular}

Data verified by V. Gabriel Garzo, MD

2006 PREGNANCY SUCCESS RATES

Age of Woman

\section{Type of Cycle}

\begin{tabular}{|c|c|c|c|c|}
\hline & & Age of & Noman & \\
\hline & $<35$ & $35-37$ & $38-40$ & $41-42^{d}$ \\
\hline & 65 & 56 & 58 & 24 \\
\hline & 63.1 & 57.1 & 36.2 & 20.8 \\
\hline & 58.5 & 50.0 & 27.6 & 16.7 \\
\hline & (45.6-70.6) & (36.3-63.7) & $(16.7-40.9)$ & $(4.7-37.4)$ \\
\hline & 60.3 & 57.1 & 34.0 & 16.7 \\
\hline & 66.7 & 59.6 & 37.2 & 20.0 \\
\hline irths ${ }^{b}$ & 45.6 & 34.0 & 25.6 & 20.0 \\
\hline & 3.1 & 12.5 & 19.0 & 0.0 \\
\hline & 1.9 & 2.1 & 2.3 & 2.7 \\
\hline & 34.1 & 43.8 & 23.8 & $0 / 5$ \\
\hline & 7.3 & 6.3 & 4.8 & $1 / 5$ \\
\hline & 31.6 & 42.9 & $5 / 16$ & $0 / 4$ \\
\hline & 20 & 10 & 11 & 7 \\
\hline & 45.0 & $4 / 10$ & $2 / 11$ & $0 / 7$ \\
\hline & 2.2 & 1.9 & 2.4 & 3.0 \\
\hline & & All Ages & ombined $^{\mathrm{e}}$ & \\
\hline & Fresh & mbryos & Frozen & nbryos \\
\hline & & & & \\
\hline & & & & \\
\hline & & & & \\
\hline
\end{tabular}

\section{Fresh Embryos from Nondonor Eggs}

Number of cycles

Percentage of cycles resulting in pregnancies ${ }^{b}$

Percentage of cycles resulting in live births ${ }^{b, c}$

(Confidence Interval)

Percentage of retrievals resulting in live births $s^{b, c}$

Percentage of transfers resulting in live births $s^{b, c}$

Percentage of transfers resulting in singleton live births ${ }^{b}$

Percentage of cancellations ${ }^{b}$

Average number of embryos transferred

Percentage of pregnancies with twins ${ }^{b}$

Percentage of pregnancies with triplets or more ${ }^{b}$

Percentage of live births having multiple infants ${ }^{b, c}$

\section{Frozen Embryos from Nondonor Eggs}

Number of transfers

Percentage of transfers resulting in live births ${ }^{\mathrm{b}, \mathrm{c}}$

Average number of embryos transferred

\section{Donor Eggs}

Number of transfers

Percentage of transfers resulting in live births $s^{\mathrm{b}, \mathrm{c}}$

Average number of embryos transferred

\section{CURRENT CLINIC SERVICES AND PROFILE}

Current Name: Reproductive Partners-UCSD Regional Fertility Center

Donor egg? Yes Gestational carriers? Yes

Donor embryo? Yes Cryopreservation? Yes

Single women? Yes

$\begin{array}{ll}\text { SART member? } & \text { Yes } \\ \text { Verified lab accreditation? } & \text { Yes } \\ \text { (See Appendix C for details.) } & \end{array}$

(See Appendix C for details.)

${ }^{a}$ Reflects patient and treatment characteristics of ART cycles performed in 2006 using fresh nondonor eggs or embryos.

${ }^{b}$ When fewer than 20 cycles are reported in an age category, rates are shown as a fraction and confidence intervals are not given. Calculating percentages from fractions may be misleading and is not encouraged.

${ }^{c}$ A multiple-infant birth is counted as one live birth.

d Clinic-specific outcome rates are unreliable for women older than 42 undergoing ART cycles using fresh or frozen embryos with nondonor eggs. Readers are urged to review national outcomes for these age groups (see page 27).

e All ages (including ages $>42$ ) are reported together because previous data show that patient age does not materially affect success with donor eggs. 


\section{REPRODUCTIVE SCIENCES CENTER \\ LA JOLLA, CALIFORNIA}

A comparison of clinic success rates may not be meaningful because patient medical characteristics and treatment approaches vary from clinic to clinic. For more details about this, along with information on how to interpret the statistics in this table, see pages 79-88.

\section{ART CYCLE PROFILE}

\section{Type of ART ${ }^{\mathrm{a}}$}

IVF

GIFT

100\% Procedural Factors:

ZIFT

$0 \%$ With ICSI

Combination

\section{$0 \%$ Unstimulated}

$0 \%$ Used gestational carrier $13 \%$

\section{Tubal factor}

Ovulatory dysfunction

Diminished ovarian reserve

Endometriosis

Uterine factor

Male factor

\section{Patient Diagnosis}

$\begin{array}{rlr}4 \% & \text { Other factor } & 2 \% \\ 0 \% & \text { Unknown factor } & 0 \% \\ 11 \% & \text { Multiple Factors: } & \\ 2 \% & \text { Female factors only } & 45 \% \\ 2 \% & \text { Female \& male factors } & 30 \% \\ 5 \% & & \end{array}$

Data verified by Samuel H. Wood, MD, PhD

2006 PREGNANCY SUCCESS RATES

\section{Age of Woman}

\section{Type of Cycle}

$\begin{array}{cccc}<35 & \mathbf{3 5 - 3 7} & \mathbf{3 8 - 4 0} & \mathbf{4 1 - 4 2}^{\mathbf{d}} \\ 2 & 5 & 9 & 4 \\ 1 / 2 & 3 / 5 & 2 / 9 & 0 / 4 \\ 1 / 2 & 2 / 5 & 2 / 9 & 0 / 4\end{array}$

\section{Fresh Embryos from Nondonor Eggs}

Number of cycles

Percentage of cycles resulting in pregnancies ${ }^{b}$

Percentage of cycles resulting in live births ${ }^{b, c}$

$1 / 2$

$2 / 5$

$2 / 9$

$0 / 4$

(Confidence Interval)

Percentage of retrievals resulting in live births $s^{b, c}$

$1 / 1$

$2 / 4$

$2 / 8$

$0 / 2$

Percentage of transfers resulting in live births $s^{b, c}$

$1 / 1$

$2 / 4$

$2 / 7$

$0 / 1$

Percentage of transfers resulting in singleton live births ${ }^{b}$

$1 / 1$

$2 / 4$

$2 / 7$

$0 / 1$

Percentage of cancellations ${ }^{b}$

$1 / 2$

$1 / 5$

$1 / 9$

$2 / 4$

Average number of embryos transferred

4.0

2.3

2.4

1.0

Percentage of pregnancies with twins ${ }^{b}$

$0 / 1$

$0 / 3$

$0 / 2$

Percentage of pregnancies with triplets or more ${ }^{\mathrm{b}}$

Percentage of live births having multiple infants ${ }^{\mathrm{b}, \mathrm{c}}$

$0 / 1$

$0 / 3$

$0 / 2$

$0 / 1$

$0 / 2$

$0 / 2$

\section{Frozen Embryos from Nondonor Eggs}

Number of transfers

Percentage of transfers resulting in live births $s^{b, c}$

Average number of embryos transferred

2

$2 / 2$

3.5

$$
1
$$

$0 / 1$

2.0
4
$2 / 4$
3.3

\section{Donor Eggs}

Number of transfers

Percentage of transfers resulting in live births $s^{b, c}$

Average number of embryos transferred

\begin{tabular}{cc}
\multicolumn{2}{c}{ All Ages Combined $^{\mathbf{e}}$} \\
Fresh Embryos & $\begin{array}{c}\text { Frozen Embryos }^{2} \\
32\end{array}$ \\
56.3 & 51.2 \\
2.3 & 3.0
\end{tabular}

\section{CURRENT CLINIC SERVICES AND PROFILE}

Current Name: Reproductive Sciences Center
Donor egg? Yes
Donor embryo? Yes
Single women? Yes

\begin{abstract}
Gestational carriers? Yes
\end{abstract}
Cryopreservation? Yes

\section{SART member? Yes}
Verified lab accreditation? Yes
(See Appendix C for details.)

\footnotetext{
${ }^{a}$ Reflects patient and treatment characteristics of ART cycles performed in 2006 using fresh nondonor eggs or embryos.

b When fewer than 20 cycles are reported in an age category, rates are shown as a fraction and confidence intervals are not given. Calculating percentages from fractions may be misleading and is not encouraged.

${ }^{c}$ A multiple-infant birth is counted as one live birth.

${ }^{d}$ Clinic-specific outcome rates are unreliable for women older than 42 undergoing ART cycles using fresh or frozen embryos with nondonor eggs. Readers are urged to review national outcomes for these age groups (see page 27).

${ }^{\mathrm{e}}$ All ages (including ages $>42$ ) are reported together because previous data show that patient age does not materially affect success with donor eggs.
} 


\section{SCRIPPS CLINIC FERTILITY CENTER \\ LA JOLLA, CALIFORNIA}

A comparison of clinic success rates may not be meaningful because patient medical characteristics and treatment approaches vary from clinic to clinic. For more details about this, along with information on how to interpret the statistics in this table, see pages 79-88.

\section{ART CYCLE PROFILE}

\section{Type of ART ${ }^{\mathrm{a}}$}

IVF

GIFT

100\% Procedural Factors:

ZIFT

Combination
$0 \%$ With ICSI

$0 \%$ Unstimulated

$0 \%$ Used gestational carrier
Tubal factor

$87 \%$

$0 \%$

$3 \%$
Ovulatory dysfunction

Diminished ovarian reserve

Endometriosis

Uterine factor

Male factor

\section{Patient Diagnosis}

7\% Other factor

3\% Unknown factor

10\% Multiple Factors:

$3 \%$ Female factors only $23 \%$

$<1 \%$ Female \& male factors $37 \%$

$13 \%$

2006 PREGNANCY SUCCESS RATES

Data verified by Jeffrey S. Rakoff, MD

\section{Type of Cycle}

\section{Age of Woman}

35-37 38-40

$41-42^{d}$
41

19.5

19.5

13.2

$(4.4-28.1)$

13.9

15.2

6.1

5.3

2.6

$2 / 7$

$1 / 7$

$3 / 5$
(8.8-34.9)

21.1

21.6

13.5

7.3

2.6

$3 / 8$

$0 / 8$

$3 / 8$
29

6.9

6.9

$(0.8-22.8)$

$8.0 \quad 0 / 10$

8.3

$0 / 9$

8.3

13.8

2.6

$0 / 2$

$0 / 2$

$0 / 2$

Percentage of live births having multiple infants ${ }^{b, c}$

\section{Frozen Embryos from Nondonor Eggs}

Number of transfers

Percentage of transfers resulting in live births ${ }^{\mathrm{b}, \mathrm{c}}$

Average number of embryos transferred

\section{Donor Eggs}

Number of transfers

Percentage of transfers resulting in live births $s^{b, c}$

Average number of embryos transferred

$\begin{array}{cccc}7 & 9 & 1 & 1 \\ 0 / 7 & 2 / 9 & 1 / 1 & 0 / 1 \\ 2.4 & 2.7 & 2.0 & 1.0\end{array}$

All Ages Combined ${ }^{\mathrm{e}}$

Fresh Embryos 5

$3 / 5$

1.8

\section{CURRENT CLINIC SERVICES AND PROFILE}

Current Name: Scripps Clinic Fertility Center

Donor egg? Yes Gestational carriers? Yes

Donor embryo? Yes

Single women? Yes

Cryopreservation? Yes

Frozen Embryos

0

${ }^{a}$ Reflects patient and treatment characteristics of ART cycles performed in 2006 using fresh nondonor eggs or embryos.

b When fewer than 20 cycles are reported in an age category, rates are shown as a fraction and confidence intervals are not given. Calculating percentages from fractions may be misleading and is not encouraged.

${ }^{c}$ A multiple-infant birth is counted as one live birth.

d Clinic-specific outcome rates are unreliable for women older than 42 undergoing ART cycles using fresh or frozen embryos with nondonor eggs. Readers are urged to review national outcomes for these age groups (see page 27).

All ages (including ages >42) are reported together because previous data show that patient age does not materially affect success with donor eggs. 


\section{MISSION REPRODUCTIVE CENTER \\ LAGUNA NIGUEL, CALIFORNIA}

A comparison of clinic success rates may not be meaningful because patient medical characteristics and treatment approaches vary from clinic to clinic. For more details about this, along with information on how to interpret the statistics in this table, see pages 79-88.

\section{ART CYCLE PROFILE}

$$
\text { Type of ART }{ }^{\mathrm{a}}
$$

\begin{tabular}{|c|c|c|c|c|c|c|c|}
\hline IVF & $100 \%$ & Procedural Factors: & & Tubal factor & $5 \%$ & Other factor & $3 \%$ \\
\hline GIFT & $0 \%$ & With ICSI & $88 \%$ & Ovulatory dysfunction & $11 \%$ & Unknown factor & $0 \%$ \\
\hline ZIFT & $0 \%$ & Unstimulated & $0 \%$ & Diminished ovarian reserve & $14 \%$ & Multiple Factors: & \\
\hline \multirow[t]{3}{*}{ Combination } & $0 \%$ & Used gestational carrier & $4 \%$ & Endometriosis & $4 \%$ & Female factors only & \\
\hline & & & & Uterine factor & $6 \%$ & Female \& male factors & \\
\hline & & & & Male factor & $13 \%$ & & \\
\hline
\end{tabular}

2006 PREGNANCY SUCCESS RATES

Data verified by Paul W. Zarutskie, MD

\section{Type of Cycle}

\section{Fresh Embryos from Nondonor Eggs}

Number of cycles

Percentage of cycles resulting in pregnancies ${ }^{b}$

Percentage of cycles resulting in live births $s^{b, c}$

(Confidence Interval)

Percentage of retrievals resulting in live births $s^{b, c}$

Percentage of transfers resulting in live births $s^{b, c}$

Percentage of transfers resulting in singleton live births ${ }^{b}$

Percentage of cancellations ${ }^{b}$

Average number of embryos transferred

Percentage of pregnancies with twins ${ }^{b}$

Percentage of pregnancies with triplets or more ${ }^{b}$

Percentage of live births having multiple infants ${ }^{b, c}$

\section{Frozen Embryos from Nondonor Eggs}

Number of transfers

Percentage of transfers resulting in live births ${ }^{\mathrm{b}, \mathrm{c}}$

Average number of embryos transferred

\section{Donor Eggs}

Number of transfers

Percentage of transfers resulting in live births ${ }^{\mathrm{b}, \mathrm{c}}$

Average number of embryos transferred

\section{Age of Woman}

35-37 38-40

$41-42^{d}$

\section{$<35$}

15

20

10

$\begin{array}{cccc}15 & 15 & 20 & 10 \\ 5 / 15 & 3 / 15 & 20.0 & 2 / 10 \\ 5 / 15 & 3 / 15 & 20.0 & 1 / 10\end{array}$

$5 / 15$

$5 / 12$

$3 / 14$

(5.7-43.7)

$3 / 12$

$3 / 12$

$4 / 17$

$1 / 9$

$0 / 15$

$2 / 12$

$4 / 15$

$1 / 4$

$4 / 15$

$1 / 4$

2.3

$1 / 15$

15.0

$1 / 10$

$2 / 5$

1.9

1.9

2.5

$0 / 5$

$1 / 3$

$1 / 4$

$0 / 2$

$2 / 5$

$0 / 3$

$1 / 3$

$0 / 4$

$0 / 2$

$0 / 4$

$0 / 1$

5

$0 / 5$

1.4
3

$0 / 3$

2.3
2

$1 / 2$

2.0
3

$0 / 3$

2.0

All Ages Combined

Fresh Embryos 7

$1 / 7$

1.9
Frozen Embryos

7

$0 / 7$

2.3

\section{CURRENT CLINIC SERVICES AND PROFILE}

Current Name: Mission Reproductive Center

Donor egg? Yes

Donor embryo? Yes

Single women? Yes

\section{Gestational carriers? Yes}

Cryopreservation? Yes

\section{SART member?}

Verified lab accreditation?

(See Appendix C for details.)

${ }^{a}$ Reflects patient and treatment characteristics of ART cycles performed in 2006 using fresh nondonor eggs or embryos.

${ }^{b}$ When fewer than 20 cycles are reported in an age category, rates are shown as a fraction and confidence intervals are not given. Calculating percentages from fractions may be misleading and is not encouraged.

${ }^{c}$ A multiple-infant birth is counted as one live birth.

d Clinic-specific outcome rates are unreliable for women older than 42 undergoing ART cycles using fresh or frozen embryos with nondonor eggs. Readers are urged to review national outcomes for these age groups (see page 27).

${ }^{e}$ All ages (including ages $>42$ ) are reported together because previous data show that patient age does not materially affect success with donor eggs. 


\section{SHER INSTITUTE OF REPRODUCTIVE MEDICINE-ORANGE COUNTY LAGUNA NIGUEL, CALIFORNIA}

A comparison of clinic success rates may not be meaningful because patient medical characteristics and treatment approaches vary from clinic to clinic. For more details about this, along with information on how to interpret the statistics in this table, see pages 79-88.

\section{ART CYCLE PROFILE}

\section{Type of ART ${ }^{\mathrm{a}}$}

IVF

GIFT

100\% Procedural Factors:

ZIFT

Combination
$0 \%$ With ICSI

$0 \%$ Unstimulated

$0 \%$ Used gestational carrier

\section{0\% Ovulatory dysfunction}

$1 \%$

$2 \%$

Endometriosis
Diminished ovarian reserve

Uterine factor

Male factor

\section{Patient Diagnosis}

$4 \%$ Other factor

2\% Unknown factor

$12 \%$

$2 \%$

20\% Multiple Factors:

$11 \%$ Female factors only $21 \%$

$<1 \%$ Female \& male factors $19 \%$

$9 \%$

\section{PREGNANCY SUCCESS RATES}

Data verified by Brian Acacio, MD

\section{Type of Cycle}

\section{Age of Woman}

35-37

38-40

$41-42^{d}$
13

$3 / 13$

$2 / 13$

(31.0-64.2)

50.0

51.4

20.0

5.3

3.0

45.0

20.0

$11 / 18$
$2 / 12$
$2 / 11$
$1 / 11$
$1 / 13$
3.8
$1 / 3$
$0 / 3$
$1 / 2$

22

31.8

18.2

(5.2-40.3)

19.0

$4 / 15$

$4 / 15$

4.5

2.5

$0 / 7$

$1 / 7$

$0 / 4$
11

$2 / 11$

$1 / 11$

Percentage of pregnancies with triplets or more
Percentage of live births having multiple infants

\section{Frozen Embryos from Nondonor Eggs}

Number of transfers

Percentage of transfers resulting in live births ${ }^{b, c}$

Average number of embryos transferred

\section{Donor Eggs}

Number of transfers

Percentage of transfers resulting in live births ${ }^{b, c}$

Average number of embryos transferred

5

$0 / 5$

2.4
1

$0 / 1$

2.0
$1 / 11$

$1 / 11$

$1 / 11$

$0 / 11$

3.7

$0 / 2$

$0 / 2$

$0 / 1$

\section{All Ages Combined}

Fresh Embryos 10

$5 / 10$

2.6
Frozen Embryos

1

$0 / 1$

2.0

\section{CURRENT CLINIC SERVICES AND PROFILE}

Current Name: Sher Institute of Reproductive Medicine-Orange County

Donor egg? Yes Gestational carriers? Yes

Donor embryo? Yes Cryopreservation? Yes

Single women? Yes

SART member?

Verified lab accreditation?

(See Appendix C for details.)

${ }^{a}$ Reflects patient and treatment characteristics of ART cycles performed in 2006 using fresh nondonor eggs or embryos.

b When fewer than 20 cycles are reported in an age category, rates are shown as a fraction and confidence intervals are not given. Calculating percentages from fractions may be misleading and is not encouraged.

${ }^{c}$ A multiple-infant birth is counted as one live birth.

d Clinic-specific outcome rates are unreliable for women older than 42 undergoing ART cycles using fresh or frozen embryos with nondonor eggs. Readers are urged to review national outcomes for these age groups (see page 27).

e All ages (including ages $>42$ ) are reported together because previous data show that patient age does not materially affect success with donor eggs. 


\section{LOMA LINDA UNIVERSITY CENTER FOR FERTILITY AND IVF LOMA LINDA, CALIFORNIA}

A comparison of clinic success rates may not be meaningful because patient medical characteristics and treatment approaches vary from clinic to clinic. For more details about this, along with information on how to interpret the statistics in this table, see pages 79-88.

\section{ART CYCLE PROFILE}

$$
\text { Type of ART }{ }^{\mathrm{a}}
$$

\begin{tabular}{|c|c|c|c|c|c|c|c|}
\hline IVF & $100 \%$ & Procedural Factors: & & Tubal factor & $14 \%$ & Other factor & $3 \%$ \\
\hline GIFT & $0 \%$ & With ICSI & $83 \%$ & Ovulatory dysfunction & $4 \%$ & Unknown factor & \\
\hline ZIFT & $0 \%$ & Unstimulated & $1 \%$ & Diminished ovarian reserve & $14 \%$ & Multiple Factors: & \\
\hline \multirow[t]{3}{*}{ Combination } & $0 \%$ & Used gestational carrier & $0 \%$ & Endometriosis & $4 \%$ & Female factors only & \\
\hline & & & & Uterine factor & $<1 \%$ & Female \& male factors & \\
\hline & & & & Male factor & $18 \%$ & & \\
\hline
\end{tabular}

2006 PREGNANCY SUCCESS RATES

Data verified by John D. Jacobson, MD

\section{Type of Cycle}

\section{Fresh Embryos from Nondonor Eggs}

Number of cycles

Percentage of cycles resulting in pregnancies ${ }^{b}$

Percentage of cycles resulting in live births $s^{b, c}$

(Confidence Interval)

Percentage of retrievals resulting in live births $s^{b, c}$

Percentage of transfers resulting in live births $s^{b, c}$

Percentage of transfers resulting in singleton live births ${ }^{b}$

Percentage of cancellations ${ }^{b}$

Average number of embryos transferred

Percentage of pregnancies with twins ${ }^{b}$

Percentage of pregnancies with triplets or more ${ }^{b}$

Percentage of live births having multiple infants ${ }^{b, c}$

\section{Frozen Embryos from Nondonor Eggs}

Number of transfers

Percentage of transfers resulting in live births $s^{b, c}$

Average number of embryos transferred

\section{Donor Eggs}

Number of transfers

Percentage of transfers resulting in live births $s^{b, c}$

Average number of embryos transferred

\section{Age of Woman}

35-37 38-40

$41-42^{d}$

\section{$<35$}

42

42.9

30

4

44.2

34.9

33.3

10.0

$1 / 4$

(24.9-45.9)

(19.6-49.5)

6.7

$1 / 4$

40.0

37.8

(0.8-22.1)

41.7

38.9

9.5

$1 / 2$

19.4

30.6

$2 / 19$

$1 / 2$

12.8

11.9

$2 / 19$

$0 / 2$

2.2

2.8

30.0

$2 / 4$

39.5

$5 / 18$

3.0

3.5

2.6

$0 / 18$

$1 / 3$

$1 / 1$

53.3

$3 / 14$

$1 / 3$

$0 / 1$

$0 / 2$

$1 / 1$

$\begin{array}{cccc}19 & 10 & 3 & 2 \\ 7 / 19 & 4 / 10 & 2 / 3 & 1 / 2 \\ 2.7 & 2.7 & 3.0 & 3.5\end{array}$

Fresh Embryos

All Ages Combined

\section{CURRENT CLINIC SERVICES AND PROFILE}

Current Name: Loma Linda University Center for Fertility and IVF

Donor egg? Yes Gestational carriers? Yes

Donor embryo? Yes Cryopreservation? Yes

Single women? Yes

$\begin{array}{cc}14 & 12 \\ 5 / 14 & 2 / 12 \\ 2.3 & 2.9\end{array}$

Embryos

${ }^{a}$ Reflects patient and treatment characteristics of ART cycles performed in 2006 using fresh nondonor eggs or embryos.

${ }^{b}$ When fewer than 20 cycles are reported in an age category, rates are shown as a fraction and confidence intervals are not given. Calculating percentages from fractions may be misleading and is not encouraged.

${ }^{c}$ A multiple-infant birth is counted as one live birth.

${ }^{d}$ Clinic-specific outcome rates are unreliable for women older than 42 undergoing ART cycles using fresh or frozen embryos with nondonor eggs. Readers are urged to review national outcomes for these age groups (see page 27).

All ages (including ages $>42$ ) are reported together because previous data show that patient age does not materially affect success with donor eggs. 


\section{REPRODUCTIVE PARTNERS-LONG BEACH \\ LONG BEACH, CALIFORNIA}

A comparison of clinic success rates may not be meaningful because patient medical characteristics and treatment approaches vary from clinic to clinic. For more details about this, along with information on how to interpret the statistics in this table, see pages 79-88.

\section{ART CYCLE PROFILE}

\begin{tabular}{|c|c|c|c|c|c|c|c|}
\hline & Type & of $A R T^{a}$ & & Pat & ent [ & iagnosis & \\
\hline IVF & $>99 \%$ & Procedural Factors: & & Tubal factor & $12 \%$ & Other factor & $15 \%$ \\
\hline GIFT & $<1 \%$ & With ICSI & $38 \%$ & Ovulatory dysfunction & $6 \%$ & Unknown factor & $9 \%$ \\
\hline ZIFT & $0 \%$ & Unstimulated & $0 \%$ & Diminished ovarian reserve & $20 \%$ & Multiple Factors: & \\
\hline Combination & $0 \%$ & Used gestational carrier & $<1 \%$ & Endometriosis & $5 \%$ & Female factors only & $5 \%$ \\
\hline & & & & Uterine factor & $4 \%$ & Female \& male factors & $8 \%$ \\
\hline & & & & Male factor & $16 \%$ & & \\
\hline
\end{tabular}

2006 PREGNANCY SUCCESS RATES

Data verified by Bill Yee, MD

\section{Type of Cycle}

\section{Fresh Embryos from Nondonor Eggs}

Number of cycles

Percentage of cycles resulting in pregnancies ${ }^{b}$

Percentage of cycles resulting in live births ${ }^{\mathrm{b}, \mathrm{c}}$

(Confidence Interval)

Percentage of retrievals resulting in live births $s^{b, c}$

Percentage of transfers resulting in live births $s^{b, c}$

Percentage of transfers resulting in singleton live births ${ }^{b}$

Percentage of cancellations ${ }^{b}$

Average number of embryos transferred

Percentage of pregnancies with twins ${ }^{b}$

Percentage of pregnancies with triplets or more ${ }^{b}$

Percentage of live births having multiple infants ${ }^{b, c}$

\section{Frozen Embryos from Nondonor Eggs}

Number of transfers

Percentage of transfers resulting in live births ${ }^{b, c}$

Average number of embryos transferred

\section{Donor Eggs}

Number of transfers

Percentage of transfers resulting in live births $s^{b, c}$

Average number of embryos transferred

\section{Age of Woman}

35-37 38-40

$41-42^{d}$

$<35$

39

35.9

33

25

50.0

33.3

39.4

8.0

41.4

(28.6-55.1)

(19.1-50.2)

27.3

(13.3-45.5)

0.0

44.4

40.6

36.0

$(0.0-13.7)$

45.3

41.9

37.5

0.0

32.1

25.8

33.3

$0 / 19$

6.9

17.9

24.2

$0 / 19$

2.1

2.4

2.8

20.0

37.9

$6 / 14$

0.0

$0 / 14$

$2 / 13$

2.6

29.2

$5 / 13$

$0 / 13$

$0 / 2$

$0 / 2$

$1 / 9$

22

40.9

7

$4 / 7$

7

$2 / 7$

6

2.0

2.4

2.7

$3 / 6$

2.5

All Ages Combined ${ }^{\mathrm{e}}$

Fresh Embryos

Frozen Embryos

20

45.0

6

2.2

$2 / 6$

2.0

\section{CURRENT CLINIC SERVICES AND PROFILE}

Current Name: Reproductive Partners-Orange County

Donor egg? Yes

Donor embryo? Yes

Single women? Yes
Gestational carriers? Yes

Cryopreservation? Yes
SART member?

Yes

Verified lab accreditation?

(See Appendix C for details.)

\footnotetext{
${ }^{a}$ Reflects patient and treatment characteristics of ART cycles performed in 2006 using fresh nondonor eggs or embryos.

b When fewer than 20 cycles are reported in an age category, rates are shown as a fraction and confidence intervals are not given. Calculating percentages from fractions may be misleading and is not encouraged.

${ }^{c}$ A multiple-infant birth is counted as one live birth.

d Clinic-specific outcome rates are unreliable for women older than 42 undergoing ART cycles using fresh or frozen embryos with nondonor eggs. Readers are urged to review national outcomes for these age groups (see page 27).

e All ages (including ages $>42$ ) are reported together because previous data show that patient age does not materially affect success with donor eggs.
} 


\section{CALIFORNIA FERTILITY PARTNERS LOS ANGELES, CALIFORNIA}

A comparison of clinic success rates may not be meaningful because patient medical characteristics and treatment approaches vary from clinic to clinic. For more details about this, along with information on how to interpret the statistics in this table, see pages 79-88.

\section{ART CYCLE PROFILE}

$$
\text { Type of ART }{ }^{\mathrm{a}}
$$

\begin{tabular}{|c|c|c|c|c|c|c|c|}
\hline IVF & $100 \%$ & Procedural Factors: & & Tubal factor & $3 \%$ & Other factor & $13 \%$ \\
\hline GIFT & $0 \%$ & With ICSI & $63 \%$ & Ovulatory dysfunction & $2 \%$ & Unknown factor & $23 \%$ \\
\hline ZIFT & $0 \%$ & Unstimulated & $0 \%$ & Diminished ovarian reserve & $10 \%$ & Multiple Factors: & \\
\hline \multirow[t]{3}{*}{ Combination } & $0 \%$ & Used gestational carrier & $6 \%$ & Endometriosis & $3 \%$ & Female factors only & \\
\hline & & & & Uterine factor & $4 \%$ & Female \& male factors & \\
\hline & & & & Male factor & $17 \%$ & & \\
\hline
\end{tabular}

2006 PREGNANCY SUCCESS RATES

Data verified by Richard P. Marrs, MD

\section{Type of Cycle}

\section{Fresh Embryos from Nondonor Eggs}

Number of cycles

Percentage of cycles resulting in pregnancies ${ }^{b}$

Percentage of cycles resulting in live births $s^{b, c}$

(Confidence Interval)

Percentage of retrievals resulting in live births $s^{b, c}$

Percentage of transfers resulting in live births $s^{b, c}$

Percentage of transfers resulting in singleton live births ${ }^{b}$

Percentage of cancellations ${ }^{b}$

Average number of embryos transferred

Percentage of pregnancies with twins ${ }^{b}$

Percentage of pregnancies with triplets or more ${ }^{b}$

Percentage of live births having multiple infants ${ }^{b, c}$

\section{Frozen Embryos from Nondonor Eggs}

Number of transfers

Percentage of transfers resulting in live births ${ }^{\mathrm{b}, \mathrm{c}}$

Average number of embryos transferred

\section{Donor Eggs}

Number of transfers

Percentage of transfers resulting in live births $s^{b, c}$

Average number of embryos transferred

\section{Age of Woman}

35-37 38-40

$41-42^{d}$

$<35$

85

28.2

127

18.9

13.4

41.7

22.4

$(27.6-53.5)$

(14.0-32.7)

(8.0-20.6)

18.9

20.0

17.6

29.1

3.5

12.5

0.0

$2 / 17$

$10 / 19$

50.0

29.2

16.7

20

27

25.9

40.0

12

25.9

2.9

3.2

$1 / 12$

2.6

All Ages Combined ${ }^{\mathrm{e}}$

Fresh Embryos

93

49.5

2.5
Frozen Embryos

90

30.0

2.7

\section{CURRENT CLINIC SERVICES AND PROFILE}

Current Name: California Fertility Partners

Donor egg? Yes

Donor embryo? No

Gestational carriers? Yes

Single women? Yes

Cryopreservation? Yes

\section{SART member?}

Yes

Verified lab accreditation?

(See Appendix C for details.)

${ }^{a}$ Reflects patient and treatment characteristics of ART cycles performed in 2006 using fresh nondonor eggs or embryos.

${ }^{b}$ When fewer than 20 cycles are reported in an age category, rates are shown as a fraction and confidence intervals are not given. Calculating percentages from fractions may be misleading and is not encouraged.

${ }^{c}$ A multiple-infant birth is counted as one live birth.

d Clinic-specific outcome rates are unreliable for women older than 42 undergoing ART cycles using fresh or frozen embryos with nondonor eggs. Readers are urged to review national outcomes for these age groups (see page 27).

${ }^{e}$ All ages (including ages $>42$ ) are reported together because previous data show that patient age does not materially affect success with donor eggs. 


\section{CEDARS SINAI MEDICAL CENTER \\ CENTER FOR FERTILITY AND REPRODUCTIVE MEDICINE LOS ANGELES, CALIFORNIA}

A comparison of clinic success rates may not be meaningful because patient medical characteristics and treatment approaches vary from clinic to clinic. For more details about this, along with information on how to interpret the statistics in this table, see pages 79-88.

\section{ART CYCLE PROFILE}

\section{Type of ART ${ }^{\mathrm{a}}$}

IVF

GIFT

ZIFT

Combination
100\% Procedural Factors:

$0 \%$ With ICSI

$0 \%$ Unstimulated

$0 \%$ Used gestational carrier

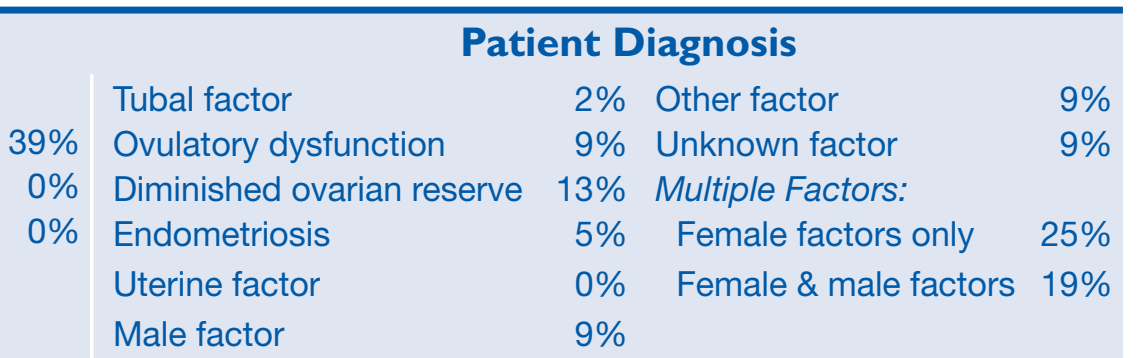

Data verified by Margareta D. Pisarska, MD

2006 PREGNANCY SUCCESS RATES

\section{Age of Woman}

\section{Type of Cycle}

$\begin{array}{cccc}\mathbf{3 5 5} & \mathbf{3 5 - 3 7} & \mathbf{3 8 - 4 0} & \mathbf{4 1 - 4 2}^{\mathbf{d}} \\ 17 & 11 & 20 & 7 \\ 7 / 17 & 7 / 11 & 25.0 & 2 / 7 \\ 6 / 17 & 6 / 11 & 15.0 & 1 / 7\end{array}$

\section{Fresh Embryos from Nondonor Eggs}

Number of cycles

Percentage of cycles resulting in pregnancies ${ }^{b}$

Percentage of cycles resulting in live births ${ }^{b, c}$

(Confidence Interval)

Percentage of retrievals resulting in live births $s^{b, c}$

Percentage of transfers resulting in live births $s^{b, c}$

Percentage of transfers resulting in singleton live births ${ }^{b}$

Percentage of cancellations ${ }^{b}$

Average number of embryos transferred

Percentage of pregnancies with twins ${ }^{b}$

Percentage of pregnancies with triplets or more ${ }^{b}$

Percentage of live births having multiple infants ${ }^{b, c}$

\section{Frozen Embryos from Nondonor Eggs}

Number of transfers

Percentage of transfers resulting in live births ${ }^{b, c}$

Average number of embryos transferred

\section{Donor Eggs}

Number of transfers

Percentage of transfers resulting in live births $s^{b, c}$

Average number of embryos transferred

$\begin{array}{cccc}6 / 12 & 6 / 9 & 3 / 16 & 1 / 6 \\ 6 / 11 & 6 / 8 & 3 / 14 & 1 / 5 \\ 3 / 11 & 5 / 8 & 1 / 14 & 1 / 5 \\ 5 / 17 & 2 / 11 & 20.0 & 1 / 7 \\ 2.7 & 2.5 & 3.4 & 2.8 \\ 5 / 7 & 1 / 7 & 2 / 5 & 1 / 2 \\ 0 / 7 & 0 / 7 & 0 / 5 & 0 / 2 \\ 3 / 6 & 1 / 6 & 2 / 3 & 0 / 1\end{array}$

3.2-37.9)

\section{All Ages Combined ${ }^{\mathrm{e}}$}

Fresh Embryos

Frozen Embryos

2

2

$0 / 2$

$0 / 2$

2.0

\begin{tabular}{|c|c|c|}
\hline 2 & 0 & 2 \\
\hline $1 / 2$ & & $0 / 2$ \\
\hline 4.5 & & 2.5 \\
\hline
\end{tabular}

\section{CURRENT CLINIC SERVICES AND PROFILE}

Current Name: Cedars Sinai Medical Center, Center for Fertility and Reproductive Medicine

Donor egg? Yes

Donor embryo? Yes

Single women? Yes

\begin{abstract}
Gestational carriers? Yes
\end{abstract}
Cryopreservation? Yes

\section{SART member?}

Verified lab accreditation?

(See Appendix C for details.)

\footnotetext{
${ }^{a}$ Reflects patient and treatment characteristics of ART cycles performed in 2006 using fresh nondonor eggs or embryos.

${ }^{b}$ When fewer than 20 cycles are reported in an age category, rates are shown as a fraction and confidence intervals are not given. Calculating percentages from fractions may be misleading and is not encouraged.

${ }^{c}$ A multiple-infant birth is counted as one live birth.

d Clinic-specific outcome rates are unreliable for women older than 42 undergoing ART cycles using fresh or frozen embryos with nondonor eggs. Readers are urged to review national outcomes for these age groups (see page 27).

e All ages (including ages $>42$ ) are reported together because previous data show that patient age does not materially affect success with donor eggs.
} 


\section{CHA FERTILITY CENTER \\ LOS ANGELES, CALIFORNIA}

A comparison of clinic success rates may not be meaningful because patient medical characteristics and treatment approaches vary from clinic to clinic. For more details about this, along with information on how to interpret the statistics in this table, see pages 79-88.

\section{ART CYCLE PROFILE}

\section{Type of ART ${ }^{\mathrm{a}}$}

IVF

GIFT

100\% Procedural Factors:

ZIFT

Combination
$0 \%$ With ICSI

$0 \%$ Unstimulated

$0 \%$ Used gestational carrier

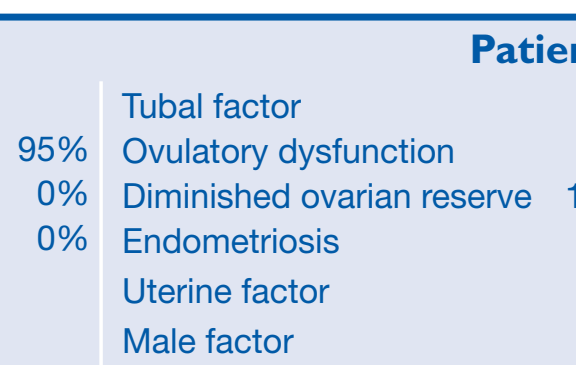

2006 PREGNANCY SUCCESS RATES

\section{Type of Cycle}

\section{Fresh Embryos from Nondonor Eggs}

Number of cycles

Percentage of cycles resulting in pregnancies ${ }^{\mathrm{b}}$

Percentage of cycles resulting in live births ${ }^{\mathrm{b}, \mathrm{c}}$

(Confidence Interval)

Percentage of retrievals resulting in live births $s^{b, c}$

Percentage of transfers resulting in live births ${ }^{b, c}$

Percentage of transfers resulting in singleton live births ${ }^{b}$

Percentage of cancellations ${ }^{b}$

Average number of embryos transferred

Percentage of pregnancies with twins ${ }^{b}$

Percentage of pregnancies with triplets or more ${ }^{b}$

Percentage of live births having multiple infants ${ }^{b, c}$

\section{Frozen Embryos from Nondonor Eggs}

Number of transfers

Percentage of transfers resulting in live births $s^{b, c}$

Average number of embryos transferred

\section{Donor Eggs}

Number of transfers

Percentage of transfers resulting in live births $s^{b, c}$

Average number of embryos transferred

(n)

1.

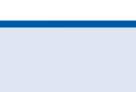

Data verified by Thomas J. Kim, MD

$4 \%$ Other factor

$1 \%$ Unknown factor

17\% Multiple Factors:

3\% Female factors only $\quad 7 \%$

$4 \%$ Female \& male factors $4 \%$

$6 \%$

\begin{tabular}{|c|c|c|c|}
\hline \multicolumn{4}{|c|}{ Age of Woman } \\
\hline$<35$ & $35-37$ & $38-40$ & $41-42^{c}$ \\
\hline 33 & 23 & 21 & 11 \\
\hline 33.3 & 47.8 & 14.3 & $4 / 11$ \\
\hline 24.2 & 34.8 & 9.5 & $2 / 11$ \\
\hline 11.1-42.3) & (16.4-57.3) & $(1.2-30.4)$ & \\
\hline 24.2 & 34.8 & 9.5 & $2 / 11$ \\
\hline 24.2 & 34.8 & 9.5 & $2 / 11$ \\
\hline 24.2 & 30.4 & 4.8 & $2 / 11$ \\
\hline 0.0 & 0.0 & 0.0 & $0 / 11$ \\
\hline 2.5 & 2.8 & 2.7 & 2.8 \\
\hline $0 / 11$ & $0 / 11$ & $0 / 3$ & $0 / 4$ \\
\hline $0 / 11$ & $1 / 11$ & $1 / 3$ & $0 / 4$ \\
\hline $0 / 8$ & $1 / 8$ & $1 / 2$ & $0 / 2$ \\
\hline 11 & 4 & 2 & 0 \\
\hline $5 / 11$ & $2 / 4$ & $2 / 2$ & \\
\hline 2.4 & 2.8 & 1.5 & \\
\hline \multicolumn{4}{|c|}{ All Ages Combined ${ }^{\mathrm{e}}$} \\
\hline \multicolumn{2}{|c|}{ Fresh Embryos } & \multicolumn{2}{|c|}{ Frozen Embryos } \\
\hline \multicolumn{2}{|c|}{73} & \multicolumn{2}{|c|}{12} \\
\hline \multicolumn{2}{|c|}{64.4} & \multicolumn{2}{|c|}{$6 / 12$} \\
\hline \multicolumn{2}{|c|}{2.0} & \multicolumn{2}{|c|}{2.6} \\
\hline
\end{tabular}

\section{CURRENT CLINIC SERVICES AND PROFILE}

Current Name: This clinic has closed or reorganized since 2006. Information on current clinic services and profile therefore is not provided here. Contact the NASS Help Desk for current information about this clinic.

\footnotetext{
${ }_{b}^{a}$ Reflects patient and treatment characteristics of ART cycles performed in 2006 using fresh nondonor eggs or embryos.

${ }^{b}$ When fewer than 20 cycles are reported in an age category, rates are shown as a fraction and confidence intervals are not given. Calculating percentages from fractions may be misleading and is not encouraged.

${ }^{c}$ A multiple-infant birth is counted as one live birth.

${ }^{d}$ Clinic-specific outcome rates are unreliable for women older than 42 undergoing ART cycles using fresh or frozen embryos with nondonor eggs. Readers are urged to review national outcomes for these age groups (see page 27).

e All ages (including ages $>42$ ) are reported together because previous data show that patient age does not materially affect success with donor eggs.
} 


\section{PACIFIC FERTILITY CENTER-LOS ANGELES LOS ANGELES, CALIFORNIA}

A comparison of clinic success rates may not be meaningful because patient medical characteristics and treatment approaches vary from clinic to clinic. For more details about this, along with information on how to interpret the statistics in this table, see pages 79-88.

\section{ART CYCLE PROFILE}

\section{Type of ART ${ }^{\mathrm{a}}$}

IVF

GIFT $100 \%$ Procedural Factors:

ZIFT

Combination
$0 \%$ With ICSI

$0 \%$ Unstimulated

$0 \%$ Used gestational carrier

\begin{tabular}{|c|c|}
\hline $67 \%$ & Ovulatory dvsfunction \\
\hline $0 \%$ & Diminished ovarian reserve \\
\hline $0 \%$ & Endometriosis \\
\hline & Uterine factor \\
\hline & Male factor \\
\hline
\end{tabular}

Patient Diagnosis

$6 \%$ Other factor

$<1 \%$ Unknown factor

0\% Multiple Factors:

$2 \% \quad$ Female factors only $\quad 4 \%$

$0 \%$ Female \& male factors $13 \%$

$9 \%$

2006 PREGNANCY SUCCESS RATES

Data verified by Vicken Sahakian, MD

\section{Type of Cycle}

\section{Fresh Embryos from Nondonor Eggs}

Number of cycles

Percentage of cycles resulting in pregnancies ${ }^{b}$

Percentage of cycles resulting in live births ${ }^{b, c}$

(Confidence Interval)

Percentage of retrievals resulting in live births $s^{b, c}$

Percentage of transfers resulting in live births ${ }^{b, c}$

Percentage of transfers resulting in singleton live births ${ }^{b}$

Percentage of cancellations ${ }^{b}$

Average number of embryos transferred

Percentage of pregnancies with twins ${ }^{b}$

Percentage of pregnancies with triplets or more ${ }^{b}$

Percentage of live births having multiple infants ${ }^{\mathrm{b}, \mathrm{c}}$

\section{Frozen Embryos from Nondonor Eggs}

Number of transfers

Percentage of transfers resulting in live births $s^{b, c}$

Average number of embryos transferred

\section{Donor Eggs}

Number of transfers

Percentage of transfers resulting in live births $s^{b, c}$

Average number of embryos transferred

\section{Age of Woman}

35-37 38-40

$41-42^{d}$

\section{$<35$}

31

27

14

52
53.8

48.4

44.4

$4 / 14$

51.9

38.7

37.0

$2 / 14$

(37.6-66.0)

51.9

(21.8-57.8)

$(19.4-57.6)$

38.7

37.0

40.0

$2 / 14$

55.1

40.0

28.0

$2 / 11$

26.7

0.0

$2 / 11$

0.0

0.0

3.4

$0 / 14$

3.2

3.6

46.4

$6 / 15$

$4 / 12$

3.0

10.7

$0 / 15$

$0 / 12$

$0 / 4$

44.4

$4 / 12$

$3 / 10$

$0 / 4$

$0 / 2$

$\begin{array}{cccc}12 & 10 & 5 & 1 \\ 4 / 12 & 2 / 10 & 1 / 5 & 0 / 1 \\ 3.6 & 3.2 & 3.6 & 3.0\end{array}$

\section{CURRENT CLINIC SERVICES AND PROFILE}

All Ages Combined ${ }^{\mathrm{e}}$

Fresh Embryos

106

50.9

3.1
Frozen Embryos 55

41.8

3.1

Current Name: Pacific Fertility Center-Los Angeles

Donor egg? No

Donor embryo? Yes

Gestational carriers? Yes

Cryopreservation? Yes

Single women? Yes

SART member?

Yes

Verified lab accreditation?

Yes

(See Appendix C for details.)

${ }^{a}$ Reflects patient and treatment characteristics of ART cycles performed in 2006 using fresh nondonor eggs or embryos.

${ }^{b}$ When fewer than 20 cycles are reported in an age category, rates are shown as a fraction and confidence intervals are not given. Calculating percentages from fractions may be misleading and is not encouraged.

${ }^{\mathrm{C}}$ A multiple-infant birth is counted as one live birth.

d Clinic-specific outcome rates are unreliable for women older than 42 undergoing ART cycles using fresh or frozen embryos with nondonor eggs. Readers are urged to review national outcomes for these age groups (see page 27).

ell ages (including ages $>42$ ) are reported together because previous data show that patient age does not materially affect success with donor eggs. 


\section{UCLA FERTILITY CENTER \\ LOS ANGELES, CALIFORNIA}

A comparison of clinic success rates may not be meaningful because patient medical characteristics and treatment approaches vary from clinic to clinic. For more details about this, along with information on how to interpret the statistics in this table, see pages 79-88.

\section{ART CYCLE PROFILE}

$$
\text { Type of ART }{ }^{\mathrm{a}}
$$

\begin{tabular}{|c|c|c|c|c|c|c|c|}
\hline IVF & $100 \%$ & Procedural Factors: & & Tubal factor & $10 \%$ & Other factor & $7 \%$ \\
\hline GIFT & $0 \%$ & With ICSI & $40 \%$ & Ovulatory dysfunction & $4 \%$ & Unknown factor & \\
\hline ZIFT & $0 \%$ & Unstimulated & $0 \%$ & Diminished ovarian reserve & $22 \%$ & Multiple Factors: & \\
\hline \multirow{3}{*}{ Combination } & $0 \%$ & Used gestational carrier & $0 \%$ & Endometriosis & $3 \%$ & Female factors only & \\
\hline & & & & Uterine factor & $0 \%$ & Female \& male factors & \\
\hline & & & & Male factor & $27 \%$ & & \\
\hline
\end{tabular}

2006 PREGNANCY SUCCESS RATES

Data verified by T.C. Jackson Wu, MD, PhD

\section{Type of Cycle}

\section{Fresh Embryos from Nondonor Eggs}

Number of cycles

Percentage of cycles resulting in pregnancies ${ }^{b}$

Percentage of cycles resulting in live births $s^{b, c}$

(Confidence Interval)

Percentage of retrievals resulting in live births $s^{b, c}$

Percentage of transfers resulting in live births $s^{b, c}$

Percentage of transfers resulting in singleton live births ${ }^{b}$

Percentage of cancellations ${ }^{b}$

Average number of embryos transferred

Percentage of pregnancies with twins ${ }^{b}$

Percentage of pregnancies with triplets or more ${ }^{b}$

Percentage of live births having multiple infants ${ }^{b, c}$

\section{Frozen Embryos from Nondonor Eggs}

Number of transfers

Percentage of transfers resulting in live births ${ }^{\mathrm{b}, \mathrm{c}}$

Average number of embryos transferred

\section{Donor Eggs}

Number of transfers

Percentage of transfers resulting in live births ${ }^{\mathrm{b}, \mathrm{c}}$

Average number of embryos transferred

\section{Age of Woman}

35-37 38-40

$41-42^{d}$

$<35$

16

$6 / 16$

$6 / 16$

22

11

46.2

34.6

(17.2-55.7)

37.5

$9 / 19$

$6 / 13$

$6 / 13$

$3 / 13$

$3 / 16$

18.2

$2 / 11$

$5 / 19$

3.4

3.5

$2 / 12$

$2 / 6$

$2 / 12$

$1 / 6$

$4 / 9$

$3 / 6$

13.6

$2 / 11$

(2.9-34.9)

$3 / 18 \quad 2 / 8$

$3 / 13$

$2 / 8$

$3 / 13$

$2 / 8$

18.2

$3 / 11$

3.3

2.9

$0 / 4$

$0 / 2$

$0 / 4$

$0 / 2$

$0 / 3$

$0 / 2$

\section{6}

$1 / 6$

2.7

\section{1}

$1 / 1$

3.0

\section{2}

$1 / 2$

1.0 . 


\section{USC REPRODUCTIVE ENDOCRINOLOGY AND INFERTILITY LOS ANGELES, CALIFORNIA}

A comparison of clinic success rates may not be meaningful because patient medical characteristics and treatment approaches vary from clinic to clinic. For more details about this, along with information on how to interpret the statistics in this table, see pages 79-88.

\section{ART CYCLE PROFILE}

\section{Type of ART ${ }^{\mathrm{a}}$}

IVF

GIFT

ZIFT

Combination
90\% Procedural Factors:

$1 \%$ With ICSI

$7 \%$ Unstimulated

$1 \%$ Used gestational carrier

\begin{tabular}{|c|c|}
\hline $46 \%$ & hyulatory dycfunction \\
\hline $0 \%$ & Ovulatory dysfunction \\
\hline $4 \%$ & Diminished ovarian reserve \\
\hline $4 \%$ & Endometriosis \\
\hline & Uterine factor \\
\hline & Male factor \\
\hline
\end{tabular}

Patient Diagnosis

$4 \%$ Other factor

4\% Unknown factor

7\% Multiple Factors:

$3 \%$ Female factors only $32 \%$

$3 \%$ Female \& male factors $20 \%$

$9 \%$

\section{PREGNANCY SUCCESS RATES}

\section{Type of Cycle}

Data verified by Richard J. Paulson, MD

\section{Age of Woman}

$<35 \quad 35-37 \quad 38-40 \quad 41-42^{d}$

$$
41-42^{d}
$$

$\begin{array}{cccc}37 & 25 & 43 & 20 \\ 29.7 & 48.0 & 37.2 & 30.0 \\ 27.0 & 48.0 & 27.9 & 15.0 \\ 13.8-44.1) & (27.8-68.7) & (15.3-43.7) & (3.2-37.9) \\ 28.6 & 54.5 & 30.8 & 3 / 19 \\ 29.4 & 60.0 & 30.8 & 3 / 18 \\ 11.8 & 45.0 & 23.1 & 3 / 18 \\ 5.4 & 12.0 & 9.3 & 5.0 \\ 3.0 & 3.2 & 3.8 & 4.8 \\ 3 / 11 & 2 / 12 & 4 / 16 & 0 / 6 \\ 3 / 11 & 3 / 12 & 1 / 16 & 0 / 6 \\ 6 / 10 & 3 / 12 & 3 / 12 & 0 / 3\end{array}$

$\begin{array}{cccc}10 & 10 & 19 & 6 \\ 1 / 10 & 1 / 10 & 4 / 19 & 3 / 6 \\ 3.2 & 3.3 & 3.4 & 4.5\end{array}$

All Ages Combined ${ }^{\mathrm{e}}$

Fresh Embryos 36 38.9 3.0
Frozen Embryos 43

30.2

3.3

Average number of embryos transferred

\section{CURRENT CLINIC SERVICES AND PROFILE}

Current Name: USC Reproductive Endocrinology and Infertility

Donor egg? Yes Gestational carriers? Yes

Donor embryo? No Cryopreservation? Yes

Single women? Yes

SART member? Yes

Verified lab accreditation? Yes

(See Appendix C for details.)

\footnotetext{
${ }^{a}$ Reflects patient and treatment characteristics of ART cycles performed in 2006 using fresh nondonor eggs or embryos.

${ }^{b}$ When fewer than 20 cycles are reported in an age category, rates are shown as a fraction and confidence intervals are not given. Calculating percentages from fractions may be misleading and is not encouraged.

${ }^{c}$ A multiple-infant birth is counted as one live birth.

${ }^{d}$ Clinic-specific outcome rates are unreliable for women older than 42 undergoing ART cycles using fresh or frozen embryos with nondonor eggs. Readers are urged to review national outcomes for these age groups (see page 27).

e All ages (including ages $>42$ ) are reported together because previous data show that patient age does not materially affect success with donor eggs.
} 


\section{REPRODUCTIVE SPECIALTY MEDICAL CENTER NEWPORT BEACH, CALIFORNIA}

A comparison of clinic success rates may not be meaningful because patient medical characteristics and treatment approaches vary from clinic to clinic. For more details about this, along with information on how to interpret the statistics in this table, see pages 79-88.

\section{ART CYCLE PROFILE}

\section{Type of ART ${ }^{\mathrm{a}}$}

IVF

GIFT

100\% Procedural Factors:

ZIFT

Combination

\author{
$0 \%$ With ICSI
}

$0 \%$ Unstimulated

0\% Used gestational carrier

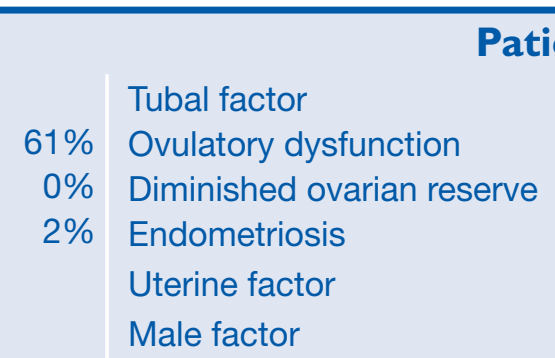

\section{Patient Diagnosis}

$9 \%$ Other factor

$0 \%$ Unknown factor

46\% Multiple Factors:

$<1 \%$ Female factors only $3 \%$

$0 \%$ Female \& male factors $13 \%$ $11 \%$

\section{PREGNANCY SUCCESS RATES}

Data verified by Beth A. Ary, MD

\section{Type of Cycle}

\section{Age of Woman}

\section{Fresh Embryos from Nondonor Eggs}

Number of cycles

Percentage of cycles resulting in pregnancies ${ }^{b}$

Percentage of cycles resulting in live births $s^{b, c}$

(Confidence Interval)

Percentage of retrievals resulting in live births $s^{b, c}$

Percentage of transfers resulting in live births $s^{b, c}$

Percentage of transfers resulting in singleton live births ${ }^{b}$

Percentage of cancellations ${ }^{b}$ 35-37

38-40

$41-42^{d}$

Average number of embryos transferred

Percentage of pregnancies with twins ${ }^{b}$ $<35$

8

18

3

Percentage of pregnancies with triplets or more ${ }^{b}$

Percentage of live births having multiple infants ${ }^{b, c}$

$\begin{array}{cccc}20 & 8 & 18 & 3 \\ 45.0 & 2 / 8 & 5 / 18 & 0 / 3 \\ 45.0 & 1 / 8 & 3 / 18 & 0 / 3\end{array}$

\section{Frozen Embryos from Nondonor Eggs}

Number of transfers

Percentage of transfers resulting in live births $s^{b, c}$

Average number of embryos transferred

$(23.1-68.5)$

$\begin{array}{cccc}9 / 19 & 1 / 8 & 3 / 16 & 0 / 3 \\ 9 / 18 & 1 / 6 & 3 / 14 & 0 / 3 \\ 4 / 18 & 1 / 6 & 3 / 14 & 0 / 3 \\ 5.0 & 0 / 8 & 2 / 18 & 0 / 3 \\ 2.9 & 2.7 & 3.8 & 3.0 \\ 5 / 9 & 0 / 2 & 0 / 5 & \\ 1 / 9 & 0 / 2 & 0 / 5 & \\ 5 / 9 & 0 / 1 & 0 / 3 & \end{array}$

\section{Donor Eggs}

$2 \quad 2$

$1 / 2$

$0 / 2$

1

2.5

3.0

$0 / 1$

1

3.0

$1 / 1$

1.0

Number of transfers

Percentage of transfers resulting in live births $s^{b, c}$

All Ages Combined ${ }^{\mathrm{e}}$

Fresh Embryos

\section{6}

69.4

2.9
Frozen Embryos 10

$1 / 10$

3.2

Average number of embryos transferred

\section{CURRENT CLINIC SERVICES AND PROFILE}

Current Name: Reproductive Specialty Medical Center

Donor egg? Yes

Donor embryo? Yes

Single women? Yes

\begin{abstract}
Gestational carriers? Yes
Cryopreservation? Yes
\end{abstract}

SART member?

Yes

Verified lab accreditation?

(See Appendix C for details.)

${ }^{a}$ Reflects patient and treatment characteristics of ART cycles performed in 2006 using fresh nondonor eggs or embryos.

${ }^{b}$ When fewer than 20 cycles are reported in an age category, rates are shown as a fraction and confidence intervals are not given. Calculating percentages from fractions may be misleading and is not encouraged.

${ }^{c}$ A multiple-infant birth is counted as one live birth.

d Clinic-specific outcome rates are unreliable for women older than 42 undergoing ART cycles using fresh or frozen embryos with nondonor eggs. Readers are urged to review national outcomes for these age groups (see page 27).

${ }^{e}$ All ages (including ages $>42$ ) are reported together because previous data show that patient age does not materially affect success with donor eggs. 


\section{SOUTHERN CALIFORNIA CENTER FOR REPRODUCTIVE MEDICINE NEWPORT BEACH, CALIFORNIA}

A comparison of clinic success rates may not be meaningful because patient medical characteristics and treatment approaches vary from clinic to clinic. For more details about this, along with information on how to interpret the statistics in this table, see pages 79-88.

\section{ART CYCLE PROFILE}

\section{Type of ART ${ }^{\mathrm{a}}$}

IVF

GIFT

$100 \%$ Procedural Factors:

ZIFT

Combination
$0 \%$ With ICSI

$0 \%$ Unstimulated

$0 \%$ Used gestational carrier

\section{$88 \%$}

$0 \%$

$1 \%$
Tubal factor

Ovulatory dysfunction

Diminished ovarian reserve

Endometriosis

Uterine factor

Male factor

\section{Patient Diagnosis}

$7 \%$ Other factor

2\% Unknown factor

26\% Multiple Factors:

$6 \%$ Female factors only $8 \%$

$<1 \%$ Female \& male factors $12 \%$

$17 \%$
$8 \%$

$14 \%$

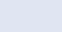

2006 PREGNANCY SUCCESS RATES

Data verified by Robert E. Anderson, MD

\section{Type of Cycle}

\section{Age of Woman}

35-37 38-40

$41-42^{d}$
69

30.4

26.1

(16.3-38.1)

28.1

30.5

27.1

7.2

3.5

19.0

0.0

$2 / 18$

33.3

$$
14
$$

35.0

$4 / 14$

3.0

3.1

$\begin{array}{cc}68 & 43 \\ 25.0 & 25.6 \\ 19.1 & 9.3\end{array}$

$(10.6-30.5)$

(2.6-22.1)

22.4

10.8

23.2

11.8

14.3

8.8

14.7

14.0

3.5

3.4

$5 / 17$

$1 / 11$

$0 / 17$

$0 / 11$

$5 / 13$

$1 / 4$

\section{Frozen Embryos from Nondonor Eggs}

Percentage of transfers resulting in live births ${ }^{b, c}$

Average number of embryos transferred

\section{Donor Eggs}

All Ages Combined ${ }^{\mathrm{e}}$

Number of transfers

Percentage of transfers resulting in live births $s^{b, c}$

Average number of embryos transferred

\section{Fresh Embryos}

43

53.5

2.8

\section{CURRENT CLINIC SERVICES AND PROFILE}

Current Name: Southern California Center for Reproductive Medicine

Donor egg? Yes Gestational carriers? Yes

Donor embryo? Yes Cryopreservation? Yes

Single women? Yes

SART member? Yes

Verified lab accreditation? Yes

(See Appendix C for details.)

${ }^{a}$ Reflects patient and treatment characteristics of ART cycles performed in 2006 using fresh nondonor eggs or embryos.

b When fewer than 20 cycles are reported in an age category, rates are shown as a fraction and confidence intervals are not given. Calculating percentages from fractions may be misleading and is not encouraged.

${ }^{c}$ A multiple-infant birth is counted as one live birth.

d Clinic-specific outcome rates are unreliable for women older than 42 undergoing ART cycles using fresh or frozen embryos with nondonor eggs. Readers are urged to review national outcomes for these age groups (see page 27).

e All ages (including ages $>42$ ) are reported together because previous data show that patient age does not materially affect success with donor eggs. 


\section{IVF-ORANGE SURGERY CENTER ORANGE, CALIFORNIA}

A comparison of clinic success rates may not be meaningful because patient medical characteristics and treatment approaches vary from clinic to clinic. For more details about this, along with information on how to interpret the statistics in this table, see pages 79-88.

\section{ART CYCLE PROFILE}

\section{Type of ART ${ }^{\mathrm{a}}$}

IVF

GIFT

100\% Procedural Factors:

ZIFT

Combination

\author{
$0 \%$ With ICSI
}

$0 \%$ Unstimulated

$0 \%$ Used gestational carrier

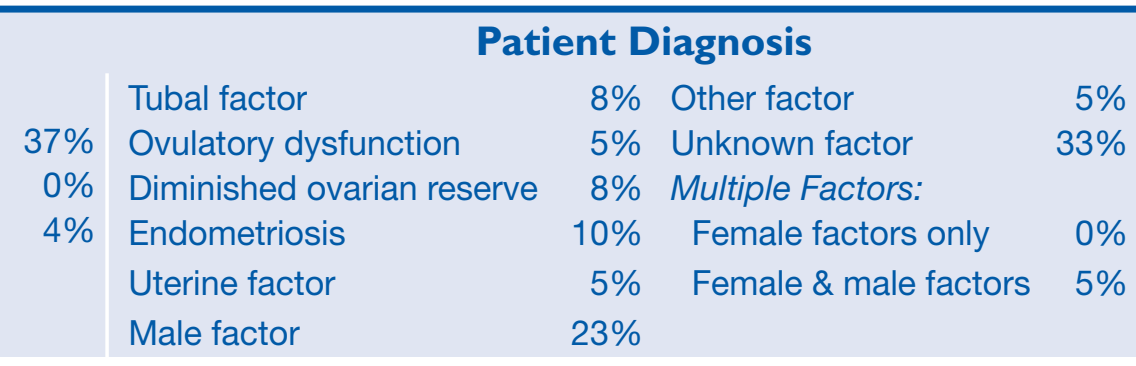

Data verified by Darush L. Mohyi, MD

2006 PREGNANCY SUCCESS RATES

\section{Age of Woman}

\section{Type of Cycle}

\section{Fresh Embryos from Nondonor Eggs}

Number of cycles

Percentage of cycles resulting in pregnancies ${ }^{b}$

Percentage of cycles resulting in live births $s^{b, c}$

(Confidence Interval)

Percentage of retrievals resulting in live births $s^{b, c}$

Percentage of transfers resulting in live births $s^{b, c}$

Percentage of transfers resulting in singleton live births ${ }^{b}$

Percentage of cancellations ${ }^{b}$

Average number of embryos transferred

Percentage of pregnancies with twins ${ }^{b}$

Percentage of pregnancies with triplets or more ${ }^{\mathrm{b}}$

Percentage of live births having multiple infants ${ }^{b, c}$

\section{Frozen Embryos from Nondonor Eggs}

Number of transfers

Percentage of transfers resulting in live births ${ }^{\mathrm{b}, \mathrm{c}}$

Average number of embryos transferred

\section{Donor Eggs}

Number of transfers

Percentage of transfers resulting in live births $s^{b, c}$

Average number of embryos transferred

$\begin{array}{ccc}3 / 13 & 1 / 9 & 1 / 5 \\ 3 / 13 & 1 / 9 & 1 / 4 \\ 3 / 13 & 1 / 9 & 1 / 4 \\ 0 / 13 & 0 / 9 & 0 / 5 \\ 3.7 & 4.6 & 4.0 \\ 0 / 3 & 0 / 2 & 0 / 1 \\ 0 / 3 & 0 / 2 & 0 / 1 \\ 0 / 3 & 0 / 1 & 0 / 1\end{array}$

3

$0 / 3$

4.0

\section{5}

$1 / 5$

6.0
$1 / 5$
$1 / 4$
$1 / 4$
$0 / 5$
4.0
$0 / 1$
$0 / 1$
$0 / 1$

Fresh Embryos

All Ages Combined ${ }^{\mathrm{e}}$

$\begin{array}{cccc}<35 & \mathbf{3 5 - 3 7} & \mathbf{3 8 - 4 0} & \mathbf{4 1 - 4 2}^{\mathbf{d}} \\ 13 & 9 & 5 & 0 \\ 3 / 13 & 2 / 9 & 1 / 5 & \\ 3 / 13 & 1 / 9 & 1 / 5 & \end{array}$

5
4
5
1
1

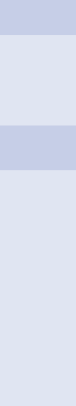

\section{CURRENT CLINIC SERVICES AND PROFILE}

Current Name: IVF-Orange Surgery Center
Donor egg? Yes
Donor embryo? No
Gestational carriers? Yes
Cryopreservation? Yes
Single women? Yes

\section{SART member? \\ (See Appendix C for details.)}
No
Verified lab accreditation?
No

\footnotetext{
${ }^{a}$ Reflects patient and treatment characteristics of ART cycles performed in 2006 using fresh nondonor eggs or embryos.

${ }^{b}$ When fewer than 20 cycles are reported in an age category, rates are shown as a fraction and confidence intervals are not given. Calculating percentages from fractions may be misleading and is not encouraged.

${ }^{c}$ A multiple-infant birth is counted as one live birth.

d Clinic-specific outcome rates are unreliable for women older than 42 undergoing ART cycles using fresh or frozen embryos with nondonor eggs. Readers are urged to review national outcomes for these age groups (see page 27).

${ }^{e}$ All ages (including ages $>42$ ) are reported together because previous data show that patient age does not materially affect success with donor eggs.
} 


\section{NOVA IN VITRO FERTILIZATION \\ PALO ALTO, CALIFORNIA}

A comparison of clinic success rates may not be meaningful because patient medical characteristics and treatment approaches vary from clinic to clinic. For more details about this, along with information on how to interpret the statistics in this table, see pages 79-88.

\section{ART CYCLE PROFILE}

\section{Type of ART ${ }^{\mathrm{a}}$}

IVF

GIFT

$100 \%$ Procedural Factors:

ZIFT

Combination
$0 \%$ With ICSI

$0 \%$ Unstimulated

$0 \%$ Used gestational carrier

\section{$49 \%$ \\ $0 \%$ \\ $0 \%$}

Tubal factor

Ovulatory dysfunction

Diminished ovarian reserve

Endometriosis

Uterine factor

Male factor

\section{Patient Diagnosis}

$14 \%$ Other factor

4\% Unknown factor

26\% Multiple Factors:

$2 \%$ Female factors only $6 \%$

$2 \%$ Female \& male factors $8 \%$ $14 \%$

2006 PREGNANCY SUCCESS RATES

Data verified by Richard J. Schmidt, MD

\section{Type of Cycle}

\section{Fresh Embryos from Nondonor Eggs}

Number of cycles

Percentage of cycles resulting in pregnancies ${ }^{b}$

Percentage of cycles resulting in live births ${ }^{b, c}$

(Confidence Interval)

Percentage of retrievals resulting in live births ${ }^{b, c}$

Percentage of transfers resulting in live births $s^{b, c}$

Percentage of transfers resulting in singleton live births ${ }^{b}$

Percentage of cancellations ${ }^{b}$

Average number of embryos transferred

Percentage of pregnancies with twins ${ }^{b}$

Percentage of pregnancies with triplets or more ${ }^{b}$

Percentage of live births having multiple infants ${ }^{b, c}$

\section{Frozen Embryos from Nondonor Eggs}

Number of transfers

Percentage of transfers resulting in live births $s^{b, c}$

Average number of embryos transferred

\section{Donor Eggs}

Number of transfers

Percentage of transfers resulting in live births $s^{b, c}$

Average number of embryos transferred

\section{Age of Woman}

35-37 38-40

$41-42^{d}$ $<35$

27

40.7

35

20

41.7

25.9

28.6

25.0

38.9

(11.1-46.3)

22.9

15.0

$(23.1-56.5)$

40.0

28.0

$(10.4-40.1)$

(3.2-37.9)

42.4

28.0

22.9

22.9

$3 / 18$

18.2

20.0

14.3

$3 / 17$

2.8

7.4

0.0

$3 / 17$

2.5

2.8

3.0

10.0

$7 / 15$

$3 / 11$

$1 / 15$

$1 / 11$

$3 / 10$

4.2

$8 / 14$

$2 / 7$

$1 / 10$

$0 / 5$

$0 / 5$

$3 / 8$

$0 / 3$

$\begin{array}{cccc}13 & 13 & 11 & 4 \\ 5 / 13 & 3 / 13 & 3 / 11 & 0 / 4 \\ 2.5 & 2.5 & 2.5 & 6.8\end{array}$

\section{CURRENT CLINIC SERVICES AND PROFILE}

All Ages Combined ${ }^{\mathrm{e}}$

Fresh Embryos 19

$11 / 19$

2.9
Frozen Embryos 18

$3 / 18$

1.7

Current Name: NOVA In Vitro Fertilization

Donor egg? Yes

Donor embryo? No

Single women? Yes

\section{Gestational carriers? Yes}

Cryopreservation? Yes
SART member?

Yes

Verified lab accreditation?

(See Appendix C for details.)

${ }^{a}$ Reflects patient and treatment characteristics of ART cycles performed in 2006 using fresh nondonor eggs or embryos.

b When fewer than 20 cycles are reported in an age category, rates are shown as a fraction and confidence intervals are not given. Calculating percentages from fractions may be misleading and is not encouraged.

${ }^{c}$ A multiple-infant birth is counted as one live birth.

d Clinic-specific outcome rates are unreliable for women older than 42 undergoing ART cycles using fresh or frozen embryos with nondonor eggs. Readers are urged to review national outcomes for these age groups (see page 27).

e All ages (including ages $>42$ ) are reported together because previous data show that patient age does not materially affect success with donor eggs. 


\section{STANFORD UNIVERSITY IVF/ART PROGRAM DEPARTMENT OF GYNECOLOGY AND OBSTETRICS PALO ALTO, CALIFORNIA}

A comparison of clinic success rates may not be meaningful because patient medical characteristics and treatment approaches vary from clinic to clinic. For more details about this, along with information on how to interpret the statistics in this table, see pages 79-88.

\section{ART CYCLE PROFILE}

\section{Type of ART ${ }^{\mathrm{a}}$}

IVF

GIFT

100\% Procedural Factors:

ZIFT

Combination

\author{
$0 \%$ With ICSI
}

$0 \%$ Unstimulated

$0 \%$ Used gestational carrier $<1 \%$

\section{Patient Diagnosis}

3\% Other factor

3\% Unknown factor

24\% Multiple Factors:

$5 \%$ Female factors only $\quad 1 \%$

$<1 \%$ Female \& male factors $38 \%$ $9 \%$

$11 \%$

$6 \%$

2006 PREGNANCY SUCCESS RATES

Data verified by Valerie Baker, MD

\section{Type of Cycle}

Uterine factor

Male factor

of Woman

\section{Fresh Embryos from Nondonor Eggs}

Number of cycles

Percentage of cycles resulting in pregnancies ${ }^{b}$

Percentage of cycles resulting in live births ${ }^{b, c}$

(Confidence Interval)

Percentage of retrievals resulting in live births $s^{b, c}$

Percentage of transfers resulting in live births $s^{b, c}$

Percentage of transfers resulting in singleton live births ${ }^{b}$

Percentage of cancellations ${ }^{b}$

Average number of embryos transferred

Percentage of pregnancies with twins ${ }^{b}$

Percentage of pregnancies with triplets or more ${ }^{b}$

Percentage of live births having multiple infants ${ }^{b, c}$

\section{Frozen Embryos from Nondonor Eggs}

Number of transfers

Percentage of transfers resulting in live births $s^{\mathrm{b}, \mathrm{c}}$

Average number of embryos transferred

83

28.9

2.0

\section{Age of Woman}

35-37

38-40

$41-42^{d}$

$\begin{array}{llll}205 & 172 & 186 & 138 \\ 32.7 & 30.8 & 22.0 & 20.3 \\ 26.8 & 21.5 & 15.1 & 10.9\end{array}$

(20.9-33.4)

(15.6-28.4)

$(10.2-21.0)$

(6.2-17.3)

29.6

22.8

16.3

17.0

13.9

12.9

24.0

17.5

7.5

3.0

22.0

4.9

17.9

27.0

2.4

20.8

1.9

40

57

15.8

2.0

30.0

1.7

$3 / 16$

2.2

\section{Donor Eggs}

Number of transfers

Percentage of transfers resulting in live births $s^{b, c}$

Average number of embryos transferred
All Ages Combined ${ }^{\mathrm{e}}$

\section{Fresh Embryos}

Frozen Embryos

$\begin{array}{cc}57 & 49 \\ 45.6 & 16.3 \\ 2.1 & 2.0\end{array}$

\section{CURRENT CLINIC SERVICES AND PROFILE}

Current Name: Stanford Fertility and Reproductive Medicine Center, Stanford University Department of Gynecology and Obstetrics
Donor egg? Yes
Gestational carriers? Yes
Donor embryo? Yes
Cryopreservation? Yes
SART member?
Yes
Verified lab accreditation?
Yes
Single women? Yes
(See Appendix C for details.)
${ }^{a}$ Reflects patient and treatment characteristics of ART cycles performed in 2006 using fresh nondonor eggs or embryos.
${ }^{b}$ When fewer than 20 cycles are reported in an age category, rates are shown as a fraction and confidence intervals are not given. Calculating percentages from fractions may be misleading and is not encouraged.
${ }^{\mathrm{C}}$ A multiple-infant birth is counted as one live birth.
d Clinic-specific outcome rates are unreliable for women older than 42 undergoing ART cycles using fresh or frozen embryos with nondonor eggs. Readers are urged to review national outcomes for these age groups (see page 27).
e All ages (including ages $>42$ ) are reported together because previous data show that patient age does not materially affect success with donor eggs. 


\section{HUNTINGTON REPRODUCTIVE CENTER \\ PASADENA, CALIFORNIA}

A comparison of clinic success rates may not be meaningful because patient medical characteristics and treatment approaches vary from clinic to clinic. For more details about this, along with information on how to interpret the statistics in this table, see pages 79-88.

\section{ART CYCLE PROFILE}

\begin{tabular}{|c|c|c|c|c|c|c|}
\hline \multicolumn{3}{|c|}{ Type of ART ${ }^{a}$} & \multicolumn{4}{|c|}{ Patient Diagnosis } \\
\hline IVF & $>99 \%$ Procedural Factors: & & Tubal factor & $6 \%$ & Other factor & $19 \%$ \\
\hline GIFT & $<1 \%$ With ICSI & $76 \%$ & Ovulatory dysfunction & $3 \%$ & Unknown factor & $12 \%$ \\
\hline ZIFT & $<1 \%$ Unstimulated & $<1 \%$ & Diminished ovarian reserve & $16 \%$ & Multiple Factors: & \\
\hline \multirow[t]{3}{*}{ Combination } & $<1 \%$ Used gestational carrier & $3 \%$ & Endometriosis & $2 \%$ & Female factors only & $9 \%$ \\
\hline & & & Uterine factor & $2 \%$ & Female \& male factors & $13 \%$ \\
\hline & & & Male factor & $19 \%$ & & \\
\hline
\end{tabular}

2006 PREGNANCY SUCCESS RATES

Data verified by Daniel A. Potter, MD

\section{Type of Cycle}

\section{Fresh Embryos from Nondonor Eggs}

Number of cycles

Percentage of cycles resulting in pregnancies ${ }^{b}$

Percentage of cycles resulting in live births ${ }^{b, c}$

(Confidence Interval)

Percentage of retrievals resulting in live births ${ }^{b, c}$

Percentage of transfers resulting in live births $s^{b, c}$

Percentage of transfers resulting in singleton live births ${ }^{b}$

Percentage of cancellations ${ }^{b}$

Average number of embryos transferred

Percentage of pregnancies with twins ${ }^{b}$

Percentage of pregnancies with triplets or more ${ }^{b}$

Percentage of live births having multiple infants ${ }^{\mathrm{b}, \mathrm{c}}$

\section{Frozen Embryos from Nondonor Eggs}

Number of transfers

Percentage of transfers resulting in live births ${ }^{b, c}$

Average number of embryos transferred

\section{Donor Eggs}

Number of transfers

Percentage of transfers resulting in live births $s^{b, c}$

Average number of embryos transferred

\section{Age of Woman}

35-37 38-40

$41-42^{d}$

$<35$

379

31.1

402

153

35.5

25.1

25.6

17.6

30.3

(20.8-29.7)

19.2

11.1

(26.4-34.4)

31.5

27.5

$(15.4-23.3)$

(6.6-17.2)

32.9

29.4

20.2

21.4

17.8

12.6

19.8

3.9

8.7

3.1

28.8

22.2

6.3

31.1

1.7

32.6

132

34.8

2.7
69

24.6

3.0
5.2

3.1

16.5

1.9

16.9

41

17.1

2.8
13.6

12.8

11.8

3.4

0.0

3.7

$1 / 17$
14

2 / 14

3.1

All Ages Combined ${ }^{\mathrm{e}}$

Fresh Embryos

218

46.3

2.6

\section{CURRENT CLINIC SERVICES AND PROFILE}

Current Name: Huntington Reproductive Center

Donor egg? Yes Gestational carriers? Yes

Donor embryo? Yes

Cryopreservation? Yes

Frozen Embryos

Single women? Yes

SART member?

Verified lab accreditation?

(See Appendix C for details.)

${ }^{a}$ Reflects patient and treatment characteristics of ART cycles performed in 2006 using fresh nondonor eggs or embryos.

b When fewer than 20 cycles are reported in an age category, rates are shown as a fraction and confidence intervals are not given. Calculating percentages from fractions may be misleading and is not encouraged.

${ }^{c}$ A multiple-infant birth is counted as one live birth.

d Clinic-specific outcome rates are unreliable for women older than 42 undergoing ART cycles using fresh or frozen embryos with nondonor eggs. Readers are urged to review national outcomes for these age groups (see page 27).

e All ages (including ages $>42$ ) are reported together because previous data show that patient age does not materially affect success with donor eggs. 


\section{PALO ALTO MEDICAL FOUNDATION \\ REPRODUCTIVE ENDOCRINOLOGY \& FERTILITY \\ PORTOLA VALLEY, CALIFORNIA}

A comparison of clinic success rates may not be meaningful because patient medical characteristics and treatment approaches vary from clinic to clinic. For more details about this, along with information on how to interpret the statistics in this table, see pages 79-88.

\section{ART CYCLE PROFILE}

$$
\text { Type of ART }{ }^{\mathrm{a}}
$$

\begin{tabular}{ll|llllr} 
IVF & $100 \%$ Procedural Factors: & & Tubal factor & $11 \%$ & Other factor & $5 \%$ \\
GIFT & $0 \%$ With ICSI & $45 \%$ & Ovulatory dysfunction & $11 \%$ & Unknown factor & $47 \%$ \\
ZIFT & $0 \%$ Unstimulated & $0 \%$ & Diminished ovarian reserve & $0 \%$ & Multiple Factors: & \\
Combination & $0 \%$ Used gestational carrier & $0 \%$ & Endometriosis & $0 \%$ & Female factors only & $3 \%$ \\
& & & Uterine factor & $5 \%$ & Female \& male factors & $5 \%$ \\
& & & Male factor & $13 \%$ & &
\end{tabular}

2006 PREGNANCY SUCCESS RATES

Data verified by Lillian M. Swiersz, MD

\section{Type of Cycle}

\section{Fresh Embryos from Nondonor Eggs}

Number of cycles

Percentage of cycles resulting in pregnancies ${ }^{b}$

Percentage of cycles resulting in live births ${ }^{b, c}$

(Confidence Interval)

Percentage of retrievals resulting in live births $s^{b, c}$

Percentage of transfers resulting in live births $s^{b, c}$

Percentage of transfers resulting in singleton live births ${ }^{b}$

Percentage of cancellations ${ }^{b}$

Average number of embryos transferred

Percentage of pregnancies with twins ${ }^{b}$

Percentage of pregnancies with triplets or more ${ }^{b}$

Percentage of live births having multiple infants ${ }^{b, c}$

\section{Frozen Embryos from Nondonor Eggs}

Number of transfers

Percentage of transfers resulting in live births ${ }^{\mathrm{b}, \mathrm{c}}$

Average number of embryos transferred

\section{Donor Eggs}

Number of transfers

Percentage of transfers resulting in live births ${ }^{\mathrm{b}, \mathrm{c}}$

Average number of embryos transferred

\section{Age of Woman}

$\begin{array}{cccc}<35 & 35-37 & 38-40 & \mathbf{4 1 - 4 2}^{\text {d }} \\ 7 & 12 & 8 & 4 \\ 3 / 7 & 2 / 12 & 0 / 8 & 0 / 4 \\ 2 / 7 & 2 / 12 & 0 / 8 & 0 / 4\end{array}$

$2 / 7$

$2 / 11$

$0 / 6$

$0 / 3$

$2 / 7$

$2 / 11$

$0 / 5$

$0 / 3$

$2 / 7$

$1 / 11$

$0 / 5$

$0 / 3$

$0 / 7$

$1 / 12$

$2 / 8$

$1 / 4$

2.1

2.6

3.0

2.7

$1 / 2$

$0 / 3$

$0 / 2$

$0 / 2$

$1 / 2$

$\begin{array}{cccc}4 & 1 & 1 & 1 \\ 2 / 4 & 0 / 1 & 0 / 1 & 0 / 1 \\ 2.3 & 2.0 & 2.0 & 1.0\end{array}$

All Ages Combined

Fresh Embryos

0
Frozen Embryos

0

\section{CURRENT CLINIC SERVICES AND PROFILE}

Current Name: Palo Alto Medical Foundation, Reproductive Endocrinology \& Fertility

Donor egg? No

Donor embryo? No

Gestational carriers? No

SART member?

Yes

Single women? Yes

Cryopreservation? Yes

Verified lab accreditation?

Yes

(See Appendix C for details.)

${ }^{a}$ Reflects patient and treatment characteristics of ART cycles performed in 2006 using fresh nondonor eggs or embryos.

${ }^{b}$ When fewer than 20 cycles are reported in an age category, rates are shown as a fraction and confidence intervals are not given. Calculating percentages from fractions may be misleading and is not encouraged.

${ }^{c}$ A multiple-infant birth is counted as one live birth.

d Clinic-specific outcome rates are unreliable for women older than 42 undergoing ART cycles using fresh or frozen embryos with nondonor eggs. Readers are urged to review national outcomes for these age groups (see page 27).

e All ages (including ages $>42$ ) are reported together because previous data show that patient age does not materially affect success with donor eggs. 


\section{REPRODUCTIVE PARTNERS-REDONDO BEACH REDONDO BEACH, CALIFORNIA}

A comparison of clinic success rates may not be meaningful because patient medical characteristics and treatment approaches vary from clinic to clinic. For more details about this, along with information on how to interpret the statistics in this table, see pages 79-88.

\section{ART CYCLE PROFILE}

\begin{tabular}{|c|c|c|c|c|c|c|c|}
\hline \multicolumn{4}{|c|}{ Type of ART ${ }^{a}$} & \multicolumn{4}{|c|}{ Patient Diagnosis } \\
\hline IVF & $100 \%$ & Procedural Factors: & & Tubal factor & $10 \%$ & Other factor & $8 \%$ \\
\hline GIFT & $0 \%$ & With ICSI & $54 \%$ & Ovulatory dysfunction & $4 \%$ & Unknown factor & $24 \%$ \\
\hline ZIFT & $0 \%$ & Unstimulated & $0 \%$ & Diminished ovarian reserve & $11 \%$ & Multiple Factors: & \\
\hline \multirow[t]{3}{*}{ Combination } & $0 \%$ & Used gestational carrier & $2 \%$ & Endometriosis & $4 \%$ & Female factors only & $7 \%$ \\
\hline & & & & Uterine factor & $2 \%$ & Female \& male factors & $11 \%$ \\
\hline & & & & Male factor & $18 \%$ & & \\
\hline
\end{tabular}

2006 PREGNANCY SUCCESS RATES

Data verified by Bill Yee, MD

\section{Type of Cycle}

\section{Fresh Embryos from Nondonor Eggs}

Number of cycles

Percentage of cycles resulting in pregnancies ${ }^{b}$

Percentage of cycles resulting in live births ${ }^{b, c}$

(Confidence Interval)

Percentage of retrievals resulting in live births ${ }^{b, c}$

Percentage of transfers resulting in live births $s^{b, c}$

Percentage of transfers resulting in singleton live births ${ }^{b}$

Percentage of cancellations ${ }^{b}$

Average number of embryos transferred

Percentage of pregnancies with twins ${ }^{b}$

Percentage of pregnancies with triplets or more ${ }^{b}$

Percentage of live births having multiple infants ${ }^{b, c}$

\section{Frozen Embryos from Nondonor Eggs}

Number of transfers

Percentage of transfers resulting in live births ${ }^{b, c}$

Average number of embryos transferred

\section{Donor Eggs}

Number of transfers

Percentage of transfers resulting in live births $s^{b, c}$

Average number of embryos transferred

\section{Age of Woman}

35-37 $38-40 \quad 41-42^{d}$

$<35$

65

78

40

62
62.9

50.8

32.1

17.5

59.7

50.8

25.6

5.0

$(46.4-71.9)$

(38.1-63.4)

(16.4-36.8)

(0.6-16.9)

62.7

62.7

55.0

29.0

29.9

6.7

39.0

56.9

25.4

7.1

41.4

11.5

7.1

4.8

7.7

2.5

25.0

2.0

48.7

2.3

36.4

20.0

3.0

0.0

0.0

8.0

$0 / 7$

37.8

27.3

15.0

$0 / 7$

$0 / 2$

$\begin{array}{cccc}12 & 21 & 13 & 5 \\ 4 / 12 & 52.4 & 3 / 13 & 1 / 5 \\ 2.1 & 2.0 & 1.8 & 2.4\end{array}$

\section{All Ages Combined}

Fresh Embryos

Frozen Embryos

$\begin{array}{cc}26 & 13 \\ 69.2 & 5 / 13 \\ 2.0 & 2.0\end{array}$

\section{CURRENT CLINIC SERVICES AND PROFILE}

Current Name: Reproductive Partners-Redondo Beach

Donor egg? Yes Gestational carriers? Yes

Donor embryo? Yes Cryopreservation? Yes

Single women? Yes

SART member? Yes

Verified lab accreditation? Yes

(See Appendix C for details.)

${ }^{a}$ Reflects patient and treatment characteristics of ART cycles performed in 2006 using fresh nondonor eggs or embryos.

b When fewer than 20 cycles are reported in an age category, rates are shown as a fraction and confidence intervals are not given. Calculating percentages from fractions may be misleading and is not encouraged.

${ }^{c}$ A multiple-infant birth is counted as one live birth.

d Clinic-specific outcome rates are unreliable for women older than 42 undergoing ART cycles using fresh or frozen embryos with nondonor eggs. Readers are urged to review national outcomes for these age groups (see page 27).

e All ages (including ages $>42$ ) are reported together because previous data show that patient age does not materially affect success with donor eggs. 


\title{
NORTHERN CALIFORNIA FERTILITY MEDICAL CENTER ROSEVILLE, CALIFORNIA
}

A comparison of clinic success rates may not be meaningful because patient medical characteristics and treatment approaches vary from clinic to clinic. For more details about this, along with information on how to interpret the statistics in this table, see pages 79-88.

\section{ART CYCLE PROFILE}

$$
\text { Type of ART }{ }^{\mathrm{a}}
$$

\begin{tabular}{lll|llll} 
IVF & $100 \%$ Procedural Factors: & & Tubal factor & $11 \%$ & Other factor & $8 \%$ \\
GIFT & $0 \%$ With ICSI & $57 \%$ & Ovulatory dysfunction & $7 \%$ & Unknown factor & $3 \%$ \\
ZIFT & $0 \%$ Unstimulated & $0 \%$ & Diminished ovarian reserve & $12 \%$ & Multiple Factors: & \\
Combination & $0 \%$ Used gestational carrier & $2 \%$ & Endometriosis & $5 \%$ & Female factors only & $10 \%$ \\
& & & Uterine factor & $3 \%$ & Female \& male factors & $14 \%$ \\
& & & Male factor & $28 \%$ & &
\end{tabular}

2006 PREGNANCY SUCCESS RATES

Data verified by John L. Gililland, MD

\section{Type of Cycle}

\section{Fresh Embryos from Nondonor Eggs}

Number of cycles

Percentage of cycles resulting in pregnancies ${ }^{b}$

Percentage of cycles resulting in live births ${ }^{b, c}$

(Confidence Interval)

Percentage of retrievals resulting in live births $s^{b, c}$

Percentage of transfers resulting in live births $s^{b, c}$

Percentage of transfers resulting in singleton live births ${ }^{b}$

Percentage of cancellations ${ }^{b}$

Average number of embryos transferred

Percentage of pregnancies with twins ${ }^{b}$

Percentage of pregnancies with triplets or more ${ }^{b}$

Percentage of live births having multiple infants ${ }^{b, c}$

\section{Frozen Embryos from Nondonor Eggs}

Number of transfers

Percentage of transfers resulting in live births ${ }^{\mathrm{b}, \mathrm{c}}$

Average number of embryos transferred

\section{Donor Eggs}

Number of transfers

Percentage of transfers resulting in live births $s^{b, c}$

Average number of embryos transferred

\section{Age of Woman}

35-37 38-40

$41-42^{d}$

$<35$

97

42.3

36.1

(31.3-45.5)

40.6

(26.6-46.5)

38.0

39.3

25.8

5.2

2.8

24.4

7.3

34.3

6.2

41.1

34.3

69

27.5

2.4

\begin{abstract}
45
\end{abstract}
17.8

2.5

98
41.8
30.6

18.9

10.8
(21.7-40.7)
31.9

(3.0-25.4)

32.3

11.4

12.1

24.7

9.1

4.1

5.4

3.0

3.4

22.0

$1 / 7$

2.4

$0 / 7$

23.3

$1 / 4$

All Ages Combined ${ }^{\mathrm{e}}$

Fresh Embryos 80

56.3

2.3
10

$1 / 10$

2.3

\section{CURRENT CLINIC SERVICES AND PROFILE}

Current Name: Northern California Fertility Medical Center

Donor egg? Yes Gestational carriers? Yes

Donor embryo? Yes Cryopreservation? Yes

Single women? Yes

SART member? Yes

Verified lab accreditation? Yes

(See Appendix C for details.)

${ }^{a}$ Reflects patient and treatment characteristics of ART cycles performed in 2006 using fresh nondonor eggs or embryos.

${ }^{b}$ When fewer than 20 cycles are reported in an age category, rates are shown as a fraction and confidence intervals are not given. Calculating percentages from fractions may be misleading and is not encouraged.

${ }^{c}$ A multiple-infant birth is counted as one live birth.

d Clinic-specific outcome rates are unreliable for women older than 42 undergoing ART cycles using fresh or frozen embryos with nondonor eggs. Readers are urged to review national outcomes for these age groups (see page 27).

${ }^{e}$ All ages (including ages $>42$ ) are reported together because previous data show that patient age does not materially affect success with donor eggs. 


\section{KAISER PERMANENTE CENTER FOR REPRODUCTIVE HEALTH-SACRAMENTO SACRAMENTO, CALIFORNIA}

A comparison of clinic success rates may not be meaningful because patient medical characteristics and treatment approaches vary from clinic to clinic. For more details about this, along with information on how to interpret the statistics in this table, see pages 79-88.

\section{ART CYCLE PROFILE}

\section{Type of ART ${ }^{\mathrm{a}}$}

IVF

GIFT 100\% Procedural Factors:

ZIFT

Combination

\author{
$0 \%$ With ICSI
}

$0 \%$ Used gestational carrier
$0 \%$ Unstimulated

\begin{tabular}{r|lrll}
\multicolumn{4}{c}{ Patient Diagnosis } \\
\hline $57 \%$ & Tubal factor & $15 \%$ & Other factor & $5 \%$ \\
$0 \%$ & Diminished ovarian reserve & $10 \%$ & Unknown factor & $5 \%$ \\
$0 \%$ & Endometriosis & $1 \%$ & Multiple Factors: & \\
& Uterine factor & $2 \%$ & Female factors only & $12 \%$ \\
& Male factor & $26 \%$ & & \\
\end{tabular}

Data verified by Kenneth Vu, MD

\section{PREGNANCY SUCCESS RATES}

\section{Type of Cycle}

\section{Age of Woman}

35-37

38-40

$41-42^{d}$

\section{$<35$}

32

43.8

11

3

Number of cycles

Percentage of cycles resulting in pregnancies ${ }^{b}$

Percentage of cycles resulting in live births ${ }^{b, c}$

(Confidence Interval)

Percentage of retrievals resulting in live births $s^{b, c}$

Percentage of transfers resulting in live births $s^{b, c}$

Percentage of transfers resulting in singleton live births ${ }^{b}$

Percentage of cancellations ${ }^{b}$

Average number of embryos transferred

Percentage of pregnancies with twins ${ }^{b}$

Percentage of pregnancies with triplets or more ${ }^{b}$

Percentage of live births having multiple infants ${ }^{b, c}$

Frozen Embryos from Nondonor Eggs

Number of transfers

Percentage of transfers resulting in live births ${ }^{b, c}$

Average number of embryos transferred

\section{Donor Eggs}

Number of transfers

Percentage of transfers resulting in live births $s^{b, c}$

Average number of embryos transferred

$\begin{array}{cccc}36 & 32 & 11 & 3 \\ 41.7 & 43.8 & 4 / 11 & 1 / 3 \\ 36.1 & 34.4 & 3 / 11 & 1 / 3 \\ 0.8-53.8) & (18.6-53.2) & & \\ 40.6 & 37.9 & 3 / 9 & 1 / 3 \\ 40.6 & 37.9 & 3 / 9 & 1 / 3 \\ 25.0 & 20.7 & 2 / 9 & 1 / 3 \\ 11.1 & 9.4 & 2 / 11 & 0 / 3 \\ 2.5 & 2.9 & 3.1 & 3.7 \\ 6 / 15 & 5 / 14 & 1 / 4 & 0 / 1 \\ 1 / 15 & 1 / 14 & 0 / 4 & 0 / 1 \\ 5 / 13 & 5 / 11 & 1 / 3 & 0 / 1\end{array}$

\section{CURRENT CLINIC SERVICES AND PROFILE}

Current Name: Kaiser Permanente Center for Reproductive Health-Sacramento

\begin{tabular}{ll|ll|lll} 
Donor egg? & Yes & Gestational carriers? & Yes & SART member? & Yes \\
\hline Donor embryo? & Yes & Cryopreservation? & Yes & Verified lab accreditation? & Pending \\
Single women? & Yes & & & (See Appendix C for details.)
\end{tabular}

${ }^{a}$ Reflects patient and treatment characteristics of ART cycles performed in 2006 using fresh nondonor eggs or embryos.

b When fewer than 20 cycles are reported in an age category, rates are shown as a fraction and confidence intervals are not given. Calculating percentages from fractions may be misleading and is not encouraged.

${ }_{\text {C }}^{c}$ A multiple-infant birth is counted as one live birth.

${ }^{d}$ Clinic-specific outcome rates are unreliable for women older than 42 undergoing ART cycles using fresh or frozen embryos with nondonor eggs. Readers are urged to review national outcomes for these age groups (see page 27 ).

e All ages (including ages $>42$ ) are reported together because previous data show that patient age does not materially affect success with donor eggs. 


\section{THE UNIVERSITY OF CALIFORNIA-DAVIS ASSISTED REPRODUCTIVE TECHNOLOGY PROGRAM SACRAMENTO, CALIFORNIA}

A comparison of clinic success rates may not be meaningful because patient medical characteristics and treatment approaches vary from clinic to clinic. For more details about this, along with information on how to interpret the statistics in this table, see pages 79-88.

\section{ART CYCLE PROFILE}

\section{Type of ART ${ }^{\mathrm{a}}$}

IVF

GIFT

ZIFT

Combination

\author{
100\% Procedural Factors:
}

$0 \%$ With ICSI

$0 \%$ Unstimulated

$0 \%$ Used gestational carrier

\section{$54 \%$ \\ $0 \%$ \\ $0 \%$}

\section{Patient Diagnosis}

Tubal factor

$11 \%$ Other factor

$0 \%$ Unknown factor

Ovulatory dysfunction

14\% Multiple Factors:

3\% Female factors only $\quad 8 \%$

$0 \%$ Female \& male factors $17 \%$

Uterine factor $33 \%$
$0 \%$

$15 \%$

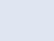

2006 PREGNANCY SUCCESS RATES

Data verified by Albert K. Wei, MD

\section{Type of Cycle}

\section{Fresh Embryos from Nondonor Eggs}

Number of cycles

Percentage of cycles resulting in pregnancies ${ }^{b}$

Percentage of cycles resulting in live births ${ }^{b, c}$

(Confidence Interval)

Percentage of retrievals resulting in live births $s^{b, c}$

Percentage of transfers resulting in live births $s^{b, c}$

Percentage of transfers resulting in singleton live births ${ }^{b}$

Percentage of cancellations ${ }^{b}$

Average number of embryos transferred

Percentage of pregnancies with twins ${ }^{b}$

Percentage of pregnancies with triplets or more ${ }^{b}$

Percentage of live births having multiple infants ${ }^{b, c}$

\section{Frozen Embryos from Nondonor Eggs}

Number of transfers

Percentage of transfers resulting in live births $s^{b, c}$

Average number of embryos transferred

\section{Donor Eggs}

Number of transfers

Percentage of transfers resulting in live births ${ }^{\mathrm{b}, \mathrm{c}}$

Average number of embryos transferred

\section{Age of Woman}

35-37 38-40

$41-42^{d}$

\section{$<35$}

13

$6 / 13$

$5 / 13$

7

4

$6 / 19$

$5 / 19$

$2 / 7$

$1 / 4$

$5 / 17$

$5 / 14$

$5 / 13$

$0 / 7$

$1 / 4$

$5 / 11$

$0 / 5$

$1 / 4$

$0 / 5$

$1 / 3$

$4 / 14$

$1 / 11$

$2 / 19$

$0 / 13$

$0 / 5$

$1 / 3$

3.1

$1 / 6$

3.4

$1 / 6$

$1 / 5$

$3 / 6$

$1 / 6$

$2 / 7$

$0 / 4$

4.2

4.3

$1 / 2$

$0 / 1$

$0 / 2$

$0 / 1$

$4 / 5$

$0 / 1$

\section{9}

$0 / 9$

3.1

$1 / 1$

2.0

\section{2 \\ $0 / 2$}

6.5

\section{CURRENT CLINIC SERVICES AND PROFILE}

Current Name: The University of California-Davis, Assisted Reproductive Technology Program
Donor egg?
Yes
Gestational carriers? No
Donor embryo? No
Cryopreservation? Yes
Single women? Yes

$\begin{array}{ll}\text { SART member? } & \text { Yes } \\ \text { Verified lab accreditation? } & \text { Yes } \\ \text { (See Appendix C for details.) } & \end{array}$

Fresh Embryos

All Ages Combined ${ }^{\mathrm{e}}$

5

$1 / 5$

Frozen Embryos

3.4

3

$0 / 3$

4.3

${ }^{a}$ Reflects patient and treatment characteristics of ART cycles performed in 2006 using fresh nondonor eggs or embryos.

b When fewer than 20 cycles are reported in an age category, rates are shown as a fraction and confidence intervals are not given. Calculating percentages from fractions may be misleading and is not encouraged.

${ }^{c}$ A multiple-infant birth is counted as one live birth.

${ }^{d}$ Clinic-specific outcome rates are unreliable for women older than 42 undergoing ART cycles using fresh or frozen embryos with nondonor eggs. Readers are urged to review national outcomes for these age groups (see page 27).

e All ages (including ages $>42$ ) are reported together because previous data show that patient age does not materially affect success with donor eggs. 


\section{THE FERTILITY AND GYNECOLOGY CENTER \\ MONTEREY BAY IVF PROGRAM \\ SALINAS, CALIFORNIA}

A comparison of clinic success rates may not be meaningful because patient medical characteristics and treatment approaches vary from clinic to clinic. For more details about this, along with information on how to interpret the statistics in this table, see pages 79-88.

\section{ART CYCLE PROFILE}

\section{Type of ART ${ }^{\mathrm{a}}$}

IVF

GIFT

100\% Procedural Factors:

ZIFT

Combination
$0 \%$ With ICSI

$0 \%$ Unstimulated

$0 \%$ Used gestational carrier

\begin{tabular}{|c|c|c|c|c|}
\hline & \multicolumn{4}{|c|}{ Patient Diagnosis } \\
\hline & Tubal factor & $17 \%$ & Other factor & $13 \%$ \\
\hline $67 \%$ & Ovulatory dysfunction & $4 \%$ & Unknown factor & $1 \%$ \\
\hline $0 \%$ & Diminished ovarian reserve & $4 \%$ & Multiple Factors: & \\
\hline $0 \%$ & Endometriosis & $1 \%$ & Female factors only & $26 \%$ \\
\hline & Uterine factor & $0 \%$ & Female \& male factors & $19 \%$ \\
\hline & Male factor & $14 \%$ & & \\
\hline
\end{tabular}

Data verified by Edward J. Ramirez, MD

2006 PREGNANCY SUCCESS RATES

\section{Type of Cycle}

\section{Fresh Embryos from Nondonor Eggs}

Number of cycles

Percentage of cycles resulting in pregnancies ${ }^{b}$

Percentage of cycles resulting in live births ${ }^{b, c}$

(Confidence Interval)

Percentage of retrievals resulting in live births $s^{b, c}$

Percentage of transfers resulting in live births $s^{b, c}$

Percentage of transfers resulting in singleton live births ${ }^{b}$

Percentage of cancellations ${ }^{b}$

Average number of embryos transferred

Percentage of pregnancies with twins ${ }^{b}$

Percentage of pregnancies with triplets or more ${ }^{b}$

Percentage of live births having multiple infants ${ }^{b, c}$

\section{Frozen Embryos from Nondonor Eggs}

Number of transfers

Percentage of transfers resulting in live births ${ }^{b, c}$

Average number of embryos transferred

\section{Donor Eggs}

Number of transfers

Percentage of transfers resulting in live births $s^{b, c}$

Average number of embryos transferred

\section{Age of Woman}

35-37 38-40

$41-42^{d}$

\section{$<35$}

9

$3 / 9$

8

5

48.1

$3 / 9$

$4 / 8$

$0 / 5$

44.4

$4 / 8$

$0 / 5$

(25.5-64.7)

44.4

$3 / 9$

$4 / 7$

$0 / 5$

48.0

$3 / 9$

$4 / 7$

$0 / 3$

32.0

$2 / 9$

$2 / 7$

$0 / 3$

0.0

$0 / 9$

$1 / 8$

$0 / 5$

2.8

3.8

$1 / 3$

4.9

1.3

$4 / 13$

$0 / 3$

$3 / 4$

$1 / 13$

$1 / 3$

$0 / 4$

$4 / 12$

$2 / 4$

$\begin{array}{cccc}5 & 1 & 0 & 1 \\ 1 / 5 & 0 / 1 & & 0 / 1 \\ 2.8 & 2.0 & & 1.0\end{array}$

\section{All Ages Combined $^{\mathrm{e}}$}

Fresh Embryos

5

$2 / 5$

2.4
Frozen Embryos

4

$0 / 4$

2.5

\section{CURRENT CLINIC SERVICES AND PROFILE}

Current Name: The Fertility and Gynecology Center, Monterey Bay IVF Program

Donor egg? Yes

Donor embryo? Yes

Single women? Yes

\begin{abstract}
Gestational carriers? Yes
Cryopreservation?
\end{abstract}

SART member?

Verified lab accreditation?

(See Appendix C for details.)

${ }^{a}$ Reflects patient and treatment characteristics of ART cycles performed in 2006 using fresh nondonor eggs or embryos.

b When fewer than 20 cycles are reported in an age category, rates are shown as a fraction and confidence intervals are not given. Calculating percentages from fractions may be misleading and is not encouraged.

${ }^{\mathrm{C}}$ A multiple-infant birth is counted as one live birth.

d Clinic-specific outcome rates are unreliable for women older than 42 undergoing ART cycles using fresh or frozen embryos with nondonor eggs. Readers are urged to review national outcomes for these age groups (see page 27).

e All ages (including ages $>42$ ) are reported together because previous data show that patient age does not materially affect success with donor eggs. 


\section{FERTILITY SPECIALISTS MEDICAL GROUP SAN DIEGO, CALIFORNIA}

A comparison of clinic success rates may not be meaningful because patient medical characteristics and treatment approaches vary from clinic to clinic. For more details about this, along with information on how to interpret the statistics in this table, see pages 79-88.

\section{ART CYCLE PROFILE}

\section{Type of ART ${ }^{\mathrm{a}}$}

IVF

GIFT

ZIFT

Combination

\section{0\% Procedural Factors:}

$0 \%$ With ICSI

$0 \%$ Unstimulated

$0 \%$ Used gestational carrier $<1 \%$

\section{Patient Diagnosis}

$4 \%$ Other factor $\quad 2 \%$

$6 \%$ Unknown factor $\quad<1 \%$

$27 \%$ Multiple Factors:

$<1 \% \quad$ Female factors only $\quad 9 \%$

$<1 \%$ Female \& male factors $29 \%$ $22 \%$

2006 PREGNANCY SUCCESS RATES

Data verified by Arlene J. Morales, MD

\section{Type of Cycle}

\section{Age of Woman}

\section{Fresh Embryos from Nondonor Eggs}

Number of cycles

Uterine factor

35-37

38-40

$41-42^{d}$

Percentage of cycles resulting in pregnancies ${ }^{b}$

Percentage of cycles resulting in live births ${ }^{b, c}$

(Confidence Interval)

Percentage of retrievals resulting in live births $s^{b, c}$

Percentage of transfers resulting in live births $s^{b, c}$ $<35$

40

30.0

26

19

92
30.4

20.0

38.5

$4 / 19$

26.1

$(9.1-35.6)$

34.6

$3 / 19$

Percentage of transfers resulting in singleton live births ${ }^{b}$

Percentage of cancellations ${ }^{b}$

(17.5-36.3)

30.0

25.0

$(17.2-55.7)$

31.2

25.8

40.9

$3 / 14$

18.2

12.9

42.9

$3 / 13$

13.0

20.0

42.9

$3 / 13$

2.6

2.4

15.4

$5 / 19$

Average number of embryos transferred

Percentage of pregnancies with twins ${ }^{b}$

32.1

$5 / 12$

3.0

2.3

14.3

$0 / 12$

$1 / 10$

$0 / 4$

Percentage of pregnancies with triplets or more ${ }^{b}$

Percentage of live births having multiple infants ${ }^{b, c}$

41.7

$4 / 8$

$0 / 10$

$0 / 4$

\section{Frozen Embryos from Nondonor Eggs}

Number of transfers

Percentage of transfers resulting in live births $s^{\mathrm{b}, \mathrm{c}}$

Average number of embryos transferred

\begin{tabular}{|c|c|c|c|}
\hline 7 & 0 & 3 & 1 \\
\hline $0 / 7$ & & $1 / 3$ & $0 / 1$ \\
\hline 2.3 & & 3.0 & 4.0 \\
\hline
\end{tabular}

\section{Donor Eggs}

All Ages Combined ${ }^{\mathrm{e}}$

Number of transfers

Percentage of transfers resulting in live births $s^{b, c}$

Fresh Embryos

62.9

2.5

\section{Frozen Embryos} 15

$2 / 15$

2.3

\section{CURRENT CLINIC SERVICES AND PROFILE}

Current Name: Fertility Specialists Medical Group

\begin{tabular}{l|l|l} 
Donor egg? Yes & Gestational carriers? Yes
\end{tabular}

Donor embryo? Yes Cryopreservation? Yes

Single women? Yes

SART member? Yes

Verified lab accreditation?

Pending

${ }^{a}$ Reflects patient and treatment characteristics of ART cycles performed in 2006 using fresh nondonor eggs or embryos.

${ }^{b}$ When fewer than 20 cycles are reported in an age category, rates are shown as a fraction and confidence intervals are not given. Calculating percentages from fractions may be misleading and is not encouraged.

${ }^{c}$ A multiple-infant birth is counted as one live birth.

d Clinic-specific outcome rates are unreliable for women older than 42 undergoing ART cycles using fresh or frozen embryos with nondonor eggs. Readers are urged to review national outcomes for these age groups (see page 27).

e All ages (including ages $>42$ ) are reported together because previous data show that patient age does not materially affect success with donor eggs. 


\section{IGO MEDICAL GROUP OF SAN DIEGO \\ SAN DIEGO, CALIFORNIA}

A comparison of clinic success rates may not be meaningful because patient medical characteristics and treatment approaches vary from clinic to clinic. For more details about this, along with information on how to interpret the statistics in this table, see pages 79-88.

\section{ART CYCLE PROFILE}

\section{Type of ART ${ }^{\mathrm{a}}$}

IVF

GIFT 100\% Procedural Factors:

ZIFT

Combination
$0 \%$ With ICSI

$0 \%$ Unstimulated

$0 \%$ Used gestational carrier

\begin{tabular}{|c|c|}
\hline $78 \%$ & Ovulatory dysfunction \\
\hline $0 \%$ & Diminished ovarian reserve \\
\hline $0 \%$ & Endometriosis \\
\hline & Uterine factor \\
\hline & Male factor \\
\hline
\end{tabular}

Patient Diagnosis

$8 \%$ Other factor

$0 \%$ Unknown factor

7\% Multiple Factors:

$4 \% \quad$ Female factors only $18 \%$

$1 \%$ Female \& male factors $26 \%$

$35 \%$

2006 PREGNANCY SUCCESS RATES

Data verified by Benito Villanueva, MD

\section{Type of Cycle}

\section{Age of Woman}

35-37 38-40

15

$4 / 15$

8

$1 / 8$

44.4

$2 / 15$

$1 / 8$

$0 / 2$

$(19.4-57.6)$

(Confidence Interval)

Percentage of retrievals resulting in live births $s^{b, c}$

Percentage of transfers resulting in live births ${ }^{b, c}$

Percentage of transfers resulting in singleton live births ${ }^{b}$

Percentage of cancellations ${ }^{b}$

Average number of embryos transferred

Percentage of pregnancies with twins ${ }^{b}$

Percentage of pregnancies with triplets or more ${ }^{b}$

Percentage of live births having multiple infants ${ }^{b, c}$

\section{Frozen Embryos from Nondonor Eggs}

Number of transfers

Percentage of transfers resulting in live births $s^{b, c}$

Average number of embryos transferred

\section{Donor Eggs}

Number of transfers

Percentage of transfers resulting in live births $s^{b, c}$

Average number of embryos transferred

$\begin{array}{ccc}2 / 13 & 1 / 6 & 0 / 2 \\ 2 / 12 & 1 / 3 & 0 / 2 \\ 2 / 12 & 1 / 3 & 0 / 2 \\ 2 / 15 & 2 / 8 & 0 / 2 \\ 2.5 & 3.3 & 1.5 \\ 0 / 4 & 0 / 1 & \\ 0 / 4 & 0 / 1 & \\ 0 / 2 & 0 / 1 & \end{array}$

$0 / 2$

40.0

20.0

7.4

2.2

$5 / 12$

$0 / 12$

$5 / 10$

$0 / 2$

$0 / 1$

$\begin{array}{ccc}6 & 5 & 2 \\ 3 / 6 & 0 / 5 & 0 / 2 \\ 2.5 & 2.4 & 2.0\end{array}$

All Ages Combined ${ }^{\mathrm{e}}$
Fresh Embryos

2

$1 / 2$

2.0
Frozen Embryos 2

$2 / 2$

2.5

\section{CURRENT CLINIC SERVICES AND PROFILE}

Current Name: IGO Medical Group of San Diego

Donor egg? Yes

Donor embryo? No

Gestational carriers? Yes

Cryopreservation? Yes

Single women? Yes

SART member?

Yes

Verified lab accreditation?

Yes

${ }^{a}$ Reflects patient and treatment characteristics of ART cycles performed in 2006 using fresh nondonor eggs or embryos.

${ }^{b}$ When fewer than 20 cycles are reported in an age category, rates are shown as a fraction and confidence intervals are not given. Calculating percentages from fractions may be misleading and is not encouraged.

${ }^{\mathrm{C}}$ A multiple-infant birth is counted as one live birth.

d Clinic-specific outcome rates are unreliable for women older than 42 undergoing ART cycles using fresh or frozen embryos with nondonor eggs. Readers are urged to review national outcomes for these age groups (see page 27).

e All ages (including ages $>42$ ) are reported together because previous data show that patient age does not materially affect success with donor eggs. 


\section{NTC INFERTILITY CLINIC SAN DIEGO, CALIFORNIA}

A comparison of clinic success rates may not be meaningful because patient medical characteristics and treatment approaches vary from clinic to clinic. For more details about this, along with information on how to interpret the statistics in this table, see pages 79-88.

\section{ART CYCLE PROFILE}

$$
\text { Type of ART }{ }^{\mathrm{a}}
$$

IVF

$$
\text { 100\% Procedural Factors: }
$$

GIFT

$0 \%$ With ICSI

ZIFT

$0 \%$ Unstimulated

Combination

\begin{tabular}{|c|c|}
\hline & Tubal factor \\
\hline $85 \%$ & Ovulatory dysfunction \\
\hline $0 \%$ & Diminished ovarian reserve \\
\hline $1 \%$ & Endometriosis \\
\hline & Uterine factor \\
\hline & Male factor \\
\hline
\end{tabular}

$0 \%$ Used gestational carrier

Patient Diagnosis

$\begin{array}{rlr}34 \% & \text { Other factor } & <1 \% \\ 3 \% & \text { Unknown factor } & 19 \% \\ 0 \% & \text { Multiple Factors: } & \\ 4 \% & \text { Female factors only } & 1 \% \\ 0 \% & \text { Female \& male factors } & 9 \% \\ 29 \% & & \end{array}$

2006 PREGNANCY SUCCESS RATES

Data verified by Larry R. Laufer, MD

\section{Type of Cycle}

\section{Age of Woman}

\section{Fresh Embryos from Nondonor Eggs}

Number of cycles

Percentage of cycles resulting in pregnancies ${ }^{b}$

Percentage of cycles resulting in live births $s^{b, c}$

(Confidence Interval)

Percentage of retrievals resulting in live births $s^{b, c}$

Percentage of transfers resulting in live births $s^{b, c}$

Percentage of transfers resulting in singleton live births ${ }^{b}$

Percentage of cancellations ${ }^{b}$

35-37

38-40

$41-42^{d}$

Average number of embryos transferred

Percentage of pregnancies with twins ${ }^{b}$ $<35$

26

42.3

39.0

42.3

24.4

(12.4-40.3)

(23.4-63.1)

47.8

47.8

27.8

21.7

11.5

0.0

2.2

2.0

$4 / 11$

20

3

Percentage of pregnancies with triplets or more ${ }^{b}$

Percentage of live births having multiple infants ${ }^{b, c}$

$6 / 16$

$2 / 11$

50.0

$0 / 3$

Frozen Embryos from Nondonor Eggs

Number of transfers

Percentage of transfers resulting in live births $s^{b, c}$

Average number of embryos transferred

$0 / 16$

$5 / 10$

$6 / 11$

30.0

$0 / 3$

(11.9-54.3)

$6 / 19 \quad 0 / 3$

$6 / 19$

$0 / 3$

$6 / 19$

$0 / 3$

5.0

$0 / 3$

2.6

3.7

$0 / 10$

$0 / 10$

$0 / 6$

30

33.3

2.2

\section{1}

$4 / 11$

1.8

\section{6 \\ $2 / 6$}

2.8

All Ages Combined ${ }^{\mathrm{e}}$
Fresh Embryos

0
Frozen Embryos

0

Number of transfers

Percentage of transfers resulting in live births $s^{b, c}$

Average number of embryos transferred

\section{CURRENT CLINIC SERVICES AND PROFILE}

Current Name: NTC Infertility Clinic
Donor egg?
No
Gestational carriers?
Yes
Cryopreservation?
Yes
SART member?
No
Donor embryo? No
Verified lab accreditation?
Yes
Single women? Yes
(See Appendix C for details.)

\footnotetext{
${ }^{a}$ Reflects patient and treatment characteristics of ART cycles performed in 2006 using fresh nondonor eggs or embryos.

${ }^{b}$ When fewer than 20 cycles are reported in an age category, rates are shown as a fraction and confidence intervals are not given. Calculating percentages from fractions may be misleading and is not encouraged.

${ }^{c}$ A multiple-infant birth is counted as one live birth.

d Clinic-specific outcome rates are unreliable for women older than 42 undergoing ART cycles using fresh or frozen embryos with nondonor eggs. Readers are urged to review national outcomes for these age groups (see page 27).

${ }^{e}$ All ages (including ages $>42$ ) are reported together because previous data show that patient age does not materially affect success with donor eggs.
} 


\section{SAN DIEGO FERTILITY CENTER \\ (SDFC) \\ SAN DIEGO, CALIFORNIA}

A comparison of clinic success rates may not be meaningful because patient medical characteristics and treatment approaches vary from clinic to clinic. For more details about this, along with information on how to interpret the statistics in this table, see pages 79-88.

\section{ART CYCLE PROFILE}

\begin{tabular}{|c|c|c|c|c|c|c|}
\hline \multicolumn{3}{|c|}{ Type of ART ${ }^{a}$} & \multicolumn{4}{|c|}{ Patient Diagnosis } \\
\hline IVF & $>99 \%$ Procedural Factors: & & Tubal factor & $2 \%$ & Other factor & $0 \%$ \\
\hline GIFT & $0 \%$ With ICSI & $90 \%$ & Ovulatory dysfunction & $<1 \%$ & Unknown factor & $2 \%$ \\
\hline ZIFT & $<1 \%$ Unstimulated & $0 \%$ & Diminished ovarian reserve & $10 \%$ & Multiple Factors: & \\
\hline \multirow[t]{3}{*}{ Combination } & $0 \%$ Used gestational carrier & $<1 \%$ & Endometriosis & $2 \%$ & Female factors only & $5 \%$ \\
\hline & & & Uterine factor & $<1 \%$ & Female \& male factors & $52 \%$ \\
\hline & & & Male factor & $25 \%$ & & \\
\hline
\end{tabular}

2006 PREGNANCY SUCCESS RATES

Data verified by William P. Hummel, MD

\section{Type of Cycle}

\section{Age of Woman}

35-37 38-40

$41-42^{d}$

$<35$

56

41.1

74

27

54.3

35.7

33.8

11.1

48.6

(23.4-49.6)

28.4

11.1

(Confidence Interval)

Percentage of retrievals resulting in live births

(36.4-60.8)

51.5

40.8

$(18.5-40.1)$

(2.4-29.2)

51.5

40.8

33.3

12.0

39.4

30.6

34.4

12.5

5.7

12.5

29.5

12.5

2.5

3.1

31.6

0.0

23.5

21.7

0.0

14.9

7.4

Percentage of pregnancies with twins ${ }^{b}$

Percentage of pregnancies with triplets or more ${ }^{b}$

Percentage of live births having multiple infants ${ }^{b, c}$

Frozen Embryos from Nondonor Eggs

Number of transfers

Percentage of transfers resulting in live births ${ }^{b, c}$

Average number of embryos transferred

\section{Donor Eggs}

41

56.1

2.6

25.0

3.1

3.8

$0 / 3$

16.0

$0 / 3$

0.0

$0 / 3$

14.3

24

54.2

2.7

All Ages Combined ${ }^{\mathrm{e}}$

Fresh Embryos

63

81.0

2.4

Number of transfers

Percentage of transfers resulting in live births $s^{b, c}$

Average number of embryos transferred

4

$2 / 4$

3.8
22

31.8

2.3

Frozen Embryos

22

68.2

2.4

\section{CURRENT CLINIC SERVICES AND PROFILE}

Current Name: San Diego Fertility Center, (SDFC)
Donor egg?
Yes
Gestational carriers?
Yes
Donor embryo? Yes
Cryopreservation? Yes
SART member?
Yes
Single women? Yes
Verified lab accreditation?
Yes
(See Appendix C for details.)

\footnotetext{
${ }^{a}$ Reflects patient and treatment characteristics of ART cycles performed in 2006 using fresh nondonor eggs or embryos.

${ }^{b}$ When fewer than 20 cycles are reported in an age category, rates are shown as a fraction and confidence intervals are not given. Calculating percentages from fractions may be misleading and is not encouraged.

${ }^{c}$ A multiple-infant birth is counted as one live birth.

d Clinic-specific outcome rates are unreliable for women older than 42 undergoing ART cycles using fresh or frozen embryos with nondonor eggs. Readers are urged to review national outcomes for these age groups (see page 27).

${ }^{e}$ All ages (including ages $>42$ ) are reported together because previous data show that patient age does not materially affect success with donor eggs.
} 


\section{XPERT FERTILITY CARE OF CALIFORNIA \\ MINH N. HO, MD, FACOG \\ SAN DIEGO, CALIFORNIA}

A comparison of clinic success rates may not be meaningful because patient medical characteristics and treatment approaches vary from clinic to clinic. For more details about this, along with information on how to interpret the statistics in this table, see pages 79-88.

\section{ART CYCLE PROFILE}

$$
\text { Type of ART }{ }^{\mathrm{a}}
$$

IVF

100\% Procedural Factors:

GIFT

$0 \%$ With ICSI

ZIFT

$0 \%$ Unstimulated

Combination

0\% Used gestational carrier

\section{$86 \%$ \\ $0 \%$ \\ $0 \%$}

\section{Patient Diagnosis}

$6 \%$ Other factor

6\% Unknown factor

Ovulatory dysfunction

23\% Multiple Factors:

Diminished ovarian reserve

$6 \%$ Female factors only

$2 \%$ Female \& male factors $12 \%$
Uterine factor

Male factor

2006 PREGNANCY SUCCESS RATES

Data verified by Minh N. Ho, MD

\section{Type of Cycle}

\section{Fresh Embryos from Nondonor Eggs}

Number of cycles

Percentage of cycles resulting in pregnancies ${ }^{b}$

Percentage of cycles resulting in live births $s^{b, c}$

(Confidence Interval)

Percentage of retrievals resulting in live births $s^{b, c}$

Percentage of transfers resulting in live births $s^{b, c}$

Percentage of transfers resulting in singleton live births ${ }^{b}$

Percentage of cancellations ${ }^{b}$

Average number of embryos transferred

Percentage of pregnancies with twins ${ }^{b}$

Percentage of pregnancies with triplets or more ${ }^{\mathrm{b}}$

Percentage of live births having multiple infants ${ }^{b, c}$

\section{Frozen Embryos from Nondonor Eggs}

Number of transfers

Percentage of transfers resulting in live births $s^{\mathrm{b}, \mathrm{c}}$

Average number of embryos transferred

\section{Donor Eggs}

Number of transfers

Percentage of transfers resulting in live births ${ }^{\mathrm{b}, \mathrm{c}}$

Average number of embryos transferred
$10 \%$

$12 \%$

$10 \%$

$15 \%$

\section{CURRENT CLINIC SERVICES AND PROFILE}

Current Name: Xpert Fertility Care of California, Minh N. Ho, MD, FACOG

Donor egg? Yes

Donor embryo? No

Single women? Yes

\section{Age of Woman}

35-37

38-40

$41-42^{d}$

\section{$<35$}

10

$4 / 10$

$4 / 10$

6

2

$12 / 15$

$12 / 15$

$3 / 6$

$0 / 2$

$12 / 15$

$12 / 15$

$4 / 10$

$3 / 6$

$0 / 2$

$4 / 10$

$3 / 6$

$0 / 2$

$3 / 6$

$0 / 1$

$9 / 15$

$3 / 10$

$0 / 15$

$0 / 10$

$2 / 6$

$0 / 1$

3.1

$3 / 12$

3.4

$0 / 6$

$0 / 2$

$0 / 12$

$0 / 4$

2.5

2.0

$3 / 12$

$1 / 4$

$1 / 3$

$0 / 3$

$1 / 4$

$1 / 3$

\section{0}

$\begin{array}{cc}5 & 2 \\ 2 / 5 & 1 / 2 \\ 3.2 & 3.0\end{array}$

1

$1 / 1$

3.0

\section{Fresh Embryos}

\section{5}

$4 / 5$

3.2
All Ages Combined

Frozen Embryos

3

$2 / 3$

2.7

${ }^{a}$ Reflects patient and treatment characteristics of ART cycles performed in 2006 using fresh nondonor eggs or embryos.

${ }^{b}$ When fewer than 20 cycles are reported in an age category, rates are shown as a fraction and confidence intervals are not given. Calculating percentages from fractions may be misleading and is not encouraged.

${ }^{c}$ A multiple-infant birth is counted as one live birth.

d Clinic-specific outcome rates are unreliable for women older than 42 undergoing ART cycles using fresh or frozen embryos with nondonor eggs. Readers are urged to review national outcomes for these age groups (see page 27).

e All ages (including ages $>42$ ) are reported together because previous data show that patient age does not materially affect success with donor eggs. 


\section{LAUREL FERTILITY CARE SAN FRANCISCO, CALIFORNIA}

A comparison of clinic success rates may not be meaningful because patient medical characteristics and treatment approaches vary from clinic to clinic. For more details about this, along with information on how to interpret the statistics in this table, see pages 79-88.

\section{ART CYCLE PROFILE}

\section{Type of ART ${ }^{\mathrm{a}}$}

IVF

GIFT

$100 \%$ Procedural Factors:

ZIFT

Combination
$0 \%$ With ICSI

$0 \%$ Unstimulated

$0 \%$ Used gestational carrier

\begin{tabular}{r|l} 
& \\
& Tubal factor \\
\hline 88\% & Ovulatory dysfunction \\
0\% & Diminished ovarian reserve \\
$4 \%$ & Endometriosis \\
& Uterine factor \\
& Male factor
\end{tabular}

Male factor
Patient Diagnosis

$4 \%$ Other factor

11\% Unknown factor

27\% Multiple Factors:

$<1 \%$ Female factors only $13 \%$

$3 \%$ Female \& male factors $9 \%$ $3 \%$

2006 PREGNANCY SUCCESS RATES

Data verified by Collin B. Smikle, MD

\section{Type of Cycle}

\section{Fresh Embryos from Nondonor Eggs}

Number of cycles

Percentage of cycles resulting in pregnancies ${ }^{b}$

Percentage of cycles resulting in live births ${ }^{b, c}$

(Confidence Interval)

Percentage of retrievals resulting in live births ${ }^{b, c}$

Percentage of transfers resulting in live births $s^{b, c}$

Percentage of transfers resulting in singleton live births ${ }^{b}$

Percentage of cancellations ${ }^{b}$

Average number of embryos transferred

Percentage of pregnancies with twins ${ }^{b}$

Percentage of pregnancies with triplets or more ${ }^{b}$

Percentage of live births having multiple infants ${ }^{\mathrm{b}, \mathrm{c}}$

\section{Frozen Embryos from Nondonor Eggs}

Number of transfers

Percentage of transfers resulting in live births $s^{b, c}$

Average number of embryos transferred

\section{Donor Eggs}

Number of transfers

Percentage of transfers resulting in live births $s^{b, c}$

Average number of embryos transferred

\section{Age of Woman}

35-37 38-40

$41-42^{d}$

\section{$<35$}

17

33

16

22
45.5

$4 / 17$

27.3

$3 / 16$

45.5

$3 / 17$

24.2

$2 / 16$

(24.4-67.8)

47.6

50.0

$3 / 16$

(11.1-42.3)

28.6

$2 / 13$

29.6

$2 / 13$

25.0

$3 / 15$

25.9

$2 / 13$

$1 / 17$

15.2

$3 / 16$

2.9

3.1

$1 / 4$

3.0

3.9

$4 / 10$

$0 / 4$

$2 / 10$

$1 / 3$

$1 / 9$

$0 / 3$

$5 / 10$

$0 / 9$

$0 / 3$

$1 / 8$

$0 / 2$

10

$0 / 10$

10

2.3

$2 / 10$

2

$1 / 2$

2

2.7

2.5

$1 / 2$

4.5

All Ages Combined ${ }^{\mathrm{e}}$

Fresh Embryos

27

66.7

2.8
Frozen Embryos 12

$4 / 12$

2.7

\section{CURRENT CLINIC SERVICES AND PROFILE}

Current Name: Laurel Fertility Care

Donor egg? Yes Gestational carriers? Yes

Donor embryo? Yes

Cryopreservation? Yes

SART member?

Yes

Single women? Yes

Verified lab accreditation?

Yes

(See Appendix C for details.)

${ }^{\text {a }}$ Reflects patient and treatment characteristics of ART cycles performed in 2006 using fresh nondonor eggs or embryos.

${ }^{b}$ When fewer than 20 cycles are reported in an age category, rates are shown as a fraction and confidence intervals are not given. Calculating percentages from fractions may be misleading and is not encouraged.

${ }^{c}$ A multiple-infant birth is counted as one live birth.

d Clinic-specific outcome rates are unreliable for women older than 42 undergoing ART cycles using fresh or frozen embryos with nondonor eggs. Readers are urged to review national outcomes for these age groups (see page 27).

e All ages (including ages $>42$ ) are reported together because previous data show that patient age does not materially affect success with donor eggs. 


\section{PACIFIC FERTILITY CENTER SAN FRANCISCO, CALIFORNIA}

A comparison of clinic success rates may not be meaningful because patient medical characteristics and treatment approaches vary from clinic to clinic. For more details about this, along with information on how to interpret the statistics in this table, see pages 79-88.

\section{ART CYCLE PROFILE}

\section{Type of ART ${ }^{\mathrm{a}}$}

IVF

GIFT

ZIFT

$>99 \%$ Procedural Factors:

Combination
$0 \%$ With ICSI

$<1 \%$ Unstimulated

$0 \%$ Used gestational carrier

\begin{tabular}{|c|c|}
\hline & Tubal factor \\
\hline $44 \%$ & Ovulatory dysfunction \\
\hline $0 \%$ & Diminished ovarian reserve \\
\hline $2 \%$ & Endometriosis \\
\hline & Uterine factor \\
\hline
\end{tabular}

Patient Diagnosis

$7 \%$ Other factor

$6 \%$ Unknown factor

29\% Multiple Factors:

$3 \%$ Female factors only $10 \%$

$1 \%$ Female \& male factors $11 \%$ $13 \%$

2006 PREGNANCY SUCCESS RATES

Data verified by Philip E. Chenette, MD

\section{Type of Cycle}

\section{Fresh Embryos from Nondonor Eggs}

Number of cycles

Percentage of cycles resulting in pregnancies ${ }^{b}$

Percentage of cycles resulting in live births $s^{b, c}$

(Confidence Interval)

Percentage of retrievals resulting in live births $s^{b, c}$

Percentage of transfers resulting in live births $s^{b, c}$

Percentage of transfers resulting in singleton live births ${ }^{b}$

Percentage of cancellations ${ }^{b}$

Average number of embryos transferred

Percentage of pregnancies with twins ${ }^{b}$

Percentage of pregnancies with triplets or more ${ }^{b}$

Percentage of live births having multiple infants ${ }^{b, c}$

\section{Frozen Embryos from Nondonor Eggs}

Number of transfers

Percentage of transfers resulting in live births $s^{b, c}$

Average number of embryos transferred

\section{Donor Eggs}

Number of transfers

Percentage of transfers resulting in live births $s^{\mathrm{b}, \mathrm{c}}$

Average number of embryos transferred

\section{Age of Woman}

35-37 38-40

$41-42^{d}$

$<35$

165

179

77

152

35.5

33.9

26.8

16.9

30.9

27.9

21.8

13.0

(23.7-38.9)

(21.2-35.4)

(16.0-28.6)

(6.4-22.6)

34.3

30.3

26.4

15.6

37.0

32.9

27.7

17.2

23.6

18.4

12.1

9.9

7.9

17.3

16.9

2.4

27.8

0.0

29.8

3.1

25.0

7.1

28.3

3.5

4.4

31.3

$5 / 13$

2.1

$0 / 13$

33.3

$3 / 10$

$\begin{array}{cccc}67 & 77 & 50 & 9 \\ 25.4 & 33.8 & 20.0 & 2 / 9 \\ 2.4 & 2.8 & 2.9 & 2.2\end{array}$

All Ages Combined ${ }^{\mathrm{e}}$ Fresh Embryos

Frozen Embryos

179

171

57.0

32.2

2.1

\section{CURRENT CLINIC SERVICES AND PROFILE}

Current Name: Pacific Fertility Center
Donor egg? Yes
Donor embryo? Yes
Gestational carriers? Yes
Cryopreservation? Yes
Single women? Yes

$\begin{array}{ll}\text { SART member? } & \text { Yes } \\ \text { Verified lab accreditation? } & \text { Yes } \\ \text { (See Appendix C for details.) } & \end{array}$

${ }^{a}$ Reflects patient and treatment characteristics of ART cycles performed in 2006 using fresh nondonor eggs or embryos.

${ }^{b}$ When fewer than 20 cycles are reported in an age category, rates are shown as a fraction and confidence intervals are not given. Calculating percentages from fractions may be misleading and is not encouraged.

c A multiple-infant birth is counted as one live birth.

${ }^{d}$ Clinic-specific outcome rates are unreliable for women older than 42 undergoing ART cycles using fresh or frozen embryos with nondonor eggs. Readers are urged to review national outcomes for these age groups (see page 27).

e All ages (including ages $>42$ ) are reported together because previous data show that patient age does not materially affect success with donor eggs. 


\section{UCSF CENTER FOR REPRODUCTIVE HEALTH SAN FRANCISCO, CALIFORNIA}

A comparison of clinic success rates may not be meaningful because patient medical characteristics and treatment approaches vary from clinic to clinic. For more details about this, along with information on how to interpret the statistics in this table, see pages 79-88.

\section{ART CYCLE PROFILE}

\section{Type of ART ${ }^{\mathrm{a}}$}

IVF

GIFT

ZIFT

Combination
100\% Procedural Factors:

$0 \%$ With ICSI

$0 \%$ Unstimulated

$0 \%$ Used gestational carrier $<1 \%$
Tubal factor

$61 \%$ Ovulatory dysfunction

Diminished ovarian reserve

Endometriosis

Uterine factor

Male factor

\section{Patient Diagnosis}

6\% Other factor

4\% Unknown factor

19\% Multiple Factors:

$23 \%$

$6 \%$

$23 \%$

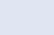

2006 PREGNANCY SUCCESS RATES

\section{Type of Cycle}

35-37 38-40 $<35$

151

43.0

37.1

Percentage of cycles resulting in live births ${ }^{b, c}$

(Confidence Interval)

Percentage of retrievals resulting in live births $s^{b, c}$

Percentage of transfers resulting in live births $s^{b, c}$

Percentage of transfers resulting in singleton live births ${ }^{b}$

Percentage of cancellations ${ }^{b}$

Average number of embryos transferred

Percentage of pregnancies with twins ${ }^{\mathrm{b}}$

Percentage of pregnancies with triplets or more ${ }^{b}$

Percentage of live births having multiple infants ${ }^{b, c}$

\section{Frozen Embryos from Nondonor Eggs}

Number of transfers

Percentage of transfers resulting in live births $s^{b, c}$

Average number of embryos transferred

\section{Donor Eggs}

Number of transfers

Percentage of transfers resulting in live births ${ }^{b, c}$

Average number of embryos transferred

\begin{tabular}{|c|c|c|c|c|}
\hline \multicolumn{5}{|l|}{ Fresh Embryos trom Nondonor Eggs } \\
\hline Number of cycles & 151 & 134 & 163 & 82 \\
\hline Percentage of cycles resulting in pregnancies ${ }^{\mathrm{b}}$ & 43.0 & 38.1 & 32.5 & 23.2 \\
\hline $\begin{array}{l}\text { Percentage of cycles resulting in live births }{ }^{b, c} \\
\text { (Confidence Interval) }\end{array}$ & $\begin{array}{c}37.1 \\
(29.4-45.3)\end{array}$ & $\begin{array}{c}29.9 \\
(22.3-38.4)\end{array}$ & $\begin{array}{c}23.9 \\
(17.6-31.2)\end{array}$ & $\begin{array}{c}13.4 \\
(6.9-22.7)\end{array}$ \\
\hline Percentage of retrievals resulting in live births $s^{b, c}$ & 40.0 & 36.0 & 28.1 & 15.3 \\
\hline Percentage of transfers resulting in live births ${ }^{b, c}$ & 41.2 & 37.0 & 29.5 & 16.2 \\
\hline Percentage of transfers resulting in singleton live births ${ }^{b}$ & 30.9 & 28.7 & 22.0 & 11.8 \\
\hline Percentage of cancellations ${ }^{b}$ & 7.3 & 17.2 & 14.7 & 12.2 \\
\hline Average number of embryos transferred & 2.2 & 2.4 & 3.2 & 4.1 \\
\hline Percentage of pregnancies with twins ${ }^{\mathrm{b}}$ & 26.2 & 19.6 & 18.9 & $3 / 19$ \\
\hline Percentage of pregnancies with triplets or more ${ }^{\mathrm{b}}$ & 0.0 & 0.0 & 5.7 & $2 / 19$ \\
\hline Percentage of live births having multiple infants ${ }^{b, c}$ & 25.0 & 22.5 & 25.6 & $3 / 11$ \\
\hline \multicolumn{5}{|l|}{ Frozen Embryos from Nondonor Eggs } \\
\hline Number of transfers & 48 & 43 & 42 & 16 \\
\hline Percentage of transfers resulting in live births $s^{b, c}$ & 25.0 & 20.9 & 9.5 & $4 / 16$ \\
\hline \multirow[t]{2}{*}{ Average number of embryos transferred } & 2.4 & 2.5 & 2.9 & 3.1 \\
\hline & \multicolumn{4}{|c|}{ All Ages Combined $^{\mathrm{e}}$} \\
\hline Donor Eggs & \multicolumn{2}{|c|}{ Fresh Embryos } & \multicolumn{2}{|c|}{ Frozen Embryos } \\
\hline Number of transfers & \multicolumn{2}{|c|}{81} & \multicolumn{2}{|c|}{38} \\
\hline Percentage of transfers resulting in live births $s^{b, c}$ & \multicolumn{2}{|c|}{58.0} & \multicolumn{2}{|c|}{50.0} \\
\hline Average number of embryos transferred & \multicolumn{2}{|c|}{2.0} & \multicolumn{2}{|c|}{2.3} \\
\hline
\end{tabular}

$1 \% \quad$ Female factors only $\quad 7 \%$

$2 \%$ Female \& male factors $9 \%$

Data verified by Victor Y. Fujimoto, MD

\section{Age of Woman}

82

23.2

13.4

38.1

163

32.5

23.9

$41-42^{d}$

\section{CURRENT CLINIC SERVICES AND PROFILE}

Current Name: UCSF Center for Reproductive Health

Donor egg? Yes Gestational carriers? Yes

Donor embryo? Yes Cryopreservation? Yes

Single women? Yes

SART member? Yes

Verified lab accreditation? Yes

(See Appendix C for details.)

${ }^{a}$ Reflects patient and treatment characteristics of ART cycles performed in 2006 using fresh nondonor eggs or embryos.

b When fewer than 20 cycles are reported in an age category, rates are shown as a fraction and confidence intervals are not given. Calculating percentages from fractions may be misleading and is not encouraged.

${ }^{c}$ A multiple-infant birth is counted as one live birth.

d Clinic-specific outcome rates are unreliable for women older than 42 undergoing ART cycles using fresh or frozen embryos with nondonor eggs. Readers are urged to review national outcomes for these age groups (see page 27).

e All ages (including ages $>42$ ) are reported together because previous data show that patient age does not materially affect success with donor eggs. 


\section{FERTILITY PHYSICIANS OF NORTHERN CALIFORNIA \\ SAN JOSE, CALIFORNIA}

A comparison of clinic success rates may not be meaningful because patient medical characteristics and treatment approaches vary from clinic to clinic. For more details about this, along with information on how to interpret the statistics in this table, see pages 79-88.

\section{ART CYCLE PROFILE}

$$
\text { Type of ART }{ }^{\mathrm{a}}
$$

IVF

$$
\text { 100\% Procedural Factors: }
$$

GIFT

$0 \%$ With ICSI

Tubal factor

Patient Diagnosis

ZIFT

$0 \%$ Unstimulated

$60 \%$

Ovulatory dysfunction

$4 \%$ Other factor

$10 \%$

2\% Unknown factor

$6 \%$

Combination

$0 \%$ Used gestational carrier $<1 \%$

Diminished ovarian reserve $12 \%$ Multiple Factors:

Endometriosis

$3 \%$ Female factors only $18 \%$

Uterine factor

$<1 \%$ Female \& male factors

$25 \%$

Male factor $19 \%$

2006 PREGNANCY SUCCESS RATES

Data verified by Karen Purcell, MD, PhD

\section{Type of Cycle}

\section{Fresh Embryos from Nondonor Eggs}

Number of cycles

Percentage of cycles resulting in pregnancies ${ }^{b}$

Percentage of cycles resulting in live births ${ }^{b, c}$

(Confidence Interval)

Percentage of retrievals resulting in live births $s^{b, c}$

Percentage of transfers resulting in live births $s^{b, c}$

Percentage of transfers resulting in singleton live births ${ }^{b}$

Percentage of cancellations ${ }^{b}$

Average number of embryos transferred

Percentage of pregnancies with twins ${ }^{b}$

Percentage of pregnancies with triplets or more ${ }^{\mathrm{b}}$

Percentage of live births having multiple infants ${ }^{b, c}$

\section{Frozen Embryos from Nondonor Eggs}

Number of transfers

Percentage of transfers resulting in live births $s^{b, c}$

Average number of embryos transferred

\section{Donor Eggs}

Number of transfers

Percentage of transfers resulting in live births $s^{b, c}$

Average number of embryos transferred

\section{Age of Woman}

35-37 38-40

$41-42^{d}$

$<35$

122

111

70

36.3

35.2

27.9

10.0

32.7

26.2

20.7

7.1

$(25.8-40.3)$

$(18.7-35.0)$

(13.6-29.5)

(2.4-15.9)

34.8

35.9

28.3

27.4

9.1

28.8

29.6

29.1

20.3

10.6

21.3

24.3

10.6

5.8

7.4

2.8

21.4

2.1

17.7

2.4

22.6

3.4

1.6

23.3

0.0

$0 / 7$

19.6

28.1

30.4

$0 / 7$

$0 / 5$

65

27.7

36

22.2

26

11.5

6

2.0

2.1

2.1

$0 / 6$

3.0

All Ages Combined ${ }^{\mathrm{e}}$

Fresh Embryos 38

63.2

2.1
Frozen Embryos 14

$3 / 14$

1.9

\section{CURRENT CLINIC SERVICES AND PROFILE}

Current Name: Fertility Physicians of Northern California

Donor egg? Yes

Donor embryo? Yes

Single women? Yes
Gestational carriers? Yes

Cryopreservation? Yes
SART member?

Yes

Verified lab accreditation?

(See Appendix C for details.)

${ }^{a}$ Reflects patient and treatment characteristics of ART cycles performed in 2006 using fresh nondonor eggs or embryos.

${ }^{b}$ When fewer than 20 cycles are reported in an age category, rates are shown as a fraction and confidence intervals are not given. Calculating percentages from fractions may be misleading and is not encouraged.

c A multiple-infant birth is counted as one live birth.

d Clinic-specific outcome rates are unreliable for women older than 42 undergoing ART cycles using fresh or frozen embryos with nondonor eggs. Readers are urged to review national outcomes for these age groups (see page 27).

${ }^{e}$ All ages (including ages $>42$ ) are reported together because previous data show that patient age does not materially affect success with donor eggs. 


\section{REPRODUCTIVE SCIENCE CENTER OF THE SAN FRANCISCO BAY AREA SAN RAMON, CALIFORNIA}

A comparison of clinic success rates may not be meaningful because patient medical characteristics and treatment approaches vary from clinic to clinic. For more details about this, along with information on how to interpret the statistics in this table, see pages 79-88.

\section{ART CYCLE PROFILE}

\section{Type of ART ${ }^{\mathrm{a}}$}

IVF

GIFT 100\% Procedural Factors:

ZIFT

Combination
$0 \%$ With ICSI

$0 \%$ Unstimulated

$0 \%$ Used gestational carrier

\section{$48 \%$}

$0 \%$

$2 \%$
Tubal factor

Ovulatory dysfunction

Diminished ovarian reserve

Endometriosis

Uterine factor

Male factor

\section{Patient Diagnosis}

9\% Other factor

7\% Unknown factor

11\% Multiple Factors:

$4 \%$ Female factors only $12 \%$

$2 \%$ Female \& male factors $15 \%$ $19 \%$

2006 PREGNANCY SUCCESS RATES

Data verified by Louis N. Weckstein, MD

\section{Type of Cycle}

Age of Woman

35-37 38-40

\section{$<35$}

267

34.8

186

31.7

25.8

31.1

Percentage of cycles resulting in live births ${ }^{b, c}$

(Confidence Interval)

Percentage of retrievals resulting in live births ${ }^{b, c}$

Percentage of transfers resulting in live births ${ }^{b, c}$

(25.6-37.0)

34.4

35.3

21.7

Percentage of cancellations ${ }^{b}$

9.7

2.2

38.7

2.2

38.6

$(19.7-32.7)$

28.4

30.4

19.6

9.1

2.5

30.5

Percentage of pregnancies with twins ${ }^{b}$

Percentage of pregnancies with triplets or more

Percentage of live births having multiple infants ${ }^{b, c}$

\section{Frozen Embryos from Nondonor Eggs}

Number of transfers

Percentage of transfers resulting in live births ${ }^{\mathrm{b}, \mathrm{c}}$

Average number of embryos transferred

\section{Donor Eggs}

Number of transfers

Percentage of transfers resulting in live births ${ }^{b, c}$

Average number of embryos transferred

\section{CURRENT CLINIC SERVICES AND PROFILE}

Current Name: Reproductive Science Center of the San Francisco Bay Area
Donor egg? Yes
Donor embryo? Yes
Gestational carriers? Yes
Cryopreservation?
SART member?
Yes
Verified lab accreditation?
Yes
Single women? Yes
(See Appendix C for details.)

\footnotetext{
${ }^{a}$ Reflects patient and treatment characteristics of ART cycles performed in 2006 using fresh nondonor eggs or embryos.

b When fewer than 20 cycles are reported in an age category, rates are shown as a fraction and confidence intervals are not given. Calculating percentages from fractions may be misleading and is not encouraged.

${ }^{c}$ A multiple-infant birth is counted as one live birth.

d Clinic-specific outcome rates are unreliable for women older than 42 undergoing ART cycles using fresh or frozen embryos with nondonor eggs. Readers are urged to review national outcomes for these age groups (see page 27).

e All ages (including ages $>42$ ) are reported together because previous data show that patient age does not materially affect success with donor eggs.
} 


\section{PARKER-ROSENMAN-RODI GYNECOLOGY AND INFERTILITY MEDICAL GROUP SANTA MONICA, CALIFORNIA}

A comparison of clinic success rates may not be meaningful because patient medical characteristics and treatment approaches vary from clinic to clinic. For more details about this, along with information on how to interpret the statistics in this table, see pages $79-88$.

\section{ART CYCLE PROFILE}

$$
\text { Type of ART }{ }^{\mathrm{a}}
$$

\begin{tabular}{lll|llll} 
IVF & $100 \%$ Procedural Factors: & & Tubal factor & $6 \%$ & Other factor & $8 \%$ \\
GIFT & $0 \%$ With ICSI & $53 \%$ & Ovulatory dysfunction & $1 \%$ & Unknown factor & $1 \%$ \\
ZIFT & $0 \%$ Unstimulated & $0 \%$ & Diminished ovarian reserve & $27 \%$ & Multiple Factors: & \\
Combination & $0 \%$ Used gestational carrier & $1 \%$ & Endometriosis & $0 \%$ & Female factors only & $17 \%$ \\
& & & Uterine factor & $0 \%$ & Female \& male factors & $33 \%$ \\
& & & Male factor & $7 \%$ & &
\end{tabular}

2006 PREGNANCY SUCCESS RATES

Data verified by Ingrid A. Rodi, MD

\section{Type of Cycle}

\section{Fresh Embryos from Nondonor Eggs}

Number of cycles

Percentage of cycles resulting in pregnancies ${ }^{b}$

Percentage of cycles resulting in live births $s^{b, c}$

(Confidence Interval)

Percentage of retrievals resulting in live births $s^{b, c}$

Percentage of transfers resulting in live births $s^{b, c}$

Percentage of transfers resulting in singleton live births ${ }^{b}$

Percentage of cancellations ${ }^{b}$

Average number of embryos transferred

Percentage of pregnancies with twins ${ }^{b}$

Percentage of pregnancies with triplets or more ${ }^{b}$

Percentage of live births having multiple infants ${ }^{b, c}$

\section{Frozen Embryos from Nondonor Eggs}

Number of transfers

Percentage of transfers resulting in live births $s^{\mathrm{b}, \mathrm{c}}$

Average number of embryos transferred

\section{Donor Eggs}

Number of transfers

Percentage of transfers resulting in live births $s^{b, c}$

Average number of embryos transferred

\section{Age of Woman}

$<35 \quad 35-37 \quad 38-40 \quad 41-42^{d}$

$\begin{array}{llcc}14 & 7 & 28 & 16 \\ 6 / 14 & 2 / 7 & 39.3 & 3 / 16 \\ 5 / 14 & 2 / 7 & 28.6 & 2 / 16\end{array}$

(13.2-48.7)

$\begin{array}{cccc}5 / 13 & 2 / 7 & 30.8 & 2 / 12 \\ 5 / 11 & 2 / 7 & 32.0 & 2 / 11 \\ 3 / 11 & 1 / 7 & 16.0 & 1 / 11 \\ 1 / 14 & 0 / 7 & 7.1 & 4 / 16 \\ 2.7 & 3.4 & 3.5 & 4.2 \\ 3 / 6 & 0 / 2 & 4 / 11 & 1 / 3 \\ 0 / 6 & 1 / 2 & 1 / 11 & 0 / 3 \\ 2 / 5 & 1 / 2 & 4 / 8 & 1 / 2\end{array}$

0

5

$1 / 5$

2.6

All Ages Combined ${ }^{\mathrm{e}}$

Fresh Embryos

Frozen Embryos

\section{5}

$3 / 5$

5

$3 / 5$

2.2

\section{CURRENT CLINIC SERVICES AND PROFILE}

Current Name: Parker-Rosenman-Rodi Gynecology and Infertility Medical Group

\begin{tabular}{ll|ll|ll} 
Donor egg? & Yes & Gestational carriers? & Yes & SART member? & Yes \\
Donor embryo? & Yes & Cryopreservation? & Yes & Verified lab accreditation? & Yes \\
Single women? & Yes & & & (See Appendix C for details.)
\end{tabular}

${ }^{a}$ Reflects patient and treatment characteristics of ART cycles performed in 2006 using fresh nondonor eggs or embryos.

b When fewer than 20 cycles are reported in an age category, rates are shown as a fraction and confidence intervals are not given. Calculating percentages from fractions may be misleading and is not encouraged.

${ }^{c}$ A multiple-infant birth is counted as one live birth.

${ }^{d}$ Clinic-specific outcome rates are unreliable for women older than 42 undergoing ART cycles using fresh or frozen embryos with nondonor eggs. Readers are urged to review national outcomes for these age groups (see page 27).

e All ages (including ages $>42$ ) are reported together because previous data show that patient age does not materially affect success with donor eggs. 


\section{ADVANCED FERTILITY ASSOCIATES MEDICAL GROUP, INC. SANTA ROSA, CALIFORNIA}

A comparison of clinic success rates may not be meaningful because patient medical characteristics and treatment approaches vary from clinic to clinic. For more details about this, along with information on how to interpret the statistics in this table, see pages 79-88.

\section{ART CYCLE PROFILE}

\section{Type of ART ${ }^{\mathrm{a}}$}

IVF

GIFT

ZIFT

Combination
100\% Procedural Factors:

$0 \%$ With ICSI

$0 \%$ Unstimulated

$0 \%$ Used gestational carrier $<1 \%$
Tubal factor

Ovulatory dysfunction

Diminished ovarian reserve

Endometriosis

Uterine factor

Male factor

\section{Patient Diagnosis}

$\begin{array}{rlr}12 \% & \text { Other factor } & 6 \% \\ 2 \% & \text { Unknown factor } & 19 \% \\ 13 \% & \text { Multiple Factors: } & \\ 2 \% & \text { Female factors only } & 7 \% \\ <1 \% & \text { Female \& male factors } & 21 \% \\ 18 \% & & \end{array}$

Data verified by Jennfer V. Ratcliffe, MD, PhD

2006 PREGNANCY SUCCESS RATES

Age of Woman

Type of Cycle
$6 \%$

$19 \%$

$\%$ $41-42^{d}$ $<35$

41

\section{7}

43.9

18

24

15

Percentage of cycles resulting in pregnancies ${ }^{b}$

Percentage of cycles resulting in live births $s^{b, c}$

(Confidence Interval)

Percentage of retrievals resulting in live births $s^{b, c}$

Percentage of transfers resulting in live births $s^{b, c}$

Percentage of transfers resulting in singleton live births ${ }^{b}$

Percentage of cancellations ${ }^{b}$

(28.5-60.3)

48.6

48.6

29.7

9.8

2.2

27.3

4.5

$7 / 18$

$6 / 18$

$5 / 18$

41.7

$4 / 15$

Average number of embryos transferred

Percentage of pregnancies with twins ${ }^{b}$

Percentage of pregnancies with triplets or more ${ }^{b}$

Percentage of live births having multiple infants ${ }^{\mathrm{b}, \mathrm{c}}$

\section{Frozen Embryos from Nondonor Eggs}

Number of transfers

Percentage of transfers resulting in live births $s^{b, c}$

Average number of embryos transferred

\section{Donor Eggs}

Number of transfers

Percentage of transfers resulting in live births $s^{b, c}$

Average number of embryos transferred

$\begin{array}{ccc} & (15.6-55.3) & \\ 5 / 17 & 33.3 & 3 / 13 \\ 5 / 17 & 36.4 & 3 / 12 \\ 3 / 17 & 13.6 & 2 / 12 \\ 1 / 18 & 0.0 & 2 / 15 \\ 2.7 & 3.6 & 3.3 \\ 2 / 6 & 4 / 10 & 0 / 4 \\ 0 / 6 & 1 / 10 & 1 / 4 \\ 2 / 5 & 5 / 8 & 1 / 3\end{array}$

$\begin{array}{cccc}14 & 10 & 8 & 3 \\ 4 / 14 & 4 / 10 & 5 / 8 & 3 / 3 \\ 2.4 & 2.7 & 2.8 & 2.3\end{array}$

All Ages Combined ${ }^{\mathrm{e}}$
Fresh Embryos 13

$8 / 13$

2.1
Frozen Embryos 15

$1 / 15$

3.0

\section{CURRENT CLINIC SERVICES AND PROFILE}

Current Name: Advanced Fertility Associates Medical Group, Inc.

Donor egg? Yes Gestational carriers? Yes

Donor embryo? Yes

Single women? Yes

Cryopreservation? Yes

${ }_{b}^{a}$ Reflects patient and treatment characteristics of ART cycles performed in 2006 using fresh nondonor eggs or embryos.

${ }^{b}$ When fewer than 20 cycles are reported in an age category, rates are shown as a fraction and confidence intervals are not given. Calculating percentages from fractions may be misleading and is not encouraged.

${ }^{c}$ A multiple-infant birth is counted as one live birth.

d Clinic-specific outcome rates are unreliable for women older than 42 undergoing ART cycles using fresh or frozen embryos with nondonor eggs. Readers are urged to review national outcomes for these age groups (see page 27).

e All ages (including ages $>42$ ) are reported together because previous data show that patient age does not materially affect success with donor eggs. 


\section{VALLEY CENTER FOR REPRODUCTIVE HEALTH \\ TINA KOOPERSMITH, MD \\ SHERMAN OAKS, CALIFORNIA}

A comparison of clinic success rates may not be meaningful because patient medical characteristics and treatment approaches vary from clinic to clinic. For more details about this, along with information on how to interpret the statistics in this table, see pages 79-88.

\section{ART CYCLE PROFILE}

$$
\text { Type of ART }{ }^{\mathrm{a}}
$$

\begin{tabular}{|c|c|c|c|c|c|c|c|}
\hline IVF & $100 \%$ & Procedural Factors: & & Tubal factor & $13 \%$ & Other factor & $0 \%$ \\
\hline GIFT & $0 \%$ & With ICSI & $49 \%$ & Ovulatory dysfunction & $8 \%$ & Unknown factor & $8 \%$ \\
\hline ZIFT & $0 \%$ & Unstimulated & $0 \%$ & Diminished ovarian reserve & $7 \%$ & Multiple Factors: & \\
\hline \multirow[t]{3}{*}{ Combination } & $0 \%$ & Used gestational carrier & $2 \%$ & Endometriosis & $9 \%$ & Female factors only & \\
\hline & & & & Uterine factor & $0 \%$ & Female \& male factors & \\
\hline & & & & Male factor & $8 \%$ & & \\
\hline
\end{tabular}

2006 PREGNANCY SUCCESS RATES

Data verified by Tina B. Koopersmith, MD

\section{Type of Cycle}

\section{Fresh Embryos from Nondonor Eggs}

Number of cycles

Percentage of cycles resulting in pregnancies ${ }^{b}$

Percentage of cycles resulting in live births ${ }^{b, c}$

(Confidence Interval)

Percentage of retrievals resulting in live births $s^{b, c}$

Percentage of transfers resulting in live births $s^{b, c}$

Percentage of transfers resulting in singleton live births ${ }^{b}$

Percentage of cancellations ${ }^{b}$

Average number of embryos transferred

Percentage of pregnancies with twins ${ }^{b}$

Percentage of pregnancies with triplets or more ${ }^{b}$

Percentage of live births having multiple infants ${ }^{b, c}$

\section{Frozen Embryos from Nondonor Eggs}

Number of transfers

Percentage of transfers resulting in live births $s^{b, c}$

Average number of embryos transferred

\section{Donor Eggs}

Number of transfers

Percentage of transfers resulting in live births $s^{b, c}$

Average number of embryos transferred

\section{Age of Woman}

35-37 38-40

$41-42^{d}$

$<35$

16

14

10

$\begin{array}{cccc}13 & 16 & 14 & 10 \\ 6 / 13 & 6 / 16 & 3 / 14 & 2 / 10 \\ 5 / 13 & 6 / 16 & 3 / 14 & 2 / 10\end{array}$

13
$6 / 13$

$6 / 16$

$3 / 14$

$2 / 10$

$\begin{array}{cccc}5 / 12 & 6 / 15 & 3 / 13 & 2 / 10 \\ 5 / 11 & 6 / 13 & 3 / 11 & 2 / 9 \\ 4 / 11 & 3 / 13 & 1 / 11 & 1 / 9 \\ 1 / 13 & 1 / 16 & 1 / 14 & 0 / 10 \\ 2.3 & 3.3 & 3.0 & 3.1 \\ 2 / 6 & 4 / 6 & 2 / 3 & 1 / 2 \\ 0 / 6 & 1 / 6 & 0 / 3 & 1 / 2 \\ 1 / 5 & 3 / 6 & 2 / 3 & 1 / 2\end{array}$

$5 / 12$

$6 / 15$

$3 / 13$

$2 / 10$

/ 9

$1 / 9$

3.1

$1 / 2$

$1 / 2$

$1 / 2$

\section{6}

$2 / 6$

3

$2 / 3$

1

$0 / 1$

2.2

3.3

1.0

All Ages Combined ${ }^{\mathrm{e}}$

Fresh Embryos 8

$3 / 8$

2.3
Frozen Embryos

4

$1 / 4$

2.3

\section{CURRENT CLINIC SERVICES AND PROFILE}

Current Name: Valley Center for Reproductive Health, Tina Koopersmith, MD

Donor egg? Yes

Donor embryo? Yes

Single women? Yes

\section{Gestational carriers? Yes}

Cryopreservation?
SART member?

Verified lab accreditation?

(See Appendix C for details.)

${ }^{a}$ Reflects patient and treatment characteristics of ART cycles performed in 2006 using fresh nondonor eggs or embryos.

${ }^{b}$ When fewer than 20 cycles are reported in an age category, rates are shown as a fraction and confidence intervals are not given. Calculating percentages from fractions may be misleading and is not encouraged.

${ }^{c}$ A multiple-infant birth is counted as one live birth.

d Clinic-specific outcome rates are unreliable for women older than 42 undergoing ART cycles using fresh or frozen embryos with nondonor eggs. Readers are urged to review national outcomes for these age groups (see page 27).

e All ages (including ages $>42$ ) are reported together because previous data show that patient age does not materially affect success with donor eggs. 


\section{THE CENTER FOR FERTILITY AND GYNECOLOGY \\ VERMESH CENTER FOR FERTILITY \\ TARZANA, CALIFORNIA}

A comparison of clinic success rates may not be meaningful because patient medical characteristics and treatment approaches vary from clinic to clinic. For more details about this, along with information on how to interpret the statistics in this table, see pages 79-88.

\section{ART CYCLE PROFILE}

\section{Type of ART ${ }^{\mathrm{a}}$}

IVF

GIFT

ZIFT

Combination
91\% Procedural Factors:

$0 \%$ With ICSI

$0 \%$ Unstimulated

9\% Used gestational carrier
Tubal factor

Ovulatory dysfunction

$<1 \%$

$<1 \%$

Endometriosis

\section{Patient Diagnosis}

$6 \%$ Other factor

1\% Unknown factor

Diminished ovarian reserve $31 \%$ Multiple Factors:

Uterine factor

$<1 \%$ Female factors only

$1 \%$ Female \& male factors $13 \%$

Male factor $11 \%$

2006 PREGNANCY SUCCESS RATES

Data verified by Eliran Mor, MD

\section{Type of Cycle}

\section{Fresh Embryos from Nondonor Eggs}

Number of cycles

Percentage of cycles resulting in pregnancies ${ }^{b}$

Percentage of cycles resulting in live births ${ }^{b, c}$

(Confidence Interval)

Percentage of retrievals resulting in live births ${ }^{b, c}$

Percentage of transfers resulting in live births $s^{b, c}$

Percentage of transfers resulting in singleton live births ${ }^{b}$

Percentage of cancellations ${ }^{b}$

Average number of embryos transferred

Percentage of pregnancies with twins ${ }^{b}$

Percentage of pregnancies with triplets or more ${ }^{b}$

Percentage of live births having multiple infants ${ }^{b, c}$

\section{Frozen Embryos from Nondonor Eggs}

Number of transfers

Percentage of transfers resulting in live births ${ }^{b, c}$

Average number of embryos transferred

\section{Donor Eggs}

Number of transfers

Percentage of transfers resulting in live births $s^{b, c}$

Average number of embryos transferred

\section{Age of Woman}

35-37 $38-40 \quad 41-42^{d}$

\section{$<35$}

33

60.6

72

53

66.2

48.5

40.3

30.6

(47.1-72.0)

60.0

(30.8-66.5)

(20.2-42.5)

30.6

30.6

26.4

0.0

3.8

27.6

3.4

13.6

34.0

18.9

38.5

0.0

2.9

7.0

35.9

10.0

$10 / 16$

22

27.3

3.4

\section{5}

$2 / 5$

3.8

22

22.7

3.5

(9.4-32.0)

18.9

18.9

17.0

0.0

4.1

$2 / 18$

$0 / 18$

$1 / 10$

All Ages Combined ${ }^{\mathrm{e}}$

Fresh Embryos

48

62.5

3.1
Frozen Embryos 30

33.3

3.9

\section{CURRENT CLINIC SERVICES AND PROFILE}

Current Name: The Center for Fertility and Gynecology, Vermesh Center for Fertility

Donor egg? Yes

Donor embryo? Yes

Single women? Yes

\begin{abstract}
Gestational carriers? Yes
Cryopreservation? Yes
\end{abstract}

SART member?

Verified lab accreditation?

(See Appendix C for details.)
7

$1 / 7$

3.1
$14 \%$

$11 \%$

$10 \%$

$3 \%$

${ }^{a}$ Reflects patient and treatment characteristics of ART cycles performed in 2006 using fresh nondonor eggs or embryos.

${ }^{b}$ When fewer than 20 cycles are reported in an age category, rates are shown as a fraction and confidence intervals are not given. Calculating percentages from fractions may be misleading and is not encouraged.

${ }^{c}$ A multiple-infant birth is counted as one live birth.

d Clinic-specific outcome rates are unreliable for women older than 42 undergoing ART cycles using fresh or frozen embryos with nondonor eggs. Readers are urged to review national outcomes for these age groups (see page 27).

e All ages (including ages $>42$ ) are reported together because previous data show that patient age does not materially affect success with donor eggs. 


\section{TREE OF LIFE CENTER \\ SNUNIT BEN-OZER, MD \\ TARZANA, CALIFORNIA}

A comparison of clinic success rates may not be meaningful because patient medical characteristics and treatment approaches vary from clinic to clinic. For more details about this, along with information on how to interpret the statistics in this table, see pages 79-88.

\section{ART CYCLE PROFILE}

$$
\text { Type of ART }{ }^{\mathrm{a}}
$$

\begin{tabular}{|c|c|c|c|c|c|c|c|}
\hline IVF & $100 \%$ & Procedural Factors: & & Tubal factor & $0 \%$ & Other factor & $0 \%$ \\
\hline GIFT & $0 \%$ & With ICSI & $81 \%$ & Ovulatory dysfunction & $0 \%$ & Unknown factor & \\
\hline ZIFT & $0 \%$ & Unstimulated & $0 \%$ & Diminished ovarian reserve & $3 \%$ & Multiple Factors: & \\
\hline \multirow[t]{3}{*}{ Combination } & $0 \%$ & Used gestational carrier & $3 \%$ & Endometriosis & $0 \%$ & Female factors only & \\
\hline & & & & Uterine factor & $2 \%$ & Female \& male factors & \\
\hline & & & & Male factor & $5 \%$ & & \\
\hline
\end{tabular}

2006 PREGNANCY SUCCESS RATES

Data verified by Snunit Ben-Ozer, MD

\section{Type of Cycle}

\section{Fresh Embryos from Nondonor Eggs}

Number of cycles

Percentage of cycles resulting in pregnancies ${ }^{b}$

Percentage of cycles resulting in live births $s^{b, c}$

(Confidence Interval)

Percentage of retrievals resulting in live births $s^{b, c}$

Percentage of transfers resulting in live births $s^{b, c}$

Percentage of transfers resulting in singleton live births ${ }^{b}$

Percentage of cancellations ${ }^{b}$

Average number of embryos transferred

Percentage of pregnancies with twins ${ }^{b}$

Percentage of pregnancies with triplets or more ${ }^{b}$

Percentage of live births having multiple infants ${ }^{b, c}$

\section{Frozen Embryos from Nondonor Eggs}

Number of transfers

Percentage of transfers resulting in live births ${ }^{\mathrm{b}, \mathrm{c}}$

Average number of embryos transferred

\section{Donor Eggs}

Number of transfers

Percentage of transfers resulting in live births ${ }^{\mathrm{b}, \mathrm{c}}$

Average number of embryos transferred

\section{Age of Woman}

35-37 38-40

$41-42^{d}$

\section{$<35$}

5

11

8

$2 / 6$

$2 / 5$

$4 / 11$

$4 / 8$

$1 / 6$

$2 / 5$

$2 / 11$

$2 / 8$

$\begin{array}{cccc}1 / 6 & 2 / 5 & 2 / 10 & 2 / 8 \\ 1 / 5 & 2 / 5 & 2 / 9 & 2 / 8 \\ 0 / 5 & 1 / 5 & 1 / 9 & 2 / 8 \\ 0 / 6 & 0 / 5 & 1 / 11 & 0 / 8 \\ 2.6 & 3.6 & 4.3 & 4.0 \\ 1 / 2 & 1 / 2 & 1 / 4 & 0 / 4 \\ 0 / 2 & 0 / 2 & 0 / 4 & 0 / 4 \\ 1 / 1 & 1 / 2 & 1 / 2 & 0 / 2\end{array}$

3

4

$2 / 4$

$2 / 3$

3.3
Fresh Embryos

All Ages Combined 12

$6 / 12$

3.1
Frozen Embryos 8

$3 / 8$

2.8

\section{CURRENT CLINIC SERVICES AND PROFILE}

Current Name: Tree of Life Center, Snunit Ben-Ozer, MD

Donor egg? Yes

Donor embryo? Yes

Single women? Yes

\section{Gestational carriers? \\ Yes}

Cryopreservation?
Yes
SART member?

Yes

Verified lab accreditation?

(See Appendix C for details.)

${ }^{a}$ Reflects patient and treatment characteristics of ART cycles performed in 2006 using fresh nondonor eggs or embryos.

b When fewer than 20 cycles are reported in an age category, rates are shown as a fraction and confidence intervals are not given. Calculating percentages from fractions may be misleading and is not encouraged.

${ }^{\mathrm{C}}$ A multiple-infant birth is counted as one live birth.

d Clinic-specific outcome rates are unreliable for women older than 42 undergoing ART cycles using fresh or frozen embryos with nondonor eggs. Readers are urged to review national outcomes for these age groups (see page 27).

e All ages (including ages $>42$ ) are reported together because previous data show that patient age does not materially affect success with donor eggs. 


\section{FERTILITY AND SURGICAL ASSOCIATES OF CALIFORNIA THOUSAND OAKS, CALIFORNIA}

A comparison of clinic success rates may not be meaningful because patient medical characteristics and treatment approaches vary from clinic to clinic. For more details about this, along with information on how to interpret the statistics in this table, see pages 79-88.

\section{ART CYCLE PROFILE}

\begin{tabular}{|c|c|c|c|c|c|c|c|}
\hline \multicolumn{4}{|c|}{ Type of ART ${ }^{a}$} & \multicolumn{4}{|c|}{ Patient Diagnosis } \\
\hline IVF & $100 \%$ & Procedural Factors: & & Tubal factor & $5 \%$ & Other factor & $16 \%$ \\
\hline GIFT & $0 \%$ & With ICSI & $67 \%$ & Ovulatory dysfunction & $3 \%$ & Unknown factor & $3 \%$ \\
\hline ZIFT & $0 \%$ & Unstimulated & $0 \%$ & Diminished ovarian reserve & $17 \%$ & Multiple Factors: & \\
\hline \multirow[t]{3}{*}{ Combination } & $0 \%$ & Used gestational carrier & $1 \%$ & Endometriosis & $2 \%$ & Female factors only & $19 \%$ \\
\hline & & & & Uterine factor & $<1 \%$ & Female \& male factors & $22 \%$ \\
\hline & & & & Male factor & $13 \%$ & & \\
\hline
\end{tabular}

2006 PREGNANCY SUCCESS RATES

Data verified by Gary Hubert, MD

\section{Type of Cycle}

\section{Fresh Embryos from Nondonor Eggs}

Number of cycles

Percentage of cycles resulting in pregnancies ${ }^{b}$

Percentage of cycles resulting in live births ${ }^{b, c}$

(Confidence Interval)

Percentage of retrievals resulting in live births $s^{b, c}$

Percentage of transfers resulting in live births $s^{b, c}$

Percentage of transfers resulting in singleton live births ${ }^{b}$

Percentage of cancellations ${ }^{b}$

Average number of embryos transferred

Percentage of pregnancies with twins ${ }^{b}$

Percentage of pregnancies with triplets or more ${ }^{b}$

Percentage of live births having multiple infants ${ }^{b, c}$

Frozen Embryos from Nondonor Eggs

Number of transfers

Percentage of transfers resulting in live births $s^{\mathrm{b}, \mathrm{c}}$

Average number of embryos transferred

\section{Donor Eggs}

Number of transfers

Percentage of transfers resulting in live births $s^{b, c}$

Average number of embryos transferred

\section{Age of Woman}

35-37 38-40 $41-42^{d}$

\section{$<35$}

77

40.3

106

80

43.9

37.7

21.7

18.8

39.4

$(26.9-49.4)$

17.0

15.0

(31.0-48.3)

41.6

41.4

(10.4-25.5)

(8.0-24.7)

43.3

43.3

20.2

22.2

17.1

18.2

32.5

28.4

14.8

16.7

5.3

9.1

16.0

12.5

2.6

3.0

3.9

4.5

22.4

32.3

30.4

$2 / 15$

5.2

6.5

25.0

34.5

4.3

$0 / 15$

$6 / 18$

$1 / 12$

$\begin{array}{cccc}36 & 21 & 12 & 6 \\ 47.2 & 28.6 & 4 / 12 & 2 / 6 \\ 2.3 & 2.4 & 2.9 & 3.8\end{array}$

\section{All Ages Combined}

Fresh Embryos

75

54.7

2.5
Frozen Embryos

49

32.7

2.7

\section{CURRENT CLINIC SERVICES AND PROFILE}

Current Name: Fertility and Surgical Associates of California

Donor egg? Yes Gestational carriers? Yes

Donor embryo? Yes Cryopreservation? Yes

Single women? Yes

SART member? Yes

Verified lab accreditation? Yes

(See Appendix C for details.)

${ }^{a}$ Reflects patient and treatment characteristics of ART cycles performed in 2006 using fresh nondonor eggs or embryos.

${ }^{b}$ When fewer than 20 cycles are reported in an age category, rates are shown as a fraction and confidence intervals are not given. Calculating percentages from fractions may be misleading and is not encouraged.

${ }^{c}$ A multiple-infant birth is counted as one live birth.

d Clinic-specific outcome rates are unreliable for women older than 42 undergoing ART cycles using fresh or frozen embryos with nondonor eggs. Readers are urged to review national outcomes for these age groups (see page 27).

e All ages (including ages $>42$ ) are reported together because previous data show that patient age does not materially affect success with donor eggs. 


\section{PACIFIC REPRODUCTIVE CENTER TORRANCE, CALIFORNIA}

A comparison of clinic success rates may not be meaningful because patient medical characteristics and treatment approaches vary from clinic to clinic. For more details about this, along with information on how to interpret the statistics in this table, see pages 79-88.

\section{ART CYCLE PROFILE}

\section{Type of ART ${ }^{\mathrm{a}}$}

IVF

GIFT

$>99 \%$ Procedural Factors:

$<1 \%$ With ICSI

ZIFT

Combination $\begin{array}{ll}0 \% \text { Unstimulated } & <1 \% \\ 0 \% \text { Used gestational carrier } & <1 \%\end{array}$

\section{Patient Diagnosis}

$\begin{array}{rlr}16 \% & \text { Other factor } & 1 \% \\ 6 \% & \text { Unknown factor } & 5 \% \\ 27 \% & \text { Multiple Factors: } & \\ 5 \% & \text { Female factors only } & 5 \% \\ 5 \% & \text { Female \& male factors } & 6 \% \\ 24 \% & & \end{array}$

Data verified by Rifaat Salem, MD, PhD

2006 PREGNANCY SUCCESS RATES

\section{Age of Woman}

\section{Type of Cycle}

35-37 $38-40 \quad 41-42^{d}$

\section{Fresh Embryos from Nondonor Eggs}

Number of cycles $<35$

89

41.6

106

51

Percentage of cycles resulting in pregnancies ${ }^{b}$

Percentage of cycles resulting in live births ${ }^{b, c}$

52.1

32.6

23.6

11.8

(Confidence Interval)

Percentage of retrievals resulting in live births $s^{b, c}$

(23.0-43.3)

18.9

3.9

Percentage of transfers resulting in live births $s^{b, c}$

(39.0-54.6)

46.7

33.0

$(11.9-27.6)$

(0.5-13.5)

47.9

34.5

19.4

4.0

Percentage of transfers resulting in singleton live births ${ }^{b}$

31.9

26.2

20.6

4.7

0.0

1.1

13.4

4.7

Percentage of cancellations ${ }^{b}$

3.4

31.0

3.6

2.8

2.0

Average number of embryos transferred

Percentage of pregnancies with twins ${ }^{b}$

4.6

21.6

4.0

4.3

Percentage of pregnancies with triplets or more ${ }^{\mathrm{b}}$

Percentage of live births having multiple infants ${ }^{b, c}$

33.3

2.7

28.0

$0 / 6$

24.1

12.0

$0 / 6$

\section{Frozen Embryos from Nondonor Eggs}

Number of transfers

Percentage of transfers resulting in live births $s^{\mathrm{b}, \mathrm{c}}$

8

Average number of embryos transferred

$2 / 8$

4

35.0

$0 / 2$

3.6

$1 / 4$

3.3

\section{3}

$1 / 3$

2

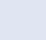

All Ages Combined

\section{Donor Eggs}

Fresh Embryos

Frozen Embryos

Number of transfers

Percentage of transfers resulting in live births $s^{b, c}$

3.8

$2 / 5$

Average number of embryos transferred

\section{CURRENT CLINIC SERVICES AND PROFILE}

Current Name: Pacific Reproductive Center
Donor egg? Yes
Donor embryo? No
Gestational carriers? Yes
Cryopreservation? Yes
Single women? Yes

$\begin{array}{ll}\text { SART member? } & \text { Yes } \\ \text { Verified lab accreditation? } & \text { Yes } \\ \text { (See Appendix C for details.) } & \end{array}$

${ }^{a}$ Reflects patient and treatment characteristics of ART cycles performed in 2006 using fresh nondonor eggs or embryos.

b When fewer than 20 cycles are reported in an age category, rates are shown as a fraction and confidence intervals are not given. Calculating percentages from fractions may be misleading and is not encouraged.

c A multiple-infant birth is counted as one live birth.

${ }^{d}$ Clinic-specific outcome rates are unreliable for women older than 42 undergoing ART cycles using fresh or frozen embryos with nondonor eggs. Readers are urged to review national outcomes for these age groups (see page 27).

e All ages (including ages $>42$ ) are reported together because previous data show that patient age does not materially affect success with donor eggs. 


\section{CONTRA COSTA OB/GYN \& INFERTILITY \\ WALNUT CREEK, CALIFORNIA}

A comparison of clinic success rates may not be meaningful because patient medical characteristics and treatment approaches vary from clinic to clinic. For more details about this, along with information on how to interpret the statistics in this table, see pages 79-88.

\section{ART CYCLE PROFILE}

\section{Type of ART ${ }^{\mathrm{a}}$}

IVF

GIFT

ZIFT

Combination
100\% Procedural Factors:

$0 \%$ With ICSI

$0 \%$ Unstimulated

$0 \%$ Used gestational carrier $100 \%$
Tubal factor

$39 \%$

$0 \%$

Ovulatory dysfunction

Diminished ovarian reserve

Endometriosis

Uterine factor

Male factor

\section{Patient Diagnosis}

$\begin{array}{rlr}18 \% & \text { Other factor } & 4 \% \\ 4 \% & \text { Unknown factor } & 14 \% \\ 11 \% & \text { Multiple Factors: } & \\ 7 \% & \text { Female factors only } & 7 \% \\ 0 \% & \text { Female \& male factors } & 11 \% \\ 25 \% & & \end{array}$

Data verified by Stephen G. Weinstein, MD

\section{Age of Woman}

Type of Cycle

$\begin{array}{cccc}<35 & \mathbf{3 5 - 3 7} & \mathbf{3 8 - 4 0} & \mathbf{4 1 - 4 2}^{\mathbf{d}} \\ 14 & 6 & 2 & 3 \\ 1 / 14 & 2 / 6 & 0 / 2 & 0 / 3 \\ 1 / 14 & 0 / 6 & 0 / 2 & 0 / 3\end{array}$

$1 / 13$

$0 / 5$

$0 / 2$

$0 / 1$

$1 / 13$

$0 / 5$

$0 / 2$

$0 / 1$

$1 / 13$

$0 / 5$

$0 / 2$

$0 / 1$

$1 / 14$

$1 / 6$

$0 / 2$

$2 / 3$

3.0

3.8

1.0

4.0

$0 / 1$

$0 / 2$

$0 / 1$

$0 / 2$

$0 / 1$

Percentage of pregnancies with triplets or more ${ }^{b}$

Percentage of live births having multiple infants ${ }^{b, c}$

\section{Frozen Embryos from Nondonor Eggs}

Number of transfers

Percentage of transfers resulting in live births $s^{b, c}$

0

$0 / 1$

Average number of embryos transferred

4.0

\section{Donor Eggs}

Number of transfers

Percentage of transfers resulting in live births $s^{b, c}$

Average number of embryos transferred

All Ages Combined ${ }^{\mathrm{e}}$

Fresh Embryos

1

$1 / 1$

4.0

\section{CURRENT CLINIC SERVICES AND PROFILE}

Current Name: Contra Costa OB/GYN \& Infertility

Donor egg? Yes Gestational carriers? Yes

Donor embryo? No Cryopreservation? Yes

Single women? Yes

SART member?

No

Verified lab accreditation?

No

${ }^{a}$ Reflects patient and treatment characteristics of ART cycles performed in 2006 using fresh nondonor eggs or embryos.

b When fewer than 20 cycles are reported in an age category, rates are shown as a fraction and confidence intervals are not given. Calculating percentages from fractions may be misleading and is not encouraged.

${ }^{\mathrm{C}}$ A multiple-infant birth is counted as one live birth.

d Clinic-specific outcome rates are unreliable for women older than 42 undergoing ART cycles using fresh or frozen embryos with nondonor eggs. Readers are urged to review national outcomes for these age groups (see page 27).

e All ages (including ages $>42$ ) are reported together because previous data show that patient age does not materially affect success with donor eggs. 


\section{ADVANCED REPRODUCTIVE MEDICINE UNIVERSITY OF COLORADO HEALTH SCIENCES CENTER AURORA, COLORADO}

A comparison of clinic success rates may not be meaningful because patient medical characteristics and treatment approaches vary from clinic to clinic. For more details about this, along with information on how to interpret the statistics in this table, see pages 79-88.

\section{ART CYCLE PROFILE}

\begin{tabular}{|c|c|c|c|c|c|c|c|}
\hline \multicolumn{4}{|c|}{ Type of ART ${ }^{\mathrm{a}}$} & \multicolumn{4}{|c|}{ Patient Diagnosis } \\
\hline IVF & $100 \%$ & Procedural Factors: & & Tubal factor & $10 \%$ & Other factor & $3 \%$ \\
\hline GIFT & $0 \%$ & With ICSI & $68 \%$ & Ovulatory dysfunction & $5 \%$ & Unknown factor & $7 \%$ \\
\hline $\mathrm{ZIFT}$ & $0 \%$ & Unstimulated & $0 \%$ & Diminished ovarian reserve & $23 \%$ & Multiple Factors: & \\
\hline Combination & $0 \%$ & Used gestational carrier & $0 \%$ & Endometriosis & $2 \%$ & Female factors only & $16 \%$ \\
\hline & & & & Uterine factor & $0 \%$ & Female \& male factors & $27 \%$ \\
\hline
\end{tabular}

2006 PREGNANCY SUCCESS RATES

Data verified by Ruben J. Alvero, MD

\section{Type of Cycle}

\section{Age of Woman}

35-37 38-40

$41-42^{d}$ $<35$

14

12

4

Number of cycles

46

52.2

50.0

$8 / 14$

$3 / 12$

$0 / 4$

Percentage of cycles resulting in live births ${ }^{b, c}$

(Confidence Interval)

Percentage of retrievals resulting in live births ${ }^{b, c}$

(34.9-65.1)

Percentage of transfers resulting in live births ${ }^{b, c}$

60.5

65.7

$6 / 14$

$3 / 12$

$0 / 4$

Percentage of transfers resulting in singleton live births ${ }^{b}$

Percentage of cancellations ${ }^{b}$

45.7

17.4

$6 / 12$

$3 / 10$

$0 / 2$

$6 / 11$

$3 / 9$

$0 / 2$

$5 / 11$

$2 / 9$

$0 / 2$

17.4

$2 / 14$

$2 / 12$

$2 / 4$

Average number of embryos transferred

Percentage of pregnancies with twins ${ }^{b}$

Percentage of pregnancies with triplets or more ${ }^{b}$

Percentage of live births having multiple infants ${ }^{b, c}$

\section{Frozen Embryos from Nondonor Eggs}

Number of transfers

Percentage of transfers resulting in live births ${ }^{b, c}$

Average number of embryos transferred

33.3

4.2
30.4

3.0

$3 / 8$

4.1

3.5

$1 / 8$

$2 / 3$

$0 / 3$

$1 / 6$

$1 / 3$
21

19.0

2.6

9
$2 / 9$

2.4
1

$0 / 1$

3.0
1

$0 / 1$

4.0

\section{Donor Eggs}

Number of transfers

Percentage of transfers resulting in live births ${ }^{b, c}$

Average number of embryos transferred

\section{Fresh Embryos}

$$
18
$$

$13 / 18$

2.1
All Ages Combined

Frozen Embryos

27

29.6

2.6

\section{CURRENT CLINIC SERVICES AND PROFILE}

Current Name: Advanced Reproductive Medicine, University of Colorado Health Sciences Center

Donor egg? Yes

Donor embryo? Yes

Single women? Yes

\begin{abstract}
Gestational carriers? Yes
Cryopreservation?
\end{abstract}

SART member?

Verified lab accreditation?

(See Appendix C for details.)

${ }^{a}$ Reflects patient and treatment characteristics of ART cycles performed in 2006 using fresh nondonor eggs or embryos.

b When fewer than 20 cycles are reported in an age category, rates are shown as a fraction and confidence intervals are not given. Calculating percentages from fractions may be misleading and is not encouraged.

${ }^{c}$ A multiple-infant birth is counted as one live birth.

d Clinic-specific outcome rates are unreliable for women older than 42 undergoing ART cycles using fresh or frozen embryos with nondonor eggs. Readers are urged to review national outcomes for these age groups (see page 27).

e All ages (including ages $>42$ ) are reported together because previous data show that patient age does not materially affect success with donor eggs. 


\section{REPRODUCTIVE MEDICINE AND FERTILITY CENTER COLORADO SPRINGS, COLORADO}

A comparison of clinic success rates may not be meaningful because patient medical characteristics and treatment approaches vary from clinic to clinic. For more details about this, along with information on how to interpret the statistics in this table, see pages 79-88.

\section{ART CYCLE PROFILE}

\section{Type of ART ${ }^{\mathrm{a}}$}

IVF

GIFT

100\% Procedural Factors:

ZIFT

Combination
$0 \%$ With ICSI

$0 \%$ Unstimulated

$0 \%$ Used gestational carrier

\begin{tabular}{|c|c|c|c|c|}
\hline & \multicolumn{4}{|c|}{ Patient Diagnosis } \\
\hline & Tubal factor & $3 \%$ & Other factor & $6 \%$ \\
\hline $86 \%$ & Ovulatory dysfunction & $5 \%$ & Unknown factor & $3 \%$ \\
\hline $0 \%$ & Diminished ovarian reserve & $12 \%$ & Multiple Factors: & \\
\hline $0 \%$ & Endometriosis & $2 \%$ & Female factors only & $8 \%$ \\
\hline & Uterine factor & $<1 \%$ & Female \& male factors & $48 \%$ \\
\hline & Male factor & $12 \%$ & & \\
\hline
\end{tabular}

2006 PREGNANCY SUCCESS RATES

Data verified by Paul C. Magarelli, MD, PhD

\section{Type of Cycle}

\section{Age of Woman}

35-37 38-40

$41-42^{d}$

\section{$<35$}

40

30

21

Number of cycles

71

27.5

23.3

4.8

Percentage of cycles resulting in live births ${ }^{b, c}$

31.0

22.5

20.0

4.8

(Confidence Interval)

Percentage of retrievals resulting in live births $s^{b, c}$

(16.9-38.6)

28.8

(10.8-38.5)

(7.7-38.6)

(0.1-23.8)

38.8

28.1

21.4

30.0

$1 / 16$

45.0

20.0

$1 / 12$

35.0

6.7

$1 / 12$

7.0

20.0

3.3

\section{$2 / 11$}

$1 / 11$

2.8

23.8

4.5

13.6

$2 / 9$

$2 / 7$

3.2

$4 / 19$

$0 / 7$

$0 / 1$

$0 / 1$

$2 / 6$

$0 / 1$

$\begin{array}{cccc}17 & 4 & 2 & 2 \\ 8 / 17 & 2 / 4 & 0 / 2 & 0 / 2 \\ 2.9 & 2.8 & 2.0 & 2.0\end{array}$

All Ages Combined ${ }^{\mathrm{e}}$

Fresh Embryos

Frozen Embryos

12

$6 / 12$

8

Number of transfers

Percentage of transfers resulting in live births $s^{b, c}$

Average number of embryos transferred

\section{CURRENT CLINIC SERVICES AND PROFILE}

Current Name: Reproductive Medicine and Fertility Center

Donor egg? Yes Gestational carriers? Yes

Donor embryo? Yes Cryopreservation? Yes

Single women? Yes

SART member? Yes

Verified lab accreditation? Yes

(See Appendix C for details.)

${ }^{a}$ Reflects patient and treatment characteristics of ART cycles performed in 2006 using fresh nondonor eggs or embryos.

${ }^{b}$ When fewer than 20 cycles are reported in an age category, rates are shown as a fraction and confidence intervals are not given. Calculating percentages from fractions may be misleading and is not encouraged.

${ }^{c}$ A multiple-infant birth is counted as one live birth.

d Clinic-specific outcome rates are unreliable for women older than 42 undergoing ART cycles using fresh or frozen embryos with nondonor eggs. Readers are urged to review national outcomes for these age groups (see page 27).

e All ages (including ages $>42$ ) are reported together because previous data show that patient age does not materially affect success with donor eggs. 


\section{ERIC H. SILVERSTEIN, MD, PROFESSIONAL LLC, DBA \\ THE FERTILITY CENTER OF COLORADO \\ COLORADO SPRINGS, COLORADO}

A comparison of clinic success rates may not be meaningful because patient medical characteristics and treatment approaches vary from clinic to clinic. For more details about this, along with information on how to interpret the statistics in this table, see pages $79-88$.

\section{ART CYCLE PROFILE}

\begin{tabular}{|c|c|c|c|c|c|c|c|}
\hline \multicolumn{4}{|c|}{ Type of ART } & \multicolumn{4}{|c|}{ Patient Diagnosis } \\
\hline IVF & $100 \%$ & Procedural Factors: & & Tubal factor & $8 \%$ & Other factor & $1 \%$ \\
\hline GIFT & $0 \%$ & With ICSI & $76 \%$ & Ovulatory dysfunction & $3 \%$ & Unknown factor & $6 \%$ \\
\hline ZIFT & $0 \%$ & Unstimulated & $0 \%$ & Diminished ovarian reserve & $16 \%$ & Multiple Factors: & \\
\hline \multirow[t]{3}{*}{ Combination } & $0 \%$ & Used gestational carrier & $0 \%$ & Endometriosis & $8 \%$ & Female factors only & $16 \%$ \\
\hline & & & & Uterine factor & $0 \%$ & Female \& male factors & $26 \%$ \\
\hline & & & & Male factor & $15 \%$ & & \\
\hline
\end{tabular}

2006 PREGNANCY SUCCESS RATES

Data verified by Eric H. Silverstein, MD

\section{Type of Cycle}

\section{Fresh Embryos from Nondonor Eggs}

Number of cycles

Percentage of cycles resulting in pregnancies ${ }^{b}$

Percentage of cycles resulting in live births ${ }^{b, c}$

(Confidence Interval)

Percentage of retrievals resulting in live births $s^{b, c}$

Percentage of transfers resulting in live births $s^{b, c}$

Percentage of transfers resulting in singleton live births ${ }^{b}$

Percentage of cancellations ${ }^{b}$

Average number of embryos transferred

Percentage of pregnancies with twins ${ }^{b}$

Percentage of pregnancies with triplets or more ${ }^{b}$

Percentage of live births having multiple infants ${ }^{b, c}$

\section{Frozen Embryos from Nondonor Eggs}

Number of transfers

Percentage of transfers resulting in live births ${ }^{\mathrm{b}, \mathrm{c}}$

Average number of embryos transferred

\section{Donor Eggs}

Number of transfers

Percentage of transfers resulting in live births $s^{\mathrm{b}, \mathrm{c}}$

Average number of embryos transferred

\section{Age of Woman}

35-37 38-40

$41-42^{d}$

\section{$<35$}

9

$4 / 9$

$4 / 9$

9

2

69.2

61.5

$(40.6-79.8)$

64.0

$4 / 8$

$4 / 9$

$0 / 2$

66.7

$4 / 8$

$3 / 7$

$3 / 6$

$0 / 2$

41.7

$4 / 8$

$3 / 6$

$0 / 2$

3.8

$1 / 9$

$2 / 9$

$0 / 2$

2.1

2.4

2.0

$0 / 2$

$6 / 18$

$0 / 4$

$0 / 4$

$1 / 18$

$0 / 4$

$0 / 4$

$6 / 16$

$0 / 4$

$0 / 3$

$\begin{array}{cccc}5 & 1 & 4 & 1 \\ 1 / 5 & 0 / 1 & 1 / 4 & 0 / 1 \\ 2.2 & 1.0 & 3.0 & 2.0\end{array}$

\section{All Ages Combined $^{\mathrm{e}}$}

Fresh Embryos

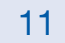

$5 / 11$

1.9
Frozen Embryos

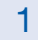

$0 / 1$

3.0

\section{CURRENT CLINIC SERVICES AND PROFILE}

Current Name: Eric H. Silverstein, MD, Professional LLC, dba The Fertility Center of Colorado

Donor egg? Yes

Donor embryo? No

Single women? Yes

\section{Gestational carriers? No}

Cryopreservation?

\section{SART member?}

Verified lab accreditation?

(See Appendix C for details.)

${ }^{a}$ Reflects patient and treatment characteristics of ART cycles performed in 2006 using fresh nondonor eggs or embryos.

${ }^{b}$ When fewer than 20 cycles are reported in an age category, rates are shown as a fraction and confidence intervals are not given. Calculating percentages from fractions may be misleading and is not encouraged.

${ }^{c}$ A multiple-infant birth is counted as one live birth.

${ }^{d}$ Clinic-specific outcome rates are unreliable for women older than 42 undergoing ART cycles using fresh or frozen embryos with nondonor eggs. Readers are urged to review national outcomes for these age groups (see page 27).

e All ages (including ages $>42$ ) are reported together because previous data show that patient age does not materially affect success with donor eggs. 


\section{COLORADO REPRODUCTIVE ENDOCRINOLOGY DENVER, COLORADO}

A comparison of clinic success rates may not be meaningful because patient medical characteristics and treatment approaches vary from clinic to clinic. For more details about this, along with information on how to interpret the statistics in this table, see pages 79-88.

\section{ART CYCLE PROFILE}

\begin{tabular}{|c|c|c|c|c|c|c|c|}
\hline \multicolumn{4}{|c|}{ Type of ART ${ }^{a}$} & \multicolumn{4}{|c|}{ Patient Diagnosis } \\
\hline IVF & $100 \%$ & Procedural Factors: & & Tubal factor & $14 \%$ & Other factor & $5 \%$ \\
\hline GIFT & $0 \%$ & With ICSI & $24 \%$ & Ovulatory dysfunction & $12 \%$ & Unknown factor & $16 \%$ \\
\hline ZIFT & $0 \%$ & Unstimulated & $0 \%$ & Diminished ovarian reserve & $21 \%$ & Multiple Factors: & \\
\hline \multirow[t]{3}{*}{ Combination } & $0 \%$ & Used gestational carrier & $0 \%$ & Endometriosis & $4 \%$ & Female factors only & $13 \%$ \\
\hline & & & & Uterine factor & $2 \%$ & Female \& male factors & $6 \%$ \\
\hline & & & & Male factor & $8 \%$ & & \\
\hline
\end{tabular}

2006 PREGNANCY SUCCESS RATES

Data verified by Susan W. Trout, MD

\section{Type of Cycle}

\section{Fresh Embryos from Nondonor Eggs}

Number of cycles

Percentage of cycles resulting in pregnancies ${ }^{b}$

Percentage of cycles resulting in live births $s^{b, c}$

(Confidence Interval)

Percentage of retrievals resulting in live births $s^{b, c}$

Percentage of transfers resulting in live births $s^{b, c}$

Percentage of transfers resulting in singleton live births ${ }^{b}$

Percentage of cancellations ${ }^{b}$

Average number of embryos transferred

Percentage of pregnancies with twins ${ }^{b}$

Percentage of pregnancies with triplets or more ${ }^{b}$

Percentage of live births having multiple infants ${ }^{\mathrm{b}, \mathrm{c}}$

\section{Frozen Embryos from Nondonor Eggs}

Number of transfers

Percentage of transfers resulting in live births $s^{b, c}$

Average number of embryos transferred

\section{Donor Eggs}

Number of transfers

Percentage of transfers resulting in live births $s^{b, c}$

Average number of embryos transferred

\section{Age of Woman}

$$
\text { 35-37 38-40 }
$$

$41-42^{d}$

$<35$

36

28

13

61
42.6

27.8

28.6

$0 / 13$

41.0

19.4

14.3

$0 / 13$

$(28.6-54.3)$

(8.2-36.0)

$(4.0-32.7)$

52.1

25.9

19.0

$0 / 7$

54.3

28.0

20.0

$0 / 6$

20.0

10.0

$0 / 6$

21.3

25.0

25.0

$6 / 13$

2.0

2.8

3.0

2.8

50.0

$3 / 10$

$2 / 8$

3.8

$0 / 10$

$0 / 8$

48.0

$2 / 7$

$2 / 4$

19

$7 / 19$

1.9

\section{9}

$4 / 9$

1.9

\section{9}

$5 / 9$

2.8

$0 / 3$

2.0

\section{CURRENT CLINIC SERVICES AND PROFILE}

Fresh Embryos

All Ages Combined

Frozen Embryos

$\begin{array}{cc}18 & 17 \\ 11 / 18 & 6 / 17 \\ 2.0 & 1.8\end{array}$

Current Name: Colorado Reproductive Endocrinology

Donor egg? Yes Gestational carriers? Yes

Donor embryo? Yes Cryopreservation? Yes

Single women? Yes

SART member? Yes

Verified lab accreditation? Yes

(See Appendix C for details.)

${ }^{a}$ Reflects patient and treatment characteristics of ART cycles performed in 2006 using fresh nondonor eggs or embryos.

${ }^{b}$ When fewer than 20 cycles are reported in an age category, rates are shown as a fraction and confidence intervals are not given. Calculating percentages from fractions may be misleading and is not encouraged.

${ }^{c}$ A multiple-infant birth is counted as one live birth.

${ }^{d}$ Clinic-specific outcome rates are unreliable for women older than 42 undergoing ART cycles using fresh or frozen embryos with nondonor eggs. Readers are urged to review national outcomes for these age groups (see page 27).

e All ages (including ages $>42$ ) are reported together because previous data show that patient age does not materially affect success with donor eggs. 


\section{COLORADO CENTER FOR REPRODUCTIVE MEDICINEO ENGLEWOOD, COLORADO}

A comparison of clinic success rates may not be meaningful because patient medical characteristics and treatment approaches vary from clinic to clinic. For more details about this, along with information on how to interpret the statistics in this table, see pages $79-88$.

\section{ART CYCLE PROFILE}

\begin{tabular}{|c|c|c|c|c|c|c|c|}
\hline & Type & of $A R T^{a}$ & & Pat & ent D & iagnosis & \\
\hline IVF & $100 \%$ & Procedural Factors: & & Tubal factor & $5 \%$ & Other factor & $12 \%$ \\
\hline GIFT & $0 \%$ & With ICSI & $78 \%$ & Ovulatory dysfunction & $5 \%$ & Unknown factor & $13 \%$ \\
\hline $\mathrm{ZIFT}$ & $0 \%$ & Unstimulated & $<1 \%$ & Diminished ovarian reserve & $30 \%$ & Multiple Factors: & \\
\hline Combination & $0 \%$ & Used gestational carrier & $2 \%$ & Endometriosis & $5 \%$ & Female factors only & $6 \%$ \\
\hline & & & & Uterine factor & $2 \%$ & Female \& male factors & $8 \%$ \\
\hline
\end{tabular}

2006 PREGNANCY SUCCESS RATES

Data verified by William B. Schoolcraft, MD

\section{Type of Cycle}

\section{Fresh Embryos from Nondonor Eggs}

Number of cycles

Percentage of cycles resulting in pregnancies ${ }^{b}$

Percentage of cycles resulting in live births ${ }^{b, c}$

(Confidence Interval)

Percentage of retrievals resulting in live births $s^{b, c}$

Percentage of transfers resulting in live births ${ }^{b, c}$

Percentage of transfers resulting in singleton live births ${ }^{b}$

Percentage of cancellations ${ }^{b}$

Average number of embryos transferred

Percentage of pregnancies with twins ${ }^{b}$

Percentage of pregnancies with triplets or more ${ }^{b}$

Percentage of live births having multiple infants ${ }^{b, c}$

\section{Frozen Embryos from Nondonor Eggs}

Number of transfers

Percentage of transfers resulting in live births ${ }^{\mathrm{b}, \mathrm{c}}$

Average number of embryos transferred

\section{Donor Eggs}

Number of transfers

Percentage of transfers resulting in live births ${ }^{\mathrm{b}, \mathrm{c}}$

Average number of embryos transferred

\section{Age of Woman}

35-37 38-40

$41-42^{d}$

$<35$

195

166

76

71.3

59.5

53.0

35.5

62.2

49.7

41.0

26.3

(56.2-67.9)

62.6

(42.5-57.0)

(33.4-48.9)

(16.9-37.7)

65.3

52.4

43.0

44.4

27.0

53.6

32.0

28.2

37.8

37.0

4.8

23.9

0.7

5.1

3.1

27.3

8.0

27.9

2.6

3.6

18.5

25.9

7.8

30.9

42.1

85

48.2

47

53.2

26

42.3

8

2.4

2.4

2.8

4 / 8

2.6

All Ages Combined ${ }^{\mathrm{e}}$

Fresh Embryos

201

79.6

2.1
Frozen Embryos 79

45.6

2.3

\section{CURRENT CLINIC SERVICES AND PROFILE}

Current Name: Colorado Center for Reproductive Medicine

Donor egg? Yes Gestational carriers? Yes

Donor embryo? Yes Cryopreservation? Yes

Single women? Yes

SART member? Yes

Verified lab accreditation? Yes

(See Appendix C for details.)

${ }_{b}^{a}$ Reflects patient and treatment characteristics of ART cycles performed in 2006 using fresh nondonor eggs or embryos.

When fewer than 20 cycles are reported in an age category, rates are shown as a fraction and confidence intervals are not given. Calculating

c percentages from fractions may be misleading and is not encouraged.

d A multiple-infant birth is counted as one live birth.

Clinic-specific outcome rates are unreliable for women older than 42 undergoing ART cycles using fresh or frozen embryos with nondonor

e eggs. Readers are urged to review national outcomes for these age groups (see page 27).

All ages (including ages $>42$ ) are reported together because previous data show that patient age does not materially affect

success with donor eggs.

- Reflects clinic performed more than 50 cycles with Preimplantation Genetic Diagnosis (PGD) in 2006 and among them more than 10 specifically for the purpose of prevention of genetic disorders. See Appendix $C$ for a complete list of clinics with symbol. 


\section{ROCKY MOUNTAIN CENTER FOR REPRODUCTIVE MEDICINE FORT COLLINS, COLORADO}

A comparison of clinic success rates may not be meaningful because patient medical characteristics and treatment approaches vary from clinic to clinic. For more details about this, along with information on how to interpret the statistics in this table, see pages 79-88.

\section{ART CYCLE PROFILE}

\begin{tabular}{|c|c|c|c|c|c|c|c|}
\hline \multicolumn{4}{|c|}{ Type of ART ${ }^{a}$} & \multicolumn{4}{|c|}{ Patient Diagnosis } \\
\hline IVF & $100 \%$ & Procedural Factors: & & Tubal factor & $14 \%$ & Other factor & $0 \%$ \\
\hline GIFT & $0 \%$ & With ICSI & $85 \%$ & Ovulatory dysfunction & $3 \%$ & Unknown factor & $14 \%$ \\
\hline ZIFT & $0 \%$ & Unstimulated & $0 \%$ & Diminished ovarian reserve & $23 \%$ & Multiple Factors: & \\
\hline \multirow[t]{3}{*}{ Combination } & $0 \%$ & Used gestational carrier & $0 \%$ & Endometriosis & $10 \%$ & Female factors only & $5 \%$ \\
\hline & & & & Uterine factor & $0 \%$ & Female \& male factors & $14 \%$ \\
\hline & & & & Male factor & $19 \%$ & & \\
\hline
\end{tabular}

2006 PREGNANCY SUCCESS RATES

Data verified by Kevin E. Bachus, MD

\section{Type of Cycle}

\section{Fresh Embryos from Nondonor Eggs}

Number of cycles

Percentage of cycles resulting in pregnancies ${ }^{b}$

Percentage of cycles resulting in live births ${ }^{b, c}$

(Confidence Interval)

Percentage of retrievals resulting in live births $s^{b, c}$

Percentage of transfers resulting in live births $s^{b, c}$

Percentage of transfers resulting in singleton live births ${ }^{b}$

Percentage of cancellations ${ }^{b}$

Average number of embryos transferred

Percentage of pregnancies with twins ${ }^{b}$

Percentage of pregnancies with triplets or more ${ }^{b}$

Percentage of live births having multiple infants ${ }^{b, c}$

\section{Frozen Embryos from Nondonor Eggs}

Number of transfers

Percentage of transfers resulting in live births ${ }^{b, c}$

Average number of embryos transferred

\section{Donor Eggs}

Number of transfers

Percentage of transfers resulting in live births $s^{b, c}$

Average number of embryos transferred

\section{Age of Woman}

35-37 38-40

$41-42^{d}$

\section{$<35$}

12

$6 / 12$

$6 / 12$

5

$3 / 5$

$2 / 5$

/

51.7

44.8

(26.4-64.3)

48.1

52.0

$6 / 12$

$2 / 5$

$2 / 5$

$1 / 5$

$4 / 12$

$0 / 5$

3.6

$1 / 3$

$0 / 3$

2.6

$2 / 6$

$0 / 6$

$2 / 6$

$1 / 2$

$1 / 1$

1

$6 / 13$

\section{5}

$4 / 5$

2.6

3

$2 / 3$

$4 / 8$

3.0

All Ages Combined ${ }^{\mathrm{e}}$

Fresh Embryos

Frozen Embryos

\section{5}

$4 / 5$

2.0
$2 / 5$

2.4

\section{CURRENT CLINIC SERVICES AND PROFILE}

Current Name: Rocky Mountain Center for Reproductive Medicine

Donor egg? Yes Gestational carriers? No

Donor embryo? No Cryopreservation? Yes

Single women? Yes

SART member? Yes

Verified lab accreditation? Yes

(See Appendix C for details.)

${ }^{a}$ Reflects patient and treatment characteristics of ART cycles performed in 2006 using fresh nondonor eggs or embryos.

${ }^{b}$ When fewer than 20 cycles are reported in an age category, rates are shown as a fraction and confidence intervals are not given. Calculating percentages from fractions may be misleading and is not encouraged.

${ }^{c}$ A multiple-infant birth is counted as one live birth.

d Clinic-specific outcome rates are unreliable for women older than 42 undergoing ART cycles using fresh or frozen embryos with nondonor eggs. Readers are urged to review national outcomes for these age groups (see page 27).

e All ages (including ages $>42$ ) are reported together because previous data show that patient age does not materially affect success with donor eggs. 


\section{CONCEPTIONS REPRODUCTIVE ASSOCIATES OF COLORADO LITTLETON, COLORADO}

A comparison of clinic success rates may not be meaningful because patient medical characteristics and treatment approaches vary from clinic to clinic. For more details about this, along with information on how to interpret the statistics in this table, see pages $79-88$.

\section{ART CYCLE PROFILE}

\begin{tabular}{|c|c|c|c|c|c|c|c|}
\hline & Type & of $A R T^{a}$ & & Pat & ent D & iagnosis & \\
\hline IVF & $100 \%$ & Procedural Factors: & & Tubal factor & $8 \%$ & Other factor & $4 \%$ \\
\hline GIFT & $0 \%$ & With ICSI & $24 \%$ & Ovulatory dysfunction & $11 \%$ & Unknown factor & $15 \%$ \\
\hline ZIFT & $0 \%$ & Unstimulated & $0 \%$ & Diminished ovarian reserve & $23 \%$ & Multiple Factors: & \\
\hline Combination & $0 \%$ & Used gestational carrier & $0 \%$ & Endometriosis & $2 \%$ & Female factors only & $10 \%$ \\
\hline & & & & Uterine factor & $<1 \%$ & Female \& male factors & $18 \%$ \\
\hline
\end{tabular}

2006 PREGNANCY SUCCESS RATES

Data verified by Bruce H. Albrecht, MD

\section{Type of Cycle}

\section{Fresh Embryos from Nondonor Eggs}

Number of cycles

Percentage of cycles resulting in pregnancies ${ }^{b}$

Percentage of cycles resulting in live births $s^{b, c}$

(Confidence Interval)

Percentage of retrievals resulting in live births ${ }^{b, c}$

Percentage of transfers resulting in live births $s^{b, c}$

Percentage of transfers resulting in singleton live births ${ }^{b}$

Percentage of cancellations ${ }^{b}$

Average number of embryos transferred

Percentage of pregnancies with twins ${ }^{b}$

Percentage of pregnancies with triplets or more ${ }^{b}$

Percentage of live births having multiple infants ${ }^{b, c}$

\section{Frozen Embryos from Nondonor Eggs}

Number of transfers

Percentage of transfers resulting in live births $s^{b, c}$

Average number of embryos transferred

\section{Donor Eggs}

Number of transfers

Percentage of transfers resulting in live births ${ }^{\mathrm{b}, \mathrm{c}}$

Average number of embryos transferred

\section{Age of Woman}

35-37 38-40

$41-42^{d}$

\section{$<35$}

64

42

16

91
54.9

51.6

47.6

$5 / 16$

46.2

43.8

38.1

$3 / 16$

(35.6-56.9)

47.2

(31.4-56.7)

(23.6-54.4)

51.9

50.0

44.4

50.9

45.7

$3 / 13$

33.3

32.7

40.0

$3 / 13$

$3 / 13$

2.2

12.5

14.3

$3 / 16$

2.1

2.5

38.0

27.3

0.0

6.1

35.7

35.7

2.5

2.8

15.0

$0 / 5$

0.0

$0 / 5$

$2 / 16$

$0 / 3$

30

56.7

13

$8 / 13$

1.8

4

2

2.0

2 / 4

$0 / 2$

All Ages Combined ${ }^{\mathrm{e}}$

Fresh Embryos

Frozen Embryos

40

70.0

12

2.0

$6 / 12$

1.7

\section{CURRENT CLINIC SERVICES AND PROFILE}

Current Name: Conceptions Reproductive Associates of Colorado

\begin{tabular}{l|l|l} 
Donor egg? Yes & Gestational carriers? Yes
\end{tabular}

Donor embryo? No Cryopreservation? Yes

Single women? Yes

SART member? Yes

Verified lab accreditation? Yes

(See Appendix C for details.)

${ }^{a}$ Reflects patient and treatment characteristics of ART cycles performed in 2006 using fresh nondonor eggs or embryos.

${ }^{b}$ When fewer than 20 cycles are reported in an age category, rates are shown as a fraction and confidence intervals are not given. Calculating percentages from fractions may be misleading and is not encouraged.

${ }^{c}$ A multiple-infant birth is counted as one live birth.

${ }^{d}$ Clinic-specific outcome rates are unreliable for women older than 42 undergoing ART cycles using fresh or frozen embryos with nondonor eggs. Readers are urged to review national outcomes for these age groups (see page 27).

e All ages (including ages $>42$ ) are reported together because previous data show that patient age does not materially affect success with donor eggs. 


\section{CONNECTICUT FERTILITY ASSOCIATES \\ BRIDGEPORT, CONNECTICUT}

A comparison of clinic success rates may not be meaningful because patient medical characteristics and treatment approaches vary from clinic to clinic. For more details about this, along with information on how to interpret the statistics in this table, see pages 79-88.

\section{ART CYCLE PROFILE}

\begin{tabular}{|c|c|c|c|c|c|c|c|}
\hline \multicolumn{4}{|c|}{ Type of ART ${ }^{a}$} & \multicolumn{4}{|c|}{ Patient Diagnosis } \\
\hline IVF & $100 \%$ & Procedural Factors: & & Tubal factor & $13 \%$ & Other factor & $15 \%$ \\
\hline GIFT & $0 \%$ & With ICSI & $87 \%$ & Ovulatory dysfunction & $7 \%$ & Unknown factor & $16 \%$ \\
\hline ZIFT & $0 \%$ & Unstimulated & $0 \%$ & Diminished ovarian reserve & $17 \%$ & Multiple Factors: & \\
\hline \multirow[t]{3}{*}{ Combination } & $0 \%$ & Used gestational carrier & $1 \%$ & Endometriosis & $8 \%$ & Female factors only & $5 \%$ \\
\hline & & & & Uterine factor & $1 \%$ & Female \& male factors & $5 \%$ \\
\hline & & & & Male factor & $14 \%$ & & \\
\hline
\end{tabular}

2006 PREGNANCY SUCCESS RATES

Data verified by Michael B. Doyle, MD

\section{Type of Cycle}

\section{Fresh Embryos from Nondonor Eggs}

Number of cycles

Percentage of cycles resulting in pregnancies ${ }^{b}$

Percentage of cycles resulting in live births $s^{b, c}$

(Confidence Interval)

Percentage of retrievals resulting in live births $s^{b, c}$

Percentage of transfers resulting in live births $s^{b, c}$

Percentage of transfers resulting in singleton live births ${ }^{b}$

Percentage of cancellations ${ }^{b}$

Average number of embryos transferred

Percentage of pregnancies with twins ${ }^{b}$

Percentage of pregnancies with triplets or more ${ }^{b}$

Percentage of live births having multiple infants ${ }^{b, c}$

\section{Frozen Embryos from Nondonor Eggs}

Number of transfers

Percentage of transfers resulting in live births ${ }^{b, c}$

Average number of embryos transferred

\section{Donor Eggs}

Number of transfers

Percentage of transfers resulting in live births $s^{b, c}$

Average number of embryos transferred

\section{Age of Woman}

35-37 38-40

$41-42^{d}$

$<35$

72

25.0

74

24

29.2

24.7

19.4

16.2

25.0

(16.2-35.0)

25.3

(11.1-30.5)

14.9

20.8

26.2

20.6

(7.7-25.0)

(7.1-42.2)

15.5

17.7

20.8

21.5

14.5

$5 / 19$

20.0

4.1

$4 / 19$

2.2

5.6

2.5

2.7

0.0

2.4

$1 / 18$

$2 / 12$

3.0

19.2

$0 / 18$

$0 / 12$

$1 / 6$

3.8

$1 / 14$

$2 / 11$

$0 / 6$

$1 / 5$

26

42.3

2.4

\section{6}

$2 / 6$

2.2

\section{3}

$0 / 3$

2.3
1

$0 / 1$

3.0

All Ages Combined ${ }^{\mathrm{e}}$

Fresh Embryos

48

60.4

2.6
Frozen Embryos 12

4 / 12

2.6

\section{CURRENT CLINIC SERVICES AND PROFILE}

Current Name: Connecticut Fertility Associates

Donor egg? Yes

Donor embryo? No

Single women? Yes

\section{Gestational carriers? Yes}

Cryopreservation? Yes
SART member?

Yes

Verified lab accreditation?

(See Appendix C for details.)

${ }^{a}$ Reflects patient and treatment characteristics of ART cycles performed in 2006 using fresh nondonor eggs or embryos.

b When fewer than 20 cycles are reported in an age category, rates are shown as a fraction and confidence intervals are not given. Calculating percentages from fractions may be misleading and is not encouraged.

${ }^{c}$ A multiple-infant birth is counted as one live birth.

d Clinic-specific outcome rates are unreliable for women older than 42 undergoing ART cycles using fresh or frozen embryos with nondonor eggs. Readers are urged to review national outcomes for these age groups (see page 27).

e All ages (including ages $>42$ ) are reported together because previous data show that patient age does not materially affect success with donor eggs. 


\section{THE CENTER FOR ADVANCED REPRODUCTIVE SERVICES \\ AT THE UNIVERSITY OF CONNECTICUT HEALTH CENTER \\ FARMINGTON, CONNECTICUT}

A comparison of clinic success rates may not be meaningful because patient medical characteristics and treatment approaches vary from clinic to clinic. For more details about this, along with information on how to interpret the statistics in this table, see pages $79-88$.

\section{ART CYCLE PROFILE}

\begin{tabular}{|c|c|c|c|c|c|c|c|}
\hline & Type & of $A R T^{a}$ & & Pati & ent $D$ & iagnosis & \\
\hline IVF & $100 \%$ & Procedural Factors: & & Tubal factor & $14 \%$ & Other factor & $10 \%$ \\
\hline GIFT & $0 \%$ & With ICSI & $68 \%$ & Ovulatory dysfunction & $8 \%$ & Unknown factor & $21 \%$ \\
\hline ZIFT & $0 \%$ & Unstimulated & $0 \%$ & Diminished ovarian reserve & $2 \%$ & Multiple Factors: & \\
\hline Combination & $0 \%$ & Used gestational carrier & $<1 \%$ & Endometriosis & $14 \%$ & Female factors only & $8 \%$ \\
\hline & & & & Uterine factor & $2 \%$ & Female \& male factors & $9 \%$ \\
\hline & & & & Male factor & $13 \%$ & & \\
\hline
\end{tabular}

2006 PREGNANCY SUCCESS RATES

Data verified by John C. Nulsen, MD

\section{Type of Cycle}

\section{Fresh Embryos from Nondonor Eggs}

Number of cycles

Percentage of cycles resulting in pregnancies ${ }^{b}$

Percentage of cycles resulting in live births ${ }^{b, c}$

(Confidence Interval)

Percentage of retrievals resulting in live births $s^{b, c}$

Percentage of transfers resulting in live births $s^{b, c}$

Percentage of transfers resulting in singleton live births ${ }^{b}$

Percentage of cancellations ${ }^{b}$

Average number of embryos transferred

Percentage of pregnancies with twins ${ }^{b}$

Percentage of pregnancies with triplets or more ${ }^{b}$

Percentage of live births having multiple infants ${ }^{b, c}$

\section{Frozen Embryos from Nondonor Eggs}

Number of transfers

Percentage of transfers resulting in live births $s^{b, c}$

Average number of embryos transferred

\section{Donor Eggs}

Number of transfers

Percentage of transfers resulting in live births $s^{b, c}$

Average number of embryos transferred

\section{Age of Woman}

35-37 $38-40 \quad 41-42^{d}$

\section{$<35$}

252

218

110

448

42.1

29.8

27.3

39.7

37.7

21.1

17.3

(35.2-44.4)

(31.7-44.0)

$(15.9-27.1)$

(10.7-25.7)

45.4

46.1

27.5

24.1

48.1

48.0

29.5

25.0

35.9

23.1

19.7

12.5

18.3

23.4

28.2

2.0

2.2

2.9

3.6

34.4

27.4

23.1

20.0

0.9

4.7

4.6

0.0

36.0

25.3

21.7

$4 / 19$

$\begin{array}{cccc}80 & 51 & 15 & 7 \\ 46.3 & 41.2 & 4 / 15 & 1 / 7 \\ 2.0 & 2.1 & 2.4 & 3.7\end{array}$

\section{All Ages Combined $^{\mathrm{e}}$}

Fresh Embryos

42

61.9

2.1
Frozen Embryos

19

$11 / 19$

1.9

\section{CURRENT CLINIC SERVICES AND PROFILE}

Current Name: The Center for Advanced Reproductive Services at the University of Connecticut Health Center

Donor egg? Yes

Donor embryo? No

Single women? Yes

\section{Gestational carriers? Yes}

Cryopreservation?

Yes
SART member?

Verified lab accreditation?

(See Appendix C for details.)

${ }^{a}$ Reflects patient and treatment characteristics of ART cycles performed in 2006 using fresh nondonor eggs or embryos.

${ }^{b}$ When fewer than 20 cycles are reported in an age category, rates are shown as a fraction and confidence intervals are not given. Calculating percentages from fractions may be misleading and is not encouraged.

${ }^{c}$ A multiple-infant birth is counted as one live birth.

d Clinic-specific outcome rates are unreliable for women older than 42 undergoing ART cycles using fresh or frozen embryos with nondonor eggs. Readers are urged to review national outcomes for these age groups (see page 27).

e All ages (including ages $>42$ ) are reported together because previous data show that patient age does not materially affect success with donor eggs. 


\section{YALE FERTILITY CENTER \\ NEW HAVEN, CONNECTICUT}

A comparison of clinic success rates may not be meaningful because patient medical characteristics and treatment approaches vary from clinic to clinic. For more details about this, along with information on how to interpret the statistics in this table, see pages 79-88.

\section{ART CYCLE PROFILE}

\begin{tabular}{|c|c|c|c|c|c|c|}
\hline \multicolumn{3}{|c|}{ Type of ART ${ }^{a}$} & \multicolumn{4}{|c|}{ Patient Diagnosis } \\
\hline IVF & $>99 \%$ Procedural Factors: & & Tubal factor & $14 \%$ & Other factor & $22 \%$ \\
\hline GIFT & $0 \%$ With ICSI & $41 \%$ & Ovulatory dysfunction & $3 \%$ & Unknown factor & $8 \%$ \\
\hline ZIFT & $<1 \%$ Unstimulated & $0 \%$ & Diminished ovarian reserve & $15 \%$ & Multiple Factors: & \\
\hline \multirow[t]{3}{*}{ Combination } & $0 \%$ Used gestational carrier & $1 \%$ & Endometriosis & $5 \%$ & Female factors only & $8 \%$ \\
\hline & & & Uterine factor & $3 \%$ & Female \& male factors & $10 \%$ \\
\hline & & & Male factor & $11 \%$ & & \\
\hline
\end{tabular}

2006 PREGNANCY SUCCESS RATES

Data verified by Pasquale Patrizio, MD

\section{Type of Cycle}

\section{Fresh Embryos from Nondonor Eggs}

Number of cycles

Percentage of cycles resulting in pregnancies ${ }^{b}$

Percentage of cycles resulting in live births $s^{b, c}$

(Confidence Interval)

Percentage of retrievals resulting in live births $s^{b, c}$

Percentage of transfers resulting in live births $s^{b, c}$

Percentage of transfers resulting in singleton live births ${ }^{b}$

Percentage of cancellations ${ }^{b}$

Average number of embryos transferred

Percentage of pregnancies with twins ${ }^{b}$

Percentage of pregnancies with triplets or more ${ }^{b}$

Percentage of live births having multiple infants ${ }^{b, c}$

\section{Frozen Embryos from Nondonor Eggs}

Number of transfers

Percentage of transfers resulting in live births ${ }^{b, c}$

Average number of embryos transferred

\section{Donor Eggs}

Number of transfers

Percentage of transfers resulting in live births $s^{b, c}$

Average number of embryos transferred

\section{Age of Woman}

35-37 38-40

$41-42^{d}$

$<35$

77

39.0

80

37

47.2

31.2

22.5

21.6

39.4

(21.1-42.7)

20.0

16.2

(30.8-48.4)

41.0

45.9

33.8

(11.9-30.4)

(6.2-32.0)

22.5

17.1

39.3

29.6

23.1

28.4

29.5

24.1

19.2

3.9

7.8

11.3

5.4

2.6

30.0

2.7

23.3

3.3

3.3

38.0

25.0

2.9

3.2

$2 / 18$

$1 / 8$

$1 / 18$

$0 / 8$

$3 / 16$

$1 / 6$

$\begin{array}{cccc}11 & 14 & 7 & 1 \\ 3 / 11 & 5 / 14 & 2 / 7 & 0 / 1 \\ 2.5 & 2.9 & 2.4 & 4.0\end{array}$

\section{All Ages Combined}

Fresh Embryos

41

48.8

2.6
Frozen Embryos

21

28.6

2.8

\section{CURRENT CLINIC SERVICES AND PROFILE}

Current Name: Yale Fertility Center

Donor egg? Yes Gestational carriers? Yes

Donor embryo? Yes Cryopreservation? Yes

Single women? Yes

SART member? Yes

Verified lab accreditation? Yes

(See Appendix C for details.)

${ }^{\text {a }}$ Reflects patient and treatment characteristics of ART cycles performed in 2006 using fresh nondonor eggs or embryos.

b When fewer than 20 cycles are reported in an age category, rates are shown as a fraction and confidence intervals are not given. Calculating percentages from fractions may be misleading and is not encouraged.

${ }^{c}$ A multiple-infant birth is counted as one live birth.

d Clinic-specific outcome rates are unreliable for women older than 42 undergoing ART cycles using fresh or frozen embryos with nondonor eggs. Readers are urged to review national outcomes for these age groups (see page 27).

e All ages (including ages $>42$ ) are reported together because previous data show that patient age does not materially affect success with donor eggs. 


\section{REPRODUCTIVE MEDICINE ASSOCIATES OF CONNECTICUT NORWALK, CONNECTICUT}

A comparison of clinic success rates may not be meaningful because patient medical characteristics and treatment approaches vary from clinic to clinic. For more details about this, along with information on how to interpret the statistics in this table, see pages $79-88$.

\section{ART CYCLE PROFILE}

\begin{tabular}{|c|c|c|c|c|c|c|c|}
\hline \multicolumn{4}{|c|}{ Type of ART ${ }^{\mathrm{a}}$} & \multicolumn{4}{|c|}{ Patient Diagnosis } \\
\hline IVF & $100 \%$ & Procedural Factors: & & Tubal factor & $9 \%$ & Other factor & $6 \%$ \\
\hline GIFT & $0 \%$ & With ICSI & $39 \%$ & Ovulatory dysfunction & $16 \%$ & Unknown factor & $16 \%$ \\
\hline $\mathrm{ZIFT}$ & $0 \%$ & Unstimulated & $<1 \%$ & Diminished ovarian reserve & $9 \%$ & Multiple Factors: & \\
\hline Combination & $0 \%$ & Used gestational carrier & $<1 \%$ & Endometriosis & $2 \%$ & Female factors only & $10 \%$ \\
\hline & & & & Uterine factor & $1 \%$ & Female \& male factors & $18 \%$ \\
\hline
\end{tabular}

2006 PREGNANCY SUCCESS RATES

Data verified by Mark P. Leondires, MD

\section{Type of Cycle}

\section{Fresh Embryos from Nondonor Eggs}

Number of cycles

Percentage of cycles resulting in pregnancies ${ }^{b}$

Percentage of cycles resulting in live births ${ }^{b, c}$

(Confidence Interval)

Percentage of retrievals resulting in live births $s^{b, c}$

Percentage of transfers resulting in live births $s^{b, c}$

Percentage of transfers resulting in singleton live births ${ }^{b}$

Percentage of cancellations ${ }^{b}$

Average number of embryos transferred

Percentage of pregnancies with twins ${ }^{b}$

Percentage of pregnancies with triplets or more ${ }^{b}$

Percentage of live births having multiple infants ${ }^{b, c}$

\section{Frozen Embryos from Nondonor Eggs}

Number of transfers

Percentage of transfers resulting in live births $s^{b, c}$

Average number of embryos transferred

\section{Donor Eggs}

Number of transfers

Percentage of transfers resulting in live births $s^{b, c}$

Average number of embryos transferred

\section{Age of Woman}

35-37 38-40

$41-42^{d}$

$<35$

162

33.3

130

25

45.6

30.2

29.2

8.0

42.3

$(35.6-49.2)$

47.6

(23.3-37.9)

38.6

24.6

8.0

\section{7}

38.9

$(17.5-32.9)$

(1.0-26.0)

38.5

31.0

30.8

33.0

$2 / 18$

$2 / 14$

11.2

21.6

20.6

$1 / 14$

2.4

2.6

24.5

6.1

22.2

7.4

20.9

20.4

20.0

28.0

3.1

3.9

34.2

$2 / 2$

2.6

$0 / 2$

37.5

$1 / 2$

20

30.0

2.4

\section{5}

$1 / 5$

2.2

\section{6 \\ $0 / 6$}

2.7
1

$0 / 1$

1.0

All Ages Combined ${ }^{\mathbf{e}}$

Fresh Embryos 20

50.0

2.4
Frozen Embryos 15

$4 / 15$

2.5

\section{CURRENT CLINIC SERVICES AND PROFILE}

Current Name: Reproductive Medicine Associates of Connecticut

Donor egg? Yes Gestational carriers? Yes

Donor embryo? Yes Cryopreservation? Yes

Single women? Yes

SART member? Yes

Verified lab accreditation? Yes

(See Appendix C for details.)

${ }^{a}$ Reflects patient and treatment characteristics of ART cycles performed in 2006 using fresh nondonor eggs or embryos.

${ }^{b}$ When fewer than 20 cycles are reported in an age category, rates are shown as a fraction and confidence intervals are not given. Calculating percentages from fractions may be misleading and is not encouraged.

${ }^{c}$ A multiple-infant birth is counted as one live birth.

d Clinic-specific outcome rates are unreliable for women older than 42 undergoing ART cycles using fresh or frozen embryos with nondonor eggs. Readers are urged to review national outcomes for these age groups (see page 27).

e All ages (including ages $>42$ ) are reported together because previous data show that patient age does not materially affect success with donor eggs. 


\section{NEW ENGLAND FERTILITY INSTITUTE \\ STAMFORD, CONNECTICUT}

A comparison of clinic success rates may not be meaningful because patient medical characteristics and treatment approaches vary from clinic to clinic. For more details about this, along with information on how to interpret the statistics in this table, see pages 79-88.

\section{ART CYCLE PROFILE}

\begin{tabular}{|c|c|c|c|c|c|c|c|}
\hline \multicolumn{4}{|c|}{ Type of ART ${ }^{a}$} & \multicolumn{4}{|c|}{ Patient Diagnosis } \\
\hline IVF & $100 \%$ & Procedural Factors: & & Tubal factor & $10 \%$ & Other factor & $5 \%$ \\
\hline GIFT & $0 \%$ & With ICSI & $61 \%$ & Ovulatory dysfunction & $6 \%$ & Unknown factor & $14 \%$ \\
\hline ZIFT & $0 \%$ & Unstimulated & $0 \%$ & Diminished ovarian reserve & $31 \%$ & Multiple Factors: & \\
\hline \multirow[t]{3}{*}{ Combination } & $0 \%$ & Used gestational carrier & $<1 \%$ & Endometriosis & $3 \%$ & Female factors only & $6 \%$ \\
\hline & & & & Uterine factor & $1 \%$ & Female \& male factors & $7 \%$ \\
\hline & & & & Male factor & $16 \%$ & & \\
\hline
\end{tabular}

\section{PREGNANCY SUCCESS RATES}

Data verified by Gad Lavy, MD

\section{Type of Cycle}

\section{Fresh Embryos from Nondonor Eggs}

Number of cycles

Percentage of cycles resulting in pregnancies ${ }^{b}$

Percentage of cycles resulting in live births $s^{b, c}$

(Confidence Interval)

Percentage of retrievals resulting in live births $s^{b, c}$

Percentage of transfers resulting in live births $s^{b, c}$

Percentage of transfers resulting in singleton live births ${ }^{b}$

Percentage of cancellations ${ }^{b}$

Average number of embryos transferred

Percentage of pregnancies with twins ${ }^{b}$

Percentage of pregnancies with triplets or more ${ }^{b}$

Percentage of live births having multiple infants ${ }^{b, c}$

\section{Frozen Embryos from Nondonor Eggs}

Number of transfers

Percentage of transfers resulting in live births ${ }^{b, c}$

Average number of embryos transferred

\section{Donor Eggs}

Number of transfers

Percentage of transfers resulting in live births $s^{b, c}$

Average number of embryos transferred

\section{Age of Woman}

35-37 38-40

$41-42^{d}$

$<35$

74

36.5

85

39

56.3

25.7

20.0

7.7

45.0

(16.2-37.2)

12.9

2.6

(33.8-56.5)

45.6

46.2

26.4

$(6.6-22.0)$

(0.1-13.5)

39.7

29.7

13.3

3.0

21.9

15.7

3.4

1.3

2.7

2.6

22.2

17.8

8.9

3.7

$5 / 19$

14.3

3.4

15.4

2.2

2.5

$1 / 3$

13.9

$3 / 17$

$0 / 3$

$0 / 17$

$0 / 1$

$\begin{array}{cccc}29 & 34 & 26 & 6 \\ 24.1 & 14.7 & 7.7 & 2 / 6 \\ 2.4 & 2.6 & 2.6 & 2.3\end{array}$

\section{All Ages Combined}

Fresh Embryos

59

61.0

2.1
Frozen Embryos

43

16.3

2.6

\section{CURRENT CLINIC SERVICES AND PROFILE}

Current Name: New England Fertility Institute

Donor egg? Yes Gestational carriers? Yes

Donor embryo? Yes Cryopreservation? Yes

Single women? Yes

SART member? Yes

Verified lab accreditation? Yes

(See Appendix C for details.)

${ }^{a}$ Reflects patient and treatment characteristics of ART cycles performed in 2006 using fresh nondonor eggs or embryos.

b When fewer than 20 cycles are reported in an age category, rates are shown as a fraction and confidence intervals are not given. Calculating percentages from fractions may be misleading and is not encouraged.

${ }^{c}$ A multiple-infant birth is counted as one live birth.

d Clinic-specific outcome rates are unreliable for women older than 42 undergoing ART cycles using fresh or frozen embryos with nondonor eggs. Readers are urged to review national outcomes for these age groups (see page 27).

e All ages (including ages $>42$ ) are reported together because previous data show that patient age does not materially affect success with donor eggs. 


\section{THE STAMFORD HOSPITAL \\ STAMFORD, CONNECTICUT}

A comparison of clinic success rates may not be meaningful because patient medical characteristics and treatment approaches vary from clinic to clinic. For more details about this, along with information on how to interpret the statistics in this table, see pages $79-88$.

\section{ART CYCLE PROFILE}

\begin{tabular}{|c|c|c|c|c|c|c|c|}
\hline \multicolumn{4}{|c|}{ Type of ART ${ }^{\mathrm{a}}$} & \multicolumn{4}{|c|}{ Patient Diagnosis } \\
\hline IVF & $100 \%$ & Procedural Factors: & & Tubal factor & $19 \%$ & Other factor & $3 \%$ \\
\hline GIFT & $0 \%$ & With ICSI & $17 \%$ & Ovulatory dysfunction & $16 \%$ & Unknown factor & $19 \%$ \\
\hline $\mathrm{ZIFT}$ & $0 \%$ & Unstimulated & $0 \%$ & Diminished ovarian reserve & $7 \%$ & Multiple Factors: & \\
\hline Combination & $0 \%$ & Used gestational carrier & $0 \%$ & Endometriosis & $7 \%$ & Female factors only & $7 \%$ \\
\hline & & & & $\begin{array}{l}\text { Uterine factor } \\
\text { Male factor }\end{array}$ & $\begin{array}{l}0 \% \\
7 \%\end{array}$ & Female \& male factors & $16 \%$ \\
\hline
\end{tabular}

2006 PREGNANCY SUCCESS RATES

Data verified by Frances W. Ginsburg, MD

\section{Type of Cycle}

\section{Fresh Embryos from Nondonor Eggs}

Number of cycles

Percentage of cycles resulting in pregnancies ${ }^{b}$

Percentage of cycles resulting in live births ${ }^{b, c}$

(Confidence Interval)

Percentage of retrievals resulting in live births $s^{b, c}$

Percentage of transfers resulting in live births $s^{b, c}$

Percentage of transfers resulting in singleton live births ${ }^{b}$

Percentage of cancellations ${ }^{b}$

Average number of embryos transferred

Percentage of pregnancies with twins ${ }^{b}$

Percentage of pregnancies with triplets or more ${ }^{\mathrm{b}}$

Percentage of live births having multiple infants ${ }^{b, c}$

\section{Frozen Embryos from Nondonor Eggs}

Number of transfers

Percentage of transfers resulting in live births ${ }^{\mathrm{b}, \mathrm{c}}$

Average number of embryos transferred

$0 / 4$

1.8

\section{Age of Woman}

$\begin{array}{cccc}<35 & \mathbf{3 5 - 3 7} & \mathbf{3 8 - 4 0} & \mathbf{4 1 - 4 2}^{\mathbf{d}} \\ 8 & 10 & 4 & 1 \\ 2 / 8 & 2 / 10 & 1 / 4 & 0 / 1 \\ 1 / 8 & 2 / 10 & 1 / 4 & 0 / 1\end{array}$

$\begin{array}{lccc}1 / 5 & 2 / 6 & 1 / 3 & 0 / 1 \\ 1 / 5 & 2 / 6 & 1 / 3 & 0 / 1 \\ 1 / 5 & 2 / 6 & 1 / 3 & 0 / 1 \\ 3 / 8 & 4 / 10 & 1 / 4 & 0 / 1 \\ 2.4 & 2.2 & 3.0 & 1.0 \\ 0 / 2 & 0 / 2 & 0 / 1 & \\ 0 / 2 & 0 / 2 & 0 / 1 & \\ 0 / 1 & 0 / 2 & 0 / 1 & \end{array}$

\section{Donor Eggs}

\section{Fresh Embryos}

4
$1 / 4$

$1 / 4$

2.8

Number of transfers

Percentage of transfers resulting in live births $s^{b, c}$

0

0

0

Average number of embryos transferred

\section{CURRENT CLINIC SERVICES AND PROFILE}

Current Name: The Stamford Hospital
Donor egg?
No
Gestational carriers?
No
Cryopreservation?
Yes
Donor embryo? No
Single women? Yes
SART member?
Yes
Verified lab accreditation?
Yes
(See Appendix C for details.)

\footnotetext{
${ }^{a}$ Reflects patient and treatment characteristics of ART cycles performed in 2006 using fresh nondonor eggs or embryos. percentages from fractions may be misleading and is not encouraged.

${ }^{c}$ A multiple-infant birth is counted as one live birth.

d Clinic-specific outcome rates are unreliable for women older than 42 undergoing ART cycles using fresh or frozen embryos with nondonor eggs. Readers are urged to review national outcomes for these age groups (see page 27).

${ }^{e}$ All ages (including ages $>42$ ) are reported together because previous data show that patient age does not materially affect success with donor eggs.
}
${ }^{b}$ When fewer than 20 cycles are reported in an age category, rates are shown as a fraction and confidence intervals are not given. Calculating

All Ages 


\section{PARK AVENUE FERTILITY AND REPRODUCTIVE MEDICINE TRUMBULL, CONNECTICUT}

A comparison of clinic success rates may not be meaningful because patient medical characteristics and treatment approaches vary from clinic to clinic. For more details about this, along with information on how to interpret the statistics in this table, see pages 79-88.

\section{ART CYCLE PROFILE}

\begin{tabular}{|c|c|c|c|c|c|c|c|}
\hline \multicolumn{4}{|c|}{ Type of ART ${ }^{a}$} & \multicolumn{4}{|c|}{ Patient Diagnosis } \\
\hline IVF & $100 \%$ & Procedural Factors: & & Tubal factor & $7 \%$ & Other factor & $4 \%$ \\
\hline GIFT & $0 \%$ & With ICSI & $87 \%$ & Ovulatory dysfunction & $7 \%$ & Unknown factor & $23 \%$ \\
\hline ZIFT & $0 \%$ & Unstimulated & $0 \%$ & Diminished ovarian reserve & $35 \%$ & Multiple Factors: & \\
\hline \multirow[t]{3}{*}{ Combination } & $0 \%$ & Used gestational carrier & $0 \%$ & Endometriosis & $3 \%$ & Female factors only & $<1 \%$ \\
\hline & & & & Uterine factor & $2 \%$ & Female \& male factors & $2 \%$ \\
\hline & & & & Male factor & $17 \%$ & & \\
\hline
\end{tabular}

2006 PREGNANCY SUCCESS RATES

Data verified by Andrew Levi, MD

\section{Type of Cycle}

\section{Fresh Embryos from Nondonor Eggs}

Number of cycles

Percentage of cycles resulting in pregnancies ${ }^{b}$

Percentage of cycles resulting in live births ${ }^{b, c}$

(Confidence Interval)

Percentage of retrievals resulting in live births $s^{b, c}$

Percentage of transfers resulting in live births $s^{b, c}$

Percentage of transfers resulting in singleton live births ${ }^{b}$

Percentage of cancellations ${ }^{b}$

Average number of embryos transferred

Percentage of pregnancies with twins ${ }^{b}$

Percentage of pregnancies with triplets or more ${ }^{b}$

Percentage of live births having multiple infants ${ }^{b, c}$

\section{Frozen Embryos from Nondonor Eggs}

Number of transfers

Percentage of transfers resulting in live births ${ }^{b, c}$

Average number of embryos transferred

\section{Donor Eggs}

Number of transfers

Percentage of transfers resulting in live births $s^{b, c}$

Average number of embryos transferred

\section{Age of Woman}

35-37 $38-40 \quad 41-42^{d}$

$<35$

43

27.9

23

10

50.0

16.3

26.1

$0 / 10$

42.9

(6.8-30.7)

17.4

$0 / 10$

(27.7-59.0)

45.0

16.7

(5.0-38.8)

48.6

17.5

18.2

$0 / 9$

35.1

12.5

20.0

$0 / 4$

4.8

2.3

15.0

$0 / 4$

2.0

2.2

4.3

$1 / 10$

38.1

$3 / 12$

2.7

3.5

0.0

$0 / 12$

$1 / 6$

$5 / 18$

$2 / 7$

$0 / 6$

$1 / 4$

4

$3 / 4$

2.8
1

$1 / 1$

3.0

\section{CURRENT CLINIC SERVICES AND PROFILE}

Current Name: Park Avenue Fertility and Reproductive Medicine

Donor egg? Yes Gestational carriers? Yes

Donor embryo? No Cryopreservation? Yes

Fresh Embryos

All Ages Combined 5

$5 / 5$

2.2

Single women? Yes

SART member?

No

Verified lab accreditation?

No

(See Appendix C for details.)

${ }^{a}$ Reflects patient and treatment characteristics of ART cycles performed in 2006 using fresh nondonor eggs or embryos.

${ }^{b}$ When fewer than 20 cycles are reported in an age category, rates are shown as a fraction and confidence intervals are not given. Calculating percentages from fractions may be misleading and is not encouraged.

${ }^{c}$ A multiple-infant birth is counted as one live birth.

d Clinic-specific outcome rates are unreliable for women older than 42 undergoing ART cycles using fresh or frozen embryos with nondonor eggs. Readers are urged to review national outcomes for these age groups (see page 27).

e All ages (including ages $>42$ ) are reported together because previous data show that patient age does not materially affect success with donor eggs. 


\section{REPRODUCTIVE ASSOCIATES OF DELAWARE NEWARK, DELAWARE}

A comparison of clinic success rates may not be meaningful because patient medical characteristics and treatment approaches vary from clinic to clinic. For more details about this, along with information on how to interpret the statistics in this table, see pages $79-88$.

\section{ART CYCLE PROFILE}

\begin{tabular}{|c|c|c|c|c|c|c|c|}
\hline \multicolumn{4}{|c|}{ Type of $A R T^{a}$} & \multicolumn{4}{|c|}{ Patient Diagnosis } \\
\hline IVF & $100 \%$ & Procedural Factors: & & Tubal factor & $9 \%$ & Other factor & $5 \%$ \\
\hline GIFT & $0 \%$ & With ICSI & $81 \%$ & Ovulatory dysfunction & $3 \%$ & Unknown factor & $3 \%$ \\
\hline ZIFT & $0 \%$ & Unstimulated & $0 \%$ & Diminished ovarian reserve & $5 \%$ & Multiple Factors: & \\
\hline \multirow[t]{3}{*}{ Combination } & $0 \%$ & Used gestational carrier & $0 \%$ & Endometriosis & $19 \%$ & Female factors only & $22 \%$ \\
\hline & & & & Uterine factor & $1 \%$ & Female \& male factors & $21 \%$ \\
\hline & & & & Male factor & $12 \%$ & & \\
\hline
\end{tabular}

2006 PREGNANCY SUCCESS RATES

Data verified by Ronald F. Feinberg, MD, PhD

\section{Type of Cycle}

\section{Fresh Embryos from Nondonor Eggs}

Number of cycles

Percentage of cycles resulting in pregnancies ${ }^{b}$

Percentage of cycles resulting in live births $s^{b, c}$

(Confidence Interval)

Percentage of retrievals resulting in live births $s^{b, c}$

Percentage of transfers resulting in live births $s^{b, c}$

Percentage of transfers resulting in singleton live births ${ }^{b}$

Percentage of cancellations ${ }^{b}$

Average number of embryos transferred

Percentage of pregnancies with twins ${ }^{b}$

Percentage of pregnancies with triplets or more ${ }^{b}$

Percentage of live births having multiple infants ${ }^{b, c}$

\section{Frozen Embryos from Nondonor Eggs}

Number of transfers

Percentage of transfers resulting in live births $s^{\mathrm{b}, \mathrm{c}}$

Average number of embryos transferred

\section{Donor Eggs}

Number of transfers

Percentage of transfers resulting in live births $s^{b, c}$

Average number of embryos transferred

\section{Age of Woman}

35-37 38-40

$41-42^{d}$

$<35$

63

38.1

37

9

45.7

31.7

35.1

$0 / 9$

39.4

(29.4-50.0)

(20.6-44.7)

21.6

$0 / 9$

44.6

40.0

(9.8-38.2)

51.4

42.6

30.8

$0 / 6$

40.3

11.7

34.0

30.8

26.9

29.7

$0 / 6$

20.6

2.3

$0 / 6$

1.8

2.0

16.7

$1 / 13$

7.0

0.0

$1 / 13$

21.6

20.0

$1 / 8$

30

53.3

20

30.0

1.9

15

$6 / 15$

$3 / 9$

2.3

1.8

All Ages Combined

Fresh Embryos

Frozen Embryos

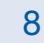

$7 / 8$

7

1.8

$4 / 7$

1.4

\section{CURRENT CLINIC SERVICES AND PROFILE}

Current Name: Reproductive Associates of Delaware

Donor egg? Yes

Donor embryo? Yes

Gestational carriers?

Yes

Cryopreservation?

SART member?

Yes

Single women? Yes

Verified lab accreditation?

Yes

(See Appendix C for details.)

${ }^{a}$ Reflects patient and treatment characteristics of ART cycles performed in 2006 using fresh nondonor eggs or embryos.

${ }^{b}$ When fewer than 20 cycles are reported in an age category, rates are shown as a fraction and confidence intervals are not given. Calculating percentages from fractions may be misleading and is not encouraged.

A multiple-infant birth is counted as one live birth.

${ }^{d}$ Clinic-specific outcome rates are unreliable for women older than 42 undergoing ART cycles using fresh or frozen embryos with nondonor eggs. Readers are urged to review national outcomes for these age groups (see page 27).

e All ages (including ages $>42$ ) are reported together because previous data show that patient age does not materially affect success with donor eggs. 


\section{THE A.R.T. INSTITUTE OF WASHINGTON, INC. WALTER REED ARMY MEDICAL CENTER WASHINGTON, DISTRICT OF COLUMBIA}

A comparison of clinic success rates may not be meaningful because patient medical characteristics and treatment approaches vary from clinic to clinic. For more details about this, along with information on how to interpret the statistics in this table, see pages 79-88.

\section{ART CYCLE PROFILE}

\section{Type of ART ${ }^{\mathrm{a}}$}

IVF

GIFT

100\% Procedural Factors:

ZIFT

Combination
$0 \%$ With ICSI

$0 \%$ Unstimulated

$0 \%$ Used gestational carrier
Tubal factor

$51 \%$ Ovulatory dysfunction

$<1 \%$ Diminished ovarian reserve

$0 \%$

\section{Patient Diagnosis}

$\begin{array}{rlr}22 \% & \text { Other factor } & 1 \% \\ 6 \% & \text { Unknown factor } & 8 \% \\ 6 \% & \text { Multiple Factors: } & \\ 5 \% & \text { Female factors only } & 13 \% \\ <1 \% & \text { Female \& male factors } & 13 \% \\ 25 \% & & \end{array}$

2006 PREGNANCY SUCCESS RATES

Data verified by James Segars, MD

Endometriosis

Uterine factor

Male factor

\section{Type of Cycle}

\section{Age of Woman}

35-37 38-40

$41-42^{d}$

\section{$<35$}

115

89

34

Number of cycles

204

55.9

44.3

33.7

29.4

47.1

36.5

18.0

17.6

(Confidence Interval)

Percentage of retrievals resulting in live births $s^{b, c}$

$(40.1-54.2)$

$(27.7-46.0)$

$(10.6-27.5)$

49.7

40.4

21.9

$(6.8-34.5)$

51.9

42.0

22.2

18.8

18.8

32.0

19.4

15.6

5.4

9.6

18.0

5.9

2.0

2.2

2.8

3.3

39.5

27.5

2.6

3.9

40.6

23.8

10.0

$2 / 10$

Percentage of pregnancies with triplets or more ${ }^{b}$

Percentage of live births having multiple infants ${ }^{b, c}$

\section{Frozen Embryos from Nondonor Eggs}

Number of transfers

Percentage of transfers resulting in live births $s^{b, c}$

Average number of embryos transferred

\section{Donor Eggs}

Number of transfers

Percentage of transfers resulting in live births $s^{b, c}$

Average number of embryos transferred

\section{4}

$5 / 14$

1.9

9
$3 / 9$
2.1

3.3

$0 / 10$

$2 / 16$

$1 / 6$

\section{All Ages Combined}

Fresh Embryos

0
Frozen Embryos

0

\section{CURRENT CLINIC SERVICES AND PROFILE}

Current Name: The A.R.T. Institute of Washington, Inc., Walter Reed Army Medical Center

Donor egg?

No

Donor embryo?

Single women?

\begin{abstract}
Gestational carriers? No
Cryopreservation?
\end{abstract}

SART member?

Verified lab accreditation?

(See Appendix C for details.)

${ }^{a}$ Reflects patient and treatment characteristics of ART cycles performed in 2006 using fresh nondonor eggs or embryos.

${ }^{b}$ When fewer than 20 cycles are reported in an age category, rates are shown as a fraction and confidence intervals are not given. Calculating percentages from fractions may be misleading and is not encouraged.

${ }^{\mathrm{C}}$ A multiple-infant birth is counted as one live birth.

d Clinic-specific outcome rates are unreliable for women older than 42 undergoing ART cycles using fresh or frozen embryos with nondonor eggs. Readers are urged to review national outcomes for these age groups (see page 27).

e All ages (including ages $>42$ ) are reported together because previous data show that patient age does not materially affect success with donor eggs. 


\section{COLUMBIA FERTILITY ASSOCIATES WASHINGTON, DISTRICT OF COLUMBIA}

A comparison of clinic success rates may not be meaningful because patient medical characteristics and treatment approaches vary from clinic to clinic. For more details about this, along with information on how to interpret the statistics in this table, see pages 79-88.

\section{ART CYCLE PROFILE}

\begin{tabular}{|c|c|c|c|c|c|c|c|}
\hline \multicolumn{4}{|c|}{ Type of ART ${ }^{a}$} & \multicolumn{4}{|c|}{ Patient Diagnosis } \\
\hline IVF & $100 \%$ & Procedural Factors: & & Tubal factor & $4 \%$ & Other factor & $10 \%$ \\
\hline GIFT & $0 \%$ & With ICSI & $57 \%$ & Ovulatory dysfunction & $4 \%$ & Unknown factor & $15 \%$ \\
\hline $\mathrm{ZIFT}$ & $0 \%$ & Unstimulated & $<1 \%$ & Diminished ovarian reserve & $20 \%$ & Multiple Factors: & \\
\hline \multirow[t]{3}{*}{ Combination } & $0 \%$ & Used gestational carrier & $0 \%$ & Endometriosis & $3 \%$ & Female factors only & $9 \%$ \\
\hline & & & & Uterine factor & $5 \%$ & Female \& male factors & $18 \%$ \\
\hline & & & & Male factor & $13 \%$ & & \\
\hline
\end{tabular}

2006 PREGNANCY SUCCESS RATES

Data verified by Safa Rifka, MD

\section{Type of Cycle}

\section{Fresh Embryos from Nondonor Eggs}

Number of cycles

Percentage of cycles resulting in pregnancies ${ }^{b}$

Percentage of cycles resulting in live births $s^{b, c}$

(Confidence Interval)

Percentage of retrievals resulting in live births $s^{b, c}$

Percentage of transfers resulting in live births $s^{b, c}$

Percentage of transfers resulting in singleton live births ${ }^{b}$

Percentage of cancellations ${ }^{b}$

Average number of embryos transferred

Percentage of pregnancies with twins ${ }^{b}$

Percentage of pregnancies with triplets or more ${ }^{b}$

Percentage of live births having multiple infants ${ }^{b, c}$

\section{Frozen Embryos from Nondonor Eggs}

Number of transfers

Percentage of transfers resulting in live births $s^{\mathrm{b}, \mathrm{c}}$

Average number of embryos transferred

\section{Donor Eggs}

Number of transfers

Percentage of transfers resulting in live births $s^{b, c}$

Average number of embryos transferred

\section{Age of Woman}

35-37 38-40

$41-42^{d}$

$<35$

78

34.6

33.3

40.2

36.8

(26.7-47.8)

(23.1-44.9)

36.6

38.2

16.2

9.0

2.9

40.7

14.8

57.7

31.3

2.6
28.6

5.7

20

30.0

2.5

\section{5}

$3 / 15$

2.2

77

36.4

29.9

(20.0-41.4)

33.3

35.4

30.8

10.4

2.8

10.7

7.1

13.0

59

18.6

15.3

(7.2-27.0)

18.0

20.5

18.2

15.3

3.5

$1 / 11$

$0 / 11$

$1 / 9$

All Ages Combined ${ }^{\mathrm{e}}$

Fresh Embryos 36

63.9

$\begin{array}{cc}15 & 5 \\ 9 / 15 & 0 / 5 \\ 2.5 & 2.4\end{array}$

\section{5}

2.4
$10 \%$

$15 \%$

$9 \%$

$8 \%$

\section{CURRENT CLINIC SERVICES AND PROFILE}

Current Name: Columbia Fertility Associates
Donor egg?
Yes
Donor embryo? Yes
Gestational carriers? Yes
Cryopreservation?
SART member?
Yes
Verified lab accreditation?
Yes
Single women? Yes
(See Appendix C for details.)
${ }^{a}$ Reflects patient and treatment characteristics of ART cycles performed in 2006 using fresh nondonor eggs or embryos.
${ }^{b}$ When fewer than 20 cycles are reported in an age category, rates are shown as a fraction and confidence intervals are not given. Calculating percentages from fractions may be misleading and is not encouraged.
c A multiple-infant birth is counted as one live birth.
${ }^{d}$ Clinic-specific outcome rates are unreliable for women older than 42 undergoing ART cycles using fresh or frozen embryos with nondonor eggs. Readers are urged to review national outcomes for these age groups (see page 27).
${ }^{e}$ All ages (including ages $>42$ ) are reported together because previous data show that patient age does not materially affect success with donor eggs. 


\section{THE GEORGE WASHINGTON UNIVERSITY MEDICAL FACULTY ASSOCIATES WASHINGTON, DISTRICT OF COLUMBIA}

A comparison of clinic success rates may not be meaningful because patient medical characteristics and treatment approaches vary from clinic to clinic. For more details about this, along with information on how to interpret the statistics in this table, see pages 79-88.

\section{ART CYCLE PROFILE}

\section{Type of ART ${ }^{\mathrm{a}}$}

IVF

GIFT

100\% Procedural Factors:

ZIFT

Combination
$0 \%$ With ICSI

$0 \%$ Unstimulated

$0 \%$ Used gestational carrier $<1 \%$
Tubal factor

Ovulatory dysfunction

Diminished ovarian reserve

Endometriosis

Uterine factor

Male factor

\section{Patient Diagnosis}

$10 \%$ Other factor

2\% Unknown factor

12\% Multiple Factors:

$1 \%$ Female factors only $1 \%$

$1 \%$ Female \& male factors $5 \%$ $13 \%$

\section{PREGNANCY SUCCESS RATES}

Data verified by Paul R. Gindoff, MD

\section{Type of Cycle}

\section{Age of Woman}

35-37 38-40

$$
41-42^{d}
$$

64

18.8

15.6

29.1

(17.6-42.9)

30.2

32.0

28.0

3.6

2.2

$3 / 19$

$0 / 19$

$2 / 16$
(7.8-26.9)

17.2

18.2

14.5

9.4

2.7

$4 / 12$

$0 / 12$

$2 / 10$
65

26.2

24.6

(14.8-36.9)

26.2

30.8

28.8

6.2

2.7

$3 / 17$

0 / 17

$1 / 16$

15

$4 / 15$

3.0

\section{9 \\ $0 / 9$}

2.9
41

9.8

7.3

(1.5-19.9)

7.9

9.1

6.1

7.3

3.2

$1 / 4$

$0 / 4$

$1 / 3$

\section{Frozen Embryos from Nondonor Eggs}

Percentage of transfers resulting in live births $s^{\mathrm{b}, \mathrm{c}}$

Average number of embryos transferred

\section{Donor Eggs}

Number of transfers

Percentage of transfers resulting in live births $s^{\mathrm{b}, \mathrm{c}}$

Average number of embryos transferred

$\begin{array}{cccc}17 & 15 & 9 & 4 \\ 4 / 17 & 4 / 15 & 0 / 9 & 1 / 4 \\ 2.5 & 3.0 & 2.9 & 3.0\end{array}$

All Ages Combined ${ }^{\mathrm{e}}$ Fresh Embryos Frozen Embryos

$\begin{array}{cc}15 & 11 \\ 4 / 15 & 2 / 11 \\ 2.4 & 2.9\end{array}$

\section{CURRENT CLINIC SERVICES AND PROFILE}

Current Name: The George Washington University Medical Faculty Associates
Donor egg?
Yes
Donor embryo? No
Gestational carriers? Yes
Cryopreservation?
SART member?
Verified lab accreditation?
Yes
Single women? Yes
Yes
(See Appendix C for details.)

\footnotetext{
${ }^{a}$ Reflects patient and treatment characteristics of ART cycles performed in 2006 using fresh nondonor eggs or embryos.

${ }^{b}$ When fewer than 20 cycles are reported in an age category, rates are shown as a fraction and confidence intervals are not given. Calculating percentages from fractions may be misleading and is not encouraged.

${ }^{c}$ A multiple-infant birth is counted as one live birth.

d Clinic-specific outcome rates are unreliable for women older than 42 undergoing ART cycles using fresh or frozen embryos with nondonor eggs. Readers are urged to review national outcomes for these age groups (see page 27).

e All ages (including ages $>42$ ) are reported together because previous data show that patient age does not materially affect success with donor eggs.
} 


\section{JAMES A. SIMON, MD, PC WASHINGTON, DISTRICT OF COLUMBIA}

A comparison of clinic success rates may not be meaningful because patient medical characteristics and treatment approaches vary from clinic to clinic. For more details about this, along with information on how to interpret the statistics in this table, see pages 79-88.

\section{ART CYCLE PROFILE}

\begin{tabular}{|c|c|c|c|c|c|c|c|}
\hline & Type & of $A R T^{a}$ & & Pati & ent D & iagnosis & \\
\hline IVF & $100 \%$ & Procedural Factors: & & Tubal factor & $8 \%$ & Other factor & $15 \%$ \\
\hline GIFT & $0 \%$ & With ICSI & $0 \%$ & Ovulatory dysfunction & $8 \%$ & Unknown factor & $0 \%$ \\
\hline ZIFT & $0 \%$ & Unstimulated & $0 \%$ & Diminished ovarian reserve & $0 \%$ & Multiple Factors: & \\
\hline Combination & $0 \%$ & Used gestational carrier & $0 \%$ & Endometriosis & $23 \%$ & Female factors only & $15 \%$ \\
\hline & & & & Uterine factor & $8 \%$ & Female \& male factors & $23 \%$ \\
\hline & & & & Male factor & $0 \%$ & & \\
\hline
\end{tabular}

2006 PREGNANCY SUCCESS RATES

Data verified by James A. Simon, MD

\section{Type of Cycle}

\section{Fresh Embryos from Nondonor Eggs}

Number of cycles

Percentage of cycles resulting in pregnancies ${ }^{b}$

Percentage of cycles resulting in live births ${ }^{b, c}$

(Confidence Interval)

Percentage of retrievals resulting in live births $s^{b, c}$

Percentage of transfers resulting in live births $s^{b, c}$

Percentage of transfers resulting in singleton live births ${ }^{b}$

Percentage of cancellations ${ }^{b}$

Average number of embryos transferred

Percentage of pregnancies with twins ${ }^{b}$

Percentage of pregnancies with triplets or more ${ }^{b}$

Percentage of live births having multiple infants ${ }^{b, c}$

\section{Frozen Embryos from Nondonor Eggs}

Number of transfers

Percentage of transfers resulting in live births ${ }^{\mathrm{b}, \mathrm{c}}$

Average number of embryos transferred

\section{Donor Eggs}

Number of transfers

Percentage of transfers resulting in live births $s^{b, c}$

Average number of embryos transferred

\section{Age of Woman}

$\begin{array}{cccc}<35 & 35-37 & 38-40 & \mathbf{4 1 - 4 2}^{\text {d }} \\ 3 & 5 & 1 & 0 \\ 0 / 3 & 1 / 5 & 0 / 1 & \\ 0 / 3 & 1 / 5 & 0 / 1 & \end{array}$

$0 / 3$

$1 / 3$

$0 / 1$

$0 / 2$

$1 / 2$

$0 / 2$

$2 / 5$

$0 / 1$

$0 / 3$

2.0

$1 / 1$

$0 / 1$

$1 / 1$

\section{2}

$0 / 2$

0

2

$1 / 2$

4.0

3.5

All Ages Combined ${ }^{\mathrm{e}}$

Fresh Embryos

0

Frozen Embryos

0

\section{CURRENT CLINIC SERVICES AND PROFILE}

Current Name: James A. Simon, MD, PC
Donor egg? Yes
Donor embryo? No
Gestational carriers? No
Cryopreservation? Yes
SART member?
Yes
Single women? Yes
Verified lab accreditation?
Yes
(See Appendix C for details.)

\footnotetext{
${ }^{a}$ Reflects patient and treatment characteristics of ART cycles performed in 2006 using fresh nondonor eggs or embryos.

b When fewer than 20 cycles are reported in an age category, rates are shown as a fraction and confidence intervals are not given. Calculating percentages from fractions may be misleading and is not encouraged.

c A multiple-infant birth is counted as one live birth.

d Clinic-specific outcome rates are unreliable for women older than 42 undergoing ART cycles using fresh or frozen embryos with nondonor eggs. Readers are urged to review national outcomes for these age groups (see page 27).

${ }^{e}$ All ages (including ages $>42$ ) are reported together because previous data show that patient age does not materially affect success with donor eggs.
} 


\section{BOCAFERTILITY \\ BOCA RATON, FLORIDA}

A comparison of clinic success rates may not be meaningful because patient medical characteristics and treatment approaches vary from clinic to clinic. For more details about this, along with information on how to interpret the statistics in this table, see pages 79-88.

\section{ART CYCLE PROFILE}

\section{Type of ART ${ }^{\mathrm{a}}$}

IVF

GIFT

100\% Procedural Factors:

ZIFT

Combination
$0 \%$ With ICSI

$0 \%$ Unstimulated

$0 \%$ Used gestational carrier

\section{$31 \%$}

$0 \%$

$2 \%$
Tubal factor

Ovulatory dysfunction

Diminished ovarian reserve

Endometriosis

Uterine factor

Male factor

\section{Patient Diagnosis}

$\begin{array}{rlr}17 \% & \text { Other factor } & 3 \% \\ 5 \% & \text { Unknown factor } & 13 \% \\ 26 \% & \text { Multiple Factors: } & \\ 3 \% & \text { Female factors only } & 9 \% \\ <1 \% & \text { Female \& male factors } & 8 \% \\ 17 \% & & \end{array}$

2006 PREGNANCY SUCCESS RATES

Data verified by Maurice (Moshe) R. Peress, MD

\section{Type of Cycle}

\section{Age of Woman}

35-37 38-40

\section{$<35$}

23

34.8

26.1

7

$4 / 7$

$4 / 7$

$41-42^{d}$

44.7

36.8

(Confidence Interval)

Percentage of retrievals resulting in live births $s^{b, c}$

Percentage of transfers resulting in live births $s^{b, c}$

$(21.8-54.0)$

$(10.2-48.4)$
28.6

40.0

28.6

$4 / 6$

$4 / 6$

$4 / 6$

$1 / 7$

2.7

8.7

2.4

$3 / 8$

$0 / 8$

$1 / 4$

$3 / 6$

$0 / 4$

$0 / 4$

16

$4 / 16$
$2 / 16$

$4 / 16$
$2 / 16$

$9 / 17$

$0 / 17$

$8 / 14$

$\begin{array}{cccc}6 & 1 & 1 & 2 \\ 1 / 6 & 0 / 1 & 0 / 1 & 0 / 2 \\ 2.2 & 3.0 & 2.0 & 1.0\end{array}$

\section{Frozen Embryos from Nondonor Eggs}

Number of transfers

Percentage of transfers resulting in live births $s^{b, c}$

Average number of embryos transferred

\section{Donor Eggs}

All Ages Combined ${ }^{\mathrm{e}}$

Number of transfers

Percentage of transfers resulting in live births $s^{b, c}$

Average number of embryos transferred

\section{Fresh Embryos}

14
$8 / 14$
2.3

Frozen Embryos

4

$0 / 4$

2.5

\section{CURRENT CLINIC SERVICES AND PROFILE}

Current Name: BocaFertility

Donor egg? Yes

Donor embryo? Yes

Single women? Yes

$\begin{array}{ll}\text { Gestational carriers? } & \text { Yes } \\ \text { Cryopreservation? } & \text { Yes }\end{array}$

SART member?

Yes

Verified lab accreditation?

Yes

(See Appendix C for details.)

${ }^{a}$ Reflects patient and treatment characteristics of ART cycles performed in 2006 using fresh nondonor eggs or embryos.

b When fewer than 20 cycles are reported in an age category, rates are shown as a fraction and confidence intervals are not given. Calculating percentages from fractions may be misleading and is not encouraged.

${ }^{c}$ A multiple-infant birth is counted as one live birth.

d Clinic-specific outcome rates are unreliable for women older than 42 undergoing ART cycles using fresh or frozen embryos with nondonor eggs. Readers are urged to review national outcomes for these age groups (see page 27).

e All ages (including ages $>42$ ) are reported together because previous data show that patient age does not materially affect success with donor eggs. 


\section{ADVANCED REPRODUCTIVE CARE CENTER, PA \\ BOYNTON BEACH, FLORIDA}

A comparison of clinic success rates may not be meaningful because patient medical characteristics and treatment approaches vary from clinic to clinic. For more details about this, along with information on how to interpret the statistics in this table, see pages $79-88$.

\section{ART CYCLE PROFILE}

\begin{tabular}{|c|c|c|c|c|c|c|c|}
\hline \multicolumn{4}{|c|}{ Type of ART } & \multicolumn{4}{|c|}{ Patient Diagnosis } \\
\hline IVF & $100 \%$ & Procedural Factors: & & Tubal factor & $9 \%$ & Other factor & $2 \%$ \\
\hline GIFT & $0 \%$ & With ICSI & $72 \%$ & Ovulatory dysfunction & $4 \%$ & Unknown factor & $4 \%$ \\
\hline ZIFT & $0 \%$ & Unstimulated & $0 \%$ & Diminished ovarian reserve & $11 \%$ & Multiple Factors: & \\
\hline \multirow[t]{3}{*}{ Combination } & $0 \%$ & Used gestational carrier & $2 \%$ & Endometriosis & $2 \%$ & Female factors only & $7 \%$ \\
\hline & & & & Uterine factor & $2 \%$ & Female \& male factors & $28 \%$ \\
\hline & & & & Male factor & $33 \%$ & & \\
\hline
\end{tabular}

2006 PREGNANCY SUCCESS RATES

Data verified by Tibor Polcz, MD

\section{Type of Cycle}

\section{Age of Woman}

35-37 38-40

$41-42^{d}$

$<35$

14

$10 / 14$

$7 / 14$

13

5

$10 / 15$

$7 / 13$

$1 / 5$

Percentage of cycles resulting in live births ${ }^{\mathrm{b}, \mathrm{c}}$

$10 / 15$

$7 / 13$

$1 / 5$

(Confidence Interval)

Percentage of retrievals resulting in live births ${ }^{\mathrm{b}, \mathrm{c}}$

Percentage of transfers resulting in live births ${ }^{\mathrm{b}, \mathrm{c}}$

$10 / 14$

$7 / 13$

$7 / 13$

$1 / 4$

$10 / 14$

$7 / 13$

$7 / 13$

$1 / 4$

Percentage of transfers resulting in singleton live births ${ }^{b}$

$2 / 14$

$5 / 13$

$6 / 13$

$1 / 4$

$1 / 15$

$1 / 14$

$0 / 13$

$1 / 5$

Average number of embryos transferred

2.7

3.0

3.2

4.0

$8 / 10$

$4 / 10$

$2 / 7$

$0 / 1$

$1 / 10$

$0 / 10$

$0 / 7$

$0 / 1$

$8 / 10$

$2 / 7$

$1 / 7$

$0 / 1$

Percentage of live births having multiple infants ${ }^{b, c}$

\section{Frozen Embryos from Nondonor Eggs}

Number of transfers

Percentage of transfers resulting in live births ${ }^{\mathrm{b}, \mathrm{c}}$

Average number of embryos transferred

4

$0 / 4$

1

2.5

$0 / 1$

1

$0 / 1$

4.0

3.0

All Ages Combined

Fresh Embryos

1

$0 / 1$

3.0

Number of transfers

Percentage of transfers resulting in live births ${ }^{\mathrm{b}, \mathrm{c}}$

Average number of embryos transferred

Frozen Embryos

0

\section{CURRENT CLINIC SERVICES AND PROFILE}

Current Name: Advanced Reproductive Care Center, PA

Donor egg? Yes

Donor embryo? Yes

Single women? Yes

\section{Gestational carriers? Yes}

Cryopreservation? Yes
SART member?

Yes

Verified lab accreditation?

(See Appendix C for details.)

${ }^{a}$ Reflects patient and treatment characteristics of ART cycles performed in 2006 using fresh nondonor eggs or embryos.

${ }^{b}$ When fewer than 20 cycles are reported in an age category, rates are shown as a fraction and confidence intervals are not given. Calculating percentages from fractions may be misleading and is not encouraged.

${ }^{c}$ A multiple-infant birth is counted as one live birth.

d Clinic-specific outcome rates are unreliable for women older than 42 undergoing ART cycles using fresh or frozen embryos with nondonor eggs. Readers are urged to review national outcomes for these age groups (see page 27).

${ }^{e}$ All ages (including ages $>42$ ) are reported together because previous data show that patient age does not materially affect success with donor eggs. 


\section{FLORIDA FERTILITY INSTITUTE CLEARWATER, FLORIDA}

A comparison of clinic success rates may not be meaningful because patient medical characteristics and treatment approaches vary from clinic to clinic. For more details about this, along with information on how to interpret the statistics in this table, see pages 79-88.

\section{ART CYCLE PROFILE}

\begin{tabular}{|c|c|c|c|c|c|c|c|}
\hline \multicolumn{4}{|c|}{ Type of ART ${ }^{a}$} & \multicolumn{4}{|c|}{ Patient Diagnosis } \\
\hline IVF & $100 \%$ & Procedural Factors: & & Tubal factor & $4 \%$ & Other factor & $1 \%$ \\
\hline GIFT & $0 \%$ & With ICSI & $84 \%$ & Ovulatory dysfunction & $3 \%$ & Unknown factor & $2 \%$ \\
\hline ZIFT & $0 \%$ & Unstimulated & $0 \%$ & Diminished ovarian reserve & $7 \%$ & Multiple Factors: & \\
\hline \multirow[t]{3}{*}{ Combination } & $0 \%$ & Used gestational carrier & $<1 \%$ & Endometriosis & $2 \%$ & Female factors only & $18 \%$ \\
\hline & & & & Uterine factor & $0 \%$ & Female \& male factors & $49 \%$ \\
\hline & & & & Male factor & $13 \%$ & & \\
\hline
\end{tabular}

\section{PREGNANCY SUCCESS RATES}

Data verified by Mark Sanchez, MD

\section{Type of Cycle}

\section{Fresh Embryos from Nondonor Eggs}

Number of cycles

Percentage of cycles resulting in pregnancies ${ }^{b}$

Percentage of cycles resulting in live births $s^{b, c}$

(Confidence Interval)

Percentage of retrievals resulting in live births $s^{b, c}$

Percentage of transfers resulting in live births $s^{b, c}$

Percentage of transfers resulting in singleton live births ${ }^{b}$

Percentage of cancellations ${ }^{b}$

Average number of embryos transferred

Percentage of pregnancies with twins ${ }^{b}$

Percentage of pregnancies with triplets or more ${ }^{b}$

Percentage of live births having multiple infants ${ }^{b, c}$

\section{Frozen Embryos from Nondonor Eggs}

Number of transfers

Percentage of transfers resulting in live births ${ }^{b, c}$

Average number of embryos transferred

$(20)$

\section{$<35$}

52

42.3

36.5

(23.6-51.0)

37.3

38.8

28.6

1.9

2.5

18.2

13.6

$5 / 19$

\section{1}

$0 / 1$

4.0

\section{Age of Woman}

$$
\text { 35-37 38-40 }
$$

$41-42^{d}$

48

29.2

20.8

(10.5-35.0)

23.3

25.6

12.8

10.4

2.6

$6 / 14$

$1 / 14$

$5 / 10$

2

$0 / 2$

3.5

25

$$
48.0
$$

9

$2 / 9$

36.0

$1 / 9$

$(18.0-57.5)$
37.5

37.5

$1 / 9$

45.0

40.0

$1 / 7$

$1 / 7$

4.0

$0 / 9$

2.6

2.4

$2 / 12$

$0 / 2$

$0 / 12$

$0 / 2$

$1 / 9$

$0 / 1$

All Ages Combined ${ }^{\mathrm{e}}$

Fresh Embryos

37

48.6

Frozen Embryos

3

2.8
$1 / 3$

2.0

Average number of embryos transferred

\section{CURRENT CLINIC SERVICES AND PROFILE}

Current Name: Florida Fertility Institute

\begin{tabular}{ll|ll} 
Donor egg? & Yes & Gestational carriers? & Yes \\
Donor embryo? & No & Cryopreservation? & Yes \\
Single women? & Yes & &
\end{tabular}

SART member?

No

Verified lab accreditation?

Yes

(See Appendix C for details.)

\footnotetext{
${ }^{a}$ Reflects patient and treatment characteristics of ART cycles performed in 2006 using fresh nondonor eggs or embryos.

b When fewer than 20 cycles are reported in an age category, rates are shown as a fraction and confidence intervals are not given. Calculating percentages from fractions may be misleading and is not encouraged.

${ }^{c}$ A multiple-infant birth is counted as one live birth.

${ }^{d}$ Clinic-specific outcome rates are unreliable for women older than 42 undergoing ART cycles using fresh or frozen embryos with nondonor eggs. Readers are urged to review national outcomes for these age groups (see page 27).

e All ages (including ages $>42$ ) are reported together because previous data show that patient age does not materially affect success with donor eggs.
} 


\section{INFERTILITY AND REPRODUCTIVE MEDICINE OF SOUTH BROWARD \\ KENNETH M. GELMAN, MD \\ COOPER CITY, FLORIDA}

A comparison of clinic success rates may not be meaningful because patient medical characteristics and treatment approaches vary from clinic to clinic. For more details about this, along with information on how to interpret the statistics in this table, see pages $79-88$.

\section{ART CYCLE PROFILE}

\begin{tabular}{|c|c|c|c|c|c|c|c|}
\hline \multicolumn{4}{|c|}{ Type of ART ${ }^{\mathrm{a}}$} & \multicolumn{4}{|c|}{ Patient Diagnosis } \\
\hline IVF & $100 \%$ & Procedural Factors: & & Tubal factor & $14 \%$ & Other factor & $6 \%$ \\
\hline GIFT & $0 \%$ & With ICSI & $90 \%$ & Ovulatory dysfunction & $19 \%$ & Unknown factor & $4 \%$ \\
\hline ZIFT & $0 \%$ & Unstimulated & $0 \%$ & Diminished ovarian reserve & $5 \%$ & Multiple Factors: & \\
\hline \multirow[t]{3}{*}{ Combination } & $0 \%$ & Used gestational carrier & $0 \%$ & Endometriosis & $1 \%$ & Female factors only & $14 \%$ \\
\hline & & & & Uterine factor & $0 \%$ & Female \& male factors & $26 \%$ \\
\hline & & & & Male factor & $11 \%$ & & \\
\hline
\end{tabular}

2006 PREGNANCY SUCCESS RATES

Data verified by Kenneth M. Gelman, MD

\section{Type of Cycle}

\section{Fresh Embryos from Nondonor Eggs}

Number of cycles

Percentage of cycles resulting in pregnancies ${ }^{b}$

Percentage of cycles resulting in live births $s^{b, c}$

(Confidence Interval)

Percentage of retrievals resulting in live births $s^{b, c}$

Percentage of transfers resulting in live births $s^{b, c}$

Percentage of transfers resulting in singleton live births ${ }^{b}$

Percentage of cancellations ${ }^{b}$

Average number of embryos transferred

Percentage of pregnancies with twins ${ }^{b}$

Percentage of pregnancies with triplets or more ${ }^{\mathrm{b}}$

Percentage of live births having multiple infants ${ }^{b, c}$

\section{Frozen Embryos from Nondonor Eggs}

Number of transfers

Percentage of transfers resulting in live births ${ }^{\mathrm{b}, \mathrm{c}}$

Average number of embryos transferred

\section{Age of Woman}

35-37 38-40

$41-42^{d}$ $<35$

22

22.7

15

5

51.5

18.2

$6 / 15$

$0 / 5$

45.5

(5.2-40.3)

$(28.1-63.6)$

$4 / 19$

$3 / 15$

$0 / 5$

46.9

46.9

$4 / 19$

$3 / 14$

$0 / 2$

28.1

$2 / 19$

$3 / 12$

$0 / 2$

3.0

13.6

$2 / 12$

$0 / 2$

3.0

2.9

$1 / 15$

$3 / 5$

$7 / 17$

$1 / 5$

3.6

2.5

$0 / 17$

$6 / 15$

$2 / 5$

$1 / 6$

$0 / 6$

$2 / 4$

$1 / 3$

\section{3}

$2 / 3$

1.7

\section{Donor Eggs}

Number of transfers

Percentage of transfers resulting in live births $s^{\mathrm{b}, \mathrm{c}}$

Average number of embryos transferred

\section{All Ages Combined ${ }^{\mathrm{e}}$ \\ Fresh Embryos \\ 0 \\ Frozen Embryos \\ 0}

\section{CURRENT CLINIC SERVICES AND PROFILE}

Current Name: Infertility and Reproductive Medicine of South Broward, Kenneth M. Gelman, MD

Donor egg? No

Donor embryo? No

Single women? Yes

\section{Gestational carriers? No}

Cryopreservation?
SART member?

Verified lab accreditation?

(See Appendix C for details.)
No

Pending

${ }^{a}$ Reflects patient and treatment characteristics of ART cycles performed in 2006 using fresh nondonor eggs or embryos.

${ }^{b}$ When fewer than 20 cycles are reported in an age category, rates are shown as a fraction and confidence intervals are not given. Calculating percentages from fractions may be misleading and is not encouraged.

${ }^{c}$ A multiple-infant birth is counted as one live birth.

${ }^{d}$ Clinic-specific outcome rates are unreliable for women older than 42 undergoing ART cycles using fresh or frozen embryos with nondonor eggs. Readers are urged to review national outcomes for these age groups (see page 27).

e All ages (including ages $>42$ ) are reported together because previous data show that patient age does not materially affect success with donor eggs. 


\section{SOUTHWEST FLORIDA FERTILITY CENTER, PA FORT MYERS, FLORIDA}

A comparison of clinic success rates may not be meaningful because patient medical characteristics and treatment approaches vary from clinic to clinic. For more details about this, along with information on how to interpret the statistics in this table, see pages 79-88.

\section{ART CYCLE PROFILE}

\begin{tabular}{|c|c|c|c|c|c|c|c|}
\hline \multicolumn{4}{|c|}{ Type of ART ${ }^{a}$} & \multicolumn{4}{|c|}{ Patient Diagnosis } \\
\hline IVF & $100 \%$ & Procedural Factors: & & Tubal factor & $3 \%$ & Other factor & $16 \%$ \\
\hline GIFT & $0 \%$ & With ICSI & $25 \%$ & Ovulatory dysfunction & $0 \%$ & Unknown factor & $8 \%$ \\
\hline ZIFT & $0 \%$ & Unstimulated & $0 \%$ & Diminished ovarian reserve & $3 \%$ & Multiple Factors: & \\
\hline \multirow[t]{3}{*}{ Combination } & $0 \%$ & Used gestational carrier & $0 \%$ & Endometriosis & $0 \%$ & Female factors only & $14 \%$ \\
\hline & & & & Uterine factor & $0 \%$ & Female \& male factors & $27 \%$ \\
\hline & & & & Male factor & $30 \%$ & & \\
\hline
\end{tabular}

\section{PREGNANCY SUCCESS RATES}

Data verified by Jacob L. Glock, MD

\section{Type of Cycle}

\section{Fresh Embryos from Nondonor Eggs}

Number of cycles

Percentage of cycles resulting in pregnancies ${ }^{b}$

Percentage of cycles resulting in live births ${ }^{b, c}$

(Confidence Interval)
Percentage of retrievals resulting in live births $s^{b, c}$

Percentage of transfers resulting in live births $s^{b, c}$

Percentage of transfers resulting in singleton live births ${ }^{b}$

Percentage of cancellations ${ }^{b}$

Average number of embryos transferred

Percentage of pregnancies with twins ${ }^{b}$

Percentage of pregnancies with triplets or more ${ }^{b}$

Percentage of live births having multiple infants ${ }^{b, c}$

\section{Frozen Embryos from Nondonor Eggs}

Number of transfers

Percentage of transfers resulting in live births $s^{b, c}$

Average number of embryos transferred

\section{Age of Woman}

35-37 38-40

\section{$<35$}

14

$6 / 14$

6

$5 / 14$

$0 / 6$

$0 / 6$

$0 / 6$

$0 / 5$

$0 / 5$

$0 / 6$

2.0

2.8

$2 / 6$

$1 / 6$

$3 / 5$

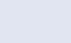

4

$0 / 4$

$0 / 4$

$0 / 4 \quad 0 / 5$

$0 / 3 \quad 0 / 3$

$0 / 3 \quad 0 / 3$

$0 / 4 \quad 0 / 5$

$2.7 \quad 2.7$

5

$0 / 5$

$0 / 5$

$(3 / 5$

1

$0 / 1$

1.0

0

0

0

\begin{tabular}{cc}
\multicolumn{2}{c}{ All Ages Combined } \\
Fresh Embryos & $\begin{array}{c}\text { Frozen Embryos } \\
4\end{array}$ \\
$1 / 4$ & 0 \\
2.8 &
\end{tabular}

\section{Donor Eggs}

Number of transfers

Percentage of transfers resulting in live births $s^{b, c}$

Average number of embryos transferred

\section{CURRENT CLINIC SERVICES AND PROFILE}

Current Name: Southwest Florida Fertility Center, PA
Donor egg?
Yes
Gestational carriers?
Yes
Cryopreservation?
SART member?
Yes
Donor embryo? No
Yes
Verified lab accreditation?
Yes
Single women? Yes
(See Appendix C for details.)

\footnotetext{
${ }^{a}$ Reflects patient and treatment characteristics of ART cycles performed in 2006 using fresh nondonor eggs or embryos.

${ }^{b}$ When fewer than 20 cycles are reported in an age category, rates are shown as a fraction and confidence intervals are not given. Calculating percentages from fractions may be misleading and is not encouraged.

${ }^{c}$ A multiple-infant birth is counted as one live birth.

${ }^{d}$ Clinic-specific outcome rates are unreliable for women older than 42 undergoing ART cycles using fresh or frozen embryos with nondonor eggs. Readers are urged to review national outcomes for these age groups (see page 27).

${ }^{e}$ All ages (including ages $>42$ ) are reported together because previous data show that patient age does not materially affect success with donor eggs.
} 


\section{SPECIALISTS IN REPRODUCTIVE MEDICINE \& SURGERY, PA \\ CRAIG R. SWEET, MD \\ FORT MYERS, FLORIDA}

A comparison of clinic success rates may not be meaningful because patient medical characteristics and treatment approaches vary from clinic to clinic. For more details about this, along with information on how to interpret the statistics in this table, see pages $79-88$.

\section{ART CYCLE PROFILE}

\begin{tabular}{|c|c|c|c|c|c|c|c|}
\hline \multicolumn{4}{|c|}{ Type of ART } & \multicolumn{4}{|c|}{ Patient Diagnosis } \\
\hline IVF & $100 \%$ & Procedural Factors: & & Tubal factor & $9 \%$ & Other factor & $9 \%$ \\
\hline GIFT & $0 \%$ & With ICSI & $84 \%$ & Ovulatory dysfunction & $6 \%$ & Unknown factor & $2 \%$ \\
\hline ZIFT & $0 \%$ & Unstimulated & $1 \%$ & Diminished ovarian reserve & $14 \%$ & Multiple Factors: & \\
\hline \multirow[t]{3}{*}{ Combination } & $0 \%$ & Used gestational carrier & $1 \%$ & Endometriosis & $8 \%$ & Female factors only & $5 \%$ \\
\hline & & & & Uterine factor & $3 \%$ & Female \& male factors & $25 \%$ \\
\hline & & & & Male factor & $20 \%$ & & \\
\hline
\end{tabular}

2006 PREGNANCY SUCCESS RATES

Data verified by Craig R. Sweet, MD

\section{Type of Cycle}

\section{Age of Woman}

\section{Fresh Embryos from Nondonor Eggs}

Number of cycles

Percentage of cycles resulting in pregnancies ${ }^{b}$

Percentage of cycles resulting in live births $s^{b, c}$

(Confidence Interval)

Percentage of retrievals resulting in live births $s^{b, c}$

Percentage of transfers resulting in live births $s^{b, c}$

Percentage of transfers resulting in singleton live births ${ }^{b}$

Percentage of cancellations ${ }^{b}$

35-37 38-40

$41-42^{d}$

$<35$

35-37

3

41
43.9

20

50.0

21

$0 / 3$

41.5

45.0

23.8

$0 / 3$

Average number of embryos transferred

Percentage of pregnancies with twins ${ }^{b}$

$(26.3-57.9)$

$(23.1-68.5)$

14.3

$(3.0-36.3)$

47.2

$9 / 18$

$3 / 16$

$0 / 2$

53.1

$9 / 13$

$3 / 12$

28.1

12.2

$3 / 13$

$3 / 12$

10.0

23.8

$1 / 3$

2.0

2.7

2.7

$9 / 18$

$5 / 10$

$2 / 5$

$4 / 18$

$1 / 10$

$0 / 5$

$8 / 17$

$6 / 9$

$0 / 3$

Percentage of pregnancies with triplets or more
Percentage of live births having multiple infants

\section{Frozen Embryos from Nondonor Eggs}

Number of transfers

Percentage of transfers resulting in live births $s^{b, c}$

Average number of embryos transferred

3

$3 / 3$

2.3

\section{2}

$1 / 2$

2.5
1

$0 / 1$

2.0

\section{Donor Eggs}

Number of transfers

Percentage of transfers resulting in live births $s^{b, c}$

Average number of embryos transferred

All Ages Combined ${ }^{\mathrm{e}}$

Fresh Embryos 16

$10 / 16$

1.9
Frozen Embryos

\section{5}

$2 / 5$

2.6

\section{CURRENT CLINIC SERVICES AND PROFILE}

Current Name: Specialists in Reproductive Medicine \& Surgery, PA, Craig R. Sweet, MD

Donor egg? Yes

Donor embryo? Yes

Single women? Yes

\begin{abstract}
Gestational carriers?
Cryopreservation?
\end{abstract}

SART member?

Verified lab accreditation?

(See Appendix C for details.)

${ }^{a}$ Reflects patient and treatment characteristics of ART cycles performed in 2006 using fresh nondonor eggs or embryos.

${ }^{b}$ When fewer than 20 cycles are reported in an age category, rates are shown as a fraction and confidence intervals are not given. Calculating percentages from fractions may be misleading and is not encouraged.

${ }^{c}$ A multiple-infant birth is counted as one live birth.

d Clinic-specific outcome rates are unreliable for women older than 42 undergoing ART cycles using fresh or frozen embryos with nondonor eggs. Readers are urged to review national outcomes for these age groups (see page 27).

e All ages (including ages $>42$ ) are reported together because previous data show that patient age does not materially affect success with donor eggs. 


\section{UNIVERSITY OF FLORIDA WOMEN'S HEALTH AT MAGNOLIA PARKE GAINESVILLE, FLORIDA}

A comparison of clinic success rates may not be meaningful because patient medical characteristics and treatment approaches vary from clinic to clinic. For more details about this, along with information on how to interpret the statistics in this table, see pages 79-88.

\section{ART CYCLE PROFILE}

\begin{tabular}{|c|c|c|c|c|c|c|c|}
\hline \multicolumn{4}{|c|}{ Type of ART ${ }^{a}$} & \multicolumn{4}{|c|}{ Patient Diagnosis } \\
\hline IVF & $100 \%$ & Procedural Factors: & & Tubal factor & $9 \%$ & Other factor & $13 \%$ \\
\hline GIFT & $0 \%$ & With ICSI & $40 \%$ & Ovulatory dysfunction & $<1 \%$ & Unknown factor & $2 \%$ \\
\hline ZIFT & $0 \%$ & Unstimulated & $0 \%$ & Diminished ovarian reserve & $4 \%$ & Multiple Factors: & \\
\hline \multirow[t]{3}{*}{ Combination } & $0 \%$ & Used gestational carrier & $2 \%$ & Endometriosis & $11 \%$ & Female factors only & $28 \%$ \\
\hline & & & & Uterine factor & $<1 \%$ & Female \& male factors & $15 \%$ \\
\hline & & & & Male factor & $17 \%$ & & \\
\hline
\end{tabular}

\section{PREGNANCY SUCCESS RATES}

\section{Type of Cycle}

\section{Fresh Embryos from Nondonor Eggs}

Number of cycles

Percentage of cycles resulting in pregnancies ${ }^{b}$

Percentage of cycles resulting in live births ${ }^{b, c}$

(Confidence Interval)

Percentage of retrievals resulting in live births $s^{b, c}$

Percentage of transfers resulting in live births ${ }^{b, c}$

Percentage of transfers resulting in singleton live births ${ }^{b}$

Percentage of cancellations ${ }^{b}$

Average number of embryos transferred

Percentage of pregnancies with twins ${ }^{b}$

Percentage of pregnancies with triplets or more ${ }^{b}$

Percentage of live births having multiple infants ${ }^{b, c}$

\section{Frozen Embryos from Nondonor Eggs}

Number of transfers

Percentage of transfers resulting in live births ${ }^{b, c}$

Average number of embryos transferred

\section{Donor Eggs}

Number of transfers

Percentage of transfers resulting in live births $s^{b, c}$

Average number of embryos transferred
Data verified by R. Stan Williams, MD

\section{Age of Woman}

35-37 38-40

$41-42^{d}$

$<35$

19

11

8

37.0

$4 / 19$

$4 / 11$

$1 / 8$

26.1

$3 / 19$

$2 / 11$

$0 / 8$

$(14.3-41.1)$

30.0

32.4

$3 / 18$

$2 / 11$

$0 / 8$

21.6

$3 / 15$

$2 / 11$

$0 / 7$

$2 / 15$

2/ 11

$0 / 7$

13.0

$1 / 19$

$0 / 11$

$0 / 8$

1.9

2.5

2.2

2.4

$5 / 17$

$2 / 4$

$0 / 4$

$0 / 1$

$0 / 17$

$0 / 4$

$0 / 4$

$0 / 1$

$4 / 12$

$1 / 3$

$0 / 2$

2

$0 / 2$

0

1.5

$0 / 2$

1.5

0

\section{CURRENT CLINIC SERVICES AND PROFILE}

Current Name: University of Florida Women's Health at Magnolia Parke

Donor egg? Yes

Donor embryo? Yes

Single women? No

\section{Gestational carriers? Yes}

Cryopreservation? Yes

\section{Fresh Embryos}

10

$2 / 10$

2.0

\section{All Ages Combined ${ }^{\mathrm{e}}$}

Frozen Embryos

0

\footnotetext{
${ }^{a}$ Reflects patient and treatment characteristics of ART cycles performed in 2006 using fresh nondonor eggs or embryos.

${ }^{b}$ When fewer than 20 cycles are reported in an age category, rates are shown as a fraction and confidence intervals are not given. Calculating percentages from fractions may be misleading and is not encouraged.

${ }^{c}$ A multiple-infant birth is counted as one live birth.

d Clinic-specific outcome rates are unreliable for women older than 42 undergoing ART cycles using fresh or frozen embryos with nondonor eggs. Readers are urged to review national outcomes for these age groups (see page 27).

e All ages (including ages $>42$ ) are reported together because previous data show that patient age does not materially affect success with donor eggs.
} 


\section{ASSISTED FERTILITY PROGRAM OF NORTH FLORIDA JACKSONVILLE, FLORIDA}

A comparison of clinic success rates may not be meaningful because patient medical characteristics and treatment approaches vary from clinic to clinic. For more details about this, along with information on how to interpret the statistics in this table, see pages $79-88$.

\section{ART CYCLE PROFILE}

\section{Type of ART ${ }^{\mathrm{a}}$}

$\begin{array}{llr}\text { IVF } & 96 \% \text { Procedural Factors: } & \\ \text { GIFT } & 0 \% \text { With ICSI } & 12 \% \\ \text { ZIFT } & 4 \% \text { Unstimulated } & 0 \% \\ \text { Combination } & 0 \% \text { Used gestational carrier } & 4 \%\end{array}$

2006 PREGNANCY SUCCESS RATES

$$
\text { Type of Cycle }
$$

\section{Fresh Embryos from Nondonor Eggs}

Number of cycles

Percentage of cycles resulting in pregnancies ${ }^{b}$

Percentage of cycles resulting in live births $s^{b, c}$

(Confidence Interval)

Percentage of retrievals resulting in live births $s^{b, c}$

Percentage of transfers resulting in live births $s^{b, c}$

Percentage of transfers resulting in singleton live births ${ }^{b}$

Percentage of cancellations ${ }^{b}$

Average number of embryos transferred

Percentage of pregnancies with twins ${ }^{b}$

Percentage of pregnancies with triplets or more ${ }^{\mathrm{b}}$

Percentage of live births having multiple infants ${ }^{b, c}$

\section{Frozen Embryos from Nondonor Eggs}

Number of transfers

Percentage of transfers resulting in live births ${ }^{\mathrm{b}, \mathrm{c}}$

Average number of embryos transferred

\section{Donor Eggs}

Number of transfers

Percentage of transfers resulting in live births $s^{b, c}$

Average number of embryos transferred

\section{Patient Diagnosis}

\section{Tubal factor}

20\% Other factor

$0 \%$ Unknown factor

$0 \%$

Ovulatory dysfunction

35\% Multiple Factors:

Diminished ovarian reserve

$11 \%$ Female factors only

$11 \%$

Uterine factor

$2 \%$

$9 \%$

Male factor

Data verified by Marwan M. Shaykh, MD

\section{Age of Woman}

$\begin{array}{cccc}<35 & 35-37 & 38-40 & \mathbf{4 1 - 4 2}^{\mathbf{d}} \\ 17 & 4 & 4 & 1 \\ 3 / 17 & 0 / 4 & 1 / 4 & 0 / 1 \\ 3 / 17 & 0 / 4 & 1 / 4 & 0 / 1\end{array}$

$\begin{array}{cccc}3 / 15 & 0 / 4 & 1 / 3 & 0 / 1 \\ 3 / 15 & 0 / 4 & 1 / 3 & 0 / 1 \\ 2 / 15 & 0 / 4 & 0 / 3 & 0 / 1 \\ 2 / 17 & 0 / 4 & 1 / 4 & 0 / 1 \\ 3.1 & 3.0 & 3.3 & 2.0 \\ 1 / 3 & & 0 / 1 & \\ 0 / 3 & & 1 / 1 & \\ 1 / 3 & & 1 / 1 & \end{array}$

1

$0 / 1$

3.0
1

$0 / 1$

2.0
0

0

All Ages Combined ${ }^{\mathrm{e}}$

Fresh Embryos

Frozen Embryos

$\begin{array}{cc}10 & 4 \\ 3 / 10 & 0 / 4 \\ 3.4 & 2.0\end{array}$

\section{CURRENT CLINIC SERVICES AND PROFILE}

Current Name: Assisted Fertility Program of North Florida

Donor egg? Yes

Donor embryo? Yes

Single women? Yes

\section{Gestational carriers? Yes}

Cryopreservation? Yes
SART member?

Verified lab accreditation?

(See Appendix C for details.)
Yes

Pending

${ }^{a}$ Reflects patient and treatment characteristics of ART cycles performed in 2006 using fresh nondonor eggs or embryos.

b When fewer than 20 cycles are reported in an age category, rates are shown as a fraction and confidence intervals are not given. Calculating percentages from fractions may be misleading and is not encouraged.

${ }^{c}$ A multiple-infant birth is counted as one live birth.

${ }^{d}$ Clinic-specific outcome rates are unreliable for women older than 42 undergoing ART cycles using fresh or frozen embryos with nondonor eggs. Readers are urged to review national outcomes for these age groups (see page 27).

e All ages (including ages $>42$ ) are reported together because previous data show that patient age does not materially affect success with donor eggs. 


\section{FLORIDA INSTITUTE FOR REPRODUCTIVE MEDICINE JACKSONVILLE, FLORIDA}

A comparison of clinic success rates may not be meaningful because patient medical characteristics and treatment approaches vary from clinic to clinic. For more details about this, along with information on how to interpret the statistics in this table, see pages 79-88.

\section{ART CYCLE PROFILE}

\begin{tabular}{|c|c|c|c|c|c|c|c|}
\hline \multicolumn{4}{|c|}{ Type of ART ${ }^{a}$} & \multicolumn{4}{|c|}{ Patient Diagnosis } \\
\hline IVF & $100 \%$ & Procedural Factors: & & Tubal factor & $6 \%$ & Other factor & $3 \%$ \\
\hline GIFT & $0 \%$ & With ICSI & $74 \%$ & Ovulatory dysfunction & $3 \%$ & Unknown factor & $5 \%$ \\
\hline ZIFT & $0 \%$ & Unstimulated & $0 \%$ & Diminished ovarian reserve & $7 \%$ & Multiple Factors: & \\
\hline \multirow[t]{3}{*}{ Combination } & $0 \%$ & Used gestational carrier & $<1 \%$ & Endometriosis & $5 \%$ & Female factors only & $8 \%$ \\
\hline & & & & Uterine factor & $<1 \%$ & Female \& male factors & $36 \%$ \\
\hline & & & & Male factor & $26 \%$ & & \\
\hline
\end{tabular}

2006 PREGNANCY SUCCESS RATES

Data verified by Kevin L. Winslow, MD

\section{Type of Cycle}

\section{Fresh Embryos from Nondonor Eggs}

Number of cycles

Percentage of cycles resulting in pregnancies ${ }^{b}$

Percentage of cycles resulting in live births ${ }^{\mathrm{b}, \mathrm{c}}$

(Confidence Interval)

Percentage of retrievals resulting in live births $s^{b, c}$

Percentage of transfers resulting in live births $s^{b, c}$

Percentage of transfers resulting in singleton live births ${ }^{b}$

Percentage of cancellations ${ }^{b}$

Average number of embryos transferred

Percentage of pregnancies with twins ${ }^{b}$

Percentage of pregnancies with triplets or more ${ }^{b}$

Percentage of live births having multiple infants ${ }^{b, c}$

\section{Frozen Embryos from Nondonor Eggs}

Number of transfers

Percentage of transfers resulting in live births $s^{\mathrm{b}, \mathrm{c}}$

Average number of embryos transferred

\section{Donor Eggs}

Number of transfers

Percentage of transfers resulting in live births $s^{b, c}$

Average number of embryos transferred

\section{Age of Woman}

35-37 38-40

$41-42^{d}$

$<35$

149

101

33

286
44.8

33.6

23.8

12.1

41.6

28.2

18.8

12.1

$(35.8-47.6)$

48.4

$(21.1-36.1)$

$(11.7-27.8)$

34.4

35.3

21.1

22.4

20.0

10.9

3.2

16.7

0.0

$2 / 19$

33.3

39.5

106

30.2

72

25.0

2.7

All Ages Combined ${ }^{\mathrm{e}}$

Fresh Embryos

64

34.4

2.5
(3.4-28.2)

14.3

14.8

11.1

15.2

3.5

$0 / 4$

$1 / 4$

$1 / 4$

\section{CURRENT CLINIC SERVICES AND PROFILE}

Current Name: Florida Institute for Reproductive Medicine

Donor egg? Yes

Donor embryo? Yes

Gestational carriers? Yes

Cryopreservation?

SART member?

Yes

Verified lab accreditation?

Yes

Single women? Yes

(See Appendix C for details.)

${ }^{a}$ Reflects patient and treatment characteristics of ART cycles performed in 2006 using fresh nondonor eggs or embryos.

${ }^{b}$ When fewer than 20 cycles are reported in an age category, rates are shown as a fraction and confidence intervals are not given. Calculating percentages from fractions may be misleading and is not encouraged.

${ }^{c}$ A multiple-infant birth is counted as one live birth.

d Clinic-specific outcome rates are unreliable for women older than 42 undergoing ART cycles using fresh or frozen embryos with nondonor eggs. Readers are urged to review national outcomes for these age groups (see page 27).

e All ages (including ages $>42$ ) are reported together because previous data show that patient age does not materially affect success with donor eggs. 


\section{JACKSONVILLE CENTER FOR REPRODUCTIVE MEDICINE \\ JACKSONVILLE, FLORIDA}

A comparison of clinic success rates may not be meaningful because patient medical characteristics and treatment approaches vary from clinic to clinic. For more details about this, along with information on how to interpret the statistics in this table, see pages $79-88$.

\section{ART CYCLE PROFILE}

\begin{tabular}{|c|c|c|c|c|c|c|}
\hline & Type of ART ${ }^{a}$ & & Pati & ent D & iagnosis & \\
\hline IVF & 100\% Procedural Factors: & & Tubal factor & $11 \%$ & Other factor & $2 \%$ \\
\hline GIFT & 0\% With ICSI & $18 \%$ & Ovulatory dysfunction & $20 \%$ & Unknown factor & $1 \%$ \\
\hline ZIFT & $0 \%$ Unstimulated & $0 \%$ & Diminished ovarian reserve & $39 \%$ & Multiple Factors: & \\
\hline Combination & $0 \%$ Used gestational carrier & $0 \%$ & Endometriosis & $8 \%$ & Female factors only & $8 \%$ \\
\hline & & & Uterine factor & $0 \%$ & Female \& male factors & $3 \%$ \\
\hline & & & Male factor & $8 \%$ & & \\
\hline
\end{tabular}

2006 PREGNANCY SUCCESS RATES

Data verified by Michael D. Fox, MD

\section{Type of Cycle}

\section{Fresh Embryos from Nondonor Eggs}

Number of cycles

Percentage of cycles resulting in pregnancies ${ }^{b}$

Percentage of cycles resulting in live births $s^{b, c}$

(Confidence Interval)

Percentage of retrievals resulting in live births $s^{b, c}$

Percentage of transfers resulting in live births $s^{b, c}$

Percentage of transfers resulting in singleton live births ${ }^{b}$

Percentage of cancellations ${ }^{b}$

Average number of embryos transferred

Percentage of pregnancies with twins ${ }^{b}$

Percentage of pregnancies with triplets or more ${ }^{b}$

Percentage of live births having multiple infants ${ }^{b, c}$

\section{Frozen Embryos from Nondonor Eggs}

Number of transfers

Percentage of transfers resulting in live births ${ }^{\mathrm{b}, \mathrm{c}}$

Average number of embryos transferred

\section{Donor Eggs}

Number of transfers

Percentage of transfers resulting in live births $s^{b, c}$

Average number of embryos transferred

\section{Age of Woman}

35-37 38-40

$41-42^{d}$

\section{$<35$}

21

46

41.3

47.6

8

$2 / 8$

$2 / 8$

$0 / 1$

34.8

47.6

$2 / 6$

$2 / 6$

47.6

37.2

$10 / 19$

$2 / 6$

24.4

$5 / 19$

$2 / 8$

2.3

2.3

0.0

$0 / 2$

$6 / 19$

$5 / 10$

$0 / 2$

$0 / 19$

$0 / 10$

$0 / 2$

$5 / 10$

3

$1 / 3$

2.7

\section{1}

$0 / 1$

3.0
1
$0 / 1$
2.0

All Ages Combined
Frozen Embryos

\section{5}

$3 / 5$

2.0

\section{2}

$1 / 2$

2.5

\section{CURRENT CLINIC SERVICES AND PROFILE}

Current Name: Jacksonville Center for Reproductive Medicine

Donor egg? Yes Gestational carriers? Yes

Donor embryo? Yes Cryopreservation? Yes

Single women? Yes

SART member? Yes

Verified lab accreditation?

Pending

${ }^{a}$ Reflects patient and treatment characteristics of ART cycles performed in 2006 using fresh nondonor eggs or embryos.

${ }^{b}$ When fewer than 20 cycles are reported in an age category, rates are shown as a fraction and confidence intervals are not given. Calculating percentages from fractions may be misleading and is not encouraged.

${ }^{c}$ A multiple-infant birth is counted as one live birth.

d Clinic-specific outcome rates are unreliable for women older than 42 undergoing ART cycles using fresh or frozen embryos with nondonor eggs. Readers are urged to review national outcomes for these age groups (see page 27).

e All ages (including ages $>42$ ) are reported together because previous data show that patient age does not materially affect success with donor eggs. 


\section{GENE F. MANKO, MD, INC. JUPITER, FLORIDA}

A comparison of clinic success rates may not be meaningful because patient medical characteristics and treatment approaches vary from clinic to clinic. For more details about this, along with information on how to interpret the statistics in this table, see pages 79-88.

\section{ART CYCLE PROFILE}

\section{Type of ART ${ }^{\mathrm{a}}$}

IVF

GIFT

100\% Procedural Factors:

ZIFT

Combination
$0 \%$ With ICSI

$0 \%$ Unstimulated

$0 \%$ Used gestational carrier

\begin{tabular}{r|l}
\hline $60 \%$ & Tubal factor \\
0vulatory dysfunction & Diminished ovarian reserve \\
$3 \%$ & Endometriosis \\
& Uterine factor \\
& Male factor
\end{tabular}

\section{Patient Diagnosis}

$\begin{array}{rlr}21 \% & \text { Other factor } & 1 \% \\ 4 \% & \text { Unknown factor } & 15 \% \\ 4 \% & \text { Multiple Factors: } & \\ 9 \% & \text { Female factors only } & 1 \% \\ 2 \% & \text { Female \& male factors } & 13 \% \\ 31 \% & & \end{array}$

2006 PREGNANCY SUCCESS RATES

Data verified by Gene F. Manko, MD

\section{Type of Cycle}

\section{Fresh Embryos from Nondonor Eggs}

Number of cycles

Percentage of cycles resulting in pregnancies ${ }^{b}$

Percentage of cycles resulting in live births ${ }^{b, c}$

(Confidence Interval)

Percentage of retrievals resulting in live births $s^{b, c}$

Percentage of transfers resulting in live births $s^{b, c}$

Percentage of transfers resulting in singleton live births ${ }^{b}$

Percentage of cancellations ${ }^{b}$

Average number of embryos transferred

Percentage of pregnancies with twins ${ }^{b}$

Percentage of pregnancies with triplets or more ${ }^{b}$

Percentage of live births having multiple infants ${ }^{b, c}$

\section{Frozen Embryos from Nondonor Eggs}

Number of transfers

Percentage of transfers resulting in live births $s^{b, c}$

Average number of embryos transferred

\section{Age of Woman}

35-37 38-40

$<35$

30

56.7

53.3

21

42.9

38.1

18

$5 / 18$

$3 / 18$

(18.1-61.6)

$(34.3-71.7)$

61.5

69.6

1.7

$5 / 17$

$4 / 16$

$41-42^{d}$

3

$0 / 3$

$0 / 3$

Average number of embryos transferred

\section{Donor Eggs}

Number of transfers

Percentage of transfers resulting in live births ${ }^{b, c}$

Average number of embryos transferred

\section{1}

$1 / 1$

2.0
$8 / 19$

$3 / 13$

$3 / 9$

$2 / 9$

$5 / 18$

1.7

$2 / 5$

$0 / 5$

$1 / 3$

$8 / 14$

$7 / 14$

9.5

1.9

$0 / 9$

$1 / 8$

\section{2}

$0 / 2$

2.0
$0 / 1$

$0 / 1$

$0 / 1$

$2 / 3$

2.0

All Ages Combined ${ }^{\mathrm{e}}$

$\begin{array}{cc}\text { Fresh Embryos } & \text { Frozen Embryos } \\ 1 & 3 \\ 1 / 1 & 1 / 3 \\ 2.0 & 1.3\end{array}$

\section{CURRENT CLINIC SERVICES AND PROFILE}

Current Name: Gene F. Manko, MD, Inc.

\begin{tabular}{|c|c|c|c|c|}
\hline Donor egg? & Yes & Gestational carriers? & Yes & SART member? \\
\hline Donor embryo? & No & Cryopreservation? & Yes & Verified lab accreditation? \\
\hline
\end{tabular}

\footnotetext{
${ }^{a}$ Reflects patient and treatment characteristics of ART cycles performed in 2006 using fresh nondonor eggs or embryos.

b When fewer than 20 cycles are reported in an age category, rates are shown as a fraction and confidence intervals are not given. Calculating percentages from fractions may be misleading and is not encouraged.

${ }^{c}$ A multiple-infant birth is counted as one live birth.

d Clinic-specific outcome rates are unreliable for women older than 42 undergoing ART cycles using fresh or frozen embryos with nondonor eggs. Readers are urged to review national outcomes for these age groups (see page 27).

e All ages (including ages $>42$ ) are reported together because previous data show that patient age does not materially affect success with donor eggs.
} 


\section{IVF FLORIDA \\ MARGATE, FLORIDA}

A comparison of clinic success rates may not be meaningful because patient medical characteristics and treatment approaches vary from clinic to clinic. For more details about this, along with information on how to interpret the statistics in this table, see pages $79-88$.

\section{ART CYCLE PROFILE}

\begin{tabular}{|c|c|c|c|c|c|c|c|}
\hline \multicolumn{4}{|c|}{ Type of ART ${ }^{\mathrm{a}}$} & \multicolumn{4}{|c|}{ Patient Diagnosis } \\
\hline IVF & $100 \%$ & Procedural Factors: & & Tubal factor & $12 \%$ & Other factor & $6 \%$ \\
\hline GIFT & $0 \%$ & With ICSI & $66 \%$ & Ovulatory dysfunction & $5 \%$ & Unknown factor & $15 \%$ \\
\hline $\mathrm{ZIFT}$ & $0 \%$ & Unstimulated & $0 \%$ & Diminished ovarian reserve & $20 \%$ & Multiple Factors: & \\
\hline \multirow[t]{3}{*}{ Combination } & $0 \%$ & Used gestational carrier & $<1 \%$ & Endometriosis & $10 \%$ & Female factors only & $7 \%$ \\
\hline & & & & Uterine factor & $3 \%$ & Female \& male factors & $8 \%$ \\
\hline & & & & Male factor & $15 \%$ & & \\
\hline
\end{tabular}

2006 PREGNANCY SUCCESS RATES

Data verified by David I. Hoffman, MD

\section{Type of Cycle}

\section{Fresh Embryos from Nondonor Eggs}

Number of cycles

Percentage of cycles resulting in pregnancies ${ }^{b}$

Percentage of cycles resulting in live births $s^{b, c}$

(Confidence Interval)

Percentage of retrievals resulting in live births $s^{b, c}$

Percentage of transfers resulting in live births $s^{b, c}$

Percentage of transfers resulting in singleton live births ${ }^{b}$

Percentage of cancellations ${ }^{b}$

Average number of embryos transferred

Percentage of pregnancies with twins ${ }^{b}$

Percentage of pregnancies with triplets or more ${ }^{b}$

Percentage of live births having multiple infants ${ }^{b, c}$

\section{Frozen Embryos from Nondonor Eggs}

Number of transfers

Percentage of transfers resulting in live births $s^{b, c}$

Average number of embryos transferred

\section{Donor Eggs}

Number of transfers

Percentage of transfers resulting in live births $s^{b, c}$

Average number of embryos transferred

\section{Age of Woman}

35-37 38-40

$41-42^{d}$

\section{$<35$}

174

35.6

26.4

148

71

43.0

36.5

(20.1-33.6)

31.1

14.1

(31.0-42.3)

40.4

31.7

(14.7-28.4

5.6

42.6

32.9

\section{8}

(1.6-13.8)

27.5

20.7

26.7

8.7

9.6

16.7

24.1

8.7

2.1

34.9

2.4

18.9

8.7

35.2

3.0

3.4

3.2

29.0

19.6

$0 / 10$

35.5

3.2

0.0

$0 / 10$

37.0

9.7

$0 / 4$

83

28.9

2.3
26

26.9

2.5

\section{7}

37.0

2.5
3

$1 / 3$

2.3

All Ages Combined ${ }^{e}$

Fresh Embryos 58

46.6

2.0
Frozen Embryos 9

$5 / 9$

2.0

\section{CURRENT CLINIC SERVICES AND PROFILE}

Current Name: IVF Florida

Donor egg? Yes

Donor embryo? No

Single women? Yes

$\begin{array}{ll}\text { Gestational carriers? } & \text { Yes } \\ \text { Cryopreservation? } & \text { Yes }\end{array}$

\section{SART member?}

Verified lab accreditation?

(See Appendix C for details.)

${ }^{a}$ Reflects patient and treatment characteristics of ART cycles performed in 2006 using fresh nondonor eggs or embryos.

${ }^{b}$ When fewer than 20 cycles are reported in an age category, rates are shown as a fraction and confidence intervals are not given. Calculating percentages from fractions may be misleading and is not encouraged.

${ }^{c}$ A multiple-infant birth is counted as one live birth.

${ }^{d}$ Clinic-specific outcome rates are unreliable for women older than 42 undergoing ART cycles using fresh or frozen embryos with nondonor eggs. Readers are urged to review national outcomes for these age groups (see page 27).

${ }^{e}$ All ages (including ages $>42$ ) are reported together because previous data show that patient age does not materially affect success with donor eggs. 


\section{FERTILITY \& REPRODUCTIVE MEDICINE CENTER FOR WOMEN MELBOURNE, FLORIDA}

A comparison of clinic success rates may not be meaningful because patient medical characteristics and treatment approaches vary from clinic to clinic. For more details about this, along with information on how to interpret the statistics in this table, see pages 79-88.

\section{ART CYCLE PROFILE}

\begin{tabular}{|c|c|c|c|c|c|c|c|}
\hline \multicolumn{4}{|c|}{ Type of ART ${ }^{a}$} & \multicolumn{4}{|c|}{ Patient Diagnosis } \\
\hline IVF & $100 \%$ & Procedural Factors: & & Tubal factor & $11 \%$ & Other factor & $2 \%$ \\
\hline GIFT & $0 \%$ & With ICSI & $36 \%$ & Ovulatory dysfunction & $5 \%$ & Unknown factor & $5 \%$ \\
\hline ZIFT & $0 \%$ & Unstimulated & $0 \%$ & Diminished ovarian reserve & $13 \%$ & Multiple Factors: & \\
\hline \multirow[t]{3}{*}{ Combination } & $0 \%$ & Used gestational carrier & $2 \%$ & Endometriosis & $5 \%$ & Female factors only & $31 \%$ \\
\hline & & & & Uterine factor & $0 \%$ & Female \& male factors & $17 \%$ \\
\hline & & & & Male factor & $13 \%$ & & \\
\hline
\end{tabular}

\section{PREGNANCY SUCCESS RATES}

Data verified by Diran Chamoun, MD

\section{Type of Cycle}

\section{Fresh Embryos from Nondonor Eggs}

Number of cycles

Percentage of cycles resulting in pregnancies ${ }^{b}$

Percentage of cycles resulting in live births $s^{b, c}$

(Confidence Interval)

Percentage of retrievals resulting in live births $s^{b, c}$

Percentage of transfers resulting in live births $s^{b, c}$

Percentage of transfers resulting in singleton live births ${ }^{b}$

Percentage of cancellations ${ }^{b}$

Average number of embryos transferred

Percentage of pregnancies with twins ${ }^{b}$

Percentage of pregnancies with triplets or more ${ }^{b}$

Percentage of live births having multiple infants ${ }^{\mathrm{b}, \mathrm{c}}$

\section{Frozen Embryos from Nondonor Eggs}

Number of transfers

Percentage of transfers resulting in live births ${ }^{b, c}$

Average number of embryos transferred

\section{Donor Eggs}

Number of transfers

Percentage of transfers resulting in live births $s^{b, c}$

Average number of embryos transferred

\section{Age of Woman}

35-37 38-40

$41-42^{d}$

\section{$<35$}

18

$10 / 18$

18

2

42.3

$8 / 18$

$4 / 18$

$0 / 2$

26.9

$4 / 18$

$0 / 2$

$(11.6-47.8)$

30.4

$8 / 18$

$4 / 12$

$0 / 2$

$7 / 19$

$8 / 17$

$4 / 11$

$0 / 1$

$3 / 19$

$5 / 17$

$2 / 11$

$0 / 1$

11.5

$0 / 18$

$6 / 18$

$0 / 2$

2.3

2.3

$4 / 11$

$4 / 10$

2.0

1.0

$1 / 11$

$4 / 7$

$1 / 10$

$1 / 4$

$1 / 4$

$3 / 8$

$2 / 4$

1

$0 / 1$

2.0

\section{1}

$0 / 1$

2.0
1

$1 / 1$

3.0

\section{CURRENT CLINIC SERVICES AND PROFILE}

Current Name: Fertility \& Reproductive Medicine Center for Women

Donor egg? Yes Gestational carriers? Yes

Donor embryo? No Cryopreservation? Yes

Fresh Embryos

All Ages Combined ${ }^{\mathrm{e}}$
Frozen Embryos

$\begin{array}{cc}14 & 1 \\ 7 / 14 & 0 / 1 \\ 2.4 & 2.0\end{array}$

Single women? Yes

SART member? $\quad$ Yes

Verified lab accreditation? Yes

(See Appendix C for details.)

${ }^{a}$ Reflects patient and treatment characteristics of ART cycles performed in 2006 using fresh nondonor eggs or embryos.

b When fewer than 20 cycles are reported in an age category, rates are shown as a fraction and confidence intervals are not given. Calculating percentages from fractions may be misleading and is not encouraged.

${ }^{c}$ A multiple-infant birth is counted as one live birth.

d Clinic-specific outcome rates are unreliable for women older than 42 undergoing ART cycles using fresh or frozen embryos with nondonor eggs. Readers are urged to review national outcomes for these age groups (see page 27).

e All ages (including ages $>42$ ) are reported together because previous data show that patient age does not materially affect success with donor eggs. 


\section{FERTILITY \& IVF CENTER OF MIAMI, INC. MIAMI, FLORIDA}

A comparison of clinic success rates may not be meaningful because patient medical characteristics and treatment approaches vary from clinic to clinic. For more details about this, along with information on how to interpret the statistics in this table, see pages $79-88$.

\section{ART CYCLE PROFILE}

\begin{tabular}{|c|c|c|c|c|c|c|c|}
\hline \multicolumn{4}{|c|}{ Type of ART ${ }^{a}$} & \multicolumn{4}{|c|}{ Patient Diagnosis } \\
\hline IVF & $100 \%$ & Procedural Factors: & & Tubal factor & $8 \%$ & Other factor & $4 \%$ \\
\hline GIFT & $0 \%$ & With ICSI & $83 \%$ & Ovulatory dysfunction & $10 \%$ & Unknown factor & $3 \%$ \\
\hline ZIFT & $0 \%$ & Unstimulated & $0 \%$ & Diminished ovarian reserve & $7 \%$ & Multiple Factors: & \\
\hline \multirow[t]{3}{*}{ Combination } & $0 \%$ & Used gestational carrier & $1 \%$ & Endometriosis & $3 \%$ & Female factors only & $9 \%$ \\
\hline & & & & Uterine factor & $<1 \%$ & Female \& male factors & $35 \%$ \\
\hline & & & & Male factor & $21 \%$ & & \\
\hline
\end{tabular}

2006 PREGNANCY SUCCESS RATES

Data verified by Michael H. Jacobs, MD

\section{Type of Cycle}

\section{Fresh Embryos from Nondonor Eggs}

Number of cycles

Percentage of cycles resulting in pregnancies ${ }^{b}$

Percentage of cycles resulting in live births $s^{b, c}$

(Confidence Interval)

Percentage of retrievals resulting in live births $s^{b, c}$

Percentage of transfers resulting in live births $s^{b, c}$

Percentage of transfers resulting in singleton live births ${ }^{b}$

Percentage of cancellations ${ }^{b}$

Average number of embryos transferred

Percentage of pregnancies with twins ${ }^{b}$

Percentage of pregnancies with triplets or more ${ }^{b}$

Percentage of live births having multiple infants ${ }^{b, c}$

\section{Frozen Embryos from Nondonor Eggs}

Number of transfers

Percentage of transfers resulting in live births ${ }^{\mathrm{b}, \mathrm{c}}$

Average number of embryos transferred

\section{Donor Eggs}

Number of transfers

Percentage of transfers resulting in live births ${ }^{\mathrm{b}, \mathrm{c}}$

Average number of embryos transferred

\section{Age of Woman}

35-37 38-40

$41-42^{d}$

\section{$<35$}

74

79

26

197

44.2

44.6

27.8

11.5

41.6

35.1

21.5

11.5

(34.7-48.8)

(24.4-47.1)

(13.1-32.2)

(2.4-30.2)

45.8

38.8

27.0

40.0

28.3

14.3

48.0

27.7

23.3

14.3

29.8

9.5

20.3

9.5

2.3

2.6

2.7

19.2

39.1

24.2

4.6

6.1

37.8

30.8

18.2

2.8

$1 / 3$

4.5

$0 / 3$

$3 / 17$

$1 / 3$

40

35.0

23

30.4

12

2

2.3

2.0

$3 / 12$

$0 / 2$

2.5

3.5

All Ages Combined ${ }^{\mathrm{e}}$

Fresh Embryos

31

54.8

2.3
Frozen Embryos

\section{5}

$4 / 5$

2.6

\section{CURRENT CLINIC SERVICES AND PROFILE}

Current Name: Fertility \& IVF Center of Miami, Inc.

Donor egg? Yes

Donor embryo? Yes

Gestational carriers? Yes

Cryopreservation? Yes

Single women? Yes

SART member?

Yes

Verified lab accreditation?

(See Appendix C for details.)

${ }^{a}$ Reflects patient and treatment characteristics of ART cycles performed in 2006 using fresh nondonor eggs or embryos.

${ }^{b}$ When fewer than 20 cycles are reported in an age category, rates are shown as a fraction and confidence intervals are not given. Calculating percentages from fractions may be misleading and is not encouraged.

${ }^{c}$ A multiple-infant birth is counted as one live birth.

d Clinic-specific outcome rates are unreliable for women older than 42 undergoing ART cycles using fresh or frozen embryos with nondonor eggs. Readers are urged to review national outcomes for these age groups (see page 27).

e All ages (including ages $>42$ ) are reported together because previous data show that patient age does not materially affect success with donor eggs. 


\section{PALMETTO FERTILITY CENTER OF SOUTH FLORIDA \\ MIAMI, FLORIDA}

A comparison of clinic success rates may not be meaningful because patient medical characteristics and treatment approaches vary from clinic to clinic. For more details about this, along with information on how to interpret the statistics in this table, see pages 79-88.

\section{ART CYCLE PROFILE}

\begin{tabular}{|c|c|c|c|c|c|c|c|}
\hline \multicolumn{4}{|c|}{ Type of ART ${ }^{a}$} & \multicolumn{4}{|c|}{ Patient Diagnosis } \\
\hline IVF & $100 \%$ & Procedural Factors: & & Tubal factor & $19 \%$ & Other factor & $0 \%$ \\
\hline GIFT & $0 \%$ & With ICSI & $67 \%$ & Ovulatory dysfunction & $7 \%$ & Unknown factor & $11 \%$ \\
\hline ZIFT & $0 \%$ & Unstimulated & $0 \%$ & Diminished ovarian reserve & $13 \%$ & Multiple Factors: & \\
\hline \multirow[t]{3}{*}{ Combination } & $0 \%$ & Used gestational carrier & $0 \%$ & Endometriosis & $<1 \%$ & Female factors only & $4 \%$ \\
\hline & & & & Uterine factor & $2 \%$ & Female \& male factors & $24 \%$ \\
\hline & & & & Male factor & $20 \%$ & & \\
\hline
\end{tabular}

2006 PREGNANCY SUCCESS RATES

Data verified by Michael D. Graubert, MD

\section{Type of Cycle}

\section{Fresh Embryos from Nondonor Eggs}

Number of cycles

Percentage of cycles resulting in pregnancies ${ }^{b}$

Percentage of cycles resulting in live births $s^{b, c}$

(Confidence Interval)

Percentage of retrievals resulting in live births $s^{b, c}$

Percentage of transfers resulting in live births $s^{b, c}$

Percentage of transfers resulting in singleton live births ${ }^{b}$

Percentage of cancellations ${ }^{b}$

Average number of embryos transferred

Percentage of pregnancies with twins ${ }^{b}$

Percentage of pregnancies with triplets or more ${ }^{b}$

Percentage of live births having multiple infants ${ }^{b, c}$

\section{Frozen Embryos from Nondonor Eggs}

Number of transfers

Percentage of transfers resulting in live births ${ }^{b, c}$

Average number of embryos transferred

\section{Donor Eggs}

Number of transfers

Percentage of transfers resulting in live births $s^{\mathrm{b}, \mathrm{c}}$

Average number of embryos transferred

\section{Age of Woman}

35-37 38-40

$41-42^{d}$

\section{$<35$}

37

13

3

46
37.0

40.5

$4 / 13$

$1 / 3$

32.6

32.4

$3 / 13$

$1 / 3$

$(19.5-48.0)$

(18.0-49.8)

36.6

35.3

$3 / 12$

$1 / 3$

38.5

38.7

$3 / 12$

$1 / 3$

30.8

32.3

$3 / 12$

$1 / 3$

10.9

8.1

$1 / 13$

$0 / 3$

3.0

3.4

2.8

4.3

$4 / 17$

$2 / 15$

$0 / 4$

$0 / 1$

$2 / 17$

$3 / 15$

$3 / 15$

$2 / 12$

$1 / 4$

$0 / 1$

$0 / 3$

$0 / 1$

$\begin{array}{cccc}7 & 5 & 1 & 1 \\ 4 / 7 & 2 / 5 & 0 / 1 & 1 / 1 \\ 2.3 & 2.2 & 2.0 & 2.0\end{array}$

\section{All Ages Combined}

Fresh Embryos

Frozen Embryos

\section{5}

$3 / 5$

5

2.2

$1 / 5$

2.2

\section{CURRENT CLINIC SERVICES AND PROFILE}

Current Name: Palmetto Fertility Center of South Florida

Donor egg? Yes Gestational carriers? Yes

Donor embryo? Yes Cryopreservation? Yes

Single women? Yes

SART member? Yes

Verified lab accreditation? Yes

(See Appendix C for details.)

${ }^{a}$ Reflects patient and treatment characteristics of ART cycles performed in 2006 using fresh nondonor eggs or embryos.

b When fewer than 20 cycles are reported in an age category, rates are shown as a fraction and confidence intervals are not given. Calculating percentages from fractions may be misleading and is not encouraged.

${ }^{c}$ A multiple-infant birth is counted as one live birth.

d Clinic-specific outcome rates are unreliable for women older than 42 undergoing ART cycles using fresh or frozen embryos with nondonor eggs. Readers are urged to review national outcomes for these age groups (see page 27).

e All ages (including ages $>42$ ) are reported together because previous data show that patient age does not materially affect success with donor eggs. 


\section{UNIVERSITY OF MIAMI INFERTILITY CENTER MIAMI, FLORIDA}

A comparison of clinic success rates may not be meaningful because patient medical characteristics and treatment approaches vary from clinic to clinic. For more details about this, along with information on how to interpret the statistics in this table, see pages $79-88$.

\section{ART CYCLE PROFILE}

\begin{tabular}{|c|c|c|c|c|c|c|c|}
\hline & Type & of $\mathrm{ART}^{\mathrm{a}}$ & & Pat & ent $D$ & iagnosis & \\
\hline IVF & $100 \%$ & Procedural Factors: & & Tubal factor & $25 \%$ & Other factor & $2 \%$ \\
\hline GIFT & $0 \%$ & With ICSI & $65 \%$ & Ovulatory dysfunction & $9 \%$ & Unknown factor & $13 \%$ \\
\hline ZIFT & $0 \%$ & Unstimulated & $0 \%$ & Diminished ovarian reserve & $9 \%$ & Multiple Factors: & \\
\hline Combination & $0 \%$ & Used gestational carrier & $0 \%$ & Endometriosis & $5 \%$ & Female factors only & $5 \%$ \\
\hline & & & & Uterine factor & $2 \%$ & Female \& male factors & $13 \%$ \\
\hline & & & & Male factor & $18 \%$ & & \\
\hline
\end{tabular}

2006 PREGNANCY SUCCESS RATES

Data verified by George R. Attia, MD

\section{Type of Cycle}

\section{Fresh Embryos from Nondonor Eggs}

Number of cycles

Percentage of cycles resulting in pregnancies ${ }^{b}$

Percentage of cycles resulting in live births $s^{b, c}$

(Confidence Interval)

Percentage of retrievals resulting in live births $s^{b, c}$

Percentage of transfers resulting in live births $s^{b, c}$

Percentage of transfers resulting in singleton live births ${ }^{b}$

Percentage of cancellations ${ }^{b}$

Average number of embryos transferred

Percentage of pregnancies with twins ${ }^{b}$

Percentage of pregnancies with triplets or more ${ }^{b}$

Percentage of live births having multiple infants ${ }^{b, c}$

\section{Frozen Embryos from Nondonor Eggs}

Number of transfers

Percentage of transfers resulting in live births $s^{\mathrm{b}, \mathrm{c}}$

Average number of embryos transferred

\section{Age of Woman}

35-37 38-40

$41-42^{d}$

\section{$<35$}

9

$6 / 9$

$6 / 9$

5

3

63.0

55.6

(35.3-74.5)

\section{7}

62.5

33.3

3.7

2.0

$9 / 17$

$1 / 17$

$7 / 15$

$6 / 9$

$6 / 9$

$4 / 9$

$0 / 9$

1.9

$4 / 6$

$0 / 6$

$2 / 6$

$2 / 5$

$2 / 5$

$1 / 3$

$1 / 3$

$2 / 3$

$1 / 2$

$2 / 3$

$1 / 2$

$2 / 3$

$1 / 2$

$2 / 5$

$1 / 3$

2.7

2.5

$0 / 2$

$0 / 1$

$0 / 2$

$0 / 1$

$0 / 2$

$0 / 1$

2

$1 / 2$

2.0

\section{1}

$0 / 1$

2.0

\section{3}

$0 / 3$

2.0

\section{Donor Eggs}

Fresh Embryos

All Ages Combined ${ }^{\mathrm{e}}$

Number of transfers

Percentage of transfers resulting in live births $s^{b, c}$

Average number of embryos transferred
1

$1 / 1$

2.0
Frozen Embryos

0

\section{CURRENT CLINIC SERVICES AND PROFILE}

Current Name: University of Miami Infertility Center

Donor egg? Yes

Donor embryo? Yes

Single women? No

\section{Gestational carriers? Yes}

Cryopreservation? Yes
SART member?

Yes

Verified lab accreditation?

(See Appendix C for details.)

${ }^{a}$ Reflects patient and treatment characteristics of ART cycles performed in 2006 using fresh nondonor eggs or embryos.

${ }^{b}$ When fewer than 20 cycles are reported in an age category, rates are shown as a fraction and confidence intervals are not given. Calculating percentages from fractions may be misleading and is not encouraged.

${ }^{c}$ A multiple-infant birth is counted as one live birth.

d Clinic-specific outcome rates are unreliable for women older than 42 undergoing ART cycles using fresh or frozen embryos with nondonor eggs. Readers are urged to review national outcomes for these age groups (see page 27).

e All ages (including ages $>42$ ) are reported together because previous data show that patient age does not materially affect success with donor eggs. 


\section{AFFORDABLE IVF \\ ORLANDO, FLORIDA}

A comparison of clinic success rates may not be meaningful because patient medical characteristics and treatment approaches vary from clinic to clinic. For more details about this, along with information on how to interpret the statistics in this table, see pages 79-88.

\section{ART CYCLE PROFILE}

\begin{tabular}{|c|c|c|c|c|c|c|c|}
\hline \multicolumn{4}{|c|}{ Type of ART ${ }^{a}$} & \multicolumn{4}{|c|}{ Patient Diagnosis } \\
\hline IVF & $100 \%$ & Procedural Factors: & & Tubal factor & $23 \%$ & Other factor & $0 \%$ \\
\hline GIFT & $0 \%$ & With ICSI & $94 \%$ & Ovulatory dysfunction & $19 \%$ & Unknown factor & $9 \%$ \\
\hline ZIFT & $0 \%$ & Unstimulated & $0 \%$ & Diminished ovarian reserve & $6 \%$ & Multiple Factors: & \\
\hline \multirow[t]{3}{*}{ Combination } & $0 \%$ & Used gestational carrier & $0 \%$ & Endometriosis & $6 \%$ & Female factors only & $10 \%$ \\
\hline & & & & Uterine factor & $<1 \%$ & Female \& male factors & $11 \%$ \\
\hline & & & & Male factor & $16 \%$ & & \\
\hline
\end{tabular}

2006 PREGNANCY SUCCESS RATES

Data verified by Mark L. Jutras, MD

\section{Type of Cycle}

\section{Fresh Embryos from Nondonor Eggs}

Number of cycles

Percentage of cycles resulting in pregnancies ${ }^{b}$

Percentage of cycles resulting in live births $s^{b, c}$

(Confidence Interval)

Percentage of retrievals resulting in live births $s^{b, c}$

Percentage of transfers resulting in live births $s^{b, c}$

Percentage of transfers resulting in singleton live births ${ }^{b}$

Percentage of cancellations ${ }^{b}$

Average number of embryos transferred

Percentage of pregnancies with twins ${ }^{b}$

Percentage of pregnancies with triplets or more ${ }^{b}$

Percentage of live births having multiple infants ${ }^{\mathrm{b}, \mathrm{c}}$

\section{Frozen Embryos from Nondonor Eggs}

Number of transfers

Percentage of transfers resulting in live births ${ }^{b, c}$

Average number of embryos transferred

\section{Donor Eggs}

Number of transfers

Percentage of transfers resulting in live births $s^{b, c}$

Average number of embryos transferred

\section{Age of Woman}

35-37 38-40

$41-42^{d}$

\section{$<35$}

20

60.0

12

7

70.6

45.0

5 / 12

$4 / 7$

62.7

(23.1-68.5)

(48.1-75.9)

$9 / 19$

3 / 12

$3 / 7$

65.3

65.3

$9 / 17$

$3 / 11$

$3 / 7$

36.7

$6 / 17$

$3 / 10$

$3 / 6$

3.9

5.0

$3 / 10$

$3 / 6$

2.0

2.0

$1 / 12$

$0 / 7$

41.7

3 / 12

0.0

$0 / 12$

2.4

3.0

43.8

$3 / 9$

$1 / 5$

$0 / 4$

$0 / 5$

$1 / 4$

$0 / 3$

$0 / 3$

$\begin{array}{cccc}5 & 4 & 2 & 1 \\ 0 / 5 & 0 / 4 & 0 / 2 & 0 / 1 \\ 2.0 & 1.5 & 2.5 & 2.0\end{array}$

All Ages Combined ${ }^{\mathrm{e}}$

Fresh Embryos

Frozen Embryos

0

0

\section{CURRENT CLINIC SERVICES AND PROFILE}

\footnotetext{
Current Name:

Affordable IVF

Donor egg? Yes Gestational carriers? Yes

Donor embryo? No Cryopreservation? Yes

Single women? No

SART member? Yes

Verified lab accreditation? Yes

(See Appendix C for details.)

${ }^{a}$ Reflects patient and treatment characteristics of ART cycles performed in 2006 using fresh nondonor eggs or embryos.

${ }^{b}$ When fewer than 20 cycles are reported in an age category, rates are shown as a fraction and confidence intervals are not given. Calculating percentages from fractions may be misleading and is not encouraged.

${ }^{c}$ A multiple-infant birth is counted as one live birth.

${ }^{d}$ Clinic-specific outcome rates are unreliable for women older than 42 undergoing ART cycles using fresh or frozen embryos with nondonor eggs. Readers are urged to review national outcomes for these age groups (see page 27).

e All ages (including ages $>42$ ) are reported together because previous data show that patient age does not materially affect success with donor eggs.
} 


\section{CENTER FOR REPRODUCTIVE MEDICINE, PA ORLANDO, FLORIDA}

A comparison of clinic success rates may not be meaningful because patient medical characteristics and treatment approaches vary from clinic to clinic. For more details about this, along with information on how to interpret the statistics in this table, see pages $79-88$.

\section{ART CYCLE PROFILE}

\begin{tabular}{|c|c|c|c|c|c|c|c|}
\hline \multicolumn{4}{|c|}{ Type of ART ${ }^{\mathrm{a}}$} & \multicolumn{4}{|c|}{ Patient Diagnosis } \\
\hline IVF & $100 \%$ & Procedural Factors: & & Tubal factor & $12 \%$ & Other factor & $2 \%$ \\
\hline GIFT & $0 \%$ & With ICSI & $47 \%$ & Ovulatory dysfunction & $14 \%$ & Unknown factor & $2 \%$ \\
\hline $\mathrm{ZIFT}$ & $0 \%$ & Unstimulated & $<1 \%$ & Diminished ovarian reserve & $1 \%$ & Multiple Factors: & \\
\hline Combination & $0 \%$ & Used gestational carrier & $2 \%$ & Endometriosis & $4 \%$ & Female factors only & $28 \%$ \\
\hline & & & & $\begin{array}{l}\text { Uterine factor } \\
\text { Male factor }\end{array}$ & $\begin{array}{r}<1 \% \\
8 \%\end{array}$ & Female \& male factors & $28 \%$ \\
\hline
\end{tabular}

2006 PREGNANCY SUCCESS RATES

Data verified by Randall A. Loy, MD

\section{Type of Cycle}

\section{Fresh Embryos from Nondonor Eggs}

Number of cycles

Percentage of cycles resulting in pregnancies ${ }^{b}$

Percentage of cycles resulting in live births $s^{b, c}$

(Confidence Interval)

Percentage of retrievals resulting in live births $s^{b, c}$

Percentage of transfers resulting in live births $s^{b, c}$

Percentage of transfers resulting in singleton live births ${ }^{b}$

Percentage of cancellations ${ }^{b}$

Average number of embryos transferred

Percentage of pregnancies with twins ${ }^{b}$

Percentage of pregnancies with triplets or more ${ }^{b}$

Percentage of live births having multiple infants ${ }^{b, c}$

\section{Frozen Embryos from Nondonor Eggs}

Number of transfers

Percentage of transfers resulting in live births $s^{b, c}$

Average number of embryos transferred

\section{Donor Eggs}

Number of transfers

Percentage of transfers resulting in live births $s^{b, c}$

Average number of embryos transferred

\section{Age of Woman}

35-37 38-40

$41-42^{d}$

\section{$<35$}

105

77

21

167

31.4

13.0

14.3

31.1

26.7

9.1

4.8

(24.2-38.8)

(18.5-36.2)

(3.7-17.8)

(0.1-23.8)

38.0

34.6

13.0

14.0

$1 / 17$

44.4

35.4

12.0

$1 / 17$

29.9

24.1

29.9

$1 / 17$

18.0

22.9

2.4

19.0

2.0

2.0

35.7

30.3

0.0

0.0

32.7

32.1

$4 / 10$

2.5

$0 / 3$

$0 / 10$

$0 / 3$

$1 / 7$

$0 / 1$

26

15.4

13

$4 / 13$

\section{5}

3

2.0

2.2

$0 / 5$

$1 / 3$

2.6

2.0

All Ages Combined ${ }^{\mathrm{e}}$

Fresh Embryos

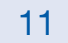

$7 / 11$

2.1
Frozen Embryos 10

$2 / 10$

2.3

\section{CURRENT CLINIC SERVICES AND PROFILE}

Current Name: Center for Reproductive Medicine, PA

Donor egg? Yes

Donor embryo? Yes

Single women? No

\section{Gestational carriers? Yes}

Cryopreservation?
SART member?

Verified lab accreditation?

(See Appendix C for details.)

${ }^{a}$ Reflects patient and treatment characteristics of ART cycles performed in 2006 using fresh nondonor eggs or embryos.

${ }^{b}$ When fewer than 20 cycles are reported in an age category, rates are shown as a fraction and confidence intervals are not given. Calculating percentages from fractions may be misleading and is not encouraged.

${ }^{c}$ A multiple-infant birth is counted as one live birth.

${ }^{d}$ Clinic-specific outcome rates are unreliable for women older than 42 undergoing ART cycles using fresh or frozen embryos with nondonor eggs. Readers are urged to review national outcomes for these age groups (see page 27).

${ }^{e}$ All ages (including ages $>42$ ) are reported together because previous data show that patient age does not materially affect success with donor eggs. 


\section{FRANK C. RIGGALL, MD, PA ORLANDO, FLORIDA}

A comparison of clinic success rates may not be meaningful because patient medical characteristics and treatment approaches vary from clinic to clinic. For more details about this, along with information on how to interpret the statistics in this table, see pages 79-88.

\section{ART CYCLE PROFILE}

\section{Type of ART ${ }^{\mathrm{a}}$}

IVF

GIFT

100\% Procedural Factors:

ZIFT

Combination
$0 \%$ With ICSI

$0 \%$ Unstimulated

$0 \%$ Used gestational carrier

\begin{tabular}{r|llll}
\multicolumn{5}{c}{ Patient Diagnosis } \\
\\
$50 \%$ & Tubal factor & $16 \%$ & Other factor & $17 \%$ \\
$0 \%$ & Dimulatory dysfunction & $8 \%$ & Unknown factor & $11 \%$ \\
$0 \%$ & Endometriosis & $17 \%$ & Multiple Factors: & \\
& Uterine factor & $4 \%$ & Female factors only & $5 \%$ \\
& Male factor & $0 \%$ & Female \& male factors & $1 \%$ \\
\end{tabular}

2006 PREGNANCY SUCCESS RATES

Data verified by Frank C. Riggall, MD

\section{Type of Cycle}

\begin{tabular}{cccc}
\multicolumn{4}{c}{ Age of Woman } \\
$<35$ & $\mathbf{3 5 - 3 7}$ & $\mathbf{3 8 - 4 0}$ & $\mathbf{4 1 - 4 2}^{\text {d }}$ \\
& & & \\
19 & 6 & 8 & 6 \\
$5 / 19$ & $5 / 6$ & $4 / 8$ & $0 / 6$ \\
$2 / 19$ & $4 / 6$ & $3 / 8$ & $0 / 6$
\end{tabular}

\section{Fresh Embryos from Nondonor Eggs}

Number of cycles

Percentage of cycles resulting in pregnancies ${ }^{b}$

Percentage of cycles resulting in live births $s^{b, c}$

(Confidence Interval)

Percentage of retrievals resulting in live births $s^{b, c}$

Percentage of transfers resulting in live births $s^{b, c}$

$2 / 13$

$2 / 12$

$0 / 12$

$6 / 19$

2.4

$2 / 5$

$0 / 5$

$2 / 2$

$4 / 5$

$4 / 5$

$3 / 5$

$1 / 6$

2.4

$1 / 5$

$0 / 5$

$1 / 4$

$3 / 5$

$3 / 5$

$2 / 5$

$3 / 8$

2.6

$1 / 4$

$0 / 4$

$1 / 3$

Percentage of pregnancies with triplets or more
Percentage of live births having multiple infants ${ }^{b, c}$

\section{Frozen Embryos from Nondonor Eggs}

Number of transfers

Percentage of transfers resulting in live births $s^{b, c}$

\section{6}

$0 / 6$

0

0

3

2.7
$0 / 5$

$0 / 3$

$0 / 3$

$1 / 6$

3.0
3.3

\section{Donor Eggs}

Number of transfers

Percentage of transfers resulting in live births $s^{b, c}$

Average number of embryos transferred

\section{Fresh Embryos}

8

$0 / 8$

2.1

\section{CURRENT CLINIC SERVICES AND PROFILE}

Current Name: Frank C. Riggall, MD, PA

Donor egg? Yes Gestational carriers? Yes

Donor embryo? Yes

Cryopreservation? Yes

Frozen Embryos

Single women? Yes

SART member?

Verified lab accreditation?

(See Appendix C for details.)

${ }^{a}$ Reflects patient and treatment characteristics of ART cycles performed in 2006 using fresh nondonor eggs or embryos.

b When fewer than 20 cycles are reported in an age category, rates are shown as a fraction and confidence intervals are not given. Calculating percentages from fractions may be misleading and is not encouraged.

${ }^{c}$ A multiple-infant birth is counted as one live birth.

d Clinic-specific outcome rates are unreliable for women older than 42 undergoing ART cycles using fresh or frozen embryos with nondonor eggs. Readers are urged to review national outcomes for these age groups (see page 27).

e All ages (including ages $>42$ ) are reported together because previous data show that patient age does not materially affect success with donor eggs. 


\section{NEW LEADERS IN INFERTILITY \& ENDOCRINOLOGY, LLC PENSACOLA, FLORIDA}

A comparison of clinic success rates may not be meaningful because patient medical characteristics and treatment approaches vary from clinic to clinic. For more details about this, along with information on how to interpret the statistics in this table, see pages $79-88$.

\section{ART CYCLE PROFILE}

\begin{tabular}{|c|c|c|c|c|c|c|c|}
\hline \multicolumn{4}{|c|}{ Type of ART } & \multicolumn{4}{|c|}{ Patient Diagnosis } \\
\hline IVF & $100 \%$ & Procedural Factors: & & Tubal factor & $10 \%$ & Other factor & $5 \%$ \\
\hline GIFT & $0 \%$ & With ICSI & $81 \%$ & Ovulatory dysfunction & $1 \%$ & Unknown factor & $8 \%$ \\
\hline ZIFT & $0 \%$ & Unstimulated & $0 \%$ & Diminished ovarian reserve & $1 \%$ & Multiple Factors: & \\
\hline \multirow[t]{3}{*}{ Combination } & $0 \%$ & Used gestational carrier & $0 \%$ & Endometriosis & $13 \%$ & Female factors only & $25 \%$ \\
\hline & & & & Uterine factor & $0 \%$ & Female \& male factors & $27 \%$ \\
\hline & & & & Male factor & $11 \%$ & & \\
\hline
\end{tabular}

2006 PREGNANCY SUCCESS RATES

Data verified by Barry A. Ripps, MD

\section{Type of Cycle}

\section{Fresh Embryos from Nondonor Eggs}

Number of cycles

Percentage of cycles resulting in pregnancies ${ }^{b}$

Percentage of cycles resulting in live births ${ }^{b, c}$

(Confidence Interval)

Percentage of retrievals resulting in live births $s^{b, c}$

Percentage of transfers resulting in live births $s^{b, c}$

Percentage of transfers resulting in singleton live births ${ }^{b}$

Percentage of cancellations ${ }^{b}$

Average number of embryos transferred

Percentage of pregnancies with twins ${ }^{b}$

Percentage of pregnancies with triplets or more ${ }^{\mathrm{b}}$

Percentage of live births having multiple infants ${ }^{b, c}$

\section{Frozen Embryos from Nondonor Eggs}

Number of transfers

Percentage of transfers resulting in live births $s^{\mathrm{b}, \mathrm{c}}$

Average number of embryos transferred

\section{Donor Eggs}

Number of transfers

Percentage of transfers resulting in live births $s^{\mathrm{b}, \mathrm{c}}$

Average number of embryos transferred

\section{Age of Woman}

35-37 38-40

$41-42^{d}$

\section{$<35$}

11

14

13

$\begin{array}{cccc}38 & 11 & 14 & 13 \\ 31.6 & 1 / 11 & 3 / 14 & 3 / 13 \\ 26.3 & 1 / 11 & 2 / 14 & 1 / 13\end{array}$

(13.4-43.1)

$\begin{array}{lccc}31.3 & 1 / 8 & 2 / 13 & 1 / 9 \\ 32.3 & 1 / 7 & 2 / 12 & 1 / 9 \\ 25.8 & 1 / 7 & 2 / 12 & 1 / 9 \\ 15.8 & 3 / 11 & 1 / 14 & 4 / 13 \\ 2.9 & 2.3 & 3.4 & 3.1 \\ 2 / 12 & 0 / 1 & 1 / 3 & 0 / 3 \\ 0 / 12 & 0 / 1 & 0 / 3 & 0 / 3 \\ 2 / 10 & 0 / 1 & 0 / 2 & 0 / 1\end{array}$

3

$0 / 3$

5

$2 / 5$

4

$0 / 4$

2.8

1.5

All Ages Combined

Fresh Embryos

Frozen Embryos

0

0

\section{CURRENT CLINIC SERVICES AND PROFILE}

Current Name: New Leaders in Infertility \& Endocrinology, LLC

Donor egg? No Gestational carriers? No

Donor embryo? Yes Cryopreservation? Yes

Single women? Yes

SART member? Yes

Verified lab accreditation?

Pending

${ }^{a}$ Reflects patient and treatment characteristics of ART cycles performed in 2006 using fresh nondonor eggs or embryos.

${ }^{b}$ When fewer than 20 cycles are reported in an age category, rates are shown as a fraction and confidence intervals are not given. Calculating percentages from fractions may be misleading and is not encouraged.

${ }^{c}$ A multiple-infant birth is counted as one live birth.

d Clinic-specific outcome rates are unreliable for women older than 42 undergoing ART cycles using fresh or frozen embryos with nondonor eggs. Readers are urged to review national outcomes for these age groups (see page 27).

e All ages (including ages $>42$ ) are reported together because previous data show that patient age does not materially affect success with donor eggs. 


\section{CENTER FOR ADVANCED REPRODUCTIVE ENDOCRINOLOGY, PA PLANTATION, FLORIDA}

A comparison of clinic success rates may not be meaningful because patient medical characteristics and treatment approaches vary from clinic to clinic. For more details about this, along with information on how to interpret the statistics in this table, see pages 79-88.

\section{ART CYCLE PROFILE}

\begin{tabular}{|c|c|c|c|c|c|c|c|}
\hline \multicolumn{4}{|c|}{ Type of ART ${ }^{a}$} & \multicolumn{4}{|c|}{ Patient Diagnosis } \\
\hline IVF & $100 \%$ & Procedural Factors: & & Tubal factor & $<1 \%$ & Other factor & $5 \%$ \\
\hline GIFT & $0 \%$ & With ICSI & $83 \%$ & Ovulatory dysfunction & $0 \%$ & Unknown factor & $0 \%$ \\
\hline ZIFT & $0 \%$ & Unstimulated & $0 \%$ & Diminished ovarian reserve & $8 \%$ & Multiple Factors: & \\
\hline \multirow[t]{3}{*}{ Combination } & $0 \%$ & Used gestational carrier & $0 \%$ & Endometriosis & $<1 \%$ & Female factors only & $21 \%$ \\
\hline & & & & Uterine factor & $5 \%$ & Female \& male factors & $53 \%$ \\
\hline & & & & Male factor & $6 \%$ & & \\
\hline
\end{tabular}

2006 PREGNANCY SUCCESS RATES

Data verified by Mick Abaé, MD

\section{Type of Cycle}

\section{Fresh Embryos from Nondonor Eggs}

Number of cycles

Percentage of cycles resulting in pregnancies ${ }^{b}$

Percentage of cycles resulting in live births ${ }^{b, c}$

(Confidence Interval)

Percentage of retrievals resulting in live births $s^{b, c}$

Percentage of transfers resulting in live births $s^{b, c}$

Percentage of cancellations ${ }^{b}$

Average number of embryos transferred

Percentage of pregnancies with twins ${ }^{b}$

Percentage of pregnancies with triplets or more ${ }^{b}$

Percentage of live births having multiple infants ${ }^{b, c}$

\section{Frozen Embryos from Nondonor Eggs}

Number of transfers

Percentage of transfers resulting in live births $s^{b, c}$

Average number of embryos transferred

\section{Donor Eggs}

Number of transfers

Percentage of transfers resulting in live births $s^{b, c}$

Average number of embryos transferred
Percentage of transfers resulting in singleton live births ${ }^{b}$

\section{Age of Woman}

$$
\text { 35-37 38-40 }
$$

$41-42^{d}$

$<35$

24

33.3

22

18.2

13.6

$1 / 5$

30.3

16.7

(13.3-45.5)

(4.7-37.4)

$(2.9-34.9)$
14.3

$1 / 5$

29.0

18.2

$1 / 5$

32.1

$4 / 16$

$3 / 16$

$1 / 3$

32.1

$3 / 16$

$1 / 16$

$1 / 3$

6.1

8.3

4.5

$0 / 5$

2.1

2.6

$2 / 8$

3.1

3.0

$1 / 10$

$0 / 8$

$2 / 4$

$0 / 1$

$0 / 10$

$0 / 9$

$1 / 4$

$0 / 4$

$0 / 1$

$2 / 3$

$0 / 1$
4

$1 / 4$

2.5
1

$0 / 1$

3.0

\section{CURRENT CLINIC SERVICES AND PROFILE}

Current Name: Center for Advanced Reproductive Endocrinology, PA

Donor egg? Yes Gestational carriers? Yes

Donor embryo? No Cryopreservation? Yes
Fresh Embryos

All Ages Combined ${ }^{\mathrm{e}}$
55.0

Frozen Embryos

2.1

\section{6}

$1 / 6$

3.0

Single women? Yes

SART member? Yes

Verified lab accreditation?

(See Appendix C for details.)

${ }^{a}$ Reflects patient and treatment characteristics of ART cycles performed in 2006 using fresh nondonor eggs or embryos.

${ }^{b}$ When fewer than 20 cycles are reported in an age category, rates are shown as a fraction and confidence intervals are not given. Calculating percentages from fractions may be misleading and is not encouraged.

${ }^{c}$ A multiple-infant birth is counted as one live birth.

d Clinic-specific outcome rates are unreliable for women older than 42 undergoing ART cycles using fresh or frozen embryos with nondonor eggs. Readers are urged to review national outcomes for these age groups (see page 27).

e All ages (including ages $>42$ ) are reported together because previous data show that patient age does not materially affect success with donor eggs. 


\section{FERTILITY CENTER AND APPLIED GENETICS OF FLORIDA, INC. SARASOTA, FLORIDA}

A comparison of clinic success rates may not be meaningful because patient medical characteristics and treatment approaches vary from clinic to clinic. For more details about this, along with information on how to interpret the statistics in this table, see pages $79-88$.

\section{ART CYCLE PROFILE}

\begin{tabular}{|c|c|c|c|c|c|c|c|}
\hline \multicolumn{4}{|c|}{ Type of ART ${ }^{a}$} & \multicolumn{4}{|c|}{ Patient Diagnosis } \\
\hline IVF & $100 \%$ & Procedural Factors: & & Tubal factor & $22 \%$ & Other factor & $12 \%$ \\
\hline GIFT & $0 \%$ & With ICSI & $56 \%$ & Ovulatory dysfunction & $5 \%$ & Unknown factor & $4 \%$ \\
\hline $\mathrm{ZIFT}$ & $0 \%$ & Unstimulated & $0 \%$ & Diminished ovarian reserve & $25 \%$ & Multiple Factors: & \\
\hline \multirow[t]{3}{*}{ Combination } & $0 \%$ & Used gestational carrier & $6 \%$ & Endometriosis & $5 \%$ & Female factors only & $<1 \%$ \\
\hline & & & & Uterine factor & $0 \%$ & Female \& male factors & $14 \%$ \\
\hline & & & & Male factor & $14 \%$ & & \\
\hline
\end{tabular}

2006 PREGNANCY SUCCESS RATES

Data verified by Julio E. Pabon, MD

\section{Type of Cycle}

\section{Fresh Embryos from Nondonor Eggs}

Number of cycles

Percentage of cycles resulting in pregnancies ${ }^{b}$

Percentage of cycles resulting in live births $s^{b, c}$

(Confidence Interval)

Percentage of retrievals resulting in live births $s^{b, c}$

Percentage of transfers resulting in live births $s^{b, c}$

Percentage of transfers resulting in singleton live births ${ }^{b}$

Percentage of cancellations ${ }^{b}$

Average number of embryos transferred

Percentage of pregnancies with twins ${ }^{b}$

Percentage of pregnancies with triplets or more ${ }^{b}$

Percentage of live births having multiple infants ${ }^{b, c}$

\section{Frozen Embryos from Nondonor Eggs}

Number of transfers

Percentage of transfers resulting in live births $s^{\mathrm{b}, \mathrm{c}}$

Average number of embryos transferred

\section{Donor Eggs}

Number of transfers

Percentage of transfers resulting in live births ${ }^{\mathrm{b}, \mathrm{c}}$

Average number of embryos transferred

\section{Age of Woman}

35-37 38-40

$41-42^{d}$

\section{$<35$}

34

18

12

62

45.2

23.5

$7 / 18$

$5 / 12$

33.9

17.6

$6 / 18$

$1 / 12$

(22.3-47.0)

$(6.8-34.5)$

42.0

25.0

$6 / 14$

$1 / 10$

45.7

27.3

$6 / 14$

$1 / 8$

32.6

27.3

$4 / 14$

$1 / 8$

19.4

29.4

$4 / 18$

$2 / 12$

2.2

2.4

2.6

3.0

25.0

7.1

$2 / 8$

$2 / 7$

$0 / 5$

28.6

$0 / 8$

$1 / 7$

$0 / 5$

$0 / 6$

$2 / 6$

$0 / 1$

3

$1 / 3$

1.7

1

$0 / 1$

2.0
0

0

\section{CURRENT CLINIC SERVICES AND PROFILE}

Current Name: Fertility Center and Applied Genetics of Florida, Inc.
Donor egg? Yes
Donor embryo? Yes
Single women? Yes
Gestational carriers? Yes
Cryopreservation? Yes

\section{All Ages Combined ${ }^{\mathrm{e}}$}

Fresh Embryos

Frozen Embryos

12

$10 / 12$ 8

2.3

$1 / 8$

2.0

\footnotetext{
${ }_{b}^{a}$ Reflects patient and treatment characteristics of ART cycles performed in 2006 using fresh nondonor eggs or embryos.

${ }^{b}$ When fewer than 20 cycles are reported in an age category, rates are shown as a fraction and confidence intervals are not given. Calculating

percentages from fractions may be misleading and is not encouraged.

c A multiple-infant birth is counted as one live birth.

${ }^{d}$ Clinic-specific outcome rates are unreliable for women older than 42 undergoing ART cycles using fresh or frozen embryos with nondonor eggs. Readers are urged to review national outcomes for these age groups (see page 27).

e All ages (including ages $>42$ ) are reported together because previous data show that patient age does not materially affect success with donor eggs.
} 


\section{SOUTH FLORIDA INSTITUTE FOR REPRODUCTIVE MEDICINE SOUTH MIAMI, FLORIDA}

A comparison of clinic success rates may not be meaningful because patient medical characteristics and treatment approaches vary from clinic to clinic. For more details about this, along with information on how to interpret the statistics in this table, see pages 79-88.

\section{ART CYCLE PROFILE}

\begin{tabular}{|c|c|c|c|c|c|c|c|}
\hline \multicolumn{4}{|c|}{ Type of ART ${ }^{a}$} & \multicolumn{4}{|c|}{ Patient Diagnosis } \\
\hline IVF & $100 \%$ & Procedural Factors: & & Tubal factor & $7 \%$ & Other factor & $10 \%$ \\
\hline GIFT & $0 \%$ & With ICSI & $66 \%$ & Ovulatory dysfunction & $<1 \%$ & Unknown factor & $<1 \%$ \\
\hline ZIFT & $0 \%$ & Unstimulated & $0 \%$ & Diminished ovarian reserve & $4 \%$ & Multiple Factors: & \\
\hline \multirow[t]{3}{*}{ Combination } & $0 \%$ & Used gestational carrier & $<1 \%$ & Endometriosis & $3 \%$ & Female factors only & $11 \%$ \\
\hline & & & & Uterine factor & $<1 \%$ & Female \& male factors & $42 \%$ \\
\hline & & & & Male factor & $22 \%$ & & \\
\hline
\end{tabular}

\section{PREGNANCY SUCCESS RATES}

Data verified by Maria Bustillo, MD

\section{Type of Cycle}

\section{Fresh Embryos from Nondonor Eggs}

Number of cycles

Percentage of cycles resulting in pregnancies ${ }^{b}$

Percentage of cycles resulting in live births $s^{b, c}$

(Confidence Interval)

Percentage of retrievals resulting in live births $s^{b, c}$

Percentage of transfers resulting in live births $s^{b, c}$

Percentage of transfers resulting in singleton live births ${ }^{b}$

Percentage of cancellations ${ }^{b}$

Average number of embryos transferred

Percentage of pregnancies with twins ${ }^{b}$

Percentage of pregnancies with triplets or more ${ }^{b}$

Percentage of live births having multiple infants ${ }^{b, c}$

\section{Frozen Embryos from Nondonor Eggs}

Number of transfers

Percentage of transfers resulting in live births ${ }^{b, c}$

Average number of embryos transferred

\section{Donor Eggs}

Number of transfers

Percentage of transfers resulting in live births $s^{b, c}$

Average number of embryos transferred

\section{Age of Woman}

35-37 38-40

$41-42^{d}$

$<35$

176

52.3

154

29.9

120

48

46.0

23.4

23.3

18.8

$(38.5-53.7)$

(16.9-30.9)

20.8

10.4

51.3

27.9

$(14.0-29.2)$

(3.5-22.7)

57.0

34.6

29.4

40.3

14.3

32.4

26.9

24.2

22.7

18.2

10.2

16.2

1.8

30.4

50.0

1.1

4.3

43.2

22.2

25

40.0

2.1

\begin{abstract}
18
\end{abstract}
$6 / 18$

1.9

29.2

27.1

2.5

$1 / 9$

$0 / 9$

$1 / 5$

50.0

0.0

40.0

3

$0 / 3$

2.7

\section{CURRENT CLINIC SERVICES AND PROFILE}

Current Name: South Florida Institute for Reproductive Medicine

Donor egg? Yes Gestational carriers? Yes

Donor embryo? Yes Cryopreservation? Yes

All Ages Combined ${ }^{\mathrm{e}}$

Fresh Embryos

69

53.6

1.9
Frozen Embryos

16
$4 / 16$
2.3

Single women? Yes

SART member? $\quad$ Yes

Verified lab accreditation? Yes

(See Appendix C for details.)

${ }^{a}$ Reflects patient and treatment characteristics of ART cycles performed in 2006 using fresh nondonor eggs or embryos.

${ }^{b}$ When fewer than 20 cycles are reported in an age category, rates are shown as a fraction and confidence intervals are not given. Calculating percentages from fractions may be misleading and is not encouraged.

${ }^{c}$ A multiple-infant birth is counted as one live birth.

d Clinic-specific outcome rates are unreliable for women older than 42 undergoing ART cycles using fresh or frozen embryos with nondonor eggs. Readers are urged to review national outcomes for these age groups (see page 27).

e All ages (including ages $>42$ ) are reported together because previous data show that patient age does not materially affect success with donor eggs. 


\section{CENTER FOR REPRODUCTIVE MEDICINE \\ TAMPA, FLORIDA}

A comparison of clinic success rates may not be meaningful because patient medical characteristics and treatment approaches vary from clinic to clinic. For more details about this, along with information on how to interpret the statistics in this table, see pages $79-88$.

\section{ART CYCLE PROFILE}

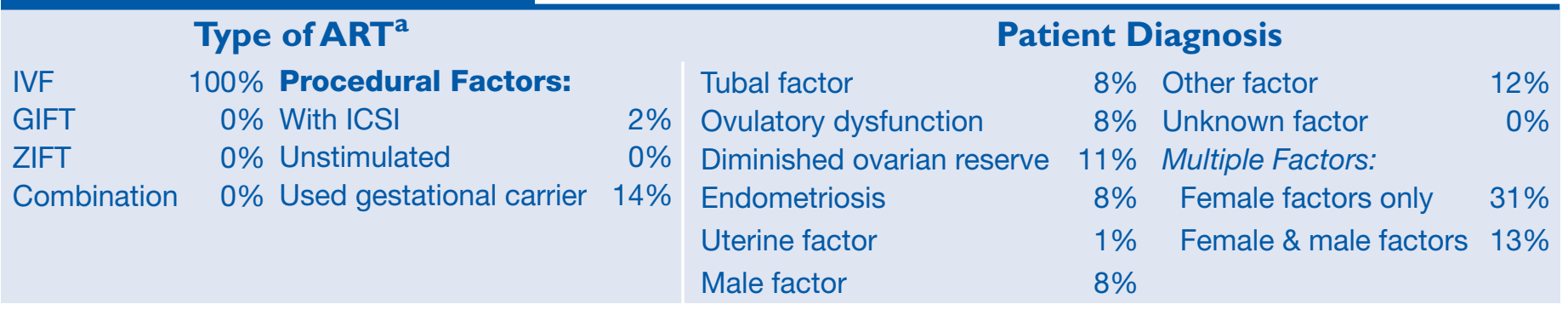

2006 PREGNANCY SUCCESS RATES

Data verified by Stephen W. Welden, MD

\section{Type of Cycle}

\section{Fresh Embryos from Nondonor Eggs}

Number of cycles

Percentage of cycles resulting in pregnancies ${ }^{b}$

Percentage of cycles resulting in live births $s^{b, c}$

(Confidence Interval)

Percentage of retrievals resulting in live births $s^{b, c}$

Percentage of transfers resulting in live births $s^{b, c}$

Percentage of transfers resulting in singleton live births ${ }^{b}$

Percentage of cancellations ${ }^{b}$

Average number of embryos transferred

Percentage of pregnancies with twins ${ }^{b}$

Percentage of pregnancies with triplets or more ${ }^{b}$

Percentage of live births having multiple infants ${ }^{b, c}$

\section{Frozen Embryos from Nondonor Eggs}

Number of transfers

Percentage of transfers resulting in live births $s^{b, c}$

Average number of embryos transferred

\section{Donor Eggs}

Number of transfers

Percentage of transfers resulting in live births $s^{\mathrm{b}, \mathrm{c}}$

Average number of embryos transferred

\section{Age of Woman}

35-37 38-40

$41-42^{d}$

$<35$

12

12

$\begin{array}{cccc}25 & 8 & 12 & 12 \\ 40.0 & 6 / 8 & 3 / 12 & 2 / 12 \\ 40.0 & 6 / 8 & 3 / 12 & 1 / 12\end{array}$

(21.1-61.3)

$\begin{array}{cccc}41.7 & 6 / 8 & 3 / 12 & 1 / 12 \\ 41.7 & 6 / 8 & 3 / 12 & 1 / 12 \\ 25.0 & 4 / 8 & 3 / 12 & 1 / 12 \\ 4.0 & 0 / 8 & 0 / 12 & 0 / 12 \\ 2.3 & 3.0 & 2.7 & 3.7 \\ 3 / 10 & 2 / 6 & 0 / 3 & 0 / 2 \\ 2 / 10 & 0 / 6 & 0 / 3 & 0 / 2 \\ 4 / 10 & 2 / 6 & 0 / 3 & 0 / 1\end{array}$

0

0

0

0

\section{CURRENT CLINIC SERVICES AND PROFILE}

Current Name: Center for Reproductive Medicine
Donor egg?
Yes
Gestational carriers?
Yes
Cryopreservation?
Yes
SART member?
No
Donor embryo? Yes
Single women? Yes
Verified lab accreditation?
No
(See Appendix C for details.)

\footnotetext{
${ }^{a}$ Reflects patient and treatment characteristics of ART cycles performed in 2006 using fresh nondonor eggs or embryos.

${ }^{b}$ When fewer than 20 cycles are reported in an age category, rates are shown as a fraction and confidence intervals are not given. Calculating percentages from fractions may be misleading and is not encouraged.

${ }^{c}$ A multiple-infant birth is counted as one live birth.

d Clinic-specific outcome rates are unreliable for women older than 42 undergoing ART cycles using fresh or frozen embryos with nondonor eggs. Readers are urged to review national outcomes for these age groups (see page 27).

${ }^{e}$ All ages (including ages $>42$ ) are reported together because previous data show that patient age does not materially affect success with donor eggs.
} 


\section{CATHERINE COWART, MD \\ REPRODUCTIVE HEALTH ASSOCIATES, PA \\ TAMPA, FLORIDA}

A comparison of clinic success rates may not be meaningful because patient medical characteristics and treatment approaches vary from clinic to clinic. For more details about this, along with information on how to interpret the statistics in this table, see pages $79-88$.

\section{ART CYCLE PROFILE}

\begin{tabular}{|c|c|c|c|c|c|c|c|}
\hline \multicolumn{4}{|c|}{ Type of ART ${ }^{a}$} & \multicolumn{4}{|c|}{ Patient Diagnosis } \\
\hline IVF & $100 \%$ & Procedural Factors: & & Tubal factor & $5 \%$ & Other factor & $0 \%$ \\
\hline GIFT & $0 \%$ & With ICSI & $59 \%$ & Ovulatory dysfunction & $<1 \%$ & Unknown factor & $7 \%$ \\
\hline ZIFT & $0 \%$ & Unstimulated & $0 \%$ & Diminished ovarian reserve & $14 \%$ & Multiple Factors: & \\
\hline \multirow[t]{3}{*}{ Combination } & $0 \%$ & Used gestational carrier & $0 \%$ & Endometriosis & $3 \%$ & Female factors only & $6 \%$ \\
\hline & & & & Uterine factor & $3 \%$ & Female \& male factors & $37 \%$ \\
\hline & & & & Male factor & $26 \%$ & & \\
\hline
\end{tabular}

2006 PREGNANCY SUCCESS RATES

Data verified by Catherine Cowart, MD

\section{Type of Cycle}

\section{Fresh Embryos from Nondonor Eggs}

Number of cycles

Percentage of cycles resulting in pregnancies ${ }^{b}$

Percentage of cycles resulting in live births $s^{b, c}$

(Confidence Interval)

Percentage of retrievals resulting in live births $s^{b, c}$

Percentage of transfers resulting in live births $s^{b, c}$

Percentage of transfers resulting in singleton live births ${ }^{b}$

Percentage of cancellations ${ }^{b}$

Average number of embryos transferred

Percentage of pregnancies with twins ${ }^{b}$

Percentage of pregnancies with triplets or more ${ }^{b}$

Percentage of live births having multiple infants ${ }^{b, c}$

\section{Frozen Embryos from Nondonor Eggs}

Number of transfers

Percentage of transfers resulting in live births $s^{b, c}$

Average number of embryos transferred

\section{Donor Eggs}

Number of transfers

Percentage of transfers resulting in live births $s^{b, c}$

Average number of embryos transferred

\section{Age of Woman}

35-37 38-40

$41-42^{d}$

\section{$<35$}

23

21.7

19

12

37
16.2

10.8

8.7

$1 / 19$

$2 / 12$

(3.0-25.4)

$(1.1-28.0)$

$0 / 19$

$2 / 12$

12.5

12.9

10.0

$0 / 13$

$2 / 10$

9.7

0.0

$0 / 13$

$2 / 10$

13.5

13.0

$0 / 13$

$2 / 10$

2.1

2.5

$6 / 19$

$2 / 12$

$3 / 6$

$0 / 6$

$2 / 5$

2.8

4.0

$1 / 4$

$1 / 5$

$0 / 1$

$0 / 2$

$0 / 1$

$0 / 2$

$2 / 2$

$0 / 2$

0

2

$0 / 2$

1

2.0

$0 / 1$

2.0

All Ages Combined ${ }^{\mathrm{e}}$

Fresh Embryos

Frozen Embryos

\section{7}

$2 / 7$

1

2.3

$0 / 1$

2.0

\section{CURRENT CLINIC SERVICES AND PROFILE}

Current Name: Catherine Cowart, MD, Reproductive Health Associates, PA

Donor egg? Yes

Donor embryo? No

Single women? Yes

\begin{abstract}
Gestational carriers? Yes
Cryopreservation? Yes
\end{abstract}

SART member?

Verified lab accreditation?

(See Appendix C for details.)

${ }^{a}$ Reflects patient and treatment characteristics of ART cycles performed in 2006 using fresh nondonor eggs or embryos.

${ }^{b}$ When fewer than 20 cycles are reported in an age category, rates are shown as a fraction and confidence intervals are not given. Calculating percentages from fractions may be misleading and is not encouraged.

${ }^{c}$ A multiple-infant birth is counted as one live birth.

d Clinic-specific outcome rates are unreliable for women older than 42 undergoing ART cycles using fresh or frozen embryos with nondonor eggs. Readers are urged to review national outcomes for these age groups (see page 27).

e All ages (including ages $>42$ ) are reported together because previous data show that patient age does not materially affect success with donor eggs. 


\section{THE REPRODUCTIVE MEDICINE GROUP \\ TAMPA, FLORIDA}

A comparison of clinic success rates may not be meaningful because patient medical characteristics and treatment approaches vary from clinic to clinic. For more details about this, along with information on how to interpret the statistics in this table, see pages $79-88$.

\section{ART CYCLE PROFILE}

\begin{tabular}{|c|c|c|c|c|c|c|c|}
\hline \multicolumn{4}{|c|}{ Type of ART ${ }^{\mathrm{a}}$} & \multicolumn{4}{|c|}{ Patient Diagnosis } \\
\hline IVF & $>99 \%$ & Procedural Factors: & & Tubal factor & $13 \%$ & Other factor & $4 \%$ \\
\hline GIFT & $0 \%$ & With ICSI & $47 \%$ & Ovulatory dysfunction & $4 \%$ & Unknown factor & $14 \%$ \\
\hline ZIFT & $<1 \%$ & Unstimulated & $<1 \%$ & Diminished ovarian reserve & $8 \%$ & Multiple Factors: & \\
\hline \multirow[t]{3}{*}{ Combination } & $<1 \%$ & Used gestational carrier & $1 \%$ & Endometriosis & $9 \%$ & Female factors only & $10 \%$ \\
\hline & & & & Uterine factor & $1 \%$ & Female \& male factors & $16 \%$ \\
\hline & & & & Male factor & $20 \%$ & & \\
\hline
\end{tabular}

2006 PREGNANCY SUCCESS RATES

Data verified by Marc Bernhisel, MD

\section{Type of Cycle}

\section{Fresh Embryos from Nondonor Eggs}

Number of cycles

Percentage of cycles resulting in pregnancies ${ }^{b}$

Percentage of cycles resulting in live births $s^{b, c}$

(Confidence Interval)

Percentage of retrievals resulting in live births $s^{b, c}$

Percentage of transfers resulting in live births $s^{b, c}$

Percentage of transfers resulting in singleton live births ${ }^{b}$

Percentage of cancellations ${ }^{b}$

Average number of embryos transferred

Percentage of pregnancies with twins ${ }^{b}$

Percentage of pregnancies with triplets or more ${ }^{b}$

Percentage of live births having multiple infants ${ }^{b, c}$

\section{Frozen Embryos from Nondonor Eggs}

Number of transfers

Percentage of transfers resulting in live births ${ }^{\mathrm{b}, \mathrm{c}}$

Average number of embryos transferred

\section{Donor Eggs}

Number of transfers

Percentage of transfers resulting in live births ${ }^{\mathrm{b}, \mathrm{c}}$

Average number of embryos transferred

\section{Age of Woman}

35-37 38-40

$41-42^{d}$

\section{$<35$}

129

119

35

209

37.2

24.4

28.6

44.0

29.5

21.8

17.1

(37.2-51.0)

48.2

(21.8-38.1)

(14.8-30.4)

(6.6-33.6)

51.4

31.1

27.4

20.0

32.5

30.6

20.7

34.6

18.8

25.9

13.8

8.6

5.4

20.2

14.3

1.9

2.1

33.3

2.7

2.8

36.3

2.1

27.6

$1 / 10$

2.0

42.1

0.0

$2 / 10$

32.6

11

$3 / 11$

$$
14
$$

$2 / 14$

15.4

$2 / 6$

\section{7 \\ $1 / 7$}

2

2.1

2.1

All Ages Combined ${ }^{\mathrm{e}}$

Fresh Embryos

60

46.7

1.9
Frozen Embryos

\section{6}

$1 / 6$

1.5

\section{CURRENT CLINIC SERVICES AND PROFILE}

Current Name: The Reproductive Medicine Group

Donor egg? Yes

Donor embryo? Yes

Single women? Yes

\section{Gestational carriers? Yes}

Cryopreservation? Yes

\section{SART member?}

Verified lab accreditation?

(See Appendix C for details.)

${ }^{a}$ Reflects patient and treatment characteristics of ART cycles performed in 2006 using fresh nondonor eggs or embryos.

${ }^{b}$ When fewer than 20 cycles are reported in an age category, rates are shown as a fraction and confidence intervals are not given. Calculating percentages from fractions may be misleading and is not encouraged.

${ }^{c}$ A multiple-infant birth is counted as one live birth.

d Clinic-specific outcome rates are unreliable for women older than 42 undergoing ART cycles using fresh or frozen embryos with nondonor eggs. Readers are urged to review national outcomes for these age groups (see page 27).

e All ages (including ages $>42$ ) are reported together because previous data show that patient age does not materially affect success with donor eggs. 


\section{F.I.R.S.T. \\ FLORIDA INSTITUTE FOR REPRODUCTIVE SCIENCES AND TECHNOLOGIES WESTON, FLORIDA}

A comparison of clinic success rates may not be meaningful because patient medical characteristics and treatment approaches vary from clinic to clinic. For more details about this, along with information on how to interpret the statistics in this table, see pages 79-88.

\section{ART CYCLE PROFILE}

\section{Type of ART ${ }^{\mathrm{a}}$}

\begin{tabular}{|c|c|c|c|c|}
\hline IVF & $91 \%$ & Procedural Factors: & & Tubal factor \\
\hline GIFT & $7 \%$ & With ICSI & $67 \%$ & Ovulatory dysfunction \\
\hline ZIFT & $2 \%$ & Unstimulated & $0 \%$ & Diminished ovarian reserve \\
\hline Combination & $0 \%$ & Used gestational carrier & $5 \%$ & $\begin{array}{l}\text { Endometriosis } \\
\text { Uterine factor } \\
\text { Male factor }\end{array}$ \\
\hline
\end{tabular}

2006 PREGNANCY SUCCESS RATES

\section{Type of Cycle}

Fresh Embryos from Nondonor Eggs
Number of cycles
Percentage of cycles resulting in pregnancies ${ }^{b}$
Percentage of cycles resulting in live births $s^{b, c}$
(Confidence Interval)
Percentage of retrievals resulting in live births $s^{b, c}$
Percentage of transfers resulting in live births $s^{b, c}$
Percentage of transfers resulting in singleton live
Percentage of cancellations
Average number of embryos transferred
Percentage of pregnancies with twins ${ }^{b}$
Percentage of pregnancies with triplets or more ${ }^{b}$
Percentage of live births having multiple infants
Frozen Embryos from Nondonor Eggs
Number of transfers
Percentage of transfers resulting in live births $s^{b, c}$
Average number of embryos transferred
Donor Eggs
Number of transfers
Percentage of transfers resulting in live births $s^{b, c}$
Average number of embryos transferred

\section{Fresh Embryos}

38

31.6

3.0

\section{Patient Diagnosis}

$\begin{array}{rlr}4 \% & \text { Other factor } & <1 \% \\ 4 \% & \text { Unknown factor } & <1 \% \\ 29 \% & \text { Multiple Factors: } & \\ 3 \% & \text { Female factors only } & 18 \% \\ 0 \% & \text { Female \& male factors } & 26 \% \\ 14 \% & & \end{array}$

Data verified by Minna R. Selub, MD

\section{Age of Woman}

$\begin{array}{cccc}\mathbf{3} 5 & \mathbf{3 5 - 3 7} & \mathbf{3 8 - 4 0} & \mathbf{4 1 - 4 2}^{\mathbf{c}} \\ 33 & 26 & 22 & 4 \\ 12.1 & 23.1 & 13.6 & 0 / 4 \\ 12.1 & 15.4 & 13.6 & 0 / 4 \\ 3.4-28.2) & (4.4-34.9) & (2.9-34.9) & \\ 12.5 & 16.7 & 13.6 & 0 / 4 \\ 12.9 & 19.0 & 3 / 16 & 0 / 4 \\ 9.7 & 9.5 & 0 / 16 & 0 / 4 \\ 3.0 & 7.7 & 0.0 & 0 / 4 \\ 3.0 & 3.2 & 2.9 & 3.0 \\ 1 / 4 & 1 / 6 & 3 / 3 & \\ 1 / 4 & 1 / 6 & 0 / 3 & \\ 1 / 4 & 2 / 4 & 3 / 3 & \end{array}$

$41-42^{d}$

4

$0 / 4$

2

$0 / 2$

3.5

2.0

\section{CURRENT CLINIC SERVICES AND PROFILE}

Current Name: F.I.R.S.T., Florida Institute for Reproductive Sciences and Technologies

Donor egg? Yes

Donor embryo? No

Single women? Yes

\section{Gestational carriers? Yes}

Cryopreservation? Yes
All Ages Combined ${ }^{\mathrm{e}}$

Frozen Embryos

3

$0 / 3$

3.0

\footnotetext{
${ }^{a}$ Reflects patient and treatment characteristics of ART cycles performed in 2006 using fresh nondonor eggs or embryos.

${ }^{b}$ When fewer than 20 cycles are reported in an age category, rates are shown as a fraction and confidence intervals are not given. Calculating percentages from fractions may be misleading and is not encouraged.

${ }^{\mathrm{C}}$ A multiple-infant birth is counted as one live birth.

${ }^{d}$ Clinic-specific outcome rates are unreliable for women older than 42 undergoing ART cycles using fresh or frozen embryos with nondonor eggs. Readers are urged to review national outcomes for these age groups (see page 27).

e All ages (including ages $>42$ ) are reported together because previous data show that patient age does not materially affect success with donor eggs.
} 


\section{FERTILITY CENTER OF ASSISTED REPRODUCTION \& ENDOCRINOLOGY WINTER PARK, FLORIDA}

A comparison of clinic success rates may not be meaningful because patient medical characteristics and treatment approaches vary from clinic to clinic. For more details about this, along with information on how to interpret the statistics in this table, see pages $79-88$.

\section{ART CYCLE PROFILE}

\begin{tabular}{|c|c|c|c|c|c|c|c|}
\hline \multicolumn{4}{|c|}{ Type of ART ${ }^{a}$} & \multicolumn{4}{|c|}{ Patient Diagnosis } \\
\hline IVF & $100 \%$ & Procedural Factors: & & Tubal factor & $7 \%$ & Other factor & $3 \%$ \\
\hline GIFT & $0 \%$ & With ICSI & $45 \%$ & Ovulatory dysfunction & $11 \%$ & Unknown factor & $12 \%$ \\
\hline ZIFT & $0 \%$ & Unstimulated & $0 \%$ & Diminished ovarian reserve & $25 \%$ & Multiple Factors: & \\
\hline Combination & $0 \%$ & Used gestational carrier & $0 \%$ & Endometriosis & $2 \%$ & Female factors only & $19 \%$ \\
\hline & & & & Uterine factor & $<1 \%$ & Female \& male factors & $14 \%$ \\
\hline
\end{tabular}

2006 PREGNANCY SUCCESS RATES

Data verified by Mark P. Trolice, MD

\section{Type of Cycle}

\section{Fresh Embryos from Nondonor Eggs}

Number of cycles

Percentage of cycles resulting in pregnancies ${ }^{b}$

Percentage of cycles resulting in live births ${ }^{b, c}$

(Confidence Interval)

Percentage of retrievals resulting in live births $s^{b, c}$

Percentage of transfers resulting in live births $s^{b, c}$

Percentage of transfers resulting in singleton live births ${ }^{b}$

Percentage of cancellations ${ }^{b}$

Average number of embryos transferred

Percentage of pregnancies with twins ${ }^{b}$

Percentage of pregnancies with triplets or more ${ }^{b}$

Percentage of live births having multiple infants ${ }^{\mathrm{b}, \mathrm{c}}$

\section{Frozen Embryos from Nondonor Eggs}

Number of transfers

Percentage of transfers resulting in live births $s^{\mathrm{b}, \mathrm{c}}$

Average number of embryos transferred

\section{Donor Eggs}

Number of transfers

Percentage of transfers resulting in live births ${ }^{\mathrm{b}, \mathrm{c}}$

Average number of embryos transferred

\section{Age of Woman}

35-37 38-40

$41-42^{d}$ $<35$

$\begin{array}{cccc}84 & 42 & 25 & 15 \\ 48.8 & 35.7 & 36.0 & 3 / 15 \\ 40.5 & 26.2 & 24.0 & 2 / 15\end{array}$

(29.9-51.7)

(13.9-42.0)

(9.4-45.1)

41.5

27.5

28.6

$2 / 13$

47.9

30.6

$6 / 17$

$2 / 11$

19.4

$6 / 17$

$1 / 11$

2.4

4.8

16.0

$2 / 15$

2.0

2.4

2.2

2.4

22.0

$5 / 15$

$0 / 9$

$1 / 3$

0.0

$1 / 15$

$0 / 9$

$0 / 3$

20.6

$4 / 11$

$0 / 6$

$1 / 2$

13

$2 / 13$

8

1.8

$2 / 8$

1

$0 / 1$

1

2.0

3.0

$0 / 1$

2.0

All Ages Combined

Fresh Embryos

22

40.9

1.9
Frozen Embryos

\section{5}

$0 / 5$

2.0

\section{CURRENT CLINIC SERVICES AND PROFILE}

Current Name: Fertility Center of Assisted Reproduction \& Endocrinology

Donor egg? Yes

Donor embryo? Yes

Single women? Yes

\section{Gestational carriers? Yes}

Cryopreservation? Yes
SART member?

Verified lab accreditation?

(See Appendix C for details.)

${ }^{a}$ Reflects patient and treatment characteristics of ART cycles performed in 2006 using fresh nondonor eggs or embryos.

${ }^{b}$ When fewer than 20 cycles are reported in an age category, rates are shown as a fraction and confidence intervals are not given. Calculating percentages from fractions may be misleading and is not encouraged.

${ }^{c}$ A multiple-infant birth is counted as one live birth.

${ }^{d}$ Clinic-specific outcome rates are unreliable for women older than 42 undergoing ART cycles using fresh or frozen embryos with nondonor eggs. Readers are urged to review national outcomes for these age groups (see page 27).

${ }^{e}$ All ages (including ages $>42$ ) are reported together because previous data show that patient age does not materially affect success with donor eggs. 


\section{ATLANTA CENTER FOR REPRODUCTIVE MEDICINE ATLANTA, GEORGIA}

A comparison of clinic success rates may not be meaningful because patient medical characteristics and treatment approaches vary from clinic to clinic. For more details about this, along with information on how to interpret the statistics in this table, see pages 79-88.

\section{ART CYCLE PROFILE}

\begin{tabular}{|c|c|c|c|c|c|c|c|}
\hline \multicolumn{4}{|c|}{ Type of ART } & \multicolumn{4}{|c|}{ Patient Diagnosis } \\
\hline IVF & $100 \%$ & Procedural Factors: & & Tubal factor & $9 \%$ & Other factor & $10 \%$ \\
\hline GIFT & $0 \%$ & With ICSI & $73 \%$ & Ovulatory dysfunction & $5 \%$ & Unknown factor & $5 \%$ \\
\hline ZIFT & $0 \%$ & Unstimulated & $0 \%$ & Diminished ovarian reserve & $12 \%$ & Multiple Factors: & \\
\hline \multirow[t]{3}{*}{ Combination } & $0 \%$ & Used gestational carrier & $0 \%$ & Endometriosis & $5 \%$ & Female factors only & $29 \%$ \\
\hline & & & & Uterine factor & $<1 \%$ & Female \& male factors & $13 \%$ \\
\hline & & & & Male factor & $12 \%$ & & \\
\hline
\end{tabular}

\section{PREGNANCY SUCCESS RATES}

\section{Type of Cycle}

\section{Fresh Embryos from Nondonor Eggs}

Number of cycles

Percentage of cycles resulting in pregnancies ${ }^{b}$

Percentage of cycles resulting in live births $s^{b, c}$

(Confidence Interval)

Percentage of retrievals resulting in live births $s^{b, c}$

Percentage of transfers resulting in live births $s^{b, c}$

Percentage of transfers resulting in singleton live births ${ }^{b}$

Percentage of cancellations ${ }^{b}$

Average number of embryos transferred

Percentage of pregnancies with twins ${ }^{b}$

Percentage of pregnancies with triplets or more ${ }^{b}$

Percentage of live births having multiple infants ${ }^{b, c}$

\section{Frozen Embryos from Nondonor Eggs}

Number of transfers

Percentage of transfers resulting in live births ${ }^{b, c}$

Average number of embryos transferred

\section{Donor Eggs}

Number of transfers

Percentage of transfers resulting in live births $s^{b, c}$

Average number of embryos transferred

Data verified by James P. Toner, MD, PhD

\section{Age of Woman}

35-37 38-40

$41-42^{d}$

$<35$

$\begin{array}{ccc}104 & 63 & 21 \\ 39.4 & 38.1 & 9.5 \\ 34.6 & 30.2 & 0.0\end{array}$

$\begin{array}{cccc}(32.0-45.6) & (25.6-44.6) & (19.2-43.0) & (0.0-16.1) \\ 42.6 & 37.5 & 32.2 & 0 / 17 \\ 45.2 & 39.1 & 36.5 & 0 / 13 \\ 30.5 & 30.4 & 30.8 & 0 / 13 \\ 9.2 & 7.7 & 6.3 & 19.0 \\ 2.3 & 2.6 & 3.2 & 3.1 \\ 29.9 & 26.8 & 25.0 & 0 / 2 \\ 5.2 & 4.9 & 4.2 & 0 / 2 \\ 32.5 & 22.2 & 3 / 19 & \end{array}$

34

18

3

3

52.9

$6 / 18$

$2 / 3$

$0 / 3$

2.2

2.2

2.3

2.0

All Ages Combined ${ }^{\mathrm{e}}$

Fresh Embryos

82

50.0

2.1
Frozen Embryos 25

36.0

1.9

\section{CURRENT CLINIC SERVICES AND PROFILE}

Current Name: Atlanta Center for Reproductive Medicine

Donor egg? Yes Gestational carriers? Yes

Donor embryo? Yes Cryopreservation? Yes

Single women? Yes

SART member? Yes

Verified lab accreditation? Yes

(See Appendix C for details.)

${ }^{a}$ Reflects patient and treatment characteristics of ART cycles performed in 2006 using fresh nondonor eggs or embryos.

${ }^{b}$ When fewer than 20 cycles are reported in an age category, rates are shown as a fraction and confidence intervals are not given. Calculating percentages from fractions may be misleading and is not encouraged.

${ }^{c}$ A multiple-infant birth is counted as one live birth.

d Clinic-specific outcome rates are unreliable for women older than 42 undergoing ART cycles using fresh or frozen embryos with nondonor eggs. Readers are urged to review national outcomes for these age groups (see page 27).

e All ages (including ages $>42$ ) are reported together because previous data show that patient age does not materially affect success with donor eggs. 


\section{EMORY REPRODUCTIVE CENTER \\ ATLANTA, GEORGIA}

A comparison of clinic success rates may not be meaningful because patient medical characteristics and treatment approaches vary from clinic to clinic. For more details about this, along with information on how to interpret the statistics in this table, see pages $79-88$.

\section{ART CYCLE PROFILE}

\begin{tabular}{|c|c|c|c|c|c|c|c|}
\hline \multicolumn{4}{|c|}{ Type of ART ${ }^{\mathrm{a}}$} & \multicolumn{4}{|c|}{ Patient Diagnosis } \\
\hline IVF & $100 \%$ & Procedural Factors: & & Tubal factor & $9 \%$ & Other factor & $1 \%$ \\
\hline GIFT & $0 \%$ & With ICSI & $57 \%$ & Ovulatory dysfunction & $5 \%$ & Unknown factor & $4 \%$ \\
\hline ZIFT & $0 \%$ & Unstimulated & $0 \%$ & Diminished ovarian reserve & $15 \%$ & Multiple Factors: & \\
\hline Combination & $0 \%$ & Used gestational carrier & $0 \%$ & Endometriosis & $2 \%$ & Female factors only & $29 \%$ \\
\hline & & & & Uterine factor & $<1 \%$ & Female \& male factors & $24 \%$ \\
\hline
\end{tabular}

2006 PREGNANCY SUCCESS RATES

Data verified by Donna R. Session, MD

\section{Type of Cycle}

\section{Fresh Embryos from Nondonor Eggs}

Number of cycles

Percentage of cycles resulting in pregnancies ${ }^{b}$

Percentage of cycles resulting in live births $s^{b, c}$

(Confidence Interval)

Percentage of retrievals resulting in live births $s^{b, c}$

Percentage of transfers resulting in live births $s^{b, c}$

Percentage of transfers resulting in singleton live births ${ }^{b}$

Percentage of cancellations ${ }^{b}$

Average number of embryos transferred

Percentage of pregnancies with twins ${ }^{b}$

Percentage of pregnancies with triplets or more ${ }^{b}$

Percentage of live births having multiple infants ${ }^{b, c}$

\section{Frozen Embryos from Nondonor Eggs}

Number of transfers

Percentage of transfers resulting in live births $s^{b, c}$

Average number of embryos transferred

\section{Donor Eggs}

Number of transfers

Percentage of transfers resulting in live births $s^{b, c}$

Average number of embryos transferred

\section{Age of Woman}

35-37 38-40

$41-42^{d}$

$<35$

28

25.0

24

8

56.8

17.9

33.3

$3 / 8$

45.5

$(30.4-61.2)$

$(6.1-36.9)$
25.0

16.7

$3 / 8$

\section{3}

$(4.7-37.4)$

54.1

25.0

$4 / 19$

$3 / 7$

43.2

20.0

$4 / 18$

$3 / 6$

11.4

28.6

$4 / 18$

$3 / 6$

2.2

24.0

0.0

20.0

2.4

$2 / 7$

20.8

$1 / 8$

4.2

2.7

$2 / 3$

$0 / 7$

$0 / 8$

$0 / 3$

$1 / 5$

$0 / 8$

$0 / 3$

$0 / 4$

4

$\begin{array}{cccc}15 & 9 & 2 & 4 \\ 3 / 15 & 4 / 9 & 0 / 2 & 0 / 4 \\ 2.0 & 2.2 & 1.5 & 2.5\end{array}$

\section{CURRENT CLINIC SERVICES AND PROFILE}

Current Name: Emory Reproductive Center
Donor egg? Yes
Donor embryo? Yes
Gestational carriers? Yes
Cryopreservation? Yes
Single women? Yes

Fresh Embryos

All Ages Combined ${ }^{\mathrm{e}}$

$\begin{array}{cc}6 & 6 \\ 3 / 6 & 1 / 6 \\ 2.2 & 2.2\end{array}$

\footnotetext{
${ }^{a}$ Reflects patient and treatment characteristics of ART cycles performed in 2006 using fresh nondonor eggs or embryos.

${ }^{b}$ When fewer than 20 cycles are reported in an age category, rates are shown as a fraction and confidence intervals are not given. Calculating

percentages from fractions may be misleading and is not encouraged.

c A multiple-infant birth is counted as one live birth.

d Clinic-specific outcome rates are unreliable for women older than 42 undergoing ART cycles using fresh or frozen embryos with nondonor eggs. Readers are urged to review national outcomes for these age groups (see page 27).

${ }^{e}$ All ages (including ages $>42$ ) are reported together because previous data show that patient age does not materially affect success with donor eggs.
} 


\section{GEORGIA REPRODUCTIVE SPECIALISTS ATLANTA, GEORGIA}

A comparison of clinic success rates may not be meaningful because patient medical characteristics and treatment approaches vary from clinic to clinic. For more details about this, along with information on how to interpret the statistics in this table, see pages 79-88.

\section{ART CYCLE PROFILE}

\begin{tabular}{|c|c|c|c|c|c|c|c|}
\hline \multicolumn{4}{|c|}{ Type of ART ${ }^{a}$} & \multicolumn{4}{|c|}{ Patient Diagnosis } \\
\hline IVF & $100 \%$ & Procedural Factors: & & Tubal factor & $11 \%$ & Other factor & $8 \%$ \\
\hline GIFT & $0 \%$ & With ICSI & $39 \%$ & Ovulatory dysfunction & $7 \%$ & Unknown factor & $13 \%$ \\
\hline ZIFT & $0 \%$ & Unstimulated & $0 \%$ & Diminished ovarian reserve & $9 \%$ & Multiple Factors: & \\
\hline \multirow[t]{3}{*}{ Combination } & $0 \%$ & Used gestational carrier & $2 \%$ & Endometriosis & $6 \%$ & Female factors only & $17 \%$ \\
\hline & & & & Uterine factor & $<1 \%$ & Female \& male factors & $13 \%$ \\
\hline & & & & Male factor & $15 \%$ & & \\
\hline
\end{tabular}

2006 PREGNANCY SUCCESS RATES

Data verified by Mark Perloe, MD

\section{Type of Cycle}

\section{Fresh Embryos from Nondonor Eggs}

Number of cycles

Percentage of cycles resulting in pregnancies ${ }^{b}$

Percentage of cycles resulting in live births ${ }^{b, c}$

(Confidence Interval)

Percentage of retrievals resulting in live births $s^{b, c}$

Percentage of transfers resulting in live births $s^{b, c}$

Percentage of transfers resulting in singleton live births ${ }^{b}$

Percentage of cancellations ${ }^{b}$

Average number of embryos transferred

Percentage of pregnancies with twins ${ }^{b}$

Percentage of pregnancies with triplets or more ${ }^{b}$

Percentage of live births having multiple infants ${ }^{\mathrm{b}, \mathrm{c}}$

\section{Frozen Embryos from Nondonor Eggs}

Number of transfers

Percentage of transfers resulting in live births ${ }^{b, c}$

Average number of embryos transferred

\section{Donor Eggs}

Number of transfers

Percentage of transfers resulting in live births $s^{b, c}$

Average number of embryos transferred

\section{Age of Woman}

35-37 38-40 $41-42^{d}$

\section{$<35$}

74

73

10

42.6

40.5

13.7

$1 / 10$

39.5

28.4

5.5

$1 / 10$

(31.0-48.5)

(18.5-40.1)

(1.5-13.4)

42.9

31.3

8.2

$1 / 7$

47.7

32.8

8.7

$1 / 6$

31.8

23.4

8.7

$0 / 6$

7.8

9.5

32.9

$3 / 10$

2.2

2.5

23.3

34.5

3.3

33.3

28.6

2.8

3.5

$1 / 10$

$1 / 1$

$0 / 10$

$0 / 1$

0 / 4

$1 / 1$

66

31.8

32

21.9

19

$4 / 19$

8

2.0

2.1

1.9

$1 / 8$

2.1

All Ages Combined ${ }^{\mathrm{e}}$

Fresh Embryos

29

65.5

2.1
Frozen Embryos 12

$8 / 12$

1.9

\section{CURRENT CLINIC SERVICES AND PROFILE}

Current Name: Georgia Reproductive Specialists

Donor egg? Yes

Donor embryo? Yes

Single women? Yes

$\begin{array}{ll}\text { Gestational carriers? } & \text { Yes } \\ \text { Cryopreservation? } & \text { Yes }\end{array}$

SART member?

Yes

Verified lab accreditation?

(See Appendix C for details.)

${ }^{a}$ Reflects patient and treatment characteristics of ART cycles performed in 2006 using fresh nondonor eggs or embryos.

b When fewer than 20 cycles are reported in an age category, rates are shown as a fraction and confidence intervals are not given. Calculating percentages from fractions may be misleading and is not encouraged.

${ }^{c}$ A multiple-infant birth is counted as one live birth.

d Clinic-specific outcome rates are unreliable for women older than 42 undergoing ART cycles using fresh or frozen embryos with nondonor eggs. Readers are urged to review national outcomes for these age groups (see page 27).

e All ages (including ages $>42$ ) are reported together because previous data show that patient age does not materially affect success with donor eggs. 


\section{REPRODUCTIVE BIOLOGY ASSOCIATES \\ ATLANTA, GEORGIA}

A comparison of clinic success rates may not be meaningful because patient medical characteristics and treatment approaches vary from clinic to clinic. For more details about this, along with information on how to interpret the statistics in this table, see pages $79-88$.

\section{ART CYCLE PROFILE}

\begin{tabular}{|c|c|c|c|c|c|c|c|}
\hline \multicolumn{4}{|c|}{ Type of ART ${ }^{\mathrm{a}}$} & \multicolumn{4}{|c|}{ Patient Diagnosis } \\
\hline IVF & $100 \%$ & Procedural Factors: & & Tubal factor & $4 \%$ & Other factor & $4 \%$ \\
\hline GIFT & $0 \%$ & With ICSI & $83 \%$ & Ovulatory dysfunction & $8 \%$ & Unknown factor & $4 \%$ \\
\hline ZIFT & $0 \%$ & Unstimulated & $0 \%$ & Diminished ovarian reserve & $16 \%$ & Multiple Factors: & \\
\hline \multirow[t]{3}{*}{ Combination } & $0 \%$ & Used gestational carrier & $0 \%$ & Endometriosis & $6 \%$ & Female factors only & $22 \%$ \\
\hline & & & & Uterine factor & $2 \%$ & Female \& male factors & $21 \%$ \\
\hline & & & & Male factor & $12 \%$ & & \\
\hline
\end{tabular}

2006 PREGNANCY SUCCESS RATES

Data verified by Daniel B. Shapiro, MD

\section{Type of Cycle}

\section{Fresh Embryos from Nondonor Eggs}

Number of cycles

Percentage of cycles resulting in pregnancies ${ }^{b}$

Percentage of cycles resulting in live births ${ }^{b, c}$

(Confidence Interval)

Percentage of retrievals resulting in live births $s^{b, c}$

Percentage of transfers resulting in live births $s^{b, c}$

Percentage of transfers resulting in singleton live births ${ }^{b}$

Percentage of cancellations ${ }^{b}$

Average number of embryos transferred

Percentage of pregnancies with twins ${ }^{b}$

Percentage of pregnancies with triplets or more ${ }^{b}$

Percentage of live births having multiple infants ${ }^{b, c}$

\section{Frozen Embryos from Nondonor Eggs}

Number of transfers

Percentage of transfers resulting in live births $s^{b, c}$

Average number of embryos transferred

\section{Donor Eggs}

Number of transfers

Percentage of transfers resulting in live births $s^{b, c}$

Average number of embryos transferred

\section{Age of Woman}

35-37 38-40

$41-42^{d}$

$<35$

234

168

89

295

39.7

26.2

5.6

35.3

34.2

20.2

3.4

$(29.8-41.0)$

39.1

(28.1-40.7)

(14.4-27.1)

(0.7-9.5)

42.3

39.4

23.0

4.6

28.9

42.8

25.4

20.9

5.7

9.8

29.4

11.9

3.8

2.3

28.4

13.2

3.1

27.0

3.4

22.6

31.7

8.6

18.2

2.9

$1 / 5$

$0 / 5$

31.3

4.5

$1 / 3$

118

38.1

66

37.9

3.1

17.6

12

3.0

All Ages Combined ${ }^{\mathrm{e}}$

Fresh Embryos

75

66.7

2.1
$6 / 12$

3.3

\section{CURRENT CLINIC SERVICES AND PROFILE}

Current Name: Reproductive Biology Associates

Donor egg? Yes

Donor embryo? Yes

Single women? Yes
Gestational carriers? Yes

Cryopreservation? Yes
Frozen Embryos

64

40.6

2.6

\footnotetext{
${ }^{a}$ Reflects patient and treatment characteristics of ART cycles performed in 2006 using fresh nondonor eggs or embryos.

${ }^{b}$ When fewer than 20 cycles are reported in an age category, rates are shown as a fraction and confidence intervals are not given. Calculating percentages from fractions may be misleading and is not encouraged.

${ }^{c}$ A multiple-infant birth is counted as one live birth.

${ }^{d}$ Clinic-specific outcome rates are unreliable for women older than 42 undergoing ART cycles using fresh or frozen embryos with nondonor eggs. Readers are urged to review national outcomes for these age groups (see page 27).

${ }^{e}$ All ages (including ages $>42$ ) are reported together because previous data show that patient age does not materially affect success with donor eggs.
} 


\section{REPRODUCTIVE MEDICINE AND INFERTILITY ASSOCIATES AUGUSTA, GEORGIA}

A comparison of clinic success rates may not be meaningful because patient medical characteristics and treatment approaches vary from clinic to clinic. For more details about this, along with information on how to interpret the statistics in this table, see pages 79-88.

\section{ART CYCLE PROFILE}

\begin{tabular}{|c|c|c|c|c|c|c|c|}
\hline \multicolumn{4}{|c|}{ Type of ART ${ }^{a}$} & \multicolumn{4}{|c|}{ Patient Diagnosis } \\
\hline IVF & $100 \%$ & Procedural Factors: & & Tubal factor & $13 \%$ & Other factor & $9 \%$ \\
\hline GIFT & $0 \%$ & With ICSI & $59 \%$ & Ovulatory dysfunction & $16 \%$ & Unknown factor & $16 \%$ \\
\hline ZIFT & $0 \%$ & Unstimulated & $0 \%$ & Diminished ovarian reserve & $19 \%$ & Multiple Factors: & \\
\hline \multirow[t]{3}{*}{ Combination } & $0 \%$ & Used gestational carrier & $0 \%$ & Endometriosis & $16 \%$ & Female factors only & $0 \%$ \\
\hline & & & & Uterine factor & $3 \%$ & Female \& male factors & $6 \%$ \\
\hline & & & & Male factor & $3 \%$ & & \\
\hline
\end{tabular}

\section{PREGNANCY SUCCESS RATES}

Data verified by Adelina M. Emmi, MD

\section{Type of Cycle}

\section{Fresh Embryos from Nondonor Eggs}

Number of cycles

Percentage of cycles resulting in pregnancies ${ }^{b}$

Percentage of cycles resulting in live births ${ }^{b, c}$

(Confidence Interval)

Percentage of retrievals resulting in live births $s^{b, c}$

Percentage of transfers resulting in live births $s^{b, c}$

Percentage of transfers resulting in singleton live births ${ }^{b}$

Percentage of cancellations ${ }^{b}$

Average number of embryos transferred

Percentage of pregnancies with twins ${ }^{b}$

Percentage of pregnancies with triplets or more ${ }^{b}$

Percentage of live births having multiple infants ${ }^{b, c}$

\section{Frozen Embryos from Nondonor Eggs}

Number of transfers

Percentage of transfers resulting in live births $s^{b, c}$

Average number of embryos transferred

\section{Donor Eggs}

Number of transfers

Percentage of transfers resulting in live births $s^{b, c}$

Average number of embryos transferred

\section{Age of Woman}

35-37 38-40

$41-42^{d}$

\section{$<35$}

2

$1 / 2$

7

5

$10 / 15$

$1 / 2$

$3 / 7$

$2 / 5$

$9 / 15$

$3 / 7$

$2 / 5$

$9 / 15$

$1 / 2$

$3 / 7$

$2 / 4$

$9 / 15$

$1 / 2$

$3 / 7$

$2 / 7$

$2 / 4$

$5 / 15$

$1 / 2$

$0 / 7$

$1 / 4$

$0 / 15$

$0 / 2$

2.9

$1 / 5$

2.5

2.5

$2 / 3$

2.3

$3 / 10$

$0 / 1$

$0 / 3$

$1 / 10$

$0 / 1$

$4 / 9$

$0 / 1$

$1 / 3$

$0 / 2$

$1 / 2$

$1 / 2$

0

$$
1
$$

$0 / 1$

2.0
Fresh Embryos

All Ages Combined ${ }^{\mathrm{e}}$

1

$0 / 1$

2.0

\section{CURRENT CLINIC SERVICES AND PROFILE}

Current Name: Reproductive Medicine and Infertility Associates

Donor egg? Yes Gestational carriers? No

Donor embryo? No Cryopreservation? Yes

Single women? Yes

SART member? $\quad$ Yes

Verified lab accreditation? Yes

(See Appendix C for details.)

${ }^{a}$ Reflects patient and treatment characteristics of ART cycles performed in 2006 using fresh nondonor eggs or embryos.

${ }^{b}$ When fewer than 20 cycles are reported in an age category, rates are shown as a fraction and confidence intervals are not given. Calculating percentages from fractions may be misleading and is not encouraged.

${ }^{c}$ A multiple-infant birth is counted as one live birth.

d Clinic-specific outcome rates are unreliable for women older than 42 undergoing ART cycles using fresh or frozen embryos with nondonor eggs. Readers are urged to review national outcomes for these age groups (see page 27).

e All ages (including ages $>42$ ) are reported together because previous data show that patient age does not materially affect success with donor eggs. 


\section{SERVY INSTITUTE FOR REPRODUCTIVE ENDOCRINOLOGY AUGUSTA, GEORGIA}

A comparison of clinic success rates may not be meaningful because patient medical characteristics and treatment approaches vary from clinic to clinic. For more details about this, along with information on how to interpret the statistics in this table, see pages $79-88$.

\section{ART CYCLE PROFILE}

\begin{tabular}{|c|c|c|c|c|c|c|c|}
\hline \multicolumn{4}{|c|}{ Type of $A R T^{a}$} & \multicolumn{4}{|c|}{ Patient Diagnosis } \\
\hline IVF & $100 \%$ & Procedural Factors: & & Tubal factor & $14 \%$ & Other factor & $0 \%$ \\
\hline GIFT & $0 \%$ & With ICSI & $31 \%$ & Ovulatory dysfunction & $5 \%$ & Unknown factor & $0 \%$ \\
\hline ZIFT & $0 \%$ & Unstimulated & $0 \%$ & Diminished ovarian reserve & $5 \%$ & Multiple Factors: & \\
\hline \multirow[t]{3}{*}{ Combination } & $0 \%$ & Used gestational carrier & $0 \%$ & Endometriosis & $0 \%$ & Female factors only & $19 \%$ \\
\hline & & & & Uterine factor & $10 \%$ & Female \& male factors & $24 \%$ \\
\hline & & & & Male factor & $24 \%$ & & \\
\hline
\end{tabular}

2006 PREGNANCY SUCCESS RATES

Data verified by Edouard Servy, MD

\section{Type of Cycle}

\section{Fresh Embryos from Nondonor Eggs}

Number of cycles

Percentage of cycles resulting in pregnancies ${ }^{b}$

Percentage of cycles resulting in live births ${ }^{b, c}$

(Confidence Interval)
Percentage of retrievals resulting in live births ${ }^{b, c}$

Percentage of transfers resulting in live births $s^{b, c}$

Percentage of transfers resulting in singleton live births ${ }^{b}$

Percentage of cancellations ${ }^{b}$

Average number of embryos transferred

Percentage of pregnancies with twins ${ }^{b}$

Percentage of pregnancies with triplets or more ${ }^{b}$

Percentage of live births having multiple infants ${ }^{b, c}$

\section{Frozen Embryos from Nondonor Eggs}

Number of transfers

Percentage of transfers resulting in live births $s^{b, c}$

Average number of embryos transferred

\section{Donor Eggs}

Number of transfers

Percentage of transfers resulting in live births ${ }^{\mathrm{b}, \mathrm{c}}$

Average number of embryos transferred

\section{Age of Woman}

$\begin{array}{cccc}<35 & 35-37 & 38-40 & \mathbf{4 1 - 4 2}^{\text {d }} \\ 10 & 4 & 0 & 1 \\ 4 / 10 & 0 / 4 & & 1 / 1 \\ 4 / 10 & 0 / 4 & & 1 / 1\end{array}$

$4 / 8$

$0 / 2$

$1 / 1$

$4 / 8$

$0 / 2$

$1 / 1$

$4 / 8$

$0 / 2$

$1 / 1$

$2 / 10$

$2 / 4$

$0 / 1$

2.4

2.0

4.0

$0 / 4$

$0 / 1$

$0 / 4$

$0 / 1$

$0 / 4$

$0 / 1$

2

$2 / 2$

2.5

1

$0 / 1$

2

$0 / 2$

1.0

2.0

All Ages Combined ${ }^{\mathrm{e}}$

Fresh Embryos

0

Frozen Embryos

0

\section{CURRENT CLINIC SERVICES AND PROFILE}

Current Name: Servy Institute for Reproductive Endocrinology

\begin{tabular}{l|l|l} 
Donor egg? Yes & Gestational carriers? No
\end{tabular}

Donor embryo? No Cryopreservation? Yes

Single women? No Nee Appendix C for details.)
(Se

${ }^{a}$ Reflects patient and treatment characteristics of ART cycles performed in 2006 using fresh nondonor eggs or embryos.

${ }^{b}$ When fewer than 20 cycles are reported in an age category, rates are shown as a fraction and confidence intervals are not given. Calculating percentages from fractions may be misleading and is not encouraged.

${ }^{c}$ A multiple-infant birth is counted as one live birth.

d Clinic-specific outcome rates are unreliable for women older than 42 undergoing ART cycles using fresh or frozen embryos with nondonor eggs. Readers are urged to review national outcomes for these age groups (see page 27).

${ }^{e}$ All ages (including ages $>42$ ) are reported together because previous data show that patient age does not materially affect success with donor eggs. 


\section{COLUMBUS CENTER FOR REPRODUCTIVE ENDOCRINOLOGY \& INFERTILITY, LLC COLUMBUS, GEORGIA}

A comparison of clinic success rates may not be meaningful because patient medical characteristics and treatment approaches vary from clinic to clinic. For more details about this, along with information on how to interpret the statistics in this table, see pages 79-88.

\section{ART CYCLE PROFILE}

\begin{tabular}{|c|c|c|c|c|c|c|c|}
\hline \multicolumn{4}{|c|}{ Type of $A R T^{a}$} & \multicolumn{4}{|c|}{ Patient Diagnosis } \\
\hline IVF & $100 \%$ & Procedural Factors: & & Tubal factor & $17 \%$ & Other factor & $0 \%$ \\
\hline GIFT & $0 \%$ & With ICSI & $34 \%$ & Ovulatory dysfunction & $28 \%$ & Unknown factor & $17 \%$ \\
\hline ZIFT & $0 \%$ & Unstimulated & $0 \%$ & Diminished ovarian reserve & $3 \%$ & Multiple Factors: & \\
\hline \multirow[t]{3}{*}{ Combination } & $0 \%$ & Used gestational carrier & $6 \%$ & Endometriosis & $3 \%$ & Female factors only & $14 \%$ \\
\hline & & & & Uterine factor & $4 \%$ & Female \& male factors & $13 \%$ \\
\hline & & & & Male factor & $3 \%$ & & \\
\hline
\end{tabular}

2006 PREGNANCY SUCCESS RATES

Data verified by Prakash J. Thiruppathi, MD

\section{Type of Cycle}

\section{Age of Woman}

35-37 38-40

$<35 \quad 35-37 \quad 38-40 \quad 41-42^{d}$

Fresh Embryos from Nondonor Eggs

Number of cycles

Percentage of cycles resulting in pregnancies ${ }^{b}$

Percentage of cycles resulting in live births $s^{\mathrm{b}, \mathrm{c}}$

(Confidence Interval)

Percentage of retrievals resulting in live births ${ }^{\mathrm{b}, \mathrm{c}}$

Percentage of transfers resulting in live births ${ }^{b, c}$

Percentage of transfers resulting in singleton live births ${ }^{b}$

Percentage of cancellations ${ }^{b}$

12

$7 / 12$

6

1

43.3

$4 / 12$

$0 / 6$

$1 / 1$

36.7

$0 / 6$

$0 / 1$

(19.9-56.1)

36.7

$4 / 10$

$0 / 5$

$0 / 1$

40.7

$4 / 9$

$0 / 5$

$0 / 1$

14.8

$4 / 9$

$0 / 5$

$0 / 1$

0.0

$2 / 12$

$1 / 6$

$0 / 1$

2.7

2.9

3.2

3.0

$7 / 13$

$0 / 7$

$0 / 13$

$1 / 7$

$7 / 11$

$0 / 4$

Percentage of pregnancies with triplets or more ${ }^{b}$

Percentage of live births having multiple infants ${ }^{\mathrm{b}, \mathrm{c}}$

Frozen Embryos from Nondonor Eggs

Number of transfers

Percentage of transfers resulting in live births ${ }^{\mathrm{b}, \mathrm{c}}$

Average number of embryos transferred

10

$2 / 10$

2

3.4

$0 / 2$

4.0

1

$0 / 1$

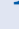

3.0

$0 / 1$

3.0

$0 / 1$

$0 / 1$

All Ages Combined ${ }^{\mathrm{e}}$

Fresh Embryos

Frozen Embryos

Donor Eggs

5

$3 / 5$

1

Percentage of transfers resulting in live births ${ }^{\mathrm{b}, \mathrm{c}}$

Average number of embryos transferred

\section{CURRENT CLINIC SERVICES AND PROFILE}

Current Name: Columbus Center for Reproductive Endocrinology \& Infertility, LLC

Donor egg? Yes

Gestational carriers? Yes

SART member?

No

Donor embryo? Yes

Cryopreservation? Yes

Verified lab accreditation?

Yes

Single women? Yes

(See Appendix C for details.)

${ }^{a}$ Reflects patient and treatment characteristics of ART cycles performed in 2006 using fresh nondonor eggs or embryos.

${ }^{b}$ When fewer than 20 cycles are reported in an age category, rates are shown as a fraction and confidence intervals are not given. Calculating percentages from fractions may be misleading and is not encouraged.

${ }^{c}$ A multiple-infant birth is counted as one live birth.

${ }^{d}$ Clinic-specific outcome rates are unreliable for women older than 42 undergoing ART cycles using fresh or frozen embryos with nondonor eggs. Readers are urged to review national outcomes for these age groups (see page 27).

e All ages (including ages $>42$ ) are reported together because previous data show that patient age does not materially affect success with donor eggs. 


\section{CENTRAL GEORGIA FERTILITY INSTITUTE \\ MACON, GEORGIA}

A comparison of clinic success rates may not be meaningful because patient medical characteristics and treatment approaches vary from clinic to clinic. For more details about this, along with information on how to interpret the statistics in this table, see pages $79-88$.

\section{ART CYCLE PROFILE}

\begin{tabular}{|c|c|c|c|c|c|c|c|}
\hline \multicolumn{4}{|c|}{ Type of ART ${ }^{\mathrm{a}}$} & \multicolumn{4}{|c|}{ Patient Diagnosis } \\
\hline IVF & $100 \%$ & Procedural Factors: & & Tubal factor & $20 \%$ & Other factor & $2 \%$ \\
\hline GIFT & $0 \%$ & With ICSI & $47 \%$ & Ovulatory dysfunction & $0 \%$ & Unknown factor & $7 \%$ \\
\hline $\mathrm{ZIFT}$ & $0 \%$ & Unstimulated & $0 \%$ & Diminished ovarian reserve & $2 \%$ & Multiple Factors: & \\
\hline Combination & $0 \%$ & Used gestational carrier & $0 \%$ & Endometriosis & $8 \%$ & Female factors only & $20 \%$ \\
\hline & & & & Uterine factor & $3 \%$ & Female \& male factors & $21 \%$ \\
\hline
\end{tabular}

2006 PREGNANCY SUCCESS RATES

Data verified by William J. Butler, MD

\section{Type of Cycle}

\section{Fresh Embryos from Nondonor Eggs}

Number of cycles

Percentage of cycles resulting in pregnancies ${ }^{b}$

Percentage of cycles resulting in live births $s^{b, c}$

(Confidence Interval)

Percentage of retrievals resulting in live births $s^{b, c}$

Percentage of transfers resulting in live births $s^{b, c}$

Percentage of transfers resulting in singleton live births ${ }^{b}$

Percentage of cancellations ${ }^{b}$

Average number of embryos transferred

Percentage of pregnancies with twins ${ }^{b}$

Percentage of pregnancies with triplets or more ${ }^{b}$

Percentage of live births having multiple infants ${ }^{b, c}$

\section{Frozen Embryos from Nondonor Eggs}

Number of transfers

Percentage of transfers resulting in live births ${ }^{\mathrm{b}, \mathrm{c}}$

Average number of embryos transferred

\section{Donor Eggs}

Number of transfers

Percentage of transfers resulting in live births ${ }^{\mathrm{b}, \mathrm{c}}$

Average number of embryos transferred

\section{Age of Woman}

35-37 38-40

$41-42^{d}$

$<35$

7

$3 / 7$

$3 / 7$

11

$3 / 11$

$3 / 11$

33.3

(17.3-52.8)

35.7

$3 / 5$

$3 / 8$

38.5

$3 / 5$

$3 / 8$

19.2

$1 / 5$

$3 / 8$

6.7

$2 / 7$

$3 / 11$

2.6

$5 / 13$

2.0

$0 / 3$

$0 / 13$

$2 / 3$

$0 / 3$

$5 / 10$

$0 / 3$

$0 / 3$
$0 / 1$

$0 / 1$

1
$0 / 1$
$0 / 1$

1

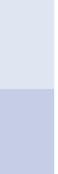

\section{CURRENT CLINIC SERVICES AND PROFILE}

Current Name: Central Georgia Fertility Institute
Donor egg?
No
Gestational carriers?
No
Donor embryo? No
Single women? Yes
Cryopreservation? Yes
${ }^{a}$ Reflects patient and treatment characteristics of ART cycles performed in 2006 using fresh nondonor eggs or embryos.
b When fewer than 20 cycles are reported in an age category, rates are shown as a fraction and confidence intervals are not given. Calculating percentages from fractions may be misleading and is not encouraged.
c A multiple-infant birth is counted as one live birth.
${ }^{d}$ Clinic-specific outcome rates are unreliable for women older than 42 undergoing ART cycles using fresh or frozen embryos with nondonor eggs. Readers are urged to review national outcomes for these age groups (see page 27).
${ }^{\mathrm{e}}$ All ages (including ages $>42$ ) are reported together because previous data show that patient age does not materially affect success with donor eggs.

\section{5}

$3 / 5$

4

2.0

$1 / 4$

1

$0 / 1$

2.0

2.0

All Ages Combined ${ }^{\mathrm{e}}$

Fresh Embryos

0
Frozen Embryos 0 


\section{GEORGIA CENTER FOR REPRODUCTIVE MEDICINE \\ SAVANNAH, GEORGIA}

A comparison of clinic success rates may not be meaningful because patient medical characteristics and treatment approaches vary from clinic to clinic. For more details about this, along with information on how to interpret the statistics in this table, see pages 79-88.

\section{ART CYCLE PROFILE}

\begin{tabular}{|c|c|c|c|c|c|c|c|}
\hline \multicolumn{4}{|c|}{ Type of ART ${ }^{a}$} & \multicolumn{4}{|c|}{ Patient Diagnosis } \\
\hline IVF & $100 \%$ & Procedural Factors: & & Tubal factor & $20 \%$ & Other factor & $2 \%$ \\
\hline GIFT & $0 \%$ & With ICSI & $37 \%$ & Ovulatory dysfunction & $12 \%$ & Unknown factor & $4 \%$ \\
\hline ZIFT & $0 \%$ & Unstimulated & $0 \%$ & Diminished ovarian reserve & $13 \%$ & Multiple Factors: & \\
\hline \multirow[t]{3}{*}{ Combination } & $0 \%$ & Used gestational carrier & $1 \%$ & Endometriosis & $4 \%$ & Female factors only & $15 \%$ \\
\hline & & & & Uterine factor & $0 \%$ & Female \& male factors & $18 \%$ \\
\hline & & & & Male factor & $13 \%$ & & \\
\hline
\end{tabular}

2006 PREGNANCY SUCCESS RATES

Data verified by Patrick L. Blohm, MD

\section{Type of Cycle}

\section{Fresh Embryos from Nondonor Eggs}

Number of cycles

Percentage of cycles resulting in pregnancies ${ }^{b}$

Percentage of cycles resulting in live births ${ }^{b, c}$

(Confidence Interval)

Percentage of retrievals resulting in live births $s^{b, c}$

Percentage of transfers resulting in live births $s^{b, c}$

Percentage of transfers resulting in singleton live births ${ }^{b}$

Percentage of cancellations ${ }^{b}$

Average number of embryos transferred

Percentage of pregnancies with twins ${ }^{b}$

Percentage of pregnancies with triplets or more ${ }^{b}$

Percentage of live births having multiple infants ${ }^{b, c}$

\section{Frozen Embryos from Nondonor Eggs}

Number of transfers

Percentage of transfers resulting in live births ${ }^{b, c}$

Average number of embryos transferred

\section{Donor Eggs}

Number of transfers

Percentage of transfers resulting in live births $s^{b, c}$

Average number of embryos transferred

\section{Age of Woman}

35-37 38-40

$41-42^{d}$

$<35$

22

68.2

11

6

58.0

59.1

$7 / 11$

$2 / 6$

54.0

(39.3-68.2)

(36.4-79.3)

61.4

59.1

$7 / 11$

$1 / 6$

65.9

39.0

59.1

$7 / 11$

$1 / 5$

$7 / 10$

$1 / 5$

31.8

$6 / 10$

$1 / 5$

12.0

0.0

$0 / 11$

$1 / 6$

2.5

2.8

2.9

2.6

31.0

$4 / 15$

$1 / 7$

$0 / 2$

10.3

$3 / 15$

40.7

$6 / 13$

$1 / 7$

$0 / 2$

$1 / 7$

$0 / 1$

14

$6 / 14$

8

$3 / 8$

3

$1 / 3$

2.1

2.0

1.7

All Ages Combined ${ }^{\mathrm{e}}$

Fresh Embryos

Frozen Embryos

12

$8 / 12$

5

2.7

$2 / 5$

2.4

\section{CURRENT CLINIC SERVICES AND PROFILE}

Current Name: Georgia Center for Reproductive Medicine

Donor egg? Yes

Donor embryo? Yes

Single women? Yes

\begin{abstract}
Gestational carriers? Yes
Cryopreservation? Yes
\end{abstract}

SART member?

Verified lab accreditation?

(See Appendix C for details.)
No

No

${ }^{a}$ Reflects patient and treatment characteristics of ART cycles performed in 2006 using fresh nondonor eggs or embryos.

${ }^{b}$ When fewer than 20 cycles are reported in an age category, rates are shown as a fraction and confidence intervals are not given. Calculating percentages from fractions may be misleading and is not encouraged.

${ }^{c}$ A multiple-infant birth is counted as one live birth.

${ }^{d}$ Clinic-specific outcome rates are unreliable for women older than 42 undergoing ART cycles using fresh or frozen embryos with nondonor eggs. Readers are urged to review national outcomes for these age groups (see page 27).

e All ages (including ages $>42$ ) are reported together because previous data show that patient age does not materially affect success with donor eggs. 


\section{ADVANCED REPRODUCTIVE CENTER OF HAWAII \\ HONOLULU, HAWAII}

A comparison of clinic success rates may not be meaningful because patient medical characteristics and treatment approaches vary from clinic to clinic. For more details about this, along with information on how to interpret the statistics in this table, see pages $79-88$.

\section{ART CYCLE PROFILE}

\begin{tabular}{|c|c|c|c|c|c|c|c|}
\hline \multicolumn{4}{|c|}{ Type of ART ${ }^{\mathrm{a}}$} & \multicolumn{4}{|c|}{ Patient Diagnosis } \\
\hline IVF & $100 \%$ & Procedural Factors: & & Tubal factor & $<1 \%$ & Other factor & $3 \%$ \\
\hline GIFT & $0 \%$ & With ICSI & $56 \%$ & Ovulatory dysfunction & $2 \%$ & Unknown factor & $0 \%$ \\
\hline ZIFT & $0 \%$ & Unstimulated & $0 \%$ & Diminished ovarian reserve & $7 \%$ & Multiple Factors: & \\
\hline Combination & $0 \%$ & Used gestational carrier & $0 \%$ & Endometriosis & $8 \%$ & Female factors only & $8 \%$ \\
\hline & & & & Uterine factor & $\begin{array}{r}<1 \% \\
8 \%\end{array}$ & Female \& male factors & $64 \%$ \\
\hline
\end{tabular}

2006 PREGNANCY SUCCESS RATES

Data verified by Christopher T. Huang, MD

\section{Type of Cycle}

\section{Fresh Embryos from Nondonor Eggs}

Number of cycles

Percentage of cycles resulting in pregnancies ${ }^{b}$

Percentage of cycles resulting in live births ${ }^{b, c}$

(Confidence Interval)

Percentage of retrievals resulting in live births $s^{b, c}$

Percentage of transfers resulting in live births $s^{b, c}$

Percentage of transfers resulting in singleton live births ${ }^{b}$

Percentage of cancellations ${ }^{b}$

Average number of embryos transferred

Percentage of pregnancies with twins ${ }^{b}$

Percentage of pregnancies with triplets or more ${ }^{b}$

Percentage of live births having multiple infants ${ }^{b, c}$

\section{Frozen Embryos from Nondonor Eggs}

Number of transfers

Percentage of transfers resulting in live births ${ }^{\mathrm{b}, \mathrm{c}}$

Average number of embryos transferred

\section{Donor Eggs}

Number of transfers

Percentage of transfers resulting in live births $s^{b, c}$

Average number of embryos transferred

\section{Age of Woman}

35-37 38-40

$41-42^{d}$

$<35$

19

31

19

$\begin{array}{cccc}14 & 19 & 31 & 19 \\ 4 / 14 & 4 / 19 & 32.3 & 7 / 19 \\ 2 / 14 & 3 / 19 & 19.4 & 5 / 19\end{array}$

$2 / 12$

$3 / 16$

28.6

$5 / 16$

$2 / 11$

$3 / 15$

28.6

$5 / 14$

$0 / 11$

$2 / 15$

19.0

$5 / 14$

$2 / 14$

$3 / 19$

32.3

$3 / 19$

3.0

3.3

$1 / 4$

$2 / 4$

3.2

2.9

$2 / 4$

$0 / 4$

$2 / 10$

$0 / 7$

$2 / 2$

$1 / 3$

$0 / 10$

$0 / 7$

$2 / 6$

$0 / 5$

\section{4}

$2 / 4$

2.0

\section{$\%$ $\%$ .}




\section{PACIFIC IN VITRO FERTILIZATION INSTITUTE HONOLULU, HAWAII}

A comparison of clinic success rates may not be meaningful because patient medical characteristics and treatment approaches vary from clinic to clinic. For more details about this, along with information on how to interpret the statistics in this table, see pages 79-88.

\section{ART CYCLE PROFILE}

\begin{tabular}{|c|c|c|c|c|c|c|c|}
\hline \multicolumn{4}{|c|}{ Type of ART ${ }^{a}$} & \multicolumn{4}{|c|}{ Patient Diagnosis } \\
\hline IVF & $100 \%$ & Procedural Factors: & & Tubal factor & $9 \%$ & Other factor & $1 \%$ \\
\hline GIFT & $0 \%$ & With ICSI & $44 \%$ & Ovulatory dysfunction & $3 \%$ & Unknown factor & $2 \%$ \\
\hline ZIFT & $0 \%$ & Unstimulated & $0 \%$ & Diminished ovarian reserve & $7 \%$ & Multiple Factors: & \\
\hline \multirow[t]{3}{*}{ Combination } & $0 \%$ & Used gestational carrier & $<1 \%$ & Endometriosis & $6 \%$ & Female factors only & $19 \%$ \\
\hline & & & & Uterine factor & $0 \%$ & Female \& male factors & $40 \%$ \\
\hline & & & & Male factor & $12 \%$ & & \\
\hline
\end{tabular}

\section{PREGNANCY SUCCESS RATES}

Data verified by Thomas S. Kosasa, MD

\section{Type of Cycle}

\section{Age of Woman}

35-37 38-40

$41-42^{d}$
59

32.2

25.4

(15.0-38.4)

26.8

30.6

16.3

5.1

3.4

$9 / 19$

$0 / 19$

$7 / 15$
42.3

15.4

45.0

18

$8 / 18$

2.1
22

45.5

2.2
46

17.4

13.0

(4.9-26.3)

15.4

17.6

11.8

15.2

3.5

$1 / 8$

$1 / 8$

$2 / 6$

10

$5 / 10$

2.4
29

3.4

3.4

$(0.1-17.8)$

4.8

5.0

5.0

27.6

4.2

$0 / 1$

$0 / 1$

$0 / 1$

All Ages Combined ${ }^{\mathbf{e}}$

Fresh Embryos 30

50.0

2.2
Frozen Embryos 9

$7 / 9$

2.0

Average number of embryos transferred

\section{CURRENT CLINIC SERVICES AND PROFILE}

Current Name: Pacific In Vitro Fertilization Institute

Donor egg? Yes Gestational carriers? Yes

Donor embryo? Yes

Single women? Yes

Cryopreservation? Yes

SART member?

Yes

Verified lab accreditation?

(See Appendix C for details.)

\footnotetext{
${ }^{a}$ Reflects patient and treatment characteristics of ART cycles performed in 2006 using fresh nondonor eggs or embryos.

${ }^{b}$ When fewer than 20 cycles are reported in an age category, rates are shown as a fraction and confidence intervals are not given. Calculating percentages from fractions may be misleading and is not encouraged.

${ }^{c}$ A multiple-infant birth is counted as one live birth.

d Clinic-specific outcome rates are unreliable for women older than 42 undergoing ART cycles using fresh or frozen embryos with nondonor eggs. Readers are urged to review national outcomes for these age groups (see page 27).

e All ages (including ages $>42$ ) are reported together because previous data show that patient age does not materially affect success with donor eggs.
} 


\section{TRIPLER ARMY MEDICAL CENTER IVF INSTITUTE \\ TRIPLER AMC, HAWAII}

A comparison of clinic success rates may not be meaningful because patient medical characteristics and treatment approaches vary from clinic to clinic. For more details about this, along with information on how to interpret the statistics in this table, see pages $79-88$.

\section{ART CYCLE PROFILE}

\begin{tabular}{|c|c|c|c|c|c|c|c|}
\hline \multicolumn{4}{|c|}{ Type of ART ${ }^{\mathrm{a}}$} & \multicolumn{4}{|c|}{ Patient Diagnosis } \\
\hline IVF & $100 \%$ & Procedural Factors: & & Tubal factor & $31 \%$ & Other factor & $3 \%$ \\
\hline GIFT & $0 \%$ & With ICSI & $68 \%$ & Ovulatory dysfunction & $5 \%$ & Unknown factor & $6 \%$ \\
\hline ZIFT & $0 \%$ & Unstimulated & $0 \%$ & Diminished ovarian reserve & $0 \%$ & Multiple Factors: & \\
\hline \multirow[t]{3}{*}{ Combination } & $0 \%$ & Used gestational carrier & $0 \%$ & Endometriosis & $3 \%$ & Female factors only & $8 \%$ \\
\hline & & & & Uterine factor & $0 \%$ & Female \& male factors & $13 \%$ \\
\hline & & & & Male factor & $33 \%$ & & \\
\hline
\end{tabular}

2006 PREGNANCY SUCCESS RATES

Data verified by Richard S. Lucidi, MD

\section{Type of Cycle}

\section{Fresh Embryos from Nondonor Eggs}

Number of cycles

Percentage of cycles resulting in pregnancies ${ }^{b}$

Percentage of cycles resulting in live births $s^{b, c}$

(Confidence Interval)

Percentage of retrievals resulting in live births $s^{b, c}$

Percentage of transfers resulting in live births $s^{b, c}$

Percentage of transfers resulting in singleton live births ${ }^{b}$

Percentage of cancellations ${ }^{b}$

Average number of embryos transferred

Percentage of pregnancies with twins ${ }^{b}$

Percentage of pregnancies with triplets or more ${ }^{b}$

Percentage of live births having multiple infants ${ }^{b, c}$

\section{Frozen Embryos from Nondonor Eggs}

Number of transfers

Percentage of transfers resulting in live births ${ }^{\mathrm{b}, \mathrm{c}}$

Average number of embryos transferred

\section{Donor Eggs}

Number of transfers

Percentage of transfers resulting in live births ${ }^{\mathrm{b}, \mathrm{c}}$

Average number of embryos transferred

\section{Age of Woman}

35-37 38-40

$41-42^{d}$

$<35$

16

$5 / 16$

$4 / 16$

11

5

33.3

29.6

$(13.8-50.2)$

32.0

32.0

$4 / 16$

$4 / 16$

$4 / 11$

$0 / 5$

8.0

$3 / 16$

$4 / 11$

$0 / 5$

7.4

$0 / 16$

2.8

$2 / 5$

$4 / 11$

$0 / 3$

2.8

$6 / 9$

$0 / 5$

$4 / 11$

$0 / 3$

$0 / 9$

$1 / 4$

$4 / 11$

$0 / 3$

$6 / 8$

$0 / 11$

$2 / 5$

3.3

2.3

$0 / 4$

$0 / 4$

$0 / 4$

\section{8}

$4 / 8$

2.0

\section{1}

$3 / 11$

2.3

2
$0 / 2$
2.5

All Ages Combined

Fresh Embryos

Frozen Embryos

0

0

\section{CURRENT CLINIC SERVICES AND PROFILE}

Current Name: Tripler Army Medical Center IVF Institute

\begin{tabular}{l|l|l} 
Donor egg? No Gestational carriers? No &
\end{tabular}

Donor embryo? No Cryopreservation? Yes

Single women? Yes

SART member? Yes

Verified lab accreditation? Yes

(See Appendix C for details.)

${ }^{a}$ Reflects patient and treatment characteristics of ART cycles performed in 2006 using fresh nondonor eggs or embryos.

${ }^{b}$ When fewer than 20 cycles are reported in an age category, rates are shown as a fraction and confidence intervals are not given. Calculating percentages from fractions may be misleading and is not encouraged.

A multiple-infant birth is counted as one live birth.

${ }^{d}$ Clinic-specific outcome rates are unreliable for women older than 42 undergoing ART cycles using fresh or frozen embryos with nondonor eggs. Readers are urged to review national outcomes for these age groups (see page 27).

e All ages (including ages $>42$ ) are reported together because previous data show that patient age does not materially affect success with donor eggs. 


\section{IDAHO CENTER FOR REPRODUCTIVE MEDICINE BOISE, IDAHO}

A comparison of clinic success rates may not be meaningful because patient medical characteristics and treatment approaches vary from clinic to clinic. For more details about this, along with information on how to interpret the statistics in this table, see pages 79-88.

\section{ART CYCLE PROFILE}

\section{Type of ART ${ }^{\mathrm{a}}$}

IVF

GIFT

$100 \%$ Procedural Factors:

ZIFT

Combination
$0 \%$ With ICSI

$0 \%$ Unstimulated

$0 \%$ Used gestational carrier

\begin{tabular}{|c|c|c|c|c|}
\hline & \multicolumn{4}{|c|}{ Patient Diagnosis } \\
\hline & Tubal factor & $7 \%$ & Other factor & $5 \%$ \\
\hline $51 \%$ & Ovulatory dysfunction & $4 \%$ & Unknown factor & $3 \%$ \\
\hline $0 \%$ & Diminished ovarian reserve & $16 \%$ & Multiple Factors: & \\
\hline $5 \%$ & Endometriosis & $3 \%$ & Female factors only & $23 \%$ \\
\hline & Uterine factor & $<1 \%$ & Female \& male factors & $27 \%$ \\
\hline & Male factor & $13 \%$ & & \\
\hline
\end{tabular}

Data verified by Cristin C. Slater, MD

2006 PREGNANCY SUCCESS RATES

\section{Age of Woman}

\section{Type of Cycle}

\section{$<35$}

\section{Fresh Embryos from Nondonor Eggs}

Number of cycles

Percentage of cycles resulting in pregnancies ${ }^{b}$

Percentage of cycles resulting in live births ${ }^{b, c}$

(Confidence Interval)

Percentage of retrievals resulting in live births $s^{b, c}$

Percentage of transfers resulting in live births $s^{b, c}$

Percentage of transfers resulting in singleton live births ${ }^{b}$

Percentage of cancellations ${ }^{b}$

Average number of embryos transferred

Percentage of pregnancies with twins ${ }^{\mathrm{b}}$

Percentage of pregnancies with triplets or more ${ }^{b}$

Percentage of live births having multiple infants ${ }^{b, c}$

\section{Frozen Embryos from Nondonor Eggs}

Number of transfers

Percentage of transfers resulting in live births $s^{b, c}$

Average number of embryos transferred
138

49.3

47.8

$(39.3-56.5)$

49.3

50.0

39.4

2.9

2.4

29.4

7.4

21.2

65

38.5

2.6
35-37

54

40.7

33.3

$(21.1-47.5)$

35.3

37.5

25.0

5.6

2.9

31.8

0.0

$6 / 18$
38-40

$41-42^{d}$

14

$4 / 14$

26.2

$3 / 14$

(10.3-36.8)

23.7

24.3

16.2

$3 / 11$

$3 / 11$

$3 / 11$

$3 / 14$

3.3

3.2

$0 / 4$

$3 / 11$

$0 / 4$

$0 / 11$

$0 / 3$

$3 / 9$

10

3

31

12.9

$2 / 10$

$0 / 3$

2.4

2.7

2.7

All Ages Combined ${ }^{\mathrm{e}}$

Fresh Embryos

45

60.0

2.3
Frozen Embryos

46

28.3

2.6

Average number of embryos transferred

\section{CURRENT CLINIC SERVICES AND PROFILE}

Current Name: Idaho Center for Reproductive Medicine

Donor egg? Yes

Donor embryo? Yes

Single women? Yes

\begin{abstract}
Gestational carriers? Yes
Cryopreservation? Yes
\end{abstract}

SART member?

Yes

Verified lab accreditation?

(See Appendix C for details.)

${ }^{a}$ Reflects patient and treatment characteristics of ART cycles performed in 2006 using fresh nondonor eggs or embryos.

b When fewer than 20 cycles are reported in an age category, rates are shown as a fraction and confidence intervals are not given. Calculating percentages from fractions may be misleading and is not encouraged.

${ }^{c}$ A multiple-infant birth is counted as one live birth.

d Clinic-specific outcome rates are unreliable for women older than 42 undergoing ART cycles using fresh or frozen embryos with nondonor eggs. Readers are urged to review national outcomes for these age groups (see page 27).

e All ages (including ages $>42$ ) are reported together because previous data show that patient age does not materially affect success with donor eggs. 


\section{RUSH-COPLEY CENTER FOR REPRODUCTIVE HEALTH AURORA, ILLINOIS}

A comparison of clinic success rates may not be meaningful because patient medical characteristics and treatment approaches vary from clinic to clinic. For more details about this, along with information on how to interpret the statistics in this table, see pages $79-88$.

\section{ART CYCLE PROFILE}

\section{Type of ART ${ }^{\mathrm{a}}$}

\begin{tabular}{lll|l} 
IVF & $98 \%$ Procedural Factors: & & Tubal factor \\
GIFT & $0 \%$ With ICSI & $62 \%$ & Ovulatory dysfunction \\
ZIFT & $1 \%$ Unstimulated & $0 \%$ & Diminished ovarian reserve \\
Combination & $1 \%$ Used gestational carrier & $0 \%$ & Endometriosis \\
& & & Uterine factor \\
& & & Male factor
\end{tabular}

2006 PREGNANCY SUCCESS RATES

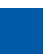

\begin{tabular}{|c|c|}
\hline & Type of ART ${ }^{a}$ \\
\hline IVF & 98\% Procedural Factors: \\
\hline GIFT & $0 \%$ With ICSI \\
\hline ZIFT & $1 \%$ Unstimulated \\
\hline Combination & $1 \%$ Used gestational carrier \\
\hline
\end{tabular}

\section{Type of Cycle}

Fresh Embryos from Nondonor Eggs

Number of cycles
Percentage of cycles resulting in pregnancies

Percentage of cycles resulting in live births $s^{b, c}$

(Confidence Interval)

Percentage of retrievals resulting in live births $s^{b, c}$

Percentage of transfers resulting in live births $s^{b, c}$

Percentage of transfers resulting in singleton live births ${ }^{b}$

Percentage of cancellations ${ }^{b}$

Average number of embryos transferred

Percentage of pregnancies with twins ${ }^{\mathrm{b}}$

Percentage of pregnancies with triplets or more ${ }^{b}$

Percentage of live births having multiple infants ${ }^{b, c}$

\section{Frozen Embryos from Nondonor Eggs}

Number of transfers

Percentage of transfers resulting in live births $s^{b, c}$

Average number of embryos transferred

\section{Donor Eggs}

Number of transfers

Percentage of transfers resulting in live births ${ }^{b, c}$

Average number of embryos transferred

\section{Patient Diagnosis}

9\% Other factor

$39 \%$

4\% Unknown factor

4\% Multiple Factors:

$6 \%$ Female factors only $\quad 8 \%$

$2 \%$ Female \& male factors $13 \%$ $8 \%$

Data verified by Zvi Binor, MD

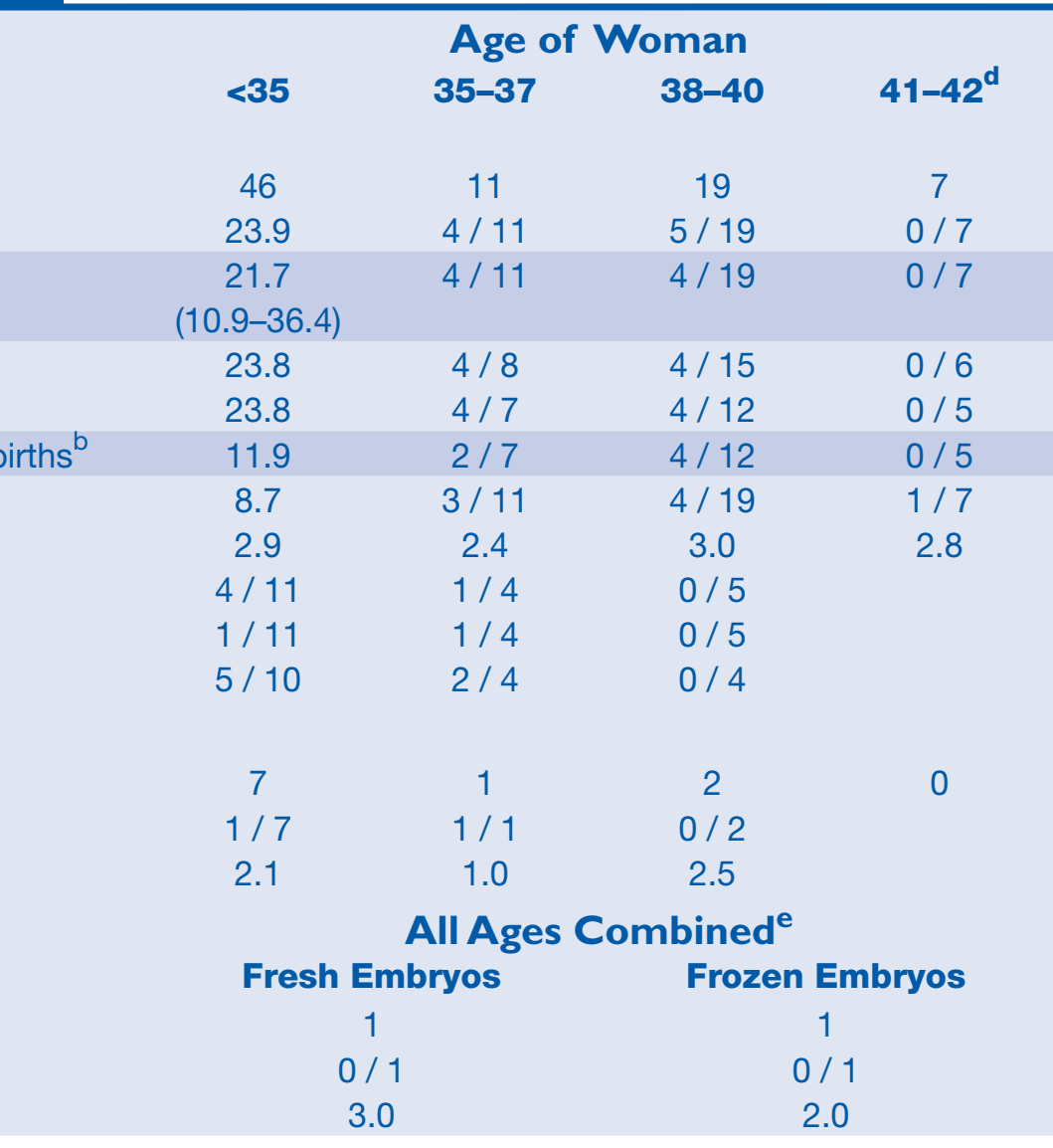

\section{CURRENT CLINIC SERVICES AND PROFILE}

Current Name: Rush-Copley Center for Reproductive Health

\begin{tabular}{ll|l|l|l|l} 
Donor egg? & Yes & Gestational carriers? & No & SART member? & Yes \\
Donor embryo? & No & Cryopreservation? & Yes & Verified lab accreditation? & Yes \\
Single women? & Yes & & & (See Appendix C for details.) &
\end{tabular}

\footnotetext{
${ }^{a}$ Reflects patient and treatment characteristics of ART cycles performed in 2006 using fresh nondonor eggs or embryos.

${ }^{b}$ When fewer than 20 cycles are reported in an age category, rates are shown as a fraction and confidence intervals are not given. Calculating percentages from fractions may be misleading and is not encouraged.

${ }^{c}$ A multiple-infant birth is counted as one live birth.

d Clinic-specific outcome rates are unreliable for women older than 42 undergoing ART cycles using fresh or frozen embryos with nondonor eggs. Readers are urged to review national outcomes for these age groups (see page 27).

${ }^{e}$ All ages (including ages $>42$ ) are reported together because previous data show that patient age does not materially affect success with donor eggs.
} 


\section{LIFE-WOMEN'S HEALTH CENTER \\ DANIEL A. ROSTEIN, MD \\ BERWYN, ILLINOIS}

A comparison of clinic success rates may not be meaningful because patient medical characteristics and treatment approaches vary from clinic to clinic. For more details about this, along with information on how to interpret the statistics in this table, see pages 79-88.

\section{ART CYCLE PROFILE}

\begin{tabular}{|c|c|c|c|c|c|c|c|}
\hline \multicolumn{4}{|c|}{ Type of ART ${ }^{a}$} & \multicolumn{4}{|c|}{ Patient Diagnosis } \\
\hline IVF & $100 \%$ & Procedural Factors: & & Tubal factor & $39 \%$ & Other factor & $8 \%$ \\
\hline GIFT & $0 \%$ & With ICSI & $56 \%$ & Ovulatory dysfunction & $15 \%$ & Unknown factor & $0 \%$ \\
\hline ZIFT & $0 \%$ & Unstimulated & $0 \%$ & Diminished ovarian reserve & $0 \%$ & Multiple Factors: & \\
\hline \multirow[t]{3}{*}{ Combination } & $0 \%$ & Used gestational carrier & $0 \%$ & Endometriosis & $15 \%$ & Female factors only & $0 \%$ \\
\hline & & & & Uterine factor & $0 \%$ & Female \& male factors & $15 \%$ \\
\hline & & & & Male factor & $8 \%$ & & \\
\hline
\end{tabular}

2006 PREGNANCY SUCCESS RATES

Data verified by Daniel A. Rostein, MD

\section{Type of Cycle}

\section{Fresh Embryos from Nondonor Eggs}

Number of cycles

Percentage of cycles resulting in pregnancies ${ }^{b}$

Percentage of cycles resulting in live births ${ }^{b, c}$

(Confidence Interval)

Percentage of retrievals resulting in live births $s^{b, c}$

Percentage of transfers resulting in live births $s^{b, c}$

Percentage of transfers resulting in singleton live births ${ }^{b}$

Percentage of cancellations ${ }^{b}$

Average number of embryos transferred

Percentage of pregnancies with twins ${ }^{b}$

Percentage of pregnancies with triplets or more ${ }^{b}$

Percentage of live births having multiple infants ${ }^{b, c}$

\section{Frozen Embryos from Nondonor Eggs}

Number of transfers

Percentage of transfers resulting in live births $s^{b, c}$

Average number of embryos transferred

\section{Donor Eggs}

Number of transfers

Percentage of transfers resulting in live births $s^{b, c}$

Average number of embryos transferred

\section{Age of Woman}

$\begin{array}{cccc}<35 & 35-37 & 38-40 & \mathbf{4 1 - 4 2}^{\mathbf{d}} \\ 5 & 2 & 2 & 0 \\ 3 / 5 & 0 / 2 & 1 / 2 & \\ 2 / 5 & 0 / 2 & 1 / 2 & \end{array}$

$\begin{array}{ccc}2 / 5 & 0 / 2 & 1 / 2 \\ 2 / 5 & 0 / 2 & 1 / 2 \\ 1 / 5 & 0 / 2 & 0 / 2 \\ 0 / 5 & 0 / 2 & 0 / 2 \\ 2.0 & 2.5 & 3.5 \\ 1 / 3 & & 1 / 1 \\ 0 / 3 & 0 / 1 \\ 1 / 2 & & 1 / 1\end{array}$

0

3

$2 / 3$

0

2.3

1

$0 / 1$

3.0

All Ages Combined ${ }^{\mathrm{e}}$

Fresh Embryos

Frozen Embryos

0

0

\section{CURRENT CLINIC SERVICES AND PROFILE}

Current Name: Life-Women's Health Center, Daniel A. Rostein, MD

Donor egg? Yes Gestational carriers? No

Donor embryo? No Cryopreservation? Yes

Single women? Yes

SART member? $\quad$ No

Verified lab accreditation? Yes

(See Appendix C for details.)

${ }^{a}$ Reflects patient and treatment characteristics of ART cycles performed in 2006 using fresh nondonor eggs or embryos.

${ }^{b}$ When fewer than 20 cycles are reported in an age category, rates are shown as a fraction and confidence intervals are not given. Calculating percentages from fractions may be misleading and is not encouraged.

${ }^{c}$ A multiple-infant birth is counted as one live birth.

d Clinic-specific outcome rates are unreliable for women older than 42 undergoing ART cycles using fresh or frozen embryos with nondonor eggs. Readers are urged to review national outcomes for these age groups (see page 27).

e All ages (including ages $>42$ ) are reported together because previous data show that patient age does not materially affect success with donor eggs. 


\section{MARTIN S. BALIN, MD, PHD \\ CHICAGO, ILLINOIS}

A comparison of clinic success rates may not be meaningful because patient medical characteristics and treatment approaches vary from clinic to clinic. For more details about this, along with information on how to interpret the statistics in this table, see pages $79-88$.

\section{ART CYCLE PROFILE}

\begin{tabular}{|c|c|c|c|c|c|c|c|}
\hline \multicolumn{4}{|c|}{ Type of $\mathrm{ART}^{\mathrm{a}}$} & \multicolumn{4}{|c|}{ Patient Diagnosis } \\
\hline IVF & $100 \%$ & Procedural Factors: & & Tubal factor & $3 \%$ & Other factor & $14 \%$ \\
\hline GIFT & $0 \%$ & With ICSI & $100 \%$ & Ovulatory dysfunction & $3 \%$ & Unknown factor & $36 \%$ \\
\hline ZIFT & $0 \%$ & Unstimulated & $0 \%$ & Diminished ovarian reserve & $0 \%$ & Multiple Factors: & \\
\hline \multirow[t]{3}{*}{ Combination } & $0 \%$ & Used gestational carrier & $0 \%$ & Endometriosis & $0 \%$ & Female factors only & $22 \%$ \\
\hline & & & & Uterine factor & $0 \%$ & Female \& male factors & $19 \%$ \\
\hline & & & & Male factor & $3 \%$ & & \\
\hline
\end{tabular}

2006 PREGNANCY SUCCESS RATES

Data verified by Martin S. Balin, MD, PhD

\section{Type of Cycle}

\section{Fresh Embryos from Nondonor Eggs}

Number of cycles

Percentage of cycles resulting in pregnancies ${ }^{b}$

Percentage of cycles resulting in live births $s^{b, c}$

(Confidence Interval)

Percentage of retrievals resulting in live births $s^{b, c}$

Percentage of transfers resulting in live births $s^{b, c}$

Percentage of transfers resulting in singleton live births ${ }^{b}$

Percentage of cancellations ${ }^{b}$

Average number of embryos transferred

Percentage of pregnancies with twins ${ }^{b}$

Percentage of pregnancies with triplets or more ${ }^{b}$

Percentage of live births having multiple infants ${ }^{b, c}$

\section{Frozen Embryos from Nondonor Eggs}

Number of transfers

Percentage of transfers resulting in live births $s^{b, c}$

Average number of embryos transferred

\section{Donor Eggs}

Number of transfers

Percentage of transfers resulting in live births $s^{b, c}$

Average number of embryos transferred

\section{Age of Woman}

$\begin{array}{cccc}<35 & \mathbf{3 5 - 3 7} & \mathbf{3 8 - 4 0} & \mathbf{4 1 - 4 2}^{\mathbf{d}} \\ 9 & 7 & 12 & 2 \\ 3 / 9 & 0 / 7 & 2 / 12 & 0 / 2 \\ 3 / 9 & 0 / 7 & 1 / 12 & 0 / 2\end{array}$

$\begin{array}{lccc}3 / 9 & 0 / 7 & 1 / 12 & 0 / 2 \\ 3 / 9 & 0 / 6 & 1 / 11 & 0 / 2 \\ 3 / 9 & 0 / 6 & 1 / 11 & 0 / 2 \\ 0 / 9 & 0 / 7 & 0 / 12 & 0 / 2 \\ 2.4 & 2.7 & 2.9 & 2.5 \\ 0 / 3 & & 0 / 2 & \\ 0 / 3 & 0 / 2 & \\ 0 / 3 & & 0 / 1\end{array}$

0

0

0

0

\section{CURRENT CLINIC SERVICES AND PROFILE}

Current Name: Martin S. Balin, MD, PhD
Donor egg? Yes
$\begin{array}{ll}\text { Donor embryo? } & \text { No } \\ \text { Single women? } & \text { Yes }\end{array}$
Gestational carriers?
Yes
SART member?
No
Cryopreservation? Yes
Verified lab accreditation?
Yes
(See Appendix C for details.)

\footnotetext{
${ }^{a}$ Reflects patient and treatment characteristics of ART cycles performed in 2006 using fresh nondonor eggs or embryos.

${ }^{b}$ When fewer than 20 cycles are reported in an age category, rates are shown as a fraction and confidence intervals are not given. Calculating percentages from fractions may be misleading and is not encouraged.

${ }^{c}$ A multiple-infant birth is counted as one live birth.

${ }^{d}$ Clinic-specific outcome rates are unreliable for women older than 42 undergoing ART cycles using fresh or frozen embryos with nondonor eggs. Readers are urged to review national outcomes for these age groups (see page 27).

${ }^{e}$ All ages (including ages $>42$ ) are reported together because previous data show that patient age does not materially affect success with donor eggs.
} 


\section{CENTER FOR REPRODUCTIVE MEDICINE \& FERTILITY \\ THE UNIVERSITY OF CHICAGO \\ CHICAGO, ILLINOIS}

A comparison of clinic success rates may not be meaningful because patient medical characteristics and treatment approaches vary from clinic to clinic. For more details about this, along with information on how to interpret the statistics in this table, see pages 79-88.

\section{ART CYCLE PROFILE}

\begin{tabular}{|c|c|c|c|c|c|c|c|}
\hline \multicolumn{4}{|c|}{ Type of ART ${ }^{a}$} & \multicolumn{4}{|c|}{ Patient Diagnosis } \\
\hline IVF & $>99 \%$ & Procedural Factors: & & Tubal factor & $10 \%$ & Other factor & $22 \%$ \\
\hline GIFT & $0 \%$ & With ICSI & $46 \%$ & Ovulatory dysfunction & $2 \%$ & Unknown factor & $22 \%$ \\
\hline ZIFT & $0 \%$ & Unstimulated & $0 \%$ & Diminished ovarian reserve & $<1 \%$ & Multiple Factors: & \\
\hline \multirow[t]{3}{*}{ Combination } & $<1 \%$ & Used gestational carrier & $<1 \%$ & Endometriosis & $4 \%$ & Female factors only & $11 \%$ \\
\hline & & & & Uterine factor & $<1 \%$ & Female \& male factors & $16 \%$ \\
\hline & & & & Male factor & $13 \%$ & & \\
\hline
\end{tabular}

2006 PREGNANCY SUCCESS RATES

Data verified by Helen Kim, MD

\section{Type of Cycle}

\section{Fresh Embryos from Nondonor Eggs}

Number of cycles

Percentage of cycles resulting in pregnancies ${ }^{b}$

Percentage of cycles resulting in live births $s^{b, c}$

(Confidence Interval)

Percentage of retrievals resulting in live births $s^{b, c}$

Percentage of transfers resulting in live births $s^{b, c}$

Percentage of transfers resulting in singleton live births ${ }^{b}$

Percentage of cancellations ${ }^{b}$

Average number of embryos transferred

Percentage of pregnancies with twins ${ }^{b}$

Percentage of pregnancies with triplets or more ${ }^{b}$

Percentage of live births having multiple infants ${ }^{b, c}$

\section{Frozen Embryos from Nondonor Eggs}

Number of transfers

Percentage of transfers resulting in live births ${ }^{b, c}$

Average number of embryos transferred

\section{Donor Eggs}

Number of transfers

Percentage of transfers resulting in live births $s^{b, c}$

Average number of embryos transferred

\section{Age of Woman}

$$
\text { 35-37 38-40 }
$$

$41-42^{d}$

$\begin{array}{cccc}44 & 33 & 25 & 24 \\ 36.4 & 27.3 & 16.0 & 8.3 \\ 31.8 & 21.2 & 12.0 & 4.2\end{array}$

(18.6-47.6)

(9.0-38.9)

(2.5-31.2)

$(0.1-21.1)$

36.8

25.0

$3 / 17$

$1 / 16$

41.2

28.0

$3 / 16$

$1 / 14$

26.5

28.0

$3 / 16$

$1 / 14$

13.6

15.2

32.0

33.3

2.3

2.4

2.6

4.0

$4 / 16$

$0 / 9$

$0 / 4$

$0 / 2$

$1 / 16$

$0 / 9$

$0 / 4$

$0 / 2$

$5 / 14$

$0 / 7$

$0 / 3$

$0 / 1$

$\begin{array}{cccc}17 & 12 & 5 & 2 \\ 1 / 17 & 2 / 12 & 0 / 5 & 0 / 2 \\ 2.6 & 3.5 & 3.6 & 4.0\end{array}$

All Ages Combined ${ }^{\mathrm{e}}$

Fresh Embryos

Frozen Embryos

9

2

$4 / 9$

$0 / 2$

2.3

4.0

\section{CURRENT CLINIC SERVICES AND PROFILE}

Current Name: Center for Reproductive Medicine \& Fertility, The University of Chicago

Donor egg? Yes

Donor embryo? No

Single women? Yes

\section{Gestational carriers? Yes}

Cryopreservation? Yes
SART member?

Verified lab accreditation?

(See Appendix C for details.)

${ }^{a}$ Reflects patient and treatment characteristics of ART cycles performed in 2006 using fresh nondonor eggs or embryos.

${ }^{b}$ When fewer than 20 cycles are reported in an age category, rates are shown as a fraction and confidence intervals are not given. Calculating percentages from fractions may be misleading and is not encouraged.

${ }^{c}$ A multiple-infant birth is counted as one live birth.

d Clinic-specific outcome rates are unreliable for women older than 42 undergoing ART cycles using fresh or frozen embryos with nondonor eggs. Readers are urged to review national outcomes for these age groups (see page 27).

e All ages (including ages $>42$ ) are reported together because previous data show that patient age does not materially affect success with donor eggs. 


\section{INSTITUTE FOR HUMAN REPRODUCTION (IHR) \\ CHICAGO, ILLINOIS}

A comparison of clinic success rates may not be meaningful because patient medical characteristics and treatment approaches vary from clinic to clinic. For more details about this, along with information on how to interpret the statistics in this table, see pages $79-88$.

\section{ART CYCLE PROFILE}

\begin{tabular}{|c|c|c|c|c|c|c|c|}
\hline \multicolumn{4}{|c|}{ Type of ART ${ }^{\mathrm{a}}$} & \multicolumn{4}{|c|}{ Patient Diagnosis } \\
\hline IVF & $100 \%$ & Procedural Factors: & & Tubal factor & $2 \%$ & Other factor & $27 \%$ \\
\hline GIFT & $0 \%$ & With ICSI & $89 \%$ & Ovulatory dysfunction & $8 \%$ & Unknown factor & $3 \%$ \\
\hline $\mathrm{ZIFT}$ & $0 \%$ & Unstimulated & $0 \%$ & Diminished ovarian reserve & $10 \%$ & Multiple Factors: & \\
\hline Combination & $0 \%$ & Used gestational carrier & $<1 \%$ & Endometriosis & $2 \%$ & Female factors only & $20 \%$ \\
\hline & & & & $\begin{array}{l}\text { Uterine factor } \\
\text { Male factor }\end{array}$ & $\begin{array}{l}0 \% \\
5 \%\end{array}$ & Female \& male factors & $24 \%$ \\
\hline
\end{tabular}

2006 PREGNANCY SUCCESS RATES

Data verified by llan Tur-Kaspa, MD

\section{Type of Cycle}

\section{Age of Woman}

35-37 38-40

$41-42^{d}$
34

35.3

31.0

(21.5-41.9)

31.8

21.6

40.7

Percentage of pregnancies with triplets or more ${ }^{b}$

Percentage of live births having multiple infants ${ }^{b, c}$

\section{Frozen Embryos from Nondonor Eggs}

Number of transfers

Percentage of transfers resulting in live births $s^{\mathrm{b}, \mathrm{c}}$

Average number of embryos transferred

\section{Donor Eggs}

Number of transfers

Percentage of transfers resulting in live births ${ }^{\mathrm{b}, \mathrm{c}}$

Average number of embryos transferred
23.5

36.5

2.3

1.8

36.7

3.3

$\begin{array}{ccc}23.5 & 21.4 & 1 / 14 \\ (10.7-41.2) & (8.3-41.0) & \\ 24.2 & 24.0 & 1 / 13 \\ 27.6 & 6 / 15 & 1 / 7 \\ 20.7 & 4 / 15 & 1 / 7 \\ 2.9 & 10.7 & 1 / 14 \\ 1.9 & 2.1 & 2.0 \\ 2 / 12 & 0 / 6 & 0 / 2 \\ 0 / 12 & 2 / 6 & 0 / 2 \\ 2 / 8 & 2 / 6 & 0 / 1\end{array}$

28

21.4

1.7
11

$3 / 11$

1.8
14

2 / 14

/ 14

/ 13

$1 / 7$

17

14

/ 2

$0 / 1$

2

$0 / 2$

1.5

\section{.}




\section{NORTHWESTERN UNIVERSITY \\ CHICAGO, ILLINOIS}

A comparison of clinic success rates may not be meaningful because patient medical characteristics and treatment approaches vary from clinic to clinic. For more details about this, along with information on how to interpret the statistics in this table, see pages 79-88.

\section{ART CYCLE PROFILE}

\begin{tabular}{|c|c|c|c|c|c|c|c|}
\hline \multicolumn{4}{|c|}{ Type of ART ${ }^{a}$} & \multicolumn{4}{|c|}{ Patient Diagnosis } \\
\hline IVF & $100 \%$ & Procedural Factors: & & Tubal factor & $6 \%$ & Other factor & $2 \%$ \\
\hline GIFT & $0 \%$ & With ICSI & $63 \%$ & Ovulatory dysfunction & $5 \%$ & Unknown factor & $27 \%$ \\
\hline ZIFT & $0 \%$ & Unstimulated & $<1 \%$ & Diminished ovarian reserve & $23 \%$ & Multiple Factors: & \\
\hline \multirow[t]{3}{*}{ Combination } & $0 \%$ & Used gestational carrier & $<1 \%$ & Endometriosis & $3 \%$ & Female factors only & $4 \%$ \\
\hline & & & & Uterine factor & $<1 \%$ & Female \& male factors & $8 \%$ \\
\hline & & & & Male factor & $20 \%$ & & \\
\hline
\end{tabular}

\section{PREGNANCY SUCCESS RATES}

\section{Type of Cycle}

\section{Fresh Embryos from Nondonor Eggs}

Number of cycles

Percentage of cycles resulting in pregnancies ${ }^{b}$

Percentage of cycles resulting in live births $s^{b, c}$

(Confidence Interval)

Percentage of retrievals resulting in live births $s^{b, c}$

Percentage of transfers resulting in live births $s^{b, c}$

Percentage of transfers resulting in singleton live births ${ }^{b}$

Percentage of cancellations ${ }^{b}$

Average number of embryos transferred

Percentage of pregnancies with twins ${ }^{b}$

Percentage of pregnancies with triplets or more ${ }^{b}$

Percentage of live births having multiple infants ${ }^{b, c}$

\section{Frozen Embryos from Nondonor Eggs}

Number of transfers

Percentage of transfers resulting in live births ${ }^{b, c}$

Average number of embryos transferred

\section{Donor Eggs}

Number of transfers

Percentage of transfers resulting in live births $s^{b, c}$

Average number of embryos transferred

Data verified by Edmond Confino, MD

\section{Age of Woman}

35-37 38-40

$41-42^{d}$

$<35$

147
35.4
25.9

136

72

47.9

25.9

22.1

16.7

44.3

(19.0-33.7)

16.2

9.7

(37.2-51.6)

47.8

49.1

29.2

$(10.4-23.5)$

(4.0-19.0)

36.0

29.9

17.9

19.0

12.3

12.7

25.2

17.2

10.9

7.2

11.6

9.6

20.8

2.2

2.2

2.7

3.1

26.9

11.5

13.3

$3 / 12$

1.1

1.9

0.0

$0 / 12$

26.7

15.8

9.1

$1 / 7$

64

31.3

44

27

13

2.8

27.3

25.9

$1 / 13$

2.5

2.7

3.1

All Ages Combined

Fresh Embryos

47

48.9

2.1
Frozen Embryos

31

32.3

2.8

\section{CURRENT CLINIC SERVICES AND PROFILE}

Current Name: Northwestern University

Donor egg? Yes Gestational carriers? Yes

Donor embryo? Yes Cryopreservation? Yes

Single women? Yes

SART member? Yes

Verified lab accreditation? Yes

(See Appendix C for details.)

${ }^{a}$ Reflects patient and treatment characteristics of ART cycles performed in 2006 using fresh nondonor eggs or embryos.

b When fewer than 20 cycles are reported in an age category, rates are shown as a fraction and confidence intervals are not given. Calculating percentages from fractions may be misleading and is not encouraged.

${ }^{c}$ A multiple-infant birth is counted as one live birth.

d Clinic-specific outcome rates are unreliable for women older than 42 undergoing ART cycles using fresh or frozen embryos with nondonor eggs. Readers are urged to review national outcomes for these age groups (see page 27).

e All ages (including ages $>42$ ) are reported together because previous data show that patient age does not materially affect success with donor eggs. 


\section{THE RINEHART-COULAM CENTER \\ CHICAGO, ILLINOIS}

A comparison of clinic success rates may not be meaningful because patient medical characteristics and treatment approaches vary from clinic to clinic. For more details about this, along with information on how to interpret the statistics in this table, see pages $79-88$.

\section{ART CYCLE PROFILE}

\begin{tabular}{|c|c|c|c|c|c|c|c|}
\hline \multicolumn{4}{|c|}{ Type of ART ${ }^{\mathrm{a}}$} & \multicolumn{4}{|c|}{ Patient Diagnosis } \\
\hline IVF & $100 \%$ & Procedural Factors: & & Tubal factor & $0 \%$ & Other factor & $45 \%$ \\
\hline GIFT & $0 \%$ & With ICSI & $96 \%$ & Ovulatory dysfunction & $8 \%$ & Unknown factor & $12 \%$ \\
\hline ZIFT & $0 \%$ & Unstimulated & $0 \%$ & Diminished ovarian reserve & $2 \%$ & Multiple Factors: & \\
\hline \multirow[t]{3}{*}{ Combination } & $0 \%$ & Used gestational carrier & $4 \%$ & Endometriosis & $0 \%$ & Female factors only & $15 \%$ \\
\hline & & & & Uterine factor & $10 \%$ & Female \& male factors & $5 \%$ \\
\hline & & & & Male factor & $3 \%$ & & \\
\hline
\end{tabular}

2006 PREGNANCY SUCCESS RATES

Data verified by Carolyn B. Coulam, MD

\section{Type of Cycle}

\section{Fresh Embryos from Nondonor Eggs}

Number of cycles

Percentage of cycles resulting in pregnancies ${ }^{b}$

Percentage of cycles resulting in live births $s^{b, c}$

(Confidence Interval)

Percentage of retrievals resulting in live births $s^{b, c}$

Percentage of transfers resulting in live births $s^{b, c}$

Percentage of transfers resulting in singleton live births ${ }^{b}$

Percentage of cancellations ${ }^{b}$

Average number of embryos transferred

Percentage of pregnancies with twins ${ }^{b}$

Percentage of pregnancies with triplets or more ${ }^{b}$

Percentage of live births having multiple infants ${ }^{b, c}$

\section{Frozen Embryos from Nondonor Eggs}

Number of transfers

Percentage of transfers resulting in live births $s^{b, c}$

Average number of embryos transferred

$0 / 2$

3.0

\section{Age of Woman}

35-37 38-40

$41-42^{d}$

$<35$

11

11

5

38.1

$3 / 11$

$2 / 11$

$0 / 5$

38.1

$3 / 11$

$1 / 11$

$0 / 5$

$(18.1-61.6)$

38.1

40.0

$3 / 11$

$1 / 11$

$0 / 4$

20.0

$3 / 8$

$1 / 10$

$0 / 4$

0.0

$3 / 8$

$1 / 10$

$0 / 4$

3.5

$0 / 11$

$0 / 11$

$1 / 5$

$3 / 8$

3.0

2.9

$1 / 2$

$1 / 8$

$0 / 3$

$0 / 2$

$4 / 8$

$0 / 3$

$0 / 1$

$0 / 3$

0

0

0

0

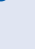

\section{Donor Eggs}

Number of transfers

Percentage of transfers resulting in live births $s^{b, c}$

Average number of embryos transferred

\begin{tabular}{cc}
\multicolumn{2}{c}{ All Ages Combined $^{\mathbf{e}}$} \\
Fresh Embryos & Frozen Embryos $^{2}$ \\
5 & 2 \\
$3 / 5$ & $0 / 2$ \\
2.8 & 2.5
\end{tabular}

\section{CURRENT CLINIC SERVICES AND PROFILE}

Current Name: The Rinehart-Coulam Center

\begin{tabular}{|c|c|c|c|c|}
\hline Donor egg? & Yes & Gestational carriers? & Yes & SART member? \\
\hline Donor embryo? & Yes & Cryopreservation? & Yes & Verified lab accreditation? \\
\hline Single women? & Yes & & & (See Appendix C for details.) \\
\hline
\end{tabular}

${ }^{a}$ Reflects patient and treatment characteristics of ART cycles performed in 2006 using fresh nondonor eggs or embryos.

${ }^{b}$ When fewer than 20 cycles are reported in an age category, rates are shown as a fraction and confidence intervals are not given. Calculating percentages from fractions may be misleading and is not encouraged.

${ }^{c}$ A multiple-infant birth is counted as one live birth.

d Clinic-specific outcome rates are unreliable for women older than 42 undergoing ART cycles using fresh or frozen embryos with nondonor eggs. Readers are urged to review national outcomes for these age groups (see page 27).

${ }^{e}$ All ages (including ages $>42$ ) are reported together because previous data show that patient age does not materially affect success with donor eggs. 


\section{RIVER NORTH IVF-FERTILITY CENTERS OF ILLINOIS CHICAGO, ILLINOIS}

A comparison of clinic success rates may not be meaningful because patient medical characteristics and treatment approaches vary from clinic to clinic. For more details about this, along with information on how to interpret the statistics in this table, see pages 79-88.

\section{ART CYCLE PROFILE}

\begin{tabular}{|c|c|c|c|c|c|c|c|}
\hline \multicolumn{4}{|c|}{ Type of ART ${ }^{\mathrm{a}}$} & \multicolumn{4}{|c|}{ Patient Diagnosis } \\
\hline IVF & $100 \%$ & Procedural Factors: & & Tubal factor & $8 \%$ & Other factor & $7 \%$ \\
\hline GIFT & $0 \%$ & With ICSI & $82 \%$ & Ovulatory dysfunction & $15 \%$ & Unknown factor & $18 \%$ \\
\hline ZIFT & $0 \%$ & Unstimulated & $0 \%$ & Diminished ovarian reserve & $12 \%$ & Multiple Factors: & \\
\hline \multirow[t]{3}{*}{ Combination } & $0 \%$ & Used gestational carrier & $<1 \%$ & Endometriosis & $4 \%$ & Female factors only & $8 \%$ \\
\hline & & & & Uterine factor & $1 \%$ & Female \& male factors & $10 \%$ \\
\hline & & & & Male factor & $17 \%$ & & \\
\hline
\end{tabular}

2006 PREGNANCY SUCCESS RATES

Data verified by Angelina Beltsos, MD

\section{Type of Cycle}

\section{Fresh Embryos from Nondonor Eggs}

Number of cycles

Percentage of cycles resulting in pregnancies ${ }^{b}$

Percentage of cycles resulting in live births $s^{b, c}$

(Confidence Interval)

Percentage of retrievals resulting in live births $s^{b, c}$

Percentage of transfers resulting in live births $s^{b, c}$

Percentage of transfers resulting in singleton live births ${ }^{b}$

Percentage of cancellations ${ }^{b}$

Average number of embryos transferred

Percentage of pregnancies with twins ${ }^{b}$

Percentage of pregnancies with triplets or more ${ }^{b}$

Percentage of live births having multiple infants ${ }^{\mathrm{b}, \mathrm{c}}$

\section{Frozen Embryos from Nondonor Eggs}

Number of transfers

Percentage of transfers resulting in live births ${ }^{b, c}$

Average number of embryos transferred

\section{Donor Eggs}

Number of transfers

Percentage of transfers resulting in live births $s^{b, c}$

Average number of embryos transferred

\section{Age of Woman}

35-37 38-40

$41-42^{d}$

$<35$

533

358

17.9

12.6

36.3

26.3

22.3

(28.2-34.2)

(18.9-26.1)

(9.3-16.5)

16.0

18.8

17.1

21.5

2.2

10.9

0.0

8.9

27.7

35.4

181

37.0

88

34.1

1.9
46

19.6

1.9
179

8.4

6.1

(3.1-10.7)

8.5

12.0

10.9

27.4

2.2

$1 / 15$

$0 / 15$

$1 / 11$
2.0
13

$2 / 13$

1.6

All Ages Combined ${ }^{\mathrm{e}}$

Fresh Embryos

106

55.7

2.2

\section{CURRENT CLINIC SERVICES AND PROFILE}

Current Name: River North IVF-Fertility Centers of Illinois

Donor egg? Yes

Donor embryo? No

Single women? Yes

\section{Gestational carriers? Yes}

Cryopreservation? Yes
Frozen Embryos 20

30.0

2.1

${ }_{b}^{a}$ Reflects patient and treatment characteristics of ART cycles performed in 2006 using fresh nondonor eggs or embryos.

b When fewer than 20 cycles are reported in an age category, rates are shown as a fraction and confidence intervals are not given. Calculating percentages from fractions may be misleading and is not encouraged.

${ }^{c}$ A multiple-infant birth is counted as one live birth.

d Clinic-specific outcome rates are unreliable for women older than 42 undergoing ART cycles using fresh or frozen embryos with nondonor eggs. Readers are urged to review national outcomes for these age groups (see page 27).

e All ages (including ages $>42$ ) are reported together because previous data show that patient age does not materially affect success with donor eggs. 


\title{
UNIVERSITY OF ILLINOIS AT CHICAGO IVF PROGRAM CHICAGO, ILLINOIS
}

A comparison of clinic success rates may not be meaningful because patient medical characteristics and treatment approaches vary from clinic to clinic. For more details about this, along with information on how to interpret the statistics in this table, see pages $79-88$.

\section{ART CYCLE PROFILE}

\begin{tabular}{|c|c|c|c|c|c|c|c|}
\hline \multicolumn{4}{|c|}{ Type of ART ${ }^{\mathrm{a}}$} & \multicolumn{4}{|c|}{ Patient Diagnosis } \\
\hline IVF & $100 \%$ & Procedural Factors: & & Tubal factor & $14 \%$ & Other factor & $10 \%$ \\
\hline GIFT & $0 \%$ & With ICSI & $73 \%$ & Ovulatory dysfunction & $3 \%$ & Unknown factor & $3 \%$ \\
\hline ZIFT & $0 \%$ & Unstimulated & $0 \%$ & Diminished ovarian reserve & $5 \%$ & Multiple Factors: & \\
\hline \multirow[t]{3}{*}{ Combination } & $0 \%$ & Used gestational carrier & $0 \%$ & Endometriosis & $11 \%$ & Female factors only & $17 \%$ \\
\hline & & & & Uterine factor & $0 \%$ & Female \& male factors & $15 \%$ \\
\hline & & & & Male factor & $21 \%$ & & \\
\hline
\end{tabular}

2006 PREGNANCY SUCCESS RATES

Data verified by Humberto Scoccia, MD

\section{Type of Cycle}

\section{Fresh Embryos from Nondonor Eggs}

Number of cycles

Percentage of cycles resulting in pregnancies ${ }^{b}$

Percentage of cycles resulting in live births $s^{b, c}$

(Confidence Interval)

Percentage of retrievals resulting in live births $s^{b, c}$

Percentage of transfers resulting in live births $s^{b, c}$

Percentage of transfers resulting in singleton live births ${ }^{b}$

Percentage of cancellations ${ }^{b}$

Average number of embryos transferred

Percentage of pregnancies with twins ${ }^{b}$

Percentage of pregnancies with triplets or more ${ }^{b}$

Percentage of live births having multiple infants ${ }^{b, c}$

\section{Frozen Embryos from Nondonor Eggs}

Number of transfers

Percentage of transfers resulting in live births ${ }^{\mathrm{b}, \mathrm{c}}$

Average number of embryos transferred

\section{Donor Eggs}

Number of transfers

Percentage of transfers resulting in live births $s^{\mathrm{b}, \mathrm{c}}$

Average number of embryos transferred

\section{Age of Woman}

35-37 38-40

$41-42^{d}$

$<35$

22

50.0

22

9

37.0

45.5

40.9

$0 / 9$

29.6

(24.4-67.8)

27.3

$0 / 9$

(18.0-43.6)

$10 / 19$

(10.7-50.2)

36.4

$10 / 18$

$6 / 17$

$0 / 5$

43.2

$9 / 18$

$6 / 16$

$0 / 4$

18.5

13.6

$4 / 16$

$0 / 4$

18.5

2.5

22.7

$4 / 9$

35.0

$1 / 11$

2.9

3.3

5.0

$0 / 11$

$3 / 9$

$6 / 16$

$1 / 10$

$1 / 9$

$2 / 6$

\section{7}

$5 / 17$

1.9

\begin{abstract}
2
\end{abstract}
$0 / 2$

2.0

3

$0 / 3$

1.7

All Ages Combined ${ }^{\mathrm{e}}$

Fresh Embryos

\section{7}

$3 / 7$

1.4
Frozen Embryos

\section{6}

$1 / 6$

3.2

\section{CURRENT CLINIC SERVICES AND PROFILE}

Current Name: University of Illinois at Chicago IVF Program

Donor egg? Yes

Donor embryo? No

Single women? Yes

\begin{abstract}
Gestational carriers?
\end{abstract}
Cryopreservation?
SART member?

Verified lab accreditation?

(See Appendix C for details.)

${ }^{a}$ Reflects patient and treatment characteristics of ART cycles performed in 2006 using fresh nondonor eggs or embryos.

${ }^{b}$ When fewer than 20 cycles are reported in an age category, rates are shown as a fraction and confidence intervals are not given. Calculating percentages from fractions may be misleading and is not encouraged.

${ }^{c}$ A multiple-infant birth is counted as one live birth.

d Clinic-specific outcome rates are unreliable for women older than 42 undergoing ART cycles using fresh or frozen embryos with nondonor eggs. Readers are urged to review national outcomes for these age groups (see page 27).

${ }^{e}$ All ages (including ages $>42$ ) are reported together because previous data show that patient age does not materially affect success with donor eggs. 


\section{WOMEN'S HEALTH CONSULTANTS \\ CHICAGO, ILLINOIS}

A comparison of clinic success rates may not be meaningful because patient medical characteristics and treatment approaches vary from clinic to clinic. For more details about this, along with information on how to interpret the statistics in this table, see pages 79-88.

\section{ART CYCLE PROFILE}

\section{Type of ART ${ }^{\mathrm{a}}$}

\begin{tabular}{lll|l} 
IVF & $96 \%$ Procedural Factors: & & Tubal factor \\
GIFT & $<1 \%$ With ICSI & $75 \%$ & Ovulatory dysfunction \\
ZIFT & $4 \%$ Unstimulated & $3 \%$ & Diminished ovarian reserve \\
Combination & $0 \%$ Used gestational carrier & $<1 \%$ & Endometriosis \\
& & & Uterine factor \\
& & & Male factor
\end{tabular}

\section{Patient Diagnosis}

$\begin{array}{rlr}2 \% & \text { Other factor } & 0 \% \\ 0 \% & \text { Unknown factor } & 1 \% \\ 2 \% & \text { Multiple Factors: } & \\ <1 \% & \text { Female factors only } & 49 \% \\ <1 \% & \text { Female \& male factors } & 42 \% \\ 4 \% & & \end{array}$

2006 PREGNANCY SUCCESS RATES

Data verified by Mary W. Molo, MD

\section{Type of Cycle}

\section{Age of Woman}

$$
\text { 35-37 38-40 }
$$$$
41-42^{d}
$$

$<35$

$$
37
$$

18.9

28.6

24.5

16.2

(13.3-38.9)

27.3

(6.2-32.0)

18.8

21.4

17.9

13.5

2.7

$1 / 7$

$0 / 7$

$1 / 6$

$\begin{array}{cc}31 & 21 \\ 22.6 & 4.8 \\ 12.9 & 0.0\end{array}$
(3.6-29.8)
16.0

18.2

18.2

19.4

2.9

$(0.0-16.1)$

$0 / 18$

$0 / 11$

$0 / 11$

14.3

3.0

$0 / 1$

$0 / 1$

$1 / 14$

$5 / 12$

$0 / 7$

$0 / 4$

Percentage of pregnancies with triplets or more
Percentage of live births having multiple infants ${ }^{b, c}$

\section{Frozen Embryos from Nondonor Eggs}

Number of transfers

Percentage of transfers resulting in live births $s^{b, c}$

Average number of embryos transferred

\begin{tabular}{cccc}
3 & 16 & 4 & 2 \\
$0 / 3$ & $1 / 16$ & $0 / 4$ & $0 / 2$ \\
3.3 & 2.6 & 2.8 & 2.5 \\
\multicolumn{4}{c}{$\begin{array}{c}\text { All Ages Combined } \\
\text { Fresh Embryos }\end{array}$} \\
$\begin{array}{c}\text { Frozen Embryos } \\
3\end{array}$ & & 0 \\
$0 / 3$ & & \\
2.7 & &
\end{tabular}

\section{Donor Eggs}

Number of transfers

Percentage of transfers resulting in live births $s^{b, c}$

Average number of embryos transferred

CURRENT CLINIC SERVICES AND PROFILE

Current Name: Women's Health Consultants
Donor egg?
Yes
Donor embryo? Yes
Gestational carriers?
Yes
Cryopreservation?
Yes
Single women? Yes
SART member?
No
Verified lab accreditation?
Yes
(See Appendix C for details.)

\footnotetext{
${ }^{a}$ Reflects patient and treatment characteristics of ART cycles performed in 2006 using fresh nondonor eggs or embryos.

${ }^{b}$ When fewer than 20 cycles are reported in an age category, rates are shown as a fraction and confidence intervals are not given. Calculating percentages from fractions may be misleading and is not encouraged.

${ }^{c}$ A multiple-infant birth is counted as one live birth.

${ }^{d}$ Clinic-specific outcome rates are unreliable for women older than 42 undergoing ART cycles using fresh or frozen embryos with nondonor eggs. Readers are urged to review national outcomes for these age groups (see page 27).

${ }^{e}$ All ages (including ages $>42$ ) are reported together because previous data show that patient age does not materially affect success with donor eggs.
} 


\section{CENTER FOR REPRODUCTIVE HEALTH/JOLIET IVF CREST HILL, ILLINOIS}

A comparison of clinic success rates may not be meaningful because patient medical characteristics and treatment approaches vary from clinic to clinic. For more details about this, along with information on how to interpret the statistics in this table, see pages $79-88$.

\section{ART CYCLE PROFILE}

\begin{tabular}{|c|c|c|c|c|c|c|c|}
\hline \multicolumn{4}{|c|}{ Type of ART } & \multicolumn{4}{|c|}{ Patient Diagnosis } \\
\hline IVF & $100 \%$ & Procedural Factors: & & Tubal factor & $12 \%$ & Other factor & $0 \%$ \\
\hline GIFT & $0 \%$ & With ICSI & $67 \%$ & Ovulatory dysfunction & $10 \%$ & Unknown factor & $18 \%$ \\
\hline ZIFT & $0 \%$ & Unstimulated & $0 \%$ & Diminished ovarian reserve & $26 \%$ & Multiple Factors: & \\
\hline \multirow[t]{3}{*}{ Combination } & $0 \%$ & Used gestational carrier & $1 \%$ & Endometriosis & $2 \%$ & Female factors only & $6 \%$ \\
\hline & & & & Uterine factor & $0 \%$ & Female \& male factors & $10 \%$ \\
\hline & & & & Male factor & $15 \%$ & & \\
\hline
\end{tabular}

2006 PREGNANCY SUCCESS RATES

Data verified by R. Scott Springer, DO

\section{Type of Cycle}

\section{Age of Woman}

35-37 38-40

$41-42^{d}$ $<35$

22

22.7

12

3

Number of cycles

57

18.2

$2 / 12$

$1 / 3$

Percentage of cycles resulting in live births ${ }^{b, c}$

(Confidence Interval)

Percentage of retrievals resulting in live births ${ }^{b, c}$

Percentage of transfers resulting in live births ${ }^{b, c}$

Percentage of transfers resulting in singleton live births ${ }^{b}$

Percentage of cancellations ${ }^{b}$

33.3

29.8

(18.4-43.4)

$(5.2-40.3)$
20.0

32.1

33.3

$1 / 12$

$0 / 3$

17.6

4 / 18

$1 / 10$

$0 / 3$

7.0

$3 / 18$

$1 / 10$

$0 / 3$

7.0

9.1

$1 / 10$

$0 / 3$

2.1

$8 / 19$

2.6

$2 / 12$

$0 / 3$

Average number of embryos transferred

Percentage of pregnancies with twins ${ }^{b}$

$0 / 19$

$1 / 5$

3.0

2.7

Percentage of pregnancies with triplets or more ${ }^{b}$

Percentage of live births having multiple infants ${ }^{b, c}$

$8 / 17$

$0 / 5$

$1 / 2$

$0 / 1$

$0 / 2$

$0 / 1$

\section{Frozen Embryos from Nondonor Eggs}

Number of transfers

Percentage of transfers resulting in live births ${ }^{b, c}$

Average number of embryos transferred

\section{Donor Eggs}

$\begin{array}{cccc}14 & 1 & 5 & 1 \\ 3 / 14 & 0 / 1 & 0 / 5 & 0 / 1 \\ 2.1 & 3.0 & 2.8 & 2.0\end{array}$

Number of transfers

Percentage of transfers resulting in live births ${ }^{\text {b,c }}$

All Ages Combined

Fresh Embryos

Frozen Embryos

$\begin{array}{cc}1 & 8 \\ 0 / 1 & 1 / 8 \\ 2.0 & 2.5\end{array}$

Average number of embryos transferred

\section{CURRENT CLINIC SERVICES AND PROFILE}

Current Name: Center for Reproductive Health/Joliet IVF

Donor egg? Yes Gestational carriers? Yes

Donor embryo? No Cryopreservation? Yes

Single women? Yes

SART member?

No

Verified lab accreditation?

Yes

(See Appendix C for details.)

${ }^{a}$ Reflects patient and treatment characteristics of ART cycles performed in 2006 using fresh nondonor eggs or embryos.

${ }^{b}$ When fewer than 20 cycles are reported in an age category, rates are shown as a fraction and confidence intervals are not given. Calculating percentages from fractions may be misleading and is not encouraged.

${ }^{c}$ A multiple-infant birth is counted as one live birth.

${ }^{d}$ Clinic-specific outcome rates are unreliable for women older than 42 undergoing ART cycles using fresh or frozen embryos with nondonor eggs. Readers are urged to review national outcomes for these age groups (see page 27).

e All ages (including ages $>42$ ) are reported together because previous data show that patient age does not materially affect success with donor eggs. 


\section{MIDWEST FERTILITY CENTER DOWNERS GROVE, ILLINOIS}

A comparison of clinic success rates may not be meaningful because patient medical characteristics and treatment approaches vary from clinic to clinic. For more details about this, along with information on how to interpret the statistics in this table, see pages 79-88.

\section{ART CYCLE PROFILE}

\begin{tabular}{|c|c|c|c|c|c|c|c|}
\hline & Type & of $A R T^{a}$ & & Pati & ent D & iagnosis & \\
\hline IVF & $>99 \%$ & Procedural Factors: & & Tubal factor & $14 \%$ & Other factor & $3 \%$ \\
\hline GIFT & $0 \%$ & With ICSI & $29 \%$ & Ovulatory dysfunction & $10 \%$ & Unknown factor & $3 \%$ \\
\hline ZIFT & $<1 \%$ & Unstimulated & $0 \%$ & Diminished ovarian reserve & $7 \%$ & Multiple Factors: & \\
\hline Combination & $0 \%$ & Used gestational carrier & $<1 \%$ & Endometriosis & $10 \%$ & Female factors only & $26 \%$ \\
\hline & & & & Uterine factor & $2 \%$ & Female \& male factors & $17 \%$ \\
\hline & & & & Male factor & $8 \%$ & & \\
\hline
\end{tabular}

2006 PREGNANCY SUCCESS RATES

\section{Type of Cycle}

\section{Fresh Embryos from Nondonor Eggs}

Number of cycles

Percentage of cycles resulting in pregnancies ${ }^{b}$

Percentage of cycles resulting in live births ${ }^{b, c}$

(Confidence Interval)

Percentage of retrievals resulting in live births $s^{b, c}$

Percentage of transfers resulting in live births $s^{b, c}$

Percentage of transfers resulting in singleton live births ${ }^{b}$

Percentage of cancellations ${ }^{b}$

Average number of embryos transferred

Percentage of pregnancies with twins ${ }^{\mathrm{b}}$

Percentage of pregnancies with triplets or more ${ }^{b}$

Percentage of live births having multiple infants ${ }^{b, c}$

\section{Frozen Embryos from Nondonor Eggs}

Number of transfers

Percentage of transfers resulting in live births $s^{\mathrm{b}, \mathrm{c}}$

Average number of embryos transferred

\section{Donor Eggs}

Number of transfers

Percentage of transfers resulting in live births $s^{b, c}$

Average number of embryos transferred

Data verified by Amos E. Madanes, MD

\section{Age of Woman}

35-37 38-40

$41-42^{d}$

$<35$

50

34

16

110

26.0

17.6

$2 / 16$

26.4

14.0

11.8

$1 / 16$

(18.4-35.6)

$(5.8-26.7)$

(3.3-27.5)

17.9

15.4

$1 / 14$

31.5

17.9

18.2

$1 / 13$

17.9

18.2

$0 / 13$

15.5

22.0

23.5

$2 / 16$

3.3

3.2

3.4

4.5

25.6

$1 / 13$

$2 / 6$

$0 / 2$

15.4

$0 / 13$

$0 / 6$

$1 / 2$

37.9

$0 / 7$

$0 / 4$

$1 / 1$

7

$1 / 7$

2.3
2

$0 / 2$

2.0
2

$1 / 2$

3.0

2.3

\section{All Ages Combined ${ }^{\mathrm{e}}$}

Fresh Embryos

$\begin{array}{cc}1 & 2 \\ 0 / 1 & 0 / 2 \\ 4.0 & 3.0\end{array}$

\section{CURRENT CLINIC SERVICES AND PROFILE}

Current Name: Midwest Fertility Center

\begin{tabular}{|c|c|c|c|c|}
\hline Donor egg? & Yes & Gestational carriers? & Yes & SART member? \\
\hline Donor embryo? & No & Cryopreservation? & Yes & Verified lab accreditation? \\
\hline Single women? & Yes & & & (See Appendix C for details.) \\
\hline
\end{tabular}

\footnotetext{
${ }^{a}$ Reflects patient and treatment characteristics of ART cycles performed in 2006 using fresh nondonor eggs or embryos.

${ }^{b}$ When fewer than 20 cycles are reported in an age category, rates are shown as a fraction and confidence intervals are not given. Calculating percentages from fractions may be misleading and is not encouraged.

${ }^{c}$ A multiple-infant birth is counted as one live birth.

d Clinic-specific outcome rates are unreliable for women older than 42 undergoing ART cycles using fresh or frozen embryos with nondonor eggs. Readers are urged to review national outcomes for these age groups (see page 27).

${ }^{e}$ All ages (including ages $>42$ ) are reported together because previous data show that patient age does not materially affect success with donor eggs.
} 


\section{THE RINEHART CENTER FOR REPRODUCTIVE MEDICINE EVANSTON, ILLINOIS}

A comparison of clinic success rates may not be meaningful because patient medical characteristics and treatment approaches vary from clinic to clinic. For more details about this, along with information on how to interpret the statistics in this table, see pages $79-88$.

\section{ART CYCLE PROFILE}

\begin{tabular}{|c|c|c|c|c|c|c|c|}
\hline \multicolumn{4}{|c|}{ Type of ART } & \multicolumn{4}{|c|}{ Patient Diagnosis } \\
\hline IVF & $100 \%$ & Procedural Factors: & & Tubal factor & $7 \%$ & Other factor & $7 \%$ \\
\hline GIFT & $0 \%$ & With ICSI & $89 \%$ & Ovulatory dysfunction & $14 \%$ & Unknown factor & $26 \%$ \\
\hline ZIFT & $0 \%$ & Unstimulated & $0 \%$ & Diminished ovarian reserve & $14 \%$ & Multiple Factors: & \\
\hline \multirow[t]{3}{*}{ Combination } & $0 \%$ & Used gestational carrier & $<1 \%$ & Endometriosis & $<1 \%$ & Female factors only & $5 \%$ \\
\hline & & & & Uterine factor & $<1 \%$ & Female \& male factors & $11 \%$ \\
\hline & & & & Male factor & $15 \%$ & & \\
\hline
\end{tabular}

2006 PREGNANCY SUCCESS RATES

$$
\text { Type of Cycle }
$$

\section{Fresh Embryos from Nondonor Eggs}

Number of cycles

Percentage of cycles resulting in pregnancies ${ }^{b}$

Percentage of cycles resulting in live births ${ }^{b, c}$

(Confidence Interval)

Percentage of retrievals resulting in live births $s^{b, c}$

Percentage of transfers resulting in live births $s^{b, c}$

Percentage of transfers resulting in singleton live births ${ }^{b}$

Percentage of cancellations ${ }^{b}$

Average number of embryos transferred

Percentage of pregnancies with twins ${ }^{b}$

Percentage of pregnancies with triplets or more ${ }^{b}$

Percentage of live births having multiple infants ${ }^{b, c}$

\section{Frozen Embryos from Nondonor Eggs}

Number of transfers

Percentage of transfers resulting in live births ${ }^{\mathrm{b}, \mathrm{c}}$

Average number of embryos transferred

\section{Donor Eggs}

Number of transfers

Percentage of transfers resulting in live births $s^{b, c}$

Average number of embryos transferred

Data verified by John S. Rinehart, MD, PhD

\section{Age of Woman}

$<35 \quad 35-37 \quad 38-40 \quad 41-42^{d}$

$\begin{array}{cccc}57 & 46 & 29 & 9 \\ 26.3 & 21.7 & 24.1 & 1 / 9 \\ 22.8 & 21.7 & 17.2 & 0 / 9\end{array}$

(12.7-35.8)

(10.9-36.4)

$(5.8-35.8)$

$19.2 \quad 0 / 8$

31.7

23.8

$5 / 16$

$0 / 5$

24.4

29.4

$4 / 16$

$0 / 5$

5.3

8.7

10.3

$1 / 9$

2.8

2.5

2.8

2.8

$4 / 15$

$3 / 10$

$0 / 7$

$0 / 1$

$1 / 15$

$0 / 10$

$1 / 7$

$0 / 1$

$3 / 13$

$3 / 10$

$1 / 5$

$\begin{array}{cccc}14 & 3 & 2 & 2 \\ 6 / 14 & 1 / 3 & 1 / 2 & 0 / 2 \\ 2.9 & 2.3 & 3.0 & 2.0\end{array}$

All Ages Combined

Fresh Embryos

24

37.5

2.8
Frozen Embryos 10

$1 / 10$

2.1

\section{CURRENT CLINIC SERVICES AND PROFILE}

Current Name: The Rinehart Center for Reproductive Medicine

Donor egg? Yes $\quad$ Gestational carriers? Yes

Donor embryo? Yes Cryopreservation? Yes

Single women? Yes

SART member? Yes

Verified lab accreditation? Yes

(See Appendix C for details.)

${ }^{a}$ Reflects patient and treatment characteristics of ART cycles performed in 2006 using fresh nondonor eggs or embryos.

${ }^{b}$ When fewer than 20 cycles are reported in an age category, rates are shown as a fraction and confidence intervals are not given. Calculating percentages from fractions may be misleading and is not encouraged.

${ }^{c}$ A multiple-infant birth is counted as one live birth.

d Clinic-specific outcome rates are unreliable for women older than 42 undergoing ART cycles using fresh or frozen embryos with nondonor eggs. Readers are urged to review national outcomes for these age groups (see page 27).

${ }^{e}$ All ages (including ages $>42$ ) are reported together because previous data show that patient age does not materially affect success with donor eggs. 


\section{ADVANCED FERTILITY CENTER OF CHICAGO GURNEE, ILLINOIS}

A comparison of clinic success rates may not be meaningful because patient medical characteristics and treatment approaches vary from clinic to clinic. For more details about this, along with information on how to interpret the statistics in this table, see pages 79-88.

\section{ART CYCLE PROFILE}

\begin{tabular}{|c|c|c|c|c|c|c|c|}
\hline & Type & of $A R T^{a}$ & & Pat & ent [ & iagnosis & \\
\hline IVF & $100 \%$ & Procedural Factors: & & Tubal factor & $8 \%$ & Other factor & $0 \%$ \\
\hline GIFT & $0 \%$ & With ICSI & $92 \%$ & Ovulatory dysfunction & $7 \%$ & Unknown factor & $7 \%$ \\
\hline ZIFT & $0 \%$ & Unstimulated & $0 \%$ & Diminished ovarian reserve & $22 \%$ & Multiple Factors: & \\
\hline Combination & $0 \%$ & Used gestational carrier & $0 \%$ & Endometriosis & $3 \%$ & Female factors only & $21 \%$ \\
\hline & & & & Uterine factor & $<1 \%$ & Female \& male factors & $17 \%$ \\
\hline & & & & Male factor & $15 \%$ & & \\
\hline
\end{tabular}

\section{PREGNANCY SUCCESS RATES}

\section{Type of Cycle}

\section{Fresh Embryos from Nondonor Eggs}

Number of cycles

Percentage of cycles resulting in pregnancies ${ }^{b}$

Percentage of cycles resulting in live births $s^{b, c}$

(Confidence Interval)

Percentage of retrievals resulting in live births $s^{b, c}$

Percentage of transfers resulting in live births $s^{b, c}$

Percentage of transfers resulting in singleton live births ${ }^{b}$

Percentage of cancellations ${ }^{b}$

Average number of embryos transferred

Percentage of pregnancies with twins ${ }^{b}$

Percentage of pregnancies with triplets or more ${ }^{b}$

Percentage of live births having multiple infants ${ }^{b, c}$

\section{Frozen Embryos from Nondonor Eggs}

Number of transfers

Percentage of transfers resulting in live births ${ }^{b, c}$

Average number of embryos transferred

\section{Donor Eggs}

Number of transfers

Percentage of transfers resulting in live births $s^{b, c}$

Average number of embryos transferred
Data verified by Richard Sherbahn, MD

\section{Age of Woman}

35-37 38-40

$41-42^{d}$

$<35$

$\begin{array}{ccc}66 & 26 & 20 \\ 39.4 & 38.5 & 15.0 \\ 27.3 & 30.8 & 10.0\end{array}$

\section{(33.9-50.5) \\ 42.4}

(17.0-39.6)

(14.3-51.8)

$(1.2-31.7)$

44.5

28.6

30.5

36.4

36.4

36.4

$2 / 19$

18.6

15.4

$2 / 18$

0.7

4.5

2.5

$1 / 18$

2.1

2.2

26.9

39.2

3.8

$2 / 10$

5.0

4.1

39.3

$7 / 18$

$0 / 10$

2.7

$2 / 3$

$0 / 3$

$0 / 8$

$1 / 2$

$\begin{array}{cccc}15 & 13 & 4 & 1 \\ 7 / 15 & 2 / 13 & 1 / 4 & 0 / 1 \\ 2.1 & 1.9 & 2.3 & 3.0\end{array}$

\section{All Ages Combined}

Fresh Embryos

Frozen Embryos

61

45.9

16

2.0

$5 / 16$

2.4

\section{CURRENT CLINIC SERVICES AND PROFILE}

Current Name: Advanced Fertility Center of Chicago

Donor egg? Yes

Donor embryo? Yes

Gestational carriers?

Yes

Cryopreservation?

Yes

Single women? Yes

SART member?

Yes

Verified lab accreditation?

Yes

(See Appendix C for details.)

\footnotetext{
${ }^{a}$ Reflects patient and treatment characteristics of ART cycles performed in 2006 using fresh nondonor eggs or embryos.

${ }^{b}$ When fewer than 20 cycles are reported in an age category, rates are shown as a fraction and confidence intervals are not given. Calculating percentages from fractions may be misleading and is not encouraged.

${ }^{c}$ A multiple-infant birth is counted as one live birth.

${ }^{d}$ Clinic-specific outcome rates are unreliable for women older than 42 undergoing ART cycles using fresh or frozen embryos with nondonor eggs. Readers are urged to review national outcomes for these age groups (see page 27).

e All ages (including ages $>42$ ) are reported together because previous data show that patient age does not materially affect success with donor eggs.
} 


\section{CHICAGO INFERTILITY ASSOCIATES HANOVER PARK, ILLINOIS}

A comparison of clinic success rates may not be meaningful because patient medical characteristics and treatment approaches vary from clinic to clinic. For more details about this, along with information on how to interpret the statistics in this table, see pages $79-88$.

\section{ART CYCLE PROFILE}

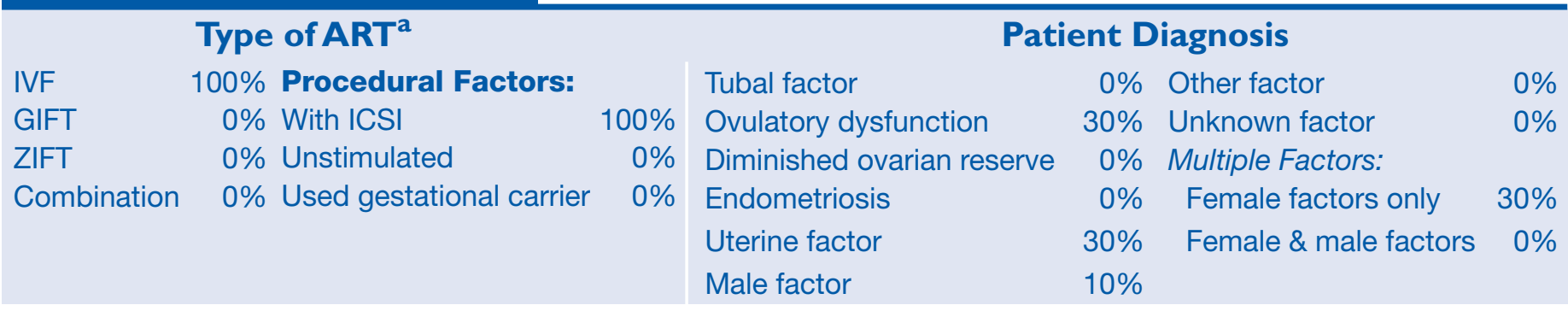

2006 PREGNANCY SUCCESS RATES

Data verified by Ketan N. Jobanputra, MD

\section{Type of Cycle}

\section{Fresh Embryos from Nondonor Eggs}

Number of cycles

Percentage of cycles resulting in pregnancies ${ }^{b}$

Percentage of cycles resulting in live births ${ }^{b, c}$

(Confidence Interval)

Percentage of retrievals resulting in live births $s^{b, c}$

Percentage of transfers resulting in live births $s^{b, c}$

Percentage of transfers resulting in singleton live births ${ }^{b}$

Percentage of cancellations ${ }^{b}$

Average number of embryos transferred

Percentage of pregnancies with twins ${ }^{b}$

Percentage of pregnancies with triplets or more ${ }^{b}$

Percentage of live births having multiple infants ${ }^{b, c}$

\section{Frozen Embryos from Nondonor Eggs}

Number of transfers

Percentage of transfers resulting in live births ${ }^{\mathrm{b}, \mathrm{c}}$

Average number of embryos transferred

\section{Donor Eggs}

Number of transfers

Percentage of transfers resulting in live births ${ }^{\mathrm{b}, \mathrm{c}}$

Average number of embryos transferred

\section{Age of Woman}

$\begin{array}{cccc}<35 & 35-37 & \mathbf{3 8 - 4 0} & \mathbf{4 1 - 4 2}^{\mathrm{d}} \\ 2 & 0 & 2 & 3 \\ 1 / 2 & & 0 / 2 & 0 / 3 \\ 1 / 2 & & 0 / 2 & 0 / 3\end{array}$

$1 / 2$

$0 / 2$

$0 / 3$

$1 / 1$

$0 / 2$

$0 / 3$

$1 / 1$

$0 / 2$

$0 / 3$

$0 / 2$

$0 / 2$

$0 / 3$

2.0

0.5

2.3

$0 / 1$

$0 / 1$

$0 / 1$
$0 \%$

$0 \%$

$0 \%$

$0 \%$

\section{CURRENT CLINIC SERVICES AND PROFILE}

Current Name: Chicago Infertility Associates

Donor egg? Yes

Donor embryo? Yes

Single women? Yes

\section{Gestational carriers? No}

Cryopreservation?
Yes

1
$0 / 1$
1.0

2

$0 / 2$

1.5

$1.0 \quad 1.5$

All Ages Combined

Fresh Embryos

0

Frozen Embryos

0

${ }_{b}^{a}$ Reflects patient and treatment characteristics of ART cycles performed in 2006 using fresh nondonor eggs or embryos.

${ }^{b}$ When fewer than 20 cycles are reported in an age category, rates are shown as a fraction and confidence intervals are not given. Calculating percentages from fractions may be misleading and is not encouraged.

c A multiple-infant birth is counted as one live birth.

d Clinic-specific outcome rates are unreliable for women older than 42 undergoing ART cycles using fresh or frozen embryos with nondonor eggs. Readers are urged to review national outcomes for these age groups (see page 27).

e All ages (including ages $>42$ ) are reported together because previous data show that patient age does not materially affect success with donor eggs. 


\section{HIGHLAND PARK IVF CENTER \\ HIGHLAND PARK, ILLINOIS}

A comparison of clinic success rates may not be meaningful because patient medical characteristics and treatment approaches vary from clinic to clinic. For more details about this, along with information on how to interpret the statistics in this table, see pages 79-88.

\section{ART CYCLE PROFILE}

\begin{tabular}{|c|c|c|c|c|c|c|c|}
\hline & Type & of $A R T^{a}$ & & Pati & ent $D$ & iagnosis & \\
\hline IVF & $100 \%$ & Procedural Factors: & & Tubal factor & $4 \%$ & Other factor & $15 \%$ \\
\hline GIFT & $0 \%$ & With ICSI & $85 \%$ & Ovulatory dysfunction & $7 \%$ & Unknown factor & $19 \%$ \\
\hline ZIFT & $0 \%$ & Unstimulated & $0 \%$ & Diminished ovarian reserve & $4 \%$ & Multiple Factors: & \\
\hline Combination & $0 \%$ & Used gestational carrier & $2 \%$ & Endometriosis & $2 \%$ & Female factors only & $30 \%$ \\
\hline & & & & Uterine factor & $<1 \%$ & Female \& male factors & $10 \%$ \\
\hline & & & & Male factor & $9 \%$ & & \\
\hline
\end{tabular}

2006 PREGNANCY SUCCESS RATES

Data verified by Edward L. Marut, MD

\section{Type of Cycle}

\section{Fresh Embryos from Nondonor Eggs}

Number of cycles

Percentage of cycles resulting in pregnancies ${ }^{b}$

Percentage of cycles resulting in live births $s^{b, c}$

(Confidence Interval)

Percentage of retrievals resulting in live births $s^{b, c}$

Percentage of transfers resulting in live births $s^{b, c}$

Percentage of transfers resulting in singleton live births ${ }^{b}$

Percentage of cancellations ${ }^{b}$

Average number of embryos transferred

Percentage of pregnancies with twins ${ }^{b}$

Percentage of pregnancies with triplets or more ${ }^{b}$

Percentage of live births having multiple infants ${ }^{b, c}$

\section{Frozen Embryos from Nondonor Eggs}

Number of transfers

Percentage of transfers resulting in live births ${ }^{b, c}$

Average number of embryos transferred

\section{Donor Eggs}

Number of transfers

Percentage of transfers resulting in live births $s^{b, c}$

Average number of embryos transferred

\section{Age of Woman}

35-37 $38-40 \quad 41-42^{d}$

\section{$<35$}

346

335

157

39.8

32.4

26.0

23.9

14.0

34.0

(21.5-31.0)

17.6

10.2

(29.3-39.0)

36.2

30.2

$(13.7-22.1)$

20.9

23.9

(5.9-16.0)

38.7

32.6

18.6

12.5

15.8

25.4

13.9

15.8

12.9

6.0

2.7

3.3

18.5

2.6

20.5

20.0

3.5

2.0

2.7

6.3

13.6

33.8

22.2

22.0

13.6

$3 / 16$

\section{CURRENT CLINIC SERVICES AND PROFILE}

Current Name: Highland Park IVF Center

\begin{tabular}{ll|lll|l} 
Donor egg? & Yes & Gestational carriers? & Yes & SART member? & Yes \\
\hline Donor embryo? & Yes & Cryopreservation? & Yes & Verified lab accreditation? & Pending \\
Single women? & Yes & & & (See Appendix C for details.)
\end{tabular}

${ }_{b}^{a}$ Reflects patient and treatment characteristics of ART cycles performed in 2006 using fresh nondonor eggs or embryos.

${ }^{b}$ When fewer than 20 cycles are reported in an age category, rates are shown as a fraction and confidence intervals are not given. Calculating percentages from fractions may be misleading and is not encouraged.

${ }^{c}$ A multiple-infant birth is counted as one live birth.

d Clinic-specific outcome rates are unreliable for women older than 42 undergoing ART cycles using fresh or frozen embryos with nondonor eggs. Readers are urged to review national outcomes for these age groups (see page 27).

${ }^{e}$ All ages (including ages $>42$ ) are reported together because previous data show that patient age does not materially affect success with donor eggs. 


\section{HINSDALE CENTER FOR REPRODUCTION HINSDALE, ILLINOIS}

A comparison of clinic success rates may not be meaningful because patient medical characteristics and treatment approaches vary from clinic to clinic. For more details about this, along with information on how to interpret the statistics in this table, see pages $79-88$.

\section{ART CYCLE PROFILE}

\begin{tabular}{|c|c|c|c|c|c|c|c|}
\hline \multicolumn{4}{|c|}{ Type of $\mathrm{ART}^{\mathrm{a}}$} & \multicolumn{4}{|c|}{ Patient Diagnosis } \\
\hline IVF & $100 \%$ & Procedural Factors: & & Tubal factor & $9 \%$ & Other factor & $21 \%$ \\
\hline GIFT & $0 \%$ & With ICSI & $74 \%$ & Ovulatory dysfunction & $4 \%$ & Unknown factor & $4 \%$ \\
\hline ZIFT & $0 \%$ & Unstimulated & $0 \%$ & Diminished ovarian reserve & $11 \%$ & Multiple Factors: & \\
\hline \multirow[t]{3}{*}{ Combination } & $0 \%$ & Used gestational carrier & $1 \%$ & Endometriosis & $3 \%$ & Female factors only & $11 \%$ \\
\hline & & & & Uterine factor & $<1 \%$ & Female \& male factors & $13 \%$ \\
\hline & & & & Male factor & $23 \%$ & & \\
\hline
\end{tabular}

2006 PREGNANCY SUCCESS RATES

Data verified by Michael J. Hickey, MD

\section{Type of Cycle}

\section{Fresh Embryos from Nondonor Eggs}

Number of cycles

Percentage of cycles resulting in pregnancies ${ }^{b}$

Percentage of cycles resulting in live births $s^{b, c}$

(Confidence Interval)

Percentage of retrievals resulting in live births $s^{b, c}$

Percentage of transfers resulting in live births $s^{b, c}$

Percentage of transfers resulting in singleton live births ${ }^{b}$

Percentage of cancellations ${ }^{b}$

Average number of embryos transferred

Percentage of pregnancies with twins ${ }^{b}$

Percentage of pregnancies with triplets or more ${ }^{b}$

Percentage of live births having multiple infants ${ }^{b, c}$

\section{Frozen Embryos from Nondonor Eggs}

Number of transfers

Percentage of transfers resulting in live births $s^{\mathrm{b}, \mathrm{c}}$

Average number of embryos transferred

\section{Donor Eggs}

Number of transfers

Percentage of transfers resulting in live births $s^{b, c}$

Average number of embryos transferred

\section{Age of Woman}

35-37 38-40

$41-42^{d}$

\section{$<35$}

20

55.0

15

4

36.0

40.0

$6 / 15$

$3 / 15$

$1 / 4$

36.0

(18.0-57.5)

(19.1-63.9)

37.5

$8 / 19$

$3 / 13$

$1 / 4$

40.9

$8 / 19$

$3 / 12$

$1 / 4$

18.2

$3 / 19$

$2 / 12$

$1 / 4$

4.0

5.0

$2 / 15$

$1 / 4$

2.4

2.7

2.4

$0 / 4$

$5 / 9$

$6 / 11$

$1 / 11$

$2 / 6$

2.0

$0 / 9$

$5 / 8$

$0 / 6$

$0 / 1$

$5 / 9$

$1 / 3$

$0 / 1$

$0 / 1$

20

35.0

4

$0 / 4$

4

$1 / 4$

2

2.3

1.8

2.8

$1 / 2$

1.5

All Ages Combined ${ }^{e}$

Fresh Embryos

5

$2 / 5$

2.6
Frozen Embryos 9

$3 / 9$

2.7

\section{CURRENT CLINIC SERVICES AND PROFILE}

Current Name: Hinsdale Center for Reproduction

Donor egg? Yes

Donor embryo? Yes

Single women? No

\section{Gestational carriers? Yes}

Cryopreservation? Yes

\section{SART member?}

Verified lab accreditation?

(See Appendix C for details.)

${ }^{a}$ Reflects patient and treatment characteristics of ART cycles performed in 2006 using fresh nondonor eggs or embryos.

${ }^{b}$ When fewer than 20 cycles are reported in an age category, rates are shown as a fraction and confidence intervals are not given. Calculating percentages from fractions may be misleading and is not encouraged.

${ }^{c}$ A multiple-infant birth is counted as one live birth.

d Clinic-specific outcome rates are unreliable for women older than 42 undergoing ART cycles using fresh or frozen embryos with nondonor eggs. Readers are urged to review national outcomes for these age groups (see page 27).

e All ages (including ages $>42$ ) are reported together because previous data show that patient age does not materially affect success with donor eggs. 


\section{REENA JABAMONI, MD, SC HOFFMAN ESTATES, ILLINOIS}

A comparison of clinic success rates may not be meaningful because patient medical characteristics and treatment approaches vary from clinic to clinic. For more details about this, along with information on how to interpret the statistics in this table, see pages 79-88.

\section{ART CYCLE PROFILE}

\section{Type of ART ${ }^{\mathrm{a}}$}

IVF

GIFT

ZIFT

Combination
100\% Procedural Factors:

$0 \%$ With ICSI

$0 \%$ Unstimulated

$0 \%$ Used gestational carrier

\begin{tabular}{r|l|} 
& Tubal factor \\
\hline $80 \%$ & Ovulatory dysfunction \\
$0 \%$ & Diminished ovarian reserve \\
$0 \%$ & Endometriosis \\
& Uterine factor \\
& Male factor
\end{tabular}

\section{Patient Diagnosis}

$\begin{array}{rlr}11 \% & \text { Other factor } & 0 \% \\ 52 \% & \text { Unknown factor } & 3 \% \\ 14 \% & \text { Multiple Factors: } & \\ 0 \% & \text { Female factors only } & 1 \% \\ 0 \% & \text { Female \& male factors } & 10 \% \\ 9 \% & & \end{array}$

2006 PREGNANCY SUCCESS RATES

Data verified by Reena Jabamoni, MD

\section{Type of Cycle}

\section{Age of Woman}

$<35 \quad 35-37 \quad 38-40 \quad 41-42^{\text {d }}$

\section{Fresh Embryos from Nondonor Eggs}

Number of cycles

11

9

10

Percentage of cycles resulting in pregnancies ${ }^{b}$

57.1

$6 / 11$

$1 / 9$

$2 / 10$

Percentage of cycles resulting in live births $s^{b, c}$

42.9

$4 / 11$

$1 / 9$

$2 / 10$

(Confidence Interval)

Percentage of retrievals resulting in live births $s^{b, c}$

(21.8-66.0)

Percentage of transfers resulting in live births $s^{b, c}$

45.0

$4 / 10$

$1 / 7$

$2 / 7$

$9 / 19$

$4 / 8$

$1 / 5$

$2 / 5$

Percentage of transfers resulting in singleton live births ${ }^{b}$

Percentage of cancellations ${ }^{b}$

$9 / 19$

$3 / 8$

$1 / 5$

$2 / 5$

4.8

$1 / 11$

$2 / 9$

$3 / 10$

1.9

2.0

2.0

1.8

Percentage of pregnancies with twins ${ }^{b}$

$1 / 12$

$2 / 6$

$0 / 1$

$0 / 2$

$1 / 12$

$0 / 6$

$0 / 1$

$0 / 2$

Percentage of pregnancies with triplets or more
Percentage of live births having multiple infants

$0 / 9$

$1 / 4$

$0 / 1$

$0 / 2$

\section{Frozen Embryos from Nondonor Eggs}

Number of transfers

Percentage of transfers resulting in live births ${ }^{b, c}$

Average number of embryos transferred

$\begin{array}{cccc}7 & 2 & 2 & 2 \\ 3 / 7 & 1 / 2 & 1 / 2 & 0 / 2 \\ 2.0 & 1.5 & 2.0 & 1.5\end{array}$

\section{Donor Eggs}

All Ages Combined ${ }^{\mathrm{e}}$

Number of transfers

Percentage of transfers resulting in live births $s^{b, c}$

Average number of embryos transferred

\section{Fresh Embryos}

\section{6}

$2 / 6$

2.0
Frozen Embryos

\section{1}

$0 / 1$

2.0

\section{CURRENT CLINIC SERVICES AND PROFILE}

Current Name: Reena Jabamoni, MD, SC

Donor egg? Yes

Donor embryo? Yes

Gestational carriers? Yes

Cryopreservation? Yes

Single women? Yes

SART member?

Yes

Verified lab accreditation?

Yes

(See Appendix C for details.)

${ }^{a}$ Reflects patient and treatment characteristics of ART cycles performed in 2006 using fresh nondonor eggs or embryos.

b When fewer than 20 cycles are reported in an age category, rates are shown as a fraction and confidence intervals are not given. Calculating percentages from fractions may be misleading and is not encouraged.

${ }^{c}$ A multiple-infant birth is counted as one live birth.

d Clinic-specific outcome rates are unreliable for women older than 42 undergoing ART cycles using fresh or frozen embryos with nondonor eggs. Readers are urged to review national outcomes for these age groups (see page 27).

e All ages (including ages $>42$ ) are reported together because previous data show that patient age does not materially affect success with donor eggs. 


\section{KARANDE AND ASSOCIATES, SC HOFFMAN ESTATES, ILLINOIS}

A comparison of clinic success rates may not be meaningful because patient medical characteristics and treatment approaches vary from clinic to clinic. For more details about this, along with information on how to interpret the statistics in this table, see pages $79-88$.

\section{ART CYCLE PROFILE}

\begin{tabular}{|c|c|c|c|c|c|c|c|}
\hline & Type & of $\mathrm{ART}^{\mathrm{a}}$ & & Pat & ent $D$ & iagnosis & \\
\hline IVF & $>99 \%$ & Procedural Factors: & & Tubal factor & $8 \%$ & Other factor & $8 \%$ \\
\hline GIFT & $<1 \%$ & With ICSI & $82 \%$ & Ovulatory dysfunction & $18 \%$ & Unknown factor & $20 \%$ \\
\hline ZIFT & $0 \%$ & Unstimulated & $0 \%$ & Diminished ovarian reserve & $18 \%$ & Multiple Factors: & \\
\hline Combination & $0 \%$ & Used gestational carrier & $1 \%$ & Endometriosis & $4 \%$ & Female factors only & $4 \%$ \\
\hline & & & & Uterine factor & $<1 \%$ & Female \& male factors & $5 \%$ \\
\hline & & & & Male factor & $16 \%$ & & \\
\hline
\end{tabular}

2006 PREGNANCY SUCCESS RATES

Data verified by Vishvanath C. Karande, MD

\section{Type of Cycle}

\section{Fresh Embryos from Nondonor Eggs}

Number of cycles

Percentage of cycles resulting in pregnancies ${ }^{b}$

Percentage of cycles resulting in live births $s^{b, c}$

(Confidence Interval)

Percentage of retrievals resulting in live births $s^{b, c}$

Percentage of transfers resulting in live births $s^{b, c}$

Percentage of transfers resulting in singleton live births ${ }^{b}$

Percentage of cancellations ${ }^{b}$

Average number of embryos transferred

Percentage of pregnancies with twins ${ }^{b}$

Percentage of pregnancies with triplets or more ${ }^{b}$

Percentage of live births having multiple infants ${ }^{b, c}$

\section{Frozen Embryos from Nondonor Eggs}

Number of transfers

Percentage of transfers resulting in live births ${ }^{\mathrm{b}, \mathrm{c}}$

Average number of embryos transferred

\section{Donor Eggs}

Number of transfers

Percentage of transfers resulting in live births ${ }^{\mathrm{b}, \mathrm{c}}$

Average number of embryos transferred

\section{Age of Woman}

35-37 38-40

$41-42^{d}$

\section{$<35$}

60

28.3

20.0

36.3

(10.8-32.3)

(29.1-43.9)

38.3

23.1

40.8

24.5

20.4

13.3

2.3

$3 / 17$

26.8

$1 / 17$

2.8

$2 / 12$

27

37.0

1.8

1

$1 / 1$

2.0

38

17

\section{7}

$2 / 17$

10.5

$1 / 17$

(2.9-24.8)

$12.5 \quad 1 / 13$

13.8

$1 / 10$

$1 / 10$

10.3

$4 / 17$

15.8

3.5

2.6

$0 / 2$

$1 / 9$

$0 / 2$

$1 / 9$

$0 / 1$

$1 / 4$

0

$3 / 6$

2.5

All Ages Combined ${ }^{\mathrm{e}}$

Fresh Embryos

23

69.6

2.0
Frozen Embryos

4

$2 / 4$

2.3

\section{CURRENT CLINIC SERVICES AND PROFILE}

Current Name: Karande and Associates, SC

Donor egg? Yes

Donor embryo? Yes

Single women? Yes

\section{Gestational carriers? Yes}

Cryopreservation? Yes
SART member?

Verified lab accreditation?

(See Appendix C for details.)

${ }^{a}$ Reflects patient and treatment characteristics of ART cycles performed in 2006 using fresh nondonor eggs or embryos.

${ }^{b}$ When fewer than 20 cycles are reported in an age category, rates are shown as a fraction and confidence intervals are not given. Calculating percentages from fractions may be misleading and is not encouraged.

${ }^{c}$ A multiple-infant birth is counted as one live birth.

d Clinic-specific outcome rates are unreliable for women older than 42 undergoing ART cycles using fresh or frozen embryos with nondonor eggs. Readers are urged to review national outcomes for these age groups (see page 27).

${ }^{e}$ All ages (including ages $>42$ ) are reported together because previous data show that patient age does not materially affect success with donor eggs. 


\section{REPRODUCTIVE HEALTH SPECIALISTS, LTD. JOLIET, ILLINOIS}

A comparison of clinic success rates may not be meaningful because patient medical characteristics and treatment approaches vary from clinic to clinic. For more details about this, along with information on how to interpret the statistics in this table, see pages 79-88.

\section{ART CYCLE PROFILE}

\begin{tabular}{|c|c|c|c|c|c|c|c|}
\hline \multicolumn{4}{|c|}{ Type of ART ${ }^{a}$} & \multicolumn{4}{|c|}{ Patient Diagnosis } \\
\hline IVF & $100 \%$ & Procedural Factors: & & Tubal factor & $2 \%$ & Other factor & $0 \%$ \\
\hline GIFT & $0 \%$ & With ICSI & $98 \%$ & Ovulatory dysfunction & $4 \%$ & Unknown factor & $22 \%$ \\
\hline ZIFT & $0 \%$ & Unstimulated & $0 \%$ & Diminished ovarian reserve & $14 \%$ & Multiple Factors: & \\
\hline \multirow[t]{3}{*}{ Combination } & $0 \%$ & Used gestational carrier & $0 \%$ & Endometriosis & $2 \%$ & Female factors only & $6 \%$ \\
\hline & & & & Uterine factor & $22 \%$ & Female \& male factors & $18 \%$ \\
\hline & & & & Male factor & $12 \%$ & & \\
\hline
\end{tabular}

2006 PREGNANCY SUCCESS RATES

Data verified by Marek W. Piekos, MD

\section{Type of Cycle}

\section{Fresh Embryos from Nondonor Eggs}

Number of cycles

Percentage of cycles resulting in pregnancies ${ }^{b}$

Percentage of cycles resulting in live births ${ }^{b, c}$

(Confidence Interval)

Percentage of retrievals resulting in live births $s^{b, c}$

Percentage of transfers resulting in live births ${ }^{b, c}$

Percentage of transfers resulting in singleton live births ${ }^{b}$

Percentage of cancellations ${ }^{b}$

Average number of embryos transferred

Percentage of pregnancies with twins ${ }^{b}$

Percentage of pregnancies with triplets or more ${ }^{b}$

Percentage of live births having multiple infants ${ }^{b, c}$

\section{Frozen Embryos from Nondonor Eggs}

Number of transfers

Percentage of transfers resulting in live births ${ }^{b, c}$

Average number of embryos transferred

\section{$<35$}

21

\section{1}

47.6

(25.7-70.2)

47.6

47.6

38.1

0.0

3.4

$2 / 12$

$1 / 12$

$2 / 10$
Age of Woman

35-37 38-40

38-40

$41-42^{d}$

13

$8 / 13$

7

4

$5 / 13$

$2 / 7$

$0 / 4$

$2 / 7$

$0 / 4$

$5 / 13$

$2 / 7$

$0 / 4$

$5 / 13$

$2 / 6$

$0 / 4$

$4 / 13$

$1 / 6$

$0 / 4$

$0 / 13$

$0 / 7$

$0 / 4$

3.2

5.7

$2 / 8$

$1 / 2$

$0 / 8$

$0 / 2$

$1 / 5$

$1 / 2$

1

$1 / 1$

3.0
1

$0 / 1$

3.0
1

$1 / 1$

4.0

\section{Donor Eggs}

Number of transfers

Percentage of transfers resulting in live births $s^{b, c}$

Average number of embryos transferred

\section{Fresh Embryos 2 \\ $1 / 2$ \\ 3.5}

\section{CURRENT CLINIC SERVICES AND PROFILE}

Current Name: Reproductive Health Specialists, Ltd.

Donor egg? Yes Gestational carriers? Yes

Donor embryo? Yes Cryopreservation? Yes

Single women? Yes

SART member? Yes

Verified lab accreditation? Yes

(See Appendix C for details.)

${ }^{a}$ Reflects patient and treatment characteristics of ART cycles performed in 2006 using fresh nondonor eggs or embryos.

${ }^{b}$ When fewer than 20 cycles are reported in an age category, rates are shown as a fraction and confidence intervals are not given. Calculating percentages from fractions may be misleading and is not encouraged.

${ }^{c}$ A multiple-infant birth is counted as one live birth.

d Clinic-specific outcome rates are unreliable for women older than 42 undergoing ART cycles using fresh or frozen embryos with nondonor eggs. Readers are urged to review national outcomes for these age groups (see page 27).

e All ages (including ages $>42$ ) are reported together because previous data show that patient age does not materially affect success with donor eggs. 


\section{IVF1 \\ NAPERVILLE, ILLINOIS}

A comparison of clinic success rates may not be meaningful because patient medical characteristics and treatment approaches vary from clinic to clinic. For more details about this, along with information on how to interpret the statistics in this table, see pages 79-88.

\section{ART CYCLE PROFILE}

\begin{tabular}{|c|c|c|c|c|c|c|}
\hline \multicolumn{3}{|c|}{ Type of ART } & \multicolumn{4}{|c|}{ Patient Diagnosis } \\
\hline IVF & $100 \%$ Procedural Factors: & & Tubal factor & $5 \%$ & Other factor & $7 \%$ \\
\hline GIFT & 0\% With ICSI & $86 \%$ & Ovulatory dysfunction & $4 \%$ & Unknown factor & $14 \%$ \\
\hline ZIFT & $0 \%$ Unstimulated & $0 \%$ & Diminished ovarian reserve & $16 \%$ & Multiple Factors: & \\
\hline \multirow[t]{3}{*}{ Combination } & $0 \%$ Used gestational carrier & $<1 \%$ & Endometriosis & $1 \%$ & Female factors only & $21 \%$ \\
\hline & & & Uterine factor & $1 \%$ & Female \& male factors & $16 \%$ \\
\hline & & & Male factor & $15 \%$ & & \\
\hline
\end{tabular}

2006 PREGNANCY SUCCESS RATES

Data verified by Randy S. Morris, MD

\section{Type of Cycle}

\section{Age of Woman}

\section{Fresh Embryos from Nondonor Eggs}

Number of cycles

Percentage of cycles resulting in pregnancies ${ }^{b}$

Percentage of cycles resulting in live births $s^{b, c}$

(Confidence Interval)

Percentage of retrievals resulting in live births $s^{b, c}$

Percentage of transfers resulting in live births $s^{b, c}$

Percentage of transfers resulting in singleton live births ${ }^{b}$

Percentage of cancellations ${ }^{b}$

35-37 $\quad 38-40$

$41-42^{d}$

$<35$

35-37

14

132

60

28

$1 / 14$

52.3

31.7

32.1

$1 / 14$

Average number of embryos transferred

Percentage of pregnancies with twins ${ }^{b}$

45.5

25.0

28.6

(36.8-54.3)

(14.7-37.9)

(13.2-48.7)

48.8

28.8

34.8

$1 / 12$

51.3

30.6

36.4

$1 / 5$

37.6

18.4

27.3

$1 / 5$

6.8

13.3

17.9

$2 / 14$

2.0

2.0

2.4

1.4

26.1

$6 / 19$

$2 / 9$

$0 / 1$

Percentage of pregnancies with triplets or more ${ }^{\mathrm{b}}$

Percentage of live births having multiple infants ${ }^{b, c}$

$1 / 19$

$0 / 9$

$0 / 1$

26.7

$6 / 15$

$2 / 8$

$0 / 1$

\section{Frozen Embryos from Nondonor Eggs}

Number of transfers

Percentage of transfers resulting in live births $s^{b, c}$

46

47.8

24

45.8

11

5

Average number of embryos transferred

1.8

1.8

$5 / 11$

$2 / 5$

1.6

2.2

\section{Donor Eggs}

All Ages Combined

Number of transfers

Fresh Embryos

$17 / 17$

2.1
Frozen Embryos

11

$5 / 11$

2.0

Average number of embryos transferred

\section{CURRENT CLINIC SERVICES AND PROFILE}

Current Name: IVF1

Donor egg? Yes

Donor embryo? Yes

Single women? Yes

\begin{abstract}
Gestational carriers? Yes
Cryopreservation? Yes
\end{abstract}

\section{SART member? Yes}

Verified lab accreditation? Yes

(See Appendix C for details.)

${ }^{a}$ Reflects patient and treatment characteristics of ART cycles performed in 2006 using fresh nondonor eggs or embryos.

${ }^{b}$ When fewer than 20 cycles are reported in an age category, rates are shown as a fraction and confidence intervals are not given. Calculating percentages from fractions may be misleading and is not encouraged.

${ }^{c}$ A multiple-infant birth is counted as one live birth.

d Clinic-specific outcome rates are unreliable for women older than 42 undergoing ART cycles using fresh or frozen embryos with nondonor eggs. Readers are urged to review national outcomes for these age groups (see page 27).

${ }^{e}$ All ages (including ages $>42$ ) are reported together because previous data show that patient age does not materially affect success with donor eggs. 


\section{CHARLES E. MILLER, MD \& ASSOCIATES \\ NAPERVILLE, ILLINOIS}

A comparison of clinic success rates may not be meaningful because patient medical characteristics and treatment approaches vary from clinic to clinic. For more details about this, along with information on how to interpret the statistics in this table, see pages 79-88.

\section{ART CYCLE PROFILE}

\begin{tabular}{|c|c|c|c|c|c|c|c|}
\hline \multicolumn{4}{|c|}{ Type of ART ${ }^{a}$} & \multicolumn{4}{|c|}{ Patient Diagnosis } \\
\hline IVF & $100 \%$ & Procedural Factors: & & Tubal factor & $5 \%$ & Other factor & $34 \%$ \\
\hline GIFT & $0 \%$ & With ICSI & $83 \%$ & Ovulatory dysfunction & $2 \%$ & Unknown factor & $0 \%$ \\
\hline ZIFT & $0 \%$ & Unstimulated & $<1 \%$ & Diminished ovarian reserve & $8 \%$ & Multiple Factors: & \\
\hline \multirow[t]{3}{*}{ Combination } & $0 \%$ & Used gestational carrier & $2 \%$ & Endometriosis & $5 \%$ & Female factors only & $12 \%$ \\
\hline & & & & Uterine factor & $4 \%$ & Female \& male factors & $15 \%$ \\
\hline & & & & Male factor & $16 \%$ & & \\
\hline
\end{tabular}

2006 PREGNANCY SUCCESS RATES

Data verified by Charles E. Miller, MD

\section{Type of Cycle}

\section{Fresh Embryos from Nondonor Eggs}

Number of cycles

Percentage of cycles resulting in pregnancies ${ }^{b}$

Percentage of cycles resulting in live births ${ }^{b, c}$

(Confidence Interval)

Percentage of retrievals resulting in live births $s^{b, c}$

Percentage of transfers resulting in live births $s^{b, c}$

Percentage of transfers resulting in singleton live births ${ }^{b}$

Percentage of cancellations ${ }^{b}$

Average number of embryos transferred

Percentage of pregnancies with twins ${ }^{b}$

Percentage of pregnancies with triplets or more ${ }^{b}$

Percentage of live births having multiple infants ${ }^{b, c}$

\section{Frozen Embryos from Nondonor Eggs}

Number of transfers

Percentage of transfers resulting in live births $s^{b, c}$

Average number of embryos transferred

\section{Donor Eggs}

Number of transfers

Percentage of transfers resulting in live births $s^{b, c}$

Average number of embryos transferred

\section{Age of Woman}

35-37 38-40

$41-42^{d}$

\section{$<35$}

98

42.9

31.6

46

34.8

26.1

46.3

(22.6-41.8)

(14.3-41.1)

37.3

35.3

41.4

27.6

26.1

3.2

$2 / 16$

$2 / 16$

4 / 12

17.2

6.9

47.4

28.6

15.3

21.4

9.5

35.5

30.4

26

42.3

2.3

14

4 / 14

33.3

2.4

All Ages Combined ${ }^{\mathrm{e}}$

Fresh Embryos

44

52.3

2.4

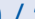

(0.8-22.8)

9.1

$2 / 18$

$1 / 18$

24.1

3.3

$1 / 5$

$0 / 5$

$1 / 2$

3

2.7

\section{CURRENT CLINIC SERVICES AND PROFILE}

Current Name: The Fertility Institute at Edward, Charles E. Miller, MD \& Associates

Donor egg? Yes

Donor embryo? Yes

Single women? Yes

\begin{abstract}
Gestational carriers? Yes
Cryopreservation? Yes
\end{abstract}

SART member?

Verified lab accreditation?

Yes

(See Appendix C for details.)

${ }^{a}$ Reflects patient and treatment characteristics of ART cycles performed in 2006 using fresh nondonor eggs or embryos.

b When fewer than 20 cycles are reported in an age category, rates are shown as a fraction and confidence intervals are not given. Calculating percentages from fractions may be misleading and is not encouraged.

${ }^{\mathrm{C}}$ A multiple-infant birth is counted as one live birth.

d Clinic-specific outcome rates are unreliable for women older than 42 undergoing ART cycles using fresh or frozen embryos with nondonor eggs. Readers are urged to review national outcomes for these age groups (see page 27).

e All ages (including ages $>42$ ) are reported together because previous data show that patient age does not materially affect success with donor eggs. 


\section{OAK BROOK FERTILITY CENTER OAK BROOK, ILLINOIS}

A comparison of clinic success rates may not be meaningful because patient medical characteristics and treatment approaches vary from clinic to clinic. For more details about this, along with information on how to interpret the statistics in this table, see pages $79-88$.

\section{ART CYCLE PROFILE}

\begin{tabular}{|c|c|c|c|c|c|c|c|}
\hline \multicolumn{4}{|c|}{ Type of ART ${ }^{\mathrm{a}}$} & \multicolumn{4}{|c|}{ Patient Diagnosis } \\
\hline IVF & $100 \%$ & Procedural Factors: & & Tubal factor & $10 \%$ & Other factor & $8 \%$ \\
\hline GIFT & $0 \%$ & With ICSI & $88 \%$ & Ovulatory dysfunction & $14 \%$ & Unknown factor & $6 \%$ \\
\hline $\mathrm{ZIFT}$ & $0 \%$ & Unstimulated & $0 \%$ & Diminished ovarian reserve & $10 \%$ & Multiple Factors: & \\
\hline \multirow[t]{3}{*}{ Combination } & $0 \%$ & Used gestational carrier & $<1 \%$ & Endometriosis & $12 \%$ & Female factors only & $12 \%$ \\
\hline & & & & Uterine factor & $0 \%$ & Female \& male factors & $19 \%$ \\
\hline & & & & Male factor & $10 \%$ & & \\
\hline
\end{tabular}

2006 PREGNANCY SUCCESS RATES

\section{Type of Cycle}

\section{Fresh Embryos from Nondonor Eggs}

Number of cycles

Percentage of cycles resulting in pregnancies ${ }^{b}$

Percentage of cycles resulting in live births $s^{b, c}$

(Confidence Interval)

Percentage of retrievals resulting in live births $s^{b, c}$

Percentage of transfers resulting in live births $s^{b, c}$

Percentage of transfers resulting in singleton live births ${ }^{b}$

Percentage of cancellations ${ }^{b}$

Average number of embryos transferred

Percentage of pregnancies with twins ${ }^{b}$

Percentage of pregnancies with triplets or more ${ }^{b}$

Percentage of live births having multiple infants ${ }^{b, c}$

\section{Frozen Embryos from Nondonor Eggs}

Number of transfers

Percentage of transfers resulting in live births $s^{b, c}$

Average number of embryos transferred

\section{Donor Eggs}

Number of transfers

Percentage of transfers resulting in live births $s^{b, c}$

Average number of embryos transferred

Data verified by W. Paul Dmowski, MD, PhD

\section{Age of Woman}

35-37 38-40

$41-42^{d}$

\section{$<35$}

21

42.9

14

3

45.2

42.9

$1 / 14$

$1 / 3$

40.3

(28.1-53.6)

$(21.8-66.0)$
45.0

$0 / 14$

$1 / 3$

40.3

$0 / 13$

$1 / 3$

53.2

$9 / 17$

$0 / 7$

$6 / 17$

$0 / 7$

$1 / 3$

31.9

4.8

$1 / 14$

$1 / 3$

0.0

2.1

2.4

$0 / 3$

2.0

39.3

$4 / 9$

$0 / 1$

2.3

3.6

$0 / 9$

$0 / 1$

$0 / 1$

40.0

$3 / 9$

$0 / 1$

$0 / 1$

$12 / 19$

\section{8}

$4 / 8$

2

$1 / 2$

2

2.2

2.4

2.0

$1 / 2$

3.0

All Ages Combined ${ }^{\mathrm{e}}$

Fresh Embryos

3

$1 / 3$

2.0
Frozen Embryos

\section{9}

$5 / 9$

2.3

\section{CURRENT CLINIC SERVICES AND PROFILE}

Current Name: Oak Brook Fertility Center
Donor egg?
Yes
Donor embryo? Yes
$\begin{array}{ll}\text { Gestational carriers? } & \text { Yes } \\ \text { Cryopreservation? } & \text { Yes }\end{array}$
SART member?
Yes
Single women? Yes
Verified lab accreditation?
Yes
(See Appendix C for details.)

\footnotetext{
${ }^{a}$ Reflects patient and treatment characteristics of ART cycles performed in 2006 using fresh nondonor eggs or embryos.

${ }^{b}$ When fewer than 20 cycles are reported in an age category, rates are shown as a fraction and confidence intervals are not given. Calculating percentages from fractions may be misleading and is not encouraged.

${ }^{c}$ A multiple-infant birth is counted as one live birth.

d Clinic-specific outcome rates are unreliable for women older than 42 undergoing ART cycles using fresh or frozen embryos with nondonor eggs. Readers are urged to review national outcomes for these age groups (see page 27).

${ }^{e}$ All ages (including ages $>42$ ) are reported together because previous data show that patient age does not materially affect success with donor eggs.
} 


\section{SHER INSTITUTE FOR REPRODUCTIVE MEDICINE-CENTRAL ILLINOIS PEORIA, ILLINOIS}

A comparison of clinic success rates may not be meaningful because patient medical characteristics and treatment approaches vary from clinic to clinic. For more details about this, along with information on how to interpret the statistics in this table, see pages 79-88.

\section{ART CYCLE PROFILE}

\begin{tabular}{|c|c|c|c|c|c|c|c|}
\hline \multicolumn{4}{|c|}{ Type of ART ${ }^{a}$} & \multicolumn{4}{|c|}{ Patient Diagnosis } \\
\hline IVF & $100 \%$ & Procedural Factors: & & Tubal factor & $8 \%$ & Other factor & $4 \%$ \\
\hline GIFT & $0 \%$ & With ICSI & $93 \%$ & Ovulatory dysfunction & $14 \%$ & Unknown factor & $6 \%$ \\
\hline ZIFT & $0 \%$ & Unstimulated & $0 \%$ & Diminished ovarian reserve & $<1 \%$ & Multiple Factors: & \\
\hline \multirow[t]{3}{*}{ Combination } & $0 \%$ & Used gestational carrier & $1 \%$ & Endometriosis & $11 \%$ & Female factors only & $25 \%$ \\
\hline & & & & Uterine factor & $<1 \%$ & Female \& male factors & $13 \%$ \\
\hline & & & & Male factor & $18 \%$ & & \\
\hline
\end{tabular}

2006 PREGNANCY SUCCESS RATES

Data verified by Peter Ahlering, MD

\section{Type of Cycle}

\section{Fresh Embryos from Nondonor Eggs}

Number of cycles

Percentage of cycles resulting in pregnancies ${ }^{b}$

Percentage of cycles resulting in live births $s^{b, c}$

(Confidence Interval)

Percentage of retrievals resulting in live births $s^{b, c}$

Percentage of transfers resulting in live births $s^{b, c}$

Percentage of transfers resulting in singleton live births ${ }^{b}$

Percentage of cancellations ${ }^{b}$

Average number of embryos transferred

Percentage of pregnancies with twins ${ }^{b}$

Percentage of pregnancies with triplets or more ${ }^{b}$

Percentage of live births having multiple infants ${ }^{\mathrm{b}, \mathrm{c}}$

\section{Frozen Embryos from Nondonor Eggs}

Number of transfers

Percentage of transfers resulting in live births ${ }^{b, c}$

Average number of embryos transferred

\section{Donor Eggs}

Number of transfers

Percentage of transfers resulting in live births $s^{b, c}$

Average number of embryos transferred

\section{Age of Woman}

35-37 38-40

$41-42^{d}$

$<35$

15

16

3

38.6

$3 / 15$

$3 / 16$

$1 / 3$

29.8

$2 / 15$

$2 / 16$

$0 / 3$

$(18.4-43.4)$

29.8

33.3

$2 / 15$

$2 / 15$

$0 / 2$

$2 / 12$

$2 / 15$

$0 / 2$

11.8

$1 / 12$

$2 / 15$

$0 / 2$

0.0

$0 / 15$

$1 / 16$

$1 / 3$

2.4

2.8

$1 / 3$

2.5

4.0

36.4

$0 / 3$

$0 / 3$

$0 / 1$

18.2

$11 / 17$

$1 / 2$

$0 / 3$

$0 / 1$

$0 / 2$

\section{2}

$2 / 12$

6

2.1

2.0

1

$0 / 1$

3.0

All Ages Combined ${ }^{\mathrm{e}}$

Fresh Embryos

3

$2 / 3$

2.7
Frozen Embryos

\section{1}

$1 / 1$

3.0

\section{CURRENT CLINIC SERVICES AND PROFILE}

Current Name: Sher Institute for Reproductive Medicine-Central Illinois

Donor egg? Yes Gestational carriers? Yes

Donor embryo? No Cryopreservation? Yes

Single women? Yes

SART member? $\quad$ No

Verified lab accreditation?

Pending

(See Appendix C for details.)

${ }^{a}$ Reflects patient and treatment characteristics of ART cycles performed in 2006 using fresh nondonor eggs or embryos.

${ }^{b}$ When fewer than 20 cycles are reported in an age category, rates are shown as a fraction and confidence intervals are not given. Calculating percentages from fractions may be misleading and is not encouraged.

${ }^{c}$ A multiple-infant birth is counted as one live birth.

d Clinic-specific outcome rates are unreliable for women older than 42 undergoing ART cycles using fresh or frozen embryos with nondonor eggs. Readers are urged to review national outcomes for these age groups (see page 27).

e All ages (including ages $>42$ ) are reported together because previous data show that patient age does not materially affect success with donor eggs. 


\section{REPRODUCTIVE HEALTH AND FERTILITY CENTER \\ ROCKFORD, ILLINOIS}

A comparison of clinic success rates may not be meaningful because patient medical characteristics and treatment approaches vary from clinic to clinic. For more details about this, along with information on how to interpret the statistics in this table, see pages $79-88$.

\section{ART CYCLE PROFILE}

\begin{tabular}{|c|c|c|c|c|c|c|c|}
\hline \multicolumn{4}{|c|}{ Type of ART ${ }^{\mathrm{a}}$} & \multicolumn{4}{|c|}{ Patient Diagnosis } \\
\hline IVF & $>99 \%$ & Procedural Factors: & & Tubal factor & $3 \%$ & Other factor & $4 \%$ \\
\hline GIFT & $<1 \%$ & With ICSI & $90 \%$ & Ovulatory dysfunction & $4 \%$ & Unknown factor & $5 \%$ \\
\hline ZIFT & $0 \%$ & Unstimulated & $0 \%$ & Diminished ovarian reserve & $4 \%$ & Multiple Factors: & \\
\hline \multirow[t]{3}{*}{ Combination } & $0 \%$ & Used gestational carrier & $0 \%$ & Endometriosis & $6 \%$ & Female factors only & $23 \%$ \\
\hline & & & & Uterine factor & $3 \%$ & Female \& male factors & $32 \%$ \\
\hline & & & & Male factor & $15 \%$ & & \\
\hline
\end{tabular}

2006 PREGNANCY SUCCESS RATES

\section{Type of Cycle}

\section{Fresh Embryos from Nondonor Eggs}

Number of cycles

Percentage of cycles resulting in pregnancies ${ }^{b}$

Percentage of cycles resulting in live births $s^{b, c}$

(Confidence Interval)

Percentage of retrievals resulting in live births $s^{b, c}$

Percentage of transfers resulting in live births $s^{b, c}$

Percentage of transfers resulting in singleton live births ${ }^{b}$

Percentage of cancellations ${ }^{b}$

Average number of embryos transferred

Percentage of pregnancies with twins ${ }^{b}$

Percentage of pregnancies with triplets or more ${ }^{b}$

Percentage of live births having multiple infants ${ }^{b, c}$

\section{Frozen Embryos from Nondonor Eggs}

Number of transfers

Percentage of transfers resulting in live births ${ }^{\mathrm{b}, \mathrm{c}}$

Average number of embryos transferred

\section{Donor Eggs}

Number of transfers

Percentage of transfers resulting in live births $s^{b, c}$

Average number of embryos transferred

Data verified by Chiravudh Sawetawan, MD

\section{Age of Woman}

35-37 38-40

$41-42^{d}$

$<35$

19

19

9

33.8

$7 / 19$

$4 / 19$

$2 / 9$

30.0

$5 / 19$

$3 / 19$

$2 / 9$

(20.3-41.3)

32.0

40.7

$5 / 19$

$3 / 16$

$2 / 9$

20.3

$5 / 16$

$3 / 13$

$2 / 8$

$5 / 16$

$3 / 13$

$1 / 8$

6.3

$0 / 19$

$3 / 19$

$0 / 9$

2.3

2.4

$0 / 7$

3.1

2.9

48.1

$0 / 7$

$1 / 4$

$0 / 2$

0.0

$0 / 5$

$0 / 4$

$1 / 2$

50.0

$0 / 3$

$1 / 2$

51

23.5

2.5

\section{4}

$1 / 14$

3.0

\section{4}

$1 / 4$

3.0
4

$0 / 4$

2.8

\section{All Ages Combined ${ }^{\mathrm{e}}$}

Fresh Embryos

6

$2 / 6$

2.0
Frozen Embryos 9

$3 / 9$

2.6

\section{CURRENT CLINIC SERVICES AND PROFILE}

Current Name: Reproductive Health and Fertility Center

Donor egg? Yes

Donor embryo? Yes

Single women? Yes

\section{Gestational carriers? Yes}

Cryopreservation? Yes
SART member?

Verified lab accreditation?

(See Appendix C for details.)

${ }^{a}$ Reflects patient and treatment characteristics of ART cycles performed in 2006 using fresh nondonor eggs or embryos.

${ }^{b}$ When fewer than 20 cycles are reported in an age category, rates are shown as a fraction and confidence intervals are not given. Calculating percentages from fractions may be misleading and is not encouraged.

${ }^{c}$ A multiple-infant birth is counted as one live birth.

d Clinic-specific outcome rates are unreliable for women older than 42 undergoing ART cycles using fresh or frozen embryos with nondonor eggs. Readers are urged to review national outcomes for these age groups (see page 27).

${ }^{e}$ All ages (including ages $>42$ ) are reported together because previous data show that patient age does not materially affect success with donor eggs. 


\section{NORTH SHORE FERTILITY, SC \\ SKOKIE, ILLINOIS}

A comparison of clinic success rates may not be meaningful because patient medical characteristics and treatment approaches vary from clinic to clinic. For more details about this, along with information on how to interpret the statistics in this table, see pages 79-88.

\section{ART CYCLE PROFILE}

\begin{tabular}{|c|c|c|c|c|c|c|c|}
\hline & Type & of $\mathrm{ART}^{\mathrm{a}}$ & & Pati & ent $D$ & iagnosis & \\
\hline IVF & $>99 \%$ & Procedural Factors: & & Tubal factor & $6 \%$ & Other factor & $2 \%$ \\
\hline GIFT & $<1 \%$ & With ICSI & $86 \%$ & Ovulatory dysfunction & $21 \%$ & Unknown factor & $<1 \%$ \\
\hline ZIFT & $<1 \%$ & Unstimulated & $0 \%$ & Diminished ovarian reserve & $29 \%$ & Multiple Factors: & \\
\hline Combination & $0 \%$ & Used gestational carrier & $<1 \%$ & Endometriosis & $2 \%$ & Female factors only & $5 \%$ \\
\hline & & & & Uterine factor & $6 \%$ & Female \& male factors & $15 \%$ \\
\hline & & & & Male factor & $14 \%$ & & \\
\hline
\end{tabular}

2006 PREGNANCY SUCCESS RATES

Data verified by Susan Davies, MD

\section{Type of Cycle}

\section{Fresh Embryos from Nondonor Eggs}

Number of cycles

Percentage of cycles resulting in pregnancies ${ }^{b}$

Percentage of cycles resulting in live births $s^{b, c}$

(Confidence Interval)

Percentage of retrievals resulting in live births ${ }^{b, c}$

Percentage of transfers resulting in live births $s^{b, c}$

Percentage of transfers resulting in singleton live births ${ }^{b}$

Percentage of cancellations ${ }^{b}$

Average number of embryos transferred

Percentage of pregnancies with twins ${ }^{b}$

Percentage of pregnancies with triplets or more ${ }^{b}$

Percentage of live births having multiple infants ${ }^{b, c}$

\section{Frozen Embryos from Nondonor Eggs}

Number of transfers

Percentage of transfers resulting in live births ${ }^{b, c}$

Average number of embryos transferred

\section{Donor Eggs}

Number of transfers

Percentage of transfers resulting in live births $s^{b, c}$

Average number of embryos transferred

\section{Age of Woman}

35-37 38-40

$41-42^{d}$

$<35$

65

27.7

23.1

39

23

33.0

25.7

(17.8-34.9)

(13.5-35.2)

23.1

12.8

0.0

26.9

24.6

(4.3-27.4)

0.0

29.8

26.3

13.5

(0.0-14.8)

13.8

21.1

15.6

0.0

4.6

6.2

2.0

9.4

$0 / 12$

2.0

$5 / 18$

5.1

$0 / 12$

47.2

$1 / 18$

2.1

13.0

2.1

2.8

53.6

$3 / 15$

$2 / 9$

$1 / 9$

$2 / 5$
4

$2 / 4$

2.8
3

$0 / 3$

2.5

All Ages Combined ${ }^{\mathrm{e}}$

Fresh Embryos

\section{1}

$4 / 11$

1.9
Frozen Embryos

6

$1 / 6$

2.5

\section{CURRENT CLINIC SERVICES AND PROFILE}

Current Name: North Shore Fertility, SC

Donor egg? Yes

Donor embryo? Yes

Single women? Yes

\section{Gestational carriers? Yes}

Cryopreservation? Yes
SART member?

Verified lab accreditation?

(See Appendix C for details.)

${ }^{a}$ Reflects patient and treatment characteristics of ART cycles performed in 2006 using fresh nondonor eggs or embryos.

${ }^{b}$ When fewer than 20 cycles are reported in an age category, rates are shown as a fraction and confidence intervals are not given. Calculating percentages from fractions may be misleading and is not encouraged.

${ }^{c}$ A multiple-infant birth is counted as one live birth.

d Clinic-specific outcome rates are unreliable for women older than 42 undergoing ART cycles using fresh or frozen embryos with nondonor eggs. Readers are urged to review national outcomes for these age groups (see page 27).

e All ages (including ages $>42$ ) are reported together because previous data show that patient age does not materially affect success with donor eggs. 


\section{REPRODUCTIVE ENDOCRINOLOGY ASSOCIATES, SC SPRINGFIELD, ILLINOIS}

A comparison of clinic success rates may not be meaningful because patient medical characteristics and treatment approaches vary from clinic to clinic. For more details about this, along with information on how to interpret the statistics in this table, see pages 79-88.

\section{ART CYCLE PROFILE}

\begin{tabular}{|c|c|c|c|c|c|c|c|}
\hline \multicolumn{4}{|c|}{ Type of $\mathrm{ART}^{\mathrm{a}}$} & \multicolumn{4}{|c|}{ Patient Diagnosis } \\
\hline IVF & $100 \%$ & Procedural Factors: & & Tubal factor & $5 \%$ & Other factor & $3 \%$ \\
\hline GIFT & $0 \%$ & With ICSI & $73 \%$ & Ovulatory dysfunction & $8 \%$ & Unknown factor & $14 \%$ \\
\hline ZIFT & $0 \%$ & Unstimulated & $0 \%$ & Diminished ovarian reserve & $3 \%$ & Multiple Factors: & \\
\hline Combination & $0 \%$ & Used gestational carrier & $0 \%$ & Endometriosis & $13 \%$ & Female factors only & $18 \%$ \\
\hline & & & & $\begin{array}{l}\text { Uterine factor } \\
\text { Male factor }\end{array}$ & $\begin{array}{l}<1 \% \\
19 \%\end{array}$ & Female \& male factors & $18 \%$ \\
\hline
\end{tabular}

2006 PREGNANCY SUCCESS RATES

Data verified by Mary Ann McRae, MD

\section{Type of Cycle}

\section{Fresh Embryos from Nondonor Eggs}

Number of cycles

Percentage of cycles resulting in pregnancies ${ }^{b}$

Percentage of cycles resulting in live births ${ }^{b, c}$

(Confidence Interval)

Percentage of retrievals resulting in live births $s^{b, c}$

Percentage of transfers resulting in live births $s^{b, c}$

Percentage of transfers resulting in singleton live births ${ }^{b}$

Percentage of cancellations ${ }^{b}$

Average number of embryos transferred

Percentage of pregnancies with twins ${ }^{b}$

Percentage of pregnancies with triplets or more ${ }^{b}$

Percentage of live births having multiple infants ${ }^{\mathrm{b}, \mathrm{c}}$

\section{Frozen Embryos from Nondonor Eggs}

Number of transfers

Percentage of transfers resulting in live births $s^{b, c}$

Average number of embryos transferred

\section{Age of Woman}

35-37 38-40

$$
41-42^{d}
$$

$<35$

34

20.6

12

0

18.9

13.2

17.6

$3 / 12$

(5.5-25.3)

17.1

(6.8-34.5)

30.0

$2 / 12$

17.5

30.0

$2 / 11$

$2 / 11$

12.5

25.0

$0 / 11$

22.6

41.2

$1 / 12$

3.5

$1 / 10$

3.3

3.9

$1 / 10$

$1 / 7$

$2 / 3$

$2 / 7$

$0 / 7$

$0 / 3$

$1 / 6$

$2 / 2$

\section{$3 \%$}

$\%$

\section{Donor Eggs}

Number of transfers

Percentage of transfers resulting in live births $s^{\mathrm{b}, \mathrm{c}}$

$9 \quad 5$

$\begin{array}{cc}9 & 5 \\ 2 / 9 & 0 / 5 \\ 3.6 & 3.0\end{array}$

All Ages Combined ${ }^{\mathrm{e}}$

Fresh Embryos

0
Frozen Embryos

0

Average number of embryos transferred

\section{CURRENT CLINIC SERVICES AND PROFILE}

Current Name: Reproductive Endocrinology Associates, SC

Donor egg? No Gestational carriers? No

Donor embryo? No Cryopreservation? Yes

Single women? No

SART member? Yes

Verified lab accreditation? Yes

(See Appendix C for details.)

${ }^{a}$ Reflects patient and treatment characteristics of ART cycles performed in 2006 using fresh nondonor eggs or embryos.

${ }^{b}$ When fewer than 20 cycles are reported in an age category, rates are shown as a fraction and confidence intervals are not given. Calculating percentages from fractions may be misleading and is not encouraged.

${ }^{c}$ A multiple-infant birth is counted as one live birth.

d Clinic-specific outcome rates are unreliable for women older than 42 undergoing ART cycles using fresh or frozen embryos with nondonor eggs. Readers are urged to review national outcomes for these age groups (see page 27).

${ }^{e}$ All ages (including ages $>42$ ) are reported together because previous data show that patient age does not materially affect success with donor eggs. 


\section{SETH LEVRANT, MD, PC \\ PARTNERS IN REPRODUCTIVE HEALTH \\ TINLEY PARK, ILLINOIS}

A comparison of clinic success rates may not be meaningful because patient medical characteristics and treatment approaches vary from clinic to clinic. For more details about this, along with information on how to interpret the statistics in this table, see pages 79-88.

\section{ART CYCLE PROFILE}

\begin{tabular}{|c|c|c|c|c|c|c|c|}
\hline \multicolumn{4}{|c|}{ Type of ART ${ }^{a}$} & \multicolumn{4}{|c|}{ Patient Diagnosis } \\
\hline IVF & $100 \%$ & Procedural Factors: & & Tubal factor & $12 \%$ & Other factor & $15 \%$ \\
\hline GIFT & $0 \%$ & With ICSI & $89 \%$ & Ovulatory dysfunction & $4 \%$ & Unknown factor & $4 \%$ \\
\hline ZIFT & $0 \%$ & Unstimulated & $0 \%$ & Diminished ovarian reserve & $1 \%$ & Multiple Factors: & \\
\hline \multirow[t]{3}{*}{ Combination } & $0 \%$ & Used gestational carrier & $0 \%$ & Endometriosis & $5 \%$ & Female factors only & $20 \%$ \\
\hline & & & & Uterine factor & $1 \%$ & Female \& male factors & $28 \%$ \\
\hline & & & & Male factor & $12 \%$ & & \\
\hline
\end{tabular}

2006 PREGNANCY SUCCESS RATES

Data verified by Seth G. Levrant, MD

\section{Type of Cycle}

\section{Fresh Embryos from Nondonor Eggs}

Number of cycles

Percentage of cycles resulting in pregnancies ${ }^{b}$

Percentage of cycles resulting in live births ${ }^{b, c}$

(Confidence Interval)

Percentage of retrievals resulting in live births $s^{b, c}$

Percentage of transfers resulting in live births $s^{b, c}$

Percentage of transfers resulting in singleton live births ${ }^{b}$

Percentage of cancellations ${ }^{b}$

Average number of embryos transferred

Percentage of pregnancies with twins ${ }^{b}$

Percentage of pregnancies with triplets or more ${ }^{b}$

Percentage of live births having multiple infants ${ }^{b, c}$

\section{Frozen Embryos from Nondonor Eggs}

Number of transfers

Percentage of transfers resulting in live births ${ }^{b, c}$

Average number of embryos transferred

\section{Donor Eggs}

Number of transfers

Percentage of transfers resulting in live births $s^{b, c}$

Average number of embryos transferred

\section{Age of Woman}

35-37 38-40

$41-42^{d}$

\section{$<35$}

15

18

2

33
33.3

33.3

$8 / 15$

$3 / 18$

$0 / 2$

$5 / 15$

$3 / 18$

$0 / 2$

(18.0-51.8)

34.4

34.4

$5 / 14$

$3 / 15$

$0 / 2$

$5 / 14$

$3 / 15$

$0 / 2$

34.4

$3 / 14$

$3 / 15$

$0 / 2$

3.0

$1 / 15$

$3 / 18$

$0 / 2$

2.3

2.7

2.5

2.5

$1 / 11$

$2 / 8$

$0 / 3$

$0 / 11$

$0 / 11$

$0 / 8$

$0 / 3$

$2 / 5$

$0 / 3$

\section{6}

$1 / 6$

2

$0 / 2$

2.5

1

$0 / 1$

2.3

All Ages Combined ${ }^{\mathrm{e}}$

Fresh Embryos

5

$3 / 5$

2.0

\section{CURRENT CLINIC SERVICES AND PROFILE}

Current Name: Seth Levrant, MD, PC, Partners in Reproductive Health

Donor egg? Yes Gestational carriers? Yes

Donor embryo? Yes Cryopreservation? Yes

Single women? Yes

SART member? Yes

Verified lab accreditation? Yes

(See Appendix C for details.)

${ }^{a}$ Reflects patient and treatment characteristics of ART cycles performed in 2006 using fresh nondonor eggs or embryos.

${ }^{b}$ When fewer than 20 cycles are reported in an age category, rates are shown as a fraction and confidence intervals are not given. Calculating percentages from fractions may be misleading and is not encouraged.

${ }^{c}$ A multiple-infant birth is counted as one live birth.

d Clinic-specific outcome rates are unreliable for women older than 42 undergoing ART cycles using fresh or frozen embryos with nondonor eggs. Readers are urged to review national outcomes for these age groups (see page 27).

e All ages (including ages $>42$ ) are reported together because previous data show that patient age does not materially affect success with donor eggs. 


\section{BONAVENTURA REPRODUCTIVE MEDICINE \\ CARMEL, INDIANA}

A comparison of clinic success rates may not be meaningful because patient medical characteristics and treatment approaches vary from clinic to clinic. For more details about this, along with information on how to interpret the statistics in this table, see pages $79-88$.

\section{ART CYCLE PROFILE}

\begin{tabular}{|c|c|c|c|c|c|c|c|}
\hline \multicolumn{4}{|c|}{ Type of $A R T^{a}$} & \multicolumn{4}{|c|}{ Patient Diagnosis } \\
\hline IVF & $100 \%$ & Procedural Factors: & & Tubal factor & $15 \%$ & Other factor & $4 \%$ \\
\hline GIFT & $0 \%$ & With ICSI & $66 \%$ & Ovulatory dysfunction & $9 \%$ & Unknown factor & $5 \%$ \\
\hline ZIFT & $0 \%$ & Unstimulated & $0 \%$ & Diminished ovarian reserve & $22 \%$ & Multiple Factors: & \\
\hline \multirow[t]{3}{*}{ Combination } & $0 \%$ & Used gestational carrier & $5 \%$ & Endometriosis & $8 \%$ & Female factors only & $8 \%$ \\
\hline & & & & Uterine factor & $1 \%$ & Female \& male factors & $13 \%$ \\
\hline & & & & Male factor & $15 \%$ & & \\
\hline
\end{tabular}

2006 PREGNANCY SUCCESS RATES

Data verified by Leo M. Bonaventura, MD

\section{Type of Cycle}

\section{Fresh Embryos from Nondonor Eggs}

Number of cycles

Percentage of cycles resulting in pregnancies ${ }^{b}$

Percentage of cycles resulting in live births $s^{b, c}$

(Confidence Interval)

Percentage of retrievals resulting in live births $s^{b, c}$

Percentage of transfers resulting in live births $s^{b, c}$

Percentage of transfers resulting in singleton live births ${ }^{b}$

Percentage of cancellations ${ }^{b}$

Average number of embryos transferred

Percentage of pregnancies with twins ${ }^{b}$

Percentage of pregnancies with triplets or more ${ }^{b}$

Percentage of live births having multiple infants ${ }^{b, c}$

\section{Frozen Embryos from Nondonor Eggs}

Number of transfers

Percentage of transfers resulting in live births $s^{b, c}$

Average number of embryos transferred

\section{Donor Eggs}

Number of transfers

Percentage of transfers resulting in live births $s^{b, c}$

Average number of embryos transferred

\section{Age of Woman}

35-37 38-40

$41-42^{d}$

$<35$

13

$1 / 13$

$1 / 13$

18

6

17.6

16.2

$0 / 18$

$0 / 6$

(8.4-27.1)

$\begin{array}{cccc}19.0 & 1 / 13 & 0 / 17 & 0 / 5 \\ 19.6 & 1 / 11 & 0 / 14 & 0 / 4 \\ 14.3 & 1 / 11 & 0 / 14 & 0 / 4 \\ 14.7 & 0 / 13 & 1 / 18 & 1 / 6 \\ 2.1 & 2.5 & 2.4 & 2.5 \\ 3 / 12 & 0 / 1 & & \\ 0 / 12 & 0 / 1 & & \\ 3 / 11 & 0 / 1 & \end{array}$

\section{8}

21.4

8

2.6

$0 / 8$

2.9
4
$0 / 4$

2.3

1

$0 / 1$

4.0

All Ages Combined ${ }^{\mathrm{e}}$

Fresh Embryos

$\begin{array}{cc}11 & 2 \\ 3 / 11 & 1 / 2 \\ 2.5 & 4.0\end{array}$

\section{CURRENT CLINIC SERVICES AND PROFILE}

Current Name: Bonaventura Reproductive Medicine

\begin{tabular}{l|l|l} 
Donor egg? Yes & Gestational carriers? Yes
\end{tabular}

Donor embryo? Yes Cryopreservation? Yes

Single women? Yes

SART member? Yes

Verified lab accreditation? Yes

(See Appendix C for details.)

${ }^{a}$ Reflects patient and treatment characteristics of ART cycles performed in 2006 using fresh nondonor eggs or embryos.

${ }^{b}$ When fewer than 20 cycles are reported in an age category, rates are shown as a fraction and confidence intervals are not given. Calculating percentages from fractions may be misleading and is not encouraged.

c A multiple-infant birth is counted as one live birth.

${ }^{d}$ Clinic-specific outcome rates are unreliable for women older than 42 undergoing ART cycles using fresh or frozen embryos with nondonor eggs. Readers are urged to review national outcomes for these age groups (see page 27).

${ }^{e}$ All ages (including ages $>42$ ) are reported together because previous data show that patient age does not materially affect success with donor eggs. 


\section{JARRETT FERTILITY GROUP \\ CARMEL, INDIANA}

A comparison of clinic success rates may not be meaningful because patient medical characteristics and treatment approaches vary from clinic to clinic. For more details about this, along with information on how to interpret the statistics in this table, see pages 79-88.

\section{ART CYCLE PROFILE}

\section{Type of ART ${ }^{\mathrm{a}}$}

\begin{tabular}{lll|lrlr} 
IVF & $98 \%$ Procedural Factors: & & Tubal factor & $6 \%$ & Other factor & $13 \%$ \\
GIFT & $<1 \%$ With ICSI & $66 \%$ & Ovulatory dysfunction & $9 \%$ & Unknown factor & $15 \%$ \\
ZIFT & $2 \%$ Unstimulated & $0 \%$ & Diminished ovarian reserve & $12 \%$ & Multiple Factors: & \\
Combination & $0 \%$ Used gestational carrier & $<1 \%$ & Endometriosis & $8 \%$ & Female factors only & $10 \%$ \\
& & & Uterine factor & $<1 \%$ & Female \& male factors & $13 \%$ \\
& & & Male factor & $13 \%$ & &
\end{tabular}

2006 PREGNANCY SUCCESS RATES

\section{Type of Cycle}

\begin{tabular}{|c|c|c|c|c|c|}
\hline \multicolumn{2}{|c|}{ 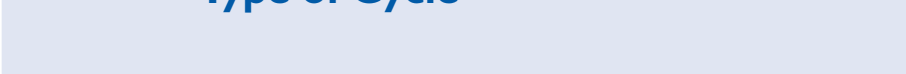 } & $<35$ & $35-37$ & $38-40$ & $41-42^{\mathrm{c}}$ \\
\hline \multicolumn{6}{|c|}{ Fresh Embryos from Nondonor Eggs } \\
\hline \multicolumn{2}{|c|}{ Number of cycles } & 187 & 80 & 47 & 18 \\
\hline \multicolumn{2}{|c|}{ Percentage of cycles resulting in pregnancies ${ }^{b}$} & 39.6 & 32.5 & 31.9 & $2 / 18$ \\
\hline \multicolumn{2}{|c|}{$\begin{array}{l}\text { Percentage of cycles resulting in live births }{ }^{b, c} \\
\text { (Confidence Interval) }\end{array}$} & $\begin{array}{c}32.6 \\
(26.0-39.8)\end{array}$ & $\begin{array}{c}25.0 \\
(16.0-35.9)\end{array}$ & $\begin{array}{c}27.7 \\
(15.6-42.6)\end{array}$ & $0 / 18$ \\
\hline \multicolumn{2}{|c|}{ Percentage of retrievals resulting in live births $s^{b, c}$} & 38.9 & 33.3 & 36.1 & $0 / 13$ \\
\hline \multicolumn{2}{|c|}{ Percentage of transfers resulting in live births ${ }^{\mathrm{b}, \mathrm{c}}$} & 40.7 & 35.1 & 37.1 & $0 / 11$ \\
\hline \multicolumn{2}{|c|}{ Percentage of transfers resulting in singleton live births ${ }^{b}$} & 24.0 & 22.8 & 25.7 & $0 / 11$ \\
\hline \multicolumn{2}{|c|}{ Percentage of cancellations ${ }^{\mathrm{b}}$} & 16.0 & 25.0 & 23.4 & $5 / 18$ \\
\hline \multicolumn{2}{|c|}{ Average number of embryos transferred } & 2.2 & 2.5 & 2.6 & 3.1 \\
\hline \multicolumn{2}{|c|}{ Percentage of pregnancies with twins ${ }^{b}$} & 33.8 & 26.9 & $2 / 15$ & $0 / 2$ \\
\hline \multicolumn{2}{|c|}{ Percentage of pregnancies with triplets or more ${ }^{b}$} & 1.4 & 0.0 & $4 / 15$ & $0 / 2$ \\
\hline \multicolumn{2}{|c|}{ Percentage of live births having multiple infants ${ }^{b, c}$} & 41.0 & 35.0 & $4 / 13$ & \\
\hline \multicolumn{6}{|c|}{ Frozen Embryos from Nondonor Eggs } \\
\hline \multicolumn{2}{|c|}{ Number of transfers } & 35 & 19 & 13 & 4 \\
\hline \multicolumn{2}{|c|}{ Percentage of transfers resulting in live births ${ }^{b, c}$} & 20.0 & $2 / 19$ & $1 / 13$ & $0 / 4$ \\
\hline \multicolumn{2}{|c|}{ Average number of embryos transferred } & 2.3 & 2.3 & 2.5 & 2.0 \\
\hline \multirow{2}{*}{\multicolumn{2}{|c|}{ Donor Eggs }} & \multicolumn{4}{|c|}{ All Ages Combined ${ }^{\mathrm{e}}$} \\
\hline & & \multicolumn{2}{|c|}{ Fresh Embryos } & \multicolumn{2}{|c|}{ Frozen Embryos } \\
\hline \multirow{3}{*}{\multicolumn{2}{|c|}{$\begin{array}{l}\text { Number of transfers } \\
\text { Percentage of transfers resulting in live births }{ }^{b, c} \\
\text { Average number of embryos transferred }\end{array}$}} & \multicolumn{2}{|c|}{39} & \multicolumn{2}{|c|}{25} \\
\hline & & \multicolumn{2}{|c|}{25.6} & \multicolumn{2}{|c|}{$\begin{array}{c}25 \\
24.0\end{array}$} \\
\hline & & \multicolumn{2}{|c|}{2.3} & \multicolumn{2}{|c|}{2.5} \\
\hline \multicolumn{6}{|c|}{ CURRENT CLINIC SERVICES AND PROFILE } \\
\hline \multicolumn{6}{|c|}{ Current Name: Jarrett Fertility Group } \\
\hline Donor egg? & \begin{tabular}{l|l} 
Yes & Gestational carriers?
\end{tabular} & Yes & SART mem & & Yes \\
\hline Donor embryo? & Cryopreservation? & Yes & Verified lab & ccreditation? & Yes \\
\hline Single women? & Yes & & (See Apper & lix C for detail & \\
\hline \multicolumn{6}{|c|}{$\begin{array}{l}\text { a Reflects patient and treatment characteristics of ART cycles performed in } 2006 \text { using fresh nondonor eggs or embryos. } \\
\text { b When fewer than } 20 \text { cycles are reported in an age category, rates are shown as a fraction and confidence intervals are not given. Calculating } \\
\text { percentages from fractions may be misleading and is not encouraged. } \\
\text { c A multiple-infant birth is counted as one live birth. } \\
\text { d Clinic-specific outcome rates are unreliable for women older than } 42 \text { undergoing ART cycles using fresh or frozen embryos with nondonor } \\
\text { eggs. Readers are urged to review national outcomes for these age groups (see page 27). } \\
\text { All ages (including ages }>42 \text { ) are reported together because previous data show that patient age does not materially affect } \\
\text { success with donor eggs. }\end{array}$} \\
\hline
\end{tabular}

Data verified by John C. Jarrett II, MD

\section{Age of Woman}

\section{Patient Diagnosis}




\section{MIDWEST FERTILITY SPECIALISTS \\ CARMEL, INDIANA}

A comparison of clinic success rates may not be meaningful because patient medical characteristics and treatment approaches vary from clinic to clinic. For more details about this, along with information on how to interpret the statistics in this table, see pages $79-88$.

\section{ART CYCLE PROFILE}

\begin{tabular}{|c|c|c|c|c|c|c|c|}
\hline \multicolumn{4}{|c|}{ Type of ART ${ }^{\mathrm{a}}$} & \multicolumn{4}{|c|}{ Patient Diagnosis } \\
\hline IVF & $>99 \%$ & Procedural Factors: & & Tubal factor & $11 \%$ & Other factor & $13 \%$ \\
\hline GIFT & $0 \%$ & With ICSI & $70 \%$ & Ovulatory dysfunction & $7 \%$ & Unknown factor & $17 \%$ \\
\hline ZIFT & $<1 \%$ & Unstimulated & $<1 \%$ & Diminished ovarian reserve & $10 \%$ & Multiple Factors: & \\
\hline \multirow[t]{3}{*}{ Combination } & $0 \%$ & Used gestational carrier & $<1 \%$ & Endometriosis & $7 \%$ & Female factors only & $5 \%$ \\
\hline & & & & Uterine factor & $1 \%$ & Female \& male factors & $8 \%$ \\
\hline & & & & Male factor & $21 \%$ & & \\
\hline
\end{tabular}

2006 PREGNANCY SUCCESS RATES

Data verified by Laura M. Reuter, MD

\section{Type of Cycle}

\section{Fresh Embryos from Nondonor Eggs}

Number of cycles

Percentage of cycles resulting in pregnancies ${ }^{b}$

Percentage of cycles resulting in live births $s^{b, c}$

(Confidence Interval)

Percentage of retrievals resulting in live births $s^{b, c}$

Percentage of transfers resulting in live births $s^{b, c}$

Percentage of transfers resulting in singleton live births ${ }^{b}$

Percentage of cancellations ${ }^{b}$

Average number of embryos transferred

Percentage of pregnancies with twins ${ }^{b}$

Percentage of pregnancies with triplets or more ${ }^{b}$

Percentage of live births having multiple infants ${ }^{b, c}$

\section{Frozen Embryos from Nondonor Eggs}

Number of transfers

Percentage of transfers resulting in live births ${ }^{\mathrm{b}, \mathrm{c}}$

Average number of embryos transferred

\section{Donor Eggs}

Number of transfers

Percentage of transfers resulting in live births $s^{b, c}$

Average number of embryos transferred

\section{Age of Woman}

35-37 38-40

$41-42^{d}$

\section{$<35$}

68

50

19

195

39.0

29.4

12.0

$3 / 19$

36.9

26.5

8.0

2/ 19

(30.1-44.1)

(16.5-38.6)

$(2.2-19.2)$

39.6

43.1

30.5

10.3

$2 / 15$

11.1

$2 / 14$

18.6

11.1

$2 / 14$

6.7

13.2

22.0

$4 / 19$

2.2

2.5

2.8

2.5

36.8

40.0

$0 / 6$

$0 / 3$

3.9

5.0

$0 / 6$

$0 / 3$

38.9

$7 / 18$

$0 / 4$

$0 / 2$

82

19.5

25

20.0

11

3

2.8

2.6

$2 / 11$

$0 / 3$

2.4

3.7

All Ages Combined ${ }^{\mathrm{e}}$

Fresh Embryos 39

35.9

2.1
Frozen Embryos 25

24.0

2.8

\section{CURRENT CLINIC SERVICES AND PROFILE}

Current Name: Midwest Fertility Specialists

Donor egg? Yes

Donor embryo? Yes

Single women? Yes

\section{Gestational carriers? Yes}

Cryopreservation? Yes
SART member?

Verified lab accreditation?

(See Appendix C for details.)

${ }^{a}$ Reflects patient and treatment characteristics of ART cycles performed in 2006 using fresh nondonor eggs or embryos.

${ }^{b}$ When fewer than 20 cycles are reported in an age category, rates are shown as a fraction and confidence intervals are not given. Calculating percentages from fractions may be misleading and is not encouraged.

${ }^{c}$ A multiple-infant birth is counted as one live birth.

${ }^{d}$ Clinic-specific outcome rates are unreliable for women older than 42 undergoing ART cycles using fresh or frozen embryos with nondonor eggs. Readers are urged to review national outcomes for these age groups (see page 27).

${ }^{e}$ All ages (including ages $>42$ ) are reported together because previous data show that patient age does not materially affect success with donor eggs. 


\section{ADVANCED REPRODUCTION INSTITUTE, LLC \\ ADVANCED FERTILITY GROUP EVANSVILLE, INDIANA}

A comparison of clinic success rates may not be meaningful because patient medical characteristics and treatment approaches vary from clinic to clinic. For more details about this, along with information on how to interpret the statistics in this table, see pages 79-88.

\section{ART CYCLE PROFILE}

\section{Type of ART ${ }^{\mathrm{a}}$}

IVF

GIFT

ZIFT

Combination
100\% Procedural Factors:

$0 \%$ With ICSI

$0 \%$ Unstimulated

$0 \%$ Used gestational carrier

\begin{tabular}{|l|lrlr}
\multicolumn{5}{c}{ Patient Diagnosis } \\
& Tubal factor & $11 \%$ & Other factor & $0 \%$ \\
49\% & Ovulatory dysfunction & $36 \%$ & Unknown factor & $<1 \%$ \\
\hline $3 \%$ & Diminished ovarian reserve & $0 \%$ & Multiple Factors: & \\
\hline & Endometriosis & $14 \%$ & Female factors only & $14 \%$ \\
& Uterine factor & $0 \%$ & Female \& male factors & $25 \%$ \\
& Male factor & $0 \%$ & &
\end{tabular}

Data verified by William L. Gentry, MD

\section{$0 \%$}

$\%$

$14 \%$

$5 \%$

2006 PREGNANCY SUCCESS RATES

Age of Woman

Type of Cycle

$<35 \quad 35-37 \quad 38-40 \quad 41-42^{d}$

\section{Fresh Embryos from Nondonor Eggs}

Number of cycles

64

42.2

17

9

11

Percentage of cycles resulting in pregnancies ${ }^{\mathrm{b}}$

Percentage of cycles resulting in live births ${ }^{b, c}$

(Confidence Interval)

Percentage of retrievals resulting in live births $s^{b, c}$

35.9

$6 / 17$

$3 / 9$

$1 / 11$

(24.3-48.9)

$4 / 17$

$2 / 9$

$0 / 11$

Percentage of transfers resulting in live births $s^{b, c}$

Percentage of transfers resulting in singleton live births ${ }^{b}$

Percentage of cancellations ${ }^{b}$

Average number of embryos transferred

Percentage of pregnancies with twins ${ }^{b}$

Percentage of pregnancies with triplets or more ${ }^{b}$

Percentage of live births having multiple infants ${ }^{b, c}$

\section{Frozen Embryos from Nondonor Eggs}

Number of transfers

Percentage of transfers resulting in live births ${ }^{b, c}$

Average number of embryos transferred

\section{Donor Eggs}

Number of transfers

Percentage of transfers resulting in live births $s^{b, c}$

Average number of embryos transferred
46.0

26.0

21.9

2.8

$\begin{array}{ccc}4 / 13 & 2 / 7 & 0 / 7 \\ 4 / 12 & 2 / 6 & 0 / 7 \\ 3 / 12 & 2 / 6 & 0 / 7 \\ 4 / 17 & 2 / 9 & 4 / 11 \\ 2.8 & 2.8 & 3.3 \\ 1 / 6 & 0 / 3 & 0 / 1 \\ 1 / 6 & 0 / 3 & 0 / 1 \\ 1 / 4 & 0 / 2 & \end{array}$

11.1

43.5

$1 / 4$

3

$0 / 3$

1

$3 / 14$

4

$1 / 4$

2.0

$0 / 1$

2.0

\section{CURRENT CLINIC SERVICES AND PROFILE}

Current Name: Advanced Reproduction Institute, LLC, Advanced Fertility Group
Donor egg?
Yes
Gestational carriers? Yes
Cryopreservation?
SART member?
Verified lab accreditation?
Yes
Donor embryo? Yes
Yes
Single women? Yes
Yes
(See Appendix C for details.)

\footnotetext{
${ }^{a}$ Reflects patient and treatment characteristics of ART cycles performed in 2006 using fresh nondonor eggs or embryos.

${ }^{b}$ When fewer than 20 cycles are reported in an age category, rates are shown as a fraction and confidence intervals are not given. Calculating percentages from fractions may be misleading and is not encouraged.

${ }^{c}$ A multiple-infant birth is counted as one live birth.

${ }^{d}$ Clinic-specific outcome rates are unreliable for women older than 42 undergoing ART cycles using fresh or frozen embryos with nondonor eggs. Readers are urged to review national outcomes for these age groups (see page 27).

e All ages (including ages $>42$ ) are reported together because previous data show that patient age does not materially affect success with donor eggs.
}

All Ages Combined ${ }^{\mathrm{e}}$ 


\section{ASSOCIATED FERTILITY \& GYNECOLOGY, PC FORT WAYNE, INDIANA}

A comparison of clinic success rates may not be meaningful because patient medical characteristics and treatment approaches vary from clinic to clinic. For more details about this, along with information on how to interpret the statistics in this table, see pages $79-88$.

\section{ART CYCLE PROFILE}

\begin{tabular}{|c|c|c|c|c|c|c|c|}
\hline \multicolumn{4}{|c|}{ Type of ART ${ }^{\mathrm{a}}$} & \multicolumn{4}{|c|}{ Patient Diagnosis } \\
\hline IVF & $100 \%$ & Procedural Factors: & & Tubal factor & $14 \%$ & Other factor & $8 \%$ \\
\hline GIFT & $0 \%$ & With ICSI & $64 \%$ & Ovulatory dysfunction & $3 \%$ & Unknown factor & $0 \%$ \\
\hline $\mathrm{ZIFT}$ & $0 \%$ & Unstimulated & $0 \%$ & Diminished ovarian reserve & $0 \%$ & Multiple Factors: & \\
\hline Combination & $0 \%$ & Used gestational carrier & $2 \%$ & Endometriosis & $3 \%$ & Female factors only & $21 \%$ \\
\hline & & & & $\begin{array}{l}\text { Uterine factor } \\
\text { Male factor }\end{array}$ & $\begin{array}{r}0 \% \\
10 \%\end{array}$ & Female \& male factors & $41 \%$ \\
\hline
\end{tabular}

2006 PREGNANCY SUCCESS RATES

Data verified by Shelby O. Cooper, MD

\section{Type of Cycle}

\section{Fresh Embryos from Nondonor Eggs}

Number of cycles

Percentage of cycles resulting in pregnancies ${ }^{b}$

Percentage of cycles resulting in live births ${ }^{b, c}$

(Confidence Interval)

Percentage of retrievals resulting in live births $s^{b, c}$

Percentage of transfers resulting in live births $s^{b, c}$

Percentage of transfers resulting in singleton live births ${ }^{b}$

Percentage of cancellations ${ }^{b}$

Average number of embryos transferred

Percentage of pregnancies with twins ${ }^{b}$

Percentage of pregnancies with triplets or more ${ }^{b}$

Percentage of live births having multiple infants ${ }^{b, c}$

\section{Frozen Embryos from Nondonor Eggs}

Number of transfers

Percentage of transfers resulting in live births $s^{b, c}$

Average number of embryos transferred

\section{Donor Eggs}

Number of transfers

Percentage of transfers resulting in live births $s^{b, c}$

Average number of embryos transferred

\section{Age of Woman}

35-37 38-40

$41-42^{d}$

$<35$

26

23.1

8

4

31.0

11.5

$1 / 8$

$0 / 4$

27.6

$(2.4-30.2)$

$(16.7-40.9)$

$3 / 18$

$1 / 8$

$0 / 4$

\section{6}

39.0

$3 / 16$

$1 / 7$

$0 / 2$

34.1

$3 / 16$

$1 / 6$

$0 / 2$

22.4

30.8

$1 / 6$

$0 / 2$

2.4

2.6

$1 / 8$

$2 / 4$

$3 / 18$

$0 / 6$

2.7

3.0

$0 / 18$

$0 / 6$

$0 / 1$

$2 / 16$

$0 / 3$

$0 / 1$

$0 / 1$

\section{3}

$2 / 13$

2.5

\section{0}

$1 / 10$

3

$1 / 3$

2.5

2.0

All Ages Combined ${ }^{\mathrm{e}}$

Fresh Embryos

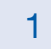

$0 / 1$

3.0
Frozen Embryos

\section{2}

$0 / 2$

1.5

\section{CURRENT CLINIC SERVICES AND PROFILE}

Current Name: Associated Fertility \& Gynecology, PC

Donor egg? Yes

Donor embryo? Yes

Single women? No

\section{Gestational carriers? Yes}

Cryopreservation? Yes

\section{SART member?}

Verified lab accreditation?

(See Appendix C for details.)

${ }^{a}$ Reflects patient and treatment characteristics of ART cycles performed in 2006 using fresh nondonor eggs or embryos.

${ }^{b}$ When fewer than 20 cycles are reported in an age category, rates are shown as a fraction and confidence intervals are not given. Calculating percentages from fractions may be misleading and is not encouraged.

${ }^{c}$ A multiple-infant birth is counted as one live birth.

d Clinic-specific outcome rates are unreliable for women older than 42 undergoing ART cycles using fresh or frozen embryos with nondonor eggs. Readers are urged to review national outcomes for these age groups (see page 27).

${ }^{e}$ All ages (including ages $>42$ ) are reported together because previous data show that patient age does not materially affect success with donor eggs. 


\section{ADVANCED FERTILITY GROUP INDIANAPOLIS, INDIANA}

A comparison of clinic success rates may not be meaningful because patient medical characteristics and treatment approaches vary from clinic to clinic. For more details about this, along with information on how to interpret the statistics in this table, see pages 79-88.

\section{ART CYCLE PROFILE}

\section{Type of ART ${ }^{\mathrm{a}}$}

IVF

GIFT

ZIFT

Combination
100\% Procedural Factors:

$0 \%$ With ICSI

$0 \%$ Unstimulated

$0 \%$ Used gestational carrier

\begin{tabular}{|c|c|c|c|c|}
\hline & \multicolumn{4}{|c|}{ Patient Diagnosis } \\
\hline & Tubal factor & $1 \%$ & Other factor & $1 \%$ \\
\hline $50 \%$ & Ovulatory dysfunction & $21 \%$ & Unknown factor & $0 \%$ \\
\hline $0 \%$ & Diminished ovarian reserve & $6 \%$ & Multiple Factors: & \\
\hline $0 \%$ & Endometriosis & $2 \%$ & Female factors only & $2 \%$ \\
\hline & Uterine factor & $0 \%$ & Female \& male factors & $7 \%$ \\
\hline & Male factor & $30 \%$ & & \\
\hline
\end{tabular}

Data verified by William L. Gentry, MD

2006 PREGNANCY SUCCESS RATES

\section{Age of Woman}

\section{Type of Cycle}

$<35 \quad 35-37 \quad 38-40 \quad 41-42^{d}$

\section{Fresh Embryos from Nondonor Eggs}

Number of cycles

Percentage of cycles resulting in pregnancies ${ }^{b}$

Percentage of cycles resulting in live births $s^{b, c}$

(Confidence Interval)

Percentage of retrievals resulting in live births $s^{b, c}$

Percentage of transfers resulting in live births $s^{b, c}$

Percentage of transfers resulting in singleton live births ${ }^{b}$

Percentage of cancellations ${ }^{b}$

\section{5}

60.0

51.4

(34.0-68.6)

\section{9}

58.1

25.8

2.9

2.1

57.1

0.0

Percentage of pregnancies with twins ${ }^{b}$

Percentage of pregnancies with triplets or more ${ }^{b}$

Percentage of live births having multiple infants ${ }^{b, c}$

\section{Frozen Embryos from Nondonor Eggs}

Number of transfers

Percentage of transfers resulting in live births ${ }^{b, c}$

Average number of embryos transferred
$10 / 18$
11
$1 / 11$

2.1
9

$4 / 9$

$3 / 9$

$3 / 7$

$3 / 7$

$0 / 7$

$2 / 9$

2.6

$2 / 4$

$1 / 4$

$3 / 3$
8

$4 / 8$

$3 / 8$

$3 / 7$

$3 / 7$

$2 / 7$

$1 / 8$

3.0

$1 / 4$

$0 / 4$

$1 / 3$

\section{9}

$1 / 9$

1.9

All Ages Combined ${ }^{\mathrm{e}}$

\section{Donor Eggs}

Number of transfers

Percentage of transfers resulting in live births $s^{b, c}$

Average number of embryos transferred

\section{Fresh Embryos}

\section{3}

$1 / 3$

2.7
7

$4 / 7$

$1 / 7$

$1 / 7$

$1 / 7$

$1 / 7$

$0 / 7$

3.0

$0 / 4$

$0 / 4$

$0 / 1$

\section{CURRENT CLINIC SERVICES AND PROFILE}

Current Name: Advanced Fertility Group
Donor egg?
Yes
Donor embryo? Yes
Gestational carriers? Yes
Cryopreservation? Yes
Single women? Yes

\footnotetext{
${ }^{a}$ Reflects patient and treatment characteristics of ART cycles performed in 2006 using fresh nondonor eggs or embryos. percentages from fractions may be misleading and is not encouraged.

${ }^{c}$ A multiple-infant birth is counted as one live birth.

d Clinic-specific outcome rates are unreliable for women older than 42 undergoing ART cycles using fresh or frozen embryos with nondonor eggs. Readers are urged to review national outcomes for these age groups (see page 27).

e All ages (including ages $>42$ ) are reported together because previous data show that patient age does not materially affect success with donor eggs.
}
${ }^{b}$ When fewer than 20 cycles are reported in an age category, rates are shown as a fraction and confidence intervals are not given. Calculating

$\begin{array}{cc}2 & 1 \\ 1 / 2 & 0 / 1 \\ 2.0 & 2.0\end{array}$

2.0 


\section{ADVANCED FERTILITY GROUP, ASSISTED FERTILITY SERVICES INDIANAPOLIS, INDIANA}

A comparison of clinic success rates may not be meaningful because patient medical characteristics and treatment approaches vary from clinic to clinic. For more details about this, along with information on how to interpret the statistics in this table, see pages $79-88$.

\section{ART CYCLE PROFILE}

\begin{tabular}{|c|c|c|c|c|c|c|c|}
\hline \multicolumn{4}{|c|}{ Type of ART ${ }^{\mathrm{a}}$} & \multicolumn{4}{|c|}{ Patient Diagnosis } \\
\hline IVF & $100 \%$ & Procedural Factors: & & Tubal factor & $23 \%$ & Other factor & $0 \%$ \\
\hline GIFT & $0 \%$ & With ICSI & $24 \%$ & Ovulatory dysfunction & $23 \%$ & Unknown factor & $0 \%$ \\
\hline $\mathrm{ZIFT}$ & $0 \%$ & Unstimulated & $0 \%$ & Diminished ovarian reserve & $0 \%$ & Multiple Factors: & \\
\hline Combination & $0 \%$ & Used gestational carrier & $0 \%$ & Endometriosis & $9 \%$ & Female factors only & $9 \%$ \\
\hline & & & & Uterine factor & $0 \%$ & Female \& male factors & $27 \%$ \\
\hline
\end{tabular}

2006 PREGNANCY SUCCESS RATES

Data verified by William L. Gentry, MD

\section{Type of Cycle}

\section{Fresh Embryos from Nondonor Eggs}

Number of cycles

Percentage of cycles resulting in pregnancies ${ }^{b}$

Percentage of cycles resulting in live births ${ }^{b, c}$

(Confidence Interval)

Percentage of retrievals resulting in live births $s^{b, c}$

Percentage of transfers resulting in live births $s^{b, c}$

Percentage of transfers resulting in singleton live births ${ }^{b}$

Percentage of cancellations ${ }^{b}$

Average number of embryos transferred

Percentage of pregnancies with twins ${ }^{b}$

Percentage of pregnancies with triplets or more ${ }^{b}$

Percentage of live births having multiple infants ${ }^{b, c}$

\section{Frozen Embryos from Nondonor Eggs}

Number of transfers

Percentage of transfers resulting in live births $s^{\mathrm{b}, \mathrm{c}}$

Average number of embryos transferred

\section{Donor Eggs}

Number of transfers

Percentage of transfers resulting in live births ${ }^{\mathrm{b}, \mathrm{c}}$

Average number of embryos transferred

\section{Age of Woman}

35-37 38-40

$41-42^{d}$

\section{$<35$}

3

$1 / 3$

2

0

$4 / 12$

$1 / 3$

$1 / 2$

$3 / 12$

$0 / 2$

$\begin{array}{ccc}3 / 10 & 1 / 2 & 0 / 2 \\ 3 / 9 & 1 / 2 & 0 / 2 \\ 1 / 9 & 1 / 2 & 0 / 2 \\ 2 / 12 & 1 / 3 & 0 / 2 \\ 2.1 & 1.5 & 2.5 \\ 3 / 4 & 0 / 1 & 0 / 1 \\ 0 / 4 & 0 / 1 & 0 / 1 \\ 2 / 3 & 0 / 1 & \end{array}$

3

$1 / 3$

0

1

$1 / 1$

2.0

2.0

All Ages Combined ${ }^{e}$

Fresh Embryos

1

$0 / 1$

2.0

\section{CURRENT CLINIC SERVICES AND PROFILE}

Current Name: Advanced Fertility Group, Assisted Fertility Services

\begin{tabular}{ll|lll|ll} 
Donor egg? & Yes & Gestational carriers? & Yes & SART member? & Yes \\
\hline Donor embryo? & Yes & Cryopreservation? & Yes & Verified lab accreditation? & Yes \\
Single women? & Yes & & & (See Appendix C for details.) &
\end{tabular}

${ }^{a}$ Reflects patient and treatment characteristics of ART cycles performed in 2006 using fresh nondonor eggs or embryos.

b When fewer than 20 cycles are reported in an age category, rates are shown as a fraction and confidence intervals are not given. Calculating percentages from fractions may be misleading and is not encouraged.

c A multiple-infant birth is counted as one live birth.

${ }^{d}$ Clinic-specific outcome rates are unreliable for women older than 42 undergoing ART cycles using fresh or frozen embryos with nondonor eggs. Readers are urged to review national outcomes for these age groups (see page 27).

e All ages (including ages $>42$ ) are reported together because previous data show that patient age does not materially affect success with donor eggs. 


\section{FAMILY BEGINNINGS, PC INDIANAPOLIS, INDIANA}

A comparison of clinic success rates may not be meaningful because patient medical characteristics and treatment approaches vary from clinic to clinic. For more details about this, along with information on how to interpret the statistics in this table, see pages 79-88.

\section{ART CYCLE PROFILE}

\begin{tabular}{|c|c|c|c|c|c|c|c|}
\hline \multicolumn{4}{|c|}{ Type of ART ${ }^{a}$} & \multicolumn{4}{|c|}{ Patient Diagnosis } \\
\hline IVF & $100 \%$ & Procedural Factors: & & Tubal factor & $21 \%$ & Other factor & $2 \%$ \\
\hline GIFT & $0 \%$ & With ICSI & $43 \%$ & Ovulatory dysfunction & $15 \%$ & Unknown factor & $20 \%$ \\
\hline ZIFT & $0 \%$ & Unstimulated & $0 \%$ & Diminished ovarian reserve & $<1 \%$ & Multiple Factors: & \\
\hline \multirow[t]{3}{*}{ Combination } & $0 \%$ & Used gestational carrier & $1 \%$ & Endometriosis & $11 \%$ & Female factors only & $2 \%$ \\
\hline & & & & Uterine factor & $0 \%$ & Female \& male factors & $9 \%$ \\
\hline & & & & Male factor & $21 \%$ & & \\
\hline
\end{tabular}

2006 PREGNANCY SUCCESS RATES

Data verified by James G. Donahue, MD

\section{Type of Cycle}

\section{Fresh Embryos from Nondonor Eggs}

Number of cycles

Percentage of cycles resulting in pregnancies ${ }^{b}$

Percentage of cycles resulting in live births ${ }^{b, c}$

(Confidence Interval)

Percentage of retrievals resulting in live births $s^{b, c}$

Percentage of transfers resulting in live births $s^{b, c}$

Percentage of transfers resulting in singleton live births ${ }^{b}$

Percentage of cancellations ${ }^{b}$

Average number of embryos transferred

Percentage of pregnancies with twins ${ }^{b}$

Percentage of pregnancies with triplets or more ${ }^{b}$

Percentage of live births having multiple infants ${ }^{b, c}$

\section{Frozen Embryos from Nondonor Eggs}

Number of transfers

Percentage of transfers resulting in live births ${ }^{b, c}$

Average number of embryos transferred

\section{Donor Eggs}

Number of transfers

Percentage of transfers resulting in live births $s^{b, c}$

Average number of embryos transferred

\section{Age of Woman}

35-37 38-40

$41-42^{d}$

\section{$<35$}

41

34.1

33

7

48.7

31.7

21.2

$1 / 7$

43.4

(18.1-48.1)

9.1

$0 / 7$

(32.1-55.3)

47.1

51.6

34.2

(1.9-24.3)

39.4

10.3

$0 / 6$

13.0

$0 / 4$

35.9

27.3

13.0

$0 / 4$

7.9

7.3

12.1

$1 / 7$

2.7

2.8

3.1

2.5

32.4

$2 / 14$

$1 / 7$

$0 / 1$

8.1

$3 / 14$

$0 / 7$

$0 / 1$

30.3

$4 / 13$

$0 / 3$

10

$5 / 10$

2.7

\section{6}

$3 / 6$

3.3

\section{3}

$1 / 3$

3.3

\section{CURRENT CLINIC SERVICES AND PROFILE}

Current Name: Family Beginnings, PC

Donor egg? Yes Gestational carriers? Yes

Donor embryo? Yes

Cryopreservation? Yes

All Ages Combined ${ }^{\mathrm{e}}$

Fresh Embryos

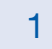

$0 / 1$

3.0
Frozen Embryos

1

$0 / 1$

4.0

Single women? Yes

SART member?

Yes

Verified lab accreditation?

(See Appendix C for details.)

${ }^{a}$ Reflects patient and treatment characteristics of ART cycles performed in 2006 using fresh nondonor eggs or embryos.

b When fewer than 20 cycles are reported in an age category, rates are shown as a fraction and confidence intervals are not given. Calculating percentages from fractions may be misleading and is not encouraged.

${ }^{c}$ A multiple-infant birth is counted as one live birth.

d Clinic-specific outcome rates are unreliable for women older than 42 undergoing ART cycles using fresh or frozen embryos with nondonor eggs. Readers are urged to review national outcomes for these age groups (see page 27).

e All ages (including ages $>42$ ) are reported together because previous data show that patient age does not materially affect success with donor eggs. 


\section{INDIANA UNIVERSITY HOSPITAL INDIANAPOLIS, INDIANA}

A comparison of clinic success rates may not be meaningful because patient medical characteristics and treatment approaches vary from clinic to clinic. For more details about this, along with information on how to interpret the statistics in this table, see pages $79-88$.

\section{ART CYCLE PROFILE}

\begin{tabular}{|c|c|c|c|c|c|c|c|}
\hline \multicolumn{4}{|c|}{ Type of ART ${ }^{\mathrm{a}}$} & \multicolumn{4}{|c|}{ Patient Diagnosis } \\
\hline IVF & $100 \%$ & Procedural Factors: & & Tubal factor & $8 \%$ & Other factor & $0 \%$ \\
\hline GIFT & $0 \%$ & With ICSI & $67 \%$ & Ovulatory dysfunction & $39 \%$ & Unknown factor & $0 \%$ \\
\hline $\mathrm{ZIFT}$ & $0 \%$ & Unstimulated & $0 \%$ & Diminished ovarian reserve & $0 \%$ & Multiple Factors: & \\
\hline Combination & $0 \%$ & Used gestational carrier & $0 \%$ & Endometriosis & $0 \%$ & Female factors only & $0 \%$ \\
\hline & & & & Uterine factor & $0 \%$ & Female \& male factors & $54 \%$ \\
\hline
\end{tabular}

2006 PREGNANCY SUCCESS RATES

Data verified by Marguerite K. Shepard, MD

\section{Type of Cycle}

\section{Fresh Embryos from Nondonor Eggs}

Number of cycles

Percentage of cycles resulting in pregnancies ${ }^{b}$

Percentage of cycles resulting in live births ${ }^{b, c}$

(Confidence Interval)

Percentage of retrievals resulting in live births $s^{b, c}$

Percentage of transfers resulting in live births $s^{b, c}$

Percentage of transfers resulting in singleton live births ${ }^{b}$

Percentage of cancellations ${ }^{b}$

Average number of embryos transferred

Percentage of pregnancies with twins ${ }^{b}$

Percentage of pregnancies with triplets or more ${ }^{b}$

Percentage of live births having multiple infants ${ }^{b, c}$

\section{Frozen Embryos from Nondonor Eggs}

Number of transfers

Percentage of transfers resulting in live births $s^{\mathrm{b}, \mathrm{c}}$

Average number of embryos transferred

\section{Age of Woman}

$\begin{array}{cccc}<35 & \mathbf{3 5 - 3 7} & \mathbf{3 8 - 4 0} & \mathbf{4 1 - 4 2}^{\mathbf{d}} \\ 6 & 4 & 1 & 1 \\ 3 / 6 & 1 / 4 & 0 / 1 & 1 / 1 \\ 2 / 6 & 1 / 4 & 0 / 1 & 1 / 1\end{array}$

$2 / 4$

$1 / 4$

$1 / 1$

$2 / 4$

$1 / 4$

$1 / 1$

$0 / 4$

$0 / 4$

$1 / 1$

$2 / 6$

$0 / 4$

2.3

$0 / 1$

2.3

$2 / 3$

$1 / 1$

2.0

$0 / 3$

$0 / 1$

$0 / 1$

$2 / 2$

$1 / 1$

$0 / 1$

$0 / 1$

\section{1}

$0 / 1$

3.0

\section{Donor Eggs}

Number of transfers

Percentage of transfers resulting in live births ${ }^{\mathrm{b}, \mathrm{c}}$

Average number of embryos transferred

\section{All Ages Combined}

Fresh Embryos

0

Frozen Embryos

0

\section{CURRENT CLINIC SERVICES AND PROFILE}

Current Name: Indiana University Hospital

\begin{tabular}{ll|lll|ll} 
Donor egg? & No & Gestational carriers? & No & SART member? & Yes \\
\hline Donor embryo? & No & Cryopreservation? & Yes & Verified lab accreditation? & Yes \\
Single women? & No & & & (See Appendix C for details.) &
\end{tabular}

${ }^{a}$ Reflects patient and treatment characteristics of ART cycles performed in 2006 using fresh nondonor eggs or embryos.

${ }^{b}$ When fewer than 20 cycles are reported in an age category, rates are shown as a fraction and confidence intervals are not given. Calculating percentages from fractions may be misleading and is not encouraged.

${ }^{c}$ A multiple-infant birth is counted as one live birth.

d Clinic-specific outcome rates are unreliable for women older than 42 undergoing ART cycles using fresh or frozen embryos with nondonor eggs. Readers are urged to review national outcomes for these age groups (see page 27).

${ }^{e}$ All ages (including ages $>42$ ) are reported together because previous data show that patient age does not materially affect success with donor eggs. 


\section{REPRODUCTIVE CARE OF INDIANA \\ INDIANAPOLIS, INDIANA}

A comparison of clinic success rates may not be meaningful because patient medical characteristics and treatment approaches vary from clinic to clinic. For more details about this, along with information on how to interpret the statistics in this table, see pages 79-88.

\section{ART CYCLE PROFILE}

\begin{tabular}{|c|c|c|c|c|c|c|c|}
\hline \multicolumn{4}{|c|}{ Type of ART ${ }^{a}$} & \multicolumn{4}{|c|}{ Patient Diagnosis } \\
\hline IVF & $100 \%$ & Procedural Factors: & & Tubal factor & $11 \%$ & Other factor & $3 \%$ \\
\hline GIFT & $0 \%$ & With ICSI & $71 \%$ & Ovulatory dysfunction & $46 \%$ & Unknown factor & $2 \%$ \\
\hline ZIFT & $0 \%$ & Unstimulated & $0 \%$ & Diminished ovarian reserve & $2 \%$ & Multiple Factors: & \\
\hline \multirow[t]{3}{*}{ Combination } & $0 \%$ & Used gestational carrier & $5 \%$ & Endometriosis & $9 \%$ & Female factors only & $14 \%$ \\
\hline & & & & Uterine factor & $11 \%$ & Female \& male factors & $2 \%$ \\
\hline & & & & Male factor & $0 \%$ & & \\
\hline
\end{tabular}

\section{PREGNANCY SUCCESS RATES}

Data verified by Michael A. Henry, MD

\section{Type of Cycle}

\section{Fresh Embryos from Nondonor Eggs}

Number of cycles

Percentage of cycles resulting in pregnancies ${ }^{b}$

Percentage of cycles resulting in live births $s^{b, c}$

(Confidence Interval)

Percentage of retrievals resulting in live births $s^{b, c}$

Percentage of transfers resulting in live births $s^{b, c}$

Percentage of transfers resulting in singleton live births ${ }^{b}$

Percentage of cancellations ${ }^{b}$

Average number of embryos transferred

Percentage of pregnancies with twins ${ }^{b}$

Percentage of pregnancies with triplets or more ${ }^{b}$

Percentage of live births having multiple infants ${ }^{b, c}$

\section{Frozen Embryos from Nondonor Eggs}

Number of transfers

Percentage of transfers resulting in live births ${ }^{b, c}$

Average number of embryos transferred

\section{Donor Eggs}

Number of transfers

Percentage of transfers resulting in live births $s^{b, c}$

Average number of embryos transferred

\section{Age of Woman}

35-37 38-40

$41-42^{d}$

\section{$<35$}

10

12

2

43.9

$7 / 10$

$3 / 12$

$0 / 2$

39.0

$4 / 10$

$2 / 12$

$0 / 2$

(24.2-55.5)

48.5

53.3

$4 / 10$

$2 / 8$

$0 / 2$

$4 / 10$

$2 / 6$

$0 / 1$

33.3

$3 / 10$

$1 / 6$

$0 / 1$

19.5

$0 / 10$

$4 / 12$

$0 / 2$

2.3

2.5

3.0

3.0

$6 / 18$

$2 / 7$

$1 / 3$

$1 / 18$

$6 / 16$

$1 / 7$

$0 / 3$

$1 / 4$

$1 / 2$

$\begin{array}{ccc}5 & 5 & 1 \\ 2 / 5 & 1 / 5 & 0 / 1 \\ 3.6 & 3.6 & 2.0\end{array}$

All Ages Combined ${ }^{\mathrm{e}}$

Fresh Embryos

Frozen Embryos

10

$3 / 10$

6

2.4

$1 / 6$

3.3

\section{CURRENT CLINIC SERVICES AND PROFILE}

Current Name: Reproductive Care of Indiana

Donor egg? Yes

Donor embryo? Yes

Single women? Yes

\section{Gestational carriers? Yes}

Cryopreservation? Yes
SART member?

Yes

Verified lab accreditation?

Yes

(See Appendix C for details.)

${ }^{a}$ Reflects patient and treatment characteristics of ART cycles performed in 2006 using fresh nondonor eggs or embryos.

b When fewer than 20 cycles are reported in an age category, rates are shown as a fraction and confidence intervals are not given. Calculating percentages from fractions may be misleading and is not encouraged.

${ }^{c}$ A multiple-infant birth is counted as one live birth.

d Clinic-specific outcome rates are unreliable for women older than 42 undergoing ART cycles using fresh or frozen embryos with nondonor eggs. Readers are urged to review national outcomes for these age groups (see page 27).

e All ages (including ages $>42$ ) are reported together because previous data show that patient age does not materially affect success with donor eggs. 


\section{REPRODUCTIVE ENDOCRINOLOGY ASSOCIATES INDIANAPOLIS, INDIANA}

A comparison of clinic success rates may not be meaningful because patient medical characteristics and treatment approaches vary from clinic to clinic. For more details about this, along with information on how to interpret the statistics in this table, see pages $79-88$.

\section{ART CYCLE PROFILE}

\begin{tabular}{|c|c|c|c|c|c|c|c|}
\hline \multicolumn{4}{|c|}{ Type of ART ${ }^{a}$} & \multicolumn{4}{|c|}{ Patient Diagnosis } \\
\hline IVF & $100 \%$ & Procedural Factors: & & Tubal factor & $11 \%$ & Other factor & $2 \%$ \\
\hline GIFT & $0 \%$ & With ICSI & $36 \%$ & Ovulatory dysfunction & $26 \%$ & Unknown factor & $2 \%$ \\
\hline ZIFT & $0 \%$ & Unstimulated & $0 \%$ & Diminished ovarian reserve & $0 \%$ & Multiple Factors: & \\
\hline \multirow[t]{3}{*}{ Combination } & $0 \%$ & Used gestational carrier & $0 \%$ & Endometriosis & $37 \%$ & Female factors only & $6 \%$ \\
\hline & & & & Uterine factor & $0 \%$ & Female \& male factors & $4 \%$ \\
\hline & & & & Male factor & $13 \%$ & & \\
\hline
\end{tabular}

2006 PREGNANCY SUCCESS RATES

Data verified by Donald L. Cline, MD

\section{Type of Cycle}

\section{Fresh Embryos from Nondonor Eggs}

Number of cycles

Percentage of cycles resulting in pregnancies ${ }^{b}$

Percentage of cycles resulting in live births $s^{b, c}$

(Confidence Interval)

Percentage of retrievals resulting in live births $s^{b, c}$

Percentage of transfers resulting in live births $s^{b, c}$

Percentage of transfers resulting in singleton live births ${ }^{b}$

Percentage of cancellations ${ }^{b}$

Average number of embryos transferred

Percentage of pregnancies with twins ${ }^{b}$

Percentage of pregnancies with triplets or more ${ }^{b}$

Percentage of live births having multiple infants ${ }^{b, c}$

\section{Frozen Embryos from Nondonor Eggs}

Number of transfers

Percentage of transfers resulting in live births $s^{b, c}$

Average number of embryos transferred

\section{Donor Eggs}

Number of transfers

Percentage of transfers resulting in live births ${ }^{\mathrm{b}, \mathrm{c}}$

Average number of embryos transferred

\section{Age of Woman}

35-37 38-40

$41-42^{d}$

$<35$

10

$1 / 10$

$1 / 10$

6

$0 / 6$

$0 / 6$

$0 / 1$

27.3

21.2

(9.0-38.9)

$\begin{array}{lccc}21.9 & 1 / 9 & 0 / 4 & 0 / 1 \\ 21.9 & 1 / 8 & 0 / 3 & 0 / 1 \\ 6.3 & 1 / 8 & 0 / 3 & 0 / 1 \\ 3.0 & 1 / 10 & 2 / 6 & 0 / 1 \\ 2.6 & 2.6 & 2.7 & 2.0 \\ 4 / 9 & 0 / 1 & & \\ 1 / 9 & 0 / 1 & & \\ 5 / 7 & 0 / 1 & & \end{array}$

0

0

2

$1 / 2$

2.0

All Ages Combined ${ }^{\mathrm{e}}$

Fresh Embryos

Frozen Embryos

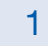

$0 / 1$

1

3.0

$0 / 1$

3.0

\section{CURRENT CLINIC SERVICES AND PROFILE}

Current Name: Reproductive Endocrinology Associates

Donor egg? Yes

Donor embryo? Yes

Single women? No
Gestational carriers? No

Cryopreservation? No
SART member?

Yes

Verified lab accreditation?

(See Appendix C for details.)

${ }^{a}$ Reflects patient and treatment characteristics of ART cycles performed in 2006 using fresh nondonor eggs or embryos.

${ }^{b}$ When fewer than 20 cycles are reported in an age category, rates are shown as a fraction and confidence intervals are not given. Calculating percentages from fractions may be misleading and is not encouraged.

${ }^{c}$ A multiple-infant birth is counted as one live birth.

d Clinic-specific outcome rates are unreliable for women older than 42 undergoing ART cycles using fresh or frozen embryos with nondonor eggs. Readers are urged to review national outcomes for these age groups (see page 27).

${ }^{e}$ All ages (including ages $>42$ ) are reported together because previous data show that patient age does not materially affect success with donor eggs. 


\section{WOMEN'S SPECIALTY HEALTH CENTERS, PC NOBLESVILLE, INDIANA}

A comparison of clinic success rates may not be meaningful because patient medical characteristics and treatment approaches vary from clinic to clinic. For more details about this, along with information on how to interpret the statistics in this table, see pages 79-88.

\section{ART CYCLE PROFILE}

\begin{tabular}{|c|c|c|c|c|c|c|c|}
\hline \multicolumn{4}{|c|}{ Type of ART ${ }^{a}$} & \multicolumn{4}{|c|}{ Patient Diagnosis } \\
\hline IVF & $100 \%$ & Procedural Factors: & & Tubal factor & $2 \%$ & Other factor & $2 \%$ \\
\hline GIFT & $0 \%$ & With ICSI & $45 \%$ & Ovulatory dysfunction & $4 \%$ & Unknown factor & $0 \%$ \\
\hline ZIFT & $0 \%$ & Unstimulated & $0 \%$ & Diminished ovarian reserve & $0 \%$ & Multiple Factors: & \\
\hline \multirow[t]{3}{*}{ Combination } & $0 \%$ & Used gestational carrier & $0 \%$ & Endometriosis & $4 \%$ & Female factors only & $49 \%$ \\
\hline & & & & Uterine factor & $0 \%$ & Female \& male factors & $36 \%$ \\
\hline & & & & Male factor & $2 \%$ & & \\
\hline
\end{tabular}

\section{PREGNANCY SUCCESS RATES}

Data verified by David S. McLaughlin, MD

\section{Type of Cycle}

\section{Fresh Embryos from Nondonor Eggs}

Number of cycles

Percentage of cycles resulting in pregnancies ${ }^{b}$

Percentage of cycles resulting in live births ${ }^{b, c}$

(Confidence Interval)

Percentage of retrievals resulting in live births $s^{b, c}$

Percentage of transfers resulting in live births $s^{b, c}$

Percentage of transfers resulting in singleton live births ${ }^{b}$

Percentage of cancellations ${ }^{b}$

Average number of embryos transferred

Percentage of pregnancies with twins ${ }^{b}$

Percentage of pregnancies with triplets or more ${ }^{b}$

Percentage of live births having multiple infants ${ }^{\mathrm{b}, \mathrm{c}}$

\section{Frozen Embryos from Nondonor Eggs}

Number of transfers

Percentage of transfers resulting in live births ${ }^{b, c}$

Average number of embryos transferred

\section{Donor Eggs}

Number of transfers

Percentage of transfers resulting in live births $s^{b, c}$

Average number of embryos transferred

\section{Age of Woman}

35-37 38-40

$41-42^{d}$

$<35$

3

$0 / 3$

$0 / 3$

5

1

42.3

38.5

$(20.2-59.4)$

45.5

$0 / 3$

$0 / 5$

$0 / 1$

45.5

$0 / 2$

$0 / 2$

$0 / 3$

$0 / 5$

$0 / 1$

13.6

15.4

2.5

$7 / 11$

$0 / 11$

$7 / 10$

$0 / 3$

$0 / 1$

$0 / 3$

$0 / 1$

$0 / 3$

$0 / 1$

$2 / 5$

$0 / 1$

2.7

2.0

$\begin{array}{cc}1 & 3 \\ 0 / 1 & 1 / 3 \\ 2.0 & 1.7\end{array}$

All Ages Combined ${ }^{\mathrm{e}}$

Fresh Embryos

Frozen Embryos

1

$0 / 1$

0

2.0

\section{CURRENT CLINIC SERVICES AND PROFILE}

Current Name: Women's Specialty Health Centers, PC

Donor egg? Yes

Donor embryo? No

Single women? Yes
Gestational carriers? No

Cryopreservation? Yes
SART member?

Yes

Verified lab accreditation?

(See Appendix C for details.)

${ }^{a}$ Reflects patient and treatment characteristics of ART cycles performed in 2006 using fresh nondonor eggs or embryos.

${ }^{b}$ When fewer than 20 cycles are reported in an age category, rates are shown as a fraction and confidence intervals are not given. Calculating percentages from fractions may be misleading and is not encouraged.

${ }^{c}$ A multiple-infant birth is counted as one live birth.

${ }^{d}$ Clinic-specific outcome rates are unreliable for women older than 42 undergoing ART cycles using fresh or frozen embryos with nondonor eggs. Readers are urged to review national outcomes for these age groups (see page 27).

e All ages (including ages $>42$ ) are reported together because previous data show that patient age does not materially affect success with donor eggs. 


\section{MID-IOWA FERTILITY, PC \\ CLIVE, IOWA}

A comparison of clinic success rates may not be meaningful because patient medical characteristics and treatment approaches vary from clinic to clinic. For more details about this, along with information on how to interpret the statistics in this table, see pages $79-88$.

\section{ART CYCLE PROFILE}

\begin{tabular}{|c|c|c|c|c|c|c|c|}
\hline \multicolumn{4}{|c|}{ Type of $A R T^{a}$} & \multicolumn{4}{|c|}{ Patient Diagnosis } \\
\hline IVF & $100 \%$ & Procedural Factors: & & Tubal factor & $12 \%$ & Other factor & $6 \%$ \\
\hline GIFT & $0 \%$ & With ICSI & $76 \%$ & Ovulatory dysfunction & $14 \%$ & Unknown factor & $9 \%$ \\
\hline ZIFT & $0 \%$ & Unstimulated & $0 \%$ & Diminished ovarian reserve & $8 \%$ & Multiple Factors: & \\
\hline Combination & $0 \%$ & Used gestational carrier & $2 \%$ & Endometriosis & $7 \%$ & Female factors only & $12 \%$ \\
\hline & & & & Uterine factor & $3 \%$ & Female \& male factors & $10 \%$ \\
\hline
\end{tabular}

2006 PREGNANCY SUCCESS RATES

Data verified by Donald C. Young, DO

\section{Type of Cycle}

\section{Fresh Embryos from Nondonor Eggs}

Number of cycles

Percentage of cycles resulting in pregnancies ${ }^{b}$

Percentage of cycles resulting in live births $s^{b, c}$

(Confidence Interval)

Percentage of retrievals resulting in live births $s^{b, c}$

Percentage of transfers resulting in live births $s^{b, c}$

Percentage of transfers resulting in singleton live births ${ }^{b}$

Percentage of cancellations ${ }^{b}$

Average number of embryos transferred

Percentage of pregnancies with twins ${ }^{b}$

Percentage of pregnancies with triplets or more ${ }^{b}$

Percentage of live births having multiple infants ${ }^{b, c}$

\section{Frozen Embryos from Nondonor Eggs}

Number of transfers

Percentage of transfers resulting in live births $s^{\mathrm{b}, \mathrm{c}}$

Average number of embryos transferred

\section{Donor Eggs}

Number of transfers

Percentage of transfers resulting in live births $s^{\mathrm{b}, \mathrm{c}}$

Average number of embryos transferred

\section{Age of Woman}

35-37 38-40

$41-42^{d}$

\section{$<35$}

43

39.5

18

5

50.7

32.6

$5 / 18$

$1 / 5$

46.5

(38.1-55.0)

(19.1-48.5)

$4 / 18$

$1 / 5$

50.8

38.9

$4 / 15$

$1 / 3$

56.9

43.8

$4 / 11$

$1 / 2$

31.3

$2 / 11$

$1 / 2$

8.5

16.3

$3 / 18$

$2 / 5$

2.1

2.1

2.4

3.5

45.8

$6 / 17$

$2 / 5$

$1 / 1$

0.0

$0 / 17$

$0 / 5$

$0 / 1$

36.4

4/ 14

$2 / 4$

$0 / 1$

23

30.4

6

2.2

$2 / 6$

2.3

\section{4}

$0 / 4$

2.5

1

$0 / 1$

3.0
Fresh Embryos

16
$7 / 16$
2.0

Frozen Embryos 4

$0 / 4$

2.5

\section{CURRENT CLINIC SERVICES AND PROFILE}

Current Name: Mid-lowa Fertility, PC

\begin{tabular}{l|l|l} 
Donor egg? Yes $\quad$ Gestational carriers? Yes &
\end{tabular}

Donor embryo? Yes Cryopreservation? Yes

Single women? Yes

\section{SART member? Yes}

Verified lab accreditation? Yes

(See Appendix C for details.)

${ }^{a}$ Reflects patient and treatment characteristics of ART cycles performed in 2006 using fresh nondonor eggs or embryos.

${ }^{b}$ When fewer than 20 cycles are reported in an age category, rates are shown as a fraction and confidence intervals are not given. Calculating percentages from fractions may be misleading and is not encouraged.

A multiple-infant birth is counted as one live birth.

${ }^{d}$ Clinic-specific outcome rates are unreliable for women older than 42 undergoing ART cycles using fresh or frozen embryos with nondonor eggs. Readers are urged to review national outcomes for these age groups (see page 27).

e All ages (including ages $>42$ ) are reported together because previous data show that patient age does not materially affect success with donor eggs. 


\section{UNIVERSITY OF IOWA HOSPITALS AND CLINICS CENTER FOR ADVANCED REPRODUCTIVE CARE IOWA CITY, IOWA}

A comparison of clinic success rates may not be meaningful because patient medical characteristics and treatment approaches vary from clinic to clinic. For more details about this, along with information on how to interpret the statistics in this table, see pages 79-88.

\section{ART CYCLE PROFILE}

\section{Type of ART ${ }^{\mathrm{a}}$}

IVF

GIFT

100\% Procedural Factors:

ZIFT

Combination
$0 \%$ With ICSI

$0 \%$ Unstimulated

$0 \%$ Used gestational carrier

\begin{tabular}{|c|c|c|c|c|}
\hline & \multicolumn{4}{|c|}{ Patient Diagnosis } \\
\hline & Tubal factor & $8 \%$ & Other factor & $10 \%$ \\
\hline $64 \%$ & Ovulatory dysfunction & $7 \%$ & Unknown factor & $13 \%$ \\
\hline $0 \%$ & Diminished ovarian reserve & $1 \%$ & Multiple Factors: & \\
\hline $0 \%$ & Endometriosis & $3 \%$ & Female factors only & $15 \%$ \\
\hline & Uterine factor & $<1 \%$ & Female \& male factors & $22 \%$ \\
\hline & Male factor & $21 \%$ & & \\
\hline
\end{tabular}

Data verified by Bradley J. Van Voorhis, MD

\section{PREGNANCY SUCCESS RATES}

\section{Type of Cycle}

\section{Age of Woman}

\section{Fresh Embryos from Nondonor Eggs}

Number of cycles

Percentage of cycles resulting in pregnancies ${ }^{b}$

Percentage of cycles resulting in live births ${ }^{b, c}$

(Confidence Interval)

Percentage of retrievals resulting in live births $s^{b, c}$

Percentage of transfers resulting in live births $s^{b, c}$

Percentage of transfers resulting in singleton live births ${ }^{b}$

Percentage of cancellations ${ }^{b}$

Average number of embryos transferred

Percentage of pregnancies with twins ${ }^{b}$

Percentage of pregnancies with triplets or more ${ }^{b}$

Percentage of live births having multiple infants ${ }^{b, c}$

\section{Frozen Embryos from Nondonor Eggs}

Number of transfers

Percentage of transfers resulting in live births $s^{b, c}$

Average number of embryos transferred

\section{Donor Eggs}

Number of transfers

Percentage of transfers resulting in live births $s^{b, c}$

Average number of embryos transferred

$<35 \quad 35-37 \quad 38-40 \quad 41-42^{\text {d }}$

$\begin{array}{cccc}168 & 87 & 41 & 22 \\ 58.3 & 46.0 & 43.9 & 13.6 \\ 51.2 & 37.9 & 29.3 & 9.1\end{array}$

$(43.4-59.0)$

55.8

$(27.7-49.0)$

$(16.1-45.5)$

$(1.1-29.2)$

61.4

42.9

31.6

$2 / 12$

33.3

$2 / 11$

50.0

31.9

25.0

$2 / 11$

8.3

11.5

7.3

45.5

1.6

1.8

2.4

2.3

21.4

1.0

20.0

$3 / 18$

$0 / 3$

18.6

7.5

$0 / 18$

$0 / 3$

30.3

$3 / 12$

$0 / 2$

68

52.9

19

1.6
$8 / 19$

1.7

\section{8}

$4 / 18$

1.8
2

$0 / 2$

1.0
All Ages Combined ${ }^{\mathrm{e}}$
Fresh Embryos 24

58.3

1.5
Frozen Embryos 14

$5 / 14$

1.7

\section{CURRENT CLINIC SERVICES AND PROFILE}

Current Name: University of lowa Hospitals and Clinics, Center for Advanced Reproductive Care

Donor egg?

Donor embryo? Yes

Single women? Yes

\begin{abstract}
Gestational carriers? No
Cryopreservation?
\end{abstract}

SART member?

Verified lab accreditation?

(See Appendix C for details.)

${ }^{a}$ Reflects patient and treatment characteristics of ART cycles performed in 2006 using fresh nondonor eggs or embryos.

${ }^{b}$ When fewer than 20 cycles are reported in an age category, rates are shown as a fraction and confidence intervals are not given. Calculating percentages from fractions may be misleading and is not encouraged.

${ }^{\mathrm{C}}$ A multiple-infant birth is counted as one live birth.

d Clinic-specific outcome rates are unreliable for women older than 42 undergoing ART cycles using fresh or frozen embryos with nondonor eggs. Readers are urged to review national outcomes for these age groups (see page 27).

ell ages (including ages $>42$ ) are reported together because previous data show that patient age does not materially affect success with donor eggs. 


\section{UNIVERSITY OF KANSAS MEDICAL CENTER \\ WOMEN'S REPRODUCTIVE CENTER \\ KANSAS CITY, KANSAS}

A comparison of clinic success rates may not be meaningful because patient medical characteristics and treatment approaches vary from clinic to clinic. For more details about this, along with information on how to interpret the statistics in this table, see pages 79-88.

\section{ART CYCLE PROFILE}

\section{Type of ART ${ }^{\mathrm{a}}$}

IVF

GIFT

ZIFT

Combination

\section{0\% Procedural Factors:}

$0 \%$ With ICSI

$0 \%$ Unstimulated

$0 \%$ Used gestational carrier

\begin{tabular}{r|l} 
& \\
& Tubal factor \\
44\% & Ovulatory dysfunction \\
$0 \%$ & Diminished ovarian reserve \\
$0 \%$ & Endometriosis \\
& Uterine factor \\
& Male factor
\end{tabular}

Patient Diagnosis

$\begin{array}{rlr}15 \% & \text { Other factor } & 0 \% \\ 6 \% & \text { Unknown factor } & 21 \% \\ 3 \% & \text { Multiple Factors: } & \\ 12 \% & \text { Female factors only } & 3 \% \\ 0 \% & \text { Female \& male factors } & 21 \% \\ 21 \% & & \end{array}$

Data verified by Linda R. Nelson, MD, PhD
2006 PREGNANCY SUCCESS RATES

\section{Type of Cycle}

\section{Age of Woman}

$\begin{array}{cccc}<35 & \mathbf{3 5 - 3 7} & \mathbf{3 8 - 4 0} & \mathbf{4 1 - 4 2}^{\mathbf{d}} \\ 13 & 5 & 2 & 2 \\ 2 / 13 & 0 / 5 & 0 / 2 & 0 / 2 \\ 1 / 13 & 0 / 5 & 0 / 2 & 0 / 2\end{array}$

$\begin{array}{cccc}1 / 8 & 0 / 3 & 0 / 2 & 0 / 1 \\ 1 / 7 & 0 / 2 & 0 / 2 & 0 / 1 \\ 1 / 7 & 0 / 2 & 0 / 2 & 0 / 1 \\ 5 / 13 & 2 / 5 & 0 / 2 & 1 / 2 \\ 2.0 & 2.5 & 3.0 & 2.0 \\ 0 / 2 & & & \\ 0 / 2 & & & \\ 0 / 1 & & & \end{array}$

$0 / 2$

$0 / 2$

$0 / 1$

\section{Frozen Embryos from Nondonor Eggs}

Number of transfers

Percentage of transfers resulting in live births $s^{b, c}$

Average number of embryos transferred

3

$1 / 3$

2.7
1

$0 / 1$

4.0
1

$0 / 1$

2.0

\section{Donor Eggs}

Number of transfers

Percentage of transfers resulting in live births $s^{b, c}$

Average number of embryos transferred

All Ages Combined ${ }^{\mathrm{e}}$

Fresh Embryos

2

$1 / 2$

3.0
Frozen Embryos

0

\section{CURRENT CLINIC SERVICES AND PROFILE}

Current Name: University of Kansas Medical Center, Women's Reproductive Center

\begin{tabular}{ll|ll|ll} 
Donor egg? & Yes & Gestational carriers? & Yes & SART member? & Yes \\
Donor embryo? & Yes & Cryopreservation? & Yes & Verified lab accreditation? & Yes \\
Single women? & Yes & & & (See Appendix C for details.) &
\end{tabular}

${ }^{a}$ Reflects patient and treatment characteristics of ART cycles performed in 2006 using fresh nondonor eggs or embryos.

b When fewer than 20 cycles are reported in an age category, rates are shown as a fraction and confidence intervals are not given. Calculating percentages from fractions may be misleading and is not encouraged.

${ }^{c}$ A multiple-infant birth is counted as one live birth.

${ }^{d}$ Clinic-specific outcome rates are unreliable for women older than 42 undergoing ART cycles using fresh or frozen embryos with nondonor eggs. Readers are urged to review national outcomes for these age groups (see page 27).

e All ages (including ages $>42$ ) are reported together because previous data show that patient age does not materially affect success with donor eggs. 


\section{MIDWEST REPRODUCTIVE CENTER, PA OLATHE, KANSAS}

A comparison of clinic success rates may not be meaningful because patient medical characteristics and treatment approaches vary from clinic to clinic. For more details about this, along with information on how to interpret the statistics in this table, see pages 79-88.

\section{ART CYCLE PROFILE}

\section{Type of ART ${ }^{\mathrm{a}}$}

IVF

GIFT

$100 \%$ Procedural Factors:

ZIFT

Combination
$0 \%$ With ICSI

$0 \%$ Unstimulated

$0 \%$ Used gestational carrier

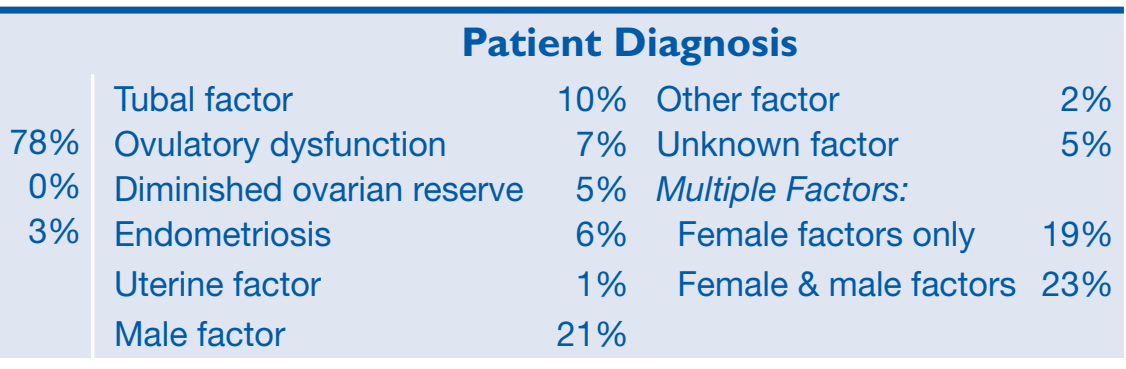

Data verified by Dan L. Gehlbach, MD

\section{PREGNANCY SUCCESS RATES}

\section{Type of Cycle}

\section{Fresh Embryos from Nondonor Eggs}

Number of cycles

Percentage of cycles resulting in pregnancies ${ }^{b}$

Percentage of cycles resulting in live births ${ }^{b, c}$

(Confidence Interval)

Percentage of retrievals resulting in live births $s^{b, c}$

Percentage of transfers resulting in live births $s^{b, c}$

Percentage of transfers resulting in singleton live births ${ }^{b}$

Percentage of cancellations ${ }^{b}$

Average number of embryos transferred

Percentage of pregnancies with twins ${ }^{\mathrm{b}}$

Percentage of pregnancies with triplets or more ${ }^{b}$

Percentage of live births having multiple infants ${ }^{\mathrm{b}, \mathrm{c}}$

\section{Frozen Embryos from Nondonor Eggs}

Number of transfers

Percentage of transfers resulting in live births $s^{b, c}$

Average number of embryos transferred

\section{Donor Eggs}

Number of transfers

Percentage of transfers resulting in live births $s^{b, c}$

Average number of embryos transferred

\section{Age of Woman}

35-37 38-40

$41-42^{d}$

\section{$<35$}

17

$4 / 17$

21

2

42.3

$3 / 17$

23.8

$0 / 2$

38.0

(26.8-50.3)

40.3

41.5

$3 / 14$

(5.4-41.9)

32.3

$3 / 13$

$4 / 17$

$0 / 2$

5.6

$0 / 13$

$4 / 17$

$0 / 1$

$3 / 17$

$4 / 17$

$0 / 1$

2.5

3.0

$2 / 4$

19.0

$0 / 1$

23.3

0.0

22.2

$1 / 4$

3.2

$0 / 5$

$0 / 5$

$3 / 3$

$0 / 4$

$\begin{array}{cccc}7 & 1 & 3 & 1 \\ 4 / 7 & 1 / 1 & 1 / 3 & 0 / 1 \\ 2.9 & 3.0 & 2.7 & 2.0\end{array}$

\section{All Ages Combined}

Fresh Embryos

6

$2 / 6$

2.8
Frozen Embryos 3

$1 / 3$

2.3

\section{CURRENT CLINIC SERVICES AND PROFILE}

Current Name: Midwest Reproductive Center, PA

Donor egg? Yes Gestational carriers? Yes

Donor embryo? Yes Cryopreservation? Yes

Single women? Yes

SART member? Yes

Verified lab accreditation?

(See Appendix C for details.)

${ }^{a}$ Reflects patient and treatment characteristics of ART cycles performed in 2006 using fresh nondonor eggs or embryos.

${ }^{b}$ When fewer than 20 cycles are reported in an age category, rates are shown as a fraction and confidence intervals are not given. Calculating percentages from fractions may be misleading and is not encouraged.

${ }^{c}$ A multiple-infant birth is counted as one live birth.

d Clinic-specific outcome rates are unreliable for women older than 42 undergoing ART cycles using fresh or frozen embryos with nondonor eggs. Readers are urged to review national outcomes for these age groups (see page 27).

e All ages (including ages $>42$ ) are reported together because previous data show that patient age does not materially affect success with donor eggs. 


\section{REPRODUCTIVE RESOURCE CENTER OF GREATER KANSAS CITY OVERLAND PARK, KANSAS}

A comparison of clinic success rates may not be meaningful because patient medical characteristics and treatment approaches vary from clinic to clinic. For more details about this, along with information on how to interpret the statistics in this table, see pages 79-88.

\section{ART CYCLE PROFILE}

\section{Type of ART ${ }^{\mathrm{a}}$}

IVF

GIFT

100\% Procedural Factors:

ZIFT

Combination

\author{
$0 \%$ With ICSI
}

$0 \%$ Unstimulated

$0 \%$ Used gestational carrier $<1 \%$

\section{Patient Diagnosis}

$6 \%$ Other factor $\quad 4 \%$

$12 \%$ Unknown factor $\quad 11 \%$

16\% Multiple Factors:

$3 \%$ Female factors only $\quad 7 \%$

$<1 \%$ Female \& male factors $27 \%$ $15 \%$

Female \& male factors 2

2006 PREGNANCY SUCCESS RATES

Data verified by Rodney Lyles, MD

\section{Type of Cycle}

Uterine factor

Male factor

of Woman

\section{Fresh Embryos from Nondonor Eggs}

Number of cycles

Percentage of cycles resulting in pregnancies ${ }^{b}$

Percentage of cycles resulting in live births ${ }^{b, c}$

(Confidence Interval)

Percentage of retrievals resulting in live births $s^{b, c}$

Percentage of transfers resulting in live births $s^{b, c}$

Percentage of transfers resulting in singleton live births ${ }^{b}$

Percentage of cancellations ${ }^{b}$

Average number of embryos transferred

Percentage of pregnancies with twins ${ }^{b}$

Percentage of pregnancies with triplets or more ${ }^{\mathrm{b}}$

Percentage of live births having multiple infants ${ }^{b, c}$

\section{Frozen Embryos from Nondonor Eggs}

Number of transfers

Percentage of transfers resulting in live births $s^{b, c}$

Average number of embryos transferred

\section{Donor Eggs}

\begin{tabular}{|c|c|c|c|}
\hline \\
\hline$<35$ & $35-37$ & $38-40$ & $41-42^{d}$ \\
\hline 196 & 64 & 44 & 1 \\
\hline 43.9 & 29.7 & 29.5 & $1 / 1$ \\
\hline 38.3 & 21.9 & 20.5 & $1 / 1$ \\
\hline 1.4-45.5) & $(12.5-34.0)$ & $(9.8-35.3)$ & \\
\hline 49.7 & 36.8 & 26.5 & $1 / 1$ \\
\hline 53.6 & 40.0 & 30.0 & $1 / 1$ \\
\hline 35.7 & 28.6 & 26.7 & $1 / 1$ \\
\hline 23.0 & 40.6 & 22.7 & $0 / 1$ \\
\hline 1.9 & 1.9 & 1.8 & 2.0 \\
\hline 30.2 & $4 / 19$ & $1 / 13$ & $0 / 1$ \\
\hline 2.3 & $0 / 19$ & $0 / 13$ & $0 / 1$ \\
\hline 33.3 & $4 / 14$ & $1 / 9$ & $0 / 1$ \\
\hline 29 & 15 & 2 & 0 \\
\hline 51.7 & $4 / 15$ & $2 / 2$ & \\
\hline 2.2 & 2.0 & 2.5 & \\
\hline & All Ages & mbined $^{e}$ & \\
\hline Fresh E & mbryos & Frozen & ibryos \\
\hline & & & \\
\hline & & & \\
\hline & & & \\
\hline
\end{tabular}

Number of transfers

Percentage of transfers resulting in live births $s^{b, c}$

Average number of embryos transferred

\section{CURRENT CLINIC SERVICES AND PROFILE}

Current Name: Reproductive Resource Center of Greater Kansas City

Donor egg? Yes

Donor embryo? No

Single women? No

\begin{abstract}
Gestational carriers? Yes
Cryopreservation? Yes
\end{abstract}

SART member? Yes

Verified lab accreditation? Yes

(See Appendix C for details.)

${ }^{a}$ Reflects patient and treatment characteristics of ART cycles performed in 2006 using fresh nondonor eggs or embryos.

${ }^{b}$ When fewer than 20 cycles are reported in an age category, rates are shown as a fraction and confidence intervals are not given. Calculating percentages from fractions may be misleading and is not encouraged.

${ }^{c}$ A multiple-infant birth is counted as one live birth.

d Clinic-specific outcome rates are unreliable for women older than 42 undergoing ART cycles using fresh or frozen embryos with nondonor eggs. Readers are urged to review national outcomes for these age groups (see page 27).

e All ages (including ages $>42$ ) are reported together because previous data show that patient age does not materially affect success with donor eggs. 


\section{REPRODUCTIVE MEDICINE \& INFERTILITY \\ SHAWNEE MISSION MEDICAL CENTER \\ SHAWNEE MISSION, KANSAS}

A comparison of clinic success rates may not be meaningful because patient medical characteristics and treatment approaches vary from clinic to clinic. For more details about this, along with information on how to interpret the statistics in this table, see pages 79-88.

\section{ART CYCLE PROFILE}

\section{Type of ART ${ }^{\mathrm{a}}$}

IVF

GIFT

100\% Procedural Factors:

ZIFT

Combination
$0 \%$ With ICSI

$0 \%$ Unstimulated

$0 \%$ Used gestational carrier

\begin{tabular}{r|l} 
& Tubal factor \\
\hline $39 \%$ & Ovulatory dysfunction \\
\hline $0 \%$ & Diminished ovarian reserve \\
4\% & Endometriosis \\
& Uterine factor \\
& Male factor
\end{tabular}

\section{Patient Diagnosis}

$13 \%$ Other factor

$4 \%$ Unknown factor

8\% Multiple Factors:

$6 \%$ Female factors only $\quad 7 \%$

$3 \%$ Female \& male factors $7 \%$ $33 \%$

\section{PREGNANCY SUCCESS RATES}

Data verified by Dan L. Stewart, MD

\section{Type of Cycle}

\section{Age of Woman}

35-37 38-40

$41-42^{d}$
19

$7 / 19$

$5 / 19$

36.0

(26.0-47.1)

42.5

45.6

30.9

15.1

2.3

30.6

5.6

32.3
$5 / 12$

$5 / 12$

$4 / 12$

$7 / 19$

2.3

$1 / 7$

$0 / 7$

$1 / 5$

Percentage of pregnancies with triplets or more ${ }^{b}$

Percentage of live births having multiple infants ${ }^{b, c}$

\section{Frozen Embryos from Nondonor Eggs}

Number of transfers

Percentage of transfers resulting in live births $s^{b, c}$

Average number of embryos transferred

\section{Donor Eggs}

Number of transfers

Percentage of transfers resulting in live births $s^{b, c}$

Average number of embryos transferred

$\begin{array}{cccc}16 & 1 & 0 & 1 \\ 8 / 16 & 1 / 1 & & 1 / 1 \\ 2.1 & 2.0 & & 2.0\end{array}$

All Ages Combined ${ }^{\mathrm{e}}$

Fresh Embryos

Frozen Embryos

2

$1 / 2$

12

$5 / 12$

2.5
4

$0 / 4$

$0 / 4$

$0 / 2$

$0 / 2$

$0 / 2$

$2 / 4$

2.0

\section{CURRENT CLINIC SERVICES AND PROFILE}

Current Name: Reproductive Medicine \& Infertility, Shawnee Mission Medical Center

Donor egg? Yes

Donor embryo? Yes

Single women? No

\begin{abstract}
Gestational carriers? Yes
Cryopreservation?
\end{abstract}

SART member?

Verified lab accreditation?

(See Appendix C for details.)

${ }^{a}$ Reflects patient and treatment characteristics of ART cycles performed in 2006 using fresh nondonor eggs or embryos.

${ }^{b}$ When fewer than 20 cycles are reported in an age category, rates are shown as a fraction and confidence intervals are not given. Calculating percentages from fractions may be misleading and is not encouraged.

${ }^{\mathrm{C}}$ A multiple-infant birth is counted as one live birth.

d Clinic-specific outcome rates are unreliable for women older than 42 undergoing ART cycles using fresh or frozen embryos with nondonor eggs. Readers are urged to review national outcomes for these age groups (see page 27).

e All ages (including ages $>42$ ) are reported together because previous data show that patient age does not materially affect success with donor eggs. 


\section{THE CENTER FOR REPRODUCTIVE MEDICINE WICHITA, KANSAS}

A comparison of clinic success rates may not be meaningful because patient medical characteristics and treatment approaches vary from clinic to clinic. For more details about this, along with information on how to interpret the statistics in this table, see pages 79-88.

\section{ART CYCLE PROFILE}

\section{Type of ART ${ }^{\mathrm{a}}$}

IVF

GIFT

100\% Procedural Factors:

ZIFT

Combination

\author{
$0 \%$ With ICSI
}

$0 \%$ Unstimulated

0\% Used gestational carrier

\begin{tabular}{|c|c|c|c|c|}
\hline & \multicolumn{4}{|c|}{ Patient Diagnosis } \\
\hline & Tubal factor & $14 \%$ & Other factor & $1 \%$ \\
\hline $65 \%$ & Ovulatory dysfunction & $7 \%$ & Unknown factor & $4 \%$ \\
\hline $0 \%$ & Diminished ovarian reserve & $4 \%$ & Multiple Factors: & \\
\hline $1 \%$ & Endometriosis & $13 \%$ & Female factors only & $12 \%$ \\
\hline & Uterine factor & $0 \%$ & Female \& male factors & $32 \%$ \\
\hline & Male factor & $14 \%$ & & \\
\hline
\end{tabular}

Data verified by David A. Grainger, MD

2006 PREGNANCY SUCCESS RATES

\section{Age of Woman}

\section{Type of Cycle}

\section{Fresh Embryos from Nondonor Eggs}

Number of cycles

Percentage of cycles resulting in pregnancies ${ }^{b}$

Percentage of cycles resulting in live births ${ }^{b, c}$

(Confidence Interval)

Percentage of retrievals resulting in live births $s^{b, c}$

Percentage of transfers resulting in live births $s^{b, c}$

Percentage of transfers resulting in singleton live births ${ }^{b}$

Percentage of cancellations ${ }^{b}$

Average number of embryos transferred

Percentage of pregnancies with twins ${ }^{b}$

Percentage of pregnancies with triplets or more ${ }^{\mathrm{b}}$

Percentage of live births having multiple infants ${ }^{b, c}$

\section{Frozen Embryos from Nondonor Eggs}

Number of transfers

Percentage of transfers resulting in live births $s^{b, c}$

Average number of embryos transferred

\section{Donor Eggs}

Number of transfers

Percentage of transfers resulting in live births $s^{b, c}$

Average number of embryos transferred

\begin{tabular}{|c|c|c|c|c|}
\hline & & Age of & Voman & \\
\hline & $<35$ & $35-37$ & $38-40$ & $41-42^{d}$ \\
\hline & 90 & 20 & 24 & 10 \\
\hline & 45.6 & 25.0 & 25.0 & $1 / 10$ \\
\hline & 36.7 & 25.0 & 16.7 & $1 / 10$ \\
\hline & $(26.8-47.5)$ & (8.7-49.1) & $(4.7-37.4)$ & \\
\hline & 39.3 & $5 / 18$ & 19.0 & $1 / 8$ \\
\hline & 39.3 & $5 / 18$ & 20.0 & $1 / 7$ \\
\hline irths ${ }^{b}$ & 26.2 & $5 / 18$ & 20.0 & $1 / 7$ \\
\hline & 6.7 & 10.0 & 12.5 & $2 / 10$ \\
\hline & 2.0 & 2.2 & 2.9 & 2.4 \\
\hline & 31.7 & $0 / 5$ & $1 / 6$ & $0 / 1$ \\
\hline & 2.4 & $0 / 5$ & $0 / 6$ & $0 / 1$ \\
\hline & 33.3 & $0 / 5$ & $0 / 4$ & $0 / 1$ \\
\hline & 17 & 1 & 1 & 2 \\
\hline & $8 / 17$ & $0 / 1$ & $1 / 1$ & $0 / 2$ \\
\hline & 2.0 & 1.0 & 3.0 & 1.0 \\
\hline & & All Ages & mbined $^{\mathrm{e}}$ & \\
\hline & Fresh & nbryos & Frozer & Abryos \\
\hline & & & & \\
\hline & & & & \\
\hline & & & & \\
\hline
\end{tabular}

\section{CURRENT CLINIC SERVICES AND PROFILE}

Current Name: The Center for Reproductive Medicine

\begin{tabular}{l|l|l} 
Donor egg? Yes & Gestational carriers? Yes
\end{tabular}

Donor embryo? Yes Cryopreservation? Yes

Single women? Yes

SART member? Yes

Verified lab accreditation? Yes

(See Appendix C for details.)

${ }^{a}$ Reflects patient and treatment characteristics of ART cycles performed in 2006 using fresh nondonor eggs or embryos.

${ }^{b}$ When fewer than 20 cycles are reported in an age category, rates are shown as a fraction and confidence intervals are not given. Calculating percentages from fractions may be misleading and is not encouraged.

${ }^{c}$ A multiple-infant birth is counted as one live birth.

d Clinic-specific outcome rates are unreliable for women older than 42 undergoing ART cycles using fresh or frozen embryos with nondonor eggs. Readers are urged to review national outcomes for these age groups (see page 27).

${ }^{e}$ All ages (including ages $>42$ ) are reported together because previous data show that patient age does not materially affect success with donor eggs. 


\section{BLUEGRASS FERTILITY CENTER \\ LEXINGTON, KENTUCKY}

A comparison of clinic success rates may not be meaningful because patient medical characteristics and treatment approaches vary from clinic to clinic. For more details about this, along with information on how to interpret the statistics in this table, see pages 79-88.

\section{ART CYCLE PROFILE}

\begin{tabular}{l|l|lrlrl}
\multicolumn{2}{c}{ Type of ART } & \multicolumn{4}{c}{ Patient Diagnosis } \\
IVF & $100 \%$ Procedural Factors: & & Tubal factor & $14 \%$ & Other factor & $8 \%$ \\
GIFT & $0 \%$ With ICSI & $48 \%$ & Ovulatory dysfunction & $4 \%$ & Unknown factor & $7 \%$ \\
ZIFT & $0 \%$ Unstimulated & $0 \%$ & Diminished ovarian reserve & $14 \%$ & Multiple Factors: & \\
Combination & $0 \%$ Used gestational carrier & $1 \%$ & Endometriosis & $12 \%$ & Female factors only & $9 \%$ \\
& & & Uterine factor & $0 \%$ & Female \& male factors & $13 \%$ \\
& & & Male factor & $20 \%$ & &
\end{tabular}

2006 PREGNANCY SUCCESS RATES

Data verified by James W. Akin, MD

\section{Type of Cycle}

\section{Fresh Embryos from Nondonor Eggs}

Number of cycles

Percentage of cycles resulting in pregnancies ${ }^{b}$

Percentage of cycles resulting in live births ${ }^{b, c}$

(Confidence Interval)

Percentage of retrievals resulting in live births $s^{b, c}$

Percentage of transfers resulting in live births $s^{b, c}$

Percentage of transfers resulting in singleton live births ${ }^{b}$

Percentage of cancellations ${ }^{b}$

Average number of embryos transferred

Percentage of pregnancies with twins ${ }^{b}$

Percentage of pregnancies with triplets or more ${ }^{b}$

Percentage of live births having multiple infants ${ }^{b, c}$

\section{Frozen Embryos from Nondonor Eggs}

Number of transfers

Percentage of transfers resulting in live births ${ }^{b, c}$

Average number of embryos transferred

\section{Donor Eggs}

Number of transfers

Percentage of transfers resulting in live births $s^{b, c}$

Average number of embryos transferred

\section{Age of Woman}

35-37 38-40

$41-42^{d}$

\section{$<35$}

34

32.4

18

6

43.8

17.6

$5 / 18$

$1 / 6$

34.8

$(25.0-45.7)$

(6.8-34.5)

$1 / 18$

$1 / 6$

37.8

39.2

20.0

$1 / 15$

$1 / 6$

29.1

17.9

$1 / 15$

$1 / 15$

$1 / 6$

7.9

11.8

$3 / 18$

$1 / 6$

2.7

2.5

2.7

$1 / 11$

23.1

$1 / 11$

$1 / 5$

$0 / 6$

2.6

25.8

$1 / 6$

$1 / 5$

$0 / 1$

3.3

$0 / 1$

$0 / 1$

$0 / 1$

20

15.0

2.4

2

$0 / 2$

2.0

2

$0 / 2$

1

3.5

$0 / 1$

1.0
Fresh Embryos 5

$1 / 5$

3.2

\section{CURRENT CLINIC SERVICES AND PROFILE}

Current Name: Bluegrass Fertility Center

Donor egg? Yes

Donor embryo? No

Single women? Yes
Gestational carriers? No

Cryopreservation? Yes
SART member?

No

Verified lab accreditation?

Yes

(See Appendix C for details.)

${ }^{a}$ Reflects patient and treatment characteristics of ART cycles performed in 2006 using fresh nondonor eggs or embryos.

b When fewer than 20 cycles are reported in an age category, rates are shown as a fraction and confidence intervals are not given. Calculating percentages from fractions may be misleading and is not encouraged.

${ }^{c}$ A multiple-infant birth is counted as one live birth.

d Clinic-specific outcome rates are unreliable for women older than 42 undergoing ART cycles using fresh or frozen embryos with nondonor eggs. Readers are urged to review national outcomes for these age groups (see page 27).

e All ages (including ages $>42$ ) are reported together because previous data show that patient age does not materially affect success with donor eggs. 


\section{FERTILITY AND ENDOCRINE ASSOCIATES \\ LOUISVILLE, KENTUCKY}

A comparison of clinic success rates may not be meaningful because patient medical characteristics and treatment approaches vary from clinic to clinic. For more details about this, along with information on how to interpret the statistics in this table, see pages $79-88$.

\section{ART CYCLE PROFILE}

\begin{tabular}{|c|c|c|c|c|c|c|c|}
\hline \multicolumn{4}{|c|}{ Type of ART ${ }^{\mathrm{a}}$} & \multicolumn{4}{|c|}{ Patient Diagnosis } \\
\hline IVF & $100 \%$ & Procedural Factors: & & Tubal factor & $2 \%$ & Other factor & $<1 \%$ \\
\hline GIFT & $0 \%$ & With ICSI & $76 \%$ & Ovulatory dysfunction & $2 \%$ & Unknown factor & $0 \%$ \\
\hline ZIFT & $0 \%$ & Unstimulated & $0 \%$ & Diminished ovarian reserve & $8 \%$ & Multiple Factors: & \\
\hline \multirow[t]{3}{*}{ Combination } & $0 \%$ & Used gestational carrier & $0 \%$ & Endometriosis & $0 \%$ & Female factors only & $23 \%$ \\
\hline & & & & Uterine factor & $0 \%$ & Female \& male factors & $62 \%$ \\
\hline & & & & Male factor & $3 \%$ & & \\
\hline
\end{tabular}

2006 PREGNANCY SUCCESS RATES

Data verified by Robert J. Homm, MD

\section{Type of Cycle}

\section{Fresh Embryos from Nondonor Eggs}

Number of cycles

Percentage of cycles resulting in pregnancies ${ }^{b}$

Percentage of cycles resulting in live births ${ }^{b, c}$

(Confidence Interval)

Percentage of retrievals resulting in live births ${ }^{b, c}$

Percentage of transfers resulting in live births $s^{b, c}$

Percentage of transfers resulting in singleton live births ${ }^{b}$

Percentage of cancellations ${ }^{b}$

Average number of embryos transferred

Percentage of pregnancies with twins ${ }^{b}$

Percentage of pregnancies with triplets or more ${ }^{b}$

Percentage of live births having multiple infants ${ }^{b, c}$

\section{Frozen Embryos from Nondonor Eggs}

Number of transfers

Percentage of transfers resulting in live births $s^{\mathrm{b}, \mathrm{c}}$

Average number of embryos transferred

\section{Donor Eggs}

Number of transfers

Percentage of transfers resulting in live births $s^{\mathrm{b}, \mathrm{c}}$

Average number of embryos transferred

\section{Age of Woman}

35-37 38-40

$41-42^{d}$ $<35$

17

$6 / 17$

12

7

52.5

4/ 17

$6 / 12$

$2 / 7$

45.0

$4 / 12$

$0 / 7$

(29.3-61.5)

47.4

$4 / 14$

$4 / 11$

$0 / 5$

50.0

$4 / 13$

$4 / 11$

$0 / 4$

33.3

$4 / 13$

$3 / 11$

$0 / 4$

5.0

$3 / 17$

$1 / 12$

$2 / 7$

2.4

2.7

2.7

3.3

28.6

$0 / 6$

$1 / 6$

$0 / 2$

0.0

$0 / 6$

$0 / 6$

$0 / 2$

$6 / 18$

$0 / 4$

$1 / 4$

21

61.9

2.5

\section{8}

$1 / 8$

1.9

\section{7}

$2 / 7$

2.6
2

$1 / 2$

2.0

All Ages Combined ${ }^{\mathrm{e}}$

Fresh Embryos 3

$2 / 3$
Frozen Embryos

0

\section{CURRENT CLINIC SERVICES AND PROFILE}

Current Name: Fertility and Endocrine Associates, Louisville Reproductive Center

Donor egg? Yes

Donor embryo? Yes

Single women? Yes

\begin{abstract}
Gestational carriers?
Cryopreservation?
\end{abstract}

SART member?

Verified lab accreditation?

(See Appendix C for details.)
No

No

${ }^{a}$ Reflects patient and treatment characteristics of ART cycles performed in 2006 using fresh nondonor eggs or embryos.

${ }^{b}$ When fewer than 20 cycles are reported in an age category, rates are shown as a fraction and confidence intervals are not given. Calculating percentages from fractions may be misleading and is not encouraged.

${ }^{c}$ A multiple-infant birth is counted as one live birth.

d Clinic-specific outcome rates are unreliable for women older than 42 undergoing ART cycles using fresh or frozen embryos with nondonor eggs. Readers are urged to review national outcomes for these age groups (see page 27).

e All ages (including ages $>42$ ) are reported together because previous data show that patient age does not materially affect success with donor eggs. 


\section{UNIVERSITY OB/GYN ASSOCIATES FERTILITY CENTER LOUISVILLE, KENTUCKY}

A comparison of clinic success rates may not be meaningful because patient medical characteristics and treatment approaches vary from clinic to clinic. For more details about this, along with information on how to interpret the statistics in this table, see pages 79-88.

\section{ART CYCLE PROFILE}

\begin{tabular}{|c|c|c|c|c|c|c|c|}
\hline \multicolumn{4}{|c|}{ Type of ART ${ }^{a}$} & \multicolumn{4}{|c|}{ Patient Diagnosis } \\
\hline IVF & $100 \%$ & Procedural Factors: & & Tubal factor & $15 \%$ & Other factor & $3 \%$ \\
\hline GIFT & $0 \%$ & With ICSI & $51 \%$ & Ovulatory dysfunction & $5 \%$ & Unknown factor & $9 \%$ \\
\hline ZIFT & $0 \%$ & Unstimulated & $0 \%$ & Diminished ovarian reserve & $5 \%$ & Multiple Factors: & \\
\hline \multirow[t]{3}{*}{ Combination } & $0 \%$ & Used gestational carrier & $2 \%$ & Endometriosis & $3 \%$ & Female factors only & $23 \%$ \\
\hline & & & & Uterine factor & $1 \%$ & Female \& male factors & $18 \%$ \\
\hline & & & & Male factor & $18 \%$ & & \\
\hline
\end{tabular}

2006 PREGNANCY SUCCESS RATES

Data verified by Steven T. Nakajima, MD

\section{Type of Cycle}

\section{Fresh Embryos from Nondonor Eggs}

Number of cycles

Percentage of cycles resulting in pregnancies ${ }^{b}$

Percentage of cycles resulting in live births ${ }^{b, c}$

(Confidence Interval)

Percentage of retrievals resulting in live births $s^{b, c}$

Percentage of transfers resulting in live births $s^{b, c}$

Percentage of transfers resulting in singleton live births ${ }^{b}$

Percentage of cancellations ${ }^{b}$

Average number of embryos transferred

Percentage of pregnancies with twins ${ }^{b}$

Percentage of pregnancies with triplets or more ${ }^{b}$

Percentage of live births having multiple infants ${ }^{b, c}$

\section{Frozen Embryos from Nondonor Eggs}

Number of transfers

Percentage of transfers resulting in live births ${ }^{b, c}$

Average number of embryos transferred

\section{Donor Eggs}

Number of transfers

Percentage of transfers resulting in live births $s^{b, c}$

Average number of embryos transferred

\section{Age of Woman}

35-37 38-40

$41-42^{d}$

\section{$<35$}

32

19

8

71
64.8

31.3

$5 / 19$

$0 / 8$

60.6

31.3

$3 / 19$

$0 / 8$

$(48.3-72.0)$

$(16.1-50.0)$

63.2

37.0

41.7

$3 / 13$

$0 / 4$

65.2

20.8

$3 / 11$

$0 / 3$

47.0

15.6

$3 / 11$

$0 / 3$

4.2

2.5

$6 / 19$

$4 / 8$

2.2

$4 / 10$

2.8

3.0

30.4

0.0

$1 / 10$

$1 / 5$

27.9

$5 / 10$

$0 / 5$

$0 / 3$

\section{CURRENT CLINIC SERVICES AND PROFILE}

Current Name: University OB/GYN Associates Fertility Center

Donor egg? Yes Gestational carriers? Yes

Donor embryo? No Cryopreservation? Yes

Single women? Yes

$\begin{array}{cccc}12 & 3 & 3 & 2 \\ 7 / 12 & 3 / 3 & 2 / 3 & 1 / 2 \\ 2.1 & 2.3 & 2.7 & 2.0\end{array}$

\section{All Ages Combined ${ }^{\mathrm{e}}$}

Fresh Embryos

8

$5 / 8$

2.4
Frozen Embryos

0

${ }_{b}^{a}$ Reflects patient and treatment characteristics of ART cycles performed in 2006 using fresh nondonor eggs or embryos.

b When fewer than 20 cycles are reported in an age category, rates are shown as a fraction and confidence intervals are not given. Calculating percentages from fractions may be misleading and is not encouraged.

${ }^{c}$ A multiple-infant birth is counted as one live birth.

${ }^{d}$ Clinic-specific outcome rates are unreliable for women older than 42 undergoing ART cycles using fresh or frozen embryos with nondonor eggs. Readers are urged to review national outcomes for these age groups (see page 27).

e All ages (including ages $>42$ ) are reported together because previous data show that patient age does not materially affect success with donor eggs. 


\section{A WOMAN'S CENTER FOR REPRODUCTIVE MEDICINE BATON ROUGE, LOUISIANA}

A comparison of clinic success rates may not be meaningful because patient medical characteristics and treatment approaches vary from clinic to clinic. For more details about this, along with information on how to interpret the statistics in this table, see pages $79-88$.

\section{ART CYCLE PROFILE}

\begin{tabular}{|c|c|c|c|c|c|c|c|}
\hline \multicolumn{4}{|c|}{ Type of ART ${ }^{a}$} & \multicolumn{4}{|c|}{ Patient Diagnosis } \\
\hline IVF & $100 \%$ & Procedural Factors: & & Tubal factor & $14 \%$ & Other factor & $11 \%$ \\
\hline GIFT & $0 \%$ & With ICSI & $85 \%$ & Ovulatory dysfunction & $7 \%$ & Unknown factor & $4 \%$ \\
\hline $\mathrm{ZIFT}$ & $0 \%$ & Unstimulated & $0 \%$ & Diminished ovarian reserve & $2 \%$ & Multiple Factors: & \\
\hline \multirow[t]{3}{*}{ Combination } & $0 \%$ & Used gestational carrier & $0 \%$ & Endometriosis & $10 \%$ & Female factors only & $23 \%$ \\
\hline & & & & Uterine factor & $2 \%$ & Female \& male factors & $12 \%$ \\
\hline & & & & Male factor & $14 \%$ & & \\
\hline
\end{tabular}

2006 PREGNANCY SUCCESS RATES

Data verified by Bobby W. Webster, MD

\section{Type of Cycle}

\section{Fresh Embryos from Nondonor Eggs}

Number of cycles

Percentage of cycles resulting in pregnancies ${ }^{b}$

Percentage of cycles resulting in live births ${ }^{b, c}$

(Confidence Interval)

Percentage of retrievals resulting in live births $s^{b, c}$

Percentage of transfers resulting in live births $s^{b, c}$

Percentage of transfers resulting in singleton live births ${ }^{b}$

Percentage of cancellations ${ }^{b}$

Average number of embryos transferred

Percentage of pregnancies with twins ${ }^{b}$

Percentage of pregnancies with triplets or more ${ }^{b}$

Percentage of live births having multiple infants ${ }^{b, c}$

\section{Frozen Embryos from Nondonor Eggs}

Number of transfers

Percentage of transfers resulting in live births $s^{b, c}$

Average number of embryos transferred

\section{Donor Eggs}

Number of transfers

Percentage of transfers resulting in live births $s^{b, c}$

Average number of embryos transferred

\section{Age of Woman}

35-37 38-40

$41-42^{d}$

$<35$

23

47.8

14

5

35.9

30.4

$7 / 14$

$1 / 5$

31.3

(20.2-44.1)

(13.2-52.9)

$6 / 14$

$1 / 5$

\section{0}

33.3

$6 / 12$

$1 / 5$

39.2

35.0

$6 / 11$

$1 / 5$

21.6

20.0

$5 / 11$

$1 / 5$

15.6

8.7

$2 / 14$

$0 / 5$

2.3

2.7

2.4

4.0

39.1

$3 / 11$

4.3

$0 / 11$

$1 / 7$

$0 / 1$

45.0

$3 / 7$

$0 / 7$

$0 / 1$

$1 / 6$

$0 / 1$

15

$1 / 15$

1.9

\section{5}

$3 / 5$

1.8
0

0

\section{CURRENT CLINIC SERVICES AND PROFILE}

Current Name: A Woman's Center for Reproductive Medicine
Donor egg?
Yes
Donor embryo? Yes
$\begin{array}{ll}\text { Gestational carriers? } & \text { Yes } \\ \text { Cryopreservation? } & \text { Yes }\end{array}$
Single women? Yes
$\begin{array}{ll}\text { SART member? } & \text { Yes } \\ \text { Verified lab accreditation? } & \text { Yes }\end{array}$
(See Appendix C for details.)

\footnotetext{
${ }^{a}$ Reflects patient and treatment characteristics of ART cycles performed in 2006 using fresh nondonor eggs or embryos.

${ }^{b}$ When fewer than 20 cycles are reported in an age category, rates are shown as a fraction and confidence intervals are not given. Calculating percentages from fractions may be misleading and is not encouraged.

${ }^{c}$ A multiple-infant birth is counted as one live birth.

d Clinic-specific outcome rates are unreliable for women older than 42 undergoing ART cycles using fresh or frozen embryos with nondonor eggs. Readers are urged to review national outcomes for these age groups (see page 27).

${ }^{e}$ All ages (including ages $>42$ ) are reported together because previous data show that patient age does not materially affect success with donor eggs.
} 


\section{OCHSNER FOUNDATION FERTILITY CLINIC JEFFERSON, LOUISIANA}

A comparison of clinic success rates may not be meaningful because patient medical characteristics and treatment approaches vary from clinic to clinic. For more details about this, along with information on how to interpret the statistics in this table, see pages 79-88.

\section{ART CYCLE PROFILE}

\begin{tabular}{l|l|lrlrl}
\multicolumn{3}{c}{ Type of ART } & \multicolumn{4}{c}{ Patient Diagnosis } \\
IVF & $100 \%$ Procedural Factors: & & Tubal factor & $11 \%$ & Other factor & $4 \%$ \\
GIFT & $0 \%$ With ICSI & $99 \%$ & Ovulatory dysfunction & $2 \%$ & Unknown factor & $1 \%$ \\
ZIFT & $0 \%$ Unstimulated & $0 \%$ & Diminished ovarian reserve & $10 \%$ & Multiple Factors: & \\
Combination & $0 \%$ Used gestational carrier & $4 \%$ & Endometriosis & $7 \%$ & Female factors only & $13 \%$ \\
& & & Uterine factor & $2 \%$ & Female \& male factors & $37 \%$ \\
& & & Male factor & $13 \%$ & &
\end{tabular}

\section{PREGNANCY SUCCESS RATES}

Data verified by Gloria Richard-Davis, MD

\section{Type of Cycle}

\section{Fresh Embryos from Nondonor Eggs}

Number of cycles

Percentage of cycles resulting in pregnancies ${ }^{b}$

Percentage of cycles resulting in live births ${ }^{b, c}$

(Confidence Interval)

Percentage of retrievals resulting in live births $s^{b, c}$

Percentage of transfers resulting in live births $s^{b, c}$

Percentage of transfers resulting in singleton live births ${ }^{b}$

Percentage of cancellations ${ }^{b}$

Average number of embryos transferred

Percentage of pregnancies with twins ${ }^{b}$

Percentage of pregnancies with triplets or more ${ }^{b}$

Percentage of live births having multiple infants ${ }^{b, c}$

\section{Frozen Embryos from Nondonor Eggs}

Number of transfers

Percentage of transfers resulting in live births ${ }^{b, c}$

Average number of embryos transferred

\section{Donor Eggs}

Number of transfers

Percentage of transfers resulting in live births $s^{b, c}$

Average number of embryos transferred

\section{Age of Woman}

35-37 38-40

$41-42^{d}$

\section{$<35$}

21

23.8

5

9

44.7

23.8

$1 / 5$

$0 / 9$

34.2

$(8.2-47.2)$
23.8

$1 / 5$

$0 / 9$

(19.6-51.4)

23.8

$1 / 5$

$0 / 9$

36.1

23.8

$1 / 4$

$0 / 8$

19.4

19.0

$1 / 4$

$0 / 8$

2.6

0.0

$0 / 5$

$0 / 9$

2.5

2.7

3.3

2.6

$3 / 17$

$1 / 5$

$0 / 1$

$3 / 17$

$6 / 13$

$0 / 5$

$0 / 1$

$1 / 5$

$0 / 1$

2

$0 / 2$

1

$0 / 1$

1

$0 / 1$

2.5

2.0

4.0

All Ages Combined ${ }^{\mathrm{e}}$ Fresh Embryos

Frozen Embryos

5

$4 / 5$

0

\section{CURRENT CLINIC SERVICES AND PROFILE}

Current Name: This clinic has closed or reorganized since 2006. Information on current clinic services and profile therefore is not provided here. Contact the NASS Help Desk for current information about this clinic.

\footnotetext{
${ }^{a}$ Reflects patient and treatment characteristics of ART cycles performed in 2006 using fresh nondonor eggs or embryos.

b When fewer than 20 cycles are reported in an age category, rates are shown as a fraction and confidence intervals are not given. Calculating percentages from fractions may be misleading and is not encouraged.

${ }^{c}$ A multiple-infant birth is counted as one live birth.

d Clinic-specific outcome rates are unreliable for women older than 42 undergoing ART cycles using fresh or frozen embryos with nondonor eggs. Readers are urged to review national outcomes for these age groups (see page 27).

e All ages (including ages $>42$ ) are reported together because previous data show that patient age does not materially affect success with donor eggs.
} 


\section{FERTILITY AND WOMEN'S HEALTH CENTER OF LOUISIANA LAFAYETTE, LOUISIANA}

A comparison of clinic success rates may not be meaningful because patient medical characteristics and treatment approaches vary from clinic to clinic. For more details about this, along with information on how to interpret the statistics in this table, see pages $79-88$.

\section{ART CYCLE PROFILE}

\begin{tabular}{|c|c|c|c|c|c|c|c|}
\hline \multicolumn{4}{|c|}{ Type of ART ${ }^{a}$} & \multicolumn{4}{|c|}{ Patient Diagnosis } \\
\hline IVF & $100 \%$ & Procedural Factors: & & Tubal factor & $34 \%$ & Other factor & $<1 \%$ \\
\hline GIFT & $0 \%$ & With ICSI & $82 \%$ & Ovulatory dysfunction & $12 \%$ & Unknown factor & $3 \%$ \\
\hline ZIFT & $0 \%$ & Unstimulated & $0 \%$ & Diminished ovarian reserve & $10 \%$ & Multiple Factors: & \\
\hline \multirow[t]{3}{*}{ Combination } & $0 \%$ & Used gestational carrier & $4 \%$ & Endometriosis & $7 \%$ & Female factors only & $4 \%$ \\
\hline & & & & Uterine factor & $2 \%$ & Female \& male factors & $5 \%$ \\
\hline & & & & Male factor & $23 \%$ & & \\
\hline
\end{tabular}

2006 PREGNANCY SUCCESS RATES

Data verified by John Storment, MD

\section{Type of Cycle}

\section{Fresh Embryos from Nondonor Eggs}

Number of cycles

Percentage of cycles resulting in pregnancies ${ }^{b}$

Percentage of cycles resulting in live births $s^{b, c}$

(Confidence Interval)

Percentage of retrievals resulting in live births $s^{b, c}$

Percentage of transfers resulting in live births $s^{b, c}$

Percentage of transfers resulting in singleton live births ${ }^{b}$

Percentage of cancellations ${ }^{b}$

Average number of embryos transferred

Percentage of pregnancies with twins ${ }^{b}$

Percentage of pregnancies with triplets or more ${ }^{b}$

Percentage of live births having multiple infants ${ }^{b, c}$

\section{Frozen Embryos from Nondonor Eggs}

Number of transfers

Percentage of transfers resulting in live births $s^{\mathrm{b}, \mathrm{c}}$

Average number of embryos transferred

\section{Donor Eggs}

Number of transfers

Percentage of transfers resulting in live births $s^{b, c}$

Average number of embryos transferred

\section{Age of Woman}

35-37 38-40

$41-42^{d}$

$<35$

17

$7 / 17$

$5 / 17$

8

2

42.0

34.0

$2 / 8$

$1 / 2$

(21.2-48.8)

34.7

34.7

$5 / 17$

$2 / 8$

$1 / 2$

\section{4}

$5 / 16$

$2 / 7$

$1 / 2$

2.0

$3 / 16$

$2 / 7$

$1 / 7$

$1 / 2$

2.0

$0 / 17$

$1 / 8$

$1 / 2$

2.4

2.4

$2 / 7$

2.9

$0 / 2$

33.3

$0 / 7$

$1 / 2$

4.0

0.0

$2 / 5$

$0 / 2$

$0 / 1$

$6 / 17$

$1 / 2$

$0 / 1$

$0 / 1$

$\begin{array}{cccc}12 & 7 & 3 & 0 \\ 2 / 12 & 1 / 7 & 0 / 3 & \\ 2.8 & 1.9 & 2.0 & \end{array}$

All Ages Combined ${ }^{\mathrm{e}}$

Fresh Embryos

\section{1}

$1 / 1$

2.0
Frozen Embryos

\section{1}

$0 / 1$

3.0

\section{CURRENT CLINIC SERVICES AND PROFILE}

Current Name: Fertility and Women's Health Center of Louisiana

Donor egg? Yes Gestational carriers? Yes

Donor embryo? Yes Cryopreservation? Yes

Single women? Yes

SART member? Yes

Verified lab accreditation? Yes

(See Appendix C for details.)

${ }^{a}$ Reflects patient and treatment characteristics of ART cycles performed in 2006 using fresh nondonor eggs or embryos.

${ }^{b}$ When fewer than 20 cycles are reported in an age category, rates are shown as a fraction and confidence intervals are not given. Calculating percentages from fractions may be misleading and is not encouraged.

${ }^{c}$ A multiple-infant birth is counted as one live birth.

d Clinic-specific outcome rates are unreliable for women older than 42 undergoing ART cycles using fresh or frozen embryos with nondonor eggs. Readers are urged to review national outcomes for these age groups (see page 27).

e All ages (including ages $>42$ ) are reported together because previous data show that patient age does not materially affect success with donor eggs. 


\section{THE FERTILITY INSTITUTE OF NEW ORLEANS \\ MANDEVILLE, LOUISIANA}

A comparison of clinic success rates may not be meaningful because patient medical characteristics and treatment approaches vary from clinic to clinic. For more details about this, along with information on how to interpret the statistics in this table, see pages 79-88.

\section{ART CYCLE PROFILE}

\begin{tabular}{|c|c|c|c|c|c|c|c|}
\hline \multicolumn{4}{|c|}{ Type of ART ${ }^{a}$} & \multicolumn{4}{|c|}{ Patient Diagnosis } \\
\hline IVF & $100 \%$ & Procedural Factors: & & Tubal factor & $22 \%$ & Other factor & $24 \%$ \\
\hline GIFT & $0 \%$ & With ICSI & $47 \%$ & Ovulatory dysfunction & $11 \%$ & Unknown factor & $5 \%$ \\
\hline ZIFT & $0 \%$ & Unstimulated & $0 \%$ & Diminished ovarian reserve & $<1 \%$ & Multiple Factors: & \\
\hline \multirow[t]{3}{*}{ Combination } & $0 \%$ & Used gestational carrier & $<1 \%$ & Endometriosis & $11 \%$ & Female factors only & $1 \%$ \\
\hline & & & & Uterine factor & $<1 \%$ & Female \& male factors & $5 \%$ \\
\hline & & & & Male factor & $20 \%$ & & \\
\hline
\end{tabular}

2006 PREGNANCY SUCCESS RATES

$$
\text { Type of Cycle }
$$

\section{Fresh Embryos from Nondonor Eggs}

Number of cycles

Percentage of cycles resulting in pregnancies ${ }^{b}$

Percentage of cycles resulting in live births $s^{b, c}$

(Confidence Interval)

Percentage of retrievals resulting in live births $s^{b, c}$

Percentage of transfers resulting in live births $s^{b, c}$

Percentage of transfers resulting in singleton live births ${ }^{b}$

Percentage of cancellations ${ }^{b}$

Average number of embryos transferred

Percentage of pregnancies with twins ${ }^{b}$

Percentage of pregnancies with triplets or more ${ }^{b}$

Percentage of live births having multiple infants ${ }^{b, c}$

\section{Frozen Embryos from Nondonor Eggs}

Number of transfers

Percentage of transfers resulting in live births ${ }^{b, c}$

Average number of embryos transferred

\section{Donor Eggs}

Number of transfers

Percentage of transfers resulting in live births $s^{b, c}$

Average number of embryos transferred
Data verified by Richard P. Dickey, MD, PhD

\section{Age of Woman}

35-37 $38-40 \quad 41-42^{d}$

$<35$

71

$\begin{array}{cc}46 & 31 \\ 17.4 & 6.5 \\ 10.9 & 3.2\end{array}$

25.4

19.7

(3.6-23.6)

$(0.1-16.7)$

(23.8-40.2)

(11.2-30.9)

12.5

3.8

37.8

27.5

14.7

4.8

21.6

5.9

4.8

9.0

18.3

13.0

16.1

2.2

2.4

2.4

2.5

42.0

$4 / 18$

4.0

$1 / 18$

$1 / 8$

$0 / 2$

45.2

$3 / 14$

$2 / 8$

$0 / 2$

$3 / 5$

$0 / 1$

28

35.7

11

$4 / 11$

5

2

1.9

1.8

$0 / 5$

$0 / 2$

1.8

1.0

\section{All Ages Combined}

Fresh Embryos

Frozen Embryos

$\begin{array}{cc}7 & 2 \\ 5 / 7 & 0 / 2 \\ 2.3 & 2.0\end{array}$

\section{CURRENT CLINIC SERVICES AND PROFILE}

Current Name: The Fertility Institute of New Orleans

Donor egg? Yes Gestational carriers? Yes

Donor embryo? Yes Cryopreservation? Yes

Single women? Yes

SART member? Yes

Verified lab accreditation? Yes

(See Appendix C for details.)

${ }^{a}$ Reflects patient and treatment characteristics of ART cycles performed in 2006 using fresh nondonor eggs or embryos.

b When fewer than 20 cycles are reported in an age category, rates are shown as a fraction and confidence intervals are not given. Calculating percentages from fractions may be misleading and is not encouraged.

${ }^{c}$ A multiple-infant birth is counted as one live birth.

d Clinic-specific outcome rates are unreliable for women older than 42 undergoing ART cycles using fresh or frozen embryos with nondonor eggs. Readers are urged to review national outcomes for these age groups (see page 27).

e All ages (including ages $>42$ ) are reported together because previous data show that patient age does not materially affect success with donor eggs. 


\section{CENTER FOR FERTILITY AND REPRODUCTIVE HEALTH SHREVEPORT, LOUISIANA}

A comparison of clinic success rates may not be meaningful because patient medical characteristics and treatment approaches vary from clinic to clinic. For more details about this, along with information on how to interpret the statistics in this table, see pages $79-88$.

\section{ART CYCLE PROFILE}

\begin{tabular}{|c|c|c|c|c|c|c|c|}
\hline \multicolumn{4}{|c|}{ Type of ART ${ }^{a}$} & \multicolumn{4}{|c|}{ Patient Diagnosis } \\
\hline IVF & $100 \%$ & Procedural Factors: & & Tubal factor & $23 \%$ & Other factor & $<1 \%$ \\
\hline GIFT & $0 \%$ & With ICSI & $56 \%$ & Ovulatory dysfunction & $10 \%$ & Unknown factor & $12 \%$ \\
\hline ZIFT & $0 \%$ & Unstimulated & $0 \%$ & Diminished ovarian reserve & $10 \%$ & Multiple Factors: & \\
\hline \multirow[t]{3}{*}{ Combination } & $0 \%$ & Used gestational carrier & $0 \%$ & Endometriosis & $14 \%$ & Female factors only & $8 \%$ \\
\hline & & & & Uterine factor & $0 \%$ & Female \& male factors & $7 \%$ \\
\hline & & & & Male factor & $15 \%$ & & \\
\hline
\end{tabular}

\section{PREGNANCY SUCCESS RATES}

Data verified by David T. Vandermolen, MD

\section{Type of Cycle}

\section{Fresh Embryos from Nondonor Eggs}

Number of cycles

Percentage of cycles resulting in pregnancies ${ }^{b}$

Percentage of cycles resulting in live births ${ }^{b, c}$

(Confidence Interval)

Percentage of retrievals resulting in live births $s^{b, c}$

Percentage of transfers resulting in live births ${ }^{b, c}$

Percentage of transfers resulting in singleton live births ${ }^{b}$

Percentage of cancellations ${ }^{b}$

Average number of embryos transferred

Percentage of pregnancies with twins ${ }^{b}$

Percentage of pregnancies with triplets or more ${ }^{b}$

Percentage of live births having multiple infants ${ }^{b, c}$

\section{Frozen Embryos from Nondonor Eggs}

Number of transfers

Percentage of transfers resulting in live births $s^{b, c}$

Average number of embryos transferred

\section{Donor Eggs}

Number of transfers

Percentage of transfers resulting in live births $s^{b, c}$

Average number of embryos transferred

\section{Age of Woman}

35-37 38-40

$41-42^{d}$

$<35$

27

59.3

12

8

42.6

48.1

$4 / 12$

$4 / 8$

35.3

(24.1-47.8)

(28.7-68.1)

42.1

42.9

59.1

$2 / 12$

$2 / 8$

28.6

16.2

59.1

$2 / 9$

$2 / 5$

40.9

$2 / 9$

$2 / 5$

18.5

$2 / 9$

$1 / 5$

2.6

34.5

3.3

$3 / 12$

$3 / 8$

34.5

$2 / 16$

4.2

4.6

0.0

$2 / 16$

$0 / 4$

$1 / 4$

33.3

$4 / 13$

$0 / 4$

$0 / 4$

$0 / 2$

$1 / 2$

9

$2 / 9$

2.6

\section{5}

$0 / 5$

2.0

\section{2 \\ $0 / 2$}

4.5
2

$0 / 2$

2.0

All Ages Combined ${ }^{\mathrm{e}}$

Fresh Embryos 2

$2 / 2$

3.0
Frozen Embryos 1

$0 / 1$

3.0

\section{CURRENT CLINIC SERVICES AND PROFILE}

Current Name: Center for Fertility and Reproductive Health

Donor egg? Yes Gestational carriers? Yes

Donor embryo? Yes Cryopreservation? Yes

Single women? Yes

SART member? Yes

Verified lab accreditation? Yes

(See Appendix C for details.)

${ }^{a}$ Reflects patient and treatment characteristics of ART cycles performed in 2006 using fresh nondonor eggs or embryos.

${ }^{b}$ When fewer than 20 cycles are reported in an age category, rates are shown as a fraction and confidence intervals are not given. Calculating percentages from fractions may be misleading and is not encouraged.

${ }^{c}$ A multiple-infant birth is counted as one live birth.

d Clinic-specific outcome rates are unreliable for women older than 42 undergoing ART cycles using fresh or frozen embryos with nondonor eggs. Readers are urged to review national outcomes for these age groups (see page 27).

e All ages (including ages $>42$ ) are reported together because previous data show that patient age does not materially affect success with donor eggs. 


\section{MAINE CENTER FOR REPRODUCTIVE HEALTH SOUTH PORTLAND, MAINE}

A comparison of clinic success rates may not be meaningful because patient medical characteristics and treatment approaches vary from clinic to clinic. For more details about this, along with information on how to interpret the statistics in this table, see pages 79-88.

\section{ART CYCLE PROFILE}

\begin{tabular}{|c|c|c|c|c|c|c|c|}
\hline \multicolumn{4}{|c|}{ Type of ART ${ }^{a}$} & \multicolumn{4}{|c|}{ Patient Diagnosis } \\
\hline IVF & $100 \%$ & Procedural Factors: & & Tubal factor & $23 \%$ & Other factor & $2 \%$ \\
\hline GIFT & $0 \%$ & With ICSI & $46 \%$ & Ovulatory dysfunction & $4 \%$ & Unknown factor & $24 \%$ \\
\hline ZIFT & $0 \%$ & Unstimulated & $0 \%$ & Diminished ovarian reserve & $1 \%$ & Multiple Factors: & \\
\hline \multirow[t]{3}{*}{ Combination } & $0 \%$ & Used gestational carrier & $0 \%$ & Endometriosis & $1 \%$ & Female factors only & $12 \%$ \\
\hline & & & & Uterine factor & $0 \%$ & Female \& male factors & $19 \%$ \\
\hline & & & & Male factor & $13 \%$ & & \\
\hline
\end{tabular}

2006 PREGNANCY SUCCESS RATES

Data verified by Beth Hartog, MD

\section{Type of Cycle}

\section{Fresh Embryos from Nondonor Eggs}

Number of cycles

Percentage of cycles resulting in pregnancies ${ }^{b}$

Percentage of cycles resulting in live births $s^{b, c}$

(Confidence Interval)

Percentage of retrievals resulting in live births $s^{b, c}$

Percentage of transfers resulting in live births $s^{b, c}$

Percentage of transfers resulting in singleton live births ${ }^{b}$

Percentage of cancellations ${ }^{b}$

Average number of embryos transferred

Percentage of pregnancies with twins ${ }^{b}$

Percentage of pregnancies with triplets or more ${ }^{b}$

Percentage of live births having multiple infants ${ }^{b, c}$

\section{Frozen Embryos from Nondonor Eggs}

Number of transfers

Percentage of transfers resulting in live births $s^{b, c}$

Average number of embryos transferred

\section{Donor Eggs}

Number of transfers

Percentage of transfers resulting in live births $s^{b, c}$

Average number of embryos transferred

\section{Age of Woman}

35-37

38-40

$41-42^{d}$

$<35$

18

17

7

50.0

$8 / 18$

$2 / 17$

$1 / 7$

47.4

$6 / 18$

$1 / 17$

$1 / 7$

$(31.0-64.2)$

48.6

$6 / 17$

$1 / 16$

$1 / 6$

51.4

$6 / 17$

$1 / 15$

$1 / 6$

37.1

$4 / 17$

$0 / 15$

$1 / 6$

2.6

$1 / 18$

$1 / 17$

$1 / 7$

2.0

2.3

2.5

2.3

$5 / 19$

$1 / 8$

$1 / 2$

$0 / 1$

$0 / 19$

$1 / 8$

$0 / 2$

$0 / 1$

$5 / 18$

$2 / 6$

$1 / 1$

$0 / 1$

\begin{tabular}{|c|c|c|c|}
\hline 5 & 0 & 1 & 1 \\
\hline $3 / 5$ & & $0 / 1$ & $0 / 1$ \\
\hline 2.2 & & 3.0 & 4.0 \\
\hline
\end{tabular}

All Ages Combined ${ }^{\mathrm{e}}$

Fresh Embryos

Frozen Embryos

0

0

\section{CURRENT CLINIC SERVICES AND PROFILE}

Current Name: Maine Center for Reproductive Health

Donor egg? No Gestational carriers? No

Donor embryo? No Cryopreservation? Yes

Single women? Yes

SART member?

Yes

Verified lab accreditation?

(See Appendix C for details.)

\footnotetext{
${ }^{a}$ Reflects patient and treatment characteristics of ART cycles performed in 2006 using fresh nondonor eggs or embryos.

b When fewer than 20 cycles are reported in an age category, rates are shown as a fraction and confidence intervals are not given. Calculating percentages from fractions may be misleading and is not encouraged.

${ }^{c}$ A multiple-infant birth is counted as one live birth.

d Clinic-specific outcome rates are unreliable for women older than 42 undergoing ART cycles using fresh or frozen embryos with nondonor eggs. Readers are urged to review national outcomes for these age groups (see page 27).

${ }^{e}$ All ages (including ages $>42$ ) are reported together because previous data show that patient age does not materially affect success with donor eggs.
} 
THE CENTER FOR ASSISTED REPRODUCTIVE TECHNOLOGY AT UNION MEMORIAL BALTIMORE, MARYLAND

A comparison of clinic success rates may not be meaningful because patient medical characteristics and treatment approaches vary from clinic to clinic. For more details about this, along with information on how to interpret the statistics in this table, see pages 79-88.

\section{ART CYCLE PROFILE}

$$
\text { Type of ART }{ }^{\mathrm{a}}
$$

\begin{tabular}{|c|c|c|c|c|c|c|c|}
\hline IVF & $100 \%$ & Procedural Factors: & & Tubal factor & $13 \%$ & Other factor & $1 \%$ \\
\hline GIFT & $0 \%$ & With ICSI & $69 \%$ & Ovulatory dysfunction & $12 \%$ & Unknown factor & $2 \%$ \\
\hline ZIFT & $0 \%$ & Unstimulated & $0 \%$ & Diminished ovarian reserve & $11 \%$ & Multiple Factors: & \\
\hline \multirow[t]{3}{*}{ Combination } & $0 \%$ & Used gestational carrier & $1 \%$ & Endometriosis & $5 \%$ & Female factors only & \\
\hline & & & & Uterine factor & $0 \%$ & Female \& male factors & \\
\hline & & & & Male factor & $14 \%$ & & \\
\hline
\end{tabular}

2006 PREGNANCY SUCCESS RATES

\section{Type of Cycle}

\section{Fresh Embryos from Nondonor Eggs}

Number of cycles

Percentage of cycles resulting in pregnancies ${ }^{b}$

Percentage of cycles resulting in live births ${ }^{b, c}$

(Confidence Interval)

Percentage of retrievals resulting in live births $s^{b, c}$

Percentage of transfers resulting in live births $s^{b, c}$

Percentage of transfers resulting in singleton live births ${ }^{b}$

Percentage of cancellations ${ }^{b}$

Average number of embryos transferred

Percentage of pregnancies with twins ${ }^{b}$

Percentage of pregnancies with triplets or more ${ }^{\mathrm{b}}$

Percentage of live births having multiple infants ${ }^{b, c}$

\section{Frozen Embryos from Nondonor Eggs}

Number of transfers

Percentage of transfers resulting in live births ${ }^{\mathrm{b}, \mathrm{c}}$

Average number of embryos transferred

\section{Donor Eggs}

Number of transfers

Percentage of transfers resulting in live births $s^{b, c}$

Average number of embryos transferred
Data verified by Nathan G. Berger, MD

\section{Age of Woman}

35-37 38-40

$41-42^{d}$

$<35$

21

23.8

19.0

22.6

$(5.4-41.9)$

24

23

62

$(12.9-35.0)$

$4 / 15$

$4 / 14$

$0 / 14$

28.6

2.6

$4 / 5$

$0 / 5$

$4 / 4$

16.7

12.5

4.3

25.5

(2.7-32.4)

$3 / 19$

21.8

$3 / 15$

(0.1-21.9)

2.3

17.4

4.3

$2 / 14$

3

$\begin{array}{cc}6 & 3 \\ 0 / 6 & 0 / 3\end{array}$

2.0

1.8

All Ages Combined ${ }^{\mathrm{e}}$
Fresh Embryos

6

$2 / 6$

2.3
5.0

$1 / 17$

$1 / 17$

13.0

2.9

$0 / 1$

$0 / 1$

$0 / 1$

\section{CURRENT CLINIC SERVICES AND PROFILE}

Current Name: The Center for Assisted Reproductive Technology at Union Memorial

\begin{tabular}{ll|ll|ll} 
Donor egg? & Yes & Gestational carriers? & Yes & SART member? & Yes \\
Donor embryo? & No & Cryopreservation? & Yes & Verified lab accreditation? & Yes \\
Single women? & Yes & & & (See Appendix C for details.)
\end{tabular}

\footnotetext{
${ }_{b}^{a}$ Reflects patient and treatment characteristics of ART cycles performed in 2006 using fresh nondonor eggs or embryos.

b When fewer than 20 cycles are reported in an age category, rates are shown as a fraction and confidence intervals are not given. Calculating percentages from fractions may be misleading and is not encouraged.

${ }_{\mathrm{d}}^{\mathrm{c}}$ A multiple-infant birth is counted as one live birth.

${ }^{d}$ Clinic-specific outcome rates are unreliable for women older than 42 undergoing ART cycles using fresh or frozen embryos with nondonor eggs. Readers are urged to review national outcomes for these age groups (see page 27).

e All ages (including ages $>42$ ) are reported together because previous data show that patient age does not materially affect success with donor eggs.
} 


\section{GBMC FERTILITY CENTER \\ BALTIMORE, MARYLAND}

A comparison of clinic success rates may not be meaningful because patient medical characteristics and treatment approaches vary from clinic to clinic. For more details about this, along with information on how to interpret the statistics in this table, see pages 79-88.

\section{ART CYCLE PROFILE}

\section{Type of ART ${ }^{\mathrm{a}}$}

IVF

GIFT $100 \%$ Procedural Factors:

ZIFT

Combination
$0 \%$ With ICSI

$0 \%$ Unstimulated

$0 \%$ Used gestational carrier $<1 \%$
Tubal factor

Ovulatory dysfunction

Diminished ovarian reserve

Endometriosis

Uterine factor

Male factor

\section{Patient Diagnosis}

$\begin{array}{rlr}11 \% & \text { Other factor } & 8 \% \\ 3 \% & \text { Unknown factor } & 13 \% \\ 8 \% & \text { Multiple Factors: } & \\ 14 \% & \text { Female factors only } & 8 \% \\ <1 \% & \text { Female \& male factors } & 6 \% \\ 28 \% & & \end{array}$

Data verified by Eugene Katz, MD

2006 PREGNANCY SUCCESS RATES

\section{Age of Woman}

\section{Type of Cycle}

\section{Fresh Embryos from Nondonor Eggs}

Number of cycles

Percentage of cycles resulting in pregnancies ${ }^{b}$

Percentage of cycles resulting in live births ${ }^{b, c}$

(Confidence Interval)

Percentage of retrievals resulting in live births $s^{b, c}$

Percentage of transfers resulting in live births $s^{b, c}$

Percentage of transfers resulting in singleton live births ${ }^{b}$

Percentage of cancellations ${ }^{b}$

Average number of embryos transferred

Percentage of pregnancies with twins ${ }^{b}$

Percentage of pregnancies with triplets or more ${ }^{b}$

Percentage of live births having multiple infants ${ }^{\mathrm{b}, \mathrm{c}}$

\section{Frozen Embryos from Nondonor Eggs}

Number of transfers

Percentage of transfers resulting in live births ${ }^{b, c}$

Average number of embryos transferred

\section{Donor Eggs}

Number of transfers

Percentage of transfers resulting in live births $s^{b, c}$

Average number of embryos transferred

35-37 38-40

\section{$<35$}

81

76

44.6

43.2

30.3

38.1

33.3

27.6

(31.4-45.2)

39.5

(23.2-44.7)

40.7

37.0

39.7

31.8

33.9

26.5

27.9

24.2

13.2

3.5

9.9

2.9

25.7

32.2

5.7

29.6

3.5

30.4

4.3

28.6

35.1

41

41.5

16

$6 / 16$

3.0

12

3.4

All Ages Combined ${ }^{\mathrm{e}}$
Fresh Embryos

\section{7}

$9 / 17$

2.1

$$
41-42^{d}
$$

28

10.7

3.6

(18.0-39.1)

(0.1-18.3)

4.2

4.8

4.8

14.3

3.7

$0 / 3$

$0 / 3$

3

Frozen Embryos

18

$8 / 18$

3.1

\section{CURRENT CLINIC SERVICES AND PROFILE}

Current Name: This clinic has closed or reorganized since 2006. Information on current clinic services and profile therefore is not provided here. Contact the NASS Help Desk for current information about this clinic.

\footnotetext{
${ }^{a}$ Reflects patient and treatment characteristics of ART cycles performed in 2006 using fresh nondonor eggs or embryos.

${ }^{b}$ When fewer than 20 cycles are reported in an age category, rates are shown as a fraction and confidence intervals are not given. Calculating percentages from fractions may be misleading and is not encouraged.

${ }^{\mathrm{C}}$ A multiple-infant birth is counted as one live birth.

d Clinic-specific outcome rates are unreliable for women older than 42 undergoing ART cycles using fresh or frozen embryos with nondonor eggs. Readers are urged to review national outcomes for these age groups (see page 27).

e All ages (including ages $>42$ ) are reported together because previous data show that patient age does not materially affect success with donor eggs.
} 


\section{UMMS-CENTER FOR ADVANCED REPRODUCTIVE TECHNOLOGIES BALTIMORE, MARYLAND}

A comparison of clinic success rates may not be meaningful because patient medical characteristics and treatment approaches vary from clinic to clinic. For more details about this, along with information on how to interpret the statistics in this table, see pages 79-88.

\section{ART CYCLE PROFILE}

$$
\text { Type of ART }{ }^{\mathrm{a}}
$$

\begin{tabular}{|c|c|c|c|c|c|c|c|}
\hline IVF & $100 \%$ & Procedural Factors: & & Tubal factor & $11 \%$ & Other factor & $4 \%$ \\
\hline GIFT & $0 \%$ & With ICSI & $55 \%$ & Ovulatory dysfunction & $4 \%$ & Unknown factor & $12 \%$ \\
\hline ZIFT & $0 \%$ & Unstimulated & $0 \%$ & Diminished ovarian reserve & $13 \%$ & Multiple Factors: & \\
\hline \multirow[t]{3}{*}{ Combination } & $0 \%$ & Used gestational carrier & $0 \%$ & Endometriosis & $0 \%$ & Female factors only & \\
\hline & & & & Uterine factor & $1 \%$ & Female \& male factors & $28 \%$ \\
\hline & & & & Male factor & $20 \%$ & & \\
\hline
\end{tabular}

2006 PREGNANCY SUCCESS RATES

Data verified by Howard D. McClamrock, MD

\section{Type of Cycle}

\section{Fresh Embryos from Nondonor Eggs}

Number of cycles

Percentage of cycles resulting in pregnancies ${ }^{b}$

Percentage of cycles resulting in live births $s^{b, c}$

(Confidence Interval)

Percentage of retrievals resulting in live births $s^{b, c}$

Percentage of transfers resulting in live births $s^{b, c}$

Percentage of transfers resulting in singleton live births ${ }^{b}$

Percentage of cancellations ${ }^{b}$

Average number of embryos transferred

Percentage of pregnancies with twins ${ }^{b}$

Percentage of pregnancies with triplets or more ${ }^{b}$

Percentage of live births having multiple infants ${ }^{b, c}$

\section{Frozen Embryos from Nondonor Eggs}

Number of transfers

Percentage of transfers resulting in live births ${ }^{\mathrm{b}, \mathrm{c}}$

Average number of embryos transferred

\section{Donor Eggs}

Number of transfers

Percentage of transfers resulting in live births ${ }^{\mathrm{b}, \mathrm{c}}$

Average number of embryos transferred

\section{Age of Woman}

35-37 38-40

$41-42^{d}$

$<35$

19

24

10

$\begin{array}{cccc}36 & 19 & 24 & 10 \\ 44.4 & 7 / 19 & 8.3 & 2 / 10 \\ 44.4 & 6 / 19 & 4.2 & 1 / 10\end{array}$

(27.9-61.9)

57.1

69.6

69.6

22.2

2.6

$1 / 16$

$0 / 16$

$0 / 16$

$6 / 14$
$6 / 13$
$6 / 13$
$5 / 19$
2.7
$0 / 7$
$0 / 7$
$0 / 6$

(0.1-21.1)

$1 / 13 \quad 1 / 9$

$1 / 10$

$1 / 6$

$1 / 10$

$1 / 6$

45.8

$1 / 10$

2.0

3.5

$0 / 2$

$0 / 2$

$0 / 2$

$0 / 2$

$0 / 1$

$0 / 1$

\section{1}

$1 / 1$

2.0

$$
1
$$

$0 / 1$

1.0

\section{1 \\ $0 / 1$}

2.0
1

$0 / 1$

4.0

\section{All Ages Combined ${ }^{\mathrm{e}}$}

Fresh Embryos

0
Frozen Embryos

\section{2}

$1 / 2$

2.5

\section{CURRENT CLINIC SERVICES AND PROFILE}

Current Name: UMMS-Center for Advanced Reproductive Technologies

Donor egg? Yes

Donor embryo? No

Single women? Yes

\section{Gestational carriers? Yes}

Cryopreservation? Yes
SART member? Yes

Verified lab accreditation? Yes

(See Appendix C for details.)

${ }^{a}$ Reflects patient and treatment characteristics of ART cycles performed in 2006 using fresh nondonor eggs or embryos.

${ }^{b}$ When fewer than 20 cycles are reported in an age category, rates are shown as a fraction and confidence intervals are not given. Calculating percentages from fractions may be misleading and is not encouraged.

${ }^{c}$ A multiple-infant birth is counted as one live birth.

d Clinic-specific outcome rates are unreliable for women older than 42 undergoing ART cycles using fresh or frozen embryos with nondonor eggs. Readers are urged to review national outcomes for these age groups (see page 27).

e All ages (including ages $>42$ ) are reported together because previous data show that patient age does not materially affect success with donor eggs. 


\section{JOHNS HOPKINS FERTILITY CENTER LUTHERVILLE, MARYLAND}

A comparison of clinic success rates may not be meaningful because patient medical characteristics and treatment approaches vary from clinic to clinic. For more details about this, along with information on how to interpret the statistics in this table, see pages 79-88.

\section{ART CYCLE PROFILE}

\section{Type of ART ${ }^{\mathrm{a}}$}

IVF

GIFT

$100 \%$ Procedural Factors:

ZIFT

Combination
$0 \%$ With ICSI

$0 \%$ Unstimulated

$0 \%$ Used gestational carrier $<1 \%$
Tubal factor

Ovulatory dysfunction

Diminished ovarian reserve

Endometriosis

Uterine factor

Male factor

\section{Patient Diagnosis}

$\begin{array}{rlr}19 \% & \text { Other factor } & 9 \% \\ 7 \% & \text { Unknown factor } & 5 \% \\ 28 \% & \text { Multiple Factors: } & \\ 12 \% & \text { Female factors only } & 4 \% \\ 0 \% & \text { Female \& male factors } & 2 \% \\ 15 \% & & \end{array}$

Data verified by Jairo E. Garcia, MD

2006 PREGNANCY SUCCESS RATES

\section{Age of Woman}

\section{Type of Cycle}

\section{Fresh Embryos from Nondonor Eggs}

Number of cycles

Percentage of cycles resulting in pregnancies ${ }^{b}$

Percentage of cycles resulting in live births $s^{b, c}$

(Confidence Interval)

Percentage of retrievals resulting in live births $s^{b, c}$

Percentage of transfers resulting in live births $s^{b, c}$

Percentage of transfers resulting in singleton live births ${ }^{b}$

Percentage of cancellations ${ }^{b}$

Average number of embryos transferred

Percentage of pregnancies with twins ${ }^{b}$

Percentage of pregnancies with triplets or more ${ }^{b}$

Percentage of live births having multiple infants ${ }^{b, c}$

\section{Frozen Embryos from Nondonor Eggs}

Number of transfers

Percentage of transfers resulting in live births ${ }^{b, c}$

Average number of embryos transferred

\section{Donor Eggs}

Number of transfers

Percentage of transfers resulting in live births $s^{b, c}$

Average number of embryos transferred

35-37 38-40

$41-42^{d}$

$<35$

47

23.4

19.1

29

33

25.0

18.3

(9.1-33.3)

27.6

24.1

6.1

(9.5-30.4)

19.0

22.0

(10.3-43.5)

0.0

21.2

25.0

28.0

$(0.0-10.6)$

11.5

16.7

$7 / 18$

0.0

3.3

12.8

$6 / 18$

$0 / 19$

$0 / 19$

2.3

2.3

$7 / 15$

$0 / 15$

$2 / 11$

$1 / 11$

$5 / 11$

$3 / 9$

13.8

30.3

2.1

$0 / 2$

$0 / 2$

$1 / 8$

$1 / 8$

$1 / 7$

$\begin{array}{cccc}18 & 12 & 10 & 3 \\ 2 / 18 & 1 / 12 & 0 / 10 & 0 / 3 \\ 2.4 & 2.3 & 2.5 & 2.3\end{array}$

All Ages Combined

Fresh Embryos

Frozen Embryos

11

$6 / 11$

5

1.9

$0 / 5$

2.0

\section{CURRENT CLINIC SERVICES AND PROFILE}

Current Name: Johns Hopkins Fertility Center

Donor egg? Yes

Donor embryo? No

Single women? Yes

\section{Gestational carriers? Yes}

Cryopreservation? Yes
SART member?

Yes

Verified lab accreditation?

(See Appendix C for details.)

${ }^{a}$ Reflects patient and treatment characteristics of ART cycles performed in 2006 using fresh nondonor eggs or embryos.

b When fewer than 20 cycles are reported in an age category, rates are shown as a fraction and confidence intervals are not given. Calculating percentages from fractions may be misleading and is not encouraged.

${ }^{c}$ A multiple-infant birth is counted as one live birth.

d Clinic-specific outcome rates are unreliable for women older than 42 undergoing ART cycles using fresh or frozen embryos with nondonor eggs. Readers are urged to review national outcomes for these age groups (see page 27).

e All ages (including ages $>42$ ) are reported together because previous data show that patient age does not materially affect success with donor eggs. 


\section{CENTER FOR REPRODUCTIVE MEDICINE ROCKVILLE, MARYLAND}

A comparison of clinic success rates may not be meaningful because patient medical characteristics and treatment approaches vary from clinic to clinic. For more details about this, along with information on how to interpret the statistics in this table, see pages 79-88.

\section{ART CYCLE PROFILE}

$$
\text { Type of ART }{ }^{\mathrm{a}}
$$

IVF

$$
\text { 100\% Procedural Factors: }
$$

GIFT

$0 \%$ With ICSI

ZIFT

$0 \%$ Unstimulated

Combination

0\% Used gestational carrier

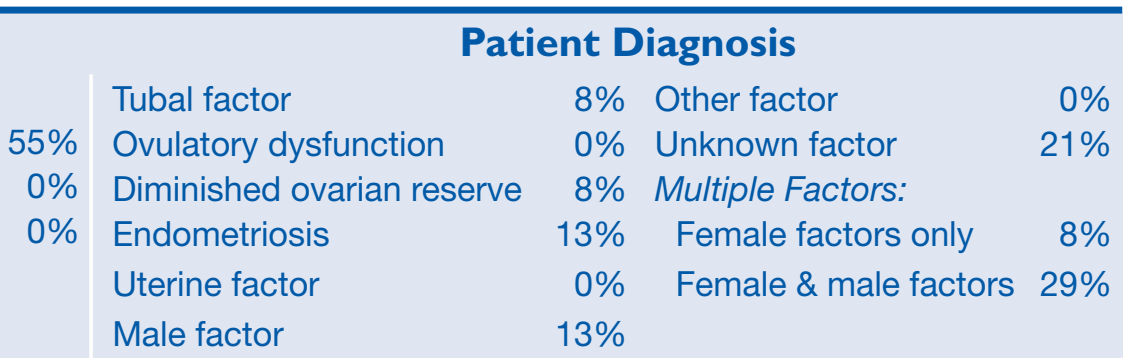

2006 PREGNANCY SUCCESS RATES

Data verified by Burt Littman, MD

\section{Type of Cycle}

\section{Fresh Embryos from Nondonor Eggs}

Number of cycles

Percentage of cycles resulting in pregnancies ${ }^{b}$

Percentage of cycles resulting in live births $s^{b, c}$

(Confidence Interval)

Percentage of retrievals resulting in live births $s^{b, c}$

Percentage of transfers resulting in live births $s^{b, c}$

Percentage of transfers resulting in singleton live births ${ }^{b}$

Percentage of cancellations ${ }^{b}$

Average number of embryos transferred

Percentage of pregnancies with twins ${ }^{b}$

Percentage of pregnancies with triplets or more ${ }^{b}$

Percentage of live births having multiple infants ${ }^{b, c}$

\section{Frozen Embryos from Nondonor Eggs}

Number of transfers

Percentage of transfers resulting in live births $s^{\mathrm{b}, \mathrm{c}}$

Average number of embryos transferred

\section{Donor Eggs}

Number of transfers

Percentage of transfers resulting in live births ${ }^{\mathrm{b}, \mathrm{c}}$

Average number of embryos transferred

\section{Age of Woman}

$\begin{array}{cccc}<35 & 35-37 & 38-40 & \mathbf{4 1 - 4 2}^{\text {d }} \\ 3 & 6 & 7 & 0 \\ 1 / 3 & 1 / 6 & 1 / 7 & \\ 1 / 3 & 0 / 6 & 1 / 7 & \end{array}$

$1 / 2$

$0 / 6$

$1 / 7$

$1 / 2$

$0 / 5$

$1 / 7$

$1 / 2$

$0 / 5$

$0 / 7$

$1 / 3$

$0 / 6$

$0 / 7$

2.0

1.6

2.3

$0 / 1$

$0 / 1$

$1 / 1$

$0 / 1$

$0 / 1$

$0 / 1$

$0 / 1$

$1 / 1$

\section{CURRENT CLINIC SERVICES AND PROFILE}

Current Name: Center for Reproductive Medicine

\begin{tabular}{l|l|l} 
Donor egg? Yes $\quad$ Gestational carriers? Yes &
\end{tabular}

Donor embryo? No Cryopreservation? Yes

$0 \quad 1 \quad 2$

$1 / 1 \quad 0 / 2$

$1.0 \quad 1.5$

\section{All Ages Combined ${ }^{\mathrm{e}}$ \\ Fresh Embryos \\ Frozen Embryos}

0 0

Single women? Yes

SART member? Yes

Verified lab accreditation? Yes

(See Appendix C for details.)

${ }^{a}$ Reflects patient and treatment characteristics of ART cycles performed in 2006 using fresh nondonor eggs or embryos.

${ }^{b}$ When fewer than 20 cycles are reported in an age category, rates are shown as a fraction and confidence intervals are not given. Calculating percentages from fractions may be misleading and is not encouraged.

${ }^{c}$ A multiple-infant birth is counted as one live birth.

d Clinic-specific outcome rates are unreliable for women older than 42 undergoing ART cycles using fresh or frozen embryos with nondonor eggs. Readers are urged to review national outcomes for these age groups (see page 27).

${ }^{e}$ All ages (including ages $>42$ ) are reported together because previous data show that patient age does not materially affect success with donor eggs. 


\section{SHADY GROVE FERTILITY REPRODUCTIVE SCIENCE CENTER ROCKVILLE, MARYLAND}

A comparison of clinic success rates may not be meaningful because patient medical characteristics and treatment approaches vary from clinic to clinic. For more details about this, along with information on how to interpret the statistics in this table, see pages 79-88.

\section{ART CYCLE PROFILE}

\section{Type of ART ${ }^{\mathrm{a}}$}

IVF

GIFT

ZIFT

Combination
100\% Procedural Factors:

$0 \%$ With ICSI

$0 \%$ Unstimulated

$0 \%$ Used gestational carrier $<1 \%$

\section{Patient Diagnosis}

$\begin{array}{rlr}11 \% & \text { Other factor } & 8 \% \\ 7 \% & \text { Unknown factor } & 25 \% \\ 13 \% & \text { Multiple Factors: } & \\ 6 \% & \text { Female factors only } & 4 \% \\ 3 \% & \text { Female \& male factors } & 5 \% \\ 19 \% & & \end{array}$

Data verified by Michael J. Levy, MD

2006 PREGNANCY SUCCESS RATES

Age of Woman

Type of Cycle
Tubal factor

Ovulatory dysfunction

Diminished ovarian reserve

Endometriosis

Uterine factor

Male factor

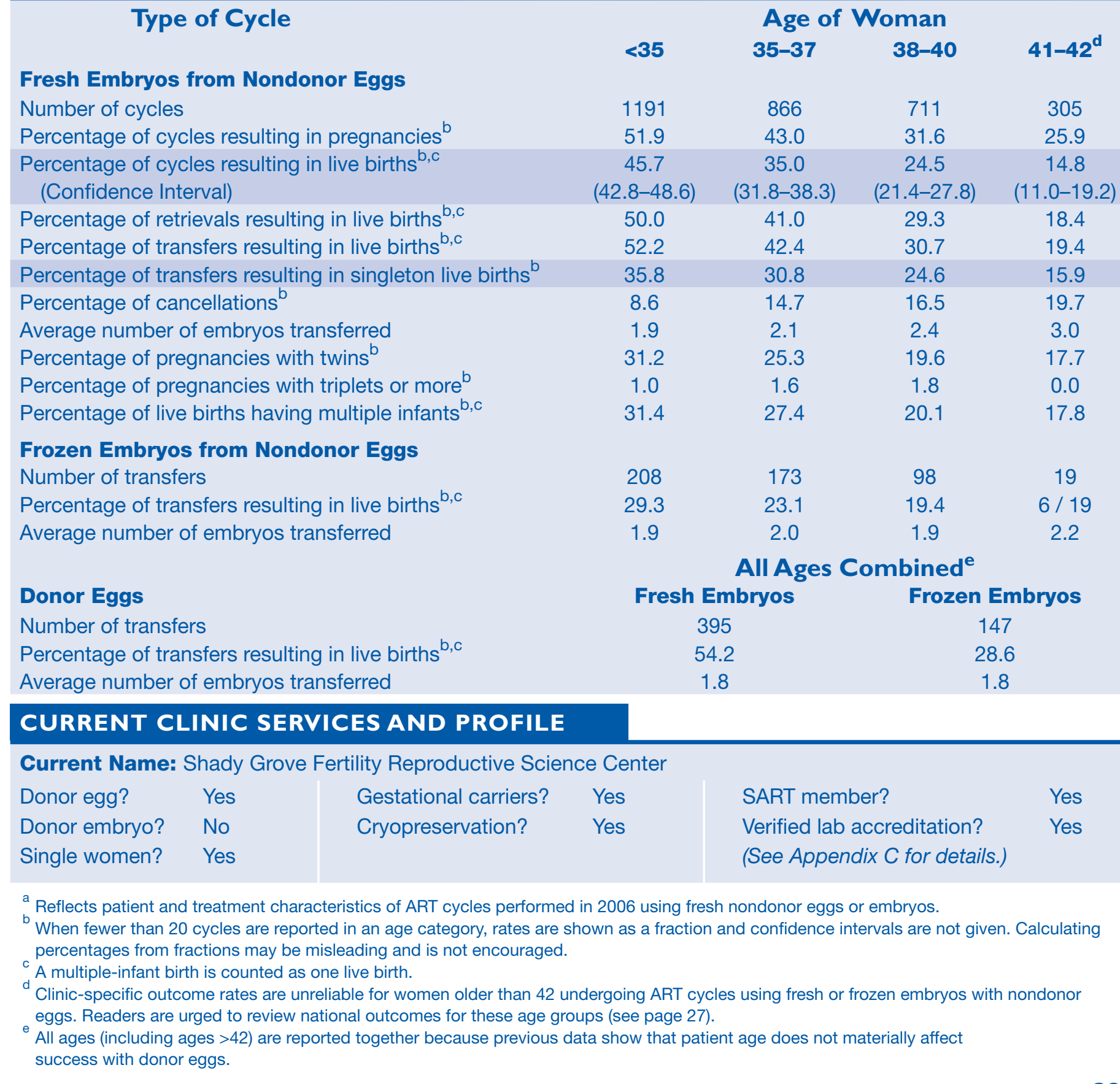




\section{FERTILITY CENTER OF MARYLAND \\ TOWSON, MARYLAND}

A comparison of clinic success rates may not be meaningful because patient medical characteristics and treatment approaches vary from clinic to clinic. For more details about this, along with information on how to interpret the statistics in this table, see pages 79-88.

\section{ART CYCLE PROFILE}

\section{Type of ART ${ }^{\mathrm{a}}$}

IVF

GIFT

ZIFT
Combination

\section{0\% Procedural Factors:}

$0 \%$ With ICSI

$0 \%$ Unstimulated

$0 \%$ Used gestational carrier

\begin{tabular}{r|l|} 
& \\
\hline $28 \%$ & Tubal factor \\
\hline$<1 \%$ & Ovulatory dysfunction \\
$0 \%$ & Endominished ovarian reserve \\
& Uterine factor \\
& Male factor
\end{tabular}

Patient Diagnosis

$\begin{array}{rlr}10 \% & \text { Other factor } & 6 \% \\ 8 \% & \text { Unknown factor } & 2 \% \\ 6 \% & \text { Multiple Factors: } & \\ 7 \% & \text { Female factors only } & 23 \% \\ 1 \% & \text { Female \& male factors } & 26 \% \\ 10 \% & & \end{array}$

Data verified by Santiago L. Padilla, MD

2006 PREGNANCY SUCCESS RATES

\section{Type of Cycle}

\section{Fresh Embryos from Nondonor Eggs}

Number of cycles

Percentage of cycles resulting in pregnancies ${ }^{b}$

Percentage of cycles resulting in live births $s^{b, c}$

(Confidence Interval)

Percentage of retrievals resulting in live births $s^{b, c}$

Percentage of transfers resulting in live births $s^{b, c}$

Percentage of transfers resulting in singleton live births ${ }^{b}$

Percentage of cancellations ${ }^{b}$

Average number of embryos transferred

Percentage of pregnancies with twins ${ }^{b}$

Percentage of pregnancies with triplets or more ${ }^{b}$

Percentage of live births having multiple infants ${ }^{b, c}$

\section{Frozen Embryos from Nondonor Eggs}

Number of transfers

Percentage of transfers resulting in live births ${ }^{\mathrm{b}, \mathrm{c}}$

Average number of embryos transferred

\section{Donor Eggs}

Number of transfers

Percentage of transfers resulting in live births $s^{b, c}$

Average number of embryos transferred

\section{Age of Woman}

35-37 38-40

$41-42^{d}$

\section{$<35$}

56

33.9

20

22

100
46.0

28.6

30.0

13.6

41.0

(17.3-42.2)

25.0

9.1

(31.3-51.3)

43.6

37.2

$(8.7-49.1)$

(1.1-29.2)

44.1

37.2

$5 / 16$

$2 / 19$

28.0

23.3

$5 / 16$

$2 / 19$

6.0

23.2

$5 / 16$

$2 / 19$

2.2

2.3

20.0

13.6

41.3

$6 / 19$

2.2

$1 / 19$

36.6

$6 / 16$

3.0

4.3

$0 / 3$

$0 / 3$

$0 / 6$

$0 / 2$

$0 / 5$

28

25.0

13

8

30.4

1.9

$5 / 13$

$3 / 8$

2.1

All Ages Combined ${ }^{\mathrm{e}}$
Fresh Embryos 10

$5 / 10$

2.1
Frozen Embryos

4

$3 / 4$

1.8

\section{CURRENT CLINIC SERVICES AND PROFILE}

Current Name: Fertility Center of Maryland

Donor egg? Yes

Donor embryo? No

Gestational carriers? Yes

Cryopreservation? Yes

Single women? Yes

SART member?

Verified lab accreditation?

(See Appendix C for details.)

${ }^{a}$ Reflects patient and treatment characteristics of ART cycles performed in 2006 using fresh nondonor eggs or embryos.

${ }^{b}$ When fewer than 20 cycles are reported in an age category, rates are shown as a fraction and confidence intervals are not given. Calculating percentages from fractions may be misleading and is not encouraged.

${ }^{c}$ A multiple-infant birth is counted as one live birth.

${ }^{d}$ Clinic-specific outcome rates are unreliable for women older than 42 undergoing ART cycles using fresh or frozen embryos with nondonor eggs. Readers are urged to review national outcomes for these age groups (see page 27).

${ }^{e}$ All ages (including ages $>42$ ) are reported together because previous data show that patient age does not materially affect success with donor eggs. 


\section{BRIGHAM AND WOMEN'S HOSPITAL ART CENTER BOSTON, MASSACHUSETTS}

A comparison of clinic success rates may not be meaningful because patient medical characteristics and treatment approaches vary from clinic to clinic. For more details about this, along with information on how to interpret the statistics in this table, see pages 79-88.

\section{ART CYCLE PROFILE}

\begin{tabular}{|c|c|c|c|c|c|c|c|}
\hline \multicolumn{4}{|c|}{ Type of $A R T^{a}$} & \multicolumn{4}{|c|}{ Patient Diagnosis } \\
\hline IVF & $>99 \%$ & Procedural Factors: & & Tubal factor & $10 \%$ & Other factor & $21 \%$ \\
\hline GIFT & $<1 \%$ & With ICSI & $39 \%$ & Ovulatory dysfunction & $5 \%$ & Unknown factor & $20 \%$ \\
\hline ZIFT & $0 \%$ & Unstimulated & $0 \%$ & Diminished ovarian reserve & $4 \%$ & Multiple Factors: & \\
\hline \multirow[t]{3}{*}{ Combination } & $0 \%$ & Used gestational carrier & $1 \%$ & Endometriosis & $5 \%$ & Female factors only & $6 \%$ \\
\hline & & & & Uterine factor & $2 \%$ & Female \& male factors & $10 \%$ \\
\hline & & & & Male factor & $19 \%$ & & \\
\hline
\end{tabular}

2006 PREGNANCY SUCCESS RATES

Data verified by Elizabeth S. Ginsburg, MD

\section{Type of Cycle}

\section{Fresh Embryos from Nondonor Eggs}

Number of cycles

Percentage of cycles resulting in pregnancies ${ }^{b}$

Percentage of cycles resulting in live births $s^{b, c}$

(Confidence Interval)

Percentage of retrievals resulting in live births $s^{b, c}$

Percentage of transfers resulting in live births $s^{b, c}$

Percentage of transfers resulting in singleton live births ${ }^{b}$

Percentage of cancellations ${ }^{b}$

Average number of embryos transferred

Percentage of pregnancies with twins ${ }^{b}$

Percentage of pregnancies with triplets or more ${ }^{b}$

Percentage of live births having multiple infants ${ }^{b, c}$

\section{Frozen Embryos from Nondonor Eggs}

Number of transfers

Percentage of transfers resulting in live births ${ }^{b, c}$

Average number of embryos transferred

\section{Donor Eggs}

Number of transfers

Percentage of transfers resulting in live births $s^{b, c}$

Average number of embryos transferred

\section{Age of Woman}

35-37 38-40

$41-42^{d}$

$<35$

408

381

177

496

37.0

27.0

24.9

38.5

30.9

19.2

(34.2-42.9)

(26.4-35.6)

(15.3-23.5)

39.8

32.6

20.5

35.3

22.1

16.1

16.4

43.1

27.5

6.6

2.8

27.2

2.2

4.9

(11.3-22.7)

17.3

19.2

3.2

1.8

26.0

0.4

23.6

2.0

22.2

27.4

17.9

5.1

3.8

20.5

0.0

6.9

$\begin{array}{cccc}95 & 59 & 28 & 8 \\ 36.8 & 27.1 & 17.9 & 2 / 8 \\ 2.1 & 2.4 & 2.6 & 3.3\end{array}$

\section{All Ages Combined}

Fresh Embryos

73

50.7

2.0
Frozen Embryos

46

45.7

2.2

\section{CURRENT CLINIC SERVICES AND PROFILE}

Current Name: Brigham and Women's Hospital ART Center

Donor egg? Yes Gestational carriers? Yes

Donor embryo? Yes Cryopreservation? Yes

Single women? Yes

SART member? Yes

Verified lab accreditation? Yes

(See Appendix C for details.)

${ }^{a}$ Reflects patient and treatment characteristics of ART cycles performed in 2006 using fresh nondonor eggs or embryos.

${ }^{b}$ When fewer than 20 cycles are reported in an age category, rates are shown as a fraction and confidence intervals are not given. Calculating percentages from fractions may be misleading and is not encouraged.

${ }^{c}$ A multiple-infant birth is counted as one live birth.

d Clinic-specific outcome rates are unreliable for women older than 42 undergoing ART cycles using fresh or frozen embryos with nondonor eggs. Readers are urged to review national outcomes for these age groups (see page 27).

e All ages (including ages $>42$ ) are reported together because previous data show that patient age does not materially affect success with donor eggs. 


\section{VINCENT IVF UNIT \\ MASSACHUSETTS GENERAL HOSPITAL \\ BOSTON, MASSACHUSETTS}

A comparison of clinic success rates may not be meaningful because patient medical characteristics and treatment approaches vary from clinic to clinic. For more details about this, along with information on how to interpret the statistics in this table, see pages $79-88$.

\section{ART CYCLE PROFILE}

\begin{tabular}{|c|c|c|c|c|c|c|c|}
\hline \multicolumn{4}{|c|}{ Type of ART ${ }^{a}$} & \multicolumn{4}{|c|}{ Patient Diagnosis } \\
\hline IVF & $100 \%$ & Procedural Factors: & & Tubal factor & $6 \%$ & Other factor & $5 \%$ \\
\hline GIFT & $0 \%$ & With ICSI & $45 \%$ & Ovulatory dysfunction & $6 \%$ & Unknown factor & $21 \%$ \\
\hline ZIFT & $0 \%$ & Unstimulated & $0 \%$ & Diminished ovarian reserve & $12 \%$ & Multiple Factors: & \\
\hline Combination & $0 \%$ & Used gestational carrier & $<1 \%$ & Endometriosis & $3 \%$ & Female factors only & $7 \%$ \\
\hline & & & & Uterine factor & $<1 \%$ & Female \& male factors & $17 \%$ \\
\hline
\end{tabular}

\section{PREGNANCY SUCCESS RATES}

Data verified by Thomas L. Toth, MD

\section{Type of Cycle}

Age of Woman

$\begin{array}{cccc}\mathbf{3} 5 & \mathbf{3 5 - 3 7} & \mathbf{3 8 - 4 0} & \mathbf{4 1 - 4 2}^{\mathbf{d}} \\ & & & \\ 162 & 121 & 103 & 32 \\ 37.7 & 41.3 & 31.1 & 21.9 \\ 35.2 & 33.9 & 25.2 & 18.8 \\ 27.9-43.1) & (25.5-43.0) & (17.2-34.8) & (7.2-36.4) \\ 38.0 & 36.9 & 26.8 & 20.7 \\ 41.9 & 38.3 & 28.3 & 21.4 \\ 35.3 & 28.0 & 20.7 & 14.3 \\ 7.4 & 8.3 & 5.8 & 9.4 \\ 1.7 & 2.0 & 2.4 & 3.4 \\ 18.0 & 28.0 & 28.1 & 3 / 7 \\ 1.6 & 0.0 & 0.0 & 0 / 7 \\ 15.8 & 26.8 & 26.9 & 2 / 6\end{array}$

$32 \quad 26$

$50.0 \quad 38.5$

1.7

\section{4}

$5 / 14$

1.9
7

$0 / 7$

2.4
1.7

Average number of embryos transferred

All Ages Combined ${ }^{\mathrm{e}}$

\section{Donor Eggs}

Number of transfers

Percentage of transfers resulting in live births $s^{b, c}$

Average number of embryos transferred

\section{Fresh Embryos}

29

55.2

1.8
Frozen Embryos

\section{6}

$1 / 6$

2.0

\section{CURRENT CLINIC SERVICES AND PROFILE}

Current Name: Vincent IVF Unit, Massachusetts General Hospital
Donor egg? Yes
Donor embryo? No
Gestational carriers? Yes
Cryopreservation?
SART member?
Verified lab accreditation?
(See Appendix $C$ for details.
Yes
Single women? Yes

${ }^{a}$ Reflects patient and treatment characteristics of ART cycles performed in 2006 using fresh nondonor eggs or embryos.

${ }^{b}$ When fewer than 20 cycles are reported in an age category, rates are shown as a fraction and confidence intervals are not given. Calculating percentages from fractions may be misleading and is not encouraged.

c A multiple-infant birth is counted as one live birth.

d Clinic-specific outcome rates are unreliable for women older than 42 undergoing ART cycles using fresh or frozen embryos with nondonor eggs. Readers are urged to review national outcomes for these age groups (see page 27).

e All ages (including ages $>42$ ) are reported together because previous data show that patient age does not materially affect success with donor eggs. 


\section{REPRODUCTIVE SCIENCE CENTER \\ LEXINGTON, MASSACHUSETTS}

A comparison of clinic success rates may not be meaningful because patient medical characteristics and treatment approaches vary from clinic to clinic. For more details about this, along with information on how to interpret the statistics in this table, see pages 79-88.

\section{ART CYCLE PROFILE}

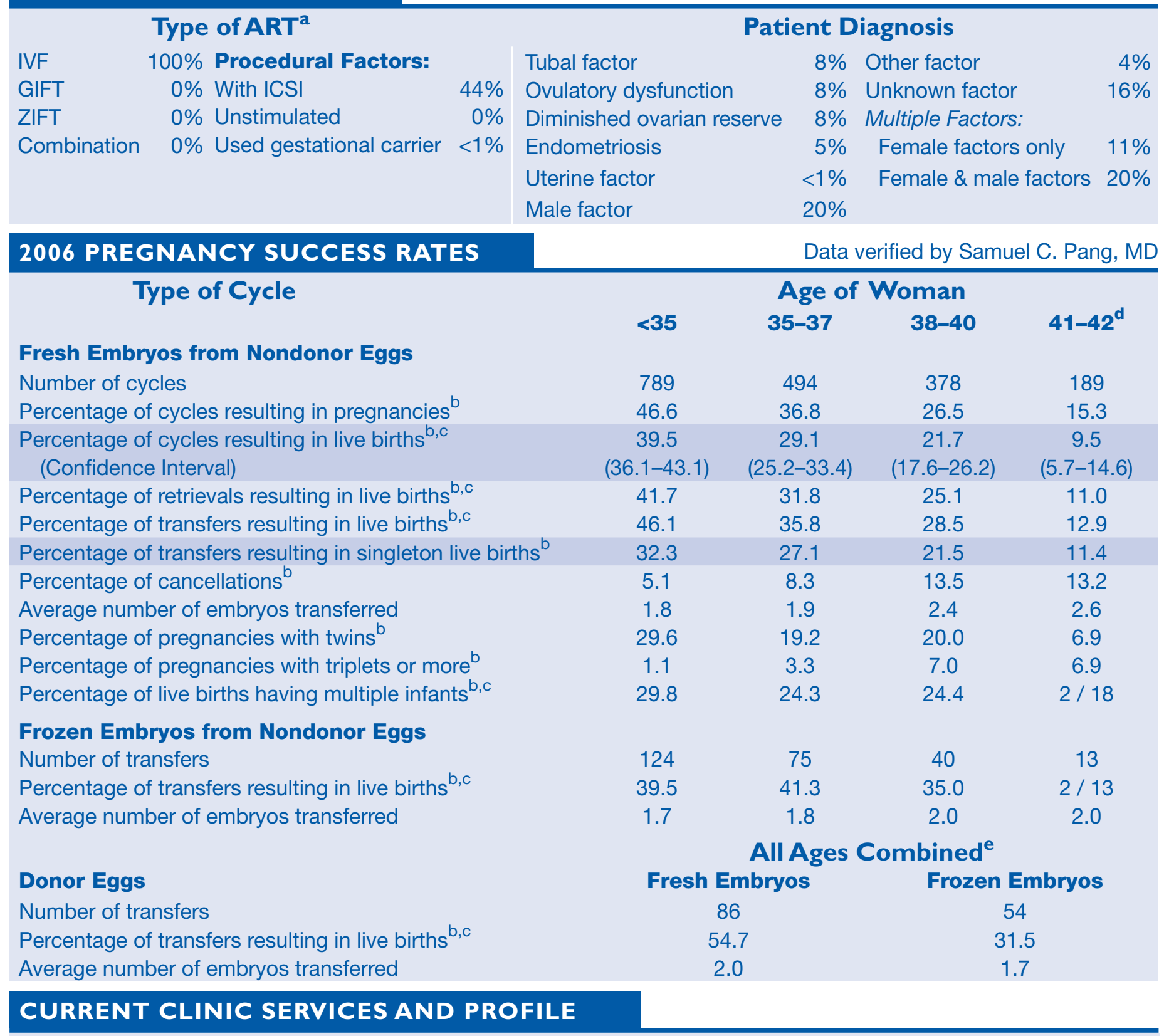

Current Name: This clinic has closed or reorganized since 2006. Information on current clinic services and profile therefore is not provided here. Contact the NASS Help Desk for current information about this clinic.

\footnotetext{
${ }^{a}$ Reflects patient and treatment characteristics of ART cycles performed in 2006 using fresh nondonor eggs or embryos.

${ }^{b}$ When fewer than 20 cycles are reported in an age category, rates are shown as a fraction and confidence intervals are not given. Calculating percentages from fractions may be misleading and is not encouraged.

${ }^{\mathrm{C}}$ A multiple-infant birth is counted as one live birth.

d Clinic-specific outcome rates are unreliable for women older than 42 undergoing ART cycles using fresh or frozen embryos with nondonor eggs. Readers are urged to review national outcomes for these age groups (see page 27).

${ }^{e}$ All ages (including ages $>42$ ) are reported together because previous data show that patient age does not materially affect success with donor eggs.
} 


\section{FERTILITY CENTERS OF NEW ENGLAND, INC. NEW ENGLAND CLINICS OF REPRODUCTIVE MEDICINE, INC. READING, MASSACHUSETTS}

A comparison of clinic success rates may not be meaningful because patient medical characteristics and treatment approaches vary from clinic to clinic. For more details about this, along with information on how to interpret the statistics in this table, see pages $79-88$.

\section{ART CYCLE PROFILE}

\begin{tabular}{|c|c|c|c|c|c|c|c|}
\hline \multicolumn{4}{|c|}{ Type of $\mathrm{ART}^{\mathrm{a}}$} & \multicolumn{4}{|c|}{ Patient Diagnosis } \\
\hline IVF & $100 \%$ & Procedural Factors: & & Tubal factor & $9 \%$ & Other factor & $8 \%$ \\
\hline GIFT & $0 \%$ & With ICSI & $57 \%$ & Ovulatory dysfunction & $7 \%$ & Unknown factor & $12 \%$ \\
\hline $\mathrm{ZIFT}$ & $0 \%$ & Unstimulated & $<1 \%$ & Diminished ovarian reserve & $7 \%$ & Multiple Factors: & \\
\hline \multirow[t]{3}{*}{ Combination } & $0 \%$ & Used gestational carrier & $<1 \%$ & Endometriosis & $5 \%$ & Female factors only & $11 \%$ \\
\hline & & & & Uterine factor & $4 \%$ & Female \& male factors & $18 \%$ \\
\hline & & & & Male factor & $21 \%$ & & \\
\hline
\end{tabular}

\section{PREGNANCY SUCCESS RATES}

Data verified by R. lan Hardy, MD, PhD

\section{Type of Cycle}

\section{Fresh Embryos from Nondonor Eggs}

Number of cycles

Percentage of cycles resulting in pregnancies ${ }^{b}$

Percentage of cycles resulting in live births $s^{b, c}$

(Confidence Interval)

Percentage of retrievals resulting in live births $s^{b, c}$

Percentage of transfers resulting in live births $s^{b, c}$

Percentage of transfers resulting in singleton live births ${ }^{b}$

Percentage of cancellations ${ }^{b}$

Average number of embryos transferred

Percentage of pregnancies with twins ${ }^{b}$

Percentage of pregnancies with triplets or more ${ }^{b}$

Percentage of live births having multiple infants ${ }^{b, c}$

\section{Frozen Embryos from Nondonor Eggs}

Number of transfers

Percentage of transfers resulting in live births ${ }^{\mathrm{b}, \mathrm{c}}$

Average number of embryos transferred

\section{Donor Eggs}

Number of transfers

Percentage of transfers resulting in live births $s^{\mathrm{b}, \mathrm{c}}$

Average number of embryos transferred

\section{Age of Woman}

35-37 38-40

$41-42^{d}$

\section{$<35$}

173

172

81

42.0

32.4

23.8

9.9

35.8

27.7

16.9

4.9

(30.8-41.0)

36.4

(21.2-35.1)

(11.6-23.3)

(1.4-12.2)

39.6

30.0

17.9

5.2

31.8

23.6

7.0

28.7

21.2

21.1

7.0

1.7

7.5

5.8

4.9

2.1

26.8

1.3

2.1

35.7

27.6

0.0

2.3

2.6

$0 / 8$

14.6

$0 / 8$

33.3

10.3

$0 / 4$
58

22.4

1.9
33

15.2

2.1
16

$3 / 16$

1.9
8

$2 / 8$

1.8
All Ages Combined ${ }^{\mathrm{e}}$
Fresh Embryos 64

57.8

2.0
Frozen Embryos 29

37.9

\section{CURRENT CLINIC SERVICES AND PROFILE}

Current Name: Fertility Centers of New England, Inc., New England Clinics of Reproductive Medicine, Inc.

Donor egg? Yes

Donor embryo? Yes

Single women? Yes

\section{Gestational carriers? Yes}

Cryopreservation?
SART member?

Verified lab accreditation?

(See Appendix C for details.)

${ }^{a}$ Reflects patient and treatment characteristics of ART cycles performed in 2006 using fresh nondonor eggs or embryos.

b When fewer than 20 cycles are reported in an age category, rates are shown as a fraction and confidence intervals are not given. Calculating percentages from fractions may be misleading and is not encouraged.

${ }^{c}$ A multiple-infant birth is counted as one live birth.

d Clinic-specific outcome rates are unreliable for women older than 42 undergoing ART cycles using fresh or frozen embryos with nondonor eggs. Readers are urged to review national outcomes for these age groups (see page 27).

e All ages (including ages $>42$ ) are reported together because previous data show that patient age does not materially affect success with donor eggs. 


\section{BAYSTATE REPRODUCTIVE MEDICINE SPRINGFIELD, MASSACHUSETTS}

A comparison of clinic success rates may not be meaningful because patient medical characteristics and treatment approaches vary from clinic to clinic. For more details about this, along with information on how to interpret the statistics in this table, see pages 79-88.

\section{ART CYCLE PROFILE}

\begin{tabular}{|c|c|c|c|c|c|c|c|}
\hline \multicolumn{4}{|c|}{ Type of ART ${ }^{a}$} & \multicolumn{4}{|c|}{ Patient Diagnosis } \\
\hline IVF & $100 \%$ & Procedural Factors: & & Tubal factor & $10 \%$ & Other factor & $4 \%$ \\
\hline GIFT & $0 \%$ & With ICSI & $54 \%$ & Ovulatory dysfunction & $11 \%$ & Unknown factor & $23 \%$ \\
\hline ZIFT & $0 \%$ & Unstimulated & $0 \%$ & Diminished ovarian reserve & $2 \%$ & Multiple Factors: & \\
\hline \multirow[t]{3}{*}{ Combination } & $0 \%$ & Used gestational carrier & $<1 \%$ & Endometriosis & $6 \%$ & Female factors only & $7 \%$ \\
\hline & & & & Uterine factor & $2 \%$ & Female \& male factors & $12 \%$ \\
\hline & & & & Male factor & $24 \%$ & & \\
\hline
\end{tabular}

2006 PREGNANCY SUCCESS RATES

Data verified by Daniel Grow, MD

\section{Type of Cycle}

\section{Fresh Embryos from Nondonor Eggs}

Number of cycles

Percentage of cycles resulting in pregnancies ${ }^{b}$

Percentage of cycles resulting in live births $s^{b, c}$

(Confidence Interval)

Percentage of retrievals resulting in live births $s^{b, c}$

Percentage of transfers resulting in live births $s^{b, c}$

Percentage of transfers resulting in singleton live births ${ }^{b}$

Percentage of cancellations ${ }^{b}$

Average number of embryos transferred

Percentage of pregnancies with twins ${ }^{b}$

Percentage of pregnancies with triplets or more ${ }^{b}$

Percentage of live births having multiple infants ${ }^{\mathrm{b}, \mathrm{c}}$

\section{Frozen Embryos from Nondonor Eggs}

Number of transfers

Percentage of transfers resulting in live births ${ }^{b, c}$

Average number of embryos transferred

\section{Donor Eggs}

Number of transfers

Percentage of transfers resulting in live births $s^{b, c}$

Average number of embryos transferred

\section{Age of Woman}

35-37 38-40

$41-42^{d}$

\section{$<35$}

77

42.9

68

47

51.1

31.2

36.8

23.4

47.3

(21.1-42.7)

25.0

12.8

(38.5-56.2)

50.0

33.8

(15.3-37.0)

(4.8-25.7)

52.1

37.5

27.4

18.2

32.8

31.3

28.3

20.0

5.3

7.8

21.7

16.7

1.8

2.3

18.2

37.3

0.0

1.5

37.1

16.7

52

19.2

33

15.2

2.2

2.0
8.8

3.0

36.0

8.0

4 / 17

25

16.0

2.9

$0 / 5$

3.2

29.8

3.8

$2 / 11$

$0 / 11$

$1 / 6$

All Ages Combined

Fresh Embryos

29

58.6

1.9
Frozen Embryos 23

26.1

2.0

\section{CURRENT CLINIC SERVICES AND PROFILE}

Current Name: Baystate Reproductive Medicine

Donor egg? Yes

Donor embryo? No

Single women? Yes

\section{Gestational carriers? Yes}

Cryopreservation? Yes
SART member?

Yes

Verified lab accreditation?

(See Appendix C for details.)

${ }^{a}$ Reflects patient and treatment characteristics of ART cycles performed in 2006 using fresh nondonor eggs or embryos.

b When fewer than 20 cycles are reported in an age category, rates are shown as a fraction and confidence intervals are not given. Calculating percentages from fractions may be misleading and is not encouraged.

${ }^{\mathrm{C}}$ A multiple-infant birth is counted as one live birth.

d Clinic-specific outcome rates are unreliable for women older than 42 undergoing ART cycles using fresh or frozen embryos with nondonor eggs. Readers are urged to review national outcomes for these age groups (see page 27).

e All ages (including ages $>42$ ) are reported together because previous data show that patient age does not materially affect success with donor eggs. 


\section{BOSTON IVF \\ WALTHAM, MASSACHUSETTS}

A comparison of clinic success rates may not be meaningful because patient medical characteristics and treatment approaches vary from clinic to clinic. For more details about this, along with information on how to interpret the statistics in this table, see pages $79-88$.

\section{ART CYCLE PROFILE}

\begin{tabular}{|c|c|c|c|c|c|c|c|}
\hline \multicolumn{4}{|c|}{ Type of $\mathrm{ART}^{\mathrm{a}}$} & \multicolumn{4}{|c|}{ Patient Diagnosis } \\
\hline IVF & $>99 \%$ & Procedural Factors: & & Tubal factor & $9 \%$ & Other factor & $26 \%$ \\
\hline GIFT & $<1 \%$ & With ICSI & $39 \%$ & Ovulatory dysfunction & $8 \%$ & Unknown factor & $28 \%$ \\
\hline $\mathrm{ZIFT}$ & $0 \%$ & Unstimulated & $0 \%$ & Diminished ovarian reserve & $0 \%$ & Multiple Factors: & \\
\hline \multirow[t]{3}{*}{ Combination } & $0 \%$ & Used gestational carrier & $<1 \%$ & Endometriosis & $3 \%$ & Female factors only & $2 \%$ \\
\hline & & & & Uterine factor & $1 \%$ & Female \& male factors & $4 \%$ \\
\hline & & & & Male factor & $19 \%$ & & \\
\hline
\end{tabular}

\section{PREGNANCY SUCCESS RATES}

Data verified by Michael M. Alper, MD

\section{Type of Cycle}

\section{Fresh Embryos from Nondonor Eggs}

Number of cycles

Percentage of cycles resulting in pregnancies ${ }^{b}$

Percentage of cycles resulting in live births ${ }^{b, c}$

(Confidence Interval)

Percentage of retrievals resulting in live births $s^{b, c}$

Percentage of transfers resulting in live births $s^{b, c}$

Percentage of transfers resulting in singleton live births ${ }^{b}$

Percentage of cancellations ${ }^{b}$

Average number of embryos transferred

Percentage of pregnancies with twins ${ }^{b}$

Percentage of pregnancies with triplets or more ${ }^{\mathrm{b}}$

Percentage of live births having multiple infants ${ }^{b, c}$

\section{Frozen Embryos from Nondonor Eggs}

Number of transfers

Percentage of transfers resulting in live births $s^{b, c}$

Average number of embryos transferred

\section{Donor Eggs}

Number of transfers

Percentage of transfers resulting in live births $s^{b, c}$

Average number of embryos transferred

\section{Age of Woman}

35-37 38-40

$41-42^{d}$

\section{$<35$}

506

34.6

498

229

43.5

29.2

25.3

17.0

37.1

(25.3-33.4)

20.3

10.9

(33.5-40.8)

38.9

32.0

$(16.8-24.1)$

(7.2-15.7)

41.8

35.2

23.1

26.6

12.6

24.8

21.6

15.1

4.7

8.7

12.2

12.7

2.0

2.1

32.6

2.6

13.5

30.1

2.9

2.9

18.3

2.9

32.6

29.7

4.0

17.9

2.6

18.8

16.0

137

25.5

77

28.6

56

11

2.0

2.2

19.6

$3 / 11$

2.1

2.5

All Ages Combined ${ }^{\mathrm{e}}$

Fresh Embryos

126

43.7

2.0
Frozen Embryos 84

27.4

1.9

\section{CURRENT CLINIC SERVICES AND PROFILE}

Current Name: Boston IVF

$\begin{array}{ll}\text { Donor egg? } & \text { Yes } \\ \text { Donor embryo? } & \text { No } \\ \text { Single women? } & \text { Yes }\end{array}$

Gestational carriers? Yes

Cryopreservation? Yes
SART member?

Yes

Verified lab accreditation?

(See Appendix C for details.)

${ }^{a}$ Reflects patient and treatment characteristics of ART cycles performed in 2006 using fresh nondonor eggs or embryos.

${ }^{b}$ When fewer than 20 cycles are reported in an age category, rates are shown as a fraction and confidence intervals are not given. Calculating percentages from fractions may be misleading and is not encouraged.

c A multiple-infant birth is counted as one live birth.

d Clinic-specific outcome rates are unreliable for women older than 42 undergoing ART cycles using fresh or frozen embryos with nondonor eggs. Readers are urged to review national outcomes for these age groups (see page 27).

${ }^{e}$ All ages (including ages $>42$ ) are reported together because previous data show that patient age does not materially affect success with donor eggs. 


\section{CENTER FOR REPRODUCTIVE MEDICINE \\ UNIVERSITY OF MICHIGAN REPRODUCTIVE ENDOCRINOLOGY AND INFERTILITY ANN ARBOR, MICHIGAN}

A comparison of clinic success rates may not be meaningful because patient medical characteristics and treatment approaches vary from clinic to clinic. For more details about this, along with information on how to interpret the statistics in this table, see pages $79-88$.

\section{ART CYCLE PROFILE}

\begin{tabular}{|c|c|c|c|c|c|c|}
\hline \multicolumn{3}{|c|}{ Type of $A R T^{a}$} & \multicolumn{4}{|c|}{ Patient Diagnosis } \\
\hline IVF & 100\% Procedural Factors: & & Tubal factor & $6 \%$ & Other factor & $1 \%$ \\
\hline GIFT & 0\% With ICSI & $49 \%$ & Ovulatory dysfunction & $<1 \%$ & Unknown factor & $3 \%$ \\
\hline ZIFT & $0 \%$ Unstimulated & $0 \%$ & Diminished ovarian reserve & $7 \%$ & Multiple Factors: & \\
\hline \multirow[t]{3}{*}{ Combination } & $0 \%$ Used gestational carrier & $0 \%$ & Endometriosis & $1 \%$ & Female factors only & $6 \%$ \\
\hline & & & Uterine factor & $0 \%$ & Female \& male factors & $47 \%$ \\
\hline & & & Male factor & $29 \%$ & & \\
\hline
\end{tabular}

2006 PREGNANCY SUCCESS RATES

Data verified by Senait Fisseha, MD

\section{Type of Cycle}

\section{Fresh Embryos from Nondonor Eggs}

Number of cycles

Percentage of cycles resulting in pregnancies ${ }^{b}$

Percentage of cycles resulting in live births ${ }^{b, c}$

(Confidence Interval)

Percentage of retrievals resulting in live births $s^{b, c}$

Percentage of transfers resulting in live births $s^{b, c}$

Percentage of transfers resulting in singleton live births ${ }^{b}$

Percentage of cancellations ${ }^{b}$

Average number of embryos transferred

Percentage of pregnancies with twins ${ }^{b}$

Percentage of pregnancies with triplets or more ${ }^{b}$

Percentage of live births having multiple infants ${ }^{b, c}$

\section{Frozen Embryos from Nondonor Eggs}

Number of transfers

Percentage of transfers resulting in live births ${ }^{b, c}$

Average number of embryos transferred

\section{Donor Eggs}

Number of transfers

Percentage of transfers resulting in live births $s^{b, c}$

Average number of embryos transferred

\section{Age of Woman}

35-37 $\quad 38-40 \quad 41-42^{d}$

\section{$<35$}

13

6

58
37.9

28

17.9

$3 / 13$

$0 / 6$

36.2

10.7

$2 / 13$

$0 / 6$

(24.0-49.9)

(2.3-28.2)

44.7

14.3

$2 / 8$

$0 / 3$

45.7

15.0

$2 / 7$

$0 / 3$

30.4

15.0

$1 / 7$

$0 / 3$

19.0

25.0

$5 / 13$

$3 / 6$

2.2

2.4

2.6

3.3

\section{3}

4.5

$0 / 5$

$1 / 3$

33.3

$0 / 5$

$0 / 3$

$0 / 3$

$1 / 2$

$\begin{array}{cccc}7 & 7 & 5 & 1 \\ 1 / 7 & 1 / 7 & 1 / 5 & 0 / 1 \\ 2.3 & 2.0 & 2.8 & 2.0\end{array}$

\section{All Ages Combined ${ }^{\mathrm{e}}$}

Fresh Embryos

11

$4 / 11$

1.9
Frozen Embryos 5

$1 / 5$

3.0

\section{CURRENT CLINIC SERVICES AND PROFILE}

Current Name: Center for Reproductive Medicine, University of Michigan Reproductive Endocrinology and Infertility

Donor egg? Yes

Donor embryo? No

Single women? Yes

\begin{abstract}
Gestational carriers? Yes
Cryopreservation?
\end{abstract}

SART member?

Verified lab accreditation?

(See Appendix C for details.)

${ }^{a}$ Reflects patient and treatment characteristics of ART cycles performed in 2006 using fresh nondonor eggs or embryos.

b When fewer than 20 cycles are reported in an age category, rates are shown as a fraction and confidence intervals are not given. Calculating percentages from fractions may be misleading and is not encouraged.

${ }^{\mathrm{C}}$ A multiple-infant birth is counted as one live birth.

d Clinic-specific outcome rates are unreliable for women older than 42 undergoing ART cycles using fresh or frozen embryos with nondonor eggs. Readers are urged to review national outcomes for these age groups (see page 27).

e All ages (including ages $>42$ ) are reported together because previous data show that patient age does not materially affect success with donor eggs. 


\section{CENTER FOR REPRODUCTIVE MEDICINE AND SURGERY, PC BIRMINGHAM, MICHIGAN}

A comparison of clinic success rates may not be meaningful because patient medical characteristics and treatment approaches vary from clinic to clinic. For more details about this, along with information on how to interpret the statistics in this table, see pages $79-88$.

\section{ART CYCLE PROFILE}

\begin{tabular}{|c|c|c|c|c|c|c|c|}
\hline \multicolumn{4}{|c|}{ Type of ART ${ }^{\mathrm{a}}$} & \multicolumn{4}{|c|}{ Patient Diagnosis } \\
\hline IVF & $100 \%$ & Procedural Factors: & & Tubal factor & $0 \%$ & Other factor & $0 \%$ \\
\hline GIFT & $0 \%$ & With ICSI & $79 \%$ & Ovulatory dysfunction & $24 \%$ & Unknown factor & $0 \%$ \\
\hline ZIFT & $0 \%$ & Unstimulated & $0 \%$ & Diminished ovarian reserve & $31 \%$ & Multiple Factors: & \\
\hline \multirow[t]{3}{*}{ Combination } & $0 \%$ & Used gestational carrier & $0 \%$ & Endometriosis & $1 \%$ & Female factors only & $10 \%$ \\
\hline & & & & Uterine factor & $0 \%$ & Female \& male factors & $29 \%$ \\
\hline & & & & Male factor & $6 \%$ & & \\
\hline
\end{tabular}

2006 PREGNANCY SUCCESS RATES

Data verified by Michael S. Mersol-Barg, MD

\section{Type of Cycle}

\section{Fresh Embryos from Nondonor Eggs}

Number of cycles

Percentage of cycles resulting in pregnancies ${ }^{b}$

Percentage of cycles resulting in live births $s^{b, c}$

(Confidence Interval)

Percentage of retrievals resulting in live births $s^{b, c}$

Percentage of transfers resulting in live births $s^{b, c}$

Percentage of transfers resulting in singleton live births ${ }^{b}$

Percentage of cancellations ${ }^{b}$

Average number of embryos transferred

Percentage of pregnancies with twins ${ }^{b}$

Percentage of pregnancies with triplets or more ${ }^{b}$

Percentage of live births having multiple infants ${ }^{b, c}$

\section{Frozen Embryos from Nondonor Eggs}

Number of transfers

Percentage of transfers resulting in live births $s^{\mathrm{b}, \mathrm{c}}$

Average number of embryos transferred

$0 / 2$

2.0

\section{Age of Woman}

$\begin{array}{cccc}<35 & \mathbf{3 5 - 3 7} & \mathbf{3 8 - 4 0} & \mathbf{4 1 - 4 2}^{\text {d }} \\ & & & \\ 28 & 17 & 12 & 3 \\ 57.1 & 6 / 17 & 5 / 12 & 0 / 3 \\ 57.1 & 6 / 17 & 3 / 12 & 0 / 3\end{array}$

(37.2-75.5)

57.1

59.3

$6 / 16$

$3 / 12$

$0 / 3$

40.7

$6 / 15$

$3 / 11$

$0 / 2$

0.0

$5 / 15$

$2 / 11$

$0 / 2$

1.9

$1 / 17$

$0 / 12$

$0 / 3$

$9 / 16$

2.1

2.1

$1 / 5$

$0 / 16$

$2 / 6$

$0 / 5$

$5 / 16$

$0 / 6$

$1 / 3$

$1 / 6$

\section{Donor Eggs}

\section{Fresh Embryos}

All Ages Combined

Number of transfers

Percentage of transfers resulting in live births $s^{b, c}$

Average number of embryos transferred

\section{6}

$3 / 6$

2.0
Frozen Embryos

\section{1}

$0 / 1$

1.0

\section{CURRENT CLINIC SERVICES AND PROFILE}

Current Name: Center for Reproductive Medicine and Surgery, PC

\begin{tabular}{l|l|l} 
Donor egg? Yes $\quad$ Gestational carriers? Yes &
\end{tabular}

Donor embryo? Yes Cryopreservation? Yes

Single women? Yes

\section{SART member? Yes}

Verified lab accreditation? Yes

(See Appendix C for details.)

${ }^{a}$ Reflects patient and treatment characteristics of ART cycles performed in 2006 using fresh nondonor eggs or embryos.

b When fewer than 20 cycles are reported in an age category, rates are shown as a fraction and confidence intervals are not given. Calculating percentages from fractions may be misleading and is not encouraged.

${ }^{c}$ A multiple-infant birth is counted as one live birth.

d Clinic-specific outcome rates are unreliable for women older than 42 undergoing ART cycles using fresh or frozen embryos with nondonor eggs. Readers are urged to review national outcomes for these age groups (see page 27).

e All ages (including ages $>42$ ) are reported together because previous data show that patient age does not materially affect success with donor eggs. 


\section{CENTER FOR REPRODUCTIVE MEDICINE \\ OAKWOOD HOSPITAL AND MEDICAL CENTER \\ DEARBORN, MICHIGAN}

A comparison of clinic success rates may not be meaningful because patient medical characteristics and treatment approaches vary from clinic to clinic. For more details about this, along with information on how to interpret the statistics in this table, see pages $79-88$.

\section{ART CYCLE PROFILE}

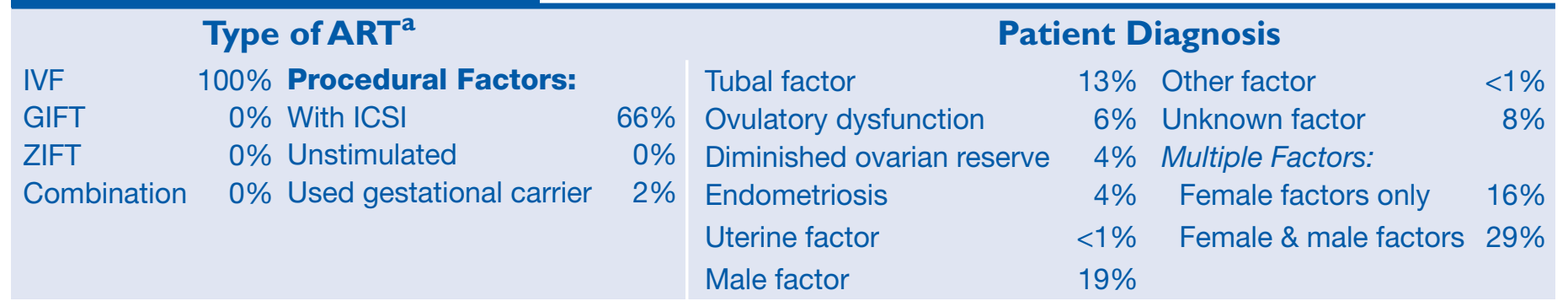

2006 PREGNANCY SUCCESS RATES

Data verified by David M. Magyar, DO

\section{Type of Cycle}

\section{Fresh Embryos from Nondonor Eggs}

Number of cycles

Percentage of cycles resulting in pregnancies ${ }^{b}$

Percentage of cycles resulting in live births $s^{b, c}$

(Confidence Interval)

Percentage of retrievals resulting in live births $s^{b, c}$

Percentage of transfers resulting in live births $s^{b, c}$

Percentage of transfers resulting in singleton live births ${ }^{b}$

Percentage of cancellations ${ }^{b}$

Average number of embryos transferred

Percentage of pregnancies with twins ${ }^{b}$

Percentage of pregnancies with triplets or more ${ }^{b}$

Percentage of live births having multiple infants ${ }^{b, c}$

\section{Frozen Embryos from Nondonor Eggs}

Number of transfers

Percentage of transfers resulting in live births ${ }^{b, c}$

Average number of embryos transferred

\section{Donor Eggs}

Number of transfers

Percentage of transfers resulting in live births $s^{b, c}$

Average number of embryos transferred

\section{Age of Woman}

35-37 38-40

$41-42^{d}$

$<35$

49

24.5

16.3

34

10

30.7

26.7

$(17.1-38.1)$

(7.3-29.7)

32.8

22.2

22.9

22.9

26.5

3.7

$1 / 12$

$0 / 12$

$0 / 8$

8.8

$1 / 10$

8.8

$0 / 10$

19.0

18.7

3.3

34.8

13.0

45.0

$(1.9-23.7)$

12.5

13.0

$0 / 4$

4.3

$0 / 4$

$0 / 4$

29.4

$6 / 10$

3.8

3.8

$1 / 3$

$0 / 1$

$1 / 3$

$0 / 1$

$2 / 3$

23

21.7

\section{7}

$1 / 7$

2.7

2.7

All Ages Combined ${ }^{\mathrm{e}}$

Fresh Embryos

20

55.0

2.9
2

$0 / 2$

5.0

\section{CURRENT CLINIC SERVICES AND PROFILE}

Current Name: This clinic has closed or reorganized since 2006. Information on current clinic services and profile therefore is not provided here. Contact the NASS Help Desk for current information about this clinic.

\footnotetext{
${ }^{a}$ Reflects patient and treatment characteristics of ART cycles performed in 2006 using fresh nondonor eggs or embryos.

b When fewer than 20 cycles are reported in an age category, rates are shown as a fraction and confidence intervals are not given. Calculating percentages from fractions may be misleading and is not encouraged.

${ }^{\mathrm{C}}$ A multiple-infant birth is counted as one live birth.

d Clinic-specific outcome rates are unreliable for women older than 42 undergoing ART cycles using fresh or frozen embryos with nondonor eggs. Readers are urged to review national outcomes for these age groups (see page 27).

e All ages (including ages $>42$ ) are reported together because previous data show that patient age does not materially affect success with donor eggs.
} 


\section{GRAND RAPIDS FERTILITY \& IVF, PC GRAND RAPIDS, MICHIGAN}

A comparison of clinic success rates may not be meaningful because patient medical characteristics and treatment approaches vary from clinic to clinic. For more details about this, along with information on how to interpret the statistics in this table, see pages $79-88$.

\section{ART CYCLE PROFILE}

\begin{tabular}{|c|c|c|c|c|c|c|c|}
\hline \multicolumn{4}{|c|}{ Type of $\mathrm{ART}^{\mathrm{a}}$} & \multicolumn{4}{|c|}{ Patient Diagnosis } \\
\hline IVF & $100 \%$ & Procedural Factors: & & Tubal factor & $11 \%$ & Other factor & $7 \%$ \\
\hline GIFT & $0 \%$ & With ICSI & $78 \%$ & Ovulatory dysfunction & $2 \%$ & Unknown factor & $8 \%$ \\
\hline ZIFT & $0 \%$ & Unstimulated & $0 \%$ & Diminished ovarian reserve & $6 \%$ & Multiple Factors: & \\
\hline Combination & $0 \%$ & Used gestational carrier & $0 \%$ & Endometriosis & $12 \%$ & Female factors only & $9 \%$ \\
\hline & & & & Uterine factor & $0 \%$ & Female \& male factors & $23 \%$ \\
\hline
\end{tabular}

2006 PREGNANCY SUCCESS RATES

Data verified by Douglas C. Daly, MD

\section{Type of Cycle}

\section{Fresh Embryos from Nondonor Eggs}

Number of cycles

Percentage of cycles resulting in pregnancies ${ }^{b}$

Percentage of cycles resulting in live births $s^{b, c}$

(Confidence Interval)

Percentage of retrievals resulting in live births $s^{b, c}$

Percentage of transfers resulting in live births $s^{b, c}$

Percentage of transfers resulting in singleton live births ${ }^{b}$

Percentage of cancellations ${ }^{b}$

Average number of embryos transferred

Percentage of pregnancies with twins ${ }^{b}$

Percentage of pregnancies with triplets or more ${ }^{\mathrm{b}}$

Percentage of live births having multiple infants ${ }^{b, c}$

\section{Frozen Embryos from Nondonor Eggs}

Number of transfers

Percentage of transfers resulting in live births $s^{b, c}$

Average number of embryos transferred

\section{Donor Eggs}

Number of transfers

Percentage of transfers resulting in live births $s^{\mathrm{b}, \mathrm{c}}$

Average number of embryos transferred

\section{Age of Woman}

35-37 38-40

$41-42^{d}$

$<35$

19

10

2

37.7

$3 / 19$

$0 / 10$

$0 / 2$

30.2

$3 / 19$

$0 / 10$

$0 / 2$

(18.3-44.3)

34.0

$3 / 11$

$0 / 10$

$0 / 2$

39.0

$3 / 9$

$0 / 8$

$0 / 2$

31.7

$2 / 9$

$0 / 8$

$0 / 2$

11.3

$8 / 19$

$0 / 10$

$0 / 2$

2.6

3.2

3.0

5.5

25.0

$1 / 3$

0.0

$0 / 3$

$3 / 16$

$1 / 3$

41

22.0

2.8

$$
14
$$

$5 / 14$

5

$1 / 5$

1

2.4

2.6

$0 / 1$

1.0

All Ages Combined ${ }^{\mathrm{e}}$

Fresh Embryos

8

$3 / 8$

2.1
Frozen Embryos 26

23.1

\section{CURRENT CLINIC SERVICES AND PROFILE}

Current Name: Grand Rapids Fertility \& IVF, PC
Donor egg?
Yes
Donor embryo? Yes
Gestational carriers? Yes
Cryopreservation?
SART member?
Yes
Single women? No
Verified lab accreditation?
Yes
(See Appendix C for details.)

\footnotetext{
${ }^{a}$ Reflects patient and treatment characteristics of ART cycles performed in 2006 using fresh nondonor eggs or embryos.

${ }^{b}$ When fewer than 20 cycles are reported in an age category, rates are shown as a fraction and confidence intervals are not given. Calculating percentages from fractions may be misleading and is not encouraged.

${ }^{c}$ A multiple-infant birth is counted as one live birth.

d Clinic-specific outcome rates are unreliable for women older than 42 undergoing ART cycles using fresh or frozen embryos with nondonor eggs. Readers are urged to review national outcomes for these age groups (see page 27).

${ }^{e}$ All ages (including ages $>42$ ) are reported together because previous data show that patient age does not materially affect success with donor eggs.
} 


\section{MICHIGAN REPRODUCTIVE \& IVF CENTER, PC GRAND RAPIDS, MICHIGAN}

A comparison of clinic success rates may not be meaningful because patient medical characteristics and treatment approaches vary from clinic to clinic. For more details about this, along with information on how to interpret the statistics in this table, see pages 79-88.

\section{ART CYCLE PROFILE}

\section{Type of ART ${ }^{\mathrm{a}}$}

$\begin{array}{llr}\text { IVF } & 98 \% \text { Procedural Factors: } & \\ \text { GIFT } & 0 \% \text { With ICSI } & 87 \% \\ \text { ZIFT } & 2 \% \text { Unstimulated } & 0 \% \\ \text { Combination } & 0 \% \text { Used gestational carrier } & <1 \%\end{array}$

\section{Patient Diagnosis}

Tubal factor

9\% Other factor

$\begin{array}{lrl}\text { Ovulatory dysfunction } & 3 \% & \text { Unknown factor } \\ \text { Diminished ovarian reserve } & 10 \% & \text { Multiple Factors: }\end{array}$

$\begin{array}{lrl}\text { Ovulatory dysfunction } & 3 \% & \text { Unknown factor } \\ \text { Diminished ovarian reserve } & 10 \% & \text { Multiple Factors: }\end{array}$

Endometriosis

Uterine factor

$10 \%$ Female factors only

$<1 \% \quad$ Female \& male factors

$33 \%$

Male factor

2006 PREGNANCY SUCCESS RATES

Data verified by William G. Dodds, MD

\section{Type of Cycle}

Age of Woman

35-37 38-40

$41-42^{d}$

\section{$<35$}

97

43.3

56

15

Number of cycles

270

55.6

36.1

33.9

$5 / 15$

Percentage of cycles resulting in live births $s^{b, c}$

47.4

(Confidence Interval)

Percentage of retrievals resulting in live births $s^{b, c}$

$(41.3-53.5)$

50.8

(26.6-46.5)

25.0

$4 / 15$

Percentage of transfers resulting in live births $s^{b, c}$

Percentage of transfers resulting in singleton live births ${ }^{b}$

Percentage of cancellations ${ }^{b}$

52.0

41.2

(14.4-38.4)

35.4

42.2

28.6

29.2

22.9

12.5

3.3

$5 / 19$

$0 / 19$

$3 / 14$

$4 / 14$

$4 / 14$

$2 / 14$

6.7

12.4

28.0

Percentage of pregnancies with twins ${ }^{b}$

Percentage of pregnancies with triplets or more ${ }^{b}$

Percentage of live births having multiple infants ${ }^{\mathrm{b}, \mathrm{c}}$

\section{Frozen Embryos from Nondonor Eggs}

Number of transfers

Percentage of transfers resulting in live births $s^{b, c}$

Average number of embryos transferred

\section{Donor Eggs}

Number of transfers

Percentage of transfers resulting in live births $s^{b, c}$

Average number of embryos transferred
5.3

32.0

121

39.7

3.0
2.9

14.3

34.3
$1 / 15$

4.4

$1 / 5$

$1 / 5$

$2 / 4$
2

$0 / 2$

2.0
All Ages Combined ${ }^{\mathrm{e}}$

$\begin{array}{cc}34 & 65 \\ 41.2 & 41.5 \\ 2.1 & 2.8\end{array}$

65

2.8

\section{CURRENT CLINIC SERVICES AND PROFILE}

Current Name: Michigan Reproductive \& IVF Center, PC

Donor egg? Yes Gestational carriers? Yes

Donor embryo? Yes

Single women? No

Cryopreservation? Yes

\section{Fresh Embryos}

$$
48
$$

25.0

3.2

${ }^{a}$ Reflects patient and treatment characteristics of ART cycles performed in 2006 using fresh nondonor eggs or embryos.

b When fewer than 20 cycles are reported in an age category, rates are shown as a fraction and confidence intervals are not given. Calculating percentages from fractions may be misleading and is not encouraged.

${ }^{c}$ A multiple-infant birth is counted as one live birth.

d Clinic-specific outcome rates are unreliable for women older than 42 undergoing ART cycles using fresh or frozen embryos with nondonor eggs. Readers are urged to review national outcomes for these age groups (see page 27).

e All ages (including ages $>42$ ) are reported together because previous data show that patient age does not materially affect success with donor eggs. 


\section{INFERTILITY AND GYNECOLOGY CENTER OF LANSING, PC LANSING, MICHIGAN}

A comparison of clinic success rates may not be meaningful because patient medical characteristics and treatment approaches vary from clinic to clinic. For more details about this, along with information on how to interpret the statistics in this table, see pages $79-88$.

\section{ART CYCLE PROFILE}

\begin{tabular}{|c|c|c|c|c|c|c|c|}
\hline \multicolumn{4}{|c|}{ Type of ART } & \multicolumn{4}{|c|}{ Patient Diagnosis } \\
\hline IVF & $100 \%$ & Procedural Factors: & & Tubal factor & $3 \%$ & Other factor & $0 \%$ \\
\hline GIFT & $0 \%$ & With ICSI & $92 \%$ & Ovulatory dysfunction & $9 \%$ & Unknown factor & $1 \%$ \\
\hline ZIFT & $0 \%$ & Unstimulated & $0 \%$ & Diminished ovarian reserve & $6 \%$ & Multiple Factors: & \\
\hline \multirow[t]{3}{*}{ Combination } & $0 \%$ & Used gestational carrier & $0 \%$ & Endometriosis & $6 \%$ & Female factors only & $16 \%$ \\
\hline & & & & Uterine factor & $0 \%$ & Female \& male factors & $49 \%$ \\
\hline & & & & Male factor & $10 \%$ & & \\
\hline
\end{tabular}

2006 PREGNANCY SUCCESS RATES

Data verified by Mohammad Mohsenian, MD

\section{Type of Cycle}

\section{Fresh Embryos from Nondonor Eggs}

Number of cycles

Percentage of cycles resulting in pregnancies ${ }^{b}$

Percentage of cycles resulting in live births $s^{b, c}$

(Confidence Interval)

Percentage of retrievals resulting in live births $s^{b, c}$

Percentage of transfers resulting in live births $s^{b, c}$

Percentage of transfers resulting in singleton live births ${ }^{b}$

Percentage of cancellations ${ }^{b}$

Average number of embryos transferred

Percentage of pregnancies with twins ${ }^{b}$

Percentage of pregnancies with triplets or more ${ }^{b}$

Percentage of live births having multiple infants ${ }^{b, c}$

\section{Frozen Embryos from Nondonor Eggs}

Number of transfers

Percentage of transfers resulting in live births $s^{\mathrm{b}, \mathrm{c}}$

Average number of embryos transferred

\section{Donor Eggs}

Number of transfers

Percentage of transfers resulting in live births $s^{b, c}$

Average number of embryos transferred

\section{Age of Woman}

35-37 38-40

$41-42^{d}$

$<35$

14

$6 / 14$

$4 / 14$

5

4

47.2

44.4

$2 / 5$

$0 / 4$

$(27.9-61.9)$

50.0

$4 / 14$

$1 / 5$

$0 / 4$

\section{0}

31.3

11.1

$4 / 14$

$1 / 5$

$0 / 3$

$3 / 14$

$1 / 5$

$0 / 3$

2.4

$0 / 14$

$1 / 5$

$0 / 3$

$6 / 17$

2.2

$0 / 5$

$1 / 4$

$0 / 17$

$1 / 6$

2.4

1.7

$6 / 16$

$0 / 6$

$0 / 2$

$0 / 2$

$1 / 4$

$0 / 1$

$11 \quad 5$

$5 / 11$

\section{5}

$0 / 5$

1

$0 / 1$

2.3

2.4

3.0

All Ages Combined ${ }^{\mathrm{e}}$

Fresh Embryos

6

$2 / 6$

2.3
Frozen Embryos 2

$0 / 2$

1.5

\section{CURRENT CLINIC SERVICES AND PROFILE}

Current Name: Infertility and Gynecology Center of Lansing, PC

Donor egg? No Gestational carriers? Yes

Donor embryo? Yes Cryopreservation? Yes

Single women? Yes

SART member? Yes

Verified lab accreditation? Yes

(See Appendix C for details.)

${ }^{a}$ Reflects patient and treatment characteristics of ART cycles performed in 2006 using fresh nondonor eggs or embryos.

${ }^{b}$ When fewer than 20 cycles are reported in an age category, rates are shown as a fraction and confidence intervals are not given. Calculating percentages from fractions may be misleading and is not encouraged.

${ }^{c}$ A multiple-infant birth is counted as one live birth.

d Clinic-specific outcome rates are unreliable for women older than 42 undergoing ART cycles using fresh or frozen embryos with nondonor eggs. Readers are urged to review national outcomes for these age groups (see page 27).

${ }^{e}$ All ages (including ages $>42$ ) are reported together because previous data show that patient age does not materially affect success with donor eggs. 


\section{MICHIGAN STATE UNIVERSITY \\ CENTER FOR ASSISTED REPRODUCTIVE TECHNOLOGY \\ LANSING, MICHIGAN}

A comparison of clinic success rates may not be meaningful because patient medical characteristics and treatment approaches vary from clinic to clinic. For more details about this, along with information on how to interpret the statistics in this table, see pages $79-88$.

\section{ART CYCLE PROFILE}

\begin{tabular}{|c|c|c|c|c|c|c|c|}
\hline \multicolumn{4}{|c|}{ Type of ART ${ }^{a}$} & \multicolumn{4}{|c|}{ Patient Diagnosis } \\
\hline IVF & $100 \%$ & Procedural Factors: & & Tubal factor & $3 \%$ & Other factor & $0 \%$ \\
\hline GIFT & $0 \%$ & With ICSI & $64 \%$ & Ovulatory dysfunction & $0 \%$ & Unknown factor & $5 \%$ \\
\hline ZIFT & $0 \%$ & Unstimulated & $0 \%$ & Diminished ovarian reserve & $3 \%$ & Multiple Factors: & \\
\hline \multirow[t]{3}{*}{ Combination } & $0 \%$ & Used gestational carrier & $0 \%$ & Endometriosis & $5 \%$ & Female factors only & $5 \%$ \\
\hline & & & & Uterine factor & $0 \%$ & Female \& male factors & $54 \%$ \\
\hline & & & & Male factor & $26 \%$ & & \\
\hline
\end{tabular}

2006 PREGNANCY SUCCESS RATES

Data verified by Harold Sauer, MD

\section{Type of Cycle}

\section{Fresh Embryos from Nondonor Eggs}

Number of cycles

Percentage of cycles resulting in pregnancies ${ }^{b}$

Percentage of cycles resulting in live births $s^{b, c}$

(Confidence Interval)

Percentage of retrievals resulting in live births $s^{b, c}$

Percentage of transfers resulting in live births $s^{b, c}$

Percentage of transfers resulting in singleton live births ${ }^{b}$

Percentage of cancellations ${ }^{b}$

Average number of embryos transferred

Percentage of pregnancies with twins ${ }^{b}$

Percentage of pregnancies with triplets or more ${ }^{b}$

Percentage of live births having multiple infants ${ }^{b, c}$

\section{Frozen Embryos from Nondonor Eggs}

Number of transfers

Percentage of transfers resulting in live births $s^{b, c}$

Average number of embryos transferred

\section{Donor Eggs}

Number of transfers

Percentage of transfers resulting in live births $s^{b, c}$

Average number of embryos transferred

\section{Age of Woman}

$<35 \quad 35-37 \quad 38-40 \quad 41-42^{\text {d }}$

11

15

$5 / 15$

$5 / 15$

5

5

$3 / 11$

$3 / 11$

$3 / 5$

$0 / 5$

$2 / 5$

$0 / 5$

$3 / 9$

$5 / 10$

$2 / 3$

$0 / 2$

$3 / 9$

$5 / 10$

$2 / 3$

$0 / 2$

$2 / 9$

$3 / 10$

$2 / 3$

$0 / 2$

$2 / 11$

$5 / 15$

$2 / 5$

$3 / 5$

2.0

2.2

2.3

1.5

$1 / 3$

$2 / 5$

$1 / 3$

$0 / 3$

$0 / 5$

$1 / 3$

$1 / 3$

$2 / 5$

$0 / 2$

0

0

0

0

All Ages
Fresh Embryos

0 bined $^{\mathrm{e}}$

Frozen Embryos

0

\section{CURRENT CLINIC SERVICES AND PROFILE}

Current Name: Michigan State University, Center for Assisted Reproductive Technology

Donor egg? Yes

Donor embryo? Yes

Single women? Yes

\section{Gestational carriers? Yes}

Cryopreservation? Yes
SART member?

Verified lab accreditation?

(See Appendix C for details.)

\footnotetext{
${ }^{a}$ Reflects patient and treatment characteristics of ART cycles performed in 2006 using fresh nondonor eggs or embryos.

${ }^{b}$ When fewer than 20 cycles are reported in an age category, rates are shown as a fraction and confidence intervals are not given. Calculating percentages from fractions may be misleading and is not encouraged.

${ }^{c}$ A multiple-infant birth is counted as one live birth.

d Clinic-specific outcome rates are unreliable for women older than 42 undergoing ART cycles using fresh or frozen embryos with nondonor eggs. Readers are urged to review national outcomes for these age groups (see page 27).

e All ages (including ages $>42$ ) are reported together because previous data show that patient age does not materially affect success with donor eggs.
} 


\section{IVF MICHIGAN \\ ROCHESTER HILLS, MICHIGAN}

A comparison of clinic success rates may not be meaningful because patient medical characteristics and treatment approaches vary from clinic to clinic. For more details about this, along with information on how to interpret the statistics in this table, see pages $79-88$.

\section{ART CYCLE PROFILE}

\section{Type of ART ${ }^{\mathrm{a}}$}

$\begin{array}{llr}\text { IVF } & 99 \% \text { Procedural Factors: } & \\ \text { GIFT } & 0 \% \text { With ICSI } & 87 \% \\ \text { ZIFT } & 1 \% \text { Unstimulated } & <1 \% \\ \text { Combination } & <1 \% \text { Used gestational carrier } & 2 \%\end{array}$

2006 PREGNANCY SUCCESS RATES

\section{Type of Cycle}

\section{Fresh Embryos from Nondonor Eggs}

Number of cycles

Percentage of cycles resulting in pregnancies ${ }^{b}$

Percentage of cycles resulting in live births ${ }^{b, c}$

(Confidence Interval)

Percentage of retrievals resulting in live births $s^{b, c}$

Percentage of transfers resulting in live births $s^{b, c}$

Percentage of transfers resulting in singleton live births ${ }^{b}$

Percentage of cancellations ${ }^{b}$

Average number of embryos transferred

Percentage of pregnancies with twins ${ }^{b}$

Percentage of pregnancies with triplets or more ${ }^{b}$

Percentage of live births having multiple infants ${ }^{b, c}$

\section{Frozen Embryos from Nondonor Eggs}

Number of transfers

Percentage of transfers resulting in live births $s^{b, c}$

Average number of embryos transferred

\section{Donor Eggs}

Number of transfers

Percentage of transfers resulting in live births $s^{b, c}$

Average number of embryos transferred

\section{Patient Diagnosis}

Tubal factor

$7 \%$ Other factor

$11 \%$ Unknown factor

$11 \%$ Multiple Factors:

Ovulatory dysfunction

$4 \%$ Female factors only $20 \%$

$2 \%$ Female \& male factors $25 \%$ $15 \%$
Male factor $\begin{array}{ll}\text { Endometriosis } & 2 \% \\ \text { Uterine factor } & 2 \%\end{array}$
$4 \%$

$3 \%$

$25 \%$

Data verified by Michael H. Fakih, MD

\section{Age of Woman}

35-37 38-40

\section{$<35$}

481

45.7

199

45.7

38.2

(34.1-43.0)

(31.4-45.3)

40.6

42.7

27.0

6.0

2.4

34.1

4.4

36.8

38.4

35.0

4.1

38.4

87

36.8

2.2
40

32.5

2.1
138

$$
28.3
$$$$
19.6
$$

(13.3-27.2)

24.8

26.0

20.2

21.0

2.8

17.9

2.6

22.2

19

$6 / 19$

2.2

\section{CURRENT CLINIC SERVICES AND PROFILE}

Current Name: IVF Michigan

$\begin{array}{ll}\text { Donor egg? } & \text { Yes } \\ \text { Donor embryo? } & \text { No } \\ \text { Single women? } & \text { Yes }\end{array}$

\section{Fresh Embryos}

All Ages Combined ${ }^{\mathrm{e}}$
Frozen Embryos

101

57.4

2.3
34

23.5

\footnotetext{
${ }_{b}^{a}$ Reflects patient and treatment characteristics of ART cycles performed in 2006 using fresh nondonor eggs or embryos.

${ }^{b}$ When fewer than 20 cycles are reported in an age category, rates are shown as a fraction and confidence intervals are not given. Calculating percentages from fractions may be misleading and is not encouraged.

${ }^{c}$ A multiple-infant birth is counted as one live birth.

${ }^{d}$ Clinic-specific outcome rates are unreliable for women older than 42 undergoing ART cycles using fresh or frozen embryos with nondonor eggs. Readers are urged to review national outcomes for these age groups (see page 27).

${ }^{e}$ All ages (including ages $>42$ ) are reported together because previous data show that patient age does not materially affect success with donor eggs.
} 


\section{UNIVERSITY WOMEN'S CARE/WAYNE STATE UNIVERSITY SOUTHFIELD, MICHIGAN}

A comparison of clinic success rates may not be meaningful because patient medical characteristics and treatment approaches vary from clinic to clinic. For more details about this, along with information on how to interpret the statistics in this table, see pages 79-88.

\section{ART CYCLE PROFILE}

\begin{tabular}{|c|c|c|c|c|c|c|c|}
\hline \multicolumn{4}{|c|}{ Type of ART ${ }^{a}$} & \multicolumn{4}{|c|}{ Patient Diagnosis } \\
\hline IVF & $100 \%$ & Procedural Factors: & & Tubal factor & $6 \%$ & Other factor & $6 \%$ \\
\hline GIFT & $0 \%$ & With ICSI & $98 \%$ & Ovulatory dysfunction & $7 \%$ & Unknown factor & $8 \%$ \\
\hline ZIFT & $0 \%$ & Unstimulated & $0 \%$ & Diminished ovarian reserve & $16 \%$ & Multiple Factors: & \\
\hline \multirow[t]{3}{*}{ Combination } & $0 \%$ & Used gestational carrier & $0 \%$ & Endometriosis & $9 \%$ & Female factors only & $9 \%$ \\
\hline & & & & Uterine factor & $0 \%$ & Female \& male factors & $21 \%$ \\
\hline & & & & Male factor & $19 \%$ & & \\
\hline
\end{tabular}

2006 PREGNANCY SUCCESS RATES

Data verified by Elizabeth E. Puscheck, MD

\section{Type of Cycle}

\section{Age of Woman}

35-37 38-40

$41-42^{d}$

$<35$

16

$8 / 16$

5

2

54.2

$7 / 16$

$0 / 5$

$0 / 2$

Percentage of cycles resulting in live births $s^{b, c}$

(Confidence Interval)

Percentage of retrievals resulting in live births ${ }^{b, c}$

50.0

(29.1-70.9)

Percentage of transfers resulting in live births ${ }^{b, c}$

50.0

$7 / 16$

$0 / 5$

$0 / 2$

Percentage of transfers resulting in singleton live births ${ }^{b}$

Percentage of cancellations ${ }^{b}$ 50.0

33.3

$7 / 16$

$0 / 5$

$0 / 2$

33.3

$4 / 16$

$0 / 4$

$0 / 1$

0.0

$0 / 16$

$0 / 4$

$0 / 1$

Average number of embryos transferred

Percentage of pregnancies with twins ${ }^{b}$

2.7

3.3

$1 / 13$

$4 / 8$

$3 / 13$

$0 / 8$

$4 / 12$

$3 / 7$

Percentage of pregnancies with triplets or more $b$

Percentage of live births having multiple infants ${ }^{b, c}$

Frozen Embryos from Nondonor Eggs

Number of transfers

Percentage of transfers resulting in live births $s^{b, c}$

Average number of embryos transferred

15

$3 / 15$

7

2.9

$3 / 7$

3.4

$0 / 2$

2.0

3.0

0

0

Fresh Embryos

5

$2 / 5$

2.0

Donor Eggs

All Ages Combined

Number of transfers

Percentage of transfers resulting in live births ${ }^{\mathrm{b}, \mathrm{c}}$

Average number of embryos transferred

\section{CURRENT CLINIC SERVICES AND PROFILE}

Current Name: University Women's Care/Wayne State University

Donor egg? Yes Gestational carriers? Yes

Donor embryo? Yes Cryopreservation? Yes

Single women? Yes

SART member? Yes

Verified lab accreditation?

(See Appendix C for details.)

${ }^{a}$ Reflects patient and treatment characteristics of ART cycles performed in 2006 using fresh nondonor eggs or embryos.

${ }^{b}$ When fewer than 20 cycles are reported in an age category, rates are shown as a fraction and confidence intervals are not given. Calculating percentages from fractions may be misleading and is not encouraged.

${ }^{c}$ A multiple-infant birth is counted as one live birth.

d Clinic-specific outcome rates are unreliable for women older than 42 undergoing ART cycles using fresh or frozen embryos with nondonor eggs. Readers are urged to review national outcomes for these age groups (see page 27).

e All ages (including ages $>42$ ) are reported together because previous data show that patient age does not materially affect success with donor eggs. 


\section{HENRY FORD REPRODUCTIVE MEDICINE \\ TROY, MICHIGAN}

A comparison of clinic success rates may not be meaningful because patient medical characteristics and treatment approaches vary from clinic to clinic. For more details about this, along with information on how to interpret the statistics in this table, see pages $79-88$.

\section{ART CYCLE PROFILE}

\begin{tabular}{|c|c|c|c|c|c|c|c|}
\hline \multicolumn{4}{|c|}{ Type of $A R T^{a}$} & \multicolumn{4}{|c|}{ Patient Diagnosis } \\
\hline IVF & $100 \%$ & Procedural Factors: & & Tubal factor & $14 \%$ & Other factor & $5 \%$ \\
\hline GIFT & $0 \%$ & With ICSI & $33 \%$ & Ovulatory dysfunction & $4 \%$ & Unknown factor & $13 \%$ \\
\hline ZIFT & $0 \%$ & Unstimulated & $0 \%$ & Diminished ovarian reserve & $0 \%$ & Multiple Factors: & \\
\hline \multirow[t]{3}{*}{ Combination } & $0 \%$ & Used gestational carrier & $0 \%$ & Endometriosis & $9 \%$ & Female factors only & $8 \%$ \\
\hline & & & & Uterine factor & $0 \%$ & Female \& male factors & $18 \%$ \\
\hline & & & & Male factor & $29 \%$ & & \\
\hline
\end{tabular}

2006 PREGNANCY SUCCESS RATES

Data verified by Ronald C. Strickler, MD

\section{Type of Cycle}

\section{Fresh Embryos from Nondonor Eggs}

Number of cycles

Percentage of cycles resulting in pregnancies ${ }^{b}$

Percentage of cycles resulting in live births ${ }^{b, c}$

(Confidence Interval)

Percentage of retrievals resulting in live births $s^{b, c}$

Percentage of transfers resulting in live births $s^{b, c}$

Percentage of transfers resulting in singleton live births ${ }^{b}$

Percentage of cancellations ${ }^{b}$

Average number of embryos transferred

Percentage of pregnancies with twins ${ }^{b}$

Percentage of pregnancies with triplets or more ${ }^{\mathrm{b}}$

Percentage of live births having multiple infants ${ }^{b, c}$

\section{Frozen Embryos from Nondonor Eggs}

Number of transfers

Percentage of transfers resulting in live births ${ }^{\mathrm{b}, \mathrm{c}}$

Average number of embryos transferred

\section{Donor Eggs}

Number of transfers

Percentage of transfers resulting in live births $s^{b, c}$

Average number of embryos transferred

CURRENT CLINIC SERVICES AND PROFILE

Current Name: Henry Ford Reproductive Medicine
Donor egg?
Yes
Gestational carriers?
No
Cryopreservation?
Yes
Donor embryo? No
Single women? Yes

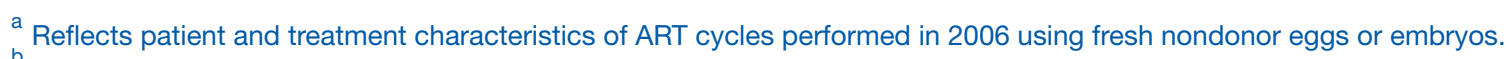
${ }^{b}$ When fewer than 20 cycles are reported in an age category, rates are shown as a fraction and confidence intervals are not given. Calculating percentages from fractions may be misleading and is not encouraged.
c A multiple-infant birth is counted as one live birth.
d Clinic-specific outcome rates are unreliable for women older than 42 undergoing ART cycles using fresh or frozen embryos with nondonor eggs. Readers are urged to review national outcomes for these age groups (see page 27).
${ }^{e}$ All ages (including ages $>42$ ) are reported together because previous data show that patient age does not materially affect success with donor eggs.

Age of Woman

$\begin{array}{cccc}<35 & 35-37 & 38-40 & \mathbf{4 1 - 4 2}^{\mathbf{d}} \\ 35 & 23 & 12 & 2 \\ 40.0 & 26.1 & 2 / 12 & 1 / 2 \\ 40.0 & 26.1 & 2 / 12 & 1 / 2\end{array}$

(23.9-57.9)

$(10.2-48.4)$

46.7

$6 / 18$

$2 / 8$

$1 / 2$

51.9

$6 / 16$

$2 / 7$

$1 / 2$

37.0

$5 / 16$

$2 / 7$

$0 / 2$

14.3

21.7

$4 / 12$

$0 / 2$

2.2

2.0

2.6

3.5

$4 / 14$

$2 / 6$

$0 / 2$

$1 / 1$

$0 / 14$

$0 / 6$

$0 / 2$

$0 / 1$

4 / 14

$1 / 6$

$0 / 2$

$1 / 1$

$\begin{array}{ccc}10 & 6 & 3 \\ 4 / 10 & 3 / 6 & 0 / 3\end{array}$

2.1

2.0

2.7

All Ages Combined ${ }^{\mathrm{e}}$

Fresh Embryos

0
Frozen Embryos

0 


\section{BRENDA L. MOSKOVITZ, MD, PC \\ TROY, MICHIGAN}

A comparison of clinic success rates may not be meaningful because patient medical characteristics and treatment approaches vary from clinic to clinic. For more details about this, along with information on how to interpret the statistics in this table, see pages 79-88.

\section{ART CYCLE PROFILE}

\begin{tabular}{|c|c|c|c|c|c|c|c|}
\hline \multicolumn{4}{|c|}{ Type of ART ${ }^{a}$} & \multicolumn{4}{|c|}{ Patient Diagnosis } \\
\hline IVF & $100 \%$ & Procedural Factors: & & Tubal factor & $5 \%$ & Other factor & $5 \%$ \\
\hline GIFT & $0 \%$ & With ICSI & $89 \%$ & Ovulatory dysfunction & $5 \%$ & Unknown factor & $25 \%$ \\
\hline ZIFT & $0 \%$ & Unstimulated & $0 \%$ & Diminished ovarian reserve & $0 \%$ & Multiple Factors: & \\
\hline \multirow[t]{3}{*}{ Combination } & $0 \%$ & Used gestational carrier & $0 \%$ & Endometriosis & $0 \%$ & Female factors only & $0 \%$ \\
\hline & & & & Uterine factor & $0 \%$ & Female \& male factors & $10 \%$ \\
\hline & & & & Male factor & $50 \%$ & & \\
\hline
\end{tabular}

2006 PREGNANCY SUCCESS RATES

Data verified by William R. Keye, MD

\section{Type of Cycle}

\section{Age of Woman}

35-37 38-40

$$
41-42^{d}
$$

$<35$

2

$2 / 2$

3

0

Percentage of cycles resulting in pregnancies ${ }^{b}$

Percentage of cycles resulting in live births $s^{b, c}$

$8 / 13$

$2 / 2$

$0 / 3$

$8 / 13$

$2 / 2$

$0 / 3$

(Confidence Interval)

Percentage of retrievals resulting in live births ${ }^{b, c}$

Percentage of transfers resulting in live births ${ }^{b, c}$

$8 / 12$

$2 / 2$

$0 / 3$

$8 / 12$

$2 / 2$

$0 / 3$

Percentage of transfers resulting in singleton live births ${ }^{b}$

Percentage of cancellations ${ }^{b}$

$3 / 12$

$0 / 2$

$0 / 3$

$1 / 13$

2.5

$0 / 3$

2.3

$1 / 2$

Percentage of pregnancies with twins ${ }^{\mathrm{b}}$

$5 / 8$

$0 / 2$

$1 / 8$

$0 / 2$

Percentage of pregnancies with triplets or more $b$

Percentage of live births having multiple infants ${ }^{\mathrm{b}, \mathrm{c}}$

$5 / 8$

\section{Frozen Embryos from Nondonor Eggs}

Number of transfers

Percentage of transfers resulting in live births ${ }^{\mathrm{b}, \mathrm{c}}$

0

0

1

$1 / 1$

Average number of embryos transferred

\section{Donor Eggs}

Number of transfers

Percentage of transfers resulting in live births ${ }^{\mathrm{b}, \mathrm{c}}$

Average number of embryos transferred

\section{All Ages Combined Fresh Embryos 1 $0 / 1$ 2.0}

\section{CURRENT CLINIC SERVICES AND PROFILE}

Current Name: Brenda L. Moskovitz, MD, PC
Donor egg?
Yes
Gestational carriers?
No
Cryopreservation?
Yes
Single women? Yes
SART member?
Yes
Verified lab accreditation?
Yes
(See Appendix C for details.)
${ }^{a}$ Reflects patient and treatment characteristics of ART cycles performed in 2006 using fresh nondonor eggs or embryos.
${ }^{b}$ When fewer than 20 cycles are reported in an age category, rates are shown as a fraction and confidence intervals are not given. Calculating percentages from fractions may be misleading and is not encouraged.
${ }^{c}$ A multiple-infant birth is counted as one live birth.
${ }^{d}$ Clinic-specific outcome rates are unreliable for women older than 42 undergoing ART cycles using fresh or frozen embryos with nondonor eggs. Readers are urged to review national outcomes for these age groups (see page 27).
e All ages (including ages $>42$ ) are reported together because previous data show that patient age does not materially affect success with donor eggs. 


\section{MICHIGAN CENTER FOR FERTILITY AND WOMEN'S HEALTH, PLC WARREN, MICHIGAN}

A comparison of clinic success rates may not be meaningful because patient medical characteristics and treatment approaches vary from clinic to clinic. For more details about this, along with information on how to interpret the statistics in this table, see pages $79-88$.

\section{ART CYCLE PROFILE}

\begin{tabular}{|c|c|c|c|c|c|c|c|}
\hline \multicolumn{4}{|c|}{ Type of ART ${ }^{\mathrm{a}}$} & \multicolumn{4}{|c|}{ Patient Diagnosis } \\
\hline IVF & $100 \%$ & Procedural Factors: & & Tubal factor & $7 \%$ & Other factor & $9 \%$ \\
\hline GIFT & $0 \%$ & With ICSI & $78 \%$ & Ovulatory dysfunction & $10 \%$ & Unknown factor & $16 \%$ \\
\hline ZIFT & $0 \%$ & Unstimulated & $0 \%$ & Diminished ovarian reserve & $17 \%$ & Multiple Factors: & \\
\hline Combination & $0 \%$ & Used gestational carrier & $0 \%$ & Endometriosis & $5 \%$ & Female factors only & $12 \%$ \\
\hline & & & & Uterine factor & $<1 \%$ & Female \& male factors & $10 \%$ \\
\hline
\end{tabular}

2006 PREGNANCY SUCCESS RATES

Data verified by Carole L. Kowalczyk, MD

\section{Type of Cycle}

\section{Fresh Embryos from Nondonor Eggs}

Number of cycles

Percentage of cycles resulting in pregnancies ${ }^{b}$

Percentage of cycles resulting in live births ${ }^{b, c}$

(Confidence Interval)

Percentage of retrievals resulting in live births $s^{b, c}$

Percentage of transfers resulting in live births $s^{b, c}$

Percentage of transfers resulting in singleton live births ${ }^{b}$

Percentage of cancellations ${ }^{b}$

Average number of embryos transferred

Percentage of pregnancies with twins ${ }^{b}$

Percentage of pregnancies with triplets or more ${ }^{\mathrm{b}}$

Percentage of live births having multiple infants ${ }^{b, c}$

\section{Frozen Embryos from Nondonor Eggs}

Number of transfers

Percentage of transfers resulting in live births ${ }^{\mathrm{b}, \mathrm{c}}$

Average number of embryos transferred

\section{Donor Eggs}

Number of transfers

Percentage of transfers resulting in live births $s^{b, c}$

Average number of embryos transferred

\section{Age of Woman}

35-37 38-40

$41-42^{d}$

$<35$

25

11

8

33.3

24.0

$2 / 11$

$0 / 8$

29.2

16.0

$1 / 11$

$0 / 8$

(12.6-51.1)

(4.5-36.1)

35.0

$4 / 19$

$1 / 7$

$0 / 7$

35.0

$4 / 19$

$1 / 6$

$0 / 7$

30.0

$4 / 19$

$0 / 6$

$0 / 7$

16.7

24.0

$4 / 11$

$1 / 8$

2.5

3.1

3.0

3.3

$0 / 8$

$0 / 6$

$1 / 2$

$1 / 8$

$0 / 6$

$0 / 2$

$1 / 7$

$0 / 4$

$1 / 1$

\section{7}

$3 / 7$

2.4

\section{3}

$1 / 3$

2.3
2

$0 / 2$

3.0
$9 \%$

$\%$

$\%$

\section{CURRENT CLINIC SERVICES AND PROFILE}

Current Name: Michigan Center for Fertility and Women's Health, PLC

Donor egg? Yes

Donor embryo? Yes

Single women? Yes

\section{Gestational carriers? Yes}

Cryopreservation? Yes

All Ages Combined ${ }^{\mathrm{e}}$

Fresh Embryos

13
$7 / 13$
2.2

Frozen Embryos 2

$2 / 2$

3.0

${ }^{a}$ Reflects patient and treatment characteristics of ART cycles performed in 2006 using fresh nondonor eggs or embryos.

${ }^{b}$ When fewer than 20 cycles are reported in an age category, rates are shown as a fraction and confidence intervals are not given. Calculating percentages from fractions may be misleading and is not encouraged.

c A multiple-infant birth is counted as one live birth.

d Clinic-specific outcome rates are unreliable for women older than 42 undergoing ART cycles using fresh or frozen embryos with nondonor eggs. Readers are urged to review national outcomes for these age groups (see page 27).

e All ages (including ages $>42$ ) are reported together because previous data show that patient age does not materially affect success with donor eggs. 


\section{THE MIDWEST CENTER FOR REPRODUCTIVE HEALTH, PA \\ MAPLE GROVE, MINNESOTA}

A comparison of clinic success rates may not be meaningful because patient medical characteristics and treatment approaches vary from clinic to clinic. For more details about this, along with information on how to interpret the statistics in this table, see pages 79-88.

\section{ART CYCLE PROFILE}

\section{Type of ART ${ }^{\mathrm{a}}$}

IVF

GIFT

ZIFT

Combination
100\% Procedural Factors:

$0 \%$ With ICSI

$0 \%$ Unstimulated

$0 \%$ Used gestational carrier
Tubal factor

$44 \%$ Ovulatory dysfunction

$<1 \%$

$<1 \%$

Endometriosis

\section{Diminished ovarian reserve}

Uterine factor

Male factor

\section{Patient Diagnosis}

$8 \%$ Other factor $\quad 5 \%$

$11 \%$ Unknown factor $\quad 9 \%$

$<1 \%$ Multiple Factors:

$5 \%$ Female factors only $\quad 7 \%$

$<1 \%$ Female \& male factors $32 \%$

$23 \%$
2006 PREGNANCY SUCCESS RATES

\section{Type of Cycle}

\section{Fresh Embryos from Nondonor Eggs}

Number of cycles

Percentage of cycles resulting in pregnancies ${ }^{b}$

Percentage of cycles resulting in live births ${ }^{b, c}$

(Confidence Interval)

Percentage of retrievals resulting in live births $s^{b, c}$

Percentage of transfers resulting in live births $s^{b, c}$

Percentage of transfers resulting in singleton live births ${ }^{b}$

Percentage of cancellations ${ }^{b}$

Average number of embryos transferred

Percentage of pregnancies with twins ${ }^{b}$

Percentage of pregnancies with triplets or more ${ }^{b}$

Percentage of live births having multiple infants ${ }^{b, c}$

\section{Frozen Embryos from Nondonor Eggs}

Number of transfers

Percentage of transfers resulting in live births $s^{b, c}$

Average number of embryos transferred

\section{Donor Eggs}

Number of transfers

Percentage of transfers resulting in live births $s^{b, c}$

Average number of embryos transferred
Data verified by Randle S. Corfman, MD, PhD

\section{Age of Woman}

35-37 38-40

$41-42^{d}$

$<35$

30

46.7

43.3

24

4

58.9

54.5

(25.5-62.6)

29.2

20.8

$0 / 4$

(44.8-63.9)

56.0

48.1

$(7.1-42.2)$

57.0

48.1

21.7

25.0

15.0

$0 / 4$

35.5

33.3

10.0

2.0

4.2

2.1

$2 / 7$

42.4

$3 / 14$

$0 / 7$

1.5

$1 / 14$

$2 / 5$
$0 / 3$

$0 / 2$

$0 / 2$

$1 / 4$

2.5
$4 / 13$

$\begin{array}{cccc}42 & 16 & 10 & 1 \\ 31.0 & 5 / 16 & 1 / 10 & 0 / 1 \\ 2.0 & 2.1 & 1.9 & 3.0\end{array}$

\section{All Ages Combined}

Fresh Embryos

20

40.0

2.0
Frozen Embryos 15

$4 / 15$

2.1

\section{CURRENT CLINIC SERVICES AND PROFILE}

Current Name: The Midwest Center for Reproductive Health, PA

Donor egg? Yes Gestational carriers? Yes

Donor embryo? Yes

Single women? Yes

Cryopreservation? Yes

SART member?

Yes

Verified lab accreditation?

Yes

(See Appendix C for details.)

${ }^{a}$ Reflects patient and treatment characteristics of ART cycles performed in 2006 using fresh nondonor eggs or embryos.

b When fewer than 20 cycles are reported in an age category, rates are shown as a fraction and confidence intervals are not given. Calculating percentages from fractions may be misleading and is not encouraged.

${ }^{\mathrm{C}}$ A multiple-infant birth is counted as one live birth.

d Clinic-specific outcome rates are unreliable for women older than 42 undergoing ART cycles using fresh or frozen embryos with nondonor eggs. Readers are urged to review national outcomes for these age groups (see page 27).

e All ages (including ages $>42$ ) are reported together because previous data show that patient age does not materially affect success with donor eggs. 


\section{CENTER FOR REPRODUCTIVE MEDICINE ADVANCED REPRODUCTIVE TECHNOLOGIES MINNEAPOLIS, MINNESOTA}

A comparison of clinic success rates may not be meaningful because patient medical characteristics and treatment approaches vary from clinic to clinic. For more details about this, along with information on how to interpret the statistics in this table, see pages $79-88$.

\section{ART CYCLE PROFILE}

\begin{tabular}{|c|c|c|c|c|c|c|c|}
\hline \multicolumn{4}{|c|}{ Type of ART } & \multicolumn{4}{|c|}{ Patient Diagnosis } \\
\hline IVF & $100 \%$ & Procedural Factors: & & Tubal factor & $9 \%$ & Other factor & $3 \%$ \\
\hline GIFT & $0 \%$ & With ICSI & $56 \%$ & Ovulatory dysfunction & $8 \%$ & Unknown factor & $12 \%$ \\
\hline ZIFT & $0 \%$ & Unstimulated & $0 \%$ & Diminished ovarian reserve & $22 \%$ & Multiple Factors: & \\
\hline \multirow[t]{3}{*}{ Combination } & $0 \%$ & Used gestational carrier & $<1 \%$ & Endometriosis & $4 \%$ & Female factors only & $6 \%$ \\
\hline & & & & Uterine factor & $1 \%$ & Female \& male factors & $11 \%$ \\
\hline & & & & Male factor & $24 \%$ & & \\
\hline
\end{tabular}

2006 PREGNANCY SUCCESS RATES

Data verified by Bruce F. Campbell, MD

\section{Type of Cycle}

\section{Fresh Embryos from Nondonor Eggs}

Number of cycles

Percentage of cycles resulting in pregnancies ${ }^{b}$

Percentage of cycles resulting in live births $s^{b, c}$

(Confidence Interval)

Percentage of retrievals resulting in live births $s^{b, c}$

Percentage of transfers resulting in live births $s^{b, c}$

Percentage of transfers resulting in singleton live births ${ }^{b}$

Percentage of cancellations ${ }^{b}$

Average number of embryos transferred

Percentage of pregnancies with twins ${ }^{b}$

Percentage of pregnancies with triplets or more ${ }^{b}$

Percentage of live births having multiple infants ${ }^{b, c}$

\section{Frozen Embryos from Nondonor Eggs}

Number of transfers

Percentage of transfers resulting in live births $s^{b, c}$

Average number of embryos transferred

\section{Donor Eggs}

Number of transfers

Percentage of transfers resulting in live births $s^{b, c}$

Average number of embryos transferred

\section{Age of Woman}

35-37 38-40

$41-42^{d}$

\section{$<35$}

142

53.5

113

40

51.3

45.1

40.7

32.5

44.7

(36.7-53.6)

28.3

22.5

(39.0-50.5)

48.6

49.6

(20.2-37.6)

(10.8-38.5)

49.8

51.6

33.0

35.2

27.3

30.5

35.5

28.6

29.0

8.0

9.2

14.2

25.8

1.9

1.9

2.0

17.5

34.4

40.8

21.7

2.8

1.3

0.0

0.0

$3 / 13$

38.8

31.3

18.8

$2 / 13$

$1 / 9$

40

32.5

25

36.0

\section{8}

3

2.0

2.1

$3 / 8$

$1 / 3$

2.1

2.0

All Ages Combined ${ }^{\mathrm{e}}$

Fresh Embryos

112

66.1

1.8
Frozen Embryos 30

33.3

2.0

\section{CURRENT CLINIC SERVICES AND PROFILE}

Current Name: Center for Reproductive Medicine, Advanced Reproductive Technologies

Donor egg? Yes

Donor embryo? Yes

Single women? Yes

\section{Gestational carriers? Yes}

Cryopreservation? Yes

\section{SART member?}

Verified lab accreditation?

(See Appendix C for details.)

${ }^{a}$ Reflects patient and treatment characteristics of ART cycles performed in 2006 using fresh nondonor eggs or embryos.

${ }^{b}$ When fewer than 20 cycles are reported in an age category, rates are shown as a fraction and confidence intervals are not given. Calculating percentages from fractions may be misleading and is not encouraged.

A multiple-infant birth is counted as one live birth.

d Clinic-specific outcome rates are unreliable for women older than 42 undergoing ART cycles using fresh or frozen embryos with nondonor eggs. Readers are urged to review national outcomes for these age groups (see page 27).

e All ages (including ages $>42$ ) are reported together because previous data show that patient age does not materially affect success with donor eggs. 


\section{REPRODUCTIVE MEDICINE CENTER \\ MINNEAPOLIS, MINNESOTA}

A comparison of clinic success rates may not be meaningful because patient medical characteristics and treatment approaches vary from clinic to clinic. For more details about this, along with information on how to interpret the statistics in this table, see pages 79-88.

\section{ART CYCLE PROFILE}

\begin{tabular}{|c|c|c|c|c|c|c|c|}
\hline \multicolumn{4}{|c|}{ Type of ART ${ }^{a}$} & \multicolumn{4}{|c|}{ Patient Diagnosis } \\
\hline IVF & $100 \%$ & Procedural Factors: & & Tubal factor & $7 \%$ & Other factor & $2 \%$ \\
\hline GIFT & $0 \%$ & With ICSI & $75 \%$ & Ovulatory dysfunction & $6 \%$ & Unknown factor & $11 \%$ \\
\hline ZIFT & $0 \%$ & Unstimulated & $0 \%$ & Diminished ovarian reserve & $3 \%$ & Multiple Factors: & \\
\hline \multirow[t]{3}{*}{ Combination } & $0 \%$ & Used gestational carrier & $0 \%$ & Endometriosis & $5 \%$ & Female factors only & $12 \%$ \\
\hline & & & & Uterine factor & $3 \%$ & Female \& male factors & $23 \%$ \\
\hline & & & & Male factor & $29 \%$ & & \\
\hline
\end{tabular}

2006 PREGNANCY SUCCESS RATES

Data verified by Mark A. Damario, MD

\section{Type of Cycle}

Age of Woman

35-37 38-40

$41-42^{d}$

$<35$

146

51.4

79

43.0

51

21

43.8

32.9

33.3

23.8

Percentage of cycles resulting in pregnancies ${ }^{b}$

(Confidence Interval)

Percentage of retrievals resulting in live births

$(35.6-52.3)$

49.2

(22.7-44.4)

27.5

14.3

Percentage of transfers resulting in live births ${ }^{b, c}$

52.5

40.6

$(15.9-41.7)$

(3.0-36.3)

37.7

44.1

32.6

$3 / 18$

11.0

33.9

34.1

$3 / 17$

Percentage of transfers resulting in singleton live births ${ }^{b}$

Percentage of cancellations ${ }^{b}$

2.1

19.0

24.4

15.7

$2 / 17$

2.1
29.3

0.0

2.3

2.5

14.3

Average number of embryos transferred

Percentage of pregnancies with twins ${ }^{b}$

Percentage of live births having multiple infants $s^{\mathrm{b}, \mathrm{c}}$

Frozen Embryos from Nondonor Eggs

Number of transfers

Percentage of transfers resulting in live births ${ }^{b, c}$

Average number of embryos transferred

\section{Donor Eggs}

28.1

17.6

$4 / 17$

2.9

0.0

$1 / 17$

$2 / 5$

23.1

4 / 14

$0 / 5$

$1 / 3$

$\begin{array}{lccc}19 & 15 & 7 & 1 \\ 6 / 19 & 5 / 15 & 1 / 7 & 0 / 1 \\ 2.0 & 2.0 & 2.1 & 3.0\end{array}$

\section{All Ages Combined}

Fresh Embryos

6

$2 / 6$

Frozen Embryos

2.2

Percentage of transfers resulting in live births $s^{b, c}$

Average number of embryos transferred

(a)

\section{1}

$0 / 1$

2.0

\section{CURRENT CLINIC SERVICES AND PROFILE}

Current Name: Reproductive Medicine Center

\begin{tabular}{|c|c|c|c|c|}
\hline Donor egg? & Yes & Gestational carriers? & Yes & SART member? \\
\hline Donor embryo? & No & Cryopreservation? & Yes & Verified lab accreditation? \\
\hline Single women? & Yes & & & (See Appendix C for details. \\
\hline
\end{tabular}

\footnotetext{
${ }^{a}$ Reflects patient and treatment characteristics of ART cycles performed in 2006 using fresh nondonor eggs or embryos.

b When fewer than 20 cycles are reported in an age category, rates are shown as a fraction and confidence intervals are not given. Calculating percentages from fractions may be misleading and is not encouraged.

${ }^{c}$ A multiple-infant birth is counted as one live birth.

${ }^{d}$ Clinic-specific outcome rates are unreliable for women older than 42 undergoing ART cycles using fresh or frozen embryos with nondonor eggs. Readers are urged to review national outcomes for these age groups (see page 27).

e All ages (including ages $>42$ ) are reported together because previous data show that patient age does not materially affect success with donor eggs.
} 


\section{MAYO CLINIC ASSISTED REPRODUCTIVE TECHNOLOGIES ROCHESTER, MINNESOTA}

A comparison of clinic success rates may not be meaningful because patient medical characteristics and treatment approaches vary from clinic to clinic. For more details about this, along with information on how to interpret the statistics in this table, see pages $79-88$.

\section{ART CYCLE PROFILE}

\begin{tabular}{|c|c|c|c|c|c|c|c|}
\hline \multicolumn{4}{|c|}{ Type of $A R T^{a}$} & \multicolumn{4}{|c|}{ Patient Diagnosis } \\
\hline IVF & $100 \%$ & Procedural Factors: & & Tubal factor & $8 \%$ & Other factor & $0 \%$ \\
\hline GIFT & $0 \%$ & With ICSI & $73 \%$ & Ovulatory dysfunction & $0 \%$ & Unknown factor & $11 \%$ \\
\hline ZIFT & $0 \%$ & Unstimulated & $0 \%$ & Diminished ovarian reserve & $7 \%$ & Multiple Factors: & \\
\hline \multirow[t]{3}{*}{ Combination } & $0 \%$ & Used gestational carrier & $0 \%$ & Endometriosis & $3 \%$ & Female factors only & $2 \%$ \\
\hline & & & & Uterine factor & $0 \%$ & Female \& male factors & $21 \%$ \\
\hline & & & & Male factor & $48 \%$ & & \\
\hline
\end{tabular}

2006 PREGNANCY SUCCESS RATES

Data verified by Charles C. Coddington, MD

\section{Type of Cycle}

\section{Fresh Embryos from Nondonor Eggs}

Number of cycles

Percentage of cycles resulting in pregnancies ${ }^{b}$

Percentage of cycles resulting in live births ${ }^{b, c}$

(Confidence Interval)

Percentage of retrievals resulting in live births $s^{b, c}$

Percentage of transfers resulting in live births $s^{b, c}$

Percentage of transfers resulting in singleton live births ${ }^{b}$

Percentage of cancellations ${ }^{b}$

Average number of embryos transferred

Percentage of pregnancies with twins ${ }^{b}$

Percentage of pregnancies with triplets or more ${ }^{b}$

Percentage of live births having multiple infants ${ }^{b, c}$

\section{Frozen Embryos from Nondonor Eggs}

Number of transfers

Percentage of transfers resulting in live births ${ }^{\mathrm{b}, \mathrm{c}}$

Average number of embryos transferred

\section{Donor Eggs}

Number of transfers

Percentage of transfers resulting in live births ${ }^{\mathrm{b}, \mathrm{c}}$

Average number of embryos transferred

\section{Age of Woman}

35-37 38-40

$41-42^{d}$

$<35$

21

38.1

13

9

44.3

38.1

$3 / 13$

$2 / 9$

32.8

(21.3-46.0)

(18.1-61.6)

36.4

$8 / 15$

$3 / 13$

$1 / 9$

39.2

$8 / 15$

$3 / 10$

$1 / 7$

29.4

$7 / 15$

$3 / 9$

$1 / 7$

9.8

28.6

$3 / 9$

$1 / 7$

2.1

2.1

$3 / 13$

$2 / 9$

25.9

0.0

$2 / 8$

1.9

2.6

25.0

$0 / 8$

$0 / 3$

$0 / 2$

$0 / 3$

$0 / 2$

$1 / 8$

$0 / 3$

$0 / 1$

34

47.1

1.9

18

$3 / 18$

1.7
12
$4 / 12$
1.7

2

$1 / 2$

2.0

\section{CURRENT CLINIC SERVICES AND PROFILE}

Current Name: Mayo Clinic Assisted Reproductive Technologies

\begin{tabular}{l|l|l} 
Donor egg? Yes & Gestational carriers? Yes
\end{tabular}

Donor embryo? No Cryopreservation? Yes

Fresh Embryos

0
Frozen Embryos 8

$2 / 8$

1.9

Single women? Yes

SART member? Yes

Verified lab accreditation? Yes

(See Appendix C for details.)

${ }^{a}$ Reflects patient and treatment characteristics of ART cycles performed in 2006 using fresh nondonor eggs or embryos.

${ }^{b}$ When fewer than 20 cycles are reported in an age category, rates are shown as a fraction and confidence intervals are not given. Calculating percentages from fractions may be misleading and is not encouraged.

A multiple-infant birth is counted as one live birth.

d Clinic-specific outcome rates are unreliable for women older than 42 undergoing ART cycles using fresh or frozen embryos with nondonor eggs. Readers are urged to review national outcomes for these age groups (see page 27).

e All ages (including ages $>42$ ) are reported together because previous data show that patient age does not materially affect success with donor eggs. 


\section{REPRODUCTIVE MEDICINE \& INFERTILITY ASSOCIATES WOODBURY, MINNESOTA}

A comparison of clinic success rates may not be meaningful because patient medical characteristics and treatment approaches vary from clinic to clinic. For more details about this, along with information on how to interpret the statistics in this table, see pages 79-88.

\section{ART CYCLE PROFILE}

\begin{tabular}{|c|c|c|c|c|c|c|c|}
\hline \multicolumn{4}{|c|}{ Type of ART ${ }^{a}$} & \multicolumn{4}{|c|}{ Patient Diagnosis } \\
\hline IVF & $100 \%$ & Procedural Factors: & & Tubal factor & $4 \%$ & Other factor & $5 \%$ \\
\hline GIFT & $0 \%$ & With ICSI & $89 \%$ & Ovulatory dysfunction & $3 \%$ & Unknown factor & $8 \%$ \\
\hline ZIFT & $0 \%$ & Unstimulated & $0 \%$ & Diminished ovarian reserve & $1 \%$ & Multiple Factors: & \\
\hline \multirow[t]{3}{*}{ Combination } & $0 \%$ & Used gestational carrier & $<1 \%$ & Endometriosis & $3 \%$ & Female factors only & $4 \%$ \\
\hline & & & & Uterine factor & $<1 \%$ & Female \& male factors & $42 \%$ \\
\hline & & & & Male factor & $30 \%$ & & \\
\hline
\end{tabular}

2006 PREGNANCY SUCCESS RATES

Data verified by Jacques P. Stassart, MD

\section{Type of Cycle}

(n)

\section{Fresh Embryos from Nondonor Eggs}

Number of cycles

Percentage of cycles resulting in pregnancies ${ }^{b}$

Percentage of cycles resulting in live births ${ }^{b, c}$

(Confidence Interval)

Percentage of retrievals resulting in live births $s^{b, c}$

Percentage of transfers resulting in live births ${ }^{b, c}$

Percentage of transfers resulting in singleton live births ${ }^{b}$

Percentage of cancellations ${ }^{b}$

Average number of embryos transferred

Percentage of pregnancies with twins ${ }^{b}$

Percentage of pregnancies with triplets or more ${ }^{b}$

Percentage of live births having multiple infants ${ }^{b, c}$

\section{Frozen Embryos from Nondonor Eggs}

Number of transfers

Percentage of transfers resulting in live births ${ }^{\mathrm{b}, \mathrm{c}}$

Average number of embryos transferred

\section{Donor Eggs}

Number of transfers

Percentage of transfers resulting in live births ${ }^{b, c}$

Average number of embryos transferred

\section{Age of Woman}

$35-37 \quad 38-40 \quad 41-42^{d}$

\section{$<35$}

104

46.2

63

26

57.7

34.6

34.9

15.4

49.0

(25.6-44.6)

25.4

15.4

(42.1-56.0)

51.0

36.4

(15.3-37.9)

(4.4-34.9)

53.1

38.3

26.2

27.6

16.0

35.9

30.9

22.4

18.2

3.8

4.8

3.2

4.5

2.0

2.2

31.7

25.0

2.5

32.4

2.1

19.4

2.7

18.2

0.0

$3 / 16$

3.8

3.0

$4 / 4$

$0 / 4$

$3 / 4$

$\begin{array}{cccc}55 & 29 & 13 & 3 \\ 34.5 & 17.2 & 3 / 13 & 0 / 3 \\ 1.9 & 2.0 & 2.2 & 1.3\end{array}$

\section{All Ages Combined}

Fresh Embryos

67

41.8

1.9
Frozen Embryos 26

19.2

2.2

\section{CURRENT CLINIC SERVICES AND PROFILE}

Current Name: Reproductive Medicine \& Infertility Associates

Donor egg? Yes Gestational carriers? Yes

Donor embryo? Yes Cryopreservation? Yes

Single women? Yes

SART member? Yes

Verified lab accreditation? Yes

(See Appendix C for details.)

\footnotetext{
${ }^{a}$ Reflects patient and treatment characteristics of ART cycles performed in 2006 using fresh nondonor eggs or embryos.

${ }^{b}$ When fewer than 20 cycles are reported in an age category, rates are shown as a fraction and confidence intervals are not given. Calculating percentages from fractions may be misleading and is not encouraged.

${ }^{c}$ A multiple-infant birth is counted as one live birth.

d Clinic-specific outcome rates are unreliable for women older than 42 undergoing ART cycles using fresh or frozen embryos with nondonor eggs. Readers are urged to review national outcomes for these age groups (see page 27).

e All ages (including ages $>42$ ) are reported together because previous data show that patient age does not materially affect success with donor eggs.
} 


\section{MISSISSIPPI FERTILITY INSTITUTE \\ JACKSON, MISSISSIPPI}

A comparison of clinic success rates may not be meaningful because patient medical characteristics and treatment approaches vary from clinic to clinic. For more details about this, along with information on how to interpret the statistics in this table, see pages 79-88.

\section{ART CYCLE PROFILE}

$$
\text { Type of ART }{ }^{\mathrm{a}}
$$

IVF

$$
\text { 100\% Procedural Factors: }
$$

GIFT

$0 \%$ With ICSI

Tubal factor

\section{Patient Diagnosis}

ZIFT

$0 \%$ Unstimulated

$70 \%$

Ovulatory dysfunction

$25 \%$ Other factor

$1 \%$ Unknown factor

$19 \%$

Combination

$0 \%$ Used gestational carrier $<1 \%$

Diminished ovarian reserve

6\% Multiple Factors:

Endometriosis

$16 \%$ Female factors only

Uterine factor

$2 \%$ Female \& male factors $11 \%$

Male factor $14 \%$

2006 PREGNANCY SUCCESS RATES

Data verified by John Isaacs, Jr., MD

\section{Type of Cycle}

\section{Age of Woman}

\section{Fresh Embryos from Nondonor Eggs}

Number of cycles

Percentage of cycles resulting in pregnancies ${ }^{b}$

Percentage of cycles resulting in live births ${ }^{b, c}$

(Confidence Interval)

Percentage of retrievals resulting in live births $s^{b, c}$

Percentage of transfers resulting in live births $s^{b, c}$

Percentage of transfers resulting in singleton live births ${ }^{b}$

Percentage of cancellations ${ }^{b}$

Average number of embryos transferred

Percentage of pregnancies with twins ${ }^{b}$

Percentage of pregnancies with triplets or more ${ }^{\mathrm{b}}$

Percentage of live births having multiple infants ${ }^{\mathrm{b}, \mathrm{c}}$

\section{Frozen Embryos from Nondonor Eggs}

Number of transfers

Percentage of transfers resulting in live births $s^{b, c}$

Average number of embryos transferred

8

$<35 \quad 35-37 \quad 38-40 \quad 41-42^{d}$

$\begin{array}{cccc}74 & 23 & 10 & 6 \\ 45.9 & 34.8 & 5 / 10 & 1 / 6 \\ 44.6 & 34.8 & 5 / 10 & 1 / 6\end{array}$

$\begin{array}{cc}44.6 & 34.8 \\ (33.0-56.6) & (16.4-57.3) \\ 47.8 & 38.1\end{array}$

$5 / 9 \quad 1 / 6$

$50.0 \quad 40.0$

$5 / 8$

$1 / 6$

37.9

35.0

$4 / 8$

$1 / 6$

6.8

8.7

$1 / 10$

$0 / 6$

2.4

2.5

2.6

2.5

32.4

$2 / 8$

$2 / 5$

$0 / 1$

0.0

$0 / 8$

$0 / 5$

$0 / 1$

24.2

$1 / 8$

$1 / 5$

$0 / 1$

\section{8}

$4 / 8$

2.0

\section{4}

$0 / 4$

1.8

$0 / 1$

4.0

All Ages Combined ${ }^{\mathrm{e}}$

\section{Donor Eggs}

Number of transfers

Percentage of transfers resulting in live births $s^{b, c}$

Average number of embryos transferred

\section{Fresh Embryos}

9

$6 / 9$

2.3
Frozen Embryos

$0 / 1$

3.0

\section{CURRENT CLINIC SERVICES AND PROFILE}

Current Name: Mississippi Fertility Institute
Donor egg?
Yes
Donor embryo? Yes
Gestational carriers? Yes
Cryopreservation? Yes
Single women? No
SART member?
Verified lab accreditation?
No
(See Appendix C for details.)
${ }^{a}$ Reflects patient and treatment characteristics of ART cycles performed in 2006 using fresh nondonor eggs or embryos.
${ }^{b}$ When fewer than 20 cycles are reported in an age category, rates are shown as a fraction and confidence intervals are not given. Calculating percentages from fractions may be misleading and is not encouraged.
c A multiple-infant birth is counted as one live birth.
${ }^{d}$ Clinic-specific outcome rates are unreliable for women older than 42 undergoing ART cycles using fresh or frozen embryos with nondonor eggs. Readers are urged to review national outcomes for these age groups (see page 27).
${ }^{e}$ All ages (including ages $>42$ ) are reported together because previous data show that patient age does not materially affect success with donor eggs. 


\section{UNIVERSITY OF MISSISSIPPI MEDICAL CENTER \\ JACKSON, MISSISSIPPI}

A comparison of clinic success rates may not be meaningful because patient medical characteristics and treatment approaches vary from clinic to clinic. For more details about this, along with information on how to interpret the statistics in this table, see pages 79-88.

\section{ART CYCLE PROFILE}

\begin{tabular}{|c|c|c|c|c|c|c|c|}
\hline \multicolumn{4}{|c|}{ Type of ART } & \multicolumn{4}{|c|}{ Patient Diagnosis } \\
\hline IVF & $100 \%$ & Procedural Factors: & & Tubal factor & $14 \%$ & Other factor & $2 \%$ \\
\hline GIFT & $0 \%$ & With ICSI & $91 \%$ & Ovulatory dysfunction & $10 \%$ & Unknown factor & $6 \%$ \\
\hline ZIFT & $0 \%$ & Unstimulated & $0 \%$ & Diminished ovarian reserve & $8 \%$ & Multiple Factors: & \\
\hline \multirow[t]{3}{*}{ Combination } & $0 \%$ & Used gestational carrier & $1 \%$ & Endometriosis & $17 \%$ & Female factors only & $15 \%$ \\
\hline & & & & Uterine factor & $2 \%$ & Female \& male factors & $15 \%$ \\
\hline & & & & Male factor & $12 \%$ & & \\
\hline
\end{tabular}

2006 PREGNANCY SUCCESS RATES

Data verified by Randall S. Hines, MD

\section{Type of Cycle}

\section{Fresh Embryos from Nondonor Eggs}

Number of cycles

Percentage of cycles resulting in pregnancies ${ }^{b}$

Percentage of cycles resulting in live births ${ }^{b, c}$

(Confidence Interval)

Percentage of retrievals resulting in live births $s^{b, c}$

Percentage of transfers resulting in live births $s^{b, c}$

Percentage of transfers resulting in singleton live births ${ }^{b}$

Percentage of cancellations ${ }^{b}$

Average number of embryos transferred

Percentage of pregnancies with twins ${ }^{b}$

Percentage of pregnancies with triplets or more ${ }^{b}$

Percentage of live births having multiple infants ${ }^{b, c}$

\section{Frozen Embryos from Nondonor Eggs}

Number of transfers

Percentage of transfers resulting in live births ${ }^{b, c}$

Average number of embryos transferred

\section{Donor Eggs}

Number of transfers

Percentage of transfers resulting in live births $s^{b, c}$

Average number of embryos transferred

\section{Age of Woman}

35-37 38-40

$41-42^{d}$

\section{$<35$}

22

40.9

9

2

24.1

20.4

36.4

$1 / 9$

$0 / 2$

(10.6-33.5)

$(17.2-59.3)$

22.4

22.9

36.4

$1 / 9$

$0 / 2$

16.7

36.4

$1 / 8$

$0 / 2$

27.3

$1 / 8$

$0 / 1$

9.3

0.0

$1 / 8$

$0 / 1$

2.9

2.8

$1 / 9$

$0 / 2$

$3 / 13$

$2 / 9$

2.3

3.0

$0 / 13$

$3 / 11$

$0 / 9$

$0 / 1$

$0 / 1$

$2 / 8$

$0 / 1$

18

$4 / 18$

2.3

\section{3}

$0 / 3$

3.0

\section{2}

$1 / 2$

2.5

\section{All Ages Combined}

Fresh Embryos

10

$5 / 10$

2.5
Frozen Embryos 5

$1 / 5$

2.0

\section{CURRENT CLINIC SERVICES AND PROFILE}

Current Name: University of Mississippi Medical Center

Donor egg? Yes

Donor embryo? Yes

Single women? No

\section{Gestational carriers? Yes}

Cryopreservation? Yes
SART member?

Yes

Verified lab accreditation?

(See Appendix C for details.)

\footnotetext{
${ }^{a}$ Reflects patient and treatment characteristics of ART cycles performed in 2006 using fresh nondonor eggs or embryos.

${ }^{b}$ When fewer than 20 cycles are reported in an age category, rates are shown as a fraction and confidence intervals are not given. Calculating percentages from fractions may be misleading and is not encouraged.

${ }^{c}$ A multiple-infant birth is counted as one live birth.

${ }^{d}$ Clinic-specific outcome rates are unreliable for women older than 42 undergoing ART cycles using fresh or frozen embryos with nondonor eggs. Readers are urged to review national outcomes for these age groups (see page 27).

e All ages (including ages $>42$ ) are reported together because previous data show that patient age does not materially affect success with donor eggs.
} 


\section{INFERTILITY INSTITUTE CHESTERFIELD, MISSOURI}

A comparison of clinic success rates may not be meaningful because patient medical characteristics and treatment approaches vary from clinic to clinic. For more details about this, along with information on how to interpret the statistics in this table, see pages $79-88$.

\section{ART CYCLE PROFILE}

\begin{tabular}{|c|c|c|c|c|c|c|}
\hline \multicolumn{3}{|c|}{ Type of ART } & \multicolumn{4}{|c|}{ Patient Diagnosis } \\
\hline IVF & $100 \%$ Procedural Factors: & & Tubal factor & $4 \%$ & Other factor & $0 \%$ \\
\hline GIFT & 0\% With ICSI & $80 \%$ & Ovulatory dysfunction & $17 \%$ & Unknown factor & $0 \%$ \\
\hline ZIFT & $0 \%$ Unstimulated & $0 \%$ & Diminished ovarian reserve & $5 \%$ & Multiple Factors: & \\
\hline \multirow[t]{3}{*}{ Combination } & $0 \%$ Used gestational carrier & $0 \%$ & Endometriosis & $1 \%$ & Female factors only & $22 \%$ \\
\hline & & & Uterine factor & $0 \%$ & Female \& male factors & $47 \%$ \\
\hline & & & Male factor & $5 \%$ & & \\
\hline
\end{tabular}

2006 PREGNANCY SUCCESS RATES

Data verified by Anthony C. Pearlstone, MD

\section{Type of Cycle}

\section{Fresh Embryos from Nondonor Eggs}

Number of cycles

Percentage of cycles resulting in pregnancies ${ }^{b}$

Percentage of cycles resulting in live births $s^{b, c}$

(Confidence Interval)

Percentage of retrievals resulting in live births $s^{b, c}$

Percentage of transfers resulting in live births $s^{b, c}$

Percentage of transfers resulting in singleton live births ${ }^{b}$

Percentage of cancellations ${ }^{b}$

Average number of embryos transferred

Percentage of pregnancies with twins ${ }^{b}$

Percentage of pregnancies with triplets or more ${ }^{b}$

Percentage of live births having multiple infants ${ }^{b, c}$

\section{Frozen Embryos from Nondonor Eggs}

Number of transfers

Percentage of transfers resulting in live births $s^{b, c}$

Average number of embryos transferred

\section{Donor Eggs}

Number of transfers

Percentage of transfers resulting in live births $s^{b, c}$

Average number of embryos transferred

\section{Age of Woman}

35-37 38-40

$41-42^{d}$

$<35$

30

53.3

16

7

50.7

43.3

$4 / 16$

$2 / 7$

40.6

(28.9-53.1)

$(25.5-62.6)$
44.8

$4 / 16$

$0 / 7$

41.8

$4 / 13$

$0 / 7$

41.8

48.1

$4 / 12$

$0 / 7$

31.3

37.0

$2 / 12$

$0 / 7$

2.9

3.3

$3 / 16$

$0 / 7$

2.4

3.1

3.7

3.6

17.1

$3 / 16$

$2 / 4$

$0 / 2$

2.9

$0 / 16$

$0 / 4$

$0 / 2$

25.0

$3 / 13$

$2 / 4$

\section{5}

$1 / 5$

2.2

\section{1}

$0 / 1$

2.0

\section{3}

$0 / 3$

3.3 . 


\section{MID-MISSOURI REPRODUCTIVE MEDICINE AND SURGERY, INC. COLUMBIA, MISSOURI}

A comparison of clinic success rates may not be meaningful because patient medical characteristics and treatment approaches vary from clinic to clinic. For more details about this, along with information on how to interpret the statistics in this table, see pages 79-88.

\section{ART CYCLE PROFILE}

\begin{tabular}{|c|c|c|c|c|c|c|c|}
\hline \multicolumn{4}{|c|}{ Type of ART ${ }^{a}$} & \multicolumn{4}{|c|}{ Patient Diagnosis } \\
\hline IVF & $100 \%$ & Procedural Factors: & & Tubal factor & $13 \%$ & Other factor & $1 \%$ \\
\hline GIFT & $0 \%$ & With ICSI & $57 \%$ & Ovulatory dysfunction & $7 \%$ & Unknown factor & $1 \%$ \\
\hline ZIFT & $0 \%$ & Unstimulated & $0 \%$ & Diminished ovarian reserve & $0 \%$ & Multiple Factors: & \\
\hline \multirow[t]{3}{*}{ Combination } & $0 \%$ & Used gestational carrier & $0 \%$ & Endometriosis & $9 \%$ & Female factors only & $13 \%$ \\
\hline & & & & Uterine factor & $0 \%$ & Female \& male factors & $23 \%$ \\
\hline & & & & Male factor & $33 \%$ & & \\
\hline
\end{tabular}

2006 PREGNANCY SUCCESS RATES

Data verified by L. L. Penney, MD

\section{Type of Cycle}

\section{Fresh Embryos from Nondonor Eggs}

Number of cycles

Percentage of cycles resulting in pregnancies ${ }^{b}$

Percentage of cycles resulting in live births ${ }^{b, c}$

(Confidence Interval)

Percentage of retrievals resulting in live births $s^{b, c}$

Percentage of transfers resulting in live births $s^{b, c}$

Percentage of transfers resulting in singleton live births ${ }^{b}$

Percentage of cancellations ${ }^{b}$

Average number of embryos transferred

Percentage of pregnancies with twins ${ }^{b}$

Percentage of pregnancies with triplets or more ${ }^{b}$

Percentage of live births having multiple infants ${ }^{b, c}$

\section{Frozen Embryos from Nondonor Eggs}

Number of transfers

Percentage of transfers resulting in live births ${ }^{b, c}$

Average number of embryos transferred

\section{Donor Eggs}

Number of transfers

Percentage of transfers resulting in live births $s^{b, c}$

Average number of embryos transferred

\section{Age of Woman}

35-37

38-40

$41-42^{d}$

\section{$<35$}

23

17.4

9

32.6

13.0

$4 / 9$

30.2

(2.8-33.6)

(17.2-46.1)

$3 / 12$

$3 / 9$

35.1

$3 / 11$

$3 / 8$

36.1

$2 / 11$

$3 / 8$

25.0

47.8

$2 / 8$

14.0

2.9

$1 / 9$

2.4

$1 / 4$

3.1

$4 / 14$

$0 / 4$

$0 / 4$

$1 / 14$

$4 / 13$

$1 / 3$

$2 / 4$

$1 / 3$

$\begin{array}{ccc}4 & 2 & 2 \\ 1 / 4 & 0 / 2 & 0 / 2 \\ 3.5 & 2.5 & 3.0\end{array}$

All Ages Combined ${ }^{\mathrm{e}}$

Fresh Embryos

Frozen Embryos

0

2

$1 / 2$

2.5

\section{CURRENT CLINIC SERVICES AND PROFILE}

Current Name: Mid-Missouri Reproductive Medicine and Surgery, Inc.

Donor egg? No Gestational carriers? Yes

Donor embryo? Yes Cryopreservation? Yes

Single women? Yes

SART member? Yes

Verified lab accreditation? Yes

(See Appendix C for details.)

${ }^{a}$ Reflects patient and treatment characteristics of ART cycles performed in 2006 using fresh nondonor eggs or embryos.

${ }^{b}$ When fewer than 20 cycles are reported in an age category, rates are shown as a fraction and confidence intervals are not given. Calculating percentages from fractions may be misleading and is not encouraged.

${ }^{c}$ A multiple-infant birth is counted as one live birth.

d Clinic-specific outcome rates are unreliable for women older than 42 undergoing ART cycles using fresh or frozen embryos with nondonor eggs. Readers are urged to review national outcomes for these age groups (see page 27).

e All ages (including ages $>42$ ) are reported together because previous data show that patient age does not materially affect success with donor eggs. 


\section{UNIVERSITY OF MISSOURI HOSPITAL AND CLINIC IVF EMBRYOLOGY LABORATORY COLUMBIA, MISSOURI}

A comparison of clinic success rates may not be meaningful because patient medical characteristics and treatment approaches vary from clinic to clinic. For more details about this, along with information on how to interpret the statistics in this table, see pages $79-88$.

\section{ART CYCLE PROFILE}

\begin{tabular}{|c|c|c|c|c|c|c|c|}
\hline \multicolumn{4}{|c|}{ Type of ART } & \multicolumn{4}{|c|}{ Patient Diagnosis } \\
\hline IVF & & Procedural Factors: & & Tubal factor & $10 \%$ & Other factor & $20 \%$ \\
\hline GIFT & $0 \%$ & With ICSI & $50 \%$ & Ovulatory dysfunction & $30 \%$ & Unknown factor & $10 \%$ \\
\hline ZIFT & $0 \%$ & Unstimulated & $0 \%$ & Diminished ovarian reserve & $0 \%$ & Multiple Factors: & \\
\hline \multirow[t]{3}{*}{ Combination } & $0 \%$ & Used gestational carrier & $0 \%$ & Endometriosis & $10 \%$ & Female factors only & $10 \%$ \\
\hline & & & & Uterine factor & $0 \%$ & Female \& male factors & $0 \%$ \\
\hline & & & & Male factor & $10 \%$ & & \\
\hline
\end{tabular}

2006 PREGNANCY SUCCESS RATES

Data verified by Danny J. Schust, MD

\section{Type of Cycle}

\section{Fresh Embryos from Nondonor Eggs}

Number of cycles

Percentage of cycles resulting in pregnancies ${ }^{b}$

Percentage of cycles resulting in live births $s^{b, c}$

(Confidence Interval)

Percentage of retrievals resulting in live births $s^{b, c}$

Percentage of transfers resulting in live births $s^{b, c}$

Percentage of transfers resulting in singleton live births ${ }^{b}$

Percentage of cancellations ${ }^{b}$

Average number of embryos transferred

Percentage of pregnancies with twins ${ }^{b}$

Percentage of pregnancies with triplets or more ${ }^{b}$

Percentage of live births having multiple infants ${ }^{b, c}$

\section{Frozen Embryos from Nondonor Eggs}

Number of transfers

Percentage of transfers resulting in live births $s^{b, c}$

Average number of embryos transferred

\section{Donor Eggs}

Number of transfers

Percentage of transfers resulting in live births $s^{\text {b,c }}$

Average number of embryos transferred

\section{Age of Woman}

$\begin{array}{cccc}<35 & 35-37 & 38-40 & \mathbf{4 1 - 4 2}^{\mathbf{d}} \\ 6 & 4 & 0 & 0 \\ 1 / 6 & 0 / 4 & & \\ 1 / 6 & 0 / 4 & & \end{array}$

$\begin{array}{ll}1 / 6 & 0 / 4 \\ 1 / 5 & 0 / 3 \\ 1 / 5 & 0 / 3 \\ 0 / 6 & 0 / 4 \\ 2.0 & 2.7 \\ 0 / 1 & \\ 0 / 1 & \\ 0 / 1 & \end{array}$

0

0

0

0

\section{CURRENT CLINIC SERVICES AND PROFILE}

Current Name: This clinic has closed or reorganized since 2006. Information on current clinic services and profile therefore is not provided here. Contact the NASS Help Desk for current information about this clinic.

${ }^{a}$ Reflects patient and treatment characteristics of ART cycles performed in 2006 using fresh nondonor eggs or embryos.

${ }^{b}$ When fewer than 20 cycles are reported in an age category, rates are shown as a fraction and confidence intervals are not given. Calculating percentages from fractions may be misleading and is not encouraged.

${ }^{c}$ A multiple-infant birth is counted as one live birth.

d Clinic-specific outcome rates are unreliable for women older than 42 undergoing ART cycles using fresh or frozen embryos with nondonor eggs. Readers are urged to review national outcomes for these age groups (see page 27).

${ }^{e}$ All ages (including ages $>42$ ) are reported together because previous data show that patient age does not materially affect success with donor eggs. 


\section{MIDWEST WOMEN'S HEALTHCARE \\ KANSAS CITY, MISSOURI}

A comparison of clinic success rates may not be meaningful because patient medical characteristics and treatment approaches vary from clinic to clinic. For more details about this, along with information on how to interpret the statistics in this table, see pages 79-88.

\section{ART CYCLE PROFILE}

\section{Type of ART ${ }^{\mathrm{a}}$}

IVF

GIFT

100\% Procedural Factors:

ZIFT

Combination
$0 \%$ With ICSI

$0 \%$ Unstimulated

$0 \%$ Used gestational carrier

\begin{tabular}{|l|llll|}
\multicolumn{5}{c}{ Patient Diagnosis } \\
\\
$82 \%$ & Tubal factor & $5 \%$ & Other factor & $0 \%$ \\
$1 \%$ & Dimininished ovarian reserve & $4 \%$ & Unknown factor & $1 \%$ \\
$0 \%$ & Endometriosis & $19 \%$ & Female factors only & $29 \%$ \\
& Uterine factor & $0 \%$ & Female \& male factors & $31 \%$ \\
& Male factor & $11 \%$ & &
\end{tabular}

2006 PREGNANCY SUCCESS RATES

Data verified by Gregory C. Starks, MD

\section{Type of Cycle}

\section{Fresh Embryos from Nondonor Eggs}

Number of cycles

Percentage of cycles resulting in pregnancies ${ }^{b}$

Percentage of cycles resulting in live births ${ }^{b, c}$

(Confidence Interval)

Percentage of retrievals resulting in live births $s^{b, c}$

Percentage of transfers resulting in live births $s^{b, c}$

Percentage of transfers resulting in singleton live births ${ }^{b}$

Percentage of cancellations ${ }^{b}$

Average number of embryos transferred

Percentage of pregnancies with twins ${ }^{b}$

Percentage of pregnancies with triplets or more ${ }^{b}$

Percentage of live births having multiple infants ${ }^{b, c}$

\section{Frozen Embryos from Nondonor Eggs}

Number of transfers

Percentage of transfers resulting in live births ${ }^{b, c}$

Average number of embryos transferred

\section{Donor Eggs}

Number of transfers

Percentage of transfers resulting in live births $s^{b, c}$

Average number of embryos transferred

\section{Age of Woman}

35-37 38-40

$41-42^{d}$

\section{$<35$}

11

$5 / 11$

$5 / 11$

16

4

68.4

63.2

$(46.0-78.2)$

68.6

70.6

$5 / 10$

$5 / 10$

$4 / 10$

$1 / 11$

1.9

$2 / 5$

$0 / 5$

$1 / 5$

$5 / 16$

$5 / 16$

$1 / 4$

7.9

2.0

42.3

0.0

37.5

3

$0 / 3$

1.3

1

$0 / 1$

1.0

$\begin{array}{cc}5 / 11 & 1 / 1 \\ 5 / 11 & 1 / 1 \\ 4 / 11 & 1 / 1 \\ 5 / 16 & 3 / 4 \\ 2.2 & 3.0 \\ 1 / 5 & 0 / 1 \\ 0 / 5 & 0 / 1 \\ 1 / 5 & 0 / 1\end{array}$

All Ages Combined ${ }^{\mathrm{e}}$

Fresh Embryos

Frozen Embryos

4

$3 / 4$

0
2

$1 / 2$

1.5
$\%$

$\%$

29\%
2.0

\section{CURRENT CLINIC SERVICES AND PROFILE}

Current Name: Midwest Women's Healthcare
Donor egg?
Yes
Gestational carriers?
No
Cryopreservation?
Yes
Donor embryo? No
Single women? Yes

$\begin{array}{ll}\text { SART member? } & \text { Yes } \\ \text { Verified lab accreditation? } & \text { Yes } \\ \text { (See Appendix C for details.) } & \end{array}$
${ }^{a}$ Reflects patient and treatment characteristics of ART cycles performed in 2006 using fresh nondonor eggs or embryos.
${ }^{b}$ When fewer than 20 cycles are reported in an age category, rates are shown as a fraction and confidence intervals are not given. Calculating percentages from fractions may be misleading and is not encouraged.
${ }^{c}$ A multiple-infant birth is counted as one live birth.
${ }^{d}$ Clinic-specific outcome rates are unreliable for women older than 42 undergoing ART cycles using fresh or frozen embryos with nondonor eggs. Readers are urged to review national outcomes for these age groups (see page 27).
e All ages (including ages $>42$ ) are reported together because previous data show that patient age does not materially affect success with donor eggs. 


\section{INFERTILITY \& IVF CENTER \\ ST. LOUIS, MISSOURI}

A comparison of clinic success rates may not be meaningful because patient medical characteristics and treatment approaches vary from clinic to clinic. For more details about this, along with information on how to interpret the statistics in this table, see pages $79-88$.

\section{ART CYCLE PROFILE}

\begin{tabular}{|c|c|c|c|c|c|c|c|}
\hline \multicolumn{4}{|c|}{ Type of ART ${ }^{\mathrm{a}}$} & \multicolumn{4}{|c|}{ Patient Diagnosis } \\
\hline IVF & $100 \%$ & Procedural Factors: & & Tubal factor & $5 \%$ & Other factor & $0 \%$ \\
\hline GIFT & $0 \%$ & With ICSI & $68 \%$ & Ovulatory dysfunction & $0 \%$ & Unknown factor & $2 \%$ \\
\hline $\mathrm{ZIFT}$ & $0 \%$ & Unstimulated & $0 \%$ & Diminished ovarian reserve & $0 \%$ & Multiple Factors: & \\
\hline \multirow[t]{3}{*}{ Combination } & $0 \%$ & Used gestational carrier & $1 \%$ & Endometriosis & $0 \%$ & Female factors only & $37 \%$ \\
\hline & & & & Uterine factor & $0 \%$ & Female \& male factors & $39 \%$ \\
\hline & & & & Male factor & $17 \%$ & & \\
\hline
\end{tabular}

2006 PREGNANCY SUCCESS RATES

Data verified by Ronald P. Wilbois, MD

\section{Type of Cycle}

\section{Fresh Embryos from Nondonor Eggs}

Number of cycles

Percentage of cycles resulting in pregnancies ${ }^{b}$

Percentage of cycles resulting in live births $s^{b, c}$

(Confidence Interval)

Percentage of retrievals resulting in live births $s^{b, c}$

Percentage of transfers resulting in live births $s^{b, c}$

Percentage of transfers resulting in singleton live births ${ }^{b}$

Percentage of cancellations ${ }^{b}$

Average number of embryos transferred

Percentage of pregnancies with twins ${ }^{b}$

Percentage of pregnancies with triplets or more ${ }^{b}$

Percentage of live births having multiple infants ${ }^{b, c}$

\section{Frozen Embryos from Nondonor Eggs}

Number of transfers

Percentage of transfers resulting in live births $s^{\mathrm{b}, \mathrm{c}}$

Average number of embryos transferred

\section{Donor Eggs}

Number of transfers

Percentage of transfers resulting in live births $s^{\mathrm{b}, \mathrm{c}}$

Average number of embryos transferred

\section{Age of Woman}

35-37 38-40

$41-42^{d}$

\section{$<35$}

11

17

7

40.0

$4 / 11$

$2 / 17$

$0 / 7$

33.3

$4 / 11$

$0 / 17$

$0 / 7$

(21.7-46.7)

$\begin{array}{cccc}37.0 & 4 / 9 & 0 / 11 & 0 / 5 \\ 41.7 & 4 / 8 & 0 / 8 & 0 / 3 \\ 33.3 & 3 / 8 & 0 / 8 & 0 / 3 \\ 10.0 & 2 / 11 & 6 / 17 & 2 / 7 \\ 2.0 & 1.8 & 2.1 & 2.3 \\ 12.5 & 1 / 4 & 0 / 2 & \\ 4.2 & 0 / 4 & 0 / 2 & \\ 20.0 & 1 / 4 & \end{array}$

18

$4 / 18$

6

1.7

$2 / 6$

5

$1 / 5$

1

2.0

2.0

$0 / 1$

1.0

All Ages Combined ${ }^{\mathrm{e}}$

Fresh Embryos 19

$8 / 19$

1.9
Frozen Embryos 15

$5 / 15$

2.1

\section{CURRENT CLINIC SERVICES AND PROFILE}

Current Name: Infertility \& IVF Center

Donor egg? Yes

Donor embryo? Yes

Single women? Yes

\section{Gestational carriers? Yes}

Cryopreservation? Yes

\section{SART member?}

Verified lab accreditation?

(See Appendix C for details.)

${ }^{a}$ Reflects patient and treatment characteristics of ART cycles performed in 2006 using fresh nondonor eggs or embryos.

${ }^{b}$ When fewer than 20 cycles are reported in an age category, rates are shown as a fraction and confidence intervals are not given. Calculating percentages from fractions may be misleading and is not encouraged.

${ }^{c}$ A multiple-infant birth is counted as one live birth.

d Clinic-specific outcome rates are unreliable for women older than 42 undergoing ART cycles using fresh or frozen embryos with nondonor eggs. Readers are urged to review national outcomes for these age groups (see page 27).

${ }^{e}$ All ages (including ages $>42$ ) are reported together because previous data show that patient age does not materially affect success with donor eggs. 
A comparison of clinic success rates may not be meaningful because patient medical characteristics and treatment approaches vary from clinic to clinic. For more details about this, along with information on how to interpret the statistics in this table, see pages 79-88.

\section{ART CYCLE PROFILE}

\begin{tabular}{|c|c|c|c|c|c|c|c|}
\hline \multicolumn{4}{|c|}{ Type of ART ${ }^{a}$} & \multicolumn{4}{|c|}{ Patient Diagnosis } \\
\hline IVF & $100 \%$ & Procedural Factors: & & Tubal factor & $17 \%$ & Other factor & $5 \%$ \\
\hline GIFT & $0 \%$ & With ICSI & $50 \%$ & Ovulatory dysfunction & $8 \%$ & Unknown factor & $13 \%$ \\
\hline ZIFT & $0 \%$ & Unstimulated & $0 \%$ & Diminished ovarian reserve & $5 \%$ & Multiple Factors: & \\
\hline \multirow[t]{3}{*}{ Combination } & $0 \%$ & Used gestational carrier & $<1 \%$ & Endometriosis & $6 \%$ & Female factors only & $14 \%$ \\
\hline & & & & Uterine factor & $<1 \%$ & Female \& male factors & $16 \%$ \\
\hline & & & & Male factor & $17 \%$ & & \\
\hline
\end{tabular}

2006 PREGNANCY SUCCESS RATES

Data verified by Randall R. Odem, MD

\section{Type of Cycle}

\section{Fresh Embryos from Nondonor Eggs}

Number of cycles

Percentage of cycles resulting in pregnancies ${ }^{b}$

Percentage of cycles resulting in live births ${ }^{b, c}$

(Confidence Interval)

Percentage of retrievals resulting in live births $s^{b, c}$

Percentage of transfers resulting in live births $s^{b, c}$

Percentage of transfers resulting in singleton live births ${ }^{b}$

Percentage of cancellations ${ }^{b}$

Average number of embryos transferred

Percentage of pregnancies with twins ${ }^{b}$

Percentage of pregnancies with triplets or more ${ }^{b}$

Percentage of live births having multiple infants ${ }^{b, c}$

Frozen Embryos from Nondonor Eggs

Number of transfers

Percentage of transfers resulting in live births $s^{\mathrm{b}, \mathrm{c}}$

Average number of embryos transferred

\section{Donor Eggs}

Number of transfers

Percentage of transfers resulting in live births $s^{b, c}$

Average number of embryos transferred

\section{Age of Woman}

35-37 38-40

$41-42^{d}$

\section{$<35$}

67

35.8

51

31

43.1

26.9

15.7

6.5

37.0

(16.8-39.1)

15.7

(30.0-44.5)

41.9

43.8

34.0

(7.0-28.6)

18.2

20.0

20.0

13.7

2.6

$0 / 8$

$0 / 8$

$0 / 8$

3.2

11.6

2.1

35.9

2.6

34.3

8.3

$8 / 18$

(0.1-16.7)

3.8

4.2

4.2

16.1

3.3

$0 / 2$

$0 / 2$

$0 / 1$

25

20.0

2.1

\begin{abstract}
16
\end{abstract}
$4 / 16$

1.9

12

3

$0 / 3$

2.0

\section{CURRENT CLINIC SERVICES AND PROFILE}

Current Name: The Infertility and Reproductive Medicine Center at Washington University School of Medicine and Barnes-Jewish Hospital

\begin{tabular}{ll|ll} 
Donor egg? & Yes & Gestational carriers? & Yes \\
Donor embryo? & No & Cryopreservation? & Yes \\
Single women? & Yes & &
\end{tabular}

All Ages Combined ${ }^{\mathrm{e}}$

Fresh Embryos

21

47.6

2.0
Frozen Embryos

\section{7}

$2 / 7$

2.6

\footnotetext{
${ }_{b}^{a}$ Reflects patient and treatment characteristics of ART cycles performed in 2006 using fresh nondonor eggs or embryos.

${ }^{b}$ When fewer than 20 cycles are reported in an age category, rates are shown as a fraction and confidence intervals are not given. Calculating percentages from fractions may be misleading and is not encouraged.

${ }^{c}$ A multiple-infant birth is counted as one live birth.

d Clinic-specific outcome rates are unreliable for women older than 42 undergoing ART cycles using fresh or frozen embryos with nondonor eggs. Readers are urged to review national outcomes for these age groups (see page 27).

All ages (including ages $>42$ ) are reported together because previous data show that patient age does not materially affect success with donor eggs.
} 


\section{INFERTILITY CENTER OF ST. LOUIS \\ ST. LUKE'S HOSPITAL \\ ST. LOUIS, MISSOURI}

A comparison of clinic success rates may not be meaningful because patient medical characteristics and treatment approaches vary from clinic to clinic. For more details about this, along with information on how to interpret the statistics in this table, see pages $79-88$.

\section{ART CYCLE PROFILE}

\section{Type of ART ${ }^{\mathrm{a}}$}

IVF

GIFT

ZIFT

Combination

\section{7\% Procedural Factors:} $<1 \%$ With ICSI

$3 \%$ Unstimulated

0\% Used gestational carrier

\begin{tabular}{|c|c|}
\hline & \\
\hline $91 \%$ & Ovulatory dysfunction \\
\hline $0 \%$ & Diminished ovarian reserve \\
\hline $1 \%$ & Endometriosis \\
\hline & Uterine factor \\
\hline & Male factor \\
\hline
\end{tabular}

Patient Diagnosis

$\begin{array}{rlr}4 \% & \text { Other factor } & 14 \% \\ 7 \% & \text { Unknown factor } & 12 \% \\ 15 \% & \text { Multiple Factors: } & \\ 2 \% & \text { Female factors only } & 2 \% \\ 3 \% & \text { Female \& male factors } & 9 \% \\ 34 \% & & \end{array}$

\section{PREGNANCY SUCCESS RATES}

\section{Type of Cycle}

\section{Fresh Embryos from Nondonor Eggs}

Number of cycles

Percentage of cycles resulting in pregnancies ${ }^{b}$

Percentage of cycles resulting in live births $s^{b, c}$

(Confidence Interval)

Percentage of retrievals resulting in live births $s^{b, c}$

Percentage of transfers resulting in live births $s^{b, c}$

Percentage of transfers resulting in singleton live births ${ }^{b}$

Percentage of cancellations ${ }^{b}$

Average number of embryos transferred

Percentage of pregnancies with twins ${ }^{b}$

Percentage of pregnancies with triplets or more ${ }^{b}$

Percentage of live births having multiple infants ${ }^{b, c}$

\section{Frozen Embryos from Nondonor Eggs}

Number of transfers

Percentage of transfers resulting in live births $s^{b, c}$

Average number of embryos transferred

\section{Donor Eggs}

Number of transfers

Percentage of transfers resulting in live births ${ }^{\mathrm{b}, \mathrm{c}}$

Average number of embryos transferred
Data verified by Sherman J. Silber, MD

\section{Age of Woman}

$\begin{array}{cccc}<35 & 35-37 & 38-40 & \mathbf{4 1 - 4 2}^{\text {d }} \\ 158 & 51 & 34 & 8 \\ 44.9 & 31.4 & 14.7 & 1 / 8 \\ 39.2 & 19.6 & 5.9 & 0 / 8\end{array}$

(31.6-47.3)

40.0

$(9.8-33.1)$

(0.7-19.7)

45.9

20.8

6.7

$0 / 7$

27.8

9.1

$0 / 4$

30.4

19.4

9.1

$0 / 4$

1.9

5.9

11.8

$1 / 8$

2.7

2.7

3.1

3.5

32.4

$3 / 16$

$0 / 5$

$0 / 1$

7.0

$2 / 16$

$0 / 5$

$0 / 1$

33.9

$3 / 10$

$0 / 2$
25

32.0

2.3

\section{8}

$4 / 8$

2.0

\section{5}

$2 / 5$

2.8

All Ages Combined ${ }^{\mathrm{e}}$

Fresh Embryos 20

40.0

2.4
Frozen Embryos 4

$0 / 4$

2.3

\section{CURRENT CLINIC SERVICES AND PROFILE}

Current Name: Infertility Center of St. Louis, St. Luke's Hospital

\begin{tabular}{ll|l} 
Donor egg? Yes & Gestational carriers? Yes
\end{tabular}

Donor embryo? Yes Cryopreservation? Yes

Single women? Yes

SART member? Yes

Verified lab accreditation? Yes

(See Appendix C for details.)

${ }^{a}$ Reflects patient and treatment characteristics of ART cycles performed in 2006 using fresh nondonor eggs or embryos.

${ }^{b}$ When fewer than 20 cycles are reported in an age category, rates are shown as a fraction and confidence intervals are not given. Calculating percentages from fractions may be misleading and is not encouraged.

${ }^{c}$ A multiple-infant birth is counted as one live birth.

d Clinic-specific outcome rates are unreliable for women older than 42 undergoing ART cycles using fresh or frozen embryos with nondonor eggs. Readers are urged to review national outcomes for these age groups (see page 27).

${ }^{e}$ All ages (including ages $>42$ ) are reported together because previous data show that patient age does not materially affect success with donor eggs. 


\section{SHER INSTITUTE FOR REPRODUCTIVE MEDICINE-ST. LOUIS \\ ST. LOUIS, MISSOURI}

A comparison of clinic success rates may not be meaningful because patient medical characteristics and treatment approaches vary from clinic to clinic. For more details about this, along with information on how to interpret the statistics in this table, see pages 79-88.

\section{ART CYCLE PROFILE}

\begin{tabular}{|c|c|c|c|c|c|c|c|}
\hline \multicolumn{4}{|c|}{ Type of ART ${ }^{a}$} & \multicolumn{4}{|c|}{ Patient Diagnosis } \\
\hline IVF & $100 \%$ & Procedural Factors: & & Tubal factor & $5 \%$ & Other factor & $27 \%$ \\
\hline GIFT & $0 \%$ & With ICSI & $96 \%$ & Ovulatory dysfunction & $7 \%$ & Unknown factor & $4 \%$ \\
\hline ZIFT & $0 \%$ & Unstimulated & $<1 \%$ & Diminished ovarian reserve & $20 \%$ & Multiple Factors: & \\
\hline \multirow[t]{3}{*}{ Combination } & $0 \%$ & Used gestational carrier & $2 \%$ & Endometriosis & $6 \%$ & Female factors only & $8 \%$ \\
\hline & & & & Uterine factor & $<1 \%$ & Female \& male factors & $7 \%$ \\
\hline & & & & Male factor & $15 \%$ & & \\
\hline
\end{tabular}

2006 PREGNANCY SUCCESS RATES

\section{Type of Cycle}

\section{Fresh Embryos from Nondonor Eggs}

Number of cycles

Percentage of cycles resulting in pregnancies ${ }^{b}$

Percentage of cycles resulting in live births ${ }^{b, c}$

(Confidence Interval)

Percentage of retrievals resulting in live births ${ }^{b, c}$

Percentage of transfers resulting in live births $s^{b, c}$

Percentage of transfers resulting in singleton live births ${ }^{b}$

Percentage of cancellations ${ }^{b}$

Average number of embryos transferred

Percentage of pregnancies with twins ${ }^{b}$

Percentage of pregnancies with triplets or more ${ }^{b}$

Percentage of live births having multiple infants ${ }^{b, c}$

\section{Frozen Embryos from Nondonor Eggs}

Number of transfers

Percentage of transfers resulting in live births ${ }^{b, c}$

Average number of embryos transferred

\section{Donor Eggs}

Number of transfers

Percentage of transfers resulting in live births $s^{b, c}$

Average number of embryos transferred

Data verified by Peter M. Ahlering, MD

\section{Age of Woman}

35-37 38-40

$41-42^{d}$

\section{$<35$}

59

64.4

49.2
$(35.9-62.5)$

48.1

40.7

(34.1-47.6)

40.7

50.0

52.7

38.2

29.8

1.7

2.6

2.5

24.0

9.6

33.0

23.7

5.3

27.6

35

37.1

6

$2 / 6$

2.7

2.5

All Ages Combined ${ }^{\mathrm{e}}$

Fresh Embryos

23

69.6

2.7
22

18.2

13.6

$(25.5-60.8)$

42.4

46.7

40.0

0.0

2.9

$3 / 19$

$0 / 19$

$2 / 14$

(2.9-34.9)

13.6

14.3

14.3

0.0

2.7

$1 / 4$

$0 / 4$

$0 / 3$

\section{6}

$3 / 6$

5

$1 / 5$

2.0

\section{CURRENT CLINIC SERVICES AND PROFILE}

Current Name: Sher Institute for Reproductive Medicine-St. Louis

Donor egg? Yes Gestational carriers? Yes

Donor embryo? Yes Cryopreservation? Yes

Single women? Yes

SART member?

No

Verified lab accreditation?

Yes

(See Appendix C for details.)

${ }^{a}$ Reflects patient and treatment characteristics of ART cycles performed in 2006 using fresh nondonor eggs or embryos.

${ }^{b}$ When fewer than 20 cycles are reported in an age category, rates are shown as a fraction and confidence intervals are not given. Calculating percentages from fractions may be misleading and is not encouraged.

${ }^{c}$ A multiple-infant birth is counted as one live birth.

d Clinic-specific outcome rates are unreliable for women older than 42 undergoing ART cycles using fresh or frozen embryos with nondonor eggs. Readers are urged to review national outcomes for these age groups (see page 27).

e All ages (including ages $>42$ ) are reported together because previous data show that patient age does not materially affect success with donor eggs. 


\section{HEARTLAND CENTER FOR REPRODUCTIVE MEDICINE, PC OMAHA, NEBRASKA}

A comparison of clinic success rates may not be meaningful because patient medical characteristics and treatment approaches vary from clinic to clinic. For more details about this, along with information on how to interpret the statistics in this table, see pages $79-88$.

\section{ART CYCLE PROFILE}

\begin{tabular}{|c|c|c|c|c|c|c|c|}
\hline \multicolumn{4}{|c|}{ Type of $A R T^{a}$} & \multicolumn{4}{|c|}{ Patient Diagnosis } \\
\hline IVF & & Procedural Factors: & & Tubal factor & $5 \%$ & Other factor & $<1 \%$ \\
\hline GIFT & $0 \%$ & With ICSI & $69 \%$ & Ovulatory dysfunction & $3 \%$ & Unknown factor & $4 \%$ \\
\hline ZIFT & $0 \%$ & Unstimulated & $0 \%$ & Diminished ovarian reserve & $7 \%$ & Multiple Factors: & \\
\hline \multirow[t]{3}{*}{ Combination } & $0 \%$ & Used gestational carrier & $1 \%$ & Endometriosis & $3 \%$ & Female factors only & $10 \%$ \\
\hline & & & & Uterine factor & $0 \%$ & Female \& male factors & $41 \%$ \\
\hline & & & & Male factor & $27 \%$ & & \\
\hline
\end{tabular}

2006 PREGNANCY SUCCESS RATES

Data verified by Victoria M. Maclin, MD

\section{Type of Cycle}

\section{Fresh Embryos from Nondonor Eggs}

Number of cycles

Percentage of cycles resulting in pregnancies ${ }^{b}$

Percentage of cycles resulting in live births ${ }^{b, c}$

(Confidence Interval)

Percentage of retrievals resulting in live births $s^{b, c}$

Percentage of transfers resulting in live births $s^{b, c}$

Percentage of transfers resulting in singleton live births ${ }^{b}$

Percentage of cancellations ${ }^{b}$

Average number of embryos transferred

Percentage of pregnancies with twins ${ }^{b}$

Percentage of pregnancies with triplets or more ${ }^{b}$

Percentage of live births having multiple infants ${ }^{b, c}$

\section{Frozen Embryos from Nondonor Eggs}

Number of transfers

Percentage of transfers resulting in live births $s^{\mathrm{b}, \mathrm{c}}$

Average number of embryos transferred

\section{Donor Eggs}

Number of transfers

Percentage of transfers resulting in live births $s^{b, c}$

Average number of embryos transferred

\section{Age of Woman}

35-37 38-40

$41-42^{d}$

\section{$<35$}

37

17

8

113

10.8

$4 / 17$

$0 / 8$

28.3

8.1

$4 / 17$

$0 / 8$

(20.2-37.6)

$(1.7-21.9)$

31.4

10.3

$4 / 16$

$0 / 6$

37.2

13.6

$4 / 13$

$0 / 6$

20.9

9.1

$3 / 13$

$0 / 6$

9.7

21.6

$1 / 17$

$2 / 8$

2.1

1.9

3.0

2.3

41.7

0.0

43.8

$0 / 4$

$1 / 4$

$1 / 4$

$0 / 4$

$1 / 3$

$1 / 4$

27

18.5

4

$0 / 4$

1

$0 / 1$

2.3

2.8

1.0

All Ages Combined ${ }^{\mathrm{e}}$

Fresh Embryos

16

$5 / 16$

2.3
Frozen Embryos 10

2 / 10

1.8

\section{CURRENT CLINIC SERVICES AND PROFILE}

Current Name: Heartland Center for Reproductive Medicine, PC

\begin{tabular}{l|l|l} 
Donor egg? Yes & Gestational carriers? Yes
\end{tabular}

Donor embryo? No Cryopreservation? Yes

Single women? Yes

SART member? Yes

Verified lab accreditation? Yes

(See Appendix C for details.)

${ }^{a}$ Reflects patient and treatment characteristics of ART cycles performed in 2006 using fresh nondonor eggs or embryos.

${ }^{b}$ When fewer than 20 cycles are reported in an age category, rates are shown as a fraction and confidence intervals are not given. Calculating percentages from fractions may be misleading and is not encouraged.

${ }^{c}$ A multiple-infant birth is counted as one live birth.

d Clinic-specific outcome rates are unreliable for women older than 42 undergoing ART cycles using fresh or frozen embryos with nondonor eggs. Readers are urged to review national outcomes for these age groups (see page 27).

e All ages (including ages $>42$ ) are reported together because previous data show that patient age does not materially affect success with donor eggs. 


\section{NEBRASKA METHODIST HOSPITAL REI OMAHA, NEBRASKA}

A comparison of clinic success rates may not be meaningful because patient medical characteristics and treatment approaches vary from clinic to clinic. For more details about this, along with information on how to interpret the statistics in this table, see pages 79-88.

\section{ART CYCLE PROFILE}

\begin{tabular}{|c|c|c|c|c|c|c|c|}
\hline \multicolumn{4}{|c|}{ Type of ART ${ }^{a}$} & \multicolumn{4}{|c|}{ Patient Diagnosis } \\
\hline IVF & $>99 \%$ & Procedural Factors: & & Tubal factor & $10 \%$ & Other factor & $8 \%$ \\
\hline GIFT & $0 \%$ & With ICSI & $81 \%$ & Ovulatory dysfunction & $7 \%$ & Unknown factor & $9 \%$ \\
\hline ZIFT & $<1 \%$ & Unstimulated & $0 \%$ & Diminished ovarian reserve & $7 \%$ & Multiple Factors: & \\
\hline Combination & $0 \%$ & Used gestational carrier & $<1 \%$ & Endometriosis & $11 \%$ & Female factors only & $12 \%$ \\
\hline & & & & $\begin{array}{l}\text { Uterine factor } \\
\text { Male factor }\end{array}$ & $\begin{array}{l}<1 \% \\
20 \%\end{array}$ & Female \& male factors & $16 \%$ \\
\hline
\end{tabular}

2006 PREGNANCY SUCCESS RATES

Data verified by Carolyn M. Doherty, MD

\section{Type of Cycle}

\section{Age of Woman}

35-37 38-40

$41-42^{d}$

$<35$

163

49.1

42.9

44

50.0

27

11

Number of cycles

Percentage of cycles resulting in live births ${ }^{b, c}$

(Confidence Interval)

Percentage of retrievals resulting in live births $s^{\mathrm{b}, \mathrm{c}}$

Percentage of transfers resulting in live births ${ }^{\mathrm{b}, \mathrm{c}}$

$(35.2-50.9)$

46.1

36.4

18.5

$3 / 11$

Percentage of transfers resulting in singleton live births ${ }^{b}$

Percentage of cancellations ${ }^{b}$

47.3

(22.4-52.2)

11.1

$2 / 11$

Average number of embryos transferred

Percentage of pregnancies with twins ${ }^{b}$

30.4

6.7

40.0

$(2.4-29.2)$

14.3

$2 / 8$

42.1

15.0

$2 / 8$

21.1

5.0

$2 / 8$

2.4

9.1

22.2

$3 / 11$

36.3

3.2

31.8

3.4

3.9

2.5

13.6

$0 / 5$

$0 / 3$

35.7

$8 / 16$

$2 / 5$

$0 / 3$

$2 / 3$

$0 / 2$

\section{Frozen Embryos from Nondonor Eggs}

Number of transfers

Percentage of transfers resulting in live births ${ }^{\mathrm{b}, \mathrm{c}}$

Average number of embryos transferred

41

48.8

2.2

\section{7}

$2 / 7$

1.7
4

$3 / 4$

2.5

\section{Donor Eggs}

Number of transfers

Percentage of transfers resulting in live births $s^{b, c}$

Average number of embryos transferred

All Ages Combined ${ }^{\mathrm{e}}$

Fresh Embryos

31

54.8

2.2
Frozen Embryos 8

$4 / 8$

1.9

\section{CURRENT CLINIC SERVICES AND PROFILE}

Current Name: Nebraska Methodist Hospital REI

Donor egg? Yes

Donor embryo? No

Single women? Yes
Gestational carriers? Yes

Cryopreservation? Yes
SART member?

Yes

Verified lab accreditation?

(See Appendix C for details.)

\footnotetext{
${ }^{a}$ Reflects patient and treatment characteristics of ART cycles performed in 2006 using fresh nondonor eggs or embryos.

b When fewer than 20 cycles are reported in an age category, rates are shown as a fraction and confidence intervals are not given. Calculating percentages from fractions may be misleading and is not encouraged.

${ }^{c}$ A multiple-infant birth is counted as one live birth.

${ }^{d}$ Clinic-specific outcome rates are unreliable for women older than 42 undergoing ART cycles using fresh or frozen embryos with nondonor eggs. Readers are urged to review national outcomes for these age groups (see page 27).

e All ages (including ages $>42$ ) are reported together because previous data show that patient age does not materially affect success with donor eggs.
} 


\section{FERTILITY CENTER OF LAS VEGAS \\ LAS VEGAS, NEVADA}

A comparison of clinic success rates may not be meaningful because patient medical characteristics and treatment approaches vary from clinic to clinic. For more details about this, along with information on how to interpret the statistics in this table, see pages $79-88$.

\section{ART CYCLE PROFILE}

\begin{tabular}{|c|c|c|c|c|c|c|c|}
\hline \multicolumn{4}{|c|}{ Type of ART } & \multicolumn{4}{|c|}{ Patient Diagnosis } \\
\hline IVF & $100 \%$ & Procedural Factors: & & Tubal factor & $11 \%$ & Other factor & $8 \%$ \\
\hline GIFT & $0 \%$ & With ICSI & $81 \%$ & Ovulatory dysfunction & $1 \%$ & Unknown factor & $9 \%$ \\
\hline ZIFT & $0 \%$ & Unstimulated & $0 \%$ & Diminished ovarian reserve & $16 \%$ & Multiple Factors: & \\
\hline \multirow[t]{3}{*}{ Combination } & $0 \%$ & Used gestational carrier & $<1 \%$ & Endometriosis & $1 \%$ & Female factors only & $12 \%$ \\
\hline & & & & Uterine factor & $0 \%$ & Female \& male factors & $25 \%$ \\
\hline & & & & Male factor & $18 \%$ & & \\
\hline
\end{tabular}

2006 PREGNANCY SUCCESS RATES

Data verified by Bruce S. Shapiro, MD

\section{Type of Cycle}

\section{Fresh Embryos from Nondonor Eggs}

Number of cycles

Percentage of cycles resulting in pregnancies ${ }^{b}$

Percentage of cycles resulting in live births ${ }^{b, c}$

(Confidence Interval)

Percentage of retrievals resulting in live births $s^{b, c}$

Percentage of transfers resulting in live births $s^{b, c}$

Percentage of transfers resulting in singleton live births ${ }^{b}$

Percentage of cancellations ${ }^{b}$

Average number of embryos transferred

Percentage of pregnancies with twins ${ }^{b}$

Percentage of pregnancies with triplets or more ${ }^{b}$

Percentage of live births having multiple infants ${ }^{b, c}$

\section{Frozen Embryos from Nondonor Eggs}

Number of transfers

Percentage of transfers resulting in live births $s^{\mathrm{b}, \mathrm{c}}$

Average number of embryos transferred

\section{Donor Eggs}

Number of transfers

Percentage of transfers resulting in live births $s^{b, c}$

Average number of embryos transferred

\section{Age of Woman}

35-37 38-40

$41-42^{d}$

$<35$

84

32.1

57

15

31.8

28.6

26.3

$2 / 15$

25.5

(19.2-39.5)

14.0

$2 / 15$

(18.9-33.0)

30.8

33.8

(6.3-25.8)

35.7

40.0

17.8

$2 / 15$

23.5

$2 / 12$

25.9

26.7

20.6

$2 / 12$

17.2

15.5

21.1

$0 / 15$

1.8

1.8

32.0

33.3

1.9

1.8

2.0

0.0

$1 / 15$

$0 / 2$

27.5

33.3

$0 / 15$

$0 / 2$

$1 / 8$

$0 / 2$

$\begin{array}{cccc}110 & 62 & 32 & 7 \\ 52.7 & 37.1 & 21.9 & 0 / 7 \\ 2.0 & 2.0 & 2.0 & 2.1\end{array}$

All Ages Combined ${ }^{\mathrm{e}}$ Fresh Embryos

Frozen Embryos

62.5

15

1.9

$9 / 15$

2.1

\section{CURRENT CLINIC SERVICES AND PROFILE}

Current Name: Fertility Center of Las Vegas

Donor egg? Yes Gestational carriers? Yes

Donor embryo? No Cryopreservation? Yes

Single women? Yes

\section{SART member? Yes}

Verified lab accreditation? Yes

(See Appendix C for details.)

${ }^{a}$ Reflects patient and treatment characteristics of ART cycles performed in 2006 using fresh nondonor eggs or embryos.

${ }^{b}$ When fewer than 20 cycles are reported in an age category, rates are shown as a fraction and confidence intervals are not given. Calculating percentages from fractions may be misleading and is not encouraged.

A multiple-infant birth is counted as one live birth.

d Clinic-specific outcome rates are unreliable for women older than 42 undergoing ART cycles using fresh or frozen embryos with nondonor eggs. Readers are urged to review national outcomes for these age groups (see page 27).

e All ages (including ages $>42$ ) are reported together because previous data show that patient age does not materially affect success with donor eggs. 


\section{NEVADA FERTILITY C.A.R.E.S. LAS VEGAS, NEVADA}

A comparison of clinic success rates may not be meaningful because patient medical characteristics and treatment approaches vary from clinic to clinic. For more details about this, along with information on how to interpret the statistics in this table, see pages 79-88.

\section{ART CYCLE PROFILE}

\section{Type of ART ${ }^{\mathrm{a}}$}

IVF

GIFT $100 \%$ Procedural Factors:

ZIFT

Combination
$0 \%$ With ICSI

$0 \%$ Unstimulated

$0 \%$ Used gestational carrier
Tubal factor

$25 \%$ Ovulatory dysfunction

$<1 \%$

$3 \%$

Endometriosis
Diminished ovarian reserve

Uterine factor

Male factor

\section{Patient Diagnosis}

$\begin{array}{rlr}11 \% & \text { Other factor } & 17 \% \\ 4 \% & \text { Unknown factor } & 20 \% \\ 25 \% & \text { Multiple Factors: } & \\ 4 \% & \text { Female factors only } & 7 \% \\ <1 \% & \text { Female \& male factors } & 7 \% \\ 6 \% & & \end{array}$

Data verified by Rachel A. McConnell, MD

\section{PREGNANCY SUCCESS RATES}

\section{Type of Cycle}

\section{Fresh Embryos from Nondonor Eggs}

Number of cycles

Percentage of cycles resulting in pregnancies ${ }^{b}$

Percentage of cycles resulting in live births ${ }^{b, c}$

(Confidence Interval)

Percentage of retrievals resulting in live births $s^{b, c}$

Percentage of transfers resulting in live births $s^{b, c}$

Percentage of transfers resulting in singleton live births ${ }^{b}$

Percentage of cancellations ${ }^{b}$

Average number of embryos transferred

Percentage of pregnancies with twins ${ }^{b}$

Percentage of pregnancies with triplets or more ${ }^{b}$

Percentage of live births having multiple infants ${ }^{b, c}$

\section{Frozen Embryos from Nondonor Eggs}

Number of transfers

Percentage of transfers resulting in live births ${ }^{b, c}$

Average number of embryos transferred

\section{Donor Eggs}

Number of transfers

Percentage of transfers resulting in live births $s^{b, c}$

Average number of embryos transferred

\section{Age of Woman}

35-37 38-40

$41-42^{d}$

$<35$

46

58.7

30

15

42.4

41.3

26.7

$2 / 15$

39.4

(27.0-56.8)

20.0

$1 / 15$

$(29.7-49.7)$

41.1

41.9

41.3

(7.7-38.6)

21.4

$1 / 14$

41.3

23.1

$1 / 9$

28.3

15.4

$1 / 9$

4.0

0.0

6.7

$1 / 15$

3.1

33.3

14.3

46.2

\section{9}

14.8

18.5

$6 / 19$

2.7

2.8

$0 / 2$

$0 / 2$

$1 / 8$

$0 / 1$
2

$1 / 2$

2.5

$\begin{array}{cccc}4 & 2 & 0 & 2 \\ 0 / 4 & 0 / 2 & & 1 / 2 \\ 2.3 & 1.5 & 2.5\end{array}$

\section{CURRENT CLINIC SERVICES AND PROFILE}

Current Name: Nevada Fertility C.A.R.E.S.

Donor egg? Yes

Donor embryo? Yes

Gestational carriers? Yes

Cryopreservation? Yes

All Ages Combined ${ }^{\mathrm{e}}$

Fresh Embryos

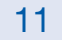

$3 / 11$

3.2
Frozen Embryos

5

$1 / 5$

3.6
SART member?

Yes

Verified lab accreditation?

Yes

(See Appendix C for details.)

${ }^{a}$ Reflects patient and treatment characteristics of ART cycles performed in 2006 using fresh nondonor eggs or embryos.

${ }^{b}$ When fewer than 20 cycles are reported in an age category, rates are shown as a fraction and confidence intervals are not given. Calculating percentages from fractions may be misleading and is not encouraged.

${ }^{\mathrm{C}}$ A multiple-infant birth is counted as one live birth.

d Clinic-specific outcome rates are unreliable for women older than 42 undergoing ART cycles using fresh or frozen embryos with nondonor eggs. Readers are urged to review national outcomes for these age groups (see page 27).

e All ages (including ages $>42$ ) are reported together because previous data show that patient age does not materially affect success with donor eggs. 


\section{SHER INSTITUTE FOR REPRODUCTIVE MEDICINE-LAS VEGAS \\ LAS VEGAS, NEVADA}

A comparison of clinic success rates may not be meaningful because patient medical characteristics and treatment approaches vary from clinic to clinic. For more details about this, along with information on how to interpret the statistics in this table, see pages $79-88$.

\section{ART CYCLE PROFILE}

\begin{tabular}{|c|c|c|c|c|c|c|c|}
\hline \multicolumn{4}{|c|}{ Type of ART ${ }^{\mathrm{a}}$} & \multicolumn{4}{|c|}{ Patient Diagnosis } \\
\hline IVF & $100 \%$ & Procedural Factors: & & Tubal factor & $8 \%$ & Other factor & $11 \%$ \\
\hline GIFT & $0 \%$ & With ICSI & $95 \%$ & Ovulatory dysfunction & $7 \%$ & Unknown factor & $11 \%$ \\
\hline $\mathrm{ZIFT}$ & $0 \%$ & Unstimulated & $0 \%$ & Diminished ovarian reserve & $30 \%$ & Multiple Factors: & \\
\hline Combination & $0 \%$ & Used gestational carrier & $4 \%$ & Endometriosis & $7 \%$ & Female factors only & $6 \%$ \\
\hline & & & & Uterine factor & $1 \%$ & Female \& male factors & $7 \%$ \\
\hline
\end{tabular}

2006 PREGNANCY SUCCESS RATES

Data verified by Jeffrey D. Fisch, MD

\section{Type of Cycle}

\section{Fresh Embryos from Nondonor Eggs}

Number of cycles

Percentage of cycles resulting in pregnancies ${ }^{b}$

Percentage of cycles resulting in live births ${ }^{b, c}$

(Confidence Interval)

Percentage of retrievals resulting in live births $s^{b, c}$

Percentage of transfers resulting in live births $s^{b, c}$

Percentage of transfers resulting in singleton live births ${ }^{b}$

Percentage of cancellations ${ }^{b}$

Average number of embryos transferred

Percentage of pregnancies with twins ${ }^{b}$

Percentage of pregnancies with triplets or more ${ }^{b}$

Percentage of live births having multiple infants ${ }^{b, c}$

\section{Frozen Embryos from Nondonor Eggs}

Number of transfers

Percentage of transfers resulting in live births $s^{b, c}$

Average number of embryos transferred

\section{Donor Eggs}

Number of transfers

Percentage of transfers resulting in live births ${ }^{\mathrm{b}, \mathrm{c}}$

Average number of embryos transferred

\section{Age of Woman}

35-37 38-40

$41-42^{d}$

\section{$<35$}

67

35.8

72

31

45.5

31.3

25.0

32.3

35.8

(20.6-43.8)

13.9

22.6

(27.3-44.9)

32.3

$(6.9-24.1)$

(9.6-41.1)

36.7

38.9

14.3

22.6

39.6

29.6

16.9

24.1

26.1

3.0

11.9

20.7

2.4

2.1

20.8

25.0

0.0

23.8

2.8

0.0

2.6

2.6

3.6

34.1

$1 / 18$

$1 / 10$

$2 / 18$

$0 / 10$

$3 / 10$

$1 / 7$

34

20.6

16

$4 / 16$

18

10

2.6

3.1

$3 / 18$

$2 / 10$

2.6

2.5

All Ages Combined ${ }^{\mathrm{e}}$

Fresh Embryos

38

42.1

2.2
Frozen Embryos 16

$3 / 16$

2.9

\section{CURRENT CLINIC SERVICES AND PROFILE}

Current Name: Sher Institute for Reproductive Medicine-Las Vegas

Donor egg? Yes Gestational carriers? Yes

\begin{tabular}{l|ll} 
Donor embryo? No Cryopreservation? Yes &
\end{tabular}

Single women? Yes

\section{SART member? Yes}

Verified lab accreditation? Yes

(See Appendix C for details.)

${ }^{a}$ Reflects patient and treatment characteristics of ART cycles performed in 2006 using fresh nondonor eggs or embryos.

${ }^{b}$ When fewer than 20 cycles are reported in an age category, rates are shown as a fraction and confidence intervals are not given. Calculating percentages from fractions may be misleading and is not encouraged.

A multiple-infant birth is counted as one live birth.

d Clinic-specific outcome rates are unreliable for women older than 42 undergoing ART cycles using fresh or frozen embryos with nondonor eggs. Readers are urged to review national outcomes for these age groups (see page 27).

e All ages (including ages $>42$ ) are reported together because previous data show that patient age does not materially affect success with donor eggs. 


\section{THE NEVADA CENTER FOR REPRODUCTIVE MEDICINE \\ RENO, NEVADA}

A comparison of clinic success rates may not be meaningful because patient medical characteristics and treatment approaches vary from clinic to clinic. For more details about this, along with information on how to interpret the statistics in this table, see pages 79-88.

\section{ART CYCLE PROFILE}

\begin{tabular}{|c|c|c|c|c|c|c|c|}
\hline \multicolumn{4}{|c|}{ Type of ART ${ }^{a}$} & \multicolumn{4}{|c|}{ Patient Diagnosis } \\
\hline IVF & $100 \%$ & Procedural Factors: & & Tubal factor & $7 \%$ & Other factor & $3 \%$ \\
\hline GIFT & $0 \%$ & With ICSI & $50 \%$ & Ovulatory dysfunction & $<1 \%$ & Unknown factor & $1 \%$ \\
\hline ZIFT & $0 \%$ & Unstimulated & $0 \%$ & Diminished ovarian reserve & $9 \%$ & Multiple Factors: & \\
\hline \multirow[t]{3}{*}{ Combination } & $0 \%$ & Used gestational carrier & $6 \%$ & Endometriosis & $<1 \%$ & Female factors only & $46 \%$ \\
\hline & & & & Uterine factor & $2 \%$ & Female \& male factors & $23 \%$ \\
\hline & & & & Male factor & $7 \%$ & & \\
\hline
\end{tabular}

\section{PREGNANCY SUCCESS RATES}

Data verified by Russell A. Foulk, MD

\section{Type of Cycle}

\section{Fresh Embryos from Nondonor Eggs}

Number of cycles

Percentage of cycles resulting in pregnancies ${ }^{b}$

Percentage of cycles resulting in live births $s^{b, c}$

(Confidence Interval)

Percentage of retrievals resulting in live births $s^{b, c}$

Percentage of transfers resulting in live births $s^{b, c}$

Percentage of transfers resulting in singleton live births ${ }^{b}$

Percentage of cancellations ${ }^{b}$

Average number of embryos transferred

Percentage of pregnancies with twins ${ }^{b}$

Percentage of pregnancies with triplets or more ${ }^{b}$

Percentage of live births having multiple infants ${ }^{b, c}$

\section{Frozen Embryos from Nondonor Eggs}

Number of transfers

Percentage of transfers resulting in live births ${ }^{b, c}$

Average number of embryos transferred

\section{Donor Eggs}

Number of transfers

Percentage of transfers resulting in live births $s^{b, c}$

Average number of embryos transferred

\section{Age of Woman}

35-37 38-40

$41-42^{d}$

$<35$

32

28.1

28

13

60.0

28.1

28.6

$3 / 13$

53.7

$(13.7-46.7)$

25.0

$1 / 13$

$(43.2-64.0)$

54.3

28.1

$(10.7-44.9)$

55.4

31.0

26.9

26.9

$1 / 13$

34.8

27.6

26.9

$1 / 13$

1.1

0.0

7.1

$1 / 13$

2.6

2.7

2.7

$0 / 13$

31.6

$2 / 9$

$0 / 8$

2.6

8.8

$0 / 9$

$0 / 8$

$1 / 3$

37.3

$1 / 9$

$0 / 7$

$0 / 3$

$0 / 1$

$\begin{array}{cccc}38 & 21 & 15 & 8 \\ 23.7 & 23.8 & 4 / 15 & 1 / 8 \\ 2.7 & 2.6 & 3.1 & 3.0\end{array}$

\section{All Ages Combined}

Fresh Embryos

60

66.7

2.7
Frozen Embryos

66

50.0

2.9

\section{CURRENT CLINIC SERVICES AND PROFILE}

Current Name: The Nevada Center for Reproductive Medicine

Donor egg? Yes Gestational carriers? Yes

Donor embryo? Yes Cryopreservation? Yes

Single women? Yes

SART member?

Yes

Verified lab accreditation?

(See Appendix C for details.)

${ }^{a}$ Reflects patient and treatment characteristics of ART cycles performed in 2006 using fresh nondonor eggs or embryos.

${ }^{b}$ When fewer than 20 cycles are reported in an age category, rates are shown as a fraction and confidence intervals are not given. Calculating percentages from fractions may be misleading and is not encouraged.

${ }^{c}$ A multiple-infant birth is counted as one live birth.

d Clinic-specific outcome rates are unreliable for women older than 42 undergoing ART cycles using fresh or frozen embryos with nondonor eggs. Readers are urged to review national outcomes for these age groups (see page 27).

e All ages (including ages $>42$ ) are reported together because previous data show that patient age does not materially affect success with donor eggs. 


\section{DARTMOUTH-HITCHCOCK MEDICAL CENTER LEBANON, NEW HAMPSHIRE}

A comparison of clinic success rates may not be meaningful because patient medical characteristics and treatment approaches vary from clinic to clinic. For more details about this, along with information on how to interpret the statistics in this table, see pages 79-88.

\section{ART CYCLE PROFILE}

\begin{tabular}{|c|c|c|c|c|c|c|c|}
\hline \multicolumn{4}{|c|}{ Type of ART ${ }^{a}$} & \multicolumn{4}{|c|}{ Patient Diagnosis } \\
\hline IVF & $100 \%$ & Procedural Factors: & & Tubal factor & $13 \%$ & Other factor & $5 \%$ \\
\hline GIFT & $0 \%$ & With ICSI & $50 \%$ & Ovulatory dysfunction & $5 \%$ & Unknown factor & $16 \%$ \\
\hline ZIFT & $0 \%$ & Unstimulated & $0 \%$ & Diminished ovarian reserve & $9 \%$ & Multiple Factors: & \\
\hline \multirow[t]{3}{*}{ Combination } & $0 \%$ & Used gestational carrier & $<1 \%$ & Endometriosis & $1 \%$ & Female factors only & $11 \%$ \\
\hline & & & & Uterine factor & $2 \%$ & Female \& male factors & $8 \%$ \\
\hline & & & & Male factor & $30 \%$ & & \\
\hline
\end{tabular}

\section{PREGNANCY SUCCESS RATES}

Data verified by Misty B. Porter, MD

\section{Type of Cycle}

\section{Fresh Embryos from Nondonor Eggs}

Number of cycles

Percentage of cycles resulting in pregnancies ${ }^{b}$

Percentage of cycles resulting in live births $s^{b, c}$

(Confidence Interval)

Percentage of retrievals resulting in live births $s^{b, c}$

Percentage of transfers resulting in live births $s^{b, c}$

Percentage of transfers resulting in singleton live births ${ }^{b}$

Percentage of cancellations ${ }^{b}$

Average number of embryos transferred

Percentage of pregnancies with twins ${ }^{b}$

Percentage of pregnancies with triplets or more ${ }^{b}$

Percentage of live births having multiple infants ${ }^{b, c}$

\section{Frozen Embryos from Nondonor Eggs}

Number of transfers

Percentage of transfers resulting in live births $s^{b, c}$

Average number of embryos transferred

\section{Donor Eggs}

Number of transfers

Percentage of transfers resulting in live births $s^{b, c}$

Average number of embryos transferred

\section{Age of Woman}

35-37 38-40

$41-42^{d}$

\section{$<35$}

28

42.9

25

20

56
48.2

35.7

40.0

35.0

42.9

(18.6-55.9)

40.0

15.0

(29.7-56.8)

45.5

(21.1-61.3)

(3.2-37.9)

46.2

48.0

47.6

47.6

$3 / 17$

38.0

47.6

47.6

$3 / 17$

7.1

21.4

33.3

$2 / 17$

2.0

2.5

18.5

$1 / 12$

0.0

$0 / 12$

20.8

$0 / 10$

16.0

15.0

3.1

3.9

$3 / 10$

$0 / 7$

$1 / 10$

$1 / 7$

$3 / 10$

$1 / 3$

18

$9 / 18$

2.0

\section{8}

$1 / 8$

2.9

\section{3}

$1 / 3$

2.3
3

$0 / 3$

3.0

All Ages Combined ${ }^{\mathrm{e}}$

Fresh Embryos

\section{7}

$3 / 7$

2.0
Frozen Embryos 13

$4 / 13$

1.9

\section{CURRENT CLINIC SERVICES AND PROFILE}

Current Name: Dartmouth-Hitchcock Medical Center

Donor egg? Yes

Donor embryo? Yes

Single women? Yes

\section{Gestational carriers? Yes}

Cryopreservation? Yes

\section{SART member?}

Verified lab accreditation?

(See Appendix C for details.)

${ }^{a}$ Reflects patient and treatment characteristics of ART cycles performed in 2006 using fresh nondonor eggs or embryos.

${ }^{b}$ When fewer than 20 cycles are reported in an age category, rates are shown as a fraction and confidence intervals are not given. Calculating percentages from fractions may be misleading and is not encouraged.

${ }^{c}$ A multiple-infant birth is counted as one live birth.

d Clinic-specific outcome rates are unreliable for women older than 42 undergoing ART cycles using fresh or frozen embryos with nondonor eggs. Readers are urged to review national outcomes for these age groups (see page 27).

e All ages (including ages $>42$ ) are reported together because previous data show that patient age does not materially affect success with donor eggs. 


\section{SHER INSTITUTE FOR REPRODUCTIVE MEDICINE-NEW JERSEY BEDMINSTER, NEW JERSEY}

A comparison of clinic success rates may not be meaningful because patient medical characteristics and treatment approaches vary from clinic to clinic. For more details about this, along with information on how to interpret the statistics in this table, see pages 79-88.

\section{ART CYCLE PROFILE}

\begin{tabular}{|c|c|c|c|c|c|c|c|}
\hline \multicolumn{4}{|c|}{ Type of ART ${ }^{a}$} & \multicolumn{4}{|c|}{ Patient Diagnosis } \\
\hline IVF & $100 \%$ & Procedural Factors: & & Tubal factor & $13 \%$ & Other factor & $4 \%$ \\
\hline GIFT & $0 \%$ & With ICSI & $86 \%$ & Ovulatory dysfunction & $5 \%$ & Unknown factor & $9 \%$ \\
\hline ZIFT & $0 \%$ & Unstimulated & $0 \%$ & Diminished ovarian reserve & $15 \%$ & Multiple Factors: & \\
\hline \multirow[t]{3}{*}{ Combination } & $0 \%$ & Used gestational carrier & $2 \%$ & Endometriosis & $3 \%$ & Female factors only & $17 \%$ \\
\hline & & & & Uterine factor & $<1 \%$ & Female \& male factors & $20 \%$ \\
\hline & & & & Male factor & $15 \%$ & & \\
\hline
\end{tabular}

2006 PREGNANCY SUCCESS RATES

$$
\text { Type of Cycle }
$$

\section{Fresh Embryos from Nondonor Eggs}

Number of cycles

Percentage of cycles resulting in pregnancies ${ }^{b}$

Percentage of cycles resulting in live births ${ }^{b, c}$

(Confidence Interval)

Percentage of retrievals resulting in live births $s^{b, c}$

Percentage of transfers resulting in live births $s^{b, c}$

Percentage of transfers resulting in singleton live births ${ }^{b}$

Percentage of cancellations ${ }^{b}$

Average number of embryos transferred

Percentage of pregnancies with twins ${ }^{b}$

Percentage of pregnancies with triplets or more ${ }^{b}$

Percentage of live births having multiple infants ${ }^{b, c}$

\section{Frozen Embryos from Nondonor Eggs}

Number of transfers

Percentage of transfers resulting in live births $s^{b, c}$

Average number of embryos transferred

\section{Donor Eggs}

Number of transfers

Percentage of transfers resulting in live births $s^{b, c}$

Average number of embryos transferred

Data verified by Alexander M. Dlugi, MD

\section{Age of Woman}

35-37 38-40

$41-42^{d}$

\section{$<35$}

59

35.6

69

35

35.0

25.4

11.6

17.1

29.1

(15.0-38.4)

7.2

11.4

(20.6-38.9)

27.8

(2.4-16.1)

(3.2-26.7)

37.5

36.6

7.9

11.9

11.8

19.5

7.1

18.2

7.8

8.5

8.7

18.2

2.2

2.4

2.3

2.9

38.9

23.8

$3 / 8$

2.1

5.6

14.3

$0 / 8$

$0 / 6$

43.3

$7 / 15$

$2 / 5$

$0 / 6$

$0 / 4$

$\begin{array}{cccc}11 & 5 & 5 & 1 \\ 0 / 11 & 1 / 5 & 0 / 5 & 0 / 1 \\ 2.2 & 2.4 & 2.4 & 1.0\end{array}$

\section{All Ages Combined}

Fresh Embryos

13

$6 / 13$

2.6
Frozen Embryos

\section{5}

$2 / 5$

3.2

\section{CURRENT CLINIC SERVICES AND PROFILE}

Current Name: Sher Institute for Reproductive Medicine-New Jersey

Donor egg? Yes Gestational carriers? Yes

Donor embryo? No Cryopreservation? Yes

Single women? Yes

SART member? Yes

Verified lab accreditation? Yes

(See Appendix C for details.)

${ }^{a}$ Reflects patient and treatment characteristics of ART cycles performed in 2006 using fresh nondonor eggs or embryos.

${ }^{b}$ When fewer than 20 cycles are reported in an age category, rates are shown as a fraction and confidence intervals are not given. Calculating percentages from fractions may be misleading and is not encouraged.

${ }^{c}$ A multiple-infant birth is counted as one live birth.

d Clinic-specific outcome rates are unreliable for women older than 42 undergoing ART cycles using fresh or frozen embryos with nondonor eggs. Readers are urged to review national outcomes for these age groups (see page 27).

e All ages (including ages $>42$ ) are reported together because previous data show that patient age does not materially affect success with donor eggs. 


\section{IVF OF NORTH JERSEY}

NORTH JERSEY CENTER FOR REPRODUCTIVE ENDOCRINOLOGY AND INFERTILITY CLIFTON, NEW JERSEY

A comparison of clinic success rates may not be meaningful because patient medical characteristics and treatment approaches vary from clinic to clinic. For more details about this, along with information on how to interpret the statistics in this table, see pages $79-88$.

\section{ART CYCLE PROFILE}

\begin{tabular}{|c|c|c|c|c|c|c|c|}
\hline \multicolumn{4}{|c|}{ Type of ART ${ }^{\mathrm{a}}$} & \multicolumn{4}{|c|}{ Patient Diagnosis } \\
\hline IVF & $100 \%$ & Procedural Factors: & & Tubal factor & $8 \%$ & Other factor & $52 \%$ \\
\hline GIFT & $0 \%$ & With ICSI & $35 \%$ & Ovulatory dysfunction & $3 \%$ & Unknown factor & $28 \%$ \\
\hline ZIFT & $0 \%$ & Unstimulated & $0 \%$ & Diminished ovarian reserve & $0 \%$ & Multiple Factors: & \\
\hline \multirow[t]{3}{*}{ Combination } & $0 \%$ & Used gestational carrier & $0 \%$ & Endometriosis & $0 \%$ & Female factors only & $2 \%$ \\
\hline & & & & Uterine factor & $2 \%$ & Female \& male factors & $0 \%$ \\
\hline & & & & Male factor & $6 \%$ & & \\
\hline
\end{tabular}

2006 PREGNANCY SUCCESS RATES

Data verified by Alfredo J. Garcia, MD

\section{Type of Cycle}

\section{Fresh Embryos from Nondonor Eggs}

Number of cycles

Percentage of cycles resulting in pregnancies ${ }^{\mathrm{b}}$

Percentage of cycles resulting in live births ${ }^{b, c}$

(Confidence Interval)

Percentage of retrievals resulting in live births $s^{b, c}$

Percentage of transfers resulting in live births $s^{b, c}$

Percentage of transfers resulting in singleton live births ${ }^{b}$

Percentage of cancellations ${ }^{b}$

Average number of embryos transferred

Percentage of pregnancies with twins ${ }^{b}$

Percentage of pregnancies with triplets or more ${ }^{b}$

Percentage of live births having multiple infants ${ }^{\mathrm{b}, \mathrm{c}}$

\section{Frozen Embryos from Nondonor Eggs}

Number of transfers

Percentage of transfers resulting in live births ${ }^{\mathrm{b}, \mathrm{c}}$

Average number of embryos transferred

\section{Donor Eggs}

Number of transfers

Percentage of transfers resulting in live births $s^{b, c}$

Average number of embryos transferred

\section{Age of Woman}

35-37 38-40

$41-42^{d}$

$<35$

12

10

4

$7 / 19$

$2 / 12$

$3 / 10$

$2 / 4$

$5 / 19$

$2 / 12$

$1 / 10$

$0 / 4$

$\begin{array}{cccc}5 / 18 & 2 / 10 & 1 / 9 & 0 / 4 \\ 5 / 18 & 2 / 10 & 1 / 5 & 0 / 4 \\ 2 / 18 & 1 / 10 & 1 / 5 & 0 / 4 \\ 1 / 19 & 2 / 12 & 1 / 10 & 0 / 4 \\ 2.1 & 2.3 & 3.4 & 2.5 \\ 2 / 7 & 1 / 2 & 0 / 3 & 0 / 2 \\ 1 / 7 & 0 / 2 & 0 / 3 & 0 / 2 \\ 3 / 5 & 1 / 2 & 0 / 1 & \end{array}$

0

1

$0 / 1$

1.0

0

0

\begin{tabular}{cc}
\multicolumn{2}{c}{ All Ages Combined $^{\mathbf{e}}$} \\
Fresh Embryos & Frozen Embryos $^{-1}$ \\
12 & 3 \\
$3 / 12$ & $1 / 3$ \\
2.0 & 2.7
\end{tabular}

\section{CURRENT CLINIC SERVICES AND PROFILE}

Current Name: Tower Fertility Center

Donor egg? Yes Gestational carriers? No

Donor embryo? No Cryopreservation? Yes

Single women? Yes

SART member? No

Verified lab accreditation? Yes

(See Appendix C for details.)

${ }^{a}$ Reflects patient and treatment characteristics of ART cycles performed in 2006 using fresh nondonor eggs or embryos.

b When fewer than 20 cycles are reported in an age category, rates are shown as a fraction and confidence intervals are not given. Calculating percentages from fractions may be misleading and is not encouraged.

${ }^{\mathrm{C}}$ A multiple-infant birth is counted as one live birth.

d Clinic-specific outcome rates are unreliable for women older than 42 undergoing ART cycles using fresh or frozen embryos with nondonor eggs. Readers are urged to review national outcomes for these age groups (see page 27).

e All ages (including ages $>42$ ) are reported together because previous data show that patient age does not materially affect success with donor eggs. 


\section{CENTER FOR ADVANCED REPRODUCTIVE MEDICINE \& FERTILITY \\ EDISON, NEW JERSEY}

A comparison of clinic success rates may not be meaningful because patient medical characteristics and treatment approaches vary from clinic to clinic. For more details about this, along with information on how to interpret the statistics in this table, see pages 79-88.

\section{ART CYCLE PROFILE}

\begin{tabular}{|c|c|c|c|c|c|c|c|}
\hline \multicolumn{4}{|c|}{ Type of ART $^{\mathrm{a}}$} & \multicolumn{4}{|c|}{ Patient Diagnosis } \\
\hline IVF & $100 \%$ & Procedural Factors: & & Tubal factor & $6 \%$ & Other factor & $1 \%$ \\
\hline GIFT & $0 \%$ & With ICSI & $64 \%$ & Ovulatory dysfunction & $11 \%$ & Unknown factor & $6 \%$ \\
\hline ZIFT & $0 \%$ & Unstimulated & $0 \%$ & Diminished ovarian reserve & $18 \%$ & Multiple Factors: & \\
\hline \multirow[t]{3}{*}{ Combination } & $0 \%$ & Used gestational carrier & $0 \%$ & Endometriosis & $2 \%$ & Female factors only & $7 \%$ \\
\hline & & & & Uterine factor & $0 \%$ & Female \& male factors & $19 \%$ \\
\hline & & & & Male factor & $31 \%$ & & \\
\hline
\end{tabular}

\section{PREGNANCY SUCCESS RATES}

\section{Type of Cycle}

\section{Fresh Embryos from Nondonor Eggs}

Number of cycles

Percentage of cycles resulting in pregnancies ${ }^{b}$

Percentage of cycles resulting in live births ${ }^{b, c}$

(Confidence Interval)

Percentage of retrievals resulting in live births $s^{b, c}$

Percentage of transfers resulting in live births $s^{b, c}$

Percentage of transfers resulting in singleton live births ${ }^{b}$

Percentage of cancellations ${ }^{b}$

Average number of embryos transferred

Percentage of pregnancies with twins ${ }^{b}$

Percentage of pregnancies with triplets or more ${ }^{b}$

Percentage of live births having multiple infants ${ }^{b, c}$

\section{Frozen Embryos from Nondonor Eggs}

Number of transfers

Percentage of transfers resulting in live births $s^{b, c}$

Average number of embryos transferred

\section{Donor Eggs}

Number of transfers

Percentage of transfers resulting in live births $s^{b, c}$

Average number of embryos transferred

Data verified by Gregory H. Corsan, MD

\section{Age of Woman}

35-37 38-40

$41-42^{d}$

\section{$<35$}

45

35.6

26

8

33.6

33.3

11.5

$3 / 8$

27.3

(20.0-49.0)

7.7

$3 / 8$

(19.8-35.9)

30.4

34.9

(0.9-25.1)

38.5

57.7

$2 / 18$

$3 / 8$

23.1

46.2

$2 / 14$

$3 / 6$

10.2

4.4

$2 / 14$

$2 / 6$

2.0

2.4

30.8

$0 / 8$

30.2

$3 / 16$

4.7

$1 / 16$

3.3

2.7

4.7
40.0

$3 / 15$

$1 / 3$

$1 / 3$

$0 / 3$

$0 / 3$

$0 / 2$

$1 / 3$

36

30.6

\section{9}

2.2

$3 / 9$

2.6

1

$1 / 1$

1

2.0

$1 / 1$

2.0

All Ages Combined ${ }^{\mathrm{e}}$

Fresh Embryos 13

$4 / 13$

1.8
Frozen Embryos

\section{7}

$1 / 7$

2.1

\section{CURRENT CLINIC SERVICES AND PROFILE}

Current Name: Center for Advanced Reproductive Medicine \& Fertility

Donor egg? Yes Gestational carriers? No

Donor embryo? Yes Cryopreservation? Yes

Single women? Yes

SART member? Yes

Verified lab accreditation? Yes

(See Appendix C for details.)

${ }^{a}$ Reflects patient and treatment characteristics of ART cycles performed in 2006 using fresh nondonor eggs or embryos.

${ }^{b}$ When fewer than 20 cycles are reported in an age category, rates are shown as a fraction and confidence intervals are not given. Calculating percentages from fractions may be misleading and is not encouraged.

${ }^{c}$ A multiple-infant birth is counted as one live birth.

d Clinic-specific outcome rates are unreliable for women older than 42 undergoing ART cycles using fresh or frozen embryos with nondonor eggs. Readers are urged to review national outcomes for these age groups (see page 27).

e All ages (including ages $>42$ ) are reported together because previous data show that patient age does not materially affect success with donor eggs. 


\section{WOMEN'S FERTILITY CENTER \\ ENGLEWOOD, NEW JERSEY}

A comparison of clinic success rates may not be meaningful because patient medical characteristics and treatment approaches vary from clinic to clinic. For more details about this, along with information on how to interpret the statistics in this table, see pages 79-88.

\section{ART CYCLE PROFILE}

\begin{tabular}{|c|c|c|c|c|c|c|c|}
\hline \multicolumn{4}{|c|}{ Type of ART ${ }^{\mathrm{a}}$} & \multicolumn{4}{|c|}{ Patient Diagnosis } \\
\hline IVF & $100 \%$ & Procedural Factors: & & Tubal factor & $5 \%$ & Other factor & $0 \%$ \\
\hline GIFT & $0 \%$ & With ICSI & $83 \%$ & Ovulatory dysfunction & $5 \%$ & Unknown factor & $14 \%$ \\
\hline ZIFT & $0 \%$ & Unstimulated & $0 \%$ & Diminished ovarian reserve & $19 \%$ & Multiple Factors: & \\
\hline \multirow[t]{3}{*}{ Combination } & $0 \%$ & Used gestational carrier & $0 \%$ & Endometriosis & $0 \%$ & Female factors only & $7 \%$ \\
\hline & & & & Uterine factor & $2 \%$ & Female \& male factors & $14 \%$ \\
\hline & & & & Male factor & $33 \%$ & & \\
\hline
\end{tabular}

2006 PREGNANCY SUCCESS RATES

Data verified by Philip R. Lesorgen, MD

\section{Type of Cycle}

\section{Age of Woman}

35-37 38-40

$41-42^{d}$ $<35$

17

13

$4 / 17$

$2 / 13$

5

7

Percentage of cycles resulting in pregnancies ${ }^{b}$

$4 / 17$

$2 / 13$

$1 / 5$

$1 / 7$

Percentage of cycles resulting in live births ${ }^{\mathrm{b}, \mathrm{c}}$

(Confidence Interval)

Percentage of retrievals resulting in live births ${ }^{\mathrm{b}, \mathrm{c}}$

$4 / 17$

$2 / 13$

$1 / 5$

$0 / 7$

Percentage of transfers resulting in live births ${ }^{\mathrm{b}, \mathrm{c}}$

$4 / 17$

$2 / 13$

$1 / 5$

$0 / 7$

Percentage of transfers resulting in singleton live births ${ }^{b}$

$3 / 17$

$0 / 13$

$1 / 4$

$0 / 6$

Percentage of cancellations ${ }^{b}$

$0 / 17$

$0 / 13$

$1 / 4$

$0 / 6$

Average number of embryos transferred

2.6

2.9

$0 / 5$

$0 / 7$

Percentage of pregnancies with twins ${ }^{b}$

$1 / 4$

$2 / 2$

3.3

3.3

$0 / 4$

$0 / 2$

$0 / 1$

$0 / 1$

Percentage of pregnancies with triplets or more ${ }^{b}$

Percentage of live births having multiple infants ${ }^{b, c}$

$1 / 4$

$2 / 2$

$0 / 1$

$0 / 1$

\section{Frozen Embryos from Nondonor Eggs}

Number of transfers

Percentage of transfers resulting in live births ${ }^{\mathrm{b}, \mathrm{c}}$

0

0

$0 / 1$

Average number of embryos transferred

\section{Donor Eggs}

Number of transfers

Percentage of transfers resulting in live births ${ }^{\mathrm{b}, \mathrm{c}}$

Average number of embryos transferred
All Ages Combined

Fresh Embryos

0

Frozen Embryos

0

\section{CURRENT CLINIC SERVICES AND PROFILE}

Current Name: Women's Fertility Center

Donor egg? No

Donor embryo? No

Gestational carriers? No

Yos

Cryopreservation?

SART member?

No

Single women? Yes

Verified lab accreditation?

Yes

(See Appendix C for details.)

${ }^{a}$ Reflects patient and treatment characteristics of ART cycles performed in 2006 using fresh nondonor eggs or embryos.

${ }^{b}$ When fewer than 20 cycles are reported in an age category, rates are shown as a fraction and confidence intervals are not given. Calculating percentages from fractions may be misleading and is not encouraged.

${ }^{c}$ A multiple-infant birth is counted as one live birth.

d Clinic-specific outcome rates are unreliable for women older than 42 undergoing ART cycles using fresh or frozen embryos with nondonor eggs. Readers are urged to review national outcomes for these age groups (see page 27).

e All ages (including ages $>42$ ) are reported together because previous data show that patient age does not materially affect success with donor eggs. 


\section{NORTH HUDSON I.V.F. CENTER FOR FERTILITY AND GYNECOLOGY ENGLEWOOD CLIFFS, NEW JERSEY}

A comparison of clinic success rates may not be meaningful because patient medical characteristics and treatment approaches vary from clinic to clinic. For more details about this, along with information on how to interpret the statistics in this table, see pages 79-88.

\section{ART CYCLE PROFILE}

\section{Type of ART ${ }^{\mathrm{a}}$}

IVF

GIFT

$100 \%$ Procedural Factors:

ZIFT

Combination
$0 \%$ With ICSI

$0 \%$ Unstimulated

$0 \%$ Used gestational carrier

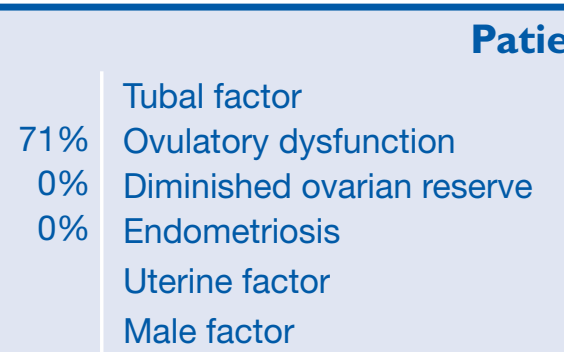

Patient Diagnosis

$\begin{array}{rlr}2 \% & \text { Other factor } & 3 \% \\ 7 \% & \text { Unknown factor } & 17 \% \\ 32 \% & \text { Multiple Factors: } & \\ 3 \% & \text { Female factors only } & 12 \% \\ 0 \% & \text { Female \& male factors } & 17 \% \\ 8 \% & & \end{array}$

Data verified by Jane E. Miller, MD

2006 PREGNANCY SUCCESS RATES

\section{Age of Woman}

Type of Cycle

$\begin{array}{cccc}<35 & \mathbf{3 5 - 3 7} & \mathbf{3 8 - 4 0} & \mathbf{4 1 - 4 2}^{\mathbf{d}} \\ 12 & 6 & 6 & 2 \\ 9 / 12 & 4 / 6 & 2 / 6 & 0 / 2 \\ 9 / 12 & 3 / 6 & 2 / 6 & 0 / 2\end{array}$

\section{Fresh Embryos from Nondonor Eggs}

Number of cycles

$9 / 12$

$3 / 6$

$2 / 6$

$0 / 2$

Percentage of cycles resulting in live births ${ }^{b, c}$

(Confidence Interval)

Percentage of retrievals resulting in live births $s^{b, c}$

$9 / 12$

$3 / 5$

$2 / 5$

$0 / 2$

Percentage of transfers resulting in live births $s^{b, c}$

$9 / 12$

$3 / 5$

$2 / 4$

$0 / 2$

Percentage of transfers resulting in singleton live births ${ }^{b}$

$6 / 12$

$2 / 5$

$1 / 4$

$0 / 2$

Percentage of cancellations ${ }^{b}$

$0 / 12$

$1 / 6$

$1 / 6$

$0 / 2$

Average number of embryos transferred

2.2

2.6

2.3

3.5

Percentage of pregnancies with twins ${ }^{b}$

$4 / 9$

$1 / 4$

$2 / 2$

Percentage of pregnancies with triplets or more ${ }^{b}$

Percentage of live births having multiple infants ${ }^{b, c}$

$0 / 9$

$3 / 9$

$1 / 4$

$0 / 2$

$1 / 3$

$1 / 2$

\section{Frozen Embryos from Nondonor Eggs}

Number of transfers

Percentage of transfers resulting in live births $s^{\mathrm{b}, \mathrm{c}}$

Average number of embryos transferred

\section{Donor Eggs}

Number of transfers

Percentage of transfers resulting in live births $s^{b, c}$

Average number of embryos transferred

$\begin{array}{cccc}4 & 1 & 0 & 1 \\ 2 / 4 & 0 / 1 & & 1 / 1 \\ 2.5 & 2.0 & & 2.0\end{array}$

All Ages Combined ${ }^{\mathrm{e}}$

Fresh Embryos 18

$13 / 18$

2.2
Frozen Embryos 6

$4 / 6$

2.3

\section{CURRENT CLINIC SERVICES AND PROFILE}

Current Name: North Hudson I.V.F., Center for Fertility and Gynecology

Donor egg? Yes Gestational carriers? Yes

Donor embryo? Yes Cryopreservation? Yes

Single women? Yes

SART member? Yes

Verified lab accreditation? Yes

(See Appendix C for details.)

${ }^{a}$ Reflects patient and treatment characteristics of ART cycles performed in 2006 using fresh nondonor eggs or embryos.

b When fewer than 20 cycles are reported in an age category, rates are shown as a fraction and confidence intervals are not given. Calculating percentages from fractions may be misleading and is not encouraged.

${ }^{c}$ A multiple-infant birth is counted as one live birth.

d Clinic-specific outcome rates are unreliable for women older than 42 undergoing ART cycles using fresh or frozen embryos with nondonor eggs. Readers are urged to review national outcomes for these age groups (see page 27).

e All ages (including ages $>42$ ) are reported together because previous data show that patient age does not materially affect success with donor eggs. 


\section{UNIVERSITY REPRODUCTIVE ASSOCIATES, PC HASBROUCK HEIGHTS, NEW JERSEY}

A comparison of clinic success rates may not be meaningful because patient medical characteristics and treatment approaches vary from clinic to clinic. For more details about this, along with information on how to interpret the statistics in this table, see pages $79-88$.

\section{ART CYCLE PROFILE}

\begin{tabular}{|c|c|c|c|c|c|c|c|}
\hline \multicolumn{4}{|c|}{ Type of $\mathrm{ART}^{\mathrm{a}}$} & \multicolumn{4}{|c|}{ Patient Diagnosis } \\
\hline IVF & $100 \%$ & Procedural Factors: & & Tubal factor & $3 \%$ & Other factor & $2 \%$ \\
\hline GIFT & $0 \%$ & With ICSI & $88 \%$ & Ovulatory dysfunction & $<1 \%$ & Unknown factor & $3 \%$ \\
\hline ZIFT & $0 \%$ & Unstimulated & $0 \%$ & Diminished ovarian reserve & $6 \%$ & Multiple Factors: & \\
\hline \multirow[t]{3}{*}{ Combination } & $0 \%$ & Used gestational carrier & $<1 \%$ & Endometriosis & $2 \%$ & Female factors only & $2 \%$ \\
\hline & & & & Uterine factor & $1 \%$ & Female \& male factors & $56 \%$ \\
\hline & & & & Male factor & $25 \%$ & & \\
\hline
\end{tabular}

2006 PREGNANCY SUCCESS RATES

Data verified by Peter G. McGovern, MD

\section{Type of Cycle}

\section{Fresh Embryos from Nondonor Eggs}

Number of cycles

Percentage of cycles resulting in pregnancies ${ }^{b}$

Percentage of cycles resulting in live births ${ }^{b, c}$

(Confidence Interval)

Percentage of retrievals resulting in live births $s^{b, c}$

Percentage of transfers resulting in live births $s^{b, c}$

Percentage of transfers resulting in singleton live births ${ }^{b}$

Percentage of cancellations ${ }^{b}$

Average number of embryos transferred

Percentage of pregnancies with twins ${ }^{b}$

Percentage of pregnancies with triplets or more ${ }^{b}$

Percentage of live births having multiple infants ${ }^{b, c}$

\section{Frozen Embryos from Nondonor Eggs}

Number of transfers

Percentage of transfers resulting in live births ${ }^{\mathrm{b}, \mathrm{c}}$

Average number of embryos transferred

\section{Donor Eggs}

Number of transfers

Percentage of transfers resulting in live births $s^{b, c}$

Average number of embryos transferred

\section{Age of Woman}

35-37 38-40

$41-42^{d}$

$<35$

47

48.9

42

15

96
63.5

42.6

33.3

$3 / 15$

51.0

(28.3-57.8)

26.2

$3 / 15$

$(40.6-61.4)$

53.3

46.5

(13.9-42.0)

53.3

47.6

26.8

$3 / 12$

37.0

28.6

27.5

$3 / 12$

4.2

8.5

25.0

$3 / 12$

2.2

29.5

4.9

2.5

39.1

30.6

4.3

40.0

2.4

$3 / 15$

2.7

2.7

$3 / 14$

$1 / 3$

$0 / 14$

$0 / 3$

$1 / 11$

$0 / 3$

8

$4 / 8$

3.3

\section{8}

$2 / 8$

3.1

\section{1}

$0 / 1$

4.0
2

$1 / 2$

4.0

All Ages Combined ${ }^{\mathrm{e}}$

Fresh Embryos

12
$4 / 12$
3.3

Frozen Embryos

1

$0 / 1$

2.0

\section{CURRENT CLINIC SERVICES AND PROFILE}

Current Name: University Reproductive Associates, PC

Donor egg? Yes

Donor embryo? No

Single women? Yes
Gestational carriers?

Cryopreservation?
Yes

Yes
SART member?

Verified lab accreditation?

(See Appendix C for details.)

${ }^{a}$ Reflects patient and treatment characteristics of ART cycles performed in 2006 using fresh nondonor eggs or embryos.

${ }^{b}$ When fewer than 20 cycles are reported in an age category, rates are shown as a fraction and confidence intervals are not given. Calculating percentages from fractions may be misleading and is not encouraged.

${ }^{c}$ A multiple-infant birth is counted as one live birth.

d Clinic-specific outcome rates are unreliable for women older than 42 undergoing ART cycles using fresh or frozen embryos with nondonor eggs. Readers are urged to review national outcomes for these age groups (see page 27).

${ }^{e}$ All ages (including ages $>42$ ) are reported together because previous data show that patient age does not materially affect success with donor eggs. 


\section{SHORE INSTITUTE FOR REPRODUCTIVE MEDICINE LAKEWOOD, NEW JERSEY}

A comparison of clinic success rates may not be meaningful because patient medical characteristics and treatment approaches vary from clinic to clinic. For more details about this, along with information on how to interpret the statistics in this table, see pages 79-88.

\section{ART CYCLE PROFILE}

\begin{tabular}{|c|c|c|c|c|c|c|c|}
\hline & Type & of $A R T^{a}$ & & Pat & ent [ & iagnosis & \\
\hline IVF & $100 \%$ & Procedural Factors: & & Tubal factor & $12 \%$ & Other factor & $11 \%$ \\
\hline GIFT & $0 \%$ & With ICSI & $23 \%$ & Ovulatory dysfunction & $6 \%$ & Unknown factor & $8 \%$ \\
\hline ZIFT & $0 \%$ & Unstimulated & $0 \%$ & Diminished ovarian reserve & $<1 \%$ & Multiple Factors: & \\
\hline Combination & $0 \%$ & Used gestational carrier & $0 \%$ & Endometriosis & $9 \%$ & Female factors only & $15 \%$ \\
\hline & & & & Uterine factor & $4 \%$ & Female \& male factors & $4 \%$ \\
\hline & & & & Male factor & $31 \%$ & & \\
\hline
\end{tabular}

2006 PREGNANCY SUCCESS RATES

Data verified by Allen Morgan, MD

\section{Type of Cycle}

\section{Fresh Embryos from Nondonor Eggs}

Number of cycles

Percentage of cycles resulting in pregnancies ${ }^{b}$

Percentage of cycles resulting in live births ${ }^{b, c}$

(Confidence Interval)

Percentage of retrievals resulting in live births $s^{b, c}$

Percentage of transfers resulting in live births $s^{b, c}$

Percentage of transfers resulting in singleton live births ${ }^{b}$

Percentage of cancellations ${ }^{b}$

Average number of embryos transferred

Percentage of pregnancies with twins ${ }^{b}$

Percentage of pregnancies with triplets or more ${ }^{b}$

Percentage of live births having multiple infants ${ }^{b, c}$

\section{Frozen Embryos from Nondonor Eggs}

Number of transfers

Percentage of transfers resulting in live births $s^{b, c}$

Average number of embryos transferred

\section{Donor Eggs}

Number of transfers

Percentage of transfers resulting in live births $s^{b, c}$

Average number of embryos transferred

\section{Age of Woman}

35-37 38-40

$41-42^{d}$

\section{$<35$}

16

$5 / 16$

$5 / 16$

21

33.3

23.8

$1 / 5$

51.4

40.5

$(24.8-57.9)$

42.9

45.5

$5 / 13$

$(8.2-47.2)$

30.3

$5 / 13$

$5 / 16$

$5 / 14$

$3 / 14$

23.8

3.1

$2 / 7$

$1 / 7$

$2 / 5$

2.8

$1 / 5$

$2 / 5$

$5 / 15$

7

$2 / 7$

2.3

\section{4}

$1 / 4$

3.3

\section{3}

$0 / 3$

2.0

\section{CURRENT CLINIC SERVICES AND PROFILE}

Current Name: Shore Institute for Reproductive Medicine

Donor egg? Yes

Donor embryo? Yes

Single women? Yes

\begin{abstract}
Gestational carriers? Yes
Cryopreservation? Yes
\end{abstract}

Fresh Embryos

All Ages Combined ${ }^{\mathrm{e}}$
Frozen Embryos 3

$1 / 2$

3.0

$1 / 3$

2.3
$0 / 4$

$0 / 4$

$0 / 4$

$1 / 5$

2.8

$0 / 1$

$0 / 1$

.

0

\footnotetext{
${ }^{a}$ Reflects patient and treatment characteristics of ART cycles performed in 2006 using fresh nondonor eggs or embryos.

${ }^{b}$ When fewer than 20 cycles are reported in an age category, rates are shown as a fraction and confidence intervals are not given. Calculating percentages from fractions may be misleading and is not encouraged.

${ }^{c}$ A multiple-infant birth is counted as one live birth.

d Clinic-specific outcome rates are unreliable for women older than 42 undergoing ART cycles using fresh or frozen embryos with nondonor eggs. Readers are urged to review national outcomes for these age groups (see page 27).

e All ages (including ages $>42$ ) are reported together because previous data show that patient age does not materially affect success with donor eggs.
} 


\section{DELAWARE VALLEY OBGYN AND INFERTILITY GROUP \\ PRINCETON IVF \\ LAWRENCEVILLE, NEW JERSEY}

A comparison of clinic success rates may not be meaningful because patient medical characteristics and treatment approaches vary from clinic to clinic. For more details about this, along with information on how to interpret the statistics in this table, see pages $79-88$.

\section{ART CYCLE PROFILE}

\begin{tabular}{|c|c|c|c|c|c|c|c|}
\hline \multicolumn{4}{|c|}{ Type of $\mathrm{ART}^{\mathrm{a}}$} & \multicolumn{4}{|c|}{ Patient Diagnosis } \\
\hline IVF & $100 \%$ & Procedural Factors: & & Tubal factor & $12 \%$ & Other factor & $2 \%$ \\
\hline GIFT & $0 \%$ & With ICSI & $41 \%$ & Ovulatory dysfunction & $7 \%$ & Unknown factor & $10 \%$ \\
\hline ZIFT & $0 \%$ & Unstimulated & $0 \%$ & Diminished ovarian reserve & $7 \%$ & Multiple Factors: & \\
\hline \multirow[t]{3}{*}{ Combination } & $0 \%$ & Used gestational carrier & $0 \%$ & Endometriosis & $<1 \%$ & Female factors only & $24 \%$ \\
\hline & & & & Uterine factor & $0 \%$ & Female \& male factors & $31 \%$ \\
\hline & & & & Male factor & $6 \%$ & & \\
\hline
\end{tabular}

2006 PREGNANCY SUCCESS RATES

Data verified by Seth G. Derman, MD

\section{Type of Cycle}

\section{Fresh Embryos from Nondonor Eggs}

Number of cycles

Percentage of cycles resulting in pregnancies ${ }^{b}$

Percentage of cycles resulting in live births ${ }^{b, c}$

(Confidence Interval)

Percentage of retrievals resulting in live births $s^{b, c}$

Percentage of transfers resulting in live births $s^{b, c}$

Percentage of transfers resulting in singleton live births ${ }^{b}$

Percentage of cancellations ${ }^{b}$

Average number of embryos transferred

Percentage of pregnancies with twins ${ }^{b}$

Percentage of pregnancies with triplets or more ${ }^{b}$

Percentage of live births having multiple infants ${ }^{b, c}$

\section{Frozen Embryos from Nondonor Eggs}

Number of transfers

Percentage of transfers resulting in live births $s^{b, c}$

Average number of embryos transferred

\section{Donor Eggs}

Number of transfers

Percentage of transfers resulting in live births $s^{\mathrm{b}, \mathrm{c}}$

Average number of embryos transferred

\section{Age of Woman}

35-37 38-40

$41-42^{d}$

\section{$<35$}

22

21

19

$\begin{array}{cccc}44 & 22 & 21 & 19 \\ 31.8 & 31.8 & 38.1 & 3 / 19 \\ 25.0 & 27.3 & 28.6 & 1 / 19\end{array}$

(13.2-40.3)

(10.7-50.2)

(11.3-52.2)

26.8

29.7

28.6

$6 / 19$

$1 / 17$

24.3

28.6

$6 / 19$

$1 / 16$

6.8

23.8

$5 / 19$

$1 / 16$

2.4

4.5

9.5

$2 / 19$

4 / 14

3.1

3.2

3.1

0 / 14

$3 / 7$

$1 / 8$

$1 / 3$

$2 / 11$

$1 / 7$

$1 / 8$

$0 / 3$

$1 / 6$

$1 / 6$

$0 / 1$

$\begin{array}{cccc}15 & 4 & 4 & 1 \\ 4 / 15 & 1 / 4 & 0 / 4 & 0 / 1 \\ 2.7 & 2.3 & 2.0 & 5.0\end{array}$

All Ages Combined

Fresh Embryos

Frozen Embryos

\section{5}

$2 / 5$

2.0
2

$1 / 2$

2.5

\section{CURRENT CLINIC SERVICES AND PROFILE}

Current Name: Delaware Valley OBGYN and Infertility Group, Princeton IVF

Donor egg? Yes

Donor embryo? Yes

Single women? Yes

\section{Gestational carriers? Yes}

Cryopreservation?
SART member?

Verified lab accreditation?

(See Appendix C for details.)

${ }^{a}$ Reflects patient and treatment characteristics of ART cycles performed in 2006 using fresh nondonor eggs or embryos.

${ }^{b}$ When fewer than 20 cycles are reported in an age category, rates are shown as a fraction and confidence intervals are not given. Calculating percentages from fractions may be misleading and is not encouraged.

${ }^{c}$ A multiple-infant birth is counted as one live birth.

d Clinic-specific outcome rates are unreliable for women older than 42 undergoing ART cycles using fresh or frozen embryos with nondonor eggs. Readers are urged to review national outcomes for these age groups (see page 27).

e All ages (including ages $>42$ ) are reported together because previous data show that patient age does not materially affect success with donor eggs. 


\section{PRINCETON CENTER FOR INFERTILITY \& REPRODUCTIVE MEDICINE LAWRENCEVILLE, NEW JERSEY}

A comparison of clinic success rates may not be meaningful because patient medical characteristics and treatment approaches vary from clinic to clinic. For more details about this, along with information on how to interpret the statistics in this table, see pages 79-88.

\section{ART CYCLE PROFILE}

\begin{tabular}{|c|c|c|c|c|c|c|c|}
\hline \multicolumn{4}{|c|}{ Type of ART ${ }^{a}$} & \multicolumn{4}{|c|}{ Patient Diagnosis } \\
\hline IVF & $100 \%$ & Procedural Factors: & & Tubal factor & $25 \%$ & Other factor & $0 \%$ \\
\hline GIFT & $0 \%$ & With ICSI & $47 \%$ & Ovulatory dysfunction & $8 \%$ & Unknown factor & $18 \%$ \\
\hline ZIFT & $0 \%$ & Unstimulated & $0 \%$ & Diminished ovarian reserve & $13 \%$ & Multiple Factors: & \\
\hline \multirow[t]{3}{*}{ Combination } & $0 \%$ & Used gestational carrier & $0 \%$ & Endometriosis & $0 \%$ & Female factors only & $1 \%$ \\
\hline & & & & Uterine factor & $0 \%$ & Female \& male factors & $12 \%$ \\
\hline & & & & Male factor & $23 \%$ & & \\
\hline
\end{tabular}

2006 PREGNANCY SUCCESS RATES

$$
\text { Type of Cycle }
$$

\section{Fresh Embryos from Nondonor Eggs}

Number of cycles

Percentage of cycles resulting in pregnancies ${ }^{b}$

Percentage of cycles resulting in live births ${ }^{b, c}$

(Confidence Interval)

Percentage of retrievals resulting in live births $s^{b, c}$

Percentage of transfers resulting in live births $s^{b, c}$

Percentage of transfers resulting in singleton live births ${ }^{b}$

Percentage of cancellations ${ }^{b}$

Average number of embryos transferred

Percentage of pregnancies with twins ${ }^{b}$

Percentage of pregnancies with triplets or more ${ }^{b}$

Percentage of live births having multiple infants ${ }^{b, c}$

\section{Frozen Embryos from Nondonor Eggs}

Number of transfers

Percentage of transfers resulting in live births $s^{\mathrm{b}, \mathrm{c}}$

Average number of embryos transferred

\section{Donor Eggs}

Number of transfers

Percentage of transfers resulting in live births $s^{\mathrm{b}, \mathrm{c}}$

Average number of embryos transferred

Data verified by Althea M. O'Shaughnessy, MD

\section{Age of Woman}

$\begin{array}{cccc}<35 & 35-37 & 38-40 & \mathbf{4 1 - 4 2}^{\mathbf{d}} \\ 16 & 9 & 11 & 5 \\ 9 / 16 & 5 / 9 & 3 / 11 & 0 / 5 \\ 9 / 16 & 4 / 9 & 3 / 11 & 0 / 5\end{array}$

$\begin{array}{cccc}9 / 16 & 4 / 8 & 3 / 9 & 0 / 5 \\ 9 / 16 & 4 / 5 & 3 / 9 & 0 / 5 \\ 7 / 16 & 3 / 5 & 2 / 9 & 0 / 5 \\ 0 / 16 & 1 / 9 & 2 / 11 & 0 / 5 \\ 2.1 & 2.6 & 2.8 & 2.8 \\ 2 / 9 & 2 / 5 & 1 / 3 & \\ 1 / 9 & 0 / 5 & 0 / 3 & \\ 2 / 9 & 1 / 4 & 1 / 3 & \end{array}$

$\begin{array}{cccc}7 & 6 & 2 & 3 \\ 2 / 7 & 1 / 6 & 1 / 2 & 1 / 3 \\ 2.3 & 2.8 & 2.5 & 3.0\end{array}$

All Ages Combined

Fresh Embryos

4

$2 / 4$

2.0
Frozen Embryos

4

$2 / 4$

1.8

\section{CURRENT CLINIC SERVICES AND PROFILE}

Current Name: Princeton Center for Infertility \& Reproductive Medicine

Donor egg? Yes Gestational carriers? Yes

Donor embryo? No Cryopreservation? Yes

Single women? Yes

SART member? $\quad$ Yes

Verified lab accreditation? Yes

(See Appendix C for details.)

${ }^{a}$ Reflects patient and treatment characteristics of ART cycles performed in 2006 using fresh nondonor eggs or embryos.

b When fewer than 20 cycles are reported in an age category, rates are shown as a fraction and confidence intervals are not given. Calculating percentages from fractions may be misleading and is not encouraged.

${ }^{c}$ A multiple-infant birth is counted as one live birth.

d Clinic-specific outcome rates are unreliable for women older than 42 undergoing ART cycles using fresh or frozen embryos with nondonor eggs. Readers are urged to review national outcomes for these age groups (see page 27).

e All ages (including ages $>42$ ) are reported together because previous data show that patient age does not materially affect success with donor eggs. 


\section{EAST COAST INFERTILITY AND IVF LITTLE SILVER, NEW JERSEY}

A comparison of clinic success rates may not be meaningful because patient medical characteristics and treatment approaches vary from clinic to clinic. For more details about this, along with information on how to interpret the statistics in this table, see pages $79-88$.

\section{ART CYCLE PROFILE}

\begin{tabular}{|c|c|c|c|c|c|c|c|}
\hline & Type & of $A R T^{a}$ & & Pati & ent D & iagnosis & \\
\hline IVF & $100 \%$ & Procedural Factors: & & Tubal factor & $4 \%$ & Other factor & $4 \%$ \\
\hline GIFT & $0 \%$ & With ICSI & $58 \%$ & Ovulatory dysfunction & $4 \%$ & Unknown factor & $<1 \%$ \\
\hline ZIFT & $0 \%$ & Unstimulated & $0 \%$ & Diminished ovarian reserve & $9 \%$ & Multiple Factors: & \\
\hline Combination & $0 \%$ & Used gestational carrier & $0 \%$ & Endometriosis & $16 \%$ & Female factors only & $13 \%$ \\
\hline & & & & $\begin{array}{l}\text { Uterine factor } \\
\text { Male factor }\end{array}$ & $\begin{array}{l}<1 \% \\
15 \%\end{array}$ & Female \& male factors & $34 \%$ \\
\hline
\end{tabular}

2006 PREGNANCY SUCCESS RATES

Data verified by Miguel Damien, MD

\section{Type of Cycle}

\section{Fresh Embryos from Nondonor Eggs}

Number of cycles

Percentage of cycles resulting in pregnancies ${ }^{b}$

Percentage of cycles resulting in live births ${ }^{b, c}$

(Confidence Interval)

Percentage of retrievals resulting in live births $s^{b, c}$

Percentage of transfers resulting in live births $s^{b, c}$

Percentage of transfers resulting in singleton live births ${ }^{b}$

Percentage of cancellations ${ }^{b}$

Average number of embryos transferred

Percentage of pregnancies with twins ${ }^{b}$

Percentage of pregnancies with triplets or more ${ }^{b}$

Percentage of live births having multiple infants ${ }^{b, c}$

\section{Frozen Embryos from Nondonor Eggs}

Number of transfers

Percentage of transfers resulting in live births $s^{b, c}$

Average number of embryos transferred

\section{Donor Eggs}

Number of transfers

Percentage of transfers resulting in live births $s^{b, c}$

Average number of embryos transferred

\section{Age of Woman}

35-37 38-40

$41-42^{d}$

\section{$<35$}

46

45.7

43

20

56
41.1

39.1

30.2

20.0

32.1

(25.1-54.6)

25.6

15.0

(20.3-46.0)

36.0

42.9

$(13.5-41.2)$

(3.2-37.9)

42.9

48.6

28.2

$3 / 19$

21.4

40.5

36.7

$3 / 14$

10.7

8.7

23.3

$2 / 14$

2.3

2.8

39.1

19.0

4.3

0.0

$9 / 18$

$3 / 18$

9.3

5.0

3.0

3.2

$4 / 13$

$1 / 4$

$0 / 13$

$0 / 4$

$4 / 11$

$1 / 3$

5

$1 / 5$

2.8

\section{6}

$2 / 6$

3.0
4

$0 / 4$

2.3 Do 


\section{INSTITUTE FOR REPRODUCTIVE MEDICINE AND SCIENCE \\ SAINT BARNABAS MEDICAL CENTER \\ LIVINGSTON, NEW JERSEY}

A comparison of clinic success rates may not be meaningful because patient medical characteristics and treatment approaches vary from clinic to clinic. For more details about this, along with information on how to interpret the statistics in this table, see pages 79-88.

\section{ART CYCLE PROFILE}

\section{Type of ART ${ }^{\mathrm{a}}$}

IVF

GIFT

$100 \%$ Procedural Factors:

ZIFT

Combination
$0 \%$ With ICSI

$0 \%$ Unstimulated

$0 \%$ Used gestational carrier $<1 \%$
Tubal factor

Ovulatory dysfunction

Diminished ovarian reserve

Endometriosis

Uterine factor

Male factor

\section{Patient Diagnosis}

$\begin{array}{rlr}4 \% & \text { Other factor } & 21 \% \\ 2 \% & \text { Unknown factor } & 3 \% \\ 4 \% & \text { Multiple Factors: } & \\ <1 \% & \text { Female factors only } & 28 \% \\ <1 \% & \text { Female \& male factors } & 30 \% \\ 6 \% & & \end{array}$

2006 PREGNANCY SUCCESS RATES

Data verified by Margaret G. Garrisi, MD

\section{Type of Cycle}

Age of Woman

$\begin{array}{llll} & 35-37 & 38-40 & 41-42^{d}\end{array}$

\section{Fresh Embryos from Nondonor Eggs}

Number of cycles

Percentage of cycles resulting in pregnancies ${ }^{b}$

Percentage of cycles resulting in live births $s^{b, c}$

(Confidence Interval)

Percentage of retrievals resulting in live births $s^{b, c}$

Percentage of transfers resulting in live births $s^{b, c}$

Percentage of transfers resulting in singleton live births ${ }^{b}$

Percentage of cancellations ${ }^{b}$

Average number of embryos transferred

Percentage of pregnancies with twins ${ }^{b}$

Percentage of pregnancies with triplets or more ${ }^{b}$

Percentage of live births having multiple infants ${ }^{b, c}$

\section{Frozen Embryos from Nondonor Eggs}

Number of transfers

Percentage of transfers resulting in live births ${ }^{b, c}$

Average number of embryos transferred

\section{Donor Eggs}

Number of transfers

Percentage of transfers resulting in live births $s^{b, c}$

Average number of embryos transferred

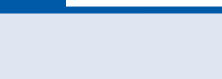

226

\section{6}

42.0

31.9

(25.8-38.4)

34.1

38.5

28.3

6.6

2.1

25.3

3.2

26.4

56

35.7

2.1
176

39.2

36.9

$(29.8-44.5)$

41.9

47.8

38.2

11.9

2.3

24.6

2.9

20.0
143

23.1

16.8

(11.1-23.9)

20.0

24.2

17.2

16.1

2.6

18.2

6.1

29.2

29

31.0

2.0

\section{5}

$6 / 15$

1.9
67

11.9

6.0

(1.7-14.6)

7.7

12.9

12.9

22.4

2.5

$0 / 8$

$0 / 8$

$0 / 4$
All Ages Combined ${ }^{\mathrm{e}}$
Fresh Embryos 56

51.8

2.1
Frozen Embryos

21

28.6

1.9

\section{CURRENT CLINIC SERVICES AND PROFILE}

Current Name: Institute for Reproductive Medicine and Science, Saint Barnabas Medical Center

Donor egg? Yes

Donor embryo? Yes

Single women? Yes

\begin{abstract}
Gestational carriers? Yes
Cryopreservation?
\end{abstract}

SART member?

Verified lab accreditation?

(See Appendix C for details.)

${ }^{a}$ Reflects patient and treatment characteristics of ART cycles performed in 2006 using fresh nondonor eggs or embryos.

${ }^{b}$ When fewer than 20 cycles are reported in an age category, rates are shown as a fraction and confidence intervals are not given. Calculating percentages from fractions may be misleading and is not encouraged.

${ }^{c}$ A multiple-infant birth is counted as one live birth.

d Clinic-specific outcome rates are unreliable for women older than 42 undergoing ART cycles using fresh or frozen embryos with nondonor eggs. Readers are urged to review national outcomes for these age groups (see page 27).

e All ages (including ages $>42$ ) are reported together because previous data show that patient age does not materially affect success with donor eggs. 


\section{COOPER INSTITUTE FOR REPRODUCTIVE HORMONAL DISORDERS MARLTON, NEW JERSEY}

A comparison of clinic success rates may not be meaningful because patient medical characteristics and treatment approaches vary from clinic to clinic. For more details about this, along with information on how to interpret the statistics in this table, see pages $79-88$.

\section{ART CYCLE PROFILE}

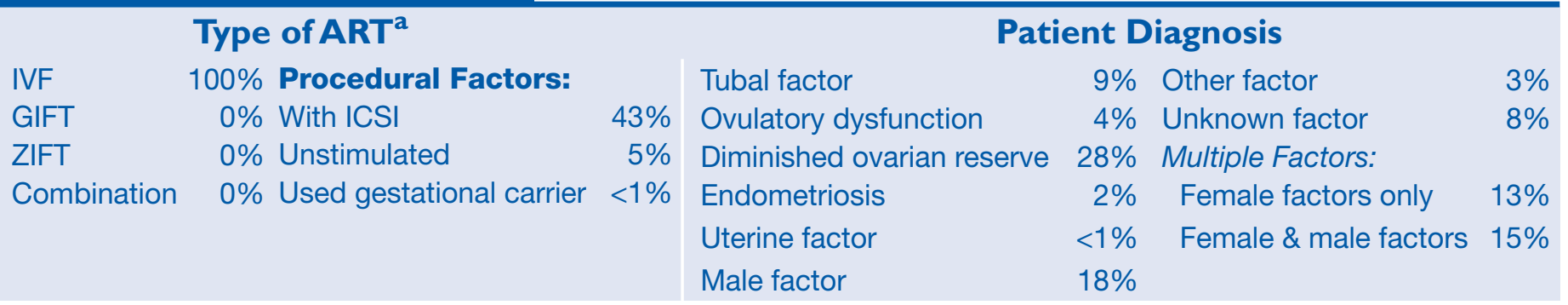

2006 PREGNANCY SUCCESS RATES

\section{Type of Cycle}

\section{Fresh Embryos from Nondonor Eggs}

Number of cycles

Percentage of cycles resulting in pregnancies ${ }^{b}$

Percentage of cycles resulting in live births ${ }^{b, c}$

(Confidence Interval)

Percentage of retrievals resulting in live births $s^{b, c}$

Percentage of transfers resulting in live births $s^{b, c}$

Percentage of transfers resulting in singleton live births ${ }^{b}$

Percentage of cancellations ${ }^{b}$

Average number of embryos transferred

Percentage of pregnancies with twins ${ }^{b}$

Percentage of pregnancies with triplets or more ${ }^{b}$

Percentage of live births having multiple infants ${ }^{b, c}$

\section{Frozen Embryos from Nondonor Eggs}

Number of transfers

Percentage of transfers resulting in live births ${ }^{\mathrm{b}, \mathrm{c}}$

Average number of embryos transferred

\section{Donor Eggs}

Number of transfers

Percentage of transfers resulting in live births ${ }^{\mathrm{b}, \mathrm{c}}$

Average number of embryos transferred

Data verified by Jerome H. Check, MD, PhD

\section{Age of Woman}

35-37 38-40 $\quad 41-42^{d}$

$<35$

185

244

174

255

23.2

15.6

9.2

23.5

19.5

11.9

5.7

$(18.5-29.2)$

$(14.0-25.9)$

$(8.1-16.6)$

(2.8-10.3)

27.5

24.8

17.3

8.4

35.7

30.5

22.3

12.3

20.3

18.5

11.1

14.5

21.6

31.1

31.6

2.4

2.5

2.4

2.3

30.6

25.6

15.8

$1 / 16$

8.3

9.3

5.3

$0 / 16$

35.0

33.3

17.2

$1 / 10$

120

59

23

14

30.0

25.4

26.1

$6 / 14$

2.5

2.5

3.0

2.9

All Ages Combined ${ }^{\mathrm{e}}$

Fresh Embryos

98

46.9

2.5

\section{Frozen Embryos}

117

33.3

\section{CURRENT CLINIC SERVICES AND PROFILE}

Current Name: Cooper Institute for Reproductive Hormonal Disorders

\begin{tabular}{l|l|l} 
Donor egg? Yes $\quad$ Gestational carriers? Yes &
\end{tabular}

Donor embryo? Yes Cryopreservation? Yes

Single women? Yes

SART member? Yes

Verified lab accreditation? Yes

(See Appendix C for details.)

${ }^{a}$ Reflects patient and treatment characteristics of ART cycles performed in 2006 using fresh nondonor eggs or embryos.

${ }^{b}$ When fewer than 20 cycles are reported in an age category, rates are shown as a fraction and confidence intervals are not given. Calculating percentages from fractions may be misleading and is not encouraged.

${ }^{c}$ A multiple-infant birth is counted as one live birth.

${ }^{d}$ Clinic-specific outcome rates are unreliable for women older than 42 undergoing ART cycles using fresh or frozen embryos with nondonor eggs. Readers are urged to review national outcomes for these age groups (see page 27).

e All ages (including ages $>42$ ) are reported together because previous data show that patient age does not materially affect success with donor eggs. 


\section{DELAWARE VALLEY INSTITUTE OF FERTILITY AND GENETICS \\ MARLTON, NEW JERSEY}

A comparison of clinic success rates may not be meaningful because patient medical characteristics and treatment approaches vary from clinic to clinic. For more details about this, along with information on how to interpret the statistics in this table, see pages 79-88.

\section{ART CYCLE PROFILE}

\begin{tabular}{|c|c|c|c|c|c|c|c|}
\hline \multicolumn{4}{|c|}{ Type of ART ${ }^{a}$} & \multicolumn{4}{|c|}{ Patient Diagnosis } \\
\hline IVF & $100 \%$ & Procedural Factors: & & Tubal factor & $0 \%$ & Other factor & $0 \%$ \\
\hline GIFT & $0 \%$ & With ICSI & $61 \%$ & Ovulatory dysfunction & $<1 \%$ & Unknown factor & $0 \%$ \\
\hline ZIFT & $0 \%$ & Unstimulated & $0 \%$ & Diminished ovarian reserve & $0 \%$ & Multiple Factors: & \\
\hline \multirow[t]{3}{*}{ Combination } & $0 \%$ & Used gestational carrier & $0 \%$ & Endometriosis & $0 \%$ & Female factors only & $16 \%$ \\
\hline & & & & Uterine factor & $0 \%$ & Female \& male factors & $84 \%$ \\
\hline & & & & Male factor & $0 \%$ & & \\
\hline
\end{tabular}

2006 PREGNANCY SUCCESS RATES

$$
\text { Type of Cycle }
$$

\section{Fresh Embryos from Nondonor Eggs}

Number of cycles

Percentage of cycles resulting in pregnancies ${ }^{b}$

Percentage of cycles resulting in live births ${ }^{b, c}$

(Confidence Interval)

Percentage of retrievals resulting in live births $s^{b, c}$

Percentage of transfers resulting in live births $s^{b, c}$

Percentage of transfers resulting in singleton live births ${ }^{b}$

Percentage of cancellations ${ }^{b}$

Average number of embryos transferred

Percentage of pregnancies with twins ${ }^{b}$

Percentage of pregnancies with triplets or more ${ }^{b}$

Percentage of live births having multiple infants ${ }^{b, c}$

\section{Frozen Embryos from Nondonor Eggs}

Number of transfers

Percentage of transfers resulting in live births $s^{b, c}$

Average number of embryos transferred

\section{Donor Eggs}

Number of transfers

Percentage of transfers resulting in live births $s^{b, c}$

Average number of embryos transferred

Data verified by George S. Taliadouros, MD

\section{Age of Woman}

35-37 38-40

$41-42^{d}$

$<35$

18

$12 / 18$

$(29.2-52.1)$

21

52.4

7

$0 / 7$

$11 / 18$

40.3

45.6

$11 / 18$

$11 / 18$

$6 / 18$

$0 / 18$

3.2

$5 / 12$

$1 / 12$

$5 / 11$

33.9

11.7

2.5

26.3

7.9

32.3

33.3

(14.6-57.0)

$7 / 17$

$7 / 15$

$5 / 15$

19.0

3.8

$3 / 11$

$1 / 11$

$2 / 7$
9

$1 / 9$

1.9
2

$0 / 2$

2.5
$0 / 7$

$0 / 5$

$0 / 5$

$0 / 5$

$2 / 7$

3.4

All Ages Combined ${ }^{\mathrm{e}}$

Fresh Embryos

\section{2}

$1 / 2$

2.5

\section{2}

$0 / 2$

2.0

\section{CURRENT CLINIC SERVICES AND PROFILE}

Current Name: Delaware Valley Institute of Fertility and Genetics

\begin{tabular}{l|l|ll} 
Donor egg? & Yes & Gestational carriers? & Yes \\
Donor embryo? & No & Cryopreservation? & Yes \\
Single women? & Yes & &
\end{tabular}

SART member?

Yes

Verified lab accreditation?

Yes

(See Appendix C for details.)

\footnotetext{
${ }^{a}$ Reflects patient and treatment characteristics of ART cycles performed in 2006 using fresh nondonor eggs or embryos.

${ }^{b}$ When fewer than 20 cycles are reported in an age category, rates are shown as a fraction and confidence intervals are not given. Calculating percentages from fractions may be misleading and is not encouraged.

${ }^{c}$ A multiple-infant birth is counted as one live birth.

d Clinic-specific outcome rates are unreliable for women older than 42 undergoing ART cycles using fresh or frozen embryos with nondonor eggs. Readers are urged to review national outcomes for these age groups (see page 27).

${ }^{e}$ All ages (including ages $>42$ ) are reported together because previous data show that patient age does not materially affect success with donor eggs.
} 


\section{SOUTH JERSEY FERTILITY CENTER \\ MARLTON, NEW JERSEY}

A comparison of clinic success rates may not be meaningful because patient medical characteristics and treatment approaches vary from clinic to clinic. For more details about this, along with information on how to interpret the statistics in this table, see pages $79-88$.

\section{ART CYCLE PROFILE}

\begin{tabular}{|c|c|c|c|c|c|c|c|}
\hline \multicolumn{4}{|c|}{ Type of ART } & \multicolumn{4}{|c|}{ Patient Diagnosis } \\
\hline IVF & $100 \%$ & Procedural Factors: & & Tubal factor & $16 \%$ & Other factor & $2 \%$ \\
\hline GIFT & $0 \%$ & With ICSI & $58 \%$ & Ovulatory dysfunction & $7 \%$ & Unknown factor & $5 \%$ \\
\hline ZIFT & $0 \%$ & Unstimulated & $0 \%$ & Diminished ovarian reserve & $8 \%$ & Multiple Factors: & \\
\hline \multirow[t]{3}{*}{ Combination } & $0 \%$ & Used gestational carrier & $0 \%$ & Endometriosis & $2 \%$ & Female factors only & $25 \%$ \\
\hline & & & & Uterine factor & $<1 \%$ & Female \& male factors & $17 \%$ \\
\hline & & & & Male factor & $18 \%$ & & \\
\hline
\end{tabular}

2006 PREGNANCY SUCCESS RATES

Data verified by Robert A. Skaf, MD

\section{Type of Cycle}

\section{Fresh Embryos from Nondonor Eggs}

Number of cycles

Percentage of cycles resulting in pregnancies ${ }^{b}$

Percentage of cycles resulting in live births ${ }^{b, c}$

(Confidence Interval)

Percentage of retrievals resulting in live births $s^{b, c}$

Percentage of transfers resulting in live births $s^{b, c}$

Percentage of transfers resulting in singleton live births ${ }^{b}$

Percentage of cancellations ${ }^{b}$

Average number of embryos transferred

Percentage of pregnancies with twins ${ }^{b}$

Percentage of pregnancies with triplets or more ${ }^{b}$

Percentage of live births having multiple infants ${ }^{b, c}$

\section{Frozen Embryos from Nondonor Eggs}

Number of transfers

Percentage of transfers resulting in live births $s^{\mathrm{b}, \mathrm{c}}$

Average number of embryos transferred

\section{Donor Eggs}

Number of transfers

Percentage of transfers resulting in live births ${ }^{\mathrm{b}, \mathrm{c}}$

Average number of embryos transferred

\section{Age of Woman}

35-37 38-40

$41-42^{d}$

$<35$

82

28.0

74

15

45.5

22.0

28.4

$4 / 15$

37.6

(13.6-32.5)

18.9

$4 / 15$

$(30.6-44.9)$

39.7

40.8

24.0

(10.7-29.7)

27.6

24.3

21.2

$4 / 13$

21.9

$4 / 11$

18.9

14.1

$4 / 11$

5.3

8.5

10.8

$2 / 15$

2.2

2.4

2.9

4.3

33.7

26.1

3.5

4.3

32.4

$4 / 18$

19.0

$0 / 4$

9.5

$0 / 4$

$5 / 14$

$0 / 4$

38

23.7

$$
19
$$

$4 / 19$

2.4

2.3

15

5

$3 / 15$

$2 / 5$

3.3

3.2

All Ages Combined ${ }^{\mathrm{e}}$

Fresh Embryos

17

2.1
Frozen Embryos

12

$0 / 12$

2.3

\section{CURRENT CLINIC SERVICES AND PROFILE}

Current Name: South Jersey Fertility Center

\begin{tabular}{l|l|l} 
Donor egg? Yes $\quad$ Gestational carriers? Yes &
\end{tabular}

Donor embryo? Yes Cryopreservation? Yes

Single women? Yes

\section{SART member? Yes}

Verified lab accreditation? Yes

(See Appendix C for details.)

${ }^{a}$ Reflects patient and treatment characteristics of ART cycles performed in 2006 using fresh nondonor eggs or embryos.

${ }^{b}$ When fewer than 20 cycles are reported in an age category, rates are shown as a fraction and confidence intervals are not given. Calculating percentages from fractions may be misleading and is not encouraged.

${ }^{c}$ A multiple-infant birth is counted as one live birth.

d Clinic-specific outcome rates are unreliable for women older than 42 undergoing ART cycles using fresh or frozen embryos with nondonor eggs. Readers are urged to review national outcomes for these age groups (see page 27).

${ }^{e}$ All ages (including ages $>42$ ) are reported together because previous data show that patient age does not materially affect success with donor eggs. 


\section{DIAMOND INSTITUTE FOR INFERTILITY \\ MILLBURN, NEW JERSEY}

A comparison of clinic success rates may not be meaningful because patient medical characteristics and treatment approaches vary from clinic to clinic. For more details about this, along with information on how to interpret the statistics in this table, see pages 79-88.

\section{ART CYCLE PROFILE}

\begin{tabular}{|c|c|c|c|c|c|c|c|}
\hline \multicolumn{4}{|c|}{ Type of ART ${ }^{a}$} & \multicolumn{4}{|c|}{ Patient Diagnosis } \\
\hline IVF & $100 \%$ & Procedural Factors: & & Tubal factor & $16 \%$ & Other factor & $<1 \%$ \\
\hline GIFT & $0 \%$ & With ICSI & $65 \%$ & Ovulatory dysfunction & $8 \%$ & Unknown factor & $9 \%$ \\
\hline ZIFT & $0 \%$ & Unstimulated & $0 \%$ & Diminished ovarian reserve & $23 \%$ & Multiple Factors: & \\
\hline \multirow[t]{3}{*}{ Combination } & $0 \%$ & Used gestational carrier & $0 \%$ & Endometriosis & $3 \%$ & Female factors only & $20 \%$ \\
\hline & & & & Uterine factor & $<1 \%$ & Female \& male factors & $14 \%$ \\
\hline & & & & Male factor & $5 \%$ & & \\
\hline
\end{tabular}

2006 PREGNANCY SUCCESS RATES

Data verified by Arie Birkenfeld, MD

\section{Type of Cycle}

\section{Fresh Embryos from Nondonor Eggs}

Number of cycles

Percentage of cycles resulting in pregnancies ${ }^{b}$

Percentage of cycles resulting in live births ${ }^{b, c}$

(Confidence Interval)

Percentage of retrievals resulting in live births $s^{b, c}$

Percentage of transfers resulting in live births $s^{b, c}$

Percentage of transfers resulting in singleton live births ${ }^{b}$

Percentage of cancellations ${ }^{b}$

Average number of embryos transferred

Percentage of pregnancies with twins ${ }^{b}$

Percentage of pregnancies with triplets or more ${ }^{b}$

Percentage of live births having multiple infants ${ }^{b, c}$

\section{Frozen Embryos from Nondonor Eggs}

Number of transfers

Percentage of transfers resulting in live births $s^{\mathrm{b}, \mathrm{c}}$

Average number of embryos transferred

\section{Donor Eggs}

Number of transfers

Percentage of transfers resulting in live births $s^{b, c}$

Average number of embryos transferred

\section{Age of Woman}

35-37 38-40

$41-42^{d}$

\section{$<35$}

121

43.8

89

37.1

29.2

(31.7-49.8)

(20.1-39.8)

31.7

32.9

22.8

7.9

2.3

36.4

0.0

30.8

44.9

47.2

1.9

30.8

27

40.7

10

2.0
$2 / 10$

2.0
69

26.1

10.1

(4.2-19.8)

11.7

13.2

9.4

13.0

2.8

$3 / 18$

$0 / 18$

$2 / 7$

15

$4 / 15$

2.3
23

21.7

17.4

(5.0-38.8)

$4 / 18$

$4 / 18$

$4 / 18$

21.7

3.3

$0 / 5$

$0 / 5$

$0 / 4$

4

$1 / 4$

1.3

All Ages Combined ${ }^{\mathrm{e}}$

Fresh Embryos

27

44.4

2.0
Frozen Embryos 18

$7 / 18$

2.1

\section{CURRENT CLINIC SERVICES AND PROFILE}

Current Name: Diamond Institute for Infertility
Donor egg?
Yes
Donor embryo? Yes
Gestational carriers? Yes
Cryopreservation?
Yes
Single women? Yes

$\begin{array}{ll}\text { SART member? } & \text { Yes } \\ \text { Verified lab accreditation? } & \text { Yes } \\ \text { (See Appendix C for details.) } & \end{array}$
a Reflects patient and treatment characteristics of ART cycles performed in 2006 using fresh nondonor eggs or embryos.
${ }^{b}$ When fewer than 20 cycles are reported in an age category, rates are shown as a fraction and confidence intervals are not given. Calculating percentages from fractions may be misleading and is not encouraged.
c A multiple-infant birth is counted as one live birth.
d Clinic-specific outcome rates are unreliable for women older than 42 undergoing ART cycles using fresh or frozen embryos with nondonor eggs. Readers are urged to review national outcomes for these age groups (see page 27).
e All ages (including ages $>42$ ) are reported together because previous data show that patient age does not materially affect success with donor eggs. 


\section{REPRODUCTIVE MEDICINE ASSOCIATES OF NEW JERSEY \\ MORRISTOWN, NEW JERSEY}

A comparison of clinic success rates may not be meaningful because patient medical characteristics and treatment approaches vary from clinic to clinic. For more details about this, along with information on how to interpret the statistics in this table, see pages $79-88$.

\section{ART CYCLE PROFILE}

\begin{tabular}{|c|c|c|c|c|c|c|c|}
\hline & Type & of $\mathrm{ART}^{\mathrm{a}}$ & & Pati & ent D & iagnosis & \\
\hline IVF & $100 \%$ & Procedural Factors: & & Tubal factor & $7 \%$ & Other factor & $20 \%$ \\
\hline GIFT & $0 \%$ & With ICSI & $57 \%$ & Ovulatory dysfunction & $10 \%$ & Unknown factor & $0 \%$ \\
\hline ZIFT & $0 \%$ & Unstimulated & $0 \%$ & Diminished ovarian reserve & $6 \%$ & Multiple Factors: & \\
\hline Combination & $0 \%$ & Used gestational carrier & $2 \%$ & Endometriosis & $3 \%$ & Female factors only & $18 \%$ \\
\hline & & & & Uterine factor & $2 \%$ & Female \& male factors & $21 \%$ \\
\hline & & & & Male factor & $15 \%$ & & \\
\hline
\end{tabular}

2006 PREGNANCY SUCCESS RATES

Data verified by Michael R. Drews, MD

\section{Type of Cycle}

\section{Fresh Embryos from Nondonor Eggs}

Number of cycles

Percentage of cycles resulting in pregnancies ${ }^{b}$

Percentage of cycles resulting in live births $s^{b, c}$

(Confidence Interval)

Percentage of retrievals resulting in live births $s^{b, c}$

Percentage of transfers resulting in live births ${ }^{b, c}$

Percentage of transfers resulting in singleton live births ${ }^{b}$

Percentage of cancellations ${ }^{b}$

Average number of embryos transferred

Percentage of pregnancies with twins ${ }^{\mathrm{b}}$

Percentage of pregnancies with triplets or more ${ }^{\mathrm{b}}$

Percentage of live births having multiple infants ${ }^{b, c}$

\section{Frozen Embryos from Nondonor Eggs}

Number of transfers

Percentage of transfers resulting in live births $s^{\mathrm{b}, \mathrm{c}}$

Average number of embryos transferred

\section{Donor Eggs}

Number of transfers

Percentage of transfers resulting in live births ${ }^{\mathrm{b}, \mathrm{c}}$

Average number of embryos transferred

\section{Age of Woman}

35-37 38-40

$41-42^{d}$ $<35$

790

484

398

204

54.2

44.4

31.9

23.5

47.0

37.0

24.9

11.3

(43.4-50.5)

(32.7-41.5)

(20.7-29.4)

(7.3-16.4)

49.9

41.7

29.7

33.1

14.5

53.8

46.0

26.8

16.3

32.5

31.9

16.3

14.9

5.9

11.4

3.0

22.1

2.3

2.6

15.7

3.2

36.2

27.9

4.7

16.7

5.1

6.5

19.2

0.0

39.6

30.7

179

38.5

96

32.3

42

23

2.0

2.2

28.6

21.7

2.1

2.2

All Ages Combined ${ }^{e}$

Fresh Embryos

190

55.3

2.3
Frozen Embryos

115

30.4

2.3

\section{CURRENT CLINIC SERVICES AND PROFILE}

Current Name: Reproductive Medicine Associates of New Jersey

Donor egg? Yes Gestational carriers? Yes

Donor embryo? Yes Cryopreservation? Yes

Single women? Yes

SART member? Yes

Verified lab accreditation? Yes

(See Appendix C for details.)

${ }^{a}$ Reflects patient and treatment characteristics of ART cycles performed in 2006 using fresh nondonor eggs or embryos.

${ }^{b}$ When fewer than 20 cycles are reported in an age category, rates are shown as a fraction and confidence intervals are not given. Calculating percentages from fractions may be misleading and is not encouraged.

${ }^{c}$ A multiple-infant birth is counted as one live birth.

${ }^{d}$ Clinic-specific outcome rates are unreliable for women older than 42 undergoing ART cycles using fresh or frozen embryos with nondonor eggs. Readers are urged to review national outcomes for these age groups (see page 27).

e All ages (including ages $>42$ ) are reported together because previous data show that patient age does not materially affect success with donor eggs. 


\section{VALLEY HOSPITAL FERTILITY CENTER \\ PARAMUS, NEW JERSEY}

A comparison of clinic success rates may not be meaningful because patient medical characteristics and treatment approaches vary from clinic to clinic. For more details about this, along with information on how to interpret the statistics in this table, see pages 79-88.

\section{ART CYCLE PROFILE}

\section{Type of ART ${ }^{\mathrm{a}}$}

IVF

GIFT

$100 \%$ Procedural Factors:

ZIFT

Combination
$0 \%$ With ICSI

$0 \%$ Unstimulated

$0 \%$ Used gestational carrier

\begin{tabular}{|c|c|c|c|c|}
\hline & \multicolumn{4}{|c|}{ Patient Diagnosis } \\
\hline & Tubal factor & $7 \%$ & Other factor & $5 \%$ \\
\hline $35 \%$ & Ovulatory dysfunction & $6 \%$ & Unknown factor & $21 \%$ \\
\hline $0 \%$ & Diminished ovarian reserve & $8 \%$ & Multiple Factors: & \\
\hline $0 \%$ & Endometriosis & $1 \%$ & Female factors only & $7 \%$ \\
\hline & Uterine factor & $2 \%$ & Female \& male factors & $25 \%$ \\
\hline & Male factor & $18 \%$ & & \\
\hline
\end{tabular}

2006 PREGNANCY SUCCESS RATES

Data verified by Ali Nasseri, MD, PhD

\section{Type of Cycle}

Age of Woman

\begin{tabular}{|c|c|c|c|c|}
\hline & & Age of & Woman & \\
\hline & $<35$ & $35-37$ & $38-40$ & $41-42^{d}$ \\
\hline & 63 & 45 & 32 & 18 \\
\hline & 60.3 & 44.4 & 28.1 & $7 / 18$ \\
\hline & 54.0 & 35.6 & 28.1 & $5 / 18$ \\
\hline & (40.9-66.6) & $(21.9-51.2)$ & $(13.7-46.7)$ & \\
\hline & 56.7 & 41.0 & 45.0 & $5 / 14$ \\
\hline & 56.7 & 42.1 & 45.0 & $5 / 14$ \\
\hline irths ${ }^{b}$ & 30.0 & 26.3 & 40.0 & $3 / 14$ \\
\hline & 4.8 & 13.3 & 37.5 & $4 / 18$ \\
\hline & 2.3 & 2.5 & 2.8 & 3.1 \\
\hline & 57.9 & 45.0 & $1 / 9$ & $2 / 7$ \\
\hline & 5.3 & 0.0 & $0 / 9$ & $0 / 7$ \\
\hline & 47.1 & $6 / 16$ & $1 / 9$ & $2 / 5$ \\
\hline & 4 & 2 & 0 & 0 \\
\hline & $1 / 4$ & $1 / 2$ & & \\
\hline & 2.8 & 2.0 & & \\
\hline & & All Ages & ombined $^{\mathrm{e}}$ & \\
\hline & Fresh I & mbryos & Frozen & Embryos \\
\hline & & 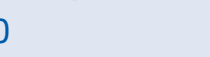 & & 0 \\
\hline
\end{tabular}

\section{Fresh Embryos from Nondonor Eggs}

Number of cycles

Percentage of cycles resulting in pregnancies ${ }^{b}$

Percentage of cycles resulting in live births ${ }^{b, c}$

(Confidence Interval)

Percentage of retrievals resulting in live births $s^{b, c}$

Percentage of transfers resulting in live births $s^{b, c}$

Percentage of transfers resulting in singleton live births ${ }^{b}$

Percentage of cancellations ${ }^{b}$

Average number of embryos transferred

Percentage of pregnancies with twins ${ }^{b}$

Percentage of pregnancies with triplets or more ${ }^{b}$

Percentage of live births having multiple infants ${ }^{b, c}$

\section{Frozen Embryos from Nondonor Eggs}

Number of transfers

Percentage of transfers resulting in live births ${ }^{b, c}$

Average number of embryos transferred

\section{Donor Eggs}

Number of transfers

Percentage of transfers resulting in live births $s^{\mathrm{b}, \mathrm{c}}$

Average number of embryos transferred

\section{CURRENT CLINIC SERVICES AND PROFILE}

Current Name: Valley Hospital Fertility Center

Donor egg? No Gestational carriers? No

Donor embryo? No

Single women? Yes

Cryopreservation? Yes

\section{SART member?}

Yes

Verified lab accreditation?

(See Appendix C for details.)

${ }^{a}$ Reflects patient and treatment characteristics of ART cycles performed in 2006 using fresh nondonor eggs or embryos.

b When fewer than 20 cycles are reported in an age category, rates are shown as a fraction and confidence intervals are not given. Calculating percentages from fractions may be misleading and is not encouraged.

${ }^{c}$ A multiple-infant birth is counted as one live birth.

d Clinic-specific outcome rates are unreliable for women older than 42 undergoing ART cycles using fresh or frozen embryos with nondonor eggs. Readers are urged to review national outcomes for these age groups (see page 27).

e All ages (including ages $>42$ ) are reported together because previous data show that patient age does not materially affect success with donor eggs. 


\section{IVF NEW JERSEY \\ SOMERSET, NEW JERSEY}

A comparison of clinic success rates may not be meaningful because patient medical characteristics and treatment approaches vary from clinic to clinic. For more details about this, along with information on how to interpret the statistics in this table, see pages $79-88$.

\section{ART CYCLE PROFILE}

\begin{tabular}{|c|c|c|c|c|c|c|c|}
\hline \multicolumn{4}{|c|}{ Type of $\mathrm{ART}^{\mathrm{a}}$} & \multicolumn{4}{|c|}{ Patient Diagnosis } \\
\hline IVF & $100 \%$ & Procedural Factors: & & Tubal factor & $6 \%$ & Other factor & $7 \%$ \\
\hline GIFT & $0 \%$ & With ICSI & $26 \%$ & Ovulatory dysfunction & $6 \%$ & Unknown factor & $9 \%$ \\
\hline ZIFT & $0 \%$ & Unstimulated & $0 \%$ & Diminished ovarian reserve & $19 \%$ & Multiple Factors: & \\
\hline \multirow[t]{3}{*}{ Combination } & $0 \%$ & Used gestational carrier & $2 \%$ & Endometriosis & $2 \%$ & Female factors only & \\
\hline & & & & Uterine factor & $<1 \%$ & Female \& male factors & $22 \%$ \\
\hline & & & & Male factor & $14 \%$ & & \\
\hline
\end{tabular}

2006 PREGNANCY SUCCESS RATES

Data verified by Michael C. Darder, MD

\section{Type of Cycle}

\section{Fresh Embryos from Nondonor Eggs}

Number of cycles

Percentage of cycles resulting in pregnancies ${ }^{b}$

Percentage of cycles resulting in live births $s^{b, c}$

(Confidence Interval)

Percentage of retrievals resulting in live births $s^{b, c}$

Percentage of transfers resulting in live births $s^{b, c}$

Percentage of transfers resulting in singleton live births ${ }^{b}$

Percentage of cancellations ${ }^{b}$

Average number of embryos transferred

Percentage of pregnancies with twins ${ }^{b}$

Percentage of pregnancies with triplets or more ${ }^{b}$

Percentage of live births having multiple infants ${ }^{b, c}$

\section{Frozen Embryos from Nondonor Eggs}

Number of transfers

Percentage of transfers resulting in live births ${ }^{\mathrm{b}, \mathrm{c}}$

Average number of embryos transferred

\section{Donor Eggs}

Number of transfers

Percentage of transfers resulting in live births $s^{\mathrm{b}, \mathrm{c}}$

Average number of embryos transferred

\section{Age of Woman}

35-37 38-40

$41-42^{d}$

\section{$<35$}

146

89

41

48.1

42.5

28.1

19.5

40.6

34.9

22.5

19.5

(34.9-46.5)

(27.2-43.3)

(14.3-32.6)

(8.8-34.9)

43.0

37.8

26.7

23.5

47.2

43.6

27.8

27.6

23.8

22.2

20.8

20.7

5.5

7.5

15.7

17.1

2.5

2.8

3.3

3.7

46.1

41.9

28.0

$2 / 8$

7.1

12.9

8.0

$1 / 8$

49.6

49.0

25.0

$2 / 8$

27

37.0

2.1
0

0

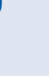

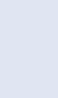

$1 / 3$

1

2.0

$0 / 1$

1.0

All Ages Combined

Fresh Embryos

102

68.6

2.1
Frozen Embryos

48.0

2.2

\section{CURRENT CLINIC SERVICES AND PROFILE}

Current Name: IVF New Jersey

Donor egg? Yes

Donor embryo? Yes

Single women? Yes

\begin{abstract}
Gestational carriers?
Yes

Cryopreservation?

Yes
\end{abstract}

\section{SART member? Yes}

Verified lab accreditation? Yes

(See Appendix C for details.)

${ }^{a}$ Reflects patient and treatment characteristics of ART cycles performed in 2006 using fresh nondonor eggs or embryos.

b When fewer than 20 cycles are reported in an age category, rates are shown as a fraction and confidence intervals are not given. Calculating percentages from fractions may be misleading and is not encouraged.

${ }^{c}$ A multiple-infant birth is counted as one live birth.

d Clinic-specific outcome rates are unreliable for women older than 42 undergoing ART cycles using fresh or frozen embryos with nondonor eggs. Readers are urged to review national outcomes for these age groups (see page 27).

e All ages (including ages $>42$ ) are reported together because previous data show that patient age does not materially affect success with donor eggs. 


\section{REPRODUCTIVE SCIENCE CENTER OF NEW JERSEY TINTON FALLS, NEW JERSEY}

A comparison of clinic success rates may not be meaningful because patient medical characteristics and treatment approaches vary from clinic to clinic. For more details about this, along with information on how to interpret the statistics in this table, see pages 79-88.

\section{ART CYCLE PROFILE}

\begin{tabular}{|c|c|c|c|c|c|c|c|}
\hline \multicolumn{4}{|c|}{ Type of ART ${ }^{a}$} & \multicolumn{4}{|c|}{ Patient Diagnosis } \\
\hline IVF & $100 \%$ & Procedural Factors: & & Tubal factor & $9 \%$ & Other factor & $2 \%$ \\
\hline GIFT & $0 \%$ & With ICSI & $53 \%$ & Ovulatory dysfunction & $3 \%$ & Unknown factor & $<1 \%$ \\
\hline ZIFT & $0 \%$ & Unstimulated & $0 \%$ & Diminished ovarian reserve & $11 \%$ & Multiple Factors: & \\
\hline \multirow[t]{3}{*}{ Combination } & $0 \%$ & Used gestational carrier & $<1 \%$ & Endometriosis & $2 \%$ & Female factors only & $25 \%$ \\
\hline & & & & Uterine factor & $0 \%$ & Female \& male factors & $31 \%$ \\
\hline & & & & Male factor & $17 \%$ & & \\
\hline
\end{tabular}

2006 PREGNANCY SUCCESS RATES

Data verified by William Ziegler, DO

\section{Type of Cycle}

\section{Fresh Embryos from Nondonor Eggs}

Number of cycles

Percentage of cycles resulting in pregnancies ${ }^{b}$

Percentage of cycles resulting in live births $s^{b, c}$

(Confidence Interval)

Percentage of retrievals resulting in live births $s^{b, c}$

Percentage of transfers resulting in live births $s^{b, c}$

Percentage of transfers resulting in singleton live births ${ }^{b}$

Percentage of cancellations ${ }^{b}$

Average number of embryos transferred

Percentage of pregnancies with twins ${ }^{b}$

Percentage of pregnancies with triplets or more ${ }^{b}$

Percentage of live births having multiple infants ${ }^{b, c}$

\section{Frozen Embryos from Nondonor Eggs}

Number of transfers

Percentage of transfers resulting in live births ${ }^{b, c}$

Average number of embryos transferred
Age of Woman

35-37 38-40

$41-42^{d}$

35

34.3

28.6

36.4

(24.9-49.1)

42.1

47.1

27.5

13.6

2.1

29.6

7.4

41.7
(14.6-46.3)

37.0

38.5

38.5

22.9

2.4

$3 / 12$

$0 / 12$

$0 / 10$
37

18.9

13.5

(4.5-28.8)

17.9

20.0

16.0

24.3

2.7

$1 / 7$

$0 / 7$

$1 / 5$
11

$6 / 11$

2.8
3

$0 / 3$

2.7
15

$3 / 15$

$0 / 15$

$0 / 11$

$0 / 10$

$0 / 10$

$4 / 15$

2.6

$1 / 3$

$0 / 3$

1

$0 / 1$

3.0

\section{Donor Eggs}

Fresh Embryos

All Ages Combined ${ }^{\mathrm{e}}$

Number of transfers

Percentage of transfers resulting in live births $s^{b, c}$

0

Frozen Embryos

0

Average number of embryos transferred

\section{CURRENT CLINIC SERVICES AND PROFILE}

Current Name: Reproductive Science Center of New Jersey

Donor egg? Yes Gestational carriers? Yes

Donor embryo? Yes Cryopreservation? Yes

Single women? Yes

SART member? Yes

Verified lab accreditation?

(See Appendix C for details.)

${ }^{a}$ Reflects patient and treatment characteristics of ART cycles performed in 2006 using fresh nondonor eggs or embryos.

${ }^{b}$ When fewer than 20 cycles are reported in an age category, rates are shown as a fraction and confidence intervals are not given. Calculating percentages from fractions may be misleading and is not encouraged.

${ }^{c}$ A multiple-infant birth is counted as one live birth.

d Clinic-specific outcome rates are unreliable for women older than 42 undergoing ART cycles using fresh or frozen embryos with nondonor eggs. Readers are urged to review national outcomes for these age groups (see page 27).

e All ages (including ages $>42$ ) are reported together because previous data show that patient age does not materially affect success with donor eggs. 


\section{DR. LOUIS R. MANARA \\ VOORHEES, NEW JERSEY}

A comparison of clinic success rates may not be meaningful because patient medical characteristics and treatment approaches vary from clinic to clinic. For more details about this, along with information on how to interpret the statistics in this table, see pages 79-88.

\section{ART CYCLE PROFILE}

\begin{tabular}{|c|c|c|c|c|c|c|c|}
\hline & Type & of $\mathrm{ART}^{\mathrm{a}}$ & & Pati & ent D & iagnosis & \\
\hline IVF & $100 \%$ & Procedural Factors: & & Tubal factor & $10 \%$ & Other factor & $2 \%$ \\
\hline GIFT & $0 \%$ & With ICSI & $42 \%$ & Ovulatory dysfunction & $4 \%$ & Unknown factor & $14 \%$ \\
\hline $\mathrm{ZIFT}$ & $0 \%$ & Unstimulated & $<1 \%$ & Diminished ovarian reserve & $9 \%$ & Multiple Factors: & \\
\hline Combination & $0 \%$ & Used gestational carrier & $2 \%$ & Endometriosis & $5 \%$ & Female factors only & $10 \%$ \\
\hline & & & & Uterine factor & $<1 \%$ & Female \& male factors & $33 \%$ \\
\hline
\end{tabular}

2006 PREGNANCY SUCCESS RATES

Data verified by Louis R. Manara, DO

\section{Type of Cycle}

\section{Age of Woman}

35-37 38-40

$<35$

26

23.1

19.2

18.8

$(6.6-39.4)$

Percentage of cycles resulting in pregnancies
Percentage of cycles resulting in live births $s^{b, c}$

(Confidence Interval)

Percentage of retrievals resulting in live births ${ }^{\mathrm{b}, \mathrm{c}}$

(8.9-32.6)

$5 / 16$

20.9

21.4

$5 / 16$

14.3

$5 / 16$

3.1

$0 / 6$

$3 / 9$

$0 / 9$

$0 / 6$

$3 / 9$

$0 / 5$
18.2

13.6

$0 / 5$

$(2.9-34.9)$

$3 / 14$

$3 / 12$

$3 / 12$

36.4

3.4

$1 / 4$

$0 / 4$

$0 / 3$

$41-42^{d}$

5

$0 / 5$

$0 / 3$

$0 / 2$

$0 / 2$

$2 / 5$

2.5

0

0

0

\section{Frozen Embryos from Nondonor Eggs}

Number of transfers

Percentage of transfers resulting in live births $s^{\mathrm{b}, \mathrm{c}}$

Average number of embryos transferred

$0 / 1$

3.0

\section{Donor Eggs}

\section{Fresh Embryos}

0

Number of transfers

Percentage of transfers resulting in live births $s^{b, c}$

Average number of embryos transferred

\section{CURRENT CLINIC SERVICES AND PROFILE}

Current Name: Dr. Louis R. Manara, The Center for Reproductive Medicine \& Fertility

Donor egg? No

Donor embryo? No

Gestational carriers? No

SART member?

Yes

Single women? Yes

Verified lab accreditation?

(See Appendix C for details.)

${ }^{a}$ Reflects patient and treatment characteristics of ART cycles performed in 2006 using fresh nondonor eggs or embryos.

${ }^{b}$ When fewer than 20 cycles are reported in an age category, rates are shown as a fraction and confidence intervals are not given. Calculating percentages from fractions may be misleading and is not encouraged.

${ }^{c}$ A multiple-infant birth is counted as one live birth.

d Clinic-specific outcome rates are unreliable for women older than 42 undergoing ART cycles using fresh or frozen embryos with nondonor eggs. Readers are urged to review national outcomes for these age groups (see page 27).

e All ages (including ages $>42$ ) are reported together because previous data show that patient age does not materially affect success with donor eggs. 


\section{NORTH JERSEY FERTILITY ASSOCIATES, LLC \\ WAYNE, NEW JERSEY}

A comparison of clinic success rates may not be meaningful because patient medical characteristics and treatment approaches vary from clinic to clinic. For more details about this, along with information on how to interpret the statistics in this table, see pages 79-88.

\section{ART CYCLE PROFILE}

\begin{tabular}{l|l|lrlrl}
\multicolumn{3}{c}{ Type of ART } & \multicolumn{4}{c}{ Patient Diagnosis } \\
IVF & $100 \%$ Procedural Factors: & & Tubal factor & $18 \%$ & Other factor & $9 \%$ \\
GIFT & $0 \%$ With ICSI & $63 \%$ & Ovulatory dysfunction & $0 \%$ & Unknown factor & $9 \%$ \\
ZIFT & $0 \%$ Unstimulated & $<1 \%$ & Diminished ovarian reserve & $8 \%$ & Multiple Factors: & \\
Combination & $0 \%$ Used gestational carrier & $0 \%$ & Endometriosis & $6 \%$ & Female factors only & $18 \%$ \\
& & & Uterine factor & $3 \%$ & Female \& male factors & $10 \%$ \\
& & & Male factor & $18 \%$ & &
\end{tabular}

2006 PREGNANCY SUCCESS RATES

Data verified by Mark X. Ransom, MD

\section{Type of Cycle}

\section{Fresh Embryos from Nondonor Eggs}

Number of cycles

Percentage of cycles resulting in pregnancies ${ }^{b}$

Percentage of cycles resulting in live births $s^{b, c}$

(Confidence Interval)

Percentage of retrievals resulting in live births $s^{b, c}$

Percentage of transfers resulting in live births $s^{b, c}$

Percentage of transfers resulting in singleton live births ${ }^{b}$

Percentage of cancellations ${ }^{b}$

Average number of embryos transferred

Percentage of pregnancies with twins ${ }^{b}$

Percentage of pregnancies with triplets or more ${ }^{b}$

Percentage of live births having multiple infants ${ }^{\mathrm{b}, \mathrm{c}}$

\section{Frozen Embryos from Nondonor Eggs}

Number of transfers

Percentage of transfers resulting in live births $s^{b, c}$

Average number of embryos transferred

\section{Donor Eggs}

Number of transfers

Percentage of transfers resulting in live births $s^{b, c}$

Average number of embryos transferred

\section{Age of Woman}

35-37 38-40

$41-42^{d}$

\section{$<35$}

27

55.6

32

6

51.2

40.7

25.0

$3 / 6$

48.8

(22.4-61.2)

18.8

$2 / 6$

(33.3-64.5)

55.3

52.4

$(7.2-36.4)$

56.8

52.4

24.0

$2 / 5$

27.0

33.3

26.1

$2 / 4$

11.6

22.2

17.4

$2 / 4$

2.5

2.6

21.9

$1 / 6$

50.0

$5 / 15$

4.5

$0 / 15$

52.4

$4 / 11$

2.7

3.3

$0 / 3$

$0 / 3$

$1 / 8$

$0 / 2$

$2 / 6$

0

0

$0 / 1$

2.0

\section{CURRENT CLINIC SERVICES AND PROFILE}

Current Name: North Jersey Fertility Associates, LLC

Donor egg? Yes

Donor embryo? Yes

Single women? Yes
Gestational carriers? No

Cryopreservation? Yes
All Ages Combined ${ }^{\mathrm{e}}$

Fresh Embryos

Frozen Embryos

\section{7}

$3 / 7$

1

$0 / 1$

2.3

2.0

\footnotetext{
${ }^{a}$ Reflects patient and treatment characteristics of ART cycles performed in 2006 using fresh nondonor eggs or embryos.

${ }^{b}$ When fewer than 20 cycles are reported in an age category, rates are shown as a fraction and confidence intervals are not given. Calculating percentages from fractions may be misleading and is not encouraged.

${ }^{c}$ A multiple-infant birth is counted as one live birth.

d Clinic-specific outcome rates are unreliable for women older than 42 undergoing ART cycles using fresh or frozen embryos with nondonor eggs. Readers are urged to review national outcomes for these age groups (see page 27).

e All ages (including ages $>42$ ) are reported together because previous data show that patient age does not materially affect success with donor eggs.
} 


\section{FERTILITY INSTITUTE OF NEW JERSEY AND NEW YORK \\ WESTWOOD, NEW JERSEY}

A comparison of clinic success rates may not be meaningful because patient medical characteristics and treatment approaches vary from clinic to clinic. For more details about this, along with information on how to interpret the statistics in this table, see pages $79-88$.

\section{ART CYCLE PROFILE}

\begin{tabular}{|c|c|c|c|c|c|c|c|}
\hline \multicolumn{4}{|c|}{ Type of $A R T^{a}$} & \multicolumn{4}{|c|}{ Patient Diagnosis } \\
\hline IVF & $100 \%$ & Procedural Factors: & & Tubal factor & $3 \%$ & Other factor & $6 \%$ \\
\hline GIFT & $0 \%$ & With ICSI & $93 \%$ & Ovulatory dysfunction & $6 \%$ & Unknown factor & $<1 \%$ \\
\hline $\mathrm{ZIFT}$ & $0 \%$ & Unstimulated & $0 \%$ & Diminished ovarian reserve & $18 \%$ & Multiple Factors: & \\
\hline \multirow[t]{3}{*}{ Combination } & $0 \%$ & Used gestational carrier & $<1 \%$ & Endometriosis & $<1 \%$ & Female factors only & $21 \%$ \\
\hline & & & & Uterine factor & $0 \%$ & Female \& male factors & $43 \%$ \\
\hline & & & & Male factor & $2 \%$ & & \\
\hline
\end{tabular}

2006 PREGNANCY SUCCESS RATES

Data verified by Daniel Navot, MD

\section{Type of Cycle}

\section{Fresh Embryos from Nondonor Eggs}

Number of cycles

Percentage of cycles resulting in pregnancies ${ }^{b}$

Percentage of cycles resulting in live births $s^{b, c}$

(Confidence Interval)

Percentage of retrievals resulting in live births $s^{b, c}$

Percentage of transfers resulting in live births $s^{b, c}$

Percentage of transfers resulting in singleton live births ${ }^{b}$

Percentage of cancellations ${ }^{b}$

Average number of embryos transferred

Percentage of pregnancies with twins ${ }^{b}$

Percentage of pregnancies with triplets or more ${ }^{b}$

Percentage of live births having multiple infants ${ }^{b, c}$

\section{Frozen Embryos from Nondonor Eggs}

Number of transfers

Percentage of transfers resulting in live births $s^{b, c}$

Average number of embryos transferred

\section{Donor Eggs}

Number of transfers

Percentage of transfers resulting in live births $s^{b, c}$

Average number of embryos transferred

\section{Age of Woman}

35-37

38-40

$41-42^{d}$

\section{$<35$}

50

34.0

46

14

52.8

28.0

41.3

$1 / 14$

45.8

(34.0-58.0)

(16.2-42.5)

32.6

$1 / 14$

46.5

29.8

(19.5-48.0)

48.5

36.8

34.9

$1 / 12$

38.5

$1 / 10$

28.9

30.8

$1 / 10$

1.4

6.0

6.5

$2 / 14$

2.3

2.9

3.1

2.7

18.4

$2 / 17$

2.6

$1 / 17$

$4 / 19$

$0 / 1$

21.2

$3 / 14$

$1 / 19$

$0 / 1$

$3 / 15$

$0 / 1$

24

33.3

2.4

\section{4}

$1 / 4$

3.0

\section{6}

$2 / 6$

2.7
3

$0 / 3$

3.0

All Ages Combined ${ }^{\mathrm{e}}$

Fresh Embryos 13

$7 / 13$

2.7
Frozen Embryos

\section{6}

$1 / 6$

2.2

\section{CURRENT CLINIC SERVICES AND PROFILE}

Current Name: Fertility Institute of New Jersey and New York

Donor egg? Yes Gestational carriers? Yes

Donor embryo? Yes Cryopreservation? Yes

Single women? Yes

SART member? Yes

Verified lab accreditation? Yes

(See Appendix C for details.)

${ }^{a}$ Reflects patient and treatment characteristics of ART cycles performed in 2006 using fresh nondonor eggs or embryos.

${ }^{b}$ When fewer than 20 cycles are reported in an age category, rates are shown as a fraction and confidence intervals are not given. Calculating percentages from fractions may be misleading and is not encouraged.

${ }^{c}$ A multiple-infant birth is counted as one live birth.

d Clinic-specific outcome rates are unreliable for women older than 42 undergoing ART cycles using fresh or frozen embryos with nondonor eggs. Readers are urged to review national outcomes for these age groups (see page 27).

${ }^{e}$ All ages (including ages $>42$ ) are reported together because previous data show that patient age does not materially affect success with donor eggs. 


\section{CENTER FOR REPRODUCTIVE MEDICINE OF NEW MEXICO ALBUQUERQUE, NEW MEXICO}

A comparison of clinic success rates may not be meaningful because patient medical characteristics and treatment approaches vary from clinic to clinic. For more details about this, along with information on how to interpret the statistics in this table, see pages 79-88.

\section{ART CYCLE PROFILE}

\begin{tabular}{|c|c|c|c|c|c|c|c|}
\hline \multicolumn{4}{|c|}{ Type of ART ${ }^{a}$} & \multicolumn{4}{|c|}{ Patient Diagnosis } \\
\hline IVF & $100 \%$ & Procedural Factors: & & Tubal factor & $2 \%$ & Other factor & $0 \%$ \\
\hline GIFT & $0 \%$ & With ICSI & $71 \%$ & Ovulatory dysfunction & $1 \%$ & Unknown factor & $8 \%$ \\
\hline ZIFT & $0 \%$ & Unstimulated & $0 \%$ & Diminished ovarian reserve & $4 \%$ & Multiple Factors: & \\
\hline \multirow[t]{3}{*}{ Combination } & $0 \%$ & Used gestational carrier & $1 \%$ & Endometriosis & $3 \%$ & Female factors only & $15 \%$ \\
\hline & & & & Uterine factor & $<1 \%$ & Female \& male factors & $49 \%$ \\
\hline & & & & Male factor & $17 \%$ & & \\
\hline
\end{tabular}

\section{PREGNANCY SUCCESS RATES}

\section{Type of Cycle}

\section{Fresh Embryos from Nondonor Eggs}

Number of cycles

Percentage of cycles resulting in pregnancies ${ }^{b}$

Percentage of cycles resulting in live births ${ }^{b, c}$

(Confidence Interval)

Percentage of retrievals resulting in live births ${ }^{b, c}$

Percentage of transfers resulting in live births $s^{b, c}$

Percentage of transfers resulting in singleton live births ${ }^{b}$

Percentage of cancellations ${ }^{b}$

Average number of embryos transferred

Percentage of pregnancies with twins ${ }^{b}$

Percentage of pregnancies with triplets or more ${ }^{b}$

Percentage of live births having multiple infants ${ }^{b, c}$

\section{Frozen Embryos from Nondonor Eggs}

Number of transfers

Percentage of transfers resulting in live births ${ }^{b, c}$

Average number of embryos transferred

\section{Donor Eggs}

Number of transfers

Percentage of transfers resulting in live births $s^{b, c}$

Average number of embryos transferred

Data verified by Douglas J. Thompson, MD

\section{Age of Woman}

$<35 \quad 35-37 \quad 38-40 \quad 41-42^{d}$

$\begin{array}{cccc}51 & 23 & 11 & 11 \\ 66.7 & 56.5 & 5 / 11 & 3 / 11 \\ 62.7 & 47.8 & 3 / 11 & 2 / 11\end{array}$

$(48.1-75.9)$

$(26.8-69.4)$

66.7

$11 / 19$

$3 / 10$

$2 / 10$

66.7

$11 / 18$

$3 / 10$

$2 / 10$

37.5

$7 / 18$

$2 / 10$

$2 / 10$

5.9

17.4

$1 / 11$

$1 / 11$

2.3

2.4

2.9

3.5

38.2

$4 / 13$

$0 / 5$

$0 / 3$

8.8

$1 / 13$

$1 / 5$

$0 / 3$

43.8

$4 / 11$

$1 / 3$

$0 / 2$

$\begin{array}{cccc}11 & 2 & 4 & 2 \\ 6 / 11 & 1 / 2 & 1 / 4 & 0 / 2 \\ 2.6 & 2.0 & 2.0 & 1.5\end{array}$

\section{All Ages Combined}

Fresh Embryos

29

72.4

2.1
Frozen Embryos 20

35.0

2.8

\section{CURRENT CLINIC SERVICES AND PROFILE}

Current Name: Center for Reproductive Medicine of New Mexico

Donor egg? Yes Gestational carriers? Yes

Donor embryo? Yes Cryopreservation? Yes

Single women? Yes

SART member? Yes

Verified lab accreditation? Yes

(See Appendix C for details.)

${ }^{a}$ Reflects patient and treatment characteristics of ART cycles performed in 2006 using fresh nondonor eggs or embryos.

b When fewer than 20 cycles are reported in an age category, rates are shown as a fraction and confidence intervals are not given. Calculating percentages from fractions may be misleading and is not encouraged.

${ }^{c}$ A multiple-infant birth is counted as one live birth.

d Clinic-specific outcome rates are unreliable for women older than 42 undergoing ART cycles using fresh or frozen embryos with nondonor eggs. Readers are urged to review national outcomes for these age groups (see page 27).

e All ages (including ages $>42$ ) are reported together because previous data show that patient age does not materially affect success with donor eggs. 


\section{ALBANY IVF, FERTILITY AND GYNECOLOGY ALBANY, NEW YORK}

A comparison of clinic success rates may not be meaningful because patient medical characteristics and treatment approaches vary from clinic to clinic. For more details about this, along with information on how to interpret the statistics in this table, see pages $79-88$.

\section{ART CYCLE PROFILE}

\begin{tabular}{|c|c|c|c|c|c|c|c|}
\hline \multicolumn{4}{|c|}{ Type of ART ${ }^{a}$} & \multicolumn{4}{|c|}{ Patient Diagnosis } \\
\hline IVF & $100 \%$ & Procedural Factors: & & Tubal factor & $9 \%$ & Other factor & $1 \%$ \\
\hline GIFT & $0 \%$ & With ICSI & $82 \%$ & Ovulatory dysfunction & $7 \%$ & Unknown factor & $8 \%$ \\
\hline ZIFT & $0 \%$ & Unstimulated & $<1 \%$ & Diminished ovarian reserve & $25 \%$ & Multiple Factors: & \\
\hline \multirow[t]{3}{*}{ Combination } & $0 \%$ & Used gestational carrier & $0 \%$ & Endometriosis & $2 \%$ & Female factors only & $14 \%$ \\
\hline & & & & Uterine factor & $0 \%$ & Female \& male factors & $12 \%$ \\
\hline & & & & Male factor & $23 \%$ & & \\
\hline
\end{tabular}

2006 PREGNANCY SUCCESS RATES

Data verified by Peter M. Horvath, MD

\section{Type of Cycle}

\section{Fresh Embryos from Nondonor Eggs}

Number of cycles

Percentage of cycles resulting in pregnancies ${ }^{b}$

Percentage of cycles resulting in live births ${ }^{b, c}$

(Confidence Interval)

Percentage of retrievals resulting in live births $s^{b, c}$

Percentage of transfers resulting in live births $s^{b, c}$

Percentage of transfers resulting in singleton live births ${ }^{b}$

Percentage of cancellations ${ }^{b}$

Average number of embryos transferred

Percentage of pregnancies with twins ${ }^{b}$

Percentage of pregnancies with triplets or more ${ }^{b}$

Percentage of live births having multiple infants ${ }^{b, c}$

\section{Frozen Embryos from Nondonor Eggs}

Number of transfers

Percentage of transfers resulting in live births ${ }^{\mathrm{b}, \mathrm{c}}$

Average number of embryos transferred

\section{Donor Eggs}

Number of transfers

Percentage of transfers resulting in live births $s^{b, c}$

Average number of embryos transferred

\section{Age of Woman}

35-37 38-40

$41-42^{d}$

$<35$

55

36.4

48

5

40.4

34.5

29.2

$2 / 5$

33.0

(22.2-48.6)

20.8

$2 / 5$

(24.3-42.7)

38.7

42.2

(10.5-35.0)

46.8

47.5

27.8

$2 / 5$

32.5

40.0

38.5

$2 / 4$

14.7

18.2

34.6

$2 / 4$

2.4

2.5

25.0

4.5

15.0

25.0

$0 / 5$

30.6

5.0

2.7

3.3

$2 / 14$

$0 / 2$

$0 / 14$

$0 / 2$

$1 / 10$

$0 / 2$

8

$2 / 8$

2.6

$3 / 19$

\section{5}

$0 / 5$

2.0

1

$0 / 8$

3.3

All Ages Combined ${ }^{\mathrm{e}}$

Fresh Embryos 15

$6 / 15$

2.5
Frozen Embryos

4

$3 / 4$

4.0

\section{CURRENT CLINIC SERVICES AND PROFILE}

Current Name: Albany IVF, Fertility and Gynecology

Donor egg? Yes

Donor embryo? No

Single women? Yes
Gestational carriers? No

Cryopreservation? Yes

${ }^{a}$ Reflects patient and treatment characteristics of ART cycles performed in 2006 using fresh nondonor eggs or embryos.

${ }^{b}$ When fewer than 20 cycles are reported in an age category, rates are shown as a fraction and confidence intervals are not given. Calculating percentages from fractions may be misleading and is not encouraged.

A multiple-infant birth is counted as one live birth.

d Clinic-specific outcome rates are unreliable for women older than 42 undergoing ART cycles using fresh or frozen embryos with nondonor eggs. Readers are urged to review national outcomes for these age groups (see page 27).

e All ages (including ages $>42$ ) are reported together because previous data show that patient age does not materially affect success with donor eggs. 


\section{THE FERTILITY INSTITUTE AT NEW YORK METHODIST HOSPITAL BROOKLYN, NEW YORK}

A comparison of clinic success rates may not be meaningful because patient medical characteristics and treatment approaches vary from clinic to clinic. For more details about this, along with information on how to interpret the statistics in this table, see pages 79-88.

\section{ART CYCLE PROFILE}

\begin{tabular}{|c|c|c|c|c|c|c|c|}
\hline \multicolumn{4}{|c|}{ Type of ART ${ }^{a}$} & \multicolumn{4}{|c|}{ Patient Diagnosis } \\
\hline IVF & $100 \%$ & Procedural Factors: & & Tubal factor & $23 \%$ & Other factor & $4 \%$ \\
\hline GIFT & $0 \%$ & With ICSI & $83 \%$ & Ovulatory dysfunction & $<1 \%$ & Unknown factor & $2 \%$ \\
\hline ZIFT & $0 \%$ & Unstimulated & $<1 \%$ & Diminished ovarian reserve & $9 \%$ & Multiple Factors: & \\
\hline \multirow[t]{3}{*}{ Combination } & $0 \%$ & Used gestational carrier & $16 \%$ & Endometriosis & $10 \%$ & Female factors only & $37 \%$ \\
\hline & & & & Uterine factor & $<1 \%$ & Female \& male factors & $12 \%$ \\
\hline & & & & Male factor & $3 \%$ & & \\
\hline
\end{tabular}

\section{PREGNANCY SUCCESS RATES}

Data verified by George D. Kofinas, MD

\section{Type of Cycle}

\section{Fresh Embryos from Nondonor Eggs}

Number of cycles

Percentage of cycles resulting in pregnancies ${ }^{b}$

Percentage of cycles resulting in live births $s^{b, c}$

(Confidence Interval)

Percentage of retrievals resulting in live births $s^{b, c}$

Percentage of transfers resulting in live births $s^{b, c}$

Percentage of transfers resulting in singleton live births ${ }^{b}$

Percentage of cancellations ${ }^{b}$

Average number of embryos transferred

Percentage of pregnancies with twins ${ }^{b}$

Percentage of pregnancies with triplets or more ${ }^{b}$

Percentage of live births having multiple infants ${ }^{b, c}$

\section{Frozen Embryos from Nondonor Eggs}

Number of transfers

Percentage of transfers resulting in live births ${ }^{b, c}$

Average number of embryos transferred

\section{Donor Eggs}

Number of transfers

Percentage of transfers resulting in live births $s^{b, c}$

Average number of embryos transferred

\section{Age of Woman}

35-37 38-40

$41-42^{d}$

$<35$

68

63

49

31.9

32.4

20.6

12.2

25.3

23.5

9.5

4.1

$(16.7-35.5)$

(14.1-35.4)

(3.6-19.6)

(0.5-14.0)

28.0

29.9

26.2

12.0

4.8

26.7

12.2

4.9

16.7

8.2

4.9

9.9

10.3

20.6

14.3

3.6

3.8

34.5

22.7

3.4

4.5

4.1

4.1

34.8

$6 / 16$

$1 / 13$

$0 / 6$

$1 / 13$

$0 / 6$

$2 / 6$

$0 / 2$

24

41.7

17

$7 / 17$

11

$1 / 11$

5

3.3

3.4

3.2

$1 / 5$

4.0

All Ages Combined ${ }^{\mathrm{e}}$

Fresh Embryos

37

54.1

3.6
Frozen Embryos

43

37.2

3.5

\section{CURRENT CLINIC SERVICES AND PROFILE}

Current Name: The Fertility Institute at New York Methodist Hospital

Donor egg? Yes Gestational carriers? No

Donor embryo? Yes Cryopreservation? Yes

Single women? Yes

SART member? Yes

Verified lab accreditation?

(See Appendix C for details.)

${ }^{a}$ Reflects patient and treatment characteristics of ART cycles performed in 2006 using fresh nondonor eggs or embryos.

b When fewer than 20 cycles are reported in an age category, rates are shown as a fraction and confidence intervals are not given. Calculating percentages from fractions may be misleading and is not encouraged.

${ }^{c}$ A multiple-infant birth is counted as one live birth.

d Clinic-specific outcome rates are unreliable for women older than 42 undergoing ART cycles using fresh or frozen embryos with nondonor eggs. Readers are urged to review national outcomes for these age groups (see page 27).

e All ages (including ages $>42$ ) are reported together because previous data show that patient age does not materially affect success with donor eggs. 


\section{GENESIS FERTILITY \& REPRODUCTIVE MEDICINE BROOKLYN, NEW YORK}

A comparison of clinic success rates may not be meaningful because patient medical characteristics and treatment approaches vary from clinic to clinic. For more details about this, along with information on how to interpret the statistics in this table, see pages $79-88$.

\section{ART CYCLE PROFILE}

\begin{tabular}{|c|c|c|c|c|c|c|c|}
\hline & Type & of $A R T^{a}$ & & Pat & ent $D$ & iagnosis & \\
\hline IVF & $100 \%$ & Procedural Factors: & & Tubal factor & $12 \%$ & Other factor & $8 \%$ \\
\hline GIFT & $0 \%$ & With ICSI & $49 \%$ & Ovulatory dysfunction & $3 \%$ & Unknown factor & $10 \%$ \\
\hline ZIFT & $0 \%$ & Unstimulated & $0 \%$ & Diminished ovarian reserve & $13 \%$ & Multiple Factors: & \\
\hline Combination & $0 \%$ & Used gestational carrier & $0 \%$ & Endometriosis & $2 \%$ & Female factors only & $5 \%$ \\
\hline & & & & $\begin{array}{l}\text { Uterine factor } \\
\text { Male factor }\end{array}$ & $\begin{array}{l}<1 \% \\
29 \%\end{array}$ & Female \& male factors & $18 \%$ \\
\hline
\end{tabular}

2006 PREGNANCY SUCCESS RATES

Data verified by Richard V. Grazi, MD

\section{Type of Cycle}

\section{Fresh Embryos from Nondonor Eggs}

Number of cycles

Percentage of cycles resulting in pregnancies ${ }^{b}$

Percentage of cycles resulting in live births $s^{b, c}$

(Confidence Interval)

Percentage of retrievals resulting in live births $s^{b, c}$

Percentage of transfers resulting in live births $s^{b, c}$

Percentage of transfers resulting in singleton live births ${ }^{b}$

Percentage of cancellations ${ }^{b}$

Average number of embryos transferred

Percentage of pregnancies with twins ${ }^{b}$

Percentage of pregnancies with triplets or more ${ }^{b}$

Percentage of live births having multiple infants ${ }^{b, c}$

\section{Frozen Embryos from Nondonor Eggs}

Number of transfers

Percentage of transfers resulting in live births ${ }^{\mathrm{b}, \mathrm{c}}$

Average number of embryos transferred

\section{Donor Eggs}

Number of transfers

Percentage of transfers resulting in live births ${ }^{\mathrm{b}, \mathrm{c}}$

Average number of embryos transferred

\section{Age of Woman}

35-37 38-40

$41-42^{d}$

\section{$<35$}

91

76

22

201

38.8

26.4

21.1

4.5

34.3

23.1

17.1

4.5

(27.8-41.3)

(14.9-33.1)

(9.4-27.5)

(0.1-22.8)

39.4

28.4

22.0

$1 / 17$

41.8

30.0

24.1

$1 / 17$

31.5

20.0

20.4

$1 / 17$

12.9

18.7

22.4

22.7

2.4

2.7

24.4

1.3

33.3

24.6

4.2

33.3

3.3

3.6

$5 / 16$

$0 / 1$

$2 / 16$

$0 / 1$

$2 / 13$

$0 / 1$

37

29.7

12

$1 / 12$

8

$2 / 8$

1

2.3

2.7

2.3

$0 / 1$

2.0

All Ages Combined ${ }^{e}$

Fresh Embryos

27

48.1

2.2
Frozen Embryos 10

$4 / 10$

2.4

\section{CURRENT CLINIC SERVICES AND PROFILE}

Current Name: Genesis Fertility \& Reproductive Medicine

Donor egg? Yes

Donor embryo? No

Single women? Yes

\section{Gestational carriers? No}

Cryopreservation?
SART member?

Yes

Verified lab accreditation?

(See Appendix C for details.)

${ }^{a}$ Reflects patient and treatment characteristics of ART cycles performed in 2006 using fresh nondonor eggs or embryos.

${ }^{b}$ When fewer than 20 cycles are reported in an age category, rates are shown as a fraction and confidence intervals are not given. Calculating percentages from fractions may be misleading and is not encouraged.

A multiple-infant birth is counted as one live birth.

d Clinic-specific outcome rates are unreliable for women older than 42 undergoing ART cycles using fresh or frozen embryos with nondonor eggs. Readers are urged to review national outcomes for these age groups (see page 27).

e All ages (including ages $>42$ ) are reported together because previous data show that patient age does not materially affect success with donor eggs. 


\section{INFERTILITY \& IVF MEDICAL ASSOCIATES OF WESTERN NEW YORK BUFFALO, NEW YORK}

A comparison of clinic success rates may not be meaningful because patient medical characteristics and treatment approaches vary from clinic to clinic. For more details about this, along with information on how to interpret the statistics in this table, see pages 79-88.

\section{ART CYCLE PROFILE}

\begin{tabular}{|c|c|c|c|c|c|c|c|}
\hline \multicolumn{4}{|c|}{ Type of ART ${ }^{a}$} & \multicolumn{4}{|c|}{ Patient Diagnosis } \\
\hline IVF & $100 \%$ & Procedural Factors: & & Tubal factor & $20 \%$ & Other factor & $<1 \%$ \\
\hline GIFT & $0 \%$ & With ICSI & $54 \%$ & Ovulatory dysfunction & $4 \%$ & Unknown factor & $19 \%$ \\
\hline ZIFT & $0 \%$ & Unstimulated & $0 \%$ & Diminished ovarian reserve & $7 \%$ & Multiple Factors: & \\
\hline \multirow[t]{3}{*}{ Combination } & $0 \%$ & Used gestational carrier & $0 \%$ & Endometriosis & $9 \%$ & Female factors only & $9 \%$ \\
\hline & & & & Uterine factor & $0 \%$ & Female \& male factors & $11 \%$ \\
\hline & & & & Male factor & $21 \%$ & & \\
\hline
\end{tabular}

2006 PREGNANCY SUCCESS RATES

Data verified by Michael W. Sullivan, MD

\section{Type of Cycle}

\section{Fresh Embryos from Nondonor Eggs}

Number of cycles

Percentage of cycles resulting in pregnancies ${ }^{b}$

Percentage of cycles resulting in live births $s^{b, c}$

(Confidence Interval)

Percentage of retrievals resulting in live births $s^{b, c}$

Percentage of transfers resulting in live births $s^{b, c}$

Percentage of transfers resulting in singleton live births ${ }^{b}$

Percentage of cancellations ${ }^{b}$

Average number of embryos transferred

Percentage of pregnancies with twins ${ }^{b}$

Percentage of pregnancies with triplets or more ${ }^{b}$

Percentage of live births having multiple infants ${ }^{b, c}$

\section{Frozen Embryos from Nondonor Eggs}

Number of transfers

Percentage of transfers resulting in live births ${ }^{b, c}$

Average number of embryos transferred

\section{Donor Eggs}

Number of transfers

Percentage of transfers resulting in live births $s^{b, c}$

Average number of embryos transferred

\section{Age of Woman}

35-37 38-40

$41-42^{d}$

$<35$

93

22.6

18.3

40

20

31.8

28.7

(21.7-36.4)

38.5

$(11.0-27.6)$

15.0

25.0

39.5

26.2

28.3

12.5

10.0

28.1

25.5

20.0

(4.2-26.8)

(1.2-31.7)

16.7

17.2

$2 / 12$

$2 / 12$

$2 / 12$

30.1

17.2

40.0

2.3

2.5

19.0

4.8

$5 / 17$

25.0

3.2

26.0

0.0

28.9

2.9

$0 / 5$

$0 / 5$

$0 / 6$

$0 / 2$

$0 / 5$

15

$9 / 15$

2.2

5

$1 / 5$

1.9

1.8

All Ages Combined ${ }^{\mathrm{e}}$

Fresh Embryos

Frozen Embryos

\section{8}

$4 / 8$

2.3
$1 / 4$

\section{4}

2.0

\section{CURRENT CLINIC SERVICES AND PROFILE}

Current Name: Infertility \& IVF Medical Associates of Western New York

Donor egg? Yes Gestational carriers? No

Donor embryo? No Cryopreservation? Yes

Single women? Yes

SART member? Yes

Verified lab accreditation?

(See Appendix C for details.)

${ }^{a}$ Reflects patient and treatment characteristics of ART cycles performed in 2006 using fresh nondonor eggs or embryos.

b When fewer than 20 cycles are reported in an age category, rates are shown as a fraction and confidence intervals are not given. Calculating percentages from fractions may be misleading and is not encouraged.

${ }^{c}$ A multiple-infant birth is counted as one live birth.

d Clinic-specific outcome rates are unreliable for women older than 42 undergoing ART cycles using fresh or frozen embryos with nondonor eggs. Readers are urged to review national outcomes for these age groups (see page 27).

e All ages (including ages $>42$ ) are reported together because previous data show that patient age does not materially affect success with donor eggs. 


\section{DIVISION OF REPRODUCTIVE ENDOCRINOLOGY \\ SUNY STONY BROOK \\ EAST SETAUKET, NEW YORK}

A comparison of clinic success rates may not be meaningful because patient medical characteristics and treatment approaches vary from clinic to clinic. For more details about this, along with information on how to interpret the statistics in this table, see pages $79-88$.

\section{ART CYCLE PROFILE}

\begin{tabular}{|c|c|c|c|c|c|c|c|}
\hline & Type & of $A R T^{a}$ & & Pat & ent D & iagnosis & \\
\hline IVF & $100 \%$ & Procedural Factors: & & Tubal factor & $5 \%$ & Other factor & $0 \%$ \\
\hline GIFT & $0 \%$ & With ICSI & $46 \%$ & Ovulatory dysfunction & $15 \%$ & Unknown factor & $3 \%$ \\
\hline ZIFT & $0 \%$ & Unstimulated & $0 \%$ & Diminished ovarian reserve & $0 \%$ & Multiple Factors: & \\
\hline Combination & $0 \%$ & Used gestational carrier & $0 \%$ & Endometriosis & $2 \%$ & Female factors only & $23 \%$ \\
\hline & & & & Uterine factor & $2 \%$ & Female \& male factors & $33 \%$ \\
\hline
\end{tabular}

2006 PREGNANCY SUCCESS RATES

Data verified by Richard A. Bronson, MD

\section{Type of Cycle}

\section{Fresh Embryos from Nondonor Eggs}

Number of cycles

Percentage of cycles resulting in pregnancies ${ }^{b}$

Percentage of cycles resulting in live births ${ }^{b, c}$

(Confidence Interval)

Percentage of retrievals resulting in live births $s^{b, c}$

Percentage of transfers resulting in live births $s^{b, c}$

Percentage of transfers resulting in singleton live births ${ }^{b}$

Percentage of cancellations ${ }^{b}$

Average number of embryos transferred

Percentage of pregnancies with twins ${ }^{b}$

Percentage of pregnancies with triplets or more ${ }^{b}$

Percentage of live births having multiple infants ${ }^{b, c}$

\section{Frozen Embryos from Nondonor Eggs}

Number of transfers

Percentage of transfers resulting in live births $s^{b, c}$

Average number of embryos transferred

\section{Donor Eggs}

Number of transfers

Percentage of transfers resulting in live births ${ }^{\mathrm{b}, \mathrm{c}}$

Average number of embryos transferred

\section{Age of Woman}

$\begin{array}{cccc}<\mathbf{3 5} & \mathbf{3 5 - 3 7} & \mathbf{3 8 - 4 0} & \mathbf{4 1 - 4 2}^{\text {d }} \\ & & & \\ 10 & 13 & 15 & 0 \\ 2 / 10 & 5 / 13 & 3 / 15 & \\ 2 / 10 & 5 / 13 & 2 / 15 & \end{array}$

$\begin{array}{ccc}2 / 9 & 5 / 10 & 2 / 12 \\ 2 / 7 & 5 / 10 & 2 / 11 \\ 1 / 7 & 4 / 10 & 1 / 11 \\ 1 / 10 & 3 / 13 & 3 / 15 \\ 2.1 & 2.4 & 2.9 \\ 1 / 2 & 2 / 5 & 0 / 3 \\ 0 / 2 & 0 / 5 & 1 / 3 \\ 1 / 2 & 1 / 5 & 1 / 2\end{array}$

$\begin{array}{cccc}10 & 4 & 4 & 1 \\ 2 / 10 & 1 / 4 & 1 / 4 & 0 / 1 \\ 2.1 & 2.0 & 3.0 & 3.0\end{array}$

All Ages Combined ${ }^{\mathrm{e}}$

Fresh Embryos

0
Frozen Embryos

0

\section{CURRENT CLINIC SERVICES AND PROFILE}

Current Name: Division of Reproductive Endocrinology, SUNY Stony Brook

Donor egg? No

Donor embryo? No

Single women? No

\section{Gestational carriers? \\ No}

Cryopreservation?
Yes
SART member?

Verified lab accreditation?

(See Appendix C for details.)

${ }^{a}$ Reflects patient and treatment characteristics of ART cycles performed in 2006 using fresh nondonor eggs or embryos.

${ }^{b}$ When fewer than 20 cycles are reported in an age category, rates are shown as a fraction and confidence intervals are not given. Calculating percentages from fractions may be misleading and is not encouraged.

c A multiple-infant birth is counted as one live birth.

d Clinic-specific outcome rates are unreliable for women older than 42 undergoing ART cycles using fresh or frozen embryos with nondonor eggs. Readers are urged to review national outcomes for these age groups (see page 27).

${ }^{e}$ All ages (including ages $>42$ ) are reported together because previous data show that patient age does not materially affect success with donor eggs. 


\section{THE NEW YORK FERTILITY CENTER \\ FLUSHING, NEW YORK}

A comparison of clinic success rates may not be meaningful because patient medical characteristics and treatment approaches vary from clinic to clinic. For more details about this, along with information on how to interpret the statistics in this table, see pages 79-88.

\section{ART CYCLE PROFILE}

\begin{tabular}{|c|c|c|c|c|c|c|c|}
\hline \multicolumn{4}{|c|}{ Type of ART ${ }^{a}$} & \multicolumn{4}{|c|}{ Patient Diagnosis } \\
\hline IVF & $100 \%$ & Procedural Factors: & & Tubal factor & $16 \%$ & Other factor & $2 \%$ \\
\hline GIFT & $0 \%$ & With ICSI & $94 \%$ & Ovulatory dysfunction & $32 \%$ & Unknown factor & $14 \%$ \\
\hline ZIFT & $0 \%$ & Unstimulated & $0 \%$ & Diminished ovarian reserve & $0 \%$ & Multiple Factors: & \\
\hline \multirow[t]{3}{*}{ Combination } & $0 \%$ & Used gestational carrier & $0 \%$ & Endometriosis & $2 \%$ & Female factors only & $14 \%$ \\
\hline & & & & Uterine factor & $5 \%$ & Female \& male factors & $9 \%$ \\
\hline & & & & Male factor & $7 \%$ & & \\
\hline
\end{tabular}

2006 PREGNANCY SUCCESS RATES

Data verified by Tony Tsai, MD

\section{Type of Cycle}

\section{Fresh Embryos from Nondonor Eggs}

Number of cycles

Percentage of cycles resulting in pregnancies ${ }^{b}$

Percentage of cycles resulting in live births ${ }^{b, c}$

(Confidence Interval)
Percentage of retrievals resulting in live births ${ }^{b, c}$

Percentage of transfers resulting in live births $s^{b, c}$

Percentage of transfers resulting in singleton live births ${ }^{b}$

Percentage of cancellations ${ }^{b}$

Average number of embryos transferred

Percentage of pregnancies with twins ${ }^{b}$

Percentage of pregnancies with triplets or more ${ }^{b}$

Percentage of live births having multiple infants ${ }^{b, c}$

\section{Frozen Embryos from Nondonor Eggs}

Number of transfers

Percentage of transfers resulting in live births $s^{b, c}$

Average number of embryos transferred

$\begin{array}{cccc}<35 & \mathbf{3 5 - 3 7} & \mathbf{3 8 - 4 0} & \mathbf{4 1 - 4 2}^{\text {d }} \\ 14 & 14 & 1 & 2 \\ 3 / 14 & 3 / 14 & 0 / 1 & 1 / 2 \\ 2 / 14 & 3 / 14 & 0 / 1 & 1 / 2\end{array}$

\section{Age of Woman}

$3 / 14$

$3 / 14$

$3 / 12$

$2 / 12$

$0 / 14$

2.3

$0 / 3$

$1 / 3$

$1 / 3$

$0 / 1$

$0 / 1$

$0 / 1$

$0 / 1$

3.0

$1 / 2$

$1 / 1$

$1 / 1$

$0 / 2$

2.2

$1 / 3$

$0 / 3$

$1 / 2$

\section{5}

$2 / 5$

$0 / 3$

2.3
5.0

$0 / 1$

$0 / 1$

$0 / 1$

\section{Donor Eggs}

\section{Fresh Embryos}

All Ages Combined ${ }^{\mathrm{e}}$

Number of transfers

Percentage of transfers resulting in live births $s^{\mathrm{b}, \mathrm{c}}$

0

Frozen Embryos

0

Average number of embryos transferred

\section{CURRENT CLINIC SERVICES AND PROFILE}

Current Name: The New York Fertility Center

Donor egg?

No

Donor embryo?

Single women?
Gestational carriers? No

Cryopreservation?
No
Yes
SART member?

Verified lab accreditation?

(See Appendix C for details.)
No

Yes

Yes

${ }^{a}$ Reflects patient and treatment characteristics of ART cycles performed in 2006 using fresh nondonor eggs or embryos.

b When fewer than 20 cycles are reported in an age category, rates are shown as a fraction and confidence intervals are not given. Calculating percentages from fractions may be misleading and is not encouraged.

${ }^{\mathrm{C}}$ A multiple-infant birth is counted as one live birth.

d Clinic-specific outcome rates are unreliable for women older than 42 undergoing ART cycles using fresh or frozen embryos with nondonor eggs. Readers are urged to review national outcomes for these age groups (see page 27).

e All ages (including ages $>42$ ) are reported together because previous data show that patient age does not materially affect success with donor eggs. 


\section{MONTEFIORE'S INSTITUTE FOR REPRODUCTIVE MEDICINE AND HEALTH HARTSDALE, NEW YORK}

A comparison of clinic success rates may not be meaningful because patient medical characteristics and treatment approaches vary from clinic to clinic. For more details about this, along with information on how to interpret the statistics in this table, see pages $79-88$.

\section{ART CYCLE PROFILE}

\begin{tabular}{|c|c|c|c|c|c|c|c|}
\hline \multicolumn{4}{|c|}{ Type of ART ${ }^{\mathrm{a}}$} & \multicolumn{4}{|c|}{ Patient Diagnosis } \\
\hline IVF & $100 \%$ & Procedural Factors: & & Tubal factor & $19 \%$ & Other factor & $1 \%$ \\
\hline GIFT & $0 \%$ & With ICSI & $71 \%$ & Ovulatory dysfunction & $2 \%$ & Unknown factor & $15 \%$ \\
\hline ZIFT & $0 \%$ & Unstimulated & $0 \%$ & Diminished ovarian reserve & $6 \%$ & Multiple Factors: & \\
\hline \multirow[t]{3}{*}{ Combination } & $0 \%$ & Used gestational carrier & $0 \%$ & Endometriosis & $<1 \%$ & Female factors only & $7 \%$ \\
\hline & & & & Uterine factor & $2 \%$ & Female \& male factors & $19 \%$ \\
\hline & & & & Male factor & $28 \%$ & & \\
\hline
\end{tabular}

2006 PREGNANCY SUCCESS RATES

Data verified by Harry J. Lieman, MD

\section{Type of Cycle}

\section{Fresh Embryos from Nondonor Eggs}

Number of cycles

Percentage of cycles resulting in pregnancies ${ }^{b}$

Percentage of cycles resulting in live births ${ }^{b, c}$

(Confidence Interval)

Percentage of retrievals resulting in live births $s^{b, c}$

Percentage of transfers resulting in live births $s^{b, c}$

Percentage of transfers resulting in singleton live births ${ }^{b}$

Percentage of cancellations ${ }^{b}$

Average number of embryos transferred

Percentage of pregnancies with twins ${ }^{b}$

Percentage of pregnancies with triplets or more ${ }^{b}$

Percentage of live births having multiple infants ${ }^{b, c}$

\section{Frozen Embryos from Nondonor Eggs}

Number of transfers

Percentage of transfers resulting in live births ${ }^{\mathrm{b}, \mathrm{c}}$

Average number of embryos transferred

\section{Donor Eggs}

Number of transfers

Percentage of transfers resulting in live births ${ }^{\mathrm{b}, \mathrm{c}}$

Average number of embryos transferred

\section{Age of Woman}

35-37 38-40

$41-42^{d}$

$<35$

47

36.2

36

11

$\begin{array}{cccc}70 & 47 & 36 & 11 \\ 42.9 & 36.2 & 19.4 & 3 / 11 \\ 31.4 & 31.9 & 13.9 & 1 / 11\end{array}$

(20.9-43.6)

(19.1-47.1)

(4.7-29.5)

33.8

34.1

17.9

$1 / 9$

37.3

37.5

20.0

$1 / 8$

25.0

20.0

$1 / 8$

7.1

6.4

22.2

$2 / 11$

2.4

2.5

3.2

3.4

23.3

$5 / 17$

$1 / 7$

$0 / 3$

3.3

$2 / 17$

$0 / 7$

$0 / 3$

27.3

$5 / 15$

$0 / 5$

$0 / 1$

\section{5}

$1 / 5$

1.6

8

$0 / 8$

3

$0 / 3$

1.6

2.0

All Ages Combined ${ }^{\mathrm{e}}$

Fresh Embryos

Frozen Embryos

0

0

\section{CURRENT CLINIC SERVICES AND PROFILE}

Current Name: Montefiore's Institute for Reproductive Medicine and Health

Donor egg? Yes

Donor embryo? Yes

Single women? Yes

\section{Gestational carriers? Yes}

Cryopreservation? Yes
SART member?

Verified lab accreditation?

(See Appendix C for details.)

${ }^{a}$ Reflects patient and treatment characteristics of ART cycles performed in 2006 using fresh nondonor eggs or embryos.

${ }^{b}$ When fewer than 20 cycles are reported in an age category, rates are shown as a fraction and confidence intervals are not given. Calculating percentages from fractions may be misleading and is not encouraged.

A multiple-infant birth is counted as one live birth.

d Clinic-specific outcome rates are unreliable for women older than 42 undergoing ART cycles using fresh or frozen embryos with nondonor eggs. Readers are urged to review national outcomes for these age groups (see page 27).

e All ages (including ages $>42$ ) are reported together because previous data show that patient age does not materially affect success with donor eggs. 


\section{NORTH SHORE UNIVERSITY HOSPITAL \\ CENTER FOR HUMAN REPRODUCTION MANHASSET, NEW YORK}

A comparison of clinic success rates may not be meaningful because patient medical characteristics and treatment approaches vary from clinic to clinic. For more details about this, along with information on how to interpret the statistics in this table, see pages 79-88.

\section{ART CYCLE PROFILE}

\begin{tabular}{|c|c|c|c|c|c|c|c|}
\hline \multicolumn{4}{|c|}{ Type of ART ${ }^{a}$} & \multicolumn{4}{|c|}{ Patient Diagnosis } \\
\hline IVF & $100 \%$ & Procedural Factors: & & Tubal factor & $11 \%$ & Other factor & $7 \%$ \\
\hline GIFT & $0 \%$ & With ICSI & $89 \%$ & Ovulatory dysfunction & $7 \%$ & Unknown factor & $19 \%$ \\
\hline ZIFT & $0 \%$ & Unstimulated & $0 \%$ & Diminished ovarian reserve & $7 \%$ & Multiple Factors: & \\
\hline \multirow[t]{3}{*}{ Combination } & $0 \%$ & Used gestational carrier & $<1 \%$ & Endometriosis & $5 \%$ & Female factors only & $4 \%$ \\
\hline & & & & Uterine factor & $3 \%$ & Female \& male factors & $7 \%$ \\
\hline & & & & Male factor & $30 \%$ & & \\
\hline
\end{tabular}

2006 PREGNANCY SUCCESS RATES

Data verified by Avner Hershlag, MD

\section{Type of Cycle}

\section{Fresh Embryos from Nondonor Eggs}

Number of cycles

Percentage of cycles resulting in pregnancies ${ }^{b}$

Percentage of cycles resulting in live births $s^{b, c}$

(Confidence Interval)

Percentage of retrievals resulting in live births $s^{b, c}$

Percentage of transfers resulting in live births $s^{b, c}$

Percentage of transfers resulting in singleton live births ${ }^{b}$

Percentage of cancellations ${ }^{b}$

Average number of embryos transferred

Percentage of pregnancies with twins ${ }^{b}$

Percentage of pregnancies with triplets or more ${ }^{b}$

Percentage of live births having multiple infants ${ }^{b, c}$

\section{Frozen Embryos from Nondonor Eggs}

Number of transfers

Percentage of transfers resulting in live births ${ }^{b, c}$

Average number of embryos transferred

\section{Donor Eggs}

Number of transfers

Percentage of transfers resulting in live births $s^{b, c}$

Average number of embryos transferred

\section{Age of Woman}

35-37 38-40

$41-42^{d}$

\section{$<35$}

142

35.9

83

47

47.2

29.6

44.6

25.5

39.9

(22.2-37.8)

42.5

32.8

37.3

23.4

44.3

35.6

(27.0-48.7)

(12.3-38.0)

43.1

43.7

25.6

28.2

24.6

29.6

25.6

6.2

9.9

13.3

8.5

2.3

2.8

33.3

3.5

3.2

26.4

3.9

4.4

28.6

31.0

35.1

$1 / 12$

2.7

$1 / 12$

32.3

$1 / 11$

82

29.3

51

13.7

2.7

2.7

All Ages Combined ${ }^{\mathrm{e}}$

Fresh Embryos

24

54.2

2.2
18

$4 / 18$

3.7

\section{CURRENT CLINIC SERVICES AND PROFILE}

Current Name: North Shore University Hospital, Center for Human Reproduction

Donor egg? Yes

Donor embryo? No

Single women? Yes

\section{Gestational carriers? Yes}

Cryopreservation? Yes
SART member?

Verified lab accreditation?

(See Appendix C for details.)
Frozen Embryos

\section{7}

$6 / 17$

2.4

${ }^{a}$ Reflects patient and treatment characteristics of ART cycles performed in 2006 using fresh nondonor eggs or embryos.

b When fewer than 20 cycles are reported in an age category, rates are shown as a fraction and confidence intervals are not given. Calculating percentages from fractions may be misleading and is not encouraged.

${ }^{c}$ A multiple-infant birth is counted as one live birth.

d Clinic-specific outcome rates are unreliable for women older than 42 undergoing ART cycles using fresh or frozen embryos with nondonor eggs. Readers are urged to review national outcomes for these age groups (see page 27).

e All ages (including ages $>42$ ) are reported together because previous data show that patient age does not materially affect success with donor eggs. 


\section{REPRODUCTIVE SPECIALISTS OF NEW YORK' \\ MINEOLA, NEW YORK}

A comparison of clinic success rates may not be meaningful because patient medical characteristics and treatment approaches vary from clinic to clinic. For more details about this, along with information on how to interpret the statistics in this table, see pages $79-88$.

\section{ART CYCLE PROFILE}

\begin{tabular}{|c|c|c|c|c|c|c|c|}
\hline \multicolumn{4}{|c|}{ Type of ART ${ }^{\mathrm{a}}$} & \multicolumn{4}{|c|}{ Patient Diagnosis } \\
\hline IVF & $>99 \%$ & Procedural Factors: & & Tubal factor & $12 \%$ & Other factor & $12 \%$ \\
\hline GIFT & $0 \%$ & With ICSI & $65 \%$ & Ovulatory dysfunction & $10 \%$ & Unknown factor & $10 \%$ \\
\hline ZIFT & $<1 \%$ & Unstimulated & $<1 \%$ & Diminished ovarian reserve & $10 \%$ & Multiple Factors: & \\
\hline \multirow[t]{3}{*}{ Combination } & $0 \%$ & Used gestational carrier & $<1 \%$ & Endometriosis & $3 \%$ & Female factors only & $7 \%$ \\
\hline & & & & Uterine factor & $2 \%$ & Female \& male factors & $15 \%$ \\
\hline & & & & Male factor & $19 \%$ & & \\
\hline
\end{tabular}

2006 PREGNANCY SUCCESS RATES

Data verified by Gabriel A. San Roman, MD

\section{Type of Cycle}

\section{Fresh Embryos from Nondonor Eggs}

Number of cycles

Percentage of cycles resulting in pregnancies ${ }^{b}$

Percentage of cycles resulting in live births ${ }^{b, c}$

(Confidence Interval)

Percentage of retrievals resulting in live births $s^{b, c}$

Percentage of transfers resulting in live births $s^{b, c}$

Percentage of transfers resulting in singleton live births ${ }^{b}$

Percentage of cancellations ${ }^{b}$

Average number of embryos transferred

Percentage of pregnancies with twins ${ }^{b}$

Percentage of pregnancies with triplets or more ${ }^{b}$

Percentage of live births having multiple infants ${ }^{b, c}$

\section{Frozen Embryos from Nondonor Eggs}

Number of transfers

Percentage of transfers resulting in live births $s^{b, c}$

Average number of embryos transferred

\section{Donor Eggs}

Number of transfers

Percentage of transfers resulting in live births ${ }^{\mathrm{b}, \mathrm{c}}$

Average number of embryos transferred

Age of Woman

35-37 $38-40 \quad 41-42^{d}$

$<35$

163

201

153

258

33.1

26.4

11.8

43.8

28.8

20.4

5.2

(37.7-50.1)

(22.0-36.4)

(15.1-26.6)

(2.3-10.0)

45.7

32.4

23.8

26.3

7.3

47.9

33.8

18.6

7.9

7.9

11.0

14.4

28.1

4.3

2.2

2.7

3.1

19.5

31.5

22.6

$0 / 18$

3.0

1.9

7.5

$0 / 18$

18.6

31.9

29.3

$0 / 8$

99

21.2

59

20.3

1.9

27

6

1.9

25.9

0 / 6

2.2

2.3

All Ages Combined ${ }^{\mathrm{e}}$

Fresh Embryos 26

46.2

1.8
Frozen Embryos 15

$2 / 15$

1.5

\section{CURRENT CLINIC SERVICES AND PROFILE}

Current Name: Reproductive Specialists of New York

Donor egg? Yes

Donor embryo? Yes

Gestational carriers?

Yes

Cryopreservation?

Yes

Single women? Yes

SART member?

Verified lab accreditation?

(See Appendix C for details.)

${ }_{b}^{a}$ Reflects patient and treatment characteristics of ART cycles performed in 2006 using fresh nondonor eggs or embryos.

When fewer than 20 cycles are reported in an age category, rates are shown as a fraction and confidence intervals are not given. Calculating

c percentages from fractions may be misleading and is not encouraged.

d A multiple-infant birth is counted as one live birth.

Clinic-specific outcome rates are unreliable for women older than 42 undergoing ART cycles using fresh or frozen embryos with nondonor

e eggs. Readers are urged to review national outcomes for these age groups (see page 27).

All ages (including ages $>42$ ) are reported together because previous data show that patient age does not materially affect

success with donor eggs.

- Reflects clinic performed more than 50 cycles with Preimplantation Genetic Diagnosis (PGD) in 2006 and among them more than 10 specifically for the purpose of prevention of genetic disorders. See Appendix $C$ for a complete list of clinics with symbol. 


\section{ADVANCED FERTILITY SERVICES NEW YORK, NEW YORK}

A comparison of clinic success rates may not be meaningful because patient medical characteristics and treatment approaches vary from clinic to clinic. For more details about this, along with information on how to interpret the statistics in this table, see pages 79-88.

\section{ART CYCLE PROFILE}

\begin{tabular}{|c|c|c|c|c|c|c|c|}
\hline \multicolumn{4}{|c|}{ Type of ART ${ }^{a}$} & \multicolumn{4}{|c|}{ Patient Diagnosis } \\
\hline IVF & $100 \%$ & Procedural Factors: & & Tubal factor & $16 \%$ & Other factor & $12 \%$ \\
\hline GIFT & $0 \%$ & With ICSI & $90 \%$ & Ovulatory dysfunction & $13 \%$ & Unknown factor & $12 \%$ \\
\hline ZIFT & $0 \%$ & Unstimulated & $0 \%$ & Diminished ovarian reserve & $12 \%$ & Multiple Factors: & \\
\hline \multirow[t]{3}{*}{ Combination } & $0 \%$ & Used gestational carrier & $0 \%$ & Endometriosis & $<1 \%$ & Female factors only & $2 \%$ \\
\hline & & & & Uterine factor & $0 \%$ & Female \& male factors & $11 \%$ \\
\hline & & & & Male factor & $22 \%$ & & \\
\hline
\end{tabular}

2006 PREGNANCY SUCCESS RATES

Data verified by Hugh D. Melnick, MD

\section{Type of Cycle}

\section{Fresh Embryos from Nondonor Eggs}

Number of cycles

Percentage of cycles resulting in pregnancies ${ }^{b}$

Percentage of cycles resulting in live births $s^{b, c}$

(Confidence Interval)

Percentage of retrievals resulting in live births $s^{b, c}$

Percentage of transfers resulting in live births $s^{b, c}$

Percentage of transfers resulting in singleton live births ${ }^{b}$

Percentage of cancellations ${ }^{b}$

Average number of embryos transferred

Percentage of pregnancies with twins ${ }^{b}$

Percentage of pregnancies with triplets or more ${ }^{b}$

Percentage of live births having multiple infants ${ }^{b, c}$

\section{Frozen Embryos from Nondonor Eggs}

Number of transfers

Percentage of transfers resulting in live births ${ }^{b, c}$

Average number of embryos transferred

\section{Donor Eggs}

Number of transfers

Percentage of transfers resulting in live births $s^{b, c}$

Average number of embryos transferred

\section{Age of Woman}

35-37 38-40

$41-42^{d}$

$<35$

72

13.9

12.5

76

28

30.2

24.5

(5.9-22.4)

10.5

7.1

(17.6-32.5)

26.4

26.8

13.2

(4.7-19.7)

7.1

17.3

14.1

11.4

(0.9-23.5)

10.9

13.8

8.7

7.2

5.6

13.8

9.5

9.5

3.3

23.8

11.9

35.3

3.5

$1 / 10$

$1 / 10$

$2 / 9$

7.9

17.9

2.8

2.8

$1 / 10$

$0 / 2$

$0 / 10$

$0 / 2$

$0 / 8$

$0 / 2$

21

4.8

10

$1 / 10$

1

2.7

$0 / 1$

3.0

All Ages Combined ${ }^{\mathrm{e}}$

Fresh Embryos

22

31.8

2.8
Frozen Embryos

25

8.0

2.6

\section{CURRENT CLINIC SERVICES AND PROFILE}

Current Name: Advanced Fertility Services

Donor egg? Yes

Donor embryo? No

Single women? Yes

\section{Gestational carriers? Yes}

Cryopreservation? Yes
SART member?

Verified lab accreditation?

(See Appendix C for details.)

${ }^{a}$ Reflects patient and treatment characteristics of ART cycles performed in 2006 using fresh nondonor eggs or embryos.

${ }^{b}$ When fewer than 20 cycles are reported in an age category, rates are shown as a fraction and confidence intervals are not given. Calculating percentages from fractions may be misleading and is not encouraged.

${ }^{c}$ A multiple-infant birth is counted as one live birth.

d Clinic-specific outcome rates are unreliable for women older than 42 undergoing ART cycles using fresh or frozen embryos with nondonor eggs. Readers are urged to review national outcomes for these age groups (see page 27).

e All ages (including ages $>42$ ) are reported together because previous data show that patient age does not materially affect success with donor eggs. 


\section{AMERICAN FERTILITY SERVICES, PC NEW YORK, NEW YORK}

A comparison of clinic success rates may not be meaningful because patient medical characteristics and treatment approaches vary from clinic to clinic. For more details about this, along with information on how to interpret the statistics in this table, see pages $79-88$.

\section{ART CYCLE PROFILE}

\begin{tabular}{|c|c|c|c|c|c|c|c|}
\hline \multicolumn{4}{|c|}{ Type of ART } & \multicolumn{4}{|c|}{ Patient Diagnosis } \\
\hline IVF & $100 \%$ & Procedural Factors: & & Tubal factor & $8 \%$ & Other factor & $12 \%$ \\
\hline GIFT & $0 \%$ & With ICSI & $85 \%$ & Ovulatory dysfunction & $2 \%$ & Unknown factor & $13 \%$ \\
\hline ZIFT & $0 \%$ & Unstimulated & $0 \%$ & Diminished ovarian reserve & $42 \%$ & Multiple Factors: & \\
\hline \multirow[t]{3}{*}{ Combination } & $0 \%$ & Used gestational carrier & $0 \%$ & Endometriosis & $1 \%$ & Female factors only & $8 \%$ \\
\hline & & & & Uterine factor & $1 \%$ & Female \& male factors & $7 \%$ \\
\hline & & & & Male factor & $5 \%$ & & \\
\hline
\end{tabular}

2006 PREGNANCY SUCCESS RATES

Data verified by Andrew Loucopoulos, MD

\section{Type of Cycle}

\section{Fresh Embryos from Nondonor Eggs}

Number of cycles

Percentage of cycles resulting in pregnancies ${ }^{b}$

Percentage of cycles resulting in live births $s^{b, c}$

(Confidence Interval)

Percentage of retrievals resulting in live births $s^{b, c}$

Percentage of transfers resulting in live births $s^{b, c}$

Percentage of transfers resulting in singleton live births ${ }^{b}$

Percentage of cancellations ${ }^{b}$

Average number of embryos transferred

Percentage of pregnancies with twins ${ }^{b}$

Percentage of pregnancies with triplets or more ${ }^{b}$

Percentage of live births having multiple infants ${ }^{b, c}$

\section{Frozen Embryos from Nondonor Eggs}

Number of transfers

Percentage of transfers resulting in live births $s^{b, c}$

Average number of embryos transferred

\section{Donor Eggs}

Number of transfers

Percentage of transfers resulting in live births ${ }^{\mathrm{b}, \mathrm{c}}$

Average number of embryos transferred

\section{Age of Woman}

35-37 38-40

$41-42^{d}$

$<35$

80

22.5

15.0

106

11.3

7.5

62

14.3

(8.0-24.7)

(3.3-14.3)

4.8

(7.4-19.8)

15.6

8.7

10.5

10.5

13.2

2.5

$0 / 12$

$0 / 12$

$0 / 8$

(1.0-13.5)

15.2

3.8

$2 / 18$

$0 / 18$

$2 / 12$

$1 / 16$

20

10.0

10

$1 / 10$

7

$0 / 7$

2.6

2.2

All Ages Combined ${ }^{\mathrm{e}}$

Fresh Embryos

31.1

2.7
Frozen Embryos

25

4.0

2.8

\section{CURRENT CLINIC SERVICES AND PROFILE}

Current Name: American Fertility Services, PC

Donor egg? Yes

Donor embryo? Yes

Gestational carriers? No

Cryopreservation?

SART member?

Yes

Single women? Yes

Verified lab accreditation?

Yes

(See Appendix C for details.)

${ }^{a}$ Reflects patient and treatment characteristics of ART cycles performed in 2006 using fresh nondonor eggs or embryos.

${ }^{b}$ When fewer than 20 cycles are reported in an age category, rates are shown as a fraction and confidence intervals are not given. Calculating percentages from fractions may be misleading and is not encouraged.

A multiple-infant birth is counted as one live birth.

d Clinic-specific outcome rates are unreliable for women older than 42 undergoing ART cycles using fresh or frozen embryos with nondonor eggs. Readers are urged to review national outcomes for these age groups (see page 27).

e All ages (including ages $>42$ ) are reported together because previous data show that patient age does not materially affect success with donor eggs. 


\section{BETH ISRAEL CENTER FOR INFERTILITY \& REPRODUCTIVE HEALTH NEW YORK, NEW YORK}

A comparison of clinic success rates may not be meaningful because patient medical characteristics and treatment approaches vary from clinic to clinic. For more details about this, along with information on how to interpret the statistics in this table, see pages 79-88.

\section{ART CYCLE PROFILE}

\begin{tabular}{|c|c|c|c|c|c|c|c|}
\hline \multicolumn{4}{|c|}{ Type of ART ${ }^{a}$} & \multicolumn{4}{|c|}{ Patient Diagnosis } \\
\hline IVF & $100 \%$ & Procedural Factors: & & Tubal factor & $10 \%$ & Other factor & $0 \%$ \\
\hline GIFT & $0 \%$ & With ICSI & $79 \%$ & Ovulatory dysfunction & $2 \%$ & Unknown factor & $12 \%$ \\
\hline ZIFT & $0 \%$ & Unstimulated & $0 \%$ & Diminished ovarian reserve & $9 \%$ & Multiple Factors: & \\
\hline \multirow[t]{3}{*}{ Combination } & $0 \%$ & Used gestational carrier & $0 \%$ & Endometriosis & $3 \%$ & Female factors only & $6 \%$ \\
\hline & & & & Uterine factor & $1 \%$ & Female \& male factors & $30 \%$ \\
\hline & & & & Male factor & $28 \%$ & & \\
\hline
\end{tabular}

2006 PREGNANCY SUCCESS RATES

Data verified by Peter Chang, MD

\section{Type of Cycle}

(n)

Fresh Embryos from Nondonor Eggs
Number of cycles

Percentage of cycles resulting in pregnancies ${ }^{b}$

Percentage of cycles resulting in live births ${ }^{b, c}$

(Confidence Interval)

Percentage of retrievals resulting in live births $\mathrm{b}^{\mathrm{b}, \mathrm{c}}$

Percentage of transfers resulting in live births $s^{b, c}$

Percentage of transfers resulting in singleton live births ${ }^{b}$

Percentage of cancellations ${ }^{b}$

Average number of embryos transferred

Percentage of pregnancies with twins ${ }^{b}$

Percentage of pregnancies with triplets or more ${ }^{b}$

Percentage of live births having multiple infants ${ }^{b, c}$

\section{Frozen Embryos from Nondonor Eggs}

Number of transfers

Percentage of transfers resulting in live births $s^{b, c}$

Average number of embryos transferred

\section{Donor Eggs}

Number of transfers

Percentage of transfers resulting in live births $s^{b, c}$

Average number of embryos transferred

\section{Age of Woman}

35-37 $38-40 \quad 41-42^{d}$

$<35$

27

29.6

47

22

41.9

18.5

23.4

9.1

27.9

(6.3-38.1)

12.8

0.0

$(15.3-43.7)$

28.6

23.8

(4.8-25.7)

(0.0-15.4)

30.8

23.8

15.4

$0 / 17$

15.4

19.0

15.8

$0 / 17$

2.3

22.2

10.5

$0 / 17$

3.4

3.2

17.0

22.7

$5 / 18$

$1 / 8$

4.4

3.6

$2 / 18$

$0 / 8$

$2 / 11$

$0 / 2$

$6 / 12$

$1 / 5$

$0 / 11$

$0 / 2$

$2 / 6$

$\begin{array}{cc}4 & 3 \\ 1 / 4 & 1 / 3 \\ 4.8 & 4.7\end{array}$

Fresh Embryos

7

$6 / 7$

0

0

3.1

2

3.5

\section{CURRENT CLINIC SERVICES AND PROFILE}

Current Name: Beth Israel Center for Infertility \& Reproductive Health

\begin{tabular}{|c|c|c|c|c|}
\hline Donor egg? & Yes & Gestational carriers? & No & SART member? \\
\hline Donor embryo? & No & Cryopreservation? & Yes & Verified lab accreditation? \\
\hline
\end{tabular}

\footnotetext{
${ }^{a}$ Reflects patient and treatment characteristics of ART cycles performed in 2006 using fresh nondonor eggs or embryos.

b When fewer than 20 cycles are reported in an age category, rates are shown as a fraction and confidence intervals are not given. Calculating percentages from fractions may be misleading and is not encouraged.

c A multiple-infant birth is counted as one live birth.

${ }^{d}$ Clinic-specific outcome rates are unreliable for women older than 42 undergoing ART cycles using fresh or frozen embryos with nondonor eggs. Readers are urged to review national outcomes for these age groups (see page 27).

e All ages (including ages $>42$ ) are reported together because previous data show that patient age does not materially affect success with donor eggs.
} 


\section{BROOKLYN/WESTSIDE FERTILITY CENTER \\ BROOKLYN FERTILITY CENTER \\ NEW YORK, NEW YORK}

A comparison of clinic success rates may not be meaningful because patient medical characteristics and treatment approaches vary from clinic to clinic. For more details about this, along with information on how to interpret the statistics in this table, see pages $79-88$.

\section{ART CYCLE PROFILE}

\begin{tabular}{|c|c|c|c|c|c|c|c|}
\hline \multicolumn{4}{|c|}{ Type of ART ${ }^{\mathrm{a}}$} & \multicolumn{4}{|c|}{ Patient Diagnosis } \\
\hline IVF & $100 \%$ & Procedural Factors: & & Tubal factor & $0 \%$ & Other factor & $0 \%$ \\
\hline GIFT & $0 \%$ & With ICSI & $100 \%$ & Ovulatory dysfunction & $0 \%$ & Unknown factor & $0 \%$ \\
\hline ZIFT & $0 \%$ & Unstimulated & $0 \%$ & Diminished ovarian reserve & $0 \%$ & Multiple Factors: & \\
\hline \multirow[t]{3}{*}{ Combination } & $0 \%$ & Used gestational carrier & $0 \%$ & Endometriosis & $0 \%$ & Female factors only & $9 \%$ \\
\hline & & & & Uterine factor & $0 \%$ & Female \& male factors & $91 \%$ \\
\hline & & & & Male factor & $0 \%$ & & \\
\hline
\end{tabular}

2006 PREGNANCY SUCCESS RATES

Data verified by Dov B. Goldstein, MD

\section{Type of Cycle}

\section{Fresh Embryos from Nondonor Eggs}

Number of cycles

Percentage of cycles resulting in pregnancies ${ }^{b}$

Percentage of cycles resulting in live births ${ }^{b, c}$

(Confidence Interval)

Percentage of retrievals resulting in live births $s^{b, c}$

Percentage of transfers resulting in live births $s^{b, c}$

Percentage of transfers resulting in singleton live births ${ }^{b}$

Percentage of cancellations ${ }^{b}$

Average number of embryos transferred

Percentage of pregnancies with twins ${ }^{b}$

Percentage of pregnancies with triplets or more ${ }^{b}$

Percentage of live births having multiple infants ${ }^{b, c}$

\section{Frozen Embryos from Nondonor Eggs}

Number of transfers

Percentage of transfers resulting in live births ${ }^{\mathrm{b}, \mathrm{c}}$

Average number of embryos transferred

\section{Donor Eggs}

Number of transfers

Percentage of transfers resulting in live births $s^{b, c}$

Average number of embryos transferred

\section{Age of Woman}

$\begin{array}{cccc}<35 & 35-37 & 38-40 & \mathbf{4 1 - 4 2}^{\text {d }} \\ 13 & 8 & 10 & 5 \\ 7 / 13 & 2 / 8 & 0 / 10 & 0 / 5 \\ 6 / 13 & 1 / 8 & 0 / 10 & 0 / 5\end{array}$

$\begin{array}{cccc}6 / 13 & 1 / 8 & 0 / 10 & 0 / 5 \\ 6 / 11 & 1 / 8 & 0 / 8 & 0 / 5 \\ 4 / 11 & 1 / 8 & 0 / 8 & 0 / 5 \\ 0 / 13 & 0 / 8 & 0 / 10 & 0 / 5 \\ 2.2 & 2.5 & 3.0 & 1.8 \\ 2 / 7 & 1 / 2 & & \\ 0 / 7 & 0 / 2 & \end{array}$

6

$0 / 6$

3

1

0

2.7

3

$0 / 1$

3.0

All Ages Combined ${ }^{\mathrm{e}}$

Fresh Embryos 5

$0 / 5$

2.4
Frozen Embryos

1

$0 / 1$

3.0

\section{CURRENT CLINIC SERVICES AND PROFILE}

Current Name: Brooklyn/Westside Fertility Center, Brooklyn Fertility Center

Donor egg? Yes

Donor embryo? No

Single women? Yes

\section{Gestational carriers? Yes}

Cryopreservation? Yes
SART member?

Verified lab accreditation?

(See Appendix C for details.)

${ }^{a}$ Reflects patient and treatment characteristics of ART cycles performed in 2006 using fresh nondonor eggs or embryos.

${ }^{b}$ When fewer than 20 cycles are reported in an age category, rates are shown as a fraction and confidence intervals are not given. Calculating percentages from fractions may be misleading and is not encouraged.

A multiple-infant birth is counted as one live birth.

d Clinic-specific outcome rates are unreliable for women older than 42 undergoing ART cycles using fresh or frozen embryos with nondonor eggs. Readers are urged to review national outcomes for these age groups (see page 27).

e All ages (including ages $>42$ ) are reported together because previous data show that patient age does not materially affect success with donor eggs. 


\section{COLUMBIA UNIVERSITY CENTER FOR WOMEN'S REPRODUCTIVE CARE NEW YORK, NEW YORK}

A comparison of clinic success rates may not be meaningful because patient medical characteristics and treatment approaches vary from clinic to clinic. For more details about this, along with information on how to interpret the statistics in this table, see pages 79-88.

\section{ART CYCLE PROFILE}

\begin{tabular}{|c|c|c|c|c|c|c|c|}
\hline \multicolumn{4}{|c|}{ Type of ART ${ }^{a}$} & \multicolumn{4}{|c|}{ Patient Diagnosis } \\
\hline IVF & $>99 \%$ & Procedural Factors: & & Tubal factor & $4 \%$ & Other factor & $2 \%$ \\
\hline GIFT & $0 \%$ & With ICSI & $54 \%$ & Ovulatory dysfunction & $2 \%$ & Unknown factor & $5 \%$ \\
\hline ZIFT & $<1 \%$ & Unstimulated & $0 \%$ & Diminished ovarian reserve & $47 \%$ & Multiple Factors: & \\
\hline \multirow[t]{3}{*}{ Combination } & $0 \%$ & Used gestational carrier & $0 \%$ & Endometriosis & $<1 \%$ & Female factors only & $3 \%$ \\
\hline & & & & Uterine factor & $2 \%$ & Female \& male factors & $15 \%$ \\
\hline & & & & Male factor & $19 \%$ & & \\
\hline
\end{tabular}

\section{PREGNANCY SUCCESS RATES}

\section{Type of Cycle}

\section{Fresh Embryos from Nondonor Eggs}

Number of cycles

Percentage of cycles resulting in pregnancies ${ }^{b}$

Percentage of cycles resulting in live births ${ }^{b, c}$

(Confidence Interval)

Percentage of retrievals resulting in live births $s^{b, c}$

Percentage of transfers resulting in live births $s^{b, c}$

Percentage of transfers resulting in singleton live births ${ }^{b}$

Percentage of cancellations ${ }^{b}$

Average number of embryos transferred

Percentage of pregnancies with twins ${ }^{b}$

Percentage of pregnancies with triplets or more ${ }^{b}$

Percentage of live births having multiple infants ${ }^{b, c}$

\section{Frozen Embryos from Nondonor Eggs}

Number of transfers

Percentage of transfers resulting in live births $s^{\mathrm{b}, \mathrm{c}}$

Average number of embryos transferred

\section{Donor Eggs}

Number of transfers

Percentage of transfers resulting in live births $s^{\mathrm{b}, \mathrm{c}}$

Average number of embryos transferred

Data verified by Michael M. Guarnaccia, MD

\section{Age of Woman}

35-37 38-40 $\quad 41-42^{d}$

$<35$

259

284

195

43.5

36.7

23.6

13.8

37.8

32.4

16.5

7.7

(32.5-43.2)

(26.8-38.5)

(12.4-21.4)

(4.4-12.4)

38.2

21.2

23.9

11.4

49.6

27.8

17.8

14.0

32.9

15.1

21.8

12.1

9.7
2.4

2.8

3.2

32.3

31.3

31.6

26.9

3.8

4.9

8.4

3.0

3.7

33.6

35.7

25.5

3.7

$2 / 15$

79

39.2

2.6
47

36.2

2.7
33

27.3

2.6

\section{6}

$1 / 6$

2.8

All Ages Combined ${ }^{\mathrm{e}}$

Fresh Embryos

89

62.9

2.3
Frozen Embryos 54

37.0

2.8

\section{CURRENT CLINIC SERVICES AND PROFILE}

Current Name: Columbia University Center for Women's Reproductive Care

Donor egg? Yes

Donor embryo? Yes

Single women? Yes

\begin{abstract}
Gestational carriers? Yes
Cryopreservation? Yes
\end{abstract}

SART member?

Verified lab accreditation?

(See Appendix C for details.)

${ }^{a}$ Reflects patient and treatment characteristics of ART cycles performed in 2006 using fresh nondonor eggs or embryos.

${ }^{b}$ When fewer than 20 cycles are reported in an age category, rates are shown as a fraction and confidence intervals are not given. Calculating percentages from fractions may be misleading and is not encouraged.

${ }^{c}$ A multiple-infant birth is counted as one live birth.

d Clinic-specific outcome rates are unreliable for women older than 42 undergoing ART cycles using fresh or frozen embryos with nondonor eggs. Readers are urged to review national outcomes for these age groups (see page 27).

e All ages (including ages $>42$ ) are reported together because previous data show that patient age does not materially affect success with donor eggs. 


\section{IVF NEW YORK \\ NEW YORK, NEW YORK}

A comparison of clinic success rates may not be meaningful because patient medical characteristics and treatment approaches vary from clinic to clinic. For more details about this, along with information on how to interpret the statistics in this table, see pages 79-88.

\section{ART CYCLE PROFILE}

\begin{tabular}{|c|c|c|c|c|c|c|c|}
\hline \multicolumn{4}{|c|}{ Type of $A R T^{a}$} & \multicolumn{4}{|c|}{ Patient Diagnosis } \\
\hline IVF & $100 \%$ & Procedural Factors: & & Tubal factor & $44 \%$ & Other factor & $0 \%$ \\
\hline GIFT & $0 \%$ & With ICSI & $73 \%$ & Ovulatory dysfunction & $17 \%$ & Unknown factor & $11 \%$ \\
\hline ZIFT & $0 \%$ & Unstimulated & $0 \%$ & Diminished ovarian reserve & $6 \%$ & Multiple Factors: & \\
\hline \multirow[t]{3}{*}{ Combination } & $0 \%$ & Used gestational carrier & $0 \%$ & Endometriosis & $17 \%$ & Female factors only & $6 \%$ \\
\hline & & & & Uterine factor & $0 \%$ & Female \& male factors & $0 \%$ \\
\hline & & & & Male factor & $0 \%$ & & \\
\hline
\end{tabular}

2006 PREGNANCY SUCCESS RATES

Data verified by Trishit K. Mukherjee, MD, PhD

\section{Type of Cycle}

\section{Age of Woman}

$<35 \quad 35-37 \quad 38-40 \quad 41-42^{d}$

\section{Fresh Embryos from Nondonor Eggs}

Number of cycles

Percentage of cycles resulting in pregnancies ${ }^{b}$

Percentage of cycles resulting in live births ${ }^{\mathrm{b}, \mathrm{c}}$

(Confidence Interval)

Percentage of retrievals resulting in live births ${ }^{\mathrm{b}, \mathrm{c}}$

Percentage of transfers resulting in live births ${ }^{\mathrm{b}, \mathrm{c}}$

$\begin{array}{cccc}4 & 1 & 6 & 2 \\ 1 / 4 & 1 / 1 & 0 / 6 & 0 / 2 \\ 1 / 4 & 1 / 1 & 0 / 6 & 0 / 2\end{array}$

Percentage of transfers resulting in singleton live births ${ }^{b}$

Percentage of cancellations ${ }^{b}$

$1 / 4$

$1 / 1$

$0 / 5$

$0 / 2$

$1 / 3$

$1 / 1$

$0 / 3$

$0 / 2$

$1 / 3$

$1 / 1$

$0 / 3$

$0 / 2$

$0 / 4$

$0 / 1$

$1 / 6$

$0 / 2$

2.7

3.0

3.0

3.0

Percentage of pregnancies with twins ${ }^{b}$

$0 / 1$

$0 / 1$

$0 / 1$

$0 / 1$

$0 / 1$

$0 / 1$

Percentage of pregnancies with triplets or more
Percentage of live births having multiple infants $\mathrm{s}^{\mathrm{b}} \mathrm{c}$

\section{Frozen Embryos from Nondonor Eggs}

Number of transfers

Percentage of transfers resulting in live births ${ }^{b, c}$

Average number of embryos transferred

$0 / 1$

3.0

1

$0 / 1$

3.0

\section{Donor Eggs}

Number of transfers

Percentage of transfers resulting in live births ${ }^{\mathrm{b}, \mathrm{c}}$

Average number of embryos transferred

\begin{tabular}{cc}
\multicolumn{2}{c}{ All Ages Combined } \\
Fresh Embryos & Frozen Embryos $^{\mathbf{e}}$ \\
1 & 0 \\
$0 / 1$ & \\
2.0 &
\end{tabular}

\section{CURRENT CLINIC SERVICES AND PROFILE}

Current Name: IVF New York

Donor egg? Yes

Donor embryo? No

Single women? Yes

$\begin{array}{ll}\text { Gestational carriers? } & \text { No } \\ \text { Cryopreservation? } & \text { Yes }\end{array}$

SART member?

Yes

Verified lab accreditation?

(See Appendix C for details.)

${ }^{a}$ Reflects patient and treatment characteristics of ART cycles performed in 2006 using fresh nondonor eggs or embryos.

${ }^{b}$ When fewer than 20 cycles are reported in an age category, rates are shown as a fraction and confidence intervals are not given. Calculating percentages from fractions may be misleading and is not encouraged.

c A multiple-infant birth is counted as one live birth.

d Clinic-specific outcome rates are unreliable for women older than 42 undergoing ART cycles using fresh or frozen embryos with nondonor eggs. Readers are urged to review national outcomes for these age groups (see page 27).

e All ages (including ages $>42$ ) are reported together because previous data show that patient age does not materially affect success with donor eggs. 


\section{MANHATTAN REPRODUCTIVE MEDICINE NEW YORK, NEW YORK}

A comparison of clinic success rates may not be meaningful because patient medical characteristics and treatment approaches vary from clinic to clinic. For more details about this, along with information on how to interpret the statistics in this table, see pages 79-88.

\section{ART CYCLE PROFILE}

\begin{tabular}{|c|c|c|c|c|c|c|}
\hline \multicolumn{3}{|c|}{ Type of ART ${ }^{a}$} & \multicolumn{4}{|c|}{ Patient Diagnosis } \\
\hline IVF & $100 \%$ Procedural Factors: & & Tubal factor & $4 \%$ & Other factor & $0 \%$ \\
\hline GIFT & 0\% With ICSI & $100 \%$ & Ovulatory dysfunction & $7 \%$ & Unknown factor & $0 \%$ \\
\hline ZIFT & $0 \%$ Unstimulated & $2 \%$ & Diminished ovarian reserve & $5 \%$ & Multiple Factors: & \\
\hline \multirow[t]{3}{*}{ Combination } & $0 \%$ Used gestational carrier & $0 \%$ & Endometriosis & $0 \%$ & Female factors only & $20 \%$ \\
\hline & & & Uterine factor & $0 \%$ & Female \& male factors & $61 \%$ \\
\hline & & & Male factor & $4 \%$ & & \\
\hline
\end{tabular}

2006 PREGNANCY SUCCESS RATES

Data verified by Hanna Jesionowska, MD

\section{Type of Cycle}

\section{Fresh Embryos from Nondonor Eggs}

Number of cycles

Percentage of cycles resulting in pregnancies ${ }^{b}$

Percentage of cycles resulting in live births $s^{b, c}$

(Confidence Interval)

Percentage of retrievals resulting in live births $s^{b, c}$

Percentage of transfers resulting in live births $s^{b, c}$

Percentage of transfers resulting in singleton live births ${ }^{b}$

Percentage of cancellations ${ }^{b}$

Average number of embryos transferred

Percentage of pregnancies with twins ${ }^{b}$

Percentage of pregnancies with triplets or more ${ }^{b}$

Percentage of live births having multiple infants ${ }^{b, c}$

\section{Frozen Embryos from Nondonor Eggs}

Number of transfers

Percentage of transfers resulting in live births ${ }^{b, c}$

Average number of embryos transferred

\section{Donor Eggs}

Number of transfers

Percentage of transfers resulting in live births $s^{b, c}$

Average number of embryos transferred

\section{Age of Woman}

35-37 38-40

$41-42^{d}$

\section{$<35$}

13

10

6

32
40.6

$5 / 13$

$1 / 10$

$0 / 6$

31.3

$5 / 13$

$1 / 10$

$0 / 6$

$(16.1-50.0)$

31.3

32.3

$5 / 13$

$1 / 10$

$0 / 6$

22.6

$5 / 13$

$1 / 9$

$0 / 6$

0.0

$4 / 13$

$1 / 9$

$0 / 6$

$0 / 13$

$0 / 10$

$0 / 6$

3.7

3.3

2.4

2.8

$5 / 13$

$1 / 5$

$0 / 1$

$0 / 13$

$0 / 5$

$0 / 1$

$3 / 10$

$1 / 5$

$0 / 1$

1

$0 / 1$

5.0

\section{$\%$}

$\%$

$20 \%$

$1 \%$

\section{CURRENT CLINIC SERVICES AND PROFILE}

Current Name: Manhattan Reproductive Medicine

Donor egg? Yes Gestational carriers? No

Donor embryo? Yes

Single women? Yes

Cryopreservation? Yes

All Ages Combined ${ }^{\mathrm{e}}$

Fresh Embryos

11

$7 / 11$

4.5

${ }^{a}$ Reflects patient and treatment characteristics of ART cycles performed in 2006 using fresh nondonor eggs or embryos.

b When fewer than 20 cycles are reported in an age category, rates are shown as a fraction and confidence intervals are not given. Calculating percentages from fractions may be misleading and is not encouraged.

${ }^{c}$ A multiple-infant birth is counted as one live birth.

d Clinic-specific outcome rates are unreliable for women older than 42 undergoing ART cycles using fresh or frozen embryos with nondonor eggs. Readers are urged to review national outcomes for these age groups (see page 27).

e All ages (including ages $>42$ ) are reported together because previous data show that patient age does not materially affect success with donor eggs. 


\section{MEDICAL OFFICES FOR HUMAN REPRODUCTION \\ CENTER FOR HUMAN REPRODUCTION (CHR) \\ NEW YORK, NEW YORK}

A comparison of clinic success rates may not be meaningful because patient medical characteristics and treatment approaches vary from clinic to clinic. For more details about this, along with information on how to interpret the statistics in this table, see pages $79-88$.

\section{ART CYCLE PROFILE}

\begin{tabular}{|c|c|c|c|c|c|c|c|}
\hline \multicolumn{4}{|c|}{ Type of ART } & \multicolumn{4}{|c|}{ Patient Diagnosis } \\
\hline IVF & $100 \%$ & Procedural Factors: & & Tubal factor & $8 \%$ & Other factor & $23 \%$ \\
\hline GIFT & $0 \%$ & With ICSI & $74 \%$ & Ovulatory dysfunction & $6 \%$ & Unknown factor & $1 \%$ \\
\hline ZIFT & $0 \%$ & Unstimulated & $2 \%$ & Diminished ovarian reserve & $18 \%$ & Multiple Factors: & \\
\hline \multirow[t]{3}{*}{ Combination } & $0 \%$ & Used gestational carrier & $0 \%$ & Endometriosis & $2 \%$ & Female factors only & $20 \%$ \\
\hline & & & & Uterine factor & $1 \%$ & Female \& male factors & $13 \%$ \\
\hline & & & & Male factor & $8 \%$ & & \\
\hline
\end{tabular}

2006 PREGNANCY SUCCESS RATES

Data verified by Norbert Gleicher, MD

\section{Type of Cycle}

\section{Fresh Embryos from Nondonor Eggs}

Number of cycles

Percentage of cycles resulting in pregnancies ${ }^{b}$

Percentage of cycles resulting in live births ${ }^{b, c}$

(Confidence Interval)

Percentage of retrievals resulting in live births $s^{b, c}$

Percentage of transfers resulting in live births $s^{b, c}$

Percentage of transfers resulting in singleton live births ${ }^{b}$

Percentage of cancellations ${ }^{b}$

Average number of embryos transferred

Percentage of pregnancies with twins ${ }^{b}$

Percentage of pregnancies with triplets or more ${ }^{b}$

Percentage of live births having multiple infants ${ }^{b, c}$

\section{Frozen Embryos from Nondonor Eggs}

Number of transfers

Percentage of transfers resulting in live births $s^{b, c}$

Average number of embryos transferred

\section{Donor Eggs}

Number of transfers

Percentage of transfers resulting in live births $s^{b, c}$

Average number of embryos transferred

\section{Age of Woman}

35-37 38-40

$41-42^{d}$

$<35$

47

21.3

19.1

41

34

32.5

24.1

(9.1-33.3)

26.8

14.7

(15.4-34.7)

26.0

22.0

22.0

14.7

29.9

23.7

(10.6-37.6)

(5.0-31.1)

17.2

25.4

15.8

30.0

20.0

7.2

12.8

20.0

20.0

2.1

2.3

12.2

14.7

14.8

$3 / 10$

2.2

2.2

3.7

$0 / 10$

$4 / 11$

$0 / 5$

15.0

$3 / 9$

$0 / 11$

$0 / 5$

$3 / 9$

$0 / 5$

20

25.0

2.4

\section{7}

2 / 17

2.6

7
$0 / 7$
3.0

1

$0 / 1$

3.0

All Ages Combined ${ }^{\mathrm{e}}$

Fresh Embryos 19

$11 / 19$

2.1
Frozen Embryos 15

$8 / 15$

2.7

\section{CURRENT CLINIC SERVICES AND PROFILE}

Current Name: Medical Offices for Human Reproduction, Center for Human Reproduction (CHR)

Donor egg? Yes

Donor embryo? Yes

Single women? Yes
Gestational carriers? No

Cryopreservation? Yes
SART member?

Verified lab accreditation?

(See Appendix C for details.)

${ }^{a}$ Reflects patient and treatment characteristics of ART cycles performed in 2006 using fresh nondonor eggs or embryos.

${ }^{b}$ When fewer than 20 cycles are reported in an age category, rates are shown as a fraction and confidence intervals are not given. Calculating percentages from fractions may be misleading and is not encouraged.

A multiple-infant birth is counted as one live birth.

d Clinic-specific outcome rates are unreliable for women older than 42 undergoing ART cycles using fresh or frozen embryos with nondonor eggs. Readers are urged to review national outcomes for these age groups (see page 27).

e All ages (including ages $>42$ ) are reported together because previous data show that patient age does not materially affect success with donor eggs. 


\section{NEW HOPE FERTILITY CENTER \\ NEW YORK, NEW YORK}

A comparison of clinic success rates may not be meaningful because patient medical characteristics and treatment approaches vary from clinic to clinic. For more details about this, along with information on how to interpret the statistics in this table, see pages 79-88.

\section{ART CYCLE PROFILE}

\begin{tabular}{|c|c|c|c|c|c|c|c|}
\hline \multicolumn{4}{|c|}{ Type of ART ${ }^{a}$} & \multicolumn{4}{|c|}{ Patient Diagnosis } \\
\hline IVF & $100 \%$ & Procedural Factors: & & Tubal factor & $14 \%$ & Other factor & $34 \%$ \\
\hline GIFT & $0 \%$ & With ICSI & $77 \%$ & Ovulatory dysfunction & $3 \%$ & Unknown factor & $15 \%$ \\
\hline ZIFT & $0 \%$ & Unstimulated & $9 \%$ & Diminished ovarian reserve & $<1 \%$ & Multiple Factors: & \\
\hline \multirow[t]{3}{*}{ Combination } & $0 \%$ & Used gestational carrier & $<1 \%$ & Endometriosis & $4 \%$ & Female factors only & $8 \%$ \\
\hline & & & & Uterine factor & $4 \%$ & Female \& male factors & $4 \%$ \\
\hline & & & & Male factor & $14 \%$ & & \\
\hline
\end{tabular}

2006 PREGNANCY SUCCESS RATES

Data verified by John J. Zhang, MD, PhD

\section{Type of Cycle}

\section{$<35$}

12.9

9.1

$(5.8-13.5)$

9.3

15.6

13.5

2.1

1.4

9.7

0.0

13.6

34.9

\section{Frozen Embryos from Nondonor Eggs}

Number of transfers

Percentage of transfers resulting in live births $s^{b, c}$

Average number of embryos transferred

\section{Age of Woman}

35-37 $38-40 \quad 41-42^{d}$

$\begin{array}{lll}194 & 260 & 236 \\ 17.0 & 7.7 & 3.4 \\ 12.9 & 5.4 & 1.3\end{array}$

(8.5-18.4)

(3.0-8.9)

$(0.3-3.7)$

5.8

1.4

24.5

14.7

3.8

23.5

12.6

3.8

2.1

6.9

11.4

1.3

1.8

1.6

3.0

5.0

$0 / 8$

0.0

5.0

$0 / 8$

4.0

$2 / 14$

$0 / 3$

\section{Donor Eggs}

Number of transfers

Percentage of transfers resulting in live births $s^{b, c}$

Average number of embryos transferred
63

1.3

\section{Fresh Embryos}

$$
40
$$

27.5

1.2

\section{0}

15.0

1.9
10

$1 / 10$

1.8

All Ages Combined ${ }^{\mathrm{e}}$

$\begin{array}{cc}12 & 20 \\ 3 / 12 & 35.0 \\ 1.4 & 1.4\end{array}$

\section{CURRENT CLINIC SERVICES AND PROFILE}

Current Name: New Hope Fertility Center

\begin{tabular}{|c|c|c|c|c|}
\hline Donor egg? & Yes & Gestational carriers? & Yes & SART member? \\
\hline Donor embryo? & Yes & Cryopreservation? & Yes & Verified lab accreditation? \\
\hline
\end{tabular}

\footnotetext{
${ }^{a}$ Reflects patient and treatment characteristics of ART cycles performed in 2006 using fresh nondonor eggs or embryos.

b When fewer than 20 cycles are reported in an age category, rates are shown as a fraction and confidence intervals are not given. Calculating percentages from fractions may be misleading and is not encouraged.

${ }^{c}$ A multiple-infant birth is counted as one live birth.

${ }^{d}$ Clinic-specific outcome rates are unreliable for women older than 42 undergoing ART cycles using fresh or frozen embryos with nondonor eggs. Readers are urged to review national outcomes for these age groups (see page 27).

e All ages (including ages $>42$ ) are reported together because previous data show that patient age does not materially affect success with donor eggs.
} 


\section{NEW YORK FERTILITY INSTITUTE NEW YORK, NEW YORK}

A comparison of clinic success rates may not be meaningful because patient medical characteristics and treatment approaches vary from clinic to clinic. For more details about this, along with information on how to interpret the statistics in this table, see pages $79-88$.

\section{ART CYCLE PROFILE}

\begin{tabular}{|c|c|c|c|c|c|c|c|}
\hline \multicolumn{4}{|c|}{ Type of ART ${ }^{\mathrm{a}}$} & \multicolumn{4}{|c|}{ Patient Diagnosis } \\
\hline IVF & $100 \%$ & Procedural Factors: & & Tubal factor & $4 \%$ & Other factor & $0 \%$ \\
\hline GIFT & $0 \%$ & With ICSI & $84 \%$ & Ovulatory dysfunction & $6 \%$ & Unknown factor & $0 \%$ \\
\hline ZIFT & $0 \%$ & Unstimulated & $3 \%$ & Diminished ovarian reserve & $22 \%$ & Multiple Factors: & \\
\hline Combination & $0 \%$ & Used gestational carrier & $2 \%$ & Endometriosis & $26 \%$ & Female factors only & $0 \%$ \\
\hline & & & & Uterine factor & $2 \%$ & Female \& male factors & $9 \%$ \\
\hline & & & & Male factor & $32 \%$ & & \\
\hline
\end{tabular}

2006 PREGNANCY SUCCESS RATES

Data verified by Majid Fateh, MD

\section{Type of Cycle}

\section{Fresh Embryos from Nondonor Eggs}

Number of cycles

Percentage of cycles resulting in pregnancies ${ }^{b}$

Percentage of cycles resulting in live births $s^{b, c}$

(Confidence Interval)

Percentage of retrievals resulting in live births $s^{b, c}$

Percentage of transfers resulting in live births $s^{b, c}$

Percentage of transfers resulting in singleton live births ${ }^{b}$

Percentage of cancellations ${ }^{b}$

Average number of embryos transferred

Percentage of pregnancies with twins ${ }^{b}$

Percentage of pregnancies with triplets or more ${ }^{b}$

Percentage of live births having multiple infants ${ }^{b, c}$

\section{Frozen Embryos from Nondonor Eggs}

Number of transfers

Percentage of transfers resulting in live births $s^{b, c}$

Average number of embryos transferred

\section{Donor Eggs}

Number of transfers

Percentage of transfers resulting in live births $s^{b, c}$

Average number of embryos transferred

\section{Age of Woman}

35-37

38-40

$41-42^{d}$

$<35$

43

41.9

48

31

45.8

39.5

37.5

25.8

40.7

(28.1-54.3)

(25.0-55.6)

31.3

12.9

42.1

44.4

41.5

(18.7-46.3)

(3.6-29.8)

38.9

43.6

33.3

34.9

14.3

33.3

30.2

15.4

3.4

4.7

6.3

15.4

3.0

3.0

3.6

9.7

14.8

$2 / 18$

0.0

$2 / 18$

$2 / 18$

3.3

12.5

$4 / 17$

$0 / 18$

$0 / 8$

$2 / 15$

$0 / 8$

$0 / 4$

$\begin{array}{cccc}2 & 1 & 2 & 1 \\ 1 / 2 & 0 / 1 & 1 / 2 & 0 / 1 \\ 3.0 & 4.0 & 2.0 & 4.0\end{array}$

All Ages Combined

Fresh Embryos

Frozen Embryos

13

$8 / 13$

16

2.7

$11 / 16$

2.8

\section{CURRENT CLINIC SERVICES AND PROFILE}

Current Name: New York Fertility Institute

Donor egg? Yes Gestational carriers? Yes

Donor embryo? Yes Cryopreservation? Yes

Single women? Yes

\section{SART member? Yes}

Verified lab accreditation? Yes

(See Appendix C for details.)

${ }^{a}$ Reflects patient and treatment characteristics of ART cycles performed in 2006 using fresh nondonor eggs or embryos.

${ }^{b}$ When fewer than 20 cycles are reported in an age category, rates are shown as a fraction and confidence intervals are not given. Calculating percentages from fractions may be misleading and is not encouraged.

A multiple-infant birth is counted as one live birth.

d Clinic-specific outcome rates are unreliable for women older than 42 undergoing ART cycles using fresh or frozen embryos with nondonor eggs. Readers are urged to review national outcomes for these age groups (see page 27).

e All ages (including ages $>42$ ) are reported together because previous data show that patient age does not materially affect success with donor eggs. 


\section{NYU FERTILITY CENTER' \\ NEW YORK UNIVERSITY SCHOOL OF MEDICINE \\ NEW YORK, NEW YORK}

A comparison of clinic success rates may not be meaningful because patient medical characteristics and treatment approaches vary from clinic to clinic. For more details about this, along with information on how to interpret the statistics in this table, see pages 79-88.

\section{ART CYCLE PROFILE}

\section{Type of ART ${ }^{\mathrm{a}}$}

IVF

GIFT

100\% Procedural Factors:

ZIFT

Combination
$0 \%$ With ICSI

$0 \%$ Unstimulated

$0 \%$ Used gestational carrier

\section{$27 \%$}

$0 \%$

$0 \%$
Tubal factor

Ovulatory dysfunction

Diminished ovarian reserve

Endometriosis

Uterine factor

Male factor

\section{Patient Diagnosis}

$\begin{array}{rll}4 \% & \text { Other factor } & 13 \% \\ 3 \% & \text { Unknown factor } & 12 \% \\ 15 \% & \text { Multiple Factors: } & \\ 3 \% & \text { Female factors only } & 20 \% \\ 3 \% & \text { Female \& male factors } & 17 \% \\ 10 \% & & \end{array}$

2006 PREGNANCY SUCCESS RATES

Data verified by James A. Grifo, MD, PhD

\section{Type of Cycle}

\section{Age of Woman}

35-37 38-40

\section{$<35$}

46.7

$(41.0-52.5)$

51.3

(27.4-39.2)

(Confidence Interval)

Percentage of retrievals resulting in live births ${ }^{b, c}$

Percentage of transfers resulting in live births ${ }^{b, c}$

Percentage of transfers resulting in singleton live births ${ }^{b}$

Percentage of cancellations ${ }^{b}$

\section{2}

35.6

8.8

2.0

Average number of embryos transferred

Percentage of pregnancies with twins ${ }^{b}$

Percentage of pregnancies with triplets or more ${ }^{b}$

Percentage of live births having multiple infants $\mathrm{s}^{\mathrm{b}, \mathrm{c}}$

\section{Frozen Embryos from Nondonor Eggs}

Number of transfers

Percentage of transfers resulting in live births $s^{\mathrm{b}, \mathrm{c}}$

Average number of embryos transferred

\section{Donor Eggs}

Number of transfers

Percentage of transfers resulting in live births $s^{\mathrm{b}, \mathrm{c}}$

Average number of embryos transferred

\section{7}

4.9

34.3

74

27.0

2.2
36.8

39.3

29.2

10.0

2.1

25.6

0.8

25.6
260

46.5

33.1

298

34.9

24.5

(19.7-29.8)

27.2

29.1

23.5

10.1

2.5

21.2

1.9

19.2

\section{9}

33.3

2.3
46

26.1

2.2

\section{All Ages Combined}

Fresh Embryos

145

62.8

2.0 $41-42^{d}$

196

23.5

14.3

$(9.7-20.0)$

17.3

19.2

15.1

17.3

3.0

21.7

0.0

21.4
Frozen Embryos 36

25.0

2.3

\section{CURRENT CLINIC SERVICES AND PROFILE}

Current Name: NYU Fertility Center, New York University School of Medicine

Donor egg?

Donor embryo?

Single women?

\begin{abstract}
Gestational carriers? Yes
Cryopreservation? Yes
\end{abstract}

SART member?

Verified lab accreditation?

(See Appendix C for details.)

\section{8}

$1 / 8$

2.8 


\section{OFFICES FOR FERTILITY AND REPRODUCTIVE MEDICINE NEW YORK, NEW YORK}

A comparison of clinic success rates may not be meaningful because patient medical characteristics and treatment approaches vary from clinic to clinic. For more details about this, along with information on how to interpret the statistics in this table, see pages $79-88$.

\section{ART CYCLE PROFILE}

\begin{tabular}{|c|c|c|c|c|c|c|c|}
\hline \multicolumn{4}{|c|}{ Type of ART ${ }^{a}$} & \multicolumn{4}{|c|}{ Patient Diagnosis } \\
\hline IVF & $100 \%$ & Procedural Factors: & & Tubal factor & $1 \%$ & Other factor & $3 \%$ \\
\hline GIFT & $0 \%$ & With ICSI & $41 \%$ & Ovulatory dysfunction & $8 \%$ & Unknown factor & $0 \%$ \\
\hline ZIFT & $0 \%$ & Unstimulated & $0 \%$ & Diminished ovarian reserve & $17 \%$ & Multiple Factors: & \\
\hline \multirow[t]{3}{*}{ Combination } & $0 \%$ & Used gestational carrier & $3 \%$ & Endometriosis & $1 \%$ & Female factors only & $21 \%$ \\
\hline & & & & Uterine factor & $0 \%$ & Female \& male factors & $41 \%$ \\
\hline & & & & Male factor & $9 \%$ & & \\
\hline
\end{tabular}

2006 PREGNANCY SUCCESS RATES

Data verified by Cecilia Schmidt-Sarosi, MD

\section{Type of Cycle}

\section{Fresh Embryos from Nondonor Eggs}

Number of cycles

Percentage of cycles resulting in pregnancies ${ }^{b}$

Percentage of cycles resulting in live births $s^{b, c}$

(Confidence Interval)

Percentage of retrievals resulting in live births $s^{b, c}$

Percentage of transfers resulting in live births $s^{b, c}$

Percentage of transfers resulting in singleton live births ${ }^{b}$

Percentage of cancellations ${ }^{b}$

Average number of embryos transferred

Percentage of pregnancies with twins ${ }^{b}$

Percentage of pregnancies with triplets or more ${ }^{b}$

Percentage of live births having multiple infants ${ }^{b, c}$

\section{Frozen Embryos from Nondonor Eggs}

Number of transfers

Percentage of transfers resulting in live births $s^{\mathrm{b}, \mathrm{c}}$

Average number of embryos transferred

\section{Donor Eggs}

Number of transfers

Percentage of transfers resulting in live births $s^{b, c}$

Average number of embryos transferred

\section{Age of Woman}

35-37 38-40

$41-42^{d}$

$<35$

22

50.0

45.5

25.0

(8.7-49.1)

(24.4-67.8)

45.5

$10 / 19$

$6 / 19$

0.0

3.8

$4 / 11$

$0 / 11$

$4 / 10$

$3 / 7$

$0 / 7$

$2 / 5$

18

$3 / 18$

3.9

9
$1 / 9$
3.2

9

3.2

All Ages Combined ${ }^{\mathrm{e}}$

Fresh Embryos

20

35.0

2.6
25

32.0

16.0

(3.8-30.7)

14.3

15.4

11.5

6.7

4.4

$0 / 7$

$1 / 7$

$1 / 4$

(4.5-36.1)

17.4

20.0

15.0

8.0

4.3

$0 / 8$

$1 / 8$

$1 / 4$

\section{CURRENT CLINIC SERVICES AND PROFILE}

Current Name: Offices for Fertility and Reproductive Medicine

Donor egg? Yes Gestational carriers? Yes

Donor embryo? Yes Cryopreservation? Yes

Single women? Yes

\section{SART member? Yes}

Verified lab accreditation? Yes

(See Appendix C for details.)

${ }^{a}$ Reflects patient and treatment characteristics of ART cycles performed in 2006 using fresh nondonor eggs or embryos.

${ }^{b}$ When fewer than 20 cycles are reported in an age category, rates are shown as a fraction and confidence intervals are not given. Calculating percentages from fractions may be misleading and is not encouraged.

A multiple-infant birth is counted as one live birth.

d Clinic-specific outcome rates are unreliable for women older than 42 undergoing ART cycles using fresh or frozen embryos with nondonor eggs. Readers are urged to review national outcomes for these age groups (see page 27).

e All ages (including ages $>42$ ) are reported together because previous data show that patient age does not materially affect success with donor eggs. 


\section{REPRODUCTIVE CARE OF NY \\ NEW YORK, NEW YORK}

A comparison of clinic success rates may not be meaningful because patient medical characteristics and treatment approaches vary from clinic to clinic. For more details about this, along with information on how to interpret the statistics in this table, see pages 79-88.

\section{ART CYCLE PROFILE}

\begin{tabular}{|c|c|c|c|c|c|c|c|}
\hline \multicolumn{4}{|c|}{ Type of ART ${ }^{a}$} & \multicolumn{4}{|c|}{ Patient Diagnosis } \\
\hline IVF & $100 \%$ & Procedural Factors: & & Tubal factor & $0 \%$ & Other factor & $0 \%$ \\
\hline GIFT & $0 \%$ & With ICSI & $43 \%$ & Ovulatory dysfunction & $0 \%$ & Unknown factor & $0 \%$ \\
\hline ZIFT & $0 \%$ & Unstimulated & $0 \%$ & Diminished ovarian reserve & $44 \%$ & Multiple Factors: & \\
\hline \multirow[t]{3}{*}{ Combination } & $0 \%$ & Used gestational carrier & $0 \%$ & Endometriosis & $0 \%$ & Female factors only & $11 \%$ \\
\hline & & & & Uterine factor & $0 \%$ & Female \& male factors & $22 \%$ \\
\hline & & & & Male factor & $22 \%$ & & \\
\hline
\end{tabular}

2006 PREGNANCY SUCCESS RATES

Data verified by Lillian D. Nash, MD

\section{Type of Cycle}

\section{Fresh Embryos from Nondonor Eggs}

Number of cycles

Percentage of cycles resulting in pregnancies ${ }^{b}$

Percentage of cycles resulting in live births ${ }^{b, c}$

(Confidence Interval)

Percentage of retrievals resulting in live births $s^{b, c}$

Percentage of transfers resulting in live births $s^{b, c}$

Percentage of transfers resulting in singleton live births ${ }^{b}$

Percentage of cancellations ${ }^{b}$

Average number of embryos transferred

Percentage of pregnancies with twins ${ }^{b}$

Percentage of pregnancies with triplets or more ${ }^{b}$

Percentage of live births having multiple infants ${ }^{b, c}$

\section{Frozen Embryos from Nondonor Eggs}

Number of transfers

Percentage of transfers resulting in live births ${ }^{b, c}$

Average number of embryos transferred

\section{Donor Eggs}

Number of transfers

Percentage of transfers resulting in live births $s^{\mathrm{b}, \mathrm{c}}$

Average number of embryos transferred

\section{Age of Woman}

$<35$

35-37

38-40

$41-42^{d}$

0

0

\section{2}

$0 / 2$

$0 / 2$

$0 / 1$

$0 / 1$

$\begin{array}{cc}0 / 2 & 0 / 1 \\ 0 / 2 & 0 / 1 \\ 0 / 2 & 0 / 1 \\ 0 / 2 & 0 / 1 \\ 2.0 & 1.0\end{array}$

\section{CURRENT CLINIC SERVICES AND PROFILE}

Current Name: Reproductive Care of NY

Donor egg? No Gestational carriers? No

Donor embryo? No Cryopreservation? Yes

Single women? Yes

SART member? Yes

Verified lab accreditation? Yes

(See Appendix C for details.)

${ }^{a}$ Reflects patient and treatment characteristics of ART cycles performed in 2006 using fresh nondonor eggs or embryos.

${ }^{b}$ When fewer than 20 cycles are reported in an age category, rates are shown as a fraction and confidence intervals are not given. Calculating percentages from fractions may be misleading and is not encouraged.

${ }^{c}$ A multiple-infant birth is counted as one live birth.

d Clinic-specific outcome rates are unreliable for women older than 42 undergoing ART cycles using fresh or frozen embryos with nondonor eggs. Readers are urged to review national outcomes for these age groups (see page 27).

e All ages (including ages $>42$ ) are reported together because previous data show that patient age does not materially affect success with donor eggs. 


\section{REPRODUCTIVE ENDOCRINOLOGY ASSOCIATES \\ OF ST. LUKE'S ROOSEVELT HOSPITAL CENTER \\ NEW YORK, NEW YORK}

A comparison of clinic success rates may not be meaningful because patient medical characteristics and treatment approaches vary from clinic to clinic. For more details about this, along with information on how to interpret the statistics in this table, see pages $79-88$.

\section{ART CYCLE PROFILE}

\begin{tabular}{|c|c|c|c|c|c|c|c|}
\hline \multicolumn{4}{|c|}{ Type of ART } & \multicolumn{4}{|c|}{ Patient Diagnosis } \\
\hline IVF & $100 \%$ & Procedural Factors: & & Tubal factor & $16 \%$ & Other factor & $7 \%$ \\
\hline GIFT & $0 \%$ & With ICSI & $87 \%$ & Ovulatory dysfunction & $15 \%$ & Unknown factor & $9 \%$ \\
\hline ZIFT & $0 \%$ & Unstimulated & $0 \%$ & Diminished ovarian reserve & $13 \%$ & Multiple Factors: & \\
\hline \multirow[t]{3}{*}{ Combination } & $0 \%$ & Used gestational carrier & $0 \%$ & Endometriosis & $4 \%$ & Female factors only & $7 \%$ \\
\hline & & & & Uterine factor & $<1 \%$ & Female \& male factors & $8 \%$ \\
\hline & & & & Male factor & $22 \%$ & & \\
\hline
\end{tabular}

2006 PREGNANCY SUCCESS RATES

Data verified by Martin Keltz, MD

\section{Type of Cycle}

\section{Fresh Embryos from Nondonor Eggs}

Number of cycles

Percentage of cycles resulting in pregnancies ${ }^{b}$

Percentage of cycles resulting in live births ${ }^{b, c}$

(Confidence Interval)

Percentage of retrievals resulting in live births $s^{b, c}$

Percentage of transfers resulting in live births $s^{b, c}$

Percentage of transfers resulting in singleton live births ${ }^{b}$

Percentage of cancellations ${ }^{b}$

Average number of embryos transferred

Percentage of pregnancies with twins ${ }^{b}$

Percentage of pregnancies with triplets or more ${ }^{b}$

Percentage of live births having multiple infants ${ }^{b, c}$

\section{Frozen Embryos from Nondonor Eggs}

Number of transfers

Percentage of transfers resulting in live births $s^{b, c}$

Average number of embryos transferred

\section{Donor Eggs}

Number of transfers

Percentage of transfers resulting in live births ${ }^{\mathrm{b}, \mathrm{c}}$

Average number of embryos transferred

\section{Age of Woman}

35-37 38-40

$41-42^{d}$

\section{$<35$}

71

66

45

133

39.4

28.8

24.4

32.3

31.0

18.2

15.6

$(24.5-41.0)$

(20.5-43.1)

(9.8-29.6)

(6.5-29.5)

33.3

35.2

32.4

19.0

16.7

34.4

20.3

17.9

23.4

15.3

17.9

3.0

4.2

4.5

6.7

2.3

2.8

25.0

3.1

3.6

37.1

10.7

$6 / 19$

$3 / 11$

4.8

31.8

$0 / 19$

$0 / 11$

37.2

$3 / 12$

$0 / 7$

26

30.8

$$
16
$$

$7 / 16$

\section{5}

$1 / 5$

1

2.4

2.6

3.4

$0 / 1$

2.0

All Ages Combined ${ }^{\mathrm{e}}$

Fresh Embryos

\section{7}

$3 / 7$

2.6
Frozen Embryos

\section{5}

$1 / 5$

2.6

\section{CURRENT CLINIC SERVICES AND PROFILE}

Current Name: Reproductive Endocrinology Associates of St. Luke's Roosevelt Hospital Center

Donor egg? Yes

Donor embryo? No

Single women? Yes

\section{Gestational carriers? No}

Cryopreservation?
SART member?

Verified lab accreditation?

(See Appendix C for details.)

${ }^{a}$ Reflects patient and treatment characteristics of ART cycles performed in 2006 using fresh nondonor eggs or embryos.

b When fewer than 20 cycles are reported in an age category, rates are shown as a fraction and confidence intervals are not given. Calculating percentages from fractions may be misleading and is not encouraged.

A multiple-infant birth is counted as one live birth.

d Clinic-specific outcome rates are unreliable for women older than 42 undergoing ART cycles using fresh or frozen embryos with nondonor eggs. Readers are urged to review national outcomes for these age groups (see page 27).

e All ages (including ages $>42$ ) are reported together because previous data show that patient age does not materially affect success with donor eggs. 


\section{REPRODUCTIVE MEDICINE ASSOCIATES OF NEW YORK, LLP NEW YORK, NEW YORK}

A comparison of clinic success rates may not be meaningful because patient medical characteristics and treatment approaches vary from clinic to clinic. For more details about this, along with information on how to interpret the statistics in this table, see pages 79-88.

\section{ART CYCLE PROFILE}

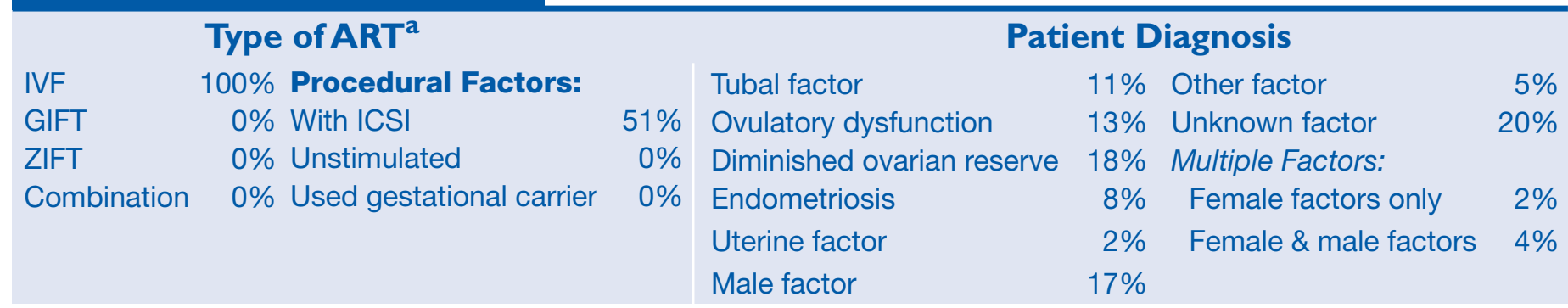

2006 PREGNANCY SUCCESS RATES

Data verified by Lawrence Grunfeld, MD

\section{Type of Cycle}

\section{Fresh Embryos from Nondonor Eggs}

Number of cycles

Percentage of cycles resulting in pregnancies ${ }^{b}$

Percentage of cycles resulting in live births ${ }^{b, c}$

(Confidence Interval)

Percentage of retrievals resulting in live births $s^{b, c}$

Percentage of transfers resulting in live births $s^{b, c}$

Percentage of transfers resulting in singleton live births ${ }^{b}$

Percentage of cancellations ${ }^{b}$

Average number of embryos transferred

Percentage of pregnancies with twins ${ }^{b}$

Percentage of pregnancies with triplets or more ${ }^{b}$

Percentage of live births having multiple infants ${ }^{b, c}$

\section{Frozen Embryos from Nondonor Eggs}

Number of transfers

Percentage of transfers resulting in live births $s^{b, c}$

Average number of embryos transferred

\section{Donor Eggs}

Number of transfers

Percentage of transfers resulting in live births $s^{b, c}$

Average number of embryos transferred

\section{Age of Woman}

35-37 38-40

$41-42^{d}$

$<35$

283

290

175

55.1

48.2

44.9

33.4

20.0

36.4

24.8

$(43.2-53.3)$

(30.8-42.3)

(20.0-30.2)

13.1

50.9

41.4

28.1

29.6

(8.5-19.1)

53.1

43.1

21.0

17.2

18.1

28.0

11.7

15.7

5.4

12.0

3.2

23.4

2.5

32.4

2.7

25.8

3.9

3.1

3.3

4.2

38.6

35.0

29.2

11.4

0.0

13.0

$\begin{array}{cccc}59 & 30 & 12 & 5 \\ 37.3 & 26.7 & 3 / 12 & 1 / 5 \\ 2.3 & 2.1 & 2.0 & 2.6\end{array}$

All Ages Combined ${ }^{\mathrm{e}}$

Fresh Embryos

Frozen Embryos

148

41

54.1

29.3

2.3

\section{CURRENT CLINIC SERVICES AND PROFILE}

Current Name: Reproductive Medicine Associates of New York, LLP

Donor egg? Yes Gestational carriers? No

Donor embryo? No Cryopreservation? Yes

Single women? Yes

SART member? Yes

Verified lab accreditation? Yes

(See Appendix C for details.)

${ }^{a}$ Reflects patient and treatment characteristics of ART cycles performed in 2006 using fresh nondonor eggs or embryos.

${ }^{b}$ When fewer than 20 cycles are reported in an age category, rates are shown as a fraction and confidence intervals are not given. Calculating percentages from fractions may be misleading and is not encouraged.

${ }^{c}$ A multiple-infant birth is counted as one live birth.

d Clinic-specific outcome rates are unreliable for women older than 42 undergoing ART cycles using fresh or frozen embryos with nondonor eggs. Readers are urged to review national outcomes for these age groups (see page 27).

e All ages (including ages $>42$ ) are reported together because previous data show that patient age does not materially affect success with donor eggs. 


\section{SHER INSTITUTES FOR REPRODUCTIVE MEDICINE-NYC NEW YORK, NEW YORK}

A comparison of clinic success rates may not be meaningful because patient medical characteristics and treatment approaches vary from clinic to clinic. For more details about this, along with information on how to interpret the statistics in this table, see pages $79-88$.

\section{ART CYCLE PROFILE}

\begin{tabular}{|c|c|c|c|c|c|c|c|}
\hline \multicolumn{4}{|c|}{ Type of ART } & \multicolumn{4}{|c|}{ Patient Diagnosis } \\
\hline IVF & $100 \%$ & Procedural Factors: & & Tubal factor & $10 \%$ & Other factor & $13 \%$ \\
\hline GIFT & $0 \%$ & With ICSI & $89 \%$ & Ovulatory dysfunction & $6 \%$ & Unknown factor & $15 \%$ \\
\hline ZIFT & $0 \%$ & Unstimulated & $0 \%$ & Diminished ovarian reserve & $30 \%$ & Multiple Factors: & \\
\hline \multirow[t]{3}{*}{ Combination } & $0 \%$ & Used gestational carrier & $0 \%$ & Endometriosis & $6 \%$ & Female factors only & $5 \%$ \\
\hline & & & & Uterine factor & $<1 \%$ & Female \& male factors & $6 \%$ \\
\hline & & & & Male factor & $9 \%$ & & \\
\hline
\end{tabular}

2006 PREGNANCY SUCCESS RATES

Data verified by Geoffrey Sher, MD

\section{Type of Cycle}

\section{Fresh Embryos from Nondonor Eggs}

Number of cycles

Percentage of cycles resulting in pregnancies ${ }^{b}$

Percentage of cycles resulting in live births $s^{b, c}$

(Confidence Interval)

Percentage of retrievals resulting in live births $s^{b, c}$

Percentage of transfers resulting in live births $s^{b, c}$

Percentage of transfers resulting in singleton live births ${ }^{b}$

Percentage of cancellations ${ }^{b}$

Average number of embryos transferred

Percentage of pregnancies with twins ${ }^{b}$

Percentage of pregnancies with triplets or more ${ }^{b}$

Percentage of live births having multiple infants ${ }^{b, c}$

\section{Frozen Embryos from Nondonor Eggs}

Number of transfers

Percentage of transfers resulting in live births $s^{\mathrm{b}, \mathrm{c}}$

Average number of embryos transferred

\section{Donor Eggs}

Number of transfers

Percentage of transfers resulting in live births ${ }^{\mathrm{b}, \mathrm{c}}$

Average number of embryos transferred

\section{Age of Woman}

35-37 38-40

$41-42^{d}$

$<35$

40

37

23

69
37.7

32.5

16.2

17.4

24.6

17.5

10.8

8.7

(15.1-36.5)

$(7.3-32.8)$
17.5

(3.0-25.4)

(1.1-28.0)

26.2

28.8

20.6

12.5

$2 / 19$

20.3

8.8

14.3

$2 / 16$

5.8

0.0

7.1

$2 / 16$

2.8

26.9

2.9

13.5

17.4

0.0

$4 / 13$

2.8

2.6

$5 / 17$

$1 / 13$

$3 / 6$

$0 / 4$

$0 / 6$

$0 / 4$

$4 / 7$

$2 / 4$

$0 / 2$

10

$3 / 10$

1

$0 / 1$

1

$0 / 1$

2.7

0.0

2.0

All Ages Combined ${ }^{\mathrm{e}}$

Fresh Embryos

32

34.4

2.5
Frozen Embryos

11

$2 / 11$

3.4

\section{CURRENT CLINIC SERVICES AND PROFILE}

Current Name: Sher Institutes for Reproductive Medicine-NYC

Donor egg? Yes $\quad$ Gestational carriers? Yes

Donor embryo? No Cryopreservation? Yes

Single women? Yes

SART member?

No

Verified lab accreditation?

Yes

(See Appendix C for details.)

${ }^{a}$ Reflects patient and treatment characteristics of ART cycles performed in 2006 using fresh nondonor eggs or embryos.

${ }^{b}$ When fewer than 20 cycles are reported in an age category, rates are shown as a fraction and confidence intervals are not given. Calculating percentages from fractions may be misleading and is not encouraged.

A multiple-infant birth is counted as one live birth.

d Clinic-specific outcome rates are unreliable for women older than 42 undergoing ART cycles using fresh or frozen embryos with nondonor eggs. Readers are urged to review national outcomes for these age groups (see page 27).

e All ages (including ages $>42$ ) are reported together because previous data show that patient age does not materially affect success with donor eggs. 


\section{WEILL MEDICAL COLLEGE OF CORNELL UNIVERSITYণ THE CENTER FOR REPRODUCTIVE MEDICINE AND INFERTILITY NEW YORK, NEW YORK}

A comparison of clinic success rates may not be meaningful because patient medical characteristics and treatment approaches vary from clinic to clinic. For more details about this, along with information on how to interpret the statistics in this table, see pages $79-88$.

\section{ART CYCLE PROFILE}

\section{Type of ART ${ }^{\mathrm{a}}$}

IVF

GIFT

ZIFT

Combination
100\% Procedural Factors:

$0 \%$ With ICSI

$0 \%$ Unstimulated

$0 \%$ Used gestational carrier
Tubal factor

$59 \%$ Ovulatory dysfunction

$<1 \%$

$0 \%$

Endometriosis

\section{Patient Diagnosis}

$7 \%$ Other factor

4\% Unknown factor

Diminished ovarian reserve $20 \%$ Multiple Factors:

$4 \%$ Female factors only $21 \%$

Uterine factor

$1 \%$ Female \& male factors $18 \%$

Male factor $16 \%$
$3 \%$

$6 \%$

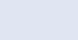

2006 PREGNANCY SUCCESS RATES

Data verified by Zev Rosenwaks, MD

\section{Type of Cycle}

Age of Woman

35-37 38-40

$41-42^{d}$
473

35.7

31.3

41.4

(37.5-45.3)

45.1

47.7

30.0

8.2

2.2

35.4

2.0

37.1

Percentage of pregnancies with triplets or more ${ }^{b}$

Percentage of live births having multiple infants ${ }^{b, c}$

\section{Frozen Embryos from Nondonor Eggs}

Number of transfers

Percentage of transfers resulting in live births ${ }^{\mathrm{b}, \mathrm{c}}$

Average number of embryos transferred
105

32.4

1.9

\section{(27.1-35.7)}

35.7

39.1

24.3

12.5

2.7

32.5

5.9

37.8
553

32.7

24.6

(21.1-28.4)

29.2

31.0

24.8

15.7

3.2

17.7

4.4

19.9

70

32.9

2.2
35

20.0

2.0

\section{Donor Eggs}

Number of transfers

Percentage of transfers resulting in live births ${ }^{b, c}$

Average number of embryos transferred

All Ages Combined ${ }^{\mathrm{e}}$

Fresh Embryos

125

48.8

2.0
Frozen Embryos 40

37.5

2.0

\section{CURRENT CLINIC SERVICES AND PROFILE}

Current Name: Weill Medical College of Cornell University, The Center for Reproductive Medicine and Infertility

Donor egg? Yes

Donor embryo? Yes

Single women? Yes

\begin{abstract}
Gestational carriers? Yes
Cryopreservation? Yes
\end{abstract}

SART member?

Verified lab accreditation?

(See Appendix C for details.)

${ }_{b}^{a}$ Reflects patient and treatment characteristics of ART cycles performed in 2006 using fresh nondonor eggs or embryos.

b When fewer than 20 cycles are reported in an age category, rates are shown as a fraction and confidence intervals are not given. Calculating

c percentages from fractions may be misleading and is not encouraged.

d A multiple-infant birth is counted as one live birth.

d Clinic-specific outcome rates are unreliable for women older than 42 undergoing ART cycles using fresh or frozen embryos with nondonor

e eggs. Readers are urged to review national outcomes for these age groups (see page 27).

All ages (including ages $>42$ ) are reported together because previous data show that patient age does not materially affect

success with donor eggs.

- Reflects clinic performed more than 50 cycles with Preimplantation Genetic Diagnosis (PGD) in 2006 and among them more than 10 specifically for the purpose of prevention of genetic disorders. See Appendix $C$ for a complete list of clinics with symbol. 


\section{EAST COAST FERTILITY \\ PLAINVIEW, NEW YORK}

A comparison of clinic success rates may not be meaningful because patient medical characteristics and treatment approaches vary from clinic to clinic. For more details about this, along with information on how to interpret the statistics in this table, see pages $79-88$.

\section{ART CYCLE PROFILE}

\begin{tabular}{|c|c|c|c|c|c|c|c|}
\hline \multicolumn{4}{|c|}{ Type of ART } & \multicolumn{4}{|c|}{ Patient Diagnosis } \\
\hline IVF & $100 \%$ & Procedural Factors: & & Tubal factor & $9 \%$ & Other factor & $4 \%$ \\
\hline GIFT & $0 \%$ & With ICSI & $66 \%$ & Ovulatory dysfunction & $7 \%$ & Unknown factor & $24 \%$ \\
\hline ZIFT & $0 \%$ & Unstimulated & $10 \%$ & Diminished ovarian reserve & $7 \%$ & Multiple Factors: & \\
\hline \multirow[t]{3}{*}{ Combination } & $0 \%$ & Used gestational carrier & $<1 \%$ & Endometriosis & $4 \%$ & Female factors only & $12 \%$ \\
\hline & & & & Uterine factor & $<1 \%$ & Female \& male factors & $17 \%$ \\
\hline & & & & Male factor & $16 \%$ & & \\
\hline
\end{tabular}

2006 PREGNANCY SUCCESS RATES

Data verified by David Kreiner, MD

\section{Type of Cycle}

\section{Fresh Embryos from Nondonor Eggs}

Number of cycles

Percentage of cycles resulting in pregnancies ${ }^{b}$

Percentage of cycles resulting in live births ${ }^{b, c}$

(Confidence Interval)

Percentage of retrievals resulting in live births $s^{b, c}$

Percentage of transfers resulting in live births $s^{b, c}$

Percentage of transfers resulting in singleton live births ${ }^{b}$

Percentage of cancellations ${ }^{b}$

Average number of embryos transferred

Percentage of pregnancies with twins ${ }^{b}$

Percentage of pregnancies with triplets or more ${ }^{b}$

Percentage of live births having multiple infants ${ }^{b, c}$

\section{Frozen Embryos from Nondonor Eggs}

Number of transfers

Percentage of transfers resulting in live births $s^{b, c}$

Average number of embryos transferred

\section{Donor Eggs}

Number of transfers

Percentage of transfers resulting in live births $s^{b, c}$

Average number of embryos transferred

\section{Age of Woman}

35-37 38-40

$41-42^{d}$

\section{$<35$}

89

38.2

77

47

136

54.4

29.2

31.2

21.3

44.1

(20.1-39.8)

23.4

14.9

$(35.6-52.9)$

45.1

30.2

(14.5-34.4)

(6.2-28.3)

45.8

30.6

24.3

25.7

17.1

36.6

22.4

20.0

18.4

2.2

3.4

3.9

18.4

2.0

2.2

23.5

2.5

12.8

17.6

0.0

0.0

26.9

20.8

2.8

20.0

0.0

$1 / 10$

$0 / 10$

4/ 18

$0 / 7$

77

39.0

40

22.5

18

11

2.0

2.1

$5 / 18$

$3 / 11$

2.1

2.7

All Ages Combined ${ }^{e}$

Fresh Embryos 15

$6 / 15$

1.9
Frozen Embryos

\section{6}

$2 / 6$

2.2

\section{CURRENT CLINIC SERVICES AND PROFILE}

Current Name: East Coast Fertility

\begin{tabular}{l|l|l} 
Donor egg? & Yes & Gestational carriers? Yes
\end{tabular}

Donor embryo? Yes Cryopreservation? Yes

Single women? Yes

\section{SART member? Yes}

Verified lab accreditation? Yes

(See Appendix C for details.)

${ }^{a}$ Reflects patient and treatment characteristics of ART cycles performed in 2006 using fresh nondonor eggs or embryos.

${ }^{b}$ When fewer than 20 cycles are reported in an age category, rates are shown as a fraction and confidence intervals are not given. Calculating percentages from fractions may be misleading and is not encouraged.

A multiple-infant birth is counted as one live birth.

d Clinic-specific outcome rates are unreliable for women older than 42 undergoing ART cycles using fresh or frozen embryos with nondonor eggs. Readers are urged to review national outcomes for these age groups (see page 27).

e All ages (including ages $>42$ ) are reported together because previous data show that patient age does not materially affect success with donor eggs. 


\section{LONG ISLAND IVF \\ PORT JEFFERSON, NEW YORK}

A comparison of clinic success rates may not be meaningful because patient medical characteristics and treatment approaches vary from clinic to clinic. For more details about this, along with information on how to interpret the statistics in this table, see pages 79-88.

\section{ART CYCLE PROFILE}

\begin{tabular}{|c|c|c|c|c|c|c|c|}
\hline \multicolumn{4}{|c|}{ Type of ART ${ }^{a}$} & \multicolumn{4}{|c|}{ Patient Diagnosis } \\
\hline IVF & $100 \%$ & Procedural Factors: & & Tubal factor & $11 \%$ & Other factor & $10 \%$ \\
\hline GIFT & $0 \%$ & With ICSI & $77 \%$ & Ovulatory dysfunction & $7 \%$ & Unknown factor & $8 \%$ \\
\hline ZIFT & $0 \%$ & Unstimulated & $0 \%$ & Diminished ovarian reserve & $10 \%$ & Multiple Factors: & \\
\hline \multirow[t]{3}{*}{ Combination } & $0 \%$ & Used gestational carrier & $<1 \%$ & Endometriosis & $7 \%$ & Female factors only & $15 \%$ \\
\hline & & & & Uterine factor & $<1 \%$ & Female \& male factors & $15 \%$ \\
\hline & & & & Male factor & $18 \%$ & & \\
\hline
\end{tabular}

2006 PREGNANCY SUCCESS RATES

Data verified by Daniel Kenigsberg, MD

\section{Type of Cycle}

\section{Fresh Embryos from Nondonor Eggs}

Number of cycles

Percentage of cycles resulting in pregnancies ${ }^{b}$

Percentage of cycles resulting in live births ${ }^{b, c}$

(Confidence Interval)

Percentage of retrievals resulting in live births $s^{b, c}$

Percentage of transfers resulting in live births $s^{b, c}$

Percentage of transfers resulting in singleton live births ${ }^{b}$

Percentage of cancellations ${ }^{b}$

Average number of embryos transferred

Percentage of pregnancies with twins ${ }^{b}$

Percentage of pregnancies with triplets or more ${ }^{b}$

Percentage of live births having multiple infants ${ }^{\mathrm{b}, \mathrm{c}}$

\section{Frozen Embryos from Nondonor Eggs}

Number of transfers

Percentage of transfers resulting in live births $s^{b, c}$

Average number of embryos transferred

\section{Donor Eggs}

Number of transfers

Percentage of transfers resulting in live births $s^{b, c}$

Average number of embryos transferred

\section{Age of Woman}

35-37 38-40

$41-42^{d}$

$<35$

125

40.8

112

63

44.1

36.0

33.9

11.1

41.0

(27.6-45.1)

39.8

25.0

4.8

(33.3-49.0)

45.8

(17.3-34.1)

(1.0-13.3)

47.8

42.5

32.2

6.3

35.5

31.1

24.1

15.2

6.4

10.6

9.6

2.8

21.1

27.5

31.0

2.0

26.7

5.3

25.0

6.4

23.8

3.1

$0 / 7$

$0 / 7$

25.8

81

38.3

43

44.2

2.3

27

29.6

11

2.5

All Ages Combined ${ }^{\mathrm{e}}$

Fresh Embryos

60

55.0

2.0
Frozen Embryos 63

28.6

2.5

\section{CURRENT CLINIC SERVICES AND PROFILE}

Current Name: Long Island IVF

$\begin{array}{ll}\text { Donor egg? } & \text { Yes } \\ \text { Donor embryo? } & \text { Yes } \\ \text { Single women? } & \text { Yes }\end{array}$

$\begin{array}{ll}\text { Gestational carriers? } & \text { Yes } \\ \text { Cryopreservation? } & \text { Yes }\end{array}$

SART member?

Verified lab accreditation?

(See Appendix C for details.)

${ }^{a}$ Reflects patient and treatment characteristics of ART cycles performed in 2006 using fresh nondonor eggs or embryos.

${ }^{b}$ When fewer than 20 cycles are reported in an age category, rates are shown as a fraction and confidence intervals are not given. Calculating percentages from fractions may be misleading and is not encouraged.

${ }^{c}$ A multiple-infant birth is counted as one live birth.

d Clinic-specific outcome rates are unreliable for women older than 42 undergoing ART cycles using fresh or frozen embryos with nondonor eggs. Readers are urged to review national outcomes for these age groups (see page 27).

e All ages (including ages $>42$ ) are reported together because previous data show that patient age does not materially affect success with donor eggs. 


\section{ROCHESTER FERTILITY CARE, PC ROCHESTER, NEW YORK}

A comparison of clinic success rates may not be meaningful because patient medical characteristics and treatment approaches vary from clinic to clinic. For more details about this, along with information on how to interpret the statistics in this table, see pages 79-88.

\section{ART CYCLE PROFILE}

\begin{tabular}{|c|c|c|c|c|c|c|c|}
\hline \multicolumn{4}{|c|}{ Type of ART ${ }^{\mathrm{a}}$} & \multicolumn{4}{|c|}{ Patient Diagnosis } \\
\hline IVF & $100 \%$ & Procedural Factors: & & Tubal factor & $11 \%$ & Other factor & $5 \%$ \\
\hline GIFT & $0 \%$ & With ICSI & $89 \%$ & Ovulatory dysfunction & $2 \%$ & Unknown factor & $1 \%$ \\
\hline $\mathrm{ZIFT}$ & $0 \%$ & Unstimulated & $0 \%$ & Diminished ovarian reserve & $14 \%$ & Multiple Factors: & \\
\hline Combination & $0 \%$ & Used gestational carrier & $0 \%$ & Endometriosis & $4 \%$ & Female factors only & $16 \%$ \\
\hline & & & & $\begin{array}{l}\text { Uterine factor } \\
\text { Male factor }\end{array}$ & $\begin{array}{r}0 \% \\
22 \%\end{array}$ & Female \& male factors & $25 \%$ \\
\hline
\end{tabular}

2006 PREGNANCY SUCCESS RATES

Data verified by Rosalind A. Hayes, MD

\section{Type of Cycle}

\section{Fresh Embryos from Nondonor Eggs}

Number of cycles

Percentage of cycles resulting in pregnancies ${ }^{b}$

Percentage of cycles resulting in live births ${ }^{b, c}$

(Confidence Interval)

Percentage of retrievals resulting in live births $s^{b, c}$

Percentage of transfers resulting in live births $s^{b, c}$

Percentage of transfers resulting in singleton live births ${ }^{b}$

Percentage of cancellations ${ }^{b}$

Average number of embryos transferred

Percentage of pregnancies with twins ${ }^{b}$

Percentage of pregnancies with triplets or more ${ }^{b}$

\section{Frozen Embryos from Nondonor Eggs}

Number of transfers

Percentage of transfers resulting in live births $s^{\mathrm{b}, \mathrm{c}}$

Average number of embryos transferred

\section{Donor Eggs}

Number of transfers

Percentage of transfers resulting in live births ${ }^{\mathrm{b}, \mathrm{c}}$

Average number of embryos transferred
Percentage of live births having multiple infants ${ }^{b, c}$

\section{Age of Woman}

35-37 38-40

$41-42^{d}$

\section{$<35$}

21

33.3

10

0

25.0

20.8

28.6

$2 / 10$

(7.1-42.2)

(11.3-52.2)

21.7

$6 / 17$

$2 / 10$

\section{7}

$6 / 16$

$2 / 9$

18.2

$5 / 16$

$2 / 9$

4.2

19.0

$0 / 9$

3.3

$1 / 6$

2.6

$1 / 10$

$0 / 6$

$1 / 7$

2.1

$1 / 5$

$0 / 7$

$1 / 2$

$1 / 2$

$1 / 6$

$2 / 2$
8

$2 / 8$

2.5
3

$2 / 3$

1.7
4

$0 / 4$

2.0

\section{CURRENT CLINIC SERVICES AND PROFILE}

Current Name: Rochester Fertility Care, PC

Donor egg? Yes

Donor embryo? Yes

Gestational carriers? Yes

Fresh Embryos

All Ages Combined ${ }^{\mathrm{e}}$

Single women? Yes

$\begin{array}{cc}11 & 9 \\ 5 / 11 & 2 / 9 \\ 3.0 & 2.7\end{array}$

\footnotetext{
${ }^{a}$ Reflects patient and treatment characteristics of ART cycles performed in 2006 using fresh nondonor eggs or embryos.

b When fewer than 20 cycles are reported in an age category, rates are shown as a fraction and confidence intervals are not given. Calculating percentages from fractions may be misleading and is not encouraged.

A multiple-infant birth is counted as one live birth.

d Clinic-specific outcome rates are unreliable for women older than 42 undergoing ART cycles using fresh or frozen embryos with nondonor eggs. Readers are urged to review national outcomes for these age groups (see page 27).

e All ages (including ages $>42$ ) are reported together because previous data show that patient age does not materially affect success with donor eggs.
} 


\section{STRONG FERTILITY AND REPRODUCTIVE SCIENCE CENTER ROCHESTER, NEW YORK}

A comparison of clinic success rates may not be meaningful because patient medical characteristics and treatment approaches vary from clinic to clinic. For more details about this, along with information on how to interpret the statistics in this table, see pages 79-88.

\section{ART CYCLE PROFILE}

\begin{tabular}{|c|c|c|c|c|c|c|c|}
\hline \multicolumn{4}{|c|}{ Type of ART ${ }^{a}$} & \multicolumn{4}{|c|}{ Patient Diagnosis } \\
\hline IVF & $100 \%$ & Procedural Factors: & & Tubal factor & $12 \%$ & Other factor & $3 \%$ \\
\hline GIFT & $0 \%$ & With ICSI & $68 \%$ & Ovulatory dysfunction & $6 \%$ & Unknown factor & $6 \%$ \\
\hline ZIFT & $0 \%$ & Unstimulated & $0 \%$ & Diminished ovarian reserve & $5 \%$ & Multiple Factors: & \\
\hline \multirow[t]{3}{*}{ Combination } & $0 \%$ & Used gestational carrier & $0 \%$ & Endometriosis & $3 \%$ & Female factors only & $18 \%$ \\
\hline & & & & Uterine factor & $<1 \%$ & Female \& male factors & $24 \%$ \\
\hline & & & & Male factor & $23 \%$ & & \\
\hline
\end{tabular}

2006 PREGNANCY SUCCESS RATES

Data verified by Vivian Lewis, MD

\section{Type of Cycle}

\section{Age of Woman}

35-37 38-40

$41-42^{d}$

$<35$

104

35.6

50

26.0

37

4

Percentage of cycles resulting in pregnancies ${ }^{b}$

Percentage of cycles resulting in live births ${ }^{b, c}$

(Confidence Interval)

Percentage of retrievals resulting in live births ${ }^{b, c}$

Percentage of transfers resulting in live births ${ }^{b, c}$

32.7

24.0

$(23.8-42.6)$

(13.1-38.2)

33.7

26.7

34.7

27.9

18.6

10.0

2.6

2.9

2.6

21.6

5.4

$2 / 13$

26.5

$2 / 13$

21.6

16.2

(6.2-32.0)

20.7

21.4

7.1

21.6

2.5

$4 / 8$

$4 / 12$

$1 / 8$

$4 / 6$

35

31.4

$$
19
$$

$7 / 19$

2.4

10
$1 / 10$
2.0

1

$0 / 1$

3.0

Average number of embryos transferred

2.5

All Ages Combined ${ }^{\mathrm{e}}$

\section{Donor Eggs}

Number of transfers

Percentage of transfers resulting in live births $s^{b, c}$

Average number of embryos transferred
Fresh Embryos 26

53.8

2.2
Frozen Embryos 14

$3 / 14$

2.1

\section{CURRENT CLINIC SERVICES AND PROFILE}

Current Name: This clinic has closed or reorganized since 2006. Information on current clinic services and profile therefore is not provided here. Contact the NASS Help Desk for current information about this clinic.

\footnotetext{
${ }^{a}$ Reflects patient and treatment characteristics of ART cycles performed in 2006 using fresh nondonor eggs or embryos.

${ }^{b}$ When fewer than 20 cycles are reported in an age category, rates are shown as a fraction and confidence intervals are not given. Calculating percentages from fractions may be misleading and is not encouraged.

${ }^{\mathrm{C}}$ A multiple-infant birth is counted as one live birth.

d Clinic-specific outcome rates are unreliable for women older than 42 undergoing ART cycles using fresh or frozen embryos with nondonor eggs. Readers are urged to review national outcomes for these age groups (see page 27).

e All ages (including ages $>42$ ) are reported together because previous data show that patient age does not materially affect success with donor eggs.
} 


\section{ISLAND REPRODUCTIVE SERVICES STATEN ISLAND, NEW YORK}

A comparison of clinic success rates may not be meaningful because patient medical characteristics and treatment approaches vary from clinic to clinic. For more details about this, along with information on how to interpret the statistics in this table, see pages $79-88$.

\section{ART CYCLE PROFILE}

\begin{tabular}{|c|c|c|c|c|c|c|c|}
\hline \multicolumn{4}{|c|}{ Type of ART ${ }^{\mathrm{a}}$} & \multicolumn{4}{|c|}{ Patient Diagnosis } \\
\hline IVF & $100 \%$ & Procedural Factors: & & Tubal factor & $<1 \%$ & Other factor & $2 \%$ \\
\hline GIFT & $0 \%$ & With ICSI & $54 \%$ & Ovulatory dysfunction & $2 \%$ & Unknown factor & $0 \%$ \\
\hline $\mathrm{ZIFT}$ & $0 \%$ & Unstimulated & $0 \%$ & Diminished ovarian reserve & $0 \%$ & Multiple Factors: & \\
\hline Combination & $0 \%$ & Used gestational carrier & $0 \%$ & Endometriosis & $2 \%$ & Female factors only & $28 \%$ \\
\hline & & & & $\begin{array}{l}\text { Uterine factor } \\
\text { Male factor }\end{array}$ & $\begin{array}{l}0 \% \\
9 \%\end{array}$ & Female \& male factors & $57 \%$ \\
\hline
\end{tabular}

2006 PREGNANCY SUCCESS RATES

Data verified by Eric S. Knochenhauer, MD

\section{Type of Cycle}

\section{Fresh Embryos from Nondonor Eggs}

Number of cycles

Percentage of cycles resulting in pregnancies ${ }^{b}$

Percentage of cycles resulting in live births ${ }^{b, c}$

(Confidence Interval)

Percentage of retrievals resulting in live births $s^{b, c}$

Percentage of transfers resulting in live births $s^{b, c}$

Percentage of transfers resulting in singleton live births ${ }^{b}$

Percentage of cancellations ${ }^{b}$

Average number of embryos transferred

Percentage of pregnancies with twins ${ }^{b}$

Percentage of pregnancies with triplets or more ${ }^{b}$

Percentage of live births having multiple infants ${ }^{b, c}$

\section{Frozen Embryos from Nondonor Eggs}

Number of transfers

Percentage of transfers resulting in live births $s^{b, c}$

Average number of embryos transferred

\section{Donor Eggs}

Number of transfers

Percentage of transfers resulting in live births ${ }^{\mathrm{b}, \mathrm{c}}$

Average number of embryos transferred

\section{Age of Woman}

$<35 \quad 35-37 \quad 38-40 \quad 41-42^{d}$

$\begin{array}{cccc}44 & 20 & 23 & 8 \\ 54.5 & 70.0 & 26.1 & 1 / 8 \\ 47.7 & 60.0 & 21.7 & 0 / 8\end{array}$

(32.5-63.3)

(36.1-80.9)

(7.5-43.7)

50.0

$12 / 18$

$5 / 17$

$0 / 5$

50.0

$12 / 18$

$5 / 17$

$0 / 5$

42.9

$8 / 18$

4 / 17

$0 / 5$

4.5

10.0

26.1

$3 / 8$

2.9

3.4

3.2

4.2

12.5

3 / 14

0.0

$2 / 14$

$3 / 6$

$0 / 1$

14.3

4 / 12

$0 / 6$

$0 / 1$

$1 / 5$

$\begin{array}{cccc}13 & 1 & 2 & 1 \\ 8 / 13 & 1 / 1 & 0 / 2 & 1 / 1 \\ 3.8 & 3.0 & 3.5 & 4.0\end{array}$

\section{CURRENT CLINIC SERVICES AND PROFILE}

Current Name: Island Reproductive Services

\begin{tabular}{l|l|l} 
Donor egg? Yes & Gestational carriers? No
\end{tabular}

Donor embryo? No Cryopreservation? Yes

All Ages Combined ${ }^{\mathrm{e}}$

Fresh Embryos

0
Frozen Embryos

0

Single women? Yes

SART member? Yes

Verified lab accreditation? Yes

(See Appendix C for details.)

${ }^{a}$ Reflects patient and treatment characteristics of ART cycles performed in 2006 using fresh nondonor eggs or embryos.

${ }^{b}$ When fewer than 20 cycles are reported in an age category, rates are shown as a fraction and confidence intervals are not given. Calculating percentages from fractions may be misleading and is not encouraged.

A multiple-infant birth is counted as one live birth.

d Clinic-specific outcome rates are unreliable for women older than 42 undergoing ART cycles using fresh or frozen embryos with nondonor eggs. Readers are urged to review national outcomes for these age groups (see page 27).

e All ages (including ages $>42$ ) are reported together because previous data show that patient age does not materially affect success with donor eggs. 


\section{GOLD COAST IVF \\ REPRODUCTIVE MEDICINE AND SURGERY CENTER \\ SYOSSET, NEW YORK}

A comparison of clinic success rates may not be meaningful because patient medical characteristics and treatment approaches vary from clinic to clinic. For more details about this, along with information on how to interpret the statistics in this table, see pages 79-88.

\section{ART CYCLE PROFILE}

\begin{tabular}{|c|c|c|c|c|c|c|c|}
\hline \multicolumn{4}{|c|}{ Type of ART ${ }^{\mathrm{a}}$} & \multicolumn{4}{|c|}{ Patient Diagnosis } \\
\hline IVF & $100 \%$ & Procedural Factors: & & Tubal factor & $5 \%$ & Other factor & $0 \%$ \\
\hline GIFT & $0 \%$ & With ICSI & $71 \%$ & Ovulatory dysfunction & $5 \%$ & Unknown factor & $3 \%$ \\
\hline ZIFT & $0 \%$ & Unstimulated & $0 \%$ & Diminished ovarian reserve & $3 \%$ & Multiple Factors: & \\
\hline \multirow[t]{3}{*}{ Combination } & $0 \%$ & Used gestational carrier & $0 \%$ & Endometriosis & $4 \%$ & Female factors only & $20 \%$ \\
\hline & & & & Uterine factor & $0 \%$ & Female \& male factors & $50 \%$ \\
\hline & & & & Male factor & $11 \%$ & & \\
\hline
\end{tabular}

\section{PREGNANCY SUCCESS RATES}

Data verified by Steven F. Palter, MD

\section{Type of Cycle}

\section{Fresh Embryos from Nondonor Eggs}

Number of cycles

Percentage of cycles resulting in pregnancies ${ }^{b}$

Percentage of cycles resulting in live births ${ }^{b, c}$

(Confidence Interval)

Percentage of retrievals resulting in live births $s^{b, c}$

Percentage of transfers resulting in live births $s^{b, c}$

Percentage of transfers resulting in singleton live births ${ }^{b}$

Percentage of cancellations ${ }^{b}$

Average number of embryos transferred

Percentage of pregnancies with twins ${ }^{b}$

Percentage of pregnancies with triplets or more ${ }^{b}$

Percentage of live births having multiple infants ${ }^{b, c}$

\section{Frozen Embryos from Nondonor Eggs}

Number of transfers

Percentage of transfers resulting in live births ${ }^{b, c}$

Average number of embryos transferred

\section{Donor Eggs}

Number of transfers

Percentage of transfers resulting in live births $s^{b, c}$

Average number of embryos transferred

\section{Age of Woman}

$\begin{array}{cccc}<35 & \mathbf{3 5 - 3 7} & \mathbf{3 8 - 4 0} & \mathbf{4 1 - 4 2}^{\mathbf{d}} \\ 28 & 18 & 14 & 4 \\ 57.1 & 10 / 18 & 7 / 14 & 0 / 4 \\ 57.1 & 7 / 18 & 6 / 14 & 0 / 4\end{array}$

(37.2-75.5)

59.3

$7 / 16$

$6 / 13$

$0 / 3$

64.0

$7 / 16$

$6 / 13$

$0 / 3$

36.0

$6 / 16$

$4 / 13$

$0 / 3$

3.6

$2 / 18$

$1 / 14$

$1 / 4$

2.8

2.7

3.8

3.0

$8 / 16$

$1 / 10$

$3 / 7$

$0 / 16$

$0 / 10$

$1 / 7$

$7 / 16$

$1 / 7$

$2 / 6$

1

$1 / 1$

4.0

\section{1}

$1 / 1$

4.0
1

$0 / 1$

5.0

\section{D}




\section{CNY FERTILITY CENTER \\ SYRACUSE, NEW YORK}

A comparison of clinic success rates may not be meaningful because patient medical characteristics and treatment approaches vary from clinic to clinic. For more details about this, along with information on how to interpret the statistics in this table, see pages $79-88$.

\section{ART CYCLE PROFILE}

\begin{tabular}{|c|c|c|c|c|c|c|c|}
\hline \multicolumn{4}{|c|}{ Type of ART } & \multicolumn{4}{|c|}{ Patient Diagnosis } \\
\hline IVF & $100 \%$ & Procedural Factors: & & Tubal factor & $13 \%$ & Other factor & $5 \%$ \\
\hline GIFT & $0 \%$ & With ICSI & $89 \%$ & Ovulatory dysfunction & $8 \%$ & Unknown factor & $12 \%$ \\
\hline ZIFT & $0 \%$ & Unstimulated & $<1 \%$ & Diminished ovarian reserve & $16 \%$ & Multiple Factors: & \\
\hline \multirow[t]{3}{*}{ Combination } & $0 \%$ & Used gestational carrier & $<1 \%$ & Endometriosis & $5 \%$ & Female factors only & $16 \%$ \\
\hline & & & & Uterine factor & $<1 \%$ & Female \& male factors & $13 \%$ \\
\hline & & & & Male factor & $12 \%$ & & \\
\hline
\end{tabular}

2006 PREGNANCY SUCCESS RATES

Data verified by Robert J. Kiltz, MD

\section{Type of Cycle}

\section{Fresh Embryos from Nondonor Eggs}

Number of cycles

Percentage of cycles resulting in pregnancies ${ }^{b}$

Percentage of cycles resulting in live births ${ }^{b, c}$

(Confidence Interval)

Percentage of retrievals resulting in live births $s^{b, c}$

Percentage of transfers resulting in live births $s^{b, c}$

Percentage of transfers resulting in singleton live births ${ }^{b}$

Percentage of cancellations ${ }^{b}$

Average number of embryos transferred

Percentage of pregnancies with twins ${ }^{b}$

Percentage of pregnancies with triplets or more ${ }^{b}$

Percentage of live births having multiple infants ${ }^{b, c}$

\section{Frozen Embryos from Nondonor Eggs}

Number of transfers

Percentage of transfers resulting in live births $s^{b, c}$

Average number of embryos transferred

\section{Donor Eggs}

Number of transfers

Percentage of transfers resulting in live births ${ }^{\mathrm{b}, \mathrm{c}}$

Average number of embryos transferred

\section{Age of Woman}

35-37 38-40

$41-42^{d}$

\section{$<35$}

234

184

89

429

41.0

35.9

26.1

12.4

35.2

26.9

19.6

5.6

$(30.7-39.9)$

(21.4-33.1)

(14.1-26.0)

$(1.8-12.6)$

37.7

39.1

30.4

22.8

6.3

25.4

20.8

25.5

7.5

6.5

6.8

21.3

4.5

2.6

2.7

14.1

11.2

31.3

31.0

2.9

3.6

5.7

35.1

7.1

16.7

$3 / 11$

0.0

$0 / 11$

31.7

16.7

$2 / 5$

141

58

12.1

37

11

7.8

2.3

10.8

$1 / 11$

2.4

All Ages Combined ${ }^{\mathrm{e}}$

Fresh Embryos

125

45.6

2.7
Frozen Embryos 33

9.1

2.5

\section{CURRENT CLINIC SERVICES AND PROFILE}

Current Name: CNY Fertility Center

\begin{tabular}{l|l|l} 
Donor egg? Yes & Gestational carriers? Yes
\end{tabular}

Donor embryo? No Cryopreservation? Yes

Single women? Yes

\section{SART member? Yes}

Verified lab accreditation? Yes

(See Appendix C for details.)

${ }^{a}$ Reflects patient and treatment characteristics of ART cycles performed in 2006 using fresh nondonor eggs or embryos.

${ }^{b}$ When fewer than 20 cycles are reported in an age category, rates are shown as a fraction and confidence intervals are not given. Calculating percentages from fractions may be misleading and is not encouraged.

A multiple-infant birth is counted as one live birth.

d Clinic-specific outcome rates are unreliable for women older than 42 undergoing ART cycles using fresh or frozen embryos with nondonor eggs. Readers are urged to review national outcomes for these age groups (see page 27).

e All ages (including ages $>42$ ) are reported together because previous data show that patient age does not materially affect success with donor eggs. 


\section{WESTCHESTER FERTILITY AND REPRODUCTIVE ENDOCRINOLOGY WHITE PLAINS, NEW YORK}

A comparison of clinic success rates may not be meaningful because patient medical characteristics and treatment approaches vary from clinic to clinic. For more details about this, along with information on how to interpret the statistics in this table, see pages 79-88.

\section{ART CYCLE PROFILE}

\begin{tabular}{l|r|lrlrl}
\multicolumn{3}{c}{ Type of ART } & \multicolumn{4}{c}{ Patient Diagnosis } \\
IVF & $100 \%$ Procedural Factors: & & Tubal factor & $14 \%$ & Other factor & $0 \%$ \\
GIFT & $0 \%$ With ICSI & $44 \%$ & Ovulatory dysfunction & $5 \%$ & Unknown factor & $6 \%$ \\
ZIFT & $0 \%$ Unstimulated & $0 \%$ & Diminished ovarian reserve & $7 \%$ & Multiple Factors: & \\
Combination & $0 \%$ Used gestational carrier & $0 \%$ & Endometriosis & $5 \%$ & Female factors only & $32 \%$ \\
& & & Uterine factor & $3 \%$ & Female \& male factors & $21 \%$ \\
& & & Male factor & $8 \%$ & &
\end{tabular}

\section{PREGNANCY SUCCESS RATES}

\section{Type of Cycle}

\section{Fresh Embryos from Nondonor Eggs}

Number of cycles

Percentage of cycles resulting in pregnancies ${ }^{b}$

Percentage of cycles resulting in live births ${ }^{b, c}$

(Confidence Interval)

Percentage of retrievals resulting in live births $s^{b, c}$

Percentage of transfers resulting in live births $s^{b, c}$

Percentage of transfers resulting in singleton live births ${ }^{b}$

Percentage of cancellations ${ }^{b}$

Average number of embryos transferred

Percentage of pregnancies with twins ${ }^{b}$

Percentage of pregnancies with triplets or more ${ }^{b}$

Percentage of live births having multiple infants ${ }^{b, c}$

\section{Frozen Embryos from Nondonor Eggs}

Number of transfers

Percentage of transfers resulting in live births $s^{b, c}$

Average number of embryos transferred

\section{Donor Eggs}

Number of transfers

Percentage of transfers resulting in live births $s^{b, c}$

Average number of embryos transferred
Data verified by Michael B. Blotner, MD

\section{Age of Woman}

35-37 38-40

$41-42^{d}$

\section{$<35$}

25

40.0

15

8

36.7

32.0

$3 / 15$

$0 / 8$

26.7

(12.3-45.9)

$(14.9-53.5)$

29.6

33.3

$2 / 15$

$0 / 8$

34.8

34.8

$2 / 12$

$0 / 2$

26.1

34.8

$2 / 12$

$0 / 2$

10.0

4.0

$2 / 12$

$0 / 2$

2.5

2.6

$3 / 15$

$6 / 8$

$2 / 11$

$2 / 10$

2.8

2.0

$0 / 3$

$1 / 10$

$0 / 11$

$2 / 8$

$0 / 8$

$0 / 3$

$0 / 2$

$\begin{array}{cccc}9 & 8 & 1 & 2 \\ 2 / 9 & 1 / 8 & 0 / 1 & 0 / 2 \\ 2.1 & 2.5 & 2.0 & 2.0\end{array}$

\section{All Ages Combined}

Fresh Embryos

0
Frozen Embryos

2

$0 / 2$

1.5

\section{CURRENT CLINIC SERVICES AND PROFILE}

Current Name: Westchester Fertility and Reproductive Endocrinology

Donor egg? Yes Gestational carriers? No

Donor embryo? No Cryopreservation? Yes

Single women? Yes

SART member? Yes

Verified lab accreditation? Yes

(See Appendix C for details.)

${ }^{a}$ Reflects patient and treatment characteristics of ART cycles performed in 2006 using fresh nondonor eggs or embryos.

b When fewer than 20 cycles are reported in an age category, rates are shown as a fraction and confidence intervals are not given. Calculating percentages from fractions may be misleading and is not encouraged.

${ }^{c}$ A multiple-infant birth is counted as one live birth.

d Clinic-specific outcome rates are unreliable for women older than 42 undergoing ART cycles using fresh or frozen embryos with nondonor eggs. Readers are urged to review national outcomes for these age groups (see page 27).

e All ages (including ages $>42$ ) are reported together because previous data show that patient age does not materially affect success with donor eggs. 


\section{NORTH CAROLINA CENTER FOR REPRODUCTIVE MEDICINE THE TALBERT FERTILITY INSTITUTE CARY, NORTH CAROLINA}

A comparison of clinic success rates may not be meaningful because patient medical characteristics and treatment approaches vary from clinic to clinic. For more details about this, along with information on how to interpret the statistics in this table, see pages 79-88.

\section{ART CYCLE PROFILE}

\begin{tabular}{|c|c|c|c|c|c|c|c|}
\hline \multicolumn{4}{|c|}{ Type of ART } & \multicolumn{4}{|c|}{ Patient Diagnosis } \\
\hline IVF & $100 \%$ & Procedural Factors: & & Tubal factor & $8 \%$ & Other factor & $12 \%$ \\
\hline GIFT & $0 \%$ & With ICSI & $55 \%$ & Ovulatory dysfunction & $8 \%$ & Unknown factor & $10 \%$ \\
\hline ZIFT & $0 \%$ & Unstimulated & $0 \%$ & Diminished ovarian reserve & $9 \%$ & Multiple Factors: & \\
\hline \multirow[t]{3}{*}{ Combination } & $0 \%$ & Used gestational carrier & $0 \%$ & Endometriosis & $9 \%$ & Female factors only & $5 \%$ \\
\hline & & & & Uterine factor & $4 \%$ & Female \& male factors & $10 \%$ \\
\hline & & & & Male factor & $24 \%$ & & \\
\hline
\end{tabular}

2006 PREGNANCY SUCCESS RATES

Data verified by Sameh K. Toma, MD

\section{Type of Cycle}

\section{Fresh Embryos from Nondonor Eggs}

Number of cycles

Percentage of cycles resulting in pregnancies ${ }^{b}$

Percentage of cycles resulting in live births ${ }^{b, c}$

(Confidence Interval)

Percentage of retrievals resulting in live births $s^{b, c}$

Percentage of transfers resulting in live births $s^{b, c}$

Percentage of transfers resulting in singleton live births ${ }^{b}$

Percentage of cancellations ${ }^{b}$

Average number of embryos transferred

Percentage of pregnancies with twins ${ }^{b}$

Percentage of pregnancies with triplets or more ${ }^{b}$

Percentage of live births having multiple infants ${ }^{b, c}$

\section{Frozen Embryos from Nondonor Eggs}

Number of transfers

Percentage of transfers resulting in live births $s^{b, c}$

Average number of embryos transferred

\section{Donor Eggs}

Number of transfers

Percentage of transfers resulting in live births ${ }^{\mathrm{b}, \mathrm{c}}$

Average number of embryos transferred

\section{Age of Woman}

35-37 38-40

$41-42^{d}$

\section{$<35$}

85

34.1

77

21

38.4

32.9

10.4

19.0

36.8

(23.1-44.0)

10.4

9.5

$(29.8-44.1)$

39.3

40.0

36.4

(4.6-19.4)

(1.2-30.4)

30.0

37.3

13.1

13.1

$2 / 16$

26.7

9.8

$2 / 14$

6.5

9.4

20.8

$1 / 14$

2.8

21.1

2.8

3.1

3.3

23.8

3.4

27.6

0.0

28.6

$3 / 8$

$1 / 4$

25.0

$0 / 8$

$0 / 4$

$2 / 8$

$1 / 2$

41

26.8

3.0

\section{3}

$4 / 13$

3.1

\section{6 \\ $1 / 6$}

2.8
2

$1 / 2$

2.5

All Ages Combined

Fresh Embryos 58

51.7

2.7
Frozen Embryos 17

$4 / 17$

2.7

\section{CURRENT CLINIC SERVICES AND PROFILE}

Current Name: North Carolina Center for Reproductive Medicine, The Talbert Fertility Institute

Donor egg? Yes

Donor embryo? Yes

Single women? Yes

\begin{abstract}
Gestational carriers? Yes
Cryopreservation?
\end{abstract}

SART member?

Verified lab accreditation?

(See Appendix C for details.)

${ }^{a}$ Reflects patient and treatment characteristics of ART cycles performed in 2006 using fresh nondonor eggs or embryos.

${ }^{b}$ When fewer than 20 cycles are reported in an age category, rates are shown as a fraction and confidence intervals are not given. Calculating percentages from fractions may be misleading and is not encouraged.

${ }^{c}$ A multiple-infant birth is counted as one live birth.

d Clinic-specific outcome rates are unreliable for women older than 42 undergoing ART cycles using fresh or frozen embryos with nondonor eggs. Readers are urged to review national outcomes for these age groups (see page 27).

e All ages (including ages $>42$ ) are reported together because previous data show that patient age does not materially affect success with donor eggs. 


\section{UNIVERSITY OF NORTH CAROLINA A.R.T. CLINIC CHAPEL HILL, NORTH CAROLINA}

A comparison of clinic success rates may not be meaningful because patient medical characteristics and treatment approaches vary from clinic to clinic. For more details about this, along with information on how to interpret the statistics in this table, see pages 79-88.

\section{ART CYCLE PROFILE}

\section{Type of ART ${ }^{\mathrm{a}}$}

IVF

GIFT $100 \%$ Procedural Factors:

ZIFT

Combination
$0 \%$ With ICSI

$0 \%$ Unstimulated

$0 \%$ Used gestational carrier

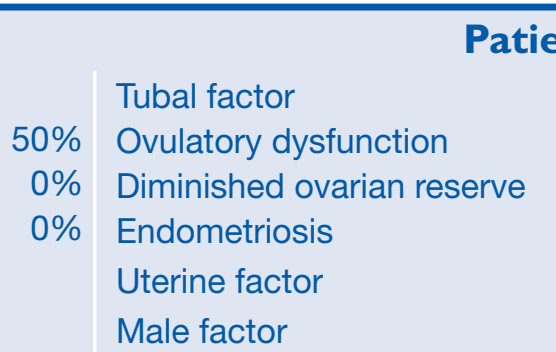

Patient Diagnosis

$\begin{array}{rlr}10 \% & \text { Other factor } & 3 \% \\ 14 \% & \text { Unknown factor } & 9 \% \\ 4 \% & \text { Multiple Factors: } & \\ 6 \% & \text { Female factors only } & 5 \% \\ 2 \% & \text { Female \& male factors } & 22 \% \\ 24 \% & & \end{array}$

Data verified by Marc A. Fritz, MD

2006 PREGNANCY SUCCESS RATES

\section{Age of Woman}

\section{Type of Cycle}

\section{Fresh Embryos from Nondonor Eggs}

Number of cycles

Percentage of cycles resulting in pregnancies ${ }^{b}$

Percentage of cycles resulting in live births ${ }^{b, c}$

(Confidence Interval)

Percentage of retrievals resulting in live births $s^{b, c}$

Percentage of transfers resulting in live births $s^{b, c}$

Percentage of transfers resulting in singleton live births ${ }^{b}$

Percentage of cancellations ${ }^{b}$

Average number of embryos transferred

Percentage of pregnancies with twins ${ }^{b}$

Percentage of pregnancies with triplets or more ${ }^{b}$

Percentage of live births having multiple infants ${ }^{b, c}$

\section{Frozen Embryos from Nondonor Eggs}

Number of transfers

Percentage of transfers resulting in live births ${ }^{b, c}$

Average number of embryos transferred

\section{Donor Eggs}

Number of transfers

Percentage of transfers resulting in live births $s^{\mathrm{b}, \mathrm{c}}$

Average number of embryos transferred

All Ages Combined ${ }^{\mathrm{e}}$
Fresh Embryos 14

$8 / 14$

2.0

$<35 \quad 35-37 \quad 38-40 \quad 41-42^{d}$

$\begin{array}{cccc}94 & 49 & 28 & 12 \\ 47.9 & 24.5 & 17.9 & 2 / 12 \\ 45.7 & 22.4 & 7.1 & 1 / 12\end{array}$

(35.4-56.3)

(11.8-36.6)

$(0.9-23.5)$

59.7

61.4

34.4

$2 / 16$

$1 / 9$

35.5

$2 / 16$

$1 / 9$

22.6

$1 / 16$

$1 / 9$

23.4

34.7

42.9

$3 / 12$

2.1

2.9

3.4

3.6

37.8

$2 / 12$

0.0

$2 / 12$

$1 / 5$

$0 / 2$

$0 / 5$

$0 / 2$

30.2

$4 / 11$

$1 / 2$

$0 / 1$

32

28.1

\section{5}

$1 / 5$

3.0

\section{5}

$2 / 5$

2.0

1

$0 / 1$

3.0

\section{CURRENT CLINIC SERVICES AND PROFILE}

Current Name: University of North Carolina A.R.T. Clinic

Donor egg? Yes

Donor embryo? No

Single women? Yes

\section{Gestational carriers? Yes}

Cryopreservation? Yes
Frozen Embryos 9

$3 / 9$

2.4 (1)

\footnotetext{
${ }^{a}$ Reflects patient and treatment characteristics of ART cycles performed in 2006 using fresh nondonor eggs or embryos.

${ }^{b}$ When fewer than 20 cycles are reported in an age category, rates are shown as a fraction and confidence intervals are not given. Calculating percentages from fractions may be misleading and is not encouraged.

${ }^{c}$ A multiple-infant birth is counted as one live birth.

d Clinic-specific outcome rates are unreliable for women older than 42 undergoing ART cycles using fresh or frozen embryos with nondonor eggs. Readers are urged to review national outcomes for these age groups (see page 27).

e All ages (including ages $>42$ ) are reported together because previous data show that patient age does not materially affect success with donor eggs.
} 


\section{INSTITUTE FOR ASSISTED REPRODUCTION CHARLOTTE, NORTH CAROLINA}

A comparison of clinic success rates may not be meaningful because patient medical characteristics and treatment approaches vary from clinic to clinic. For more details about this, along with information on how to interpret the statistics in this table, see pages 79-88.

\section{ART CYCLE PROFILE}

\begin{tabular}{|c|c|c|c|c|c|c|c|}
\hline \multicolumn{4}{|c|}{ Type of ART } & \multicolumn{4}{|c|}{ Patient Diagnosis } \\
\hline IVF & $100 \%$ & Procedural Factors: & & Tubal factor & $18 \%$ & Other factor & $15 \%$ \\
\hline GIFT & $0 \%$ & With ICSI & $81 \%$ & Ovulatory dysfunction & $9 \%$ & Unknown factor & $15 \%$ \\
\hline ZIFT & $0 \%$ & Unstimulated & $<1 \%$ & Diminished ovarian reserve & $10 \%$ & Multiple Factors: & \\
\hline \multirow[t]{3}{*}{ Combination } & $0 \%$ & Used gestational carrier & $1 \%$ & Endometriosis & $12 \%$ & Female factors only & $<1 \%$ \\
\hline & & & & Uterine factor & $1 \%$ & Female \& male factors & $<1 \%$ \\
\hline & & & & Male factor & $20 \%$ & & \\
\hline
\end{tabular}

2006 PREGNANCY SUCCESS RATES

Data verified by Jack L. Crain, MD

\section{Type of Cycle}

\section{Fresh Embryos from Nondonor Eggs}

Number of cycles

Percentage of cycles resulting in pregnancies ${ }^{b}$

Percentage of cycles resulting in live births $s^{b, c}$

(Confidence Interval)

Percentage of retrievals resulting in live births $s^{b, c}$

Percentage of transfers resulting in live births $s^{b, c}$

Percentage of transfers resulting in singleton live births ${ }^{b}$

Percentage of cancellations ${ }^{b}$

Average number of embryos transferred

Percentage of pregnancies with twins ${ }^{b}$

Percentage of pregnancies with triplets or more ${ }^{b}$

Percentage of live births having multiple infants ${ }^{b, c}$

\section{Frozen Embryos from Nondonor Eggs}

Number of transfers

Percentage of transfers resulting in live births ${ }^{\mathrm{b}, \mathrm{c}}$

Average number of embryos transferred

\section{Donor Eggs}

Number of transfers

Percentage of transfers resulting in live births ${ }^{\mathrm{b}, \mathrm{c}}$

Average number of embryos transferred

\section{Age of Woman}

35-37 38-40

$41-42^{d}$

\section{$<35$}

154

87

26

54.4

45.5

37.9

26.9

48.1

37.7

31.0

11.5

$(42.1-54.0)$

(30.0-45.8)

(21.5-41.9)

(2.4-30.2)

51.3

43.0

37.0

13.6

56.6

47.2

39.1

$3 / 18$

35.1

32.5

30.4

$3 / 18$

6.3

12.3

16.1

15.4

2.1

2.3

36.8

32.9

2.7

2.4

3.2

0.0

21.2

$0 / 7$

38.0

31.0

3.0

$0 / 7$

22.2

$0 / 3$

69

43.5

29

37.9

13

$1 / 13$

2

2.0

1.8

1.8

$0 / 2$

2.5

All Ages Combined ${ }^{\mathrm{e}}$

Fresh Embryos 38

55.3

2.1
Frozen Embryos 15

$6 / 15$

1.7

\section{CURRENT CLINIC SERVICES AND PROFILE}

Current Name: Institute for Assisted Reproduction

Donor egg? Yes

Donor embryo? No

Gestational carriers? Yes

Cryopreservation? Yes

Single women? Yes

SART member?

Yes

Verified lab accreditation?

(See Appendix C for details.)

${ }^{a}$ Reflects patient and treatment characteristics of ART cycles performed in 2006 using fresh nondonor eggs or embryos.

${ }^{b}$ When fewer than 20 cycles are reported in an age category, rates are shown as a fraction and confidence intervals are not given. Calculating percentages from fractions may be misleading and is not encouraged.

${ }^{c}$ A multiple-infant birth is counted as one live birth.

d Clinic-specific outcome rates are unreliable for women older than 42 undergoing ART cycles using fresh or frozen embryos with nondonor eggs. Readers are urged to review national outcomes for these age groups (see page 27).

${ }^{e}$ All ages (including ages $>42$ ) are reported together because previous data show that patient age does not materially affect success with donor eggs. 


\section{PROGRAM FOR ASSISTED REPRODUCTION, CAROLINAS MEDICAL CENTER CHARLOTTE, NORTH CAROLINA}

A comparison of clinic success rates may not be meaningful because patient medical characteristics and

treatment approaches vary from clinic to clinic. For more details about this, along with information on how to interpret the statistics in this table, see pages $79-88$.

\section{ART CYCLE PROFILE}

\begin{tabular}{|c|c|c|c|c|c|c|c|}
\hline \multicolumn{4}{|c|}{ Type of ART ${ }^{a}$} & \multicolumn{4}{|c|}{ Patient Diagnosis } \\
\hline IVF & $>99 \%$ & Procedural Factors: & & Tubal factor & $5 \%$ & Other factor & $6 \%$ \\
\hline GIFT & $<1 \%$ & With ICSI & $67 \%$ & Ovulatory dysfunction & $6 \%$ & Unknown factor & $11 \%$ \\
\hline ZIFT & $0 \%$ & Unstimulated & $0 \%$ & Diminished ovarian reserve & $2 \%$ & Multiple Factors: & \\
\hline \multirow[t]{3}{*}{ Combination } & $0 \%$ & Used gestational carrier & $0 \%$ & Endometriosis & $4 \%$ & Female factors only & $26 \%$ \\
\hline & & & & Uterine factor & $0 \%$ & Female \& male factors & $26 \%$ \\
\hline & & & & Male factor & $15 \%$ & & \\
\hline
\end{tabular}

2006 PREGNANCY SUCCESS RATES

Data verified by Bradley S. Hurst, MD

\section{Type of Cycle}

Age of Woman

35-37 38-40

\section{Fresh Embryos from Nondonor Eggs}

Number of cycles

Percentage of cycles resulting in pregnancies ${ }^{b}$

Percentage of cycles resulting in live births ${ }^{b, c}$

(Confidence Interval)

Percentage of retrievals resulting in live births $s^{b, c}$

Percentage of transfers resulting in live births $s^{b, c}$

Percentage of transfers resulting in singleton live births ${ }^{b}$

Percentage of cancellations ${ }^{b}$

$<35$

$\begin{array}{cccc}68 & 34 & 23 & 8 \\ 44.1 & 35.3 & 21.7 & 1 / 8 \\ 39.7 & 23.5 & 17.4 & 0 / 8\end{array}$

$(28.0-52.3)$

41.5

$(10.7-41.2)$

(5.0-38.8)

41.5

26.7

26.7

$4 / 19$

$0 / 7$

27.7

26.7

$4 / 19$

$4 / 19$

$0 / 7$

4.4

11.8

17.4

3.0

$4 / 12$

Percentage of pregnancies with twins ${ }^{\mathrm{b}}$

Percentage of pregnancies with triplets or more ${ }^{b}$

Percentage of live births having multiple infants ${ }^{b, c}$

Frozen Embryos from Nondonor Eggs

Number of transfers

Percentage of transfers resulting in live births $s^{\mathrm{b}, \mathrm{c}}$

Average number of embryos transferred

\section{Donor Eggs}

Number of transfers

Percentage of transfers resulting in live births $s^{b, c}$

Average number of embryos transferred
33.3

\section{3}

33.3

$0 / 12$

$0 / 8$

$0 / 5$

$0 / 5$

$0 / 4$

20

45.0

2.1

\section{4}

$3 / 14$

2.4
10

$1 / 10$

2.7
All Ages Combined ${ }^{\mathrm{e}}$
Fresh Embryos 5

$3 / 5$

2.0
Frozen Embryos 8

$2 / 8$

2.5

\section{CURRENT CLINIC SERVICES AND PROFILE}

Current Name: Program for Assisted Reproduction, Carolinas Medical Center

\begin{tabular}{|c|c|c|c|c|}
\hline Donor egg? & Yes & Gestational carriers? & Yes & SART member? \\
\hline Donor embryo? & Yes & Cryopreservation? & Yes & Verified lab accreditation? \\
\hline Single women? & Yes & & & (See Appendix C for details. \\
\hline
\end{tabular}

\footnotetext{
${ }^{a}$ Reflects patient and treatment characteristics of ART cycles performed in 2006 using fresh nondonor eggs or embryos.

b When fewer than 20 cycles are reported in an age category, rates are shown as a fraction and confidence intervals are not given. Calculating percentages from fractions may be misleading and is not encouraged.

${ }^{c}$ A multiple-infant birth is counted as one live birth.

${ }^{d}$ Clinic-specific outcome rates are unreliable for women older than 42 undergoing ART cycles using fresh or frozen embryos with nondonor eggs. Readers are urged to review national outcomes for these age groups (see page 27).

e All ages (including ages $>42$ ) are reported together because previous data show that patient age does not materially affect success with donor eggs.
} 


\section{DUKE FERTILITY CENTER \\ DUKE UNIVERSITY MEDICAL CENTER \\ DURHAM, NORTH CAROLINA}

A comparison of clinic success rates may not be meaningful because patient medical characteristics and treatment approaches vary from clinic to clinic. For more details about this, along with information on how to interpret the statistics in this table, see pages 79-88.

\section{ART CYCLE PROFILE}

\begin{tabular}{|c|c|c|c|c|c|c|c|}
\hline \multicolumn{4}{|c|}{ Type of ART } & \multicolumn{4}{|c|}{ Patient Diagnosis } \\
\hline IVF & & Procedural Factors: & & Tubal factor & $13 \%$ & Other factor & $6 \%$ \\
\hline GIFT & $0 \%$ & With ICSI & $62 \%$ & Ovulatory dysfunction & $17 \%$ & Unknown factor & $36 \%$ \\
\hline ZIFT & $0 \%$ & Unstimulated & $0 \%$ & Diminished ovarian reserve & $14 \%$ & Multiple Factors: & \\
\hline \multirow[t]{3}{*}{ Combination } & $0 \%$ & Used gestational carrier & $0 \%$ & Endometriosis & $12 \%$ & Female factors only & $0 \%$ \\
\hline & & & & Uterine factor & $2 \%$ & Female \& male factors & $<1 \%$ \\
\hline & & & & Male factor & $1 \%$ & & \\
\hline
\end{tabular}

2006 PREGNANCY SUCCESS RATES

Data verified by David K. Walmer, MD, PhD

\section{Type of Cycle}

\section{Fresh Embryos from Nondonor Eggs}

Number of cycles

Percentage of cycles resulting in pregnancies ${ }^{b}$

Percentage of cycles resulting in live births ${ }^{b, c}$

(Confidence Interval)

Percentage of retrievals resulting in live births $s^{b, c}$

Percentage of transfers resulting in live births $s^{b, c}$

Percentage of transfers resulting in singleton live births ${ }^{b}$

Percentage of cancellations ${ }^{b}$

Average number of embryos transferred

Percentage of pregnancies with twins ${ }^{b}$

Percentage of pregnancies with triplets or more ${ }^{b}$

Percentage of live births having multiple infants ${ }^{b, c}$

\section{Frozen Embryos from Nondonor Eggs}

Number of transfers

Percentage of transfers resulting in live births $s^{b, c}$

Average number of embryos transferred

\section{Donor Eggs}

Number of transfers

Percentage of transfers resulting in live births $s^{b, c}$

Average number of embryos transferred

\section{Age of Woman}

35-37 38-40

$41-42^{d}$

\section{$<35$}

77

32.5

42

5

46.2

26.0

31.0

$2 / 5$

38.5

$(16.6-37.2)$

28.6

$1 / 5$

(29.6-47.9)

41.3

30.8

(15.7-44.6)

45.0

31.3

40.0

40.0

$1 / 3$

26.0

25.0

33.3

$1 / 3$

6.8

15.6

28.6

$1 / 3$

2.4

2.7

24.0

37.0

0.0

9.3

42.2

20.0

\section{7}

$2 / 5$

2.3

$1 / 13$

$0 / 2$

$2 / 13$

$0 / 2$

$2 / 12$

$0 / 1$

41

36.6

2.9

$$
16
$$

$7 / 16$

10

3

2.6

$1 / 10$

$0 / 3$

2.5

2.7

All Ages Combined ${ }^{\mathrm{e}}$

Fresh Embryos

29

62.1

2.6
Frozen Embryos

29

48.3

2.9

\section{CURRENT CLINIC SERVICES AND PROFILE}

Current Name: This clinic has closed or reorganized since 2006. Information on current clinic services and profile therefore is not provided here. Contact the NASS Help Desk for current information about this clinic.

${ }^{a}$ Reflects patient and treatment characteristics of ART cycles performed in 2006 using fresh nondonor eggs or embryos.

b When fewer than 20 cycles are reported in an age category, rates are shown as a fraction and confidence intervals are not given. Calculating percentages from fractions may be misleading and is not encouraged.

${ }^{c}$ A multiple-infant birth is counted as one live birth.

${ }^{d}$ Clinic-specific outcome rates are unreliable for women older than 42 undergoing ART cycles using fresh or frozen embryos with nondonor eggs. Readers are urged to review national outcomes for these age groups (see page 27).

e All ages (including ages $>42$ ) are reported together because previous data show that patient age does not materially affect success with donor eggs. 


\section{EAST CAROLINA UNIVERSITY \\ GREENVILLE, NORTH CAROLINA}

A comparison of clinic success rates may not be meaningful because patient medical characteristics and treatment approaches vary from clinic to clinic. For more details about this, along with information on how to interpret the statistics in this table, see pages 79-88.

\section{ART CYCLE PROFILE}

\section{Type of ART ${ }^{\mathrm{a}}$}

IVF

GIFT

$100 \%$ Procedural Factors:

ZIFT

Combination
$0 \%$ Unstimulated

$0 \%$ Used gestational carrier
$0 \%$ With ICSI

\begin{tabular}{r|l} 
& Tubal factor \\
\hline $46 \%$ & Ovulatory dysfunction \\
$0 \%$ & Diminished ovarian reserve \\
$0 \%$ & Endometriosis \\
& Uterine factor \\
& Male factor
\end{tabular}

\section{Patient Diagnosis}

$\begin{array}{rlr}24 \% & \text { Other factor } & 0 \% \\ 7 \% & \text { Unknown factor } & 13 \% \\ 6 \% & \text { Multiple Factors: } & \\ 5 \% & \text { Female factors only } & 22 \% \\ 0 \% & \text { Female \& male factors } & 8 \% \\ 16 \% & & \end{array}$

Data verified by Clifford C. Hayslip, MD

\section{PREGNANCY SUCCESS RATES}

\section{Type of Cycle}

\section{Fresh Embryos from Nondonor Eggs}

Number of cycles

Percentage of cycles resulting in pregnancies ${ }^{b}$

Percentage of cycles resulting in live births ${ }^{b, c}$

(Confidence Interval)

Percentage of retrievals resulting in live births $s^{b, c}$

Percentage of transfers resulting in live births $s^{b, c}$

Percentage of transfers resulting in singleton live births ${ }^{b}$

Percentage of cancellations ${ }^{b}$

Average number of embryos transferred

Percentage of pregnancies with twins ${ }^{b}$

Percentage of pregnancies with triplets or more ${ }^{b}$

Percentage of live births having multiple infants ${ }^{\mathrm{b}, \mathrm{c}}$

\section{Frozen Embryos from Nondonor Eggs}

Number of transfers

Percentage of transfers resulting in live births $s^{b, c}$

Average number of embryos transferred

\section{7}

$2 / 17$

2.6

\section{Age of Woman}

35-37 38-40

$41-42^{d}$

18

$6 / 18$

$5 / 18$

6

4

37.5

$5 / 18$

$5 / 17$

$3 / 6$

$1 / 4$

(18.6-49.1)

33.3

34.2

$5 / 14$

$2 / 6$

$2 / 6$

$1 / 6$

$0 / 6$

2.8

$1 / 3$

$0 / 3$

$1 / 2$

2.3

$0 / 6$

$1 / 5$

6

$0 / 6$

1

$0 / 1$

2.0

4.0

All Ages Combined ${ }^{\mathrm{e}}$

Fresh Embryos

4

$2 / 4$

2.5
Frozen Embryos

2

$0 / 2$

1.5

Average number of embryos transferred

\section{CURRENT CLINIC SERVICES AND PROFILE}

Current Name: East Carolina University

\begin{tabular}{ll|ll} 
Donor egg? & Yes & Gestational carriers? & No \\
Donor embryo? & No & Cryopreservation? & Yes \\
Single women? & Yes & &
\end{tabular}

SART member?

Yes

Verified lab accreditation?

(See Appendix C for details.)

${ }^{a}$ Reflects patient and treatment characteristics of ART cycles performed in 2006 using fresh nondonor eggs or embryos.

b When fewer than 20 cycles are reported in an age category, rates are shown as a fraction and confidence intervals are not given. Calculating percentages from fractions may be misleading and is not encouraged.

${ }^{\mathrm{C}}$ A multiple-infant birth is counted as one live birth.

d Clinic-specific outcome rates are unreliable for women older than 42 undergoing ART cycles using fresh or frozen embryos with nondonor eggs. Readers are urged to review national outcomes for these age groups (see page 27).

ell ages (including ages $>42$ ) are reported together because previous data show that patient age does not materially affect success with donor eggs. 


\section{CAROLINA CONCEPTIONS \\ RALEIGH, NORTH CAROLINA}

A comparison of clinic success rates may not be meaningful because patient medical characteristics and treatment approaches vary from clinic to clinic. For more details about this, along with information on how to interpret the statistics in this table, see pages 79-88.

\section{ART CYCLE PROFILE}

\begin{tabular}{|c|c|c|c|c|c|c|c|}
\hline & Type & of $A R T^{a}$ & & Pati & ent $\mathbf{D}$ & iagnosis & \\
\hline IVF & $100 \%$ & Procedural Factors: & & Tubal factor & $2 \%$ & Other factor & $0 \%$ \\
\hline GIFT & $0 \%$ & With ICSI & $71 \%$ & Ovulatory dysfunction & $5 \%$ & Unknown factor & $73 \%$ \\
\hline ZIFT & $0 \%$ & Unstimulated & $37 \%$ & Diminished ovarian reserve & $17 \%$ & Multiple Factors: & \\
\hline Combination & $0 \%$ & Used gestational carrier & $0 \%$ & Endometriosis & $2 \%$ & Female factors only & $0 \%$ \\
\hline & & & & Uterine factor & $0 \%$ & Female \& male factors & $0 \%$ \\
\hline & & & & Male factor & $0 \%$ & & \\
\hline
\end{tabular}

2006 PREGNANCY SUCCESS RATES

Data verified by Bill Meyer, MD

\section{Type of Cycle}

\section{Fresh Embryos from Nondonor Eggs}

Number of cycles

Percentage of cycles resulting in pregnancies ${ }^{b}$

Percentage of cycles resulting in live births $s^{b, c}$

(Confidence Interval)

Percentage of retrievals resulting in live births $s^{b, c}$

Percentage of transfers resulting in live births $s^{b, c}$

Percentage of transfers resulting in singleton live births ${ }^{b}$

Percentage of cancellations ${ }^{b}$

Average number of embryos transferred

Percentage of pregnancies with twins ${ }^{b}$

Percentage of pregnancies with triplets or more ${ }^{b}$

Percentage of live births having multiple infants ${ }^{b, c}$

\section{Frozen Embryos from Nondonor Eggs}

Number of transfers

Percentage of transfers resulting in live births ${ }^{\mathrm{b}, \mathrm{c}}$

Average number of embryos transferred

$\begin{array}{cccc}<35 & 35-37 & 38-40 & \mathbf{4 1 - 4 2}^{\text {d }} \\ 16 & 8 & 9 & 2 \\ 6 / 16 & 2 / 8 & 1 / 9 & 0 / 2 \\ 6 / 16 & 1 / 8 & 1 / 9 & 0 / 2\end{array}$

$\begin{array}{cccc}6 / 16 & 1 / 8 & 1 / 9 & 0 / 2 \\ 6 / 16 & 1 / 8 & 1 / 7 & 0 / 2 \\ 6 / 16 & 0 / 8 & 1 / 7 & 0 / 2 \\ 0 / 16 & 0 / 8 & 0 / 9 & 0 / 2 \\ 2.2 & 2.3 & 3.3 & 4.0 \\ 1 / 6 & 1 / 2 & 0 / 1 & \\ 1 / 6 & 0 / 2 & 0 / 1 & \\ 0 / 6 & 1 / 1 & 0 / 1 & \end{array}$

\section{1}

$0 / 1$

3.0
0

\section{Fresh Embryos}

0
$0 \%$

\%

$0 \%$

$0 \%$

\section{Donor Eggs}

Number of transfers

Percentage of transfers resulting in live births $s^{b, c}$

Average number of embryos transferred

\section{CURRENT CLINIC SERVICES AND PROFILE}

Current Name: Carolina Conceptions

\begin{tabular}{ll|ll|l|l} 
Donor egg? & Yes & Gestational carriers? & No & SART member? & Yes \\
\hline Donor embryo? & No & Cryopreservation? & Yes & Verified lab accreditation? & Yes \\
Single women? & Yes & & & (See Appendix C for details.) &
\end{tabular}

${ }^{a}$ Reflects patient and treatment characteristics of ART cycles performed in 2006 using fresh nondonor eggs or embryos.

${ }^{b}$ When fewer than 20 cycles are reported in an age category, rates are shown as a fraction and confidence intervals are not given. Calculating percentages from fractions may be misleading and is not encouraged.

${ }^{c}$ A multiple-infant birth is counted as one live birth.

d Clinic-specific outcome rates are unreliable for women older than 42 undergoing ART cycles using fresh or frozen embryos with nondonor eggs. Readers are urged to review national outcomes for these age groups (see page 27).

e All ages (including ages $>42$ ) are reported together because previous data show that patient age does not materially affect success with donor eggs. 


\section{WAKE FOREST UNIVERSITY CENTER FOR REPRODUCTIVE MEDICINE WINSTON-SALEM, NORTH CAROLINA}

A comparison of clinic success rates may not be meaningful because patient medical characteristics and treatment approaches vary from clinic to clinic. For more details about this, along with information on how to interpret the statistics in this table, see pages $79-88$.

\section{ART CYCLE PROFILE}

\section{Type of ART ${ }^{\mathrm{a}}$}

IVF

GIFT

$100 \%$ Procedural Factors:

ZIFT

Combination
$0 \%$ With ICSI

$0 \%$ Unstimulated

$0 \%$ Used gestational carrier

\begin{tabular}{r|l}
\hline $74 \%$ & Tubal factor \\
Ovulatory dysfunction & Diminished ovarian reserve \\
$0 \%$ & Endometriosis \\
& Uterine factor \\
& Male factor
\end{tabular}

\section{Patient Diagnosis}

$\begin{array}{rlr}17 \% & \text { Other factor } & 4 \% \\ 4 \% & \text { Unknown factor } & 4 \% \\ 4 \% & \text { Multiple Factors: } & \\ 9 \% & \text { Female factors only } & 17 \% \\ 0 \% & \text { Female \& male factors } & 22 \% \\ 19 \% & & \end{array}$

Data verified by Tamer M. Yalcinkaya, MD

2006 PREGNANCY SUCCESS RATES

\section{Age of Woman}

\section{Type of Cycle}

$<35 \quad 35-37 \quad 38-40 \quad 41-42^{\text {d }}$

\section{Fresh Embryos from Nondonor Eggs}

Number of cycles

62

43.5

33

42.4

22

8

Percentage of cycles resulting in pregnancies ${ }^{b}$

Percentage of cycles resulting in live births ${ }^{\mathrm{b}, \mathrm{c}}$

37.1

39.4

40.9

$0 / 8$

(Confidence Interval)

Percentage of retrievals resulting in live births $s^{b, c}$

(25.2-50.3)

(22.9-57.9)

31.8

$0 / 8$

Percentage of transfers resulting in live births $s^{b, c}$

41.1

42.6

41.9

$(13.9-54.9)$

Percentage of transfers resulting in singleton live births ${ }^{b}$

24.1

48.1

$7 / 19$

$0 / 8$

Percentage of cancellations ${ }^{\mathrm{b}}$

9.7

29.6

$7 / 17$

$0 / 7$

Average number of embryos transferred

Percentage of pregnancies with twins ${ }^{\mathrm{b}}$

2.7

6.1

$3 / 17$

$0 / 7$

Percentage of pregnancies with triplets or more ${ }^{b}$

Percentage of live births having multiple infants ${ }^{b, c}$

\section{3}

3.7

43.5

2.4

13.6

$0 / 8$

2.8

2.4

\section{Frozen Embryos from Nondonor Eggs}

Number of transfers

Percentage of transfers resulting in live births ${ }^{b, c}$

Average number of embryos transferred

\section{Donor Eggs}

Number of transfers

Percentage of transfers resulting in live births $s^{b, c}$

Average number of embryos transferred

\section{$5 / 14$}

$1 / 14$

$4 / 9$

$0 / 9$

$5 / 13$

\section{CURRENT CLINIC SERVICES AND PROFILE}

Current Name: Wake Forest University Center for Reproductive Medicine

\begin{tabular}{|c|c|c|c|c|}
\hline Donor egg? & Yes & Gestational carriers? & No & SART member? \\
\hline $\begin{array}{l}\text { Donor embryo? } \\
\text { Single women? }\end{array}$ & $\begin{array}{l}\text { Yes } \\
\text { Yes }\end{array}$ & Cryopreservation? & Yes & $\begin{array}{l}\text { Verified lab accreditation? } \\
\text { (See Appendix C for details.) }\end{array}$ \\
\hline
\end{tabular}

${ }_{b}^{a}$ Reflects patient and treatment characteristics of ART cycles performed in 2006 using fresh nondonor eggs or embryos.

b When fewer than 20 cycles are reported in an age category, rates are shown as a fraction and confidence intervals are not given. Calculating percentages from fractions may be misleading and is not encouraged.

${ }^{c}$ A multiple-infant birth is counted as one live birth.

d Clinic-specific outcome rates are unreliable for women older than 42 undergoing ART cycles using fresh or frozen embryos with nondonor eggs. Readers are urged to review national outcomes for these age groups (see page 27).

e All ages (including ages $>42$ ) are reported together because previous data show that patient age does not materially affect success with donor eggs. 


\section{MERITCARE REPRODUCTIVE MEDICINE \\ FARGO, NORTH DAKOTA}

A comparison of clinic success rates may not be meaningful because patient medical characteristics and treatment approaches vary from clinic to clinic. For more details about this, along with information on how to interpret the statistics in this table, see pages $79-88$.

\section{ART CYCLE PROFILE}

\begin{tabular}{|c|c|c|c|c|c|c|c|}
\hline \multicolumn{4}{|c|}{ Type of ART ${ }^{\mathrm{a}}$} & \multicolumn{4}{|c|}{ Patient Diagnosis } \\
\hline IVF & $100 \%$ & Procedural Factors: & & Tubal factor & $14 \%$ & Other factor & $4 \%$ \\
\hline GIFT & $0 \%$ & With ICSI & $84 \%$ & Ovulatory dysfunction & $17 \%$ & Unknown factor & $8 \%$ \\
\hline $\mathrm{ZIFT}$ & $0 \%$ & Unstimulated & $0 \%$ & Diminished ovarian reserve & $2 \%$ & Multiple Factors: & \\
\hline Combination & $0 \%$ & Used gestational carrier & $0 \%$ & Endometriosis & $12 \%$ & Female factors only & $4 \%$ \\
\hline & & & & Uterine factor & $3 \%$ & Female \& male factors & $15 \%$ \\
\hline
\end{tabular}

2006 PREGNANCY SUCCESS RATES

Data verified by Steffen P. Christensen, MD

\section{Type of Cycle}

\section{Fresh Embryos from Nondonor Eggs}

Number of cycles

Percentage of cycles resulting in pregnancies ${ }^{b}$

Percentage of cycles resulting in live births $s^{b, c}$

(Confidence Interval)

Percentage of retrievals resulting in live births $s^{b, c}$

Percentage of transfers resulting in live births $s^{b, c}$

Percentage of transfers resulting in singleton live births ${ }^{b}$

Percentage of cancellations ${ }^{b}$

Average number of embryos transferred

Percentage of pregnancies with twins ${ }^{b}$

Percentage of pregnancies with triplets or more ${ }^{b}$

Percentage of live births having multiple infants ${ }^{b, c}$

\section{Frozen Embryos from Nondonor Eggs}

Number of transfers

Percentage of transfers resulting in live births $s^{b, c}$

Average number of embryos transferred

\section{Donor Eggs}

Number of transfers

Percentage of transfers resulting in live births ${ }^{\mathrm{b}, \mathrm{c}}$

Average number of embryos transferred

\section{Age of Woman}

35-37 38-40

$41-42^{d}$

$<35$

17

$4 / 17$

$4 / 17$

6

5

23.2

21.7

(12.7-33.3)

22.4

23.8

$4 / 12$

$1 / 6$

$0 / 5$

11.1

$4 / 12$

$4 / 12$

$5 / 17$

$1 / 6$

$0 / 5$

2.9

2.8

$0 / 4$

$1 / 6$

$0 / 1$

2.4

$7 / 16$

$0 / 4$

$1 / 4$

$1 / 4$

$0 / 6$

$4 / 5$

$2 / 16$

$8 / 15$

$0 / 4$

3.3

$0 / 1$

$0 / 1$

$0 / 1$
$4 \%$

$8 \%$

$4 \%$

\%

\section{CURRENT CLINIC SERVICES AND PROFILE}

Current Name: MeritCare Reproductive Medicine

\begin{tabular}{|c|c|c|c|c|}
\hline Donor egg? & Yes & Gestational carriers? & Yes & SART member? \\
\hline Donor embryo? & Yes & Cryopreservation? & Yes & Verified lab accreditation? \\
\hline Single women? & Yes & & & (See Appendix C for details.) \\
\hline
\end{tabular}

${ }_{b}^{a}$ Reflects patient and treatment characteristics of ART cycles performed in 2006 using fresh nondonor eggs or embryos.

${ }^{b}$ When fewer than 20 cycles are reported in an age category, rates are shown as a fraction and confidence intervals are not given. Calculating percentages from fractions may be misleading and is not encouraged.

${ }^{c}$ A multiple-infant birth is counted as one live birth.

d Clinic-specific outcome rates are unreliable for women older than 42 undergoing ART cycles using fresh or frozen embryos with nondonor eggs. Readers are urged to review national outcomes for these age groups (see page 27).

e All ages (including ages $>42$ ) are reported together because previous data show that patient age does not materially affect success with donor eggs. 


\section{FERTILITY UNLIMITED, INC. NORTHEASTERN OHIO FERTILITY CENTER AKRON, OHIO}

A comparison of clinic success rates may not be meaningful because patient medical characteristics and treatment approaches vary from clinic to clinic. For more details about this, along with information on how to interpret the statistics in this table, see pages 79-88.

\section{ART CYCLE PROFILE}

\begin{tabular}{|c|c|c|c|c|c|c|c|}
\hline \multicolumn{4}{|c|}{ Type of ART ${ }^{a}$} & \multicolumn{4}{|c|}{ Patient Diagnosis } \\
\hline IVF & $100 \%$ & Procedural Factors: & & Tubal factor & $24 \%$ & Other factor & $4 \%$ \\
\hline GIFT & $0 \%$ & With ICSI & $50 \%$ & Ovulatory dysfunction & $2 \%$ & Unknown factor & $2 \%$ \\
\hline ZIFT & $0 \%$ & Unstimulated & $3 \%$ & Diminished ovarian reserve & $6 \%$ & Multiple Factors: & \\
\hline \multirow[t]{3}{*}{ Combination } & $0 \%$ & Used gestational carrier & $3 \%$ & Endometriosis & $11 \%$ & Female factors only & $26 \%$ \\
\hline & & & & Uterine factor & $0 \%$ & Female \& male factors & $18 \%$ \\
\hline & & & & Male factor & $9 \%$ & & \\
\hline
\end{tabular}

2006 PREGNANCY SUCCESS RATES

Data verified by Nicholas J. Spirtos, DO

\section{Type of Cycle}

\section{Fresh Embryos from Nondonor Eggs}

Number of cycles

Percentage of cycles resulting in pregnancies ${ }^{b}$

Percentage of cycles resulting in live births ${ }^{b, c}$

(Confidence Interval)

Percentage of retrievals resulting in live births ${ }^{b, c}$

Percentage of transfers resulting in live births $s^{b, c}$

Percentage of transfers resulting in singleton live births ${ }^{b}$

Percentage of cancellations ${ }^{b}$

Average number of embryos transferred

Percentage of pregnancies with twins ${ }^{b}$

Percentage of pregnancies with triplets or more ${ }^{b}$

Percentage of live births having multiple infants ${ }^{\mathrm{b}, \mathrm{c}}$

\section{Frozen Embryos from Nondonor Eggs}

Number of transfers

Percentage of transfers resulting in live births $s^{b, c}$

Average number of embryos transferred

\section{Donor Eggs}

Number of transfers

Percentage of transfers resulting in live births $s^{b, c}$

Average number of embryos transferred

\section{Age of Woman}

35-37 38-40

$41-42^{d}$ $<35$

5

$0 / 5$

6

5

45.5

$0 / 5$

$2 / 6$

$0 / 5$

45.5

$2 / 6$

$0 / 5$

$(24.4-67.8)$

47.6

$0 / 5$

$2 / 6$

$0 / 4$

47.6

$0 / 5$

$2 / 6$

$0 / 4$

33.3

$0 / 5$

$0 / 6$

$0 / 4$

4.5

$0 / 5$

$0 / 6$

$1 / 5$

2.8

2.6

3.7

2.5

$2 / 10$

$2 / 2$

$1 / 10$

$0 / 2$

$3 / 10$

$2 / 2$

\section{5}

$0 / 5$

2.0

\begin{tabular}{cc}
\multicolumn{2}{c}{ All Ages Combined } \\
Fresh Embryos & Frozen Embryos \\
6 & 6 \\
$2 / 6$ & $1 / 6$ \\
2.8 & 2.3
\end{tabular}

\section{CURRENT CLINIC SERVICES AND PROFILE}

Current Name: Fertility Unlimited, Inc., Northeastern Ohio Fertility Center

\begin{tabular}{ll|lll|ll} 
Donor egg? & Yes & Gestational carriers? & Yes & SART member? & Yes \\
\hline Donor embryo? & Yes & Cryopreservation? & Yes & Verified lab accreditation? & Pending \\
Single women? & Yes & & & (See Appendix C for details.) &
\end{tabular}

${ }^{a}$ Reflects patient and treatment characteristics of ART cycles performed in 2006 using fresh nondonor eggs or embryos.

b When fewer than 20 cycles are reported in an age category, rates are shown as a fraction and confidence intervals are not given. Calculating percentages from fractions may be misleading and is not encouraged.

${ }^{c}$ A multiple-infant birth is counted as one live birth.

${ }^{d}$ Clinic-specific outcome rates are unreliable for women older than 42 undergoing ART cycles using fresh or frozen embryos with nondonor eggs. Readers are urged to review national outcomes for these age groups (see page 27).

e All ages (including ages $>42$ ) are reported together because previous data show that patient age does not materially affect success with donor eggs. 


\section{REPRODUCTIVE GYNECOLOGY \\ AKRON, OHIO}

A comparison of clinic success rates may not be meaningful because patient medical characteristics and treatment approaches vary from clinic to clinic. For more details about this, along with information on how to interpret the statistics in this table, see pages 79-88.

\section{ART CYCLE PROFILE}

\begin{tabular}{|c|c|c|c|c|c|c|c|}
\hline \multicolumn{4}{|c|}{ Type of ART } & \multicolumn{4}{|c|}{ Patient Diagnosis } \\
\hline IVF & $100 \%$ & Procedural Factors: & & Tubal factor & $12 \%$ & Other factor & $<1 \%$ \\
\hline GIFT & $0 \%$ & With ICSI & $75 \%$ & Ovulatory dysfunction & $1 \%$ & Unknown factor & $2 \%$ \\
\hline ZIFT & $0 \%$ & Unstimulated & $0 \%$ & Diminished ovarian reserve & $3 \%$ & Multiple Factors: & \\
\hline \multirow[t]{3}{*}{ Combination } & $0 \%$ & Used gestational carrier & $2 \%$ & Endometriosis & $7 \%$ & Female factors only & $14 \%$ \\
\hline & & & & Uterine factor & $1 \%$ & Female \& male factors & $44 \%$ \\
\hline & & & & Male factor & $15 \%$ & & \\
\hline
\end{tabular}

2006 PREGNANCY SUCCESS RATES

Data verified by Richard W. Moretuzzo, MD

\section{Type of Cycle}

\section{Fresh Embryos from Nondonor Eggs}

Number of cycles

Percentage of cycles resulting in pregnancies ${ }^{b}$

Percentage of cycles resulting in live births ${ }^{b, c}$

(Confidence Interval)

Percentage of retrievals resulting in live births $s^{b, c}$

Percentage of transfers resulting in live births $s^{b, c}$

Percentage of transfers resulting in singleton live births ${ }^{b}$

Percentage of cancellations ${ }^{b}$

Average number of embryos transferred

Percentage of pregnancies with twins ${ }^{b}$

Percentage of pregnancies with triplets or more ${ }^{b}$

Percentage of live births having multiple infants ${ }^{b, c}$

\section{Frozen Embryos from Nondonor Eggs}

Number of transfers

Percentage of transfers resulting in live births $s^{\mathrm{b}, \mathrm{c}}$

Average number of embryos transferred

\section{Donor Eggs}

Number of transfers

Percentage of transfers resulting in live births ${ }^{\mathrm{b}, \mathrm{c}}$

Average number of embryos transferred

\section{Age of Woman}

35-37 38-40

$41-42^{d}$

\section{$<35$}

39

33.3

30

7

52.2

28.2

33.3

$3 / 7$

42.6

$(15.0-44.9)$

23.3

$3 / 7$

(33.4-52.2)

43.8

31.4

(9.9-42.3)

45.0

31.4

26.9

$3 / 6$

25.7

25.7

28.0

$3 / 6$

2.6

10.3

16.0

$2 / 6$

3.0

2.9

13.3

$1 / 7$

38.3

$1 / 13$

3.1

3.0

10.0

$2 / 13$

$3 / 10$

$1 / 3$

42.9

$2 / 11$

$0 / 10$

$0 / 3$

$3 / 7$

$1 / 3$

29

48.3

12

$7 / 12$

8

2.7

$3 / 8$

1

2.7

All Ages Combined ${ }^{\mathrm{e}}$

Fresh Embryos

14

$8 / 14$

2.9
Frozen Embryos

\section{8}

$3 / 8$

3.0

\section{CURRENT CLINIC SERVICES AND PROFILE}

Current Name: Reproductive Gynecology

\begin{tabular}{ll|ll} 
Donor egg? & Yes & Gestational carriers? & Yes \\
Donor embryo? & Yes & Cryopreservation? & Yes
\end{tabular}

Single women? Yes

$\begin{array}{ll}\text { SART member? } & \text { Yes } \\ \text { Verified lab accreditation? } & \text { Yes } \\ \text { (See Appendix C for details.) } & \end{array}$

${ }^{a}$ Reflects patient and treatment characteristics of ART cycles performed in 2006 using fresh nondonor eggs or embryos.

${ }^{b}$ When fewer than 20 cycles are reported in an age category, rates are shown as a fraction and confidence intervals are not given. Calculating percentages from fractions may be misleading and is not encouraged.

${ }^{c}$ A multiple-infant birth is counted as one live birth.

d Clinic-specific outcome rates are unreliable for women older than 42 undergoing ART cycles using fresh or frozen embryos with nondonor eggs. Readers are urged to review national outcomes for these age groups (see page 27).

${ }^{e}$ All ages (including ages $>42$ ) are reported together because previous data show that patient age does not materially affect success with donor eggs. 


\section{BETHESDA CENTER FOR REPRODUCTIVE HEALTH \& FERTILITY CINCINNATI, OHIO}

A comparison of clinic success rates may not be meaningful because patient medical characteristics and treatment approaches vary from clinic to clinic. For more details about this, along with information on how to interpret the statistics in this table, see pages 79-88.

\section{ART CYCLE PROFILE}

\begin{tabular}{|c|c|c|c|c|c|c|c|}
\hline \multicolumn{4}{|c|}{ Type of $A R T^{a}$} & \multicolumn{4}{|c|}{ Patient Diagnosis } \\
\hline IVF & $100 \%$ & Procedural Factors: & & Tubal factor & $13 \%$ & Other factor & $5 \%$ \\
\hline GIFT & $0 \%$ & With ICSI & $50 \%$ & Ovulatory dysfunction & $6 \%$ & Unknown factor & $13 \%$ \\
\hline ZIFT & $0 \%$ & Unstimulated & $0 \%$ & Diminished ovarian reserve & $27 \%$ & Multiple Factors: & \\
\hline \multirow[t]{3}{*}{ Combination } & $0 \%$ & Used gestational carrier & $0 \%$ & Endometriosis & $4 \%$ & Female factors only & $4 \%$ \\
\hline & & & & Uterine factor & $<1 \%$ & Female \& male factors & $15 \%$ \\
\hline & & & & Male factor & $12 \%$ & & \\
\hline
\end{tabular}

\section{PREGNANCY SUCCESS RATES}

\section{Type of Cycle}

\section{Fresh Embryos from Nondonor Eggs}

Number of cycles

Percentage of cycles resulting in pregnancies ${ }^{b}$

Percentage of cycles resulting in live births ${ }^{b, c}$

(Confidence Interval)

Percentage of retrievals resulting in live births $s^{b, c}$

Percentage of transfers resulting in live births ${ }^{b, c}$

Percentage of transfers resulting in singleton live births ${ }^{b}$

Percentage of cancellations ${ }^{b}$

Average number of embryos transferred

Percentage of pregnancies with twins ${ }^{b}$

Percentage of pregnancies with triplets or more ${ }^{b}$

Percentage of live births having multiple infants ${ }^{b, c}$

\section{Frozen Embryos from Nondonor Eggs}

Number of transfers

Percentage of transfers resulting in live births ${ }^{b, c}$

Average number of embryos transferred

\section{Donor Eggs}

Number of transfers

Percentage of transfers resulting in live births $s^{b, c}$

Average number of embryos transferred

Data verified by Glen E. Hofmann, MD, PhD

\section{Age of Woman}

$<35 \quad 35-37 \quad 38-40 \quad 41-42^{d}$

$\begin{array}{cccc}86 & 30 & 32 & 13 \\ 45.3 & 33.3 & 21.9 & 0 / 13 \\ 41.9 & 20.0 & 6.3 & 0 / 13\end{array}$

$(31.3-53.0)$

(7.7-38.6)

$(0.8-20.8)$

$\begin{array}{llll}50.0 & 28.6 & 8.7 & 0 / 8\end{array}$

50.7

$6 / 19$

8.7

$0 / 7$

22.5

$4 / 19$

4.3

$0 / 7$

16.3

30.0

28.1

$5 / 13$

2.1

2.1

2.4

2.9

53.8

$2 / 10$

$2 / 7$

2.6

$0 / 10$

$0 / 7$

55.6

$2 / 6$

$1 / 2$

29

17.2

1.8

\section{7}

$2 / 7$

1.6

\section{7 \\ $0 / 7$ \\ 1.6}

All Ages Combined ${ }^{\mathrm{e}}$
Frozen Embryos

$\begin{array}{cc}21 & 21 \\ 71.4 & 57.1 \\ 2.0 & 1.9\end{array}$

\section{CURRENT CLINIC SERVICES AND PROFILE}

Current Name: Bethesda Center for Reproductive Health \& Fertility

Donor egg? Yes Gestational carriers? Yes

Donor embryo? Yes Cryopreservation? Yes

Single women? Yes

SART member? $\quad$ Yes

Verified lab accreditation? Yes

(See Appendix C for details.)

${ }^{a}$ Reflects patient and treatment characteristics of ART cycles performed in 2006 using fresh nondonor eggs or embryos.

${ }^{b}$ When fewer than 20 cycles are reported in an age category, rates are shown as a fraction and confidence intervals are not given. Calculating percentages from fractions may be misleading and is not encouraged.

${ }^{c}$ A multiple-infant birth is counted as one live birth.

d Clinic-specific outcome rates are unreliable for women older than 42 undergoing ART cycles using fresh or frozen embryos with nondonor eggs. Readers are urged to review national outcomes for these age groups (see page 27).

e All ages (including ages $>42$ ) are reported together because previous data show that patient age does not materially affect success with donor eggs. 


\section{CENTER FOR REPRODUCTIVE HEALTH \\ CINCINNATI, OHIO}

A comparison of clinic success rates may not be meaningful because patient medical characteristics and treatment approaches vary from clinic to clinic. For more details about this, along with information on how to interpret the statistics in this table, see pages $79-88$.

\section{ART CYCLE PROFILE}

\begin{tabular}{|c|c|c|c|c|c|c|c|}
\hline \multicolumn{4}{|c|}{ Type of ART } & \multicolumn{4}{|c|}{ Patient Diagnosis } \\
\hline IVF & $100 \%$ & Procedural Factors: & & Tubal factor & $11 \%$ & Other factor & $3 \%$ \\
\hline GIFT & $0 \%$ & With ICSI & $48 \%$ & Ovulatory dysfunction & $7 \%$ & Unknown factor & $8 \%$ \\
\hline ZIFT & $0 \%$ & Unstimulated & $0 \%$ & Diminished ovarian reserve & $10 \%$ & Multiple Factors: & \\
\hline \multirow[t]{3}{*}{ Combination } & $0 \%$ & Used gestational carrier & $<1 \%$ & Endometriosis & $5 \%$ & Female factors only & $13 \%$ \\
\hline & & & & Uterine factor & $0 \%$ & Female \& male factors & $22 \%$ \\
\hline & & & & Male factor & $21 \%$ & & \\
\hline
\end{tabular}

2006 PREGNANCY SUCCESS RATES

Data verified by Daniel B. Williams, MD

\section{Type of Cycle}

\section{Fresh Embryos from Nondonor Eggs}

Number of cycles

Percentage of cycles resulting in pregnancies ${ }^{b}$

Percentage of cycles resulting in live births ${ }^{b, c}$

(Confidence Interval)

Percentage of retrievals resulting in live births $s^{b, c}$

Percentage of transfers resulting in live births $s^{b, c}$

Percentage of transfers resulting in singleton live births ${ }^{b}$

Percentage of cancellations ${ }^{b}$

Average number of embryos transferred

Percentage of pregnancies with twins ${ }^{b}$

Percentage of pregnancies with triplets or more ${ }^{b}$

Percentage of live births having multiple infants ${ }^{b, c}$

\section{Frozen Embryos from Nondonor Eggs}

Number of transfers

Percentage of transfers resulting in live births $s^{\mathrm{b}, \mathrm{c}}$

Average number of embryos transferred

\section{Donor Eggs}

Number of transfers

Percentage of transfers resulting in live births $s^{b, c}$

Average number of embryos transferred

\section{Age of Woman}

35-37 38-40

$41-42^{d}$

$<35$

23

30.4

26.1

42.9

(10.2-48.4)

20

4

$\begin{array}{cccc}70 & 23 & 20 & 4 \\ 45.7 & 30.4 & 25.0 & 2 / 4 \\ 42.9 & 26.1 & 15.0 & 1 / 4\end{array}$

(31.1-55.3)

$6 / 19$

(3.2-37.9)

47.6

$6 / 18$

$3 / 17$

$3 / 15$

$1 / 4$

49.2

$3 / 18$

$2 / 15$

$1 / 4$

39.3

17.4

15.0

$1 / 4$

2.1

2.7

2.6

$0 / 4$

18.8

$3 / 7$

$0 / 5$

3.8

0.0

$0 / 7$

$1 / 5$

$0 / 2$

20.0

$3 / 6$

$1 / 3$

$0 / 2$

$0 / 1$

18

$10 / 18$

6

$2 / 6$

2

$1 / 2$

2.3

2.5

3.5

All Ages Combined ${ }^{\mathrm{e}}$

Fresh Embryos 19

$12 / 19$

2.2
Frozen Embryos 8

$1 / 8$

2.1

\section{CURRENT CLINIC SERVICES AND PROFILE}

Current Name: Center for Reproductive Health
Donor egg?
Yes
Donor embryo? Yes
Gestational carriers? Yes
Cryopreservation?
Yes
Single women? Yes

\section{SART member? \\ Verified lab accreditation? \\ (See Appendix C for details.)}
Yes
Yes

\footnotetext{
${ }^{a}$ Reflects patient and treatment characteristics of ART cycles performed in 2006 using fresh nondonor eggs or embryos.

${ }^{b}$ When fewer than 20 cycles are reported in an age category, rates are shown as a fraction and confidence intervals are not given. Calculating percentages from fractions may be misleading and is not encouraged.

c A multiple-infant birth is counted as one live birth.

${ }^{d}$ Clinic-specific outcome rates are unreliable for women older than 42 undergoing ART cycles using fresh or frozen embryos with nondonor eggs. Readers are urged to review national outcomes for these age groups (see page 27).

${ }^{e}$ All ages (including ages $>42$ ) are reported together because previous data show that patient age does not materially affect success with donor eggs.
} 


\section{INSTITUTE FOR REPRODUCTIVE HEALTH CINCINNATI, OHIO}

A comparison of clinic success rates may not be meaningful because patient medical characteristics and treatment approaches vary from clinic to clinic. For more details about this, along with information on how to interpret the statistics in this table, see pages 79-88.

\section{ART CYCLE PROFILE}

\begin{tabular}{|c|c|c|c|c|c|c|c|}
\hline \multicolumn{4}{|c|}{ Type of ART ${ }^{a}$} & \multicolumn{4}{|c|}{ Patient Diagnosis } \\
\hline IVF & $>99 \%$ & Procedural Factors: & & Tubal factor & $8 \%$ & Other factor & $4 \%$ \\
\hline GIFT & $<1 \%$ & With ICSI & $54 \%$ & Ovulatory dysfunction & $8 \%$ & Unknown factor & $6 \%$ \\
\hline ZIFT & $0 \%$ & Unstimulated & $0 \%$ & Diminished ovarian reserve & $8 \%$ & Multiple Factors: & \\
\hline \multirow[t]{3}{*}{ Combination } & $0 \%$ & Used gestational carrier & $1 \%$ & Endometriosis & $12 \%$ & Female factors only & $16 \%$ \\
\hline & & & & Uterine factor & $1 \%$ & Female \& male factors & $21 \%$ \\
\hline & & & & Male factor & $18 \%$ & & \\
\hline
\end{tabular}

\section{PREGNANCY SUCCESS RATES}

\section{Type of Cycle}

\section{Fresh Embryos from Nondonor Eggs}

Number of cycles

Percentage of cycles resulting in pregnancies ${ }^{b}$

Percentage of cycles resulting in live births ${ }^{b, c}$

(Confidence Interval)

Percentage of retrievals resulting in live births $s^{b, c}$

Percentage of transfers resulting in live births $s^{b, c}$

Percentage of transfers resulting in singleton live births ${ }^{b}$

Percentage of cancellations ${ }^{b}$

Average number of embryos transferred

Percentage of pregnancies with twins ${ }^{b}$

Percentage of pregnancies with triplets or more ${ }^{b}$

Percentage of live births having multiple infants ${ }^{b, c}$

\section{Frozen Embryos from Nondonor Eggs}

Number of transfers

Percentage of transfers resulting in live births $s^{\mathrm{b}, \mathrm{c}}$

Average number of embryos transferred

\section{Donor Eggs}

Number of transfers

Percentage of transfers resulting in live births $s^{b, c}$

Average number of embryos transferred

Data verified by Sherif G. Awadalla, MD

\section{Age of Woman}

35-37 38-40

$41-42^{d}$

$<35$

115
40.9
31.3

77

14

46.3

39.0

31.3

35.1

$4 / 14$

$(33.7-44.5)$

(23.0-40.6)

23.4

$2 / 14$

42.2

43.5

34.3

$(14.5-34.4)$

29.9

36.4

27.3

$2 / 12$

28.6

$2 / 11$

25.3

20.6

$2 / 11$

7.6

8.7

14.3

$2 / 14$

2.1

27.6

2.8

23.4

3.2

3.9

2.0

0.0

31.3

30.6

18.5

$0 / 4$

0.0

$1 / 4$

$5 / 18$

$0 / 2$

$\begin{array}{cccc}114 & 43 & 21 & 4 \\ 33.3 & 30.2 & 38.1 & 0 / 4 \\ 2.4 & 2.6 & 2.8 & 3.5\end{array}$

\section{All Ages Combined}

Fresh Embryos 58

44.8

2.3
Frozen Embryos 35

37.1

2.9

\section{CURRENT CLINIC SERVICES AND PROFILE}

Current Name: Institute for Reproductive Health

Donor egg? Yes Gestational carriers? Yes

Donor embryo? Yes Cryopreservation? Yes

Single women? Yes

SART member? Yes

Verified lab accreditation? Yes

(See Appendix C for details.)

${ }^{a}$ Reflects patient and treatment characteristics of ART cycles performed in 2006 using fresh nondonor eggs or embryos.

b When fewer than 20 cycles are reported in an age category, rates are shown as a fraction and confidence intervals are not given. Calculating percentages from fractions may be misleading and is not encouraged.

${ }^{c}$ A multiple-infant birth is counted as one live birth.

d Clinic-specific outcome rates are unreliable for women older than 42 undergoing ART cycles using fresh or frozen embryos with nondonor eggs. Readers are urged to review national outcomes for these age groups (see page 27).

e All ages (including ages $>42$ ) are reported together because previous data show that patient age does not materially affect success with donor eggs. 


\section{CLEVELAND CLINIC FERTILITY CENTER \\ CLEVELAND, OHIO}

A comparison of clinic success rates may not be meaningful because patient medical characteristics and treatment approaches vary from clinic to clinic. For more details about this, along with information on how to interpret the statistics in this table, see pages $79-88$.

\section{ART CYCLE PROFILE}

\begin{tabular}{|c|c|c|c|c|c|c|c|}
\hline \multicolumn{4}{|c|}{ Type of ART ${ }^{a}$} & \multicolumn{4}{|c|}{ Patient Diagnosis } \\
\hline IVF & $100 \%$ & Procedural Factors: & & Tubal factor & $14 \%$ & Other factor & $5 \%$ \\
\hline GIFT & $0 \%$ & With ICSI & $77 \%$ & Ovulatory dysfunction & $10 \%$ & Unknown factor & $25 \%$ \\
\hline $\mathrm{ZIFT}$ & $0 \%$ & Unstimulated & $0 \%$ & Diminished ovarian reserve & $8 \%$ & Multiple Factors: & \\
\hline \multirow[t]{3}{*}{ Combination } & $0 \%$ & Used gestational carrier & $1 \%$ & Endometriosis & $6 \%$ & Female factors only & $4 \%$ \\
\hline & & & & Uterine factor & $1 \%$ & Female \& male factors & $9 \%$ \\
\hline & & & & Male factor & $18 \%$ & & \\
\hline
\end{tabular}

2006 PREGNANCY SUCCESS RATES

Data verified by James Goldfarb, MD

\section{Type of Cycle}

\section{Fresh Embryos from Nondonor Eggs}

Number of cycles

Percentage of cycles resulting in pregnancies ${ }^{b}$

Percentage of cycles resulting in live births $s^{b, c}$

(Confidence Interval)

Percentage of retrievals resulting in live births $s^{b, c}$

Percentage of transfers resulting in live births $s^{b, c}$

Percentage of transfers resulting in singleton live births ${ }^{b}$

Percentage of cancellations ${ }^{b}$

Average number of embryos transferred

Percentage of pregnancies with twins ${ }^{b}$

Percentage of pregnancies with triplets or more ${ }^{b}$

Percentage of live births having multiple infants ${ }^{b, c}$

\section{Frozen Embryos from Nondonor Eggs}

Number of transfers

Percentage of transfers resulting in live births ${ }^{\mathrm{b}, \mathrm{c}}$

Average number of embryos transferred

\section{Donor Eggs}

Number of transfers

Percentage of transfers resulting in live births $s^{b, c}$

Average number of embryos transferred

\section{Age of Woman}

35-37 38-40

$41-42^{d}$

$<35$

164

101

44

261

49.8

37.8

26.7

13.6

46.4

32.9

16.8

$(40.2-52.6)$

(25.8-40.7)

$(10.1-25.6)$

23.9

42.9

23.9

23.9

29.7

9.1

56.8

28.6

22.0

3.1

7.4

37.1

3.2

33.3

0.0

$0 / 17$

(2.5-21.7)

14.3

16.7

12.5

36.4

3.3

$2 / 6$

$1 / 6$

32.2

76

19.7

41

9.8

13

28.6

2.1

2.3

$2 / 13$

2.1

All Ages Combined ${ }^{\mathrm{e}}$

Fresh Embryos

Frozen Embryos

40

22

57.5

22.7

2.1

\section{CURRENT CLINIC SERVICES AND PROFILE}

Current Name: Cleveland Clinic Fertility Center
Donor egg? Yes
Donor embryo? Yes
Gestational carriers? Yes
Cryopreservation? Yes
Single women? Yes

$\begin{array}{ll}\text { SART member? } & \text { Yes } \\ \text { Verified lab accreditation? } & \text { Yes } \\ \text { (See Appendix C for details.) } & \end{array}$

${ }^{a}$ Reflects patient and treatment characteristics of ART cycles performed in 2006 using fresh nondonor eggs or embryos.

b When fewer than 20 cycles are reported in an age category, rates are shown as a fraction and confidence intervals are not given. Calculating percentages from fractions may be misleading and is not encouraged.

c A multiple-infant birth is counted as one live birth.

${ }^{d}$ Clinic-specific outcome rates are unreliable for women older than 42 undergoing ART cycles using fresh or frozen embryos with nondonor eggs. Readers are urged to review national outcomes for these age groups (see page 27).

e All ages (including ages $>42$ ) are reported together because previous data show that patient age does not materially affect success with donor eggs. 


\section{MACDONALD FERTILITY AND IVF PROGRAM UNIVERSITY HOSPITALS, MACDONALD WOMEN'S HOSPITAL CLEVELAND, OHIO}

A comparison of clinic success rates may not be meaningful because patient medical characteristics and treatment approaches vary from clinic to clinic. For more details about this, along with information on how to interpret the statistics in this table, see pages 79-88.

\section{ART CYCLE PROFILE}

\begin{tabular}{|c|c|c|c|c|c|c|c|}
\hline \multicolumn{4}{|c|}{ Type of ART ${ }^{a}$} & \multicolumn{4}{|c|}{ Patient Diagnosis } \\
\hline IVF & $100 \%$ & Procedural Factors: & & Tubal factor & $9 \%$ & Other factor & $4 \%$ \\
\hline GIFT & $0 \%$ & With ICSI & $64 \%$ & Ovulatory dysfunction & $2 \%$ & Unknown factor & $2 \%$ \\
\hline ZIFT & $0 \%$ & Unstimulated & $<1 \%$ & Diminished ovarian reserve & $6 \%$ & Multiple Factors: & \\
\hline \multirow[t]{3}{*}{ Combination } & $0 \%$ & Used gestational carrier & $0 \%$ & Endometriosis & $9 \%$ & Female factors only & $13 \%$ \\
\hline & & & & Uterine factor & $4 \%$ & Female \& male factors & $30 \%$ \\
\hline & & & & Male factor & $22 \%$ & & \\
\hline
\end{tabular}

2006 PREGNANCY SUCCESS RATES

Data verified by William W. Hurd, MD

\section{Type of Cycle}

\section{Fresh Embryos from Nondonor Eggs}

Number of cycles

Percentage of cycles resulting in pregnancies ${ }^{b}$

Percentage of cycles resulting in live births ${ }^{b, c}$

(Confidence Interval)

Percentage of retrievals resulting in live births $s^{b, c}$

Percentage of transfers resulting in live births $s^{b, c}$

Percentage of transfers resulting in singleton live births ${ }^{b}$

Percentage of cancellations ${ }^{b}$

Average number of embryos transferred

Percentage of pregnancies with twins ${ }^{b}$

Percentage of pregnancies with triplets or more ${ }^{b}$

Percentage of live births having multiple infants ${ }^{b, c}$

\section{Frozen Embryos from Nondonor Eggs}

Number of transfers

Percentage of transfers resulting in live births $s^{b, c}$

Average number of embryos transferred

\section{Donor Eggs}

Number of transfers

Percentage of transfers resulting in live births $s^{b, c}$

Average number of embryos transferred

\section{Age of Woman}

35-37 38-40

$41-42^{d}$

\section{$<35$}

29

58.6

31

20

44.6

35.6

55.2

38.7

30.0

$(26.4-45.8)$

39.6

(35.7-73.6)

25.8

15.0

40.0

61.5

(11.9-44.6)

(3.2-37.9)

23.3

64.0

38.1

40.0

$3 / 17$

40.0

35.0

$3 / 16$

9.9

10.3

32.3

$3 / 16$

2.5

2.6

35.6

0.0

$7 / 17$

41.7

$1 / 17$

$6 / 16$

3.2

15.0

2.9

$0 / 6$

$0 / 6$

$1 / 12$

$0 / 3$ 


\section{METROHEALTH MEDICAL CENTER METROHEALTH FERTILITY CENTER CLEVELAND, OHIO}

A comparison of clinic success rates may not be meaningful because patient medical characteristics and treatment approaches vary from clinic to clinic. For more details about this, along with information on how to interpret the statistics in this table, see pages $79-88$.

\section{ART CYCLE PROFILE}

\begin{tabular}{|c|c|c|c|c|c|c|c|}
\hline \multicolumn{4}{|c|}{ Type of ART ${ }^{\mathrm{a}}$} & \multicolumn{4}{|c|}{ Patient Diagnosis } \\
\hline IVF & $100 \%$ & Procedural Factors: & & Tubal factor & $0 \%$ & Other factor & $38 \%$ \\
\hline GIFT & $0 \%$ & With ICSI & $80 \%$ & Ovulatory dysfunction & $0 \%$ & Unknown factor & $0 \%$ \\
\hline ZIFT & $0 \%$ & Unstimulated & $0 \%$ & Diminished ovarian reserve & $0 \%$ & Multiple Factors: & \\
\hline \multirow[t]{3}{*}{ Combination } & $0 \%$ & Used gestational carrier & $0 \%$ & Endometriosis & $0 \%$ & Female factors only & $0 \%$ \\
\hline & & & & Uterine factor & $0 \%$ & Female \& male factors & $50 \%$ \\
\hline & & & & Male factor & $13 \%$ & & \\
\hline
\end{tabular}

2006 PREGNANCY SUCCESS RATES

Data verified by Khalid M. Ataya, MD

\section{Type of Cycle}

\section{Fresh Embryos from Nondonor Eggs}

Number of cycles

Percentage of cycles resulting in pregnancies ${ }^{b}$

Percentage of cycles resulting in live births ${ }^{b, c}$

(Confidence Interval)

Percentage of retrievals resulting in live births $s^{b, c}$

Percentage of transfers resulting in live births $s^{b, c}$

Percentage of transfers resulting in singleton live births ${ }^{b}$

Percentage of cancellations ${ }^{b}$

Average number of embryos transferred

Percentage of pregnancies with twins ${ }^{b}$

Percentage of pregnancies with triplets or more ${ }^{b}$

Percentage of live births having multiple infants ${ }^{b, c}$

\section{Frozen Embryos from Nondonor Eggs}

Number of transfers

Percentage of transfers resulting in live births $s^{b, c}$

Average number of embryos transferred

\section{Donor Eggs}

Number of transfers

Percentage of transfers resulting in live births ${ }^{\mathrm{b}, \mathrm{c}}$

Average number of embryos transferred

\section{Age of Woman}

$\begin{array}{cccc}<35 & 35-37 & 38-40 & \mathbf{4 1 - 4 2}^{\text {d }} \\ 2 & 3 & 0 & 0 \\ 0 / 2 & 1 / 3 & & \\ 0 / 2 & 0 / 3 & & \end{array}$

$0 / 1 \quad 0 / 3$

$0 / 1 \quad 0 / 3$

$0 / 1 \quad 0 / 3$

$1 / 2 \quad 0 / 3$

$3.0 \quad 3.0$

$1 / 1$

$0 / 1$

12

$0 / 2$

$3.0 \quad 2.5$

All Ages Combined ${ }^{\mathrm{e}}$

Fresh Embryos

0

Frozen Embryos

0

\section{CURRENT CLINIC SERVICES AND PROFILE}

Current Name: MetroHealth Medical Center, MetroHealth Fertility Center

\begin{tabular}{ll|l|l|l|l} 
Donor egg? & No & Gestational carriers? & No & SART member? & Yes \\
\hline Donor embryo? & Yes & Cryopreservation? & Yes & Verified lab accreditation? & Yes \\
Single women? & Yes & & & (See Appendix C for details.) &
\end{tabular}

${ }^{a}$ Reflects patient and treatment characteristics of ART cycles performed in 2006 using fresh nondonor eggs or embryos.

b When fewer than 20 cycles are reported in an age category, rates are shown as a fraction and confidence intervals are not given. Calculating percentages from fractions may be misleading and is not encouraged.

${ }^{c}$ A multiple-infant birth is counted as one live birth.

${ }^{d}$ Clinic-specific outcome rates are unreliable for women older than 42 undergoing ART cycles using fresh or frozen embryos with nondonor eggs. Readers are urged to review national outcomes for these age groups (see page 27).

e All ages (including ages $>42$ ) are reported together because previous data show that patient age does not materially affect success with donor eggs. 


\section{OHIO REPRODUCTIVE MEDICINE COLUMBUS, OHIO}

A comparison of clinic success rates may not be meaningful because patient medical characteristics and treatment approaches vary from clinic to clinic. For more details about this, along with information on how to interpret the statistics in this table, see pages 79-88.

\section{ART CYCLE PROFILE}

\begin{tabular}{|c|c|c|c|c|c|c|c|}
\hline \multicolumn{4}{|c|}{ Type of ART ${ }^{a}$} & \multicolumn{4}{|c|}{ Patient Diagnosis } \\
\hline IVF & $>99 \%$ & Procedural Factors: & & Tubal factor & $20 \%$ & Other factor & $5 \%$ \\
\hline GIFT & $<1 \%$ & With ICSI & $40 \%$ & Ovulatory dysfunction & $3 \%$ & Unknown factor & $25 \%$ \\
\hline ZIFT & $0 \%$ & Unstimulated & $0 \%$ & Diminished ovarian reserve & $9 \%$ & Multiple Factors: & \\
\hline \multirow[t]{3}{*}{ Combination } & $0 \%$ & Used gestational carrier & $<1 \%$ & Endometriosis & $8 \%$ & Female factors only & $3 \%$ \\
\hline & & & & Uterine factor & $<1 \%$ & Female \& male factors & $5 \%$ \\
\hline & & & & Male factor & $23 \%$ & & \\
\hline
\end{tabular}

2006 PREGNANCY SUCCESS RATES

Data verified by Grant Schmidt, MD, PhD

\section{Type of Cycle}

\section{Fresh Embryos from Nondonor Eggs}

Number of cycles

Percentage of cycles resulting in pregnancies ${ }^{b}$

Percentage of cycles resulting in live births ${ }^{b, c}$

(Confidence Interval)

Percentage of retrievals resulting in live births $s^{b, c}$

Percentage of transfers resulting in live births $s^{b, c}$

Percentage of transfers resulting in singleton live births ${ }^{b}$

Percentage of cancellations ${ }^{b}$

Average number of embryos transferred

Percentage of pregnancies with twins ${ }^{b}$

Percentage of pregnancies with triplets or more ${ }^{b}$

Percentage of live births having multiple infants ${ }^{\mathrm{b}, \mathrm{c}}$

\section{Frozen Embryos from Nondonor Eggs}

Number of transfers

Percentage of transfers resulting in live births ${ }^{b, c}$

Average number of embryos transferred

\section{Donor Eggs}

Number of transfers

Percentage of transfers resulting in live births $s^{b, c}$

Average number of embryos transferred

\section{Age of Woman}

35-37 38-40

$41-42^{d}$

$<35$

98

36.7

67

31

45.1

29.6

32.8

25.8

38.7

(20.8-39.7)

25.4

19.4

(32.5-45.3)

41.2

42.9

34.5

(15.5-37.5)

(7.5-37.5)

27.9

30.4

17.9

25.0

37.7

26.0

9.0

27.3

33.5

14.3

3.2

36.4

27.8

0.0

31.0

4.5

$7 / 17$

22.7

22.6

4.2

$1 / 8$

$0 / 8$

$1 / 6$
25

8.0

2.1

\section{3}

$1 / 13$

2.2

\section{6}

$1 / 6$

2.7

All Ages Combined ${ }^{\mathrm{e}}$

Fresh Embryos

Frozen Embryos

$\begin{array}{cc}31 & 12 \\ 41.9 & 4 / 12 \\ 2.2 & 2.6\end{array}$

\section{CURRENT CLINIC SERVICES AND PROFILE}

Current Name: Ohio Reproductive Medicine
Donor egg?
Yes
Donor embryo? Yes
Gestational carriers? Yes
Cryopreservation?
Yes
SART member?
Yes
Verified lab accreditation?
Yes
Single women? Yes
(See Appendix C for details.)

\footnotetext{
${ }^{a}$ Reflects patient and treatment characteristics of ART cycles performed in 2006 using fresh nondonor eggs or embryos.

${ }^{b}$ When fewer than 20 cycles are reported in an age category, rates are shown as a fraction and confidence intervals are not given. Calculating percentages from fractions may be misleading and is not encouraged.

${ }^{c}$ A multiple-infant birth is counted as one live birth.

d Clinic-specific outcome rates are unreliable for women older than 42 undergoing ART cycles using fresh or frozen embryos with nondonor eggs. Readers are urged to review national outcomes for these age groups (see page 27).

e All ages (including ages $>42$ ) are reported together because previous data show that patient age does not materially affect success with donor eggs.
} 


\section{KETTERING REPRODUCTIVE MEDICINE KETTERING, OHIO}

A comparison of clinic success rates may not be meaningful because patient medical characteristics and treatment approaches vary from clinic to clinic. For more details about this, along with information on how to interpret the statistics in this table, see pages $79-88$.

\section{ART CYCLE PROFILE}

\begin{tabular}{|c|c|c|c|c|c|c|c|}
\hline \multicolumn{4}{|c|}{ Type of ART ${ }^{\mathrm{a}}$} & \multicolumn{4}{|c|}{ Patient Diagnosis } \\
\hline IVF & $100 \%$ & Procedural Factors: & & Tubal factor & $19 \%$ & Other factor & $2 \%$ \\
\hline GIFT & $0 \%$ & With ICSI & $61 \%$ & Ovulatory dysfunction & $7 \%$ & Unknown factor & $2 \%$ \\
\hline $\mathrm{ZIFT}$ & $0 \%$ & Unstimulated & $0 \%$ & Diminished ovarian reserve & $13 \%$ & Multiple Factors: & \\
\hline Combination & $0 \%$ & Used gestational carrier & $0 \%$ & Endometriosis & $13 \%$ & Female factors only & $0 \%$ \\
\hline & & & & $\begin{array}{l}\text { Uterine factor } \\
\text { Male factor }\end{array}$ & $\begin{array}{r}0 \% \\
25 \%\end{array}$ & Female \& male factors & $18 \%$ \\
\hline
\end{tabular}

2006 PREGNANCY SUCCESS RATES

Data verified by Thomas H. Burwinkel, MD

\section{Type of Cycle}

\section{Fresh Embryos from Nondonor Eggs}

Number of cycles

Percentage of cycles resulting in pregnancies ${ }^{b}$

Percentage of cycles resulting in live births $s^{b, c}$

(Confidence Interval)

Percentage of retrievals resulting in live births $s^{b, c}$

Percentage of transfers resulting in live births $s^{b, c}$

Percentage of transfers resulting in singleton live births ${ }^{b}$

Percentage of cancellations ${ }^{b}$

Average number of embryos transferred

Percentage of pregnancies with twins ${ }^{b}$

Percentage of pregnancies with triplets or more ${ }^{b}$

Percentage of live births having multiple infants ${ }^{b, c}$

\section{Frozen Embryos from Nondonor Eggs}

Number of transfers

Percentage of transfers resulting in live births ${ }^{\mathrm{b}, \mathrm{c}}$

Average number of embryos transferred

\section{Donor Eggs}

Number of transfers

Percentage of transfers resulting in live births $s^{b, c}$

Average number of embryos transferred

\section{Age of Woman}

35-37 38-40

$41-42^{d}$

$<35$

20

15.0

9

5

32.7

10.0

$2 / 9$

$0 / 5$

30.6

(18.3-45.4)

(1.2-31.7)

32.6

$2 / 19$

$2 / 9$

$0 / 5$

35.7

$2 / 18$

$2 / 8$

$0 / 4$

23.8

$1 / 18$

$2 / 8$

$0 / 3$

6.1

5.0

$1 / 8$

$0 / 3$

2.1

3.0

$1 / 9$

$1 / 5$

$6 / 16$

$1 / 3$

3.1

$1 / 2$

$1 / 16$

$0 / 3$

$0 / 2$

$5 / 15$

$1 / 2$

$1 / 2$

$\begin{array}{cccc}43 & 18 & 3 & 3 \\ 37.2 & 2 / 18 & 1 / 3 & 0 / 3 \\ 2.1 & 2.9 & 2.3 & 2.7\end{array}$

\section{All Ages Combined $^{\mathrm{e}}$}

Fresh Embryos

Frozen Embryos

$\begin{array}{cc}15 & 2 \\ 5 / 15 & 0 / 2 \\ 2.1 & 2.5\end{array}$

\section{CURRENT CLINIC SERVICES AND PROFILE}

Current Name: Kettering Reproductive Medicine

\begin{tabular}{ll|lll|ll} 
Donor egg? & Yes & Gestational carriers? & Yes & SART member? & Yes \\
Donor embryo? & Yes & Cryopreservation? & Yes & Verified lab accreditation? & Yes \\
Single women? & No & & & (See Appendix C for details.) &
\end{tabular}

${ }^{a}$ Reflects patient and treatment characteristics of ART cycles performed in 2006 using fresh nondonor eggs or embryos.

${ }^{b}$ When fewer than 20 cycles are reported in an age category, rates are shown as a fraction and confidence intervals are not given. Calculating percentages from fractions may be misleading and is not encouraged.

${ }^{c}$ A multiple-infant birth is counted as one live birth.

${ }^{d}$ Clinic-specific outcome rates are unreliable for women older than 42 undergoing ART cycles using fresh or frozen embryos with nondonor eggs. Readers are urged to review national outcomes for these age groups (see page 27).

${ }^{e}$ All ages (including ages $>42$ ) are reported together because previous data show that patient age does not materially affect success with donor eggs. 


\section{FERTILITY CENTER OF NORTHWESTERN OHIO \\ TOLEDO, OHIO}

A comparison of clinic success rates may not be meaningful because patient medical characteristics and treatment approaches vary from clinic to clinic. For more details about this, along with information on how to interpret the statistics in this table, see pages 79-88.

\section{ART CYCLE PROFILE}

\begin{tabular}{|c|c|c|c|c|c|c|c|}
\hline \multicolumn{4}{|c|}{ Type of ART ${ }^{a}$} & \multicolumn{4}{|c|}{ Patient Diagnosis } \\
\hline IVF & $100 \%$ & Procedural Factors: & & Tubal factor & $19 \%$ & Other factor & $0 \%$ \\
\hline GIFT & $0 \%$ & With ICSI & $30 \%$ & Ovulatory dysfunction & $9 \%$ & Unknown factor & $4 \%$ \\
\hline ZIFT & $0 \%$ & Unstimulated & $<1 \%$ & Diminished ovarian reserve & $5 \%$ & Multiple Factors: & \\
\hline \multirow[t]{3}{*}{ Combination } & $0 \%$ & Used gestational carrier & $0 \%$ & Endometriosis & $0 \%$ & Female factors only & $18 \%$ \\
\hline & & & & Uterine factor & $<1 \%$ & Female \& male factors & $25 \%$ \\
\hline & & & & Male factor & $20 \%$ & & \\
\hline
\end{tabular}

2006 PREGNANCY SUCCESS RATES

Data verified by Joseph V. Karnitis, MD

\section{Type of Cycle}

\section{Fresh Embryos from Nondonor Eggs}

Number of cycles

Percentage of cycles resulting in pregnancies ${ }^{b}$

Percentage of cycles resulting in live births ${ }^{b, c}$

(Confidence Interval)

Percentage of retrievals resulting in live births $s^{b, c}$

Percentage of transfers resulting in live births $s^{b, c}$

Percentage of transfers resulting in singleton live births ${ }^{b}$

Percentage of cancellations ${ }^{b}$

Average number of embryos transferred

Percentage of pregnancies with twins ${ }^{b}$

Percentage of pregnancies with triplets or more ${ }^{b}$

Percentage of live births having multiple infants ${ }^{b, c}$

\section{Frozen Embryos from Nondonor Eggs}

Number of transfers

Percentage of transfers resulting in live births ${ }^{b, c}$

Average number of embryos transferred

\section{Donor Eggs}

Number of transfers

Percentage of transfers resulting in live births $s^{b, c}$

Average number of embryos transferred

\section{Age of Woman}

35-37 38-40

$41-42^{d}$

\section{$<35$}

29

24.1

12

4

26.7

17.2

$4 / 12$

$2 / 4$

20.0

(9.6-34.6)

(5.8-35.8)

28.1

29.0

$5 / 15$

$2 / 12$

$0 / 4$

\section{4}

28.9

$5 / 13$

$2 / 10$

$0 / 3$

$4 / 13$

$2 / 8$

$0 / 3$

3.0

48.3

$1 / 8$

$0 / 3$

$2 / 12$

2.5

$2 / 12$

$1 / 4$

$2 / 12$

$1 / 7$

3.3

3.3

$3 / 9$

$0 / 7$

$1 / 4$

$2 / 2$

$0 / 4$

$0 / 2$

$1 / 5$

$1 / 2$

\begin{tabular}{|c|c|c|c|}
\hline 7 & 0 & 1 & 1 \\
\hline $1 / 7$ & & $1 / 1$ & $0 / 1$ \\
\hline 2.6 & & 2.0 & 2.0 \\
\hline
\end{tabular}

\section{All Ages Combined}

Fresh Embryos

3

$1 / 3$

2.7

\section{CURRENT CLINIC SERVICES AND PROFILE}

Current Name: Fertility Center of Northwestern Ohio

Donor egg? Yes Gestational carriers? Yes

Donor embryo? Yes Cryopreservation? Yes

Single women? Yes

SART member? Yes

Verified lab accreditation? Yes

(See Appendix C for details.)

${ }^{a}$ Reflects patient and treatment characteristics of ART cycles performed in 2006 using fresh nondonor eggs or embryos.

${ }^{b}$ When fewer than 20 cycles are reported in an age category, rates are shown as a fraction and confidence intervals are not given. Calculating percentages from fractions may be misleading and is not encouraged.

${ }^{c}$ A multiple-infant birth is counted as one live birth.

d Clinic-specific outcome rates are unreliable for women older than 42 undergoing ART cycles using fresh or frozen embryos with nondonor eggs. Readers are urged to review national outcomes for these age groups (see page 27).

e All ages (including ages $>42$ ) are reported together because previous data show that patient age does not materially affect success with donor eggs. 


\section{HENRY G. BENNETT, JR., FERTILITY INSTITUTE OKLAHOMA CITY, OKLAHOMA}

A comparison of clinic success rates may not be meaningful because patient medical characteristics and treatment approaches vary from clinic to clinic. For more details about this, along with information on how to interpret the statistics in this table, see pages $79-88$.

\section{ART CYCLE PROFILE}

\begin{tabular}{|c|c|c|c|c|c|c|}
\hline & Type of $A R T^{a}$ & & Pat & ent $D$ & iagnosis & \\
\hline IVF & $100 \%$ Procedural Factors: & & Tubal factor & $17 \%$ & Other factor & $<1 \%$ \\
\hline GIFT & 0\% With ICSI & $39 \%$ & Ovulatory dysfunction & $17 \%$ & Unknown factor & $7 \%$ \\
\hline ZIFT & $0 \%$ Unstimulated & $0 \%$ & Diminished ovarian reserve & $5 \%$ & Multiple Factors: & \\
\hline Combination & $0 \%$ Used gestational carrier & $0 \%$ & Endometriosis & $6 \%$ & Female factors only & $12 \%$ \\
\hline & & & Uterine factor & $<1 \%$ & Female \& male factors & $16 \%$ \\
\hline
\end{tabular}

2006 PREGNANCY SUCCESS RATES

Data verified by Eli Reshef, MD

\section{Type of Cycle}

\section{Fresh Embryos from Nondonor Eggs}

Number of cycles

Percentage of cycles resulting in pregnancies ${ }^{b}$

Percentage of cycles resulting in live births ${ }^{b, c}$

(Confidence Interval)

Percentage of retrievals resulting in live births $s^{b, c}$

Percentage of transfers resulting in live births $s^{b, c}$

Percentage of transfers resulting in singleton live births ${ }^{b}$

Percentage of cancellations ${ }^{b}$

Average number of embryos transferred

Percentage of pregnancies with twins ${ }^{b}$

Percentage of pregnancies with triplets or more ${ }^{b}$

Percentage of live births having multiple infants ${ }^{b, c}$

\section{Frozen Embryos from Nondonor Eggs}

Number of transfers

Percentage of transfers resulting in live births $s^{b, c}$

Average number of embryos transferred

\section{Donor Eggs}

Number of transfers

Percentage of transfers resulting in live births ${ }^{\mathrm{b}, \mathrm{c}}$

Average number of embryos transferred

\section{Age of Woman}

35-37 38-40

$41-42^{d}$ $<35$

125

62.4

43

41.9

34

6

59.2

34.9

29.4

$1 / 6$

$(50.1-67.9)$

(21.0-50.9)

61.7

38.5

26.5

$1 / 6$

\section{7}

40.5

(12.9-44.4)

34.7

32.4

32.1

36.0

$1 / 5$

4.0

9.3

32.0

$1 / 4$

$1 / 4$

2.1

2.6

17.6

$1 / 6$

42.3

$4 / 18$

2.4

2.3

3.8

$0 / 18$

44.6

$3 / 15$

$2 / 10$

$0 / 1$

$0 / 10$

$0 / 1$

$1 / 9$

$0 / 1$

11

$0 / 11$

2.4

\section{6}

$1 / 6$

2.8
1

$0 / 1$

2.0
$<1 \%$

\section{CURRENT CLINIC SERVICES AND PROFILE}

Current Name: Henry G. Bennett, Jr., Fertility Institute

Donor egg? Yes

Donor embryo? Yes

Gestational carriers?

No

Fresh Embryos

All Ages Combined

Single women? No

Cryopreservation? Yes

Frozen Embryos

$\begin{array}{cc}26 & 11 \\ 46.2 & 3 / 11 \\ 2.2 & 3.0\end{array}$

\footnotetext{
${ }^{a}$ Reflects patient and treatment characteristics of ART cycles performed in 2006 using fresh nondonor eggs or embryos.

${ }^{b}$ When fewer than 20 cycles are reported in an age category, rates are shown as a fraction and confidence intervals are not given. Calculating percentages from fractions may be misleading and is not encouraged.

A multiple-infant birth is counted as one live birth.

d Clinic-specific outcome rates are unreliable for women older than 42 undergoing ART cycles using fresh or frozen embryos with nondonor eggs. Readers are urged to review national outcomes for these age groups (see page 27).

e All ages (including ages $>42$ ) are reported together because previous data show that patient age does not materially affect success with donor eggs.
} 


\section{CENTER FOR REPRODUCTIVE HEALTH, PC OKLAHOMA CITY, OKLAHOMA}

A comparison of clinic success rates may not be meaningful because patient medical characteristics and treatment approaches vary from clinic to clinic. For more details about this, along with information on how to interpret the statistics in this table, see pages 79-88.

\section{ART CYCLE PROFILE}

\begin{tabular}{|c|c|c|c|c|c|c|c|}
\hline \multicolumn{4}{|c|}{ Type of ART ${ }^{a}$} & \multicolumn{4}{|c|}{ Patient Diagnosis } \\
\hline IVF & $100 \%$ & Procedural Factors: & & Tubal factor & $24 \%$ & Other factor & $7 \%$ \\
\hline GIFT & $0 \%$ & With ICSI & $78 \%$ & Ovulatory dysfunction & $2 \%$ & Unknown factor & $5 \%$ \\
\hline ZIFT & $0 \%$ & Unstimulated & $0 \%$ & Diminished ovarian reserve & $4 \%$ & Multiple Factors: & \\
\hline \multirow[t]{3}{*}{ Combination } & $0 \%$ & Used gestational carrier & $0 \%$ & Endometriosis & $1 \%$ & Female factors only & $13 \%$ \\
\hline & & & & Uterine factor & $0 \%$ & Female \& male factors & $24 \%$ \\
\hline & & & & Male factor & $18 \%$ & & \\
\hline
\end{tabular}

\section{PREGNANCY SUCCESS RATES}

\section{Type of Cycle}

\section{Fresh Embryos from Nondonor Eggs}

Number of cycles

Percentage of cycles resulting in pregnancies ${ }^{b}$

Percentage of cycles resulting in live births ${ }^{b, c}$

(Confidence Interval)

Percentage of retrievals resulting in live births $s^{b, c}$

Percentage of transfers resulting in live births $s^{b, c}$

Percentage of transfers resulting in singleton live births ${ }^{b}$

Percentage of cancellations ${ }^{b}$

Average number of embryos transferred

Percentage of pregnancies with twins ${ }^{b}$

Percentage of pregnancies with triplets or more ${ }^{b}$

Percentage of live births having multiple infants ${ }^{b, c}$

\section{Frozen Embryos from Nondonor Eggs}

Number of transfers

Percentage of transfers resulting in live births $s^{b, c}$

Average number of embryos transferred

\section{Donor Eggs}

Number of transfers

Percentage of transfers resulting in live births $s^{\mathrm{b}, \mathrm{c}}$

Average number of embryos transferred
Data verified by Karl R. Hansen, MD, PhD

\section{Age of Woman}

$<35 \quad 35-37 \quad 38-40 \quad 41-42^{\text {d }}$

$\begin{array}{ccc}43 & 6 & 9 \\ 51.2 & 4 / 6 & 5 / 9 \\ 51.2 & 4 / 6 & 3 / 9\end{array}$

(35.5-66.7)

$\begin{array}{ccc}64.7 & 4 / 5 & 3 / 9 \\ 64.7 & 4 / 5 & 3 / 9 \\ 41.2 & 4 / 5 & 3 / 9 \\ 20.9 & 1 / 6 & 0 / 9 \\ 2.1 & 2.4 & 2.7 \\ 50.0 & 0 / 4 & 1 / 5 \\ 0.0 & 0 / 4 & 0 / 5 \\ 36.4 & 0 / 4 & 0 / 3\end{array}$

8

$4 / 8$

1

$0 / 1$

0

1

$0 / 1$

1.8

2.0

\section{CURRENT CLINIC SERVICES AND PROFILE}

Current Name: Center for Reproductive Health, PC

Donor egg? Yes Gestational carriers? Yes

Donor embryo? Yes Cryopreservation? Yes

All Ages Combined ${ }^{\mathrm{e}}$

Fresh Embryos

10

$5 / 10$

2.0
Frozen Embryos

2

$1 / 2$

2.0

Single women? Yes

SART member? Yes

Verified lab accreditation?

(See Appendix C for details.)

${ }^{a}$ Reflects patient and treatment characteristics of ART cycles performed in 2006 using fresh nondonor eggs or embryos.

${ }^{b}$ When fewer than 20 cycles are reported in an age category, rates are shown as a fraction and confidence intervals are not given. Calculating percentages from fractions may be misleading and is not encouraged.

${ }^{c}$ A multiple-infant birth is counted as one live birth.

d Clinic-specific outcome rates are unreliable for women older than 42 undergoing ART cycles using fresh or frozen embryos with nondonor eggs. Readers are urged to review national outcomes for these age groups (see page 27).

e All ages (including ages $>42$ ) are reported together because previous data show that patient age does not materially affect success with donor eggs. 


\section{TULSA CENTER FOR FERTILITY \& WOMEN'S HEALTH TULSA, OKLAHOMA}

A comparison of clinic success rates may not be meaningful because patient medical characteristics and treatment approaches vary from clinic to clinic. For more details about this, along with information on how to interpret the statistics in this table, see pages $79-88$.

\section{ART CYCLE PROFILE}

\begin{tabular}{|c|c|c|c|c|c|c|c|}
\hline \multicolumn{4}{|c|}{ Type of ART } & \multicolumn{4}{|c|}{ Patient Diagnosis } \\
\hline IVF & $100 \%$ & Procedural Factors: & & Tubal factor & $12 \%$ & Other factor & $20 \%$ \\
\hline GIFT & $0 \%$ & With ICSI & $57 \%$ & Ovulatory dysfunction & $6 \%$ & Unknown factor & $7 \%$ \\
\hline ZIFT & $0 \%$ & Unstimulated & $0 \%$ & Diminished ovarian reserve & $<1 \%$ & Multiple Factors: & \\
\hline \multirow[t]{3}{*}{ Combination } & $0 \%$ & Used gestational carrier & $0 \%$ & Endometriosis & $4 \%$ & Female factors only & $7 \%$ \\
\hline & & & & Uterine factor & $0 \%$ & Female \& male factors & $20 \%$ \\
\hline & & & & Male factor & $23 \%$ & & \\
\hline
\end{tabular}

2006 PREGNANCY SUCCESS RATES

Data verified by Stanley G. Prough, MD

\section{Type of Cycle}

\section{Fresh Embryos from Nondonor Eggs}

Number of cycles

Percentage of cycles resulting in pregnancies ${ }^{b}$

Percentage of cycles resulting in live births ${ }^{b, c}$

(Confidence Interval)

Percentage of retrievals resulting in live births $s^{b, c}$

Percentage of transfers resulting in live births $s^{b, c}$

Percentage of transfers resulting in singleton live births ${ }^{b}$

Percentage of cancellations ${ }^{b}$

Average number of embryos transferred

Percentage of pregnancies with twins ${ }^{b}$

Percentage of pregnancies with triplets or more ${ }^{b}$

Percentage of live births having multiple infants ${ }^{b, c}$

\section{Frozen Embryos from Nondonor Eggs}

Number of transfers

Percentage of transfers resulting in live births $s^{b, c}$

Average number of embryos transferred

\section{Donor Eggs}

Number of transfers

Percentage of transfers resulting in live births $s^{b, c}$

Average number of embryos transferred

\section{Age of Woman}

35-37 38-40

$41-42^{d}$

$<35$

42

143

32.2

28.7

14.3

14.3

(21.4-36.8)

32.0

$(5.4-28.5)$

34.7

19.4

20.0

13.3

26.2

2.3

$1 / 6$

$1 / 6$

$2 / 6$

36.6

10

$6 / 10$

2.5

34.4

2.4

All Ages Combined ${ }^{\mathrm{e}}$

Fresh Embryos

$\begin{array}{cc}28 & 7 \\ 28.6 & 1 / 7 \\ 21.4 & 1 / 7\end{array}$

(8.3-41.0)

23.1

25.0

$1 / 6$

25.0

$1 / 6$

$0 / 6$

7.1

$1 / 7$

2.3

2.3

$2 / 8$

$1 / 1$

$0 / 8$

$0 / 1$

$0 / 6$

$1 / 1$

Frozen Embryos

2.1

31.8

2.4

\section{CURRENT CLINIC SERVICES AND PROFILE}

Current Name: Tulsa Fertility Center

Donor egg? Yes

Donor embryo? Yes

Single women? No
Gestational carriers? No

Cryopreservation? Yes
SART member?

Yes

Verified lab accreditation?

(See Appendix C for details.)

${ }^{a}$ Reflects patient and treatment characteristics of ART cycles performed in 2006 using fresh nondonor eggs or embryos.

${ }^{b}$ When fewer than 20 cycles are reported in an age category, rates are shown as a fraction and confidence intervals are not given. Calculating percentages from fractions may be misleading and is not encouraged.

A multiple-infant birth is counted as one live birth.

d Clinic-specific outcome rates are unreliable for women older than 42 undergoing ART cycles using fresh or frozen embryos with nondonor eggs. Readers are urged to review national outcomes for these age groups (see page 27).

e All ages (including ages $>42$ ) are reported together because previous data show that patient age does not materially affect success with donor eggs. 


\section{THE FERTILITY CENTER OF OREGON \\ EUGENE, OREGON}

A comparison of clinic success rates may not be meaningful because patient medical characteristics and treatment approaches vary from clinic to clinic. For more details about this, along with information on how to interpret the statistics in this table, see pages 79-88.

\section{ART CYCLE PROFILE}

\begin{tabular}{|c|c|c|c|c|c|c|c|}
\hline \multicolumn{4}{|c|}{ Type of ART ${ }^{a}$} & \multicolumn{4}{|c|}{ Patient Diagnosis } \\
\hline IVF & $100 \%$ & Procedural Factors: & & Tubal factor & $18 \%$ & Other factor & $2 \%$ \\
\hline GIFT & $0 \%$ & With ICSI & $80 \%$ & Ovulatory dysfunction & $3 \%$ & Unknown factor & $9 \%$ \\
\hline ZIFT & $0 \%$ & Unstimulated & $0 \%$ & Diminished ovarian reserve & $8 \%$ & Multiple Factors: & \\
\hline \multirow[t]{3}{*}{ Combination } & $0 \%$ & Used gestational carrier & $0 \%$ & Endometriosis & $4 \%$ & Female factors only & $22 \%$ \\
\hline & & & & Uterine factor & $0 \%$ & Female \& male factors & $15 \%$ \\
\hline & & & & Male factor & $19 \%$ & & \\
\hline
\end{tabular}

2006 PREGNANCY SUCCESS RATES

Data verified by Douglas J. Austin, MD

\section{Type of Cycle}

\section{Fresh Embryos from Nondonor Eggs}

Number of cycles

Percentage of cycles resulting in pregnancies ${ }^{b}$

Percentage of cycles resulting in live births $s^{b, c}$

(Confidence Interval)

Percentage of retrievals resulting in live births $s^{b, c}$

Percentage of transfers resulting in live births $s^{b, c}$

Percentage of transfers resulting in singleton live births ${ }^{b}$

Percentage of cancellations ${ }^{b}$

Average number of embryos transferred

Percentage of pregnancies with twins ${ }^{b}$

Percentage of pregnancies with triplets or more ${ }^{b}$

Percentage of live births having multiple infants ${ }^{b, c}$

\section{Frozen Embryos from Nondonor Eggs}

Number of transfers

Percentage of transfers resulting in live births ${ }^{b, c}$

Average number of embryos transferred

\section{Donor Eggs}

Number of transfers

Percentage of transfers resulting in live births $s^{b, c}$

Average number of embryos transferred

\section{Age of Woman}

35-37 38-40

$41-42^{d}$

\section{$<35$}

22

50.0

10

6

46.9

45.5

$0 / 10$

$3 / 6$

40.6

(23.7-59.4)

(24.4-67.8)

$0 / 10$

$2 / 6$

41.9

41.9

50.0

$0 / 10$

$2 / 6$

25.8

30.0

$0 / 10$

$2 / 6$

3.1

9.1

$0 / 10$

$2 / 6$

2.8

3.0

$0 / 10$

$0 / 6$

$6 / 15$

$4 / 11$

3.8

4.7

$0 / 15$

$1 / 11$

$1 / 3$

$5 / 13$

$4 / 10$

$0 / 3$

$0 / 2$

26

23.1

11

$1 / 11$

8

$2 / 8$

2.7

2.6

3.0

All Ages Combined ${ }^{\mathrm{e}}$

Fresh Embryos 16

$10 / 16$

2.1
Frozen Embryos

9

$5 / 9$

2.7

\section{CURRENT CLINIC SERVICES AND PROFILE}

Current Name: The Fertility Center of Oregon

Donor egg? Yes

Donor embryo? No

Single women? Yes
Gestational carriers? Yes

Cryopreservation? Yes
SART member?

Verified lab accreditation?

(See Appendix C for details.)
No

No

${ }^{a}$ Reflects patient and treatment characteristics of ART cycles performed in 2006 using fresh nondonor eggs or embryos.

b When fewer than 20 cycles are reported in an age category, rates are shown as a fraction and confidence intervals are not given. Calculating percentages from fractions may be misleading and is not encouraged.

${ }^{c}$ A multiple-infant birth is counted as one live birth.

d Clinic-specific outcome rates are unreliable for women older than 42 undergoing ART cycles using fresh or frozen embryos with nondonor eggs. Readers are urged to review national outcomes for these age groups (see page 27).

e All ages (including ages $>42$ ) are reported together because previous data show that patient age does not materially affect success with donor eggs. 


\section{NORTHWEST FERTILITY CENTER \\ EUGENE M. STOELK, MD \\ PORTLAND, OREGON}

A comparison of clinic success rates may not be meaningful because patient medical characteristics and treatment approaches vary from clinic to clinic. For more details about this, along with information on how to interpret the statistics in this table, see pages 79-88.

\section{ART CYCLE PROFILE}

\section{Type of ART ${ }^{\mathrm{a}}$}

IVF

GIFT

ZIFT

Combination

\author{
100\% Procedural Factors:
}

$0 \%$ With ICSI

$0 \%$ Unstimulated

$0 \%$ Used gestational carrier

\begin{tabular}{|c|c|}
\hline & Tubal factor \\
\hline $40 \%$ & Ovulatory dysfunction \\
\hline $0 \%$ & Diminished ovarian reserve \\
\hline $3 \%$ & Endometriosis \\
\hline & Uterine factor \\
\hline
\end{tabular}

Patient Diagnosis

$15 \%$ Other factor

3\% Unknown factor

$2 \%$

4\% Multiple Factors:

$4 \%$ Female factors only $25 \%$

$2 \%$ Female \& male factors $34 \%$ $10 \%$
2006 PREGNANCY SUCCESS RATES

\section{Type of Cycle}

\section{Fresh Embryos from Nondonor Eggs}

Number of cycles

Percentage of cycles resulting in pregnancies ${ }^{b}$

Percentage of cycles resulting in live births ${ }^{b, c}$

(Confidence Interval)

Percentage of retrievals resulting in live births $s^{b, c}$

Percentage of transfers resulting in live births ${ }^{b, c}$

Percentage of transfers resulting in singleton live births ${ }^{b}$

Percentage of cancellations ${ }^{b}$

Average number of embryos transferred

Percentage of pregnancies with twins ${ }^{b}$

Percentage of pregnancies with triplets or more ${ }^{b}$

Percentage of live births having multiple infants ${ }^{b, c}$

\section{Frozen Embryos from Nondonor Eggs}

Number of transfers

Percentage of transfers resulting in live births $s^{\mathrm{b}, \mathrm{c}}$

Average number of embryos transferred

\section{Donor Eggs}

Number of transfers

Percentage of transfers resulting in live births ${ }^{\mathrm{b}, \mathrm{c}}$

Average number of embryos transferred

Data verified by Eugene M. Stoelk, MD

\section{Age of Woman}

35-37 38-40

$$
41-42^{d}
$$

\section{$<35$}

16

13

2

$\begin{array}{cccc}45 & 16 & 13 & 2 \\ 37.8 & 7 / 16 & 6 / 13 & 1 / 2 \\ 37.8 & 7 / 16 & 4 / 13 & 1 / 2\end{array}$

(23.8-53.5)

39.5

43.6

$7 / 16$

$4 / 13$

$1 / 2$

$7 / 16$

$4 / 13$

$1 / 2$

23.1

$4 / 16$

$4 / 13$

$0 / 2$

4.4

$0 / 16$

$0 / 13$

$0 / 2$

2.2

2.9

3.8

2.0

$8 / 17$

$4 / 7$

$0 / 6$

$1 / 1$

$0 / 17$

$0 / 7$

$0 / 6$

$0 / 1$

$8 / 17$

$3 / 7$

$0 / 4$

$1 / 1$

32

31.3

12

$2 / 12$

3

$1 / 3$

2.7

3.1

3.7

All Ages Combined ${ }^{\mathrm{e}}$

Fresh Embryos

19

$9 / 19$

2.4
Frozen Embryos 38

13.2

2.8

\section{CURRENT CLINIC SERVICES AND PROFILE}

Current Name: Northwest Fertility Center, Eugene M. Stoelk, MD

\begin{tabular}{l|l|l} 
Donor egg? Yes & Gestational carriers? Yes
\end{tabular}

Donor embryo? No Cryopreservation? Yes

Single women? Yes

SART member? Yes

Verified lab accreditation? Yes

(See Appendix C for details.)

${ }^{a}$ Reflects patient and treatment characteristics of ART cycles performed in 2006 using fresh nondonor eggs or embryos.

${ }^{b}$ When fewer than 20 cycles are reported in an age category, rates are shown as a fraction and confidence intervals are not given. Calculating percentages from fractions may be misleading and is not encouraged.

${ }^{c}$ A multiple-infant birth is counted as one live birth.

d Clinic-specific outcome rates are unreliable for women older than 42 undergoing ART cycles using fresh or frozen embryos with nondonor eggs. Readers are urged to review national outcomes for these age groups (see page 27).

e All ages (including ages $>42$ ) are reported together because previous data show that patient age does not materially affect success with donor eggs. 


\section{PORTLAND CENTER FOR REPRODUCTIVE MEDICINE PORTLAND, OREGON}

A comparison of clinic success rates may not be meaningful because patient medical characteristics and treatment approaches vary from clinic to clinic. For more details about this, along with information on how to interpret the statistics in this table, see pages 79-88.

\section{ART CYCLE PROFILE}

\section{Type of ART ${ }^{\mathrm{a}}$}

IVF

GIFT 100\% Procedural Factors:

ZIFT

Combination
$0 \%$ With ICSI

$0 \%$ Unstimulated

$0 \%$ Used gestational carrier
Tubal factor

$61 \%$ Ovulatory dysfunction

$<1 \%$

$1 \%$

\section{Patient Diagnosis}

8\% Other factor

4\% Unknown factor

29\% Multiple Factors:

$5 \%$ Female factors only $\quad 9 \%$

$1 \%$ Female \& male factors $15 \%$ $14 \%$

2006 PREGNANCY SUCCESS RATES

Data verified by Robert K. Matteri, MD

\section{Type of Cycle}

\section{Age of Woman}

35-37 38-40

38-40

$41-42^{d}$

\section{$<35$}

58.0

52.7

$(43.8-61.5)$

55.2

58.5

31.4

4.6

2.2

51.3

3.9

46.4

Percentage of pregnancies with triplets or more

Percentage of live births having multiple infants ${ }^{\mathrm{b}, \mathrm{c}}$

\section{Frozen Embryos from Nondonor Eggs}

Number of transfers

Percentage of transfers resulting in live births $s^{b, c}$

Average number of embryos transferred

$\begin{array}{cccc}17 & 19 & 4 & 1 \\ 4 / 17 & 4 / 19 & 2 / 4 & 1 / 1 \\ 2.6 & 2.6 & 3.0 & 2.0\end{array}$

24

16.7

12.5

41.1

45.0

(2.7-32.4)

13.0

15.0

10.0

29.6

0.0

2.5

32.0

4.0

30.4

$3 / 19$

$1 / 4$

4.2

3.4

$0 / 4$

$1 / 3$

\section{Donor Eggs}

All Ages Combined ${ }^{\mathrm{e}}$

Number of transfers

Fresh Embryos

Frozen Embryos

Percentage of transfers resulting in live births $s^{b, c}$

Average number of embryos transferred

27

44.4

\section{CURRENT CLINIC SERVICES AND PROFILE}

Current Name: Oregon Reproductive Medicine

Donor egg? Yes Gestational carriers? Yes

Donor embryo? Yes Cryopreservation? Yes

Single women? Yes

$\begin{array}{cc}95 & 27 \\ 74.7 & 44.4 \\ 2.0 & 2.5\end{array}$

\footnotetext{
${ }^{a}$ Reflects patient and treatment characteristics of ART cycles performed in 2006 using fresh nondonor eggs or embryos.

b When fewer than 20 cycles are reported in an age category, rates are shown as a fraction and confidence intervals are not given. Calculating percentages from fractions may be misleading and is not encouraged.

${ }^{\mathrm{C}}$ A multiple-infant birth is counted as one live birth.

${ }^{d}$ Clinic-specific outcome rates are unreliable for women older than 42 undergoing ART cycles using fresh or frozen embryos with nondonor eggs. Readers are urged to review national outcomes for these age groups (see page 27).

e All ages (including ages $>42$ ) are reported together because previous data show that patient age does not materially affect success with donor eggs.
} 


\section{UNIVERSITY FERTILITY CONSULTANTS \\ OREGON HEALTH \& SCIENCE UNIVERSITY \\ PORTLAND, OREGON}

A comparison of clinic success rates may not be meaningful because patient medical characteristics and treatment approaches vary from clinic to clinic. For more details about this, along with information on how to interpret the statistics in this table, see pages 79-88.

\section{ART CYCLE PROFILE}

\begin{tabular}{|c|c|c|c|c|c|c|c|}
\hline \multicolumn{4}{|c|}{ Type of ART ${ }^{a}$} & \multicolumn{4}{|c|}{ Patient Diagnosis } \\
\hline IVF & $100 \%$ & Procedural Factors: & & Tubal factor & $12 \%$ & Other factor & $26 \%$ \\
\hline GIFT & $0 \%$ & With ICSI & $60 \%$ & Ovulatory dysfunction & $4 \%$ & Unknown factor & $8 \%$ \\
\hline ZIFT & $0 \%$ & Unstimulated & $0 \%$ & Diminished ovarian reserve & $3 \%$ & Multiple Factors: & \\
\hline \multirow[t]{3}{*}{ Combination } & $0 \%$ & Used gestational carrier & $1 \%$ & Endometriosis & $5 \%$ & Female factors only & $5 \%$ \\
\hline & & & & Uterine factor & $<1 \%$ & Female \& male factors & $14 \%$ \\
\hline & & & & Male factor & $22 \%$ & & \\
\hline
\end{tabular}

2006 PREGNANCY SUCCESS RATES

Data verified by Marsha J. Gorrill, MD

\section{Type of Cycle}

\section{Fresh Embryos from Nondonor Eggs}

Number of cycles

Percentage of cycles resulting in pregnancies ${ }^{b}$

Percentage of cycles resulting in live births ${ }^{b, c}$

(Confidence Interval)

Percentage of retrievals resulting in live births $s^{b, c}$

Percentage of transfers resulting in live births $s^{b, c}$

Percentage of transfers resulting in singleton live births ${ }^{b}$

Percentage of cancellations ${ }^{b}$

Average number of embryos transferred

Percentage of pregnancies with twins ${ }^{b}$

Percentage of pregnancies with triplets or more ${ }^{\mathrm{b}}$

Percentage of live births having multiple infants ${ }^{b, c}$

\section{Frozen Embryos from Nondonor Eggs}

Number of transfers

Percentage of transfers resulting in live births ${ }^{\mathrm{b}, \mathrm{c}}$

Average number of embryos transferred

\section{Donor Eggs}

Number of transfers

Percentage of transfers resulting in live births $s^{b, c}$

Average number of embryos transferred

\section{Age of Woman}

35-37 38-40

$41-42^{d}$

$<35$

86

30.2

29.1

71

23

48.6

43.8

(35.5-52.3)

52.5

(19.8-39.9)

16.9

17.4

56.3

36.2

(5.0-21.0)

13.0

31.3

41.7

14.8

(2.8-33.6)

16.7

23.3

16.0

$3 / 16$

$3 / 15$

12.0

$3 / 15$

19.8

23.9

30.4

2.0

2.3

41.4

1.4

42.3

2.5

3.1

1.4
44.4

7.7

44.0

$2 / 12$

$0 / 4$

$0 / 12$

$0 / 4$

$2 / 8$

$0 / 3$

82

30.5

$$
50
$$

36.0

2.6

\section{1}

10

2.3

All Ages Combined ${ }^{\mathrm{e}}$

Fresh Embryos

32

78.1

2.0
$1 / 10$

2.2

\section{CURRENT CLINIC SERVICES AND PROFILE}

Current Name: University Fertility Consultants, Oregon Health \& Science University
Donor egg? Yes
Donor embryo? Yes
$\begin{array}{ll}\text { Gestational carriers? } & \text { Yes } \\ \text { Cryopreservation? } & \text { Yes }\end{array}$
SART member?
Verified lab accredi
Yes
Single women? Yes
(See Appendix C for details.)

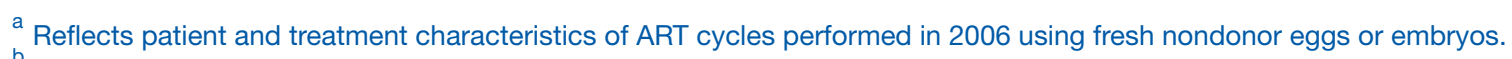

${ }^{b}$ When fewer than 20 cycles are reported in an age category, rates are shown as a fraction and confidence intervals are not given. Calculating percentages from fractions may be misleading and is not encouraged.

c A multiple-infant birth is counted as one live birth.

${ }^{d}$ Clinic-specific outcome rates are unreliable for women older than 42 undergoing ART cycles using fresh or frozen embryos with nondonor eggs. Readers are urged to review national outcomes for these age groups (see page 27).

${ }^{e}$ All ages (including ages $>42$ ) are reported together because previous data show that patient age does not materially affect success with donor eggs.
} 


\section{TOLL CENTER FOR REPRODUCTIVE SCIENCES ABINGTON, PENNSYLVANIA}

A comparison of clinic success rates may not be meaningful because patient medical characteristics and treatment approaches vary from clinic to clinic. For more details about this, along with information on how to interpret the statistics in this table, see pages 79-88.

\section{ART CYCLE PROFILE}

\begin{tabular}{|c|c|c|c|c|c|c|c|}
\hline \multicolumn{4}{|c|}{ Type of ART ${ }^{a}$} & \multicolumn{4}{|c|}{ Patient Diagnosis } \\
\hline IVF & $>99 \%$ & Procedural Factors: & & Tubal factor & $9 \%$ & Other factor & $10 \%$ \\
\hline GIFT & $0 \%$ & With ICSI & $77 \%$ & Ovulatory dysfunction & $6 \%$ & Unknown factor & $2 \%$ \\
\hline ZIFT & $<1 \%$ & Unstimulated & $0 \%$ & Diminished ovarian reserve & $12 \%$ & Multiple Factors: & \\
\hline \multirow[t]{3}{*}{ Combination } & $0 \%$ & Used gestational carrier & $1 \%$ & Endometriosis & $7 \%$ & Female factors only & $14 \%$ \\
\hline & & & & Uterine factor & $<1 \%$ & Female \& male factors & $18 \%$ \\
\hline & & & & Male factor & $21 \%$ & & \\
\hline
\end{tabular}

2006 PREGNANCY SUCCESS RATES

$$
\text { Type of Cycle }
$$

\section{Fresh Embryos from Nondonor Eggs}

Number of cycles

Percentage of cycles resulting in pregnancies ${ }^{b}$

Percentage of cycles resulting in live births ${ }^{b, c}$

(Confidence Interval)

Percentage of retrievals resulting in live births $s^{b, c}$

Percentage of transfers resulting in live births $s^{b, c}$

Percentage of transfers resulting in singleton live births ${ }^{b}$

Percentage of cancellations ${ }^{b}$

Average number of embryos transferred

Percentage of pregnancies with twins ${ }^{b}$

Percentage of pregnancies with triplets or more ${ }^{b}$

Percentage of live births having multiple infants ${ }^{b, c}$

\section{Frozen Embryos from Nondonor Eggs}

Number of transfers

Percentage of transfers resulting in live births $s^{b, c}$

Average number of embryos transferred

\section{Donor Eggs}

Number of transfers

Percentage of transfers resulting in live births $s^{b, c}$

Average number of embryos transferred

Data verified by Stephen G. Somkuti, MD, PhD

\section{Age of Woman}

35-37 $38-40 \quad 41-42^{d}$

\section{$<35$}

86

58

22

48.4

25.6

29.3

13.6

45.7

23.3

22.4

13.6

(38.3-53.1)

$(14.8-33.6)$

(12.5-35.3)

(2.9-34.9)

47.7

25.3

27.1

$3 / 19$

51.5

28.6

31.0

$3 / 16$

39.3

20.0

21.4

$2 / 16$

4.3

8.1

17.2

13.6

2.3

2.5

2.9

2.7

22.5

3.4

27.3

$5 / 17$

$1 / 3$

23.8

13.6

$1 / 17$

$0 / 3$

30.0

$4 / 13$

$1 / 3$

48

33.3

$$
16
$$

$1 / 16$

7

$2 / 7$

1

2.0

2.3

2.0

$0 / 1$

2.0

All Ages Combined ${ }^{\mathrm{e}}$

Fresh Embryos 10

$6 / 10$

2.0
Frozen Embryos 15

$4 / 15$

2.4

\section{CURRENT CLINIC SERVICES AND PROFILE}

Current Name: Toll Center for Reproductive Sciences

Donor egg? Yes

Donor embryo? Yes

Single women? Yes

\section{Gestational carriers? Yes}

Cryopreservation? Yes
SART member?

Yes

Verified lab accreditation?

(See Appendix C for details.)

${ }^{a}$ Reflects patient and treatment characteristics of ART cycles performed in 2006 using fresh nondonor eggs or embryos.

${ }^{b}$ When fewer than 20 cycles are reported in an age category, rates are shown as a fraction and confidence intervals are not given. Calculating percentages from fractions may be misleading and is not encouraged.

${ }^{c}$ A multiple-infant birth is counted as one live birth.

d Clinic-specific outcome rates are unreliable for women older than 42 undergoing ART cycles using fresh or frozen embryos with nondonor eggs. Readers are urged to review national outcomes for these age groups (see page 27).

e All ages (including ages $>42$ ) are reported together because previous data show that patient age does not materially affect success with donor eggs. 


\section{INFERTILITY SOLUTIONS, PC \\ ALLENTOWN, PENNSYLVANIA}

A comparison of clinic success rates may not be meaningful because patient medical characteristics and treatment approaches vary from clinic to clinic. For more details about this, along with information on how to interpret the statistics in this table, see pages $79-88$.

\section{ART CYCLE PROFILE}

\begin{tabular}{|c|c|c|c|c|c|c|}
\hline \multicolumn{3}{|c|}{ Type of $A R T^{a}$} & \multicolumn{4}{|c|}{ Patient Diagnosis } \\
\hline IVF & 100\% Procedural Factors: & & Tubal factor & $9 \%$ & Other factor & $2 \%$ \\
\hline GIFT & 0\% With ICSI & $90 \%$ & Ovulatory dysfunction & $12 \%$ & Unknown factor & $8 \%$ \\
\hline $\mathrm{ZIFT}$ & $0 \%$ Unstimulated & $0 \%$ & Diminished ovarian reserve & $13 \%$ & Multiple Factors: & \\
\hline \multirow[t]{3}{*}{ Combination } & $0 \%$ Used gestational carrier & $0 \%$ & Endometriosis & $0 \%$ & Female factors only & $24 \%$ \\
\hline & & & Uterine factor & $0 \%$ & Female \& male factors & $19 \%$ \\
\hline & & & Male factor & $14 \%$ & & \\
\hline
\end{tabular}

2006 PREGNANCY SUCCESS RATES

Data verified by Bruce I. Rose, MD, PhD

\section{Type of Cycle}

\section{Fresh Embryos from Nondonor Eggs}

Number of cycles

Percentage of cycles resulting in pregnancies ${ }^{b}$

Percentage of cycles resulting in live births ${ }^{b, c}$

(Confidence Interval)

Percentage of retrievals resulting in live births $s^{b, c}$

Percentage of transfers resulting in live births $s^{b, c}$

Percentage of transfers resulting in singleton live births ${ }^{b}$

Percentage of cancellations ${ }^{b}$

Average number of embryos transferred

Percentage of pregnancies with twins ${ }^{b}$

Percentage of pregnancies with triplets or more ${ }^{b}$

Percentage of live births having multiple infants ${ }^{b, c}$

\section{Frozen Embryos from Nondonor Eggs}

Number of transfers

Percentage of transfers resulting in live births ${ }^{\mathrm{b}, \mathrm{c}}$

Average number of embryos transferred

\section{Donor Eggs}

Number of transfers

Percentage of transfers resulting in live births $s^{b, c}$

Average number of embryos transferred

\section{Age of Woman}

35-37 38-40

$41-42^{d}$

$<35$

22

36.4

21

3

31.3

22.7
$(7.8-45.4)$

28.6

$1 / 3$

31.3

23.8

$0 / 3$

(16.1-50.0)

23.8
25.0

$(8.2-47.2)$

34.5

25.0

$0 / 3$

24.1

25.0

25.0

$0 / 3$

3.1

4.5

10.0

$0 / 3$

2.8

3.0

4.8

$0 / 3$

$1 / 10$

$0 / 8$

2.9

3.0

$2 / 10$

$0 / 8$

$3 / 6$

$0 / 1$

$3 / 10$

$0 / 5$

$0 / 6$

$0 / 1$

$3 / 5$

$\begin{array}{cc}4 & 2 \\ 0 / 4 & 1 / 2 \\ 2.8 & 3.5\end{array}$

\section{CURRENT CLINIC SERVICES AND PROFILE}

Current Name: Infertility Solutions, PC
Donor egg?
Yes
Donor embryo? Yes
Gestational carriers? Yes
Cryopreservation? Yes
Single women? Yes
SART member?
Yes
Verified lab accreditation?
Yes
(See Appendix C for details.)

\footnotetext{
${ }^{a}$ Reflects patient and treatment characteristics of ART cycles performed in 2006 using fresh nondonor eggs or embryos.

${ }^{b}$ When fewer than 20 cycles are reported in an age category, rates are shown as a fraction and confidence intervals are not given. Calculating percentages from fractions may be misleading and is not encouraged.

${ }^{c}$ A multiple-infant birth is counted as one live birth.

d Clinic-specific outcome rates are unreliable for women older than 42 undergoing ART cycles using fresh or frozen embryos with nondonor eggs. Readers are urged to review national outcomes for these age groups (see page 27).

${ }^{e}$ All ages (including ages $>42$ ) are reported together because previous data show that patient age does not materially affect success with donor eggs.
}

Fresh Embryos

All Ages Combined ${ }^{\mathrm{e}}$ 


\section{REPROTECH IVF PROGRAM \\ ALLENTOWN, PENNSYLVANIA}

A comparison of clinic success rates may not be meaningful because patient medical characteristics and treatment approaches vary from clinic to clinic. For more details about this, along with information on how to interpret the statistics in this table, see pages 79-88.

\section{ART CYCLE PROFILE}

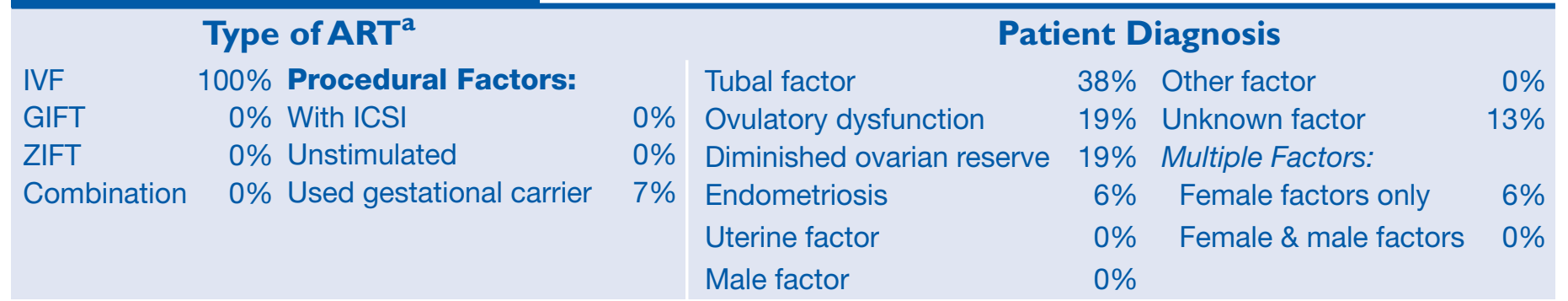

2006 PREGNANCY SUCCESS RATES

Data verified by Eric R. Rittenhouse, MD

\section{Type of Cycle}

Age of Woman

$\begin{array}{cccc}<35 & 35-37 & 38-40 & \mathbf{4 1 - 4 2}^{\mathbf{d}} \\ 5 & 3 & 3 & 3 \\ 0 / 5 & 0 / 3 & 0 / 3 & 0 / 3 \\ 0 / 5 & 0 / 3 & 0 / 3 & 0 / 3\end{array}$

\section{Fresh Embryos from Nondonor Eggs}

Number of cycles

Percentage of cycles resulting in pregnancies ${ }^{b}$

Percentage of cycles resulting in live births ${ }^{\mathrm{b}, \mathrm{c}}$

(Confidence Interval)

\begin{tabular}{|c|c|c|c|c|}
\hline Percentage of retrievals resulting in live births ${ }^{b, c}$ & $0 / 4$ & $0 / 2$ & $0 / 2$ & $0 / 2$ \\
\hline Percentage of transfers resulting in live births ${ }^{\mathrm{b}, \mathrm{c}}$ & $0 / 2$ & & & $0 / 1$ \\
\hline Percentage of transfers resulting in singleton live births ${ }^{b}$ & $0 / 2$ & & & $0 / 1$ \\
\hline Percentage of cancellations ${ }^{\mathrm{b}}$ & $1 / 5$ & $1 / 3$ & $1 / 3$ & $1 / 3$ \\
\hline Average number of embryos transferred & 3.0 & & & 1.0 \\
\hline
\end{tabular}

Percentage of pregnancies with twins ${ }^{b}$

Percentage of pregnancies with triplets or more ${ }^{b}$

Percentage of live births having multiple infants ${ }^{b, c}$

\section{Frozen Embryos from Nondonor Eggs}

Number of transfers

Percentage of transfers resulting in live births ${ }^{\mathrm{b}, \mathrm{c}}$

Average number of embryos transferred

\section{Donor Eggs}

Number of transfers

Percentage of transfers resulting in live births ${ }^{b, c}$

Average number of embryos transferred

\section{CURRENT CLINIC SERVICES AND PROFILE}

Current Name: Reprotech IVF Program

Donor egg? No Gestational carriers? Yes

Donor embryo? No

Single women? Yes

Cryopreservation? Yes

\section{Fresh Embryos}

0
All Ages Combined ${ }^{\mathrm{e}}$

Frozen Embryos

1

$0 / 1$

2.0

\footnotetext{
${ }^{a}$ Reflects patient and treatment characteristics of ART cycles performed in 2006 using fresh nondonor eggs or embryos.

b When fewer than 20 cycles are reported in an age category, rates are shown as a fraction and confidence intervals are not given. Calculating percentages from fractions may be misleading and is not encouraged.

${ }^{c}$ A multiple-infant birth is counted as one live birth.

d Clinic-specific outcome rates are unreliable for women older than 42 undergoing ART cycles using fresh or frozen embryos with nondonor eggs. Readers are urged to review national outcomes for these age groups (see page 27).

${ }^{e}$ All ages (including ages $>42$ ) are reported together because previous data show that patient age does not materially affect success with donor eggs.
} 


\section{FAMILY FERTILITY CENTER \\ BETHLEHEM, PENNSYLVANIA}

A comparison of clinic success rates may not be meaningful because patient medical characteristics and treatment approaches vary from clinic to clinic. For more details about this, along with information on how to interpret the statistics in this table, see pages $79-88$.

\section{ART CYCLE PROFILE}

\begin{tabular}{|c|c|c|c|c|c|c|c|}
\hline \multicolumn{4}{|c|}{ Type of ART ${ }^{\mathrm{a}}$} & \multicolumn{4}{|c|}{ Patient Diagnosis } \\
\hline IVF & $100 \%$ & Procedural Factors: & & Tubal factor & $7 \%$ & Other factor & $3 \%$ \\
\hline GIFT & $0 \%$ & With ICSI & $67 \%$ & Ovulatory dysfunction & $3 \%$ & Unknown factor & $1 \%$ \\
\hline $\mathrm{ZIFT}$ & $0 \%$ & Unstimulated & $0 \%$ & Diminished ovarian reserve & $6 \%$ & Multiple Factors: & \\
\hline Combination & $0 \%$ & Used gestational carrier & $1 \%$ & Endometriosis & $0 \%$ & Female factors only & $19 \%$ \\
\hline & & & & Uterine factor & $0 \%$ & Female \& male factors & $44 \%$ \\
\hline
\end{tabular}

2006 PREGNANCY SUCCESS RATES

Data verified by H. Christina Lee, MD

\section{Type of Cycle}

\section{Fresh Embryos from Nondonor Eggs}

Number of cycles

Percentage of cycles resulting in pregnancies ${ }^{b}$

Percentage of cycles resulting in live births $s^{b, c}$

(Confidence Interval)

Percentage of retrievals resulting in live births $s^{b, c}$

Percentage of transfers resulting in live births $s^{b, c}$

Percentage of transfers resulting in singleton live births ${ }^{b}$

Percentage of cancellations ${ }^{b}$

Average number of embryos transferred

Percentage of pregnancies with twins ${ }^{b}$

Percentage of pregnancies with triplets or more ${ }^{b}$

Percentage of live births having multiple infants ${ }^{b, c}$

\section{Frozen Embryos from Nondonor Eggs}

Number of transfers

Percentage of transfers resulting in live births ${ }^{\mathrm{b}, \mathrm{c}}$

Average number of embryos transferred

\section{Donor Eggs}

Number of transfers

Percentage of transfers resulting in live births $s^{b, c}$

Average number of embryos transferred

\section{Age of Woman}

35-37 38-40

$41-42^{d}$

\section{$<35$}

18

$5 / 18$

$4 / 18$

11

$5 / 11$

$5 / 11$

/

39.1
34.8

(21.4-50.2)

35.6

$4 / 18$

$5 / 10$

$5 / 9$

$4 / 9$

$1 / 11$

$3 / 17$

3.1

$2 / 5$

2.5

$1 / 5$

$0 / 5$

$0 / 18$

$0 / 5$

$1 / 5$

$5 / 16$

$1 / 4$

0

0

$1 / 3 \quad 0 / 1$

2.0

1.0

\section{CURRENT CLINIC SERVICES AND PROFILE}

Current Name: Family Fertility Center

\begin{tabular}{ll|ll} 
Donor egg? & Yes & Gestational carriers? & Yes \\
Donor embryo? & No & Cryopreservation? & Yes
\end{tabular}

Fresh Embryos

All Ages Combined ${ }^{\mathrm{e}}$

$1 / 4$

Frozen Embryos

1.8

$1 / 4$

2.0

Single women? Yes

\section{SART member? Yes}

Verified lab accreditation?

(See Appendix C for details.)

${ }^{a}$ Reflects patient and treatment characteristics of ART cycles performed in 2006 using fresh nondonor eggs or embryos.

${ }^{b}$ When fewer than 20 cycles are reported in an age category, rates are shown as a fraction and confidence intervals are not given. Calculating percentages from fractions may be misleading and is not encouraged.

${ }^{c}$ A multiple-infant birth is counted as one live birth.

d Clinic-specific outcome rates are unreliable for women older than 42 undergoing ART cycles using fresh or frozen embryos with nondonor eggs. Readers are urged to review national outcomes for these age groups (see page 27).

e All ages (including ages $>42$ ) are reported together because previous data show that patient age does not materially affect success with donor eggs. 


\section{MAIN LINE FERTILITY AND REPRODUCTIVE MEDICINE BRYN MAWR, PENNSYLVANIA}

A comparison of clinic success rates may not be meaningful because patient medical characteristics and treatment approaches vary from clinic to clinic. For more details about this, along with information on how to interpret the statistics in this table, see pages 79-88.

\section{ART CYCLE PROFILE}

\begin{tabular}{|c|c|c|c|c|c|c|c|}
\hline \multicolumn{4}{|c|}{ Type of ART ${ }^{a}$} & \multicolumn{4}{|c|}{ Patient Diagnosis } \\
\hline IVF & $100 \%$ & Procedural Factors: & & Tubal factor & $6 \%$ & Other factor & $5 \%$ \\
\hline GIFT & $0 \%$ & With ICSI & $17 \%$ & Ovulatory dysfunction & $12 \%$ & Unknown factor & $6 \%$ \\
\hline ZIFT & $0 \%$ & Unstimulated & $<1 \%$ & Diminished ovarian reserve & $27 \%$ & Multiple Factors: & \\
\hline \multirow[t]{3}{*}{ Combination } & $0 \%$ & Used gestational carrier & $<1 \%$ & Endometriosis & $1 \%$ & Female factors only & $10 \%$ \\
\hline & & & & Uterine factor & $4 \%$ & Female \& male factors & $17 \%$ \\
\hline & & & & Male factor & $12 \%$ & & \\
\hline
\end{tabular}

2006 PREGNANCY SUCCESS RATES

$$
\text { Type of Cycle }
$$

\section{Fresh Embryos from Nondonor Eggs}

Number of cycles

Percentage of cycles resulting in pregnancies ${ }^{b}$

Percentage of cycles resulting in live births $s^{b, c}$

(Confidence Interval)

Percentage of retrievals resulting in live births $s^{b, c}$

Percentage of transfers resulting in live births $s^{b, c}$

Percentage of transfers resulting in singleton live births ${ }^{b}$

Percentage of cancellations ${ }^{b}$

Average number of embryos transferred

Percentage of pregnancies with twins ${ }^{b}$

Percentage of pregnancies with triplets or more ${ }^{b}$

Percentage of live births having multiple infants ${ }^{b, c}$

\section{Frozen Embryos from Nondonor Eggs}

Number of transfers

Percentage of transfers resulting in live births ${ }^{b, c}$

Average number of embryos transferred

\section{Donor Eggs}

Number of transfers

Percentage of transfers resulting in live births $s^{b, c}$

Average number of embryos transferred

Data verified by Michael J. Glassner, MD

\section{Age of Woman}

35-37 38-40

$41-42^{d}$

\section{$<35$}

117

82

47

37.1

35.0

32.9

30.8

28.0

8.5

36.0

(22.6-40.0)

$(18.7-39.1)$

6.4

(28.9-43.5)

34.0

34.8

(1.3-17.5)

42.1

38.3

40.4

7.3

25.7

26.6

29.8

9.1

5.6

9.4

19.5

9.1

2.5

2.8

3.2

12.8

36.4

22.0

29.6

2.8

4.5

7.3

0.0

$0 / 4$

39.1

30.6

26.1

$0 / 4$

$0 / 3$

73

34.2

$$
40
$$

17.5

2.7

21

14

2.3

All Ages Combined ${ }^{\mathrm{e}}$

Fresh Embryos

24

41.7

2.2
$2 / 14$

2.6

\section{CURRENT CLINIC SERVICES AND PROFILE}

Current Name: Main Line Fertility and Reproductive Medicine

Donor egg? Yes Gestational carriers? Yes

Donor embryo? Yes Cryopreservation? Yes

Single women? Yes

SART member? Yes

Verified lab accreditation?

(See Appendix C for details.)

${ }^{a}$ Reflects patient and treatment characteristics of ART cycles performed in 2006 using fresh nondonor eggs or embryos.

${ }^{b}$ When fewer than 20 cycles are reported in an age category, rates are shown as a fraction and confidence intervals are not given. Calculating percentages from fractions may be misleading and is not encouraged.

${ }^{c}$ A multiple-infant birth is counted as one live birth.

d Clinic-specific outcome rates are unreliable for women older than 42 undergoing ART cycles using fresh or frozen embryos with nondonor eggs. Readers are urged to review national outcomes for these age groups (see page 27).

e All ages (including ages $>42$ ) are reported together because previous data show that patient age does not materially affect success with donor eggs. 


\section{GEISINGER MEDICAL CENTER FERTILITY PROGRAM DANVILLE, PENNSYLVANIA}

A comparison of clinic success rates may not be meaningful because patient medical characteristics and treatment approaches vary from clinic to clinic. For more details about this, along with information on how to interpret the statistics in this table, see pages $79-88$.

\section{ART CYCLE PROFILE}

\begin{tabular}{|c|c|c|c|c|c|c|c|}
\hline \multicolumn{4}{|c|}{ Type of ART } & \multicolumn{4}{|c|}{ Patient Diagnosis } \\
\hline IVF & $100 \%$ & Procedural Factors: & & Tubal factor & $8 \%$ & Other factor & $36 \%$ \\
\hline GIFT & $0 \%$ & With ICSI & $57 \%$ & Ovulatory dysfunction & $11 \%$ & Unknown factor & $0 \%$ \\
\hline ZIFT & $0 \%$ & Unstimulated & $0 \%$ & Diminished ovarian reserve & $34 \%$ & Multiple Factors: & \\
\hline \multirow[t]{3}{*}{ Combination } & $0 \%$ & Used gestational carrier & $0 \%$ & Endometriosis & $1 \%$ & Female factors only & $0 \%$ \\
\hline & & & & Uterine factor & $4 \%$ & Female \& male factors & $0 \%$ \\
\hline & & & & Male factor & $7 \%$ & & \\
\hline
\end{tabular}

2006 PREGNANCY SUCCESS RATES

Data verified by Jennifer Gell, MD

\section{Type of Cycle}

\section{Fresh Embryos from Nondonor Eggs}

Number of cycles

Percentage of cycles resulting in pregnancies ${ }^{b}$

Percentage of cycles resulting in live births ${ }^{b, c}$

(Confidence Interval)

Percentage of retrievals resulting in live births $s^{b, c}$

Percentage of transfers resulting in live births $s^{b, c}$

Percentage of transfers resulting in singleton live births ${ }^{b}$

Percentage of cancellations ${ }^{b}$

Average number of embryos transferred

Percentage of pregnancies with twins ${ }^{b}$

Percentage of pregnancies with triplets or more ${ }^{b}$

Percentage of live births having multiple infants ${ }^{b, c}$

\section{Frozen Embryos from Nondonor Eggs}

Number of transfers

Percentage of transfers resulting in live births ${ }^{\mathrm{b}, \mathrm{c}}$

Average number of embryos transferred

\section{Donor Eggs}

Number of transfers

Percentage of transfers resulting in live births $s^{b, c}$

Average number of embryos transferred

\section{Age of Woman}

35-37 38-40

$41-42^{d}$

$<35$

9

10

2

46.4

$2 / 9$

$1 / 10$

$0 / 2$

39.3

$1 / 9$

$1 / 10$

$0 / 2$

(21.5-59.4)

47.8

$1 / 6$

$1 / 9$

47.8

$1 / 6$

$1 / 9$

34.8

$1 / 6$

$1 / 9$

$3 / 9$

$1 / 10$

3.0

2.9

2.9

$1 / 2$

$0 / 1$

$3 / 13$

$0 / 2$

$0 / 1$

$3 / 11$

$0 / 1$

$0 / 1$ 年 


\section{ADVANCED CENTER FOR INFERTILITY AND REPRODUCTIVE MEDICINE, RPC HARRISBURG, PENNSYLVANIA}

A comparison of clinic success rates may not be meaningful because patient medical characteristics and treatment approaches vary from clinic to clinic. For more details about this, along with information on how to interpret the statistics in this table, see pages 79-88.

\section{ART CYCLE PROFILE}

\begin{tabular}{|c|c|c|c|c|c|c|c|}
\hline \multicolumn{4}{|c|}{ Type of ART ${ }^{a}$} & \multicolumn{4}{|c|}{ Patient Diagnosis } \\
\hline IVF & $100 \%$ & Procedural Factors: & & Tubal factor & $4 \%$ & Other factor & $6 \%$ \\
\hline GIFT & $0 \%$ & With ICSI & $88 \%$ & Ovulatory dysfunction & $1 \%$ & Unknown factor & $0 \%$ \\
\hline ZIFT & $0 \%$ & Unstimulated & $0 \%$ & Diminished ovarian reserve & $6 \%$ & Multiple Factors: & \\
\hline \multirow[t]{3}{*}{ Combination } & $0 \%$ & Used gestational carrier & $0 \%$ & Endometriosis & $7 \%$ & Female factors only & $30 \%$ \\
\hline & & & & Uterine factor & $8 \%$ & Female \& male factors & $36 \%$ \\
\hline & & & & Male factor & $3 \%$ & & \\
\hline
\end{tabular}

2006 PREGNANCY SUCCESS RATES

Data verified by Eric P. Fiedler, MD

\section{Type of Cycle}

\section{Fresh Embryos from Nondonor Eggs}

Number of cycles

Percentage of cycles resulting in pregnancies ${ }^{b}$

Percentage of cycles resulting in live births ${ }^{b, c}$

(Confidence Interval)

Percentage of retrievals resulting in live births $s^{b, c}$

Percentage of transfers resulting in live births $s^{b, c}$

Percentage of transfers resulting in singleton live births ${ }^{b}$

Percentage of cancellations ${ }^{b}$

Average number of embryos transferred

Percentage of pregnancies with twins ${ }^{b}$

Percentage of pregnancies with triplets or more ${ }^{b}$

Percentage of live births having multiple infants ${ }^{b, c}$

\section{Frozen Embryos from Nondonor Eggs}

Number of transfers

Percentage of transfers resulting in live births $s^{b, c}$

Average number of embryos transferred

\section{Donor Eggs}

Number of transfers

Percentage of transfers resulting in live births $s^{b, c}$

Average number of embryos transferred

\section{Age of Woman}

$<35 \quad 35-37 \quad 38-40 \quad 41-42^{\text {d }}$

11

9

$4 / 9$

4

$2 / 4$

$3 / 11$

$3 / 9$

$1 / 4$

$\begin{array}{ccc}3 / 11 & 3 / 9 & 1 / 4 \\ 3 / 7 & 3 / 6 & 1 / 4 \\ 2 / 7 & 3 / 6 & 1 / 4 \\ 0 / 11 & 0 / 9 & 0 / 4 \\ 1.9 & 1.7 & 1.8 \\ 2 / 4 & 0 / 4 & 0 / 2 \\ 0 / 4 & 1 / 4 & 0 / 2 \\ 1 / 3 & 0 / 3 & 0 / 1\end{array}$

14

$5 / 14$

9

$0 / 9$

1.9

2

$1 / 2$

1.9

All Ages Combined ${ }^{\mathrm{e}}$

Fresh Embryos 10

$6 / 10$

2.0
Frozen Embryos 10

$2 / 10$

1.9

\section{CURRENT CLINIC SERVICES AND PROFILE}

Current Name: Advanced Center for Infertility and Reproductive Medicine, RPC

Donor egg? Yes

Donor embryo? Yes

Single women? Yes

\section{Gestational carriers? Yes}

Cryopreservation? Yes
SART member?

Verified lab accreditation?

(See Appendix C for details.)
No

No

${ }^{a}$ Reflects patient and treatment characteristics of ART cycles performed in 2006 using fresh nondonor eggs or embryos.

${ }^{b}$ When fewer than 20 cycles are reported in an age category, rates are shown as a fraction and confidence intervals are not given. Calculating percentages from fractions may be misleading and is not encouraged.

${ }^{c}$ A multiple-infant birth is counted as one live birth.

d Clinic-specific outcome rates are unreliable for women older than 42 undergoing ART cycles using fresh or frozen embryos with nondonor eggs. Readers are urged to review national outcomes for these age groups (see page 27).

e All ages (including ages $>42$ ) are reported together because previous data show that patient age does not materially affect success with donor eggs. 


\section{PENN STATE MILTON S. HERSHEY MEDICAL CENTER HERSHEY, PENNSYLVANIA}

A comparison of clinic success rates may not be meaningful because patient medical characteristics and treatment approaches vary from clinic to clinic. For more details about this, along with information on how to interpret the statistics in this table, see pages $79-88$.

\section{ART CYCLE PROFILE}

\begin{tabular}{|c|c|c|c|c|c|c|c|}
\hline & Type & of $A R T^{a}$ & & Pat & ent $D$ & iagnosis & \\
\hline IVF & $100 \%$ & Procedural Factors: & & Tubal factor & $17 \%$ & Other factor & $1 \%$ \\
\hline GIFT & $0 \%$ & With ICSI & $86 \%$ & Ovulatory dysfunction & $11 \%$ & Unknown factor & $23 \%$ \\
\hline ZIFT & $0 \%$ & Unstimulated & $0 \%$ & Diminished ovarian reserve & $2 \%$ & Multiple Factors: & \\
\hline Combination & $0 \%$ & Used gestational carrier & $0 \%$ & Endometriosis & $4 \%$ & Female factors only & $3 \%$ \\
\hline & & & & Uterine factor & $0 \%$ & Female \& male factors & $9 \%$ \\
\hline & & & & Male factor & $30 \%$ & & \\
\hline
\end{tabular}

2006 PREGNANCY SUCCESS RATES

Data verified by William C. Dodson, MD

\section{Type of Cycle}

\section{Fresh Embryos from Nondonor Eggs}

Number of cycles

Percentage of cycles resulting in pregnancies ${ }^{b}$

Percentage of cycles resulting in live births ${ }^{b, c}$

(Confidence Interval)

Percentage of retrievals resulting in live births $s^{b, c}$

Percentage of transfers resulting in live births $s^{b, c}$

Percentage of transfers resulting in singleton live births ${ }^{b}$

Percentage of cancellations ${ }^{b}$

Average number of embryos transferred

Percentage of pregnancies with twins ${ }^{b}$

Percentage of pregnancies with triplets or more ${ }^{b}$

Percentage of live births having multiple infants ${ }^{b, c}$

\section{Frozen Embryos from Nondonor Eggs}

Number of transfers

Percentage of transfers resulting in live births ${ }^{\mathrm{b}, \mathrm{c}}$

Average number of embryos transferred

\section{Donor Eggs}

Number of transfers

Percentage of transfers resulting in live births ${ }^{\mathrm{b}, \mathrm{c}}$

Average number of embryos transferred

\section{Age of Woman}

35-37 38-40

$41-42^{d}$

\section{$<35$}

10

$2 / 10$

7

0

\section{3}

$2 / 10$

$4 / 7$

22.0

$0 / 7$

$(10.6-37.6)$

$\begin{array}{ccc}23.1 & 2 / 8 & 0 / 7 \\ 27.3 & 2 / 5 & 0 / 6 \\ 21.2 & 2 / 5 & 0 / 6 \\ 4.9 & 2 / 10 & 0 / 7 \\ 2.2 & 2.8 & 2.8 \\ 1 / 12 & 0 / 2 & 0 / 4 \\ 1 / 12 & 0 / 2 & 0 / 4 \\ 2 / 9 & 0 / 2\end{array}$

\section{CURRENT CLINIC SERVICES AND PROFILE}

Current Name: Penn State Milton S. Hershey Medical Center

Donor egg? Yes Gestational carriers? No

Donor embryo? No Cryopreservation? Yes

Single women? No

$\begin{array}{cccc}12 & 5 & 4 & 1 \\ 3 / 12 & 1 / 5 & 0 / 4 & 0 / 1 \\ 1.9 & 2.2 & 2.3 & 2.0\end{array}$

\section{All Ages Combined $^{\mathrm{e}}$}

Fresh Embryos

3

$0 / 3$

2.0
Frozen Embryos

\section{2}

$1 / 2$

2.0

\footnotetext{
${ }^{a}$ Reflects patient and treatment characteristics of ART cycles performed in 2006 using fresh nondonor eggs or embryos.

${ }^{b}$ When fewer than 20 cycles are reported in an age category, rates are shown as a fraction and confidence intervals are not given. Calculating percentages from fractions may be misleading and is not encouraged.

${ }^{c}$ A multiple-infant birth is counted as one live birth.

d Clinic-specific outcome rates are unreliable for women older than 42 undergoing ART cycles using fresh or frozen embryos with nondonor eggs. Readers are urged to review national outcomes for these age groups (see page 27).

${ }^{e}$ All ages (including ages $>42$ ) are reported together because previous data show that patient age does not materially affect success with donor eggs.
} 


\section{NORTHERN FERTILITY AND REPRODUCTIVE ASSOCIATES, PC MEADOWBROOK, PENNSYLVANIA}

A comparison of clinic success rates may not be meaningful because patient medical characteristics and treatment approaches vary from clinic to clinic. For more details about this, along with information on how to interpret the statistics in this table, see pages 79-88.

\section{ART CYCLE PROFILE}

\section{Type of ART ${ }^{\mathrm{a}}$}

IVF

GIFT 100\% Procedural Factors:

ZIFT

Combination
$0 \%$ With ICSI

$0 \%$ Unstimulated

$0 \%$ Used gestational carrier $<1 \%$
Tubal factor

Ovulatory dysfunction

Diminished ovarian reserve

Endometriosis

Uterine factor

Male factor

\section{Patient Diagnosis}

$\begin{array}{rlr}5 \% & \text { Other factor } & 2 \% \\ 8 \% & \text { Unknown factor } & 7 \% \\ 13 \% & \text { Multiple Factors: } & \\ 4 \% & \text { Female factors only } & 23 \% \\ 0 \% & \text { Female \& male factors } & 22 \% \\ 16 \% & & \end{array}$

2006 PREGNANCY SUCCESS RATES

Data verified by Martin F. Freedman, MD

\section{Type of Cycle}

\section{Age of Woman}

35-37

38-40

$41-42^{d}$

$<35$

34

50.0

37

8

Number of cycles

Percentage of cycles resulting in pregnancies ${ }^{b}$

Percentage of cycles resulting in live births ${ }^{b, c}$

(Confidence Interval)

Percentage of retrievals resulting in live births $s^{b, c}$

Percentage of transfers resulting in live births $s^{b, c}$

Percentage of transfers resulting in singleton live births ${ }^{b}$

Percentage of cancellations ${ }^{b}$

63
33.3

47.1

28.6

$(17.9-41.3)$

(29.8-64.9)

16.2

$3 / 8$

32.1

32.1

48.5

10.8

$3 / 8$

\section{8}

53.3

(3.0-25.4)

11.8

$3 / 6$

12.5

$3 / 6$

26.7

9.4

$3 / 6$

11.1

2.9

8.1

$2 / 8$

2.3

2.8

19.0

$6 / 17$

0.0

$3 / 17$

$3 / 18$

$8 / 16$

3.6

4.3

$0 / 3$

$0 / 3$

$0 / 6$

$0 / 3$

Percentage of live births having multiple infants ${ }^{b, c}$

\section{Frozen Embryos from Nondonor Eggs}

Number of transfers

Percentage of transfers resulting in live births $s^{b, c}$

Average number of embryos transferred

14
$5 / 14$
2.6

\section{0}

$3 / 10$

$1 / 4$

2.7

3

$1 / 3$

3.3

\section{All Ages Combined ${ }^{\mathrm{e}}$}

Fresh Embryos

Frozen Embryos

12
$4 / 12$
2.4

6

$1 / 6$

2.0

Number of transfers

Percentage of transfers resulting in live births $s^{b, c}$

Average number of embryos transferred

2.4

0

\section{CURRENT CLINIC SERVICES AND PROFILE}

Current Name: Northern Fertility and Reproductive Associates, PC

Donor egg? Yes Gestational carriers? Yes

Donor embryo? Yes Cryopreservation? Yes

Single women? Yes

SART member? $\quad$ Yes

Verified lab accreditation? Yes

(See Appendix C for details.)

${ }^{a}$ Reflects patient and treatment characteristics of ART cycles performed in 2006 using fresh nondonor eggs or embryos.

b When fewer than 20 cycles are reported in an age category, rates are shown as a fraction and confidence intervals are not given. Calculating percentages from fractions may be misleading and is not encouraged.

${ }^{c}$ A multiple-infant birth is counted as one live birth.

d Clinic-specific outcome rates are unreliable for women older than 42 undergoing ART cycles using fresh or frozen embryos with nondonor eggs. Readers are urged to review national outcomes for these age groups (see page 27).

e All ages (including ages $>42$ ) are reported together because previous data show that patient age does not materially affect success with donor eggs. 


\section{JEFFERSON IVF \\ PHILADELPHIA, PENNSYLVANIA}

A comparison of clinic success rates may not be meaningful because patient medical characteristics and treatment approaches vary from clinic to clinic. For more details about this, along with information on how to interpret the statistics in this table, see pages $79-88$.

\section{ART CYCLE PROFILE}

\begin{tabular}{|c|c|c|c|c|c|c|}
\hline & Type of ART ${ }^{a}$ & & Pat & ent $D$ & iagnosis & \\
\hline IVF & 100\% Procedural Factors: & & Tubal factor & $33 \%$ & Other factor & $0 \%$ \\
\hline GIFT & 0\% With ICSI & $0 \%$ & Ovulatory dysfunction & $40 \%$ & Unknown factor & $0 \%$ \\
\hline ZIFT & $0 \%$ Unstimulated & $0 \%$ & Diminished ovarian reserve & $0 \%$ & Multiple Factors: & \\
\hline Combination & $0 \%$ Used gestational carrier & $0 \%$ & Endometriosis & $7 \%$ & Female factors only & $0 \%$ \\
\hline & & & Uterine factor & $0 \%$ & Female \& male factors & $7 \%$ \\
\hline & & & Male factor & $13 \%$ & & \\
\hline
\end{tabular}

2006 PREGNANCY SUCCESS RATES

Data verified by Gregory T. Fossum, MD

\section{Type of Cycle}

\section{Fresh Embryos from Nondonor Eggs}

Number of cycles

Percentage of cycles resulting in pregnancies ${ }^{b}$

Percentage of cycles resulting in live births $s^{b, c}$

(Confidence Interval)

Percentage of retrievals resulting in live births $s^{b, c}$

Percentage of transfers resulting in live births $s^{b, c}$

Percentage of transfers resulting in singleton live births ${ }^{b}$

Percentage of cancellations ${ }^{b}$

Average number of embryos transferred

Percentage of pregnancies with twins ${ }^{b}$

Percentage of pregnancies with triplets or more ${ }^{b}$

Percentage of live births having multiple infants ${ }^{b, c}$

\section{Frozen Embryos from Nondonor Eggs}

Number of transfers

Percentage of transfers resulting in live births ${ }^{\mathrm{b}, \mathrm{c}}$

Average number of embryos transferred

\section{Donor Eggs}

Number of transfers

Percentage of transfers resulting in live births ${ }^{\mathrm{b}, \mathrm{c}}$

Average number of embryos transferred

\section{Age of Woman}

$\begin{array}{cccc}<35 & 35-37 & 38-40 & \mathbf{4 1 - 4 2}^{\mathbf{d}} \\ 6 & 2 & 2 & 1 \\ 1 / 6 & 1 / 2 & 0 / 2 & 1 / 1 \\ 1 / 6 & 0 / 2 & 0 / 2 & 0 / 1\end{array}$

$\begin{array}{cccc}1 / 4 & 0 / 1 & 0 / 1 & 0 / 1 \\ 1 / 4 & 0 / 1 & 0 / 1 & 0 / 1 \\ 1 / 4 & 0 / 1 & 0 / 1 & 0 / 1 \\ 2 / 6 & 1 / 2 & 1 / 2 & 0 / 1 \\ 2.8 & 3.0 & 1.0 & 3.0 \\ 0 / 1 & 1 / 1 & & 0 / 1 \\ 0 / 1 & 0 / 1 & & 0 / 1 \\ 0 / 1 & & & \end{array}$

2

$1 / 2$

1

$0 / 1$

0

2.0
1

$0 / 1$

6.0

\section{CURRENT CLINIC SERVICES AND PROFILE}

Current Name: Jefferson IVF

Donor egg? Yes

Donor embryo? No

Single women? Yes

\begin{abstract}
Gestational carriers? No
Cryopreservation? Yes
\end{abstract}

All Ages Combined ${ }^{\mathrm{e}}$

Fresh Embryos

0
Frozen Embryos

0

${ }_{b}^{a}$ Reflects patient and treatment characteristics of ART cycles performed in 2006 using fresh nondonor eggs or embryos.

b When fewer than 20 cycles are reported in an age category, rates are shown as a fraction and confidence intervals are not given. Calculating percentages from fractions may be misleading and is not encouraged.

c A multiple-infant birth is counted as one live birth.

${ }^{d}$ Clinic-specific outcome rates are unreliable for women older than 42 undergoing ART cycles using fresh or frozen embryos with nondonor eggs. Readers are urged to review national outcomes for these age groups (see page 27).

e All ages (including ages $>42$ ) are reported together because previous data show that patient age does not materially affect success with donor eggs. 


\section{UNIVERSITY OF PENNSYLVANIA \\ PENN FERTILITY CARE \\ PHILADELPHIA, PENNSYLVANIA}

A comparison of clinic success rates may not be meaningful because patient medical characteristics and treatment approaches vary from clinic to clinic. For more details about this, along with information on how to interpret the statistics in this table, see pages 79-88.

\section{ART CYCLE PROFILE}

\section{Type of ART ${ }^{\mathrm{a}}$}

IVF

GIFT

ZIFT

Combination
100\% Procedural Factors:

$0 \%$ With ICSI

$0 \%$ Unstimulated

$0 \%$ Used gestational carrier

\begin{tabular}{|l|l|}
\hline $20 \%$ & Tubal factor \\
\hline $0 \%$ & Ovulatory dysfunction \\
$2 \%$ & Endometriosis \\
& Uterine factor \\
& Male factor
\end{tabular}

\section{Patient Diagnosis}

$7 \%$ Other factor

5\% Unknown factor

9\% Multiple Factors:

$6 \%$ Female factors only $27 \%$

$2 \%$ Female \& male factors $13 \%$ $14 \%$
$6 \%$

$12 \%$

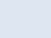

2006 PREGNANCY SUCCESS RATES

\section{Type of Cycle}

Data verified by Christos B. Coutifaris, MD, PhD

\section{Fresh Embryos from Nondonor Eggs}

Number of cycles

Percentage of cycles resulting in pregnancies ${ }^{b}$

Percentage of cycles resulting in live births $s^{b, c}$

(Confidence Interval)

Percentage of retrievals resulting in live births ${ }^{b, c}$

Percentage of transfers resulting in live births $s^{b, c}$

Percentage of transfers resulting in singleton live births ${ }^{b}$

Percentage of cancellations ${ }^{b}$

Average number of embryos transferred

Percentage of pregnancies with twins ${ }^{b}$

Percentage of pregnancies with triplets or more ${ }^{b}$

Percentage of live births having multiple infants ${ }^{\mathrm{b}, \mathrm{c}}$

\section{Frozen Embryos from Nondonor Eggs}

Number of transfers

Percentage of transfers resulting in live births $s^{b, c}$

Average number of embryos transferred

\section{Donor Eggs}

Number of transfers

Percentage of transfers resulting in live births $s^{b, c}$

Average number of embryos transferred

\section{Age of Woman}

35-37 $38-40 \quad 41-42^{d}$

$<35$

96

63

26

36.4

36.5

33.3

15.4

27.2

31.3

28.6

11.5

(20.2-35.0)

$(22.2-41.5)$

29.1

32.8

36.6

$(17.9-41.3)$

(2.4-30.2)

31.6

34.0

15.0

42.9

26.4

15.0

27.1

9.5

15.0

6.6

14.6

2.7

37.1

5.7

36.7

39.0

3.0

23.1

3.7

$0 / 4$

$0 / 4$

0.0

$0 / 3$

\section{CURRENT CLINIC SERVICES AND PROFILE}

Current Name: University of Pennsylvania, Penn Fertility Care

Donor egg? Yes Gestational carriers? Yes

Donor embryo? Yes Cryopreservation? Yes

$\begin{array}{llll}37 & 8 & 7 & 3\end{array}$

$\begin{array}{llll}43.2 & 4 / 8 & 3 / 7 & 1 / 3\end{array}$

2.8

2.9

2.4

2.0

All Ages Combined ${ }^{\mathrm{e}}$

Fresh Embryos

27

44.4

2.3
Frozen Embryos

19

$5 / 19$

2.4

Single women? Yes

SART member? Yes

Verified lab accreditation?

(See Appendix C for details.)

${ }^{a}$ Reflects patient and treatment characteristics of ART cycles performed in 2006 using fresh nondonor eggs or embryos.

${ }^{b}$ When fewer than 20 cycles are reported in an age category, rates are shown as a fraction and confidence intervals are not given. Calculating percentages from fractions may be misleading and is not encouraged.

${ }^{\mathrm{C}}$ A multiple-infant birth is counted as one live birth.

d Clinic-specific outcome rates are unreliable for women older than 42 undergoing ART cycles using fresh or frozen embryos with nondonor eggs. Readers are urged to review national outcomes for these age groups (see page 27).

e All ages (including ages $>42$ ) are reported together because previous data show that patient age does not materially affect success with donor eggs. 


\section{JONES INSTITUTE AT WEST PENN ALLEGHENY HEALTH SYSTEM \\ PITTSBURGH, PENNSYLVANIA}

A comparison of clinic success rates may not be meaningful because patient medical characteristics and treatment approaches vary from clinic to clinic. For more details about this, along with information on how to interpret the statistics in this table, see pages $79-88$.

\section{ART CYCLE PROFILE}

\begin{tabular}{|c|c|c|c|c|c|c|c|}
\hline \multicolumn{4}{|c|}{ Type of ART ${ }^{\mathrm{a}}$} & \multicolumn{4}{|c|}{ Patient Diagnosis } \\
\hline IVF & $100 \%$ & Procedural Factors: & & Tubal factor & $16 \%$ & Other factor & $6 \%$ \\
\hline GIFT & $0 \%$ & With ICSI & $59 \%$ & Ovulatory dysfunction & $2 \%$ & Unknown factor & $20 \%$ \\
\hline ZIFT & $0 \%$ & Unstimulated & $0 \%$ & Diminished ovarian reserve & $2 \%$ & Multiple Factors: & \\
\hline \multirow[t]{3}{*}{ Combination } & $0 \%$ & Used gestational carrier & $0 \%$ & Endometriosis & $6 \%$ & Female factors only & $8 \%$ \\
\hline & & & & Uterine factor & $0 \%$ & Female \& male factors & $17 \%$ \\
\hline & & & & Male factor & $22 \%$ & & \\
\hline
\end{tabular}

2006 PREGNANCY SUCCESS RATES

Data verified by Scott W. Kauma, MD

\section{Type of Cycle}

\section{Fresh Embryos from Nondonor Eggs}

Number of cycles

Percentage of cycles resulting in pregnancies ${ }^{b}$

Percentage of cycles resulting in live births $s^{b, c}$

(Confidence Interval)

Percentage of retrievals resulting in live births $s^{b, c}$

Percentage of transfers resulting in live births $s^{b, c}$

Percentage of transfers resulting in singleton live births ${ }^{b}$

Percentage of cancellations ${ }^{b}$

Average number of embryos transferred

Percentage of pregnancies with twins ${ }^{b}$

Percentage of pregnancies with triplets or more ${ }^{b}$

Percentage of live births having multiple infants ${ }^{b, c}$

\section{Frozen Embryos from Nondonor Eggs}

Number of transfers

Percentage of transfers resulting in live births $s^{\mathrm{b}, \mathrm{c}}$

Average number of embryos transferred

\section{Donor Eggs}

Number of transfers

Percentage of transfers resulting in live births ${ }^{\mathrm{b}, \mathrm{c}}$

Average number of embryos transferred

\section{Age of Woman}

35-37 38-40

$41-42^{d}$

$<35$

28

39.3

19

4

$\begin{array}{cccc}43 & 28 & 19 & 4 \\ 44.2 & 39.3 & 6 / 19 & 0 / 4 \\ 41.9 & 32.1 & 4 / 19 & 0 / 4\end{array}$

(27.0-57.9)

(15.9-52.4)

$47.4 \quad 45.0$

$4 / 13 \quad 0 / 3$

$50.0 \quad 45.0$

$4 / 13$

$0 / 3$

33.3

25.0

$3 / 13$

$0 / 3$

11.6

28.6

$6 / 19$

$1 / 4$

2.5

3.0

3.2

4.3

$9 / 19$

$2 / 11$

$2 / 6$

$0 / 19$

$2 / 11$

$0 / 6$

$6 / 18$

$4 / 9$

$1 / 4$

\section{6}

$2 / 6$

2.3
4

$2 / 4$

3.3
4

$2 / 4$

3.5

All Ages Combined ${ }^{\mathrm{e}}$

Fresh Embryos

1

$1 / 1$

2.0
Frozen Embryos

0

\section{CURRENT CLINIC SERVICES AND PROFILE}

Current Name: Jones Institute at West Penn Allegheny Health System

\begin{tabular}{ll|ll} 
Donor egg? & Yes & Gestational carriers? & Yes \\
Donor embryo? & No & Cryopreservation? & Yes
\end{tabular}

Single women? Yes

$\begin{array}{ll}\text { SART member? } & \text { Yes } \\ \text { Verified lab accreditation? } & \text { Yes } \\ \text { (See Appendix C for details.) } & \end{array}$

${ }^{a}$ Reflects patient and treatment characteristics of ART cycles performed in 2006 using fresh nondonor eggs or embryos.

${ }^{b}$ When fewer than 20 cycles are reported in an age category, rates are shown as a fraction and confidence intervals are not given. Calculating percentages from fractions may be misleading and is not encouraged.

${ }^{c}$ A multiple-infant birth is counted as one live birth.

d Clinic-specific outcome rates are unreliable for women older than 42 undergoing ART cycles using fresh or frozen embryos with nondonor eggs. Readers are urged to review national outcomes for these age groups (see page 27).

${ }^{e}$ All ages (including ages $>42$ ) are reported together because previous data show that patient age does not materially affect success with donor eggs. 


\section{REPRODUCTIVE HEALTH SPECIALISTS, INC. PITTSBURGH, PENNSYLVANIA}

A comparison of clinic success rates may not be meaningful because patient medical characteristics and treatment approaches vary from clinic to clinic. For more details about this, along with information on how to interpret the statistics in this table, see pages 79-88.

\section{ART CYCLE PROFILE}

\begin{tabular}{|c|c|c|c|c|c|c|c|}
\hline \multicolumn{4}{|c|}{ Type of ART ${ }^{a}$} & \multicolumn{4}{|c|}{ Patient Diagnosis } \\
\hline IVF & $100 \%$ & Procedural Factors: & & Tubal factor & $6 \%$ & Other factor & $8 \%$ \\
\hline GIFT & $0 \%$ & With ICSI & $49 \%$ & Ovulatory dysfunction & $3 \%$ & Unknown factor & $26 \%$ \\
\hline ZIFT & $0 \%$ & Unstimulated & $0 \%$ & Diminished ovarian reserve & $7 \%$ & Multiple Factors: & \\
\hline \multirow[t]{3}{*}{ Combination } & $0 \%$ & Used gestational carrier & $2 \%$ & Endometriosis & $7 \%$ & Female factors only & $7 \%$ \\
\hline & & & & Uterine factor & $<1 \%$ & Female \& male factors & $11 \%$ \\
\hline & & & & Male factor & $25 \%$ & & \\
\hline
\end{tabular}

2006 PREGNANCY SUCCESS RATES

Data verified by Judith L. Albert, MD

\section{Type of Cycle}

\section{Fresh Embryos from Nondonor Eggs}

Number of cycles

Percentage of cycles resulting in pregnancies ${ }^{b}$

Percentage of cycles resulting in live births $s^{b, c}$

(Confidence Interval)

Percentage of retrievals resulting in live births $s^{b, c}$

Percentage of transfers resulting in live births $s^{b, c}$

Percentage of transfers resulting in singleton live births ${ }^{b}$

Percentage of cancellations ${ }^{b}$

Average number of embryos transferred

Percentage of pregnancies with twins ${ }^{b}$

Percentage of pregnancies with triplets or more ${ }^{b}$

Percentage of live births having multiple infants ${ }^{b, c}$

\section{Frozen Embryos from Nondonor Eggs}

Number of transfers

Percentage of transfers resulting in live births ${ }^{b, c}$

Average number of embryos transferred

\section{Donor Eggs}

Number of transfers

Percentage of transfers resulting in live births $s^{b, c}$

Average number of embryos transferred

\section{Age of Woman}

35-37 38-40

$41-42^{d}$

\section{$<35$}

51

40

12

76
59.2

47.1

37.5

$0 / 12$

51.3

33.3

25.0

$0 / 12$

(39.6-63.0)

$(20.8-47.9)$

$(12.7-41.2)$

52.7

35.4

27.0

$0 / 11$

54.9

37.8

31.3

$0 / 8$

39.4

22.2

15.6

$0 / 8$

2.6

5.9

2.0

29.2

7.5

$1 / 12$

1.8

4.2

2.3

2.4

0.0

28.2

$7 / 17$

$5 / 15$

$0 / 15$

$5 / 10$

21

14.3

1.7

9
$3 / 9$
1.8

12

$1 / 12$

2.0

$0 / 1$

1.0

\section{CURRENT CLINIC SERVICES AND PROFILE}

Current Name: Reproductive Health Specialists, Inc.

Donor egg? Yes

Donor embryo? No

Single women? Yes

All Ages Combined ${ }^{\mathrm{e}}$

Fresh Embryos

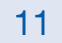

$7 / 11$

1.7
Frozen Embryos

4

$0 / 4$

1.5

${ }^{a}$ Reflects patient and treatment characteristics of ART cycles performed in 2006 using fresh nondonor eggs or embryos.

${ }^{b}$ When fewer than 20 cycles are reported in an age category, rates are shown as a fraction and confidence intervals are not given. Calculating percentages from fractions may be misleading and is not encouraged.

${ }^{c}$ A multiple-infant birth is counted as one live birth.

d Clinic-specific outcome rates are unreliable for women older than 42 undergoing ART cycles using fresh or frozen embryos with nondonor eggs. Readers are urged to review national outcomes for these age groups (see page 27).

e All ages (including ages $>42$ ) are reported together because previous data show that patient age does not materially affect success with donor eggs. 


\section{UNIVERSITY OF PITTSBURGH PHYSICIANS \\ CENTER FOR FERTILITY AND REPRODUCTIVE ENDOCRINOLOGY \\ PITTSBURGH, PENNSYLVANIA}

A comparison of clinic success rates may not be meaningful because patient medical characteristics and treatment approaches vary from clinic to clinic. For more details about this, along with information on how to interpret the statistics in this table, see pages $79-88$.

\section{ART CYCLE PROFILE}

\begin{tabular}{|c|c|c|c|c|c|c|c|}
\hline \multicolumn{4}{|c|}{ Type of ART ${ }^{\mathrm{a}}$} & \multicolumn{4}{|c|}{ Patient Diagnosis } \\
\hline IVF & $100 \%$ & Procedural Factors: & & Tubal factor & $10 \%$ & Other factor & $17 \%$ \\
\hline GIFT & $0 \%$ & With ICSI & $50 \%$ & Ovulatory dysfunction & $2 \%$ & Unknown factor & $6 \%$ \\
\hline ZIFT & $0 \%$ & Unstimulated & $<1 \%$ & Diminished ovarian reserve & $26 \%$ & Multiple Factors: & \\
\hline \multirow[t]{3}{*}{ Combination } & $0 \%$ & Used gestational carrier & $1 \%$ & Endometriosis & $6 \%$ & Female factors only & $5 \%$ \\
\hline & & & & Uterine factor & $<1 \%$ & Female \& male factors & $9 \%$ \\
\hline & & & & Male factor & $18 \%$ & & \\
\hline
\end{tabular}

2006 PREGNANCY SUCCESS RATES

Data verified by Anthony N. Wakim, MD

\section{Type of Cycle}

\section{Fresh Embryos from Nondonor Eggs}

Number of cycles

Percentage of cycles resulting in pregnancies ${ }^{b}$

Percentage of cycles resulting in live births $s^{b, c}$

(Confidence Interval)

Percentage of retrievals resulting in live births $s^{b, c}$

Percentage of transfers resulting in live births $s^{b, c}$

Percentage of transfers resulting in singleton live births ${ }^{b}$

Percentage of cancellations ${ }^{b}$

Average number of embryos transferred

Percentage of pregnancies with twins ${ }^{b}$

Percentage of pregnancies with triplets or more ${ }^{b}$

Percentage of live births having multiple infants ${ }^{b, c}$

\section{Frozen Embryos from Nondonor Eggs}

Number of transfers

Percentage of transfers resulting in live births $s^{b, c}$

Average number of embryos transferred

\section{Donor Eggs}

Number of transfers

Percentage of transfers resulting in live births $s^{b, c}$

Average number of embryos transferred

\section{Age of Woman}

35-37 38-40

$41-42^{d}$

$<35$

49

22.4

37

11

31.3

22.4

18.9

$1 / 11$

28.9

(19.5-39.9)

(11.8-36.6)

10.8

$1 / 11$

31.6

23.9

(3.0-25.4)

$35.3 \quad 27.5$

13.3

$1 / 6$

15.4

$1 / 6$

22.5

11.5

$1 / 6$

8.4

6.1

18.9

$5 / 11$

2.2

2.7

2.7

2.7

42.3

$0 / 11$

$1 / 7$

$0 / 1$

0.0

$2 / 11$

$0 / 7$

$0 / 1$

37.5

$2 / 11$

$1 / 4$

$0 / 1$

52

30.8

$$
26
$$

19.2

20

6

2.7

2.5

30.0

$1 / 6$

2.9

2.8

All Ages Combined ${ }^{\mathrm{e}}$

Fresh Embryos

31

48.4

2.0
Frozen Embryos

31

32.3

2.7

\section{CURRENT CLINIC SERVICES AND PROFILE}

Current Name: University of Pittsburgh Physicians, Center for Fertility and Reproductive Endocrinology

Donor egg? Yes

Donor embryo? Yes

Single women? Yes

\section{Gestational carriers? Yes}

Cryopreservation? Yes
SART member?

Verified lab accreditation?

(See Appendix C for details.)

${ }^{a}$ Reflects patient and treatment characteristics of ART cycles performed in 2006 using fresh nondonor eggs or embryos.

${ }^{b}$ When fewer than 20 cycles are reported in an age category, rates are shown as a fraction and confidence intervals are not given. Calculating percentages from fractions may be misleading and is not encouraged.

${ }^{c}$ A multiple-infant birth is counted as one live birth.

d Clinic-specific outcome rates are unreliable for women older than 42 undergoing ART cycles using fresh or frozen embryos with nondonor eggs. Readers are urged to review national outcomes for these age groups (see page 27).

e All ages (including ages $>42$ ) are reported together because previous data show that patient age does not materially affect success with donor eggs. 


\section{REPRODUCTIVE ENDOCRINOLOGY AND FERTILITY CENTER UPLAND, PENNSYLVANIA}

A comparison of clinic success rates may not be meaningful because patient medical characteristics and treatment approaches vary from clinic to clinic. For more details about this, along with information on how to interpret the statistics in this table, see pages 79-88.

\section{ART CYCLE PROFILE}

\begin{tabular}{|c|c|c|c|c|c|c|c|}
\hline \multicolumn{4}{|c|}{ Type of ART ${ }^{a}$} & \multicolumn{4}{|c|}{ Patient Diagnosis } \\
\hline IVF & $100 \%$ & Procedural Factors: & & Tubal factor & $8 \%$ & Other factor & $13 \%$ \\
\hline GIFT & $0 \%$ & With ICSI & $64 \%$ & Ovulatory dysfunction & $2 \%$ & Unknown factor & $0 \%$ \\
\hline ZIFT & $0 \%$ & Unstimulated & $0 \%$ & Diminished ovarian reserve & $1 \%$ & Multiple Factors: & \\
\hline \multirow[t]{3}{*}{ Combination } & $0 \%$ & Used gestational carrier & $0 \%$ & Endometriosis & $6 \%$ & Female factors only & $24 \%$ \\
\hline & & & & Uterine factor & $<1 \%$ & Female \& male factors & $37 \%$ \\
\hline & & & & Male factor & $9 \%$ & & \\
\hline
\end{tabular}

2006 PREGNANCY SUCCESS RATES

Data verified by Albert El-Roeiy, MD

\section{Type of Cycle}

\section{Fresh Embryos from Nondonor Eggs}

Number of cycles

Percentage of cycles resulting in pregnancies ${ }^{b}$

Percentage of cycles resulting in live births $s^{b, c}$

(Confidence Interval)

Percentage of retrievals resulting in live births $s^{b, c}$

Percentage of transfers resulting in live births $s^{b, c}$

Percentage of transfers resulting in singleton live births ${ }^{b}$

Percentage of cancellations ${ }^{b}$

Average number of embryos transferred

Percentage of pregnancies with twins ${ }^{b}$

Percentage of pregnancies with triplets or more ${ }^{b}$

Percentage of live births having multiple infants ${ }^{b, c}$

\section{Frozen Embryos from Nondonor Eggs}

Number of transfers

Percentage of transfers resulting in live births ${ }^{b, c}$

Average number of embryos transferred

\section{Donor Eggs}

Number of transfers

Percentage of transfers resulting in live births $s^{\mathrm{b}, \mathrm{c}}$

Average number of embryos transferred

\section{Age of Woman}

35-37 38-40 $\quad 41-42^{d}$

\section{$<35$}

32

37.5

23

9

30.6

31.3

26.1

$0 / 9$

29.2

$(16.1-50.0)$

21.7

(19.0-41.1)

37.0

(7.5-43.7)

33.3

$10 / 19$

23.8

$0 / 9$

44.7

$7 / 19$

$5 / 18$

$0 / 7$

31.9

15.6

$3 / 18$

$0 / 6$

12.5

3.0

3.1

31.8

$3 / 12$

8.7

$0 / 6$

9.1

$1 / 12$

3.4

$2 / 9$

28.6

$3 / 10$

$0 / 6$

$2 / 6$

$2 / 5$

13

$5 / 13$

3.1
2

$1 / 2$

3.5

All Ages Combined ${ }^{\mathrm{e}}$

Fresh Embryos

8

$2 / 8$

3.0
Frozen Embryos

4

$1 / 4$

3.0

\section{CURRENT CLINIC SERVICES AND PROFILE}

Current Name: Reproductive Endocrinology and Fertility Center

Donor egg? Yes Gestational carriers? No

Donor embryo? No Cryopreservation? Yes

Single women? Yes

SART member? $\quad$ Yes

Verified lab accreditation? Yes

(See Appendix C for details.)

${ }^{a}$ Reflects patient and treatment characteristics of ART cycles performed in 2006 using fresh nondonor eggs or embryos.

${ }^{b}$ When fewer than 20 cycles are reported in an age category, rates are shown as a fraction and confidence intervals are not given. Calculating percentages from fractions may be misleading and is not encouraged.

${ }^{c}$ A multiple-infant birth is counted as one live birth.

d Clinic-specific outcome rates are unreliable for women older than 42 undergoing ART cycles using fresh or frozen embryos with nondonor eggs. Readers are urged to review national outcomes for these age groups (see page 27).

e All ages (including ages $>42$ ) are reported together because previous data show that patient age does not materially affect success with donor eggs. 


\section{REPRODUCTIVE SCIENCE INSTITUTE OF SUBURBAN PHILADELPHIA WAYNE, PENNSYLVANIA}

A comparison of clinic success rates may not be meaningful because patient medical characteristics and treatment approaches vary from clinic to clinic. For more details about this, along with information on how to interpret the statistics in this table, see pages $79-88$.

\section{ART CYCLE PROFILE}

\begin{tabular}{|c|c|c|c|c|c|c|c|}
\hline \multicolumn{4}{|c|}{ Type of ART ${ }^{a}$} & \multicolumn{4}{|c|}{ Patient Diagnosis } \\
\hline IVF & $100 \%$ & Procedural Factors: & & Tubal factor & $4 \%$ & Other factor & $<1 \%$ \\
\hline GIFT & $0 \%$ & With ICSI & $53 \%$ & Ovulatory dysfunction & $12 \%$ & Unknown factor & $1 \%$ \\
\hline $\mathrm{ZIFT}$ & $0 \%$ & Unstimulated & $0 \%$ & Diminished ovarian reserve & $36 \%$ & Multiple Factors: & \\
\hline \multirow[t]{3}{*}{ Combination } & $0 \%$ & Used gestational carrier & $<1 \%$ & Endometriosis & $<1 \%$ & Female factors only & $17 \%$ \\
\hline & & & & Uterine factor & $<1 \%$ & Female \& male factors & $20 \%$ \\
\hline & & & & Male factor & $9 \%$ & & \\
\hline
\end{tabular}

2006 PREGNANCY SUCCESS RATES

Data verified by Abraham K. Munabi, MD

\section{Type of Cycle}

\section{Fresh Embryos from Nondonor Eggs}

Number of cycles

Percentage of cycles resulting in pregnancies ${ }^{b}$

Percentage of cycles resulting in live births ${ }^{b, c}$

(Confidence Interval)

Percentage of retrievals resulting in live births $s^{b, c}$

Percentage of transfers resulting in live births $s^{b, c}$

Percentage of transfers resulting in singleton live births ${ }^{b}$

Percentage of cancellations ${ }^{b}$

Average number of embryos transferred

Percentage of pregnancies with twins ${ }^{b}$

Percentage of pregnancies with triplets or more ${ }^{b}$

Percentage of live births having multiple infants ${ }^{b, c}$

\section{Frozen Embryos from Nondonor Eggs}

Number of transfers

Percentage of transfers resulting in live births $s^{b, c}$

Average number of embryos transferred

\section{Donor Eggs}

Number of transfers

Percentage of transfers resulting in live births ${ }^{\mathrm{b}, \mathrm{c}}$

Average number of embryos transferred

\section{Age of Woman}

35-37 38-40

$41-42^{d}$

$<35$

47

27.7

39

12

39.0

17.0

23.1

$0 / 12$

30.5

$(19.2-43.9)$

$(7.6-30.8)$

15.4

$0 / 12$

\section{6}

36.0

21.6

(5.9-30.5)

17.1

$0 / 8$

24.2

18.2

$0 / 8$

26.0

18.2

15.2

$0 / 8$

11.9

21.3

10.3

$4 / 12$

2.8

3.0

3.7

3.8

17.4

$2 / 13$

$1 / 9$

4.3

$0 / 13$

$0 / 9$

$5 / 18$

$2 / 8$

$1 / 6$

13

$3 / 13$

2.8

\section{1}

$5 / 11$

2.8

\section{3 \\ $1 / 3$}

4.0
2

$0 / 2$

3.5

\section{CURRENT CLINIC SERVICES AND PROFILE}

Current Name: Reproductive Science Institute of Suburban Philadelphia

Donor egg? Yes

Donor embryo? Yes

Single women? Yes

\section{Gestational carriers? Yes}

Cryopreservation? Yes
All Ages Combined ${ }^{\mathrm{e}}$
Fresh Embryos 28

50.0

2.4

\section{Frozen Embryos} 20

30.0

\footnotetext{
${ }^{a}$ Reflects patient and treatment characteristics of ART cycles performed in 2006 using fresh nondonor eggs or embryos.

${ }^{b}$ When fewer than 20 cycles are reported in an age category, rates are shown as a fraction and confidence intervals are not given. Calculating percentages from fractions may be misleading and is not encouraged.

${ }^{c}$ A multiple-infant birth is counted as one live birth.

d Clinic-specific outcome rates are unreliable for women older than 42 undergoing ART cycles using fresh or frozen embryos with nondonor eggs. Readers are urged to review national outcomes for these age groups (see page 27).

${ }^{e}$ All ages (including ages $>42$ ) are reported together because previous data show that patient age does not materially affect success with donor eggs.
} 


\section{WOMEN'S CLINIC, LTD. WEST READING, PENNSYLVANIA}

A comparison of clinic success rates may not be meaningful because patient medical characteristics and treatment approaches vary from clinic to clinic. For more details about this, along with information on how to interpret the statistics in this table, see pages 79-88.

\section{ART CYCLE PROFILE}

\begin{tabular}{|c|c|c|c|c|c|c|c|}
\hline \multicolumn{4}{|c|}{ Type of ART ${ }^{a}$} & \multicolumn{4}{|c|}{ Patient Diagnosis } \\
\hline IVF & $100 \%$ & Procedural Factors: & & Tubal factor & $9 \%$ & Other factor & $0 \%$ \\
\hline GIFT & $0 \%$ & With ICSI & $48 \%$ & Ovulatory dysfunction & $2 \%$ & Unknown factor & $2 \%$ \\
\hline ZIFT & $0 \%$ & Unstimulated & $0 \%$ & Diminished ovarian reserve & $2 \%$ & Multiple Factors: & \\
\hline \multirow[t]{3}{*}{ Combination } & $0 \%$ & Used gestational carrier & $0 \%$ & Endometriosis & $7 \%$ & Female factors only & $25 \%$ \\
\hline & & & & Uterine factor & $0 \%$ & Female \& male factors & $34 \%$ \\
\hline & & & & Male factor & $18 \%$ & & \\
\hline
\end{tabular}

2006 PREGNANCY SUCCESS RATES

Data verified by Vincent A. Pellegrini, MD

\section{Type of Cycle}

\section{Fresh Embryos from Nondonor Eggs}

Number of cycles

Percentage of cycles resulting in pregnancies ${ }^{b}$

Percentage of cycles resulting in live births $s^{b, c}$

(Confidence Interval)

Percentage of retrievals resulting in live births $s^{b, c}$

Percentage of transfers resulting in live births $s^{b, c}$

Percentage of transfers resulting in singleton live births ${ }^{b}$

Percentage of cancellations ${ }^{b}$

Average number of embryos transferred

Percentage of pregnancies with twins ${ }^{b}$

Percentage of pregnancies with triplets or more ${ }^{b}$

Percentage of live births having multiple infants ${ }^{\mathrm{b}, \mathrm{c}}$

\section{Frozen Embryos from Nondonor Eggs}

Number of transfers

Percentage of transfers resulting in live births $s^{b, c}$

Average number of embryos transferred

\section{Donor Eggs}

Number of transfers

Percentage of transfers resulting in live births $s^{b, c}$

Average number of embryos transferred

\section{Age of Woman}

$<35 \quad 35-37 \quad 38-40 \quad 41-42^{\text {d }}$

$\begin{array}{cccc}23 & 10 & 8 & 3 \\ 47.8 & 4 / 10 & 6 / 8 & 0 / 3 \\ 39.1 & 3 / 10 & 4 / 8 & 0 / 3\end{array}$

$(19.7-61.5)$

$\begin{array}{cccc}9 / 18 & 3 / 9 & 4 / 8 & 0 / 3 \\ 9 / 16 & 3 / 9 & 4 / 8 & 0 / 3 \\ 4 / 16 & 2 / 9 & 3 / 8 & 0 / 3 \\ 21.7 & 1 / 10 & 0 / 8 & 0 / 3 \\ 2.9 & 2.9 & 3.4 & 3.3 \\ 6 / 11 & 1 / 4 & 2 / 6 & \\ 0 / 11 & 0 / 4 & 0 / 6 & \\ 5 / 9 & 1 / 3 & 1 / 4 & \end{array}$

0

0

0

0

\section{CURRENT CLINIC SERVICES AND PROFILE}

Current Name: Women's Clinic, Ltd.

\begin{tabular}{ll|l|l|l|l} 
Donor egg? & No & Gestational carriers? & No & SART member? & Yes \\
\hline Donor embryo? & No & Cryopreservation? & No & Verified lab accreditation? & Yes \\
\hline Single women? & No & & & (See Appendix C for details.) &
\end{tabular}

${ }_{b}^{a}$ Reflects patient and treatment characteristics of ART cycles performed in 2006 using fresh nondonor eggs or embryos.

${ }^{b}$ When fewer than 20 cycles are reported in an age category, rates are shown as a fraction and confidence intervals are not given. Calculating percentages from fractions may be misleading and is not encouraged.

${ }^{c}$ A multiple-infant birth is counted as one live birth.

d Clinic-specific outcome rates are unreliable for women older than 42 undergoing ART cycles using fresh or frozen embryos with nondonor eggs. Readers are urged to review national outcomes for these age groups (see page 27).

${ }^{e}$ All ages (including ages $>42$ ) are reported together because previous data show that patient age does not materially affect success with donor eggs. 


\section{FERTILITY AND GYNECOLOGY ASSOCIATES \\ WILLOW GROVE, PENNSYLVANIA}

A comparison of clinic success rates may not be meaningful because patient medical characteristics and treatment approaches vary from clinic to clinic. For more details about this, along with information on how to interpret the statistics in this table, see pages $79-88$.

\section{ART CYCLE PROFILE}

\begin{tabular}{|c|c|c|c|c|c|c|c|}
\hline \multicolumn{4}{|c|}{ Type of ART ${ }^{a}$} & \multicolumn{4}{|c|}{ Patient Diagnosis } \\
\hline IVF & $100 \%$ & Procedural Factors: & & Tubal factor & $12 \%$ & Other factor & $7 \%$ \\
\hline GIFT & $0 \%$ & With ICSI & $39 \%$ & Ovulatory dysfunction & $0 \%$ & Unknown factor & $17 \%$ \\
\hline ZIFT & $0 \%$ & Unstimulated & $0 \%$ & Diminished ovarian reserve & $29 \%$ & Multiple Factors: & \\
\hline \multirow[t]{3}{*}{ Combination } & $0 \%$ & Used gestational carrier & $0 \%$ & Endometriosis & $10 \%$ & Female factors only & $0 \%$ \\
\hline & & & & Uterine factor & $0 \%$ & Female \& male factors & $7 \%$ \\
\hline & & & & Male factor & $19 \%$ & & \\
\hline
\end{tabular}

2006 PREGNANCY SUCCESS RATES

Data verified by Leonore C. Huppert, MD

\section{Type of Cycle}

\section{Fresh Embryos from Nondonor Eggs}

Number of cycles

Percentage of cycles resulting in pregnancies ${ }^{b}$

Percentage of cycles resulting in live births ${ }^{b, c}$

(Confidence Interval)

Percentage of retrievals resulting in live births $s^{b, c}$

Percentage of transfers resulting in live births $s^{b, c}$

Percentage of transfers resulting in singleton live births ${ }^{b}$

Percentage of cancellations ${ }^{b}$

Average number of embryos transferred

Percentage of pregnancies with twins ${ }^{b}$

Percentage of pregnancies with triplets or more ${ }^{b}$

Percentage of live births having multiple infants ${ }^{b, c}$

\section{Frozen Embryos from Nondonor Eggs}

Number of transfers

Percentage of transfers resulting in live births $s^{\mathrm{b}, \mathrm{c}}$

Average number of embryos transferred

$<35$

Age of Woman

35-37 38-40

$41-42^{d}$

11

$6 / 11$

3

$5 / 11$

$2 / 3$

$2 / 3$

\section{4}

2

$5 / 11$

$2 / 3$

$2 / 3$

$2 / 3$

$0 / 3$

3.0

$1 / 2$

$0 / 2$

$0 / 2$

$2 / 4$

$2 / 4$

$1 / 2$

$5 / 10$

$0 / 11$

2.3

$2 / 6$

$0 / 6$

$2 / 5$

$2 / 4$

$2 / 4$

$2 / 4$

$0 / 4$

3.8

$2 / 2$

$0 / 2$

$0 / 2$

\section{7}

$5 / 7$

2.4

\section{2}

$1 / 2$

3.0

$0 / 2$

\section{年}




\section{THE FERTILITY CENTER, LLC YORK, PENNSYLVANIA}

A comparison of clinic success rates may not be meaningful because patient medical characteristics and treatment approaches vary from clinic to clinic. For more details about this, along with information on how to interpret the statistics in this table, see pages 79-88.

\section{ART CYCLE PROFILE}

\begin{tabular}{|c|c|c|c|c|c|c|c|}
\hline \multicolumn{4}{|c|}{ Type of ART ${ }^{a}$} & \multicolumn{4}{|c|}{ Patient Diagnosis } \\
\hline IVF & $100 \%$ & Procedural Factors: & & Tubal factor & $7 \%$ & Other factor & $10 \%$ \\
\hline GIFT & $0 \%$ & With ICSI & $100 \%$ & Ovulatory dysfunction & $16 \%$ & Unknown factor & $7 \%$ \\
\hline ZIFT & $0 \%$ & Unstimulated & $0 \%$ & Diminished ovarian reserve & $14 \%$ & Multiple Factors: & \\
\hline \multirow[t]{3}{*}{ Combination } & $0 \%$ & Used gestational carrier & $7 \%$ & Endometriosis & $0 \%$ & Female factors only & $3 \%$ \\
\hline & & & & Uterine factor & $0 \%$ & Female \& male factors & $14 \%$ \\
\hline & & & & Male factor & $29 \%$ & & \\
\hline
\end{tabular}

2006 PREGNANCY SUCCESS RATES

Data verified by Robert B. Filer, MD

\section{Type of Cycle}

\section{Fresh Embryos from Nondonor Eggs}

Number of cycles

Percentage of cycles resulting in pregnancies ${ }^{b}$

Percentage of cycles resulting in live births $s^{b, c}$

(Confidence Interval)
Percentage of retrievals resulting in live births $s^{b, c}$

Percentage of transfers resulting in live births $s^{b, c}$

Percentage of transfers resulting in singleton live births ${ }^{b}$

Percentage of cancellations ${ }^{b}$

Average number of embryos transferred

Percentage of pregnancies with twins ${ }^{b}$

Percentage of pregnancies with triplets or more ${ }^{b}$

Percentage of live births having multiple infants ${ }^{b, c}$

\section{Frozen Embryos from Nondonor Eggs}

Number of transfers

Percentage of transfers resulting in live births $s^{b, c}$

Average number of embryos transferred

\section{Donor Eggs}

Number of transfers

Percentage of transfers resulting in live births $s^{b, c}$

Average number of embryos transferred

\section{Age of Woman}

35-37 38-40

$41-42^{d}$

\section{$<35$}

7

$2 / 7$

3

$9 / 19$

$2 / 7$

$1 / 3$

$0 / 3$

$9 / 19$

$2 / 7$

$0 / 3$

$0 / 2$

$0 / 2$

$0 / 3$

3.0

$0 / 1$

$0 / 1$

$0 / 9$

$1 / 9$

$0 / 2$

$0 / 2$

$1 / 2$
$0 / 1$

$0 / 1$

\section{3}

$2 / 3$

3.0

All Ages Combined

Fresh Embryos 4

$1 / 4$

3.0
Frozen Embryos

\section{8}

$0 / 8$

3.4

\section{CURRENT CLINIC SERVICES AND PROFILE}

Current Name: The Fertility Center, LLC

Donor egg? Yes

Donor embryo? Yes

Single women? Yes

\section{Gestational carriers? Yes}

Cryopreservation? Yes
SART member?

Verified lab accreditation?

(See Appendix C for details.)
No

No

${ }^{a}$ Reflects patient and treatment characteristics of ART cycles performed in 2006 using fresh nondonor eggs or embryos.

b When fewer than 20 cycles are reported in an age category, rates are shown as a fraction and confidence intervals are not given. Calculating percentages from fractions may be misleading and is not encouraged.

${ }^{\mathrm{C}}$ A multiple-infant birth is counted as one live birth.

d Clinic-specific outcome rates are unreliable for women older than 42 undergoing ART cycles using fresh or frozen embryos with nondonor eggs. Readers are urged to review national outcomes for these age groups (see page 27).

ell ages (including ages $>42$ ) are reported together because previous data show that patient age does not materially affect success with donor eggs. 


\section{PEDRO J. BEAUCHAMP, MD \\ BAYAMON, PUERTO RICO}

A comparison of clinic success rates may not be meaningful because patient medical characteristics and treatment approaches vary from clinic to clinic. For more details about this, along with information on how to interpret the statistics in this table, see pages $79-88$.

\section{ART CYCLE PROFILE}

\begin{tabular}{|c|c|c|c|c|c|c|c|}
\hline \multicolumn{4}{|c|}{ Type of ART ${ }^{\mathrm{a}}$} & \multicolumn{4}{|c|}{ Patient Diagnosis } \\
\hline IVF & $100 \%$ & Procedural Factors: & & Tubal factor & $13 \%$ & Other factor & $<1 \%$ \\
\hline GIFT & $0 \%$ & With ICSI & $87 \%$ & Ovulatory dysfunction & $3 \%$ & Unknown factor & $0 \%$ \\
\hline ZIFT & $0 \%$ & Unstimulated & $0 \%$ & Diminished ovarian reserve & $<1 \%$ & Multiple Factors: & \\
\hline \multirow[t]{3}{*}{ Combination } & $0 \%$ & Used gestational carrier & $1 \%$ & Endometriosis & $3 \%$ & Female factors only & $21 \%$ \\
\hline & & & & Uterine factor & $0 \%$ & Female \& male factors & $49 \%$ \\
\hline & & & & Male factor & $12 \%$ & & \\
\hline
\end{tabular}

2006 PREGNANCY SUCCESS RATES

Data verified by Pedro J. Beauchamp, MD

\section{Type of Cycle}

\section{Fresh Embryos from Nondonor Eggs}

Number of cycles

Percentage of cycles resulting in pregnancies ${ }^{b}$

Percentage of cycles resulting in live births ${ }^{b, c}$

(Confidence Interval)

Percentage of retrievals resulting in live births $s^{b, c}$

Percentage of transfers resulting in live births $s^{b, c}$

Percentage of transfers resulting in singleton live births ${ }^{b}$

Percentage of cancellations ${ }^{b}$

Average number of embryos transferred

Percentage of pregnancies with twins ${ }^{b}$

Percentage of pregnancies with triplets or more ${ }^{b}$

Percentage of live births having multiple infants ${ }^{b, c}$

\section{Frozen Embryos from Nondonor Eggs}

Number of transfers

Percentage of transfers resulting in live births $s^{\mathrm{b}, \mathrm{c}}$

Average number of embryos transferred

\section{Donor Eggs}

Number of transfers

Percentage of transfers resulting in live births $s^{b, c}$

Average number of embryos transferred

\section{Age of Woman}

35-37 38-40

$41-42^{d}$

\section{$<35$}

48

41.7

47

13

63
54.0

39.6

34.0

$4 / 13$

41.3

(25.8-54.7)

19.1

$1 / 13$

(29.0-54.4)

43.3

41.3

(9.1-33.3)

44.1

42.2

23.1

$1 / 13$

28.8

33.3

25.0

$1 / 12$

4.8

4.2

11.1

$1 / 12$

2.8

2.7

17.0

$0 / 13$

32.4

20.0

5.9

10.0

3.2

3.3

34.6

$4 / 19$

$5 / 16$

$0 / 4$

$2 / 16$

$0 / 4$

$5 / 9$

$0 / 1$

\section{1}

$0 / 1$

6.0

1

$0 / 1$

1

$0 / 1$

4.0

4.0

All Ages Combined ${ }^{\mathrm{e}}$

Fresh Embryos 3

$1 / 3$

3.0

\section{CURRENT CLINIC SERVICES AND PROFILE}

Current Name: Pedro J. Beauchamp, MD
Donor egg?
Yes
Donor embryo? No
$\begin{array}{ll}\text { Gestational carriers? } & \text { Yes } \\ \text { Cryopreservation? } & \text { Yes }\end{array}$
Single women? No
SART member? Yes
Verified lab accreditation?
(See Appendix C for details.)

\footnotetext{
${ }^{a}$ Reflects patient and treatment characteristics of ART cycles performed in 2006 using fresh nondonor eggs or embryos.

${ }^{b}$ When fewer than 20 cycles are reported in an age category, rates are shown as a fraction and confidence intervals are not given. Calculating percentages from fractions may be misleading and is not encouraged.

${ }^{c}$ A multiple-infant birth is counted as one live birth.

${ }^{d}$ Clinic-specific outcome rates are unreliable for women older than 42 undergoing ART cycles using fresh or frozen embryos with nondonor eggs. Readers are urged to review national outcomes for these age groups (see page 27).

${ }^{e}$ All ages (including ages $>42$ ) are reported together because previous data show that patient age does not materially affect success with donor eggs.
} 


\section{GREFI \\ GYNECOLOGY, REPRODUCTIVE ENDOCRINOLOGY \& FERTILITY INSTITUTE SANTURCE, PUERTO RICO}

A comparison of clinic success rates may not be meaningful because patient medical characteristics and treatment approaches vary from clinic to clinic. For more details about this, along with information on how to interpret the statistics in this table, see pages 79-88.

\section{ART CYCLE PROFILE}

\begin{tabular}{|c|c|c|c|c|c|c|c|}
\hline \multicolumn{4}{|c|}{ Type of ART ${ }^{a}$} & \multicolumn{4}{|c|}{ Patient Diagnosis } \\
\hline IVF & $100 \%$ & Procedural Factors: & & Tubal factor & $28 \%$ & Other factor & $4 \%$ \\
\hline GIFT & $0 \%$ & With ICSI & $32 \%$ & Ovulatory dysfunction & $1 \%$ & Unknown factor & $3 \%$ \\
\hline ZIFT & $0 \%$ & Unstimulated & $0 \%$ & Diminished ovarian reserve & $16 \%$ & Multiple Factors: & \\
\hline \multirow[t]{3}{*}{ Combination } & $0 \%$ & Used gestational carrier & $0 \%$ & Endometriosis & $12 \%$ & Female factors only & $0 \%$ \\
\hline & & & & Uterine factor & $3 \%$ & Female \& male factors & $16 \%$ \\
\hline & & & & Male factor & $17 \%$ & & \\
\hline
\end{tabular}

2006 PREGNANCY SUCCESS RATES

Data verified by Rosa lleana Cruz, MD

\section{Type of Cycle}

\section{Fresh Embryos from Nondonor Eggs}

Number of cycles

Percentage of cycles resulting in pregnancies ${ }^{b}$

Percentage of cycles resulting in live births ${ }^{b, c}$

(Confidence Interval)

Percentage of retrievals resulting in live births $s^{b, c}$

Percentage of transfers resulting in live births $s^{b, c}$

Percentage of transfers resulting in singleton live births ${ }^{b}$

Percentage of cancellations ${ }^{b}$

Average number of embryos transferred

Percentage of pregnancies with twins ${ }^{b}$

Percentage of pregnancies with triplets or more ${ }^{b}$

Percentage of live births having multiple infants ${ }^{b, c}$

\section{Frozen Embryos from Nondonor Eggs}

Number of transfers

Percentage of transfers resulting in live births $s^{b, c}$

Average number of embryos transferred

\section{Donor Eggs}

Number of transfers

Percentage of transfers resulting in live births $s^{b, c}$

Average number of embryos transferred

\section{Age of Woman}

35-37 38-40

$41-42^{d}$

$<35$

7

$2 / 7$

$2 / 7$

8

6

16.7

13.9

$2 / 7$

$3 / 8$

$1 / 6$

$(4.7-29.5)$

$\begin{array}{cccc}15.2 & 2 / 7 & 2 / 8 & 0 / 6 \\ 17.2 & 2 / 6 & 2 / 8 & 0 / 6 \\ 6.9 & 1 / 6 & 1 / 8 & 0 / 6 \\ 8.3 & 0 / 7 & 0 / 8 & 0 / 6 \\ 2.8 & 3.2 & 2.9 & 3.5 \\ 4 / 6 & 1 / 2 & 2 / 3 & 0 / 1 \\ 0 / 6 & 0 / 2 & 0 / 3 & 0 / 1 \\ 3 / 5 & 1 / 2 & 1 / 2 & \end{array}$

2

$0 / 2$

3.0

\section{CURRENT CLINIC SERVICES AND PROFILE}

Current Name: GREFI, Gynecology, Reproductive Endocrinology \& Fertility Institute

\begin{tabular}{ll|l|l|l|l} 
Donor egg? & Yes & Gestational carriers? & No & SART member? & Yes \\
\hline Donor embryo? & Yes & Cryopreservation? & Yes & Verified lab accreditation? & Yes \\
Single women? & Yes & & & (See Appendix C for details.) &
\end{tabular}

${ }_{b}^{a}$ Reflects patient and treatment characteristics of ART cycles performed in 2006 using fresh nondonor eggs or embryos.

b When fewer than 20 cycles are reported in an age category, rates are shown as a fraction and confidence intervals are not given. Calculating percentages from fractions may be misleading and is not encouraged.

${ }_{\text {C }}^{c}$ A multiple-infant birth is counted as one live birth.

d Clinic-specific outcome rates are unreliable for women older than 42 undergoing ART cycles using fresh or frozen embryos with nondonor eggs. Readers are urged to review national outcomes for these age groups (see page 27).

e All ages (including ages $>42$ ) are reported together because previous data show that patient age does not materially affect success with donor eggs. 
A comparison of clinic success rates may not be meaningful because patient medical characteristics and treatment approaches vary from clinic to clinic. For more details about this, along with information on how to interpret the statistics in this table, see pages 79-88.

\section{ART CYCLE PROFILE}

\begin{tabular}{|c|c|c|c|c|c|c|c|}
\hline \multicolumn{4}{|c|}{ Type of ART ${ }^{a}$} & \multicolumn{4}{|c|}{ Patient Diagnosis } \\
\hline IVF & $100 \%$ & Procedural Factors: & & Tubal factor & $13 \%$ & Other factor & $9 \%$ \\
\hline GIFT & $0 \%$ & With ICSI & $57 \%$ & Ovulatory dysfunction & $9 \%$ & Unknown factor & $28 \%$ \\
\hline ZIFT & $0 \%$ & Unstimulated & $<1 \%$ & Diminished ovarian reserve & $2 \%$ & Multiple Factors: & \\
\hline \multirow[t]{3}{*}{ Combination } & $0 \%$ & Used gestational carrier & $0 \%$ & Endometriosis & $4 \%$ & Female factors only & $4 \%$ \\
\hline & & & & Uterine factor & $3 \%$ & Female \& male factors & $8 \%$ \\
\hline & & & & Male factor & $20 \%$ & & \\
\hline
\end{tabular}

2006 PREGNANCY SUCCESS RATES

Data verified by Gary Frishman, MD

\section{Type of Cycle}

\section{Fresh Embryos from Nondonor Eggs}

Number of cycles

Percentage of cycles resulting in pregnancies ${ }^{b}$

Percentage of cycles resulting in live births ${ }^{b, c}$

(Confidence Interval)

Percentage of retrievals resulting in live births $s^{b, c}$

Percentage of transfers resulting in live births $s^{b, c}$

Percentage of transfers resulting in singleton live births ${ }^{b}$

Percentage of cancellations ${ }^{b}$

Average number of embryos transferred

Percentage of pregnancies with twins ${ }^{b}$

Percentage of pregnancies with triplets or more ${ }^{\mathrm{b}}$

Percentage of live births having multiple infants ${ }^{b, c}$

Frozen Embryos from Nondonor Eggs

Number of transfers

Percentage of transfers resulting in live births $s^{b, c}$

Average number of embryos transferred

\section{Donor Eggs}

Number of transfers

Percentage of transfers resulting in live births $s^{b, c}$

Average number of embryos transferred

\section{Age of Woman}

35-37 38-40

$41-42^{d}$

$<35$

148

130

71

278

37.2

23.8

9.9

38.1

33.8

15.4

5.6

(32.4-44.1)

(26.2-42.0)

$(9.7-22.8)$

17.5

(1.6-13.8)

39.4

38.5

20.2

6.2

41.4

21.5

13.1

7.8

30.1

6.1

12.3

7.8

2.0

29.0

2.4

2.3

38.2

7.3

27.4

44.0

2.6

8.5

2.8

22.6

$1 / 7$

3.2

$0 / 7$

35.0

$0 / 4$

47

25.5

16

20

11

2.4

$1 / 16$

35.0

$0 / 11$

2.3

2.2

2.6

All Ages Combined ${ }^{\mathrm{e}}$

Fresh Embryos

52

36.5

2.0
Frozen Embryos 18

$4 / 18$

2.5

\section{CURRENT CLINIC SERVICES AND PROFILE}

Current Name: Women and Infants' Division of Reproductive Medicine and Infertility

Donor egg? Yes

Donor embryo? Yes

Single women? Yes
Gestational carriers? Yes

Cryopreservation? Yes
SART member? Yes

Verified lab accreditation?

(See Appendix C for details.)

${ }^{a}$ Reflects patient and treatment characteristics of ART cycles performed in 2006 using fresh nondonor eggs or embryos.

${ }^{b}$ When fewer than 20 cycles are reported in an age category, rates are shown as a fraction and confidence intervals are not given. Calculating percentages from fractions may be misleading and is not encouraged.

A multiple-infant birth is counted as one live birth.

d Clinic-specific outcome rates are unreliable for women older than 42 undergoing ART cycles using fresh or frozen embryos with nondonor eggs. Readers are urged to review national outcomes for these age groups (see page 27).

e All ages (including ages $>42$ ) are reported together because previous data show that patient age does not materially affect success with donor eggs. 


\section{PIEDMONT REPRODUCTIVE ENDOCRINOLOGY GROUP, PA GREENVILLE, SOUTH CAROLINA}

A comparison of clinic success rates may not be meaningful because patient medical characteristics and treatment approaches vary from clinic to clinic. For more details about this, along with information on how to interpret the statistics in this table, see pages 79-88.

\section{ART CYCLE PROFILE}

\section{Type of ART ${ }^{\mathrm{a}}$}

IVF

GIFT $100 \%$ Procedural Factors:

ZIFT

Combination
$0 \%$ With ICSI

$0 \%$ Unstimulated

$0 \%$ Used gestational carrier

\begin{tabular}{|c|c|c|c|c|}
\hline & \multicolumn{4}{|c|}{ Patient Diagnosis } \\
\hline & Tubal factor & $6 \%$ & Other factor & $2 \%$ \\
\hline $85 \%$ & Ovulatory dysfunction & $33 \%$ & Unknown factor & $2 \%$ \\
\hline $0 \%$ & Diminished ovarian reserve & $4 \%$ & Multiple Factors: & \\
\hline $0 \%$ & Endometriosis & $4 \%$ & Female factors only & $28 \%$ \\
\hline & Uterine factor & $0 \%$ & Female \& male factors & $18 \%$ \\
\hline & Male factor & $3 \%$ & & \\
\hline
\end{tabular}

Data verified by John E. Nichols, MD

2006 PREGNANCY SUCCESS RATES

\section{Age of Woman}

Type of Cycle

$\begin{array}{cccc}<35 & \mathbf{3 5 - 3 7} & \mathbf{3 8 - 4 0} & \mathbf{4 1 - 4 2}^{\mathbf{d}} \\ 52 & 12 & 11 & 2 \\ 46.2 & 7 / 12 & 4 / 11 & 2 / 2 \\ 42.3 & 6 / 12 & 2 / 11 & 1 / 2\end{array}$

\section{Fresh Embryos from Nondonor Eggs}

Number of cycles

Percentage of cycles resulting in pregnancies ${ }^{b}$

Percentage of cycles resulting in live births ${ }^{b, c}$

(Confidence Interval)

(28.7-56.8)

Percentage of retrievals resulting in live births $s^{b, c}$

Percentage of transfers resulting in live births $s^{b, c}$

Percentage of transfers resulting in singleton live births ${ }^{b}$

Percentage of cancellations ${ }^{b}$

\section{9}

44.9

28.6

Average number of embryos transferred

Percentage of pregnancies with twins ${ }^{\mathrm{b}}$

Percentage of pregnancies with triplets or more ${ }^{b}$

Percentage of live births having multiple infants ${ }^{b, c}$

\section{Frozen Embryos from Nondonor Eggs}

Number of transfers

Percentage of transfers resulting in live births $s^{b, c}$

Average number of embryos transferred

\section{Donor Eggs}

Number of transfers

Percentage of transfers resulting in live births $s^{b, c}$

Average number of embryos transferred
5.8

2.2

33.3

4.2

36.4

16

$3 / 16$

2.6
$6 / 11$

$6 / 11$

$5 / 11$

$1 / 12$

2.5

$1 / 7$

$0 / 7$

$1 / 6$
$2 / 9$

$2 / 9$

$2 / 9$

$2 / 11$

2.4

$1 / 4$

$0 / 4$

$0 / 2$

4

$1 / 4$

2.3
2

$2 / 2$

2.5
$1 / 2$

$1 / 2$

$1 / 2$

$0 / 2$

4.0

$0 / 2$

$0 / 2$

$0 / 1$

All Ages Combined ${ }^{\mathrm{e}}$

Fresh Embryos 12

$4 / 12$

2.1
Frozen Embryos 10

$4 / 10$

2.7

\section{CURRENT CLINIC SERVICES AND PROFILE}

Current Name: Piedmont Reproductive Endocrinology Group, PA

Donor egg? Yes Gestational carriers? Yes

Donor embryo? Yes Cryopreservation? Yes

Single women? Yes

SART member? Yes

Verified lab accreditation? Yes

(See Appendix C for details.)

${ }^{a}$ Reflects patient and treatment characteristics of ART cycles performed in 2006 using fresh nondonor eggs or embryos.

${ }^{b}$ When fewer than 20 cycles are reported in an age category, rates are shown as a fraction and confidence intervals are not given. Calculating percentages from fractions may be misleading and is not encouraged.

${ }^{c}$ A multiple-infant birth is counted as one live birth.

d Clinic-specific outcome rates are unreliable for women older than 42 undergoing ART cycles using fresh or frozen embryos with nondonor eggs. Readers are urged to review national outcomes for these age groups (see page 27).

e All ages (including ages $>42$ ) are reported together because previous data show that patient age does not materially affect success with donor eggs. 


\section{UNIVERSITY MEDICAL GROUP, DEPARTMENT OF OBSTETRICS AND GYNECOLOGY REPRODUCTIVE ENDOCRINOLOGY AND INFERTILITY GREENVILLE, SOUTH CAROLINA}

A comparison of clinic success rates may not be meaningful because patient medical characteristics and treatment approaches vary from clinic to clinic. For more details about this, along with information on how to interpret the statistics in this table, see pages 79-88.

\section{ART CYCLE PROFILE}

\begin{tabular}{|c|c|c|c|c|c|c|c|}
\hline \multicolumn{4}{|c|}{ Type of ART ${ }^{\mathrm{a}}$} & \multicolumn{4}{|c|}{ Patient Diagnosis } \\
\hline IVF & $100 \%$ & Procedural Factors: & & Tubal factor & $7 \%$ & Other factor & $3 \%$ \\
\hline GIFT & $0 \%$ & With ICSI & $70 \%$ & Ovulatory dysfunction & $21 \%$ & Unknown factor & $2 \%$ \\
\hline ZIFT & $0 \%$ & Unstimulated & $0 \%$ & Diminished ovarian reserve & $<1 \%$ & Multiple Factors: & \\
\hline \multirow[t]{3}{*}{ Combination } & $0 \%$ & Used gestational carrier & $<1 \%$ & Endometriosis & $17 \%$ & Female factors only & $21 \%$ \\
\hline & & & & Uterine factor & $0 \%$ & Female \& male factors & $12 \%$ \\
\hline & & & & Male factor & $17 \%$ & & \\
\hline
\end{tabular}

2006 PREGNANCY SUCCESS RATES

Data verified by Bruce A. Lessey, MD, PhD

\section{Type of Cycle}

\section{Age of Woman}

Fresh Embryos from Nondonor Eggs

35-37

38-40

$41-42^{d}$ $<35$

23

14

2

Number of cycles

Percentage of cycles resulting in pregnancies ${ }^{b}$

Percentage of cycles resulting in live births $s^{b, c}$

(Confidence Interval)

Percentage of retrievals resulting in live births $s^{b, c}$

Percentage of transfers resulting in live births $s^{b, c}$

Percentage of transfers resulting in singleton live births ${ }^{b}$

Percentage of cancellations ${ }^{b}$

\section{5}

$(28.0-52.9)$

Average number of embryos transferred

Percentage of pregnancies with twins ${ }^{b}$

Percentage of pregnancies with triplets or more ${ }^{b}$

Percentage of live births having multiple infants ${ }^{b, c}$

\section{Frozen Embryos from Nondonor Eggs}

Number of transfers

Percentage of transfers resulting in live births $s^{\mathrm{b}, \mathrm{c}}$

Average number of embryos transferred

\section{Donor Eggs}

Number of transfers

Percentage of transfers resulting in live births $s^{b, c}$

Average number of embryos transferred
53.8

40.0

43.3

48.1

29.6

7.7

2.0

43.5

30.4

(13.2-52.9)

$7 / 19$

$7 / 18$

$5 / 18$

17.4

2.3

28.6

2.9

38.5

$2 / 10$

$1 / 10$

$2 / 7$

22

36.4

2.2

\section{0}

$3 / 10$

2.2
$5 / 14$

$5 / 14$

$1 / 2$

$1 / 2$

$\begin{array}{ll}5 / 13 & 1 / 2 \\ 5 / 11 & 1 / 2 \\ 4 / 11 & 1 / 2 \\ 1 / 14 & 0 / 2 \\ 2.5 & 3.0 \\ 0 / 5 & 0 / 1 \\ 1 / 5 & 0 / 1 \\ 1 / 5 & 0 / 1\end{array}$

Fresh Embryos

All Ages Combined ${ }^{\mathrm{e}}$

$6 / 9$

Frozen Embryos

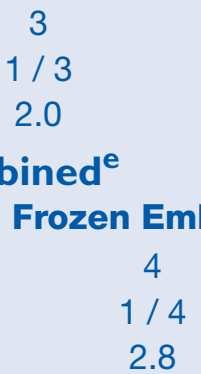

\section{CURRENT CLINIC SERVICES AND PROFILE}

Current Name: University Medical Group, Department of Obstetrics and Gynecology, Reproductive Endocrinology and Infertility
Donor egg? Yes
Gestational carriers? Yes
Donor embryo? No
Cryopreservation? Yes
SART member?
Yes
Verified lab accreditation?
Yes
Single women? Yes
(See Appendix C for details.)

${ }^{a}$ Reflects patient and treatment characteristics of ART cycles performed in 2006 using fresh nondonor eggs or embryos.

${ }^{b}$ When fewer than 20 cycles are reported in an age category, rates are shown as a fraction and confidence intervals are not given. Calculating percentages from fractions may be misleading and is not encouraged.

${ }^{c}$ A multiple-infant birth is counted as one live birth.

d Clinic-specific outcome rates are unreliable for women older than 42 undergoing ART cycles using fresh or frozen embryos with nondonor eggs. Readers are urged to review national outcomes for these age groups (see page 27).

e All ages (including ages $>42$ ) are reported together because previous data show that patient age does not materially affect success with donor eggs. 


\section{SOUTHEASTERN FERTILITY CENTER, PA MOUNT PLEASANT, SOUTH CAROLINA}

A comparison of clinic success rates may not be meaningful because patient medical characteristics and treatment approaches vary from clinic to clinic. For more details about this, along with information on how to interpret the statistics in this table, see pages 79-88.

\section{ART CYCLE PROFILE}

\section{Type of ART ${ }^{\mathrm{a}}$}

IVF

GIFT 100\% Procedural Factors:

ZIFT

Combination
$0 \%$ With ICSI

$0 \%$ Unstimulated

$0 \%$ Used gestational carrier $<1 \%$
Tubal factor

Ovulatory dysfunction

Diminished ovarian reserve

Endometriosis

Uterine factor

Male factor

\section{Patient Diagnosis}

$\begin{array}{rlr}14 \% & \text { Other factor } & 3 \% \\ 4 \% & \text { Unknown factor } & 8 \% \\ 19 \% & \text { Multiple Factors: } & \\ 5 \% & \text { Female factors only } & 16 \% \\ <1 \% & \text { Female \& male factors } & 15 \% \\ 16 \% & & \end{array}$

\section{Age of Woman}

\section{Type of Cycle}

\section{Fresh Embryos from Nondonor Eggs}

Number of cycles

Percentage of cycles resulting in pregnancies ${ }^{b}$

Percentage of cycles resulting in live births ${ }^{b, c}$

(Confidence Interval)

Percentage of retrievals resulting in live births $s^{b, c}$

Percentage of transfers resulting in live births $s^{b, c}$

Percentage of transfers resulting in singleton live births ${ }^{b}$

Percentage of cancellations ${ }^{b}$

Average number of embryos transferred

Percentage of pregnancies with twins ${ }^{\mathrm{b}}$

Percentage of pregnancies with triplets or more ${ }^{b}$

Percentage of live births having multiple infants ${ }^{\mathrm{b}, \mathrm{c}}$

\section{Frozen Embryos from Nondonor Eggs}

Number of transfers

Percentage of transfers resulting in live births $s^{b, c}$

Average number of embryos transferred

\section{Donor Eggs}

Number of transfers

Percentage of transfers resulting in live births $s^{b, c}$

Average number of embryos transferred

All Ages Combined ${ }^{\mathrm{e}}$
Fresh Embryos

62

64.5

2.0

35-37 $38-40 \quad 41-42^{d}$

\section{$<35$}

70

31

12

45.3

42.9

29.0

$2 / 12$

38.4

37.1

19.4

$2 / 12$

(30.8-46.4)

41.2

(25.9-49.5)

(7.5-37.5)

38.2

22.2

$2 / 7$

42.7

39.4

25.0

$2 / 6$

25.8

16.7

$2 / 6$

6.9

2.9

12.9

$5 / 12$

2.1

2.2

36.7

2.5

3.2

38.9

3.3

$2 / 9$

$0 / 2$

2.8

34.6

$0 / 9$

$0 / 2$

37.7

$2 / 6$

$0 / 2$

41

46.3

15

$8 / 15$

6

$2 / 6$

1

2.0

2.0

2.0

$0 / 1$

3.0
Frozen Embryos

21

42.9

2.0

\section{CURRENT CLINIC SERVICES AND PROFILE}

Current Name: Southeastern Fertility Center, PA

Donor egg? Yes

Donor embryo? Yes

Single women? Yes

\section{Gestational carriers? Yes}

Cryopreservation? Yes
SART member?

Yes

Verified lab accreditation?

(See Appendix C for details.)

${ }^{a}$ Reflects patient and treatment characteristics of ART cycles performed in 2006 using fresh nondonor eggs or embryos.

${ }^{b}$ When fewer than 20 cycles are reported in an age category, rates are shown as a fraction and confidence intervals are not given. Calculating percentages from fractions may be misleading and is not encouraged.

${ }^{\mathrm{C}}$ A multiple-infant birth is counted as one live birth.

d Clinic-specific outcome rates are unreliable for women older than 42 undergoing ART cycles using fresh or frozen embryos with nondonor eggs. Readers are urged to review national outcomes for these age groups (see page 27).

ell ages (including ages $>42$ ) are reported together because previous data show that patient age does not materially affect success with donor eggs. 


\section{ADVANCED FERTILITY \& REPRODUCTIVE ENDOCRINOLOGY WEST COLUMBIA, SOUTH CAROLINA}

A comparison of clinic success rates may not be meaningful because patient medical characteristics and treatment approaches vary from clinic to clinic. For more details about this, along with information on how to interpret the statistics in this table, see pages 79-88.

\section{ART CYCLE PROFILE}

\begin{tabular}{|c|c|c|c|c|c|c|c|}
\hline \multicolumn{4}{|c|}{ Type of $A R T^{a}$} & \multicolumn{4}{|c|}{ Patient Diagnosis } \\
\hline IVF & $100 \%$ & Procedural Factors: & & Tubal factor & $4 \%$ & Other factor & $2 \%$ \\
\hline GIFT & $0 \%$ & With ICSI & $98 \%$ & Ovulatory dysfunction & $4 \%$ & Unknown factor & $2 \%$ \\
\hline ZIFT & $0 \%$ & Unstimulated & $0 \%$ & Diminished ovarian reserve & $7 \%$ & Multiple Factors: & \\
\hline \multirow[t]{3}{*}{ Combination } & $0 \%$ & Used gestational carrier & $0 \%$ & Endometriosis & $2 \%$ & Female factors only & $3 \%$ \\
\hline & & & & Uterine factor & $0 \%$ & Female \& male factors & $55 \%$ \\
\hline & & & & Male factor & $21 \%$ & & \\
\hline
\end{tabular}

2006 PREGNANCY SUCCESS RATES

Data verified by Gail F. Whitman-Elia, MD

\section{Type of Cycle}

\section{Fresh Embryos from Nondonor Eggs}

Number of cycles

Percentage of cycles resulting in pregnancies ${ }^{b}$

Percentage of cycles resulting in live births ${ }^{b, c}$

(Confidence Interval)

Percentage of retrievals resulting in live births $s^{b, c}$

Percentage of transfers resulting in live births $s^{b, c}$

Percentage of transfers resulting in singleton live births ${ }^{b}$

Percentage of cancellations ${ }^{b}$

Average number of embryos transferred

Percentage of pregnancies with twins ${ }^{b}$

Percentage of pregnancies with triplets or more ${ }^{b}$

Percentage of live births having multiple infants ${ }^{b, c}$

\section{Frozen Embryos from Nondonor Eggs}

Number of transfers

Percentage of transfers resulting in live births ${ }^{\mathrm{b}, \mathrm{c}}$

Average number of embryos transferred

\section{Donor Eggs}

Number of transfers

Percentage of transfers resulting in live births $s^{b, c}$

Average number of embryos transferred

\section{Age of Woman}

35-37 38-40

$41-42^{d}$

$<35$

34

47.1

28

13

60.4

29.4

35.7

$4 / 13$

52.1

$(41.6-62.4)$

(15.1-47.5)

28.6

$3 / 13$

\section{1}

54.3

29.4

(13.2-48.7)

35.9

29.4

29.6

30.8

$3 / 12$

20.6

26.9

$3 / 11$

0.0

0.0

3.6

2/ 11

2.4

2.7

3.3

$1 / 13$

32.8

$3 / 16$

6.9

$1 / 16$

34.0

$3 / 10$

$1 / 10$

3.7

$1 / 4$

$0 / 10$

$0 / 4$

$1 / 8$

$1 / 3$

18

$4 / 18$

2.2
0

0

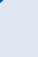

\section{6 \\ $1 / 6$}

2.3

All Ages Combined ${ }^{\mathrm{e}}$

Fresh Embryos

9

$2 / 9$

2.0
Frozen Embryos

\section{3}

$0 / 3$

2.7

\section{CURRENT CLINIC SERVICES AND PROFILE}

Current Name: Advanced Fertility \& Reproductive Endocrinology

Donor egg? Yes Gestational carriers? Yes

Donor embryo? Yes Cryopreservation? Yes

Single women? Yes

SART member? Yes

Verified lab accreditation? Yes

(See Appendix C for details.)

${ }^{a}$ Reflects patient and treatment characteristics of ART cycles performed in 2006 using fresh nondonor eggs or embryos.

${ }^{b}$ When fewer than 20 cycles are reported in an age category, rates are shown as a fraction and confidence intervals are not given. Calculating percentages from fractions may be misleading and is not encouraged.

${ }^{c}$ A multiple-infant birth is counted as one live birth.

d Clinic-specific outcome rates are unreliable for women older than 42 undergoing ART cycles using fresh or frozen embryos with nondonor eggs. Readers are urged to review national outcomes for these age groups (see page 27).

e All ages (including ages $>42$ ) are reported together because previous data show that patient age does not materially affect success with donor eggs. 


\section{SIOUX VALLEY CLINIC OB-GYN, LTD. SIOUX FALLS, SOUTH DAKOTA}

A comparison of clinic success rates may not be meaningful because patient medical characteristics and treatment approaches vary from clinic to clinic. For more details about this, along with information on how to interpret the statistics in this table, see pages 79-88.

\section{ART CYCLE PROFILE}

\begin{tabular}{|c|c|c|c|c|c|c|c|}
\hline \multicolumn{4}{|c|}{ Type of ART ${ }^{\mathrm{a}}$} & \multicolumn{4}{|c|}{ Patient Diagnosis } \\
\hline IVF & $100 \%$ & Procedural Factors: & & Tubal factor & $12 \%$ & Other factor & $4 \%$ \\
\hline GIFT & $0 \%$ & With ICSI & $62 \%$ & Ovulatory dysfunction & $8 \%$ & Unknown factor & $3 \%$ \\
\hline ZIFT & $0 \%$ & Unstimulated & $0 \%$ & Diminished ovarian reserve & $9 \%$ & Multiple Factors: & \\
\hline \multirow[t]{3}{*}{ Combination } & $0 \%$ & Used gestational carrier & $0 \%$ & Endometriosis & $7 \%$ & Female factors only & $6 \%$ \\
\hline & & & & Uterine factor & $0 \%$ & Female \& male factors & $30 \%$ \\
\hline & & & & Male factor & $22 \%$ & & \\
\hline
\end{tabular}

2006 PREGNANCY SUCCESS RATES

Data verified by Keith A. Hansen, MD

\section{Type of Cycle}

\section{Fresh Embryos from Nondonor Eggs}

Number of cycles

Percentage of cycles resulting in pregnancies ${ }^{b}$

Percentage of cycles resulting in live births ${ }^{b, c}$

(Confidence Interval)

Percentage of retrievals resulting in live births $s^{b, c}$

Percentage of transfers resulting in live births $s^{b, c}$

Percentage of transfers resulting in singleton live births ${ }^{b}$

Percentage of cancellations ${ }^{b}$

Average number of embryos transferred

Percentage of pregnancies with twins ${ }^{b}$

Percentage of pregnancies with triplets or more ${ }^{b}$

Percentage of live births having multiple infants ${ }^{b, c}$

\section{Frozen Embryos from Nondonor Eggs}

Number of transfers

Percentage of transfers resulting in live births ${ }^{b, c}$

Average number of embryos transferred

\section{Donor Eggs}

Number of transfers

Percentage of transfers resulting in live births $s^{b, c}$

Average number of embryos transferred

\section{Age of Woman}

35-37 38-40

$41-42^{d}$

\section{$<35$}

21

38.1

15

5

44.4

33.3

$5 / 15$

$0 / 5$

39.4

$(14.6-57.0)$

$(29.7-49.7)$

35.0

$3 / 15$

$0 / 5$

41.1

42.4

35.0

$3 / 13$

$0 / 4$

26.1

25.0

$3 / 13$

$0 / 4$

4.0

4.8

$3 / 13$

$0 / 4$

2.2

31.8

2.8

$2 / 15$

$1 / 5$

4.5

38.5

$4 / 8$

2.4

$0 / 5$

$0 / 8$

$0 / 5$

$2 / 7$

$0 / 3$

35

8.6

12

$3 / 12$

3

2.9

$0 / 3$

2.0

All Ages Combined ${ }^{\mathrm{e}}$

Fresh Embryos 5

$1 / 5$

1.8
Frozen Embryos

5

$0 / 5$

2.6

\section{CURRENT CLINIC SERVICES AND PROFILE}

Current Name: Sioux Valley Clinic OB-GYN, Ltd.

Donor egg? Yes Gestational carriers? Yes

Donor embryo? Yes Cryopreservation? Yes

Single women? Yes

SART member? Yes

Verified lab accreditation? Yes

(See Appendix C for details.)

${ }^{a}$ Reflects patient and treatment characteristics of ART cycles performed in 2006 using fresh nondonor eggs or embryos.

${ }^{b}$ When fewer than 20 cycles are reported in an age category, rates are shown as a fraction and confidence intervals are not given. Calculating percentages from fractions may be misleading and is not encouraged.

${ }^{c}$ A multiple-infant birth is counted as one live birth.

d Clinic-specific outcome rates are unreliable for women older than 42 undergoing ART cycles using fresh or frozen embryos with nondonor eggs. Readers are urged to review national outcomes for these age groups (see page 27).

e All ages (including ages $>42$ ) are reported together because previous data show that patient age does not materially affect success with donor eggs. 


\section{FERTILITY CENTER, LLC CHATTANOOGA, TENNESSEE}

A comparison of clinic success rates may not be meaningful because patient medical characteristics and treatment approaches vary from clinic to clinic. For more details about this, along with information on how to interpret the statistics in this table, see pages $79-88$.

\section{ART CYCLE PROFILE}

\begin{tabular}{|c|c|c|c|c|c|c|c|}
\hline & Type & of $A R T^{a}$ & & Pati & ent D & iagnosis & \\
\hline IVF & $100 \%$ & Procedural Factors: & & Tubal factor & $15 \%$ & Other factor & $7 \%$ \\
\hline GIFT & $0 \%$ & With ICSI & $76 \%$ & Ovulatory dysfunction & $4 \%$ & Unknown factor & $11 \%$ \\
\hline $\mathrm{ZIFT}$ & $0 \%$ & Unstimulated & $0 \%$ & Diminished ovarian reserve & $8 \%$ & Multiple Factors: & \\
\hline Combination & $0 \%$ & Used gestational carrier & $<1 \%$ & Endometriosis & $7 \%$ & Female factors only & $12 \%$ \\
\hline & & & & Uterine factor & $1 \%$ & Female \& male factors & $16 \%$ \\
\hline
\end{tabular}

2006 PREGNANCY SUCCESS RATES

Data verified by Barry W. Donesky, MD

\section{Type of Cycle}

\section{Fresh Embryos from Nondonor Eggs}

Number of cycles

Percentage of cycles resulting in pregnancies ${ }^{b}$

Percentage of cycles resulting in live births ${ }^{b, c}$

(Confidence Interval)

Percentage of retrievals resulting in live births $s^{b, c}$

Percentage of transfers resulting in live births ${ }^{b, c}$

Percentage of transfers resulting in singleton live births ${ }^{b}$

Percentage of cancellations ${ }^{b}$

Average number of embryos transferred

Percentage of pregnancies with twins ${ }^{b}$

Percentage of pregnancies with triplets or more ${ }^{b}$

Percentage of live births having multiple infants ${ }^{b, c}$

\section{Frozen Embryos from Nondonor Eggs}

Number of transfers

Percentage of transfers resulting in live births $s^{b, c}$

Average number of embryos transferred

\section{Donor Eggs}

Number of transfers

Percentage of transfers resulting in live births $s^{b, c}$

Average number of embryos transferred

\section{Age of Woman}

35-37 38-40

$41-42^{d}$

$<35$

33

21.2

14

7

26.2

15.2

$2 / 14$

$0 / 7$

24.6

$(14.5-37.3)$

$(5.1-31.9)$

26.8

20.8

$1 / 14$

$0 / 7$

33.3

23.8

$1 / 12$

$0 / 5$

15.6

19.0

$1 / 7$

$0 / 4$

8.2

27.3

$1 / 7$

$0 / 4$

2.0

2.3

$2 / 14$

$2 / 7$

$7 / 16$

$2 / 7$

2.1

2.5

$2 / 16$

$8 / 15$

$0 / 7$

$0 / 2$

$0 / 2$

$1 / 5$

$0 / 1$

20

45.0

2.0

\section{3}

$1 / 3$

1.7

\section{3}

$1 / 3$

2.3 . 


\section{CENTER FOR APPLIED REPRODUCTIVE SCIENCE JOHNSON CITY, TENNESSEE}

A comparison of clinic success rates may not be meaningful because patient medical characteristics and treatment approaches vary from clinic to clinic. For more details about this, along with information on how to interpret the statistics in this table, see pages 79-88.

\section{ART CYCLE PROFILE}

\begin{tabular}{|c|c|c|c|c|c|c|c|}
\hline \multicolumn{4}{|c|}{ Type of ART ${ }^{a}$} & \multicolumn{4}{|c|}{ Patient Diagnosis } \\
\hline IVF & $100 \%$ & Procedural Factors: & & Tubal factor & $12 \%$ & Other factor & $<1 \%$ \\
\hline GIFT & $0 \%$ & With ICSI & $28 \%$ & Ovulatory dysfunction & $17 \%$ & Unknown factor & $<1 \%$ \\
\hline ZIFT & $0 \%$ & Unstimulated & $0 \%$ & Diminished ovarian reserve & $4 \%$ & Multiple Factors: & \\
\hline \multirow[t]{3}{*}{ Combination } & $0 \%$ & Used gestational carrier & $0 \%$ & Endometriosis & $5 \%$ & Female factors only & $28 \%$ \\
\hline & & & & Uterine factor & $0 \%$ & Female \& male factors & $29 \%$ \\
\hline & & & & Male factor & $5 \%$ & & \\
\hline
\end{tabular}

\section{PREGNANCY SUCCESS RATES}

\section{Type of Cycle}

\section{Fresh Embryos from Nondonor Eggs}

Number of cycles

Percentage of cycles resulting in pregnancies ${ }^{b}$

Percentage of cycles resulting in live births ${ }^{b, c}$

(Confidence Interval)

Percentage of retrievals resulting in live births $s^{b, c}$

Percentage of transfers resulting in live births $s^{b, c}$

Percentage of transfers resulting in singleton live births ${ }^{b}$

Percentage of cancellations ${ }^{b}$

Average number of embryos transferred

Percentage of pregnancies with twins ${ }^{b}$

Percentage of pregnancies with triplets or more ${ }^{b}$

Percentage of live births having multiple infants ${ }^{b, c}$

\section{Frozen Embryos from Nondonor Eggs}

Number of transfers

Percentage of transfers resulting in live births $s^{b, c}$

Average number of embryos transferred

\section{Donor Eggs}

Number of transfers

Percentage of transfers resulting in live births $s^{b, c}$

Average number of embryos transferred
Data verified by Samuel S. Thatcher, MD, PhD

\section{Age of Woman}

35-37 $38-40 \quad 41-42^{d}$

\section{$<35$}

45

28.9

31

9

43.2

24.4

25.8

$1 / 9$

38.9

(12.9-39.5)

22.6

$1 / 9$

(29.1-49.5)

43.5

29.7

(9.6-41.1)

48.1

32.4

29.2

$1 / 8$

36.4

23.5

$7 / 18$

$1 / 7$

10.5

17.8

$6 / 18$

$1 / 7$

1.9

1.9

22.6

$1 / 9$

22.0

$3 / 13$

1.8

1.9

2.4

$0 / 13$

$0 / 8$

$0 / 1$

24.3

$3 / 11$

$1 / 8$

$0 / 1$

$1 / 7$

$0 / 1$

$\begin{array}{cccc}12 & 5 & 3 & 1 \\ 8 / 12 & 1 / 5 & 1 / 3 & 0 / 1 \\ 2.0 & 1.8 & 2.0 & 2.0\end{array}$

All Ages Combined

Fresh Embryos

Frozen Embryos

2

$2 / 2$

9

2.0

$2 / 9$

1.7

\section{CURRENT CLINIC SERVICES AND PROFILE}

Current Name: Center for Applied Reproductive Science

Donor egg? Yes Gestational carriers? No

Donor embryo? Yes Cryopreservation? Yes

Single women? Yes

SART member?

No

Verified lab accreditation?

No

(See Appendix C for details.)

${ }^{a}$ Reflects patient and treatment characteristics of ART cycles performed in 2006 using fresh nondonor eggs or embryos.

${ }^{b}$ When fewer than 20 cycles are reported in an age category, rates are shown as a fraction and confidence intervals are not given. Calculating percentages from fractions may be misleading and is not encouraged.

${ }^{c}$ A multiple-infant birth is counted as one live birth.

d Clinic-specific outcome rates are unreliable for women older than 42 undergoing ART cycles using fresh or frozen embryos with nondonor eggs. Readers are urged to review national outcomes for these age groups (see page 27).

e All ages (including ages $>42$ ) are reported together because previous data show that patient age does not materially affect success with donor eggs. 


\section{EAST TENNESSEE IVF, FERTILITY, AND ANDROLOGY CENTER KNOXVILLE, TENNESSEE}

A comparison of clinic success rates may not be meaningful because patient medical characteristics and treatment approaches vary from clinic to clinic. For more details about this, along with information on how to interpret the statistics in this table, see pages $79-88$.

\section{ART CYCLE PROFILE}

\begin{tabular}{|c|c|c|c|c|c|c|c|}
\hline \multicolumn{4}{|c|}{ Type of ART ${ }^{\mathrm{a}}$} & \multicolumn{4}{|c|}{ Patient Diagnosis } \\
\hline IVF & $100 \%$ & Procedural Factors: & & Tubal factor & $12 \%$ & Other factor & $0 \%$ \\
\hline GIFT & $0 \%$ & With ICSI & $30 \%$ & Ovulatory dysfunction & $8 \%$ & Unknown factor & $0 \%$ \\
\hline ZIFT & $0 \%$ & Unstimulated & $0 \%$ & Diminished ovarian reserve & $4 \%$ & Multiple Factors: & \\
\hline \multirow[t]{3}{*}{ Combination } & $0 \%$ & Used gestational carrier & $0 \%$ & Endometriosis & $19 \%$ & Female factors only & $0 \%$ \\
\hline & & & & Uterine factor & $8 \%$ & Female \& male factors & $35 \%$ \\
\hline & & & & Male factor & $15 \%$ & & \\
\hline
\end{tabular}

2006 PREGNANCY SUCCESS RATES

Data verified by Gayla S. Harris, MD

\section{Type of Cycle}

\section{Fresh Embryos from Nondonor Eggs}

Number of cycles

Percentage of cycles resulting in pregnancies ${ }^{b}$

Percentage of cycles resulting in live births $s^{b, c}$

(Confidence Interval)

Percentage of retrievals resulting in live births $s^{b, c}$

Percentage of transfers resulting in live births $s^{b, c}$

Percentage of transfers resulting in singleton live births ${ }^{b}$

Percentage of cancellations ${ }^{b}$

Average number of embryos transferred

Percentage of pregnancies with twins ${ }^{b}$

Percentage of pregnancies with triplets or more ${ }^{b}$

Percentage of live births having multiple infants ${ }^{b, c}$

\section{Frozen Embryos from Nondonor Eggs}

Number of transfers

Percentage of transfers resulting in live births $s^{\mathrm{b}, \mathrm{c}}$

Average number of embryos transferred

\section{Donor Eggs}

Number of transfers

Percentage of transfers resulting in live births $s^{\mathrm{b}, \mathrm{c}}$

Average number of embryos transferred

\section{Age of Woman}

35-37 38-40

$41-42^{d}$

$<35$

1

$0 / 1$

$0 / 1$

5

2

$7 / 15$

$7 / 15$

$2 / 5$

$2 / 5$

$0 / 2$

$7 / 15$

$0 / 1$

$2 / 5$

$0 / 2$

$7 / 15$

$0 / 1$

$2 / 5$

$0 / 2$

$5 / 15$

$0 / 1$

$2 / 5$

$0 / 2$

$0 / 15$

$0 / 1$

$0 / 5$

$0 / 2$

2.2

3.0

2.4

$0 / 2$

$2 / 7$

$0 / 2$

$0 / 7$

$0 / 2$

$2 / 7$

$0 / 2$

0

0

0

\section{CURRENT CLINIC SERVICES AND PROFILE}

Current Name: East Tennessee IVF, Fertility, and Andrology Center
Donor egg?
Gestational carriers?
Yes
Cryopreservation?
Yes
Donor embryo? Yes
Single women? No

$\begin{array}{ll}\text { SART member? } & \text { Yes } \\ \text { Verified lab accreditation? } & \text { Pending } \\ \text { (See Appendix C for details.) } & \end{array}$

All Ages Combined ${ }^{\mathrm{e}}$

$2 / 3$

Frozen Embryos

2.0

${ }^{a}$ Reflects patient and treatment characteristics of ART cycles performed in 2006 using fresh nondonor eggs or embryos.

${ }^{b}$ When fewer than 20 cycles are reported in an age category, rates are shown as a fraction and confidence intervals are not given. Calculating percentages from fractions may be misleading and is not encouraged.

${ }^{c}$ A multiple-infant birth is counted as one live birth.

${ }^{d}$ Clinic-specific outcome rates are unreliable for women older than 42 undergoing ART cycles using fresh or frozen embryos with nondonor eggs. Readers are urged to review national outcomes for these age groups (see page 27).

${ }^{e}$ All ages (including ages $>42$ ) are reported together because previous data show that patient age does not materially affect success with donor eggs. 


\section{SOUTHEASTERN FERTILITY CENTER KNOXVILLE, TENNESSEE}

A comparison of clinic success rates may not be meaningful because patient medical characteristics and treatment approaches vary from clinic to clinic. For more details about this, along with information on how to interpret the statistics in this table, see pages 79-88.

\section{ART CYCLE PROFILE}

\begin{tabular}{|c|c|c|c|c|c|c|c|}
\hline \multicolumn{4}{|c|}{ Type of ART ${ }^{a}$} & \multicolumn{4}{|c|}{ Patient Diagnosis } \\
\hline IVF & $100 \%$ & Procedural Factors: & & Tubal factor & $4 \%$ & Other factor & $3 \%$ \\
\hline GIFT & $0 \%$ & With ICSI & $50 \%$ & Ovulatory dysfunction & $0 \%$ & Unknown factor & $5 \%$ \\
\hline ZIFT & $0 \%$ & Unstimulated & $0 \%$ & Diminished ovarian reserve & $4 \%$ & Multiple Factors: & \\
\hline \multirow[t]{3}{*}{ Combination } & $0 \%$ & Used gestational carrier & $0 \%$ & Endometriosis & $4 \%$ & Female factors only & $12 \%$ \\
\hline & & & & Uterine factor & $0 \%$ & Female \& male factors & $24 \%$ \\
\hline & & & & Male factor & $44 \%$ & & \\
\hline
\end{tabular}

2006 PREGNANCY SUCCESS RATES

Data verified by Jeffrey A. Keenan, MD

\section{Type of Cycle}

\section{Fresh Embryos from Nondonor Eggs}

Number of cycles

Percentage of cycles resulting in pregnancies ${ }^{b}$

Percentage of cycles resulting in live births ${ }^{b, c}$

(Confidence Interval)
Percentage of retrievals resulting in live births $s^{b, c}$

Percentage of transfers resulting in live births $s^{b, c}$

Percentage of transfers resulting in singleton live births ${ }^{b}$

Percentage of cancellations ${ }^{b}$

Average number of embryos transferred

Percentage of pregnancies with twins ${ }^{b}$

Percentage of pregnancies with triplets or more ${ }^{b}$

Percentage of live births having multiple infants ${ }^{b, c}$

\section{Frozen Embryos from Nondonor Eggs}

Number of transfers

Percentage of transfers resulting in live births $s^{b, c}$

Average number of embryos transferred

$\begin{array}{cccc}<35 & \mathbf{3 5 - 3 7} & \mathbf{3 8 - 4 0} & \mathbf{4 1 - 4 2}^{\text {d }} \\ 14 & 3 & 5 & 0 \\ 8 / 14 & 2 / 3 & 1 / 5 & \\ 8 / 14 & 2 / 3 & 1 / 5 & \end{array}$

$\begin{array}{ccc}8 / 14 & 2 / 3 & 1 / 5 \\ 8 / 13 & 2 / 3 & 1 / 4 \\ 6 / 13 & 1 / 3 & 1 / 4 \\ 0 / 14 & 0 / 3 & 0 / 5 \\ 2.5 & 2.0 & 3.8 \\ 3 / 8 & 1 / 2 & 0 / 1 \\ 0 / 8 & 0 / 2 & 0 / 1 \\ 2 / 8 & 1 / 2 & 0 / 1\end{array}$

5

$1 / 5$

2.6
1

$0 / 1$

3.0
$1 / 5$
$1 / 4$
$1 / 4$
$0 / 5$
3.8
$0 / 1$
$0 / 1$
$0 / 1$

2

$1 / 2$

3.5

\section{Donor Eggs}

Fresh Embryos

All Ages Combined ${ }^{\mathrm{e}}$

Number of transfers

Percentage of transfers resulting in live births $s^{b, c}$

Average number of embryos transferred

\section{1}

$1 / 1$

2.0
Frozen Embryos

39

48.7

2.8

\section{CURRENT CLINIC SERVICES AND PROFILE}

Current Name: Southeastern Fertility Center
Donor egg?
Yes
Gestational carriers?
No
Cryopreservation?
Yes
Single women? No
SART member?
Verified lab accreditation?
(See Appendix C for details.)
${ }^{a}$ Reflects patient and treatment characteristics of ART cycles performed in 2006 using fresh nondonor eggs or embryos.
${ }^{b}$ When fewer than 20 cycles are reported in an age category, rates are shown as a fraction and confidence intervals are not given. Calculating percentages from fractions may be misleading and is not encouraged.
c A multiple-infant birth is counted as one live birth.
d Clinic-specific outcome rates are unreliable for women older than 42 undergoing ART cycles using fresh or frozen embryos with nondonor eggs. Readers are urged to review national outcomes for these age groups (see page 27).
e All ages (including ages $>42$ ) are reported together because previous data show that patient age does not materially affect success with donor eggs. 


\section{KUTTEH KE FERTILITY ASSOCIATES OF MEMPHIS, PLLC MEMPHIS, TENNESSEE}

A comparison of clinic success rates may not be meaningful because patient medical characteristics and treatment approaches vary from clinic to clinic. For more details about this, along with information on how to interpret the statistics in this table, see pages $79-88$.

\section{ART CYCLE PROFILE}

\begin{tabular}{|c|c|c|c|c|c|c|c|}
\hline \multicolumn{4}{|c|}{ Type of ART ${ }^{a}$} & \multicolumn{4}{|c|}{ Patient Diagnosis } \\
\hline IVF & $100 \%$ & Procedural Factors: & & Tubal factor & $7 \%$ & Other factor & $1 \%$ \\
\hline GIFT & $0 \%$ & With ICSI & $48 \%$ & Ovulatory dysfunction & $8 \%$ & Unknown factor & $4 \%$ \\
\hline ZIFT & $0 \%$ & Unstimulated & $0 \%$ & Diminished ovarian reserve & $8 \%$ & Multiple Factors: & \\
\hline \multirow[t]{3}{*}{ Combination } & $0 \%$ & Used gestational carrier & $0 \%$ & Endometriosis & $8 \%$ & Female factors only & $24 \%$ \\
\hline & & & & Uterine factor & $<1 \%$ & Female \& male factors & $30 \%$ \\
\hline & & & & Male factor & $10 \%$ & & \\
\hline
\end{tabular}

2006 PREGNANCY SUCCESS RATES

Data verified by Raymond W. Ke, MD

\section{Type of Cycle}

\section{Fresh Embryos from Nondonor Eggs}

Number of cycles

Percentage of cycles resulting in pregnancies ${ }^{b}$

Percentage of cycles resulting in live births $s^{b, c}$

(Confidence Interval)

Percentage of retrievals resulting in live births $s^{b, c}$

Percentage of transfers resulting in live births ${ }^{b, c}$

Percentage of transfers resulting in singleton live births ${ }^{b}$

Percentage of cancellations ${ }^{b}$

Average number of embryos transferred

Percentage of pregnancies with twins ${ }^{b}$

Percentage of pregnancies with triplets or more ${ }^{b}$

Percentage of live births having multiple infants ${ }^{b, c}$

\section{Frozen Embryos from Nondonor Eggs}

Number of transfers

Percentage of transfers resulting in live births ${ }^{\mathrm{b}, \mathrm{c}}$

Average number of embryos transferred

\section{Donor Eggs}

Number of transfers

Percentage of transfers resulting in live births $s^{b, c}$

Average number of embryos transferred

\section{Age of Woman}

35-37 38-40

$41-42^{d}$

\section{$<35$}

42

33.3

28

14

50.4

31.0

25.0

$1 / 14$

45.1

(17.6-47.1)

25.0

$1 / 14$

(36.5-54.0)

48.0

35.1

$(10.7-44.9)$

50.0

36.1

26.9

$1 / 10$

30.4

$1 / 9$

27.8

26.1

$1 / 9$

6.0

11.9

7.1

$4 / 14$

2.2

2.5

2.6

2.3

37.3

$4 / 14$

$1 / 7$

$0 / 1$

4.5

$1 / 14$

$0 / 7$

$0 / 1$

38.3

$3 / 13$

$1 / 7$

$0 / 1$

12

$10 / 12$

2.0

\section{6}

$3 / 6$

1.3
5
$2 / 5$
2.4

1

$1 / 1$

2.0

All Ages Combined ${ }^{\mathrm{e}}$

Fresh Embryos

10

$5 / 10$

2.1
Frozen Embryos

2

$0 / 2$

2.5

\section{CURRENT CLINIC SERVICES AND PROFILE}

Current Name: Kutteh Ke Fertility Associates of Memphis, PLLC

\begin{tabular}{l|l|l} 
Donor egg? Yes & Gestational carriers? No
\end{tabular}

Donor embryo? Yes Cryopreservation? Yes

Single women? No

SART member? Yes

Verified lab accreditation? Yes

(See Appendix C for details.)

${ }^{a}$ Reflects patient and treatment characteristics of ART cycles performed in 2006 using fresh nondonor eggs or embryos.

${ }^{b}$ When fewer than 20 cycles are reported in an age category, rates are shown as a fraction and confidence intervals are not given. Calculating percentages from fractions may be misleading and is not encouraged.

${ }^{c}$ A multiple-infant birth is counted as one live birth.

d Clinic-specific outcome rates are unreliable for women older than 42 undergoing ART cycles using fresh or frozen embryos with nondonor eggs. Readers are urged to review national outcomes for these age groups (see page 27).

${ }^{e}$ All ages (including ages $>42$ ) are reported together because previous data show that patient age does not materially affect success with donor eggs. 


\section{THE CENTER FOR REPRODUCTIVE HEALTH \\ NASHVILLE, TENNESSEE}

A comparison of clinic success rates may not be meaningful because patient medical characteristics and treatment approaches vary from clinic to clinic. For more details about this, along with information on how to interpret the statistics in this table, see pages 79-88.

\section{ART CYCLE PROFILE}

\begin{tabular}{|c|c|c|c|c|c|c|c|}
\hline \multicolumn{4}{|c|}{ Type of ART ${ }^{a}$} & \multicolumn{4}{|c|}{ Patient Diagnosis } \\
\hline IVF & $100 \%$ & Procedural Factors: & & Tubal factor & $2 \%$ & Other factor & $3 \%$ \\
\hline GIFT & $0 \%$ & With ICSI & $74 \%$ & Ovulatory dysfunction & $12 \%$ & Unknown factor & $0 \%$ \\
\hline ZIFT & $0 \%$ & Unstimulated & $0 \%$ & Diminished ovarian reserve & $4 \%$ & Multiple Factors: & \\
\hline \multirow[t]{3}{*}{ Combination } & $0 \%$ & Used gestational carrier & $0 \%$ & Endometriosis & $3 \%$ & Female factors only & $14 \%$ \\
\hline & & & & Uterine factor & $0 \%$ & Female \& male factors & $53 \%$ \\
\hline & & & & Male factor & $11 \%$ & & \\
\hline
\end{tabular}

2006 PREGNANCY SUCCESS RATES

Data verified by Jaime M. Vasquez, MD

\section{Type of Cycle}

\section{Fresh Embryos from Nondonor Eggs}

Number of cycles

Percentage of cycles resulting in pregnancies ${ }^{b}$

Percentage of cycles resulting in live births $s^{b, c}$

(Confidence Interval)

Percentage of retrievals resulting in live births $s^{b, c}$

Percentage of transfers resulting in live births $s^{b, c}$

Percentage of transfers resulting in singleton live births ${ }^{b}$

Percentage of cancellations ${ }^{b}$

Average number of embryos transferred

Percentage of pregnancies with twins ${ }^{b}$

Percentage of pregnancies with triplets or more ${ }^{b}$

Percentage of live births having multiple infants ${ }^{b, c}$

\section{Frozen Embryos from Nondonor Eggs}

Number of transfers

Percentage of transfers resulting in live births ${ }^{b, c}$

Average number of embryos transferred

\section{Donor Eggs}

Number of transfers

Percentage of transfers resulting in live births $s^{b, c}$

Average number of embryos transferred

\section{Age of Woman}

35-37 38-40

$41-42^{d}$

$<35$

17

$9 / 17$

6

0

\section{0}

40.5

$8 / 17$

$2 / 6$

$(25.6-56.7)$

42.5

43.6

$8 / 14$

$2 / 6$

25.6

4.8

3.2

19.0

28.6

$7 / 17$

$8 / 14$

$2 / 6$

$5 / 14$

$2 / 5$

$2 / 5$

$3 / 17$

$0 / 6$

3.5

$1 / 9$

3.4

$0 / 2$

$2 / 9$

$0 / 2$

$3 / 8$

$0 / 2$

9

$1 / 9$

3.2
0

\section{Fresh Embryos}

All Ages Combined ${ }^{\mathrm{e}}$
$10 / 16$

3.5
Frozen Embryos

6

$1 / 6$

3.5

\section{CURRENT CLINIC SERVICES AND PROFILE}

Current Name: The Center for Reproductive Health

Donor egg? Yes

Donor embryo? Yes

Single women? Yes

\section{Gestational carriers? Yes}

Cryopreservation? Yes
SART member?

Yes

Verified lab accreditation?

(See Appendix C for details.)

${ }^{a}$ Reflects patient and treatment characteristics of ART cycles performed in 2006 using fresh nondonor eggs or embryos.

${ }^{b}$ When fewer than 20 cycles are reported in an age category, rates are shown as a fraction and confidence intervals are not given. Calculating percentages from fractions may be misleading and is not encouraged.

${ }^{c}$ A multiple-infant birth is counted as one live birth.

d Clinic-specific outcome rates are unreliable for women older than 42 undergoing ART cycles using fresh or frozen embryos with nondonor eggs. Readers are urged to review national outcomes for these age groups (see page 27).

e All ages (including ages $>42$ ) are reported together because previous data show that patient age does not materially affect success with donor eggs. 


\section{NASHVILLE FERTILITY CENTER \\ NASHVILLE, TENNESSEE}

A comparison of clinic success rates may not be meaningful because patient medical characteristics and treatment approaches vary from clinic to clinic. For more details about this, along with information on how to interpret the statistics in this table, see pages $79-88$.

\section{ART CYCLE PROFILE}

\begin{tabular}{|c|c|c|c|c|c|c|c|}
\hline \multicolumn{4}{|c|}{ Type of ART } & \multicolumn{4}{|c|}{ Patient Diagnosis } \\
\hline IVF & $100 \%$ & Procedural Factors: & & Tubal factor & $7 \%$ & Other factor & $2 \%$ \\
\hline GIFT & $0 \%$ & With ICSI & $66 \%$ & Ovulatory dysfunction & $4 \%$ & Unknown factor & $4 \%$ \\
\hline ZIFT & $0 \%$ & Unstimulated & $0 \%$ & Diminished ovarian reserve & $8 \%$ & Multiple Factors: & \\
\hline \multirow[t]{3}{*}{ Combination } & $0 \%$ & Used gestational carrier & $0 \%$ & Endometriosis & $6 \%$ & Female factors only & $31 \%$ \\
\hline & & & & Uterine factor & $<1 \%$ & Female \& male factors & $25 \%$ \\
\hline & & & & Male factor & $14 \%$ & & \\
\hline
\end{tabular}

2006 PREGNANCY SUCCESS RATES

Data verified by George A. Hill, MD

\section{Type of Cycle}

\section{Fresh Embryos from Nondonor Eggs}

Number of cycles

Percentage of cycles resulting in pregnancies ${ }^{b}$

Percentage of cycles resulting in live births ${ }^{b, c}$

(Confidence Interval)

Percentage of retrievals resulting in live births $s^{b, c}$

Percentage of transfers resulting in live births $s^{b, c}$

Percentage of transfers resulting in singleton live births ${ }^{b}$

Percentage of cancellations ${ }^{b}$

Average number of embryos transferred

Percentage of pregnancies with twins ${ }^{b}$

Percentage of pregnancies with triplets or more ${ }^{b}$

Percentage of live births having multiple infants ${ }^{b, c}$

\section{Frozen Embryos from Nondonor Eggs}

Number of transfers

Percentage of transfers resulting in live births ${ }^{\mathrm{b}, \mathrm{c}}$

Average number of embryos transferred

\section{Donor Eggs}

Number of transfers

Percentage of transfers resulting in live births $s^{b, c}$

Average number of embryos transferred

\section{Age of Woman}

35-37 38-40

$41-42^{d}$

$<35$

72

34.7

58

17

46.0

31.9

24.1

$0 / 17$

38.9

(21.4-44.0)

17.2

$0 / 17$

(32.1-46.1)

43.5

41.8

(8.6-29.4)

46.1

45.1

24.4

$0 / 12$

26.3

29.4

25.6

$0 / 10$

10.6

23.6

20.5

$0 / 10$

2.2

2.4

29.3

$5 / 17$

36.3

32.0

3.0

3.5

5.5

42.9

4.0

$5 / 14$

34.8

$1 / 14$

$2 / 10$

71

32.4

33

39.4

9

$1 / 9$

2

2.6

2.3

2.7

$0 / 2$

All Ages Combined ${ }^{\mathrm{e}}$

Fresh Embryos

Frozen Embryos

67.9

41

2.3

41.5

2.6

\section{CURRENT CLINIC SERVICES AND PROFILE}

Current Name: Nashville Fertility Center

Donor egg? Yes

Donor embryo? Yes

Gestational carriers? Yes

Cryopreservation? Yes

Single women? Yes

SART member?

Yes

Verified lab accreditation?

(See Appendix C for details.)

${ }^{a}$ Reflects patient and treatment characteristics of ART cycles performed in 2006 using fresh nondonor eggs or embryos.

${ }^{b}$ When fewer than 20 cycles are reported in an age category, rates are shown as a fraction and confidence intervals are not given. Calculating percentages from fractions may be misleading and is not encouraged.

${ }^{c}$ A multiple-infant birth is counted as one live birth.

${ }^{d}$ Clinic-specific outcome rates are unreliable for women older than 42 undergoing ART cycles using fresh or frozen embryos with nondonor eggs. Readers are urged to review national outcomes for these age groups (see page 27).

${ }^{e}$ All ages (including ages $>42$ ) are reported together because previous data show that patient age does not materially affect success with donor eggs. 


\section{TEXAS FERTILITY CENTER \\ DRS. VAUGHN, SILVERBERG AND HANSARD \\ AUSTIN, TEXAS}

A comparison of clinic success rates may not be meaningful because patient medical characteristics and treatment approaches vary from clinic to clinic. For more details about this, along with information on how to interpret the statistics in this table, see pages 79-88.

\section{ART CYCLE PROFILE}

\begin{tabular}{|c|c|c|c|c|c|c|c|}
\hline \multicolumn{4}{|c|}{ Type of ART ${ }^{a}$} & \multicolumn{4}{|c|}{ Patient Diagnosis } \\
\hline IVF & $100 \%$ & Procedural Factors: & & Tubal factor & $11 \%$ & Other factor & $10 \%$ \\
\hline GIFT & $0 \%$ & With ICSI & $36 \%$ & Ovulatory dysfunction & $5 \%$ & Unknown factor & $6 \%$ \\
\hline ZIFT & $0 \%$ & Unstimulated & $0 \%$ & Diminished ovarian reserve & $12 \%$ & Multiple Factors: & \\
\hline \multirow[t]{3}{*}{ Combination } & $0 \%$ & Used gestational carrier & $<1 \%$ & Endometriosis & $15 \%$ & Female factors only & $17 \%$ \\
\hline & & & & Uterine factor & $<1 \%$ & Female \& male factors & $12 \%$ \\
\hline & & & & Male factor & $12 \%$ & & \\
\hline
\end{tabular}

2006 PREGNANCY SUCCESS RATES

Data verified by Kaylen Silverberg, MD

\section{Type of Cycle}

\section{Fresh Embryos from Nondonor Eggs}

Number of cycles

Percentage of cycles resulting in pregnancies ${ }^{b}$

Percentage of cycles resulting in live births $s^{b, c}$

(Confidence Interval)

Percentage of retrievals resulting in live births $s^{b, c}$

Percentage of transfers resulting in live births $s^{b, c}$

Percentage of transfers resulting in singleton live births ${ }^{b}$

Percentage of cancellations ${ }^{b}$

Average number of embryos transferred

Percentage of pregnancies with twins ${ }^{b}$

Percentage of pregnancies with triplets or more ${ }^{b}$

Percentage of live births having multiple infants ${ }^{\mathrm{b}, \mathrm{c}}$

\section{Frozen Embryos from Nondonor Eggs}

Number of transfers

Percentage of transfers resulting in live births ${ }^{b, c}$

Average number of embryos transferred

\section{Donor Eggs}

Number of transfers

Percentage of transfers resulting in live births $s^{b, c}$

Average number of embryos transferred

\section{Age of Woman}

35-37 38-40

$<35$

126

87

29.9

19.5

31.7

(11.8-29.4)

25.0

26.2

20.0

21.8

2.9

19.2

0.0

$4 / 17$

40.0

43.0

41.7

6.3

40.0

55

36.4

1.8
43

16.3

1.8
16

$7 / 16$

2.0 $41-42^{d}$

34

29.4

8.8

(1.9-23.7)

10.7

11.5

11.5

17.6

3.1

$1 / 10$

$0 / 10$

$0 / 3$

\section{All Ages Combined ${ }^{\mathrm{e}}$}

Fresh Embryos 49

67.3

2.1
Frozen Embryos 15

$2 / 15$

1.9

\section{CURRENT CLINIC SERVICES AND PROFILE}

Current Name: Texas Fertility Center, Drs. Vaughn, Silverberg and Hansard

Donor egg? Yes

Donor embryo? No

Single women? Yes

\begin{abstract}
Gestational carriers? Yes
Cryopreservation? Yes
\end{abstract}

SART member?

Verified lab accreditation?

(See Appendix C for details.)
5

$2 / 5$

1.8

\footnotetext{
${ }^{a}$ Reflects patient and treatment characteristics of ART cycles performed in 2006 using fresh nondonor eggs or embryos.

${ }^{b}$ When fewer than 20 cycles are reported in an age category, rates are shown as a fraction and confidence intervals are not given. Calculating percentages from fractions may be misleading and is not encouraged.

${ }^{c}$ A multiple-infant birth is counted as one live birth.

d Clinic-specific outcome rates are unreliable for women older than 42 undergoing ART cycles using fresh or frozen embryos with nondonor eggs. Readers are urged to review national outcomes for these age groups (see page 27).

e All ages (including ages $>42$ ) are reported together because previous data show that patient age does not materially affect success with donor eggs.
} 


\section{JEFFREY T. YOUNGKIN, MD \\ AUSTIN FERTILITY CENTER \\ AUSTIN, TEXAS}

A comparison of clinic success rates may not be meaningful because patient medical characteristics and treatment approaches vary from clinic to clinic. For more details about this, along with information on how to interpret the statistics in this table, see pages $79-88$.

\section{ART CYCLE PROFILE}

\begin{tabular}{|c|c|c|c|c|c|c|c|}
\hline & Type & of $A R T^{a}$ & & Pat & ent $D$ & iagnosis & \\
\hline IVF & $100 \%$ & Procedural Factors: & & Tubal factor & $18 \%$ & Other factor & $18 \%$ \\
\hline GIFT & $0 \%$ & With ICSI & $43 \%$ & Ovulatory dysfunction & $18 \%$ & Unknown factor & $6 \%$ \\
\hline ZIFT & $0 \%$ & Unstimulated & $0 \%$ & Diminished ovarian reserve & $0 \%$ & Multiple Factors: & \\
\hline Combination & $0 \%$ & Used gestational carrier & $0 \%$ & Endometriosis & $6 \%$ & Female factors only & $24 \%$ \\
\hline & & & & Uterine factor & $0 \%$ & Female \& male factors & $6 \%$ \\
\hline
\end{tabular}

2006 PREGNANCY SUCCESS RATES

Data verified by Jeffrey T. Youngkin, MD

\section{Type of Cycle}

\section{Fresh Embryos from Nondonor Eggs}

Number of cycles

Percentage of cycles resulting in pregnancies ${ }^{b}$

Percentage of cycles resulting in live births ${ }^{b, c}$

(Confidence Interval)

Percentage of retrievals resulting in live births $s^{b, c}$

Percentage of transfers resulting in live births $s^{b, c}$

Percentage of transfers resulting in singleton live births ${ }^{b}$

Percentage of cancellations ${ }^{b}$

Average number of embryos transferred

Percentage of pregnancies with twins ${ }^{b}$

Percentage of pregnancies with triplets or more ${ }^{b}$

Percentage of live births having multiple infants ${ }^{b, c}$

\section{Frozen Embryos from Nondonor Eggs}

Number of transfers

Percentage of transfers resulting in live births $s^{b, c}$

Average number of embryos transferred

$\begin{array}{cccc}<35 & \mathbf{3 5 - 3 7} & \mathbf{3 8 - 4 0} & \mathbf{4 1 - 4 2}^{\text {d }} \\ 7 & 2 & 4 & 1 \\ 5 / 7 & 1 / 2 & 2 / 4 & 0 / 1 \\ 3 / 7 & 1 / 2 & 2 / 4 & 0 / 1\end{array}$

\section{Age of Woman}

$\begin{array}{llll}3 / 7 & 1 / 2 & 2 / 4 & 0 / 1\end{array}$

$\begin{array}{llll}3 / 7 & 1 / 2 & 2 / 4 & 0 / 1\end{array}$

$\begin{array}{llll}3 / 7 & 1 / 2 & 2 / 4 & 0 / 1\end{array}$

$\begin{array}{llll}0 / 7 & 0 / 2 & 0 / 4 & 0 / 1\end{array}$

$\begin{array}{llll}2.1 & 1.5 & 3.8 & 4.0\end{array}$

$1 / 5 \quad 0 / 1 \quad 1 / 2$

$0 / 5 \quad 0 / 1 \quad 0 / 2$

$0 / 3 \quad 0 / 1 \quad 0 / 2$

$0 / 1 \quad 1 / 2$

$2.0 \quad 2.0$

\section{Donor Eggs}

All Ages Combined

Number of transfers

Fresh Embryos

0
Frozen Embryos

0

Percentage of transfers resulting in live births $s^{b, c}$

Average number of embryos transferred

\section{CURRENT CLINIC SERVICES AND PROFILE}

Current Name: Jeffrey T. Youngkin, MD, Austin Fertility Center

Donor egg? No Gestational carriers? No

Donor embryo? No Cryopreservation? Yes

Single women? No

SART member? Yes

Verified lab accreditation? Yes

(See Appendix C for details.)

${ }^{a}$ Reflects patient and treatment characteristics of ART cycles performed in 2006 using fresh nondonor eggs or embryos.

${ }^{b}$ When fewer than 20 cycles are reported in an age category, rates are shown as a fraction and confidence intervals are not given. Calculating percentages from fractions may be misleading and is not encouraged.

A multiple-infant birth is counted as one live birth.

d Clinic-specific outcome rates are unreliable for women older than 42 undergoing ART cycles using fresh or frozen embryos with nondonor eggs. Readers are urged to review national outcomes for these age groups (see page 27).

e All ages (including ages $>42$ ) are reported together because previous data show that patient age does not materially affect success with donor eggs. 


\section{CENTER FOR ASSISTED REPRODUCTION \\ BEDFORD, TEXAS}

A comparison of clinic success rates may not be meaningful because patient medical characteristics and treatment approaches vary from clinic to clinic. For more details about this, along with information on how to interpret the statistics in this table, see pages 79-88.

\section{ART CYCLE PROFILE}

\begin{tabular}{|c|c|c|c|c|c|c|}
\hline \multicolumn{3}{|c|}{ Type of ART ${ }^{a}$} & \multicolumn{4}{|c|}{ Patient Diagnosis } \\
\hline IVF & $100 \%$ Procedural Factors: & & Tubal factor & $10 \%$ & Other factor & $14 \%$ \\
\hline GIFT & $0 \%$ With ICSI & $64 \%$ & Ovulatory dysfunction & $7 \%$ & Unknown factor & $10 \%$ \\
\hline ZIFT & $0 \%$ Unstimulated & $<1 \%$ & Diminished ovarian reserve & $3 \%$ & Multiple Factors: & \\
\hline \multirow[t]{3}{*}{ Combination } & $0 \%$ Used gestational carrier & $3 \%$ & Endometriosis & $3 \%$ & Female factors only & $8 \%$ \\
\hline & & & Uterine factor & $3 \%$ & Female \& male factors & $22 \%$ \\
\hline & & & Male factor & $20 \%$ & & \\
\hline
\end{tabular}

2006 PREGNANCY SUCCESS RATES

Data verified by Kevin J. Doody, MD

\section{Type of Cycle}

\section{Fresh Embryos from Nondonor Eggs}

Number of cycles

Percentage of cycles resulting in pregnancies ${ }^{b}$

Percentage of cycles resulting in live births $s^{b, c}$

(Confidence Interval)

Percentage of retrievals resulting in live births $s^{b, c}$

Percentage of transfers resulting in live births $s^{b, c}$

Percentage of transfers resulting in singleton live births ${ }^{b}$

Percentage of cancellations ${ }^{b}$

Average number of embryos transferred

Percentage of pregnancies with twins ${ }^{b}$

Percentage of pregnancies with triplets or more ${ }^{b}$

Percentage of live births having multiple infants ${ }^{b, c}$

\section{Frozen Embryos from Nondonor Eggs}

Number of transfers

Percentage of transfers resulting in live births ${ }^{b, c}$

Average number of embryos transferred

\section{Donor Eggs}

Number of transfers

Percentage of transfers resulting in live births $s^{b, c}$

Average number of embryos transferred

\section{Age of Woman}

$$
\text { 35-37 38-40 } \quad 41-42^{d}
$$

\section{$<35$}

81

44

23

41.1

37.0

36.4

13.0

33.7

29.6

31.8

8.7

(27.2-40.6)

(20.0-40.8)

(18.6-47.6)

(1.1-28.0)

34.7

30.8

34.1

8.7

36.2

33.8

40.0

$2 / 14$

22.3

29.6

37.1

$2 / 14$

3.0

3.7

1.9

10.0

6.8

0.0

1.9

0.0

2.0

2.1

2.4

38.2

12.5

$2 / 16$

$0 / 3$

$0 / 16$

$0 / 3$

$1 / 14$

$0 / 2$

102

40.2

38

36.8

20

9

2.0

2.0

20.0

$3 / 9$

2.2

All Ages Combined

Fresh Embryos 38

52.6

1.9
Frozen Embryos

49

30.6

1.9

\section{CURRENT CLINIC SERVICES AND PROFILE}

Current Name: Center for Assisted Reproduction

Donor egg? Yes Gestational carriers? Yes

Donor embryo? Yes Cryopreservation? Yes

Single women? Yes

SART member? Yes

Verified lab accreditation?

(See Appendix C for details.)

${ }^{a}$ Reflects patient and treatment characteristics of ART cycles performed in 2006 using fresh nondonor eggs or embryos.

${ }^{b}$ When fewer than 20 cycles are reported in an age category, rates are shown as a fraction and confidence intervals are not given. Calculating percentages from fractions may be misleading and is not encouraged.

${ }^{c}$ A multiple-infant birth is counted as one live birth.

d Clinic-specific outcome rates are unreliable for women older than 42 undergoing ART cycles using fresh or frozen embryos with nondonor eggs. Readers are urged to review national outcomes for these age groups (see page 27).

e All ages (including ages $>42$ ) are reported together because previous data show that patient age does not materially affect success with donor eggs. 


\section{TRINITY INVITRO FERTILIZATION PROGRAM \\ CARROLLTON, TEXAS}

A comparison of clinic success rates may not be meaningful because patient medical characteristics and treatment approaches vary from clinic to clinic. For more details about this, along with information on how to interpret the statistics in this table, see pages $79-88$.

\section{ART CYCLE PROFILE}

\begin{tabular}{|c|c|c|c|c|c|c|c|}
\hline \multicolumn{4}{|c|}{ Type of ART ${ }^{\mathrm{a}}$} & \multicolumn{4}{|c|}{ Patient Diagnosis } \\
\hline IVF & $100 \%$ & Procedural Factors: & & Tubal factor & $0 \%$ & Other factor & $19 \%$ \\
\hline GIFT & $0 \%$ & With ICSI & $33 \%$ & Ovulatory dysfunction & $0 \%$ & Unknown factor & $0 \%$ \\
\hline ZIFT & $0 \%$ & Unstimulated & $0 \%$ & Diminished ovarian reserve & $6 \%$ & Multiple Factors: & \\
\hline \multirow[t]{3}{*}{ Combination } & $0 \%$ & Used gestational carrier & $11 \%$ & Endometriosis & $0 \%$ & Female factors only & $13 \%$ \\
\hline & & & & Uterine factor & $0 \%$ & Female \& male factors & $63 \%$ \\
\hline & & & & Male factor & $0 \%$ & & \\
\hline
\end{tabular}

2006 PREGNANCY SUCCESS RATES

Data verified by W. F. Howard, MD

\section{Type of Cycle}

\section{Fresh Embryos from Nondonor Eggs}

Number of cycles

Percentage of cycles resulting in pregnancies ${ }^{b}$

Percentage of cycles resulting in live births $s^{b, c}$

(Confidence Interval)

Percentage of retrievals resulting in live births $s^{b, c}$

Percentage of transfers resulting in live births $s^{b, c}$

Percentage of transfers resulting in singleton live births ${ }^{b}$

Percentage of cancellations ${ }^{b}$

Average number of embryos transferred

Percentage of pregnancies with twins ${ }^{b}$

Percentage of pregnancies with triplets or more ${ }^{b}$

Percentage of live births having multiple infants ${ }^{b, c}$

\section{Frozen Embryos from Nondonor Eggs}

Number of transfers

Percentage of transfers resulting in live births ${ }^{\mathrm{b}, \mathrm{c}}$

Average number of embryos transferred

\section{Donor Eggs}

Number of transfers

Percentage of transfers resulting in live births $s^{b, c}$

Average number of embryos transferred

\section{Age of Woman}

$\begin{array}{cccc}<35 & 35-37 & 38-40 & \mathbf{4 1 - 4 2}^{d} \\ 6 & 3 & 0 & 0 \\ 2 / 6 & 1 / 3 & & \\ 2 / 6 & 0 / 3 & & \end{array}$

$\begin{array}{cc}2 / 6 & 0 / 1 \\ 2 / 6 & 0 / 1 \\ 1 / 6 & 0 / 1 \\ 0 / 6 & 2 / 3 \\ 1.8 & 2.0 \\ 1 / 2 & 1 / 1 \\ 1 / 2 & 0 / 1 \\ 1 / 2 & \end{array}$

$2 / 6 \quad 0 / 1$

$0 / 1$

$2 / 3$

2.0

$1 / 1$

/ 1

2

$1 / 2$

1.5

\section{1}

$0 / 1$

2.0
0

$0 / 1$

2.0

\section{CURRENT CLINIC SERVICES AND PROFILE}

Current Name: Trinity InVitro Fertilization Program

\begin{tabular}{l|l|l} 
Donor egg? Yes $\quad$ Gestational carriers? Yes &
\end{tabular}

\begin{tabular}{l|ll} 
Donor embryo? No Cryopreservation? Yes &
\end{tabular}

Fresh Embryos

All Ages Combined

Single women? Yes

2

$2 / 2$

2.0

${ }^{a}$ Reflects patient and treatment characteristics of ART cycles performed in 2006 using fresh nondonor eggs or embryos.

${ }^{b}$ When fewer than 20 cycles are reported in an age category, rates are shown as a fraction and confidence intervals are not given. Calculating percentages from fractions may be misleading and is not encouraged.

A multiple-infant birth is counted as one live birth.

d Clinic-specific outcome rates are unreliable for women older than 42 undergoing ART cycles using fresh or frozen embryos with nondonor eggs. Readers are urged to review national outcomes for these age groups (see page 27).

e All ages (including ages $>42$ ) are reported together because previous data show that patient age does not materially affect success with donor eggs. 


\section{DALLAS-FORT WORTH FERTILITY ASSOCIATES DALLAS, TEXAS}

A comparison of clinic success rates may not be meaningful because patient medical characteristics and treatment approaches vary from clinic to clinic. For more details about this, along with information on how to interpret the statistics in this table, see pages 79-88.

\section{ART CYCLE PROFILE}

\section{Type of ART ${ }^{\mathrm{a}}$}

IVF

GIFT

ZIFT

Combination
100\% Procedural Factors:

$0 \%$ With ICSI

$0 \%$ Unstimulated

$0 \%$ Used gestational carrier $<1 \%$
Tubal factor

Ovulatory dysfunction

Diminished ovarian reserve

Endometriosis

Uterine factor

Male factor

\section{Patient Diagnosis}

$\begin{array}{rlr}3 \% & \text { Other factor } & 4 \% \\ 7 \% & \text { Unknown factor } & 2 \% \\ 21 \% & \text { Multiple Factors: } & \\ 1 \% & \text { Female factors only } & 18 \% \\ <1 \% & \text { Female \& male factors } & 35 \% \\ 10 \% & & \end{array}$

2006 PREGNANCY SUCCESS RATES

Data verified by Samuel J. Chantilis, MD

\section{Type of Cycle}

\section{Age of Woman}

$\begin{array}{llll}35-37 & 38-40 & 41-42^{\text {d }}\end{array}$

\section{Fresh Embryos from Nondonor Eggs}

Number of cycles

140

92

69

35

Percentage of cycles resulting in pregnancies ${ }^{b}$

Percentage of cycles resulting in live births ${ }^{b, c}$

60.0

51.1

44.9

37.1

(Confidence Interval)

Percentage of retrievals resulting in live births $s^{b, c}$

52.1

47.8

33.3

22.9

Percentage of transfers resulting in live births $s^{b, c}$

$(43.5-60.7)$

58.4

(37.3-58.5)

(22.4-45.7)

(10.4-40.1)

59.3

52.4

41.1

28.6

40.7

55.0

41.8

33.3

41.3

34.5

33.3

10.7

8.7

18.8

20.0

2.0

2.4

2.5

3.0

36.9

23.4

12.9

$3 / 13$

1.2

4.3

6.5

$0 / 13$

31.5

25.0

17.4

$0 / 8$

\section{Frozen Embryos from Nondonor Eggs}

Number of transfers

Percentage of transfers resulting in live births ${ }^{b, c}$

Average number of embryos transferred

$11 / 17$

1.7

\section{2}

$5 / 12$

\section{5}

$1 / 5$

1.8

2.0

All Ages Combined ${ }^{\mathrm{e}}$

Fresh Embryos 66

68.2

1.9
Frozen Embryos 13

$7 / 13$

1.7

Average number of embryos transferred

\section{CURRENT CLINIC SERVICES AND PROFILE}

Current Name: Dallas-Fort Worth Fertility Associates

\begin{tabular}{ll|ll} 
Donor egg? & Yes & Gestational carriers? & Yes \\
Donor embryo? & Yes & Cryopreservation? & Yes \\
Single women? & No & &
\end{tabular}

SART member?

Yes

Verified lab accreditation?

Yes

(See Appendix C for details.)

${ }^{a}$ Reflects patient and treatment characteristics of ART cycles performed in 2006 using fresh nondonor eggs or embryos.

${ }^{b}$ When fewer than 20 cycles are reported in an age category, rates are shown as a fraction and confidence intervals are not given. Calculating percentages from fractions may be misleading and is not encouraged.

${ }^{c}$ A multiple-infant birth is counted as one live birth.

d Clinic-specific outcome rates are unreliable for women older than 42 undergoing ART cycles using fresh or frozen embryos with nondonor eggs. Readers are urged to review national outcomes for these age groups (see page 27).

e All ages (including ages $>42$ ) are reported together because previous data show that patient age does not materially affect success with donor eggs. 


\section{FERTILITY SPECIALISTS OF DALLAS, PA \\ DALLAS, TEXAS}

A comparison of clinic success rates may not be meaningful because patient medical characteristics and treatment approaches vary from clinic to clinic. For more details about this, along with information on how to interpret the statistics in this table, see pages $79-88$.

\section{ART CYCLE PROFILE}

\begin{tabular}{|c|c|c|c|c|c|c|c|}
\hline \multicolumn{4}{|c|}{ Type of ART ${ }^{a}$} & \multicolumn{4}{|c|}{ Patient Diagnosis } \\
\hline IVF & $100 \%$ & Procedural Factors: & & Tubal factor & $14 \%$ & Other factor & $0 \%$ \\
\hline GIFT & $0 \%$ & With ICSI & $32 \%$ & Ovulatory dysfunction & $12 \%$ & Unknown factor & $4 \%$ \\
\hline $\mathrm{ZIFT}$ & $0 \%$ & Unstimulated & $0 \%$ & Diminished ovarian reserve & $16 \%$ & Multiple Factors: & \\
\hline \multirow[t]{3}{*}{ Combination } & $0 \%$ & Used gestational carrier & $0 \%$ & Endometriosis & $4 \%$ & Female factors only & $12 \%$ \\
\hline & & & & Uterine factor & $0 \%$ & Female \& male factors & $25 \%$ \\
\hline & & & & Male factor & $14 \%$ & & \\
\hline
\end{tabular}

2006 PREGNANCY SUCCESS RATES

Data verified by Jerald S. Goldstein, MD

\section{Type of Cycle}

\section{Fresh Embryos from Nondonor Eggs}

Number of cycles

Percentage of cycles resulting in pregnancies ${ }^{b}$

Percentage of cycles resulting in live births $s^{b, c}$

(Confidence Interval)

Percentage of retrievals resulting in live births $s^{b, c}$

Percentage of transfers resulting in live births $s^{b, c}$

Percentage of transfers resulting in singleton live births ${ }^{b}$

Percentage of cancellations ${ }^{b}$

Average number of embryos transferred

Percentage of pregnancies with twins ${ }^{b}$

Percentage of pregnancies with triplets or more ${ }^{b}$

Percentage of live births having multiple infants ${ }^{b, c}$

\section{Frozen Embryos from Nondonor Eggs}

Number of transfers

Percentage of transfers resulting in live births ${ }^{\mathrm{b}, \mathrm{c}}$

Average number of embryos transferred

\section{Age of Woman}

35-37 38-40

$41-42^{d}$

$<35$

5

$3 / 5$

$2 / 5$

5

3

45.9

45.9

(29.5-63.1)

50.0

$2 / 5$

$0 / 5$

$0 / 3$

51.5

27.3

8.1

$2 / 5$

$1 / 5$

$0 / 5$

2.6

$0 / 3$

$8 / 17$

$1 / 3$

$1 / 17$

$1 / 2$

$0 / 5$

$0 / 3$

$8 / 17$

$0 / 3$

$0 / 1$

$0 / 3$

$0 / 1$

$0 / 3$

$0 / 1$

$2 / 5$

$2 / 3$

3.7

2.0

2

$1 / 2$

2

$1 / 2$

2.0

2.0

All Ages Combined ${ }^{\mathrm{e}}$

\section{Donor Eggs}

Number of transfers

Percentage of transfers resulting in live births $s^{b, c}$

Average number of embryos transferred

\section{CURRENT CLINIC SERVICES AND PROFILE}

Current Name: Fertility Specialists of Dallas, PA

\begin{tabular}{ll|lll|ll} 
Donor egg? & Yes & Gestational carriers? & Yes & SART member? & Yes \\
\hline Donor embryo? & Yes & Cryopreservation? & Yes & Verified lab accreditation? & Yes \\
Single women? & No & & & (See Appendix C for details.) &
\end{tabular}

${ }^{a}$ Reflects patient and treatment characteristics of ART cycles performed in 2006 using fresh nondonor eggs or embryos.

${ }^{b}$ When fewer than 20 cycles are reported in an age category, rates are shown as a fraction and confidence intervals are not given. Calculating percentages from fractions may be misleading and is not encouraged.

A multiple-infant birth is counted as one live birth.

d Clinic-specific outcome rates are unreliable for women older than 42 undergoing ART cycles using fresh or frozen embryos with nondonor eggs. Readers are urged to review national outcomes for these age groups (see page 27).

e All ages (including ages $>42$ ) are reported together because previous data show that patient age does not materially affect success with donor eggs. 


\section{SHER INSTITUTE FOR REPRODUCTIVE MEDICINE-DALLAS DALLAS, TEXAS}

A comparison of clinic success rates may not be meaningful because patient medical characteristics and treatment approaches vary from clinic to clinic. For more details about this, along with information on how to interpret the statistics in this table, see pages 79-88.

\section{ART CYCLE PROFILE}

\begin{tabular}{|c|c|c|c|c|c|c|c|}
\hline \multicolumn{4}{|c|}{ Type of ART ${ }^{a}$} & \multicolumn{4}{|c|}{ Patient Diagnosis } \\
\hline IVF & $100 \%$ & Procedural Factors: & & Tubal factor & $9 \%$ & Other factor & $26 \%$ \\
\hline GIFT & $0 \%$ & With ICSI & $86 \%$ & Ovulatory dysfunction & $8 \%$ & Unknown factor & $6 \%$ \\
\hline ZIFT & $0 \%$ & Unstimulated & $0 \%$ & Diminished ovarian reserve & $14 \%$ & Multiple Factors: & \\
\hline \multirow[t]{3}{*}{ Combination } & $0 \%$ & Used gestational carrier & $4 \%$ & Endometriosis & $4 \%$ & Female factors only & $8 \%$ \\
\hline & & & & Uterine factor & $3 \%$ & Female \& male factors & $18 \%$ \\
\hline & & & & Male factor & $3 \%$ & & \\
\hline
\end{tabular}

2006 PREGNANCY SUCCESS RATES

Data verified by Walid Saleh, MD

\section{Type of Cycle}

\section{Fresh Embryos from Nondonor Eggs}

Number of cycles

Percentage of cycles resulting in pregnancies ${ }^{b}$

Percentage of cycles resulting in live births $s^{b, c}$

(Confidence Interval)

Percentage of retrievals resulting in live births $s^{b, c}$

Percentage of transfers resulting in live births $s^{b, c}$

Percentage of transfers resulting in singleton live births ${ }^{b}$

Percentage of cancellations ${ }^{b}$

Average number of embryos transferred

Percentage of pregnancies with twins ${ }^{b}$

Percentage of pregnancies with triplets or more ${ }^{b}$

Percentage of live births having multiple infants ${ }^{\mathrm{b}, \mathrm{c}}$

\section{Frozen Embryos from Nondonor Eggs}

Number of transfers

Percentage of transfers resulting in live births ${ }^{b, c}$

Average number of embryos transferred

\section{Donor Eggs}

Number of transfers

Percentage of transfers resulting in live births $s^{b, c}$

Average number of embryos transferred

\section{Age of Woman}

35-37

38-40

$41-42^{d}$

$<35$

28

42.9

35.7
$18.6-55$

22

4

41.3

37.0

22.7

$0 / 4$

$(23.2-52.5)$

40.5

37.0

13.6

$0 / 4$

53.1

$10 / 19$

(2.9-34.9)

25.0

$6 / 19$

$3 / 17$

$0 / 3$

$3 / 15$

$0 / 3$

8.7

3.6

$1 / 15$

$0 / 3$

2.3

2.1

22.7

$1 / 4$

$10 / 19$

$4 / 12$

2.5

2.3

$1 / 19$

$9 / 17$

$0 / 12$

$3 / 5$

$0 / 5$

$4 / 10$

$2 / 3$

\begin{tabular}{|c|c|c|c|}
\hline 6 & 0 & 2 & 1 \\
\hline $4 / 6$ & & $0 / 2$ & $0 / 1$ \\
\hline 2.3 & & 2.5 & 3.0 \\
\hline
\end{tabular}

\section{All Ages Combined}

Fresh Embryos

8

$3 / 8$

2.4
Frozen Embryos 8

$1 / 8$

2.8

\section{CURRENT CLINIC SERVICES AND PROFILE}

Current Name: Sher Institute for Reproductive Medicine-Dallas

Donor egg? Yes Gestational carriers? Yes

Donor embryo? No Cryopreservation? Yes

Single women? Yes

SART member? $\quad$ Yes

Verified lab accreditation?

(See Appendix C for details.)

${ }^{a}$ Reflects patient and treatment characteristics of ART cycles performed in 2006 using fresh nondonor eggs or embryos.

${ }^{b}$ When fewer than 20 cycles are reported in an age category, rates are shown as a fraction and confidence intervals are not given. Calculating percentages from fractions may be misleading and is not encouraged.

${ }^{c}$ A multiple-infant birth is counted as one live birth.

d Clinic-specific outcome rates are unreliable for women older than 42 undergoing ART cycles using fresh or frozen embryos with nondonor eggs. Readers are urged to review national outcomes for these age groups (see page 27).

e All ages (including ages $>42$ ) are reported together because previous data show that patient age does not materially affect success with donor eggs. 


\section{TEXAS CENTER FOR REPRODUCTIVE HEALTH \\ DALLAS, TEXAS}

A comparison of clinic success rates may not be meaningful because patient medical characteristics and treatment approaches vary from clinic to clinic. For more details about this, along with information on how to interpret the statistics in this table, see pages $79-88$.

\section{ART CYCLE PROFILE}

\begin{tabular}{|c|c|c|c|c|c|c|c|}
\hline & Type & of $\mathrm{ART}^{\mathrm{a}}$ & & Pati & ent D & iagnosis & \\
\hline IVF & $100 \%$ & Procedural Factors: & & Tubal factor & $9 \%$ & Other factor & $3 \%$ \\
\hline GIFT & $0 \%$ & With ICSI & $83 \%$ & Ovulatory dysfunction & $4 \%$ & Unknown factor & $2 \%$ \\
\hline ZIFT & $0 \%$ & Unstimulated & $0 \%$ & Diminished ovarian reserve & $8 \%$ & Multiple Factors: & \\
\hline Combination & $0 \%$ & Used gestational carrier & $<1 \%$ & Endometriosis & $10 \%$ & Female factors only & $18 \%$ \\
\hline & & & & Uterine factor & $<1 \%$ & Female \& male factors & $23 \%$ \\
\hline
\end{tabular}

2006 PREGNANCY SUCCESS RATES

Data verified by Michael Putman, MD

\section{Type of Cycle}

\section{Fresh Embryos from Nondonor Eggs}

Number of cycles

Percentage of cycles resulting in pregnancies ${ }^{b}$

Percentage of cycles resulting in live births ${ }^{b, c}$

(Confidence Interval)

Percentage of retrievals resulting in live births $s^{b, c}$

Percentage of transfers resulting in live births $s^{b, c}$

Percentage of transfers resulting in singleton live births ${ }^{b}$

Percentage of cancellations ${ }^{b}$

Average number of embryos transferred

Percentage of pregnancies with twins ${ }^{b}$

Percentage of pregnancies with triplets or more ${ }^{b}$

Percentage of live births having multiple infants ${ }^{b, c}$

\section{Frozen Embryos from Nondonor Eggs}

Number of transfers

Percentage of transfers resulting in live births $s^{b, c}$

Average number of embryos transferred

\section{Donor Eggs}

Number of transfers

Percentage of transfers resulting in live births $s^{b, c}$

Average number of embryos transferred

\section{Age of Woman}

35-37 38-40

$41-42^{d}$

\section{$<35$}

27

48.1

14

7

56.3

37.0

$1 / 14$

$0 / 7$

48.4

$(19.4-57.6)$
37.0

$1 / 14$

$0 / 7$

$(35.8-61.3)$

50.0

$1 / 13$

$0 / 6$

52.5

40.0

$1 / 10$

$0 / 5$

33.9

20.0

$1 / 10$

$0 / 5$

3.1

0.0

$1 / 14$

$1 / 7$

2.2

2.8

3.2

2.2

36.1

$3 / 13$

$0 / 1$

2.8

$2 / 13$

$0 / 1$

35.5

$5 / 10$

$0 / 1$

29

51.7

11

$6 / 11$

2

$0 / 2$

2

2.2

2.1

3.0

$1 / 2$

4.5

All Ages Combined ${ }^{\mathrm{e}}$

Fresh Embryos 10

$6 / 10$

2.0
Frozen Embryos

4

$2 / 4$

2.5

\section{CURRENT CLINIC SERVICES AND PROFILE}

Current Name: Texas Center for Reproductive Health

Donor egg? Yes

Donor embryo? Yes

Single women? Yes

\section{Gestational carriers? \\ Yes}

Cryopreservation?
Yes

${ }^{a}$ Reflects patient and treatment characteristics of ART cycles performed in 2006 using fresh nondonor eggs or embryos.

${ }^{b}$ When fewer than 20 cycles are reported in an age category, rates are shown as a fraction and confidence intervals are not given. Calculating percentages from fractions may be misleading and is not encouraged.

A multiple-infant birth is counted as one live birth.

d Clinic-specific outcome rates are unreliable for women older than 42 undergoing ART cycles using fresh or frozen embryos with nondonor eggs. Readers are urged to review national outcomes for these age groups (see page 27).

e All ages (including ages $>42$ ) are reported together because previous data show that patient age does not materially affect success with donor eggs. 


\section{THE WOMEN'S PLACE \\ DALLAS, TEXAS}

A comparison of clinic success rates may not be meaningful because patient medical characteristics and treatment approaches vary from clinic to clinic. For more details about this, along with information on how to interpret the statistics in this table, see pages 79-88.

\section{ART CYCLE PROFILE}

\begin{tabular}{|c|c|c|c|c|c|c|c|}
\hline \multicolumn{4}{|c|}{ Type of ART ${ }^{a}$} & \multicolumn{4}{|c|}{ Patient Diagnosis } \\
\hline IVF & $100 \%$ & Procedural Factors: & & Tubal factor & $20 \%$ & Other factor & $0 \%$ \\
\hline GIFT & $0 \%$ & With ICSI & $46 \%$ & Ovulatory dysfunction & $16 \%$ & Unknown factor & $12 \%$ \\
\hline ZIFT & $0 \%$ & Unstimulated & $0 \%$ & Diminished ovarian reserve & $0 \%$ & Multiple Factors: & \\
\hline \multirow[t]{3}{*}{ Combination } & $0 \%$ & Used gestational carrier & $0 \%$ & Endometriosis & $0 \%$ & Female factors only & $12 \%$ \\
\hline & & & & Uterine factor & $0 \%$ & Female \& male factors & $36 \%$ \\
\hline & & & & Male factor & $4 \%$ & & \\
\hline
\end{tabular}

2006 PREGNANCY SUCCESS RATES

Data verified by Lisa A. King-Hatley, MD

\section{Type of Cycle}

\section{Fresh Embryos from Nondonor Eggs}

Number of cycles

Percentage of cycles resulting in pregnancies ${ }^{b}$

Percentage of cycles resulting in live births ${ }^{b, c}$

(Confidence Interval)
Percentage of retrievals resulting in live births ${ }^{b, c}$

Percentage of transfers resulting in live births $s^{b, c}$

Percentage of transfers resulting in singleton live births ${ }^{b}$

Percentage of cancellations ${ }^{b}$

Average number of embryos transferred

Percentage of pregnancies with twins ${ }^{b}$

Percentage of pregnancies with triplets or more ${ }^{b}$

Percentage of live births having multiple infants ${ }^{b, c}$

\section{Frozen Embryos from Nondonor Eggs}

Number of transfers

Percentage of transfers resulting in live births $s^{b, c}$

Average number of embryos transferred

\section{Donor Eggs}

Number of transfers

Percentage of transfers resulting in live births $s^{b, c}$

Average number of embryos transferred

\section{Age of Woman}

$<35 \quad 35-37 \quad 38-40 \quad 41-42^{\text {d }}$

11

$1 / 11$

5

$1 / 11$

$1 / 5$

$1 / 5$

5

1

$1 / 5$

$0 / 1$

$1 / 5$

$0 / 1$

$\begin{array}{lll}1 / 11 & 1 / 5 & 1 / 3 \\ 1 / 11 & 1 / 5 & 1 / 3 \\ 1 / 11 & 1 / 5 & 1 / 3 \\ 0 / 11 & 0 / 5 & 2 / 5 \\ 2.5 & 2.4 & 1.3 \\ 0 / 1 & 0 / 1 & 0 / 1 \\ 0 / 1 & 0 / 1 & 0 / 1 \\ 0 / 1 & 0 / 1 & 0 / 1\end{array}$

$1 / 3$

$1 / 3$

$1 / 3$

$2 / 5$

$1 / 1$

1.3

$0 / 1$

$0 / 1$

3

$1 / 3$

2.3

0

0

0

\section{All Ages Combined ${ }^{\mathrm{e}}$}

Fresh Embryos

Frozen Embryos

0 0

\section{CURRENT CLINIC SERVICES AND PROFILE}

Current Name: The Women's Place

Donor egg? No Gestational carriers? Yes

Donor embryo? No

Single women? No

Cryopreservation? Yes

\section{SART member?}

Verified lab accreditation?

(See Appendix C for details.)

\footnotetext{
${ }^{a}$ Reflects patient and treatment characteristics of ART cycles performed in 2006 using fresh nondonor eggs or embryos.

b When fewer than 20 cycles are reported in an age category, rates are shown as a fraction and confidence intervals are not given. Calculating percentages from fractions may be misleading and is not encouraged.

${ }^{c}$ A multiple-infant birth is counted as one live birth.

d Clinic-specific outcome rates are unreliable for women older than 42 undergoing ART cycles using fresh or frozen embryos with nondonor eggs. Readers are urged to review national outcomes for these age groups (see page 27).

${ }^{e}$ All ages (including ages $>42$ ) are reported together because previous data show that patient age does not materially affect success with donor eggs.
} 


\section{SOUTHWEST CENTER FOR REPRODUCTIVE HEALTH, PA \\ EL PASO, TEXAS}

A comparison of clinic success rates may not be meaningful because patient medical characteristics and treatment approaches vary from clinic to clinic. For more details about this, along with information on how to interpret the statistics in this table, see pages $79-88$.

\section{ART CYCLE PROFILE}

\section{Type of ART ${ }^{\mathrm{a}}$}

$\begin{array}{llr}\text { IVF } & 99 \% \text { Procedural Factors: } & \\ \text { GIFT } & 0 \% \text { With ICSI } & 75 \% \\ \text { ZIFT } & 0 \% \text { Unstimulated } & 0 \% \\ \text { Combination } & 1 \% \text { Used gestational carrier } & 33 \%\end{array}$

\section{Patient Diagnosis}

Tubal factor

Ovulatory dysfunction

$18 \%$ Other factor

4\% Unknown factor

4\% Multiple Factors:

Diminished ovarian reserve

Endometriosis

$8 \%$ Female factors only $16 \%$

Uterine factor

$3 \%$ Female \& male factors $23 \%$

Male factor $8 \%$
$7 \%$

$8 \%$

2006 PREGNANCY SUCCESS RATES

Data verified by Luis S. Noble, MD

\section{Type of Cycle}

\section{Fresh Embryos from Nondonor Eggs}

Number of cycles

Percentage of cycles resulting in pregnancies ${ }^{b}$

Percentage of cycles resulting in live births $s^{b, c}$

(Confidence Interval)

Percentage of retrievals resulting in live births $s^{b, c}$

Percentage of transfers resulting in live births $s^{b, c}$

Percentage of transfers resulting in singleton live births ${ }^{b}$

Percentage of cancellations ${ }^{b}$

Average number of embryos transferred

Percentage of pregnancies with twins ${ }^{b}$

Percentage of pregnancies with triplets or more ${ }^{b}$

Percentage of live births having multiple infants ${ }^{b, c}$

\section{Frozen Embryos from Nondonor Eggs}

Number of transfers

Percentage of transfers resulting in live births $s^{\mathrm{b}, \mathrm{c}}$

Average number of embryos transferred

\section{Donor Eggs}

Number of transfers

Percentage of transfers resulting in live births $s^{\mathrm{b}, \mathrm{c}}$

Average number of embryos transferred

\section{Age of Woman}

35-37 38-40

$41-42^{d}$ $<35$

26

42.3

16

3

54.3

26.9

$7 / 16$

$1 / 3$

45.7

$(11.6-47.8)$

$4 / 16$

$1 / 3$

(28.8-63.4)

45.7

33.3

$4 / 15$

$1 / 3$

48.5

$7 / 19$

$4 / 14$

$1 / 3$

39.4

$4 / 19$

$2 / 14$

$1 / 3$

0.0

19.2

$1 / 16$

$0 / 3$

1.9

2.3

$4 / 19$

$4 / 11$

$0 / 19$

$0 / 11$

$3 / 16$

$3 / 7$

2.6

3.0

$0 / 1$

$0 / 1$

$1 / 7$

$0 / 1$

$2 / 4$

\section{8}

$2 / 8$

2.0

4

$2 / 4$

$5 / 9$

1.9

All Age

2.0

\section{CURRENT CLINIC SERVICES AND PROFILE}

Current Name: Southwest Center for Reproductive Health, PA

Donor egg? Yes Gestational carriers? Yes

Donor embryo? Yes Cryopreservation? Yes

Fresh Embryos

$\begin{array}{cc}14 & 2 \\ 4 / 14 & 0 / 2 \\ 2.0 & 2.0\end{array}$

Single women? Yes

\section{SART member? Yes}

Verified lab accreditation?

(See Appendix C for details.)

${ }^{a}$ Reflects patient and treatment characteristics of ART cycles performed in 2006 using fresh nondonor eggs or embryos.

${ }^{b}$ When fewer than 20 cycles are reported in an age category, rates are shown as a fraction and confidence intervals are not given. Calculating percentages from fractions may be misleading and is not encouraged.

A multiple-infant birth is counted as one live birth.

d Clinic-specific outcome rates are unreliable for women older than 42 undergoing ART cycles using fresh or frozen embryos with nondonor eggs. Readers are urged to review national outcomes for these age groups (see page 27).

e All ages (including ages $>42$ ) are reported together because previous data show that patient age does not materially affect success with donor eggs. 


\section{CENTER FOR WOMEN'S MEDICINE \\ HOUSTON, TEXAS}

A comparison of clinic success rates may not be meaningful because patient medical characteristics and treatment approaches vary from clinic to clinic. For more details about this, along with information on how to interpret the statistics in this table, see pages 79-88.

\section{ART CYCLE PROFILE}

\begin{tabular}{|c|c|c|c|c|c|c|c|}
\hline \multicolumn{4}{|c|}{ Type of ART ${ }^{a}$} & \multicolumn{4}{|c|}{ Patient Diagnosis } \\
\hline IVF & $100 \%$ & Procedural Factors: & & Tubal factor & $14 \%$ & Other factor & $3 \%$ \\
\hline GIFT & $0 \%$ & With ICSI & $92 \%$ & Ovulatory dysfunction & $11 \%$ & Unknown factor & $<1 \%$ \\
\hline ZIFT & $0 \%$ & Unstimulated & $0 \%$ & Diminished ovarian reserve & $3 \%$ & Multiple Factors: & \\
\hline \multirow[t]{3}{*}{ Combination } & $0 \%$ & Used gestational carrier & $2 \%$ & Endometriosis & $7 \%$ & Female factors only & $25 \%$ \\
\hline & & & & Uterine factor & $1 \%$ & Female \& male factors & $26 \%$ \\
\hline & & & & Male factor & $9 \%$ & & \\
\hline
\end{tabular}

2006 PREGNANCY SUCCESS RATES

Data verified by Michael A. Allon, MD

\section{Type of Cycle}

\section{Fresh Embryos from Nondonor Eggs}

Number of cycles

Percentage of cycles resulting in pregnancies ${ }^{b}$

Percentage of cycles resulting in live births $s^{b, c}$

(Confidence Interval)

Percentage of retrievals resulting in live births $s^{b, c}$

Percentage of transfers resulting in live births $s^{b, c}$

Percentage of transfers resulting in singleton live births ${ }^{b}$

Percentage of cancellations ${ }^{b}$

Average number of embryos transferred

Percentage of pregnancies with twins ${ }^{b}$

Percentage of pregnancies with triplets or more ${ }^{b}$

Percentage of live births having multiple infants ${ }^{b, c}$

\section{Frozen Embryos from Nondonor Eggs}

Number of transfers

Percentage of transfers resulting in live births ${ }^{b, c}$

Average number of embryos transferred

\section{Donor Eggs}

Number of transfers

Percentage of transfers resulting in live births $s^{b, c}$

Average number of embryos transferred

\section{Age of Woman}

35-37 38-40

$41-42^{d}$

\section{$<35$}

37

43.2

17

4

44.8

32.4

$5 / 17$

$0 / 4$

39.7

(27.0-53.4)

(18.0-49.8)

41.1

45.1

34.3

$5 / 17$

$0 / 4$

25.5

36.4

$5 / 16$

$0 / 3$

27.3

$5 / 14$

$0 / 3$

3.4

5.4

$4 / 14$

$0 / 3$

2.9

2.8

$1 / 17$

$1 / 4$

42.3

$3 / 16$

3.1

$1 / 5$

3.8

43.5

$0 / 16$

$0 / 5$

$3 / 12$

$1 / 5$

15

$5 / 15$

2.9

\section{3}

$0 / 3$

3.0

\section{All Ages Combined ${ }^{\mathrm{e}}$}

Fresh Embryos 16

$4 / 16$

3.1
Frozen Embryos 5

$3 / 5$

3.0

\section{CURRENT CLINIC SERVICES AND PROFILE}

Current Name: Center for Women's Medicine

Donor egg? Yes

Donor embryo? Yes

Single women? Yes

\section{Gestational carriers? Yes}

Cryopreservation? Yes
SART member?

Verified lab accreditation?

(See Appendix C for details.)

\footnotetext{
${ }^{a}$ Reflects patient and treatment characteristics of ART cycles performed in 2006 using fresh nondonor eggs or embryos.

${ }^{b}$ When fewer than 20 cycles are reported in an age category, rates are shown as a fraction and confidence intervals are not given. Calculating percentages from fractions may be misleading and is not encouraged.

${ }^{c}$ A multiple-infant birth is counted as one live birth.

d Clinic-specific outcome rates are unreliable for women older than 42 undergoing ART cycles using fresh or frozen embryos with nondonor eggs. Readers are urged to review national outcomes for these age groups (see page 27).

e All ages (including ages $>42$ ) are reported together because previous data show that patient age does not materially affect success with donor eggs.
} 


\section{COOPER INSTITUTE FOR ADVANCED REPRODUCTIVE MEDICINE HOUSTON, TEXAS}

A comparison of clinic success rates may not be meaningful because patient medical characteristics and treatment approaches vary from clinic to clinic. For more details about this, along with information on how to interpret the statistics in this table, see pages $79-88$.

\section{ART CYCLE PROFILE}

\begin{tabular}{|c|c|c|c|c|c|c|c|}
\hline \multicolumn{4}{|c|}{ Type of ART ${ }^{a}$} & \multicolumn{4}{|c|}{ Patient Diagnosis } \\
\hline IVF & $100 \%$ & Procedural Factors: & & Tubal factor & $22 \%$ & Other factor & $4 \%$ \\
\hline GIFT & $0 \%$ & With ICSI & $85 \%$ & Ovulatory dysfunction & $1 \%$ & Unknown factor & $0 \%$ \\
\hline ZIFT & $0 \%$ & Unstimulated & $0 \%$ & Diminished ovarian reserve & $8 \%$ & Multiple Factors: & \\
\hline \multirow[t]{3}{*}{ Combination } & $0 \%$ & Used gestational carrier & $4 \%$ & Endometriosis & $5 \%$ & Female factors only & $7 \%$ \\
\hline & & & & Uterine factor & $0 \%$ & Female \& male factors & $37 \%$ \\
\hline & & & & Male factor & $16 \%$ & & \\
\hline
\end{tabular}

2006 PREGNANCY SUCCESS RATES

Data verified by C. James Chuong, MD

\section{Type of Cycle}

\section{Fresh Embryos from Nondonor Eggs}

Number of cycles

Percentage of cycles resulting in pregnancies ${ }^{b}$

Percentage of cycles resulting in live births ${ }^{b, c}$

(Confidence Interval)

Percentage of retrievals resulting in live births $s^{b, c}$

Percentage of transfers resulting in live births $s^{b, c}$

Percentage of transfers resulting in singleton live births ${ }^{b}$

Percentage of cancellations ${ }^{b}$

Average number of embryos transferred

Percentage of pregnancies with twins ${ }^{b}$

Percentage of pregnancies with triplets or more ${ }^{b}$

Percentage of live births having multiple infants ${ }^{b, c}$

\section{Frozen Embryos from Nondonor Eggs}

Number of transfers

Percentage of transfers resulting in live births ${ }^{\mathrm{b}, \mathrm{c}}$

Average number of embryos transferred

\section{Donor Eggs}

Number of transfers

Percentage of transfers resulting in live births $s^{b, c}$

Average number of embryos transferred

\section{Age of Woman}

35-37 38-40

$41-42^{d}$ $<35$

6

21
28.6

$1 / 6$

7

4

19.0

$1 / 6$

$2 / 7$

$0 / 4$

(5.4-41.9)

$20.0 \quad 1 / 6$

$2 / 7$

$0 / 4$

$4 / 18$

$1 / 6$

$2 / 7$

$0 / 3$

$3 / 18$

$1 / 6$

$2 / 6$

$0 / 3$

4.8

$0 / 6$

$2 / 6$

$0 / 3$

3.7

4.2

$0 / 7$

$1 / 4$

$2 / 6$

$0 / 1$

3.3

$0 / 2$

$0 / 6$

$0 / 1$

$0 / 2$

$1 / 4$

$0 / 1$

$0 / 2$

\section{5}

$1 / 5$

3.0

\section{2}

$1 / 2$

3.5

2
$0 / 2$
4.5

All Ages Combined

Fresh Embryos

15
$6 / 15$
4.3

Frozen Embryos 2

$1 / 2$

5.0

\section{CURRENT CLINIC SERVICES AND PROFILE}

Current Name: Cooper Institute for Advanced Reproductive Medicine

\begin{tabular}{l|l|l} 
Donor egg? Yes & Gestational carriers? Yes
\end{tabular}

Donor embryo? Yes Cryopreservation? Yes

Single women? Yes

SART member? Yes

Verified lab accreditation?

(See Appendix C for details.)

${ }^{a}$ Reflects patient and treatment characteristics of ART cycles performed in 2006 using fresh nondonor eggs or embryos.

${ }^{b}$ When fewer than 20 cycles are reported in an age category, rates are shown as a fraction and confidence intervals are not given. Calculating percentages from fractions may be misleading and is not encouraged.

A multiple-infant birth is counted as one live birth.

d Clinic-specific outcome rates are unreliable for women older than 42 undergoing ART cycles using fresh or frozen embryos with nondonor eggs. Readers are urged to review national outcomes for these age groups (see page 27).

e All ages (including ages $>42$ ) are reported together because previous data show that patient age does not materially affect success with donor eggs. 


\section{FERTILITY SPECIALISTS OF HOUSTON \\ HOUSTON, TEXAS}

A comparison of clinic success rates may not be meaningful because patient medical characteristics and treatment approaches vary from clinic to clinic. For more details about this, along with information on how to interpret the statistics in this table, see pages 79-88.

\section{ART CYCLE PROFILE}

\section{Type of ART ${ }^{\mathrm{a}}$}

IVF

GIFT

100\% Procedural Factors:

ZIFT

Combination
$0 \%$ With ICSI

$0 \%$ Unstimulated

$0 \%$ Used gestational carrier

\begin{tabular}{|c|c|}
\hline \multirow[b]{2}{*}{$76 \%$} & Tubal factor \\
\hline & Ovulatory dysfunction \\
\hline $0 \%$ & Diminished ovarian reserve \\
\hline $1 \%$ & Endometriosis \\
\hline & Uterine factor \\
\hline & Male factor \\
\hline
\end{tabular}

Patient Diagnosis

$\begin{array}{lll}4 \% & \text { Other factor } & 14 \% \\ 3 \% & \text { Unknown factor } & <1 \% \\ 1 \% & \text { Multiple Factors: } & \\ 7 \% & \text { Female factors only } & 13 \% \\ 1 \% & \text { Female \& male factors } & 43 \% \\ 13 \% & & \end{array}$

2006 PREGNANCY SUCCESS RATES

Data verified by George M. Grunert, MD

\section{Type of Cycle}

\section{Age of Woman}

35-37 38-40

\section{$<35$}

246

41.9

32.9

(27.1-39.2)

37.9

40.9

24.7

13.0

2.1

35.9

2.9

39.5

Percentage of pregnancies with triplets or more ${ }^{b}$

Percentage of live births having multiple infants ${ }^{b, c}$

\section{Frozen Embryos from Nondonor Eggs}

Number of transfers

Percentage of transfers resulting in live births ${ }^{\mathrm{b}, \mathrm{c}}$

Average number of embryos transferred

\section{Donor Eggs}

Number of transfers

Percentage of transfers resulting in live births ${ }^{b, c}$

Average number of embryos transferred

All Ages Combined ${ }^{\mathrm{e}}$
Fresh Embryos

61

49.2

2.0

$\begin{array}{ccc}153 & 119 & 43 \\ 34.6 & 17.6 & 11.6 \\ 23.5 & 12.6 & 7.0 \\ 17.1-31.1) & (7.2-19.9) & (1.5-19.1) \\ 29.3 & 16.9 & 9.7 \\ 32.7 & 19.2 & 12.5 \\ 20.9 & 12.8 & 8.3 \\ 19.6 & 25.2 & 27.9 \\ 2.3 & 2.3 & 2.0 \\ 28.3 & 33.3 & 1 / 5 \\ 1.9 & 4.8 & 0 / 5 \\ 36.1 & 5 / 15 & 1 / 3\end{array}$

$\begin{array}{cccc}46 & 28 & 13 & 7 \\ 19.6 & 10.7 & 2 / 13 & 2 / 7 \\ 2.0 & 2.0 & 1.8 & 1.7\end{array}$

Frozen Embryos 26

38.5

2.5

\section{CURRENT CLINIC SERVICES AND PROFILE}

Current Name: Fertility Specialists of Houston

$\begin{array}{llll}\text { Donor egg? } & \text { Yes } & \text { Gestational carriers? } & \text { Yes } \\ \text { Donor embryo? } & \text { Yes } & \text { Cryopreservation? } & \text { Yes }\end{array}$

Single women? Yes

SART member? Yes

Verified lab accreditation? Yes

(See Appendix C for details.)

${ }^{a}$ Reflects patient and treatment characteristics of ART cycles performed in 2006 using fresh nondonor eggs or embryos.

${ }^{b}$ When fewer than 20 cycles are reported in an age category, rates are shown as a fraction and confidence intervals are not given. Calculating percentages from fractions may be misleading and is not encouraged.

${ }^{\mathrm{C}}$ A multiple-infant birth is counted as one live birth.

d Clinic-specific outcome rates are unreliable for women older than 42 undergoing ART cycles using fresh or frozen embryos with nondonor eggs. Readers are urged to review national outcomes for these age groups (see page 27).

e All ages (including ages $>42$ ) are reported together because previous data show that patient age does not materially affect success with donor eggs. 


\section{HOUSTON INFERTILITY CLINIC \\ SONJA KRISTIANSEN, MD \\ HOUSTON, TEXAS}

A comparison of clinic success rates may not be meaningful because patient medical characteristics and treatment approaches vary from clinic to clinic. For more details about this, along with information on how to interpret the statistics in this table, see pages $79-88$.

\section{ART CYCLE PROFILE}

\begin{tabular}{|c|c|c|c|c|c|c|c|}
\hline \multicolumn{4}{|c|}{ Type of ART ${ }^{\mathrm{a}}$} & \multicolumn{4}{|c|}{ Patient Diagnosis } \\
\hline IVF & $100 \%$ & Procedural Factors: & & Tubal factor & $9 \%$ & Other factor & $14 \%$ \\
\hline GIFT & $0 \%$ & With ICSI & $91 \%$ & Ovulatory dysfunction & $3 \%$ & Unknown factor & $12 \%$ \\
\hline ZIFT & $0 \%$ & Unstimulated & $0 \%$ & Diminished ovarian reserve & $8 \%$ & Multiple Factors: & \\
\hline \multirow[t]{3}{*}{ Combination } & $0 \%$ & Used gestational carrier & $5 \%$ & Endometriosis & $1 \%$ & Female factors only & $2 \%$ \\
\hline & & & & Uterine factor & $2 \%$ & Female \& male factors & $8 \%$ \\
\hline & & & & Male factor & $39 \%$ & & \\
\hline
\end{tabular}

2006 PREGNANCY SUCCESS RATES

Data verified by Sonja B. Kristiansen, MD

\section{Type of Cycle}

\section{Fresh Embryos from Nondonor Eggs}

Number of cycles

Percentage of cycles resulting in pregnancies ${ }^{b}$

Percentage of cycles resulting in live births $s^{b, c}$

(Confidence Interval)

Percentage of retrievals resulting in live births ${ }^{b, c}$

Percentage of transfers resulting in live births $s^{b, c}$

Percentage of transfers resulting in singleton live births ${ }^{b}$

Percentage of cancellations ${ }^{b}$

Average number of embryos transferred

Percentage of pregnancies with twins ${ }^{b}$

Percentage of pregnancies with triplets or more ${ }^{b}$

Percentage of live births having multiple infants ${ }^{b, c}$

\section{Frozen Embryos from Nondonor Eggs}

Number of transfers

Percentage of transfers resulting in live births ${ }^{\mathrm{b}, \mathrm{c}}$

Average number of embryos transferred

\section{Donor Eggs}

Number of transfers

Percentage of transfers resulting in live births $s^{b, c}$

Average number of embryos transferred

\section{Age of Woman}

35-37 38-40

$41-42^{d}$

$<35$

13

18

4

58.1

$5 / 13$

$6 / 18$

$1 / 4$

51.6

$3 / 13$

$5 / 18$

$1 / 4$

(33.1-69.8)

53.3

53.3

$3 / 13$

$5 / 17$

$1 / 4$

43.3

$3 / 10$

$5 / 17$

$1 / 2$

$2 / 10$

$4 / 17$

$1 / 2$

3.2

$0 / 13$

$1 / 18$

$0 / 4$

2.2

2.1

$1 / 5$

2.2

3.0

$1 / 18$

$0 / 5$

$1 / 6$

$0 / 1$

$2 / 18$

$1 / 3$

$0 / 6$

$0 / 1$

$3 / 16$

$1 / 5$

$0 / 1$

$\begin{array}{cccc}7 & 8 & 2 & 1 \\ 3 / 7 & 3 / 8 & 0 / 2 & 0 / 1 \\ 1.9 & 1.9 & 1.0 & 1.0\end{array}$

Fresh Embryos

All Ages Combined 3

$2 / 3$

2.3
Frozen Embryos

\section{6}

$1 / 6$

3.8

\section{CURRENT CLINIC SERVICES AND PROFILE}

Current Name: Houston Infertility Clinic, Sonja Kristiansen, MD

Donor egg? Yes Gestational carriers? Yes

Donor embryo? Yes Cryopreservation? Yes

Single women? Yes

SART member? Yes

Verified lab accreditation? Yes

(See Appendix C for details.)

${ }^{a}$ Reflects patient and treatment characteristics of ART cycles performed in 2006 using fresh nondonor eggs or embryos.

${ }^{b}$ When fewer than 20 cycles are reported in an age category, rates are shown as a fraction and confidence intervals are not given. Calculating percentages from fractions may be misleading and is not encouraged.

A multiple-infant birth is counted as one live birth.

d Clinic-specific outcome rates are unreliable for women older than 42 undergoing ART cycles using fresh or frozen embryos with nondonor eggs. Readers are urged to review national outcomes for these age groups (see page 27).

e All ages (including ages $>42$ ) are reported together because previous data show that patient age does not materially affect success with donor eggs. 


\section{HOUSTON IVF \\ HOUSTON, TEXAS}

A comparison of clinic success rates may not be meaningful because patient medical characteristics and treatment approaches vary from clinic to clinic. For more details about this, along with information on how to interpret the statistics in this table, see pages 79-88.

\section{ART CYCLE PROFILE}

\begin{tabular}{|c|c|c|c|c|c|c|c|}
\hline \multicolumn{4}{|c|}{ Type of ART ${ }^{a}$} & \multicolumn{4}{|c|}{ Patient Diagnosis } \\
\hline IVF & $100 \%$ & Procedural Factors: & & Tubal factor & $6 \%$ & Other factor & $8 \%$ \\
\hline GIFT & $0 \%$ & With ICSI & $97 \%$ & Ovulatory dysfunction & $9 \%$ & Unknown factor & $25 \%$ \\
\hline ZIFT & $0 \%$ & Unstimulated & $0 \%$ & Diminished ovarian reserve & $16 \%$ & Multiple Factors: & \\
\hline \multirow[t]{3}{*}{ Combination } & $0 \%$ & Used gestational carrier & $2 \%$ & Endometriosis & $9 \%$ & Female factors only & $12 \%$ \\
\hline & & & & Uterine factor & $2 \%$ & Female \& male factors & $6 \%$ \\
\hline & & & & Male factor & $8 \%$ & & \\
\hline
\end{tabular}

\section{PREGNANCY SUCCESS RATES}

\section{Type of Cycle}

\section{Fresh Embryos from Nondonor Eggs}

Number of cycles

Percentage of cycles resulting in pregnancies ${ }^{b}$

Percentage of cycles resulting in live births ${ }^{b, c}$

(Confidence Interval)

Percentage of retrievals resulting in live births $s^{b, c}$

Percentage of transfers resulting in live births $s^{b, c}$

Percentage of transfers resulting in singleton live births ${ }^{b}$

Percentage of cancellations ${ }^{b}$

Average number of embryos transferred

Percentage of pregnancies with twins ${ }^{b}$

Percentage of pregnancies with triplets or more ${ }^{b}$

Percentage of live births having multiple infants ${ }^{b, c}$

\section{Frozen Embryos from Nondonor Eggs}

Number of transfers

Percentage of transfers resulting in live births $s^{b, c}$

Average number of embryos transferred

\section{Donor Eggs}

Number of transfers

Percentage of transfers resulting in live births $s^{b, c}$

Average number of embryos transferred

Data verified by Timothy N. Hickman, MD

\section{Age of Woman}

35-37 38-40 $41-42^{d}$

\section{$<35$}

76

42

27

54.5

52.6

33.3

25.9

50.8

47.4

28.6

14.8

$(41.9-59.6)$

$(35.8-59.2)$

$(15.7-44.6)$

(4.2-33.7)

51.5

48.0

29.3

29.3

14.8

51.5

48.6

19.5

14.8

26.9

35.1

2.4

7.4

1.5

1.3

3.4

0.0

2.5

44.4

22.5

$2 / 14$

3.9

1.4

5.0

$2 / 14$

$3 / 7$

47.8

27.8

4 / 12

$0 / 7$

$2 / 4$

14

$6 / 14$

2.6

\section{4}

$2 / 4$

2.5
1

$0 / 1$

3.0
2

$0 / 2$

2.5

All Ages Combined ${ }^{\mathrm{e}}$

Fresh Embryos 50

64.0

2.0
Frozen Embryos 5

$4 / 5$

2.6

\section{CURRENT CLINIC SERVICES AND PROFILE}

Current Name: Houston IVF

Donor egg? Yes

Donor embryo? Yes

Single women? Yes

$\begin{array}{ll}\text { Gestational carriers? } & \text { Yes } \\ \text { Cryopreservation? } & \text { Yes }\end{array}$

SART member?

Verified lab accreditation?

(See Appendix C for details.)

\footnotetext{
${ }^{a}$ Reflects patient and treatment characteristics of ART cycles performed in 2006 using fresh nondonor eggs or embryos.

b When fewer than 20 cycles are reported in an age category, rates are shown as a fraction and confidence intervals are not given. Calculating percentages from fractions may be misleading and is not encouraged.

${ }^{c}$ A multiple-infant birth is counted as one live birth.

d Clinic-specific outcome rates are unreliable for women older than 42 undergoing ART cycles using fresh or frozen embryos with nondonor eggs. Readers are urged to review national outcomes for these age groups (see page 27).

e All ages (including ages $>42$ ) are reported together because previous data show that patient age does not materially affect success with donor eggs.
} 


\section{NORTH HOUSTON CENTER FOR REPRODUCTIVE MEDICINE, PA \\ (NHCRM) \\ HOUSTON, TEXAS}

A comparison of clinic success rates may not be meaningful because patient medical characteristics and treatment approaches vary from clinic to clinic. For more details about this, along with information on how to interpret the statistics in this table, see pages $79-88$.

\section{ART CYCLE PROFILE}

\begin{tabular}{|c|c|c|c|c|c|c|c|}
\hline \multicolumn{4}{|c|}{ Type of ART } & \multicolumn{4}{|c|}{ Patient Diagnosis } \\
\hline IVF & $100 \%$ & Procedural Factors: & & Tubal factor & $1 \%$ & Other factor & $4 \%$ \\
\hline GIFT & $0 \%$ & With ICSI & $89 \%$ & Ovulatory dysfunction & $3 \%$ & Unknown factor & $9 \%$ \\
\hline ZIFT & $0 \%$ & Unstimulated & $0 \%$ & Diminished ovarian reserve & $0 \%$ & Multiple Factors: & \\
\hline \multirow[t]{3}{*}{ Combination } & $0 \%$ & Used gestational carrier & $0 \%$ & Endometriosis & $1 \%$ & Female factors only & $12 \%$ \\
\hline & & & & Uterine factor & $0 \%$ & Female \& male factors & $60 \%$ \\
\hline & & & & Male factor & $10 \%$ & & \\
\hline
\end{tabular}

2006 PREGNANCY SUCCESS RATES

Data verified by Dorothy J. Roach, MD

\section{Type of Cycle}

\section{Fresh Embryos from Nondonor Eggs}

Number of cycles

Percentage of cycles resulting in pregnancies ${ }^{b}$

Percentage of cycles resulting in live births ${ }^{b, c}$

(Confidence Interval)

Percentage of retrievals resulting in live births $s^{b, c}$

Percentage of transfers resulting in live births $s^{b, c}$

Percentage of transfers resulting in singleton live births ${ }^{b}$

Percentage of cancellations ${ }^{b}$

Average number of embryos transferred

Percentage of pregnancies with twins ${ }^{b}$

Percentage of pregnancies with triplets or more ${ }^{b}$

Percentage of live births having multiple infants ${ }^{b, c}$

\section{Frozen Embryos from Nondonor Eggs}

Number of transfers

Percentage of transfers resulting in live births $s^{b, c}$

Average number of embryos transferred

\section{Donor Eggs}

Number of transfers

Percentage of transfers resulting in live births $s^{b, c}$

Average number of embryos transferred

\section{Age of Woman}

35-37 38-40

$41-42^{d}$

$<35$

13

$8 / 13$

14

4

78.1

$7 / 13$

$5 / 14$

$2 / 4$

65.6

$4 / 14$

$1 / 4$

$(46.8-81.4)$

65.6

$7 / 13$

$4 / 13$

$1 / 4$

67.7

$7 / 13$

$4 / 12$

$1 / 4$

25.8

$4 / 13$

$2 / 12$

$1 / 4$

0.0

$0 / 13$

$1 / 14$

$0 / 4$

2.5

2.7

$3 / 8$

2.3

2.8

44.0

12.0

$0 / 8$

$2 / 5$

$0 / 2$

61.9

$3 / 7$

$0 / 5$

$0 / 2$

$2 / 4$

$0 / 1$

4

$1 / 4$

3.5

\begin{abstract}
1
\end{abstract}
$0 / 1$

2.0

\section{2}

$2 / 2$

3.5 


\section{THE WOMEN'S SPECIALISTS OF HOUSTON HOUSTON, TEXAS}

A comparison of clinic success rates may not be meaningful because patient medical characteristics and treatment approaches vary from clinic to clinic. For more details about this, along with information on how to interpret the statistics in this table, see pages 79-88.

\section{ART CYCLE PROFILE}

\begin{tabular}{|c|c|c|c|c|c|c|c|}
\hline \multicolumn{4}{|c|}{ Type of ART ${ }^{a}$} & \multicolumn{4}{|c|}{ Patient Diagnosis } \\
\hline IVF & $100 \%$ & Procedural Factors: & & Tubal factor & $4 \%$ & Other factor & $0 \%$ \\
\hline GIFT & $0 \%$ & With ICSI & $82 \%$ & Ovulatory dysfunction & $0 \%$ & Unknown factor & $0 \%$ \\
\hline ZIFT & $0 \%$ & Unstimulated & $0 \%$ & Diminished ovarian reserve & $0 \%$ & Multiple Factors: & \\
\hline \multirow[t]{3}{*}{ Combination } & $0 \%$ & Used gestational carrier & $12 \%$ & Endometriosis & $0 \%$ & Female factors only & $9 \%$ \\
\hline & & & & Uterine factor & $13 \%$ & Female \& male factors & $74 \%$ \\
\hline & & & & Male factor & $0 \%$ & & \\
\hline
\end{tabular}

2006 PREGNANCY SUCCESS RATES

Data verified by Michael J. Heard, MD

\section{Type of Cycle}

\section{Fresh Embryos from Nondonor Eggs}

Number of cycles

Percentage of cycles resulting in pregnancies ${ }^{b}$

Percentage of cycles resulting in live births ${ }^{b, c}$

(Confidence Interval)
Percentage of retrievals resulting in live births $s^{b, c}$

Percentage of transfers resulting in live births $s^{b, c}$

Percentage of transfers resulting in singleton live births ${ }^{b}$

Percentage of cancellations ${ }^{b}$

Average number of embryos transferred

Percentage of pregnancies with twins ${ }^{b}$

Percentage of pregnancies with triplets or more ${ }^{b}$

Percentage of live births having multiple infants ${ }^{b, c}$

\section{Frozen Embryos from Nondonor Eggs}

Number of transfers

Percentage of transfers resulting in live births $s^{b, c}$

Average number of embryos transferred

\section{Donor Eggs}

Number of transfers

Percentage of transfers resulting in live births $s^{b, c}$

Average number of embryos transferred

\section{Age of Woman}

$\begin{array}{llll}35-37 & 38-40 & 41-42^{\text {d }}\end{array}$

$\begin{array}{cccc}6 & 1 & 5 & 4 \\ 6 / 6 & 1 / 1 & 1 / 5 & 1 / 4 \\ 5 / 6 & 1 / 1 & 1 / 5 & 1 / 4\end{array}$

$5 / 6$

$1 / 1$

$1 / 5$

$1 / 4$

$5 / 6$

$1 / 1$

$1 / 5$

$1 / 3$

$3 / 6$

$1 / 1$

$0 / 5$

$0 / 3$

$0 / 6$

$0 / 1$

$0 / 5$

$0 / 4$

2.3

3.0

2.6

3.7

$2 / 6$

$0 / 1$

$1 / 1$

$1 / 1$

$0 / 6$

$0 / 1$

$0 / 1$

$0 / 1$

$2 / 5$

$0 / 1$

$1 / 1$

$1 / 1$

0

0

0

0

\section{CURRENT CLINIC SERVICES AND PROFILE}

Current Name: The Women's Specialists of Houston

Donor egg? Yes Gestational carriers? Yes

Donor embryo? Yes Cryopreservation? Yes

Single women? Yes

\begin{tabular}{cc}
\multicolumn{2}{c}{ All Ages Combined } \\
Fresh Embryos & $\begin{array}{c}\text { Frozen Embryos } \\
\text { Fros }^{2}\end{array}$ \\
$3 / 6$ & 0 \\
2.5 &
\end{tabular}

\footnotetext{
${ }^{a}$ Reflects patient and treatment characteristics of ART cycles performed in 2006 using fresh nondonor eggs or embryos.

${ }^{b}$ When fewer than 20 cycles are reported in an age category, rates are shown as a fraction and confidence intervals are not given. Calculating percentages from fractions may be misleading and is not encouraged.

${ }^{c}$ A multiple-infant birth is counted as one live birth.

${ }^{d}$ Clinic-specific outcome rates are unreliable for women older than 42 undergoing ART cycles using fresh or frozen embryos with nondonor eggs. Readers are urged to review national outcomes for these age groups (see page 27).

e All ages (including ages $>42$ ) are reported together because previous data show that patient age does not materially affect success with donor eggs.
} 


\section{ADVANCED REPRODUCTIVE CARE CENTER OF IRVING IRVING, TEXAS}

A comparison of clinic success rates may not be meaningful because patient medical characteristics and treatment approaches vary from clinic to clinic. For more details about this, along with information on how to interpret the statistics in this table, see pages $79-88$.

\section{ART CYCLE PROFILE}

\begin{tabular}{|c|c|c|c|c|c|c|c|}
\hline \multicolumn{4}{|c|}{ Type of ART } & \multicolumn{4}{|c|}{ Patient Diagnosis } \\
\hline IVF & $100 \%$ & Procedural Factors: & & Tubal factor & $15 \%$ & Other factor & $9 \%$ \\
\hline GIFT & $0 \%$ & With ICSI & $55 \%$ & Ovulatory dysfunction & $3 \%$ & Unknown factor & $11 \%$ \\
\hline ZIFT & $0 \%$ & Unstimulated & $0 \%$ & Diminished ovarian reserve & $10 \%$ & Multiple Factors: & \\
\hline \multirow[t]{3}{*}{ Combination } & $0 \%$ & Used gestational carrier & $1 \%$ & Endometriosis & $3 \%$ & Female factors only & $14 \%$ \\
\hline & & & & Uterine factor & $2 \%$ & Female \& male factors & $21 \%$ \\
\hline & & & & Male factor & $12 \%$ & & \\
\hline
\end{tabular}

2006 PREGNANCY SUCCESS RATES

Data verified by Sy Q. Le, MD

\section{Type of Cycle}

\section{Fresh Embryos from Nondonor Eggs}

Number of cycles

Percentage of cycles resulting in pregnancies ${ }^{b}$

Percentage of cycles resulting in live births $s^{b, c}$

(Confidence Interval)

Percentage of retrievals resulting in live births $s^{b, c}$

Percentage of transfers resulting in live births $s^{b, c}$

Percentage of transfers resulting in singleton live births ${ }^{b}$

Percentage of cancellations ${ }^{b}$

Average number of embryos transferred

Percentage of pregnancies with twins ${ }^{b}$

Percentage of pregnancies with triplets or more ${ }^{b}$

Percentage of live births having multiple infants ${ }^{b, c}$

\section{Frozen Embryos from Nondonor Eggs}

Number of transfers

Percentage of transfers resulting in live births $s^{b, c}$

Average number of embryos transferred

\section{Donor Eggs}

Number of transfers

Percentage of transfers resulting in live births ${ }^{\mathrm{b}, \mathrm{c}}$

Average number of embryos transferred

\section{Age of Woman}

35-37 38-40

$41-42^{d}$

\section{$<35$}

140

49.3

43.6

$(35.2-52.2)$

46.9

48.8

33.6

7.1

2.0

27.5

1.4

31.1

55

40.0

34

12

36.4

41.2

$3 / 12$

(23.8-50.4)

32.4

$3 / 12$

41.7

$(17.4-50.5)$

43.5

33.3

35.5

$3 / 10$

19.6

35.5

$3 / 10$

$3 / 10$

12.7

2.9

$2 / 12$

2.2

50.0

4.5

55.0

2.3

2.1

$0 / 14$

$1 / 3$

$0 / 14$

$0 / 3$

$0 / 11$

$0 / 3$

39

28.2

2.0

\section{7}

$2 / 17$

\section{9}

$1 / 9$

2

2.1

2.0

$2 / 2$

2.5

All Ages Combined

Fresh Embryos 23

73.9

2.0
Frozen Embryos

11

$3 / 11$

1.9

\section{CURRENT CLINIC SERVICES AND PROFILE}

Current Name: Advanced Reproductive Care Center of Irving

Donor egg? Yes Gestational carriers? Yes

Donor embryo? Yes Cryopreservation? Yes

Single women? Yes

SART member? Yes

Verified lab accreditation? Yes

(See Appendix C for details.)

${ }^{a}$ Reflects patient and treatment characteristics of ART cycles performed in 2006 using fresh nondonor eggs or embryos.

${ }^{b}$ When fewer than 20 cycles are reported in an age category, rates are shown as a fraction and confidence intervals are not given. Calculating percentages from fractions may be misleading and is not encouraged.

A multiple-infant birth is counted as one live birth.

d Clinic-specific outcome rates are unreliable for women older than 42 undergoing ART cycles using fresh or frozen embryos with nondonor eggs. Readers are urged to review national outcomes for these age groups (see page 27).

e All ages (including ages $>42$ ) are reported together because previous data show that patient age does not materially affect success with donor eggs. 


\section{WILFORD HALL MEDICAL CENTER LACKLAND AFB, TEXAS}

A comparison of clinic success rates may not be meaningful because patient medical characteristics and treatment approaches vary from clinic to clinic. For more details about this, along with information on how to interpret the statistics in this table, see pages 79-88.

\section{ART CYCLE PROFILE}

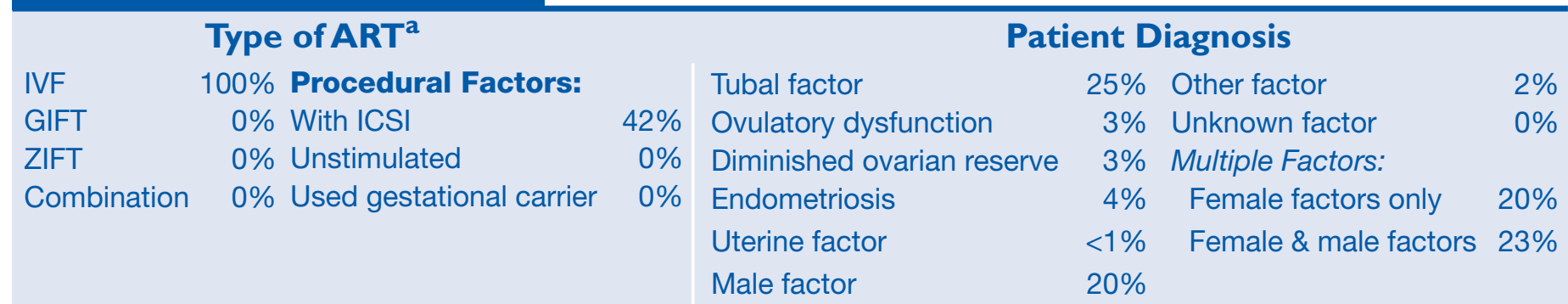

2006 PREGNANCY SUCCESS RATES

Data verified by Anthony M. Propst, MD

\section{Type of Cycle}

\section{Fresh Embryos from Nondonor Eggs}

Number of cycles

Percentage of cycles resulting in pregnancies ${ }^{b}$

Percentage of cycles resulting in live births $s^{b, c}$

(Confidence Interval)

Percentage of retrievals resulting in live births $s^{b, c}$

Percentage of transfers resulting in live births $s^{b, c}$

Percentage of transfers resulting in singleton live births ${ }^{b}$

Percentage of cancellations ${ }^{b}$

Average number of embryos transferred

Percentage of pregnancies with twins ${ }^{b}$

Percentage of pregnancies with triplets or more ${ }^{b}$

Percentage of live births having multiple infants ${ }^{\mathrm{b}, \mathrm{c}}$

\section{Frozen Embryos from Nondonor Eggs}

Number of transfers

Percentage of transfers resulting in live births $s^{b, c}$

Average number of embryos transferred

\section{Donor Eggs}

Number of transfers

Percentage of transfers resulting in live births $s^{b, c}$

Average number of embryos transferred

\section{Age of Woman}

35-37 38-40

$41-42^{d}$

$<35$

42

47.6

15

0

54.8

46.2

$(35.8-56.9)$

45.2

$8 / 15$

$5 / 15$

49.4

(29.8-61.3)

50.0

45.2

$5 / 13$

34.9

45.2

$5 / 13$

33.3

$5 / 13$

6.5

0.0

$2 / 15$

2.2

2.4

2.7

33.3

0.0

50.0

$1 / 8$

30.2

0.0

$0 / 8$

$5 / 19$

$0 / 5$
0

0

0

\section{CURRENT CLINIC SERVICES AND PROFILE}

Current Name: Wilford Hall Medical Center

Donor egg? No Gestational carriers? No

Donor embryo? No

Single women? No

Cryopreservation? Yes

\section{Fresh Embryos}

0
$\%$

$\%$

$20 \%$

$3 \%$ 


\section{TEXAS FERTILITY \\ LEWISVILLE, TEXAS}

A comparison of clinic success rates may not be meaningful because patient medical characteristics and treatment approaches vary from clinic to clinic. For more details about this, along with information on how to interpret the statistics in this table, see pages $79-88$.

\section{ART CYCLE PROFILE}

\begin{tabular}{|c|c|c|c|c|c|c|c|}
\hline \multicolumn{4}{|c|}{ Type of ART ${ }^{a}$} & \multicolumn{4}{|c|}{ Patient Diagnosis } \\
\hline IVF & $100 \%$ & Procedural Factors: & & Tubal factor & $12 \%$ & Other factor & $4 \%$ \\
\hline GIFT & $0 \%$ & With ICSI & $65 \%$ & Ovulatory dysfunction & $0 \%$ & Unknown factor & $0 \%$ \\
\hline $\mathrm{ZIFT}$ & $0 \%$ & Unstimulated & $0 \%$ & Diminished ovarian reserve & $0 \%$ & Multiple Factors: & \\
\hline \multirow[t]{3}{*}{ Combination } & $0 \%$ & Used gestational carrier & $0 \%$ & Endometriosis & $0 \%$ & Female factors only & $12 \%$ \\
\hline & & & & Uterine factor & $0 \%$ & Female \& male factors & $72 \%$ \\
\hline & & & & Male factor & $0 \%$ & & \\
\hline
\end{tabular}

2006 PREGNANCY SUCCESS RATES

Data verified by Barry R. Jacobs, MD

\section{Type of Cycle}

\section{Fresh Embryos from Nondonor Eggs}

Number of cycles

Percentage of cycles resulting in pregnancies ${ }^{b}$

Percentage of cycles resulting in live births $s^{b, c}$

(Confidence Interval)

Percentage of retrievals resulting in live births $s^{b, c}$

Percentage of transfers resulting in live births $s^{b, c}$

Percentage of transfers resulting in singleton live births ${ }^{b}$

Percentage of cancellations ${ }^{b}$

Average number of embryos transferred

Percentage of pregnancies with twins ${ }^{b}$

Percentage of pregnancies with triplets or more ${ }^{b}$

Percentage of live births having multiple infants ${ }^{b, c}$

\section{Frozen Embryos from Nondonor Eggs}

Number of transfers

Percentage of transfers resulting in live births ${ }^{\mathrm{b}, \mathrm{c}}$

Average number of embryos transferred

\section{Age of Woman}

$\begin{array}{cccc}<35 & 35-37 & 38-40 & \mathbf{4 1 - 4 2}^{\text {d }} \\ 17 & 2 & 1 & 0 \\ 5 / 17 & 1 / 2 & 0 / 1 & \\ 5 / 17 & 1 / 2 & 0 / 1 & \end{array}$

$$
41-42^{d}
$$

$\begin{array}{ccc}5 / 13 & 1 / 2 & 0 / 1 \\ 5 / 13 & 1 / 2 & 0 / 1 \\ 3 / 13 & 1 / 2 & 0 / 1 \\ 4 / 17 & 0 / 2 & 0 / 1 \\ 2.1 & 2.0 & 2.0 \\ 2 / 5 & 0 / 1 & \\ 0 / 5 & 0 / 1 & \\ 2 / 5 & 0 / 1\end{array}$

2

$1 / 2$

2.0
$0 / 1$

$0 / 1$

$0 / 1$

$0 / 1$

2.0
$0 / 5-0 / 1$

All Ages Combined ${ }^{\mathrm{e}}$

Fresh Embryos

Frozen Embryos

Donor Eggs

2

$0 / 2$

1

Percentage of transfers resulting in live births $s^{b, c}$

Average number of embryos transferred

$1 / 1$

2.0

\section{CURRENT CLINIC SERVICES AND PROFILE}

Current Name: Texas Fertility

Donor egg? Yes

Donor embryo? Yes

Single women? Yes

$\begin{array}{ll}\text { Gestational carriers? } & \text { Yes } \\ \text { Cryopreservation? } & \text { Yes }\end{array}$

${ }^{a}$ Reflects patient and treatment characteristics of ART cycles performed in 2006 using fresh nondonor eggs or embryos.

${ }^{b}$ When fewer than 20 cycles are reported in an age category, rates are shown as a fraction and confidence intervals are not given. Calculating percentages from fractions may be misleading and is not encouraged.

A multiple-infant birth is counted as one live birth.

d Clinic-specific outcome rates are unreliable for women older than 42 undergoing ART cycles using fresh or frozen embryos with nondonor eggs. Readers are urged to review national outcomes for these age groups (see page 27).

e All ages (including ages $>42$ ) are reported together because previous data show that patient age does not materially affect success with donor eggs. 


\section{CENTER FOR FERTILITY \& REPRODUCTIVE SURGERY \\ TEXAS TECH UNIVERSITY HEALTH SCIENCES CENTER \\ LUBBOCK, TEXAS}

A comparison of clinic success rates may not be meaningful because patient medical characteristics and treatment approaches vary from clinic to clinic. For more details about this, along with information on how to interpret the statistics in this table, see pages 79-88.

\section{ART CYCLE PROFILE}

\begin{tabular}{|c|c|c|c|c|c|c|c|}
\hline \multicolumn{4}{|c|}{ Type of ART ${ }^{a}$} & \multicolumn{4}{|c|}{ Patient Diagnosis } \\
\hline IVF & $100 \%$ & Procedural Factors: & & Tubal factor & $28 \%$ & Other factor & $0 \%$ \\
\hline GIFT & $0 \%$ & With ICSI & $47 \%$ & Ovulatory dysfunction & $0 \%$ & Unknown factor & $6 \%$ \\
\hline ZIFT & $0 \%$ & Unstimulated & $0 \%$ & Diminished ovarian reserve & $0 \%$ & Multiple Factors: & \\
\hline \multirow[t]{3}{*}{ Combination } & $0 \%$ & Used gestational carrier & $0 \%$ & Endometriosis & $22 \%$ & Female factors only & $0 \%$ \\
\hline & & & & Uterine factor & $0 \%$ & Female \& male factors & $6 \%$ \\
\hline & & & & Male factor & $39 \%$ & & \\
\hline
\end{tabular}

2006 PREGNANCY SUCCESS RATES

Data verified by Sami I. Jabara, MD

\section{Type of Cycle}

\section{Fresh Embryos from Nondonor Eggs}

Number of cycles

Percentage of cycles resulting in pregnancies ${ }^{b}$

Percentage of cycles resulting in live births ${ }^{b, c}$

(Confidence Interval)

Percentage of retrievals resulting in live births $s^{b, c}$

Percentage of transfers resulting in live births $s^{b, c}$

Percentage of transfers resulting in singleton live births ${ }^{b}$

Percentage of cancellations ${ }^{b}$

Average number of embryos transferred

Percentage of pregnancies with twins ${ }^{b}$

Percentage of pregnancies with triplets or more ${ }^{b}$

Percentage of live births having multiple infants ${ }^{b, c}$

\section{Frozen Embryos from Nondonor Eggs}

Number of transfers

Percentage of transfers resulting in live births ${ }^{b, c}$

Average number of embryos transferred

\section{Donor Eggs}

Number of transfers

Percentage of transfers resulting in live births $s^{b, c}$

Average number of embryos transferred

\section{Age of Woman}

$\begin{array}{cccc}<35 & 35-37 & 38-40 & \mathbf{4 1 - 4 2}^{\mathbf{d}} \\ 10 & 2 & 2 & 1 \\ 6 / 10 & 0 / 2 & 1 / 2 & 0 / 1 \\ 6 / 10 & 0 / 2 & 0 / 2 & 0 / 1\end{array}$

$\begin{array}{cccc}6 / 10 & 0 / 2 & 0 / 2 & 0 / 1 \\ 6 / 10 & 0 / 2 & 0 / 2 & 0 / 1 \\ 3 / 10 & 0 / 2 & 0 / 2 & 0 / 1 \\ 0 / 10 & 0 / 2 & 0 / 2 & 0 / 1 \\ 2.2 & 3.0 & 3.0 & 4.0 \\ 3 / 6 & & 0 / 1 & \\ 0 / 6 & & 0 / 1 & \\ 3 / 6 & & & \end{array}$

$3 / 6$

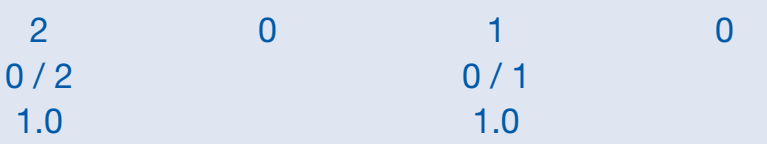

\section{All Ages Combined}

Fresh Embryos

Frozen Embryos

0

0

\section{CURRENT CLINIC SERVICES AND PROFILE}

Current Name: Center for Fertility \& Reproductive Surgery, Texas Tech University Health Sciences Center

Donor egg? No

Donor embryo? No

Single women? Yes

\begin{abstract}
Gestational carriers? No
Cryopreservation?
\end{abstract}

SART member?

Verified lab accreditation?

(See Appendix C for details.)

${ }^{a}$ Reflects patient and treatment characteristics of ART cycles performed in 2006 using fresh nondonor eggs or embryos.

${ }^{b}$ When fewer than 20 cycles are reported in an age category, rates are shown as a fraction and confidence intervals are not given. Calculating percentages from fractions may be misleading and is not encouraged.

${ }^{\mathrm{C}}$ A multiple-infant birth is counted as one live birth.

d Clinic-specific outcome rates are unreliable for women older than 42 undergoing ART cycles using fresh or frozen embryos with nondonor eggs. Readers are urged to review national outcomes for these age groups (see page 27).

e All ages (including ages $>42$ ) are reported together because previous data show that patient age does not materially affect success with donor eggs. 


\section{THE CENTRE FOR REPRODUCTIVE MEDICINE LUBBOCK, TEXAS}

A comparison of clinic success rates may not be meaningful because patient medical characteristics and treatment approaches vary from clinic to clinic. For more details about this, along with information on how to interpret the statistics in this table, see pages $79-88$.

\section{ART CYCLE PROFILE}

\begin{tabular}{|c|c|c|c|c|c|c|c|}
\hline \multicolumn{4}{|c|}{ Type of ART ${ }^{\mathrm{a}}$} & \multicolumn{4}{|c|}{ Patient Diagnosis } \\
\hline IVF & $100 \%$ & Procedural Factors: & & Tubal factor & $10 \%$ & Other factor & $4 \%$ \\
\hline GIFT & $0 \%$ & With ICSI & $14 \%$ & Ovulatory dysfunction & $4 \%$ & Unknown factor & $2 \%$ \\
\hline $\mathrm{ZIFT}$ & $0 \%$ & Unstimulated & $0 \%$ & Diminished ovarian reserve & $4 \%$ & Multiple Factors: & \\
\hline \multirow[t]{3}{*}{ Combination } & $0 \%$ & Used gestational carrier & $0 \%$ & Endometriosis & $9 \%$ & Female factors only & $28 \%$ \\
\hline & & & & Uterine factor & $0 \%$ & Female \& male factors & $27 \%$ \\
\hline & & & & Male factor & $12 \%$ & & \\
\hline
\end{tabular}

2006 PREGNANCY SUCCESS RATES

Data verified by Janelle O. Dorsett, MD

\section{Type of Cycle}

\section{Fresh Embryos from Nondonor Eggs}

Number of cycles

Percentage of cycles resulting in pregnancies ${ }^{b}$

Percentage of cycles resulting in live births ${ }^{b, c}$

(Confidence Interval)

Percentage of retrievals resulting in live births $s^{b, c}$

Percentage of transfers resulting in live births $s^{b, c}$

Percentage of transfers resulting in singleton live births ${ }^{b}$

Percentage of cancellations ${ }^{b}$

Average number of embryos transferred

Percentage of pregnancies with twins ${ }^{b}$

Percentage of pregnancies with triplets or more ${ }^{b}$

Percentage of live births having multiple infants ${ }^{b, c}$

\section{Frozen Embryos from Nondonor Eggs}

Number of transfers

Percentage of transfers resulting in live births ${ }^{\mathrm{b}, \mathrm{c}}$

Average number of embryos transferred

\section{Donor Eggs}

Number of transfers

Percentage of transfers resulting in live births $s^{b, c}$

Average number of embryos transferred

\section{Age of Woman}

35-37 38-40

$41-42^{d}$

$<35$

19

$8 / 19$

$7 / 19$

10

4

58.9

58.9

$7 / 10$

$1 / 4$

$(45.0-71.9)$

63.5

70.2

$7 / 17$

$7 / 10$

$0 / 4$

\section{1}

7.1

1.7

30.3

0.0

27.3

\section{$7 / 15$}

$6 / 15$

$2 / 19$

1.9

$1 / 8$

$0 / 8$

$1 / 7$

$7 / 10$

$7 / 8$

$0 / 4$

$7 / 8$

$0 / 3$

$0 / 3$

$0 / 10$

$0 / 4$

1.9

2.0

$0 / 7$

$0 / 1$

$1 / 1$

$0 / 7$

$0 / 7$

\section{7}

$1 / 7$

1.7

\section{4}

$1 / 4$

1.3

\section{3}

$0 / 3$

2.0

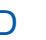




\section{REPRODUCTIVE INSTITUTE OF SOUTH TEXAS \\ MCALLEN, TEXAS}

A comparison of clinic success rates may not be meaningful because patient medical characteristics and treatment approaches vary from clinic to clinic. For more details about this, along with information on how to interpret the statistics in this table, see pages 79-88.

\section{ART CYCLE PROFILE}

\begin{tabular}{|c|c|c|c|c|c|c|c|}
\hline \multicolumn{4}{|c|}{ Type of ART ${ }^{a}$} & \multicolumn{4}{|c|}{ Patient Diagnosis } \\
\hline IVF & $100 \%$ & Procedural Factors: & & Tubal factor & $7 \%$ & Other factor & $1 \%$ \\
\hline GIFT & $0 \%$ & With ICSI & $51 \%$ & Ovulatory dysfunction & $3 \%$ & Unknown factor & $1 \%$ \\
\hline ZIFT & $0 \%$ & Unstimulated & $0 \%$ & Diminished ovarian reserve & $4 \%$ & Multiple Factors: & \\
\hline \multirow[t]{3}{*}{ Combination } & $0 \%$ & Used gestational carrier & $0 \%$ & Endometriosis & $0 \%$ & Female factors only & $30 \%$ \\
\hline & & & & Uterine factor & $0 \%$ & Female \& male factors & $41 \%$ \\
\hline & & & & Male factor & $11 \%$ & & \\
\hline
\end{tabular}

2006 PREGNANCY SUCCESS RATES

Data verified by Esteban O. Brown, MD

\section{Type of Cycle}

\section{Age of Woman}

35-37 38-40

$41-42^{d}$

\section{$<35$}

10

$4 / 10$

11

1

50.0

36.7

$3 / 10$

$5 / 11$

$0 / 1$

(19.9-56.1)

(Confidence Interval)

Percentage of retrievals resulting in live births $s^{b, c}$

Percentage of transfers resulting in live births $s^{b, c}$

36.7

42.3

$3 / 10$

$1 / 11$

$0 / 1$

Percentage of transfers resulting in singleton live births ${ }^{b}$

Percentage of cancellations ${ }^{b}$

7.7

0.0

$3 / 9$

$1 / 11$

$0 / 1$

$1 / 9$

$1 / 10$

$0 / 1$

3.3

$0 / 10$

$1 / 10$

$0 / 1$

Average number of embryos transferred

Percentage of pregnancies with twins ${ }^{b}$

Percentage of pregnancies with triplets or more ${ }^{b}$

Percentage of live births having multiple infants ${ }^{b, c}$

\section{Frozen Embryos from Nondonor Eggs}

Number of transfers

Percentage of transfers resulting in live births ${ }^{b, c}$

Average number of embryos transferred

\section{$7 / 15$}

$3 / 15$

3.4

$1 / 4$

$0 / 11$

$0 / 1$

$9 / 11$

$1 / 4$

3.4

1.0

$0 / 5$

$0 / 5$

$2 / 3$

$0 / 1$
8

$3 / 8$

3.3

\section{3}

$1 / 3$

3.3
1

$0 / 1$

4.0

\section{Donor Eggs}

Fresh Embryos

All Ages Combined

Number of transfers

Percentage of transfers resulting in live births $s^{b, c}$

Average number of embryos transferred

\section{3}

$1 / 3$

2.7
Frozen Embryos

2

$0 / 2$

2.0

\section{CURRENT CLINIC SERVICES AND PROFILE}

Current Name: Reproductive Institute of South Texas

Donor egg? Yes

Donor embryo? No

Single women? Yes

\section{Gestational carriers? Yes}

Cryopreservation? Yes
SART member?

Verified lab accreditation?

(See Appendix C for details.)
No

Yes

${ }^{a}$ Reflects patient and treatment characteristics of ART cycles performed in 2006 using fresh nondonor eggs or embryos.

b When fewer than 20 cycles are reported in an age category, rates are shown as a fraction and confidence intervals are not given. Calculating percentages from fractions may be misleading and is not encouraged.

${ }^{c}$ A multiple-infant birth is counted as one live birth.

d Clinic-specific outcome rates are unreliable for women older than 42 undergoing ART cycles using fresh or frozen embryos with nondonor eggs. Readers are urged to review national outcomes for these age groups (see page 27).

e All ages (including ages $>42$ ) are reported together because previous data show that patient age does not materially affect success with donor eggs. 


\section{DALLAS IVF \\ PLANO, TEXAS}

A comparison of clinic success rates may not be meaningful because patient medical characteristics and treatment approaches vary from clinic to clinic. For more details about this, along with information on how to interpret the statistics in this table, see pages $79-88$.

\section{ART CYCLE PROFILE}

\begin{tabular}{|c|c|c|c|c|c|c|c|}
\hline \multicolumn{4}{|c|}{ Type of ART ${ }^{\mathrm{a}}$} & \multicolumn{4}{|c|}{ Patient Diagnosis } \\
\hline IVF & $100 \%$ & Procedural Factors: & & Tubal factor & $4 \%$ & Other factor & $6 \%$ \\
\hline GIFT & $0 \%$ & With ICSI & $47 \%$ & Ovulatory dysfunction & $7 \%$ & Unknown factor & $10 \%$ \\
\hline $\mathrm{ZIFT}$ & $0 \%$ & Unstimulated & $0 \%$ & Diminished ovarian reserve & $17 \%$ & Multiple Factors: & \\
\hline Combination & $0 \%$ & Used gestational carrier & $<1 \%$ & Endometriosis & $2 \%$ & Female factors only & $4 \%$ \\
\hline & & & & Uterine factor & $0 \%$ & Female \& male factors & $30 \%$ \\
\hline
\end{tabular}

2006 PREGNANCY SUCCESS RATES

Data verified by Brian D. Barnett, MD

\section{Type of Cycle}

\section{Fresh Embryos from Nondonor Eggs}

Number of cycles

Percentage of cycles resulting in pregnancies ${ }^{b}$

Percentage of cycles resulting in live births ${ }^{b, c}$

(Confidence Interval)

Percentage of retrievals resulting in live births $s^{b, c}$

Percentage of transfers resulting in live births $s^{b, c}$

Percentage of transfers resulting in singleton live births ${ }^{b}$

Percentage of cancellations ${ }^{b}$

Average number of embryos transferred

Percentage of pregnancies with twins ${ }^{b}$

Percentage of pregnancies with triplets or more ${ }^{b}$

Percentage of live births having multiple infants ${ }^{b, c}$

\section{Frozen Embryos from Nondonor Eggs}

Number of transfers

Percentage of transfers resulting in live births $s^{b, c}$

Average number of embryos transferred

\section{Donor Eggs}

Number of transfers

Percentage of transfers resulting in live births $s^{b, c}$

Average number of embryos transferred

\section{Age of Woman}

35-37 38-40

$41-42^{d}$

$<35$

51
52.9
49.0

41

20

67.8

63.5

49.0

41.5

25.0

(54.0-72.3)

(34.8-63.4)

29.3

20.0

66.4

51.0

(16.1-45.5)

(5.7-43.7)

67.6

51.0

33.3

$4 / 19$

47.2

36.7

36.4

$4 / 19$

24.2

$2 / 19$

4.3

3.9

12.2

5.0

2.0

2.3

33.3

40.7

0.0

0.0

30.1

28.0

2.7

3.0

$6 / 17$

$2 / 5$

$0 / 17$

$1 / 5$

$4 / 12$

$2 / 4$

17

$10 / 17$

1.6

\section{5}

$5 / 5$

1.6

8
$3 / 8$
1.4

1

$1 / 1$

1.0

All Ages Combined ${ }^{\mathrm{e}}$

Fresh Embryos

13

$10 / 13$

2.0
Frozen Embryos

\section{3}

$3 / 3$

2.0

\section{CURRENT CLINIC SERVICES AND PROFILE}

Current Name: Dallas IVF

Donor egg? Yes

Donor embryo? Yes

Single women? No

$\begin{array}{ll}\text { Gestational carriers? } & \text { Yes } \\ \text { Cryopreservation? } & \text { Yes }\end{array}$

\section{SART member?}

Verified lab accreditation?

(See Appendix C for details.)

${ }^{a}$ Reflects patient and treatment characteristics of ART cycles performed in 2006 using fresh nondonor eggs or embryos.

${ }^{b}$ When fewer than 20 cycles are reported in an age category, rates are shown as a fraction and confidence intervals are not given. Calculating percentages from fractions may be misleading and is not encouraged.

A multiple-infant birth is counted as one live birth.

d Clinic-specific outcome rates are unreliable for women older than 42 undergoing ART cycles using fresh or frozen embryos with nondonor eggs. Readers are urged to review national outcomes for these age groups (see page 27).

e All ages (including ages $>42$ ) are reported together because previous data show that patient age does not materially affect success with donor eggs. 


\section{FERTILITY CENTER OF SAN ANTONIO SAN ANTONIO, TEXAS}

A comparison of clinic success rates may not be meaningful because patient medical characteristics and treatment approaches vary from clinic to clinic. For more details about this, along with information on how to interpret the statistics in this table, see pages 79-88.

\section{ART CYCLE PROFILE}

\begin{tabular}{|c|c|c|c|c|c|c|c|}
\hline \multicolumn{4}{|c|}{ Type of ART ${ }^{a}$} & \multicolumn{4}{|c|}{ Patient Diagnosis } \\
\hline IVF & $100 \%$ & Procedural Factors: & & Tubal factor & $12 \%$ & Other factor & $5 \%$ \\
\hline GIFT & $0 \%$ & With ICSI & $64 \%$ & Ovulatory dysfunction & $7 \%$ & Unknown factor & $7 \%$ \\
\hline ZIFT & $0 \%$ & Unstimulated & $0 \%$ & Diminished ovarian reserve & $7 \%$ & Multiple Factors: & \\
\hline \multirow[t]{3}{*}{ Combination } & $0 \%$ & Used gestational carrier & $4 \%$ & Endometriosis & $5 \%$ & Female factors only & $13 \%$ \\
\hline & & & & Uterine factor & $3 \%$ & Female \& male factors & $17 \%$ \\
\hline & & & & Male factor & $24 \%$ & & \\
\hline
\end{tabular}

2006 PREGNANCY SUCCESS RATES

Data verified by Joseph E. Martin, MD

\section{Type of Cycle}

\section{$<35$}

163

52.1

44.2

(36.4-52.1)

47.4

50.7

30.3

6.7

2.1

38.8

3.5

40.3

86

36.0

1.8

\section{Frozen Embryos from Nondonor Eggs}

Number of transfers

Percentage of transfers resulting in live births ${ }^{\mathrm{b}, \mathrm{c}}$

Average number of embryos transferred

\section{Fresh Embryos}

Age of Woman

35-37 $38-40 \quad 41-42^{d}$

\section{Donor Eggs}

$10 / 16$

80

36.3

60

31

33.3

29.0

Number of transfers

Percentage of transfers resulting in live births $s^{b, c}$

Average number of embryos transferred
23.8

$(14.9-34.6)$
28.4

21.7

2.1

25.0

28.9

24.4

13.3

2.6

10.0

0.0

$2 / 13$

12.9

(3.6-29.8)

16.0

18.2

18.2

19.4

2.8

$0 / 9$

$0 / 9$

0.0

$8 / 19$

63

27.0

24

25.0

7

1.8

1.8

$3 / 7$

1.4

All Ages Combined ${ }^{\mathrm{e}}$

Frozen Embryos

16
$7 / 16$
1.8

(12.1-34.2)

\section{CURRENT CLINIC SERVICES AND PROFILE}

Current Name: Fertility Center of San Antonio

\begin{tabular}{ll|ll|l|l} 
Donor egg? & Yes & Gestational carriers? & Yes & SART member? & Yes \\
\hline Donor embryo? & No & Cryopreservation? & Yes & Verified lab accreditation? & Yes \\
\hline Single women? & Yes & & & (See Appendix C for details.) &
\end{tabular}

\footnotetext{
${ }^{a}$ Reflects patient and treatment characteristics of ART cycles performed in 2006 using fresh nondonor eggs or embryos.

${ }^{b}$ When fewer than 20 cycles are reported in an age category, rates are shown as a fraction and confidence intervals are not given. Calculating percentages from fractions may be misleading and is not encouraged.

${ }^{c}$ A multiple-infant birth is counted as one live birth.

${ }^{d}$ Clinic-specific outcome rates are unreliable for women older than 42 undergoing ART cycles using fresh or frozen embryos with nondonor eggs. Readers are urged to review national outcomes for these age groups (see page 27).

e All ages (including ages $>42$ ) are reported together because previous data show that patient age does not materially affect success with donor eggs.
} 


\section{INSTITUTE FOR WOMEN'S HEALTH \\ ADVANCED FERTILITY LABORATORY \\ SAN ANTONIO, TEXAS}

A comparison of clinic success rates may not be meaningful because patient medical characteristics and treatment approaches vary from clinic to clinic. For more details about this, along with information on how to interpret the statistics in this table, see pages $79-88$.

\section{ART CYCLE PROFILE}

\begin{tabular}{|c|c|c|c|c|c|c|c|}
\hline \multicolumn{4}{|c|}{ Type of $\mathrm{ART}^{\mathrm{a}}$} & \multicolumn{4}{|c|}{ Patient Diagnosis } \\
\hline IVF & $100 \%$ & Procedural Factors: & & Tubal factor & $23 \%$ & Other factor & $8 \%$ \\
\hline GIFT & $0 \%$ & With ICSI & $75 \%$ & Ovulatory dysfunction & $15 \%$ & Unknown factor & 29 \\
\hline $\mathrm{ZIFT}$ & $0 \%$ & Unstimulated & $0 \%$ & Diminished ovarian reserve & $2 \%$ & Multiple Factors: & \\
\hline \multirow[t]{3}{*}{ Combination } & $0 \%$ & Used gestational carrier & $2 \%$ & Endometriosis & $5 \%$ & Female factors only & 139 \\
\hline & & & & Uterine factor & $3 \%$ & Female \& male factors & $13 \%$ \\
\hline & & & & Male factor & $15 \%$ & & \\
\hline
\end{tabular}

2006 PREGNANCY SUCCESS RATES

Data verified by Joseph R. Garza, MD

\section{Type of Cycle}

\section{Fresh Embryos from Nondonor Eggs}

Number of cycles

Percentage of cycles resulting in pregnancies ${ }^{b}$

Percentage of cycles resulting in live births ${ }^{b, c}$

(Confidence Interval)

Percentage of retrievals resulting in live births $s^{b, c}$

Percentage of transfers resulting in live births $s^{b, c}$

Percentage of transfers resulting in singleton live births ${ }^{b}$

Percentage of cancellations ${ }^{b}$

Average number of embryos transferred

Percentage of pregnancies with twins ${ }^{b}$

Percentage of pregnancies with triplets or more ${ }^{\mathrm{b}}$

Percentage of live births having multiple infants ${ }^{b, c}$

\section{Frozen Embryos from Nondonor Eggs}

Number of transfers

Percentage of transfers resulting in live births $s^{\mathrm{b}, \mathrm{c}}$

Average number of embryos transferred

\section{Donor Eggs}

Number of transfers

Percentage of transfers resulting in live births ${ }^{\mathrm{b}, \mathrm{c}}$

Average number of embryos transferred

\section{Age of Woman}

35-37 38-40

$41-42^{d}$

\section{$<35$}

9

13

4

$\begin{array}{cccc}18 & 9 & 13 & 4 \\ 6 / 18 & 4 / 9 & 6 / 13 & 0 / 4 \\ 5 / 18 & 4 / 9 & 5 / 13 & 0 / 4\end{array}$

$5 / 14$

$4 / 8$

$5 / 9$

$0 / 1$

$5 / 14$

$4 / 8$

$5 / 9$

$0 / 1$

$3 / 14$

$3 / 8$

$5 / 9$

$0 / 1$

4/ 18

$1 / 9$

$4 / 13$

$3 / 4$

3.1

3.3

3.4

5.0

$2 / 6$

$1 / 4$

$0 / 6$

$0 / 6$

$0 / 4$

$0 / 6$

$2 / 5$

$1 / 4$

$0 / 5$

3

$0 / 3$

1.7

\section{5}

$1 / 5$

2.0
1

$0 / 1$

4.0
1

$0 / 1$

2.0

All Ages Combined ${ }^{\mathrm{e}}$

Fresh Embryos

1

$0 / 1$

3.0

\section{CURRENT CLINIC SERVICES AND PROFILE}

Current Name: Institute for Women's Health, Advanced Fertility Laboratory

Donor egg? Yes

Donor embryo? Yes

Single women? Yes

\begin{abstract}
Gestational carriers? Yes
Cryopreservation? Yes
\end{abstract}

Frozen Embryos

0

${ }^{a}$ Reflects patient and treatment characteristics of ART cycles performed in 2006 using fresh nondonor eggs or embryos.

${ }^{\mathrm{b}}$ When fewer than 20 cycles are reported in an age category, rates are shown as a fraction and confidence intervals are not given. Calculating percentages from fractions may be misleading and is not encouraged.

A multiple-infant birth is counted as one live birth.

d Clinic-specific outcome rates are unreliable for women older than 42 undergoing ART cycles using fresh or frozen embryos with nondonor eggs. Readers are urged to review national outcomes for these age groups (see page 27).

e All ages (including ages $>42$ ) are reported together because previous data show that patient age does not materially affect success with donor eggs. 


\section{PERINATAL AND FERTILITY SPECIALISTS, PA SAN ANTONIO, TEXAS}

A comparison of clinic success rates may not be meaningful because patient medical characteristics and treatment approaches vary from clinic to clinic. For more details about this, along with information on how to interpret the statistics in this table, see pages 79-88.

\section{ART CYCLE PROFILE}

\begin{tabular}{l|l|lrlrl}
\multicolumn{3}{c}{ Type of ART } & \multicolumn{4}{c}{ Patient Diagnosis } \\
IVF & $100 \%$ Procedural Factors: & & Tubal factor & $13 \%$ & Other factor & $0 \%$ \\
GIFT & $0 \%$ With ICSI & $80 \%$ & Ovulatory dysfunction & $0 \%$ & Unknown factor & $0 \%$ \\
ZIFT & $0 \%$ Unstimulated & $0 \%$ & Diminished ovarian reserve & $13 \%$ & Multiple Factors: & \\
Combination & $0 \%$ Used gestational carrier & $0 \%$ & Endometriosis & $25 \%$ & Female factors only & $38 \%$ \\
& & & Uterine factor & $0 \%$ & Female \& male factors & $13 \%$ \\
& & & Male factor & $0 \%$ & &
\end{tabular}

2006 PREGNANCY SUCCESS RATES

Data verified by Gerard M. Honore, MD, PhD

\section{Type of Cycle}

\section{Fresh Embryos from Nondonor Eggs}

Number of cycles

Percentage of cycles resulting in pregnancies ${ }^{b}$

Percentage of cycles resulting in live births ${ }^{b, c}$

(Confidence Interval)

Percentage of retrievals resulting in live births $s^{b, c}$

Percentage of transfers resulting in live births $s^{b, c}$

Percentage of transfers resulting in singleton live births ${ }^{b}$

Percentage of cancellations ${ }^{b}$

Average number of embryos transferred

Percentage of pregnancies with twins ${ }^{b}$

Percentage of pregnancies with triplets or more ${ }^{b}$

Percentage of live births having multiple infants ${ }^{b, c}$

\section{Frozen Embryos from Nondonor Eggs}

Number of transfers

Percentage of transfers resulting in live births ${ }^{b, c}$

Average number of embryos transferred

\section{Donor Eggs}

Number of transfers

Percentage of transfers resulting in live births $s^{b, c}$

Average number of embryos transferred

\section{Age of Woman}

$<35 \quad 35-37 \quad 38-40 \quad 41-42^{\text {d }}$

$\begin{array}{cccc}4 & 1 & 0 & 0 \\ 3 / 4 & 0 / 1 & & \\ 3 / 4 & 0 / 1 & & \end{array}$

$\begin{array}{ll}3 / 3 & 0 / 1 \\ 3 / 3 & 0 / 1 \\ 3 / 3 & 0 / 1 \\ 1 / 4 & 0 / 1 \\ 3.3 & 4.0 \\ 0 / 3 & \\ 0 / 3 & \\ 0 / 3 & \end{array}$

1

1

$0 / 1$

3.0

3.0

$0 / 1$

$0 / 1$

$0 / 1$

$0 / 1$

4.0

\section{All Ages Combined ${ }^{\mathrm{e}}$}

Fresh Embryos

1

$1 / 1$

3.0

\section{CURRENT CLINIC SERVICES AND PROFILE}

Current Name: Perinatal and Fertility Specialists, PA

Donor egg? Yes Gestational carriers? Yes

Donor embryo? Yes Cryopreservation? Yes

Single women? Yes

SART member? No

Verified lab accreditation?

(See Appendix C for details.)

${ }^{a}$ Reflects patient and treatment characteristics of ART cycles performed in 2006 using fresh nondonor eggs or embryos.

${ }^{b}$ When fewer than 20 cycles are reported in an age category, rates are shown as a fraction and confidence intervals are not given. Calculating percentages from fractions may be misleading and is not encouraged.

${ }^{c}$ A multiple-infant birth is counted as one live birth.

d Clinic-specific outcome rates are unreliable for women older than 42 undergoing ART cycles using fresh or frozen embryos with nondonor eggs. Readers are urged to review national outcomes for these age groups (see page 27).

e All ages (including ages $>42$ ) are reported together because previous data show that patient age does not materially affect success with donor eggs. 


\section{SOUTH TEXAS FERTILITY CENTER \\ SAN ANTONIO, TEXAS}

A comparison of clinic success rates may not be meaningful because patient medical characteristics and treatment approaches vary from clinic to clinic. For more details about this, along with information on how to interpret the statistics in this table, see pages 79-88.

\section{ART CYCLE PROFILE}

\begin{tabular}{|c|c|c|c|c|c|c|c|}
\hline \multicolumn{4}{|c|}{ Type of ART ${ }^{\mathrm{a}}$} & \multicolumn{4}{|c|}{ Patient Diagnosis } \\
\hline IVF & $100 \%$ & Procedural Factors: & & Tubal factor & $20 \%$ & Other factor & $2 \%$ \\
\hline GIFT & $0 \%$ & With ICSI & $20 \%$ & Ovulatory dysfunction & $6 \%$ & Unknown factor & $21 \%$ \\
\hline $\mathrm{ZIFT}$ & $0 \%$ & Unstimulated & $0 \%$ & Diminished ovarian reserve & $8 \%$ & Multiple Factors: & \\
\hline Combination & $0 \%$ & Used gestational carrier & $0 \%$ & Endometriosis & $4 \%$ & Female factors only & $8 \%$ \\
\hline & & & & $\begin{array}{l}\text { Uterine factor } \\
\text { Male factor }\end{array}$ & $\begin{array}{l}<1 \% \\
15 \%\end{array}$ & Female \& male factors & $16 \%$ \\
\hline
\end{tabular}

2006 PREGNANCY SUCCESS RATES

\section{Type of Cycle}

\section{Fresh Embryos from Nondonor Eggs}

Number of cycles

Percentage of cycles resulting in pregnancies ${ }^{b}$

Percentage of cycles resulting in live births ${ }^{b, c}$

(Confidence Interval)

Percentage of retrievals resulting in live births $s^{b, c}$

Percentage of transfers resulting in live births $s^{b, c}$

Percentage of transfers resulting in singleton live births ${ }^{b}$

Percentage of cancellations ${ }^{b}$

Average number of embryos transferred

Percentage of pregnancies with twins ${ }^{b}$

Percentage of pregnancies with triplets or more ${ }^{b}$

Percentage of live births having multiple infants ${ }^{\mathrm{b}, \mathrm{c}}$

\section{Frozen Embryos from Nondonor Eggs}

Number of transfers

Percentage of transfers resulting in live births $s^{b, c}$

Average number of embryos transferred

\section{Donor Eggs}

Number of transfers

Percentage of transfers resulting in live births ${ }^{\mathrm{b}, \mathrm{c}}$

Average number of embryos transferred

Data verified by Robert G. Brzyski, MD, PhD

\section{Age of Woman}

35-37 38-40

$41-42^{d}$

$<35$

16

$4 / 16$

8

6

25.7

$3 / 16$

$2 / 8$

$0 / 6$

22.9

$2 / 8$

$0 / 6$

$(10.4-40.1)$

26.7

28.6

$3 / 15$

$2 / 6$

$0 / 3$

14.3

$3 / 13$

$2 / 6$

$0 / 3$

14.3

$3 / 13$

$2 / 6$

$0 / 3$

2.2

$1 / 16$

$2 / 8$

$3 / 6$

$4 / 9$

2.2

2.8

2.0

$0 / 9$

$0 / 4$

$1 / 2$

$4 / 8$

$0 / 4$

$0 / 2$

$0 / 3$

$0 / 2$

12

$4 / 12$

\section{5}

2.3

$1 / 5$

2.6

\section{1}

$0 / 1$

2.0

4

$0 / 4$

2.5

All Ages Combined

Fresh Embryos

5

$1 / 5$

2.2
Frozen Embryos

\section{8}

$1 / 8$

2.0

\section{CURRENT CLINIC SERVICES AND PROFILE}

Current Name: South Texas Fertility Center, University of Texas Health Science Center-San Antonio

Donor egg? Yes

Donor embryo? Yes

Single women? Yes

\section{Gestational carriers? \\ Yes}

Cryopreservation?
Yes
SART member?

Verified lab accreditation?

(See Appendix C for details.)

${ }^{a}$ Reflects patient and treatment characteristics of ART cycles performed in 2006 using fresh nondonor eggs or embryos.

${ }^{b}$ When fewer than 20 cycles are reported in an age category, rates are shown as a fraction and confidence intervals are not given. Calculating percentages from fractions may be misleading and is not encouraged.

A multiple-infant birth is counted as one live birth.

d Clinic-specific outcome rates are unreliable for women older than 42 undergoing ART cycles using fresh or frozen embryos with nondonor eggs. Readers are urged to review national outcomes for these age groups (see page 27).

e All ages (including ages $>42$ ) are reported together because previous data show that patient age does not materially affect success with donor eggs. 


\section{HOUSTON FERTILITY INSTITUTE \\ TOMBALL, TEXAS}

A comparison of clinic success rates may not be meaningful because patient medical characteristics and treatment approaches vary from clinic to clinic. For more details about this, along with information on how to interpret the statistics in this table, see pages 79-88.

\section{ART CYCLE PROFILE}

\begin{tabular}{|c|c|c|c|c|c|c|c|}
\hline \multicolumn{4}{|c|}{ Type of ART ${ }^{a}$} & \multicolumn{4}{|c|}{ Patient Diagnosis } \\
\hline IVF & $100 \%$ & Procedural Factors: & & Tubal factor & $11 \%$ & Other factor & $3 \%$ \\
\hline GIFT & $0 \%$ & With ICSI & $95 \%$ & Ovulatory dysfunction & $7 \%$ & Unknown factor & $15 \%$ \\
\hline ZIFT & $0 \%$ & Unstimulated & $0 \%$ & Diminished ovarian reserve & $17 \%$ & Multiple Factors: & \\
\hline \multirow[t]{3}{*}{ Combination } & $0 \%$ & Used gestational carrier & $1 \%$ & Endometriosis & $5 \%$ & Female factors only & $14 \%$ \\
\hline & & & & Uterine factor & $2 \%$ & Female \& male factors & $12 \%$ \\
\hline & & & & Male factor & $15 \%$ & & \\
\hline
\end{tabular}

2006 PREGNANCY SUCCESS RATES

Data verified by Inderbir S. Gill, MD

\section{Type of Cycle}

\section{Fresh Embryos from Nondonor Eggs}

Number of cycles

Percentage of cycles resulting in pregnancies ${ }^{b}$

Percentage of cycles resulting in live births $s^{b, c}$

(Confidence Interval)

Percentage of retrievals resulting in live births $s^{b, c}$

Percentage of transfers resulting in live births $s^{b, c}$

Percentage of transfers resulting in singleton live births ${ }^{b}$

Percentage of cancellations ${ }^{b}$

Average number of embryos transferred

Percentage of pregnancies with twins ${ }^{b}$

Percentage of pregnancies with triplets or more ${ }^{b}$

Percentage of live births having multiple infants ${ }^{b, c}$

\section{Frozen Embryos from Nondonor Eggs}

Number of transfers

Percentage of transfers resulting in live births ${ }^{b, c}$

Average number of embryos transferred

\section{Donor Eggs}

Number of transfers

Percentage of transfers resulting in live births $s^{b, c}$

Average number of embryos transferred

\section{Age of Woman}

35-37 38-40

$41-42^{d}$

\section{$<35$}

53

60.4

34

11

52.1

45.8

54.7

23.5

$2 / 11$

(37.4-54.3)

$(40.4-68.4)$

14.7

$0 / 11$

47.1

48.9

55.8

(5.0-31.1)

16.1

$0 / 10$

58.0

16.1

$0 / 7$

29.3

42.0

9.7

$0 / 7$

2.8

1.9

8.8

$1 / 11$

2.8

3.0

25.0

9.4

27.6

3.1

3.3

23.0

40.0

$2 / 8$

$0 / 2$

$1 / 8$

$0 / 2$

$2 / 5$

34

35.3

2.8
12

\section{8}

$2 / 8$

3.1

3.0

All Ages Combined ${ }^{\mathrm{e}}$

Fresh Embryos

47

51.1

2.9
Frozen Embryos 8

$4 / 8$

3.1

\section{CURRENT CLINIC SERVICES AND PROFILE}

Current Name: Houston Fertility Institute

Donor egg? Yes Gestational carriers? Yes

Donor embryo? Yes Cryopreservation? Yes

Single women? Yes

SART member? Yes

Verified lab accreditation?

(See Appendix C for details.)

${ }^{a}$ Reflects patient and treatment characteristics of ART cycles performed in 2006 using fresh nondonor eggs or embryos.

${ }^{b}$ When fewer than 20 cycles are reported in an age category, rates are shown as a fraction and confidence intervals are not given. Calculating percentages from fractions may be misleading and is not encouraged.

${ }^{c}$ A multiple-infant birth is counted as one live birth.

d Clinic-specific outcome rates are unreliable for women older than 42 undergoing ART cycles using fresh or frozen embryos with nondonor eggs. Readers are urged to review national outcomes for these age groups (see page 27).

e All ages (including ages $>42$ ) are reported together because previous data show that patient age does not materially affect success with donor eggs. 


\section{CENTER OF REPRODUCTIVE MEDICINE (CORM) \\ WEBSTER, TEXAS}

A comparison of clinic success rates may not be meaningful because patient medical characteristics and treatment approaches vary from clinic to clinic. For more details about this, along with information on how to interpret the statistics in this table, see pages $79-88$.

\section{ART CYCLE PROFILE}

\begin{tabular}{|c|c|c|c|c|c|c|c|}
\hline \multicolumn{4}{|c|}{ Type of ART ${ }^{a}$} & \multicolumn{4}{|c|}{ Patient Diagnosis } \\
\hline IVF & $100 \%$ & Procedural Factors: & & Tubal factor & $20 \%$ & Other factor & $7 \%$ \\
\hline GIFT & $0 \%$ & With ICSI & $62 \%$ & Ovulatory dysfunction & $16 \%$ & Unknown factor & $1 \%$ \\
\hline ZIFT & $0 \%$ & Unstimulated & $0 \%$ & Diminished ovarian reserve & $24 \%$ & Multiple Factors: & \\
\hline \multirow[t]{3}{*}{ Combination } & $0 \%$ & Used gestational carrier & $1 \%$ & Endometriosis & $11 \%$ & Female factors only & $0 \%$ \\
\hline & & & & Uterine factor & $5 \%$ & Female \& male factors & $0 \%$ \\
\hline & & & & Male factor & $18 \%$ & & \\
\hline
\end{tabular}

2006 PREGNANCY SUCCESS RATES

Data verified by Vicki L. Schnell, MD

\section{Type of Cycle}

\section{Fresh Embryos from Nondonor Eggs}

Number of cycles

Percentage of cycles resulting in pregnancies ${ }^{b}$

Percentage of cycles resulting in live births $s^{b, c}$

(Confidence Interval)

Percentage of retrievals resulting in live births $s^{b, c}$

Percentage of transfers resulting in live births $s^{b, c}$

Percentage of transfers resulting in singleton live births ${ }^{b}$

Percentage of cancellations ${ }^{b}$

Average number of embryos transferred

Percentage of pregnancies with twins ${ }^{b}$

Percentage of pregnancies with triplets or more ${ }^{b}$

Percentage of live births having multiple infants ${ }^{b, c}$

\section{Frozen Embryos from Nondonor Eggs}

Number of transfers

Percentage of transfers resulting in live births ${ }^{\mathrm{b}, \mathrm{c}}$

Average number of embryos transferred

\section{Donor Eggs}

Number of transfers

Percentage of transfers resulting in live births $s^{b, c}$

Average number of embryos transferred

\section{Age of Woman}

35-37 38-40

$41-42^{d}$

$<35$

28

50.0

42.9

20

10

30.5

29.3

(24.5-62.8)

20.0

$2 / 10$

(19.7-40.4)

32.4

44.4

15.0

$2 / 10$

32.9

44.4

(3.2-37.9)

$3 / 17$

$2 / 7$

20.5

29.6

$3 / 16$

$2 / 7$

9.8

3.6

$3 / 16$

$2 / 7$

2.2

2.2

15.0

$3 / 10$

36.0

$5 / 14$

8.0

$1 / 14$

37.5

$4 / 12$

3.0

4.1

$1 / 4$

$0 / 2$

$0 / 4$

$0 / 2$

$0 / 3$

$0 / 2$

31

25.8

2.6
14

$4 / 14$

2.7

\section{2}

$1 / 2$

3.0 


\section{REPRODUCTIVE CARE CENTER \\ SALT LAKE CITY, UTAH}

A comparison of clinic success rates may not be meaningful because patient medical characteristics and treatment approaches vary from clinic to clinic. For more details about this, along with information on how to interpret the statistics in this table, see pages 79-88.

\section{ART CYCLE PROFILE}

\begin{tabular}{|c|c|c|c|c|c|c|c|}
\hline \multicolumn{4}{|c|}{ Type of ART ${ }^{a}$} & \multicolumn{4}{|c|}{ Patient Diagnosis } \\
\hline IVF & $100 \%$ & Procedural Factors: & & Tubal factor & $10 \%$ & Other factor & $<1 \%$ \\
\hline GIFT & $0 \%$ & With ICSI & $47 \%$ & Ovulatory dysfunction & $9 \%$ & Unknown factor & $6 \%$ \\
\hline ZIFT & $0 \%$ & Unstimulated & $0 \%$ & Diminished ovarian reserve & $4 \%$ & Multiple Factors: & \\
\hline \multirow[t]{3}{*}{ Combination } & $0 \%$ & Used gestational carrier & $0 \%$ & Endometriosis & $8 \%$ & Female factors only & $9 \%$ \\
\hline & & & & Uterine factor & $0 \%$ & Female \& male factors & $25 \%$ \\
\hline & & & & Male factor & $29 \%$ & & \\
\hline
\end{tabular}

2006 PREGNANCY SUCCESS RATES

Data verified by Keith L. Blauer, MD

\section{Type of Cycle}

(2)

\section{Fresh Embryos from Nondonor Eggs \\ Number of cycles \\ Percentage of cycles resulting in pregnancies ${ }^{b}$ \\ Percentage of cycles resulting in live births ${ }^{\mathrm{b}, \mathrm{c}}$}

(Confidence Interval)

Percentage of retrievals resulting in live births ${ }^{b, c}$

Percentage of transfers resulting in live births ${ }^{b, c}$

Percentage of transfers resulting in singleton live births ${ }^{b}$

Percentage of cancellations ${ }^{b}$

Average number of embryos transferred

Percentage of pregnancies with twins ${ }^{b}$

Percentage of pregnancies with triplets or more ${ }^{b}$

Percentage of live births having multiple infants ${ }^{b, c}$

\section{Frozen Embryos from Nondonor Eggs}

Number of transfers

Percentage of transfers resulting in live births ${ }^{\mathrm{b}, \mathrm{c}}$

Average number of embryos transferred

\section{Donor Eggs}

Number of transfers

Percentage of transfers resulting in live births ${ }^{\mathrm{b}, \mathrm{c}}$

Average number of embryos transferred

\section{Age of Woman}

35-37 38-40

$41-42^{d}$

\section{$<35$}

31

48.4

28

32.1

17.9

4

48.7

41.9

(32.9-47.2)

(24.5-60.9)

$(6.1-36.9)$
19.2

$2 / 4$

42.3

48.1

2 / 4

43.8

48.1

20.8

$2 / 3$

30.7

29.6

8.3

$2 / 3$

5.7

12.9

7.1

$0 / 3$

2.3

2.6

3.0

$1 / 4$

35.1

$6 / 15$

2.1

$0 / 15$

29.9

$5 / 13$

$1 / 9$

3.3

$1 / 2$

$2 / 9$

$1 / 2$

$3 / 5$

$2 / 2$

33

36.4

2.3

\section{7}

$2 / 7$

2.7

\section{9}

$1 / 9$

All Ages Combined

Fresh Embryos 10

$5 / 10$

2.1
Frozen Embryos 4

$3 / 4$

3.3

\section{CURRENT CLINIC SERVICES AND PROFILE}

Current Name: Reproductive Care Center

\begin{tabular}{ll|ll} 
Donor egg? & Yes & Gestational carriers? & Yes \\
Donor embryo? & Yes & Cryopreservation? & Yes \\
Single women? & No & &
\end{tabular}

SART member?

Yes

Verified lab accreditation?

Yes

(See Appendix C for details.)

\footnotetext{
${ }^{a}$ Reflects patient and treatment characteristics of ART cycles performed in 2006 using fresh nondonor eggs or embryos.

${ }^{b}$ When fewer than 20 cycles are reported in an age category, rates are shown as a fraction and confidence intervals are not given. Calculating percentages from fractions may be misleading and is not encouraged.

${ }^{c}$ A multiple-infant birth is counted as one live birth.

d Clinic-specific outcome rates are unreliable for women older than 42 undergoing ART cycles using fresh or frozen embryos with nondonor eggs. Readers are urged to review national outcomes for these age groups (see page 27).

e All ages (including ages $>42$ ) are reported together because previous data show that patient age does not materially affect success with donor eggs.
} 


\section{UTAH CENTER FOR REPRODUCTIVE MEDICINE \\ SALT LAKE CITY, UTAH}

A comparison of clinic success rates may not be meaningful because patient medical characteristics and treatment approaches vary from clinic to clinic. For more details about this, along with information on how to interpret the statistics in this table, see pages $79-88$.

\section{ART CYCLE PROFILE}

\begin{tabular}{|c|c|c|c|c|c|c|}
\hline & Type of ART ${ }^{a}$ & & Pati & ent D & iagnosis & \\
\hline IVF & $100 \%$ Procedural Factors: & & Tubal factor & $8 \%$ & Other factor & $4 \%$ \\
\hline GIFT & 0\% With ICSI & $54 \%$ & Ovulatory dysfunction & $3 \%$ & Unknown factor & $8 \%$ \\
\hline ZIFT & $0 \%$ Unstimulated & $0 \%$ & Diminished ovarian reserve & $8 \%$ & Multiple Factors: & \\
\hline Combination & $0 \%$ Used gestational carrier & $<1 \%$ & Endometriosis & $7 \%$ & Female factors only & $9 \%$ \\
\hline & & & Uterine factor & $<1 \%$ & Female \& male factors & $28 \%$ \\
\hline
\end{tabular}

2006 PREGNANCY SUCCESS RATES

Data verified by Harry H. Hatasaka, MD

\section{Type of Cycle}

\section{Fresh Embryos from Nondonor Eggs}

Number of cycles

Percentage of cycles resulting in pregnancies ${ }^{b}$

Percentage of cycles resulting in live births $s^{b, c}$

(Confidence Interval)

Percentage of retrievals resulting in live births $s^{b, c}$

Percentage of transfers resulting in live births $s^{b, c}$

Percentage of transfers resulting in singleton live births ${ }^{b}$

Percentage of cancellations ${ }^{b}$

Average number of embryos transferred

Percentage of pregnancies with twins ${ }^{b}$

Percentage of pregnancies with triplets or more ${ }^{b}$

Percentage of live births having multiple infants ${ }^{b, c}$

\section{Frozen Embryos from Nondonor Eggs}

Number of transfers

Percentage of transfers resulting in live births ${ }^{\mathrm{b}, \mathrm{c}}$

Average number of embryos transferred

\section{Donor Eggs}

Number of transfers

Percentage of transfers resulting in live births ${ }^{\mathrm{b}, \mathrm{c}}$

Average number of embryos transferred

\section{Age of Woman}

35-37 38-40

$41-42^{d}$

$<35$

60

40.0

36.7

49

19

\section{6}

48.6

$(41.9-55.4)$

$(24.6-50.1)$
45.8

32.7

$1 / 19$

57.4

60.3

48.9

26.5

$0 / 19$

34.6

15.3

2.1

47.1

0.8

42.6

31.1

20.0

(14.9-41.1)

33.3

34.2

$0 / 12$

28.9

$0 / 11$

$0 / 11$

20.4

$7 / 19$

2.1

33.3

0.0

36.4

2.2

2.6

$4 / 16$

$0 / 1$

$0 / 16$

$0 / 1$

$2 / 13$

40

30.0

2.2

16
$6 / 16$
2.3

16

6

3

2.3

$0 / 6$

$0 / 3$

2.0

3.0

All Ages Combined ${ }^{\mathrm{e}}$

Fresh Embryos

32

53.1

2.1
Frozen Embryos

9

$1 / 9$

2.6

\section{CURRENT CLINIC SERVICES AND PROFILE}

Current Name: Utah Center for Reproductive Medicine

Donor egg? Yes Gestational carriers? Yes

Donor embryo? No Cryopreservation? Yes

Single women? Yes

SART member? Yes

Verified lab accreditation? Yes

(See Appendix C for details.)

${ }^{a}$ Reflects patient and treatment characteristics of ART cycles performed in 2006 using fresh nondonor eggs or embryos.

${ }^{b}$ When fewer than 20 cycles are reported in an age category, rates are shown as a fraction and confidence intervals are not given. Calculating percentages from fractions may be misleading and is not encouraged.

${ }^{c}$ A multiple-infant birth is counted as one live birth.

d Clinic-specific outcome rates are unreliable for women older than 42 undergoing ART cycles using fresh or frozen embryos with nondonor eggs. Readers are urged to review national outcomes for these age groups (see page 27).

${ }^{e}$ All ages (including ages $>42$ ) are reported together because previous data show that patient age does not materially affect success with donor eggs. 


\section{VERMONT CENTER FOR REPRODUCTIVE MEDICINE BURLINGTON, VERMONT}

A comparison of clinic success rates may not be meaningful because patient medical characteristics and treatment approaches vary from clinic to clinic. For more details about this, along with information on how to interpret the statistics in this table, see pages 79-88.

\section{ART CYCLE PROFILE}

\begin{tabular}{|c|c|c|c|c|c|c|c|}
\hline \multicolumn{4}{|c|}{ Type of ART ${ }^{a}$} & \multicolumn{4}{|c|}{ Patient Diagnosis } \\
\hline IVF & $100 \%$ & Procedural Factors: & & Tubal factor & $18 \%$ & Other factor & $<1 \%$ \\
\hline GIFT & $0 \%$ & With ICSI & $39 \%$ & Ovulatory dysfunction & $4 \%$ & Unknown factor & $16 \%$ \\
\hline ZIFT & $0 \%$ & Unstimulated & $0 \%$ & Diminished ovarian reserve & $7 \%$ & Multiple Factors: & \\
\hline \multirow[t]{3}{*}{ Combination } & $0 \%$ & Used gestational carrier & $<1 \%$ & Endometriosis & $6 \%$ & Female factors only & $4 \%$ \\
\hline & & & & Uterine factor & $3 \%$ & Female \& male factors & $8 \%$ \\
\hline & & & & Male factor & $32 \%$ & & \\
\hline
\end{tabular}

\section{PREGNANCY SUCCESS RATES}

\section{Type of Cycle}

\section{Fresh Embryos from Nondonor Eggs}

Number of cycles

Percentage of cycles resulting in pregnancies ${ }^{b}$

Percentage of cycles resulting in live births $s^{b, c}$

(Confidence Interval)

Percentage of retrievals resulting in live births $s^{b, c}$

Percentage of transfers resulting in live births ${ }^{b, c}$

Percentage of transfers resulting in singleton live births ${ }^{b}$

Percentage of cancellations ${ }^{b}$

Average number of embryos transferred

Percentage of pregnancies with twins ${ }^{b}$

Percentage of pregnancies with triplets or more ${ }^{b}$

Percentage of live births having multiple infants ${ }^{b, c}$

\section{Frozen Embryos from Nondonor Eggs}

Number of transfers

Percentage of transfers resulting in live births ${ }^{b, c}$

Average number of embryos transferred

\section{Donor Eggs}

Number of transfers

Percentage of transfers resulting in live births $s^{b, c}$

Average number of embryos transferred
Data verified by Peter R. Casson, MD

\section{Age of Woman}

35-37 38-40

$41-42^{d}$

\section{$<35$}

38

31.6

21

6

46.0

28.9

28.6

$0 / 6$

42.0

$(15.4-45.9)$

23.8

$0 / 6$

$(28.2-56.8)$

40.7

(8.2-47.2)

47.7

42.3

$5 / 19$

$0 / 3$

50.0

30.8

$5 / 16$

$0 / 3$

35.7

28.9

$4 / 16$

$0 / 3$

12.0

2.2

9.5

$3 / 6$

2.0

$3 / 12$

2.6

1.3

26.1

$0 / 12$

$0 / 6$

4.3

28.6

$3 / 11$

$1 / 6$

$1 / 5$

$\begin{array}{ccc}7 & 7 & 2 \\ 2 / 7 & 1 / 7 & 0 / 2 \\ 2.1 & 2.4 & 2.0\end{array}$

\section{CURRENT CLINIC SERVICES AND PROFILE}

Current Name: Vermont Center for Reproductive Medicine

Donor egg? Yes Gestational carriers? Yes

Donor embryo? No Cryopreservation? Yes

All Ages Combined ${ }^{\mathrm{e}}$

Fresh Embryos

6

$3 / 6$

2.0
Frozen Embryos

3

$2 / 3$

2.3

Single women? Yes

SART member? Yes

Verified lab accreditation?

(See Appendix C for details.)

${ }^{a}$ Reflects patient and treatment characteristics of ART cycles performed in 2006 using fresh nondonor eggs or embryos.

${ }^{b}$ When fewer than 20 cycles are reported in an age category, rates are shown as a fraction and confidence intervals are not given. Calculating percentages from fractions may be misleading and is not encouraged.

${ }^{c}$ A multiple-infant birth is counted as one live birth.

d Clinic-specific outcome rates are unreliable for women older than 42 undergoing ART cycles using fresh or frozen embryos with nondonor eggs. Readers are urged to review national outcomes for these age groups (see page 27).

e All ages (including ages $>42$ ) are reported together because previous data show that patient age does not materially affect success with donor eggs. 


\section{NANCY DURSO, MD, PC METRO FERTILITY CARE ALEXANDRIA, VIRGINIA}

A comparison of clinic success rates may not be meaningful because patient medical characteristics and treatment approaches vary from clinic to clinic. For more details about this, along with information on how to interpret the statistics in this table, see pages $79-88$.

\section{ART CYCLE PROFILE}

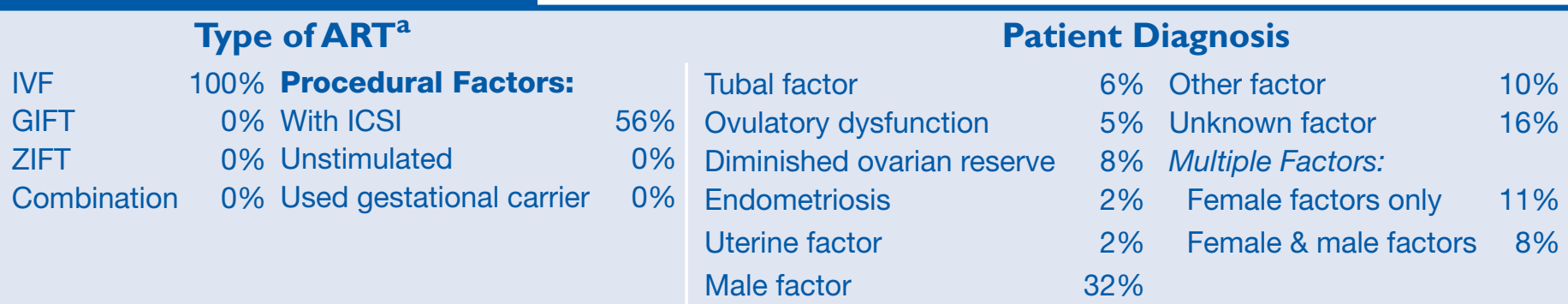

2006 PREGNANCY SUCCESS RATES

Data verified by Nancy M. Durso, MD

Male factor

\section{Type of Cycle}

Fresh Embryos from Nondonor Eggs

Number of cycles

Percentage of cycles resulting in pregnancies ${ }^{b}$

Percentage of cycles resulting in live births $s^{b, c}$

(Confidence Interval)

Percentage of retrievals resulting in live births $s^{b, c}$

Percentage of transfers resulting in live births $s^{b, c}$

ge of Woman

$\begin{array}{cccc}<35 & 35-37 & 38-40 & \mathbf{4 1 - 4 2}^{\text {d }} \\ & & & \\ 15 & 25 & 11 & 11 \\ 7 / 15 & 24.0 & 3 / 11 & 3 / 11 \\ 4 / 15 & 20.0 & 2 / 11 & 2 / 11\end{array}$

(6.8-40.7)

\begin{tabular}{|c|c|c|c|}
\hline $4 / 15$ & 22.7 & $2 / 8$ & $2 / 8$ \\
\hline $4 / 15$ & 23.8 & $2 / 8$ & $2 / 7$ \\
\hline $3 / 15$ & 14.3 & $1 / 8$ & $1 / 7$ \\
\hline $0 / 15$ & 12.0 & $3 / 11$ & $3 / 11$ \\
\hline 2.3 & 2.5 & 2.5 & 3.9 \\
\hline $1 / 7$ & $2 / 6$ & $1 / 3$ & $1 / 3$ \\
\hline $0 / 7$ & $0 / 6$ & $0 / 3$ & $0 / 3$ \\
\hline $1 / 4$ & $2 / 5$ & $1 / 2$ & $1 / 2$ \\
\hline
\end{tabular}

\section{Frozen Embryos from Nondonor Eggs}

Number of transfers

Percentage of transfers resulting in live births $s^{\mathrm{b}, \mathrm{c}}$

Average number of embryos transferred
7

$1 / 7$

2.6
4

$1 / 4$

3.0

\section{3}

$0 / 3$

2.0
2

$0 / 2$

3.5

\section{Donor Eggs}

Fresh Embryos

All Ages Combined ${ }^{\mathrm{e}}$
Number of transfers

Percentage of transfers resulting in live births $s^{b, c}$

Average number of embryos transferred

\section{Frozen Embryos}

0

\section{CURRENT CLINIC SERVICES AND PROFILE}

Current Name: Nancy Durso, MD, PC, Metro Fertility Care

Donor egg? Yes Gestational carriers? Yes

Donor embryo? Yes Cryopreservation? Yes

Single women? Yes

${ }^{a}$ Reflects patient and treatment characteristics of ART cycles performed in 2006 using fresh nondonor eggs or embryos.

${ }^{b}$ When fewer than 20 cycles are reported in an age category, rates are shown as a fraction and confidence intervals are not given. Calculating percentages from fractions may be misleading and is not encouraged.

${ }^{c}$ A multiple-infant birth is counted as one live birth.

d Clinic-specific outcome rates are unreliable for women older than 42 undergoing ART cycles using fresh or frozen embryos with nondonor eggs. Readers are urged to review national outcomes for these age groups (see page 27).

e All ages (including ages $>42$ ) are reported together because previous data show that patient age does not materially affect success with donor eggs. 


\section{WASHINGTON FERTILITY CENTER \\ ANNANDALE, VIRGINIA}

A comparison of clinic success rates may not be meaningful because patient medical characteristics and treatment approaches vary from clinic to clinic. For more details about this, along with information on how to interpret the statistics in this table, see pages 79-88.

\section{ART CYCLE PROFILE}

\section{Type of ART ${ }^{\mathrm{a}}$}

IVF

GIFT

ZIFT

Combination
100\% Procedural Factors:

$0 \%$ With ICSI

$0 \%$ Unstimulated

$0 \%$ Used gestational carrier

\section{$53 \%$ \\ $0 \%$ \\ $0 \%$}

Tubal factor

Ovulatory dysfunction

Diminished ovarian reserve

Endometriosis

Uterine factor

Male factor

\section{Patient Diagnosis}

$\begin{array}{rlr}10 \% & \text { Other factor } & 17 \% \\ 1 \% & \text { Unknown factor } & 8 \% \\ 13 \% & \text { Multiple Factors: } & \\ 2 \% & \text { Female factors only } & 4 \% \\ 3 \% & \text { Female \& male factors } & 10 \% \\ 32 \% & & \end{array}$

Data verified by Pierre Asmar, MD

2006 PREGNANCY SUCCESS RATES

\section{Age of Woman}

\section{Type of Cycle}

\section{Fresh Embryos from Nondonor Eggs}

Number of cycles

Percentage of cycles resulting in pregnancies ${ }^{b}$

Percentage of cycles resulting in live births ${ }^{b, c}$

(Confidence Interval)

Percentage of retrievals resulting in live births $s^{b, c}$

Percentage of transfers resulting in live births $s^{b, c}$

Percentage of transfers resulting in singleton live births ${ }^{b}$

Percentage of cancellations ${ }^{b}$

Average number of embryos transferred

Percentage of pregnancies with twins ${ }^{b}$

Percentage of pregnancies with triplets or more ${ }^{b}$

Percentage of live births having multiple infants ${ }^{b, c}$

\section{Frozen Embryos from Nondonor Eggs}

Number of transfers

Percentage of transfers resulting in live births ${ }^{b, c}$

Average number of embryos transferred

\section{Donor Eggs}

Number of transfers

Percentage of transfers resulting in live births $s^{b, c}$

Average number of embryos transferred

35-37 38-40

$41-42^{d}$

\section{$<35$}

52

28.8

24

9

28.2

21.2

29.2

$1 / 9$

17.9

(11.1-34.7)

22.0

25.0

22.7

3.8

2.0

$5 / 15$

$0 / 15$

$1 / 11$

$2 / 14$

0

$0 / 2$

2.0

25.0

$(9.8-46.7)$

28.6

$6 / 17$

$5 / 17$

12.5

1.9

$1 / 7$

$0 / 7$

$1 / 6$

1

$0 / 1$

2.0 


\section{DOMINION FERTILITY AND ENDOCRINOLOGY \\ ARLINGTON, VIRGINIA}

A comparison of clinic success rates may not be meaningful because patient medical characteristics and treatment approaches vary from clinic to clinic. For more details about this, along with information on how to interpret the statistics in this table, see pages $79-88$.

\section{ART CYCLE PROFILE}

\begin{tabular}{|c|c|c|c|c|c|c|c|}
\hline \multicolumn{4}{|c|}{ Type of ART } & \multicolumn{4}{|c|}{ Patient Diagnosis } \\
\hline IVF & $100 \%$ & Procedural Factors: & & Tubal factor & $5 \%$ & Other factor & $13 \%$ \\
\hline GIFT & $0 \%$ & With ICSI & $54 \%$ & Ovulatory dysfunction & $5 \%$ & Unknown factor & $4 \%$ \\
\hline ZIFT & $0 \%$ & Unstimulated & $0 \%$ & Diminished ovarian reserve & $18 \%$ & Multiple Factors: & \\
\hline \multirow[t]{3}{*}{ Combination } & $0 \%$ & Used gestational carrier & $<1 \%$ & Endometriosis & $<1 \%$ & Female factors only & $22 \%$ \\
\hline & & & & Uterine factor & $0 \%$ & Female \& male factors & $22 \%$ \\
\hline & & & & Male factor & $11 \%$ & & \\
\hline
\end{tabular}

2006 PREGNANCY SUCCESS RATES

\section{Type of Cycle}

\section{Fresh Embryos from Nondonor Eggs}

Number of cycles

Percentage of cycles resulting in pregnancies ${ }^{b}$

Percentage of cycles resulting in live births $s^{b, c}$

(Confidence Interval)

Percentage of retrievals resulting in live births $s^{b, c}$

Percentage of transfers resulting in live births $s^{b, c}$

Percentage of transfers resulting in singleton live births ${ }^{b}$

Percentage of cancellations ${ }^{b}$

Average number of embryos transferred

Percentage of pregnancies with twins ${ }^{b}$

Percentage of pregnancies with triplets or more ${ }^{b}$

Percentage of live births having multiple infants ${ }^{b, c}$

\section{Frozen Embryos from Nondonor Eggs}

Number of transfers

Percentage of transfers resulting in live births $s^{b, c}$

Average number of embryos transferred

\section{Donor Eggs}

Number of transfers

Percentage of transfers resulting in live births ${ }^{\mathrm{b}, \mathrm{c}}$

Average number of embryos transferred
Data verified by Michael DiMattina, MD

\section{Age of Woman}

35-37 38-40

$41-42^{d}$

\section{$<35$}

43

37.2

34

15

\section{7}

32.6

32.4

$2 / 15$

41.9

$(19.1-48.5)$

23.5

$1 / 15$

(31.3-53.0)

46.2

33.3

$(10.7-41.2)$

49.3

35.9

24.2

27.6

$1 / 12$

28.2

13.8

$1 / 12$

35.6

2.3

2.9

$1 / 12$

9.3

2.8

3.9

$3 / 15$

34.1

$9 / 16$

0.0

$0 / 16$

$6 / 11$

4.3

27.8

$3 / 14$

$1 / 11$

$0 / 2$

$0 / 2$

$4 / 8$

$0 / 1$
45

35.6

2.1

\section{4}

50.0

2.5

\section{8 \\ $2 / 8$}

2.3
2

$2 / 2$

2.0
All Ages Combined
Fresh Embryos 35

62.9

2.1
Frozen Embryos 25

24.0

\section{CURRENT CLINIC SERVICES AND PROFILE}

Current Name: Dominion Fertility and Endocrinology

Donor egg? Yes

Donor embryo? Yes

Single women? Yes

$\begin{array}{ll}\text { Gestational carriers? } & \text { Yes } \\ \text { Cryopreservation? } & \text { Yes }\end{array}$

SART member?

Yes

Verified lab accreditation?

(See Appendix C for details.)

${ }^{a}$ Reflects patient and treatment characteristics of ART cycles performed in 2006 using fresh nondonor eggs or embryos.

${ }^{b}$ When fewer than 20 cycles are reported in an age category, rates are shown as a fraction and confidence intervals are not given. Calculating percentages from fractions may be misleading and is not encouraged.

${ }^{\mathrm{C}}$ A multiple-infant birth is counted as one live birth.

d Clinic-specific outcome rates are unreliable for women older than 42 undergoing ART cycles using fresh or frozen embryos with nondonor eggs. Readers are urged to review national outcomes for these age groups (see page 27).

${ }^{e}$ All ages (including ages $>42$ ) are reported together because previous data show that patient age does not materially affect success with donor eggs. 


\section{REPRODUCTIVE MEDICINE AND SURGERY CENTER OF VIRGINIA, PLC CHARLOTTESVILLE, VIRGINIA}

A comparison of clinic success rates may not be meaningful because patient medical characteristics and treatment approaches vary from clinic to clinic. For more details about this, along with information on how to interpret the statistics in this table, see pages 79-88.

\section{ART CYCLE PROFILE}

\begin{tabular}{|c|c|c|c|c|c|c|c|}
\hline \multicolumn{4}{|c|}{ Type of ART ${ }^{a}$} & \multicolumn{4}{|c|}{ Patient Diagnosis } \\
\hline IVF & $100 \%$ & Procedural Factors: & & Tubal factor & $14 \%$ & Other factor & $2 \%$ \\
\hline GIFT & $0 \%$ & With ICSI & $62 \%$ & Ovulatory dysfunction & $5 \%$ & Unknown factor & $5 \%$ \\
\hline ZIFT & $0 \%$ & Unstimulated & $0 \%$ & Diminished ovarian reserve & $4 \%$ & Multiple Factors: & \\
\hline \multirow[t]{3}{*}{ Combination } & $0 \%$ & Used gestational carrier & $0 \%$ & Endometriosis & $8 \%$ & Female factors only & $9 \%$ \\
\hline & & & & Uterine factor & $0 \%$ & Female \& male factors & $25 \%$ \\
\hline & & & & Male factor & $28 \%$ & & \\
\hline
\end{tabular}

2006 PREGNANCY SUCCESS RATES

Data verified by Christopher D. Williams, MD

\section{Type of Cycle}

\section{Fresh Embryos from Nondonor Eggs}

Number of cycles

Percentage of cycles resulting in pregnancies ${ }^{b}$

Percentage of cycles resulting in live births ${ }^{b, c}$

(Confidence Interval)

Percentage of retrievals resulting in live births $s^{b, c}$

Percentage of transfers resulting in live births $s^{b, c}$

Percentage of transfers resulting in singleton live births ${ }^{b}$

Percentage of cancellations ${ }^{b}$

Average number of embryos transferred

Percentage of pregnancies with twins ${ }^{b}$

Percentage of pregnancies with triplets or more ${ }^{b}$

Percentage of live births having multiple infants ${ }^{b, c}$

\section{Frozen Embryos from Nondonor Eggs}

Number of transfers

Percentage of transfers resulting in live births ${ }^{b, c}$

Average number of embryos transferred

\section{Donor Eggs}

Number of transfers

Percentage of transfers resulting in live births $s^{b, c}$

Average number of embryos transferred

\section{Age of Woman}

35-37 38-40

$41-42^{d}$

\section{$<35$}

30

10

2

52
44.2

43.3

$4 / 10$

$0 / 2$

34.6

33.3

$4 / 10$

$0 / 2$

(22.0-49.1)

(17.3-52.8)

36.7

41.7

$4 / 8$

$0 / 2$

37.5

41.7

$4 / 7$

$0 / 1$

22.9

33.3

$2 / 7$

$0 / 1$

5.8

20.0

$2 / 10$

$0 / 2$

2.0

2.3

3.3

4.0

30.4

$5 / 13$

$3 / 4$

4.3

$1 / 13$

$0 / 4$

$7 / 18$

$2 / 10$

$2 / 4$

5

$4 / 5$

3

$0 / 3$

0

2

1.7

$0 / 2$

2.0

All Ages Combined ${ }^{\mathrm{e}}$

Fresh Embryos

5

$4 / 5$

2.0
Frozen Embryos

\section{1}

$1 / 1$

3.0

\section{CURRENT CLINIC SERVICES AND PROFILE}

Current Name: Reproductive Medicine and Surgery Center of Virginia, PLC

Donor egg? Yes

Donor embryo? Yes

Single women? Yes

\begin{abstract}
Gestational carriers? Yes
\end{abstract}
Cryopreservation? Yes
SART member?

Verified lab accreditation?

Yes

(See Appendix C for details.)

${ }^{a}$ Reflects patient and treatment characteristics of ART cycles performed in 2006 using fresh nondonor eggs or embryos.

b When fewer than 20 cycles are reported in an age category, rates are shown as a fraction and confidence intervals are not given. Calculating percentages from fractions may be misleading and is not encouraged.

${ }^{c}$ A multiple-infant birth is counted as one live birth.

d Clinic-specific outcome rates are unreliable for women older than 42 undergoing ART cycles using fresh or frozen embryos with nondonor eggs. Readers are urged to review national outcomes for these age groups (see page 27).

e All ages (including ages $>42$ ) are reported together because previous data show that patient age does not materially affect success with donor eggs. 


\section{GENETICS \& IVF INSTITUTE \\ FAIRFAX, VIRGINIA}

A comparison of clinic success rates may not be meaningful because patient medical characteristics and treatment approaches vary from clinic to clinic. For more details about this, along with information on how to interpret the statistics in this table, see pages $79-88$.

\section{ART CYCLE PROFILE}

\begin{tabular}{|c|c|c|c|c|c|c|c|}
\hline & Type & of $A R T^{a}$ & & Pati & ent D & iagnosis & \\
\hline IVF & $100 \%$ & Procedural Factors: & & Tubal factor & $3 \%$ & Other factor & $19 \%$ \\
\hline GIFT & $0 \%$ & With ICSI & $70 \%$ & Ovulatory dysfunction & $3 \%$ & Unknown factor & $2 \%$ \\
\hline $\mathrm{ZIFT}$ & $0 \%$ & Unstimulated & $0 \%$ & Diminished ovarian reserve & $15 \%$ & Multiple Factors: & \\
\hline Combination & $0 \%$ & Used gestational carrier & $<1 \%$ & Endometriosis & $<1 \%$ & Female factors only & $7 \%$ \\
\hline & & & & $\begin{array}{l}\text { Uterine factor } \\
\text { Male factor }\end{array}$ & $\begin{array}{l}<1 \% \\
18 \%\end{array}$ & Female \& male factors & $32 \%$ \\
\hline
\end{tabular}

2006 PREGNANCY SUCCESS RATES

Data verified by Stephen R. Lincoln, MD

\section{Type of Cycle}

\section{Fresh Embryos from Nondonor Eggs}

Number of cycles

Percentage of cycles resulting in pregnancies ${ }^{b}$

Percentage of cycles resulting in live births ${ }^{b, c}$

(Confidence Interval)

Percentage of retrievals resulting in live births $s^{b, c}$

Percentage of transfers resulting in live births $s^{b, c}$

Percentage of transfers resulting in singleton live births ${ }^{b}$

Percentage of cancellations ${ }^{b}$

Average number of embryos transferred

Percentage of pregnancies with twins ${ }^{b}$

Percentage of pregnancies with triplets or more ${ }^{b}$

Percentage of live births having multiple infants ${ }^{b, c}$

\section{Frozen Embryos from Nondonor Eggs}

Number of transfers

Percentage of transfers resulting in live births ${ }^{\mathrm{b}, \mathrm{c}}$

Average number of embryos transferred

\section{Donor Eggs}

Number of transfers

Percentage of transfers resulting in live births ${ }^{\mathrm{b}, \mathrm{c}}$

Average number of embryos transferred

\section{Age of Woman}

35-37 38-40

$41-42^{d}$

$<35$

97

97
21.6

89

41

29.0

18.6

11.2

22.0

24.5

(18.0-32.1)

(11.4-27.7)

9.0

14.6

25.7

27.0

20.0

$(4.0-16.9)$

(5.6-29.2)

17.0

22.2

9.6

17.1

4.5

18.5

11.0

20.7

11.0

17.2

2.4

33.3

0.0

7.2

6.7

14.6

2.5

14.3

2.5

2.8

36.8

14.3

$0 / 10$

$1 / 9$

$0 / 10$

$0 / 9$

$0 / 8$

$1 / 6$

$3 / 18$

40

12.5

20

15.0

20

5

2.0

1.9

5.0

$1 / 5$

2.1

3.6

All Ages Combined ${ }^{\mathrm{e}}$

Fresh Embryos

148

36.5

2.2
Frozen Embryos

106

15.1

2.3

\section{CURRENT CLINIC SERVICES AND PROFILE}

Current Name: Genetics \& IVF Institute
Donor egg? Yes
Donor embryo? Yes
$\begin{array}{ll}\text { Gestational carriers? } & \text { Yes } \\ \text { Cryopreservation? } & \text { Yes }\end{array}$
SART member?
Yes
Single women? Yes
Verified lab accreditation?
Yes
(See Appendix C for details.)

\footnotetext{
${ }^{a}$ Reflects patient and treatment characteristics of ART cycles performed in 2006 using fresh nondonor eggs or embryos.

b When fewer than 20 cycles are reported in an age category, rates are shown as a fraction and confidence intervals are not given. Calculating percentages from fractions may be misleading and is not encouraged.

${ }^{c}$ A multiple-infant birth is counted as one live birth.

d Clinic-specific outcome rates are unreliable for women older than 42 undergoing ART cycles using fresh or frozen embryos with nondonor eggs. Readers are urged to review national outcomes for these age groups (see page 27).

${ }^{e}$ All ages (including ages $>42$ ) are reported together because previous data show that patient age does not materially affect success with donor eggs.
} 


\section{THE MUASHER CENTER FOR FERTILITY AND IVF \\ FAIRFAX, VIRGINIA}

A comparison of clinic success rates may not be meaningful because patient medical characteristics and treatment approaches vary from clinic to clinic. For more details about this, along with information on how to interpret the statistics in this table, see pages 79-88.

\section{ART CYCLE PROFILE}

\begin{tabular}{|c|c|c|c|c|c|c|c|}
\hline \multicolumn{4}{|c|}{ Type of ART } & \multicolumn{4}{|c|}{ Patient Diagnosis } \\
\hline IVF & $100 \%$ & Procedural Factors: & & Tubal factor & $8 \%$ & Other factor & $3 \%$ \\
\hline GIFT & $0 \%$ & With ICSI & $41 \%$ & Ovulatory dysfunction & $10 \%$ & Unknown factor & $12 \%$ \\
\hline ZIFT & $0 \%$ & Unstimulated & $0 \%$ & Diminished ovarian reserve & $8 \%$ & Multiple Factors: & \\
\hline \multirow[t]{3}{*}{ Combination } & $0 \%$ & Used gestational carrier & $2 \%$ & Endometriosis & $5 \%$ & Female factors only & $10 \%$ \\
\hline & & & & Uterine factor & $1 \%$ & Female \& male factors & $31 \%$ \\
\hline & & & & Male factor & $12 \%$ & & \\
\hline
\end{tabular}

2006 PREGNANCY SUCCESS RATES

Data verified by Suheil J. Muasher, MD

\section{Type of Cycle}

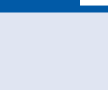

\section{Fresh Embryos from Nondonor Eggs}

Number of cycles

Percentage of cycles resulting in pregnancies ${ }^{b}$

Percentage of cycles resulting in live births ${ }^{\mathrm{b}, \mathrm{c}}$

(Confidence Interval)

Percentage of retrievals resulting in live births ${ }^{b, c}$

Percentage of transfers resulting in live births ${ }^{\mathrm{b}, \mathrm{c}}$

Percentage of transfers resulting in singleton live births ${ }^{b}$

Percentage of cancellations ${ }^{b}$

Average number of embryos transferred

Percentage of pregnancies with twins ${ }^{b}$

Percentage of pregnancies with triplets or more ${ }^{b}$

Percentage of live births having multiple infants ${ }^{b, c}$

\section{Frozen Embryos from Nondonor Eggs}

Number of transfers

Percentage of transfers resulting in live births ${ }^{\text {b,c }}$

Average number of embryos transferred

\section{Donor Eggs}

Number of transfers

Percentage of transfers resulting in live births ${ }^{b, c}$

Average number of embryos transferred

\section{Age of Woman}

35-37 38-40

$41-42^{d}$ $<35$

33

21.2

15.2
$(5.1-31.9)$

25

9

25.5

25.5

28.0

$0 / 9$

(14.7-39.0)

25.9

16.1

12.0

$0 / 9$

25.9

16.7

(2.5-31.2)

13.6

$0 / 8$

14.8

16.7

15.0

$0 / 7$

1.8

6.1

15.0

$0 / 7$

2.6

3.0

12.0

$1 / 9$

4 / 14

$0 / 7$

3.0

3.0

$2 / 14$

$6 / 14$

$1 / 7$

$0 / 7$

$0 / 7$

$0 / 5$

$0 / 3$

\section{6}

$1 / 6$

2.2

\begin{tabular}{cc}
\multicolumn{2}{c}{ All Ages Combined } \\
Fresh Embryos & Frozen Embryos \\
7 & 2 \\
$2 / 7$ & $1 / 2$ \\
3.0 & 2.5
\end{tabular}

0

0

0

\section{CURRENT CLINIC SERVICES AND PROFILE}

Current Name: The Muasher Center for Fertility and IVF

\begin{tabular}{ll|ll} 
Donor egg? & Yes & Gestational carriers? & Yes \\
Donor embryo? & No & Cryopreservation? & Yes \\
Single women? & Yes & &
\end{tabular}

Single women? Yes

${ }_{b}^{a}$ Reflects patient and treatment characteristics of ART cycles performed in 2006 using fresh nondonor eggs or embryos.

b When fewer than 20 cycles are reported in an age category, rates are shown as a fraction and confidence intervals are not given. Calculating percentages from fractions may be misleading and is not encouraged.

c A multiple-infant birth is counted as one live birth.

${ }^{d}$ Clinic-specific outcome rates are unreliable for women older than 42 undergoing ART cycles using fresh or frozen embryos with nondonor eggs. Readers are urged to review national outcomes for these age groups (see page 27).

${ }^{e}$ All ages (including ages $>42$ ) are reported together because previous data show that patient age does not materially affect success with donor eggs. 


\section{JONES INSTITUTE FOR REPRODUCTIVE MEDICINE \\ NORFOLK, VIRGINIA}

A comparison of clinic success rates may not be meaningful because patient medical characteristics and treatment approaches vary from clinic to clinic. For more details about this, along with information on how to interpret the statistics in this table, see pages $79-88$.

\section{ART CYCLE PROFILE}

\begin{tabular}{|c|c|c|c|c|c|c|c|}
\hline \multicolumn{4}{|c|}{ Type of ART ${ }^{\mathrm{a}}$} & \multicolumn{4}{|c|}{ Patient Diagnosis } \\
\hline IVF & $100 \%$ & Procedural Factors: & & Tubal factor & $15 \%$ & Other factor & $7 \%$ \\
\hline GIFT & $0 \%$ & With ICSI & $69 \%$ & Ovulatory dysfunction & $6 \%$ & Unknown factor & $5 \%$ \\
\hline $\mathrm{ZIFT}$ & $0 \%$ & Unstimulated & $0 \%$ & Diminished ovarian reserve & $11 \%$ & Multiple Factors: & \\
\hline Combination & $0 \%$ & Used gestational carrier & $<1 \%$ & Endometriosis & $5 \%$ & Female factors only & $12 \%$ \\
\hline & & & & Uterine factor & $1 \%$ & Female \& male factors & $23 \%$ \\
\hline
\end{tabular}

2006 PREGNANCY SUCCESS RATES

Data verified by Laurel A. Stadtmauer, MD

\section{Type of Cycle}

\section{Fresh Embryos from Nondonor Eggs}

Number of cycles

Percentage of cycles resulting in pregnancies ${ }^{b}$

Percentage of cycles resulting in live births $s^{b, c}$

(Confidence Interval)

Percentage of retrievals resulting in live births $s^{b, c}$

Percentage of transfers resulting in live births $s^{b, c}$

Percentage of transfers resulting in singleton live births ${ }^{b}$

Percentage of cancellations ${ }^{b}$

Average number of embryos transferred

Percentage of pregnancies with twins ${ }^{b}$

Percentage of pregnancies with triplets or more ${ }^{b}$

Percentage of live births having multiple infants ${ }^{b, c}$

\section{Frozen Embryos from Nondonor Eggs}

Number of transfers

Percentage of transfers resulting in live births $s^{\mathrm{b}, \mathrm{c}}$

Average number of embryos transferred

\section{Donor Eggs}

Number of transfers

Percentage of transfers resulting in live births $s^{b, c}$

Average number of embryos transferred

\section{Age of Woman}

35-37 38-40

$41-42^{d}$

$<35$

40

32.5

27.5

26.1

(14.6-43.9)

(17.3-36.6)

26.4

28.4

30.6

31.4

28.6

10.0

2.4

$1 / 13$

$0 / 13$

$1 / 11$

34.8

2.1
55.2

0.0

14

$5 / 14$

2.5

22.2

2.2

All Ages Combined ${ }^{\mathrm{e}}$
Fresh Embryos

34

44.1

2.1
14

$2 / 14$

21.4

$2 / 14$
(8.9-30.4)

20.8

$2 / 10$

23.3

$2 / 10$

$2 / 10$

$4 / 14$

14.3

2.3

2.5

$0 / 2$

$0 / 12$

$0 / 2$

$0 / 12$

$0 / 2$

$0 / 10$

15

4

$2 / 15$

$0 / 4$

2.2

2.8
Frozen Embryos

20.8

2.3

\section{CURRENT CLINIC SERVICES AND PROFILE}

Current Name: Jones Institute for Reproductive Medicine

Donor egg? Yes

Donor embryo? Yes

Single women? Yes

\section{Gestational carriers? Yes}

Cryopreservation? Yes

\footnotetext{
${ }^{a}$ Reflects patient and treatment characteristics of ART cycles performed in 2006 using fresh nondonor eggs or embryos.

${ }^{b}$ When fewer than 20 cycles are reported in an age category, rates are shown as a fraction and confidence intervals are not given. Calculating percentages from fractions may be misleading and is not encouraged.

c A multiple-infant birth is counted as one live birth.

${ }^{d}$ Clinic-specific outcome rates are unreliable for women older than 42 undergoing ART cycles using fresh or frozen embryos with nondonor eggs. Readers are urged to review national outcomes for these age groups (see page 27).

${ }^{e}$ All ages (including ages $>42$ ) are reported together because previous data show that patient age does not materially affect success with donor eggs.
} 


\section{VIRGINIA CENTER FOR REPRODUCTIVE MEDICINE \\ RESTON, VIRGINIA}

A comparison of clinic success rates may not be meaningful because patient medical characteristics and treatment approaches vary from clinic to clinic. For more details about this, along with information on how to interpret the statistics in this table, see pages $79-88$.

\section{ART CYCLE PROFILE}

\begin{tabular}{|c|c|c|c|c|c|c|c|}
\hline \multicolumn{4}{|c|}{ Type of ART ${ }^{\mathrm{a}}$} & \multicolumn{4}{|c|}{ Patient Diagnosis } \\
\hline IVF & $100 \%$ & Procedural Factors: & & Tubal factor & $6 \%$ & Other factor & $7 \%$ \\
\hline GIFT & $0 \%$ & With ICSI & $92 \%$ & Ovulatory dysfunction & $2 \%$ & Unknown factor & $2 \%$ \\
\hline ZIFT & $0 \%$ & Unstimulated & $0 \%$ & Diminished ovarian reserve & $16 \%$ & Multiple Factors: & \\
\hline \multirow[t]{3}{*}{ Combination } & $0 \%$ & Used gestational carrier & $3 \%$ & Endometriosis & $2 \%$ & Female factors only & $11 \%$ \\
\hline & & & & Uterine factor & $0 \%$ & Female \& male factors & $41 \%$ \\
\hline & & & & Male factor & $14 \%$ & & \\
\hline
\end{tabular}

2006 PREGNANCY SUCCESS RATES

Data verified by Fady I. Sharara, MD

\section{Type of Cycle}

\section{Fresh Embryos from Nondonor Eggs}

Number of cycles

Percentage of cycles resulting in pregnancies ${ }^{b}$

Percentage of cycles resulting in live births ${ }^{b, c}$

(Confidence Interval)

Percentage of retrievals resulting in live births $s^{b, c}$

Percentage of transfers resulting in live births $s^{b, c}$

Percentage of transfers resulting in singleton live births ${ }^{b}$

Percentage of cancellations ${ }^{b}$

Average number of embryos transferred

Percentage of pregnancies with twins ${ }^{b}$

Percentage of pregnancies with triplets or more ${ }^{b}$

Percentage of live births having multiple infants ${ }^{b, c}$

\section{Frozen Embryos from Nondonor Eggs}

Number of transfers

Percentage of transfers resulting in live births ${ }^{b, c}$

Average number of embryos transferred

\section{Donor Eggs}

Number of transfers

Percentage of transfers resulting in live births $s^{b, c}$

Average number of embryos transferred

\section{Age of Woman}

35-37 38-40

$41-42^{d}$

\section{$<35$}

30

46.7

23

13

69.8

33.3

39.1

$4 / 13$

58.1

$(17.3-52.8)$

30.4

$2 / 13$

$(42.1-73.0)$

58.1

35.7

$(13.2-52.9)$

59.5

35.7

31.8

$2 / 12$

31.0

21.4

31.8

$2 / 12$

0.0

6.7

22.7

$1 / 12$

2.0

2.0

4.3

$1 / 13$

50.0

$4 / 14$

0.0

$1 / 14$

48.0

$4 / 10$

2.5

2.8

$0 / 4$

$1 / 4$

$0 / 9$

$1 / 2$

$2 / 7$

0

0

$\begin{array}{cc}1 & 2 \\ 2.1 & 1 / 2\end{array}$

2.0

2.5

All Ages Combined ${ }^{\mathrm{e}}$

Fresh Embryos

7

$3 / 7$

2.0
Frozen Embryos 3

$1 / 3$

2.3

\section{CURRENT CLINIC SERVICES AND PROFILE}

Current Name: Virginia Center for Reproductive Medicine

Donor egg? Yes

Donor embryo? Yes

Single women? Yes

\begin{abstract}
Gestational carriers? Yes
Cryopreservation? Yes
\end{abstract}

SART member?

Yes

Verified lab accreditation?

(See Appendix C for details.)

${ }^{a}$ Reflects patient and treatment characteristics of ART cycles performed in 2006 using fresh nondonor eggs or embryos.

b When fewer than 20 cycles are reported in an age category, rates are shown as a fraction and confidence intervals are not given. Calculating percentages from fractions may be misleading and is not encouraged.

${ }^{c}$ A multiple-infant birth is counted as one live birth.

${ }^{d}$ Clinic-specific outcome rates are unreliable for women older than 42 undergoing ART cycles using fresh or frozen embryos with nondonor eggs. Readers are urged to review national outcomes for these age groups (see page 27).

e All ages (including ages $>42$ ) are reported together because previous data show that patient age does not materially affect success with donor eggs. 


\section{FERTILITY INSTITUTE OF VIRGINIA \\ RICHMOND, VIRGINIA}

A comparison of clinic success rates may not be meaningful because patient medical characteristics and treatment approaches vary from clinic to clinic. For more details about this, along with information on how to interpret the statistics in this table, see pages $79-88$.

\section{ART CYCLE PROFILE}

\begin{tabular}{|c|c|c|c|c|c|c|c|}
\hline \multicolumn{4}{|c|}{ Type of ART ${ }^{\mathrm{a}}$} & \multicolumn{4}{|c|}{ Patient Diagnosis } \\
\hline IVF & $100 \%$ & Procedural Factors: & & Tubal factor & $22 \%$ & Other factor & $1 \%$ \\
\hline GIFT & $0 \%$ & With ICSI & $78 \%$ & Ovulatory dysfunction & $7 \%$ & Unknown factor & $14 \%$ \\
\hline ZIFT & $0 \%$ & Unstimulated & $0 \%$ & Diminished ovarian reserve & $9 \%$ & Multiple Factors: & \\
\hline \multirow[t]{3}{*}{ Combination } & $0 \%$ & Used gestational carrier & $0 \%$ & Endometriosis & $14 \%$ & Female factors only & $3 \%$ \\
\hline & & & & Uterine factor & $<1 \%$ & Female \& male factors & $8 \%$ \\
\hline & & & & Male factor & $23 \%$ & & \\
\hline
\end{tabular}

2006 PREGNANCY SUCCESS RATES

Data verified by Michael C. Edelstein, MD

\section{Type of Cycle}

\section{Fresh Embryos from Nondonor Eggs}

Number of cycles

Percentage of cycles resulting in pregnancies ${ }^{b}$

Percentage of cycles resulting in live births $s^{b, c}$

(Confidence Interval)

Percentage of retrievals resulting in live births $s^{b, c}$

Percentage of transfers resulting in live births ${ }^{b, c}$

Percentage of transfers resulting in singleton live births ${ }^{b}$

Percentage of cancellations ${ }^{b}$

Average number of embryos transferred

Percentage of pregnancies with twins ${ }^{b}$

Percentage of pregnancies with triplets or more ${ }^{b}$

Percentage of live births having multiple infants ${ }^{b, c}$

\section{Frozen Embryos from Nondonor Eggs}

Number of transfers

Percentage of transfers resulting in live births $s^{b, c}$

Average number of embryos transferred

\section{Donor Eggs}

Number of transfers

Percentage of transfers resulting in live births $s^{b, c}$

Average number of embryos transferred

\section{Age of Woman}

35-37 38-40

$41-42^{d}$

$<35$

55

50.9

36.4

38

12

46.5

39.4

$(23.8-50.4)$
37.7

39.5

$3 / 12$

(28.0-51.7)

40.6

31.6

$2 / 12$

43.8

38.5

(17.5-48.7)

26.6

28.8

31.6

$2 / 12$

31.6

$2 / 11$

2.8

3.6

21.1

$2 / 11$

2.4

2.8

30.3

28.6

3.0

0.0

39.3

25.0

0.0

$0 / 12$

3.0

3.9

$5 / 15$

$0 / 3$

$1 / 15$

$0 / 3$

$4 / 12$

$0 / 2$

34

41.2

2.8

\section{8}

35.7

3.1

\section{9}

$2 / 9$

2.8

All Ages Combined ${ }^{\mathrm{e}}$
Fresh Embryos

8

$4 / 8$

2.3
Frozen Embryos

\section{6}

$4 / 6$

2.8

\section{CURRENT CLINIC SERVICES AND PROFILE}

Current Name: Fertility Institute of Virginia
Donor egg? Yes
Donor embryo? Yes
Gestational carriers? Yes
Cryopreservation? Yes
Single women? Yes

\section{SART member? \\ Verified lab accreditation? \\ (See Appendix C for details.)}
Yes
Yes

\footnotetext{
${ }^{a}$ Reflects patient and treatment characteristics of ART cycles performed in 2006 using fresh nondonor eggs or embryos.

${ }^{b}$ When fewer than 20 cycles are reported in an age category, rates are shown as a fraction and confidence intervals are not given. Calculating percentages from fractions may be misleading and is not encouraged.

${ }^{c}$ A multiple-infant birth is counted as one live birth.

${ }^{d}$ Clinic-specific outcome rates are unreliable for women older than 42 undergoing ART cycles using fresh or frozen embryos with nondonor eggs. Readers are urged to review national outcomes for these age groups (see page 27).

${ }^{e}$ All ages (including ages $>42$ ) are reported together because previous data show that patient age does not materially affect success with donor eggs.
} 


\section{LIFESOURCE FERTILITY CENTER RICHMOND, VIRGINIA}

A comparison of clinic success rates may not be meaningful because patient medical characteristics and treatment approaches vary from clinic to clinic. For more details about this, along with information on how to interpret the statistics in this table, see pages 79-88.

\section{ART CYCLE PROFILE}

\begin{tabular}{|c|c|c|c|c|c|c|c|}
\hline \multicolumn{4}{|c|}{ Type of ART ${ }^{a}$} & \multicolumn{4}{|c|}{ Patient Diagnosis } \\
\hline IVF & $100 \%$ & Procedural Factors: & & Tubal factor & $6 \%$ & Other factor & $1 \%$ \\
\hline GIFT & $0 \%$ & With ICSI & $76 \%$ & Ovulatory dysfunction & $2 \%$ & Unknown factor & $4 \%$ \\
\hline ZIFT & $0 \%$ & Unstimulated & $1 \%$ & Diminished ovarian reserve & $7 \%$ & Multiple Factors: & \\
\hline \multirow[t]{3}{*}{ Combination } & $0 \%$ & Used gestational carrier & $0 \%$ & Endometriosis & $1 \%$ & Female factors only & $5 \%$ \\
\hline & & & & Uterine factor & $1 \%$ & Female \& male factors & $49 \%$ \\
\hline & & & & Male factor & $23 \%$ & & \\
\hline
\end{tabular}

\section{PREGNANCY SUCCESS RATES}

\section{Type of Cycle}

\section{Fresh Embryos from Nondonor Eggs}

Number of cycles

Percentage of cycles resulting in pregnancies ${ }^{b}$

Percentage of cycles resulting in live births $s^{b, c}$

(Confidence Interval)

Percentage of retrievals resulting in live births $s^{b, c}$

Percentage of transfers resulting in live births $s^{b, c}$

Percentage of transfers resulting in singleton live births ${ }^{b}$

Percentage of cancellations ${ }^{b}$

Average number of embryos transferred

Percentage of pregnancies with twins ${ }^{b}$

Percentage of pregnancies with triplets or more ${ }^{b}$

Percentage of live births having multiple infants ${ }^{b, c}$

\section{Frozen Embryos from Nondonor Eggs}

Number of transfers

Percentage of transfers resulting in live births ${ }^{b, c}$

Average number of embryos transferred

\section{Donor Eggs}

Number of transfers

Percentage of transfers resulting in live births $s^{b, c}$

Average number of embryos transferred

Data verified by Joseph G. Gianfortoni, MD

\section{Age of Woman}

$<35 \quad 35-37 \quad 38-40 \quad 41-42^{\text {d }}$

34

23

18

6

44.1

43.5

$4 / 18$

$4 / 6$

38.2

34.8

$2 / 18$

$3 / 6$

(22.2-56.4)

(16.4-57.3)

38.2

$8 / 18$

$2 / 15$

$3 / 5$

40.6

$8 / 16$

$2 / 15$

$3 / 5$

21.9

$8 / 16$

2/15

$2 / 5$

0.0

21.7

$3 / 18$

$1 / 6$

2.0

2.4

2.6

3.8

$6 / 15$

$0 / 10$

$0 / 4$

$1 / 4$

$0 / 15$

$0 / 10$

$0 / 4$

$0 / 4$

$6 / 13$

$0 / 8$

$0 / 2$

$1 / 3$

16

$3 / 16$

2.3

\section{7}

$7 / 17$

2.7

\section{6}

$1 / 6$

2.3

All Ages Combined
Frozen Embryos

\section{2}

$1 / 2$

2.5
11

$2 / 11$

2.3

\section{CURRENT CLINIC SERVICES AND PROFILE}

Current Name: LifeSource Fertility Center
Donor egg?
Yes
Gestational carriers?
Yes
Cryopreservation?
Yes
Donor embryo? Yes
Single women? Yes
SART member?
Yes
Verified lab accreditation?
Yes
(See Appendix C for details.)

\footnotetext{
${ }^{a}$ Reflects patient and treatment characteristics of ART cycles performed in 2006 using fresh nondonor eggs or embryos.

${ }^{b}$ When fewer than 20 cycles are reported in an age category, rates are shown as a fraction and confidence intervals are not given. Calculating percentages from fractions may be misleading and is not encouraged.

${ }^{c}$ A multiple-infant birth is counted as one live birth.

d Clinic-specific outcome rates are unreliable for women older than 42 undergoing ART cycles using fresh or frozen embryos with nondonor eggs. Readers are urged to review national outcomes for these age groups (see page 27).

e All ages (including ages $>42$ ) are reported together because previous data show that patient age does not materially affect success with donor eggs.
} 


\section{THE RICHMOND CENTER FOR FERTILITY AND ENDOCRINOLOGY RICHMOND, VIRGINIA}

A comparison of clinic success rates may not be meaningful because patient medical characteristics and treatment approaches vary from clinic to clinic. For more details about this, along with information on how to interpret the statistics in this table, see pages $79-88$.

\section{ART CYCLE PROFILE}

\begin{tabular}{|c|c|c|c|c|c|c|c|}
\hline \multicolumn{4}{|c|}{ Type of ART ${ }^{a}$} & \multicolumn{4}{|c|}{ Patient Diagnosis } \\
\hline IVF & $100 \%$ & Procedural Factors: & & Tubal factor & $12 \%$ & Other factor & $0 \%$ \\
\hline GIFT & $0 \%$ & With ICSI & $72 \%$ & Ovulatory dysfunction & $3 \%$ & Unknown factor & $8 \%$ \\
\hline ZIFT & $0 \%$ & Unstimulated & $<1 \%$ & Diminished ovarian reserve & $11 \%$ & Multiple Factors: & \\
\hline \multirow[t]{3}{*}{ Combination } & $0 \%$ & Used gestational carrier & $4 \%$ & Endometriosis & $11 \%$ & Female factors only & $6 \%$ \\
\hline & & & & Uterine factor & $5 \%$ & Female \& male factors & $19 \%$ \\
\hline & & & & Male factor & $26 \%$ & & \\
\hline
\end{tabular}

2006 PREGNANCY SUCCESS RATES

Data verified by Sanford M. Rosenberg, MD

\section{Type of Cycle}

\section{Fresh Embryos from Nondonor Eggs}

Number of cycles

Percentage of cycles resulting in pregnancies ${ }^{b}$

Percentage of cycles resulting in live births ${ }^{b, c}$

(Confidence Interval)

Percentage of retrievals resulting in live births $s^{b, c}$

Percentage of transfers resulting in live births $s^{b, c}$

Percentage of transfers resulting in singleton live births ${ }^{b}$

Percentage of cancellations ${ }^{b}$

Average number of embryos transferred

Percentage of pregnancies with twins ${ }^{b}$

Percentage of pregnancies with triplets or more ${ }^{b}$

Percentage of live births having multiple infants ${ }^{b, c}$

\section{Frozen Embryos from Nondonor Eggs}

Number of transfers

Percentage of transfers resulting in live births $s^{\mathrm{b}, \mathrm{c}}$

Average number of embryos transferred

\section{Donor Eggs}

Number of transfers

Percentage of transfers resulting in live births ${ }^{\mathrm{b}, \mathrm{c}}$

Average number of embryos transferred

\section{Age of Woman}

35-37 38-40

$41-42^{d}$ $<35$

40

40.0

24

4

44.1

35.0

33.3

$3 / 4$

42.6

$(30.7-55.2)$

(20.6-51.7)

20.8

$2 / 4$

\section{9}

43.8

$(7.1-42.2)$

47.5

45.2

22.7

$2 / 4$

31.1

25.8

22.7

$2 / 4$

2.9

20.0

9.1

$2 / 4$

2.3

3.0

8.3

$0 / 4$

36.7

$5 / 16$

3.3

34.5

$1 / 16$

$6 / 14$

3.0

3.8

$0 / 3$

$0 / 3$

$0 / 8$

$0 / 2$

$3 / 5$

0

25

8

$2 / 8$

10

$1 / 10$

2.7

3.5

2.6

All Ages Combined ${ }^{\mathrm{e}}$

Fresh Embryos 3

$1 / 3$

2.0
Frozen Embryos 18

$4 / 18$

2.6

\section{CURRENT CLINIC SERVICES AND PROFILE}

Current Name: The Richmond Center for Fertility and Endocrinology

Donor egg? Yes Gestational carriers? Yes

Donor embryo? Yes Cryopreservation? Yes

Single women? Yes

SART member? Yes

Verified lab accreditation? Yes

(See Appendix C for details.)

${ }^{a}$ Reflects patient and treatment characteristics of ART cycles performed in 2006 using fresh nondonor eggs or embryos.

${ }^{b}$ When fewer than 20 cycles are reported in an age category, rates are shown as a fraction and confidence intervals are not given. Calculating percentages from fractions may be misleading and is not encouraged.

${ }^{c}$ A multiple-infant birth is counted as one live birth.

${ }^{d}$ Clinic-specific outcome rates are unreliable for women older than 42 undergoing ART cycles using fresh or frozen embryos with nondonor eggs. Readers are urged to review national outcomes for these age groups (see page 27).

e All ages (including ages $>42$ ) are reported together because previous data show that patient age does not materially affect success with donor eggs. 


\section{THE NEW HOPE CENTER FOR REPRODUCTIVE MEDICINE VIRGINIA BEACH, VIRGINIA}

A comparison of clinic success rates may not be meaningful because patient medical characteristics and treatment approaches vary from clinic to clinic. For more details about this, along with information on how to interpret the statistics in this table, see pages 79-88.

\section{ART CYCLE PROFILE}

\begin{tabular}{|c|c|c|c|c|c|c|c|}
\hline \multicolumn{4}{|c|}{ Type of ART ${ }^{a}$} & \multicolumn{4}{|c|}{ Patient Diagnosis } \\
\hline IVF & $100 \%$ & Procedural Factors: & & Tubal factor & $9 \%$ & Other factor & $2 \%$ \\
\hline GIFT & $0 \%$ & With ICSI & $54 \%$ & Ovulatory dysfunction & $10 \%$ & Unknown factor & $<1 \%$ \\
\hline ZIFT & $0 \%$ & Unstimulated & $0 \%$ & Diminished ovarian reserve & $7 \%$ & Multiple Factors: & \\
\hline \multirow[t]{3}{*}{ Combination } & $0 \%$ & Used gestational carrier & $<1 \%$ & Endometriosis & $5 \%$ & Female factors only & $41 \%$ \\
\hline & & & & Uterine factor & $2 \%$ & Female \& male factors & $20 \%$ \\
\hline & & & & Male factor & $4 \%$ & & \\
\hline
\end{tabular}

\section{PREGNANCY SUCCESS RATES}

\section{Type of Cycle}

\section{Fresh Embryos from Nondonor Eggs}

Number of cycles

Percentage of cycles resulting in pregnancies ${ }^{b}$

Percentage of cycles resulting in live births ${ }^{b, c}$

(Confidence Interval)

Percentage of retrievals resulting in live births $s^{b, c}$

Percentage of transfers resulting in live births $s^{b, c}$

Percentage of transfers resulting in singleton live births ${ }^{b}$

Percentage of cancellations ${ }^{b}$

Average number of embryos transferred

Percentage of pregnancies with twins ${ }^{b}$

Percentage of pregnancies with triplets or more ${ }^{b}$

Percentage of live births having multiple infants ${ }^{b, c}$

\section{Frozen Embryos from Nondonor Eggs}

Number of transfers

Percentage of transfers resulting in live births ${ }^{b, c}$

Average number of embryos transferred

\section{Donor Eggs}

Number of transfers

Percentage of transfers resulting in live births $s^{b, c}$

Average number of embryos transferred

Data verified by Robin L. Poe-Zeigler, MD

\section{Age of Woman}

$<35 \quad 35-37 \quad 38-40 \quad 41-42^{d}$

$\begin{array}{cccc}109 & 53 & 38 & 18 \\ 51.4 & 43.4 & 31.6 & 4 / 18 \\ 44.0 & 30.2 & 15.8 & 2 / 18\end{array}$

$(34.5-53.9)$

$(18.3-44.3)$

$(6.0-31.3)$

49.5

52.7

35.6

17.1

$2 / 15$

39.0

18.8

$2 / 13$

31.9

19.5

18.8

$2 / 13$

11.0

15.1

7.9

$3 / 18$

2.2

2.8

2.8

2.8

42.9

21.7

$1 / 12$

$0 / 4$

1.8

13.0

$1 / 12$

$0 / 4$

39.6

$8 / 16$

$0 / 6$

$0 / 2$

38

50.0

2.4

\section{9}

$3 / 9$

2.0

\section{7}

$2 / 7$

3.3
1

$0 / 1$

3.0

\section{CURRENT CLINIC SERVICES AND PROFILE}

Current Name: The New Hope Center for Reproductive Medicine
Donor egg?
Yes
Donor embryo? Yes
Gestational carriers? Yes
Cryopreservation?
SART member?
Yes
Verified lab accreditation?
Yes
Single women? Yes
(See Appendix C for details.)

\footnotetext{
${ }^{a}$ Reflects patient and treatment characteristics of ART cycles performed in 2006 using fresh nondonor eggs or embryos.

${ }^{b}$ When fewer than 20 cycles are reported in an age category, rates are shown as a fraction and confidence intervals are not given. Calculating percentages from fractions may be misleading and is not encouraged.

${ }^{c}$ A multiple-infant birth is counted as one live birth.

d Clinic-specific outcome rates are unreliable for women older than 42 undergoing ART cycles using fresh or frozen embryos with nondonor eggs. Readers are urged to review national outcomes for these age groups (see page 27).

${ }^{e}$ All ages (including ages $>42$ ) are reported together because previous data show that patient age does not materially affect success with donor eggs.
} 


\section{FRANCISCO M. IRIANNI INFERTILITY CLINIC WINCHESTER, VIRGINIA}

A comparison of clinic success rates may not be meaningful because patient medical characteristics and treatment approaches vary from clinic to clinic. For more details about this, along with information on how to interpret the statistics in this table, see pages $79-88$.

\section{ART CYCLE PROFILE}

\begin{tabular}{|c|c|c|c|c|c|c|c|}
\hline \multicolumn{4}{|c|}{ Type of ART ${ }^{\mathrm{a}}$} & \multicolumn{4}{|c|}{ Patient Diagnosis } \\
\hline IVF & $100 \%$ & Procedural Factors: & & Tubal factor & $8 \%$ & Other factor & $0 \%$ \\
\hline GIFT & $0 \%$ & With ICSI & $54 \%$ & Ovulatory dysfunction & $0 \%$ & Unknown factor & $0 \%$ \\
\hline ZIFT & $0 \%$ & Unstimulated & $0 \%$ & Diminished ovarian reserve & $5 \%$ & Multiple Factors: & \\
\hline \multirow[t]{3}{*}{ Combination } & $0 \%$ & Used gestational carrier & $0 \%$ & Endometriosis & $0 \%$ & Female factors only & $38 \%$ \\
\hline & & & & Uterine factor & $0 \%$ & Female \& male factors & $50 \%$ \\
\hline & & & & Male factor & $0 \%$ & & \\
\hline
\end{tabular}

2006 PREGNANCY SUCCESS RATES

Data verified by Francisco M. Irianni, MD

\section{Type of Cycle}

\section{Fresh Embryos from Nondonor Eggs}

Number of cycles

Percentage of cycles resulting in pregnancies ${ }^{b}$

Percentage of cycles resulting in live births ${ }^{b, c}$

(Confidence Interval)
Percentage of retrievals resulting in live births ${ }^{b, c}$

Percentage of transfers resulting in live births $s^{b, c}$

Percentage of transfers resulting in singleton live births ${ }^{b}$

Percentage of cancellations ${ }^{b}$

Average number of embryos transferred

Percentage of pregnancies with twins ${ }^{b}$

Percentage of pregnancies with triplets or more ${ }^{b}$

Percentage of live births having multiple infants ${ }^{b, c}$

\section{Frozen Embryos from Nondonor Eggs}

Number of transfers

Percentage of transfers resulting in live births ${ }^{\mathrm{b}, \mathrm{c}}$

Average number of embryos transferred

\section{Donor Eggs}

Number of transfers

Percentage of transfers resulting in live births $s^{b, c}$

Average number of embryos transferred

\section{Age of Woman}

35-37 38-40

$41-42^{d}$

\section{$<35$}

3

$0 / 12$

$2 / 3$

7

$1 / 7$

$0 / 12$

$2 / 3$

$1 / 7$

$0 / 8 \quad 2 / 3 \quad 1 / 6$

$0 / 8$

$2 / 3$

$1 / 6$

$0 / 8$

$0 / 3$

$1 / 6$

$4 / 12$

$0 / 3$

$1 / 7$

2.3

3.0

2.3

$1 / 2$

$0 / 1$

$1 / 2$

$0 / 1$

$2 / 2$

$0 / 1$

\section{CURRENT CLINIC SERVICES AND PROFILE}

Current Name: Francisco M. Irianni Infertility Clinic

Donor egg? Yes

Donor embryo? No

Single women? No

\section{Gestational carriers? Yes}

Cryopreservation? Yes

\section{5}

$1 / 5$

2.8
0

\section{All Ages Combined ${ }^{\mathrm{e}}$}

Fresh Embryos

4

$0 / 4$

2.8
Frozen Embryos

4

$1 / 4$

2.5

\footnotetext{
${ }^{a}$ Reflects patient and treatment characteristics of ART cycles performed in 2006 using fresh nondonor eggs or embryos.

${ }^{b}$ When fewer than 20 cycles are reported in an age category, rates are shown as a fraction and confidence intervals are not given. Calculating percentages from fractions may be misleading and is not encouraged.

${ }^{c}$ A multiple-infant birth is counted as one live birth.

d Clinic-specific outcome rates are unreliable for women older than 42 undergoing ART cycles using fresh or frozen embryos with nondonor eggs. Readers are urged to review national outcomes for these age groups (see page 27).

e All ages (including ages $>42$ ) are reported together because previous data show that patient age does not materially affect success with donor eggs.
} 


\section{OVERLAKE REPRODUCTIVE HEALTH INC., PS BELLEVUE, WASHINGTON}

A comparison of clinic success rates may not be meaningful because patient medical characteristics and treatment approaches vary from clinic to clinic. For more details about this, along with information on how to interpret the statistics in this table, see pages 79-88.

\section{ART CYCLE PROFILE}

\section{Type of ART ${ }^{\mathrm{a}}$}

IVF

GIFT

ZIFT

Combination
100\% Procedural Factors:

$0 \%$ With ICSI

$0 \%$ Unstimulated

$0 \%$ Used gestational carrier $<1 \%$
Tubal factor

Ovulatory dysfunction

Diminished ovarian reserve

Endometriosis

Uterine factor

Male factor

\section{Patient Diagnosis}

$4 \%$ Other factor

5\% Unknown factor

16\% Multiple Factors:

$<1 \% \quad$ Female factors only $31 \%$

$<1 \%$ Female \& male factors $39 \%$

$1 \%$
$1 \%$

$<1 \%$

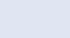

2006 PREGNANCY SUCCESS RATES

Data verified by Kevin M. Johnson, MD

\section{Type of Cycle}

\section{Age of Woman}

$\begin{array}{llll}<35 & 35-37 & 38-40 & 41-42^{d}\end{array}$

\section{Fresh Embryos from Nondonor Eggs}

Number of cycles

21

19

11

Percentage of cycles resulting in pregnancies ${ }^{b}$

46.3

47.6

$3 / 19$

$1 / 11$

Percentage of cycles resulting in live births $s^{b, c}$

40.7

38.1

$2 / 19$

$1 / 11$

(Confidence Interval)

Percentage of retrievals resulting in live births $s^{b, c}$

$(27.6-55.0)$

(18.1-61.6)

Percentage of transfers resulting in live births $s^{b, c}$

43.1

$8 / 18$

$2 / 17$

$1 / 11$

52.4

$8 / 17$

$2 / 14$

$1 / 10$

Percentage of transfers resulting in singleton live births ${ }^{b}$

31.0

$4 / 17$

$1 / 14$

$1 / 10$

Percentage of cancellations ${ }^{b}$

5.6

14.3

$2 / 19$

$0 / 11$

Average number of embryos transferred

Percentage of pregnancies with twins ${ }^{b}$

2.3

2.5

2.1

3.1

Percentage of pregnancies with triplets or more ${ }^{b}$

Percentage of live births having multiple infants ${ }^{b, c}$

36.0

$4 / 10$

$1 / 3$

$0 / 1$

0.0

$0 / 10$

$0 / 3$

$0 / 1$

40.9

$4 / 8$

$1 / 2$

$0 / 1$

\section{Frozen Embryos from Nondonor Eggs}

Number of transfers

Percentage of transfers resulting in live births $s^{b, c}$

Average number of embryos transferred

6

$1 / 6$

1.5

\section{3}

$2 / 3$

2.0
1

$0 / 1$

1.0

\section{Donor Eggs}

Fresh Embryos

All Ages Combined ${ }^{\mathrm{e}}$

Number of transfers

Percentage of transfers resulting in live births $s^{b, c}$

Average number of embryos transferred

Frozen Embryos

9
$8 / 13$
$3 / 9$

2.1

\section{CURRENT CLINIC SERVICES AND PROFILE}

Current Name: Overlake Reproductive Health Inc., PS

Donor egg? Yes Gestational carriers? Yes

Donor embryo? Yes Cryopreservation? Yes

Single women? Yes

SART member?

Yes

Verified lab accreditation?

Yes

(See Appendix C for details.)

${ }^{a}$ Reflects patient and treatment characteristics of ART cycles performed in 2006 using fresh nondonor eggs or embryos.

${ }^{b}$ When fewer than 20 cycles are reported in an age category, rates are shown as a fraction and confidence intervals are not given. Calculating percentages from fractions may be misleading and is not encouraged.

${ }^{c}$ A multiple-infant birth is counted as one live birth.

d Clinic-specific outcome rates are unreliable for women older than 42 undergoing ART cycles using fresh or frozen embryos with nondonor eggs. Readers are urged to review national outcomes for these age groups (see page 27).

e All ages (including ages $>42$ ) are reported together because previous data show that patient age does not materially affect success with donor eggs. 


\section{WASHINGTON CENTER FOR REPRODUCTIVE MEDICINE BELLEVUE, WASHINGTON}

A comparison of clinic success rates may not be meaningful because patient medical characteristics and treatment approaches vary from clinic to clinic. For more details about this, along with information on how to interpret the statistics in this table, see pages $79-88$.

\section{ART CYCLE PROFILE}

\begin{tabular}{|c|c|c|c|c|c|c|c|}
\hline \multicolumn{4}{|c|}{ Type of ART ${ }^{\mathrm{a}}$} & \multicolumn{4}{|c|}{ Patient Diagnosis } \\
\hline IVF & $100 \%$ & Procedural Factors: & & Tubal factor & $9 \%$ & Other factor & $10 \%$ \\
\hline GIFT & $0 \%$ & With ICSI & $85 \%$ & Ovulatory dysfunction & $2 \%$ & Unknown factor & $11 \%$ \\
\hline ZIFT & $0 \%$ & Unstimulated & $0 \%$ & Diminished ovarian reserve & $24 \%$ & Multiple Factors: & \\
\hline \multirow[t]{3}{*}{ Combination } & $0 \%$ & Used gestational carrier & $0 \%$ & Endometriosis & $1 \%$ & Female factors only & $1 \%$ \\
\hline & & & & Uterine factor & $<1 \%$ & Female \& male factors & $15 \%$ \\
\hline & & & & Male factor & $26 \%$ & & \\
\hline
\end{tabular}

2006 PREGNANCY SUCCESS RATES

Data verified by James I. Kustin, MD

\section{Type of Cycle}

\section{Fresh Embryos from Nondonor Eggs}

Number of cycles

Percentage of cycles resulting in pregnancies ${ }^{b}$

Percentage of cycles resulting in live births ${ }^{b, c}$

(Confidence Interval)

Percentage of retrievals resulting in live births $s^{b, c}$

Percentage of transfers resulting in live births $s^{b, c}$

Percentage of transfers resulting in singleton live births ${ }^{b}$

Percentage of cancellations ${ }^{b}$

Average number of embryos transferred

Percentage of pregnancies with twins ${ }^{b}$

Percentage of pregnancies with triplets or more ${ }^{b}$

Percentage of live births having multiple infants ${ }^{b, c}$

\section{Frozen Embryos from Nondonor Eggs}

Number of transfers

Percentage of transfers resulting in live births ${ }^{\mathrm{b}, \mathrm{c}}$

Average number of embryos transferred

\section{Donor Eggs}

Number of transfers

Percentage of transfers resulting in live births $s^{b, c}$

Average number of embryos transferred

\section{Age of Woman}

35-37 38-40

$41-42^{d}$

$<35$

24

29.2

12

2

27.9

20.8

$5 / 12$

$0 / 2$

27.9

$(7.1-42.2)$

(15.3-43.7)

23.8
$5 / 17$

$4 / 12$

$0 / 2$

29.3

32.4

$5 / 17$

$4 / 12$

$0 / 1$

16.2

12.5

$4 / 9$

$0 / 1$

4.7

2.4

$4 / 9$

$0 / 1$

2.6

$5 / 12$

$1 / 7$

$0 / 12$

$1 / 2$

$1 / 12$

$6 / 12$

$0 / 7$

3.0

3.0

$1 / 5$

$0 / 5$

$0 / 5$

$0 / 4$

$\begin{array}{cccc}11 & 13 & 3 & 1 \\ 5 / 11 & 3 / 13 & 0 / 3 & 0 / 1 \\ 2.3 & 2.7 & 3.0 & 2.0\end{array}$

Fresh Embryos

All Ages Combined ${ }^{\mathrm{e}}$
Frozen Embryos

$\begin{array}{cc}15 & 16 \\ 5 / 15 & 4 / 16 \\ 2.4 & 3.1\end{array}$

\section{CURRENT CLINIC SERVICES AND PROFILE}

Current Name: Washington Center for Reproductive Medicine

\begin{tabular}{l|l|l} 
Donor egg? Yes & Gestational carriers? Yes
\end{tabular}

Donor embryo? Yes Cryopreservation? Yes

Single women? Yes

SART member? Yes

Verified lab accreditation? Yes

(See Appendix C for details.)

${ }^{a}$ Reflects patient and treatment characteristics of ART cycles performed in 2006 using fresh nondonor eggs or embryos.

${ }^{b}$ When fewer than 20 cycles are reported in an age category, rates are shown as a fraction and confidence intervals are not given. Calculating percentages from fractions may be misleading and is not encouraged.

A multiple-infant birth is counted as one live birth.

d Clinic-specific outcome rates are unreliable for women older than 42 undergoing ART cycles using fresh or frozen embryos with nondonor eggs. Readers are urged to review national outcomes for these age groups (see page 27).

e All ages (including ages $>42$ ) are reported together because previous data show that patient age does not materially affect success with donor eggs. 


\section{BELLINGHAM IVF \& FERTILITY CARE BELLINGHAM, WASHINGTON}

A comparison of clinic success rates may not be meaningful because patient medical characteristics and treatment approaches vary from clinic to clinic. For more details about this, along with information on how to interpret the statistics in this table, see pages 79-88.

\section{ART CYCLE PROFILE}

\section{Type of ART ${ }^{\mathrm{a}}$}

IVF

GIFT $100 \%$ Procedural Factors:

ZIFT

Combination
$0 \%$ With ICSI

$0 \%$ Unstimulated

$0 \%$ Used gestational carrier

\begin{tabular}{|c|c|c|c|c|}
\hline & \multicolumn{4}{|c|}{ Patient Diagnosis } \\
\hline & Tubal factor & $2 \%$ & Other factor & $0 \%$ \\
\hline $71 \%$ & Ovulatory dysfunction & $2 \%$ & Unknown factor & $0 \%$ \\
\hline $0 \%$ & Diminished ovarian reserve & $9 \%$ & Multiple Factors: & \\
\hline $0 \%$ & Endometriosis & $0 \%$ & Female factors only & $4 \%$ \\
\hline & Uterine factor & $0 \%$ & Female \& male factors & $69 \%$ \\
\hline & Male factor & $15 \%$ & & \\
\hline
\end{tabular}

Data verified by Emmett F. Branigan, MD

2006 PREGNANCY SUCCESS RATES

\section{Type of Cycle}

\section{Fresh Embryos from Nondonor Eggs}

Number of cycles

Percentage of cycles resulting in pregnancies ${ }^{b}$

Percentage of cycles resulting in live births ${ }^{b, c}$

(Confidence Interval)

Percentage of retrievals resulting in live births $s^{b, c}$

Percentage of transfers resulting in live births ${ }^{b, c}$

Percentage of transfers resulting in singleton live births ${ }^{b}$

Percentage of cancellations ${ }^{b}$

Average number of embryos transferred

Percentage of pregnancies with twins ${ }^{b}$

Percentage of pregnancies with triplets or more ${ }^{b}$

Percentage of live births having multiple infants ${ }^{b, c}$

\section{Frozen Embryos from Nondonor Eggs}

Number of transfers

Percentage of transfers resulting in live births ${ }^{b, c}$

Average number of embryos transferred

\section{Donor Eggs}

Number of transfers

Percentage of transfers resulting in live births $s^{b, c}$

Average number of embryos transferred

\section{Age of Woman}

35-37 38-40

$41-42^{d}$

\section{$<35$}

13

8

8

69.0

$8 / 13$

$1 / 8$

$1 / 8$

55.2

$7 / 13$

$0 / 8$

$0 / 8$

(35.7-73.6)

57.1

59.3

$7 / 13$

$0 / 7$

$0 / 8$

$7 / 13$

$0 / 7$

$0 / 8$

55.6

$7 / 13$

$0 / 7$

$0 / 8$

3.4

$0 / 13$

$1 / 8$

$0 / 8$

2.2

2.5

$0 / 8$

2.6

2.5

5.0

$0 / 8$

$0 / 1$

$0 / 1$

0.0

$0 / 7$

$0 / 1$

$0 / 1$

$1 / 16$

(

$\begin{array}{ccc}8 & 4 & 4 \\ 3 / 8 & 2 / 4 & 0 / 4 \\ 2.3 & 2.0 & 2.5\end{array}$

All Ages Combined

Fresh Embryos

Frozen Embryos

2.0

$4 / 15$

2.0

\section{CURRENT CLINIC SERVICES AND PROFILE}

Current Name: Bellingham IVF \& Fertility Care

Donor egg? Yes

Donor embryo? No

Single women? Yes

\section{Gestational carriers? No}

Cryopreservation?

Yes
SART member?

Verified lab accreditation?

(See Appendix C for details.)
No

No

\footnotetext{
${ }^{a}$ Reflects patient and treatment characteristics of ART cycles performed in 2006 using fresh nondonor eggs or embryos.

b When fewer than 20 cycles are reported in an age category, rates are shown as a fraction and confidence intervals are not given. Calculating percentages from fractions may be misleading and is not encouraged.

${ }^{c}$ A multiple-infant birth is counted as one live birth.

${ }^{d}$ Clinic-specific outcome rates are unreliable for women older than 42 undergoing ART cycles using fresh or frozen embryos with nondonor eggs. Readers are urged to review national outcomes for these age groups (see page 27).

e All ages (including ages $>42$ ) are reported together because previous data show that patient age does not materially affect success with donor eggs.
} 


\section{NORTHWEST CENTER FOR REPRODUCTIVE SCIENCES KIRKLAND, WASHINGTON}

A comparison of clinic success rates may not be meaningful because patient medical characteristics and treatment approaches vary from clinic to clinic. For more details about this, along with information on how to interpret the statistics in this table, see pages $79-88$.

\section{ART CYCLE PROFILE}

\begin{tabular}{|c|c|c|c|c|c|c|c|}
\hline \multicolumn{4}{|c|}{ Type of $A R T^{a}$} & \multicolumn{4}{|c|}{ Patient Diagnosis } \\
\hline IVF & $100 \%$ & Procedural Factors: & & Tubal factor & $2 \%$ & Other factor & $6 \%$ \\
\hline GIFT & $0 \%$ & With ICSI & $32 \%$ & Ovulatory dysfunction & $9 \%$ & Unknown factor & $9 \%$ \\
\hline ZIFT & $0 \%$ & Unstimulated & $<1 \%$ & Diminished ovarian reserve & $20 \%$ & Multiple Factors: & \\
\hline \multirow[t]{3}{*}{ Combination } & $0 \%$ & Used gestational carrier & $0 \%$ & Endometriosis & $3 \%$ & Female factors only & $18 \%$ \\
\hline & & & & Uterine factor & $2 \%$ & Female \& male factors & $18 \%$ \\
\hline & & & & Male factor & $13 \%$ & & \\
\hline
\end{tabular}

2006 PREGNANCY SUCCESS RATES

Data verified by Michael S. Opsahl, MD

\section{Type of Cycle}

\section{Fresh Embryos from Nondonor Eggs}

Number of cycles

Percentage of cycles resulting in pregnancies ${ }^{b}$

Percentage of cycles resulting in live births ${ }^{b, c}$

(Confidence Interval)

Percentage of retrievals resulting in live births $s^{b, c}$

Percentage of transfers resulting in live births $s^{b, c}$

Percentage of transfers resulting in singleton live births ${ }^{b}$

Percentage of cancellations ${ }^{b}$

Average number of embryos transferred

Percentage of pregnancies with twins ${ }^{b}$

Percentage of pregnancies with triplets or more ${ }^{b}$

Percentage of live births having multiple infants ${ }^{b, c}$

\section{Frozen Embryos from Nondonor Eggs}

Number of transfers

Percentage of transfers resulting in live births $s^{b, c}$

Average number of embryos transferred

\section{Donor Eggs}

Number of transfers

Percentage of transfers resulting in live births ${ }^{\mathrm{b}, \mathrm{c}}$

Average number of embryos transferred

\section{Age of Woman}

35-37 38-40

$41-42^{d}$

$<35$

49

49.0

51

13

65.1

42.9

49.0

$1 / 13$

61.4

(28.8-57.8)

35.3

$0 / 13$

$(50.1-71.9)$

67.1

47.7

(22.4-49.9)

72.9

48.8

43.9

$0 / 8$

28.6

34.9

46.2

$0 / 8$

8.4

10.2

30.8

$0 / 8$

2.7

3.1

19.6

$5 / 13$

55.6

25.0

3.8

3.0

9.3

8.3

60.8

28.6

36.0

$0 / 1$

8.0

$0 / 1$

$6 / 18$

8

$4 / 8$

2.4

\section{2}

$1 / 2$

1.5

\section{5}

$1 / 5$

1.6
1

$0 / 1$

3.0

\section{CURRENT CLINIC SERVICES AND PROFILE}

Current Name: Northwest Center for Reproductive Sciences

\begin{tabular}{l|l|l} 
Donor egg? Yes & Gestational carriers? Yes
\end{tabular}

Donor embryo? Yes Cryopreservation? Yes

Fresh Embryos

All Ages Combined ${ }^{\mathrm{e}}$

Single women? Yes

$\begin{array}{cc}26 & 7 \\ 76.9 & 3 / 7 \\ 2.1 & 1.7\end{array}$

${ }_{b}^{a}$ Reflects patient and treatment characteristics of ART cycles performed in 2006 using fresh nondonor eggs or embryos.

${ }^{b}$ When fewer than 20 cycles are reported in an age category, rates are shown as a fraction and confidence intervals are not given. Calculating percentages from fractions may be misleading and is not encouraged.

A multiple-infant birth is counted as one live birth.

d Clinic-specific outcome rates are unreliable for women older than 42 undergoing ART cycles using fresh or frozen embryos with nondonor eggs. Readers are urged to review national outcomes for these age groups (see page 27).

e All ages (including ages $>42$ ) are reported together because previous data show that patient age does not materially affect success with donor eggs. 


\section{OLYMPIA WOMEN'S HEALTH \\ OLYMPIA, WASHINGTON}

A comparison of clinic success rates may not be meaningful because patient medical characteristics and treatment approaches vary from clinic to clinic. For more details about this, along with information on how to interpret the statistics in this table, see pages 79-88.

\section{ART CYCLE PROFILE}

\section{Type of ART ${ }^{\mathrm{a}}$}

IVF

GIFT

100\% Procedural Factors:

ZIFT

Combination
$0 \%$ With ICSI

$0 \%$ Unstimulated

$0 \%$ Used gestational carrier

\section{$0 \%$ \\ $0 \%$ \\ $0 \%$}

Tubal factor

Ovulatory dysfunction

Diminished ovarian reserve

Endometriosis

Uterine factor

Male factor

\section{Patient Diagnosis}

$\begin{array}{rlr}21 \% & \text { Other factor } & 14 \% \\ 7 \% & \text { Unknown factor } & 24 \% \\ 0 \% & \text { Multiple Factors: } & \\ 10 \% & \text { Female factors only } & 0 \% \\ 0 \% & \text { Female \& male factors } & 14 \% \\ 10 \% & & \end{array}$

Data verified by James F. Moruzzi, MD, PhD

2006 PREGNANCY SUCCESS RATES

Age of Woman

Type of Cycle

$\begin{array}{cccc}<35 & 35-37 & 38-40 & \mathbf{4 1 - 4 2}^{\mathbf{d}} \\ 13 & 2 & 3 & 2 \\ 5 / 13 & 1 / 2 & 1 / 3 & 2 / 2 \\ 4 / 13 & 1 / 2 & 1 / 3 & 2 / 2\end{array}$

\section{Fresh Embryos from Nondonor Eggs}

Number of cycles

$5 / 13$

$1 / 2$

$1 / 3$

$2 / 2$

Percentage of cycles resulting in live births ${ }^{\mathrm{b}, \mathrm{c}}$

(Confidence Interval)

Percentage of retrievals resulting in live births $s^{b, c}$

$4 / 11$

$1 / 2$

$1 / 3$

$2 / 2$

$4 / 9$

$1 / 2$

$1 / 3$

$2 / 2$

Percentage of transfers resulting in singleton live births ${ }^{b}$

$2 / 9$

$1 / 2$

$1 / 3$

$2 / 2$

$2 / 13$

$0 / 2$

$0 / 3$

$0 / 2$

Average number of embryos transferred

1.9

Percentage of pregnancies with twins ${ }^{b}$

$3 / 5$

1.5

2.7

2.5

$1 / 5$

$0 / 1$

$0 / 1$

$0 / 2$

$2 / 4$

$0 / 1$

$0 / 1$

$0 / 2$

Percentage of live births having multiple infants ${ }^{b, c}$

\section{Frozen Embryos from Nondonor Eggs}

Number of transfers

Percentage of transfers resulting in live births ${ }^{b, c}$

Average number of embryos transferred

3

$2 / 3$

$0 / 1$

$0 / 1$

$0 / 2$

2.0

0

2

$0 / 2$

1.5

All Ages Combined ${ }^{\mathrm{e}}$

Fresh Embryos

Frozen Embryos

3

$3 / 3$

Number of transfers

Percentage of transfers resulting in live births $s^{b, c}$

Average number of embryos transferred

\section{CURRENT CLINIC SERVICES AND PROFILE}

Current Name: Olympia Women's Health
Donor egg?
Yes
Donor embryo? Yes
Gestational carriers? Yes
Cryopreservation?
Yes
Single women? Yes
SART member?
Yes
Verified lab accreditation?
Yes
(See Appendix C for details.)
${ }^{a}$ Reflects patient and treatment characteristics of ART cycles performed in 2006 using fresh nondonor eggs or embryos.
${ }^{b}$ When fewer than 20 cycles are reported in an age category, rates are shown as a fraction and confidence intervals are not given. Calculating percentages from fractions may be misleading and is not encouraged.
${ }^{c}$ A multiple-infant birth is counted as one live birth.
d Clinic-specific outcome rates are unreliable for women older than 42 undergoing ART cycles using fresh or frozen embryos with nondonor eggs. Readers are urged to review national outcomes for these age groups (see page 27).
e All ages (including ages $>42$ ) are reported together because previous data show that patient age does not materially affect success with donor eggs. 


\section{PACIFIC NORTHWEST FERTILITY AND IVF SPECIALISTS SEATTLE, WASHINGTON}

A comparison of clinic success rates may not be meaningful because patient medical characteristics and treatment approaches vary from clinic to clinic. For more details about this, along with information on how to interpret the statistics in this table, see pages $79-88$.

\section{ART CYCLE PROFILE}

\begin{tabular}{|c|c|c|c|c|c|c|c|}
\hline \multicolumn{4}{|c|}{ Type of ART ${ }^{\mathrm{a}}$} & \multicolumn{4}{|c|}{ Patient Diagnosis } \\
\hline IVF & $100 \%$ & Procedural Factors: & & Tubal factor & $1 \%$ & Other factor & $39 \%$ \\
\hline GIFT & $0 \%$ & With ICSI & $85 \%$ & Ovulatory dysfunction & $5 \%$ & Unknown factor & $12 \%$ \\
\hline ZIFT & $0 \%$ & Unstimulated & $0 \%$ & Diminished ovarian reserve & $17 \%$ & Multiple Factors: & \\
\hline \multirow[t]{3}{*}{ Combination } & $0 \%$ & Used gestational carrier & $2 \%$ & Endometriosis & $2 \%$ & Female factors only & $5 \%$ \\
\hline & & & & Uterine factor & $<1 \%$ & Female \& male factors & $8 \%$ \\
\hline & & & & Male factor & $13 \%$ & & \\
\hline
\end{tabular}

2006 PREGNANCY SUCCESS RATES

Data verified by Lorna A. Marshall, MD

\section{Type of Cycle}

\section{Fresh Embryos from Nondonor Eggs}

Number of cycles

Percentage of cycles resulting in pregnancies ${ }^{b}$

Percentage of cycles resulting in live births ${ }^{b, c}$

(Confidence Interval)

Percentage of retrievals resulting in live births $s^{b, c}$

Percentage of transfers resulting in live births $s^{b, c}$

Percentage of transfers resulting in singleton live births ${ }^{b}$

Percentage of cancellations ${ }^{b}$

Average number of embryos transferred

Percentage of pregnancies with twins ${ }^{b}$

Percentage of pregnancies with triplets or more ${ }^{b}$

Percentage of live births having multiple infants ${ }^{b, c}$

\section{Frozen Embryos from Nondonor Eggs}

Number of transfers

Percentage of transfers resulting in live births $s^{b, c}$

Average number of embryos transferred

\section{Donor Eggs}

Number of transfers

Percentage of transfers resulting in live births ${ }^{\mathrm{b}, \mathrm{c}}$

Average number of embryos transferred

\section{Age of Woman}

35-37 38-40

$41-42^{d}$

\section{$<35$}

93

31.2

73

33

41.8

24.7

28.8

27.3

32.0

(16.4-34.8)

21.9

15.2

(23.8-41.0)

27.7

(13.1-33.1)

(5.1-31.9)

33.9

35.1

28.8

25.8

16.7

22.5

21.3

26.7

17.9

5.7

10.8

18.3

14.3

2.2

2.4

31.4

20.7

15.1

9.1

3.9

3.4

35.9

26.1

2.8

4.0

$1 / 9$

33.3

$0 / 9$

0.0

$1 / 5$

$5 / 16$

12

61
29.5

34

23

$3 / 12$

2.0

20.6

26.1

2.3

2.5

All Ages Combined ${ }^{\mathrm{e}}$

Fresh Embryos

84

63.1

1.9
Frozen Embryos 55

47.3

2.3

\section{CURRENT CLINIC SERVICES AND PROFILE}

Current Name: Pacific Northwest Fertility and IVF Specialists

\begin{tabular}{l|l|l} 
Donor egg? & Yes & Gestational carriers? Yes
\end{tabular}

Donor embryo? Yes Cryopreservation? Yes

Single women? Yes

SART member? Yes

Verified lab accreditation? Yes

(See Appendix C for details.)

${ }^{a}$ Reflects patient and treatment characteristics of ART cycles performed in 2006 using fresh nondonor eggs or embryos.

${ }^{b}$ When fewer than 20 cycles are reported in an age category, rates are shown as a fraction and confidence intervals are not given. Calculating percentages from fractions may be misleading and is not encouraged.

${ }^{c}$ A multiple-infant birth is counted as one live birth.

d Clinic-specific outcome rates are unreliable for women older than 42 undergoing ART cycles using fresh or frozen embryos with nondonor eggs. Readers are urged to review national outcomes for these age groups (see page 27).

e All ages (including ages $>42$ ) are reported together because previous data show that patient age does not materially affect success with donor eggs. 


\section{SEATTLE REPRODUCTIVE MEDICINE \\ INTEGRAMED AMERICA \\ SEATTLE, WASHINGTON}

A comparison of clinic success rates may not be meaningful because patient medical characteristics and treatment approaches vary from clinic to clinic. For more details about this, along with information on how to interpret the statistics in this table, see pages 79-88.

\section{ART CYCLE PROFILE}

\section{Type of ART ${ }^{\mathrm{a}}$}

IVF

GIFT

$100 \%$ Procedural Factors:

ZIFT

Combination
$0 \%$ With ICSI

$0 \%$ Unstimulated

$0 \%$ Used gestational carrier

\begin{tabular}{|c|c|c|c|c|}
\hline & \multicolumn{4}{|c|}{ Patient Diagnosis } \\
\hline & Tubal factor & $12 \%$ & Other factor & $5 \%$ \\
\hline $56 \%$ & Ovulatory dysfunction & $4 \%$ & Unknown factor & $12 \%$ \\
\hline $0 \%$ & Diminished ovarian reserve & $16 \%$ & Multiple Factors: & \\
\hline $0 \%$ & Endometriosis & $5 \%$ & Female factors only & $10 \%$ \\
\hline & Uterine factor & $1 \%$ & Female \& male factors & $13 \%$ \\
\hline & Male factor & $22 \%$ & & \\
\hline
\end{tabular}

Data verified by Nancy A. Klein, MD

\section{PREGNANCY SUCCESS RATES}

\section{Age of Woman}

\section{Type of Cycle}

\section{Fresh Embryos from Nondonor Eggs}

Number of cycles

Percentage of cycles resulting in pregnancies ${ }^{b}$

Percentage of cycles resulting in live births ${ }^{b, c}$

(Confidence Interval)

Percentage of retrievals resulting in live births $s^{b, c}$

Percentage of transfers resulting in live births $s^{b, c}$

Percentage of transfers resulting in singleton live births ${ }^{b}$

Percentage of cancellations ${ }^{b}$

Average number of embryos transferred

Percentage of pregnancies with twins ${ }^{b}$

Percentage of pregnancies with triplets or more ${ }^{b}$

Percentage of live births having multiple infants ${ }^{b, c}$

\section{Frozen Embryos from Nondonor Eggs}

Number of transfers

Percentage of transfers resulting in live births ${ }^{b, c}$

Average number of embryos transferred

\section{Donor Eggs}

Number of transfers

Percentage of transfers resulting in live births $s^{b, c}$

Average number of embryos transferred

67

43

25

35.8

20.9

24.0

2.1

2.3

1.8

All Ages Combined ${ }^{\mathrm{e}}$
Fresh Embryos

105

61.9

1.9

$\begin{array}{ccc}\mathbf{3 5 - 3 7} & \mathbf{3 8 - 4 0} & \mathbf{4 1 - 4 2}^{\mathbf{d}} \\ & & \\ 152 & 138 & 47 \\ 46.7 & 36.2 & 23.4 \\ 35.5 & 26.1 & 17.0 \\ 27.9-43.7) & (19.0-34.2) & (7.6-30.8) \\ 39.1 & 29.5 & 20.0 \\ 40.9 & 32.1 & 21.6 \\ 31.1 & 25.0 & 16.2 \\ 9.2 & 11.6 & 14.9 \\ 2.1 & 2.5 & 2.7 \\ 22.5 & 24.0 & 2 / 11 \\ 1.4 & 4.0 & 0 / 11 \\ 24.1 & 22.2 & 2 / 8\end{array}$

15

$3 / 15$

1.9

Frozen Embryos

19

$5 / 19$

2.5

\section{CURRENT CLINIC SERVICES AND PROFILE}

Current Name: Seattle Reproductive Medicine, Integramed America

Donor egg? Yes Gestational carriers? Yes

Donor embryo? Yes Cryopreservation? Yes

Single women? Yes

SART member? Yes

Verified lab accreditation? Yes

(See Appendix C for details.)

${ }^{a}$ Reflects patient and treatment characteristics of ART cycles performed in 2006 using fresh nondonor eggs or embryos.

${ }^{b}$ When fewer than 20 cycles are reported in an age category, rates are shown as a fraction and confidence intervals are not given. Calculating percentages from fractions may be misleading and is not encouraged.

${ }^{c}$ A multiple-infant birth is counted as one live birth.

d Clinic-specific outcome rates are unreliable for women older than 42 undergoing ART cycles using fresh or frozen embryos with nondonor eggs. Readers are urged to review national outcomes for these age groups (see page 27).

e All ages (including ages $>42$ ) are reported together because previous data show that patient age does not materially affect success with donor eggs. 


\section{THE CENTER FOR REPRODUCTIVE ENDOCRINOLOGY AND FERTILITY SPOKANE, WASHINGTON}

A comparison of clinic success rates may not be meaningful because patient medical characteristics and treatment approaches vary from clinic to clinic. For more details about this, along with information on how to interpret the statistics in this table, see pages $79-88$.

\section{ART CYCLE PROFILE}

\begin{tabular}{|c|c|c|c|c|c|c|c|}
\hline \multicolumn{4}{|c|}{ Type of ART ${ }^{a}$} & \multicolumn{4}{|c|}{ Patient Diagnosis } \\
\hline IVF & $100 \%$ & Procedural Factors: & & Tubal factor & $8 \%$ & Other factor & $2 \%$ \\
\hline GIFT & $0 \%$ & With ICSI & $70 \%$ & Ovulatory dysfunction & $6 \%$ & Unknown factor & $8 \%$ \\
\hline ZIFT & $0 \%$ & Unstimulated & $0 \%$ & Diminished ovarian reserve & $14 \%$ & Multiple Factors: & \\
\hline \multirow[t]{3}{*}{ Combination } & $0 \%$ & Used gestational carrier & $0 \%$ & Endometriosis & $5 \%$ & Female factors only & $5 \%$ \\
\hline & & & & Uterine factor & $0 \%$ & Female \& male factors & $20 \%$ \\
\hline & & & & Male factor & $32 \%$ & & \\
\hline
\end{tabular}

2006 PREGNANCY SUCCESS RATES

Data verified by Edwin Robins, MD

\section{Type of Cycle}

\section{Fresh Embryos from Nondonor Eggs}

Number of cycles

Percentage of cycles resulting in pregnancies ${ }^{b}$

Percentage of cycles resulting in live births $s^{b, c}$

(Confidence Interval)

Percentage of retrievals resulting in live births $s^{b, c}$

Percentage of transfers resulting in live births $s^{b, c}$

Percentage of transfers resulting in singleton live births ${ }^{b}$

Percentage of cancellations ${ }^{b}$

Average number of embryos transferred

Percentage of pregnancies with twins ${ }^{b}$

Percentage of pregnancies with triplets or more ${ }^{b}$

Percentage of live births having multiple infants ${ }^{b, c}$

\section{Frozen Embryos from Nondonor Eggs}

Number of transfers

Percentage of transfers resulting in live births ${ }^{\mathrm{b}, \mathrm{c}}$

Average number of embryos transferred

\section{Donor Eggs}

Number of transfers

Percentage of transfers resulting in live births ${ }^{\mathrm{b}, \mathrm{c}}$

Average number of embryos transferred

\section{Age of Woman}

35-37 38-40

$41-42^{d}$

\section{$<35$}

51

43.1

26

2

54.6

39.2

38.5

$0 / 2$

48.1

(25.8-53.9)

26.9

$0 / 2$

(38.4-58.0)

50.0

45.5

$(11.6-47.8)$

54.7

51.3

31.8

$0 / 1$

36.8

35.9

$7 / 19$

$0 / 1$

3.7

13.7

$6 / 19$

$0 / 1$

2.1

2.2

15.4

$1 / 2$

32.2

36.4

2.5

2.0

1.7

0.0

$1 / 10$

32.7

30.0

$0 / 10$

$1 / 7$

32

37.5

2.3

\section{0}

$2 / 10$

6

$0 / 6$

1

2.5

2.3

$0 / 1$

2.0

All Ages Combined ${ }^{\mathrm{e}}$

Fresh Embryos 28

78.6

2.0
Frozen Embryos 19

$5 / 19$

2.4

\section{CURRENT CLINIC SERVICES AND PROFILE}

Current Name: The Center for Reproductive Endocrinology and Fertility

\begin{tabular}{l|l|l} 
Donor egg? Yes & Gestational carriers? Yes
\end{tabular}

Donor embryo? No Cryopreservation? Yes

Single women? Yes

SART member? Yes

Verified lab accreditation? Yes

(See Appendix C for details.)

${ }^{a}$ Reflects patient and treatment characteristics of ART cycles performed in 2006 using fresh nondonor eggs or embryos.

${ }^{b}$ When fewer than 20 cycles are reported in an age category, rates are shown as a fraction and confidence intervals are not given. Calculating percentages from fractions may be misleading and is not encouraged.

A multiple-infant birth is counted as one live birth.

d Clinic-specific outcome rates are unreliable for women older than 42 undergoing ART cycles using fresh or frozen embryos with nondonor eggs. Readers are urged to review national outcomes for these age groups (see page 27).

e All ages (including ages $>42$ ) are reported together because previous data show that patient age does not materially affect success with donor eggs. 


\section{GYFT CLINIC, PLLC TACOMA, WASHINGTON}

A comparison of clinic success rates may not be meaningful because patient medical characteristics and treatment approaches vary from clinic to clinic. For more details about this, along with information on how to interpret the statistics in this table, see pages 79-88.

\section{ART CYCLE PROFILE}

\begin{tabular}{|c|c|c|c|c|c|c|c|}
\hline \multicolumn{4}{|c|}{ Type of ART ${ }^{a}$} & \multicolumn{4}{|c|}{ Patient Diagnosis } \\
\hline IVF & $100 \%$ & Procedural Factors: & & Tubal factor & $22 \%$ & Other factor & $2 \%$ \\
\hline GIFT & $0 \%$ & With ICSI & $42 \%$ & Ovulatory dysfunction & $6 \%$ & Unknown factor & $11 \%$ \\
\hline ZIFT & $0 \%$ & Unstimulated & $0 \%$ & Diminished ovarian reserve & $20 \%$ & Multiple Factors: & \\
\hline \multirow[t]{3}{*}{ Combination } & $0 \%$ & Used gestational carrier & $0 \%$ & Endometriosis & $6 \%$ & Female factors only & $11 \%$ \\
\hline & & & & Uterine factor & $0 \%$ & Female \& male factors & $13 \%$ \\
\hline & & & & Male factor & $9 \%$ & & \\
\hline
\end{tabular}

2006 PREGNANCY SUCCESS RATES

Data verified by Joseph A. Robinette, MD

\section{Type of Cycle}

\section{Fresh Embryos from Nondonor Eggs}

Number of cycles

Percentage of cycles resulting in pregnancies ${ }^{b}$

Percentage of cycles resulting in live births $s^{b, c}$

(Confidence Interval)

Percentage of retrievals resulting in live births $s^{b, c}$

Percentage of transfers resulting in live births $s^{b, c}$

Percentage of transfers resulting in singleton live births ${ }^{b}$

Percentage of cancellations ${ }^{b}$

Average number of embryos transferred

Percentage of pregnancies with twins ${ }^{b}$

Percentage of pregnancies with triplets or more ${ }^{b}$

Percentage of live births having multiple infants ${ }^{\mathrm{b}, \mathrm{c}}$

\section{Frozen Embryos from Nondonor Eggs}

Number of transfers

Percentage of transfers resulting in live births ${ }^{b, c}$

Average number of embryos transferred

\section{Donor Eggs}

Number of transfers

Percentage of transfers resulting in live births $s^{b, c}$

Average number of embryos transferred

\section{Age of Woman}

35-37 38-40

$41-42^{d}$

\section{$<35$}

14

$6 / 14$

$6 / 14$

8

2

53.8

42.3

(23.4-63.1)

42.3

42.3

$6 / 13$

$2 / 8$

$0 / 2$

23.1

0.0

$6 / 13$

$2 / 8$

$0 / 2$

4.8

$3 / 13$

$1 / 14$

$2 / 8$

$0 / 2$

$2 / 8$

$0 / 1$

$1 / 8$

$0 / 1$

$4 / 14$

4.0

$0 / 8$

$0 / 2$

$3 / 14$

$2 / 6$

3.9

3.0

$5 / 11$

$2 / 6$

$1 / 2$

$0 / 2$

$3 / 6$

$1 / 2$

2

$0 / 2$

3.0

1

$0 / 1$

4.0

\section{CURRENT CLINIC SERVICES AND PROFILE}

\begin{tabular}{cc}
\multicolumn{2}{c}{ All Ages Combined } \\
Fresh Embryos & Frozen Embryos $^{\mathbf{e}}$ \\
7 & 1 \\
$3 / 7$ & $0 / 1$ \\
4.4 & 3.0
\end{tabular}

\section{Current Name: GYFT Clinic, PLLC}

Donor egg? Yes Gestational carriers? Yes

Donor embryo? No Cryopreservation? Yes

Single women? Yes

SART member? Yes

Verified lab accreditation? Yes

(See Appendix C for details.)

${ }^{a}$ Reflects patient and treatment characteristics of ART cycles performed in 2006 using fresh nondonor eggs or embryos.

${ }^{b}$ When fewer than 20 cycles are reported in an age category, rates are shown as a fraction and confidence intervals are not given. Calculating percentages from fractions may be misleading and is not encouraged.

${ }^{c}$ A multiple-infant birth is counted as one live birth.

d Clinic-specific outcome rates are unreliable for women older than 42 undergoing ART cycles using fresh or frozen embryos with nondonor eggs. Readers are urged to review national outcomes for these age groups (see page 27).

e All ages (including ages $>42$ ) are reported together because previous data show that patient age does not materially affect success with donor eggs. 


\section{CABELL HUNTINGTON HOSPITAL CENTER FOR ADVANCED REPRODUCTIVE MEDICINE HUNTINGTON, WEST VIRGINIA}

A comparison of clinic success rates may not be meaningful because patient medical characteristics and treatment approaches vary from clinic to clinic. For more details about this, along with information on how to interpret the statistics in this table, see pages 79-88.

\section{ART CYCLE PROFILE}

\begin{tabular}{|c|c|c|c|c|c|c|c|}
\hline \multicolumn{4}{|c|}{ Type of ART ${ }^{a}$} & \multicolumn{4}{|c|}{ Patient Diagnosis } \\
\hline IVF & $100 \%$ & Procedural Factors: & & Tubal factor & $24 \%$ & Other factor & $6 \%$ \\
\hline GIFT & $0 \%$ & With ICSI & $25 \%$ & Ovulatory dysfunction & $6 \%$ & Unknown factor & $3 \%$ \\
\hline $\mathrm{ZIFT}$ & $0 \%$ & Unstimulated & $0 \%$ & Diminished ovarian reserve & $3 \%$ & Multiple Factors: & \\
\hline \multirow[t]{3}{*}{ Combination } & $0 \%$ & Used gestational carrier & $0 \%$ & Endometriosis & $21 \%$ & Female factors only & $21 \%$ \\
\hline & & & & Uterine factor & $0 \%$ & Female \& male factors & $12 \%$ \\
\hline & & & & Male factor & $3 \%$ & & \\
\hline
\end{tabular}

2006 PREGNANCY SUCCESS RATES

Data verified by William N. Burns, MD

\section{Type of Cycle}

\section{Fresh Embryos from Nondonor Eggs}

Number of cycles

Percentage of cycles resulting in pregnancies ${ }^{b}$

Percentage of cycles resulting in live births $s^{b, c}$

(Confidence Interval)

Percentage of retrievals resulting in live births $s^{b, c}$

Percentage of transfers resulting in live births $s^{b, c}$

Percentage of transfers resulting in singleton live births ${ }^{b}$

Percentage of cancellations ${ }^{b}$

Average number of embryos transferred

Percentage of pregnancies with twins ${ }^{b}$

Percentage of pregnancies with triplets or more ${ }^{\mathrm{b}}$

Percentage of live births having multiple infants ${ }^{b, c}$

\section{Frozen Embryos from Nondonor Eggs}

Number of transfers

Percentage of transfers resulting in live births $s^{\mathrm{b}, \mathrm{c}}$

Average number of embryos transferred

\section{Donor Eggs}

Number of transfers

Percentage of transfers resulting in live births $s^{b, c}$

Average number of embryos transferred

\section{Age of Woman}

35-37 38-40

$41-42^{d}$

$<35$

7

$5 / 7$

5

2

$7 / 14$

$5 / 7$

$0 / 5$

$0 / 5$

$0 / 2$

$7 / 14$

$5 / 6$

$0 / 5$

$0 / 5$

$0 / 5$

$0 / 5$

1.8

$0 / 2$

$7 / 12$

$5 / 5$

$5 / 5$

$1 / 7$

2.6

$0 / 5$

$2 / 7$

$0 / 5$

$0 / 7$

$0 / 5$

$1 / 7$

0

0

$0 / 1$

2.0
$0 / 1$

$0 / 1$

$0 / 1$

$1 / 2$

2.0

\section{CURRENT CLINIC SERVICES AND PROFILE}

Current Name: Cabell Huntington Hospital, Center for Advanced Reproductive Medicine
Donor egg?
Yes
Donor embryo? No
Gestational carriers? No
Cryopreservation?
No
Yes
SART member?
Verified lab accreditation?
No
Single women? Yes
(See Appendix C for details.)

\footnotetext{
${ }^{a}$ Reflects patient and treatment characteristics of ART cycles performed in 2006 using fresh nondonor eggs or embryos.

${ }^{b}$ When fewer than 20 cycles are reported in an age category, rates are shown as a fraction and confidence intervals are not given. Calculating percentages from fractions may be misleading and is not encouraged.

${ }^{c}$ A multiple-infant birth is counted as one live birth.

${ }^{d}$ Clinic-specific outcome rates are unreliable for women older than 42 undergoing ART cycles using fresh or frozen embryos with nondonor eggs. Readers are urged to review national outcomes for these age groups (see page 27).

${ }^{e}$ All ages (including ages $>42$ ) are reported together because previous data show that patient age does not materially affect success with donor eggs.
} 


\section{WEST VIRGINIA UNIVERSITY CENTER FOR REPRODUCTIVE MEDICINE MORGANTOWN, WEST VIRGINIA}

A comparison of clinic success rates may not be meaningful because patient medical characteristics and treatment approaches vary from clinic to clinic. For more details about this, along with information on how to interpret the statistics in this table, see pages 79-88.

\section{ART CYCLE PROFILE}

\begin{tabular}{l|l|lrlrl}
\multicolumn{3}{c}{ Type of ART } & \multicolumn{4}{c}{ Patient Diagnosis } \\
IVF & $100 \%$ Procedural Factors: & & Tubal factor & $5 \%$ & Other factor & $<1 \%$ \\
GIFT & $0 \%$ With ICSI & $71 \%$ & Ovulatory dysfunction & $<1 \%$ & Unknown factor & $3 \%$ \\
ZIFT & $0 \%$ Unstimulated & $0 \%$ & Diminished ovarian reserve & $4 \%$ & Multiple Factors: & \\
Combination & $0 \%$ Used gestational carrier & $0 \%$ & Endometriosis & $2 \%$ & Female factors only & $17 \%$ \\
& & & Uterine factor & $0 \%$ & Female \& male factors & $56 \%$ \\
& & & Male factor & $12 \%$ & &
\end{tabular}

2006 PREGNANCY SUCCESS RATES

Data verified by Roger C. Toffle, MD

\section{Type of Cycle}

\section{Fresh Embryos from Nondonor Eggs}

Number of cycles

Percentage of cycles resulting in pregnancies ${ }^{b}$

Percentage of cycles resulting in live births $s^{b, c}$

(Confidence Interval)

Percentage of retrievals resulting in live births $s^{b, c}$

Percentage of transfers resulting in live births $s^{b, c}$

Percentage of transfers resulting in singleton live births ${ }^{b}$

Percentage of cancellations ${ }^{b}$

Average number of embryos transferred

Percentage of pregnancies with twins ${ }^{b}$

Percentage of pregnancies with triplets or more ${ }^{b}$

Percentage of live births having multiple infants ${ }^{b, c}$

\section{Frozen Embryos from Nondonor Eggs}

Number of transfers

Percentage of transfers resulting in live births ${ }^{b, c}$

Average number of embryos transferred

\section{Donor Eggs}

Number of transfers

Percentage of transfers resulting in live births $s^{b, c}$

Average number of embryos transferred

\section{Age of Woman}

35-37 38-40 $41-42^{d}$

\section{$<35$}

19

19

2

46.9

$2 / 19$

$1 / 19$

$0 / 2$

42.9

$1 / 19$

$1 / 19$

$0 / 2$

(28.8-57.8)

$\begin{array}{llll}45.7 & 1 / 18 & 1 / 15 & 0 / 1\end{array}$

47.7

$1 / 15$

$1 / 14$

$0 / 1$

34.1

$1 / 15$

$1 / 14$

$0 / 1$

6.1

$1 / 19$

$4 / 19$

$1 / 2$

2.7

2.4

$0 / 2$

2.1

$1 / 1$

0.0

$0 / 2$

$0 / 1$

28.6

$0 / 1$

$0 / 1$

$12 \quad 2 \quad 1$

$4 / 12$

2.5

$1 / 2$

1

1.0

$0 / 1$

9.0

All Ages Combined ${ }^{\mathrm{e}}$

Fresh Embryos

Frozen Embryos

0

\section{CURRENT CLINIC SERVICES AND PROFILE}

Current Name: West Virginia University Center for Reproductive Medicine

Donor egg? No

Donor embryo? Yes

Single women? Yes

\begin{abstract}
Gestational carriers? No
Cryopreservation?

Yes
\end{abstract}

SART member?

Verified lab accreditation?

(See Appendix C for details.)

${ }^{a}$ Reflects patient and treatment characteristics of ART cycles performed in 2006 using fresh nondonor eggs or embryos.

${ }^{b}$ When fewer than 20 cycles are reported in an age category, rates are shown as a fraction and confidence intervals are not given. Calculating percentages from fractions may be misleading and is not encouraged.

${ }^{c}$ A multiple-infant birth is counted as one live birth.

d Clinic-specific outcome rates are unreliable for women older than 42 undergoing ART cycles using fresh or frozen embryos with nondonor eggs. Readers are urged to review national outcomes for these age groups (see page 27).

e All ages (including ages $>42$ ) are reported together because previous data show that patient age does not materially affect success with donor eggs. 


\section{THE WOMEN'S CENTER AT AURORA BAYCARE MEDICAL CENTER REPRODUCTIVE ENDOCRINOLOGY AND FERTILITY GREEN BAY, WISCONSIN}

A comparison of clinic success rates may not be meaningful because patient medical characteristics and treatment approaches vary from clinic to clinic. For more details about this, along with information on how to interpret the statistics in this table, see pages $79-88$.

\section{ART CYCLE PROFILE}

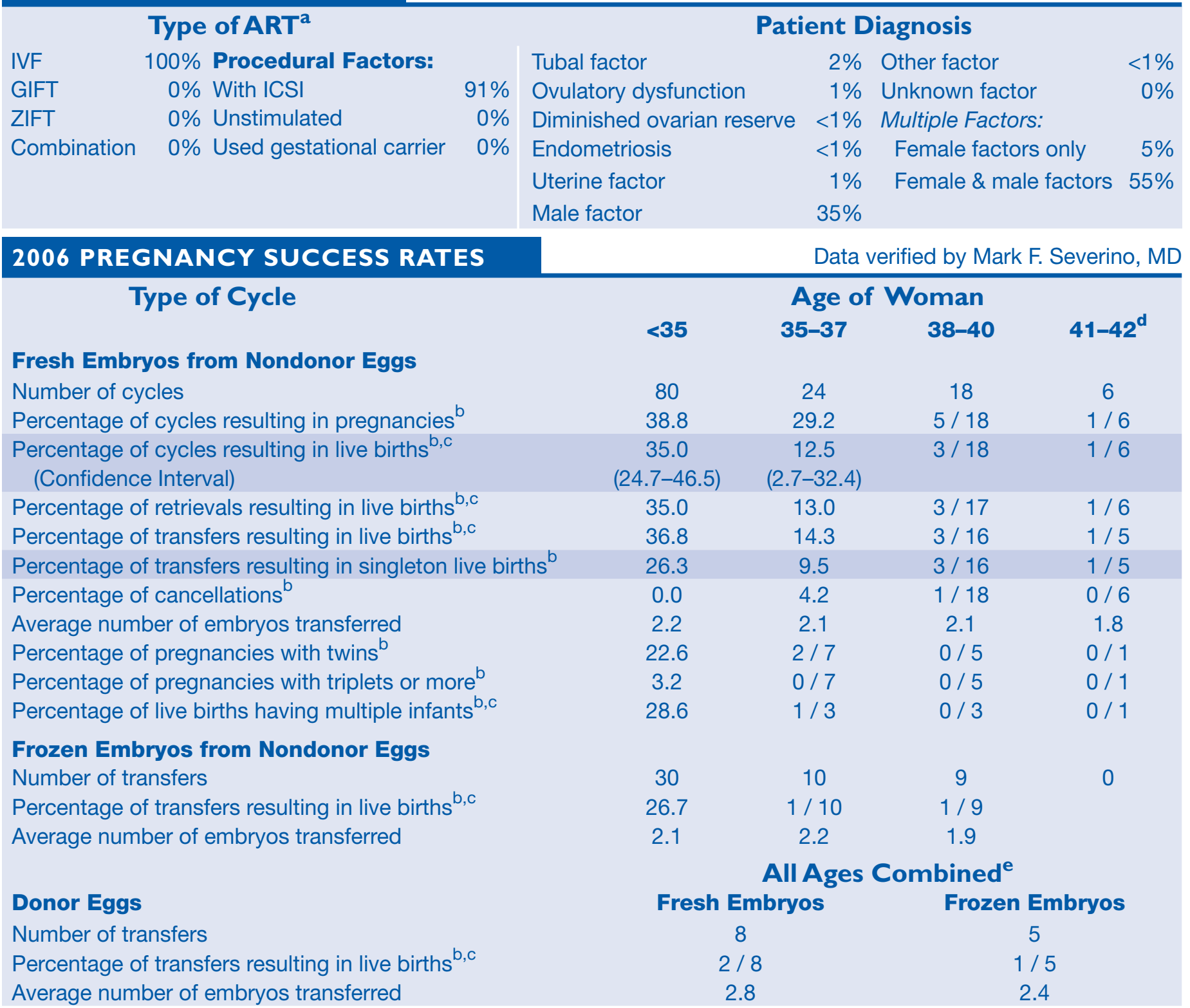

\section{CURRENT CLINIC SERVICES AND PROFILE}

Current Name: Aurora Health Care-Aurora Fertility Services, Green Bay, The Women's Center at Aurora BayCare Medical Center

\begin{tabular}{ll|lll|l|l} 
Donor egg? & Yes & Gestational carriers? & Yes & SART member? & Yes \\
\hline Donor embryo? & Yes & Cryopreservation? & Yes & Verified lab accreditation? & Yes \\
Single women? & Yes & & & (See Appendix C for details.) &
\end{tabular}

${ }^{a}$ Reflects patient and treatment characteristics of ART cycles performed in 2006 using fresh nondonor eggs or embryos.

${ }^{b}$ When fewer than 20 cycles are reported in an age category, rates are shown as a fraction and confidence intervals are not given. Calculating percentages from fractions may be misleading and is not encouraged.

${ }^{\mathrm{C}}$ A multiple-infant birth is counted as one live birth.

d Clinic-specific outcome rates are unreliable for women older than 42 undergoing ART cycles using fresh or frozen embryos with nondonor eggs. Readers are urged to review national outcomes for these age groups (see page 27).

e All ages (including ages $>42$ ) are reported together because previous data show that patient age does not materially affect success with donor eggs. 


\section{UNIVERSITY OF WISCONSIN-MADISON \\ REPRODUCTIVE ENDOCRINOLOGY AND INFERTILITY \\ MADISON, WISCONSIN}

A comparison of clinic success rates may not be meaningful because patient medical characteristics and treatment approaches vary from clinic to clinic. For more details about this, along with information on how to interpret the statistics in this table, see pages 79-88.

\section{ART CYCLE PROFILE}

\section{Type of ART ${ }^{\mathrm{a}}$}

IVF

GIFT

100\% Procedural Factors:

ZIFT

Combination
$0 \%$ With ICSI

$0 \%$ Unstimulated

$0 \%$ Used gestational carrier

\begin{tabular}{r|l} 
& \\
& Tubal factor \\
$75 \%$ & Ovulatory dysfunction \\
$0 \%$ & Diminished ovarian reserve \\
$7 \%$ & Endometriosis \\
& Uterine factor \\
& Male factor
\end{tabular}

\section{Patient Diagnosis}

$3 \%$ Other factor

2\% Unknown factor

19\% Multiple Factors:

$0 \%$ Female factors only $6 \%$

$3 \%$ Female \& male factors $34 \%$ $25 \%$
$4 \%$

$5 \%$

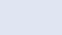

2006 PREGNANCY SUCCESS RATES

Data verified by Steven Lindheim, MD

\section{Type of Cycle}

\section{Age of Woman}

$\begin{array}{llll}35-37 & 38-40 & 41-42^{d}\end{array}$

\section{Fresh Embryos from Nondonor Eggs}

Number of cycles

$45 \quad 22$

Percentage of cycles resulting in pregnancies ${ }^{b}$

Percentage of cycles resulting in live births $s^{b, c}$

22

15

6

(Confidence Interval)

Percentage of retrievals resulting in live births $s^{b, c}$

37.8

40.9

$7 / 15$

$0 / 6$

Percentage of transfers resulting in live births $s^{b, c}$

(23.8-53.5)

40.9

$3 / 15$

$0 / 6$

Percentage of transfers resulting in singleton live births ${ }^{b}$

Percentage of cancellations ${ }^{b}$

41.5

(20.7-63.6)

42.5

$9 / 19$

$3 / 14$

$0 / 5$

30.0

$9 / 17$

$3 / 14$

$0 / 5$

Average number of embryos transferred

8.9

$7 / 17$

$2 / 14$

$0 / 5$

13.6

$1 / 15$

$1 / 6$

2.2

2.2

3.3

3.8

Percentage of pregnancies with twins ${ }^{b}$

$6 / 19$

$3 / 9$

$1 / 7$

$0 / 19$

$0 / 9$

$0 / 7$

$5 / 17$

$2 / 9$

$1 / 3$

Percentage of pregnancies with triplets or more
Percentage of live births having multiple infants

\section{Frozen Embryos from Nondonor Eggs}

Number of transfers

Percentage of transfers resulting in live births $s^{b, c}$

Average number of embryos transferred

20

30.0

\section{8}

2.6

$2 / 8$

2.4

\section{3}

$0 / 3$

1

All Ages Combined ${ }^{\mathrm{e}}$

\section{Donor Eggs}

Fresh Embryos

2.2

6

Number of transfers

Percentage of transfers resulting in live births $s^{b, c}$

Average number of embryos transferred

Frozen Embryos

21

33.3

3.1

\section{CURRENT CLINIC SERVICES AND PROFILE}

Current Name: University of Wisconsin-Madison, Reproductive Endocrinology and Infertility

Donor egg? Yes

Donor embryo? Yes

Single women? Yes

\begin{abstract}
Gestational carriers? Yes
Cryopreservation?
\end{abstract}

SART member?

Verified lab accreditation?

(See Appendix C for details.)

${ }^{a}$ Reflects patient and treatment characteristics of ART cycles performed in 2006 using fresh nondonor eggs or embryos.

${ }^{b}$ When fewer than 20 cycles are reported in an age category, rates are shown as a fraction and confidence intervals are not given. Calculating percentages from fractions may be misleading and is not encouraged.

${ }^{c}$ A multiple-infant birth is counted as one live birth.

d Clinic-specific outcome rates are unreliable for women older than 42 undergoing ART cycles using fresh or frozen embryos with nondonor eggs. Readers are urged to review national outcomes for these age groups (see page 27).

e All ages (including ages $>42$ ) are reported together because previous data show that patient age does not materially affect success with donor eggs. 


\section{ADVANCED INSTITUTE OF FERTILITY MILWAUKEE, WISCONSIN}

A comparison of clinic success rates may not be meaningful because patient medical characteristics and treatment approaches vary from clinic to clinic. For more details about this, along with information on how to interpret the statistics in this table, see pages $79-88$.

\section{ART CYCLE PROFILE}

\begin{tabular}{|c|c|c|c|c|c|c|c|}
\hline \multicolumn{4}{|c|}{ Type of ART ${ }^{\mathrm{a}}$} & \multicolumn{4}{|c|}{ Patient Diagnosis } \\
\hline IVF & $100 \%$ & Procedural Factors: & & Tubal factor & $4 \%$ & Other factor & $14 \%$ \\
\hline GIFT & $0 \%$ & With ICSI & $83 \%$ & Ovulatory dysfunction & $2 \%$ & Unknown factor & $2 \%$ \\
\hline ZIFT & $0 \%$ & Unstimulated & $0 \%$ & Diminished ovarian reserve & $9 \%$ & Multiple Factors: & \\
\hline \multirow[t]{3}{*}{ Combination } & $0 \%$ & Used gestational carrier & $1 \%$ & Endometriosis & $4 \%$ & Female factors only & $6 \%$ \\
\hline & & & & Uterine factor & $4 \%$ & Female \& male factors & $35 \%$ \\
\hline & & & & Male factor & $21 \%$ & & \\
\hline
\end{tabular}

2006 PREGNANCY SUCCESS RATES

\section{Type of Cycle}

\section{Fresh Embryos from Nondonor Eggs}

Number of cycles

Percentage of cycles resulting in pregnancies ${ }^{b}$

Percentage of cycles resulting in live births ${ }^{b, c}$

(Confidence Interval)

Percentage of retrievals resulting in live births $s^{b, c}$

Percentage of transfers resulting in live births $s^{b, c}$

Percentage of transfers resulting in singleton live births ${ }^{b}$

Percentage of cancellations ${ }^{b}$

Average number of embryos transferred

Percentage of pregnancies with twins ${ }^{b}$

Percentage of pregnancies with triplets or more ${ }^{b}$

Percentage of live births having multiple infants ${ }^{b, c}$

\section{Frozen Embryos from Nondonor Eggs}

Number of transfers

Percentage of transfers resulting in live births $s^{b, c}$

Average number of embryos transferred

\section{Donor Eggs}

Number of transfers

Percentage of transfers resulting in live births $s^{b, c}$

Average number of embryos transferred

Data verified by K. Paul Katayama, MD, PhD

\section{Age of Woman}

35-37 38-40

$41-42^{d}$

\section{$<35$}

19

14

2

48.6

$6 / 19$

$3 / 14$

$1 / 2$

42.9

$6 / 19$

$0 / 14$

$1 / 2$

(26.3-60.6)

44.1

44.1

$6 / 19$

$0 / 14$

$1 / 2$

$6 / 19$

$0 / 14$

$1 / 2$

26.5

$3 / 19$

$0 / 14$

$1 / 2$

2.9

$0 / 19$

$0 / 14$

$0 / 2$

3.1

3.3

4.0

2.5

$3 / 17$

$2 / 6$

$0 / 3$

$0 / 1$

$3 / 17$

$1 / 6$

$0 / 3$

$0 / 1$

$6 / 15$

$3 / 6$

$0 / 1$

$\begin{array}{cccc}12 & 15 & 5 & 2 \\ 4 / 12 & 2 / 15 & 0 / 5 & 1 / 2 \\ 2.4 & 1.8 & 1.8 & 2.5\end{array}$

Fresh Embryos

All Ages Combined
Frozen Embryos

9

$5 / 9$

2.0
15

$5 / 15$

2.6

\section{CURRENT CLINIC SERVICES AND PROFILE}

Current Name: Advanced Institute of Fertility

Donor egg? Yes

Donor embryo? Yes

Single women? Yes

\section{Gestational carriers? Yes}

Cryopreservation? Yes

\section{SART member?}

Verified lab accreditation?

(See Appendix C for details.)

${ }^{a}$ Reflects patient and treatment characteristics of ART cycles performed in 2006 using fresh nondonor eggs or embryos.

${ }^{b}$ When fewer than 20 cycles are reported in an age category, rates are shown as a fraction and confidence intervals are not given. Calculating percentages from fractions may be misleading and is not encouraged.

${ }^{c}$ A multiple-infant birth is counted as one live birth.

d Clinic-specific outcome rates are unreliable for women older than 42 undergoing ART cycles using fresh or frozen embryos with nondonor eggs. Readers are urged to review national outcomes for these age groups (see page 27).

e All ages (including ages $>42$ ) are reported together because previous data show that patient age does not materially affect success with donor eggs. 


\section{FROEDTERT \& MEDICAL COLLEGE OF WISCONSIN \\ REPRODUCTIVE MEDICINE CLINIC \\ MILWAUKEE, WISCONSIN}

A comparison of clinic success rates may not be meaningful because patient medical characteristics and treatment approaches vary from clinic to clinic. For more details about this, along with information on how to interpret the statistics in this table, see pages 79-88.

\section{ART CYCLE PROFILE}

\begin{tabular}{|c|c|c|c|c|c|c|c|}
\hline & Type & of $A R T^{a}$ & & Pat & ent [ & iagnosis & \\
\hline IVF & $100 \%$ & Procedural Factors: & & Tubal factor & $9 \%$ & Other factor & $3 \%$ \\
\hline GIFT & $0 \%$ & With ICSI & $83 \%$ & Ovulatory dysfunction & $13 \%$ & Unknown factor & $13 \%$ \\
\hline ZIFT & $0 \%$ & Unstimulated & $<1 \%$ & Diminished ovarian reserve & $13 \%$ & Multiple Factors: & \\
\hline Combination & $0 \%$ & Used gestational carrier & $<1 \%$ & Endometriosis & $5 \%$ & Female factors only & $5 \%$ \\
\hline & & & & Uterine factor & $<1 \%$ & Female \& male factors & $15 \%$ \\
\hline & & & & Male factor & $25 \%$ & & \\
\hline
\end{tabular}

2006 PREGNANCY SUCCESS RATES

Data verified by Estil Strawn, Jr., MD

\section{Type of Cycle}

\section{Fresh Embryos from Nondonor Eggs}

Number of cycles

Percentage of cycles resulting in pregnancies ${ }^{b}$

Percentage of cycles resulting in live births ${ }^{b, c}$

(Confidence Interval)

Percentage of retrievals resulting in live births $s^{b, c}$

Percentage of transfers resulting in live births $s^{b, c}$

Percentage of transfers resulting in singleton live births ${ }^{b}$

Percentage of cancellations ${ }^{b}$

Average number of embryos transferred

Percentage of pregnancies with twins ${ }^{b}$

Percentage of pregnancies with triplets or more ${ }^{b}$

Percentage of live births having multiple infants ${ }^{b, c}$

\section{Frozen Embryos from Nondonor Eggs}

Number of transfers

Percentage of transfers resulting in live births $s^{b, c}$

Average number of embryos transferred

\section{Donor Eggs}

Number of transfers

Percentage of transfers resulting in live births $s^{b, c}$

Average number of embryos transferred

\section{Age of Woman}

35-37 38-40

$41-42^{d}$

$<35$

46

37.0

26.1

42.4

$(32.5-52.8)$

(14.3-41.1)

45.7

46.7

30.0

30.8

25.6

13.0

2.1

$3 / 17$

$0 / 17$

$2 / 12$

0.0

31.0

30

16.7

1.8

\section{4}

$3 / 14$

2.1

$\begin{array}{cc}39 & 7 \\ 15.4 & 1 / 7 \\ 15.4 & 1 / 7\end{array}$

(5.9-30.5)

$\begin{array}{ll}18.2 & 1 / 6 \\ 19.4 & 1 / 5 \\ 19.4 & 0 / 5 \\ 15.4 & 1 / 7 \\ 2.4 & 2.8 \\ 0 / 6 & 1 / 1 \\ 0 / 6 & 0 / 1 \\ 0 / 6 & 1 / 1\end{array}$

$\begin{array}{cc}11 & 1 \\ 4 / 11 & 1 / 1 \\ 2.0 & 2.0\end{array}$

All Ages Combined ${ }^{\mathrm{e}}$

Fresh Embryos 14

$6 / 14$

1.9

\section{CURRENT CLINIC SERVICES AND PROFILE}

Current Name: Froedtert \& Medical College of Wisconsin, Reproductive Medicine Clinic

Donor egg? Yes

Donor embryo? Yes

Single women? Yes

\section{Gestational carriers? Yes}

Cryopreservation? Yes
SART member?

Verified lab accreditation?

(See Appendix C for details.)
Frozen Embryos 20

25.0

2.0

\footnotetext{
${ }^{a}$ Reflects patient and treatment characteristics of ART cycles performed in 2006 using fresh nondonor eggs or embryos.

${ }^{b}$ When fewer than 20 cycles are reported in an age category, rates are shown as a fraction and confidence intervals are not given. Calculating percentages from fractions may be misleading and is not encouraged.

${ }^{c}$ A multiple-infant birth is counted as one live birth.

${ }^{d}$ Clinic-specific outcome rates are unreliable for women older than 42 undergoing ART cycles using fresh or frozen embryos with nondonor eggs. Readers are urged to review national outcomes for these age groups (see page 27).

e All ages (including ages $>42$ ) are reported together because previous data show that patient age does not materially affect success with donor eggs.
} 


\section{REPRODUCTIVE SPECIALTY CENTER \\ IVF COLUMBIA \\ MILWAUKEE, WISCONSIN}

A comparison of clinic success rates may not be meaningful because patient medical characteristics and treatment approaches vary from clinic to clinic. For more details about this, along with information on how to interpret the statistics in this table, see pages $79-88$.

\section{ART CYCLE PROFILE}

\begin{tabular}{|c|c|c|c|c|c|c|}
\hline & Type of ART ${ }^{a}$ & & Pati & ent D & iagnosis & \\
\hline IVF & $100 \%$ Procedural Factors: & & Tubal factor & $15 \%$ & Other factor & $5 \%$ \\
\hline GIFT & 0\% With ICSI & $33 \%$ & Ovulatory dysfunction & $3 \%$ & Unknown factor & $3 \%$ \\
\hline ZIFT & $0 \%$ Unstimulated & $0 \%$ & Diminished ovarian reserve & $7 \%$ & Multiple Factors: & \\
\hline Combination & $0 \%$ Used gestational carrier & $0 \%$ & Endometriosis & $20 \%$ & Female factors only & $9 \%$ \\
\hline & & & Uterine factor & $1 \%$ & Female \& male factors & $9 \%$ \\
\hline & & & Male factor & $28 \%$ & & \\
\hline
\end{tabular}

2006 PREGNANCY SUCCESS RATES

Data verified by Grace M. Janik, MD

$$
\text { Type of Cycle }
$$

Age of Woman

$<35 \quad 35-37 \quad 38-40 \quad 41-42^{\text {d }}$

Fresh Embryos from Nondonor Eggs

Number of cycles

Percentage of cycles resulting in pregnancies ${ }^{b}$

Percentage of cycles resulting in live births ${ }^{b, c}$

(Confidence Interval)

Percentage of retrievals resulting in live births $s^{b, c}$

Percentage of transfers resulting in live births $s^{b, c}$

Percentage of transfers resulting in singleton live births ${ }^{b}$

Percentage of cancellations ${ }^{b}$

\section{1}

42.9

22

45.5

19

4

38.1

40.9

$4 / 19$

$2 / 4$

$(18.1-61.6)$

$(20.7-63.6)$

38.1

38.1

$9 / 19$

$4 / 19$

$1 / 4$

28.6

$9 / 18$

$4 / 18$

$1 / 4$

$6 / 18$

$4 / 18$

$1 / 4$

0.0

13.6

$4 / 18$

$1 / 4$

2.4

2.6

$1 / 19$

$0 / 4$

$3 / 9$

$3 / 10$

2.6

3.0

$0 / 9$

$0 / 10$

$0 / 4$

$1 / 2$

$2 / 8$

$3 / 9$

$0 / 4$

$0 / 2$

Percentage of pregnancies with triplets or more
Percentage of live births having multiple infants

\section{Frozen Embryos from Nondonor Eggs}

Number of transfers

Percentage of transfers resulting in live births $s^{\mathrm{b}, \mathrm{c}}$

Average number of embryos transferred

4
$0 / 4$
2.3

5

$0 / 4$

$0 / 1$

$0 / 5$

2.6

\section{3}

$1 / 3$

2.0

All Ages Combined ${ }^{\mathrm{e}}$

Fresh Embryos

0

Frozen Embryos

0

Number of transfers

Percentage of transfers resulting in live births $s^{b, c}$

Average number of embryos transferred

\section{CURRENT CLINIC SERVICES AND PROFILE}

Current Name: Reproductive Specialty Center, IVF Columbia

Donor egg? No Gestational carriers? No

Donor embryo? No Cryopreservation? Yes

Single women? Yes

SART member? Yes

Verified lab accreditation? Yes

(See Appendix C for details.)

${ }^{a}$ Reflects patient and treatment characteristics of ART cycles performed in 2006 using fresh nondonor eggs or embryos.

${ }^{b}$ When fewer than 20 cycles are reported in an age category, rates are shown as a fraction and confidence intervals are not given. Calculating percentages from fractions may be misleading and is not encouraged.

${ }^{c}$ A multiple-infant birth is counted as one live birth.

d Clinic-specific outcome rates are unreliable for women older than 42 undergoing ART cycles using fresh or frozen embryos with nondonor eggs. Readers are urged to review national outcomes for these age groups (see page 27).

${ }^{e}$ All ages (including ages $>42$ ) are reported together because previous data show that patient age does not materially affect success with donor eggs. 


\section{WOMEN'S HEALTH CARE, SC \\ WAUKESHA, WISCONSIN}

A comparison of clinic success rates may not be meaningful because patient medical characteristics and treatment approaches vary from clinic to clinic. For more details about this, along with information on how to interpret the statistics in this table, see pages 79-88.

\section{ART CYCLE PROFILE}

\section{Type of ART ${ }^{\mathrm{a}}$}

IVF

GIFT 100\% Procedural Factors:

ZIFT

Combination

$0 \%$ With ICSI
$0 \%$ Unstimulated

$0 \%$ Used gestational carrier

\begin{tabular}{|c|c|c|c|c|}
\hline & \multicolumn{4}{|c|}{ Patient Diagnosis } \\
\hline & Tubal factor & $2 \%$ & Other factor & $0 \%$ \\
\hline $54 \%$ & Ovulatory dysfunction & $16 \%$ & Unknown factor & $21 \%$ \\
\hline $0 \%$ & Diminished ovarian reserve & $0 \%$ & Multiple Factors: & \\
\hline $0 \%$ & Endometriosis & $5 \%$ & Female factors only & $14 \%$ \\
\hline & Uterine factor & $0 \%$ & Female \& male factors & $19 \%$ \\
\hline & Male factor & $23 \%$ & & \\
\hline
\end{tabular}

2006 PREGNANCY SUCCESS RATES

Data verified by Matthew A. Meyer, MD

\section{Type of Cycle}

\begin{tabular}{|c|c|c|c|}
\hline$<35$ & 35-37 & $38-40$ & $41-42^{d}$ \\
\hline 16 & 7 & 3 & 0 \\
\hline / / 16 & $3 / 7$ & $1 / 3$ & \\
\hline 16 & $2 / 7$ & $1 / 3$ & \\
\hline
\end{tabular}

$\begin{array}{ccc}4 / 15 & 2 / 7 & 1 / 2 \\ 4 / 14 & 2 / 5 & 1 / 2 \\ 4 / 14 & 1 / 5 & 0 / 2 \\ 1 / 16 & 0 / 7 & 1 / 3 \\ 1.9 & 1.8 & 2.0 \\ 0 / 4 & 1 / 3 & 1 / 1 \\ 0 / 4 & 0 / 3 & 0 / 1 \\ 0 / 4 & 1 / 2 & 1 / 1\end{array}$

\section{Frozen Embryos from Nondonor Eggs}

Number of transfers

Percentage of transfers resulting in live births $s^{b, c}$

Average number of embryos transferred

\section{8}

$1 / 8$

3

1.6

$0 / 3$

1.7

3
$0 / 3$
2.0

1

$0 / 1$

1.0

\section{Donor Eggs}

Number of transfers

Percentage of transfers resulting in live births $s^{b, c}$

Average number of embryos transferred

All Ages Combined ${ }^{\mathrm{e}}$

Fresh Embryos

0
Frozen Embryos

0

\section{CURRENT CLINIC SERVICES AND PROFILE}

Current Name: Women's Health Care, SC
Donor egg?
Yes
Gestational carriers?
No
Cryopreservation?
Yes
Single women? Yes
SART member?
Yes
Verified lab accreditation?
Yes
(See Appendix C for details.)

\footnotetext{
${ }^{a}$ Reflects patient and treatment characteristics of ART cycles performed in 2006 using fresh nondonor eggs or embryos.

${ }^{b}$ When fewer than 20 cycles are reported in an age category, rates are shown as a fraction and confidence intervals are not given. Calculating percentages from fractions may be misleading and is not encouraged.

${ }^{c}$ A multiple-infant birth is counted as one live birth.

${ }^{d}$ Clinic-specific outcome rates are unreliable for women older than 42 undergoing ART cycles using fresh or frozen embryos with nondonor eggs. Readers are urged to review national outcomes for these age groups (see page 27).

e All ages (including ages $>42$ ) are reported together because previous data show that patient age does not materially affect success with donor eggs.
} 


\section{AURORA HEALTH CARE-AURORA FERTILITY SERVICES, WEST ALLIS WEST ALLIS, WISCONSIN}

A comparison of clinic success rates may not be meaningful because patient medical characteristics and treatment approaches vary from clinic to clinic. For more details about this, along with information on how to interpret the statistics in this table, see pages $79-88$.

\section{ART CYCLE PROFILE}

\begin{tabular}{|c|c|c|c|c|c|c|c|}
\hline \multicolumn{4}{|c|}{ Type of ART ${ }^{\mathrm{a}}$} & \multicolumn{4}{|c|}{ Patient Diagnosis } \\
\hline IVF & $100 \%$ & Procedural Factors: & & Tubal factor & $0 \%$ & Other factor & $20 \%$ \\
\hline GIFT & $0 \%$ & With ICSI & $50 \%$ & Ovulatory dysfunction & $0 \%$ & Unknown factor & $0 \%$ \\
\hline ZIFT & $0 \%$ & Unstimulated & $0 \%$ & Diminished ovarian reserve & $0 \%$ & Multiple Factors: & \\
\hline \multirow[t]{3}{*}{ Combination } & $0 \%$ & Used gestational carrier & $0 \%$ & Endometriosis & $0 \%$ & Female factors only & $0 \%$ \\
\hline & & & & Uterine factor & $0 \%$ & Female \& male factors & $60 \%$ \\
\hline & & & & Male factor & $20 \%$ & & \\
\hline
\end{tabular}

2006 PREGNANCY SUCCESS RATES

Data verified by Mark F. Severino, MD

\section{Type of Cycle}

\section{Fresh Embryos from Nondonor Eggs}

Number of cycles

Percentage of cycles resulting in pregnancies ${ }^{b}$

Percentage of cycles resulting in live births ${ }^{b, c}$

(Confidence Interval)

Percentage of retrievals resulting in live births $s^{b, c}$

Percentage of transfers resulting in live births $s^{b, c}$

Percentage of transfers resulting in singleton live births ${ }^{b}$

Percentage of cancellations ${ }^{b}$

Average number of embryos transferred

Percentage of pregnancies with twins ${ }^{b}$

Percentage of pregnancies with triplets or more ${ }^{\mathrm{b}}$

Percentage of live births having multiple infants ${ }^{b, c}$

\section{Frozen Embryos from Nondonor Eggs}

Number of transfers

Percentage of transfers resulting in live births ${ }^{\mathrm{b}, \mathrm{c}}$

Average number of embryos transferred

\section{Donor Eggs}

Number of transfers

Percentage of transfers resulting in live births ${ }^{\mathrm{b}, \mathrm{c}}$

Average number of embryos transferred

\section{Age of Woman}

$\begin{array}{cccc}<35 & 35-37 & 38-40 & \mathbf{4 1 - 4 2}^{\mathbf{d}} \\ 1 & 0 & 1 & 0 \\ 1 / 1 & & 0 / 1 & \\ 1 / 1 & & 0 / 1 & \end{array}$

$1 / 1$

$1 / 1$

$0 / 1$

$0 / 1$

$1 / 1$

2.0

$1 / 1$

$0 / 1$

$1 / 1$

\section{CURRENT CLINIC SERVICES AND PROFILE}

Current Name: Aurora Health Care-Aurora Fertility Services, West Allis

\begin{tabular}{ll|ll|l|l} 
Donor egg? & Yes & Gestational carriers? & Yes & SART member? & Yes \\
\hline Donor embryo? & Yes & Cryopreservation? & Yes & Verified lab accreditation? & Yes \\
Single women? & Yes & & & (See Appendix C for details.) &
\end{tabular}

${ }^{a}$ Reflects patient and treatment characteristics of ART cycles performed in 2006 using fresh nondonor eggs or embryos.

b When fewer than 20 cycles are reported in an age category, rates are shown as a fraction and confidence intervals are not given. Calculating percentages from fractions may be misleading and is not encouraged.

${ }_{\mathrm{d}}^{\mathrm{c}}$ A multiple-infant birth is counted as one live birth.

${ }^{d}$ Clinic-specific outcome rates are unreliable for women older than 42 undergoing ART cycles using fresh or frozen embryos with nondonor eggs. Readers are urged to review national outcomes for these age groups (see page 27).

e All ages (including ages $>42$ ) are reported together because previous data show that patient age does not materially affect success with donor eggs. 
Appendix A

Technical Notes 



\section{APPENDIX A: TECHNICAL NOTES}

\section{How to Interpret a Confidence Interval}

\section{What is a confidence interval?}

Simply speaking, confidence intervals are a useful way to consider margin of error, a statistic often used in voter polls to indicate the range within which a value is likely to be correct (e.g., $30 \%$ of the voters favor a particular candidate with a margin of error of plus or minus 3.5\%). Similarly, in this report, confidence intervals are used to provide a range that we can be quite confident contains the success rate for a particular clinic during a particular time.

\section{Why do we need to consider confidence intervals if we already know the exact success rates for each clinic in 2006?}

No success rate or statistic is absolute. Suppose a clinic performed 100 cycles among women younger than 35 in 2006 and had a success rate of 20\% with a confidence interval of 12\%-28\%. The $20 \%$ success rate tells us that the average chance of success for women younger than 35 treated at this clinic in 2006 was $20 \%$. How likely is it that the clinic could repeat this performance? For example, if the same clinic performed another 100 cycles under similar clinical conditions on women with similar characteristics, would the success rate again be $20 \%$ ? The confidence interval tells us that the success rate would likely fall between $12 \%$ and $28 \%$.

\section{Why does the size of the confidence interval vary for different clinics?}

The size of the confidence interval gives us a realistic sense of how secure we feel about the success rate. If the clinic had performed only 20 cycles instead of 100 among women younger than 35 and still had a $20 \%$ success rate (4 successes out of 20 cycles), the confidence interval would be much larger (between $3 \%$ and $37 \%$ ) because the success or failure of each individual cycle would be more significant. For example, if just one more cycle had resulted in a live birth, the success rate would have been substantially higher-25\%, or 5 successes out of 20 cycles. Likewise, if just one more cycle had not been successful, the success rate would have been substantially lower $-15 \%$, or 3 out of 20 cycles. Compare this scenario to the original example of the clinic that performed 100 cycles and had a $20 \%$ success rate. If just one more cycle had resulted in a live birth, the success rate would have changed only slightly, from $20 \%$ to $21 \%$, and if one more cycle had not been successful, the success rate would have fallen to only $19 \%$. Thus, our confidence in a $20 \%$ success rate depends on how many cycles were performed.

\section{Why should confidence intervals be considered when success rates from different clinics are being compared?}

Confidence intervals should be considered because success rates can be misleading. For example, if Clinic A performs 20 cycles in a year and 8 cycles result in a live birth, its live birth rate would be $40 \%$. If Clinic B performs 600 cycles and 180 result in a live birth, the percentage of cycles that resulted in a live birth would be $30 \%$. We might be tempted to say that Clinic A has a better success rate than Clinic B. However, because Clinic A performed few cycles, its success rate would have a wide $95 \%$ confidence interval of $18.5 \%-61.5 \%$. On the other hand, because Clinic B performed a large number of cycles, its success rate would have a relatively narrow 
confidence interval of $26.2 \%-33.8 \%$. Thus, Clinic A could have a rate as low as $18.5 \%$ and Clinic B could have a rate as high as $33.8 \%$ if each clinic repeated its treatment with similar patients under similar clinical conditions. Moreover, Clinic B's rate is much more likely to be reliable because the size of its confidence interval is much smaller than Clinic A's.

Even though one clinic's success rate may appear higher than another's based on the confidence intervals, these confidence intervals are only one indication that the success rate may be better. Other factors also must be considered when comparing rates from two clinics. For example, some clinics see more than the average number of patients with difficult infertility problems, whereas others discourage patients with a low probability of success. For further information on important factors to consider when using the tables to assess a clinic, refer to pages 79-81. 


\section{Findings from Validation Visits for 2006 ART Data}

Clinic site visits for validation of 2006 ART data were conducted April through June 2008. During each visit, data reported by the clinic were compared with information recorded in patients' charts. Records for 1,644 cycles at 35 clinics were randomly selected for validation. These selected cycles included 533 cycles that resulted in a pregnancy and 434 cycles that resulted in a live-birth delivery.

Discrepancy rates are listed on the next page for key data items that were validated for each of the selected cycles. Review of the discrepancies indicated that in the majority of cases, the error did not affect the success rates (included in the national summary table and in the individual clinic tables). In addition to fully validating data for the randomly selected 1,644 cycles, during each visit the validation team also reviewed the documentation for every live birth that had been reported to CDC. There were no cases found in which a live birth had been reported erroneously. In all, validation indicated that the clinic success rates presented in this report are valid. 


\section{Discrepancy Rates by Data Fields Selected for Validation \\ Discrepancy Rate* \\ Data Field Name \\ (Confidence Interval $^{\dagger}$ ) \\ Comments}

\begin{tabular}{lcl}
\hline Patient date of birth & $2.0 \%$ & Nearly one-third of the discrepancies resulted in a \\
& change of Age Group Category (see Clinic Summary \\
& Table classification) and differed only by one age \\
& category.
\end{tabular}

Diagnosis of infertility

$16.7 \% \quad$ For approximately one-third of the total 283

(13.2-20.2) discrepancies, multiple causes of infertility were found in the patient's chart, but only a single cause was reported. For 44 discrepancies, multiple causes were reported but only a single cause was found in the patient's chart.

Type of ART (i.e., fresh vs.

$<1 \%$

frozen; donor vs. nondonor)

Use of ICSI

$1.6 \%$ For about three-fourths of these discrepancies, use of

(0.8-2.3) ICSI was indicated in the patient's chart but was not reported.

Number of embryos

$1.6 \% \quad$ Nearly all discrepancies differed by one or two

transferred

$(0.6-2.7)$

embryos.

Outcome of ART treatment

$<1 \%$

(i.e., pregnant vs. not

pregnant)

Number of fetal hearts on

ultrasound

$1.3 \%$

$(0.5-2.1)$

Of the discrepancies, six cases resulted in a change

ultrasound

in categorization of single- versus multiple-fetus pregnancy.

Pregnancy outcome

$1.1 \%$

(i.e., miscarriage, stillbirth,

$(0.5-1.8)$

In most of these discrepancies, there was no

and live birth

information on pregnancy outcome in the patient's chart, or spontaneous abortion was reported as induced abortion, or vice versa.

Number of infants born

$1.1 \%$

$(0.7-1.4)$

In more than half of the discrepancies, there was no information on the number of infants born in the patient's chart. In one case, a twin delivery was recorded in the patient's chart when a singleton delivery was reported.

Cycle cancellation

$<1 \%$

Notes: ART = assisted reproductive technology; ICSI = intracytoplasmic sperm injection.

*Discrepancy rates estimate the proportion of all treatment cycles with differences for a particular data item. The discrepancy-rate calculations weight the data from validated cycles to reflect the overall number of cycles performed at each clinic. Thus, findings from larger clinical practices were weighted more heavily than findings from smaller practices.

${ }^{\dagger}$ This table shows a range, called the $95 \%$ confidence interval, that conveys the reliability of the discrepancy rate. For a more general explanation of confidence intervals, see pages 519-520. 
Appendix B

Glossary of Terms 



\section{APPENDIX B: GLOSSARY OF TERMS USED IN THIS REPORT}

Adverse outcome. A pregnancy that does not result in a live birth. The adverse outcomes reported for ART procedures are miscarriages, induced abortions, and stillbirths.

\section{American Society for Reproductive Medicine (ASRM). Professional society whose affiliate organization, the Society for Assisted Reproductive Technology (SART), is composed of clinics and programs that provide ART.}

ART (assisted reproductive technology). All treatments or procedures that involve surgically removing eggs from a woman's ovaries and combining the eggs with sperm to help a woman become pregnant. The types of ART are in vitro fertilization (IVF), gamete intrafallopian transfer (GIFT), and zygote intrafallopian transfer (ZIFT).

ART cycle. A process in which (1) an ART procedure is carried out, (2) a woman has undergone ovarian stimulation or monitoring with the intent of having an ART procedure, or (3) frozen embryos have been thawed with the intent of transferring them to a woman. A cycle begins when a woman begins taking fertility drugs or having her ovaries monitored for follicle production.

Canceled cycle. An ART cycle in which ovarian stimulation was carried out but was stopped before eggs were retrieved or, in the case of frozen embryo cycles, before embryos were transferred. Cycles are canceled for many reasons: eggs may not develop, the patient may become ill, or the patient may choose to stop treatment.

Combination cycle. A cycle that uses more than one ART procedure. Combination cycles usually involve IVF plus either GIFT or ZIFT.

Cryopreservation. The practice of freezing extra embryos from a couple's ART cycle for potential future use.

Diminished ovarian reserve. This diagnosis means that the ability of the ovary to produce eggs is reduced. Reasons include congenital, medical, or surgical causes or advanced age.

Donor egg cycle. An embryo is formed from the egg of one woman (the donor) and then transferred to another woman who is unable to use her own eggs (the recipient). The donor relinquishes all parental rights to any resulting offspring.
Donor embryo. An embryo that is donated by a couple who previously underwent ART treatment and had extra embryos available.

Ectopic pregnancy. A pregnancy in which the fertilized egg implants in a location outside of the uterus-usually in the fallopian tube, the ovary, or the abdominal cavity. Ectopic pregnancy is a dangerous condition that must receive prompt medical treatment.

Egg. A female reproductive cell, also called an oocyte or ovum.

Egg retrieval (also called oocyte retrieval). A procedure to collect the eggs contained in the ovarian follicles.

Egg transfer (also called oocyte transfer). The transfer of retrieved eggs into a woman's fallopian tubes through laparoscopy. This procedure is used only in GIFT.

Embryo. An egg that has been fertilized by a sperm and has undergone one or more divisions.

Embryo transfer. Placement of embryos into a woman's uterus through the cervix after IVF: in ZIFT, the embryos are placed in a woman's fallopian tube.

Endometriosis. A medical condition that involves the presence of tissue similar to the uterine lining in abnormal locations. This condition can affect both fertilization of the egg and embryo implantation.

Fertility Clinic Success Rate and Certification Act of 1992 (FCSRCA). Law passed by the United States Congress in 1992 requiring all clinics performing ART in the United States to annually report their success rate data to CDC.

Fertilization. The penetration of the egg by the sperm and the resulting combining of genetic material that develops into an embryo.

Fetus. The unborn offspring from the eighth week after conception to the moment of birth.

Follicle. A structure in the ovaries that contains a developing egg.

Fresh eggs, sperm, or embryos. Eggs, sperm, or embryos that have not been frozen. Fresh embryos, however, may have been conceived using either fresh or frozen sperm. 
Frozen embryo cycle. An ART cycle in which frozen (cryopreserved) embryos are thawed and transferred to the woman.

Gamete. A reproductive cell, either a sperm or an egg.

GIFT (gamete intrafallopian transfer). An ART procedure that involves removing eggs from the woman's ovary, combining them with sperm, and using a laparoscope to place the unfertilized eggs and sperm into the woman's fallopian tube through small incisions in her abdomen.

Gestation. The period of time from conception to birth.

\section{Gestational carrier (also called a gestational} surrogate). A woman who gestates, or carries, an embryo that was formed from the egg of another woman. The gestational carrier usually has a contractual obligation to return the infant to its intended parents.

Gestational sac. A fluid-filled structure that develops within the uterus early in pregnancy. In a normal pregnancy, a gestational sac contains a developing fetus.

ICSI (intracytoplasmic sperm injection). A procedure in which a single sperm is injected directly into an egg; this procedure is most commonly used to overcome male infertility problems.

Induced or therapeutic abortion. A surgical or other medical procedure used to end a pregnancy.

IUI (intrauterine insemination). A medical procedure that involves placing sperm into a woman's uterus to facilitate fertilization. IUI is not considered an ART procedure because it does not involve the manipulation of eggs.

IVF (in vitro fertilization). An ART procedure that involves removing eggs from a woman's ovaries and fertilizing them outside her body. The resulting embryos are then transferred into the woman's uterus through the cervix.

Laparoscopy. A surgical procedure in which a fiber-optic instrument (a laparoscope) is inserted through a small incision in the abdomen to view the inside of the pelvis.

Live birth. The delivery of one or more infants with any signs of life.
Male factor. Any cause of infertility due to low sperm count or problems with sperm function that makes it difficult for a sperm to fertilize an egg under normal conditions.

Miscarriage (also called spontaneous abortion). A pregnancy ending in the spontaneous loss of the embryo or fetus before 20 weeks of gestation.

Multifetal pregnancy reduction. A procedure used to decrease the number of fetuses a woman carries and improve the chances that the remaining fetuses will develop into healthy infants. Multifetal reductions that occur naturally are referred to as spontaneous reductions.

Multiple factors, female only. A diagnostic category used when more than one female cause of infertility is diagnosed.

Multiple factors, female and male. A diagnostic category used when one or more female causes and male factor infertility are diagnosed.

Multiple-fetus pregnancy. A pregnancy with two or more fetuses, determined by the number of fetal hearts observed on an ultrasound performed early in pregnancy (usually in the first trimester).

Multiple-infant birth. A pregnancy that results in the birth of more than one infant.

NASS (National ART Surveillance System). Webbased data collection system used by all ART clinics to report data for each ART procedure to CDC.

Oocyte. The female reproductive cell, also called an egg.

Other causes of infertility. These include immunological problems, chromosomal abnormalities, cancer chemotherapy, and serious illnesses.

Ovarian monitoring. The use of ultrasound and/or blood or urine tests to monitor follicle development and hormone production.

Ovarian stimulation. The use of drugs (oral or injected) to stimulate the ovaries to develop follicles and eggs.

Ovulatory dysfunction. A diagnostic category used when a woman's ovaries are not producing eggs normally. It includes polycystic ovary syndrome and multiple ovarian cysts. 
PGD (Preimplantation Genetic Diagnosis). A technique combining the recent significant advances in molecular genetics and assisted reproductive technology. PGD allows physicians to identify various genetic diseases in the embryo (fertilized egg with several divisions) prior to implantation, that is, before the pregnancy is established. It is of special value for those who are at risk of having children with serious genetic problems.

Pregnancy (clinical). A pregnancy documented by ultrasound that shows a gestational sac in the uterus. For ART data collection purposes, pregnancy is defined as a clinical pregnancy rather than a chemical pregnancy (i.e., a positive pregnancy test).

Singleton. A single live-born infant.

Society for Assisted Reproductive Technology

(SART). An affiliate of the American Society for Reproductive Medicine composed of clinics and programs that provide ART.

Sperm. The male reproductive cell.

Spontaneous abortion. See Miscarriage.

Stillbirth. The birth of an infant after 20 or more weeks of gestation that shows no signs of life.

Stimulated cycle. An ART cycle in which a woman receives oral or injected fertility drugs to stimulate her ovaries to produce more follicles.
Thawed embryo cycle. Same as frozen embryo cycle.

Tubal factor. A diagnostic category used when the woman's fallopian tubes are blocked or damaged, making it difficult for the egg to be fertilized or for an embryo to travel to the uterus.

Ultrasound. A technique used in ART for visualizing the follicles in the ovaries, the gestational sac, or the fetus.

Unexplained cause of infertility. A diagnostic category used when no cause of infertility is found in either the woman or the man.

Unstimulated cycle. An ART cycle in which the woman does not receive drugs to stimulate her ovaries to produce more follicles. Instead, follicles develop naturally.

Uterine factor. A structural or functional disorder of the uterus that results in reduced fertility.

ZIFT (zygote intrafallopian transfer). An ART procedure in which eggs are collected from a woman's ovary and fertilized outside her body. A laparoscope is then used to place the resulting zygote (fertilized egg) into the woman's fallopian tube through a small incision in her abdomen. 



\section{Appendix C}

\section{ART Clinics}





\section{APPENDIX C: ART CLINICS, 2006}

\section{Reporting ART Clinics for 2006, by State}

If the clinic name has changed since 2006, the current name is listed in italics directly under the 2006 name. If the clinic location has changed since 2006, the clinic is listed alphabetically by the current city and state.

Clinic names preceded by the $\$$ symbol have reorganized or closed since 2006 . Reorganization is defined as a change in ownership or affiliation or a change in at least two of the three key staff positions (practice director, medical director, or laboratory director). Contact the NASS Help Desk for current clinic information at 1-888-650-0822 or NASS@Westat.com.

Clinic names preceded by the symbol performed more than 50 cycles with Preimplantation Genetic Diagnosis (PGD) in 2006 and among them more than 10 cycles specifically for the purpose of prevention of genetic disorders.

Explanation of abbreviations for accrediting agencies used throughout this list:

$\mathrm{CAP} / \mathrm{ASRM}=$ College of American Pathologists/American Society for Reproductive Medicine, Reproductive Laboratory Accreditation Program

$\mathrm{JCAHO}=$ Joint Commission on Accreditation of Healthcare Organizations

NYSTB = New York State Tissue Bank Program

PLEASE NOTE that CDC does not oversee any of these accreditation programs. For further information on how to contact accrediting organizations directly, see page 88.

\section{ALABAMA}

Alabama Fertility Specialists

2700 Hwy 280, Suite 370

Birmingham AL 35223

Telephone: (205) 874-0000; Fax: (205) 874-7021

Lab Name: Alabama Fertility Specialists Laboratory Accreditation: CAP/ASRM

ART Fertility Program of Alabama 2006 Brookwood Medical Center Dr, Suite 508 Birmingham AL 35209

Telephone: (205) 870-9784; Fax: (205) 870-0698

Lab Name: ART Program of Alabama IVF/

Andrology Laboratory

Accreditation: CAP/ASRM

University of Alabama at Birmingham 2000 6th Ave South, Kirklin Clinic-OB/GYN Birmingham AL 35233

Telephone: (205) 801-8212; Fax: (205) 326-9440

Lab Name: University of Alabama at Birmingham

Gamete Biology Laboratory

Accreditation: CAP/ASRM
Huntsville Reproductive Medicine, PC

185 Chateau Dr, Suite 301

Huntsville AL 35801

Telephone: (256) 213-2229; Fax: (256) 213-9978

Lab Name: Huntsville Reproductive Medicine, PC

Accreditation: None

Center for Reproductive Medicine

3 Mobile Infirmary Cir, Suite 213

Mobile AL 36607

Telephone: (251) 438-4200; Fax: (251) 438-4211

Lab Name: Center for Reproductive Medicine

Accreditation: CAP/ASRM

University of South Alabama IVF and ART Program Reproductive Endocrinology and Infertility Division 251 Cox St, Suite 100

Mobile AL 36604

Telephone: (251) 415-1491; Fax: (251) 415-1552

Lab Name: University of South Alabama In Vitro

Fertilization \& Andrology Laboratory

Accreditation: CAP/ASRM 


\section{ALASKA}

Peninsula Medical Center

John Nels Anderson, MD

265 N. Binkley St

Soldotna AK 99669

Telephone: (907) 262-4161; Fax: (907) 262-1545

Lab Name: Peninsula Medical Center,

John Nels Anderson, MD

Accreditation: None

\section{ARIZONA}

West Valley Fertility Center

17612 N. 59th Ave, Suite 100

Glendale AZ 85308

Telephone: (602) 993-8636; Fax: (602) 993-2528

Lab Name: West Valley Fertility Center

ART Laboratory

Accreditation: CAP/ASRM

Arizona Reproductive Medicine Specialists

1701 E. Thomas Rd

Bldg 1, Suite 101

Phoenix AZ 85016

Telephone: (602) 343-2767; Fax: (602) 343-2766

Lab Name: Arizona Reproductive

Medicine Specialists

Accreditation: CAP/ASRM

Southwest Fertility Center

3125 N. 32nd St, Suite 200

Phoenix AZ 85018

Telephone: (602) 956-7481; Fax: (602) 956-7591

Lab Name: Southwest Fertility Center Laboratory

Accreditation: CAP/ASRM

Advanced Fertility Care

9819 N. 95th St, Suite 105

Scottsdale AZ 85258

Telephone: (480) 874-2229; Fax: (480) 874-2231

Lab Name: Arizona Advanced

Reproductive Laboratory

Accreditation: CAP/ASRM

Arizona Associates for Reproductive Health

8573 E. Princess Dr, Suite 101

Scottsdale AZ 85255

Telephone: (480) 946-9900; Fax: (480) 946-9914

Lab Name: Arizona Associates for Reproductive

Health ART Laboratories

Accreditation: CAP/ASRM
Arizona Center for Fertility Studies

8997 E. Desert Cove Ave, 2nd Floor

Scottsdale AZ 85260

Telephone: (480) 860-4792; Fax: (480) 860-6819

Lab Name: Scottsdale Healthcare Institute for

Reproductive Studies Fertility Laboratory

Accreditation: CAP/ASRM, JCAHO

IVF Phoenix

9817 N. 95th St, Suite 105

Scottsdale AZ 85258

Telephone: (602) 765-2229; Fax: (602) 493-6641

Lab Name: IVF Phoenix Laboratory

Accreditation: CAP/ASRM

Fertility Treatment Center

2155 E. Conference Dr, Suite 115

Tempe AZ 85284

Telephone: (480) 831-2445; Fax: (480) 897-1283

Lab Name: Fertility Treatment Center

ART Laboratory

Accreditation: CAP/ASRM

Arizona Center for Reproductive Endocrinology and Infertility

5190 E. Farness Dr, Suite 114

Tucson AZ 85712

Telephone: (520) 326-0001; Fax: (520) 326-7451

Lab Name: Arizona Center for Reproductive

Endocrinology \& Infertility

Accreditation: CAP/ASRM

Reproductive Health Center

4518 E. Camp Lowell Dr

Tucson AZ 85712

Telephone: (520) 733-0083; Fax: (520) 733-0771

Lab Name: Reproductive Health Center

Accreditation: JCAHO

\section{ARKANSAS}

Arkansas Fertility Center

9101 Kanis Rd, Suite 300

Little Rock AR 72205

Telephone: (501) 801-1200; Fax: (501) 801-1207

Lab Name: Arkansas Fertility Center

Accreditation: CAP/ASRM 


\section{CALIFORNIA}

Lifestart Fertility Center

Anita Singh, MD

29525 Canwood St, Suite 220

Agoura Hills CA 91301

Telephone: (818) 889-4532; Fax: (818) 889-4536

Lab Name: ART Reproductive Center

Accreditation: CAP/ASRM

Garfield Fertility Center

320 S. Garfield Ave, Suite 226

Alhambra CA 91801

Telephone: (626) 943-9536; Fax: (626) 943-9529

Lab Name: ART Reproductive Center

Accreditation: CAP/ASRM

Alta Bates In Vitro Fertilization Program

2999 Regent St, Suite 101A

Berkeley CA 94705

Telephone: (510) 649-0440; Fax: (510) 649-8700

Lab Name: Alta Bates Summit Medical Center,

Alta Bates IVF Program

Accreditation: CAP/ASRM

Center for Reproductive Health \& Gynecology

(CRH\&G)

99 N. La Cienega Blvd, Suite 109

Beverly Hills CA 90211

Telephone: (310) 360-7584; Fax: (310) 360-9827

Lab Name: Center for Reproductive Health and Gynecology

Accreditation: CAP/ASRM

Southern California Reproductive Center

Hal Danzer, MD

450 N. Roxbury Dr, Suite 500

Beverly Hills CA 90210

Telephone: (310) 277-2393; Fax: (310) 274-5112

Lab Name: ART Reproductive Center

Accreditation: CAP/ASRM

Southern California Reproductive Center

Mark W. Surrey, MD

450 N. Roxbury Dr, Suite 500

Beverly Hills CA 90210

Telephone: (310) 277-2393; Fax: (310) 274-5112

Lab Name: ART Reproductive Center

Accreditation: CAP/ASRM
West Coast IVF Clinic, Inc.

250 N. Robertson Blvd, Suite 403

Beverly Hills CA 90211

Telephone: (310) 285-0333; Fax: (310) 285-0334

Lab Name: LA IVF Lab, LLC

Accreditation: JCAHO

Fertility Care of Orange County

203 N. Brea Blvd, Suite 100

Brea CA 92821

Telephone: (714) 256-0777; Fax: (714) 245-0105

Lab Name: Southern California Institute for

Reproductive Sciences

Accreditation: CAP/ASRM

Lab Name: Huntington Reproductive Center

Gamete Laboratory

Accreditation: CAP/ASRM

Central California IVF Program

Women's Specialty and Fertility Center

722 Medical Center Dr East, Suite 105

Clovis CA 93611

Telephone: (559) 299-7700; Fax: (559) 297-9679

Lab Name: Community Medical Center-Fresno

Accreditation: JCAHO

Zouves Fertility Center

901 Campus Dr, Suite 214

Daly City CA 94015

Telephone: (650) 301-4933; Fax: (650) 301-4939

Lab Name: Zouves Fertility Center

Accreditation: CAP/ASRM

California IVF: Davis Fertility Center, Inc.

1550 Drew Ave, Suite 100

Davis CA 95616

Telephone: (530) 771-0177; Fax: (530) 771-0135

Lab Name: California IVF: Davis Fertility Center, Inc.

Accreditation: None

The Fertility Institutes-California, Nevada

16030 Ventura Blvd, Suite 404

Encino CA 91436

Telephone: (818) 728-4600; Fax: (818) 728-4616

Lab Name: The Fertility Institutes-

California, Nevada ART Reproductive Center

Accreditation: CAP/ASRM (Pend)

Lab Name: ART Reproductive Center

Accreditation: CAP/ASRM 
West Coast Fertility Centers

11160 Warner Ave, Suite 411

Fountain Valley CA 92708

Telephone: (714) 513-1399; Fax: (714) 513-1393

Lab Name: West Coast Fertility Center

Accreditation: CAP/ASRM

Xpert Fertility Care of California

Minh N. Ho, MD, FACOG

11180 Warner Ave, Suite 461

Fountain Valley CA 92708

Telephone: (714) 429-5848; Fax: (714) 545-5675

Lab Name: Pacific Reproductive Center-Irvine

Accreditation: None

Lab Name: Zarutskie Fertility \& Endocrine Institute

Accreditation: None

Kaiser Permanente Center for Reproductive Health 39141 Civic Center Dr, Suite 350

Fremont CA 94538

Telephone: (510) 248-6900; Fax: (510) 248-6981

Lab Name: Kaiser Permanente Center for

Reproductive Health

Accreditation: CAP/ASRM, JCAHO

Kathleen L. Kornafel, MD, PhD

1560 E. Chevy Chase Dr, Suite 200

Glendale CA 91206

Telephone: (818) 242-9933; Fax: (818) 242-9937

Lab Name: ART Reproductive Center

Accreditation: CAP/ASRM

Sher Institute of Reproductive Medicine-

Los Angeles

1520 E. Chevy Chase Dr, Suite 101

Glendale CA 91206

Telephone: (818) 291-1985; Fax: (818) 291-1986

Lab Name: Sher Institute for Reproductive

Medicine-Los Angeles Laboratory

Accreditation: CAP/ASRM, JCAHO

\$Marin Reproductive Medical Associates, Inc.

1100 S. Eliseo Dr, Suite 107

Greenbrae CA 94904

Telephone: (415) 464-8688; Fax: (415) 464-8042

Contact the NASS Help Desk for current

clinic information.
Coastal Fertility Medical Center, Inc.

4900 Barranca Pkwy, Suite 103

Irvine CA 92604

Telephone: (949) 726-0600; Fax: (949) 726-0601

Lab Name: Coastal Fertility Medical Center, Inc.,

Reproductive Specialty Laboratories, Inc.

Accreditation: CAP/ASRM

Fertility Center of Southern California

2192 Martin St, Suite 110

Irvine CA 92612

Telephone: (949) 955-0072; Fax: (949) 955-0077

Lab Name: Southern California Institute for

Reproductive Sciences

Accreditation: CAP/ASRM

Reproductive Fertility Center

16300 Sand Canyon Ave, Suite 901

Irvine CA 92618

Telephone: (949) 453-8600; Fax: (949) 453-8601

Lab Name: Reproductive Fertility Center

Accreditation: CAP/ASRM (Pend)

Reproductive Partners-UCSD Regional

Fertility Center

9850 Genesee Ave, Suite 800

La Jolla CA 92037

Telephone: (858) 552-9177; Fax: (858) 552-9188

Lab Name: Reproductive Partners Medical Group,

Inc.-La Jolla Laboratory

Accreditation: CAP/ASRM

Reproductive Sciences Center

4150 Regents Park Row, Suite 280

La Jolla CA 92037

Telephone: (858) 625-0125; Fax: (858) 625-0131

Lab Name: Reproductive Science Center

IVF Laboratory

Accreditation: CAP/ASRM

Scripps Clinic Fertility Center

10666 N. Torrey Pines Rd

La Jolla CA 92037

Telephone: (858) 554-8630; Fax: (858) 554-9092

Lab Name: Scripps Clinic Torrey Pines Fertility

Center Laboratory

Accreditation: CAP/ASRM, JCAHO 
Mission Reproductive Center

25500 Rancho Niguel Rd, Suite 280

Laguna Niguel CA 92677

Telephone: (949) 448-7818; Fax: (949) 448-7819

Lab Name: Mission Reproductive Center Laboratory

Accreditation: CAP/ASRM

Sher Institute of Reproductive Medicine-

$$
\text { Orange County }
$$

27882 Forbes Rd, Suite 200

Laguna Niguel CA 92677

Telephone: (949) 249-9200; Fax: (949) 249-9203

Lab Name: Sher Institute for Reproductive

Medicine-Orange County

Accreditation: CAP/ASRM (Pend), JCAHO

Loma Linda University Center for Fertility and IVF

Department of Gynecology and Obstetrics

11370 Anderson St, Suite 3950

Loma Linda CA 92354

Telephone: (909) 558-2851; Fax: (909) 558-2450

Lab Name: Loma Linda University Health Care

Fertility Science Laboratory

Accreditation: CAP/ASRM

California Fertility Partners

11818 Wilshire Blvd, Suite 300

Los Angeles CA 90025

Telephone: (310) 828-4008; Fax: (310) 828-3310

Lab Name: California Fertility Partners Reproductive

Technology Laboratories

Accreditation: CAP/ASRM

Cedars Sinai Medical Center

Center for Fertility and Reproductive Medicine

8700 Beverly Blvd, Suite 3611

Los Angeles CA 90048

Telephone: (310) 423-9964; Fax: (310) 423-9704

Lab Name: ART Reproductive Center

Accreditation: CAP/ASRM

\$CHA Fertility Center

5455 Wilshire Blvd, Suite 1904

Los Angeles CA 90036

Telephone: (323) 525-3377; Fax: (323) 525-3376

Contact the NASS Help Desk for current

clinic information.
Pacific Fertility Center-Los Angeles

10921 Wilshire Blvd, Suite 700

Los Angeles CA 90024

Telephone: (310) 209-7700; Fax: (310) 209-7799

Lab Name: Pacific Fertility Medical Center-

Los Angeles

Accreditation: CAP/ASRM

UCLA Fertility Center

Department of Obstetrics and Gynecology

200 Medical Plaza, Suite 430

Los Angeles CA 90095

Telephone: (310) 825-9500; Fax: (310) 206-9731

Lab Name: ART Reproductive Center

Accreditation: CAP/ASRM

USC Reproductive Endocrinology and Infertility

1127 Wilshire Blvd, Suite 1400

Los Angeles CA 90017

Telephone: (213) 975-9990; Fax: (213) 975-9997

Lab Name: USC Reproductive Endocrinology and

Infertility Laboratory

Accreditation: CAP/ASRM

Reproductive Specialty Medical Center

1441 Avocado Ave, Suite 203

Newport Beach CA 92660

Telephone: (949) 640-7200; Fax: (949) 720-0203

Lab Name: Reproductive Specialty Medical Center

Accreditation: JCAHO

Southern California Center for

Reproductive Medicine

361 Hospital Rd, Suite 333

Newport Beach CA 92663

Telephone: (949) 642-8727; Fax: (949) 642-5413

Lab Name: Southern California Institute for

Reproductive Sciences

Accreditation: CAP/ASRM

IVF-Orange Surgery Center

431 S. Batavia St, Suite 102

Orange CA 92868

Telephone: (714) 771-7800; Fax: (714) 289-9900

Lab Name: IVF-Orange Surgery Center

Accreditation: None 
NOVA In Vitro Fertilization

1681 El Camino Real

Palo Alto CA 94306

Telephone: (650) 322-0500; Fax: (650) 322-5404

Lab Name: NOVA In Vitro Fertilization

Main Laboratory

Accreditation: CAP/ASRM

Stanford University IVF/ART Program

Department of Gynecology and Obstetrics

Stanford Fertility and Reproductive Medicine Center

Stanford University Department of Gynecology and Obstetrics

900 Welch Rd, Suite 350

Palo Alto CA 94304

Telephone: (650) 736-4036; Fax: (650) 498-7294

Lab Name: Stanford University Hospitals and Clinics IVF/REI Laboratory

Accreditation: CAP/ASRM, JCAHO

Huntington Reproductive Center

333 S. Arroyo Pkwy, 3rd Floor

Pasadena CA 91105

Telephone: (626) 440-9161; Fax: (626) 440-0138

Lab Name: Huntington Reproductive Center

Gamete Laboratory

Accreditation: CAP/ASRM

Palo Alto Medical Foundation

Reproductive Endocrinology \& Fertility

3220 Alpine Rd

Portola Valley CA 94028

Telephone: (650) 853-2200; Fax: (650) 853-2237

Lab Name: Fertility \& Reproductive Health Institute

IVF Laboratory

Accreditation: CAP/ASRM

Reproductive Partners-Redondo Beach

510 N. Prospect Ave, Suite 202

Redondo Beach CA 90277

Telephone: (310) 318-3010; Fax: (310) 798-7304

Lab Name: Reproductive Partners Medical

Group, Inc.-Redondo Beach

Accreditation: CAP/ASRM

Northern California Fertility Medical Center

1130 Conroy Ln, Suite 100

Roseville CA 95661

Telephone: (916) 773-2229; Fax: (916) 773-8391

Lab Name: Northern California Fertility

Medical Center

Accreditation: CAP/ASRM
Kaiser Permanente Center for Reproductive

Health-Sacramento

1650 Response Rd, Suite 1A

Sacramento CA 95815

Telephone: (916) 614-5200; Fax: (916) 614-5045

Lab Name: Kaiser Permanente Center for

Reproductive Health-Sacramento

Accreditation: CAP/ASRM (Pend)

The University of California-Davis

Assisted Reproductive Technology Program

2521 Stockton Blvd, Suite 4200

Sacramento CA 95817

Telephone: (916) 734-6106; Fax: (916) 734-6150

Lab Name: UC Davis Medical Center Assisted

Reproductive Technology Program

Accreditation: CAP/ASRM

The Fertility and Gynecology Center

Monterey Bay IVF Program

212 San Jose St, Suite 201

Salinas CA 93901

Telephone: (831) 769-0161; Fax: (831) 759-0939

Lab Name: Fertility and Gynecology Center

Accreditation: CAP/ASRM

Fertility Specialists Medical Group

8010 Frost St, Plaza Level

San Diego CA 92123

Telephone: (858) 505-5500; Fax: (858) 505-5555

Lab Name: San Diego Center for

Reproductive Surgery

Accreditation: CAP/ASRM (Pend)

IGO Medical Group of San Diego

9339 Genesee Ave, Suite 220

San Diego CA 92121

Telephone: (858) 455-7520; Fax: (858) 455-5461

Lab Name: Reproductive Science Center

IVF Laboratory

Accreditation: CAP/ASRM

NTC Infertility Clinic

2650 Stockton Rd, Bldg 624

San Diego CA 92106

Telephone: (619) 524-6218; Fax: (619) 524-6241

Lab Name: Reproductive Partners Medical

Group, Inc.-La Jolla Laboratory

Accreditation: CAP/ASRM 
San Diego Fertility Center

(SDFC)

11515 El Camino Real, Suite 100

San Diego CA 92130

Telephone: (858) 794-6363; Fax: (858) 794-6360

Lab Name: SDFC IVF \& Andrology Laboratories

Accreditation: CAP/ASRM

Laurel Fertility Care

1700 California St, Suite 570

San Francisco CA 94109

Telephone: (415) 673-9199; Fax: (415) 673-8796

Lab Name: Laurel Fertility Care

Accreditation: CAP/ASRM

Pacific Fertility Center

55 Francisco St, Suite 500

San Francisco CA 94133

Telephone: (415) 834-3000; Fax: (415) 834-3080

Lab Name: Pacific Fertility Center IVF Laboratory

Accreditation: CAP/ASRM

UCSF Center for Reproductive Health

2356 Sutter St, 7th Floor

San Francisco CA 94115

Telephone: (415) 353-3040; Fax: (415) 353-7744

Lab Name: UCSF Center for Reproductive Health

Accreditation: CAP/ASRM, JCAHO

Fertility Physicians of Northern California

2581 Samaritan Dr, Suite 302

San Jose CA 95124

Telephone: (408) 358-2500; Fax: (408) 876-4735

Lab Name: Fertility \& Reproductive Health Institute

IVF Laboratory

Accreditation: CAP/ASRM

Reproductive Science Center of the San Francisco

Bay Area

3160 Crow Canyon Rd, Suite 150

San Ramon CA 94583

Telephone: (925) 867-1800; Fax: (925) 275-3862

Lab Name: Reproductive Science Center of the

San Francisco Bay Area

Accreditation: CAP/ASRM
Parker-Rosenman-Rodi Gynecology and Infertility Medical Group

1450 Tenth St, Suite 404

Santa Monica CA 90401

Telephone: (310) 451-8144; Fax: (310) 451-3414

Lab Name: Pacific Fertility Medical Center-

Los Angeles

Accreditation: CAP/ASRM

Advanced Fertility Associates Medical Group, Inc. 1111 Sonoma Ave, Suite 214

Santa Rosa CA 95405

Telephone: (707) 575-5831; Fax: (707) 575-4379

Lab Name: Advanced Fertility Associates Medical

Group, Inc.

Accreditation: CAP/ASRM

Valley Center for Reproductive Health

Tina Koopersmith, MD

13320 Riverside Dr, Suite 220

Sherman Oaks CA 91423

Telephone: (818) 986-1648; Fax: (818) 986-1653

Lab Name: ART Reproductive Center

Accreditation: CAP/ASRM

The Center for Fertility and Gynecology

Vermesh Center for Fertility

18370 Burbank Blvd, Suite 301

Tarzana CA 91356

Telephone: (818) 881-9800; Fax: (818) 881-1857

Lab Name: A.R.T Medical Group, Inc., Laboratory

Accreditation: CAP/ASRM

Tree of Life Center

Snunit Ben-Ozer, MD

18370 Burbank Blvd, Suite 514

Tarzana CA 91356

Telephone: (818) 344-8522; Fax: (818) 344-3992

Lab Name: ART Reproductive Center

Accreditation: CAP/ASRM

Fertility and Surgical Associates of California

325 Rolling Oaks Dr, Suite 110

Thousand Oaks CA 91361

Telephone: (805) 778-1122; Fax: (805) 778-0855

Lab Name: Fertility and Surgical Associates

of California

Accreditation: CAP/ASRM 
Pacific Reproductive Center

3720 Lomita Blvd, Suite 200

Torrance CA 90505

Telephone: (310) 376-7000; Fax: (310) 373-0319

Lab Name: Pacific Reproductive Center-Torrance

Accreditation: CAP/ASRM

Contra Costa OB/GYN \& Infertility

240 La Casa Via, Suite 100

Walnut Creek CA 94598

Telephone: (925) 932-2565; Fax: (925) 930-8568

Lab Name: Ygnacio Andrology

Accreditation: None

Reproductive Partners-Long Beach

Reproductive Partners-Orange County

13950 Milton Ave, Suite 100

Westminster CA 92683

Telephone: (714) 702-3000; Fax: (714) 702-3039

Lab Name: Reproductive Partners Medical Group-

Long Beach IVF \& Andrology Laboratory

Accreditation: CAP/ASRM

Lab Name: Reproductive Partners Medical

Group, Inc.-Redondo Beach

Accreditation: CAP/ASRM

\section{COLORADO}

Advanced Reproductive Medicine

University of Colorado Health Sciences Center

Anschutz Outpatient Pavilion

1635 N. Ursula St

Aurora CO 80010

Telephone: (720) 848-1690; Fax: (720) 848-1678

Lab Name: University of Colorado Hospital

IVF Clinical Laboratory

Accreditation: CAP/ASRM, JCAHO

Reproductive Medicine and Fertility Center

3225 International Cir, Suite 100

Colorado Springs CO 80910

Telephone: (719) 475-2229; Fax: (719) 475-2227

Lab Name: Reproductive Medicine and Fertility

Center of Southern Colorado,

Accreditation: CAP/ASRM

Eric H. Silverstein, MD, Professional LLC, dba

The Fertility Center of Colorado

6160 Tutt Blvd, Suite 210

Colorado Springs CO 80923

Telephone: (719) 636-0080; Fax: (719) 636-3030

Lab Name: The Fertility Center of Colorado

Accreditation: CAP/ASRM
Colorado Reproductive Endocrinology

4600 E. Hale Pkwy, Suite 350

Denver CO 80220

Telephone: (303) 321-7115; Fax: (303) 321-9519

Lab Name: Colorado Reproductive Endocrinology

Accreditation: CAP/ASRM

Rocky Mountain Center for Reproductive Medicine 1080 E. Elizabeth St

Fort Collins CO 80524

Telephone: (970) 493-6353; Fax: (970) 493-6366

Lab Name: Rocky Mountain Center for

Reproductive Medicine IVF/Embryology

Laboratory

Accreditation: CAP/ASRM

Conceptions Reproductive Associates of Colorado

271 W. County Line Rd

Littleton CO 80129

Telephone: (303) 794-0045; Fax: (303) 794-2054

Lab Name: Conceptions Embryology Laboratory

Accreditation: CAP/ASRM

DColorado Center for Reproductive Medicine 10290 RidgeGate Cir

Lone Tree CO 80124

Telephone: (303) 788-8300; Fax: (303) 788-8310

Lab Name: Fertility Laboratories of Colorado

Accreditation: CAP/ASRM

\section{CONNECTICUT}

Connecticut Fertility Associates

4920 Main St, Suite 301

Bridgeport CT 06606

Telephone: (203) 373-1200; Fax: (203) 365-6516

Lab Name: Connecticut Fertility

Associates Laboratory

Accreditation: CAP/ASRM

The Center for Advanced Reproductive Services at the University of Connecticut Health Center

Dowling South Bldg, 263 Farmington Ave

Farmington CT 06030

Telephone: (860) 679-4580; Fax: (860) 679-3639

Lab Name: University of Connecticut Health

Center Laboratory

Accreditation: CAP/ASRM 
Yale Fertility Center

150 Sargent Dr, 2nd Floor

New Haven CT 06511

Telephone: (203) 785-4708; Fax: (203) 764-5669

Lab Name: Yale New Haven Hospital

Accreditation: CAP/ASRM, JCAHO

Reproductive Medicine Associates of Connecticut 10 Glover Ave

Norwalk CT 06850

Telephone: (203) 750-7400; Fax: (203) 846-9579

Lab Name: Reproductive Medicine Associates of

Connecticut Embryology Laboratory

Accreditation: CAP/ASRM

New England Fertility Institute

1275 Summer St, Suite 201

Stamford CT 06905

Telephone: (203) 325-3200; Fax: (203) 323-3130

Lab Name: New England Fertility Institute

ART-IVF Laboratory

Accreditation: CAP/ASRM

The Stamford Hospital

30 Shelburne Rd

Stamford CT 06904

Telephone: (203) 276-7559; Fax: (203) 276-7259

Lab Name: New England Fertility Institute

ART-IVF Laboratory

Accreditation: CAP/ASRM

Park Avenue Fertility and Reproductive Medicine 5520 Park Ave

Trumbull CT 06611

Telephone: (203) 372-6700; Fax: (203) 372-6706

Lab Name: Park Avenue Fertility and

Reproductive Medicine

Accreditation: None

\section{DELAWARE}

Reproductive Associates of Delaware

4735 Ogletown-Stanton Rd

Medical Arts Pavilion 2, Suite 3217

Newark DE 19713

Telephone: (302) 623-4242; Fax: (302) 623-4241

Lab Name: Reproductive Associates of

Delaware Laboratory

Accreditation: CAP/ASRM

\section{DISTRICT OF COLUMBIA}

The A.R.T. Institute of Washington, Inc.

Walter Reed Army Medical Center

6900 Georgia Ave N.W., Ward 43, Bldg 2, Rm 4304

Washington DC 20307

Telephone: (202) 782-6198; Fax: (202) 782-4833

Lab Name: The A.R.T. Institute of Washington, Inc.

Accreditation: CAP/ASRM, JCAHO

Columbia Fertility Associates

2440 M St N.W., Suite 401

Washington DC 20037

Telephone: (202) 293-6567; Fax: (202) 778-6190

Lab Name: Columbia Fertility Associates

IVF Center Laboratory

Accreditation: JCAHO

The George Washington University Medical

Faculty Associates

2150 Pennsylvania Ave N.W., Suite 6-300

Washington DC 20037

Telephone: (202) 741-2520; Fax: (202) 741-2518

Lab Name: Medical Faculty Associates, Inc.

Accreditation: CAP/ASRM

James A. Simon, MD, PC

1850 M St N.W., Suite 450

Washington DC 20036

Telephone: (202) 293-1000; Fax: (202) 463-6150

Lab Name: Columbia Fertility Associates

IVF Center Laboratory

Accreditation: JCAHO

\section{FLORIDA}

BocaFertility

875 Meadows Rd, Suite 334

Boca Raton FL 33486

Telephone: (561) 368-5500; Fax: (561) 368-4793

Lab Name: BocaFertility IVF Laboratory

Accreditation: CAP/ASRM

Advanced Reproductive Care Center, PA

10301 Hagen Ranch Rd, Suite 6

Boynton Beach FL 33437

Telephone: (561) 736-6006; Fax: (561) 736-5788

Lab Name: Advanced Reproductive Care Center, PA

Accreditation: JCAHO 
Florida Fertility Institute

2454 McMullen Booth Rd, Suite 601

Clearwater FL 33759

Telephone: (727) 796-7705; Fax: (727) 796-8764

Lab Name: Florida Fertility Institute

Accreditation: JCAHO

Infertility and Reproductive Medicine of

South Broward

Kenneth M. Gelman, MD

9900 Stirling Rd, Suite 300

Cooper City FL 33024

Telephone: (954) 432-2228; Fax: (954) 432-7277

Lab Name: Infertility and Reproductive Medicine of South Broward

Accreditation: CAP/ASRM (Pend)

Southwest Florida Fertility Center, PA

13685 Doctor's Way, Suite 330

Fort Myers FL 33912

Telephone: (239) 561-3430; Fax: (239) 561-6980

Lab Name: Southwest Florida Fertility Center, PA

Accreditation: CAP/ASRM

Specialists in Reproductive Medicine \& Surgery, PA

Craig R. Sweet, MD

12611 World Plaza Ln, Bldg 53

Fort Myers FL 33907

Telephone: (239) 275-81 18; Fax: (239) 275-5914

Lab Name: Specialists in Reproductive Medicine and Surgery, PA

Accreditation: JCAHO

University of Florida Women's Health at Magnolia Parke

3951 N.W. 48th Terrace, Suite 101

Gainesville FL 32606

Telephone: (352) 265-6200; Fax: (352) 265-9103

Lab Name: Shands at the University of Florida IVF and Andrology Laboratory

Accreditation: CAP/ASRM

Assisted Fertility Program of North Florida

3627 University Blvd South, Suite 450

Jacksonville FL 32216

Telephone: (904) 398-1473; Fax: (904) 399-3436

Lab Name: North Florida Reproductive Laboratory

Accreditation: CAP/ASRM (Pend)
Florida Institute for Reproductive Medicine

Baptist Medical Center Pavilion

836 Prudential Dr, Suite 902

Jacksonville FL 32207

Telephone: (904) 399-5620; Fax: (904) 399-5645

Lab Name: Florida Institute for Reproductive

Medicine IVF Laboratory

Accreditation: CAP/ASRM

Jacksonville Center for Reproductive Medicine 3627 University Blvd South, Suite 200

Jacksonville FL 32216

Telephone: (904) 493-2229; Fax: (904) 396-4546

Lab Name: North Florida Reproductive Laboratory

Accreditation: CAP/ASRM (Pend)

Gene F. Manko, MD, Inc.

600 Heritage Dr, Suite 200

Jupiter FL 33458

Telephone: (561) 354-1525; Fax: (561) 354-1526

Lab Name: South Florida Institute for Reproductive

Medicine-Jupiter Palm Beach Affiliate

Accreditation: CAP/ASRM

Center for Reproductive Medicine

18944 N. Dale Mabry Hwy

Lutz FL 33548

Telephone: (813) 386-0618; Fax: (813) 386-0622

Lab Name: Bill Clark

Accreditation: None

IVF Florida

2960 N. State Rd 7, Suite 300

Margate FL 33063

Telephone: (954) 247-6200; Fax: (954) 247-6296

Lab Name: IVF Florida Reproductive Associates

Accreditation: CAP/ASRM

Fertility \& Reproductive Medicine Center for Women

95 Bulldog Blvd, Suite 204

Melbourne FL 32901

Telephone: (321) 724-4410; Fax: (321) 956-9957

Lab Name: Fertility and Reproductive Medicine

Center for Women

Accreditation: JCAHO 
Fertility \& IVF Center of Miami, Inc.

8950 N. Kendall Dr, Suite 103

Miami FL 33176

Telephone: (305) 596-4013; Fax: (305) 596-4557

Lab Name: Fertility \& IVF Center of Miami Assisted Reproduction Laboratory

Accreditation: CAP/ASRM

Palmetto Fertility Center of South Florida

7100 W. 20th Ave, Suite 205

Miami FL 33016

Telephone: (305) 558-0808; Fax: (305) 558-0806

Lab Name: Palmetto Fertility Lab, Inc.

Accreditation: CAP/ASRM

University of Miami Infertility Center

1400 N.W. 12th Ave, Suite 5

Miami FL 33136

Telephone: (305) 243-8642; Fax: (305) 324-0363

Lab Name: University of Miami Infertility Center

Accreditation: CAP/ASRM

Center for Reproductive Medicine, PA

3435 Pinehurst Ave

Orlando FL 32804

Telephone: (407) 740-0909; Fax: (407) 740-7262

Lab Name: Center for Reproductive Medicine IVF Laboratory

Accreditation: CAP/ASRM

Frank C. Riggall, MD, PA

2501 N. Orange Ave, Suite 209S

Orlando FL 32804

Telephone: (407) 898-0254; Fax: (407) 898-6224

Lab Name: Center for Reproductive Medicine

IVF Laboratory

Accreditation: CAP/ASRM

Lab Name: Fertility C.A.R.E. Laboratory

Accreditation: CAP/ASRM

New Leaders in Infertility \& Endocrinology, LLC

4400 Bayou Blvd, Suite 36

Pensacola FL 32503

Telephone: (850) 857-3733; Fax: (850) 857-0670

Lab Name: ART Lab at New LIFE

Accreditation: CAP/ASRM (Pend)
Center for Advanced Reproductive

Endocrinology, PA

201 N. Pine Island Rd

Plantation FL 33324

Telephone: (954) 584-2273; Fax: (954) 587-9630

Lab Name: Lab for Implantation, Fertilization \&

Embryology (LIFE), LC

Accreditation: CAP/ASRM

Fertility Center and Applied Genetics of Florida, Inc. 5664 Bee Ridge Rd, Suites 103 \& 202

Sarasota FL 34233

Telephone: (941) 342-1568; Fax: (941) 342-8296

Lab Name: Fertility Center \& Applied Genetics of

Florida, Inc.

Accreditation: JCAHO

South Florida Institute for Reproductive Medicine 7300 S.W. 62nd Pl, 4th Floor

South Miami FL 33143

Telephone: (305) 662-7901; Fax: (305) 662-7910

Lab Name: South Florida Institute for

Reproductive Medicine-Miami

Accreditation: CAP/ASRM

Catherine Cowart, MD

Reproductive Health Associates, PA

2919 Swann Ave, Suite 307

Tampa FL 33609

Telephone: (813) 872-0018; Fax: (813) 876-1149

The Reproductive Medicine Group

5245 E. Fletcher Ave

Tampa FL 33617

Telephone: (813) 676-8844; Fax: (813) 676-8815

Lab Name: Reproductive Medicine Group

ART Laboratories, Inc.

Accreditation: CAP/ASRM

\section{F.I.R.S.T.}

Florida Institute for Reproductive Sciences and Technologies

2300 N. Commerce Pkwy, Suite 313

Weston FL 33326

Telephone: (954) 217-3456; Fax: (954) 217-3462

Lab Name: Florida Institute for

Reproductive Sciences \& Technologies

Accreditation: JCAHO 
Fertility Center of Assisted Reproduction

\& Endocrinology

5931 Brick Ct

Winter Park FL 32792

Telephone: (407) 672-1106; Fax: (407) 678-2790

Lab Name: Fertility C.A.R.E. Laboratory

Accreditation: CAP/ASRM

\section{GEORGIA}

Atlanta Center for Reproductive Medicine 5909 Peachtree Dunwoody Rd, Suite 720

Atlanta GA 30328

Telephone: (770) 928-2276; Fax: (770) 592-2092

Lab Name: Atlanta Center for

Reproductive Medicine

Accreditation: CAP/ASRM

Emory Reproductive Center

550 Peachtree St, Suite 1800

Atlanta GA 30308

Telephone: (404) 686-1593; Fax: (404) 686-4956

Lab Name: Emory Center for

Reproductive Medicine

Accreditation: JCAHO

Georgia Reproductive Specialists

5445 Meridian Mark Rd, Suite 270

Atlanta GA 30342

Telephone: (404) 843-2229; Fax: (404) 843-0812

Lab Name: Georgia Reproductive Specialists

Accreditation: JCAHO

Reproductive Biology Associates

1150 Lake Hearn Dr, Suite 600

Atlanta GA 30342

Telephone: (404) 843-3064; Fax: (404) 256-1528

Lab Name: Reproductive Biology Associates

IVF Laboratory

Accreditation: CAP/ASRM

Reproductive Medicine and Infertility Associates

810 Chafee St

Augusta GA 30904

Telephone: (706) 722-4434; Fax: (706) 722-9647

Lab Name: MCGH/PPG Reproductive

Laboratories, LLC

Accreditation: CAP/ASRM
Servy Institute for Reproductive Endocrinology

812 Chafee Ave

Augusta GA 30904

Telephone: (706) 724-0228; Fax: (706) 722-2387

Lab Name: MCGH/PPG Reproductive

Laboratories, LLC

Accreditation: CAP/ASRM

Columbus Center for Reproductive Endocrinology

\& Infertility, LLC

2323 Whittlesey Rd

Columbus GA 31909

Telephone: (706) 653-6344; Fax: (706) 653-8933

Lab Name: Columbus Center for Reproductive

Endocrinology \& Infertility Laboratory

Accreditation: CAP/ASRM

Central Georgia Fertility Institute

4075 Elnora Dr

Macon GA 31210

Telephone: (478) 757-7888; Fax: (478) 757-7887

Lab Name: Central Georgia Fertility Institute

Accreditation: JCAHO

Georgia Center for Reproductive Medicine

5354 Reynolds St, Suite 510

Savannah GA 31405

Telephone: (912) 352-8588; Fax: (912) 352-8893

Lab Name: Georgia Center for

Reproductive Medicine

Accreditation: None

\section{HAWAII}

Advanced Reproductive Center of Hawaii

1319 Punahou St, Suite 520

Honolulu HI 96826

Telephone: (808) 949-6611; Fax: (808) 949-6610

Lab Name: Pacific IVF Institute

Accreditation: CAP/ASRM

Pacific In Vitro Fertilization Institute

Kapi olani Medical Center for Women and Children 1319 Punahou St, Suite 980

Honolulu HI 96826

Telephone: (808) 946-2226; Fax: (808) 943-1563

Lab Name: Pacific IVF Institute

Accreditation: CAP/ASRM 
Tripler Army Medical Center IVF Institute

Department of OB/GYN

1 Jarrett White Rd

Tripler AMC HI 96859

Telephone: (808) 433-4558; Fax: (808) 433-1552

Lab Name: Pacific IVF Institute

Accreditation: CAP/ASRM

\section{IDAHO}

Idaho Center for Reproductive Medicine

111 Main St, Suite 100

Boise ID 83702

Telephone: (208) 342-5900; Fax: (208) 342-2088

Lab Name: Idaho Reproductive Labs, Inc.

Accreditation: JCAHO

\section{ILLINOIS}

Rush-Copley Center for Reproductive Health Rush-Copley Medical Center

2020 Ogden Ave, Suite 250

Aurora IL 60504

Telephone: (630) 978-6254; Fax: (630) 499-2487

Lab Name: Rush-Copley Medical Center

Accreditation: JCAHO

Life-Women's Health Center

Daniel A. Rostein, MD

6425 W. Cermark Rd, Suite 202

Berwyn IL 60402

Telephone: (708) 484-0500; Fax: (708) 484-4259

Lab Name: Chicago Fertility Laboratories, Inc.

Accreditation: JCAHO

Martin S. Balin, MD, PhD

2825 N. Halsted St

Chicago IL 60657

Telephone: (800) 241-7133; Fax: (773) 871-5221

Lab Name: Reproductive Genetics Institute IVF

Accreditation: CAP/ASRM, NYSTB (Pend)

Center for Reproductive Medicine \& Fertility

The University of Chicago

333 S. Desplaines St, Suite 201

Chicago IL 60661

Telephone: (773) 702-6642; Fax: (773) 702-5848

Lab Name: Center for Reproductive Medicine

\& Fertility

Accreditation: CAP/ASRM
O Institute for Human Reproduction (IHR)

2825 N. Halsted St

Chicago IL 60657

Telephone: (773) 472-4949; Fax: (773) 935-3691

Lab Name: Reproductive Genetics Institute IVF

Accreditation: CAP/ASRM, NYSTB (Pend)

Northwestern University

675 N. St. Clair St, Suite 14-219

Chicago IL 60611

Telephone: (312) 695-1364; Fax: (312) 695-4924

Lab Name: Northwestern Medical Faculty

Foundation, Inc. IVF \& Andrology Laboratories

Accreditation: CAP/ASRM

River North IVF-Fertility Centers of Illinois

900 N. Kingsbury, Suite RW6

Chicago IL 60610

Telephone: (312) 222-8200; Fax: (773) 385-8740

Lab Name: Fertility Centers of Illinois, River North

IVF Center

Accreditation: CAP/ASRM

University of Illinois at Chicago IVF Program

1801 W. Taylor St, Suite 4A

Chicago IL 60612

Telephone: (312) 996-9820; Fax: (312) 355-3161

Lab Name: University of Illinois at Chicago

IVF Program

Accreditation: CAP/ASRM

Women's Health Consultants

1725 W. Harrison St, Suite 408E

Chicago IL 60612

Telephone: (312) 997-2229; Fax: (312) 997-2354

Lab Name: Rush Center for Advanced Reproductive

Care Andrology Lab

Accreditation: JCAHO

Center for Reproductive Health/Joliet IVF

2246 Weber Rd

Crest Hill IL 60403

Telephone: (815) 725-4161; Fax: (815) 725-4341

Lab Name: Center for Reproductive Health/

Joliet IVF, LLC

Accreditation: CAP/ASRM

Midwest Fertility Center

4333 Main St

Downers Grove IL 60515

Telephone: (630) 810-0212; Fax: (630) 810-1027

Lab Name: Midwest Fertility Center ART Laboratory

Accreditation: CAP/ASRM 
The Rinehart Center for Reproductive Medicine 2500 Ridge Ave, Suite 200

Evanston IL 60201

Telephone: (847) 869-7777; Fax: (847) 869-7782

Lab Name: The Rinehart Center for

Reproductive Medicine

Accreditation: CAP/ASRM

The Rinehart-Coulam Center

2500 Ridge Ave, Suite 200

Evanston IL 60201

Telephone: (847) 869-7777; Fax: (847) 869-7782

Lab Name: The Rinehart Center for

Reproductive Medicine

Accreditation: CAP/ASRM

Advanced Fertility Center of Chicago

30 Tower Ct, Suite F

Gurnee IL 60031

Telephone: (847) 662-1818; Fax: (847) 662-3001

Lab Name: Advanced Fertility Center of Chicago

Accreditation: CAP/ASRM

Chicago Infertility Associates

Alexian Brother's Hospital Pavilion

1515 W. Lake St, Suite 208

Hanover Park IL 60133

Telephone: (630) 540-9317; Fax: (630) 540-2262

Lab Name: Reproductive Genetics Institute IVF

Accreditation: CAP/ASRM, NYSTB (Pend)

Highland Park IVF Center

767 Park Ave West, B400

Highland Park IL 60035

Telephone: (847) 266-3535; Fax: (847) 266-8838

Lab Name: Gamete Resources

Accreditation: JCAHO (Pend)

Hinsdale Center for Reproduction

$121 \mathrm{~N}$. Elm St

Hinsdale IL 60521

Telephone: (630) 856-3535; Fax: (630) 856-3545

Lab Name: Hinsdale Center for Reproduction

Accreditation: CAP/ASRM

Reena Jabamoni, MD, SC

1585 Barrington Rd, Suite 401

Hoffman Estates IL 60194

Telephone: (847) 843-7090; Fax: (847) 843-0584

Lab Name: Reproductive Genetics Institute

Accreditation: CAP/ASRM, NYSTB (Pend)
Karande and Associates, SC

1585 N. Barrington Rd, Suite 406

Hoffman Estates IL 60194

Telephone: (847) 884-8884; Fax: (847) 884-8093

Lab Name: Karande and Associates, SC

Accreditation: CAP/ASRM

Reproductive Health Specialists, Ltd.

744 Essington Rd

Joliet IL 60435

Telephone: (815) 730-1100; Fax: (815) 730-1066

Lab Name: Reproductive Health Specialists, Ltd.

IVF/Andrology Laboratory

Accreditation: CAP/ASRM

IVF1

636 Raymond Dr, Suite 303

Naperville IL 60563

Telephone: (630) 357-6540; Fax: (630) 357-6435

Lab Name: Reproductive Genetics Institute

Accreditation: CAP/ASRM, NYSTB (Pend)

Charles E. Miller, MD \& Associates

The Fertility Institute at Edward

Charles E. Miller, MD \& Associates

120 Osler Dr

Naperville IL 60540

Telephone: (630) 428-2229; Fax: (630) 428-0336

Lab Name: Charles E. Miller, MD \&

Associates Laboratory

Accreditation: CAP/ASRM

Oak Brook Fertility Center

2425 W. 22nd St, Suite 102

Oak Brook IL 60523

Telephone: (630) 954-0054; Fax: (630) 954-0064

Lab Name: Chicago Fertility Laboratories, Inc.

Accreditation: JCAHO

Sher Institute for Reproductive Medicine-

Central Illinois

5401 N. Knoxville Ave, Suite 110

Peoria IL 61614

Telephone: (309) 689-0411; Fax: (309) 689-0784

Lab Name: Sher Institute for Reproductive

Medicine-Central Illinois

Accreditation: CAP/ASRM (Pend) 
Reproductive Health and Fertility Center

973 Featherstone Rd, Suite 100

Rockford IL 61107

Telephone: (815) 986-3737; Fax: (815) 986-3748

Lab Name: Fertility and Reproductive

Endocrinology Specialists

Accreditation: CAP/ASRM

North Shore Fertility, SC

4250 Dempster St

Skokie IL 60076

Telephone: (847) 763-8850; Fax: (847) 763-8851

Lab Name: North Shore Fertility, SC, IVF Laboratory

Accreditation: CAP/ASRM, JCAHO, NYSTB

Reproductive Endocrinology Associates, SC

340 W. Miller St

Springfield IL 62702

Telephone: (217) 523-4700; Fax: (217) 523-9025

Lab Name: Reproductive Endocrinology Associates

Accreditation: CAP/ASRM

Seth Levrant, MD, PC

Partners in Reproductive Health

16345 S. Harlem Ave, Suite 1W

Tinley Park IL 60477

Telephone: (708) 532-7017; Fax: (708) 845-5287

Lab Name: In Vitro Lab, Seth Levrant, MD, PC

Accreditation: CAP/ASRM

\section{INDIANA}

Bonaventura Reproductive Medicine

11725 Illinois St, Suite 345

Carmel IN 46032

Telephone: (317) 814-4570; Fax: (317) 814-4571

Lab Name: Heartland Laboratories

Accreditation: CAP/ASRM

Jarrett Fertility Group

11725 Illinois St, Suite 515

Carmel IN 46032

Telephone: (317) 814-4110; Fax: (317) 814-4114

Lab Name: Heartland Laboratories

Accreditation: CAP/ASRM

Midwest Fertility Specialists

12188-A N. Meridian St, Suite 250

Carmel IN 46032

Telephone: (317) 571-1637; Fax: (317) 571-9483

Lab Name: Midwest Fertility Specialists

Accreditation: JCAHO
Advanced Reproduction Institute, LLC

Advanced Fertility Group

1222 Professional Blvd

Evansville IN 47714

Telephone: (812) 469-4920; Fax: (812) 469-4930

Lab Name: Advanced Reproduction Institute, LLC

Accreditation: JCAHO

Associated Fertility \& Gynecology, PC

7910 W. Jefferson Blvd, Suite 301

Fort Wayne IN 46804

Telephone: (260) 432-6250; Fax: (260) 436-7220

Lab Name: Associated Fertility \& Gynecology

Laboratory, PC

Accreditation: CAP/ASRM

Advanced Fertility Group

201 N. Pennsylvania Pkwy, Suite 205

Indianapolis IN 46280

Telephone: (317) 817-1300; Fax: (317) 817-1306

Lab Name: Center for Reproductive Biology of

Indiana, LLC

Accreditation: JCAHO

Advanced Fertility Group, Assisted Fertility Services 8040 Clearvista Pkwy, Suite 510

Indianapolis IN 46256

Telephone: (317) 621-2497; Fax: (317) 621-7285

Lab Name: Community Hospital East

Accreditation: JCAHO

Family Beginnings, PC

7440 N. Shadeland Ave, Suite 212

Indianapolis IN 46250

Telephone: (317) 595-3665; Fax: (317) 595-3666

Lab Name: Family Beginnings, PC

Accreditation: CAP/ASRM

Indiana University Hospital

550 N. University Blvd, Room 2440

Indianapolis IN 46202

Telephone: (317) 274-4875; Fax: (317) 278-3787

Lab Name: Center for Reproductive Biology of

Indiana, LLC

Accreditation: JCAHO

Reproductive Care of Indiana

201 Pennsylvania Pkwy, Suite 310

Indianapolis IN 46280

Telephone: (317) 817-1800; Fax: (317) 817-1810

Lab Name: Center for Reproductive Biology of Indiana, LLC

Accreditation: JCAHO 
Reproductive Endocrinology Associates

2020 W. 86th St, Suite 310

Indianapolis IN 46260

Telephone: (317) 872-1515; Fax: (317) 879-2784

Lab Name: Community Hospital East

Accreditation: JCAHO

Women's Specialty Health Centers, PC

9660 E. 146th St, Suite 300

Noblesville IN 46060

Telephone: (317) 774-1200; Fax: (317) 774-1222

Lab Name: Follas Center for Reproductive

Medicine Laboratory

Accreditation: CAP/ASRM

\section{IOWA}

Mid-lowa Fertility, PC

1371 N.W. 121 st St

Clive IA 50325

Telephone: (515) 222-3060; Fax: (515) 222-9563

Lab Name: Mid-lowa Fertility, PC, Main Laboratory

Accreditation: CAP/ASRM

University of lowa Hospitals and Clinics

Center for Advanced Reproductive Care

Department of Obstetrics and Gynecology

200 Hawkins Dr

lowa City IA 52242

Telephone: (319) 356-8483; Fax: (319) 353-6659

Lab Name: University of Iowa Hospital \& Clinics IVF

\& Reproductive Testing

Accreditation: CAP/ASRM

\section{KANSAS}

University of Kansas Medical Center

Women's Reproductive Center

KU Main Hospital

3901 Rainbow Blvd, 5th Floor

Kansas City KS 66160

Telephone: (913) 588-6272; Fax: (913) 588-6258

Lab Name: University of Kansas Medical Center

Embryology Laboratory

Accreditation: CAP/ASRM

Midwest Reproductive Center, PA

20375 W. 151 st St, Bldg 1, Suite 403

Olathe KS 66061

Telephone: (913) 780-4300; Fax: (913) 780-4250

Lab Name: Midwest Reproductive Center

Accreditation: None
Reproductive Resource Center of Greater

Kansas City

12200 W. 106th St, Suite 120

Overland Park KS 66215

Telephone: (913) 894-2323; Fax: (913) 894-0841

Lab Name: Reproductive Resource Center

IVF Laboratory

Accreditation: CAP/ASRM

Reproductive Medicine \& Infertility

Shawnee Mission Medical Center

8800 W. 75th St, Suite 101

Shawnee Mission KS 66204

Telephone: (913) 432-7161; Fax: (913) 432-6158

Lab Name: Shawnee Mission Medical Center,

Reproductive Medicine \& Infertility

Accreditation: CAP/ASRM

The Center for Reproductive Medicine

9300 E. 29th St North, Suite 102

Wichita KS 67226

Telephone: (316) 687-2112; Fax: (316) 687-1260

Lab Name: The Center for Reproductive Medicine

CRM Laboratories

Accreditation: CAP/ASRM

\section{KENTUCKY}

Bluegrass Fertility Center

1760 Nicholasville Rd, Suite 501

Lexington KY 40503

Telephone: (859) 260-1515; Fax: (859) 260-1425

Lab Name: Bluegrass Fertility Center Laboratory

Accreditation: CAP/ASRM

Fertility and Endocrine Associates

Fertility and Endocrine Associates

Louisville Reproductive Center

4121 Dutchman's Ln, Suites 414 and 416

Louisville KY 40207

Telephone: (502) 897-2144; Fax: (502) 897-1773

Lab Name: Louisville Reproductive Center

Accreditation: None

University OB/GYN Associates Fertility Center

315 E. Broadway, Suite 1105

Louisville KY 40202

Telephone: (502) 271-5999; Fax: (502) 271-5984

Lab Name: University OB/GYN Associates, PSC

Accreditation: CAP/ASRM (Pend), JCAHO 


\section{LOUISIANA}

A Woman's Center for Reproductive Medicine 9000 Airline Hwy, Suite 670

Baton Rouge LA 70815

Telephone: (225) 926-6886; Fax: (225) 922-3730

Lab Name: A Woman's Center for

Reproductive Medicine

Accreditation: CAP/ASRM

\$Ochsner Foundation Fertility Clinic

1221 S. Clearview Pkwy, Bldg A, 1st Floor

Jefferson LA 70121

Telephone: (504) 842-4584; Fax: (504) 842-4156

Contact the NASS Help Desk for current clinic information.

Fertility and Women's Health Center of Louisiana 4630 Ambassador Caffery Pkwy, Suite 206

Lafayette LA 70508

Telephone: (337) 989-8795; Fax: (337) 989-9728

Lab Name: Fertility and Women's Health Center of Louisiana

Accreditation: CAP/ASRM (Pend), JCAHO

The Fertility Institute of New Orleans

800 N. Causeway Blvd, Suite 2C

Mandeville LA 70448

Telephone: (985) 892-7621; Fax: (985) 892-9245

Lab Name: Lakeside Hospital Fertility Institute of New Orleans

Accreditation: CAP/ASRM

Center for Fertility and Reproductive Health

2401 Greenwood Rd

Shreveport LA 71103

Telephone: (318) 212-8270; Fax: (318) 212-8230

Lab Name: Willis-Knighton Health Center Fertility \&

Reproductive Health Laboratory

Accreditation: CAP/ASRM

\section{MAINE}

Maine Center for Reproductive Health

778 Main St, Suite 2

South Portland ME 04106

Telephone: (207) 775-1255; Fax: (207) 775-1299

Lab Name: Maine Center for Reproductive Health

Accreditation: CAP/ASRM (Pend)

\section{MARYLAND}

The Center for Assisted Reproductive Technology at Union Memorial

201 E. University Pkwy, 33rd St Bldg, Suite 474

Baltimore MD 21218

Telephone: (410) 554-2271; Fax: (410) 554-2091

Lab Name: Union Memorial Hospital Center for ART

Accreditation: CAP/ASRM

\$GBMC Fertility Center

6569 N. Charles St, Suite 406

Baltimore MD 21204

Telephone: (443) 849-2484; Fax: (443) 849-3067

Contact the NASS Help Desk for current clinic information.

UMMS-Center for Advanced Reproductive Technologies

405 W. Redwood St, 3rd Floor

Baltimore MD 21201

Telephone: (410) 328-2304; Fax: (410) 328-8389

Lab Name: UMMS-Center for Advanced

Reproductive Technologies

Accreditation: CAP/ASRM

Johns Hopkins Fertility Center

10753 Falls Rd, Suite 335

Lutherville MD 21093

Telephone: (410) 847-3650; Fax: (410) 583-2792

Lab Name: The Johns Hopkins at

Greenspring Station

Accreditation: JCAHO

Center for Reproductive Medicine

9711 Medical Center Dr, Suite 214

Rockville MD 20850

Telephone: (301) 424-1904; Fax: (301) 424-1902

Lab Name: Medical Faculty Associates, Inc.

Accreditation: CAP/ASRM

Shady Grove Fertility Reproductive Science Center 15001 Shady Grove Rd, Suite 400

Rockville MD 20850

Telephone: (301) 340-1188; Fax: (301) 340-1612

Lab Name: Shady Grove Fertility Reproductive

Science Center

Accreditation: JCAHO 
Fertility Center of Maryland

110 West Rd, Suite 102

Towson MD 21204

Telephone: (410) 296-6400; Fax: (410) 296-6405

Lab Name: Fertility Center of Maryland, Inc.

Accreditation: JCAHO

\section{MASSACHUSETTS}

Brigham and Women's Hospital ART Center

Brigham and Women's Hospital

75 Francis St

Boston MA 02115

Telephone: (617) 732-5570; Fax: (617) 975-0825

Lab Name: Brigham and Women's Hospital Center for Assisted Reproduction

Accreditation: CAP/ASRM, JCAHO

Vincent IVF Unit

Massachusetts General Hospital

55 Fruit St, Yawkey 10A

Boston MA 02114

Telephone: (617) 726-6942; Fax: (617) 724-8882

Lab Name: Massachusetts General Hospital,

Vincent IVF Unit

Accreditation: CAP/ASRM, JCAHO

§Reproductive Science Center

1 Forbes Rd

Lexington MA 02421

Telephone: (781) 674-1200; Fax: (781) 674-2442

Contact the NASS Help Desk for current clinic information.

Fertility Centers of New England, Inc.

New England Clinics of Reproductive Medicine, Inc.

20 Pond Meadow Dr, Suite 207

Reading MA 01867

Telephone: (781) 942-7000; Fax: (781) 942-7200

Lab Name: New England Clinic of Reproductive

Medicine, Inc.

Accreditation: CAP/ASRM

Lab Name: Portsmouth Regional Hospital

Pathology Laboratory

Accreditation: CAP/ASRM
Baystate Reproductive Medicine

Chestnut Surgical Center

759 Chestnut St

Springfield MA 01199

Telephone: (413) 794-1950; Fax: (413) 794-1857

Lab Name: Baystate Medical Center

Reproductive Biology Lab

Accreditation: CAP/ASRM

Boston IVF

130 Second Ave

Waltham MA 02451

Telephone: (781) 434-6400; Fax: (781) 434-6464

Lab Name: Boston IVF, Inc.

Accreditation: CAP/ASRM

\section{MICHIGAN}

Center for Reproductive Medicine

University of Michigan Reproductive Endocrinology and Infertility

475 Market PI, Suite B

Ann Arbor MI 48108

Telephone: (734) 763-4323; Fax: (734) 763-7682

Lab Name: University of Michigan ART Laboratories

Accreditation: CAP/ASRM

Center for Reproductive Medicine and Surgery, PC 300 Park St, Suite 460

Birmingham MI 48009

Telephone: (248) 593-6990; Fax: (248) 593-5925

Lab Name: SMART Labs

Accreditation: CAP/ASRM

\$Center for Reproductive Medicine

Oakwood Hospital and Medical Center

Michigan Comprehensive Fertility Center

18181 Oakwood Blvd, Suite 109

Dearborn MI 48124

Telephone: (313) 299-6635; Fax: (313) 299-6658

Contact the NASS Help Desk for current

clinic information.

Grand Rapids Fertility \& IVF, PC

1900 Wealthy St S.E., Suite 315

Grand Rapids MI 49506

Telephone: (616) 774-2030; Fax: (616) 774-2053

Lab Name: Grand Rapids Fertility \& IVF, PC

Accreditation: CAP/ASRM 
Michigan Reproductive \& IVF Center, PC

630 Kenmoor Ave, Suite 100

Grand Rapids MI 49546

Telephone: (616) 988-2229; Fax: (616) 988-2009

Lab Name: Michigan Reproductive \& IVF Center, PC

Accreditation: JCAHO

Infertility and Gynecology Center of Lansing, PC

1200 E. Michigan Ave, Suite 305

Lansing MI 48912

Telephone: (517) 484-4900; Fax: (517) 339-7553

Lab Name: Sparrow Hospital, Sparrow

Fertility Services

Accreditation: CAP/ASRM

Michigan State University

Center for Assisted Reproductive Technology

1200 E. Michigan Ave, Suite 700

Lansing MI 48912

Telephone: (517) 364-5888; Fax: (571) 364-5889

Lab Name: Sparrow Hospital, Sparrow

Fertility Services

Accreditation: CAP/ASRM

IVF Michigan

3950 S. Rochester Rd, Suite 2300

Rochester Hills MI 48307

Telephone: (248) 844-8845; Fax: (248) 844-9039

Lab Name: IVF Michigan IVF/Andrology Laboratory

Accreditation: CAP/ASRM

University Women's Care/Wayne State University

26400 W. 12 Mile Rd, Suite 140

Southfield MI 48034

Telephone: (248) 352-8200; Fax: (248) 356-8255

Lab Name: University OBGYN, Inc. Assisted

Reproductive Technology Laboratories

Accreditation: CAP/ASRM

Henry Ford Reproductive Medicine

1500 W. Big Beaver, Suite 105

Troy MI 48084

Telephone: (248) 637-4050; Fax: (248) 637-0115

Lab Name: Henry Ford Health System, Henry Ford

Reproductive Medicine

Accreditation: CAP/ASRM

Brenda L. Moskovitz, MD, PC

415 E. Maple Rd, Suite 101

Troy MI 48083

Telephone: (248) 524-1001; Fax: (248) 851-6522

Lab Name: SMART Labs

Accreditation: CAP/ASRM
Michigan Center for Fertility and

Women's Health, PLC

4700 Thirteen Mile Rd

Warren MI 48092

Telephone: (586) 576-0431; Fax: (586) 576-0924

Lab Name: Michigan Center for Fertility and

Women's Health

Accreditation: None

\section{MINNESOTA}

The Midwest Center for Reproductive Health, PA

Arbor Lakes Medical Bldg

12000 Elm Creek Blvd North, Suite 350

Maple Grove MN 55369

Telephone: (763) 494-7700; Fax: (763) 494-7706

Lab Name: Midwest Center for Reproductive Health

Assisted Reproductive Technology

Accreditation: CAP/ASRM

Center for Reproductive Medicine

Advanced Reproductive Technologies

2800 Chicago Ave South, Suite 300

Minneapolis MN 55407

Telephone: (612) 863-5390; Fax: (612) 863-2697

Lab Name: Center for Reproductive Medicine

Embryology Laboratory

Accreditation: CAP/ASRM

Reproductive Medicine Center

606 24th Ave South, Suite 500

Minneapolis MN 55454

Telephone: (612) 372-7037; Fax: (612) 372-7022

Lab Name: University of Minnesota Physicians

Reproductive Medicine Center

Accreditation: CAP/ASRM

Mayo Clinic Assisted Reproductive Technologies

200 First St S.W., Charlton Bldg, Desk 3A

Rochester MN 55905

Telephone: (507) 284-4520; Fax: (507) 284-1774

Lab Name: Mayo Clinic Fertility Testing

and IVF Laboratories

Accreditation: CAP/ASRM

Reproductive Medicine \& Infertility Associates

Woodbury Medical ARTS BIdg

2101 Woodwinds Dr, Suite 100

Woodbury MN 55125

Telephone: (651) 222-6050; Fax: (651) 222-5975

Lab Name: Reproductive Medicine \& Infertility

Associates Reproductive Biology Laboratory

Accreditation: CAP/ASRM 


\section{MISSISSIPPI}

Mississippi Fertility Institute

501 Marshall St, Suite 600

Jackson MS 39202

Telephone: (601) 948-3874; Fax: (601) 948-6544

Lab Name: Women's Specialty Center, Mississippi Fertility Institute

Accreditation: JCAHO

University of Mississippi Medical Center

Department of Ob/Gyn, Division of Reproductive Endocrinology and Fertility

2500 N. State St

Jackson MS 39216

Telephone: (601) 984-5330; Fax: (601) 984-5965

Lab Name: University of Mississippi

Medical Center, OB-GYN Department,

IVF \& Andrology Laboratory

Accreditation: CAP/ASRM

\section{MISSOURI}

Infertility Institute

The Fertility Center at Missouri Baptist Medical Center

226 S. Woods Mill Rd, Suite 39 West

Chesterfield MO 63017

Telephone: (314) 205-8809; Fax: (314) 205-8776

Lab Name: The Fertility Center at Missouri Baptist Medical Center

Accreditation: CAP/ASRM

Mid-Missouri Reproductive Medicine and

Surgery, Inc.

1502 E. Broadway, Suite 106

Columbia MO 65201

Telephone: (573) 443-4511; Fax: (573) 443-7860

Lab Name: Mid-Missouri Reproductive Medicine and Surgery, Inc., Laboratory

Accreditation: CAP/ASRM

§University of Missouri Hospital and Clinic

IVF Embryology Laboratory

Department of Obstetrics, Gynecology and Women's Health

1 Hospital Dr

Columbia MO 65212

Telephone: (573) 882-1725; Fax: (573) 882-9010

Contact the NASS Help Desk for current clinic information.
Midwest Women's Healthcare

6400 Prospect, Suite 598

Kansas City MO 64132

Telephone: (816) 444-6888; Fax: (816) 444-8430

Lab Name: Research Medical Center IVF Laboratory

Accreditation: CAP/ASRM

Infertility \& IVF Center

3009 N. Ballas Rd, Suite 359C

St. Louis MO 63131

Telephone: (314) 872-9200; Fax: (314) 872-9040

Lab Name: Infertility \& Gynecologic Medicine, Inc., Infertility \& IVF Center

Accreditation: CAP/ASRM

The Infertility and Reproductive Medicine Center at Washington University School of Medicine and Barnes-Jewish Hospital

Barnes-Jewish Hospital, North Campus

4444 Forest Park Ave, Suite 3100

St. Louis MO 63108

Telephone: (314) 286-2400; Fax: (314) 286-2455

Lab Name: Barnes-Jewish Hospital Infertility \&

Reproductive Medicine

Accreditation: CAP/ASRM, JCAHO

Infertility Center of St. Louis

St. Luke's Hospital

224 S. Woods Mill Rd, Suite 730

St. Louis MO 63017

Telephone: (314) 576-1400; Fax: (314) 576-1442

Lab Name: St. Luke's Hospital Assisted

Reproductive Technology Laboratory

Accreditation: CAP/ASRM

Sher Institute for Reproductive Medicine-St. Louis 456 N. New Ballas Rd, Suite 101

St. Louis MO 63141

Telephone: (314) 983-9000; Fax: (314) 983-9023

Lab Name: Sher Institute for

Reproductive Medicine-St. Louis

Accreditation: CAP/ASRM

\section{NEBRASKA}

Heartland Center for Reproductive Medicine, PC 7308 S. 142 nd St

Omaha NE 68138

Telephone: (402) 717-4200; Fax: (402) 717-4230

Lab Name: Heartland Center for Reproductive

Medicine, PC

Accreditation: CAP/ASRM 
Nebraska Methodist Hospital REI

8111 Dodge St, Suite 237

Omaha NE 68114

Telephone: (402) 354-5210; Fax: (402) 354-5221

Lab Name: Nebraska Methodist Hospital

Andrology/Embryology Laboratory

Accreditation: CAP/ASRM, JCAHO

\section{NEVADA}

Fertility Center of Las Vegas

8851 W. Sahara Ave, Suite 100

Las Vegas NV 89117

Telephone: (702) 254-1777; Fax: (702) 254-1213

Lab Name: Fertility Center of Las Vegas Laboratory

Accreditation: CAP/ASRM

Nevada Fertility C.A.R.E.S.

653 Town Center Dr, Suite 206

Las Vegas NV 89144

Telephone: (702) 341-6616; Fax: (702) 341-6617

Lab Name: Nevada Fertility C.A.R.E.S.

Accreditation: CAP/ASRM

Sher Institute for Reproductive Medicine-Las Vegas

3121 S. Maryland Pkwy, Suite 300

Las Vegas NV 89109

Telephone: (702) 892-9696; Fax: (702) 892-9666

Lab Name: Sher Institute for

Reproductive Medicine-Las Vegas

Accreditation: CAP/ASRM

The Nevada Center for Reproductive Medicine

645 Sierra Rose Dr, Suite 205

Reno NV 89511

Telephone: (775) 828-1200; Fax: (775) 828-1785

Lab Name: Nevada Center for

Reproductive Medicine

Accreditation: JCAHO

\section{NEW HAMPSHIRE}

Dartmouth-Hitchcock Medical Center

1 Medical Center Dr

Lebanon $\mathrm{NH} 03756$

Telephone: (603) 653-9240; Fax: (603) 650-0905

Lab Name: Dartmouth-Hitchcock Medical Center

Mary Hitchcock Reproductive

Sciences Laboratory

Accreditation: CAP/ASRM

\section{NEW JERSEY}

Sher Institute for Reproductive Medicine-

New Jersey

One Robertson Dr

Bedminster NJ 07921

Telephone: (908) 781-0666; Fax: (908) 781-6377

Lab Name: Sher Institute for Reproductive

Medicine-New Jersey

Accreditation: CAP/ASRM

IVF of North Jersey

North Jersey Center for Reproductive Endocrinology and Infertility

Tower Fertility Center

1035 Route 46 East

Clifton NJ 07013

Telephone: (973) 470-0303; Fax: (973) 916-0488

Lab Name: IVF of North Jersey, PA Laboratory

Accreditation: CAP/ASRM

Center for Advanced Reproductive Medicine

\& Fertility

Four Ethel Rd, Suite 405A

Edison NJ 08817

Telephone: (732) 339-9300; Fax: (732) 339-9400

Lab Name: Center for Advanced Reproductive

Medicine \& Fertility

Accreditation: JCAHO

Women's Fertility Center

106 Grand Ave

Englewood NJ 07631

Telephone: (201) 569-6979; Fax: (201) 569-0269

Lab Name: Fertility Institute of Northern New Jersey

IVF Laboratory

Accreditation: CAP/ASRM

North Hudson I.V.F.

Center for Fertility and Gynecology

385 Sylvan Ave

Englewood Cliffs NJ 07632

Telephone: (201) 871-1999; Fax: (201) 871-1031

Lab Name: North Hudson IVF Center

Accreditation: CAP/ASRM

University Reproductive Associates, PC

214 Terrace Ave

Hasbrouck Heights NJ 07604

Telephone: (201) 288-6330; Fax: (201) 288-6331

Lab Name: University Reproductive Associates, PC,

Center for Reproductive Medicine

Accreditation: CAP/ASRM 
Shore Institute for Reproductive Medicine

475 Route 70, Suite 201

Lakewood NJ 08701

Telephone: (732) 363-4777; Fax: (732) 363-2004

Lab Name: Shore Area IVF Laboratories, PC

Accreditation: CAP/ASRM

Delaware Valley OBGYN and Infertility Group

Princeton IVF

2 Princess Rd, Suite C

Lawrenceville NJ 08648

Telephone: (609) 896-0777; Fax: (609) 896-3266

Lab Name: Delaware Valley OBGYN and Infertility

Group, Princeton IVF

Accreditation: CAP/ASRM

Princeton Center for Infertility \&

Reproductive Medicine

3131 Princeton Pike, Bldg 4, Suite 204

Lawrenceville NJ 08648

Telephone: (609) 895-1114; Fax: (609) 895-1 196

Lab Name: Cooper Institute for Reproductive

Hormonal Disorders

Accreditation: CAP/ASRM

East Coast Infertility and IVF

200 White Rd, Suite 214

Little Silver NJ 07739

Telephone: (732) 758-6511; Fax: (732) 758-1048

Lab Name: East Coast Infertility and IVF

Accreditation: CAP/ASRM

Institute for Reproductive Medicine and Science

Saint Barnabas Medical Center, East Wing

94 Old Short Hills Rd, Suite 403

Livingston NJ 07039

Telephone: (973) 322-8286; Fax: (973) 322-8890

Lab Name: Institute for Reproductive Medicine and

Science at Saint Barnabas Medical Center

Accreditation: CAP/ASRM

Cooper Institute for Reproductive

Hormonal Disorders

8002E Greentree Commons

Marlton NJ 08053

Telephone: (856) 751-5575; Fax: (856) 751-7289

Lab Name: Cooper Institute for Reproductive

Hormonal Disorders

Accreditation: CAP/ASRM
Delaware Valley Institute of Fertility and Genetics 6000 Sagemore Dr, Suite 6102

Marlton NJ 08053

Telephone: (856) 988-0072; Fax: (856) 988-0056

Lab Name: Delaware Valley Institute of Fertility \&

Genetics Reproductive Laboratories

Accreditation: CAP/ASRM

South Jersey Fertility Center

400 Lippincott Dr, Suite 130

Marlton NJ 08053

Telephone: (856) 596-2233; Fax: (856) 596-2411

Lab Name: South Jersey Fertility Center

Accreditation: JCAHO

Diamond Institute for Infertility

89 Millburn Ave

Millburn NJ 07041

Telephone: (973) 761-5600; Fax: (973) 761-5100

Lab Name: Diamond Institute for Infertility IVF

Laboratory

Accreditation: CAP/ASRM

Reproductive Medicine Associates of New Jersey 111 Madison Ave, Suite 100

Morristown NJ 07962

Telephone: (973) 971-4600; Fax: (973) 290-8370

Lab Name: Reproductive Medicine Associates of

New Jersey Embryology Laboratory

Accreditation: CAP/ASRM

Valley Hospital Fertility Center

1 Valley Health Plaza

Paramus NJ 07652

Telephone: (201) 634-5400; Fax: (201) 634-5506

Lab Name: Valley Hospital Fertility Center

Accreditation: CAP/ASRM

IVF New Jersey

81 Veronica Ave

Somerset NJ 08873

Telephone: (732) 220-9060; Fax: (732) 545-1164

Lab Name: IVF New Jersey Embryology Laboratory

Accreditation: CAP/ASRM

Reproductive Science Center of New Jersey

4000 Route 66, Suite 125

Tinton Falls NJ 07753

Telephone: (732) 918-2500; Fax: (732) 918-2504

Lab Name: Reproductive Science Center of

New Jersey

Accreditation: CAP/ASRM (Pend) 
Dr. Louis R. Manara

Dr. Louis R. Manara

The Center for Reproductive Medicine \& Fertility

200A Route 73

Voorhees NJ 08043

Telephone: (856) 767-0009; Fax: (856) 767-0990

Lab Name: Center for Reproductive Medicine and Fertility

Accreditation: CAP/ASRM (Pend)

North Jersey Fertility Associates, LLC

57 Willowbrooks Blvd, Suite 301

Wayne NJ 07470

Telephone: (973) 754-4055; Fax: (973) 754-4058

Lab Name: North Jersey Fertility Associates,

LLC, Laboratory

Accreditation: CAP/ASRM

Fertility Institute of New Jersey and New York

400 Old Hook Rd, Suite 2-3

Westwood NJ 07675

Telephone: (201) 666-4200; Fax: (201) 666-2262

Lab Name: Fertility Institute of Northern New Jersey

IVF Laboratory

Accreditation: CAP/ASRM

\section{NEW MEXICO}

Center for Reproductive Medicine of New Mexico

Presbyterian Professional Bldg

201 Cedar St S.E., Suite S1-20

Albuquerque NM 87106

Telephone: (505) 247-3333; Fax: (505) 224-7476

Lab Name: Center for Reproductive Medicine of

New Mexico In Vitro Fertilization and Andrology Accreditation: CAP/ASRM

\section{NEW YORK}

Albany IVF, Fertility and Gynecology

349 Northern Blvd

Albany NY 12204

Telephone: (518) 434-9759; Fax: (518) 436-9822

Lab Name: Albany IVF Fertility \& Gynecology

Accreditation: NYSTB

The Fertility Institute at New York

Methodist Hospital

506 Sixth St

Brooklyn NY 11215

Telephone: (718) 780-5065; Fax: (718) 780-5085

Lab Name: The Fertility Institute at New York

Methodist Hospital

Accreditation: NYSTB
Genesis Fertility \& Reproductive Medicine

1355 84th St

Brooklyn NY 11228

Telephone: (718) 283-8600; Fax: (718) 283-6580

Lab Name: Brooklyn IVF Maimonides

Medical Center

Accreditation: NYSTB

Infertility \& IVF Medical Associates of Western

New York

4510 Main St

Buffalo NY 14226

Telephone: (716) 839-3057; Fax: (716) 839-1477

Lab Name: Infertility and IVF Medical Associates

of Western New York

Accreditation: NYSTB

Division of Reproductive Endocrinology

SUNY Stony Brook

University Physicians at Stony Brook

6 Technology Dr

East Setauket NY 11733

Telephone: (631) 444-5174; Fax: (631) 444-5175

Lab Name: John T. Mather Memorial Hospital

IVF Laboratory

Accreditation: CAP/ASRM, NYSTB

The New York Fertility Center

42-31 Colden St, Suite 202/208

Flushing NY 11355

Telephone: (718) 261-9068; Fax: (718) 261-9067

Lab Name: New Hope Fertility Center

Accreditation: NYSTB

Montefiore's Institute for Reproductive Medicine and Health

141 S. Central Ave, Suite 201

Hartsdale NY 10530

Telephone: (914) 997-1060; Fax: (914) 997-1099

Lab Name: Institute for Reproductive Medicine and

Health of Montefiore Medical Center

Accreditation: CAP/ASRM, JCAHO, NYSTB

North Shore University Hospital

Center for Human Reproduction

300 Community Dr

Manhasset NY 11030

Telephone: (516) 562-2229; Fax: (516) 562-1710

Lab Name: North Shore University Hospital Center

for Human Reproduction

Accreditation: CAP/ASRM, JCAHO, NYSTB 
Deproductive Specialists of New York 200 Old Country Rd, Suite 330

Mineola NY 11501

Telephone: (516) 739-2100; Fax: (516) 739-2179

Lab Name: Reproductive Specialists of New York

Accreditation: NYSTB

Advanced Fertility Services

1625 Third Ave

New York NY 10128

Telephone: (212) 369-8700; Fax: (212) 722-5587

Lab Name: Advanced Fertility Services, PC

Accreditation: NYSTB

American Fertility Services, PC

115 E. 57th St, Suite 500

New York NY 10022

Telephone: (212) 750-3330; Fax: (212) 750-3334

Lab Name: American Fertility Services, PC

Accreditation: CAP/ASRM (Pend), NYSTB

Beth Israel Center for Infertility \&

Reproductive Health

10 Union Square East, Suite 2E

New York NY 10003

Telephone: (212) 844-8587; Fax: (212) 844-6184

Lab Name: New York Medical Services for

Reproductive Medicine

Accreditation: NYSTB

Brooklyn/Westside Fertility Center

Brooklyn Fertility Center

55 Central Park West, Suite 1C

New York NY 10023

Telephone: (212) 721-4545; Fax: (212) 721-4598

Lab Name: Brooklyn Fertility Center

Accreditation: NYSTB

Columbia University Center for Women's

Reproductive Care

1790 Broadway, 2nd Floor

New York NY 10019

Telephone: (646) 756-3874; Fax: (646) 756-8283

Lab Name: Center for Women's Reproductive Care

Accreditation: NYSTB

IVF New York

230 Central Park South

New York NY 10019

Telephone: (212) 582-4094; Fax: (212) 246-3430

Lab Name: IVF New York

Accreditation: NYSTB
Manhattan Reproductive Medicine

159 E. 74th St, Suite 1C

New York NY 10021

Telephone: (212) 794-0080; Fax: (212) 794-0066

Lab Name: Manhattan Reproductive Medicine, PC

Accreditation: NYSTB

Medical Offices for Human Reproduction

Center for Human Reproduction (CHR)

21 E. 69th St

New York NY 10021

Telephone: (212) 994-4400; Fax: (212) 994-4499

Lab Name: Medical Offices for

Human Reproduction-New York

Accreditation: NYSTB

New Hope Fertility Center

784 Park Ave

New York NY 10021

Telephone: (212) 517-7676; Fax: (212) 396-0600

Lab Name: New Hope Fertility Center

Accreditation: NYSTB

New York Fertility Institute

1016 5th Ave

New York NY 10028

Telephone: (212) 734-5555; Fax: (212) 734-6059

Lab Name: New York Fertility Institute

Reproductive Laboratory

Accreditation: CAP/ASRM, NYSTB

O NYU Fertility Center

New York University School of Medicine

660 First Ave, 5th Floor

New York NY 10016

Telephone: (212) 263-8990; Fax: (212) 263-7853

Lab Name: NYU Fertility Center

Accreditation: NYSTB

Offices for Fertility and Reproductive Medicine

51 E. 67th St

New York NY 10021

Telephone: (212) 535-5350; Fax: (212) 535-5080

Lab Name: Offices for Fertility and

Reproductive Medicine, PC

Accreditation: NYSTB

Reproductive Care of NY

330 W. 58th St, Suite 613

New York NY 10019

Telephone: (212) 247-3111; Fax: (212) 247-3255

Lab Name: IVF New York

Accreditation: NYSTB 
Reproductive Endocrinology Associates of

St. Luke's Roosevelt Hospital Center

425 W. 59th St, Suite 5A

New York NY 10019

Telephone: (212) 523-7751; Fax: (212) 523-8348

Lab Name: Continuum Reproductive Center

Accreditation: NYSTB

Reproductive Medicine Associates of

New York, LLP

635 Madison Ave, 10th Floor

New York NY 10022

Telephone: (212) 756-5777; Fax: (212) 756-5770

Lab Name: Reproductive Medicine Associates

of New York, LLP

Accreditation: NYSTB

Sher Institutes for Reproductive Medicine-NYC

425 5th Ave

New York NY 10016

Telephone: (646) 792-7476; Fax: (646) 274-0600

Lab Name: Sher Institute for

Reproductive Medicine-NYC

Accreditation: NYSTB

O Weill Medical College of Cornell University

The Center for Reproductive Medicine and Infertility 1305 York Ave, 6th Floor

New York NY 10021

Telephone: (646) 962-2764; Fax: (646) 962-0359

Lab Name: Weill Medical College of

Cornell University Infertility Laboratory

Accreditation: NYSTB

East Coast Fertility

1074 Old Country Rd

Plainview NY 11803

Telephone: (516) 939-2229; Fax: (516) 939-2252

Lab Name: East Coast Fertility

Accreditation: CAP/ASRM (Pend), NYSTB

Long Island IVF

625 Belle Terre Rd, Suite 200

Port Jefferson NY 11777

Telephone: (631) 331-7575; Fax: (631) 331-1332

Lab Name: John T. Mather Memorial Hospital

IVF Laboratory

Accreditation: CAP/ASRM, NYSTB
Rochester Fertility Care, PC

1561 Long Pond Rd, Suite 410

Rochester NY 14626

Telephone: (585) 453-7760; Fax: (585) 453-7771

Lab Name: Strong Fertility and

Reproductive Science Center

Accreditation: NYSTB

\$Strong Fertility and Reproductive Science Center 500 Red Creek Dr, Suite 220

Rochester NY 14623

Telephone: (585) 487-3378; Fax: (585) 334-8998

Contact the NASS Help Desk for current

clinic information.

Island Reproductive Services

1110 South Ave, Suite 305

Staten Island NY 10314

Telephone: (718) 761-6000; Fax: (718) 761-6066

Lab Name: North Shore University Hospital Center for Human Reproduction

Accreditation: CAP/ASRM, JCAHO, NYSTB

Lab Name: Brooklyn IVF Maimonides

Medical Center

Accreditation: NYSTB

Gold Coast IVF

Reproductive Medicine and Surgery Center

243 Jericho Turnpike

Syosset NY 11791

Telephone: (516) 682-8900; Fax: (516) 682-8901

Lab Name: North Shore University Hospital Center for Human Reproduction

Accreditation: CAP/ASRM, JCAHO, NYSTB

CNY Fertility Center

195 Intrepid Ln

Syracuse NY 13205

Telephone: (315) 469-8700; Fax: (315) 469-6789

Lab Name: CNY Fertility Center-Latham

Accreditation: NYSTB

Lab Name: CNY Fertility Center-Syracuse

Accreditation: NYSTB

Westchester Fertility and

Reproductive Endocrinology

136 S. Broadway, Suite 100

White Plains NY 10605

Telephone: (914) 949-6677; Fax: (914) 949-5758

Lab Name: Westchester IVF

Accreditation: NYSTB 


\section{NORTH CAROLINA}

North Carolina Center for Reproductive Medicine

The Talbert Fertility Institute

400 Ashville Ave, Suite 200

Cary NC 27511

Telephone: (919) 233-1680; Fax: (919) 233-1685

Lab Name: North Carolina Center for Reproductive Medicine, North Carolina Reproductive Laboratories

Accreditation: CAP/ASRM

University of North Carolina A.R.T. Clinic

UNC School of Medicine/ CB\#7570

Department of OB/GYN

Chapel Hill NC 27599

Telephone: (919) 966-1150; Fax: (919) 966-1259

Lab Name: UNC Hospitals Reproductive

Endocrinology \& Fertility

Accreditation: CAP/ASRM, JCAHO

Institute for Assisted Reproduction

1524 E. Morehead St

Charlotte NC 28207

Telephone: (704) 343-3400; Fax: (704) 343-3428

Lab Name: Reproductive Endocrinology Associates of Charlotte, Institute for Assisted Reproduction

Accreditation: CAP/ASRM

Program for Assisted Reproduction, Carolinas Medical Center

Carolinas Medical Center Women's Institute

1025 Morehead Medical Dr, Suite 500

Charlotte NC 28204

Telephone: (704) 355-3153; Fax: (704) 355-1941

Lab Name: Carolinas Medical Center Andrology and ART Laboratories

Accreditation: CAP/ASRM

§Duke Fertility Center

Duke University Medical Center

5704 Fayetteville Rd

Durham NC 27713

Telephone: (919) 572-4673; Fax: (919) 484-0682

Contact the NASS Help Desk for current

clinic information.
East Carolina University

ECU Women's Physicians, 2160 Herbert Ct

Greenville NC 27834

Telephone: (252) 744-3849; Fax: (252) 744-2016

Lab Name: ECU Women's Physicians Assisted

Reproductive Technology Laboratory

Accreditation: CAP/ASRM

Affordable IVF

9800 W. Kincey Ave, Suite 160

Huntersville NC 28078

Telephone: (704) 947-9000; Fax: (704) 992-1900

Lab Name: Affordable IVF

Accreditation: CAP/ASRM

Carolina Conceptions

2601 Lake Dr, Suite 301

Raleigh NC 27607

Telephone: (919) 782-5911; Fax: (919) 861-6439

Lab Name: Carolina Conceptions Laboratory

Accreditation: CAP/ASRM

Wake Forest University Center for Reproductive

Medicine

CompRehab Plaza, 131 Miller St, 2nd Floor

Winston-Salem NC 27103

Telephone: (336) 716-6476; Fax: (336) 716-0194

Lab Name: Wake Forest University Reproduction

Medicine Laboratory

Accreditation: CAP/ASRM (Pend)

\section{NORTH DAKOTA}

MeritCare Reproductive Medicine

1111 Harwood Dr South, Suite 743

Fargo ND 58122

Telephone: (701) 234-2700; Fax: (701) 234-2783

Lab Name: MeritCare Medical Group

Reproductive Medicine Laboratory

Accreditation: CAP/ASRM

\section{OHIO}

Fertility Unlimited, Inc.

Northeastern Ohio Fertility Center

468 E. Market St

Akron $\mathrm{OH} 44304$

Telephone: (330) 376-8353; Fax: (330) 376-4807

Lab Name: Fertility Unlimited, Inc.

Accreditation: JCAHO (Pend) 
Reproductive Gynecology

95 Arch St, Suite 250

Akron $\mathrm{OH} 44304$

Telephone: (330) 375-7722; Fax: (330) 375-3986

Lab Name: Reproductive Gynecology

Laboratories, LLC

Accreditation: JCAHO

Cleveland Clinic Fertility Center

26900 Cedar Rd, Suite 220 South

Beachwood $\mathrm{OH} 44122$

Telephone: (216) 839-3150; Fax: (216) 839-3195

Lab Name: Cleveland Clinic Foundation, Cleveland

Clinic Fertility Center

Accreditation: CAP/ASRM, JCAHO

Bethesda Center for Reproductive Health \& Fertility

Bethesda Hospital

10506 Montgomery Rd, Suite 303

Cincinnati $\mathrm{OH} 45242$

Telephone: (513) 745-1675; Fax: (513) 745-1676

Lab Name: Reproductive Studies Laboratory

Accreditation: JCAHO

Center for Reproductive Health

2123 Auburn Ave, Suite A44

Cincinnati $\mathrm{OH} 45219$

Telephone: (513) 585-0752; Fax: (513) 585-0808

Lab Name: The Christ Hospital

Accreditation: JCAHO

Institute for Reproductive Health

3805 Edwards Rd, Suite 450

Cincinnati OH 45209

Telephone: (513) 924-5550; Fax: (513) 924-5549

Lab Name: Institute for Reproductive Health

ART Laboratory

Accreditation: CAP/ASRM

Lab Name: The Christ Hospital

Accreditation: JCAHO

MacDonald Fertility and IVF Program

University Hospitals, MacDonald Women's Hospital

MacDonald Fertility and IVF

11100 Euclid Ave, Suite 1200

Cleveland $\mathrm{OH} 44106$

Telephone: (216) 844-1514; Fax: (216) 844-7098

Lab Name: MacDonald Fertility and IVF Program

Accreditation: CAP/ASRM
MetroHealth Medical Center

MetroHealth Fertility Center

Department of Obstetrics \& Gynecology

2500 MetroHealth $\mathrm{Dr}$

Cleveland $\mathrm{OH} 44109$

Telephone: (216) 778-5990; Fax: (216) 778-8642

Lab Name: Cleveland Clinic Foundation, Cleveland

Clinic Fertility Center

Accreditation: CAP/ASRM, JCAHO

Ohio Reproductive Medicine

4830 E. Knightsbridge Blvd

Columbus $\mathrm{OH} 43214$

Telephone: (614) 451-2280; Fax: (614) 451-4352

Lab Name: Reproductive Diagnostics, Inc.

Accreditation: CAP/ASRM

Kettering Reproductive Medicine

3533 Southern Blvd, Suite 4100

Kettering $\mathrm{OH} 45429$

Telephone: (937) 395-8444; Fax: (937) 395-8450

Lab Name: Kettering Medical Center Reproductive

Medicine Laboratory

Accreditation: CAP/ASRM

Fertility Center of Northwestern Ohio

2142 N. Cove Blvd

Toledo OH 43606

Telephone: (419) 291-8835; Fax: (419) 479-6005

Lab Name: The Toledo Hospital, The Fertility Center of NW Ohio

Accreditation: CAP/ASRM

\section{OKLAHOMA}

Henry G. Bennett, Jr., Fertility Institute

3433 N.W. 56th St, Suite 200

Oklahoma City OK 73112

Telephone: (405) 949-6060; Fax: (405) 949-6872

Lab Name: Integris Baptist Medical Center, Bennett

Fertility Institute Reproductive Services

Accreditation: CAP/ASRM, JCAHO

Center for Reproductive Health, PC

1000 N. Lincoln Blvd, Suite 300

Oklahoma City OK 73104

Telephone: (405) 271-1616; Fax: (405) 271-9222

Lab Name: OU Physicians, Department of

OB/GYN ART Laboratory

Accreditation: CAP/ASRM 
Tulsa Center for Fertility \& Women's Health

Tulsa Fertility Center

115 E. 15th St

Tulsa OK 74119

Telephone: (918) 584-2870; Fax: (918) 587-3602

Lab Name: Tulsa Fertility Center Laboratory

Accreditation: CAP/ASRM

\section{OREGON}

The Fertility Center of Oregon

590 Country Club Pkwy, Suite A

Eugene OR 97401

Telephone: (541) 683-1559; Fax: (541) 683-1709

Lab Name: Fertility Center of Oregon

Embryology Laboratory

Accreditation: None

Northwest Fertility Center

Eugene M. Stoelk, MD

1750 S.W. Harbor Way, Suite 200

Portland OR 97201

Telephone: (503) 227-7799; Fax: (503) 227-5452

Lab Name: Oregon Health Sciences University

Andrology/Embryology

Accreditation: CAP/ASRM

Portland Center for Reproductive Medicine

Oregon Reproductive Medicine

2222 N.W. Lovejoy, Suite 304

Portland OR 97210

Telephone: (503) 274-4994; Fax: (503) 274-4946

Lab Name: The Reproductive Medicine Laboratory

Accreditation: JCAHO

University Fertility Consultants

Oregon Health \& Science University

OHSU Center for Health \& Healing

3303 S.W. Bond Ave, 10th Floor

Portland OR 97239

Telephone: (503) 418-3700; Fax: (503) 418-3708

Lab Name: Oregon Health Sciences University

Andrology/Embryology

Accreditation: CAP/ASRM

\section{PENNSYLVANIA}

Toll Center for Reproductive Sciences

1200 Old York Rd

Abington PA 19001

Telephone: (215) 481-2349; Fax: (215) 481-7550

Lab Name: Abington Memorial Hospital, Toll Center for Reproductive Sciences

Accreditation: CAP/ASRM, JCAHO
Infertility Solutions, PC

1275 S. Cedar Crest Blvd, Suite 3

Allentown PA 18103

Telephone: (610) 776-1217; Fax: (610) 776-4149

Lab Name: Infertility Solutions, PC

Accreditation: JCAHO

Reprotech IVF Program

440 S. 15th St

Allentown PA 18102

Telephone: (610) 437-7000; Fax: (610) 437-6381

Lab Name: Reprotech, Inc.

Accreditation: None

Family Fertility Center

95 Highland Ave, Suite 100

Bethlehem PA 18017

Telephone: (610) 868-8600; Fax: (610) 868-8700

Lab Name: Family Fertility Center

Accreditation: CAP/ASRM

Main Line Fertility and Reproductive Medicine 130 S. Bryn Mawr Ave, Suite 1000

D Wing, Ground Floor

Bryn Mawr PA 19010

Telephone: (610) 527-0800; Fax: (610) 527-9868

Lab Name: Main Line Fertility Center Laboratory

Accreditation: CAP/ASRM, JCAHO

\$Geisinger Medical Center Fertility Program

100 N. Academy Ave

Danville PA 17822

Telephone: (570) 271-5620; Fax: (570) 271-5629

Contact the NASS Help Desk for current clinic information.

Advanced Center for Infertility and Reproductive Medicine, RPC

2708 Commerce Dr, Suite 100

Harrisburg PA 17110

Telephone: (717) 545-9300; Fax: (717) 540-3700

Lab Name: Central Penn Reproductive

Laboratory, LLC

Accreditation: None

Penn State Milton S. Hershey Medical Center 500 University $\mathrm{Dr}$

Hershey PA 17033

Telephone: (717) 531-8478; Fax: (717) 531-6286

Lab Name: Penn State Milton S. Hershey

Medical Center

Accreditation: JCAHO 
Northern Fertility and Reproductive Associates, PC 1650 Huntingdon Pike, Suite 154

Meadowbrook PA 19046

Telephone: (215) 938-1515; Fax: (215) 938-8756

Lab Name: Reproductive Science Institute of

Suburban Philadelphia

Accreditation: CAP/ASRM, JCAHO

Jefferson IVF

834 Chestnut St, Suite 300

Philadelphia PA 19107

Telephone: (215) 955-4018; Fax: (215) 955-7258

Lab Name: Main Line Fertility Center Laboratory

Accreditation: CAP/ASRM

University of Pennsylvania

Penn Fertility Care

3701 Market St, Suite 730

Philadelphia PA 19104

Telephone: (215) 662-6560; Fax: (215) 349-5512

Lab Name: Penn Fertility Care at Limerick

Accreditation: CAP/ASRM, JCAHO

Jones Institute at West Penn Allegheny

Health System

4815 Liberty Ave, Suite 330

Pittsburgh PA 15224

Telephone: (412) 578-5588; Fax: (412) 605-6544

Lab Name: Jones Institute at West Penn Allegheny

Health System AGH Outpatient Surgery Center

Accreditation: CAP/ASRM

Reproductive Health Specialists, Inc.

665 Rodi Rd

Rodi Plaza, Bldg 2, 2nd Floor

Pittsburgh PA 15235

Telephone: (412) 731-8000; Fax: (412) 731-8399

Lab Name: Reproductive Health Specialists, Inc.

Accreditation: CAP/ASRM

University of Pittsburgh Physicians

Center for Fertility and Reproductive Endocrinology

Magee Womens Hospital

300 Halket St, Suite 5150

Pittsburgh PA 15213

Telephone: (412) 641-1600; Fax: (412) 641-1133

Lab Name: Center for Fertility and Reproductive

Endocrinology IVF Laboratory

Accreditation: CAP/ASRM
Reproductive Endocrinology and Fertility Center

Crozer-Chester Medical Center

Ambulatory Care Pavilion

1 Medical Center Blvd, Suite 531

Upland PA 19013

Telephone: (610) 447-2727; Fax: (610) 447-6549

Lab Name: Crozer-Chester Medical Center

Andrology/IVF Laboratory

Accreditation: CAP/ASRM

Reproductive Science Institute of

Suburban Philadelphia

945 Chesterbrook Blvd

Wayne PA 19087

Telephone: (610) 964-9663; Fax: (610) 964-0536

Lab Name: Reproductive Science Institute of

Suburban Philadelphia

Accreditation: CAP/ASRM, JCAHO

Women's Clinic, Ltd.

301 S. 7th Ave, Suite 245

West Reading PA 19611

Telephone: (610) 374-2214; Fax: (610) 374-8852

Lab Name: Fertility Medical Labs, Ltd.

Accreditation: CAP/ASRM

Fertility and Gynecology Associates

Executive Mews, 2300 Computer Rd

Willow Grove PA 19090

Telephone: (215) 706-4090; Fax: (215) 706-4072

Lab Name: Abington Memorial Hospital, Toll Center for Reproductive Sciences

Accreditation: CAP/ASRM, JCAHO

The Fertility Center, LLC

130 Leader Heights Rd

York PA 17403

Telephone: (717) 747-3099; Fax: (717) 747-3214

Lab Name: The Fertility Center, LLC

Accreditation: None

\section{PUERTO RICO}

Pedro J. Beauchamp, MD

Dr. Arturo Cadilla Bldg

100 Paseo San Pablo, Suite 503

Bayamon PR 00959

Telephone: (787) 798-0100; Fax: (787) 740-7250

Lab Name: Dr Pedro J. Beauchamp Fertility

Center Laboratory

Accreditation: JCAHO 
GREFI

Gynecology, Reproductive Endocrinology \&

Fertility Institute

1519 Ponce de Leon Ave, First Bank Bldg,

Suite 705

Santurce PR 00910

Telephone: (787) 721-3544; Fax: (787) 848-0979

Lab Name: GREFI Laboratory-San Juan

Accreditation: CAP/ASRM

Lab Name: GREFI Laboratory-Ponce

Accreditation: CAP/ASRM

\section{RHODE ISLAND}

Women and Infants' Division of Reproductive Medicine and Infertility

One Blackstone Place

Providence RI 02905

Telephone: (401) 453-7500; Fax: (401) 453-7598

Lab Name: Women and Infants Hospital

IVF Laboratory

Accreditation: CAP/ASRM

\section{SOUTH CAROLINA}

Piedmont Reproductive Endocrinology Group, PA 17 Caledon Ct, Suite C

Greenville SC 29615

Telephone: (864) 232-7734; Fax: (864) 232-7099

Lab Name: Piedmont Reproductive Endocrinology

Group, PA, Embryology Laboratory

Accreditation: CAP/ASRM

University Medical Group, Department of

Obstetrics and Gynecology

Reproductive Endocrinology and Infertility

890 W. Faris Rd, Suite 470

Greenville SC 29605

Telephone: (864) 455-1675; Fax: (864) 455-3095

Lab Name: Greenville Hospital System

Reproductive Endocrinology and Infertility

Accreditation: CAP/ASRM, JCAHO

Southeastern Fertility Center, PA

1375 Hospital Dr

Mount Pleasant SC 29464

Telephone: (843) 881-3900; Fax: (843) 881-4729

Lab Name: Southeastern Fertility Center, PA

Embryology Laboratory

Accreditation: CAP/ASRM
Advanced Fertility \& Reproductive Endocrinology 2728 Sunset Blvd, Suite 305

West Columbia SC 29169

Telephone: (803) 939-1515; Fax: (803) 939-0977

Lab Name: Advanced Fertility \& Reproductive

Endocrinology Institute Laboratory

Accreditation: CAP/ASRM

\section{SOUTH DAKOTA}

Sioux Valley Clinic OB-GYN, Ltd.

1500 W. 22nd St, MB3, Suite 102B

Sioux Falls SD 57105

Telephone: (605) 328-7700; Fax: (605) 328-8831

Lab Name: Sanford Women's Health, Advanced

Reproductive Laboratory

Accreditation: CAP/ASRM

\section{TENNESSEE}

Fertility Center, LLC

1624 Gunbarrel Rd

Chattanooga TN 37421

Telephone: (423) 899-0500; Fax: (423) 899-2411

Lab Name: Fertility Center of Chattanooga

Accreditation: JCAHO

Center for Applied Reproductive Science

408 N. State of Franklin Rd, Suite 31

Johnson City TN 37604

Telephone: (423) 461-8880; Fax: (423) 461-8887

Lab Name: Center for Applied

Reproductive Science

Accreditation: None

East Tennessee IVF, Fertility, and Andrology Center 1924 Alcoa Hwy, Bldg B, Suite 304

Knoxville TN 37920

Telephone: (865) 549-4575; Fax: (865) 549-4577

Lab Name: East Tennessee IVF, Fertility and

Andrology Center

Accreditation: JCAHO (Pend)

Southeastern Fertility Center

10810 Parkside Dr, Suite 304

Knoxville TN 37934

Telephone: (865) 218-6600; Fax: (865) 218-6666

Lab Name: Southeastern Fertility Center

Accreditation: None 
Kutteh Ke Fertility Associates of Memphis, PLLC 80 Humphreys Center, Suite 307

Memphis TN 38120

Telephone: (901) 747-2229; Fax: (901) 747-4446

Lab Name: Memphis Fertility Laboratory, Inc.

Accreditation: CAP/ASRM

The Center for Reproductive Health

2011 Murphy Ave, Suite 605

Nashville TN 37203

Telephone: (615) 321-8899; Fax: (615) 321-8877

Lab Name: Fertility Laboratories of Nashville, Inc.

Accreditation: CAP/ASRM

Nashville Fertility Center

345 23rd Ave North, Suite 401

Nashville TN 37203

Telephone: (615) 321-4740; Fax: (615) 320-0240

Lab Name: Reproductive Specialty Laboratory of

Middle Tennessee, LLC

Accreditation: CAP/ASRM

\section{TEXAS}

Texas Fertility Center

Drs. Vaughn, Silverberg and Hansard

6500 N. Mopac Expressway, Bldg 1, Suite 1200

Austin TX 78731

Telephone: (512) 451-0149; Fax: (512) 451-0977

Lab Name: St. David's Hospital ART Laboratory

Accreditation: JCAHO

Jeffrey T. Youngkin, MD

Austin Fertility Center

805 E. 32nd St, Suite 201

Austin TX 78705

Telephone: (512) 478-3188; Fax: (512) 478-5092

Lab Name: Austin IVF

Accreditation: CAP/ASRM (Pend), JCAHO

Center for Assisted Reproduction

1701 Park Place Ave

Bedford TX 76022

Telephone: (817) 540-1157; Fax: (817) 267-0522

Lab Name: Center for Assisted Reproduction

IVF Laboratory

Accreditation: CAP/ASRM
Texas Fertility

4323 N. Josey Ln, Plaza I, Suite 201

Carrollton TX 75010

Telephone: (972) 394-9590; Fax: (972) 394-9579

Lab Name: Trinity Medical Center IVF Laboratory

Accreditation: CAP/ASRM

Trinity InVitro Fertilization Program

Trinity Medical Center Plaza III

4325 N. Josey Ln, Suite 111

Carrollton TX 75010

Telephone: (972) 394-3699; Fax: (972) 394-6517

Lab Name: Trinity Medical Center IVF Laboratory

Accreditation: CAP/ASRM

Dallas-Fort Worth Fertility Associates

8160 Walnut Hill Ln, Suite 328

Dallas TX 75231

Telephone: (214) 363-5965; Fax: (214) 363-0639

Lab Name: Presbyterian Hospital of Dallas

ARTS Program

Accreditation: CAP/ASRM, JCAHO

Fertility Specialists of Dallas, PA

8230 Walnut Hill Ln, Suite 300

Dallas TX 75231

Telephone: (214) 750-5500; Fax: (214) 750-5540

Lab Name: Presbyterian Hospital of Dallas

ARTS Program

Accreditation: CAP/ASRM, JCAHO

Sher Institute for Reproductive Medicine-Dallas

7777 Forest Ln, Suite C638

Dallas TX 75230

Telephone: (972) 566-6686; Fax: (972) 566-6670

Lab Name: Sher Institute for Reproductive

Medicine-Dallas ART Laboratory

Accreditation: CAP/ASRM

Texas Center for Reproductive Health

3600 Gaston Ave, Barnett Tower 504

Dallas TX 75246

Telephone: (214) 821-2274; Fax: (214) 821-2373

Lab Name: Texas Center for Reproductive Health

Accreditation: CAP/ASRM

The Women's Place

950 Scotland Dr

DeSoto TX 75115

Telephone: (972) 709-9777; Fax: (972) 709-8300

Lab Name: The Fertility Center at

Methodist Charlton

Accreditation: CAP/ASRM 
Southwest Center for Reproductive Health, PA

700 S. Mesa Hills

El Paso TX 79912

Telephone: (915) 842-9998; Fax: (915) 842-9972

Lab Name: Southwest Center for

Reproductive Health, PA

Accreditation: None

Center for Women's Medicine

10901 Katy Freeway

Houston TX 77079

Telephone: (713) 467-4488; Fax: (713) 467-9499

Lab Name: Center for Women's

Medicine Laboratory

Accreditation: CAP/ASRM

Cooper Institute for Advanced

Reproductive Medicine

7500 Beechnut St, Suite 308

Houston TX 77074

Telephone: (713) 771-9771; Fax: (713) 771-9773

Lab Name: Cooper Reproductive Laboratory

Accreditation: None

Fertility Specialists of Houston

7900 Fannin St, Suite 3100

Houston TX 77054

Telephone: (713) 512-7914; Fax: (713) 512-7853

Lab Name: Obstetrical and Gynecological

Associates Laboratories

Accreditation: CAP/ASRM

Houston Infertility Clinic

Sonja Kristiansen, MD

9055 Katy Freeway, Suite 450

Houston TX 77024

Telephone: (713) 862-6181; Fax: (713) 464-2810

Lab Name: Center for Women's

Medicine Laboratory

Accreditation: CAP/ASRM

Houston IVF

920 Frostwood, Suite 720

Houston TX 77024

Telephone: (713) 465-1211; Fax: (713) 550-1475

Lab Name: Houston IVF Laboratory

Accreditation: CAP/ASRM
North Houston Center for Reproductive

Medicine, PA

(NHCRM)

530 Wells Fargo Dr, Suite 116

Houston TX 77090

Telephone: (281) 444-4784; Fax: (281) 444-0429

Lab Name: North Houston Fertility Laboratory, Inc.

Accreditation: CAP/ASRM

The Women's Specialists of Houston

6624 Fannin St, Suite 1800

Houston TX 77030

Telephone: (713) 425-3783; Fax: (713) 425-3077

Lab Name: Center for Women's

Medicine Laboratory

Accreditation: CAP/ASRM

Lab Name: Obstetrical and Gynecological

Associates Laboratories

Accreditation: CAP/ASRM

Advanced Reproductive Care Center of Irving

7501 Las Colinas Blvd, Suite 200A

Irving TX 75063

Telephone: (972) 506-9986; Fax: (972) 506-0044

Lab Name: Advanced Reproductive Care Center of

Irving, Advanced Reproductive Laboratory, LP

Accreditation: CAP/ASRM

Wilford Hall Medical Center

Department of Obstetrics \& Gynecology

2200 Bergquist Dr, Suite 1

Lackland AFB TX 78236

Telephone: (210) 292-4016; Fax: (210) 292-6084

Lab Name: Wilford Hall Medical Center IVF/

Embryology Infertility Clinic

Accreditation: CAP/ASRM

Center for Fertility \& Reproductive Surgery

Texas Tech University Health Sciences Center

3502 9th St, Suite 150

Lubbock TX 79415

Telephone: (806) 743-4256; Fax: (806) 743-4462

Lab Name: Texas Tech University Health Sciences

Center IVF Laboratory

Accreditation: CAP/ASRM

The Centre for Reproductive Medicine

3405 22nd St, Suite 300

Lubbock TX 79410

Telephone: (806) 788-1212; Fax: (806) 788-1253

Lab Name: The Centre for Reproductive Medicine

Accreditation: CAP/ASRM 
Reproductive Institute of South Texas

110 E. Savannah, Bldg B, Suite 103

McAllen TX 78503

Telephone: (956) 687-2693; Fax: (956) 687-2829

Lab Name: Reproductive Institute of

South Texas Laboratory

Accreditation: CAP/ASRM

Dallas IVF

6124 W. Parker Rd, Suite 334

Plano TX 75093

Telephone: (972) 981-8700; Fax: (972) 981-8708

Lab Name: Presbyterian Hospital of Plano Assisted

Reproduction Technologies

Accreditation: CAP/ASRM, JCAHO

Fertility Center of San Antonio

4499 Medical Dr, Suite 200

San Antonio TX 78229

Telephone: (210) 692-0577; Fax: (210) 692-1210

Lab Name: Fertility Center of San Antonio, Inc.

Accreditation: CAP/ASRM

Institute for Women's Health

Advanced Fertility Laboratory

502 Madison Oak Dr, Suite 230

San Antonio TX 78258

Telephone: (210) 616-0680; Fax: (210) 616-0684

Lab Name: Institute for Womens Health Advanced Fertility Laboratory

Accreditation: JCAHO

Perinatal and Fertility Specialists, PA

502 Madison Oak, Suite 210

San Antonio TX 78258

Telephone: (210) 481-3000; Fax: (210) 481-3222

Lab Name: Institute for Womens Health Advanced

Fertility Laboratory

Accreditation: JCAHO

South Texas Fertility Center

South Texas Fertility Center

University of Texas Health Science Center-

San Antonio

8122 Datapoint, Suite 1300

San Antonio TX 78229

Telephone: (210) 567-7575; Fax: (210) 567-7538

Lab Name: University of Texas Health Science

Center, San Antonio South Texas Women's

Health Center

Accreditation: CAP/ASRM
Houston Fertility Institute

13414 Medical Complex Dr, Suite 7

Tomball TX 77375

Telephone: (281) 357-1881; Fax: (281) 357-1865

Lab Name: Tomball Regional Hospital In Vitro

Fertilization Laboratory

Accreditation: CAP/ASRM

Lab Name: Houston Fertility Institute, Houston

Fertility Laboratory

Accreditation: CAP/ASRM

Center of Reproductive Medicine (CORM)

1015 Medical Center Blvd, Suite 2100

Webster TX 77598

Telephone: (281) 332-0073; Fax: (281) 332-1860

Lab Name: Center of Reproductive Medicine

Accreditation: CAP/ASRM

\section{UTAH}

Utah Center for Reproductive Medicine

675 Arapeen Way, Suite 205

Salt Lake City UT 84108

Telephone: (801) 581-4838; Fax: (801) 585-2231

Lab Name: University of Utah School of Medicine

Andrology/Embryology Laboratory

Accreditation: CAP/ASRM

Reproductive Care Center

10150 Petunia Way

Sandy UT 84092

Telephone: (801) 878-8888; Fax: (801) 878-8890

Lab Name: Reproductive Care Center Andrology and Embryology Laboratory

Accreditation: CAP/ASRM

\section{VERMONT}

Vermont Center for Reproductive Medicine

FAHC-Reproductive Endocrinology \& Infertility

111 Colchester Ave, ACC MP-4

Burlington VT 05401

Telephone: (802) 847-0986; Fax: (802) 847-0111

Lab Name: Fletcher Allen Health Care Vermont

Center for Reproductive Medicine

Accreditation: CAP/ASRM, JCAHO 


\section{VIRGINIA}

Nancy Durso, MD, PC

Metro Fertility Care

6355 Walker Ln, Suite 500

Alexandria VA 22310

Telephone: (703) 313-6997; Fax: (703) 719-7632

Lab Name: Medical Faculty Associates, Inc.

Accreditation: CAP/ASRM

Lab Name: The Muasher Center for Fertility and IVF

Accreditation: CAP/ASRM

Washington Fertility Center

4316 Evergreen Ln

Annandale VA 22003

Telephone: (703) 658-3100; Fax: (703) 658-3103

Lab Name: Washington Fertility Center, Washington

Reproductive Laboratories

Accreditation: CAP/ASRM

Dominion Fertility and Endocrinology

46 S. Glebe Rd, Suite 301

Arlington VA 22204

Telephone: (703) 920-3890; Fax: (703) 892-6037

Lab Name: Dominion Fertility and Endocrinology

Main Laboratory

Accreditation: CAP/ASRM

Reproductive Medicine and Surgery Center of

Virginia, PLC

595 Peter Jefferson Pkwy, Suite 390

Charlottesville VA 22911

Telephone: (434) 982-8520; Fax: (434) 982-8521

Lab Name: Martha Jefferson Reproductive

Technology Laboratory

Accreditation: JCAHO

Genetics \& IVF Institute

3015 Williams Dr

Fairfax VA 22031

Telephone: (703) 698-7355; Fax: (703) 204-4617

Lab Name: Genetics \& IVF Institute

Embryology Laboratory

Accreditation: CAP/ASRM

The Muasher Center for Fertility and IVF

8501 Arlington Blvd, Suite 500

Fairfax VA 22031

Telephone: (703) 876-6311; Fax: (703) 876-6317

Lab Name: The Muasher Center for Fertility and IVF Accreditation: CAP/ASRM
Jones Institute for Reproductive Medicine

601 Colley Ave, Suite 251

Norfolk VA 23507

Telephone: (757) 446-7116; Fax: (757) 446-8998

Lab Name: Jones Institute for Reproductive

Medicine Embryology Laboratory

Accreditation: CAP/ASRM

Virginia Center for Reproductive Medicine

11150 Sunset Hills Rd, Suite 100

Reston VA 20190

Telephone: (703) 437-7722; Fax: (703) 437-0066

Lab Name: Virginia Center for

Reproductive Medicine

Accreditation: CAP/ASRM

Fertility Institute of Virginia

10710 Midlothian Turnpike, Suite 331

Richmond VA 23235

Telephone: (804) 379-9000; Fax: (804) 379-9031

Lab Name: Virginia IVF and Andrology Center

Accreditation: CAP/ASRM

LifeSource Fertility Center

7603 Forest Ave, Suite 204

Richmond VA 23229

Telephone: (804) 673-2273; Fax: (804) 285-3109

Lab Name: Virginia IVF and Andrology Center

Accreditation: CAP/ASRM

The Richmond Center for Fertility and Endocrinology

Courtyard Office Bldg

7603 Forest Ave, Suite 301

Richmond VA 23229

Telephone: (804) 285-9700; Fax: (804) 285-9745

Lab Name: Virginia IVF and Andrology Center

Accreditation: CAP/ASRM

The New Hope Center for Reproductive Medicine 1181 First Colonial Rd, Suite 100

Virginia Beach VA 23454

Telephone: (757) 496-5370; Fax: (757) 481-3354

Lab Name: The New Hope Center for Reproductive

Medicine Laboratory

Accreditation: CAP/ASRM

Francisco M. Irianni Infertility Clinic

1820 W. Plaza Dr

Winchester VA 22601

Telephone: (540) 662-6092; Fax: (540) 667-2476

Lab Name: Medical Faculty Associates, Inc.

Accreditation: CAP/ASRM 


\section{WASHINGTON}

Overlake Reproductive Health Inc., PS

1135 116th Ave N.E., Suite 640

Bellevue WA 98004

Telephone: (425) 646-4700; Fax: (425) 646-1076

Lab Name: Overlake Health Care Association

Accreditation: JCAHO

Washington Center for Reproductive Medicine

1370 116th Ave N.E., Suite 100

Bellevue WA 98004

Telephone: (425) 462-6100; Fax: (425) 635-0742

Lab Name: Eastside Fertility Laboratory

Accreditation: CAP/ASRM

Bellingham IVF \& Fertility Care

2980 Squalicum Pkwy, Suite 103

Bellingham WA 98225

Telephone: (360) 715-8124; Fax: (360) 715-8126

Lab Name: Bellingham IVF \& Infertility Care

Accreditation: None

Northwest Center for Reproductive Sciences

12333 N.E. 130th Ln, Suite 220

Kirkland WA 98034

Telephone: (425) 284-4400; Fax: (425) 899-9803

Lab Name: Northwest Center for

Reproductive Sciences

Accreditation: None

Olympia Women's Health

403 E. Black Hills Ln N.W.

Olympia WA 98502

Telephone: (360) 786-1515; Fax: (360) 754-7476

Lab Name: Olympia Women's Health

Accreditation: CAP/ASRM

Pacific Northwest Fertility and IVF Specialists

1101 Madison Ave, Suite 1050

Seattle WA 98104

Telephone: (206) 515-0000; Fax: (205) 150-001

Lab Name: Pacific Northwest Fertility Laboratory

Accreditation: CAP/ASRM

Seattle Reproductive Medicine

Integramed America

1505 Westlake Ave North, Suite 400

Seattle WA 98109

Telephone: (206) 301-5000; Fax: (206) 285-1119

Lab Name: Seattle Reproductive Medicine, SRM Laboratory

Accreditation: CAP/ASRM
The Center for Reproductive Endocrinology

and Fertility

508 W. 6th Ave, Suite 500

Spokane WA 99204

Telephone: (509) 462-7070; Fax: (509) 444-3894

Lab Name: Center for Reproductive Endocrinology and Fertility

Accreditation: JCAHO

GYFT Clinic, PLLC

502 S. M St, Suite 200

Tacoma WA 98405

Telephone: (206) 475-5433; Fax: (206) 473-6715

Lab Name: GYFT Clinic Reproductive

Assays Laboratory

Accreditation: CAP/ASRM

\section{WEST VIRGINIA}

Cabell Huntington Hospital

Center for Advanced Reproductive Medicine

1340 Hal Greer Blvd

Huntington WV 25701

Telephone: (304) 691-1484; Fax: (304) 691-1410

Lab Name: Cabell Huntington Hospital

Accreditation: JCAHO

West Virginia University Center for

Reproductive Medicine

1322 Pineview Dr, Suite 2

Morgantown WV 26505

Telephone: (304) 598-3100; Fax: (304) 598-8301

Lab Name: West Virginia University, Department of

OB GYN, Center for Reproductive Medicine

Accreditation: CAP/ASRM

\section{WISCONSIN}

The Women's Center at Aurora BayCare Medical Center

Reproductive Endocrinology and Fertility

Aurora Health Care-Aurora Fertility Services, Green Bay

The Women's Center at Aurora BayCare

Medical Center

2845 Greenbrier Rd, Suite 350

Green Bay WI 54308

Telephone: (920) 288-8500; Fax: (920) 288-8570

Lab Name: Aurora Health Care-Aurora

Fertility Services

Accreditation: CAP/ASRM 
University of Wisconsin-Madison

Reproductive Endocrinology and Infertility

600 Highland Ave

Madison WI 53792

Telephone: (608) 265-0237; Fax: (608) 262-9862

Lab Name: University of Wisconsin Hospitals Clinics

ART and Andrology Laboratory

Accreditation: CAP/ASRM, JCAHO

Advanced Institute of Fertility

2801 W. Kinnickinnic River Pkwy, Suite 535

Milwaukee WI 53215

Telephone: (414) 645-5437; Fax: (414) 645-5401

Lab Name: Advanced Institute of Fertility

Accreditation: CAP/ASRM

Froedtert \& Medical College of Wisconsin

Reproductive Medicine Clinic

9200 W. Wisconsin Ave, Floor 5P

Milwaukee WI 53226

Telephone: (414) 805-7370; Fax: (414) 805-7240

Lab Name: Froedtert Hospital Reproductive

Medicine Clinic

Accreditation: CAP/ASRM
Reproductive Specialty Center

IVF Columbia

2015 E. Newport Ave, Suite 707

Milwaukee WI 53211

Telephone: (414) 289-9668; Fax: (414) 289-0974

Lab Name: Reproductive Specialty Center,

IVF Columbia

Accreditation: CAP/ASRM

Women's Health Care, SC

721 American Ave, Suite 304

Waukesha WI 53188

Telephone: (262) 549-2229; Fax: (262) 549-1657

Lab Name: Advanced Institute of Fertility

Accreditation: CAP/ASRM

Aurora Health Care-Aurora Fertility Services, West Allis

West Allis Memorial Hospital

8901 West Lincoln Ave, 2nd Floor

West Allis WI 53227

Telephone: (414) 329-4300; Fax: (414) 329-4399

Lab Name: Aurora Health Care-Aurora Fertility

Services, West Allis Memorial Hospital

Accreditation: CAP/ASRM 


\section{Nonreporting ART Clinics for 2006, by State}

The clinics listed below provided ART services throughout 2006 and accordingly were required to submit ART cycle data under the provisions of the Fertility Clinic Success Rate and Certification Act passed by the U.S. Congress. These clinics either failed to submit data or did not provide verification by the clinic medical director that the tabulated success rates were correct, as required for publication.

Consumers who are aware of a clinic that was in operation in 2006 but is not included in this report's lists of either reporting or nonreporting clinics are encouraged to contact us with the complete name, mailing address, and telephone number of the clinic, by e-mail at ccdinfo@cdc.gov (Subject: ART) or by regular mail at CDC, ATTN: ARTE team; 4770 Buford Highway, N.E.; Mail Stop K-34; Atlanta GA 30341-3717. Providing this information will help ensure that clinics that should be in the report will be included in upcoming years.

Clinic names preceded by the †symbol have closed since 2006.

†UAMS Women's Health Center

Division of Reproductive Endocrinology

5800 W. 10th St, Suite 705

Little Rock AR 72205

Telephone: (501) 296-1800; Fax: (501) 296-1710

Tyler Medical Clinic

9301 Wilshire Blvd, Suite 208

Beverly Hills CA 90210

Telephone: (310) 278-7590; Fax: (310) 278-7599

Gil N. Mileikowsky, MD

5363 Balboa Blvd, Suite 245

Encino CA 91316

Telephone: (818) 981-1888; Fax: (818) 981-1994

Hope IVF and Fertility Center

2500 Alton Pkwy, Suite 201

Irvine CA 92606

Telephone: (949) 387-3888; Fax: (949) 387-3907

La Jolla IVF

9850 Genesee Ave, Suite 610

La Jolla CA 92037

Telephone: (858) 558-2221; Fax: (858) 558-2263

Northridge Center for Reproductive Medicine 18546 Roscoe Blvd, Suite 240

Northridge CA 91324

Telephone: (818) 886-0600; Fax: (818) 701-8100
Sher Institute for Reproductive Medicine-Sacramento

2288 Auburn Blvd, Suite 204

Sacramento CA 95821

Telephone: (916) 568-2125; Fax: (916) 567-1360

† Issa Shamonki, MD

2001 Santa Monica Blvd, Suite 770W

Santa Monica CA 90404

Telephone: (310) 829-4781; Fax: (310) 828-3874

†San Antonio Fertility Center

510 N. 13th Ave, Suite 201

Upland CA 91786

Telephone: (909) 949-4858; Fax: (909) 985-7137

Reproductive Genetics In Vitro

455 S. Hudson St, Level 3

Denver CO 80246

Telephone: (303) 399-5393; Fax: (303) 399-9160

Delaware Institute for Reproductive Medicine, PA 4745 Ogletown-Stanton Rd, Suite 111

Newark DE 19713

Telephone: (302) 738-4600; Fax: (302) 738-3508

Palm Beach Fertility Center

9970 Central Park Blvd, Suite 300

Boca Raton FL 33428

Telephone: (561) 477-7728; Fax: (561) 477-7035 
†Fertility Institute of Northwest Florida

1110 Gulf Breeze Pkwy, Suite 202

Gulf Breeze FL 32561

Telephone: (850) 934-3900; Fax: (850) 932-3753

USF, Department of OB/GYN

Division of REI

4 Columbia Dr, Suite 500

Tampa FL 33606

Telephone: (813) 259-8500; Fax: (813) 259-8593

IVF Hawaii

Queen's Physicians Office Bldg II

1329 Lusitana St, Suite 607

Honolulu HI 96813

Telephone: (808) 538-6655; Fax: (808) 537-5500

Hawaii Center for Reproductive Medicine and Surgery

642 Ulukahiki St, Suite 300

Kailua HI 96734

Telephone: (808) 261-4166; Fax: (808) 261-4086

Center for Women's Care

1725 W. Harrison St, Suite 739

Chicago IL 60612

Telephone: (312) 563-9389; Fax: (312) 563-9549

Chicago Women's Wellness Center, LLC

845 N. Michigan Ave, Suite 935E

Chicago IL 60611

Telephone: (312) 642-6777; Fax: (312) 642-8383

Advanced Reproductive Health Centers, Ltd.

Chicago IVF

10811 W. 143rd St, Suite 120

Orland Park IL 60467

Telephone: (708) 403-4210; Fax: (708) 403-5272

† McFarland Clinic, PC

Assisted Reproduction Program

1215 Duff Ave

Ames IA 50010

Telephone: (515) 239-4414; Fax: (515) 239-4786

Kentucky Center for Reproductive Medicine

310 S. Limestone St

Lexington KY 40508

Telephone: (859) 226-7254; Fax: (859) 226-0026
Kentucky Fertility, Gynecology \& Obstetrics

141 N. Eagle Creek Dr, Suite 203

Lexington KY 40509

Telephone: (859) 263-9600; Fax: (859) 276-2236

Fertility Clinic, Tulane University Hospital and Clinic 1415 Tulane Ave, Suite HC-15

New Orleans LA 70112

Telephone: (504) 988-2342; Fax: (504) 988-2316

Siu Ng-Wagner, MD

9333 Sprinklewood Ln

Potomac MD 20854

Telephone: (301) 838-9711; Fax: (301) 838-9712

† William Beaumont Fertility Center

Center for Conception and Reproductive Medicine 3535 W. Thirteen Mile Rd, Suite 344

Royal Oak MI 48073

Telephone: (248) 551-0515; Fax: (248) 551-3616

Luana J. Kyselka, MD, PC

2877 Crooks Rd, Suite D

Troy MI 48084

Telephone: (248) 643-6634; Fax: (248) 643-7165

Douglas S. Rabin, MD

33-00 Broadway, Suite 303

Fair Lawn NJ 07410

Telephone: (201) 703-9555; Fax: (201) 475-5678

†RWJMS In Vitro Fertilization Program

University Center for Reproductive Endocrinology and Fertility

303 George St, Suite 250

New Brunswick NJ 08901

Telephone: (732) 235-7300; Fax: (732) 235-7318

Thomas Annos, MD

40 Farley Pl

Short Hills NJ 07078

Telephone: (973) 467-0099; Fax: (973) 467-3631

†Leading Institute for Fertility Enhancement (LIFE)

130 Everett Rd

Albany NY 12205

Telephone: (518) 482-1008; Fax: (518) 489-6210

†Queens Fertility \& Gynecological, PC

10848 70th Rd, Suite 2F

Forest Hills NY 11375

Telephone: (718) 793-7752; Fax: (718) 520-5056 
Brandeis Center for Reproductive Health

137 W. 96th St

New York NY 10025

Telephone: (646) 245-5358; Fax: (718) 963-6363

†The Center for Fertility \& Advanced Reproductive Medicine at Bellevue Woman's Hospital

2210 Troy Rd

Niskayuna NY 12309

Telephone: (518) 346-9544; Fax: (518) 347-3392

University OB/GYN Associates

725 Irving Ave, Suite 600

Syracuse NY 13212

Telephone: (315) 464-7249; Fax: (315) 464-4615

† Reproductive Medicine and IVF

1321 Millersport Hwy, Suite 102

Williamsville NY 14221

Telephone: (716) 634-4351; Fax: (716) 773-7927

† Reproductive Consultants

2500 Blue Ridge Rd, Suite 300

Raleigh NC 27607

Telephone: (919) 881-7795; Fax: (919) 881-7796

Junaelo Institute of Reproductive Medicine

4256 Fulton Dr N.W., Suite B

Canton $\mathrm{OH} 44718$

Telephone: (330) 497-9400; Fax: (330) 497-9406

†Fertility Center at the Medical University of Ohio 3120 Glendale Ave

Toledo $\mathrm{OH} 43614$

Telephone: (419) 383-3030; Fax: (419) 383-6530

The Reproductive Center

900 Sahara $\mathrm{Tr}$

Youngstown $\mathrm{OH} 44514$

Telephone: (330) 965-8390; Fax: (330) 965-8391

Reproductive Endocrinology and Infertility Specialists

401 N. 17th St, Suite 303

Allentown PA 18104

Telephone: (610) 969-4522; Fax: (610) 969-3049
†Pennsylvania Reproductive Associates

Women's Institute for Fertility, Endocrinology, and Menopause

819 Locust St

Philadelphia PA 19462

Telephone: (215) 922-3173; Fax: (215) 627-7554

Centro de Fertilidad del Caribe

Torre San Francisco, Suite 606

Ave de Diego 369

San Juan PR 00923

Telephone: (787) 763-2773; Fax: (787) 763-2773

Appalachian Fertility \& Endocrinology Center

2204 Pavilion Dr, Suite 307

Kingsport TN 37660

Telephone: (423) 392-6330; Fax: (423) 392-6053

†Harold Brumley, MD

1301 W. 38th St, Suite 109

Austin TX 78705

Telephone: (512) 451-8211; Fax: (512) 450-1146

Steven Farmer, MD

3001 Airport Freeway

Bedford TX 76021

Telephone: (817) 571-6863; Fax: (817) 540-5775

UTMB Women's Healthcare Group

1804 FM 646 West, Suite N

Dickinson TX 77539

Telephone: (800) 509-2229; Fax: (281) 534-2770

Office of Frank Deleon, MD

1325 Pennsylvania Ave, Suite 690

Fort Worth TX 76104

Telephone: (817) 878-5270; Fax: (817) 878-5294

† Baylor Assisted Reproductive Technology Center 6550 Fannin St, Suite 821

Houston TX 77030

Telephone: (713) 798-8230; Fax: (713) 798-8231

†Center for Women's Health

7400 Fannin St, Suite 1130

Houston TX 77054

Telephone: (713) 797-9200; Fax: (713) 797-9276 
Alfred J. Rodriguez, MD

Texas IVF

6200 W. Parker Rd, Suite 215

Plano TX 75093

Telephone: (972) 981-7800; Fax: (972) 981-7814

Fertility Concepts

4499 Medical Dr, Suite 380

Methodist Plaza

San Antonio TX 78229

Telephone: (210) 614-3303; Fax: (210) 615-1052

Scott \& White

IVF Clinic

2401 S. 31 st St

Temple TX 76508

Telephone: (254) 724-2111; Fax: (254) 724-1046

Center for Advanced Reproductive Medicine

376 E. 400 South

Springville UT 84663

Telephone: (801) 489-9670; Fax: (801) 491-8659
†Southwest Virginia Fertility Center

2850 Keagy Rd, Suite 200

Salem VA 24153

Telephone: (540) 776-4989; Fax: (540) 776-4957

Beach Center for Fertility, Endocrinology and IVF

844 First Colonial Rd, Suite 202

Virginia Beach VA 23451

Telephone: (757) 428-0002; Fax: (757) 428-4555

Pacific Gynecology Specialists

1101 Madison St, Suite 1500

Seattle WA 98104

Telephone: (206) 215-3200; Fax: (206) 965-1737

†Gundersen/Lutheran Medical Center

1900 South Ave

La Crosse WI 54601

Telephone: (608) 775-2306; Fax: (608) 775-2993 
Appendix D

National Consumer

Organizations 



\section{APPENDIX D: NATIONAL CONSUMER ORGANIZATIONS}

The following national consumer organizations offer support to people experiencing infertility:

The American Fertility Association

305 Madison Ave, Suite 449

New York NY 10165

Telephone: (888) 917-3777; Fax: (718) 621-2444

www.theafa.org

National Women's Health Information Center

(NWHIC)

U.S. Department of Health and Human Services

Office of Women's Health

Telephone: (800) 994-9662; TDD: (888) 220-5446

www.womenshealth.gov
RESOLVE: The National Infertility Association

1760 Old Meadow Rd, Suite 500

McLean VA 22102

Telephone: (703) 556-7172; Fax: (703) 506-3266

www.resolve.org 


Centers for Disease Control

AND Prevention

Safer - Healthier - People $^{\text {tM }}$ 